\title{
Oil and Gas Field Code Master List
}

\section{4}

January 1995

\section{Energy Information Administration Office of Oil and Gas}

U.S. Department of Energy

Washington, D.C. 20585

This report was prepared by the Energy Information Administration, the independent statistical and analytical agency within the Department of Energy. The information contained herein should not be construed as advocating or reflecting any policy position of the Department of Energy or any other organization. 


\section{Preface}

The Oil and Gas Field Code Master List 1994 is the thirteenth annual listing of all identified oil and gas fields in the United States. It is updated with field information collected through October 1994. The purpose of this publication is to provide codes for easy identification of domestic fields. Standardization on these field codes fosters consistency of field identification by government and industry.

The use of field names and codes listed in this publication is required on survey forms and other reports regarding field-specific data collected by the Energy Information Administration (EIA) and the Federal Energy Regulatory Commission (FERC). The surveys currently using these field codes are the following: Form EIA-23, "Annual Survey of Domestic Oil and Gas Reserves" and the Forms FERC 8 and EIA-191, both entitled "Underground Gas Storage Report".
A glossary of key terms is provided to assist readers in more fully understanding the information in this Field Code Master List.

EIA gratefully acknowledges the assistance provided by the various State organizations and trade associations in verifying the existence of fields and confirming their officially recognized names.

General information regarding this publication may be obtained from Craig $H$. Cranston (202) 586-6083, Chief, Reserves and Production Branch, Reserves and Natural Gas Division, in the Energy Information Administration's Office of Oil and Gas. Detailed information regarding the preparation of this document may be obtained from Robert F. King (202) 586-4787, Reserves and Production Branch. 


\section{DISCLAIMER}

This report was prepared as an account of work sponsored by an agency of the United States Government. Neither the United States Government nor any agency thereof, nor any of their employees, make any warranty, express or implied, or assumes any legal liability or responsibility for the accuracy, completeness, or usefulness of any information, apparatus, product, or process disclosed, or represents that its use would not infringe privately owned rights. Reference herein to any specific commercial product, process, or service by trade name, trademark, manufacturer, or otherwise does not necessarily constitute or imply its endorsement, recommendation, or favoring by the United States Government or any agency thereof. The views and opinions of authors expressed herein do not necessarily state or reflect those of the United States Government or any agency thereof. 


\section{DISCLAIMER}

Portions of this document may be illegible in electronic image products. Images are produced from the best available original document. 


\section{Contents}

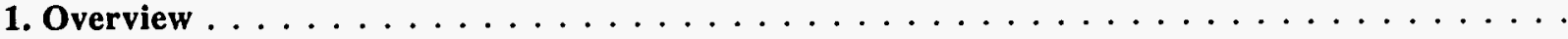

Introduction

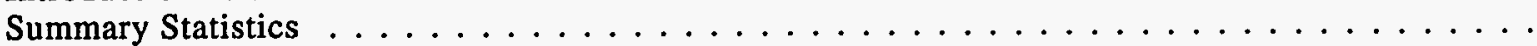

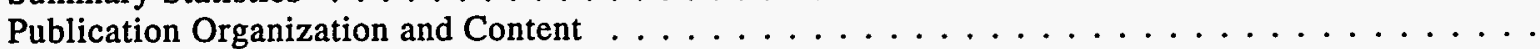

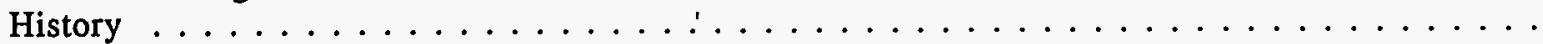

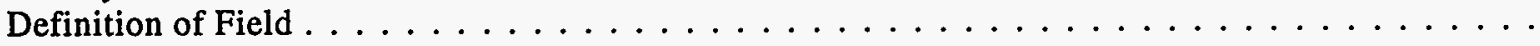

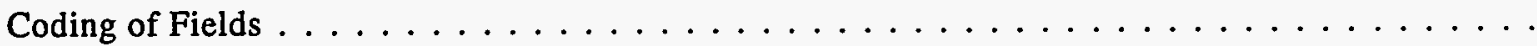

General Field Naming Conventions

2. Methodology for Field Code Assignments $\ldots \ldots \ldots \ldots \ldots \ldots \ldots \ldots \ldots \ldots \ldots \ldots \ldots$

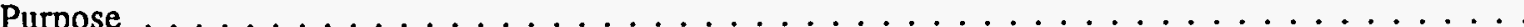

General System Overview $\ldots \ldots \ldots \ldots \ldots \ldots \ldots \ldots \ldots \ldots \ldots \ldots \ldots$

Field Information Research $\ldots \ldots \ldots \ldots \ldots \ldots \ldots \ldots \ldots \ldots \ldots \ldots \ldots$

State Source Review Procedures $\ldots \ldots \ldots \ldots \ldots \ldots \ldots \ldots \ldots$

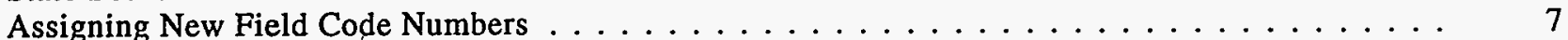

Field Alias Procedures $\ldots \ldots \ldots \ldots \ldots \ldots \ldots \ldots \ldots \ldots \ldots \ldots$

Offshore Code Assignments $\ldots \ldots \ldots \ldots \ldots \ldots \ldots \ldots \ldots \ldots \ldots$

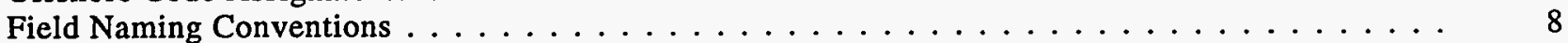

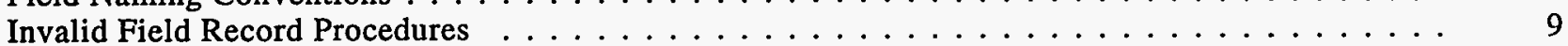

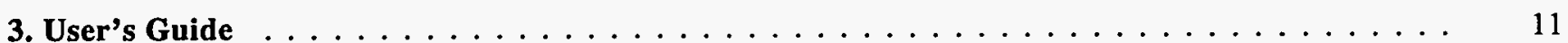

Field Code Master List $\ldots \ldots \ldots \ldots \ldots \ldots \ldots \ldots \ldots \ldots \ldots$

Field Code Index $\ldots \ldots \ldots \ldots \ldots \ldots \ldots \ldots \ldots \ldots \ldots \ldots \ldots$

Coalbed Methane Field List $\ldots \ldots \ldots \ldots \ldots \ldots \ldots \ldots \ldots \ldots$

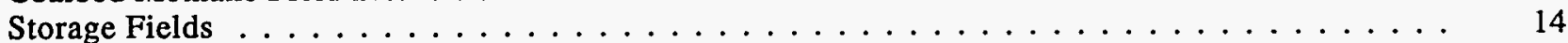

Fields Located in Multiple Jurisdictions $\ldots \ldots \ldots \ldots \ldots \ldots \ldots \ldots \ldots$

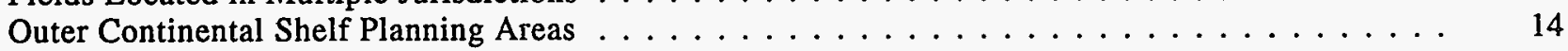

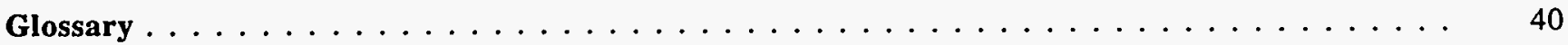

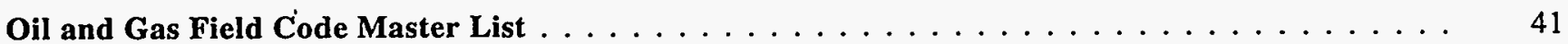

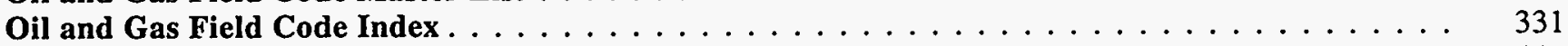

Oil and Gas Invalid Field Record List $\ldots \ldots \ldots \ldots \ldots \ldots \ldots$

\section{Tables}

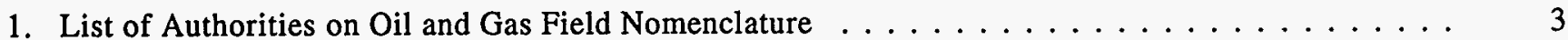

2. State Abbreviations and Geographic Subdivision Codes . . . . . . . . . . . . . . . . . 16

3. U.S. Geological Survey Alaska Quadrangles and Associated Codes . . . . . . . . . . . . . . 17

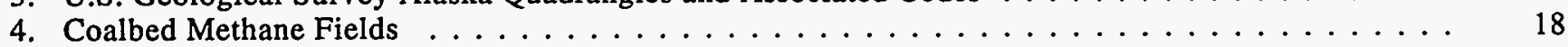

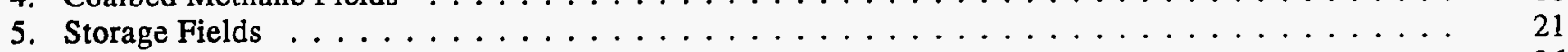

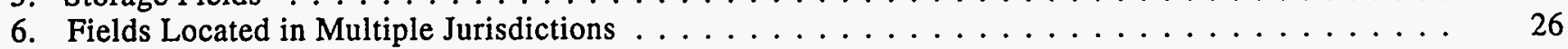




\section{Figures}

1. Field Code Processing Flow Chart $\ldots \ldots \ldots \ldots \ldots \ldots \ldots \ldots \ldots \ldots \ldots \ldots$

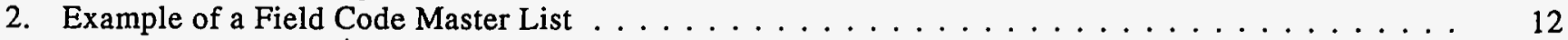

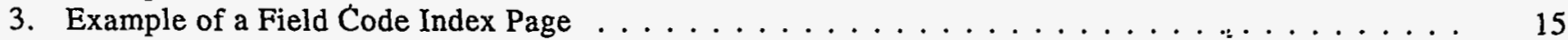

4. Subdivisions of Alaska and U.S. Geological Survey Quadrangles . . . . . . . . . . . . . . 32

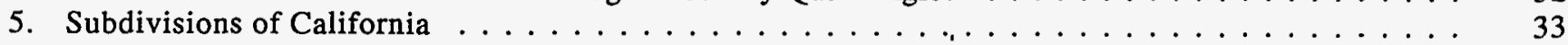

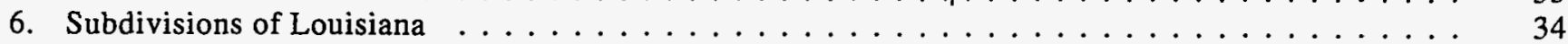

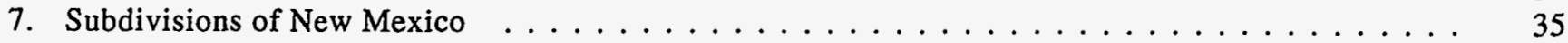

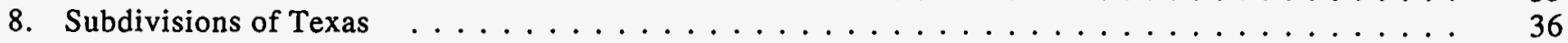

9. Western Planning Area, Gulf of Mexico Outer Continental Shelf Region . . . . . . . . . . . . . 37

10. Central Planning Area, Gulf of Mexico Outer Continental Shelf Region . . . . . . . . . . . . . . . 38

11. Eastern Planning Area, Gulf of Mexico Outer Continental Shelf Region . . . . . . . . . . . . 39 


\section{Overview}

\section{Introduction}

This is the thirteenth annual edition of the Energy Information Administration's (EIA) Oil and Gas Field Code Master List. It reflects data collected through October 1994 and provides standardized field name spellings and codes for all identified oil and/or gas fields in the United States. The master field name spellings and codes are to be used by respondents when filing the following Department of Energy (DOE) forms:

- Form EIA-23, "Annual Survey of Domestic Oil and Gas Reserves", filed by oil and gas well operators (field codes are required from larger operators only).

- Forms FERC 8 and EIA-191, "Underground Gas Storage Report", filed by natural gas producers and distributors who operate underground natural gas storage facilities.

Other Federal and State government agencies, as well as industry, use the EIA Oil and Gas Field Code Master List as the standard for field identification. A machinereadable version of the Oil and Gas Field Code Master List is available from the National Technical Information Service, 5285 Port Royal Road, Springfield, Virginia 22161, (703) 487-4650.

In order for the Master List to be useful, it must be accurate and remain current. To accomplish this, EIA constantly reviews and revises this list. The EIA welcomes all comments, corrections, and additions to the Master List. All such information should be given to the EIA Field Code Coordinator at (214) 953-1858.

EIA gratefully acknowledges the assistance provided by numerous State organizations and trade associations in verifying the existence of fields and their official nomenclature.

\section{Summary Statistics}

There are 56,754 field records in this year's Oil and Gas Field Code Master List (FCML). This amounts to 331 more than in last year's report. As it is maintained by EIA, the Master List includes the following:

- Field records for each State and county in which a field resides.

- Field records for each offshore area block in the Gulf of Mexico in which a field resides.
- Field records for each alias field name (see definition of alias below).

- Fields crossing State boundaries that may be assigned different names by the respective State naming authorities.

Taking into consideration the double-counting of fields under such circumstances, EIA can identify 45,992 distinct fields in the United States as of October 1994. This count includes fields that no longer produce oil or gas. Of this number, 382 are fields used in whole or in part for oil or gas storage. The following is a summary count by hydrocarbon type of distinct fields in the United States:

\begin{tabular}{|c|c|}
\hline Field Type & Number \\
\hline U.S. oil and gas distinct fields. . & 45,992 \\
\hline Fields with oil . & 34,067 \\
\hline Fields with gas. . . . . . . . . . . & 31,436 \\
\hline Fields with associated-dissolved gas..... & 15,640 \\
\hline Fields with nonassociated gas . . . . . . . . & 20,993 \\
\hline Fields with unspecified hydrocarbon .... & 146 \\
\hline
\end{tabular}

Note: The sum of these fields is greater than the total number of distinct fields, since many fields have more than one type of hydrocarbon present.

EIA has assigned a unique six-digit field code to each field name. A listing of all field names and the States in which they occur, ordered by field code, is presented in the Field Code Index. The number of entries in the Field Code Index, 51,461, represents the number of field names that have been recognized by State naming authorities. Of these, 44,989 are currently considered official, while 6,472 are no longer valid and have been designated as alias names by EIA.

In addition, this report contains an Invalid Field Record List of 25 records that have been removed from the FCML since last year's report. These records were found to be either technically incorrect or to represent field names which were never recognized by State naming authorities. 


\section{Publication Organization and Content}

Chapter 2 provides details on the methodology used to assign field codes and the procedures followed to standardize field names. Chapter 3, the User's Guide, and the Glossary provide users with the definitions and explanations needed to utilize this publication. The FCML follows the Glossary. It is organized by State, with fields sorted alphabetically by name within each individual State. Fields in the Federal Offshore Outer Continental Shelf are listed separately at the end after Wyoming. Each field name entry contains the field code, field name, geographical information, and other related information. The Field Code Index, which follows the Master List, is an abbreviated listing sorted by field code with field names and the State or States in which each field is located. This listing is intended for use as a quick reference. The Master List must be consulted for more detailed information. The Invalid Field Record List identifies those field records which were determined to be invalid since the previous report.

\section{History}

The EIA FCML evolved from the Federal Power Commission's Field/Plant Code List (FPC Field Code List). The FPC Field Code List, originally developed over 20 years ago, had a unique code assigned to each field on the list. That is, two fields having identical names in separate States had separate six-digit field codes. However, some respondents to Form FPC 15, "Interstate Pipeline's Annual Report of Gas Supply", began using the first code given in the list for a field name, regardless of the State involved. With few respondents utilizing computerized edits on their submissions at that time, miscoding of fields became a problem. The solution applied to this problem was to recode the fields on the list so that any fields with identical names were assigned the same six-digit code (a field name code) but were differentiated by the State and county codes incorporated in the full field code. For example, $145385 \mathrm{KS} 101$ is the field code for the CLARK field in Kansas and 145385TX285 is the field code for the CLARK field in Texas, as 145385 is the field name code for CLARK.

The FPC Field Code List, originally designed to handle data relating to interstate gas fields, was expanded over the years, as resources permitted, to include the names of oil fields and intrastate gas fields. Six-digit codes were assigned in ascending order to alphabetically sorted field names. Codes from the FPC Field Code List were used in filing Form FPC 15 and Form FPC 8, "Underground Gas Storage Report".

After the establishment of the DOE in 1977, the requirement to gather annual, verifiable oil and gas reserves estimates led to the development of Form EIA-23, "Annual Survey of Domestic Oil and Gas Reserves". Form EIA-23 collects certain data by field, and the use of the FPC Field Code List aided the reporting and processing of these data. As use of the Master List expanded by way of the Form EIA-23 program, additional work to verify and update the code list was necessary to keep the list current. In 1981, the correlation between the code number sequence and the alphabetical field name sequence was dropped. This change precluded the necessity of periodically reassigning field codes in order to maintain the list in parallel numeric and alphabetic order.

A procedure was developed for the 1988 Field Code Master List publication which determined the actual count of distinct oil and gas fields in the United States. A part of this procedure was to aggregate the separate county portions of existing fields into their recognized entirety, even if the field crossed a State boundary. This procedure is time-consuming and does entail manual intervention, so that it is accomplished on a yearly cycle.

\section{Definition Of "Field"}

A field is defined as:

\begin{abstract}
"An area consisting of a single reservoir or multiple reservoirs all grouped on, or related to, the same individual geological structural feature and/or stratigraphic condition. There may be two or more reservoirs in a field which are separated vertically by intervening impervious strata, or laterally by local geologic barriers, or by both."
\end{abstract}

This definition is not used by all States in their designation of fields; consequently, areas classified as individual fields by some States may be found combined in this Master List.

\section{Coding Of Fields}

As noted above, the six-digit field name code is common to a specific field name, regardless of whether one or several fields exist with that particular name. In order to uniquely identify a particular field, the field name code must be coupled with the corresponding State abbreviation and county code.

Most codes on the Master List remain in a numerically ascending order by alphabetized field name. However, field names added since 1981 have been assigned the first available (numerically lowest) unused code. Fields located in the Federal Offshore area and large State offshore blocks of the Gulf of Mexico will continue to be represented by codes above 800000 , according to their area, subarea, and block number.

Records that appear on the list with field name codes in light italics are alias records. The correct field name and field name code appear as part of the alias field name record after the label "Alias For:". Alias field 
names are not currently recognized by State naming authorities, and alias field names and their codes should not be used in DOE filings.

Records that were on the Field Code Master List last year but which were subsequently found to be invalid are entered on a separate Invalid Field Record List. These records were incorrectly placed on the FCML or when complete information was not available. Field names and codes on the Invalid Field Record List should not be used in DOE filings with the indicated State and county. Note, however, that the identified field name and field name code may still be valid for a different State/county combination.

\section{General Field Naming Conventions}

Field name spellings in the Master List reflect a number of conventions and conditions. In most instances, the 26-character name reflects the conventions imposed by the data block length in the DOE forms and by the State naming authority, usually the oil and gas regulatory agency. Field identification for a well, lease, block, unit, or section is the responsibility of the State naming authority. A listing of these agencies appears in Table 1. In the absence of a State authority, field names which have come into general acceptance in an area may be listed. In the Appalachian Region, field area names are often used. Detailed information is provided in Chapter 2, Methodology for Field Code Assignments, concerning the field naming conventions followed

Table 1. List of Authorities on Oil and Gas Field Nomenclature

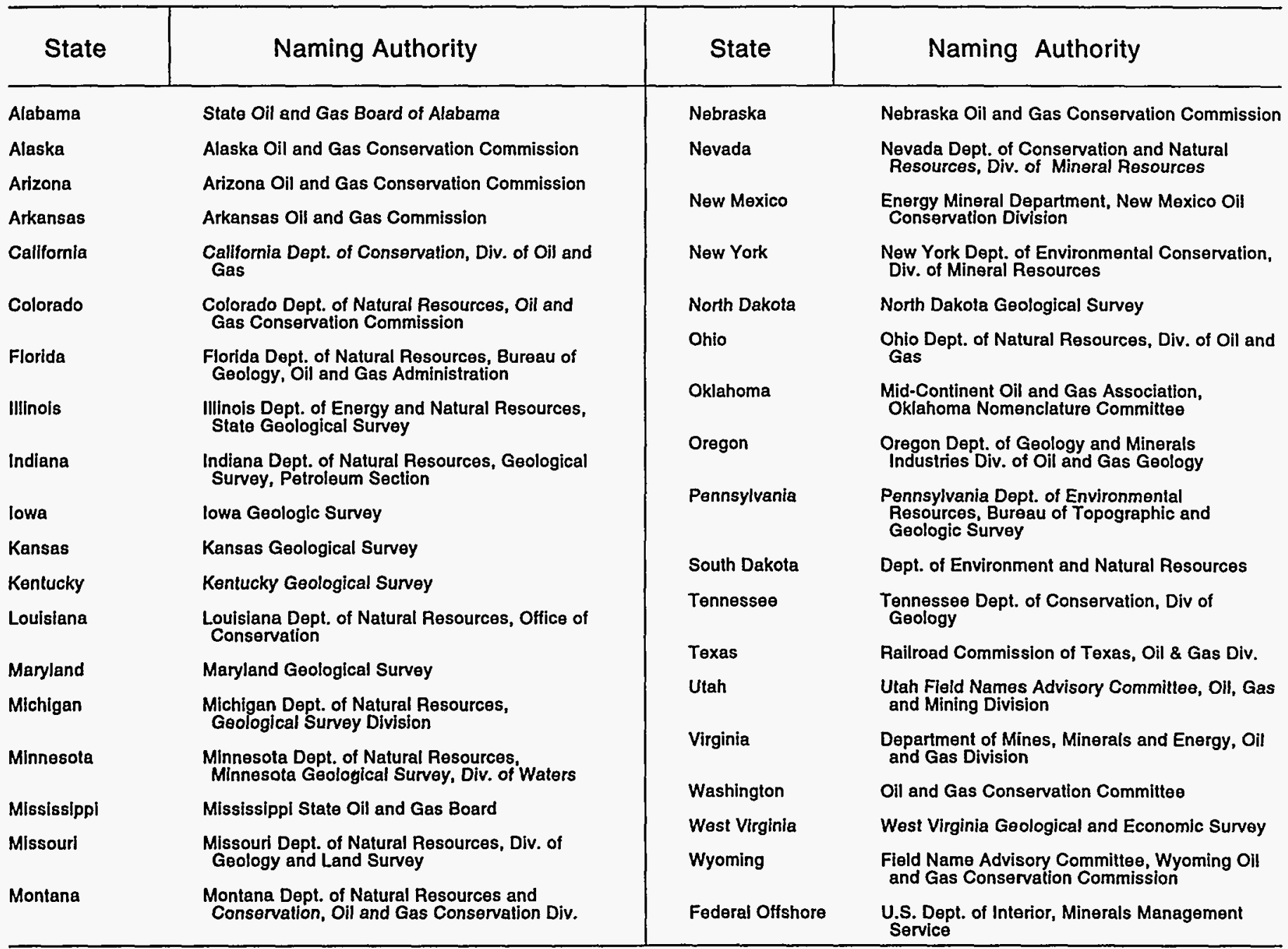

Source: Energy Information Administration, Office of Oil and Gas 


\section{Methodology for Field Code Assignments}

\section{Purpose}

The purpose of the Field Code Master List effort is to provide the Energy Information Administration (EIA) and others with standardized field name spellings and codes for all crude oil and natural gas fields throughout the United States. The codes are compiled on the computerized Field Code Master File (FCMF), which is updated on a quarterly cycle. The Oil and Gas Field Code Master List, derived from the FCMF, is published annually. The first edition was released by EIA in December 1982. Computer tapes containing the latest field code information are delivered to the National Technical Information Service in January of each year. Printouts of newly added fields and changes to existing fields become available in June of each year and may be requested in writing from Robert F. King, EI-443, Forrestal Bldg., U.S. Dept. of Energy, Washington, DC 20585.

\section{General System Overview}

Figure 1 presents a flow chart of the activities necessary to process new field information. All new field information received by EIA goes through this cycle, which is designed to process field information received from respondents filing Forms EIA-23, and FERC 8 and EIA-191, as well as from other sources such as State publications.

The system incorporates both a Field Code Working File (FCWF) and the FCMF. The FCWF contains all new, or unverified, field information. It exists for two reasons:

- The large size of the FCMF makes it relatively difficult to manipulate.

- It is useful in tracking the progress made toward verifying the large number of in process fields, while at the same time assuring that redundant activities are not taking place.

The FCWF is updated as information about the field is obtained. Quarterly, and more often when warranted by the number of completed transactions, all fully resolved records are extracted from the working file and used to update the FCMF. Once the master file update is completed, the resolved records are deleted from the FCWF.

\section{Field Information Research}

Geologists and petroleum engineers are responsible for supervising research and final resolution of field information. Listings of the FCWF are produced by region for review. There are several possible explanations why field information under review is not already contained on the FCMF. That information could reflect the following:

- A relatively recent field discovery.

- A recently discovered extension of an old field into a new county or State.

- An alias used for the official name.

- An error exists in the reported information (e.g., a reporting error on the Form EIA-23).

The official recognition of a new field discovery by a State field naming authority is a prerequisite for the assignment of an official EIA field code. Table 1 on page 3 lists these naming authorities. Information regarding State recognition is obtained through official State publications and computer tapes, or through other contact with the State agencies.

If the field name in question has not been officially recognized, several sources of information exist for further investigation into the third and fourth possibilities listed above. These include:

- Analysis of State data files (the Texas and New Mexico files, for example, contain detailed information for each field and operator.)

- Review of other oil and gas publications.

- Telephone contact with the source of the information (e.g., a respondent to Forms FERC 8 and EIA-191, or Form EIA-23).

\section{State Source Review Procedures}

State sources provide most of the field names. Field information is processed on a routine basis from State sources so that EIA field codes will hopefully be assigned as this information is needed. 
Figure 1. Field Code Processing Flow Chart

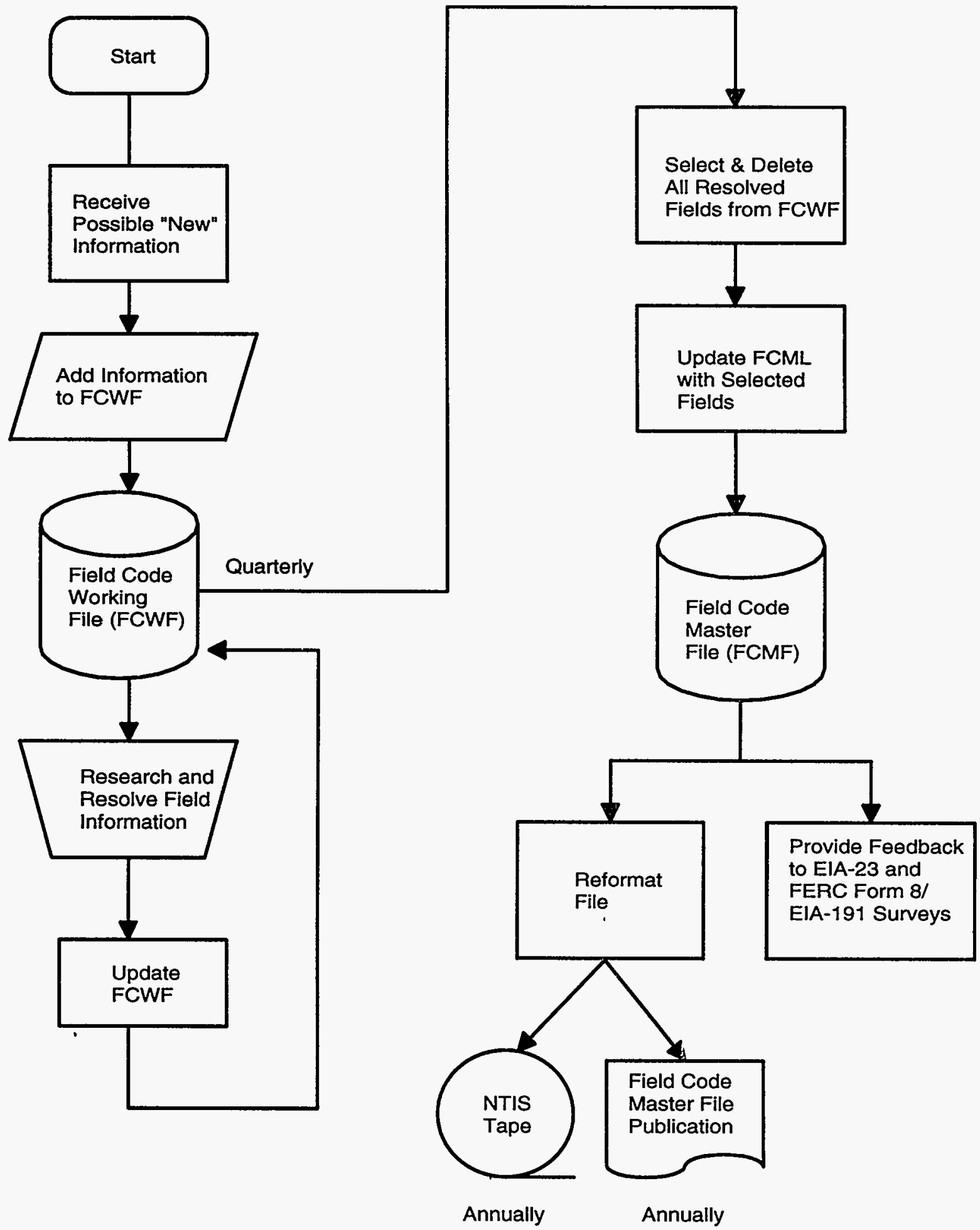

Source: Energy Information Administration, Office of Oil and Gas 
As State publications are released, they are routinely reviewed, and their information regarding new fields is compared to the information on the FCMF. If the FCMF does not contain the new field information, the working file is updated to reflect it. Prior to updating the working file, entries on it are reviewed in order to identify any research which might be in progress on the same field. Information added to the working file from State publications is added as resolved information, ready to be entered on the FCMF, since the States are the final authority for this information.

The quality and quantity of information available through the State publications varies. Some States publish new field information relatively frequently (e.g., monthly) in a format that is easily reviewed for incorporation into the FCMF. Routine processing of State sources begins with these publications. Additional State publications, such as State geological papers, are also included in these routine reviews.

Periodically, all FCMF records for a State are compared to the most recent State publication. Some States do not publish field information, or the field information is not carried in the latest publication but in a previous one. In an overall review of the FCMF, when a particular field is not found in any available State publication, the source NISP (not in State publication) is initially coded into the source section of the FCMF. When an earlier State source reports "No Production", "No Reports", or some similar remark for one or more years, and does not show the field in subsequent sources, the field is still considered to be official, whether it is producing at this time or not. When a field is found in an older State source in some sort of combination or in subordination with another field name, the State is contacted and asked to clarify the status of the field. This research resolves most of the NISP records, allowing them to be finally categorized as a master field record with a State naming authority source, as an alias field record, or as an invalid field record. Certain field records represented field names assigned by non-State Naming Authority publications which were coded prior to initiation of production of the field, which subsequently was abandoned without any recorded production. These records have been classified as invalid.

\section{Assigning New Field Code Numbers}

A sequential listing of available field code numbers starting with 000101 , has been produced for use by the Field Code Coordinator when new field codes are assigned. The first available code (numerically lowest) is used for a new field, regardless of name spelling, with the following exception: codes 800000-999998 are excluded from sequential code assignments, as they are reserved for offshore Gulf of Mexico fields.

\section{Field Alias Procedures}

A field alias is an unofficial name for an officially recognized field. Each name designated as an alias is cross-referenced to the official name. An official name is the current, State-sanctioned name. An unofficial name is one which no longer is, or never was, a State-sanctioned name.

A field alias may reflect the following possibilities:

- An alternate name for the official name.

- A field that has been renamed to something else.

- A field that has been combined, consolidated, or merged into another field.

- A field that has split into two or more new fields and the old name is no longer used.

For the combined or renamed types of aliases, both the official and alias names are on the FCMF.

For alternate name aliases, the official name must be on the FCMF in all cases. The alias name will be on the FCMF in the following cases:

- A representative from the State agency is aware of the alias name as an alternative for the official name.

- The alias name was submitted by a Form FERC 15 "Interstate Pipeline's Annual Report of Gas Supply" respondent.

- Two or more operators report the same alias.

- The alias is already on the FCMF for some reason.

An alternate name alias which does not meet one of these four criteria normally would not be added to the FCMF.

\section{Field Alias Records That Have Several Official Names}

Certain alias field records result from the splitting of a field into two or more fields, with none of the resulting fields using the original field name. For example, Field A was split into three fields: Field B, Field C, and Field D. Fields B, C, and D would be listed as field records on the Master List. Field A would be listed as a field alias record referencing Field B, but not Fields $C$ and D and would show SPLIT as its reason for alias. Because of space limitations, the appropriate State naming Authority or the Field Code Coordinator must be contacted to determine names of 
the other fields created out of Field A when it was split, as well as how many others were created.

\section{Fields With More Than One Name Change}

The current official name may be the result of several name changes. For instance, in Oklahoma, RINGWOOD NE was combined with RINGWOOD NORTH in 1965 , which was subsequently combined into RINGWOOD in 1966. The listing for RINGWOOD NE would show the correct field name as RINGWOOD. If multiple names were involved, the alias field name record would list only the current (correct) field name. (For example, in Oklahoma, HENNESSEY SE is an alias for HENNESSEY EAST which has since been changed first to DOVER-HENNESSEY and finally to SOONER TREND. The correct name for the alias HENNESSEY SE, HENNESSEY EAST and DOVER-HENNESSEY is SOONER TREND.) Information on the chain of successive field names may be obtained from the appropriate State naming authority or the Field Code Coordinator.

\section{Offshore Code Assignments}

Offshore fields on the Federal Gulf of Mexico Outer Continental Shelf receive a field code that is determined by the lease block (or blocks) for which they are named by the U.S. Minerals Management Service (MMS). The last three digits of the code are the block number. For instance, East Cameron (offshore area prefix code 824) Block 071, receives the field code of 824071. If several blocks are included in one field, the field code reflects the number of the block for which the field is named. The other blocks included in the field are aliases to the official name. When the MMS has named two fields with the same basic block number i.e., Ship Shoal Blk 113 field and Ship Shoal Blk 113A field, for the second designated field the basic block number is combined with the prefix code of an adjacent area within which this block number would not occur.

There are cases where a particular OCS lease block contains portions of two or more fields. If one of the fields is named for the block concerned, there will be an entry in the REMARKS section indicating PBI, or Part of Block In, followed by the field code of the other included field. If the block concerned is only an alias to all the fields involved, it will be designated in MULTI to highlight that more than one field is included within the lease block.

Fields in large offshore State blocks in the Gulf of Mexico are assigned codes in the manner of offshore Federal fields. Special prefixes, generally unassigned prefixes for area surveys, are used for the small State block fields. A special prefix has been set aside for High Island-State. This prefix is used only with the small block fields. For State blocks that exceed 999, an un- used three-digit prefix ending in 1 is used, such as SOUTH PADRE IS BLK 1068 with code 951068.

Federal offshore fields in areas other than the Gulf of Mexico are given names in the same manner as for onshore fields, and are coded with the next sequential code.

\section{Field Naming Conventions}

\section{Use of Compass Directions in Field Names}

As a general rule, a compass direction used as part of a field name is placed at the end of the name. For example, the field named West Davenport by a State source bears the name DAVENPORT WEST on the FCMF. A field named after a known landmark, such as the town of East Davenport for example, bears the name EAST DAVENPORT on the FCMF.

If the field DAVENPORT WEST is then combined with other wells or fields and the word District, or some similar word, is added to the name, the name will appear on the FCMF as DAVENPORT WEST DISTRICT. This enables users to distinguish between two fields, one named as a result of forming a district with the DAVENPORT WEST field as its nucleus, e.g., DAVENPORT WEST DISTRICT, and the other which is a new district to the west of DAVENPORT DISTRICT, e.g., DAVENPORT DISTRICT WEST.

Abbreviations are not used in connection with onshore field names except for noncardinal compass points, such as NW for Northwest or SE for Southeast, and for names of combined fields in certain States which use all the individual names of the former fields to form the new field name. Cardinal compass points are spelled out in onshore field names.

Offshore field names in the Gulf of Mexico usually consist of an offshore area name and block number specified by the U.S. Minerals Management Service (MMS). Example: EAST CAMERON BLOCK 299. The Field Code Master List has retained the subarea identifiers such as EAST CAMERON SOUTH ADDITION BLOCK 299 field which yields a 37-character field name. Such lengthy offshore area names must be abbreviated to fit within the 26 characters available. When this is necessary, the following standard abbreviations are used:

$\begin{array}{lllll}\text { North } \ldots & \text { N } & \text { North Addition .. } & \text { NA } \\ \text { South } \ldots & \text { S } & \text { South Addition .. } & \text { SA } \\ \text { East . . } & \text { E } & \text { East Addition ... } & \text { EA } \\ \text { West . . } & \text { W } & \text { West Addition ... } & \text { WA } \\ \text { Block } & \ldots & \text { BLK } & \text { South Extension .. } & \text { SX } \\ \text { Island ... } & \text { IS } & \text { East Extension . . } & \text { EX }\end{array}$


For example, High Island East Addition South Extension Block A376 is abbreviated HIGH IS EA SX BLK A376.

\section{Special Naming Conventions}

Some States regard reservoirs as fields and keep their records on that basis (e.g., Texas and New Mexico). The Master List does not follow the State conventions in these instances. For example, in Texas, PARKER (PENNSYLVANIAN) and PARKER (WOLFCAMP) are considered to be separate fields by the State. PARKER is actually the name of the field and PENNSYLVANIAN and WOLFCAMP are the names of reservoirs in the field. The Texas Railroad Commission requires that the reservoir name appear in parentheses after the field name in its publications. In the Master List, you will only find PARKER listed as the field.

The MMS quarterly publication, OCS Operations Field Names Master List, Gulf of Mexico OCS Region, (FNML) is the primary source for the Federal offshore Fields in the Gulf of Mexico. This publication, and the MMS computer file on which it is based, does not use the subarea identifiers, such as the SOUTH ADDITION, although maps of the region still carry these subareas. That is, where the MMS FNML carries the name WEST CAMERON BLOCK 617, most maps and the Field Code Master List call the field WEST CAMERON SA BLK 617 with SA the abbreviation for SOUTH ADDITION.

\section{Reused Field Names}

Some States occasionally reuse field names for areas other than the original field location. This problem is handled on the FCMF by indicating (OLD) and (NEW) after the field name, with each being assigned a different field code. This procedure facilitates computerized usage of the database.

\section{Invalid Field Record Procedures}

Field records are removed from the FCML when they are found to be incorrect for one of the following reasons:

- The field name as it appears was never approved by the State naming authority for that State and county; i.e., it could have been a misspelled field name or the name of a producing unit, well, lease or offshore platform.

- County or State location data are incorrect.

- Two separate field codes were assigned to the same field name.

When research on a NISP field record determines that one of those reasons applies to a field or field alias record, the record type is changed to INVALID. Invalid field records appear in the Invalid Field Record List section of the FCML publication for the year in which they have been designated INVALID. They will not appear in subsequent years. A cumulative list of INVALID records may be obtained by contacting the Field Code Coordinator. The National Technical Information Service machine-readable version of the EIA Oil and Gas Field Code Master List includes a separate and cumulative Invalid Field Record List. 


\section{User's Guide}

\section{Field Code Master List}

Fields in the Field Code Master List (FCML) are sorted alphabetically by State and alphabetically by field name within a State. Fields in the Federal Offshore are listed separately. Fields that occur in multiple States are listed in each and are further sublisted by county.

The layout of the FCML is shown in Figure 2. Each field record is one line long except for records with a "REMARK", which is on a second line. Each alias field record is two lines long. A brief description of each data item follows.

\section{Master Field Record}

Item 1, FIELD CODE. The six-digit field code assigned to this field name.

Item 2, FIELD NAME. This is the field name (26-character limit).

Item 3, STATE POSTAL ABBREVIATION AND STATE SUBDIVISION CODE. This is a four-character code indicating the State and State subdivision. The first two positions are the State postal abbreviation. The last two positions are the two-digit subdivision code, used only in Alaska, California, Louisiana, New Mexico, and Texas, and in offshore areas to differentiate between State and Federal waters. Table 2 on page 16 is a listing of these codes. Figures 4 through 8 , at the end of the Users' Guide, are subdivision maps for the aforementioned States.

Item 4, COUNTY CODE. This is a three-character code for the county or parish. For all States except Alaska this is the Federal Information Processing Standards (FIPS) county code, as presented in FIPS publication 6-3 dated December 15, 1979 and its amendments. As Alaska has no cqunties, the FCML uses the U.S. Geological Survey $1^{0} \times 3^{\circ}$ quadrangles for Alaska and the three-digit pseudo-county codes assigned to them by the American Petroleum Institute. Codes used for fields located in State or Federal offshore areas are: Offshore-State, 990; Offshore-Federal, 995; OffshoreGeneral, 999.

Item 5, COUNTY NAME. This is the county or parish name (23-character limit) as defined in FIPS publication 6-3 for all State onshore areas except Alaska, For Alaska, the name associated with the USGS $1^{\circ} \times 3^{\circ}$ quadrangle is used. Table 3 on page 17 is a listing of the pseudo-county codes and names for Alaska. If the field is in an offshore area, see the list in Item 4 above.

Item 6, FIELD DISCOVERY YEAR. This is the fourdigit year of first discovery of oil or gas in this field, if it is known. In the case of combined fields, this is the earliest date among the formerly separate fields.

Item 7, FIELD TYPE. This is a three-character block giving the type of hydrocarbon found in the field using the symbols defined below.

\begin{tabular}{|c|c|}
\hline Symbol & Meaning of Symbol \\
\hline ONA & $\begin{array}{l}\text { Oil, nonassociated gas, and associated- } \\
\text { dissolved gas are present. }\end{array}$ \\
\hline ON & $\begin{array}{l}\text { Oil and nonassociated gas present; } \\
\text { associated-dissolved gas absent. }\end{array}$ \\
\hline $\mathbf{N}$ & $\begin{array}{l}\text { Nonassociated gas present; } \\
\text { oil and associated-dissolved gas absent. }\end{array}$ \\
\hline 0 & $\begin{array}{l}\text { Oil present; nonassociated gas and associated-dis } \\
\text { solved gas absent. }\end{array}$ \\
\hline OA & $\begin{array}{l}\text { Oil and associated-dissolved gas present; } \\
\text { nonassociated gas absent. }\end{array}$ \\
\hline Blank & Type of hydrocarbon is unknown. \\
\hline
\end{tabular}

Item 8, REMARKS. This is a 23-character entry specifying an attribute of the field. 
Figure 2. Example of a Field Code Master List

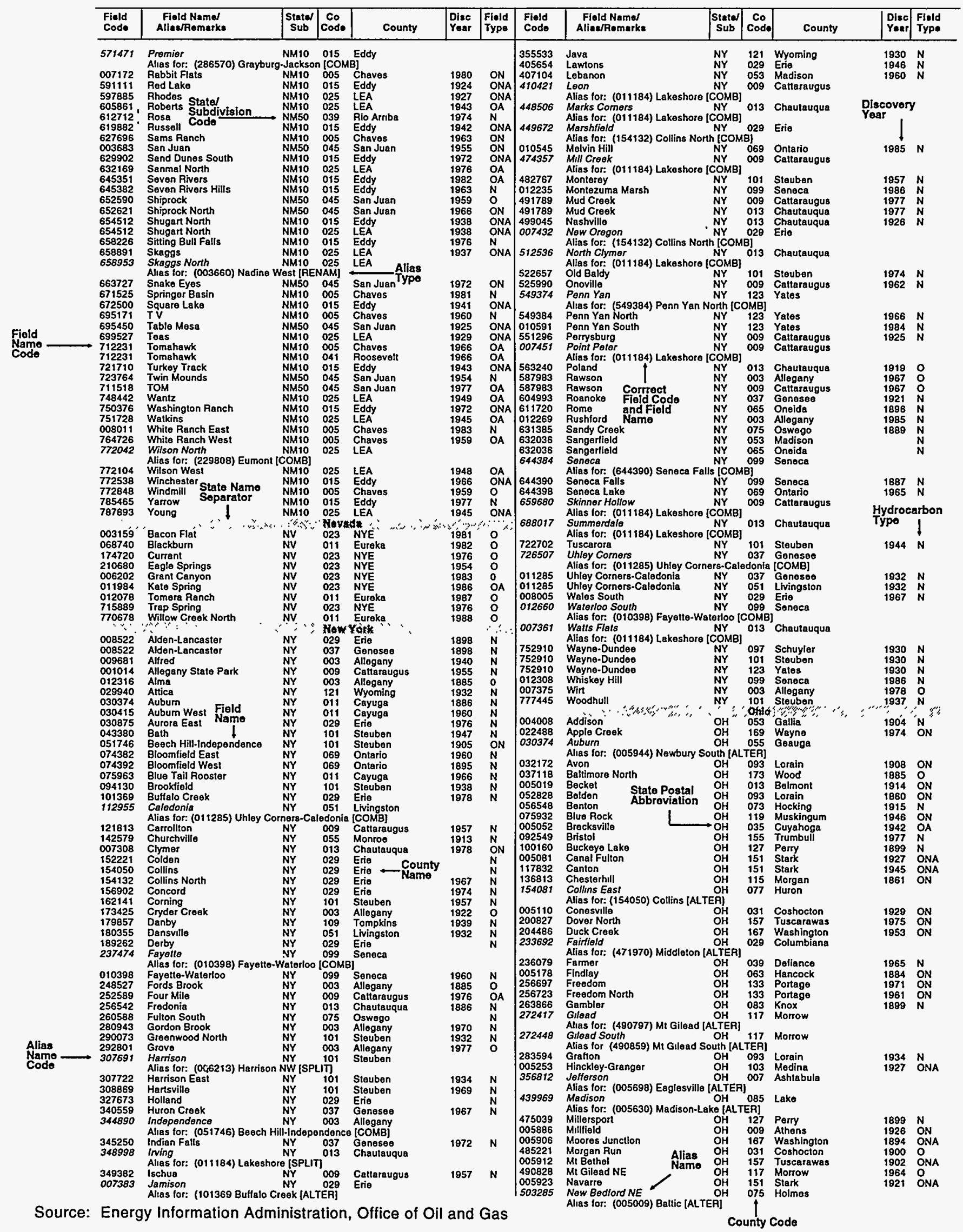


Common remarks are described below:

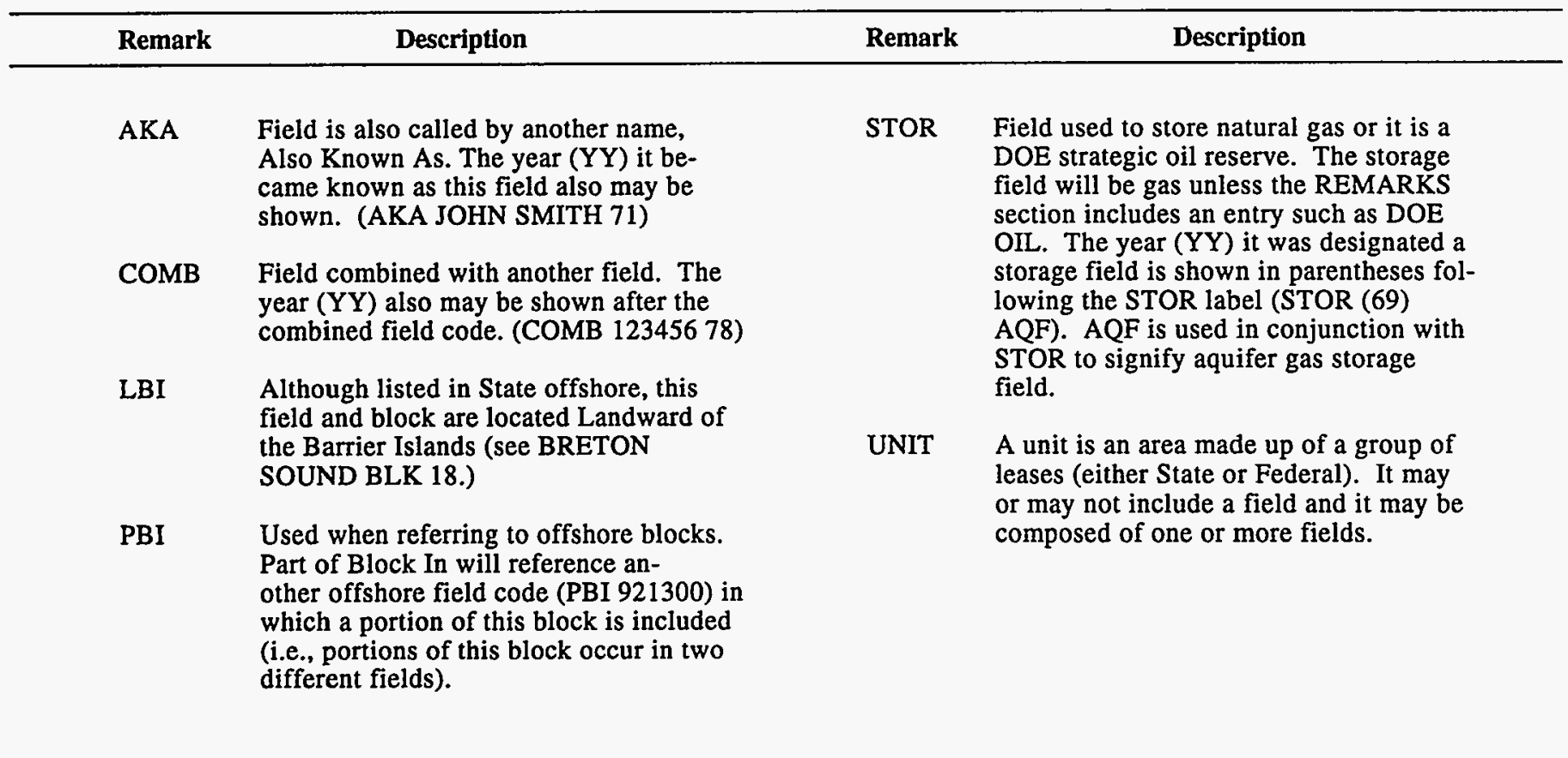

\section{Alias Field Record}

Item 1, ALIAS FIELD CODE. The six-digit field name code assigned to this alias field name, printed in light italics.

Item 2, ALIAS FIELD NAME. This is the alias field name (26-character limit), printed in italics.

Item 3, STATE POSTAL ABBREVIATION and STATE SUBDIVISION CODE. Same as Item 3 under Master Field Record.

Item 4, COUNTY CODE. Same as Item 4 under Master Field Record.

Item 5, COUNTY NAME. Same as Item 5 under Master Field Record.

Item 6, CROSS-REFERENCE. This is the identification of the master field code and field name which should be used in place of the alias name listed in Item 2 of this record. This item consists of the label "Alias For:" followed by the correct six-digit field name code and the correct (i.e., current) field name.

Item 7, ALIAS TYPE. This code (five-character limit) indicating type of alias is present only for alias fields. The types of alias codes are as follows:
ALTER This field name is an alternative for the official field name and should not be used in DOE forms. The correct name and code are listed after "Alias For:".

BLOCK This offshore field extends into more than one offshore block. This offshore block name is not the one for which the field is named. The block for which the field is named is given after "Alias For:".

COMB This field has been combined with another field which is listed after "Alias For:".

MULTI This offshore lease block has portions of two or more fields in it. In the Field Code Master List, the record is listed once for each field to which it is aliased so that appropriate cross reference is made.

RENAM This field has been renamed. The new name is listed after "Alias For:".

SPLIT This field has been split into two or more fields, none of which retains this name. One of the new fields is listed after Alias For:". Contact the Field Code Coordinator for the additional fields and information relating to this alias field.

Included on the following page is a listing of the State names and the page of the Oil and Gas Field Code Master List on which the first field in those States occurs. 


\begin{tabular}{lclclclc}
\hline $\begin{array}{l}\text { First Field } \\
\text { In State }\end{array}$ & $\begin{array}{r}\text { On } \\
\text { Page }\end{array}$ & $\begin{array}{l}\text { First Field } \\
\text { In State }\end{array}$ & $\begin{array}{c}\text { On } \\
\text { Page }\end{array}$ & $\begin{array}{l}\text { First Field } \\
\text { In State }\end{array}$ & $\begin{array}{c}\text { On } \\
\text { Page }\end{array}$ & $\begin{array}{l}\text { First Field } \\
\text { In State }\end{array}$ & $\begin{array}{c}\text { On } \\
\text { Page }\end{array}$ \\
\hline Alabama & 42 & Kansas & 66 & Nevada & 145 & Texas & 201 \\
Alaska & 43 & Kentucky & 103 & New Mexico & 145 & Utah & 298 \\
Arizona & 44 & Louisiana & 114 & New York & 151 & Virginia & 299 \\
Arkansas & 44 & Maryland & 125 & North Dakota & 153 & Washington & 299 \\
California & 46 & Michigan & 125 & Ohio & 155 & West Virginia & 300 \\
Colorado & 49 & Minnesota & 133 & Oklahoma & 161 & Wyoming & 304 \\
Forida & 56 & Mississippi & 133 & Oregon & 196 & F.O.-California & 311 \\
Illinois & 56 & Missouri & 137 & Pennsylvania & 196 & F.O.-Louisiana & 312 \\
Indiana & 61 & Montana & 138 & South Dakota & 200 & F.O.-Other Gulf & 325 \\
Iowa & 66 & Nebraska & 141 & Tennessec & 200 & F.O.-Texas & 325 \\
\hline
\end{tabular}

\section{Field Code Index}

In the Field Code Index, the field codes, State abbreviations, and associated field names are listed in ascending field code order. If a field name occurs in more than one State, the names are sorted in alphabetic State abbreviation order.

The field codes are arranged in four columns per page, as shown in Figure 3. As the six-digit field code relates directly to the particular field name, there is only one listing in this section per State/field code combination, regardless of the number of counties within that State in which it occurs.

The Index is intended for quick reference only. All detailed information is included on the Master List.

\section{Coalbed Methane Field List}

A dramatic rise in coalbed methane's share of natural gas production has heightened interest in those fields with coalbed methane potential. In Table 4, p. 18, Coalbed Methane Fields, the field name, field code, county code and name and state are given for those fields currently productive or with drilling activity.

\section{Storage Fields}

Storage fields have been developed in many States. Some were developed in existing, official oil and/or gas fields. All storage fields are listed in Table 5 . In certain States, the storage portion of a field or pool has been given a different name. In this case, the storage field name is carried on the FCML as an alias to the official field name. The storage field names which are aliases are listed in both Table 5 and the FCML in italics. These alias field names and codes in italics should only be used in filing Forms FERC 8 and EIA-191, "Underground Gas Storage Report".

\section{Fields Located in Multiple Jurisdictions}

Table 6, Fields Located in Multiple Jurisdictions, is compiled to reflect those oil and/or gas fields which cross State boundaries. In developing the summary statistics on page 1 , a field is only counted once, no matter how many counties or States it is in.

\section{Outer Continental Shelf Planning Areas}

Gulf of Mexico Outer Continental Shelf statistics published by the Minerals Management Service (MMS) follow the boundary lines of their Western, Central and Eastern Planning areas, shown respectively in Figures 9, 10 , and 11. In order that reports developed from the U.S. Crude Oil, Natural Gas, and Natural Gas Liquids Reserves 1992 track the reporting by MMS, fields currently found in the Garden Banks leasing area (GB), and those which may be found in GB or in the Keathley Canyon leasing area, will henceforth be listed in the FCML as Texas Federal Offshore rather than LouisianaSouth Federal Offshore. 
Figure 3. Example of a Field Code Index Page

\begin{tabular}{|c|c|c|c|c|c|c|c|c|c|c|c|}
\hline $\begin{array}{l}\text { Flold } \\
\text { codo }\end{array}$ & State & Fleld Namo & $\begin{array}{l}\text { Field } \\
\text { Code }\end{array}$ & State & Fiold Name & $\begin{array}{l}\text { Field } \\
\text { Codo }\end{array}$ & State & Fleld Name & $\begin{array}{l}\text { Field } \\
\text { Coda } \\
\end{array}$ & Stato & Field Name \\
\hline 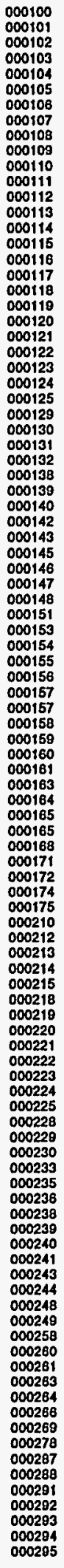 & 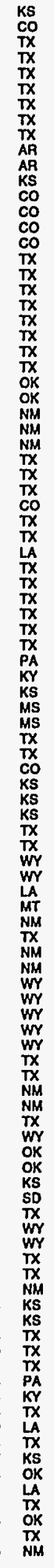 & 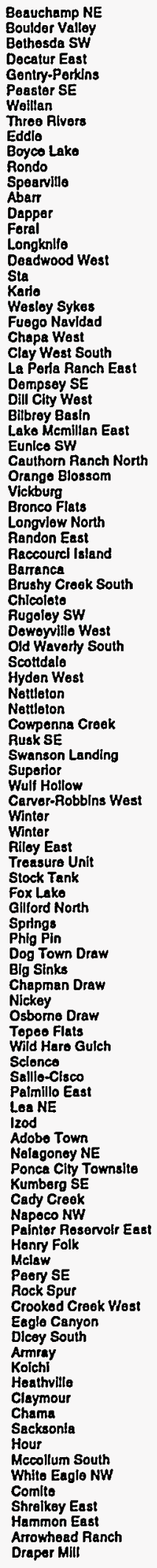 & 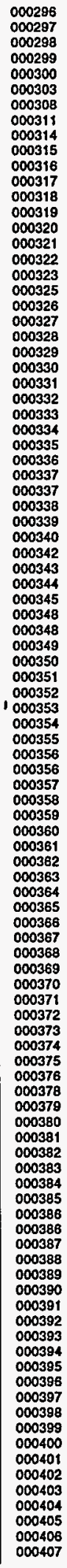 & 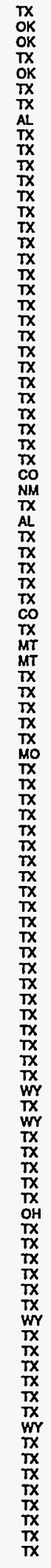 & 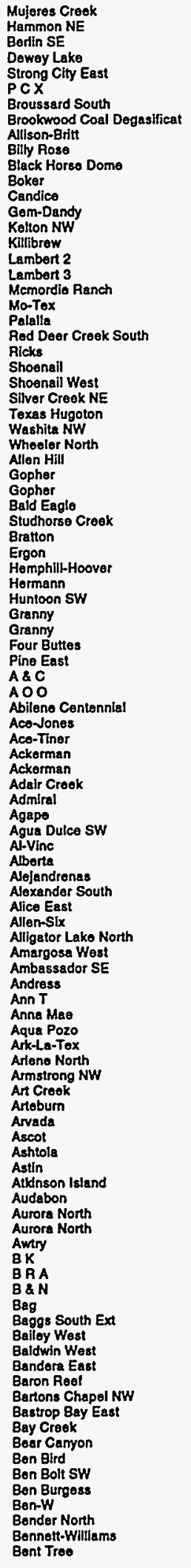 & 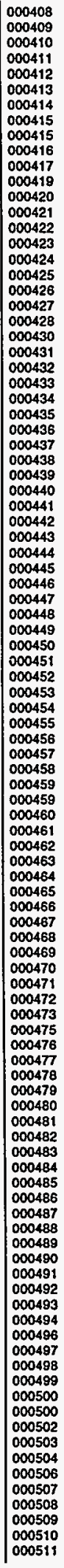 & 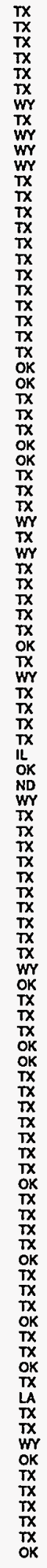 & 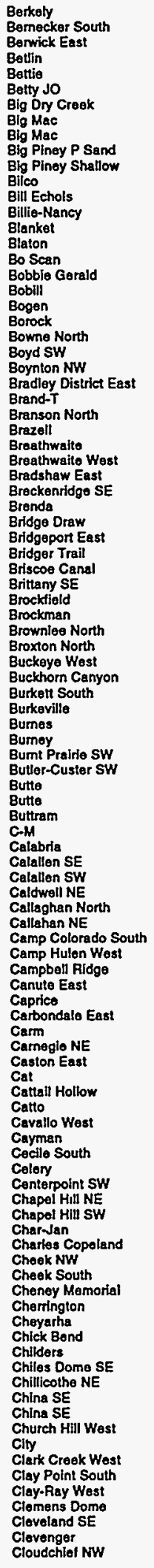 & 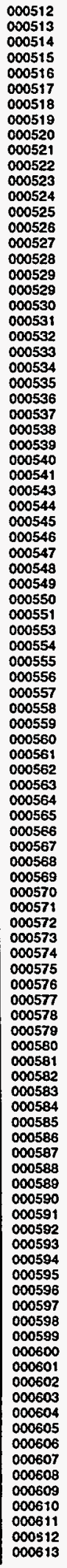 & 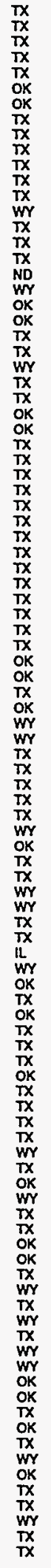 & 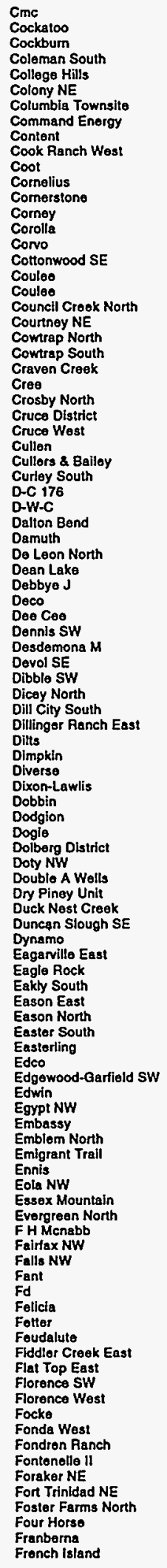 \\
\hline
\end{tabular}

Source: Energy Information Administration, Office of Oil and Gas 
Table 2. State Abbreviations and Geographic Subdivision Codes

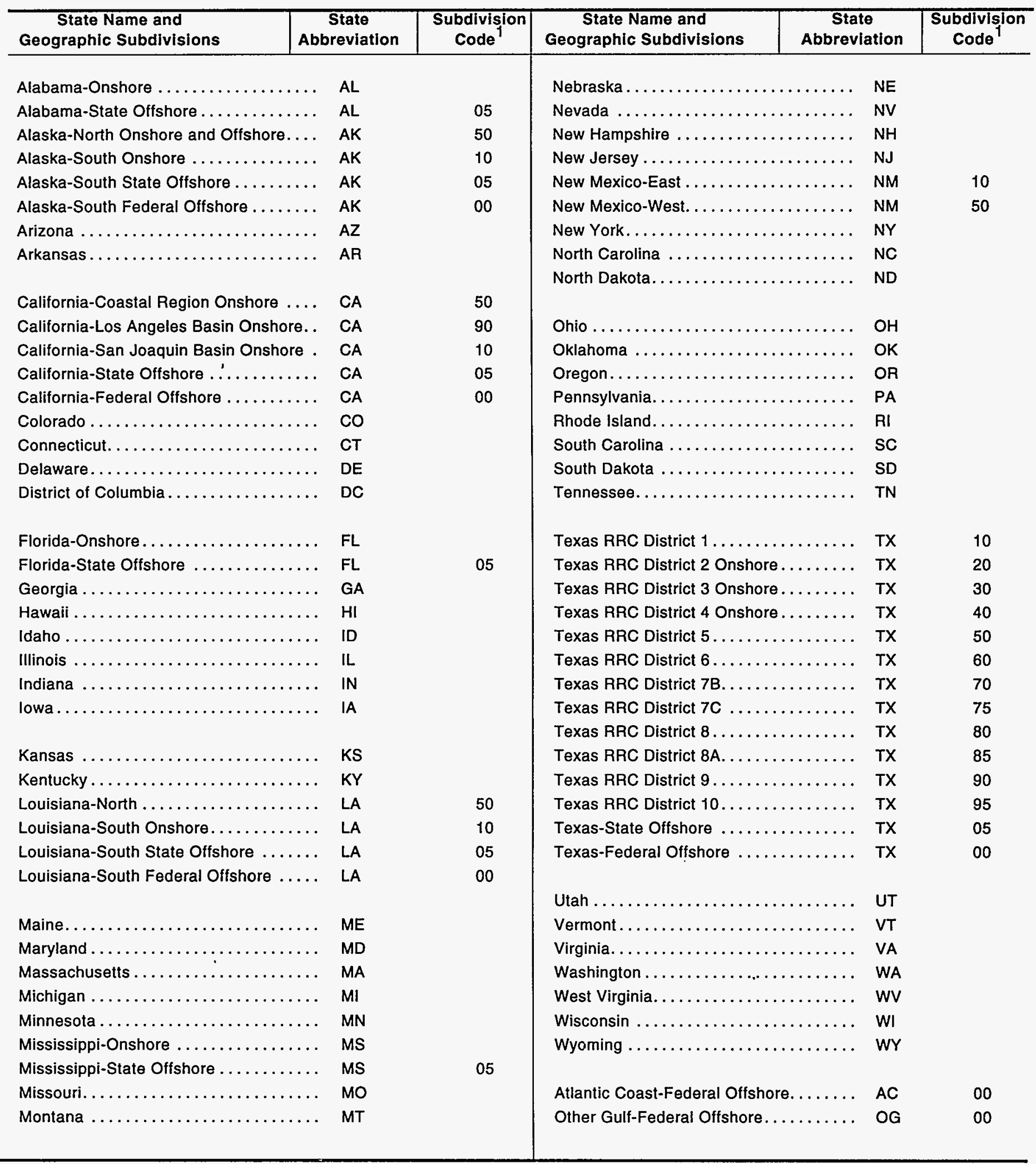

\footnotetext{
${ }^{1}$ If no subdivision code is specified, there is no subdivision.
}

Sources: National Bureau of Standards, U.S. Department of Commerce, FIPS PUB 6-3, Counties and County Equivalents of the States of the United States and the District of Columbia. Energy Information Administration, Office of Oil and Gas. 


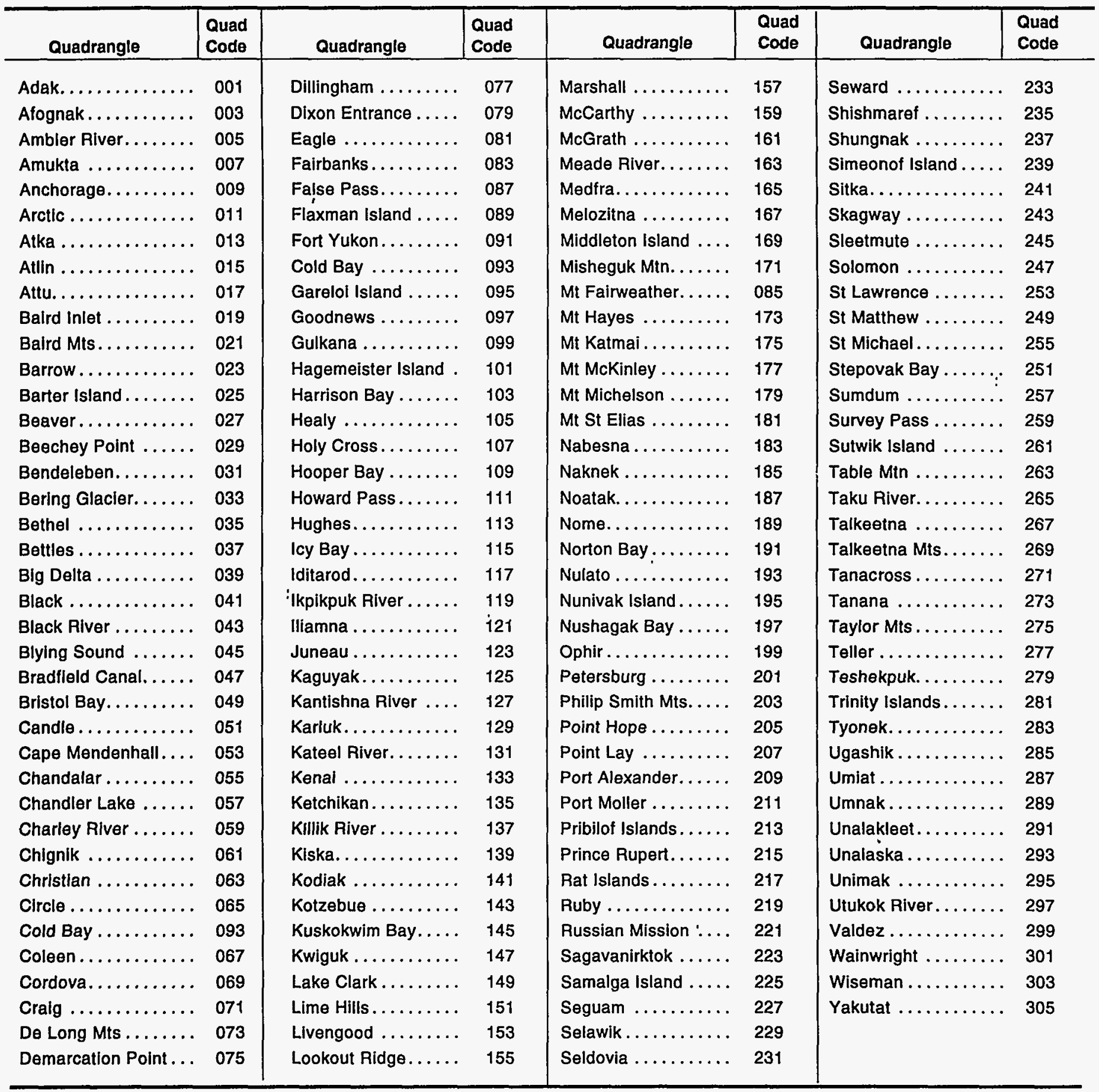

Note: The State of Alaska does not have counties that can be used for field identification. Areas identified by $U$. S. Geological Survey quadrangles have been assigned codes by the American Petroleum Institute for use as pseudo-counties. These three-digit codes are analogous to the usual "county code".

Source: The API Well Number and Standard State and County Numeric Codes Including Offshore Waters. API Bulletin D12A, January 1979. American Petroleum Institule, Washington, DC. 


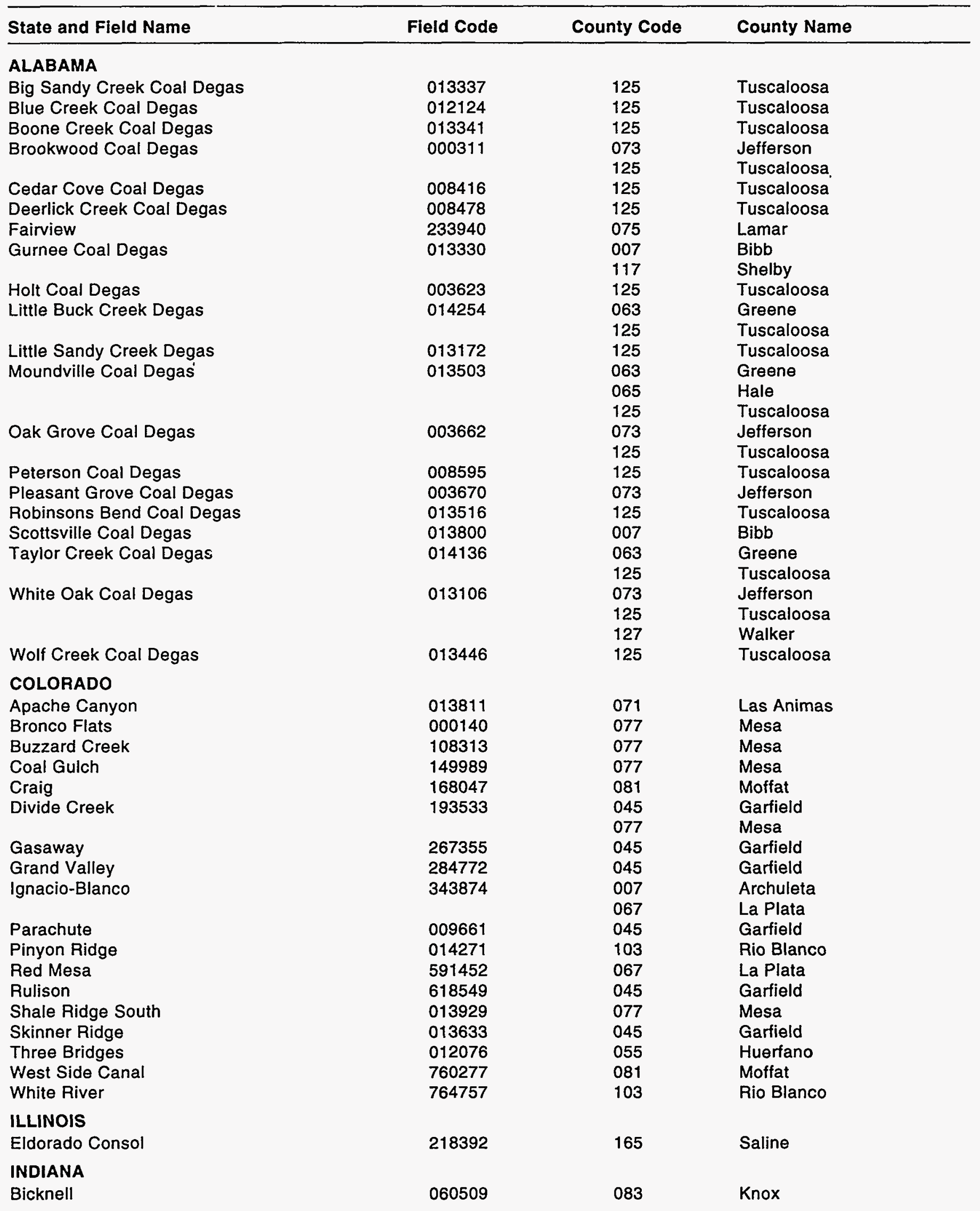


Table 4. Coalbed Methane Fields (continued)

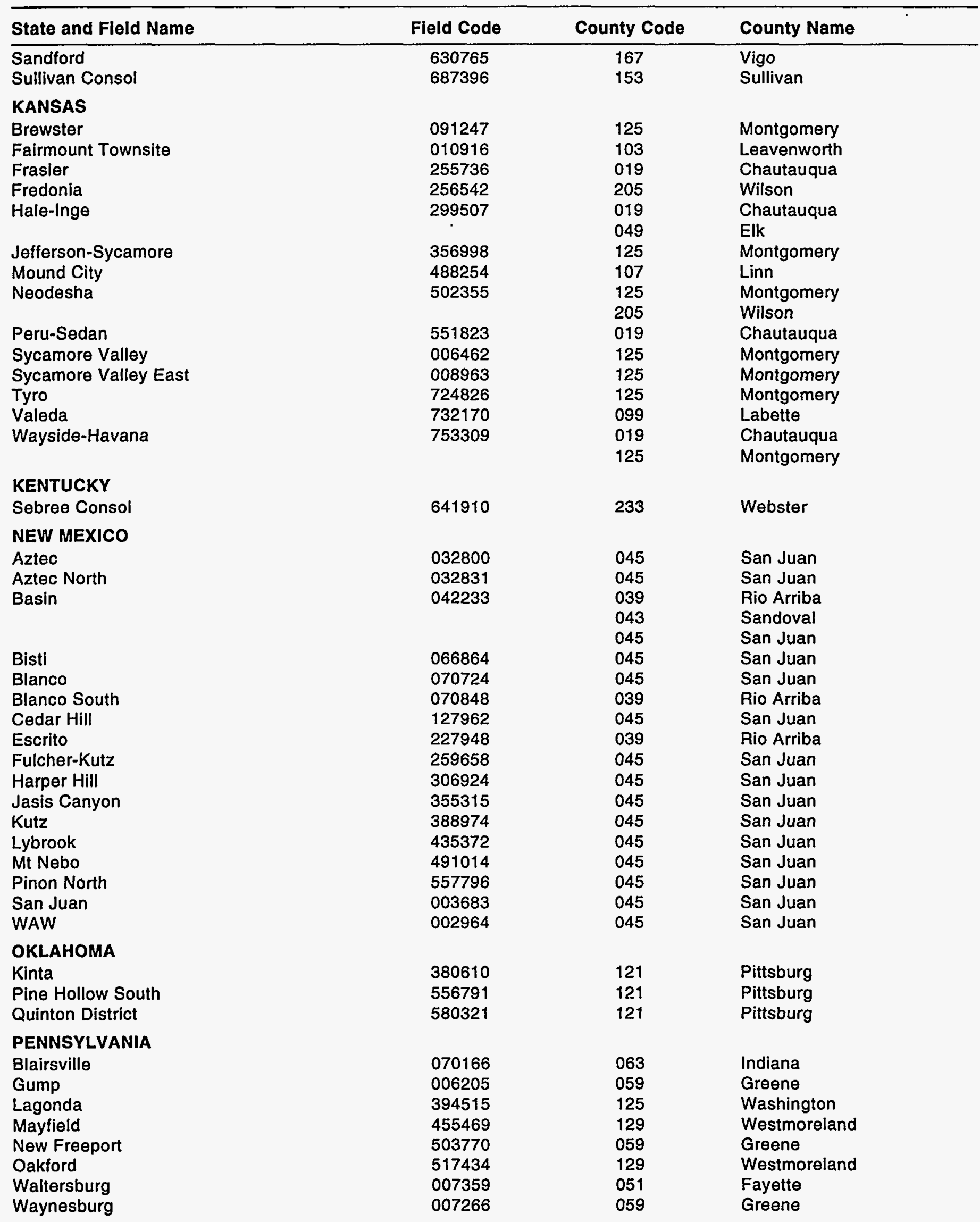


Table 4. Coalbed Methane Fields (continued)

\begin{tabular}{|c|c|c|c|}
\hline State and Field Name & Field Code & County Code & County Name \\
\hline \multicolumn{4}{|l|}{ UTAH } \\
\hline Castlegate & 014836 & 007 & Carbon \\
\hline Drunkards Wash & 014840 & 007 & Carbon \\
\hline \multicolumn{4}{|l|}{ VIRGINIA } \\
\hline Glick & 277145 & 027 & Buchanan \\
\hline Keen Mountain & 001394 & 027 & Buchanan \\
\hline Nora & 511254 & 051 & -Dickenson \\
\hline & & 167 & Russell \\
\hline & & 195 & Wise \\
\hline Oakwood & 518178 & 027 & Buchanan \\
\hline \multicolumn{4}{|l|}{ WEST VIRGINIA } \\
\hline Big Run-Birchfield & 063361 & 103 & Wetzel \\
\hline \multicolumn{4}{|l|}{ WYOMING } \\
\hline Bone Pile & 080017 & 005 & Campbell \\
\hline Breen & 090658 & 005 & Campbell \\
\hline Dixon & 193998 & 007 & Carbon \\
\hline Hartzog Draw & 308943 & 005 & Campbell \\
\hline Kingsbury Creek & 380145 & 005 & Campbell \\
\hline Oedekoven & 520069 & 005 & Campbell \\
\hline Rawhide Butte & 013282 & 005 & Campbell \\
\hline Recluse & 589685 & 005 & Campbell \\
\hline Wolff & 000853 & 005 & Campbell \\
\hline
\end{tabular}

Source: Energy Information Administration, Office of Oil and Gas 
Table 5. Storage Fields*

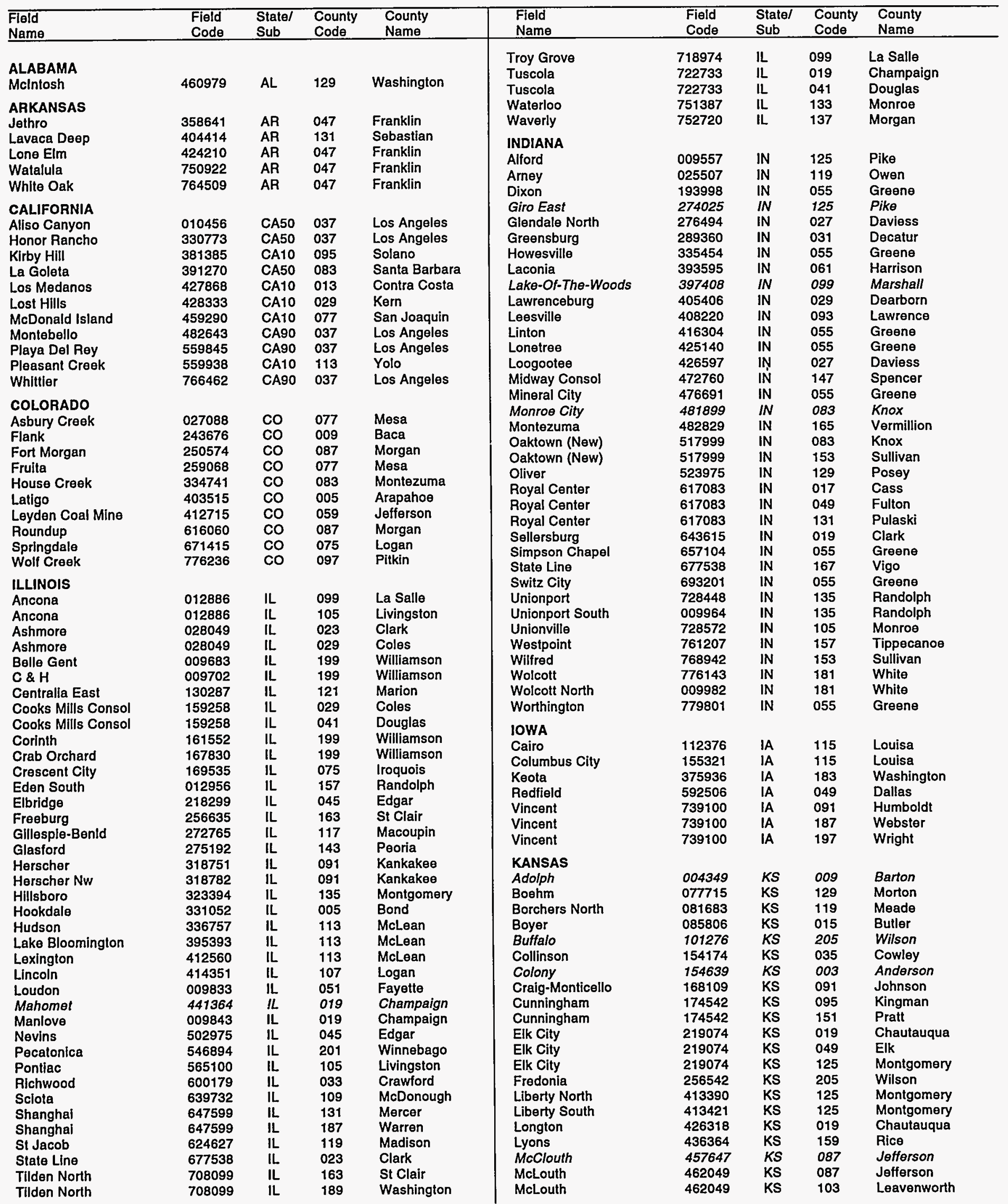

*Alias field names are in italics. 
Table 5. Storage Fields* (Continued)

\begin{tabular}{|c|c|c|c|c|c|c|c|c|c|}
\hline $\begin{array}{l}\text { Field } \\
\text { Name } \\
\end{array}$ & $\begin{array}{l}\text { Field } \\
\text { Code } \\
\end{array}$ & $\begin{array}{l}\text { State/ } \\
\text { Sub }\end{array}$ & $\begin{array}{l}\text { County } \\
\text { Code }\end{array}$ & $\begin{array}{l}\text { County } \\
\text { Name }\end{array}$ & $\begin{array}{l}\text { Field } \\
\text { Name } \\
\end{array}$ & $\begin{array}{l}\text { Field } \\
\text { Code }\end{array}$ & $\begin{array}{l}\text { State/ } \\
\text { Sub }\end{array}$ & $\begin{array}{l}\text { County } \\
\text { Code }\end{array}$ & $\begin{array}{l}\text { County } \\
\text { Name }\end{array}$ \\
\hline Piqua & 558155 & KS & 001 & Allen & Coldwater & 152252 & MI & 073 & Isabella \\
\hline Piqua & 558155 & KS & 207 & Woodson & Collin & 153988 & MI & 147 & St Clair \\
\hline Welda North & 756526 & KS & 003 & Anderson & Columbus & 155290 & MI & 147 & St Clair \\
\hline Welda South & 756557 & KS & 003 & Anderson & Columbus West & 155538 & MI & 147 & St Clair \\
\hline KENTUCKY & & & & & Cranberry Lake & 168233 & M! & 035 & Clare \\
\hline Adair North & 053171 & KY & 091 & Hancock & Cranberry Lake & 168233 & MI & 113 & Missaukee \\
\hline Anton & 021244 & $\mathrm{KY}$ & 107 & Hopkins & Croton & 172387 & MI & 123 & Newaygo \\
\hline Ballardsville & 036808 & $\mathrm{KY}$ & 185 & Oldham & Excelsior $06-27 \mathrm{~N}-6 \mathrm{~W}$ & .009735 & MI & 079 & Kalkaska \\
\hline Bon Harbor & 079544 & $K Y$ & 059 & Daviess & Four Corners & 252434 & MI & 147 & St Clair \\
\hline Canmer & 009704 & KY & 099 & Hart & Freeman-Lincoln & 256790 & MI & 035 & Clare \\
\hline Cecilia & 004841 & KY & 093 & Hardin & Goodwell & 280385 & MI & 123 & Newaygo \\
\hline Center & 129264 & KY & 009 & Barren & Hamilton North & 301584 & MI & 035 & Clare \\
\hline Center & 129264 & $\mathrm{KY}$ & 087 & Green & Hessen & 319123 & MI & 147 & St Clair \\
\hline Center & 129264 & $\mathrm{KY}$ & 169 & Metcalfe & Howell & 335361 & MI & 093 & Livingston \\
\hline Crofton East & 009721 & KY & 047 & Christian & Ira & 347913 & MI & 147 & St Clair \\
\hline Dixie & 193812 & $\mathrm{KY}$ & 101 & Henderson & Kalkaska 01-27N-7W & 009799 & MI & 079 & Kalkaska \\
\hline Doe Run & 194968 & KY & 163 & Meade & Lacey Station & 393525 & MI & 015 & Barry \\
\hline Eagle Creek & 210463 & KY & 077 & Gallatin & Lee 02-1S-5W & 003265 & MI & 025 & Calhoun \\
\hline Eagle Creek & 210463 & KY & 081 & Grant & Lee 04-1S-5W & 009822 & MI & 025 & Calhoun \\
\hline Eagle Creek & 210463 & $\mathrm{KY}$ & 187 & Owen & Le日 11-1S-5W & 003273 & MI & 025 & Calhoun \\
\hline Flint Hill & 245385 & $K Y$ & 093 & Hardin & Lee 16 & 009823 & MI & 025 & Calhoun \\
\hline Flint Hill Consol & 012752 & KY & 093 & Hardin & Lenox & 410204 & MI & 099 & Macomb \\
\hline Graham Lake & 283842 & $K Y$ & 177 & Muhlenberg & Leonard & 410731 & MI & 125 & Oakland \\
\hline Grandview & 284927 & KY & 059 & Daviess & Marion(Winterfield) & 447874 & MI & 035 & Clare \\
\hline Greenville & 289577 & KY & 177 & Muhlenberg & Marion(Winterfield) & 447874 & MI & 133 & Osceola \\
\hline Hawesville Nw & 011146 & KY & 091 & Hancock & Marsac Creek & 449207 & MI & 147 & St Clair \\
\hline Hickory School & 320852 & $K Y$ & 059 & Daviess & Marysville-Morton & 009849 & MI & 147 & St Clair \\
\hline Kettle Island & 005569 & KY & 013 & Bell & Muttonville & 495943 & MI & 099 & Macomb \\
\hline Kirkwood Springs & 381788 & KY & 107 & Hopkins & Northville & 513052 & MI & 125 & Oakland \\
\hline Magnolia & 440775 & KY & 087 & Green & Northville & 513052 & MI & 161 & Washtenaw \\
\hline Magnolia & 440775 & KY & 099 & Hart & Northville & 513052 & MI & 163 & Wayne \\
\hline Magnolia & 440775 & KY & 123 & LaRue & Orient & 527889 & MI & 035 & Clare \\
\hline Midland & 472280 & KY & 177 & Muhlenberg & Orient & 527889 & MI & 133 & Osceola \\
\hline Mt Olive Church & 009874 & KY & 227 & Warren & Overisel & 531485 & MI & 005 & Allegan \\
\hline Muldraugh & 493184 & KY & 163 & Meade & Partello & 541445 & MI & 025 & Calhoun \\
\hline Owensboro & 532074 & KY & 059 & Daviess & Puttygut & 575943 & MI & 147 & St Clair \\
\hline Owensboro West & 532167 & KY & 059 & Daviess & Rapid River $35-28 \mathrm{~N}-7 \mathrm{~W}$ & 003354 & MI & 079 & Kalkaska \\
\hline St Charles & 673074 & KY & 107 & Hopkins & Ray & 588018 & MI & 099 & Macomb \\
\hline LOUISIANA & & & & & Reed City & 593157 & MI & 085 & Lake \\
\hline Bayou Choctaw & 045581 & LA10 & 047 & Iberville & Reed City & 593157 & MI & 133 & Osceola \\
\hline Bayou Choctaw & 045581 & LA1O & 121 & West Baton & Riverside & 603992 & MI & 035 & Clare \\
\hline & & & & $\begin{array}{l}\text { West Baton } \\
\text { Rouge }\end{array}$ & Riverside & 603992 & MI & 113 & Missauke日 \\
\hline Beat Creek & 048643 & LA50 & 013 & Bienville & Salem & $\begin{array}{l}6113 \% 9 \\
625030\end{array}$ & $\begin{array}{l}M I \\
M I\end{array}$ & $\begin{array}{l}073 \\
005\end{array}$ & $\begin{array}{l}\text { Isabella } \\
\text { Alleaan }\end{array}$ \\
\hline Epps & 226762 & LA50 & 035 & East Carroll & Salem & 625030 & $M !$ & 139 & Otlawa \\
\hline Epps & 226762 & LA50 & 123 & West Carroll & Shaver & & & & \\
\hline Garden Island Bay & 265340 & LA10 & 075 & Plaquemines & (Sumner-New Haven) & 648622 & MI & 057 & Gratiot \\
\hline Hackberry West & 298329 & LA10 & 023 & Cameron & Shaver & & & & \\
\hline Hester & 319154 & LA10 & 093 & St James & (Sumner-New Haven) & 648622 & MI & 117 & Montcalm \\
\hline Holly & 328510 & LA50 & 031 & De Soto & Six Lakes & 658449 & MI & 073 & Isabella \\
\hline Lake Bistineau & 395331 & LA50 & 013 & Bienville & Six Lakes & 658449 & MI & 107 & Mecosta \\
\hline Lake Bistineau & 395331 & LA50 & 015 & Bossier & Six Lakes & 658449 & MI & 117 & Montcalm \\
\hline Lake Bistineau & 395331 & LA50 & 119 & Webster & Swan Creek & 691651 & MI & 147 & St Clair \\
\hline Ruston & 620502 & LA50 & 061 & Lincoln & WC Taggart(Six Lakes) & 743079 & $M I$ & 107 & Mecosta \\
\hline Sulphur Mines & 687737 & LA10 & 019 & Calcasieu & W C Taggart(Six Lakes) & 743079 & $M I$ & 117 & Montcalm \\
\hline Unionville & 728572 & LA50 & 061 & Lincoln & Wintield & 773189 & Ml & 107 & Mecosta \\
\hline Unionville & 728572 & LA50 & 111 & Union & Winfield & 773189 & MI & 117 & Montcalm \\
\hline Washington & 750240 & LA10 & 097 & St Landry & Woodville & 778406 & MI & 123 & Newaygo \\
\hline Weeks Island & 754914 & LA10 & 045 & Iberia & MINNESOTA & & & & \\
\hline Weeks Island & 754914 & LA10 & 101 & St Mary & $\begin{array}{l}\text { MINNESOTA } \\
\text { Waterville-Waseca }\end{array}$ & 751666 & MN & 079 & Le Sugur \\
\hline MARYLAND & & & & & Waterville-Waseca & 751666 & MN & 131 & Rice \\
\hline Accident & 002210 & MD & 023 & Garrett & Watervilie-Waseca & 751666 & $M N$ & 161 & Waseca \\
\hline Brandywine & 088891 & MD & 033 & Prince Georges & MISSISSIPPI & & & & \\
\hline MICHIGAN & & & & & Amory & 016408 & MS & 095 & Monroe \\
\hline Austin & 030901 & MI & 107 & Mecosta & Eminence & 223786 & MS & 031 & Covington \\
\hline Belle River Mills & 053820 & MI & 147 & St Clair & Jackson & 353006 & MS & 049 & Hinds \\
\hline Broomfield Deerfield & 074351 & MI & 073 & Isabella & Jackson & 353006 & MS & 121 & Rankin \\
\hline Capac & 118576 & MI & 147 & St Clair & Muldon & 492967 & MS & 095 & Monroe \\
\hline Charlton $01-30 \mathrm{~N}-1 \mathrm{~W}$ & 002860 & MI & 137 & Otsego & MISSOURI & & & & \\
\hline Chester Sec 15 & 003494 & MI & 137 & Otsego & Florissant & 0.02175 & MO & 183 & St Charles \\
\hline Clarence 19-1S-4W & 009710 & M! & $\begin{array}{l}025 \\
079\end{array}$ & $\begin{array}{l}\text { Calhoun } \\
\text { Kalkaska }\end{array}$ & Florissant & 002175 & MO & 189 & St Louis \\
\hline
\end{tabular}

*Alias field names are in italics. 
Table 5. Storage Fields ${ }^{\star}$ (Continued)

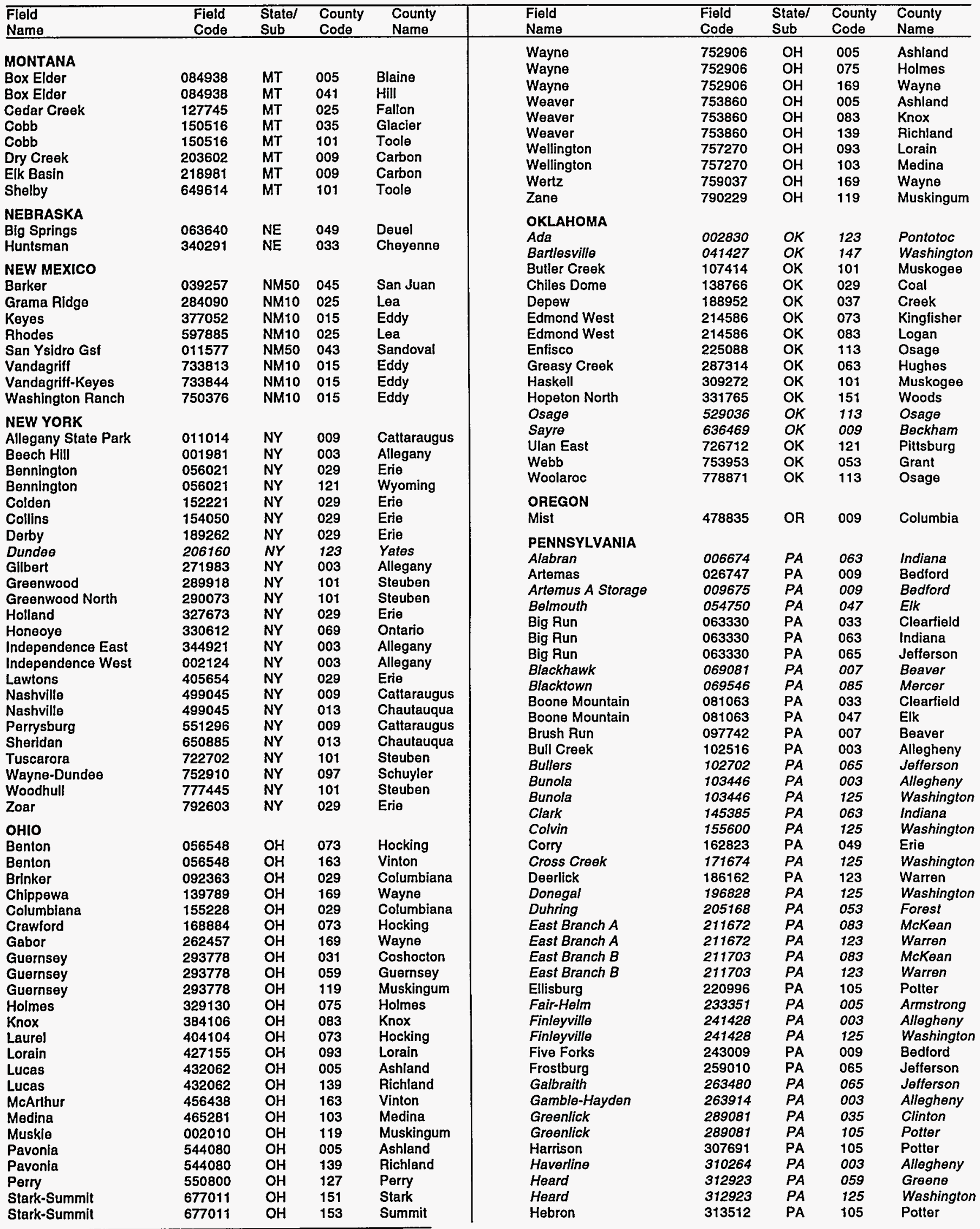

*Alias field names are in italics. 
Table 5. Storage Fields* (Continued)

\begin{tabular}{|c|c|c|c|c|c|c|c|c|c|}
\hline $\begin{array}{l}\text { Field } \\
\text { Name } \\
\end{array}$ & $\begin{array}{l}\text { Field } \\
\text { Code }\end{array}$ & $\begin{array}{l}\text { State/ } \\
\text { Sub }\end{array}$ & $\begin{array}{l}\text { County } \\
\text { Code }\end{array}$ & $\begin{array}{l}\text { County } \\
\text { Name }\end{array}$ & $\begin{array}{l}\text { Field } \\
\text { Name }\end{array}$ & $\begin{array}{l}\text { Field } \\
\text { Code } \\
\end{array}$ & $\begin{array}{l}\text { State/ } \\
\text { Sub }\end{array}$ & $\begin{array}{l}\text { County } \\
\text { Code }\end{array}$ & $\begin{array}{l}\text { County } \\
\text { Name }\end{array}$ \\
\hline Henderson & 315837 & $P A$ & 085 & Mercer & Clemens $\mathrm{Ne}$ & 147891 & TX30 & 039 & Brazoria \\
\hline Henderson & 315837 & $P A$ & 121 & Venango & Cliffside & 148547 & TX95 & 375 & Potter \\
\hline Holbrook & 327239 & $P A$ & 059 & Greene & De Leon & 183713 & TX70 & 093 & Comanche \\
\hline Hughes & 337532 & $P A$ & 019 & Butler & Hawkins & 310450 & $\mathrm{~T} \times 60$ & 499 & Wood \\
\hline Hunters Cave & 339950 & $P A$ & 059 & Greone & Hilbig & 322309 & $\mathrm{TX} 10$ & 021 & Bastrop \\
\hline lowa & 347711 & $P A$ & 065 & Jefferson & Hill & 322712 & TX70 & 133 & Eastland \\
\hline Inwin & 349153 & $P A$ & 129 & Westmoreland & Janellen & 354742 & TX70 & 049 & Brown \\
\hline Keasey & 370945 & $P A$ & 019 & Butler & La-Pan & 391797 & TX90 & 077 & Clay \\
\hline Keelor & 371213 & $P A$ & 083 & McKean & Lake Dallas & 396106 & $T \times 90$ & 121 & Denton \\
\hline Kinter & 380765 & $P A$ & 063 & Indiana & Lansing North & 401810 & TX60 & 203 & Harrison \\
\hline Kirby & 381292 & $P A$ & 059 & Greene & Lone Camp & 424179 & $\mathrm{~T} \times 70$ & 363 & Palo Pinto \\
\hline Leidy & 408747 & PA & 035 & Clinton & Loop & 426876 & TX85 & 165 & Gaines \\
\hline Leidy & 408747 & PA & 105 & Potter & Milsap & 009871 & TX70 & 367 & Parker \\
\hline Majorsville & 441705 & $P A$ & 059 & Greene & New York City & 505145 & TX90 & 077 & Clay \\
\hline Majorsville & 441705 & $P A$ & 125 & Washington & Pecan Station & 546832 & TX75 & 451 & Tom Green \\
\hline Markle & 448308 & $P A$ & 065 & Jefferson & Pottsville South & 568851 & TX70 & 193 & Hamilton \\
\hline McKinley & 461429 & $P A$ & 047 & $E / k$ & Tri-Cities & 716897 & $\mathrm{~T} \times 50$ & 213 & Henderson \\
\hline Meade Run & 463638 & $P A$ & 083 & McKean & View & 738604 & $\mathrm{TX70}$ & 441 & Taylor \\
\hline Meoker & 465622 & $P A$ & 117 & Tioga & Worsham-Steed & 779584 & TX90 & 237 & Jack \\
\hline Mohan Run & 480876 & $P A$ & 047 & $E / k$ & Yoast & 787149 & $\mathrm{~T} \times 10$ & 021 & Bastrop \\
\hline Mt Royal & 491169 & $P A$ & 003 & Allegheny & UTAH & & & & \\
\hline Munderf & 493897 & $P A$ & 065 & Jefferson & Bridaer Lake & & UT & & Summit \\
\hline Murrysvillo & 494982 & $P A$ & 003 & Allegheny & Briager Lake & $\begin{array}{l}091743 \\
131078\end{array}$ & UIT & 043 & Summit \\
\hline Neubert & 502851 & $P A$ & 019 & Butler & Cnalk Creek & 131078 & 01 & 043 & Summit \\
\hline Oakford & 517434 & PA & 129 & Westmoreland & Clay Basin & 146315 & UT & 009 & Daggett \\
\hline Owls Nest & 532477 & $P A$ & 047 & $E / k$ & Coalville & 150268 & UT & 043 & Summit \\
\hline Palmer Storago & 536981 & $P A$ & 117 & Tioga & VIRGINIA & & & & \\
\hline Patton & 542933 & $P A$ & 129 & Westmoreland & Early Grove & 211424 & VA & 169 & Scott \\
\hline Portman & 567146 & PA & 019 & Butler & Early Grove & 211424 & VA & 191 & Washington \\
\hline Pratt & 571347 & PA & 059 & Greene & WASHINGTON & & & & \\
\hline Pratt & 571347 & PA & 125 & Washington & WASHING ION & & & & \\
\hline Queen & 579391 & PA & 053 & Forest & Jackson Prairie & 353192 & WA & 041 & LewIS \\
\hline Queen & 579391 & PA & 123 & Warren & WEST VIRGINIA & & & & \\
\hline Rager Mountain & 582872 & PA & 021 & Cambria & Aspinall-Finster & 028700 & WV & 041 & Lewis \\
\hline Rush Run & 619479 & $P A$ & 051 & Fayette & Augusta & 030591 & WV & 027 & Hampshire \\
\hline Sabinsville & 623108 & PA & 105 & Potter & Big Run-Birchfield & 063361 & WV & 103 & Wotzel \\
\hline Sabinsville & 623108 & $\mathrm{PA}$ & 117 & Tioga & Blue Creek (Falling Rock) & 075064 & WV & 039 & Kanawha \\
\hline Saltsburg & 627155 & PA & 063 & Indiana & Bridgeport-Pruntytown & 091681 & WV & 033 & Harrison \\
\hline Saxonburg & 636376 & PA & 019 & Butler & Bridgeport-Pruntytown & 091681 & WV & 091 & Taylor \\
\hline Sharon & 647971 & $P A$ & 105 & Potter & Browns Creek & 096052 & $w V$ & 039 & Kanawha \\
\hline South Bend & 666548 & $P A$ & 005 & Armstrong & Browns Creek & 096052 & WV & 079 & Putnam \\
\hline South Bend & 666548 & $P A$ & 063 & Indiana & Burdett-St Albans & 104035 & WV & 039 & Kanawha \\
\hline Sprankle & 670764 & $P A$ & 065 & Jefferson & Burdett-St Albans & 104035 & WV & 079 & Putnam \\
\hline St Marys & 674345 & PA & 047 & Elk & Burning Springs South & 105430 & WV & 105 & Wirt \\
\hline State Line & 677538 & PA & 105 & Potter & Cameron-Kausooth & 004358 & WV & 051 & Marshall \\
\hline Summit & 688357 & $P A$ & 049 & Erio & Coco & 150919 & WV & 039 & Kanawha \\
\hline Swarts & 692023 & $P A$ & 059 & Greene & Comet & 155941 & $W V$ & 091 & Taylor \\
\hline Swarts West & 692034 & $P A$ & 059 & Greene & Conings & 157615 & WV & 017 & Doddridge \\
\hline Swede Hill & 692147 & $P A$ & 083 & McKean & Conings & 157615 & WV & 021 & Gilmer \\
\hline Tamarack & 696938 & $P A$ & 035 & Clinton & Craddock & 167954 & WV & 083 & Randolph \\
\hline Tepe & 701449 & $P A$ & 003 & Allegheny & Craddock & 167954 & WV & 097 & Upshur \\
\hline Tioga & 709091 & PA & 117 & Tioga & Derricks Creek & 189324 & WV & 039 & Kanawha \\
\hline Trafford & 715192 & $P A$ & 129 & Westmoreland & Elk-Poca (Slssonville) & 219529 & WV & 035 & Jackson \\
\hline Truittsburg & 719222 & $P A$ & 031 & Clarion & Elk-Poca (Sissonville) & 219529 & WV & 039 & Kanawha \\
\hline Wadsworth-North Oakland & 743970 & PA & 019 & Butler & Elk-Poca (Sissonville) & 219529 & WV & 079 & Putnam \\
\hline Webster & 754542 & PA & 129 & Westmoreland & Fink Creok (Dry Fork) & 241304 & WV & 041 & Lewls \\
\hline Wellendorf & 757146 & $P A$ & 083 & McKean & Fink-Kennedy & 241335 & WV & 017 & Doddridge \\
\hline Wharton & 762308 & $P A$ & 023 & Cameron & Fink-Kennedy & 241335 & $W V$ & 033 & Harrison \\
\hline Wharton & 762308 & $P A$ & 105 & Potter & Fink-Kennedy & 241335 & $W V$ & 041 & Lewis \\
\hline Whitehorn Creek & 010238 & PA & 129 & Westmoreland & Five Mile-Carpenter & 243102 & WV & 039 & Kanawha \\
\hline Wolf Creek & 776236 & PA & 121 & Venango & Gauley Mountain & 267851 & WV & 019 & Fayette \\
\hline & & & & & Gauley Mountain & 267851 & WV & 067 & Nicholas \\
\hline TEXAS & & & & & Glady & 274789 & WV & 075 & Pocahontas \\
\hline Ambassador & 015230 & TX90 & 077 & Clay & Glady & 274789 & WV & 083 & Randolph \\
\hline Bammel & 037242 & TX30 & 201 & Harris & Grapevine A \& B & 285795 & $W V$ & 039 & Kanawha \\
\hline Bethel Dome & 009684 & $T \times 60$ & 001 & Anderson & Herbert & 317697 & WV & 099 & Wayne \\
\hline Bibb East & 060292 & TX70 & 093 & Comanche & $\begin{array}{l}\text { Heroent } \\
\text { Hunt }\end{array}$ & 339392 & WV & 039 & Kanawha \\
\hline Big Hill & 061780 & TX30 & 245 & Jefferson & $\begin{array}{l}\text { Hunt } \\
\text { Hurricane Creok }\end{array}$ & 340601 & WV & 079 & $\begin{array}{l}\text { Nanawna } \\
\text { Putnam }\end{array}$ \\
\hline Bryan Mound & 098160 & $\mathrm{TX} 30$ & 039 & Brazoria & Kausooth & 370371 & $W V$ & 051 & Marshall \\
\hline Bryson South & 099028 & TX90 & 237 & Jack & Lake & 394866 & $W V$ & 079 & Putnam \\
\hline Burnall & 105089 & $T \times 20$ & 025 & $B e \theta$ & $\begin{array}{l}\text { Lake } \\
\text { Lanham }\end{array}$ & 401624 & WV & 039 & Kanawha \\
\hline Burnell & 105089 & $T \times 20$ & 255 & Karnes & $\begin{array}{l}\text { Lanham } \\
\text { Lanham }\end{array}$ & $\begin{array}{l}401624 \\
401624\end{array}$ & $W V$ & 079 & $\begin{array}{l}\text { Nunawna } \\
\text { Putnam }\end{array}$ \\
\hline Butler & 107383 & $T \times 50$ & 161 & Freestone & Little Cacapon & 418024 & WV & 027 & Hampshire \\
\hline Clear Lake West & 147307 & $\mathrm{TX} 30$ & 201 & Harris & Liverpool & 420613 & WV & 035 & Jackson \\
\hline
\end{tabular}

*Alias field names are in italics. 
Table 5. Storage Fields* (Continued)

\begin{tabular}{|c|c|c|c|c|}
\hline $\begin{array}{l}\text { Fiold } \\
\text { Name }\end{array}$ & $\begin{array}{l}\text { Field } \\
\text { Code }\end{array}$ & $\begin{array}{l}\text { Statel } \\
\text { Sub }\end{array}$ & $\begin{array}{l}\text { County } \\
\text { Code }\end{array}$ & $\begin{array}{l}\text { County } \\
\text { Name }\end{array}$ \\
\hline $\begin{array}{l}\text { Liverpool } \\
\text { Liverpool } \\
\text { Logansport } \\
\text { Lynn Camp } \\
\text { Majorsville } \\
\text { Manninglon } \\
\text { Maple Lake } \\
\text { Maple Lake } \\
\text { Murphy Creek } \\
\text { (Freemansburg) } \\
\text { Murphy Creek } \\
\text { (Freemansburg) } \\
\text { Newberne } \\
\text { Rockport } \\
\text { Rockport } \\
\text { Rockport } \\
\text { Sllver HIII } \\
\text { Sinklng Creek } \\
\text { Slab Fork } \\
\text { Terra Alta } \\
\text { Victory } \\
\text { Victory } \\
\text { Weston-Jane Lew } \\
\text { Weston-Jane Lew } \\
\text { Wilbur } \\
\text { Wilbur } \\
\text { X- } 1\end{array}$ & $\begin{array}{l}420613 \\
420613 \\
423063 \\
436178 \\
441705 \\
444340 \\
445487 \\
445487 \\
\\
494610 \\
\\
494610 \\
505393 \\
608868 \\
608868 \\
608868 \\
656279 \\
657612 \\
659945 \\
701821 \\
738077 \\
738077 \\
760959 \\
760959 \\
767795 \\
767795 \\
782253\end{array}$ & $\begin{array}{l}\text { WV } \\
W V \\
W V \\
W V \\
W V \\
W V \\
W V \\
W V \\
W V \\
W V \\
W V \\
W V \\
W V \\
W V \\
W V \\
W V \\
W V \\
W V\end{array}$ & $\begin{array}{l}017 \\
041 \\
021 \\
035 \\
105 \\
107 \\
103 \\
021 \\
081 \\
077 \\
051 \\
103 \\
033 \\
041 \\
017 \\
095 \\
079\end{array}$ & $\begin{array}{l}\text { Roane } \\
\text { Wirt } \\
\text { Marion } \\
\text { Wetzel } \\
\text { Marshall } \\
\text { Marion } \\
\text { Harrison } \\
\text { Taylor } \\
\text { Doddridge } \\
\text { Lewis } \\
\text { Gilmer } \\
\text { Jackson } \\
\text { Wirt } \\
\text { Wood } \\
\text { Wetzel } \\
\text { Gilmer } \\
\text { Raleigh } \\
\text { Preston } \\
\text { Marshall } \\
\text { Wetzel } \\
\text { Harrison } \\
\text { Lewis } \\
\text { Doddridge } \\
\text { Tyler } \\
\text { Putnam }\end{array}$ \\
\hline $\begin{array}{l}\text { WYOMING } \\
\text { Billy Creok } \\
\text { Brady } \\
\text { Bunker Hill } \\
\text { Chimney Butte } \\
\text { Chimney Butte } \\
\text { Elk Basin } \\
\text { Kirk } \\
\text { Oil Springs }\end{array}$ & $\begin{array}{l}064880 \\
087651 \\
103353 \\
139262 \\
139262 \\
218981 \\
381509 \\
520968\end{array}$ & $\begin{array}{l}\text { WY } \\
\text { WY } \\
\text { WY } \\
\text { WY } \\
\text { WY } \\
\text { WY } \\
\text { WY } \\
\text { WY }\end{array}$ & $\begin{array}{l}019 \\
037 \\
007 \\
023 \\
035 \\
029 \\
013 \\
007\end{array}$ & $\begin{array}{l}\text { Johnson } \\
\text { Sweetwater } \\
\text { Carbon } \\
\text { Lincoln } \\
\text { Sublette } \\
\text { Park } \\
\text { Fremont } \\
\text { Carbon }\end{array}$ \\
\hline
\end{tabular}

*Alias field names are in italics. 
Table 6. Fields Located in Multiple Jurisdictions

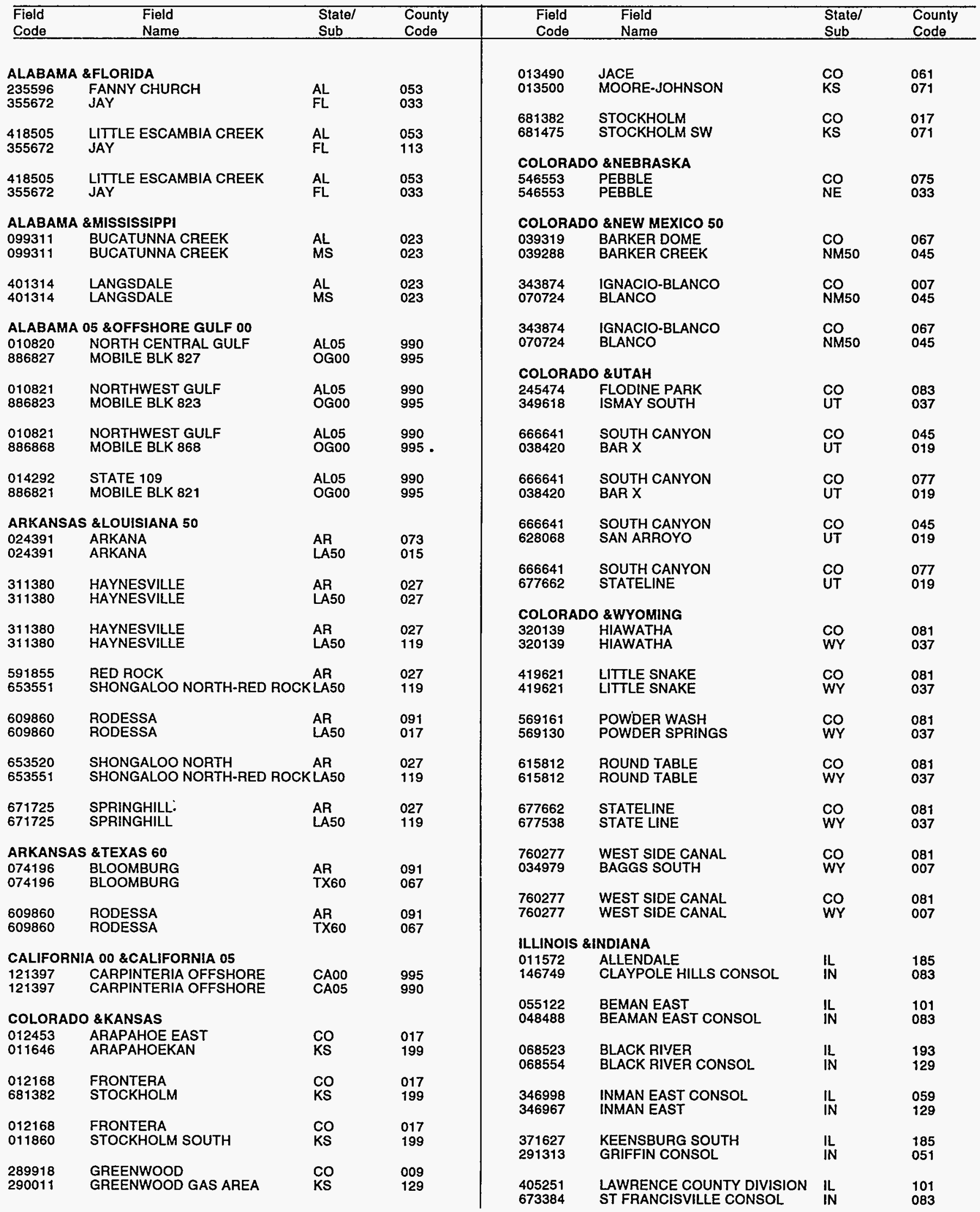


Table 6. Fields Located in Multiple Jurisdictions (Continued)

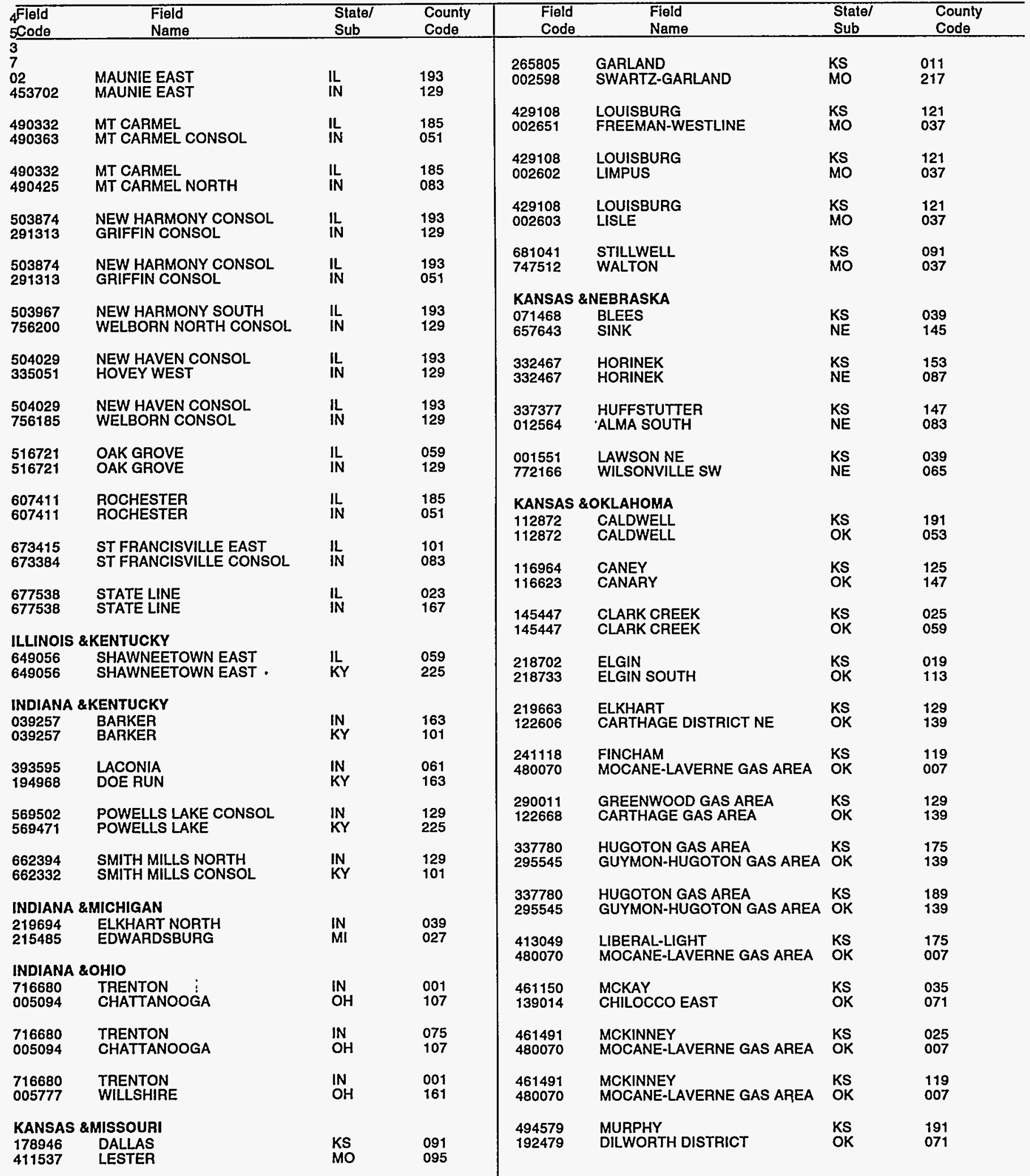


Table 6. Fields Located in Multiple Jurisdictions (Continued)

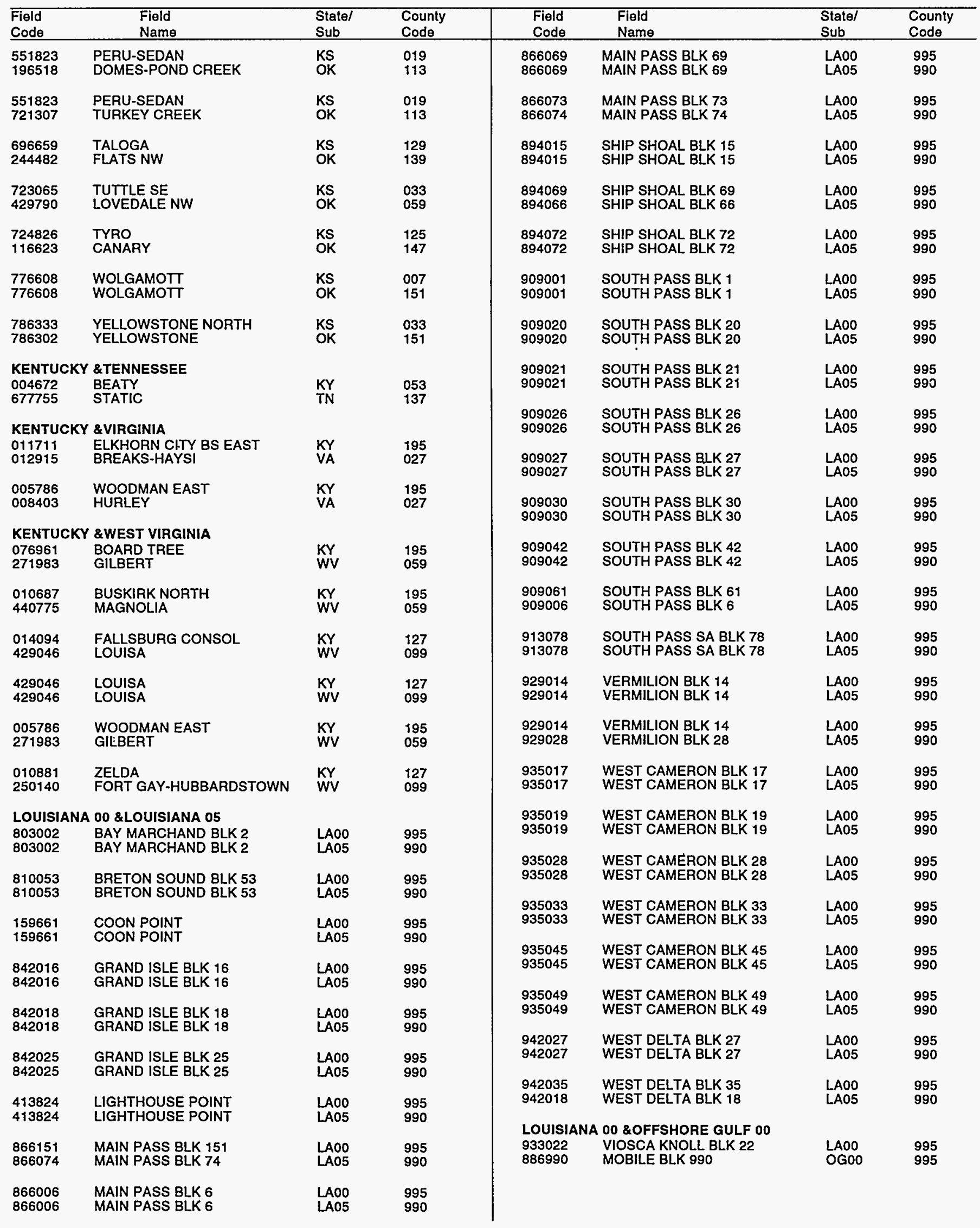


Table 6. Fields Located in Multiple Jurisdictions (Continued)

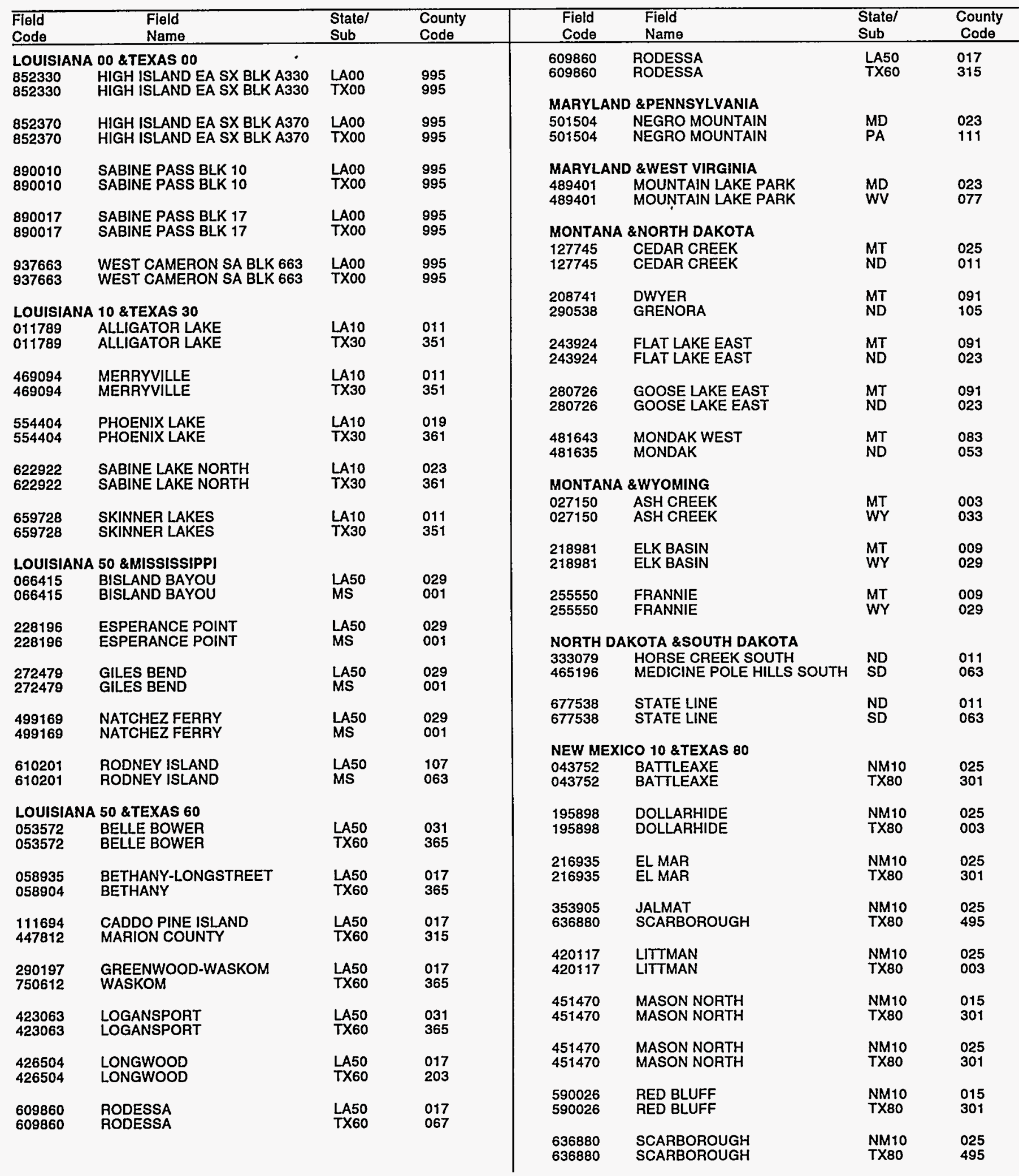


Table 6. Fields Located in Multiple Jurisdictions (Continued)

\begin{tabular}{|c|c|c|c|c|c|c|c|}
\hline $\begin{array}{l}\text { Field } \\
\text { Code } \\
\end{array}$ & $\begin{array}{l}\text { Field } \\
\text { Name }\end{array}$ & $\begin{array}{l}\text { Statel } \\
\text { Sub }\end{array}$ & $\begin{array}{l}\text { County } \\
\text { Code }\end{array}$ & $\begin{array}{l}\text { Field } \\
\text { Code } \\
\end{array}$ & $\begin{array}{l}\text { Field } \\
\text { Name } \\
\end{array}$ & $\begin{array}{l}\text { State/ } \\
\text { Sub }\end{array}$ & $\begin{array}{l}\text { County } \\
\text { Code }\end{array}$ \\
\hline $\begin{array}{l}677662 \\
677662\end{array}$ & $\begin{array}{l}\text { STATELINE } \\
\text { STATELINE }\end{array}$ & $\begin{array}{l}\text { NM10 } \\
\text { TX80 }\end{array}$ & $\begin{array}{l}025 \\
003\end{array}$ & $\begin{array}{l}284927 \\
056331\end{array}$ & $\begin{array}{l}\text { GRANDVIEW } \\
\text { BENS RUN }\end{array}$ & $\begin{array}{l}\text { OH } \\
\text { WV }\end{array}$ & $\begin{array}{l}167 \\
095\end{array}$ \\
\hline $\begin{array}{l}093727 \\
093727\end{array}$ & $\begin{array}{l}\text { CO } 10 \text { \&TEXAS } 85 \\
\text { BRONCO } \\
\text { BRONCO }\end{array}$ & $\begin{array}{l}\text { NM10 } \\
\text { TX85 }\end{array}$ & $\begin{array}{l}025 \\
501\end{array}$ & $\begin{array}{l}292584 \\
292584\end{array}$ & $\begin{array}{l}\text { GROUNDHOG CREEK } \\
\text { GROUNDHOG CREEK }\end{array}$ & $\begin{array}{l}\text { OH } \\
\text { WV }\end{array}$ & $\begin{array}{l}105 \\
035\end{array}$ \\
\hline $\begin{array}{l}325720 \\
325720\end{array}$ & $\begin{array}{l}\text { HOBBS EAST } \\
\text { HOBBS EAST }\end{array}$ & $\begin{array}{l}\text { NM10 } \\
\text { TX85 }\end{array}$ & $\begin{array}{l}025 \\
165\end{array}$ & $\begin{array}{l}292584 \\
602752\end{array}$ & $\begin{array}{l}\text { GROUNDHOG CREEK } \\
\text { RIPLEY NORTH }\end{array}$ & $\begin{array}{l}\text { OH } \\
\text { WV }\end{array}$ & $\begin{array}{l}105 \\
035\end{array}$ \\
\hline $\begin{array}{l}420117 \\
420117\end{array}$ & $\begin{array}{l}\text { LITTMAN } \\
\text { LITTMAN }\end{array}$ & $\begin{array}{l}\text { NM10 } \\
\text { TX85 }\end{array}$ & $\begin{array}{l}025 \\
165\end{array}$ & $\begin{array}{l}315775 \\
077064\end{array}$ & $\begin{array}{l}\text { HENDERSHOT } \\
\text { BOAZ }\end{array}$ & OH & $\begin{array}{l}167 \\
107\end{array}$ \\
\hline \multicolumn{4}{|c|}{ NEW YORK \&PENNSYLVANIA } & $\begin{array}{l}407104 \\
502510\end{array}$ & $\begin{array}{l}\text { LEBANON } \\
\text { NEPTUNE }\end{array}$ & $\stackrel{\text { OH }}{\text { WV }}$ & $\begin{array}{l}105 \\
035\end{array}$ \\
\hline $\begin{array}{l}086938 \\
086938\end{array}$ & $\begin{array}{l}\text { BRADFORD } \\
\text { BRADFORD }\end{array}$ & $\begin{array}{l}\text { NY } \\
\text { PA }\end{array}$ & $\begin{array}{l}009 \\
083\end{array}$ & $\begin{array}{l}420644 \\
136565\end{array}$ & $\begin{array}{l}\text { LIVERPOOL EAST } \\
\text { CHESTER-PUGHTOWN }\end{array}$ & $\begin{array}{l}\text { OH } \\
\text { WV }\end{array}$ & $\begin{array}{l}029 \\
029\end{array}$ \\
\hline $\begin{array}{l}094130 \\
094130\end{array}$ & $\begin{array}{l}\text { BROOKFIELD } \\
\text { BROOKFIELD }\end{array}$ & $\begin{array}{l}\text { NY } \\
\text { PA }\end{array}$ & $\begin{array}{l}101 \\
117\end{array}$ & $\begin{array}{l}430875 \\
077064\end{array}$ & $\begin{array}{l}\text { LOWER NEWPORT } \\
\text { BOAZ }\end{array}$ & $\begin{array}{l}\text { OH } \\
\text { WV }\end{array}$ & $\begin{array}{l}167 \\
107\end{array}$ \\
\hline $\begin{array}{l}107176 \\
686683\end{array}$ & $\begin{array}{l}\text { BUSTI } \\
\text { SUGAR GROVE }\end{array}$ & $\begin{array}{l}\text { NY } \\
\text { PA }\end{array}$ & $\begin{array}{l}013 \\
123\end{array}$ & $\begin{array}{l}005882 \\
077064\end{array}$ & $\begin{array}{l}\text { MILE RUN } \\
\text { BOAZ }\end{array}$ & $\begin{array}{l}\text { OH } \\
\text { WV }\end{array}$ & $\begin{array}{l}167 \\
107\end{array}$ \\
\hline $\begin{array}{l}307722 \\
307691\end{array}$ & $\begin{array}{l}\text { HARRISON EAST } \\
\text { HARRISON }\end{array}$ & $\begin{array}{l}\text { NY } \\
\text { PA }\end{array}$ & $\begin{array}{l}101 \\
105\end{array}$ & $\begin{array}{l}005906 \\
077064\end{array}$ & $\begin{array}{l}\text { MOORES JUNCTION } \\
\text { BOAZ }\end{array}$ & $\begin{array}{l}\text { OH } \\
\text { WV }\end{array}$ & $\begin{array}{l}167 \\
107\end{array}$ \\
\hline $\begin{array}{l}442573 \\
759626\end{array}$ & $\begin{array}{l}\text { MALLORY } \\
\text { WEST BRANCH }\end{array}$ & $\begin{array}{l}\text { NY } \\
\text { PA }\end{array}$ & $\begin{array}{l}009 \\
083\end{array}$ & $\begin{array}{l}005945 \\
674345\end{array}$ & $\begin{array}{l}\text { NEWELL RUN } \\
\text { ST MARYS }\end{array}$ & $\underset{\text { WV }}{\text { OH }}$ & $\begin{array}{l}167 \\
073\end{array}$ \\
\hline $\begin{array}{l}599404 \\
652414\end{array}$ & $\begin{array}{l}\text { RICHBURG } \\
\text { SHINGLEHOUSE }\end{array}$ & $\begin{array}{l}\text { NY } \\
\text { PA }\end{array}$ & $\begin{array}{l}003 \\
083\end{array}$ & $\begin{array}{l}005968 \\
503688\end{array}$ & $\begin{array}{l}\text { OLIVE-MEIGS } \\
\text { NEW ENGLAND }\end{array}$ & $\begin{array}{l}\text { OH } \\
\text { WV }\end{array}$ & $\begin{array}{l}105 \\
107\end{array}$ \\
\hline $\begin{array}{l}599404 \\
652414\end{array}$ & $\begin{array}{l}\text { RICHBURG } \\
\text { SHINGLEHOUSE }\end{array}$ & $\begin{array}{l}\text { NY } \\
\text { PA }\end{array}$ & $\begin{array}{l}003 \\
105\end{array}$ & $\begin{array}{l}005980 \\
276711\end{array}$ & $\begin{array}{l}\text { PATTON RUN } \\
\text { GLENN RUN }\end{array}$ & $\begin{array}{l}\text { OH } \\
\text { WV }\end{array}$ & $\begin{array}{l}013 \\
069\end{array}$ \\
\hline $\begin{array}{l}647971 \\
652414\end{array}$ & $\begin{array}{l}\text { SHARON } \\
\text { SHINGLEHOUSE }\end{array}$ & $\begin{array}{l}\text { NY } \\
\text { PA }\end{array}$ & $\begin{array}{l}003 \\
105\end{array}$ & $\begin{array}{l}006036 \\
340105\end{array}$ & $\begin{array}{l}\text { PROCTORVILLE } \\
\text { HUNTINGTON }\end{array}$ & $\underset{\text { WH }}{\text { OH }}$ & $\begin{array}{l}087 \\
011\end{array}$ \\
\hline $\begin{array}{l}675523 \\
675523\end{array}$ & $\begin{array}{l}\text { STAGECOACH } \\
\text { STAGECOACH }\end{array}$ & $\begin{array}{l}\text { NY } \\
\text { PA }\end{array}$ & $\begin{array}{l}107 \\
015\end{array}$ & $\begin{array}{l}006036 \\
340105\end{array}$ & $\begin{array}{l}\text { PROCTORVILLE } \\
\text { HUNTINGTON }\end{array}$ & $\underset{\text { WV }}{\mathrm{OH}}$ & $\begin{array}{l}087 \\
099\end{array}$ \\
\hline $\begin{array}{l}677538 \\
677538\end{array}$ & $\begin{array}{l}\text { STATE LINE } \\
\text { STATE LINE }\end{array}$ & $\begin{array}{l}\text { NY } \\
\text { PA }\end{array}$ & $\begin{array}{l}003 \\
105\end{array}$ & $\begin{array}{l}575633 \\
503688\end{array}$ & $\begin{array}{l}\text { PUTNAM } \\
\text { NEW ENGLAND }\end{array}$ & OH & $\begin{array}{l}009 \\
107\end{array}$ \\
\hline $\begin{array}{l}\text { OHIO \& } \\
107011 \\
157832\end{array}$ & $\begin{array}{l}\text { BUSYLVANIA } \\
\text { CONNEAUT }\end{array}$ & $\begin{array}{l}\mathrm{OH} \\
\mathrm{PA}\end{array}$ & $\begin{array}{l}007 \\
039\end{array}$ & $\begin{array}{l}006417 \\
056331\end{array}$ & $\begin{array}{l}\text { SHEETS RUN } \\
\text { BENS RUN }\end{array}$ & $\begin{array}{l}\text { OH } \\
\text { WV }\end{array}$ & $\begin{array}{l}167 \\
095\end{array}$ \\
\hline $\begin{array}{l}005001 \\
340105\end{array}$ & $\begin{array}{l}\text { ST VIRGINIA } \\
\text { ATHALIA } \\
\text { HUNTINGTON }\end{array}$ & $\begin{array}{l}\text { OH } \\
\text { WV }\end{array}$ & $\begin{array}{l}087 \\
011\end{array}$ & $\begin{array}{l}006417 \\
056331\end{array}$ & $\begin{array}{l}\text { SHEETS RUN } \\
\text { BENS RUN }\end{array}$ & $\underset{W V}{\mathrm{OH}}$ & $\begin{array}{l}167 \\
073\end{array}$ \\
\hline $\begin{array}{l}005001 \\
340105\end{array}$ & $\begin{array}{l}\text { ATHALIA } \\
\text { HUNTINGTON }\end{array}$ & $\begin{array}{l}\text { OH } \\
\text { WV }\end{array}$ & $\begin{array}{l}087 \\
099\end{array}$ & $\begin{array}{l}658139 \\
658139 \\
691364\end{array}$ & $\begin{array}{l}\text { SISTERSVILLE } \\
\text { SISTERSVILLE } \\
\text { SUTTON }\end{array}$ & $\begin{array}{l}\text { OH } \\
\text { WV } \\
\text { OH }\end{array}$ & $\begin{array}{l}111 \\
095 \\
105\end{array}$ \\
\hline $\begin{array}{l}039505 \\
540174\end{array}$ & $\begin{array}{l}\text { BARLOW } \\
\text { PARKERSBURG }\end{array}$ & $\begin{array}{l}\mathrm{OH} \\
\mathrm{WV}\end{array}$ & $\begin{array}{l}167 \\
107\end{array}$ & OKLAH & $\begin{array}{l}\text { NEW HAVEN } \\
\text { A \&TEXAS } 90\end{array}$ & WV & 053 \\
\hline $\begin{array}{l}054409 \\
674345\end{array}$ & $\begin{array}{l}\text { BELLS RUN } \\
\text { ST MARYS }\end{array}$ & OH & $\begin{array}{l}167 \\
073\end{array}$ & $\begin{array}{l}302824 \\
302824\end{array}$ & $\begin{array}{l}\text { HANDY SE } \\
\text { HANDY SE }\end{array}$ & $\begin{array}{l}\text { OK } \\
\text { TX90 }\end{array}$ & $\begin{array}{l}095 \\
181\end{array}$ \\
\hline $\begin{array}{l}082830 \\
674345\end{array}$ & $\begin{array}{l}\text { BOSWORTH } \\
\text { ST MARYS }\end{array}$ & $\begin{array}{l}\text { OH } \\
\text { WV }\end{array}$ & $\begin{array}{l}167 \\
073\end{array}$ & $\begin{array}{l}641879 \\
482395\end{array}$ & $\begin{array}{l}\text { SEAY } \\
\text { MONTAGUE COUNTY REGULAR }\end{array}$ & $\begin{array}{l}\text { OK } \\
\text { TX90 }\end{array}$ & $\begin{array}{l}067 \\
337\end{array}$ \\
\hline $\begin{array}{l}005056 \\
686435\end{array}$ & $\begin{array}{l}\text { BRILLIANT } \\
\text { SUCKERROD }\end{array}$ & $\underset{W V}{\mathrm{OH}}$ & $\begin{array}{l}081 \\
009\end{array}$ & $\begin{array}{l}658325 \\
658325\end{array}$ & $\begin{array}{l}\text { SIVELLS BEND } \\
\text { SIVELLS BEND }\end{array}$ & $\begin{array}{l}\text { OK } \\
\text { TX90 }\end{array}$ & $\begin{array}{l}085 \\
097\end{array}$ \\
\hline $\begin{array}{l}104872 \\
276711\end{array}$ & $\begin{array}{l}\text { BURLINGTON } \\
\text { GLENN RUN }\end{array}$ & $\stackrel{\text { OH }}{W V}$ & $\begin{array}{l}013 \\
069\end{array}$ & $\begin{array}{l}\text { OKLAH } \\
036467 \\
011479\end{array}$ & $\begin{array}{l}\text { A \&TEXAS } 95 \\
\text { BALKO SOUTH } \\
\text { ALLEN-PARKER }\end{array}$ & $\begin{array}{l}\text { OK } \\
\text { TX95 }\end{array}$ & $\begin{array}{l}007 \\
357\end{array}$ \\
\hline $\begin{array}{l}005096 \\
562837\end{array}$ & $\begin{array}{l}\text { CHESIRE } \\
\text { POINT PLEASANT }\end{array}$ & $\begin{array}{l}\mathrm{OH} \\
\mathrm{WV}\end{array}$ & $\begin{array}{l}053 \\
053\end{array}$ & $\begin{array}{l}116065 \\
551389\end{array}$ & $\begin{array}{l}\text { CAMRICK DISTRICT } \\
\text { PERRYTON NORTH }\end{array}$ & $\begin{array}{l}\text { OK } \\
\text { TX95 }\end{array}$ & $\begin{array}{l}007 \\
357\end{array}$ \\
\hline $\begin{array}{l}284927 \\
056331\end{array}$ & $\begin{array}{l}\text { GRANDVIEW } \\
\text { BENS RUN }\end{array}$ & $\underset{\text { WH }}{\mathrm{OH}}$ & $\begin{array}{l}167 \\
073\end{array}$ & & & & \\
\hline
\end{tabular}


Table 6. Fields Located in Multiple Jurisdictions (Continued)

\begin{tabular}{|c|c|c|c|c|c|c|c|}
\hline $\begin{array}{l}\text { Fleld } \\
\text { Code } \\
\end{array}$ & $\begin{array}{l}\text { Field } \\
\text { Name } \\
\end{array}$ & $\begin{array}{l}\text { Statel } \\
\text { Sub }\end{array}$ & $\begin{array}{l}\text { County } \\
\text { Code }\end{array}$ & $\begin{array}{l}\text { Field } \\
\text { Code } \\
\end{array}$ & $\begin{array}{l}\text { Field } \\
\text { Name } \\
\end{array}$ & $\begin{array}{l}\text { State/ } \\
\text { Sub }\end{array}$ & $\begin{array}{l}\text { County } \\
\text { Code }\end{array}$ \\
\hline $\begin{array}{l}295545 \\
000333\end{array}$ & $\begin{array}{l}\text { GUYMON-HUGOTON GAS AREA } \\
\text { TEXAS HUGOTON }\end{array}$ & $\underset{T \times 95}{O K}$ & $\begin{array}{l}139 \\
195\end{array}$ & $\begin{array}{l}503770 \\
054502\end{array}$ & $\begin{array}{l}\text { NEW FREEPORT } \\
\text { BELLTON }\end{array}$ & $\stackrel{P A}{W V}$ & $\begin{array}{l}059 \\
051\end{array}$ \\
\hline $\begin{array}{l}321565 \\
321565\end{array}$ & $\begin{array}{l}\text { HIGGINS SOUTH } \\
\text { HIGGINS SOUTH }\end{array}$ & $\begin{array}{l}\text { OK } \\
\text { TX95 }\end{array}$ & $\begin{array}{l}045 \\
295\end{array}$ & $\begin{array}{l}503770 \\
607597\end{array}$ & $\begin{array}{l}\text { NEW FREEPORT } \\
\text { ROCK CAMP }\end{array}$ & PA & $\begin{array}{l}059 \\
103\end{array}$ \\
\hline $\begin{array}{l}321565 \\
321565\end{array}$ & $\begin{array}{l}\text { HIGGINS SOUTH } \\
\text { HIGGINS SOUTH }\end{array}$ & $\underset{T \times 95}{\text { OK }}$ & $\begin{array}{l}045 \\
211\end{array}$ & $\begin{array}{l}007450 \\
124404\end{array}$ & $\begin{array}{l}\text { POINT MARION } \\
\text { CASSVILLE-BOWLBY }\end{array}$ & PA & $\begin{array}{l}059 \\
061\end{array}$ \\
\hline $\begin{array}{l}455562 \\
475752\end{array}$ & $\begin{array}{l}\text { MAYFIELD WEST } \\
\text { MILLS RANCH }\end{array}$ & $\begin{array}{l}\text { OK } \\
\text { TX95 }\end{array}$ & $\begin{array}{l}009 \\
483\end{array}$ & $\begin{array}{l}662890 \\
662890\end{array}$ & $\begin{array}{l}\text { SMITHS FERRY } \\
\text { SMITHS FERRY }\end{array}$ & $\begin{array}{l}\text { PA } \\
\text { WV }\end{array}$ & $\begin{array}{l}007 \\
029\end{array}$ \\
\hline $\begin{array}{l}480070 \\
247567\end{array}$ & $\begin{array}{l}\text { MOCANE-LAVERNE GAS AREA } \\
\text { FOLLETT }\end{array}$ & $\begin{array}{l}\text { OK } \\
\text { TX95 }\end{array}$ & $\begin{array}{l}007 \\
295\end{array}$ & $\begin{array}{l}721514 \\
721803\end{array}$ & $\begin{array}{l}\text { TURKEY FOOT } \\
\text { TURKEYFOOT }\end{array}$ & $\stackrel{P A}{W V}$ & $\begin{array}{l}125 \\
029\end{array}$ \\
\hline $\begin{array}{l}\text { PENNSY } \\
007281 \\
445595\end{array}$ & $\begin{array}{l}\text { ANIA \&WEST VIRGINIA } \\
\text { ASHTREE } \\
\text { MAPLE-WADESTOWN }\end{array}$ & PA & $\begin{array}{l}059 \\
061\end{array}$ & $\begin{array}{l}765353 \\
124404 \\
\text { TEXAS O }\end{array}$ & $\begin{array}{l}\text { WHITELY CREEK } \\
\text { CASSVILLE-BOWLBY } \\
\text { STEXAS } 05\end{array}$ & $\begin{array}{l}P A \\
W V\end{array}$ & $\begin{array}{l}059 \\
061\end{array}$ \\
\hline $\begin{array}{l}121862 \\
267324\end{array}$ & $\begin{array}{l}\text { CARSON } \\
\text { GAS VALLEY }\end{array}$ & $\stackrel{P A}{W V}$ & $\begin{array}{l}007 \\
029\end{array}$ & $\begin{array}{l}805437 \\
805438\end{array}$ & $\begin{array}{l}\text { BRAZOS BLK } 437 \\
\text { BRAZOS BLK } 438\end{array}$ & $\begin{array}{l}\text { TX00 } \\
\text { TX05 }\end{array}$ & $\begin{array}{l}995 \\
990\end{array}$ \\
\hline $\begin{array}{l}241424 \\
441705\end{array}$ & $\begin{array}{l}\text { FINLEY WEST } \\
\text { MAJORSVILLE }\end{array}$ & $\stackrel{P A}{W V}$ & $\begin{array}{l}059 \\
051\end{array}$ & $\begin{array}{l}805479 \\
805479\end{array}$ & $\begin{array}{l}\text { BRAZOS BLK } 479 \\
\text { BRAZOS BLK } 479\end{array}$ & $\begin{array}{l}\text { TX00 } \\
\text { TX05 }\end{array}$ & $\begin{array}{l}995 \\
990\end{array}$ \\
\hline $\begin{array}{l}007381 \\
445595\end{array}$ & $\begin{array}{l}\text { HOOVERS RUN } \\
\text { MAPLE-WADESTOWN }\end{array}$ & PA & $\begin{array}{l}059 \\
061\end{array}$ & \multicolumn{2}{|c|}{$\begin{array}{ll}\text { UTAH \&WYOMING } \\
002380 & \text { ANSCHUTZ RANCH EAST } \\
002380 & \text { ANSCHUTZ RANCH EAST }\end{array}$} & $\begin{array}{l}\text { UT } \\
\text { WY }\end{array}$ & $\begin{array}{l}043 \\
041\end{array}$ \\
\hline $\begin{array}{l}374107 \\
721803\end{array}$ & $\begin{array}{l}\text { KENDALL } \\
\text { TURKEYFOOT }\end{array}$ & $\begin{array}{l}\text { PA } \\
\text { WV }\end{array}$ & $\begin{array}{l}007 \\
029\end{array}$ & \multicolumn{2}{|c|}{ VIRGINIA \&WEST VIRGINIA } & 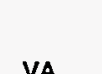 & 195 \\
\hline $\begin{array}{l}458484 \\
004358\end{array}$ & $\begin{array}{l}\text { MCCRACKEN } \\
\text { CAMERON-KAUSOOTH }\end{array}$ & $\begin{array}{l}\text { PA } \\
\text { WV }\end{array}$ & $\begin{array}{l}059 \\
051\end{array}$ & 061408 & BIG CREEK & $w V$ & 047 \\
\hline $\begin{array}{l}458484 \\
608868\end{array}$ & $\begin{array}{l}\text { MCCRACKEN } \\
\text { ROCKPORT }\end{array}$ & $\begin{array}{l}\text { PA } \\
\text { WV }\end{array}$ & $\begin{array}{l}059 \\
051\end{array}$ & $\begin{array}{l}277145 \\
004501 \\
\\
008403 \\
271983\end{array}$ & $\begin{array}{l}\text { GLICK } \\
\text { ROCKRIDGE } \\
\text { HURLEY } \\
\text { GILBERT }\end{array}$ & $\begin{array}{l}\text { VA } \\
W V \\
\text { VA } \\
\text { WV }\end{array}$ & $\begin{array}{l}027 \\
047 \\
\\
027 \\
059\end{array}$ \\
\hline
\end{tabular}


Figure 4. Subdivisions of Alaska and U.S. Geological Survey Quadrangles

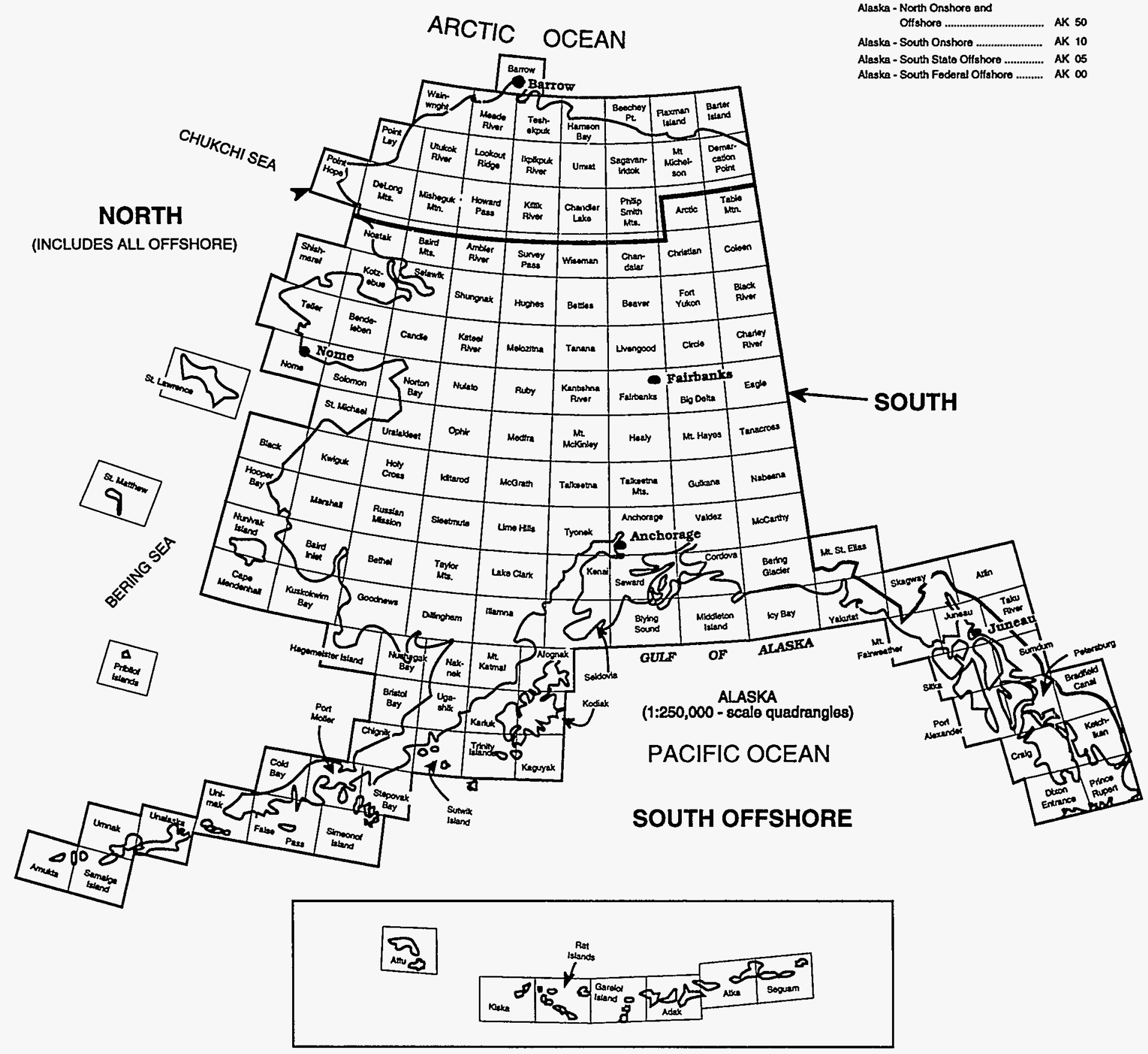

Source: After U.S. Geological Survey 
Figure 5. Subdivisions of California

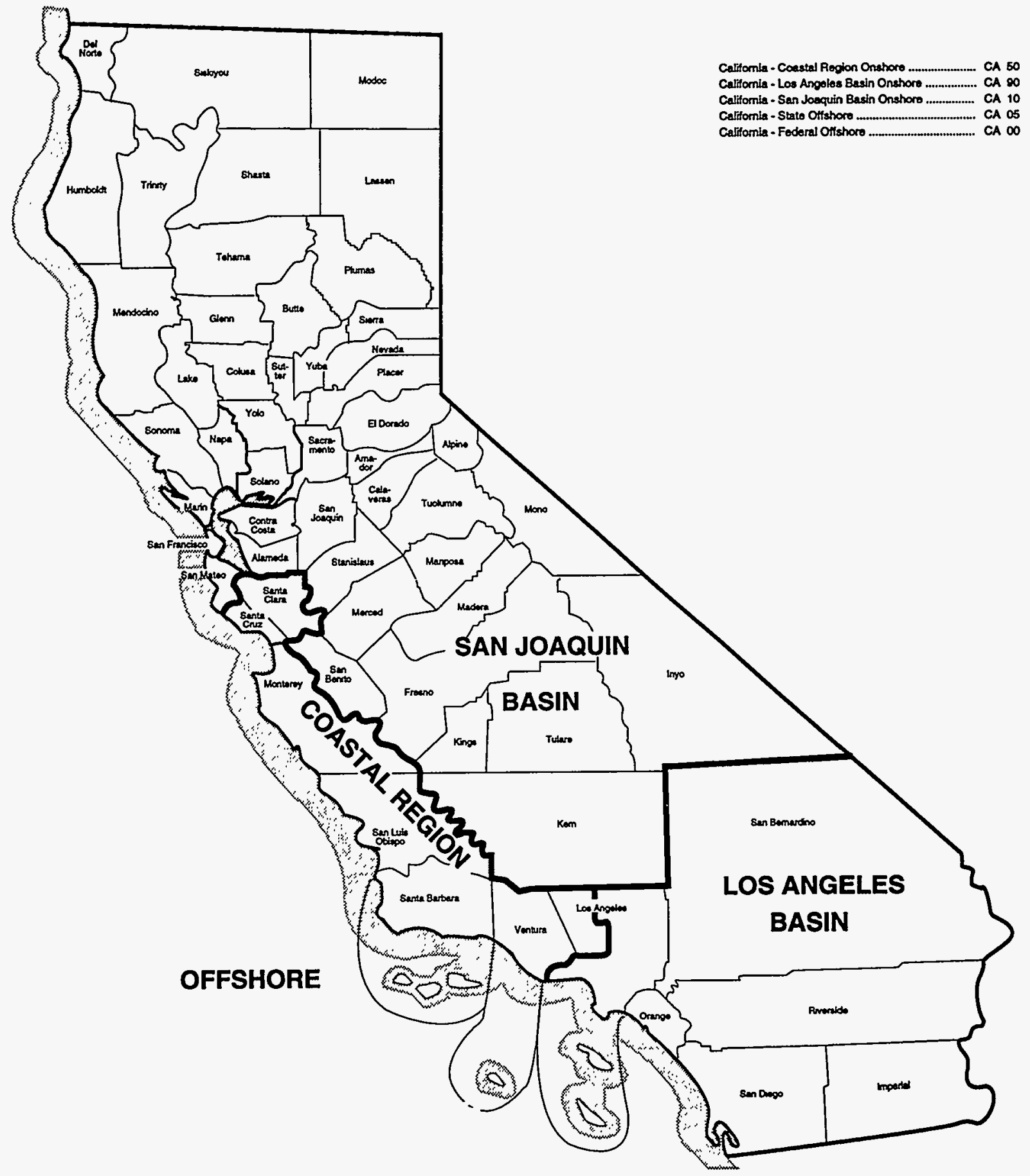

Source: Energy Information Administration, Office of Oil and Gas 


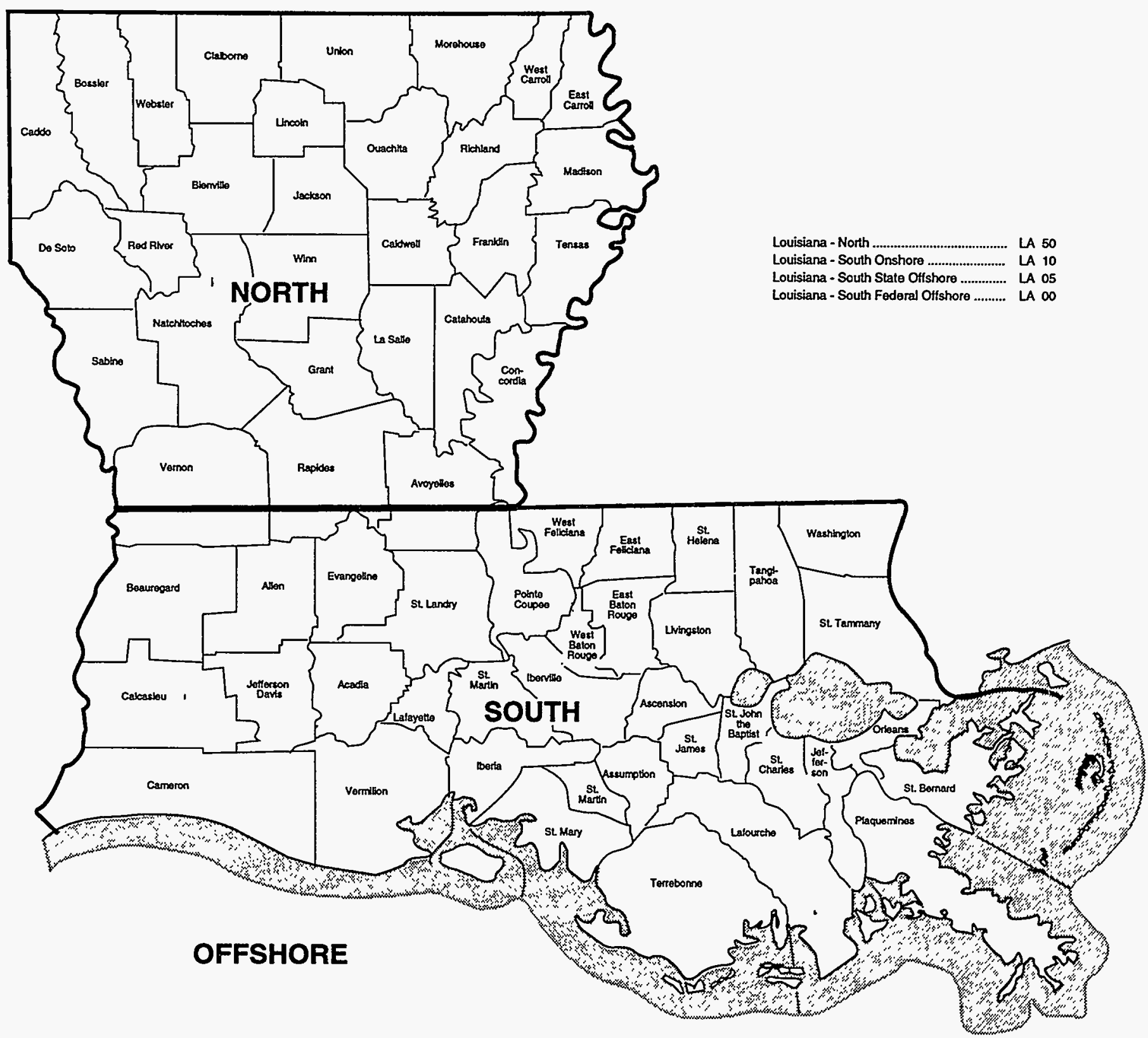

Source: Energy Information Administration, Office of Oil and Gas 
Figure 7. Subdivisions of New Mexico

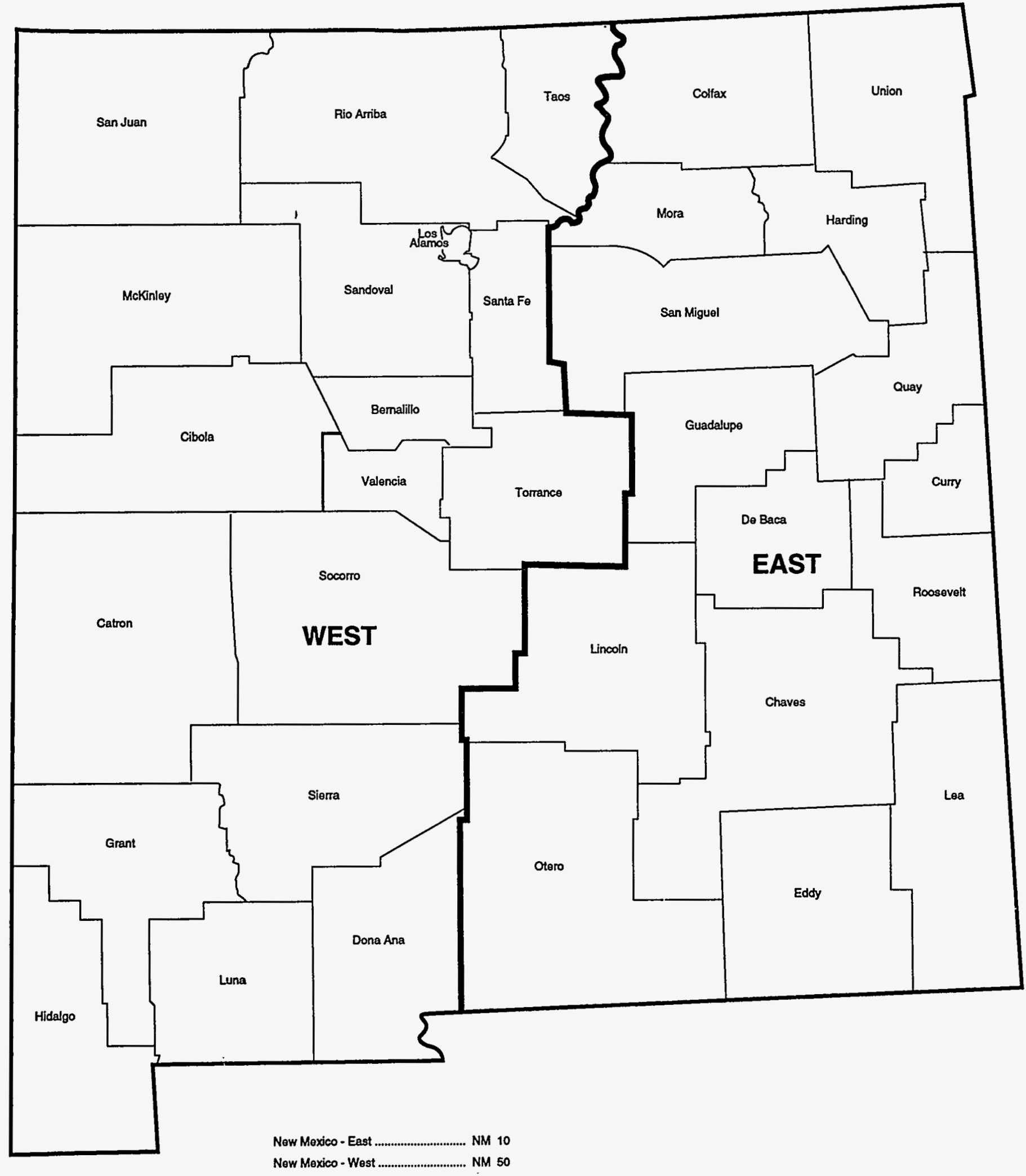

Source: Energy Information Administration, Office of Oil and Gas 
Figure 8. Subdivisions of Texas

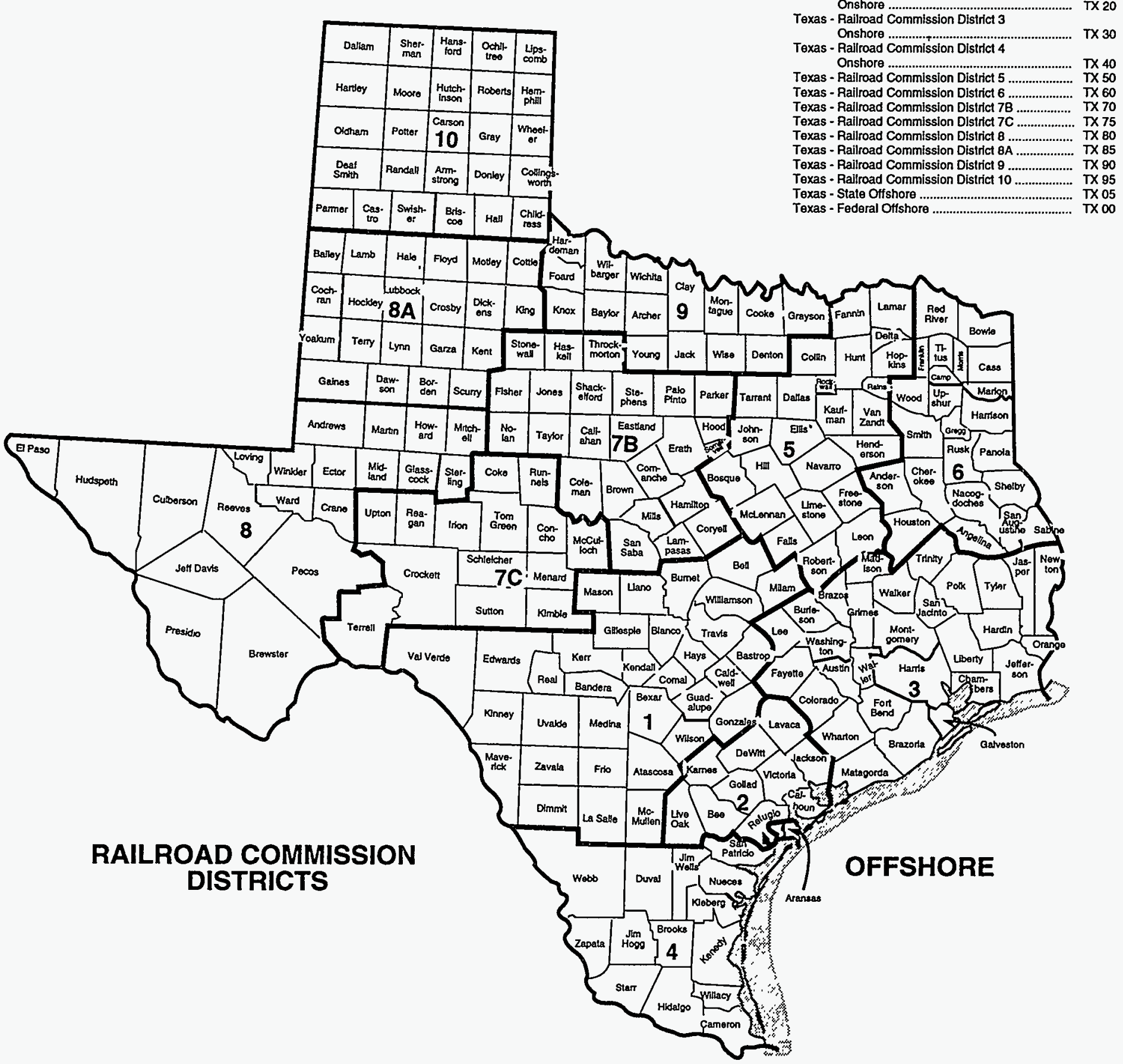

Source: Energy Information Administration, Office of Oil and Gas 
Figure 9. Western Planning Area, Gulf of Mexico Outer Continental Shelf Region

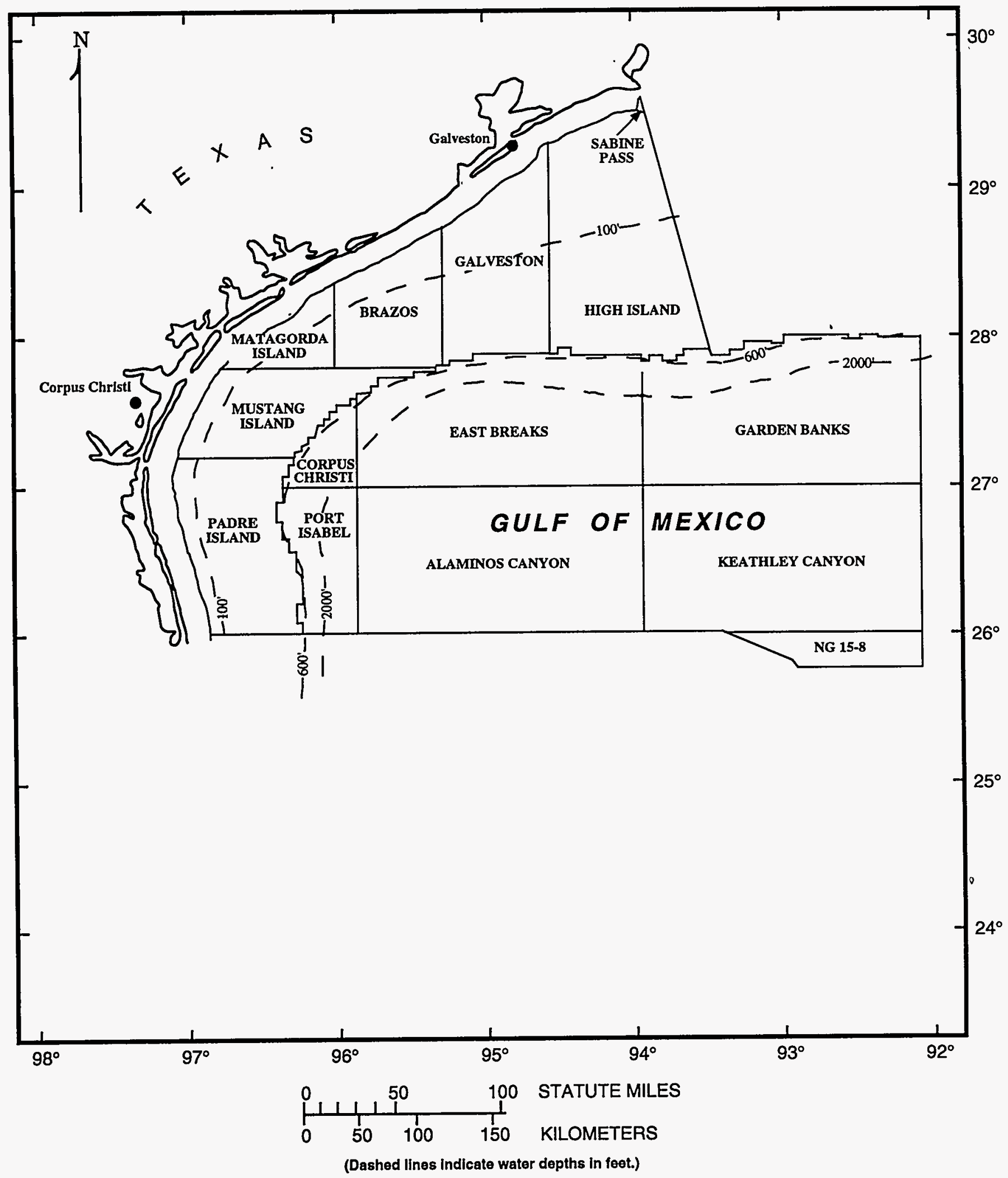

Source: After Minerals Management Service, U.S. Department of the Interior 


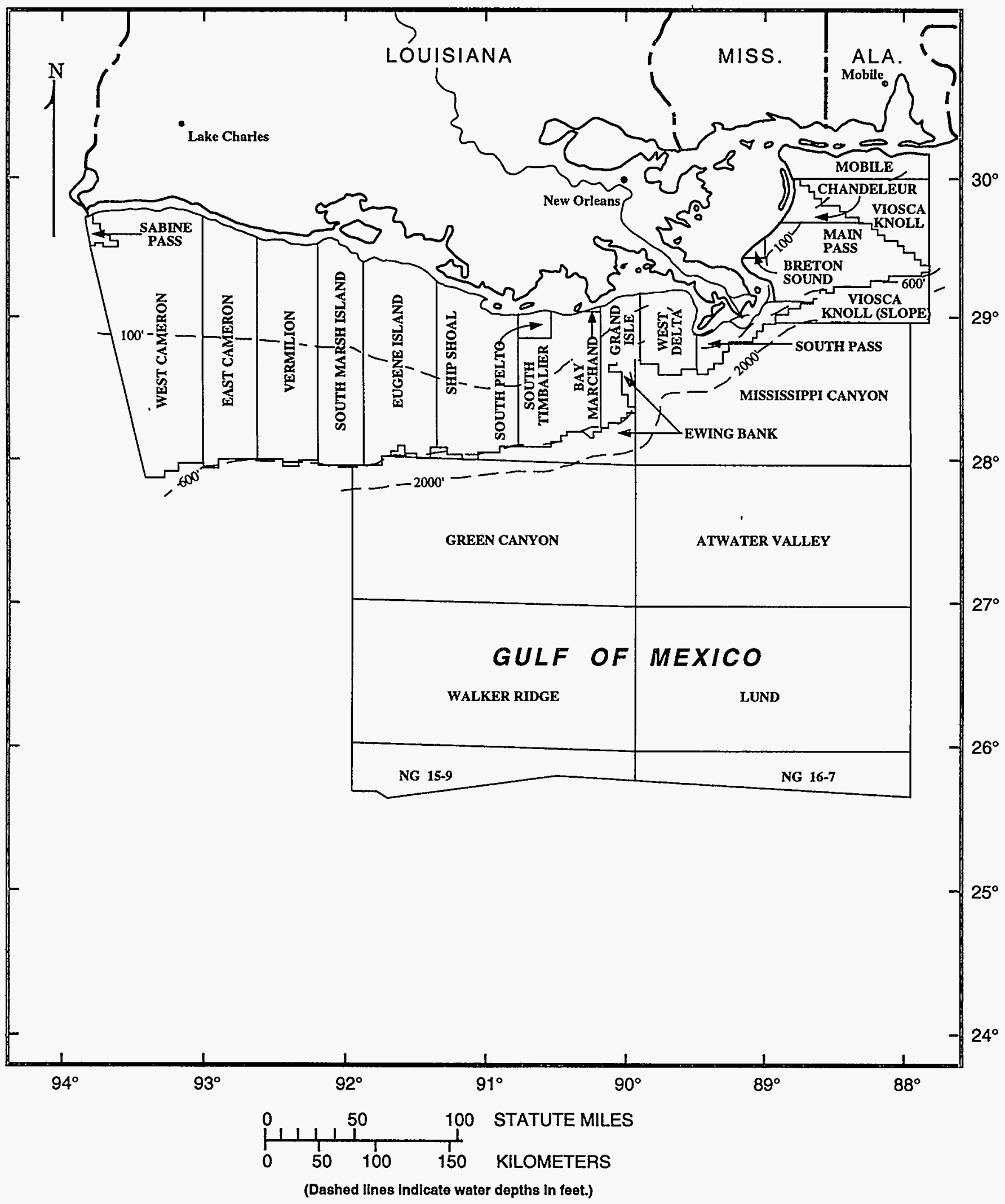

Source: After Minerals Management Service, U.S. Department of the Interior 
Figure 11. Eastern Planning Area, Gulf of Mexico Outer Continental Shelf Region

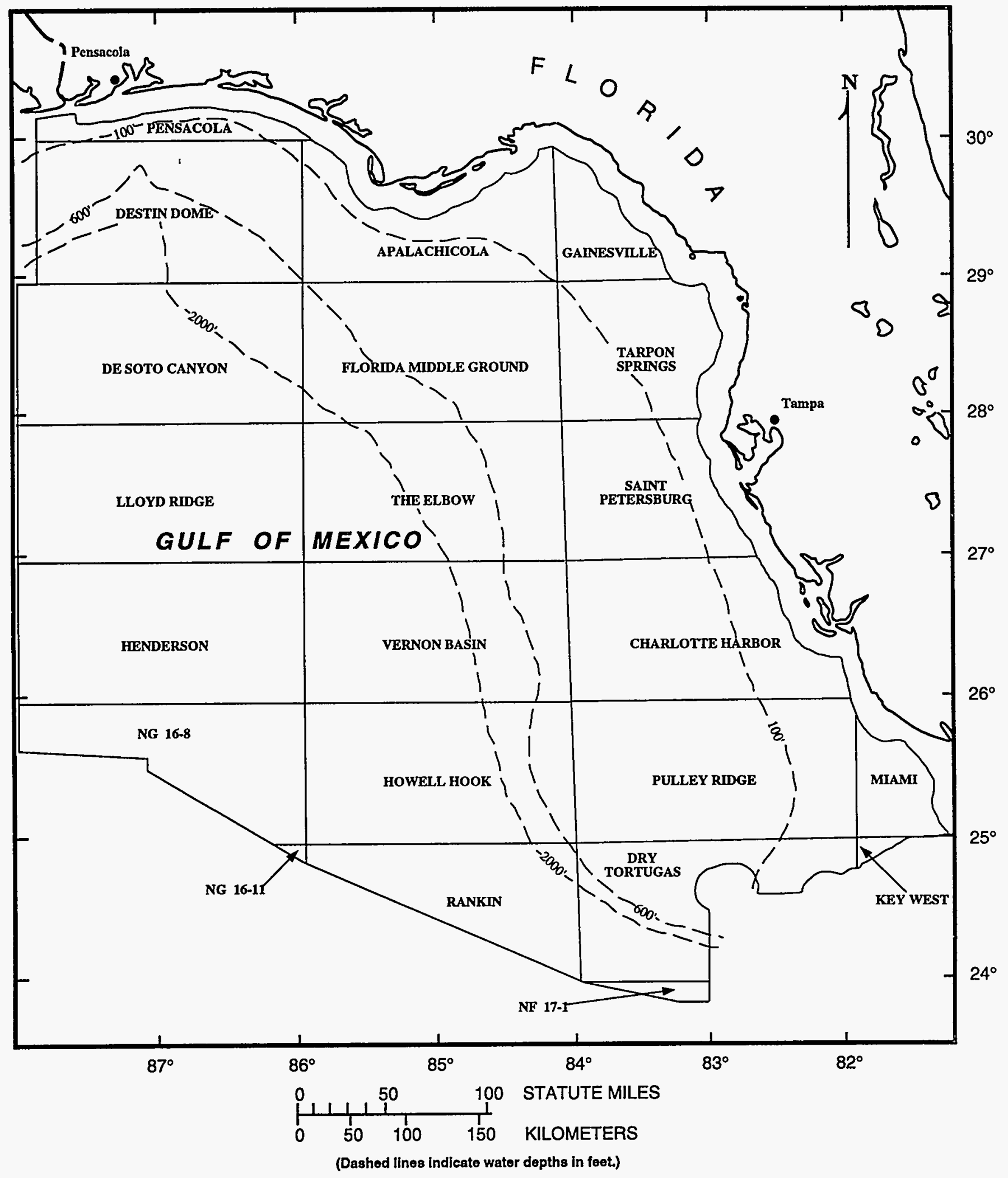

Source: After Minerals Management Service, U.S. Department of the Interior 


\section{Glossary}

This Glossary defines many of the technical terms used in this report.

Crude Oil: A mixture of hydrocarbons that exist in the liquid phase in natural underground reservoirs and remain liquid at atmospheric pressure after passing through surface separating facilities. Crude oil may also include:

- Small amounts of hydrocarbons that exist in the gaseous phase in natural underground reservoirs but are liquid at atmospheric pressure after being recovered from oil well (casinghead) gas in lease separators, and that subsequently are commingled with the crude stream without being separately measured.

- Small amounts of nonhydrocarbons produced with the oil.

Field: An area consisting of a single reservoir or multiple reservoirs all grouped on or related to the same individual geological structural feature and/or stratigraphic condition. There may be two or more reservoirs in a field which are separated vertically by intervening impervious strata, or laterally by local geologic barriers, or by both. (See Reservoir.)

Field Area: A geographic area encompassing two or more pools that have a common gathering and metering system, the reserves of which are reported as a single unit. This concept applies primarily to the Appalachian region. (See Pool.)
Field Discovery Year: The calendar year in which a field was first recognized as containing economically recoverable accumulations of oil and/or gas.

Natural Gas: A mixture of hydrocarbon compounds and small quantities of various nonhydrocarbons existing in the gaseous phase or in solution with crude oil in natural underground reservoirs at reservoir conditions. The principal hydrocarbons usually contained in the mixture are methane, ethane, propane, butane, and pentane. Typical nonhydrocarbon gases which may be present in reservoir natural gas are carbon dioxide, helium, hydrogen sulfide, and nitrogen. Under reservoir conditions, natural gas and the liquefiable portions occur either in a single gaseous phase in the reservoir or in solution with crude oil and are not distinguishable at the time as separate substances.

Pool: In general, a reservoir. In certain situations a pool may consist of more than one reservoir. (See Field Area.)

Reservoir: A porous and permeable underground formation containing an individual and separate natural accumulation of producible hydrocarbons (oil and/or gas) which is confined by impermeable rock or water barriers and is characterized by a single natural pressure system.

Subdivision: A prescribed portion of a given State of other geographical region defined in this publication for statistical reporting purposes. 


\section{Oil and Gas \\ Field Code Master List}


ALABAMA

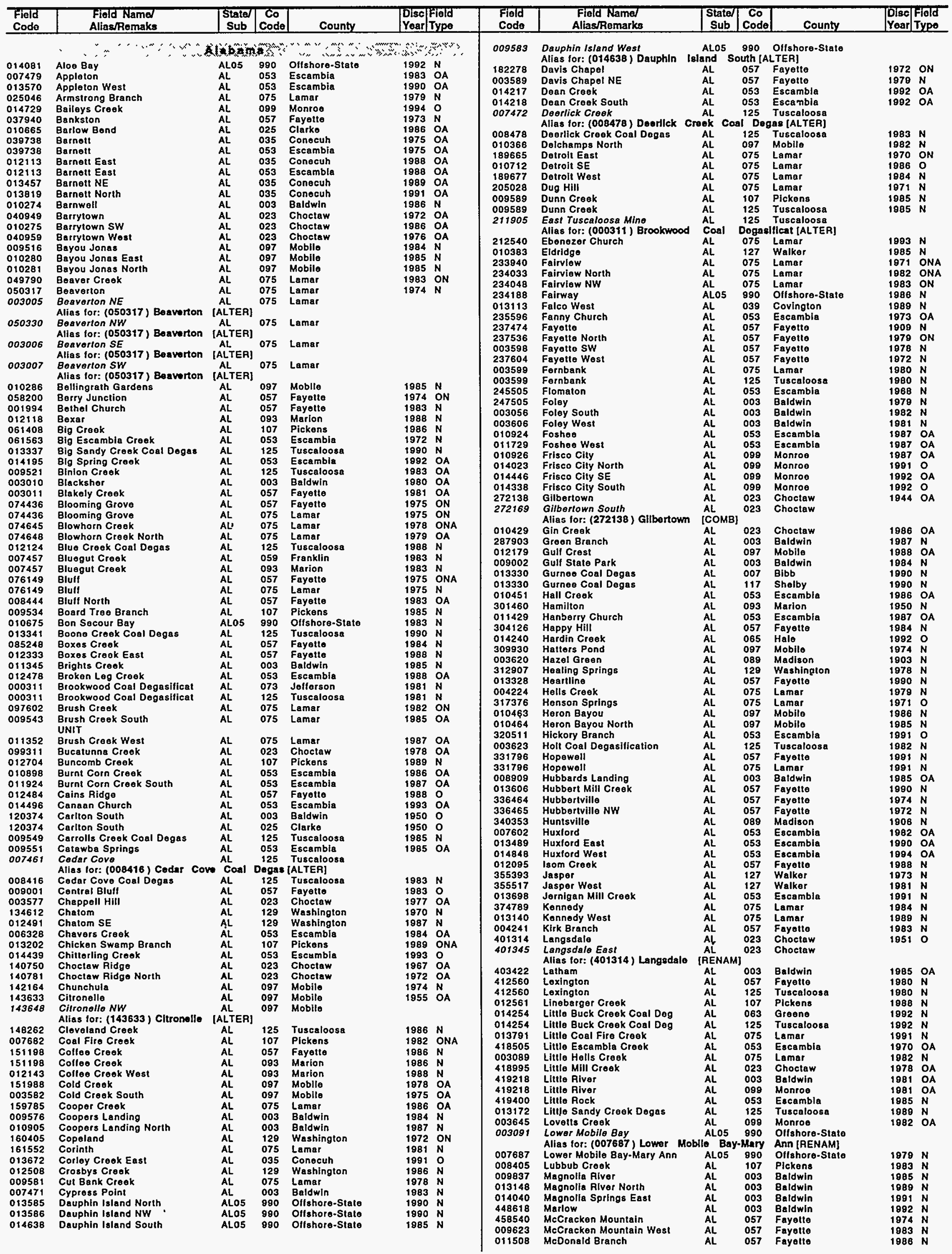


A LASKA

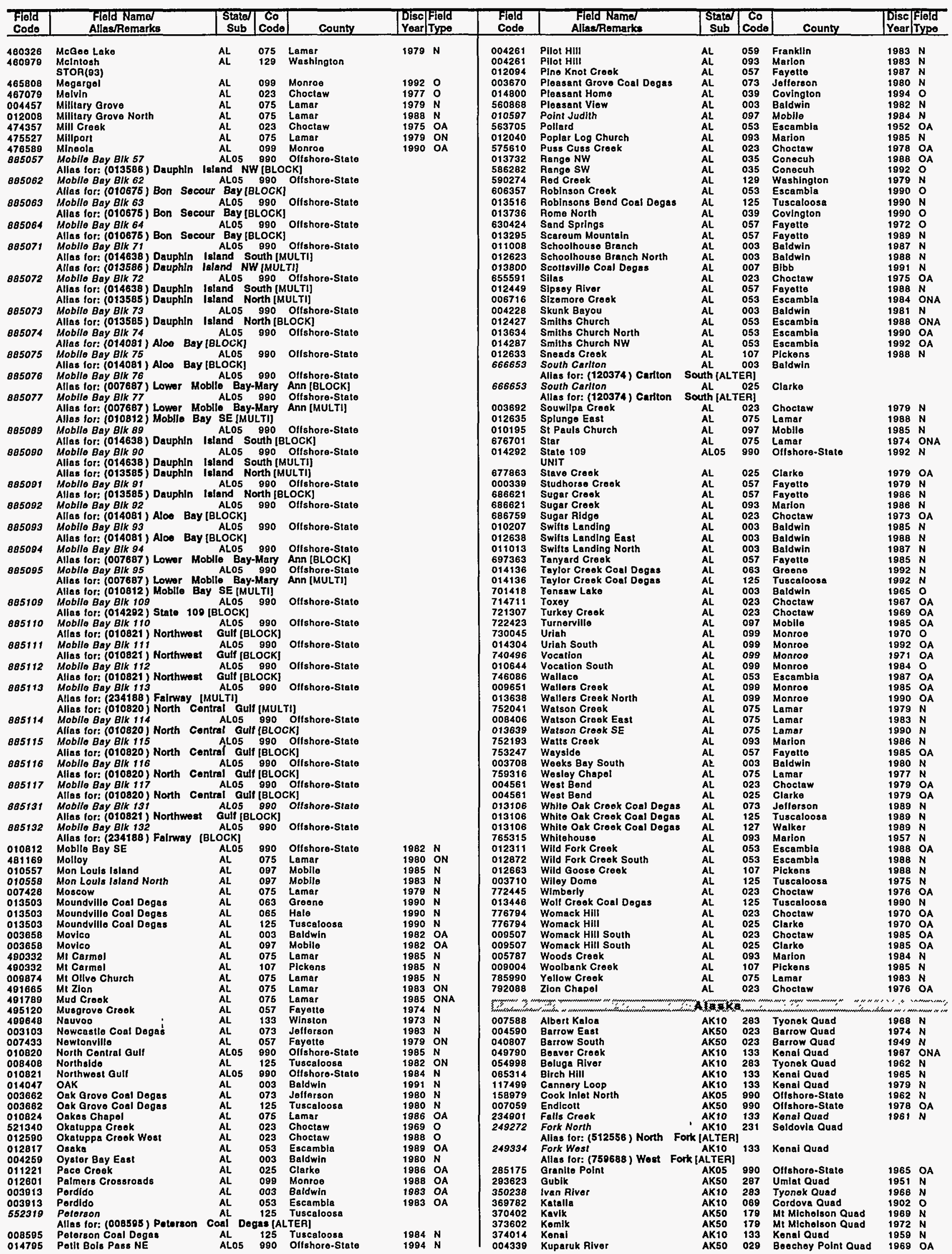


A LASKA

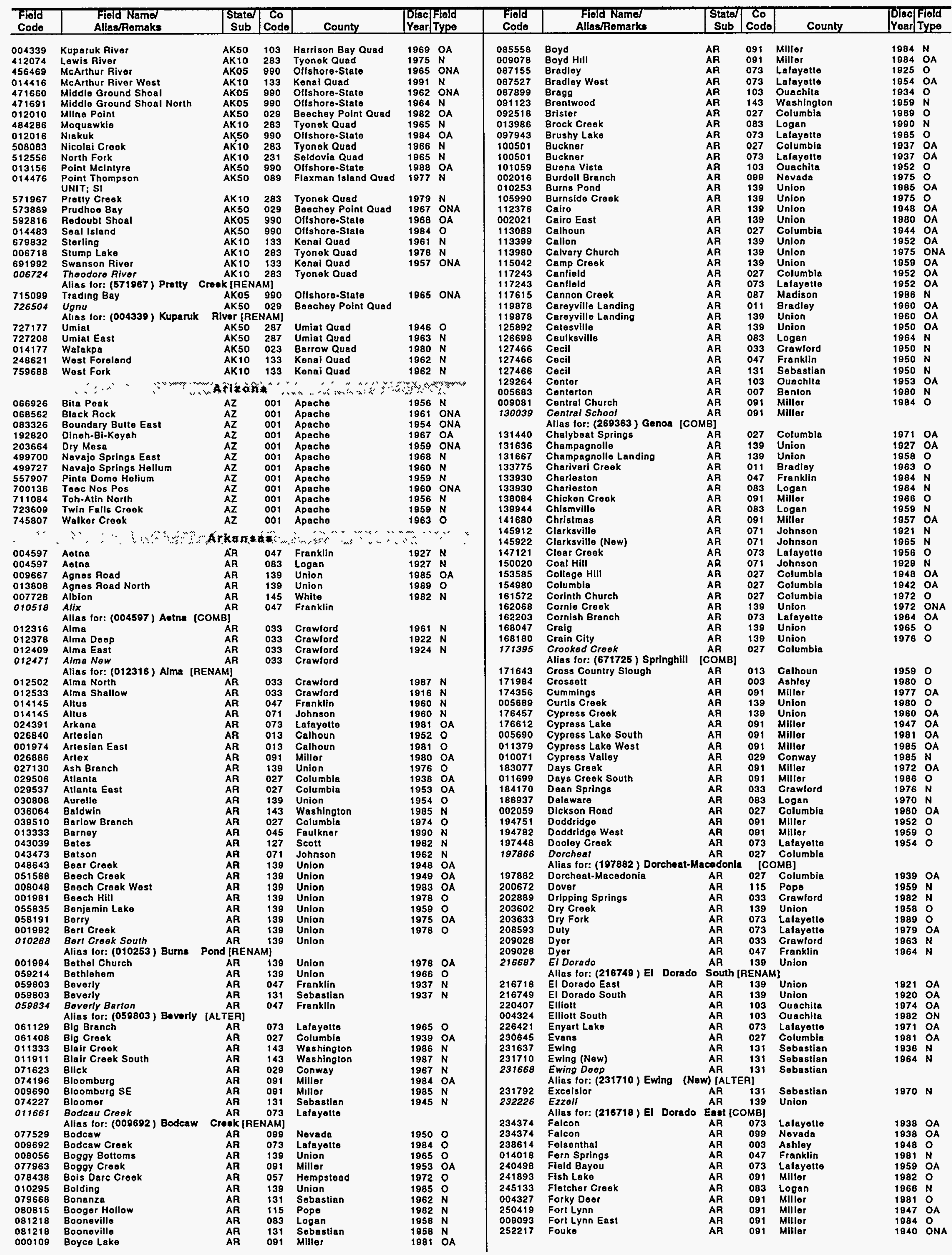


ARKANSAS

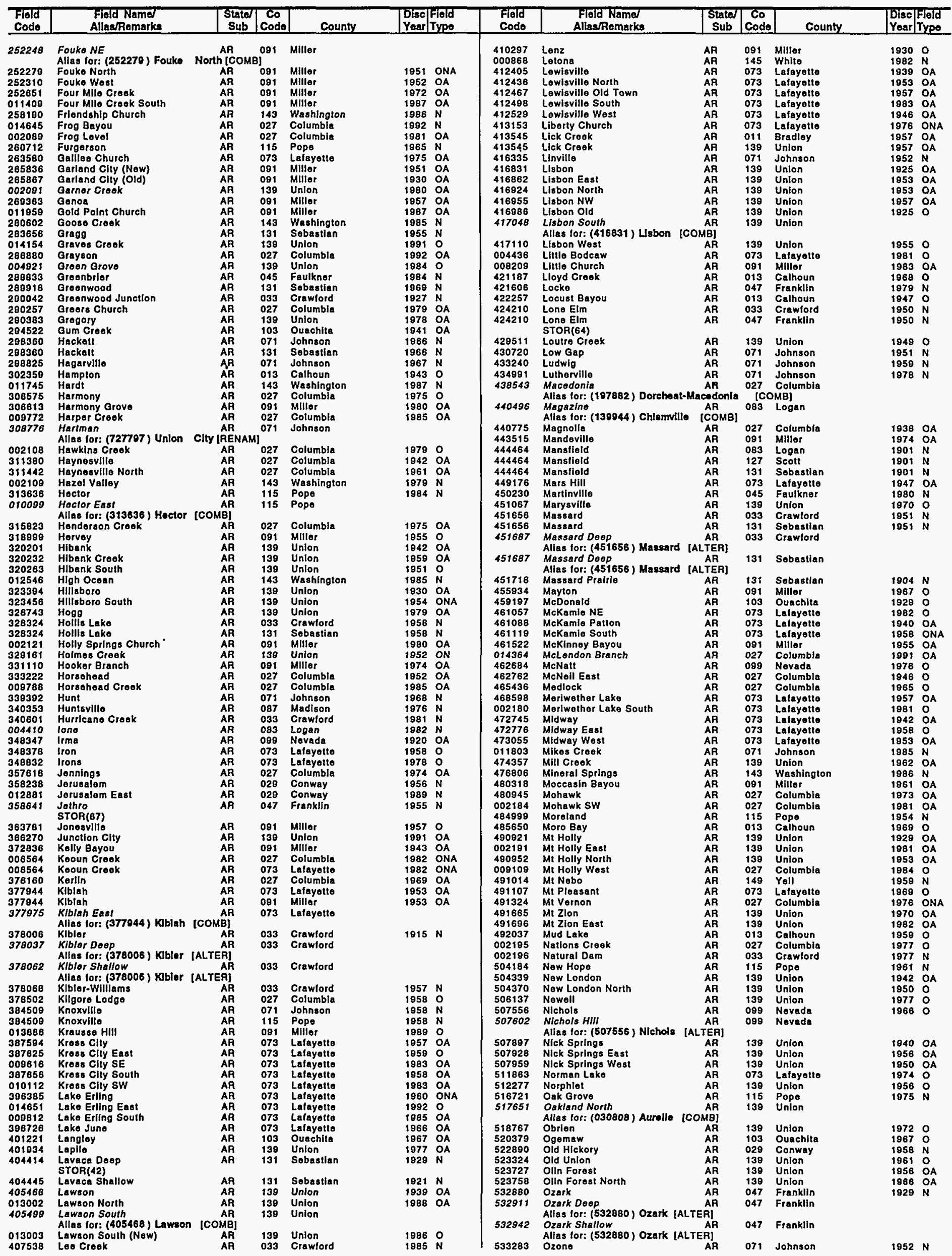




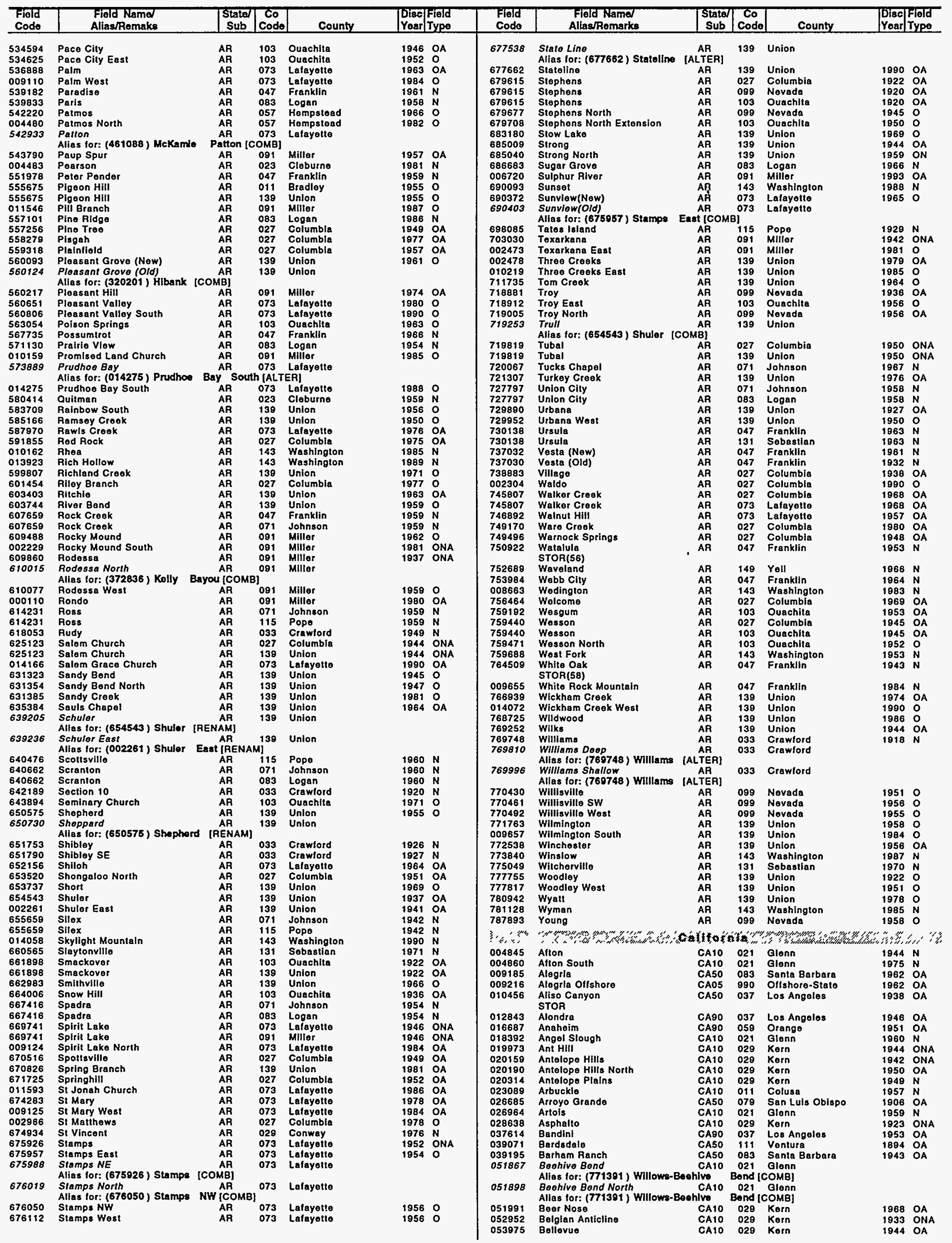


CALIFORNIA

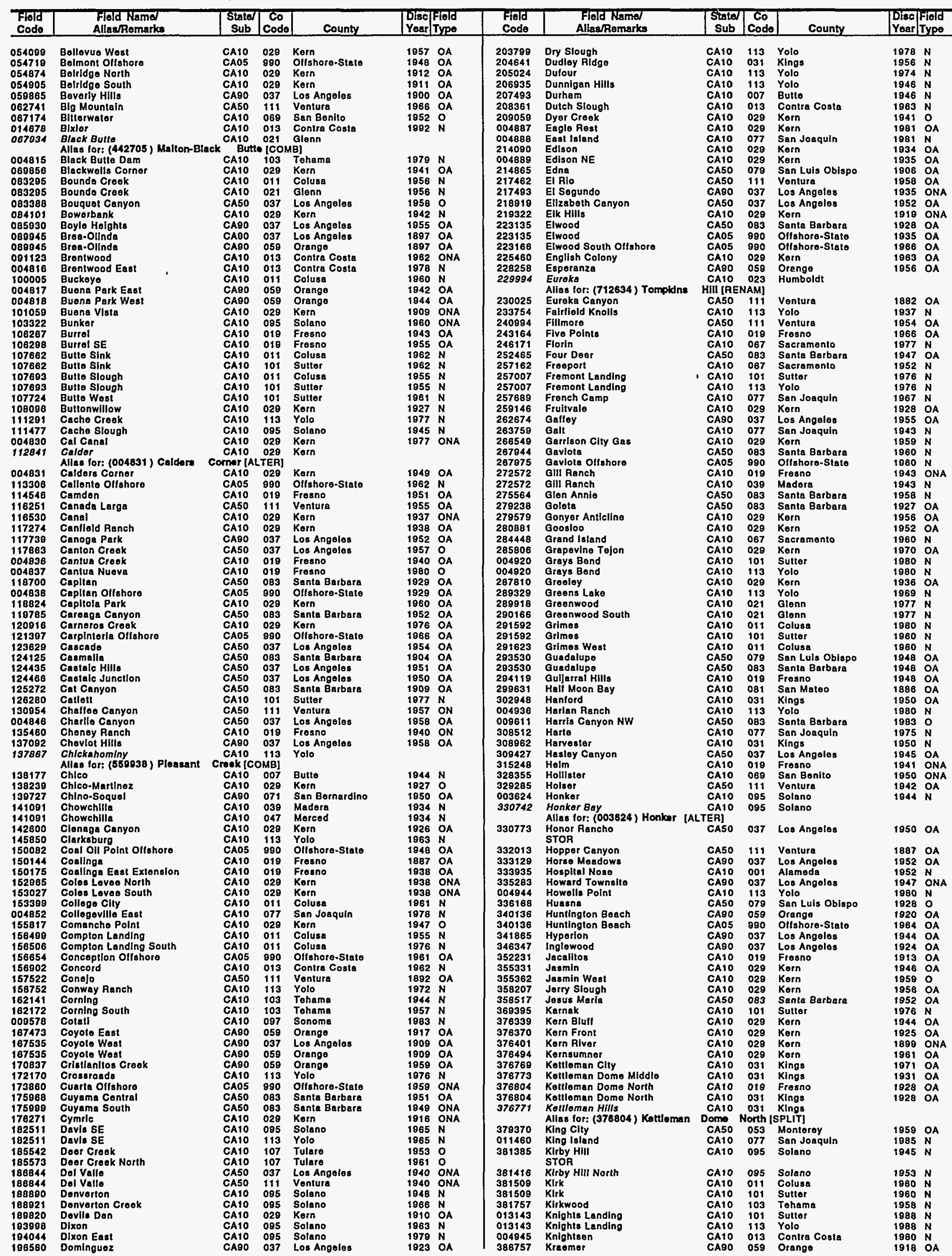


CALIFORNIA

\begin{tabular}{|c|}
\hline $\begin{array}{l}\text { Field } \\
\text { Code } \\
\end{array}$ \\
\hline $\begin{array}{l}004947 \\
004946 \\
387718 \\
391270\end{array}$ \\
\hline $\begin{array}{l}391456 \\
391735\end{array}$ \\
\hline $\begin{array}{l}391735 \\
398741\end{array}$ \\
\hline $\begin{array}{l}3987411 \\
398772\end{array}$ \\
\hline 009816 \\
\hline $\begin{array}{l}004949 \\
402399\end{array}$ \\
\hline 402957 \\
\hline 403019 \\
\hline $\begin{array}{l}403186 \\
403236\end{array}$ \\
\hline $\begin{array}{l}403236 \\
403453\end{array}$ \\
\hline $\begin{array}{l}403453 \\
403484\end{array}$ \\
\hline $\begin{array}{l}403484 \\
405189\end{array}$ \\
\hline 405189 \\
\hline 408375 \\
\hline 413173 \\
\hline 413328 \\
\hline $\begin{array}{l}415777 \\
011487\end{array}$ \\
\hline 420427 \\
\hline 421063 \\
\hline $\begin{array}{l}421063 \\
422629\end{array}$ \\
\hline 422632 \\
\hline 010520 \\
\hline $\begin{array}{r}423838 \\
424799\end{array}$ \\
\hline $\begin{array}{l}424799 \\
004951\end{array}$ \\
\hline 425295 \\
\hline 425301 \\
\hline 425388 \\
\hline 427062 \\
\hline 427611 \\
\hline 427651 \\
\hline 427682 \\
\hline 427713 \\
\hline 427837 \\
\hline 427868 \\
\hline 004952 \\
\hline 428333 \\
\hline 004953 \\
\hline $\begin{array}{l}435806 \\
436302\end{array}$ \\
\hline $\begin{array}{l}436302 \\
439969\end{array}$ \\
\hline 441271 \\
\hline 441271 \\
\hline $\begin{array}{l}441581 \\
442697\end{array}$ \\
\hline 442697 \\
\hline 442697 \\
\hline \\
\hline 442705 \\
\hline 442705 \\
\hline 451098 \\
\hline \\
\hline 457678 \\
\hline $\begin{array}{l}458236 \\
459228\end{array}$ \\
\hline 459228 \\
\hline 459290 \\
\hline 461646 \\
\hline 462421 \\
\hline 468257 \\
\hline \\
\hline 013712 \\
\hline 469057 \\
\hline 469063 \\
\hline 469063 \\
\hline 469559 \\
\hline 473024 \\
\hline 473024 \\
\hline 474450 \\
\hline 474450 \\
\hline 478449 \\
\hline 480690 \\
\hline 481155 \\
\hline 482085 \\
\hline 482426 \\
\hline 482426 \\
\hline 482643 \\
\hline 004954 \\
\hline 483387 \\
\hline 483387 \\
\hline 484162 \\
\hline 484175 \\
\hline 484410 \\
\hline 489184 \\
\hline 489432 \\
\hline 493618 \\
\hline 498704 \\
\hline 506156 \\
\hline 004955 \\
\hline 506168 \\
\hline 506540 \\
\hline 506695 \\
\hline 506726 \\
\hline 508114 \\
\hline 516659 \\
\hline 516969 \\
\hline 517682 \\
\hline 517756 \\
\hline 517930 \\
\hline 517155 \\
\hline 518395 \\
\hline
\end{tabular}


COLORADO

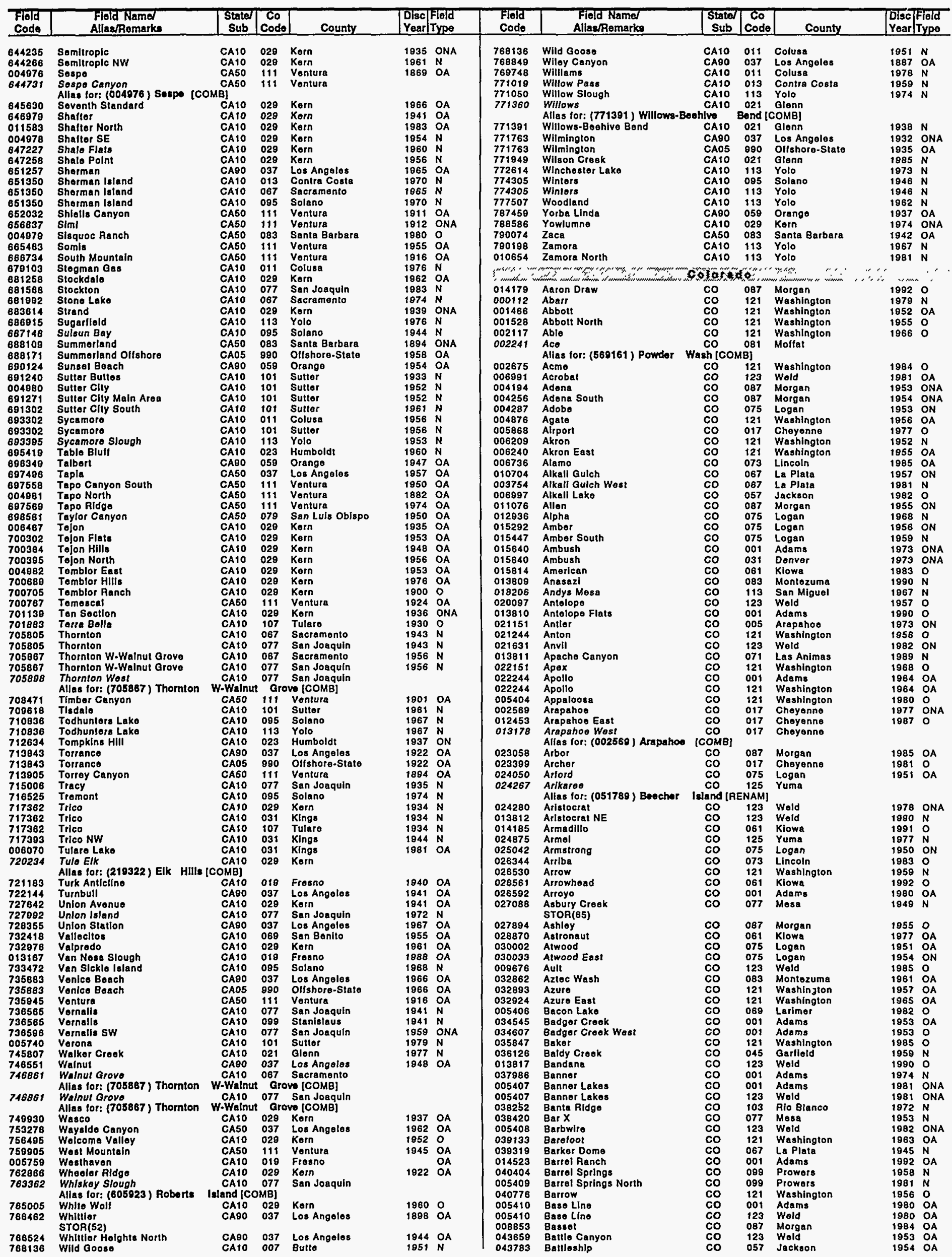


COLORADO

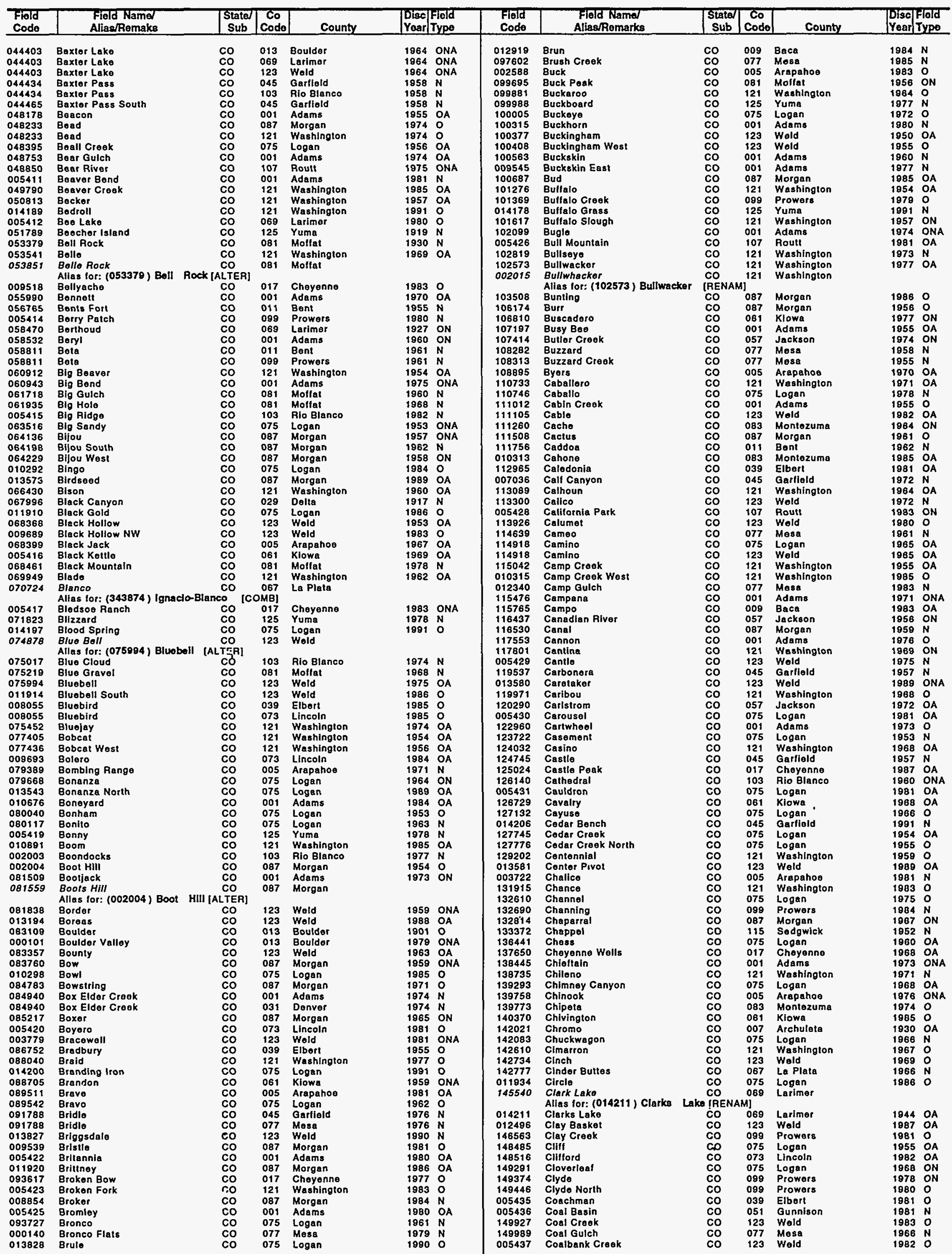


COLORADO

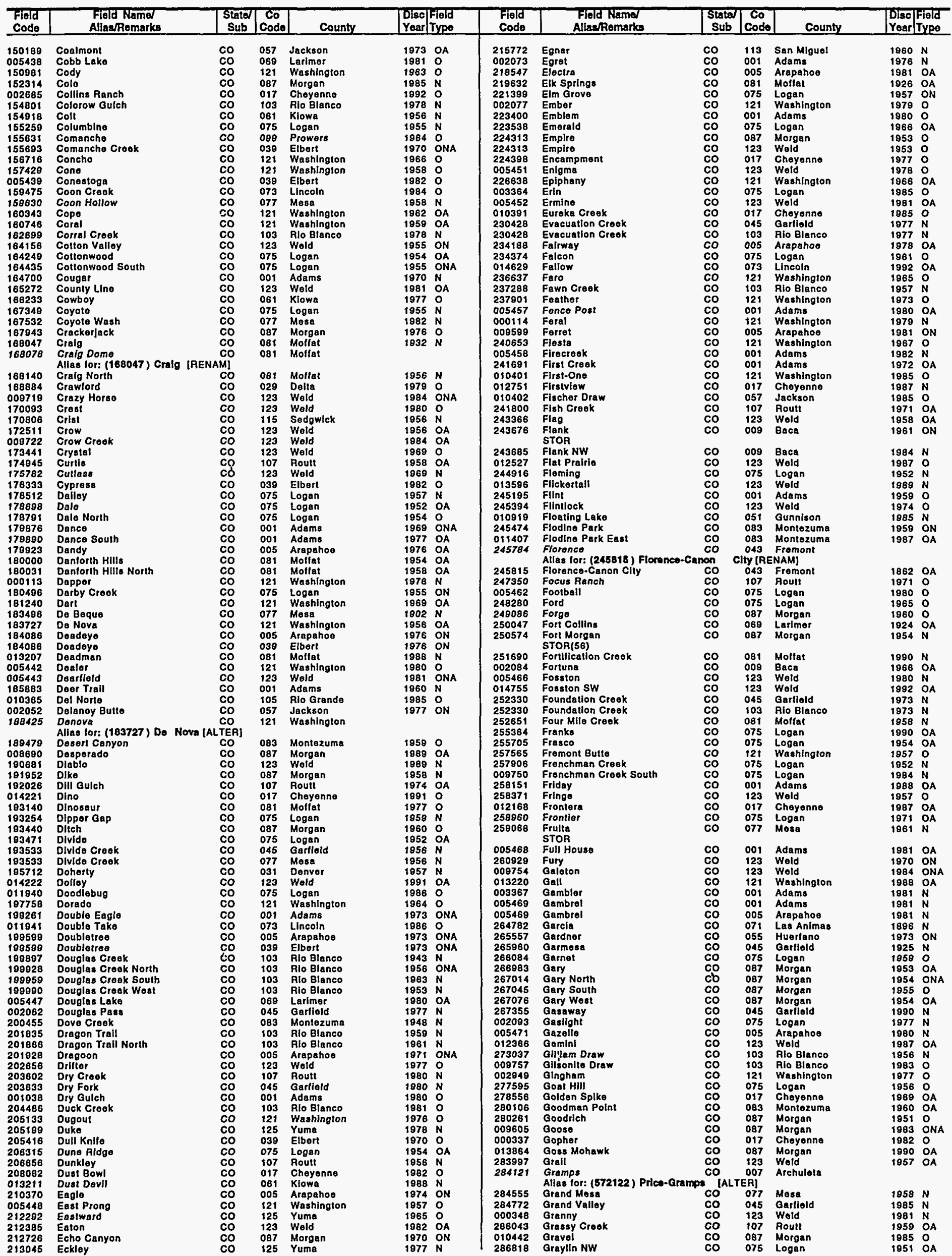


COLORADO

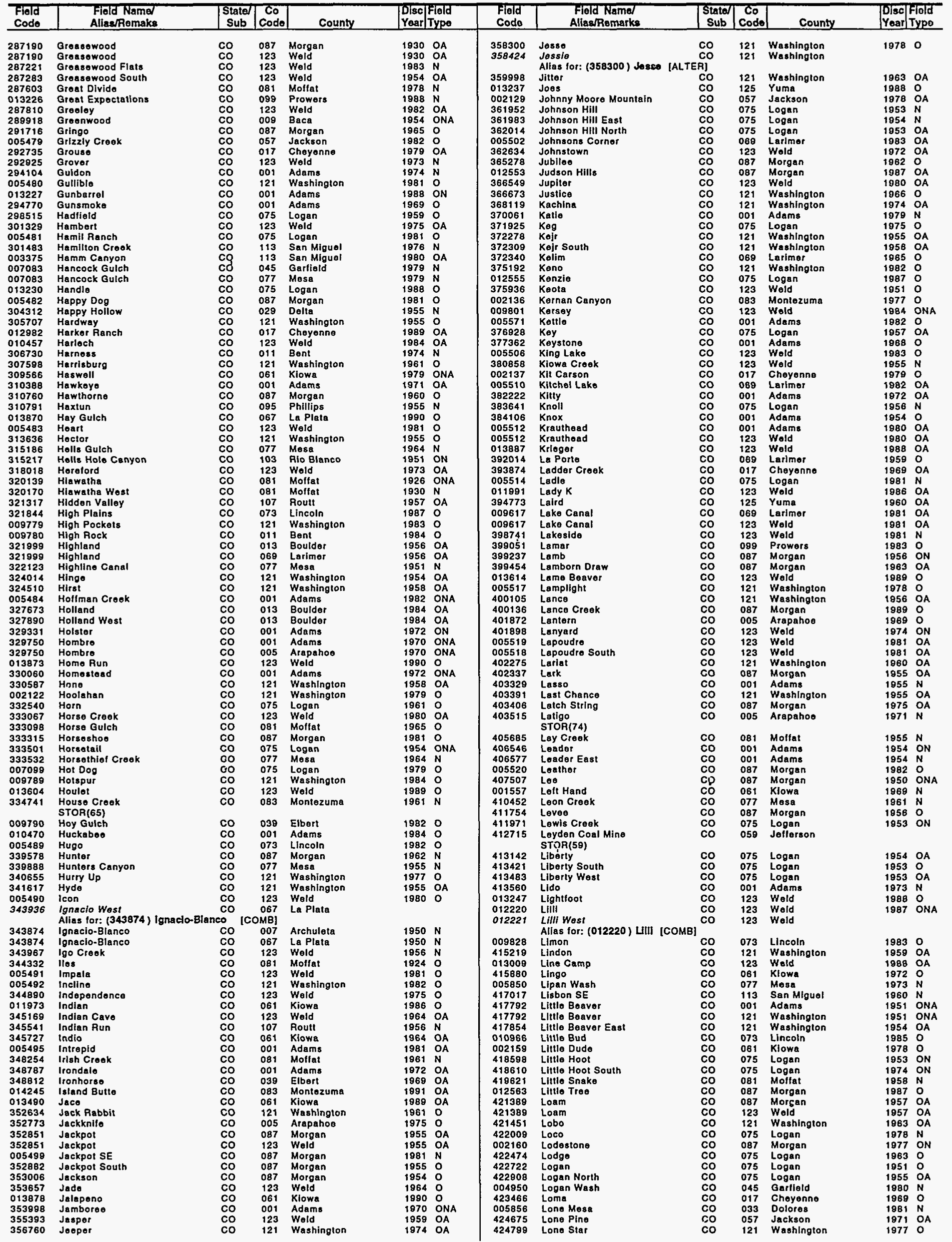


COLORADO

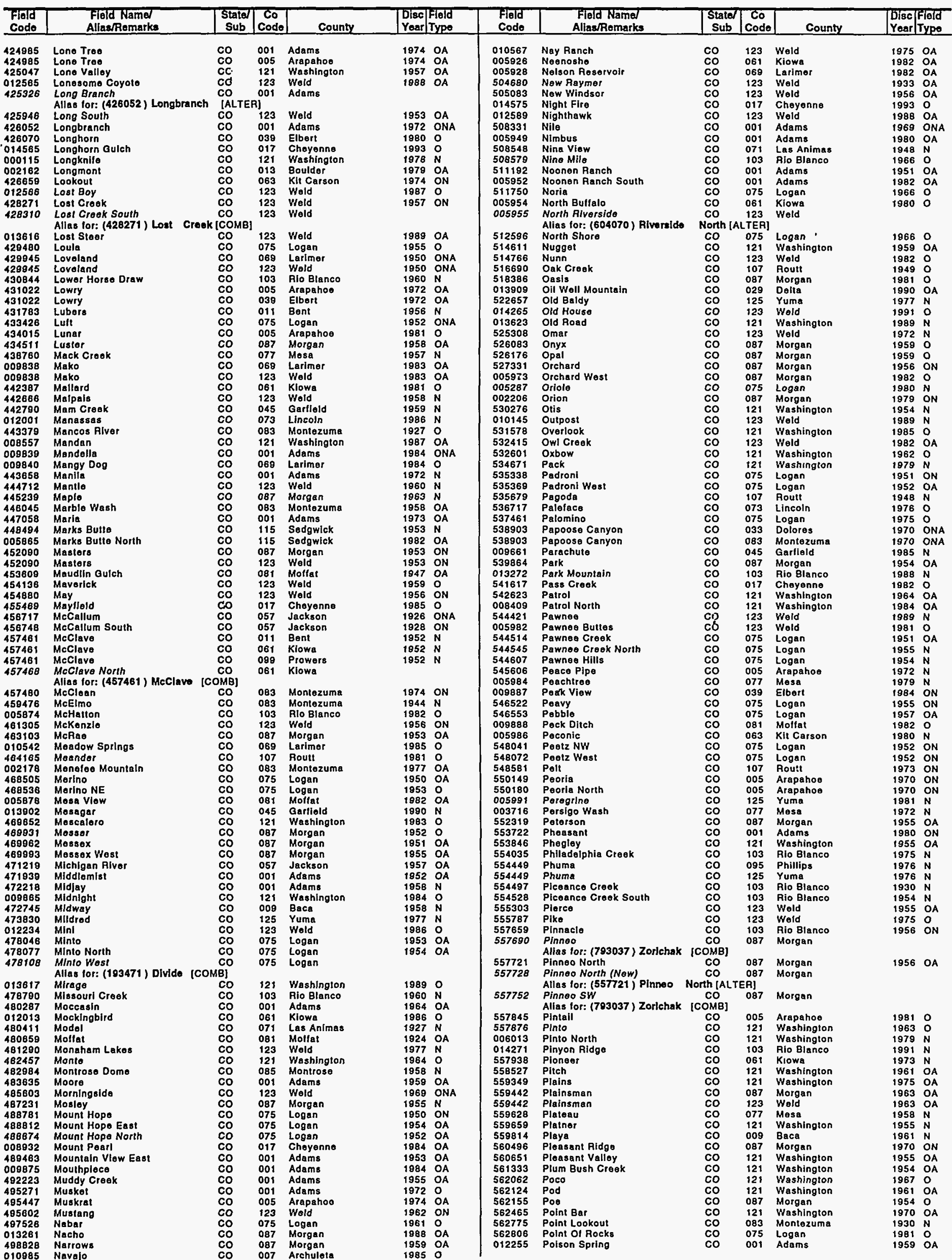




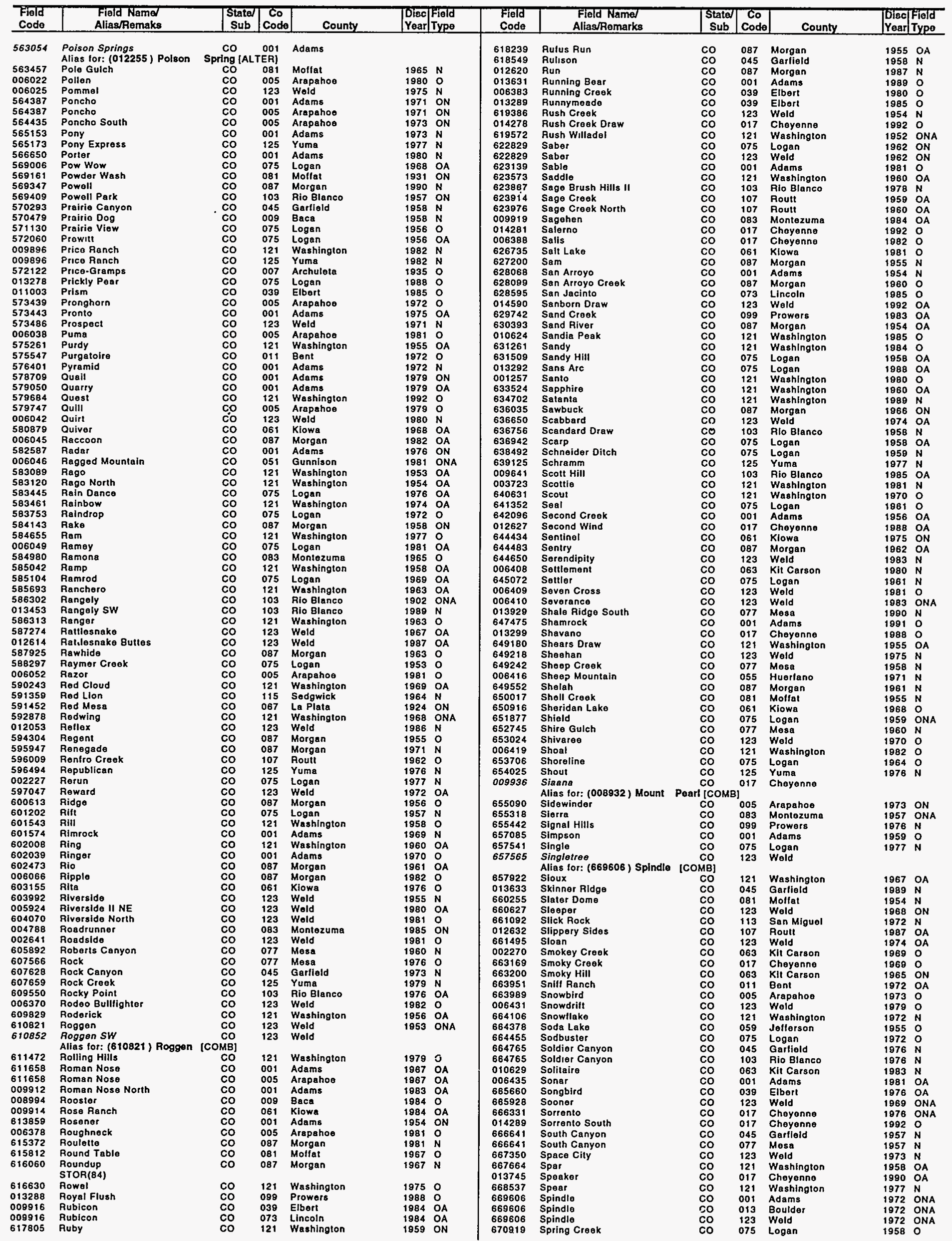


FLORIDA

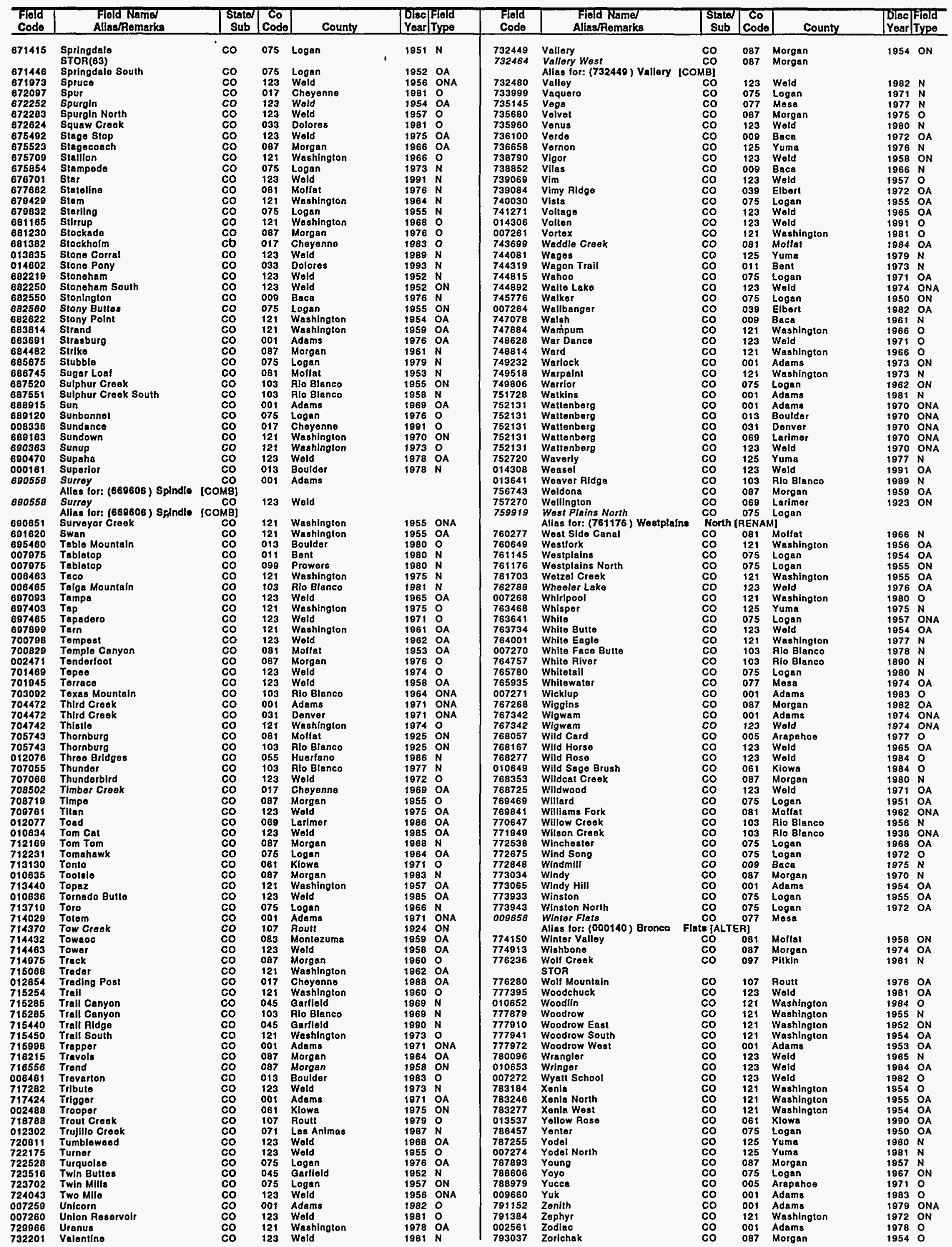


FLORIDA

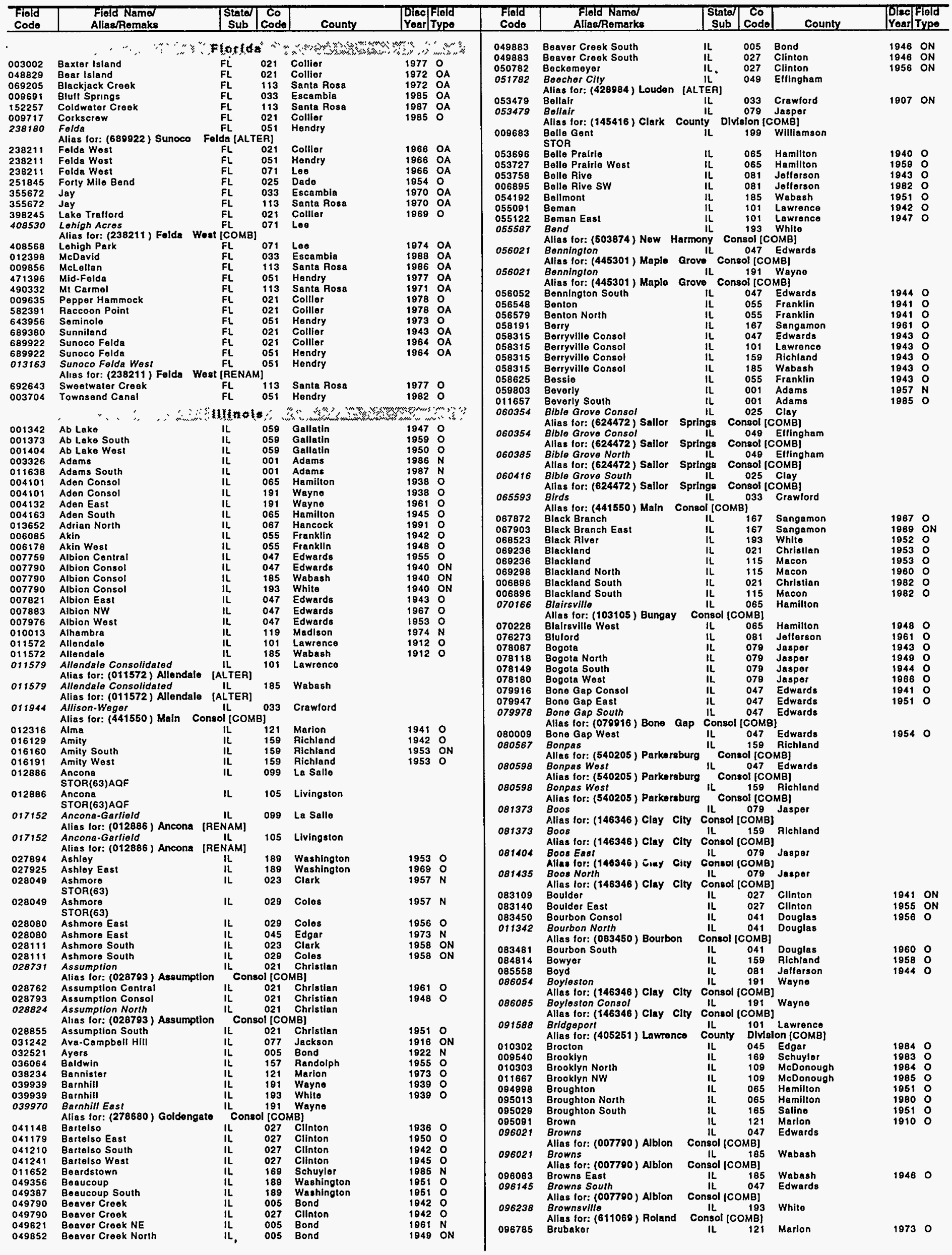


ILLINOIS

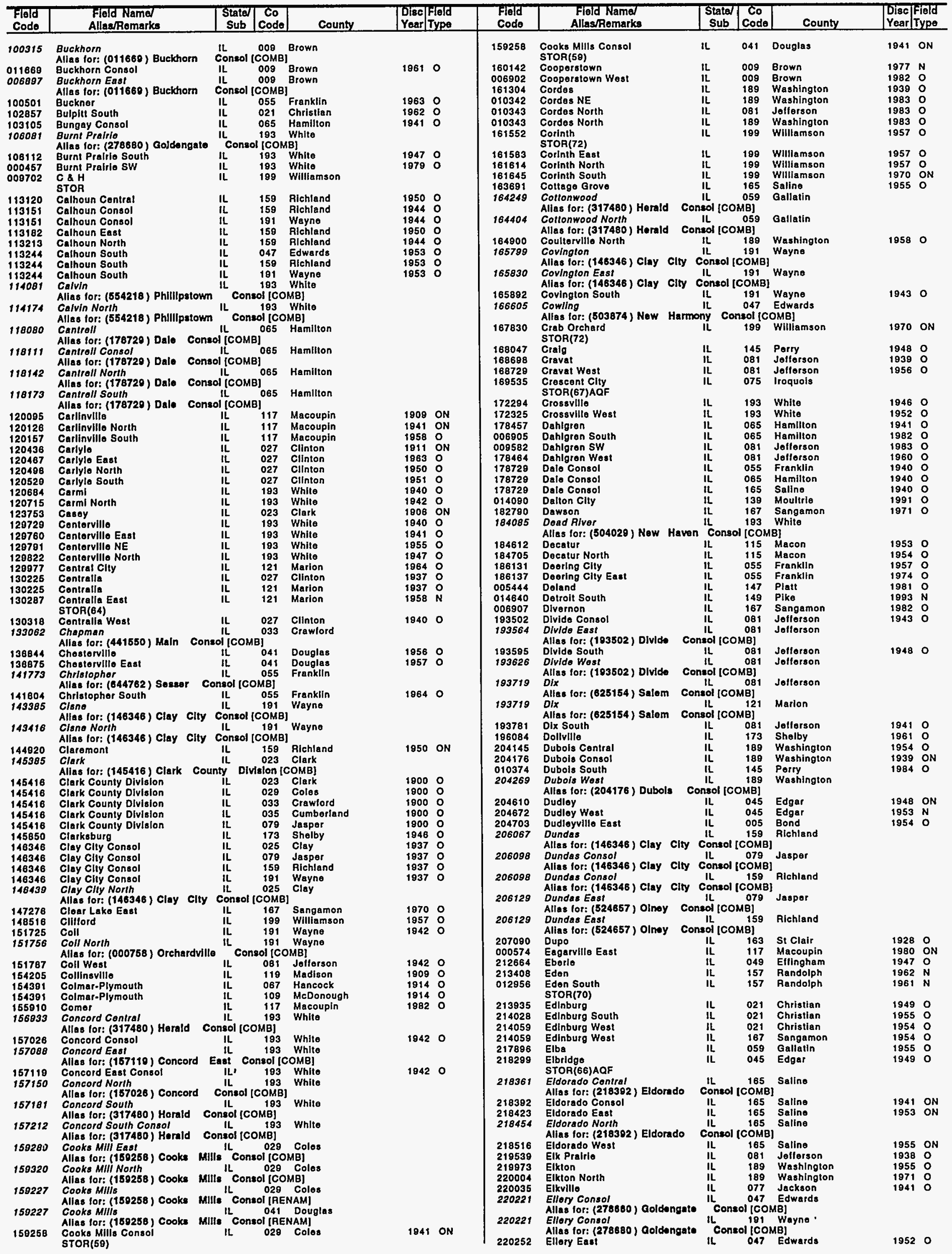




\section{ILLINOIS}

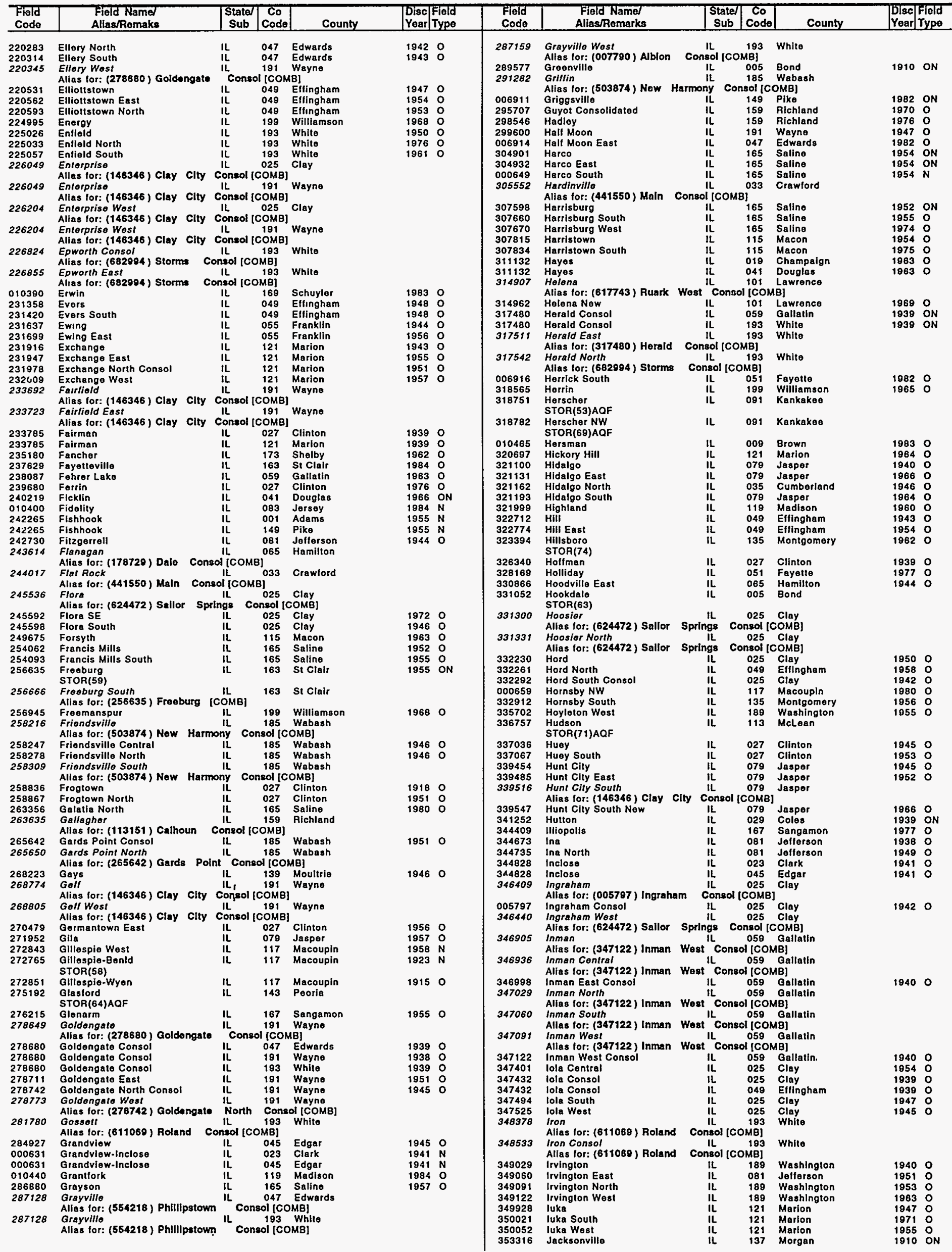




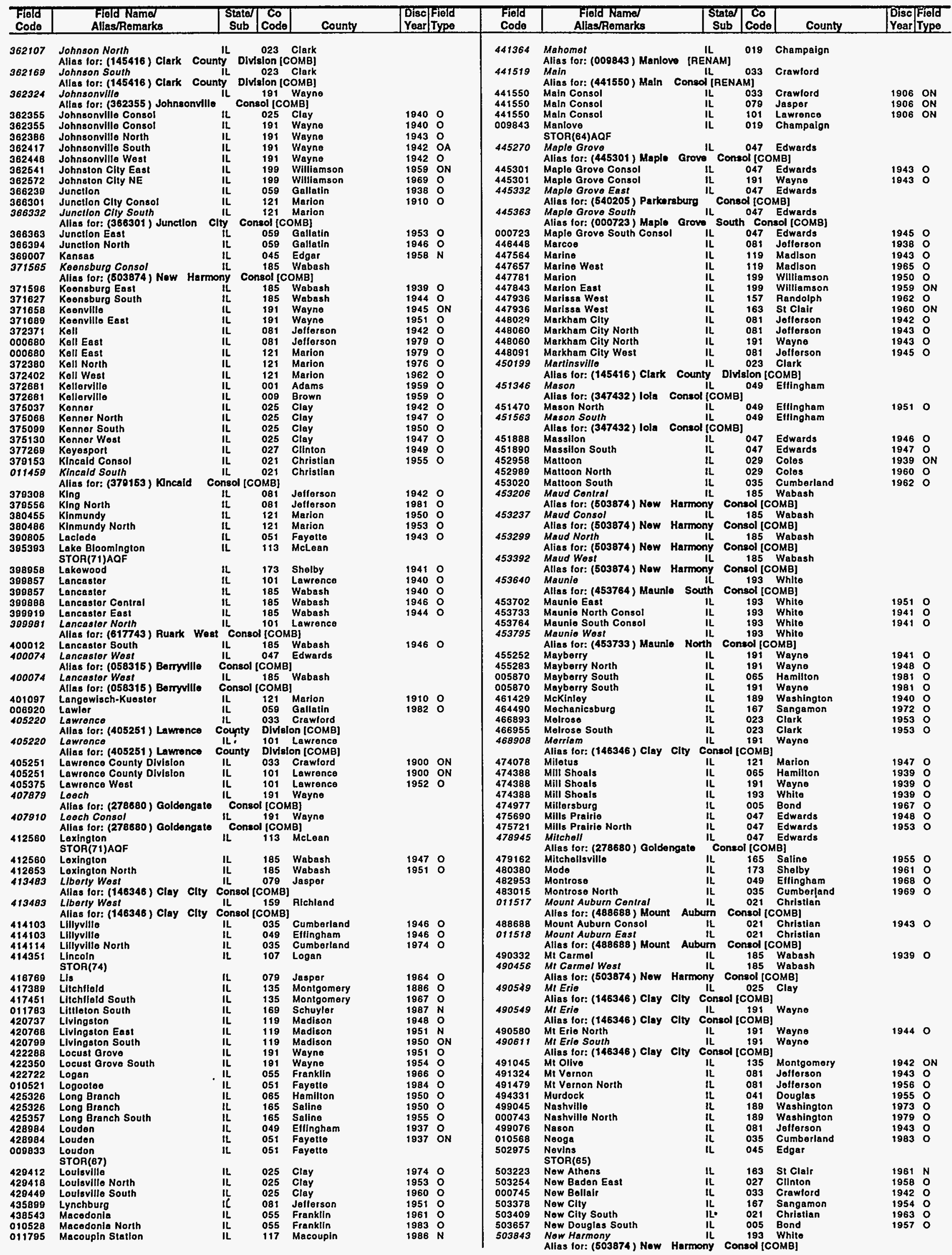


ILLINOIS

\begin{tabular}{|c|c|c|c|c|}
\hline $\begin{array}{l}\text { Fold } \\
\text { Code }\end{array}$ & $\begin{array}{l}\text { Field Namd } \\
\text { Alias/Remaks }\end{array}$ & $\begin{array}{c}\text { Stated } \\
\text { Sub }\end{array}$ & $\mid \begin{array}{c}\text { Co } \\
\text { Coda }\end{array}$ & County \\
\hline $\begin{array}{l}503874 \\
503874\end{array}$ & $\begin{array}{l}\text { Now Hermony Consol } \\
\text { Now Harmony Consol }\end{array}$ & $\begin{array}{l}\text { IL } \\
\text { iL }\end{array}$ & $\begin{array}{l}047 \\
185\end{array}$ & $\begin{array}{l}\text { Edwards } \\
\text { Wabash }\end{array}$ \\
\hline 503874 & Naw Harmony Consol & IL & $193 \mathrm{~V}$ & White \\
\hline $\begin{array}{l}503967 \\
503998\end{array}$ & $\begin{array}{l}\text { New Harmony South } \\
\text { Now Haven }\end{array}$ & IL & $\begin{array}{l}193 \mathrm{~V} \\
193\end{array}$ & $\begin{array}{l}\text { White } \\
\text { White }\end{array}$ \\
\hline & Altas for: $(504028)$ Now Haven & Consol & ol [сомв & \\
\hline 504029 & Now Haven Consol & IL & $193 \mathrm{~V} r(\mathrm{r}-\mathrm{r}-\mathrm{r}$ & White \\
\hline $\begin{array}{l}504060 \\
504091\end{array}$ & $\begin{array}{l}\text { Now Havan North } \\
\text { Alias for: (1571t9) Concord Eas } \\
\text { New Haven West }\end{array}$ & $\begin{array}{l}\text { IL } \\
\text { ast Con } \\
\text { IL }\end{array}$ & $\begin{array}{c}193 \\
\text { insol } \\
059\end{array}$ & $\begin{array}{l}\text { White } \\
\text { OMB] } \\
\text { Gallatin }\end{array}$ \\
\hline 504091 & & Consol & ol ICOMB & \\
\hline 504122 & $\begin{array}{l}\text { Now Habron } \\
\text { Alias for: (441550) Main Consol }\end{array}$ & IL & 033 & Crawford \\
\hline 504153 & Now Hebron East & IL & 033 & Crawford \\
\hline 504463 & $\begin{array}{l}\text { New Momphis } \\
\text { Alies for: (011811) Naw Memph }\end{array}$ & his con & $\begin{array}{l}027 \\
\text { onsol }[\mathrm{CO}\end{array}$ & $\begin{array}{l}\text { Clinton } \\
\text { OMB] }\end{array}$ \\
\hline $\begin{array}{l}011811 \\
504494\end{array}$ & $\begin{array}{l}\text { Now Momphis Consol } \\
\text { Now Memphis East }\end{array}$ & IL & 027 & $\begin{array}{l}\text { Clinton } \\
\text { Washington }\end{array}$ \\
\hline 504525 & $\begin{array}{l}\text { Alias for: }(521407) \text { Okawvillo } \\
\text { Now Memphis North } \\
\text { Alias for: (011811) Now Memph }\end{array}$ & $\begin{array}{l}\text { North C } \\
\text { IL Con } \\
\text { hls Con }\end{array}$ & $\begin{array}{l}\text { Consol l } \\
027 \\
\text { onsol [co }\end{array}$ & $\begin{array}{l}\text { [COMB] } \\
\text { Clinton } \\
\text { OMB] }\end{array}$ \\
\hline 504556 & New Memphis South & IL & 027 & Clinton \\
\hline 504556 & New Memphis South & IL & 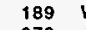 & Washington \\
\hline 506819 & Newton & IL & 079 & Jasper \\
\hline 506912 & Newton North & IL & 079 & Jasper \\
\hline 507005 & Newton Wost & IL & 079 & Jasper \\
\hline 509642 & $\begin{array}{l}\text { Noble } \\
\text { Alies lor, (146346) Clay Clty c }\end{array}$ & $\begin{array}{l}\text { IL } \\
\text { Consol IC }\end{array}$ & $\begin{array}{c}025 \\
{[\mathrm{COMB}]}\end{array}$ & Clay \\
\hline 509642 & $\begin{array}{l}\text { Alles for. }(146340) \text { Ciay City } \\
\text { Noblo }\end{array}$ & IL & 159 & Richland \\
\hline & Allas for (146346) Clay CIty C & Consol lc & [COMB] & \\
\hline 509766 & $\begin{array}{l}\text { Noblo North } \\
\text { Alias for: (146346) Clay City c }\end{array}$ & IL & 159 & Richland \\
\hline 509859 & $\begin{array}{l}\text { Alias for: (146346) Clay City C } \\
\text { Noblo South }\end{array}$ & Consol lc & [COMB] & \\
\hline & $\begin{array}{l}\text { Noblo South } \\
\text { Allas for: (146346) Clay Clty C }\end{array}$ & IL & COMB & Aichland \\
\hline 509921 & Noblo West & IL & 025 & Clay \\
\hline 512370 & Norris City & IL & 193 & White \\
\hline & Alias for: $(611069)$ Roland Con & onaol [CO & OMB] & \\
\hline 000749 & Norris City Wast & IL & 193 & White \\
\hline 011520 & $\begin{array}{l}\text { North Cuty } \\
\text { Altas for: (644762) Sesser Con }\end{array}$ & $\begin{array}{l}\text { IL } \\
\text { onsol [Co }\end{array}$ & $\begin{array}{r}055 \\
\text { OMB] }\end{array}$ & Franklin \\
\hline 516721 & Oak Grove & IL & 059 & Gallatin \\
\hline $\begin{array}{l}517000 \\
517000\end{array}$ & $\begin{array}{l}\text { Oak Point } \\
\text { Oak Point }\end{array}$ & $\begin{array}{l}\text { IL } \\
\text { IL }\end{array}$ & $\begin{array}{l}023 \\
079\end{array}$ & $\begin{array}{l}\text { Clark } \\
\text { Jasper }\end{array}$ \\
\hline 517031 & Oak Point West & IL & 023 & Clark \\
\hline 517031 & Oak Point Wost & IL & 035 & Cumberland \\
\hline 517186 & Oakdalo & IL & 081 & Jafferson \\
\hline 517217 & Oakdale North & IL & 081 & Jafferson \\
\hline 517682 & Oakloy & IL & 115 & Macon \\
\hline 518736 & $\begin{array}{l}\text { Oblong } \\
\text { Alias for: (441550) Maln Conso }\end{array}$ & IL İome & MB] & Crawtord \\
\hline 519263 & Oconee & IL. & 173 & Shelby \\
\hline 519821 & Odın & IL & 121 & Marion \\
\hline 521371 & Okewville & IL & 189 & Washington \\
\hline 521402 & $\begin{array}{l}\text { Okawvillo North } \\
\text { Alias for: }(521407) \text { Okawvillo } \mathrm{N}\end{array}$ & North c & $\begin{array}{c}189 \\
\text { Consol i }\end{array}$ & $\begin{array}{l}\text { Washington } \\
\text { [COMB] }\end{array}$ \\
\hline 521407 & Okawville North Consol & IL & 189 & Washington \\
\hline 523169 & Old Aiploy & II & 005 & Bond \\
\hline 523200 & Otd Ripley North & II & 005 & Bond \\
\hline 011818 & Old Rlpley West & IL & 005 & Bond \\
\hline 524657 & Oiney Consol & II & 079 & Jasper \\
\hline 524657 & OIney Consol & IL & 159 & Richland \\
\hline 524688 & OIney East & IL & 159 & Richland \\
\hline & Alias for: $(524657)$ Olney Cons & 1801 [COM & $M B 1$ & \\
\hline 524781 & OInoy South & IL. & 159 & Richland \\
\hline 525184 & Omahs & IL & 059 & Gallatin \\
\hline 525215 & Omeha East & IL & 059 & Gallatin \\
\hline 525246 & Omaha South & IL & 059 & Gallatin \\
\hline 525246 & Omaha South & II & 165 & Saline \\
\hline 525277 & Omaha West & IL & 059 & Gallatin \\
\hline $\mathbf{5 2 5 2 7 7}$ & Omaha Wost & IL & 165 & Saline \\
\hline 525339 & Omega & IL & 121 & Marion \\
\hline 011819 & Omaga NW & IL & 121 & Marion \\
\hline 526238 & Opdyke & IL & 081 & Jefferson \\
\hline 527393 & Orchardville & IL & 191 & Wayne \\
\hline & Alias for: $(000758)$ Orchardville & Cons & $28001[\mathrm{CO}$ & $M B]$ \\
\hline 000758 & Orchardville Consol & $\mathrm{IL}$ & $\begin{array}{l}191 \\
191\end{array}$ & $\begin{array}{l}\text { Wayne } \\
\text { Wayno }\end{array}$ \\
\hline 527424 & $\begin{array}{l}\text { Orcherdville North } \\
\text { Orient }\end{array}$ & IL & $\begin{array}{l}191 \\
055\end{array}$ & $\begin{array}{l}\text { Wayno } \\
\text { Franklin }\end{array}$ \\
\hline $\begin{array}{l}527889 \\
527920\end{array}$ & $\begin{array}{l}\text { Orient } \\
\text { Orient North }\end{array}$ & IL & 055 & Franklin \\
\hline 529687 & Oskaloosa & IL & 025 & Clay \\
\hline 529718 & Oskaloosa East & IL & 025 & Clay \\
\hline 529749 & Oskaloosa South & IL & 025 & Clay \\
\hline 537632 & Pana & IL & 021 & Christian \\
\hline 000765 & Pana Cantral & IL & 021 & Christian \\
\hline 537646 & Pana South & IL. & 021 & Christian \\
\hline 537663 & Panama & IL & 005 & Bond \\
\hline 537653 & Panama & IL & 135 & Montgomery \\
\hline 537973 & Pankeyville & IL & 165 & Saline \\
\hline 538004 & Pankeyville East & IL & 165 & Saline \\
\hline 539957 & $\begin{array}{l}\text { Parker } \\
\text { Alias for: (441550) Main Conso }\end{array}$ & It & $\mathrm{MB]}$ & Crawlord \\
\hline 540174 & Parkersbuig & IL & 159 & Richland \\
\hline & Alias lor: (540205) Parkersburg & 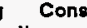 & neo & \\
\hline 540205 & Parkersburg Consol & IL & 047 & Edwards \\
\hline 540205 & Parkersburg Consol & IL & 159 & Richland \\
\hline 540236 & $\begin{array}{l}\text { Parkersburg North } \\
\text { Alias tor: (540205) Parkersburg }\end{array}$ & IL Cons & $\begin{array}{l}159 \\
\mathrm{nsol} \\
\text { [CON }\end{array}$ & $\begin{array}{l}\text { Alehland } \\
\mathrm{MBl}\end{array}$ \\
\hline 540267 & Parkersburg South & IL & 047 & Edwards \\
\hline 540298 & Parkersburg West & IL & 047 & Edwards \\
\hline 540298 & Parkersburg Wost & IL & 159 & Richland \\
\hline 540887 & Parnell & IL & 039 & De witt \\
\hline 541724 & Passport & II & 025 & Clay \\
\hline & Alias for. (005797) Ingraham & Consol I & [COMB] & \\
\hline $\begin{array}{l}541755 \\
541786\end{array}$ & $\begin{array}{l}\text { Passport North } \\
\text { Passport South }\end{array}$ & IL & $\begin{array}{l}159 \\
025\end{array}$ & $\begin{array}{l}\text { Richland } \\
\text { Clay }\end{array}$ \\
\hline 541786 & Passport South & IL & 159 & Richland \\
\hline 541817 & Passport Wost & iI. & 025 & Clay \\
\hline 542251 & Patoka & IL & 027 & Clinton \\
\hline 542251 & Patoka & IL & 121 & Marion \\
\hline 542282 & Patoka East & II & 121 & Marion \\
\hline 542375 & Patoka South & IL & 121 & Marion \\
\hline 542406 & Patoka West & IL & 051 & Fayetto \\
\hline
\end{tabular}


INDIANA

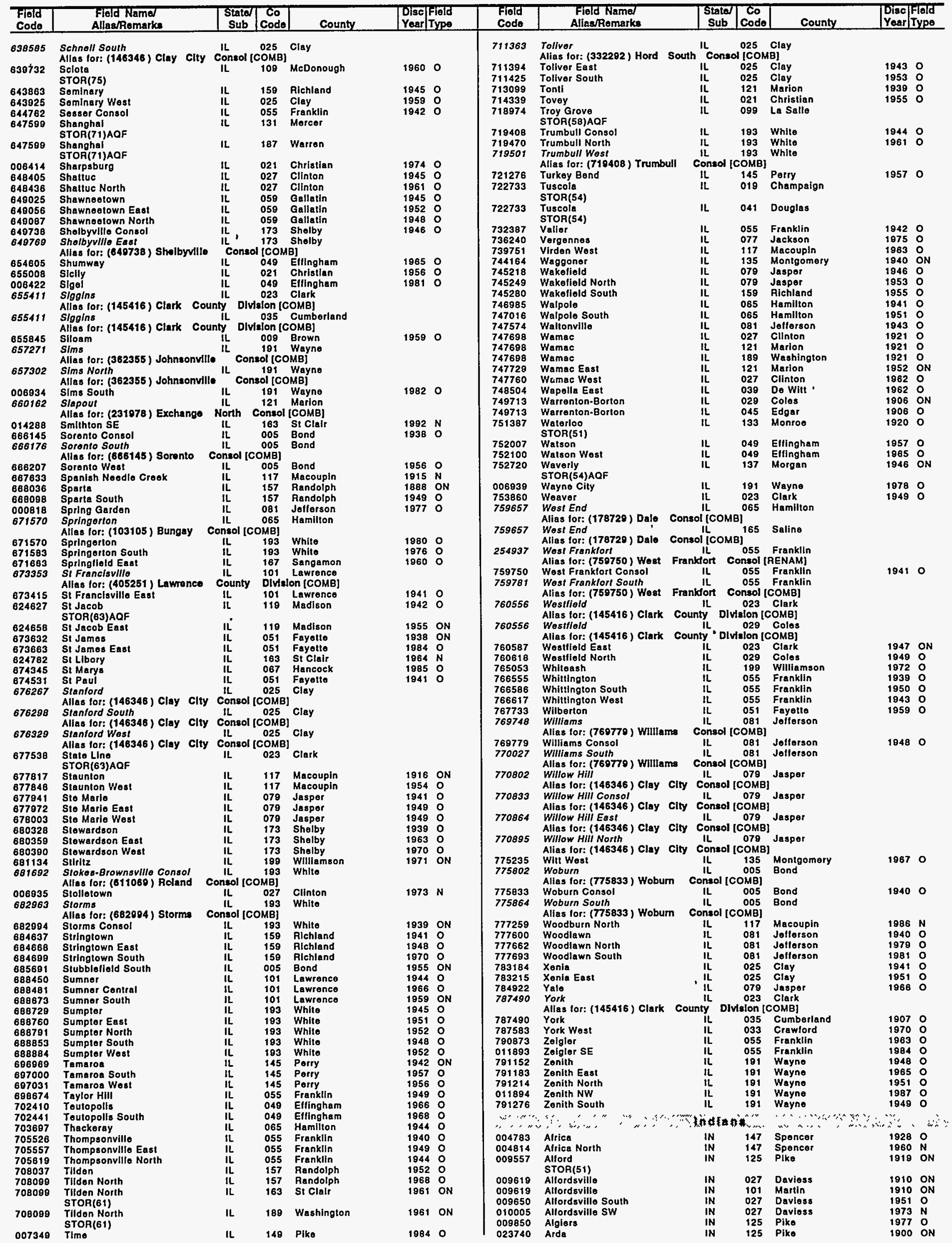


INDIANA

\begin{tabular}{|c|c|c|}
\hline $\begin{array}{l}\text { Frald } \\
\text { Code } \\
\end{array}$ & $\begin{array}{l}\text { Field Name } \\
\text { Alias/Remaks }\end{array}$ & $\begin{array}{c}\text { Statel } \\
\text { Sub }\end{array}$ \\
\hline $\begin{array}{l}024639 \\
025042\end{array}$ & $\begin{array}{l}\text { Arlington } \\
\text { Armstrong }\end{array}$ & $\begin{array}{l}\text { IN, } \\
\text { IN, }\end{array}$ \\
\hline 025507 & $\begin{array}{l}\text { Arnoy } \\
\text { STOR }\end{array}$ & in \\
\hline 010267 & Arnoy North & IN \\
\hline 026716 & Ant & IN \\
\hline 026902 & $\begin{array}{l}\text { Arthur } \\
\text { Alias for: (5 }\end{array}$ & IN \\
\hline 027305 & $\begin{array}{l}\text { Alias for: }(517530 \\
\text { Ash Iron Springs }\end{array}$ & City Cons \\
\hline 003000 & Ashboro East & IN \\
\hline $\begin{array}{l}027770 \\
027894\end{array}$ & $\begin{array}{l}\text { Ashlend } \\
\text { Ashley }\end{array}$ & IN \\
\hline 027894 & $\begin{array}{l}\text { Ashley } \\
\text { Auburn }\end{array}$ & IN \\
\hline $\begin{array}{l}030374 \\
030405\end{array}$ & $\begin{array}{l}\text { Auburn } \\
\text { Auburn East }\end{array}$ & $\begin{array}{l}\text { IN } \\
\text { IN }\end{array}$ \\
\hline 011904 & Aydoville & IN \\
\hline 036064 & Baldwin & IN \\
\hline 039257 & Barker & IN \\
\hline 040559 & $\begin{array}{l}\text { Barretr-Mitcholl } \\
\text { Alias for: (254341) Franciseo }\end{array}$ & in \\
\hline 040590 & $\begin{array}{l}\text { Barrett-Mitcholl Consol } \\
\text { Alias for: (254341) Franclsco }\end{array}$ & $\begin{array}{l}\text { IN } \\
\text { South }\end{array}$ \\
\hline 040621 & $\begin{array}{l}\text { Barrolt-Mitchall East } \\
\text { Alias for: (254341) Franclaco }\end{array}$ & $\begin{array}{l}\text { IN } \\
\text { South }\end{array}$ \\
\hline 040852 & $\begin{array}{l}\text { Barrett-Mitcholl North } \\
\text { Alias for: (254341) Franclsco }\end{array}$ & IN \\
\hline 040683 & $\begin{array}{l}\text { Alias for: }(254341) \text { Franclsco } \\
\text { Barrolt-Mitchell South } \\
\text { Alies for: }(762649) \text { Wheatonville }\end{array}$ & South \\
\hline 040714 & $\begin{array}{l}\text { Alias for: }(762649) \text { Wheatonville } \\
\text { Barrett-Mitchell West }\end{array}$ & ${ }_{\text {IN }}^{\text {IN }}$ Con \\
\hline $\begin{array}{l}041675 \\
041675\end{array}$ & $\begin{array}{l}\text { Bartlottsville } \\
\text { Bartlettsvilio }\end{array}$ & IN \\
\hline 012683 & Bartlottsvillo North & IN \\
\hline 010027 & $\begin{array}{l}\text { Boaman East } \\
\text { Allas for: }(048488) \text { Beaman Ea }\end{array}$ & East Con \\
\hline 048488 & Beamen East Consol & in \\
\hline 048519 & $\begin{array}{l}\text { Beaman South } \\
\text { Allas for: (048488) Beaman Ea }\end{array}$ & East. Con \\
\hline 053107 & Belknap & IN \\
\hline 053138 & Bolknap East & IN \\
\hline $\begin{array}{l}056021 \\
056703\end{array}$ & & IN \\
\hline $\begin{array}{l}056703 \\
060509\end{array}$ & Bentonvillo & IN \\
\hline $\begin{array}{l}060509 \\
060540\end{array}$ & $\begin{array}{l}\text { Bicknell } \\
\text { Bicknall East }\end{array}$ & IN \\
\hline 065655 & $\begin{array}{l}\text { Bicknell East } \\
\text { Birdsaye }\end{array}$ & IN \\
\hline 065655 & Birdsoye & IN \\
\hline 065702 & Birdsoye (New) & IN \\
\hline & $\begin{array}{l}\text { Alias for: (065655) Blrdseyo If } \\
\text { Birdseye (New) }\end{array}$ & [RENAM] \\
\hline 065702 & $\begin{array}{l}\text { Birdseye (New) } \\
\text { Alias for: (065655) Birdseyo }\end{array}$ & $\begin{array}{l}\text { IN } \\
\text { [RENAM] }\end{array}$ \\
\hline 065686 & Birdsayo East & IN \\
\hline & Alias for: (065655) Blrdsoye ic & [COMB] \\
\hline $\begin{array}{l}065717 \\
065748\end{array}$ & $\begin{array}{l}\text { Birdsoyo North } \\
\text { Birdsoye West }\end{array}$ & $\begin{array}{l}\text { IN } \\
\text { IN }\end{array}$ \\
\hline 068027 & Black Chapel & in \\
\hline 068058 & Black Chapel South & IN \\
\hline 068523 & $\begin{array}{l}\text { Black River } \\
\text { Alias for: (06855) Black Rive }\end{array}$ & ver Cons. \\
\hline 068554 & Black River Consol & IN \\
\hline 069081 & Blackhawk & IN \\
\hline 070166 & Blairsvillo & in \\
\hline 070197 & Blairsvillo East & iN \\
\hline 009015 & Blairsville South & IN \\
\hline 076676 & Blythe Chapel & IN \\
\hline 081311 & Boonville & IN \\
\hline 007020 & Boonville South & IN \\
\hline 084600 & Bowling Grean & IN \\
\hline 084604 & Bowling Green South & IN \\
\hline 088310 & Bramble & IN \\
\hline 014323 & Bramble East & IN \\
\hline 088581 & Branchvillo & IN \\
\hline 090813 & Bromen & IN \\
\hline 013342 & Bremen South & IN \\
\hline 091160 & Bretzville & IN \\
\hline 092580 & Bristow & IN \\
\hline 092735 & Bristow North & IN \\
\hline 092766 & Bristow South & IN \\
\hline 092828 & Bristow West & IN \\
\hline 093138 & Broad Ripplo & IN \\
\hline 093169 & Broad Ripple South & IN \\
\hline 084626 & Brooksburg & IN \\
\hline 084626 & Brooksburg & IN \\
\hline 007460 & Brookville & IN \\
\hline 100563 & Buckskin & IN \\
\hline 100594 & Buckskin Wost & IN \\
\hline & Alias lor: (762649) Wheatonville & Ille \\
\hline 101970 & Buffaloville & IN \\
\hline 101989 & Burkin & IN \\
\hline & Alias for: $(111167)$ Caborn & Consol [C \\
\hline 102020 & Bufkin Wost & IN \\
\hline 103353 & Bunker Hill & IN \\
\hline 107383 & Butler & IN \\
\hline 111196 & Caborn & IN \\
\hline & Alias for: (111167) Cabom & Consol IR \\
\hline 111167 & Caborn Consol & IN \\
\hline 111198 & $\begin{array}{l}\text { Caborn South } \\
\text { Alias for: (111167) Caborn }\end{array}$ & IN \\
\hline & $\begin{array}{l}\text { Alias for: (1111167) Cabom } \\
\text { Caborn West }\end{array}$ & onsol [C \\
\hline 111229 & Caborn West & IN \\
\hline$\$ 17491$ & Cennalburg (New) & IN \\
\hline 003021 & Capohart & IN \\
\hline 120188 & Carlisle & IN \\
\hline $\begin{array}{l}009021 \\
102204\end{array}$ & $\begin{array}{l}\text { Carlisle North } \\
\text { Carlisle West }\end{array}$ & $\begin{array}{l}\text { IN } \\
\text { IN }\end{array}$ \\
\hline 125764 & $\begin{array}{l}\text { Cates } \\
\text { Catol }\end{array}$ & IN \\
\hline 126233 & Cathlinett & IN \\
\hline & Alias for: (674903) St Thomas & as Conso \\
\hline 126357 & Cato & IN \\
\hline 128805 & Celina & IN \\
\hline 128728 & Centerville & IN \\
\hline 132101 & ndlor & IN \\
\hline 141339 & Chrisney North & IN \\
\hline 141370 & Chrisney South & IN \\
\hline $\begin{array}{l}146439 \\
146284\end{array}$ & $\begin{array}{l}\text { Clay City North } \\
\text { Claybank }\end{array}$ & $\begin{array}{l}\text { IN } \\
\text { IN }\end{array}$ \\
\hline 146284 & Claybank & IN \\
\hline
\end{tabular}


INDIANA

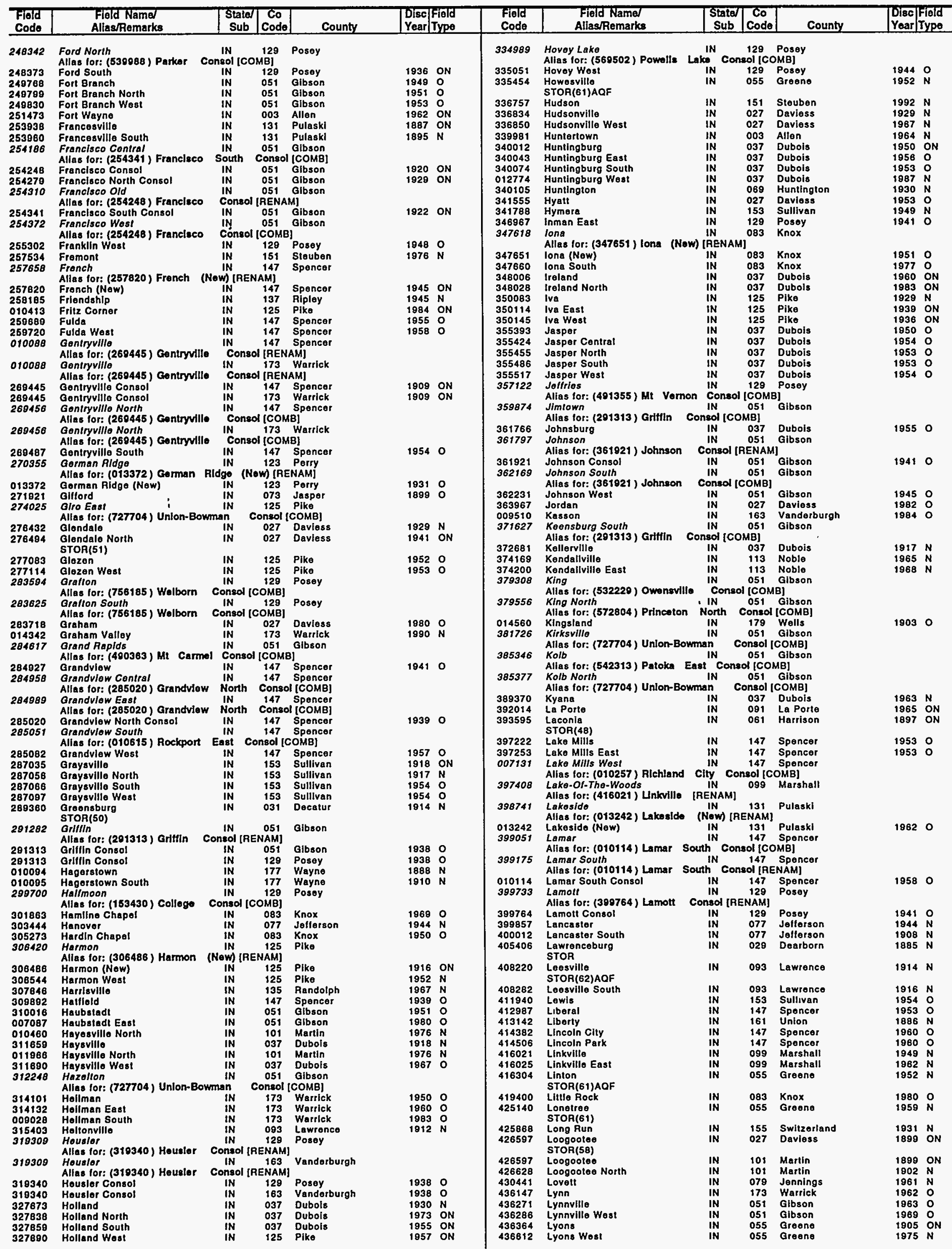




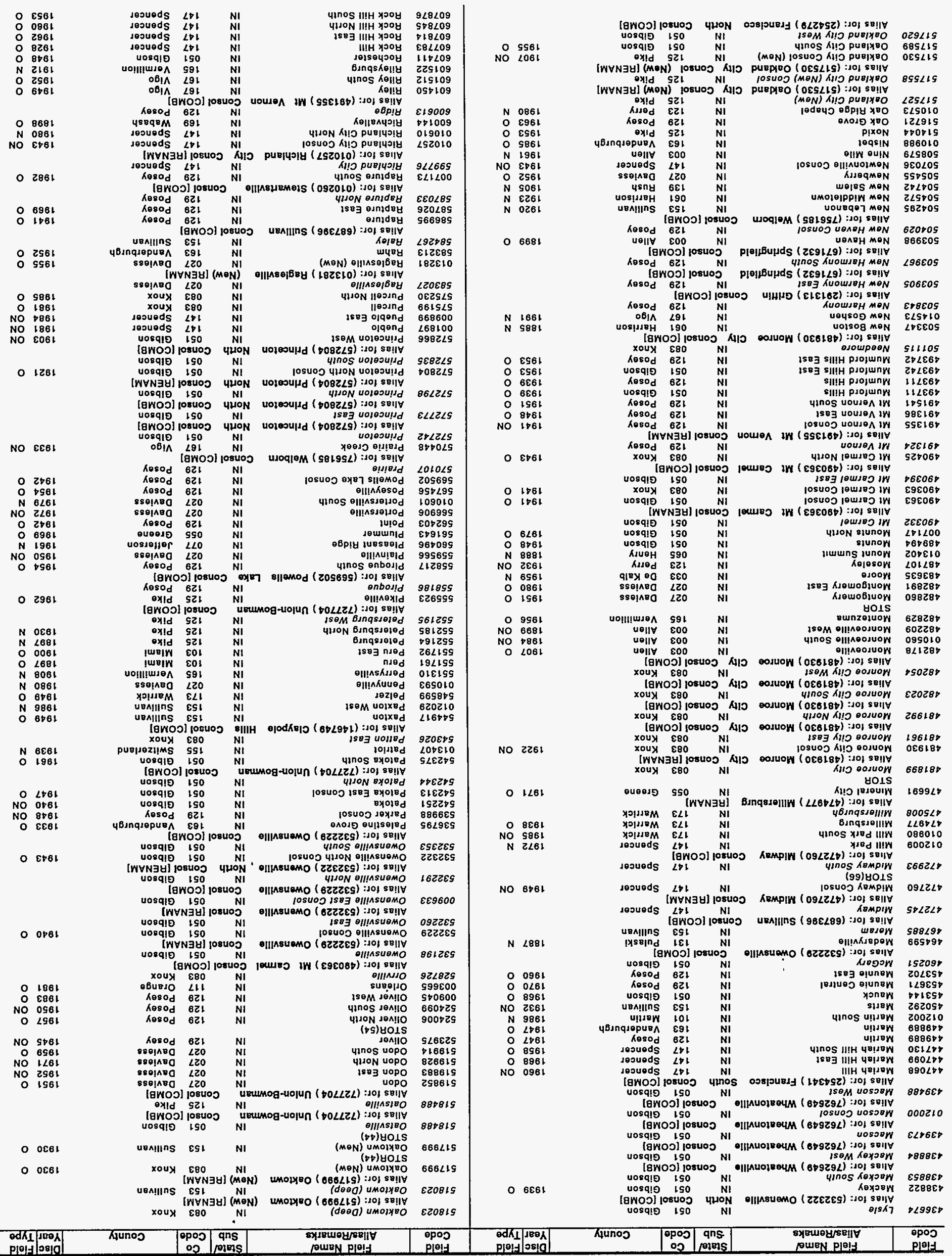


INDIANA

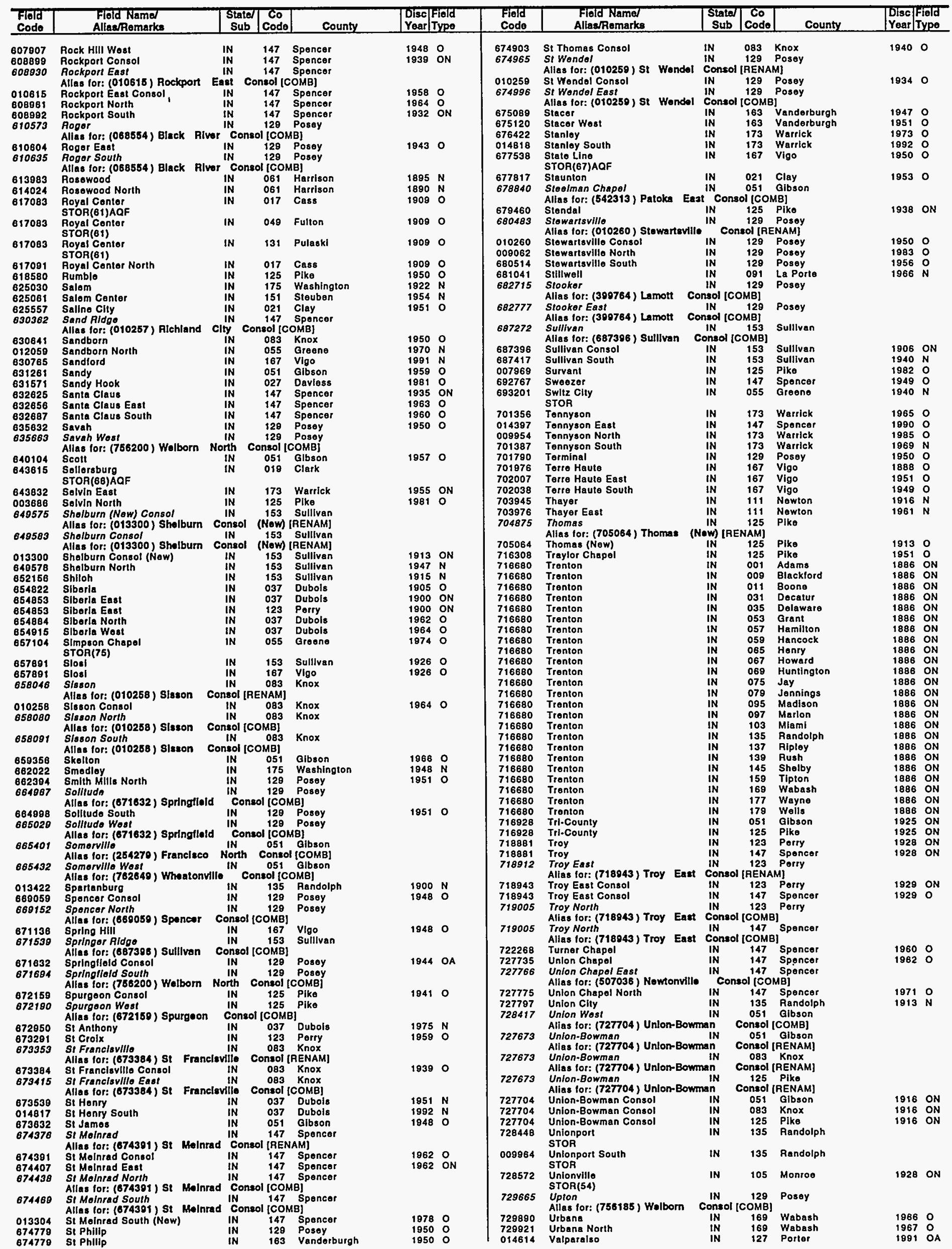


INDIANA

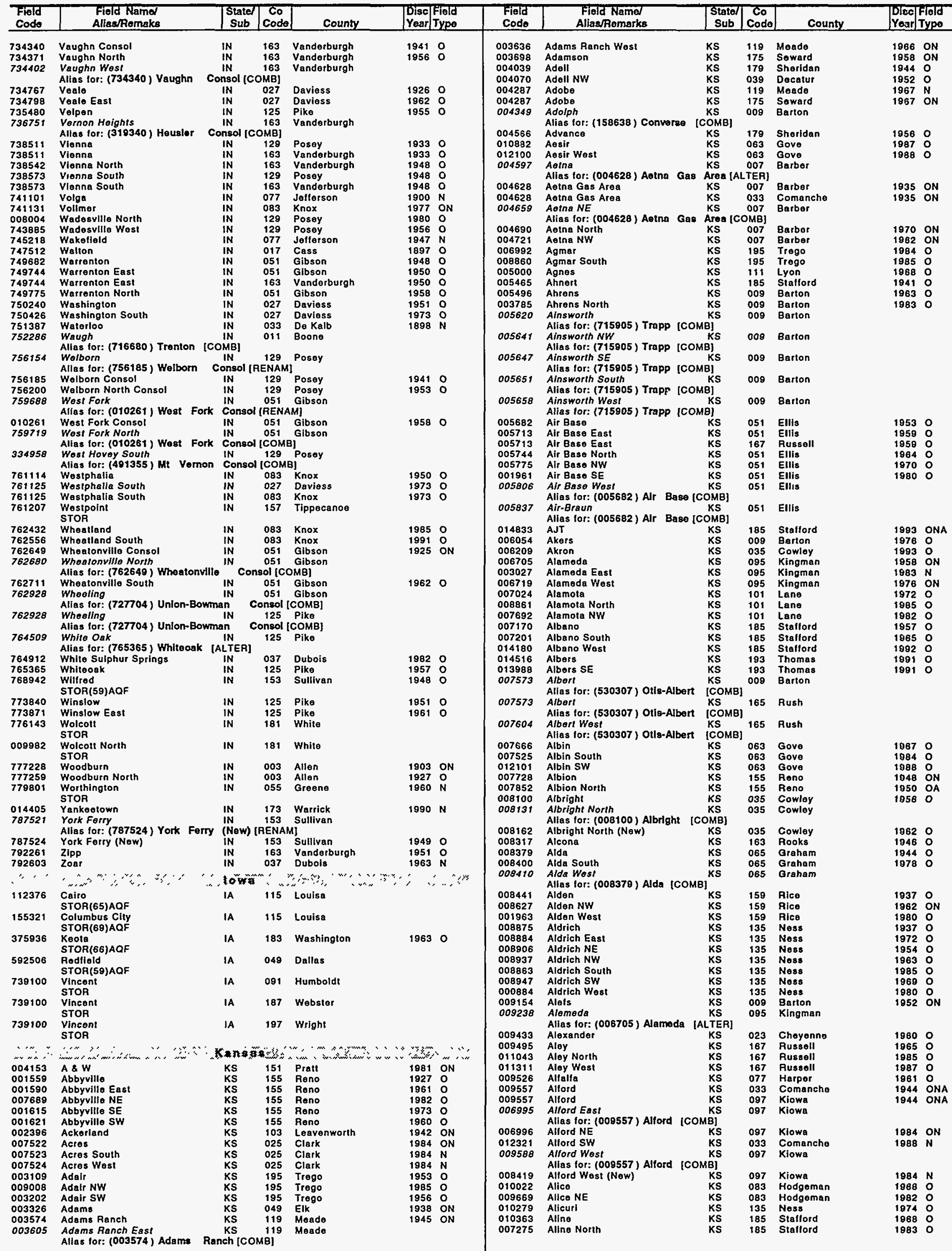


KANSAS

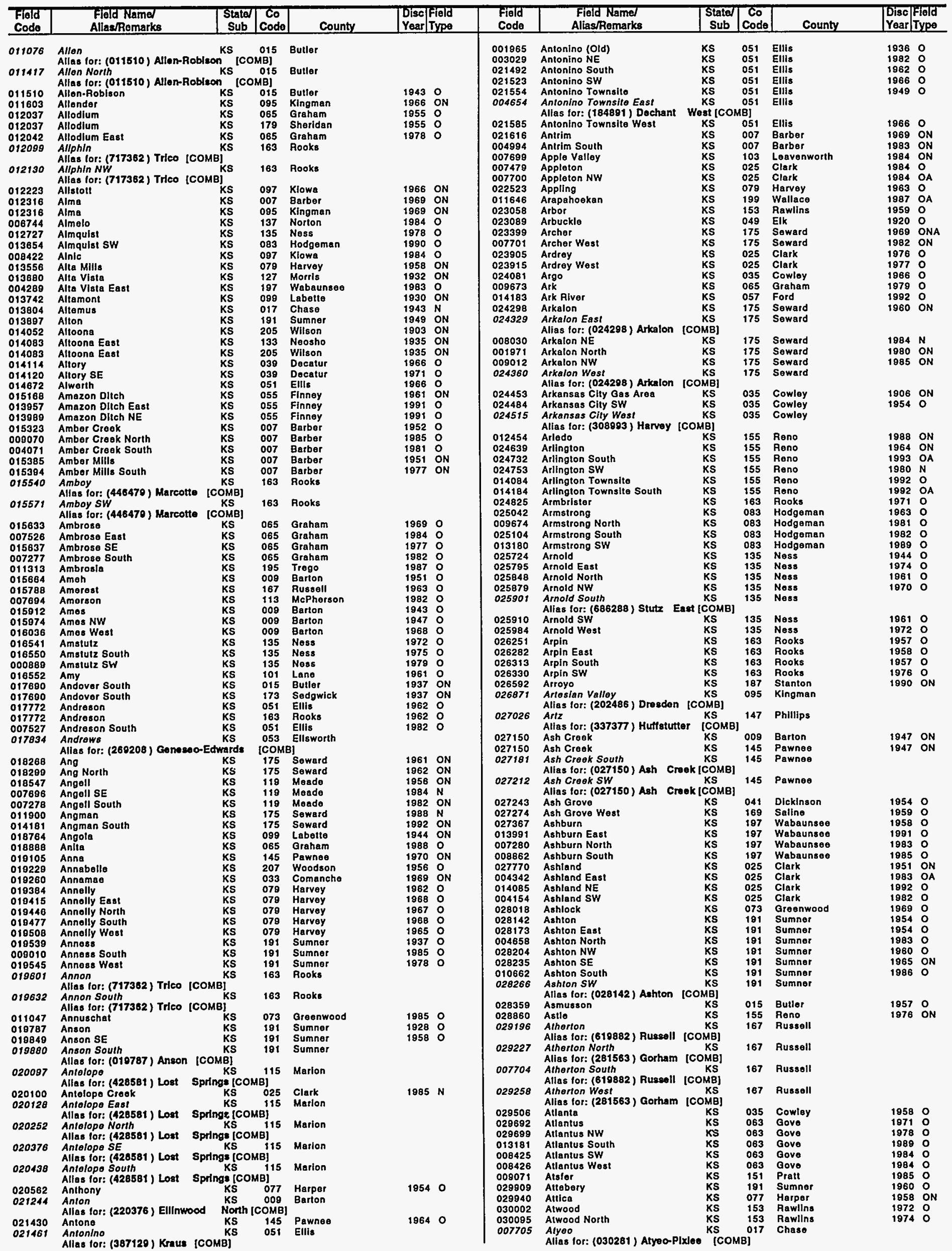




\section{KANSAS}

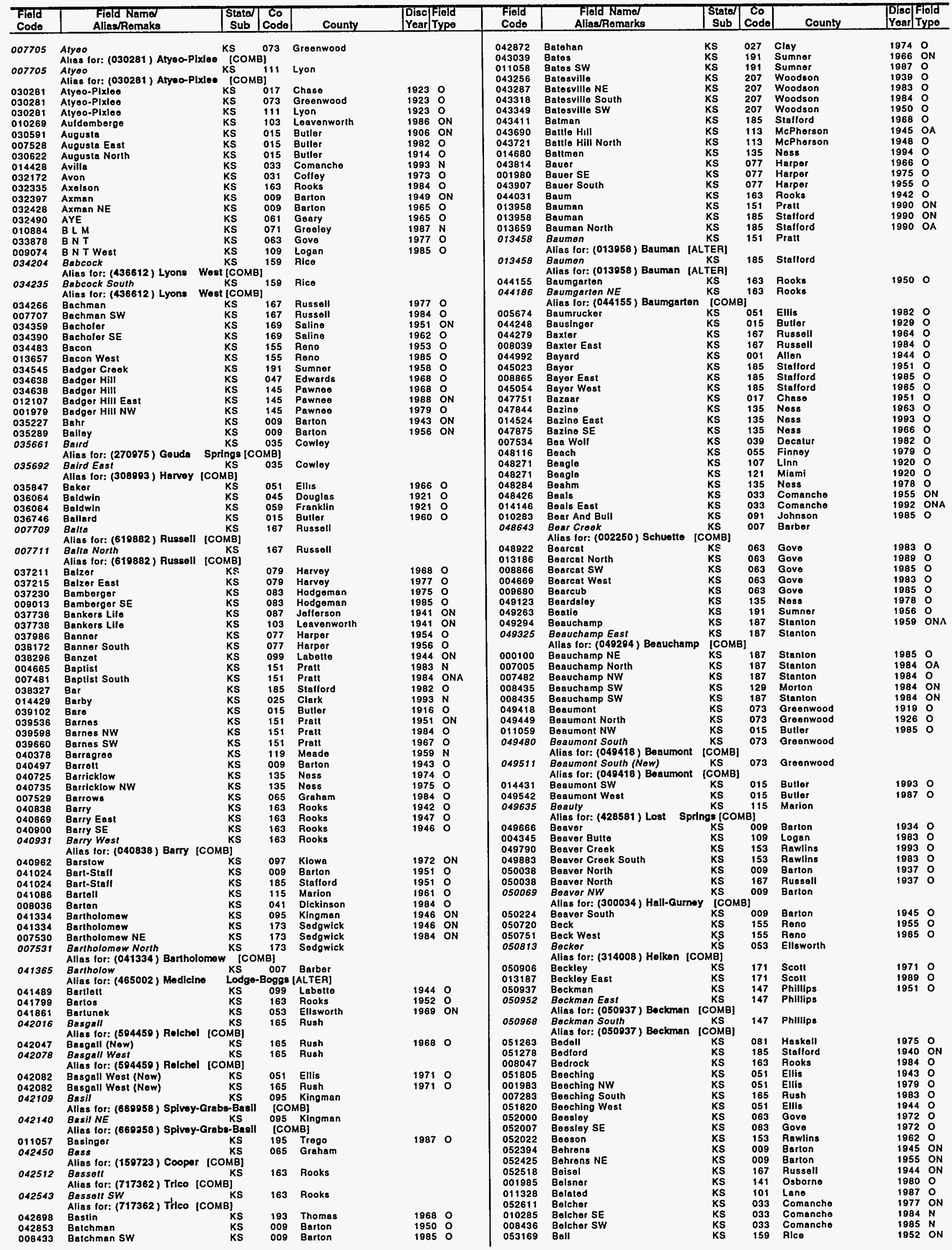


KANSAS

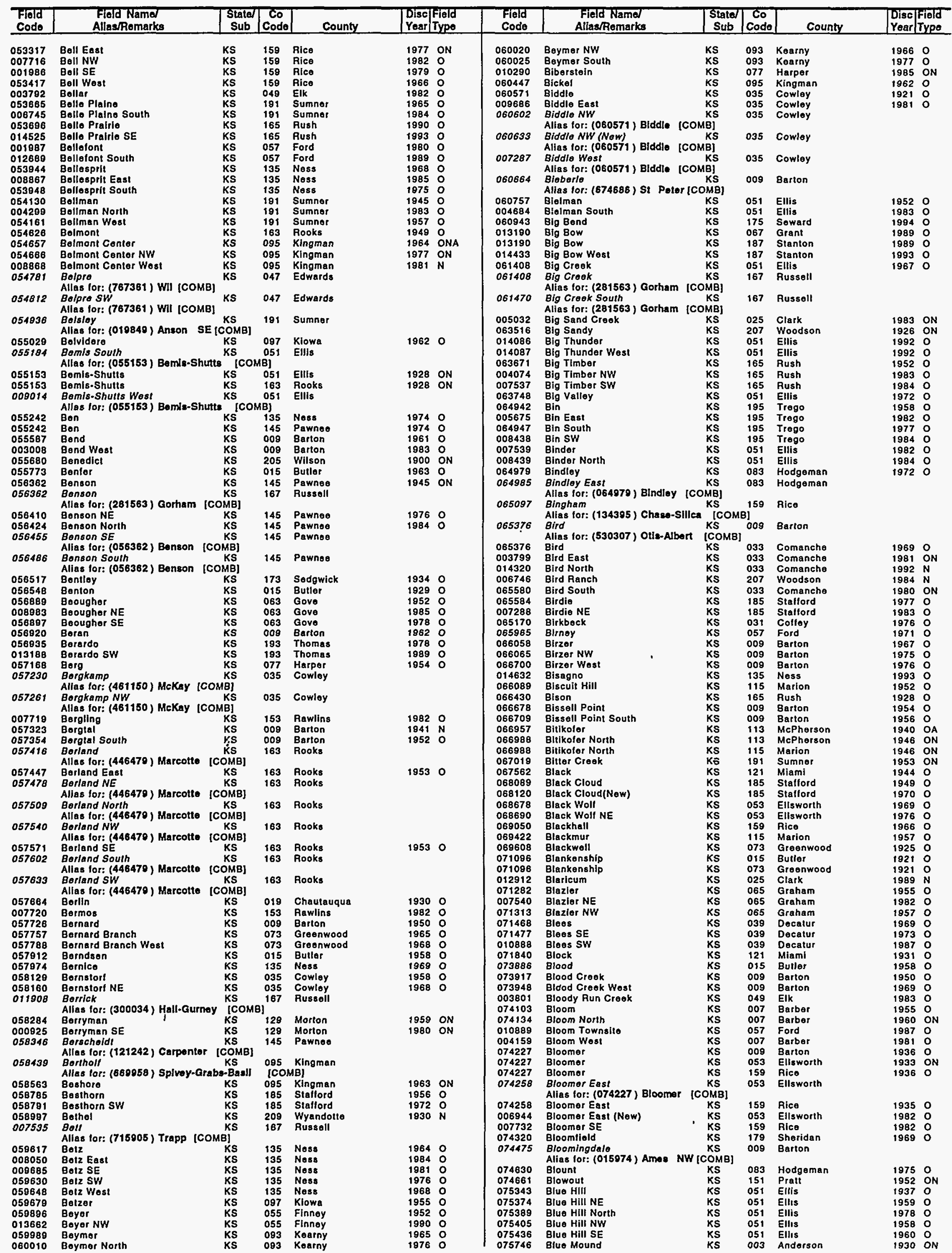


KANSAS

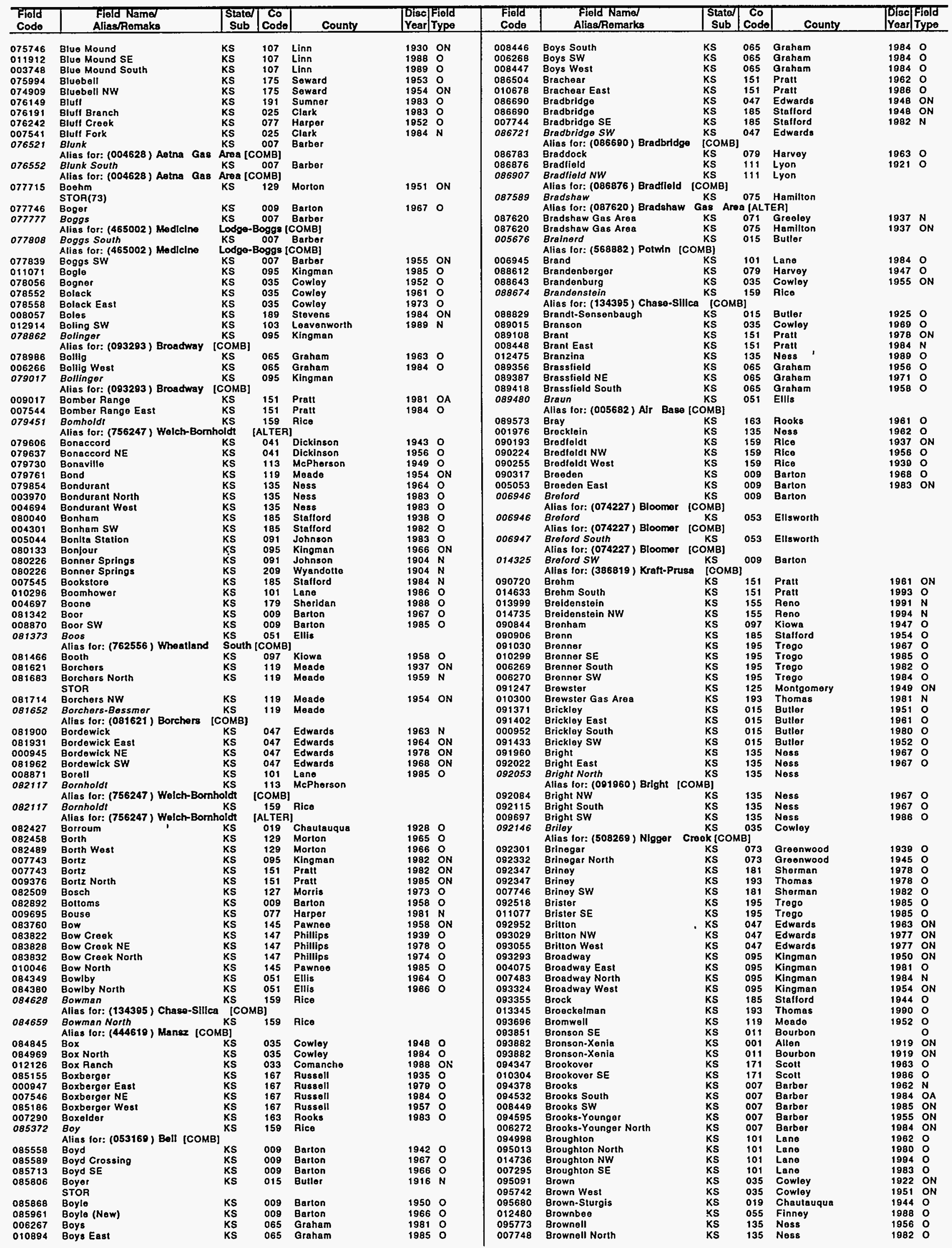


KANSAS

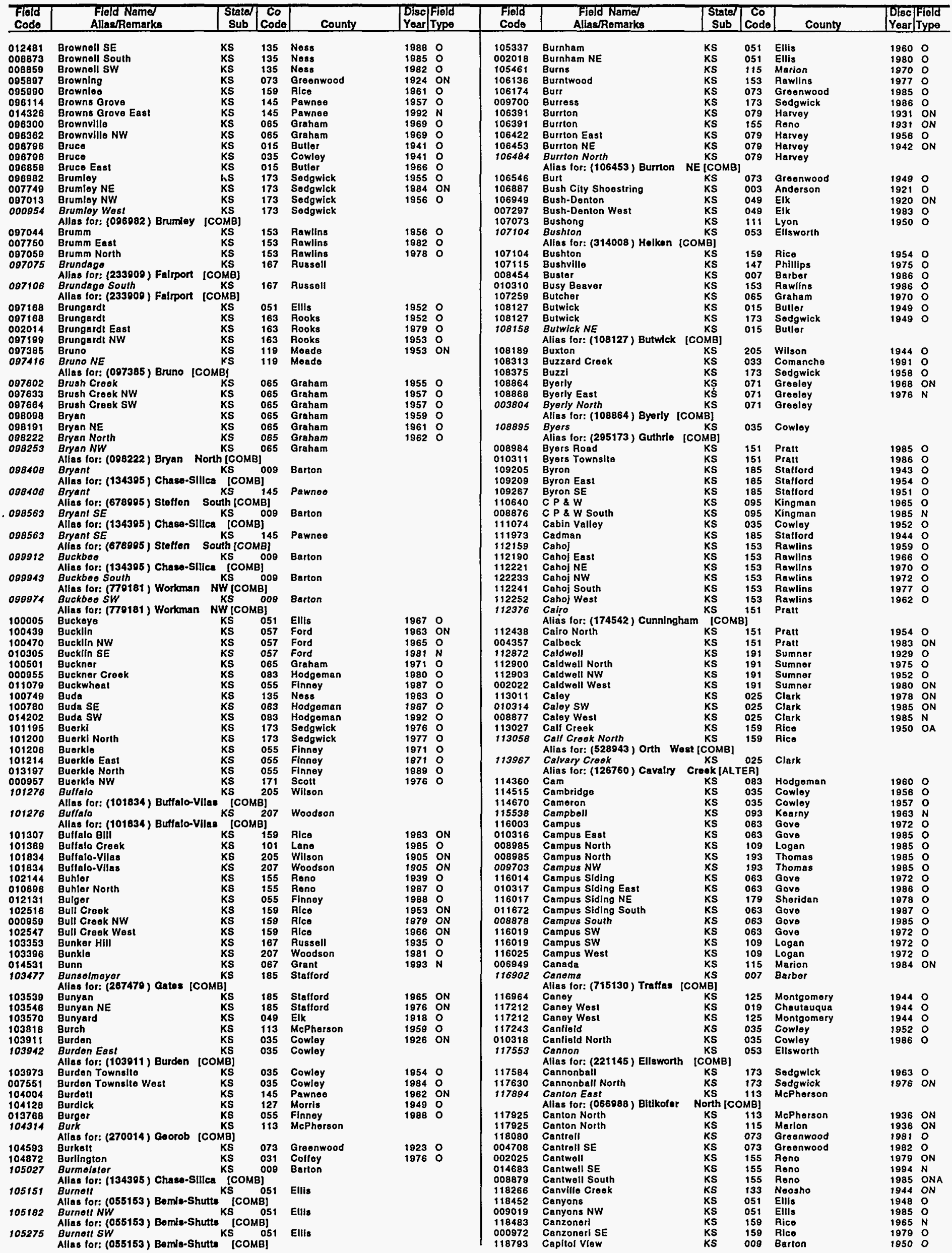


KANSAS

\begin{tabular}{|c|c|c|}
\hline $\begin{array}{l}\text { Ficld } \\
\text { Code }\end{array}$ & $\begin{array}{l}\text { Field Name } \\
\text { Alias/Remaks }\end{array}$ & $\begin{array}{c}\text { Statel } \\
\text { Sub }\end{array}$ \\
\hline $\begin{array}{l}119289 \\
119304\end{array}$ & Car & ks \\
\hline $\begin{array}{l}119304 \\
119311\end{array}$ & Car sw & ks \\
\hline & $\begin{array}{l}\text { Car West } \\
\text { Alías for: (119304) Car SW [C }\end{array}$ & KS \\
\hline 119382 & $\begin{array}{l}\text { Alias for: (119304) Car SW [C } \\
\text { Caraway }\end{array}$ & \\
\hline 119816 & $\begin{array}{l}\text { Caraway } \\
\text { Caray }\end{array}$ & $\begin{array}{l}\text { KS } \\
\text { KS }\end{array}$ \\
\hline 002027 & Carl & KS \\
\hline 004709 & Carlson & KS \\
\hline 120684 & $\begin{array}{l}\text { Carmi } \\
\text { Alıas for. (349959) luka-Carmi }\end{array}$ & $\begin{array}{l}\text { KS } \\
\text { [COMB] }\end{array}$ \\
\hline 011086 & Carmi Corner & KS \\
\hline $\begin{array}{l}120746 \\
121211\end{array}$ & Cermichsel & ks \\
\hline 121211 & Carothers & KS \\
\hline 121242 & Carpentor & Ks \\
\hline 121242 & Carponter & KS \\
\hline 121273 & $\begin{array}{l}\text { Carpenter North } \\
\text { Alias for: (121242) Carpenter }\end{array}$ & $\begin{array}{l}\text { KS } \\
\text { [COMB] }\end{array}$ \\
\hline 121304 & Carpenter SE & KS \\
\hline 121304 & $\begin{array}{l}\text { Alias tor: }(121242) \text { Carpenter } \\
\text { Carpenter SE }\end{array}$ & $\begin{array}{l}\text { [COMB] } \\
\mathrm{KS}\end{array}$ \\
\hline & $\begin{array}{l}\text { Carpenler SE } \\
\text { Alıas for: (121242) Carpenter }\end{array}$ & [COMB] \\
\hline 121366 & Carpontor Wost & KS \\
\hline 007753 & $\begin{array}{l}\text { Alias for: (121242) Carpenter } \\
\text { Carrol J }\end{array}$ & (СоM8) \\
\hline & $\begin{array}{l}\text { Carrol J } \\
\text { Carroll }\end{array}$ & KS \\
\hline 121707 & Carroll & KS \\
\hline $1217+3$ & Carroll East & ks \\
\hline 121738 & $\begin{array}{l}\text { Carroll North } \\
\text { Alias tor: }(121707) \text { Carroll loc }\end{array}$ & $\begin{array}{l}\text { KS } \\
\text { OMB] }\end{array}$ \\
\hline 121769 & Carroll SE & Ks \\
\hline 121800 & Carroll sw & ks \\
\hline $\begin{array}{l}122048 \\
002029\end{array}$ & $\begin{array}{l}\text { Carter } \\
\text { Garthur }\end{array}$ & $\begin{array}{l}\text { KS } \\
\text { KS }\end{array}$ \\
\hline 122955 & Cartmill & KS \\
\hline 004614 & Gartmill North & ks \\
\hline 010322 & Cartmill NW & KS \\
\hline 004076 & Cartmill South & $\begin{array}{l}\text { KS } \\
\text { KS }\end{array}$ \\
\hline $\begin{array}{l}010323 \\
123102\end{array}$ & $\begin{array}{l}\text { Carimill West } \\
\text { Carver-Robbins }\end{array}$ & $\begin{array}{l}\text { KS } \\
\text { KS }\end{array}$ \\
\hline 007754 & Carver-Robbins East & KS \\
\hline 123133 & $\begin{array}{l}\text { Carver-Robbins NE } \\
\text { Alias for: }(123102) \text { Carver-Rob }\end{array}$ & $\begin{array}{c}\text { KS } \\
\text { bbins }\end{array}$ \\
\hline 000164 & Carvar-Robbins West & KS \\
\hline 000164 & Carver-Robbins West & KS \\
\hline $\begin{array}{l}123164 \\
123681\end{array}$ & $\begin{array}{l}\text { Carwood } \\
\text { Case }\end{array}$ & KS \\
\hline 123691 & Caso & KS \\
\hline 123722 & Casament & KS \\
\hline 007553 & Casoment East & KS \\
\hline 123753 & Casoy & KS \\
\hline 011088 & Cash City & KS \\
\hline 011088 & Cash City & ks \\
\hline $\begin{array}{l}124063 \\
124094\end{array}$ & $\begin{array}{l}\text { Caskay } \\
\text { Casloy }\end{array}$ & $\begin{array}{l}\text { KS } \\
\text { KS }\end{array}$ \\
\hline & Alias lor: (093293) Brosdway & [COMB] \\
\hline 124652 & Caster & ks \\
\hline 125055 & Castle Rock & KS \\
\hline 007554 & Castle Rock North & KS \\
\hline 125158 & Castleton & KS \\
\hline 125165 & Castleton South & KS \\
\hline 125985 & Catharine & ks \\
\hline 125991 & Catharine East & KS \\
\hline & Alias for: (125085) Catharine & [COMB] \\
\hline 125995 & Catharine East (Now) & KS \\
\hline $\begin{array}{l}126016 \\
126047\end{array}$ & $\begin{array}{l}\text { Catharine NW } \\
\text { Cathorino South }\end{array}$ & $\begin{array}{l}\text { KS } \\
\text { KS }\end{array}$ \\
\hline $\begin{array}{l}126047 \\
126078\end{array}$ & $\begin{array}{l}\text { Catharine South } \\
\text { Gatharine Townsite }\end{array}$ & $\begin{array}{l}\text { KS } \\
\text { KS }\end{array}$ \\
\hline 126109 & Catheart & KS \\
\hline 010324 & Cante & KS \\
\hline 126760 & Cavalry Croak & KS \\
\hline 004304 & Cavalty Craek SE & KS \\
\hline 126822 & Cava & KS \\
\hline 126946 & Cava Wost & Ks \\
\hline 127590 & Codar & KS \\
\hline 127714 & Codar Bluft & KS \\
\hline 003809 & Cedar Blufl East & KS \\
\hline 127745 & Codar Crook & KS \\
\hline 008459 & Cedar Draw & KS \\
\hline 128303 & Codar View & KS \\
\hline 128305 & Codar Viow East & KS \\
\hline 128312 & Codar View NE & KS \\
\hline $\begin{array}{l}128320 \\
008077\end{array}$ & $\begin{array}{l}\text { Codar View SE } \\
\text { Codar Vlew SW }\end{array}$ & $\begin{array}{l}\text { KS } \\
\text { KS }\end{array}$ \\
\hline 008880 & $\begin{array}{l}\text { Codar Vlaw SW } \\
\text { Codar View West }\end{array}$ & KS \\
\hline 128582 & Cedarvale & KS \\
\hline 128582 & Cedarvale & ks \\
\hline 128613 & Codarvale North & KS \\
\hline 128613 & Codarvale North & xs \\
\hline 007555 & Cedarvale NW & KS \\
\hline 128644 & Cedarvale South & ks \\
\hline & & [COMB] \\
\hline $\begin{array}{l}007556 \\
007762\end{array}$ & $\begin{array}{l}\text { Cedarvalo South (Now) } \\
\text { Codaryalo Sw }\end{array}$ & $\begin{array}{l}\text { KS } \\
\text { KS }\end{array}$ \\
\hline $\begin{array}{l}007762 \\
128675\end{array}$ & $\begin{array}{l}\text { Cadarvalo SW } \\
\text { Cedarvale Wost }\end{array}$ & KS \\
\hline $\begin{array}{l}128675 \\
128892\end{array}$ & $\begin{array}{l}\text { Cedarvale Wost } \\
\text { Colia }\end{array}$ & KS \\
\hline 004710 & Celis South & ks \\
\hline 129202 & $\begin{array}{l}\text { Centennial } \\
\text { Alies for: (283718) Graham }\end{array}$ & $\begin{array}{c}\text { KS } \\
\text { сомB! }\end{array}$ \\
\hline 129202 & Contennial & ks \\
\hline 129233 & $\begin{array}{l}\text { Contennial North } \\
\text { Altas for: (283718) Graham ic }\end{array}$ & $\begin{array}{c}\mathrm{KS} \\
\text { COMB! }\end{array}$ \\
\hline 129264 & Center & $\mathrm{ks}$ \\
\hline 129295 & Conter Hill & KS \\
\hline 129357 & Conter NW & KS \\
\hline 129543 & $\begin{array}{l}\text { Center West } \\
\text { Alias for: (538159) Panoma }\end{array}$ & Ga: Ases \\
\hline 129698 & Centerview & $\mathrm{kS}$ \\
\hline 129729 & Centerville & KS \\
\hline 129729 & Conterville & KS \\
\hline & Centropolis & \\
\hline $\begin{array}{l}130349 \\
130380\end{array}$ & $\begin{array}{l}\text { Cophas } \\
\text { Cephas North }\end{array}$ & $\begin{array}{l}\text { KS } \\
\text { KS }\end{array}$ \\
\hline 008986 & $\begin{array}{l}\text { Corcanada } \\
\text { Alies tor: }(009555) \text { Chiof IR }\end{array}$ & $\begin{array}{l}\text { KS } \\
\text { NAM] }\end{array}$ \\
\hline
\end{tabular}


KANSAS

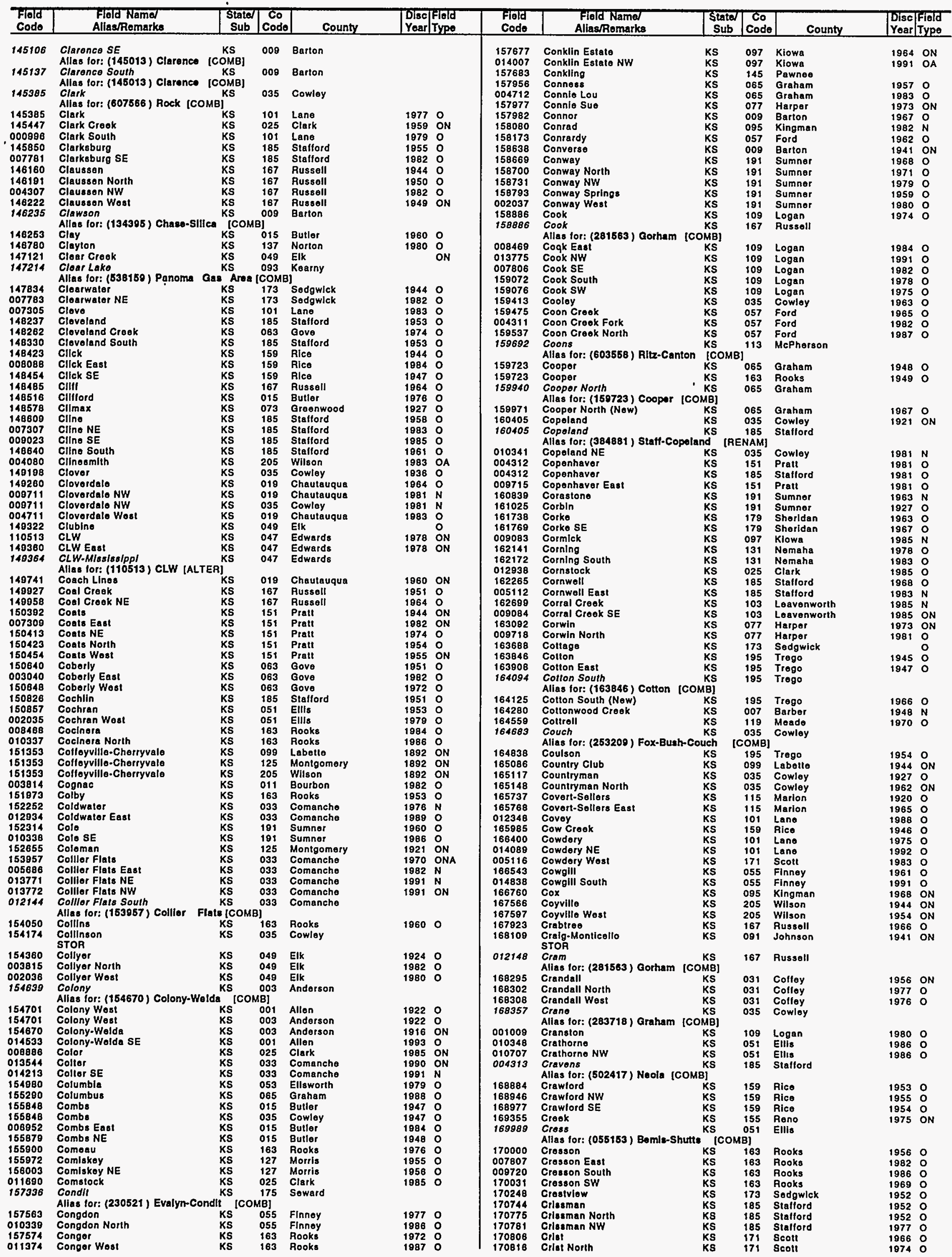


KANSAS

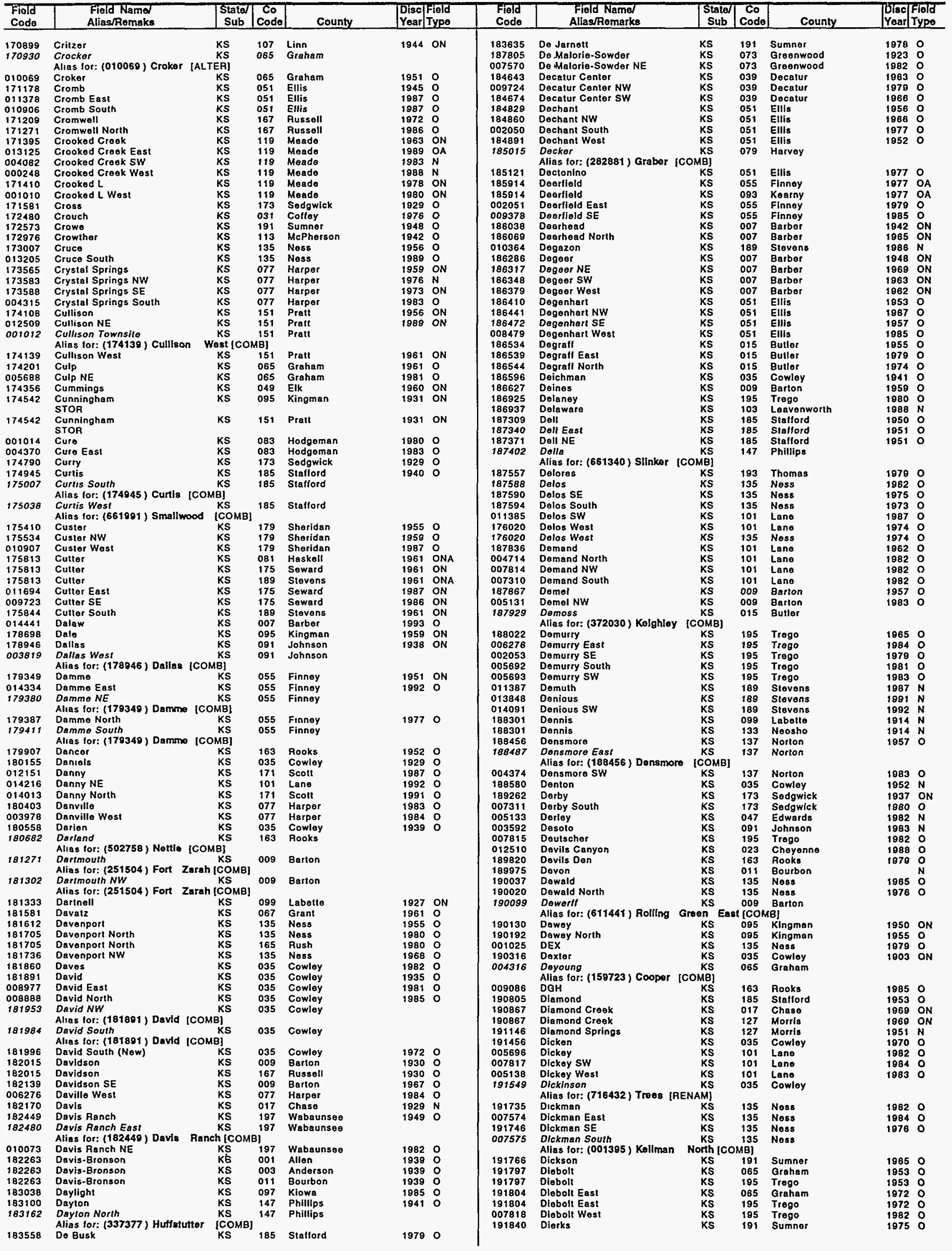


KANSAS

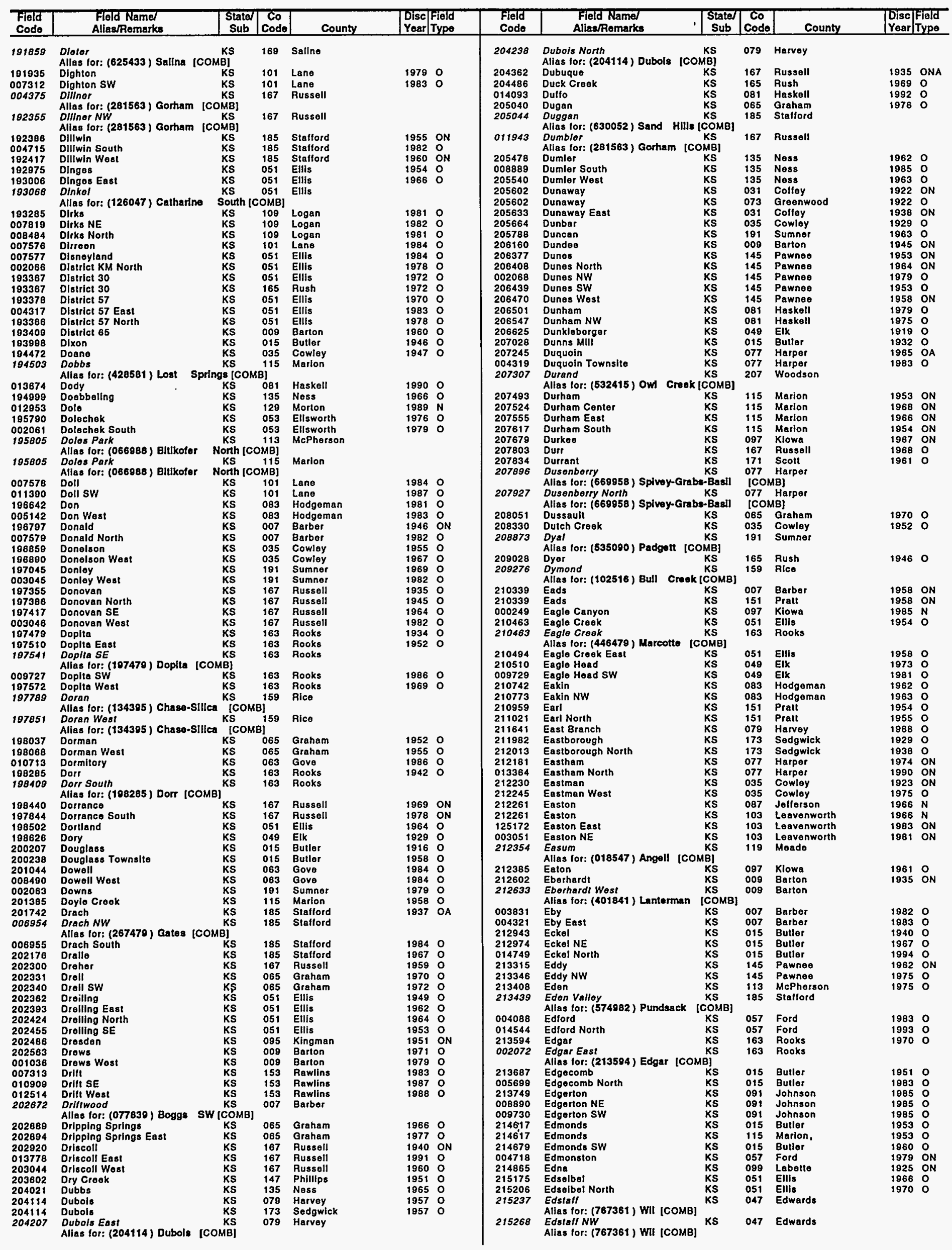


KANSAS

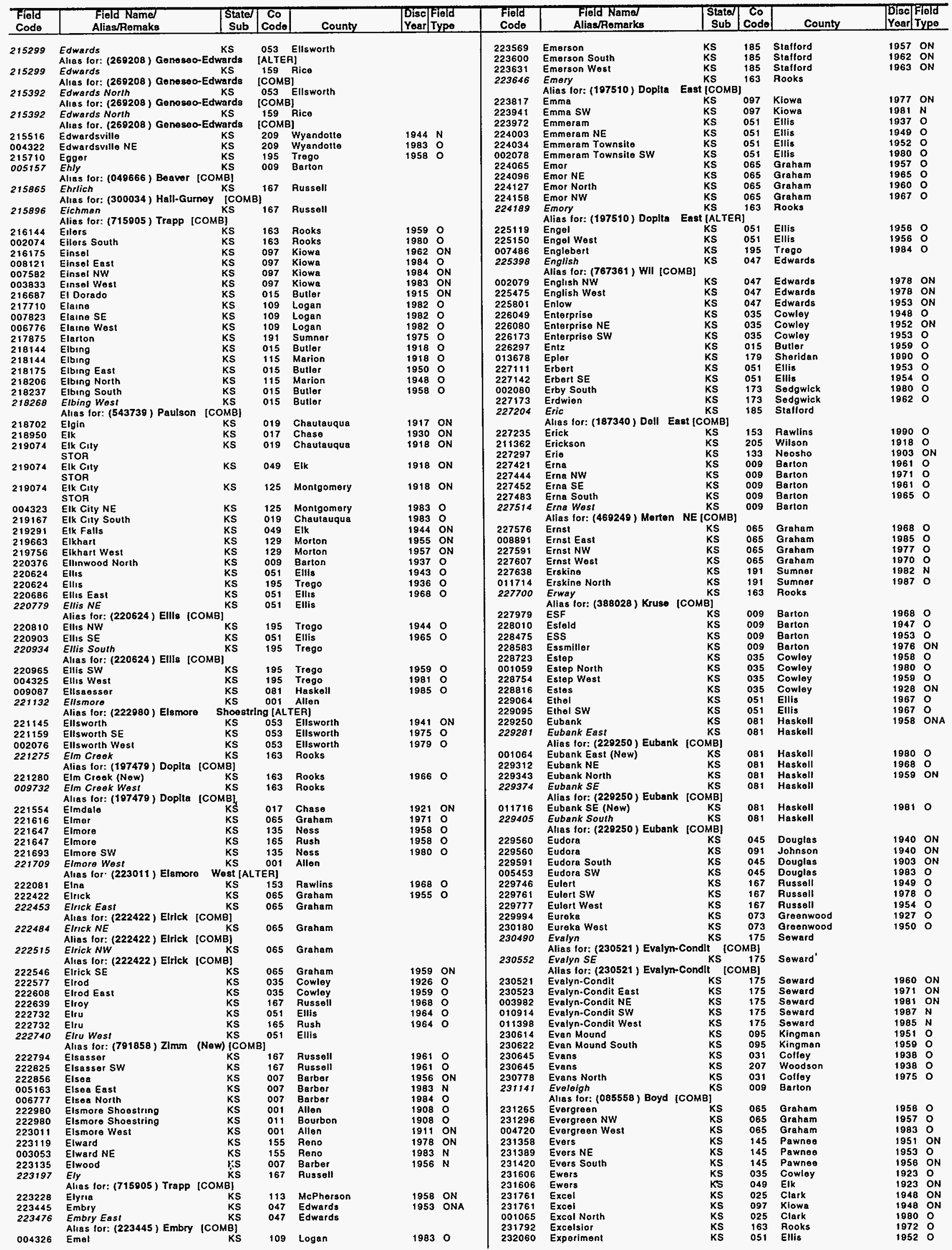


KANSAS

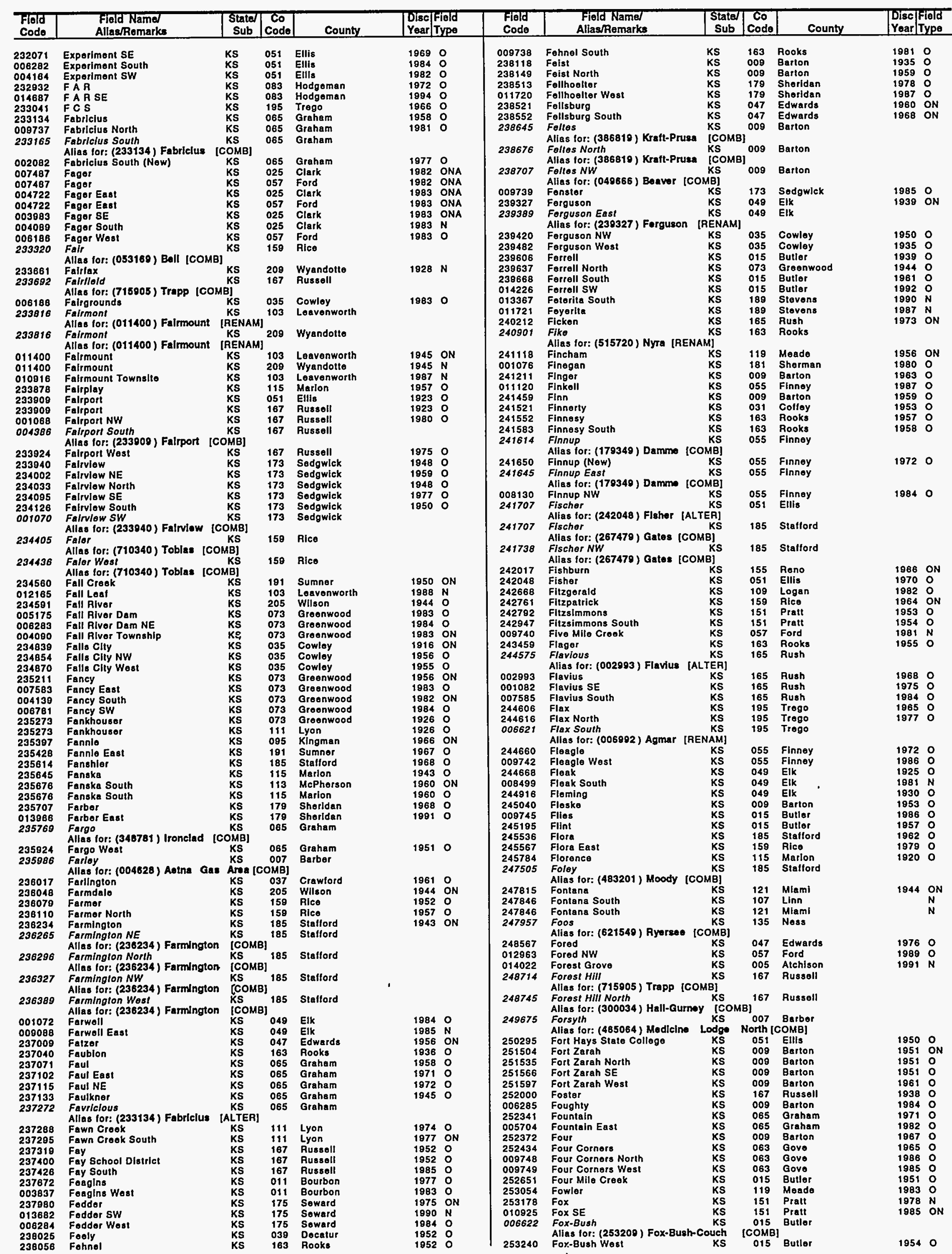




\section{KANSAS}

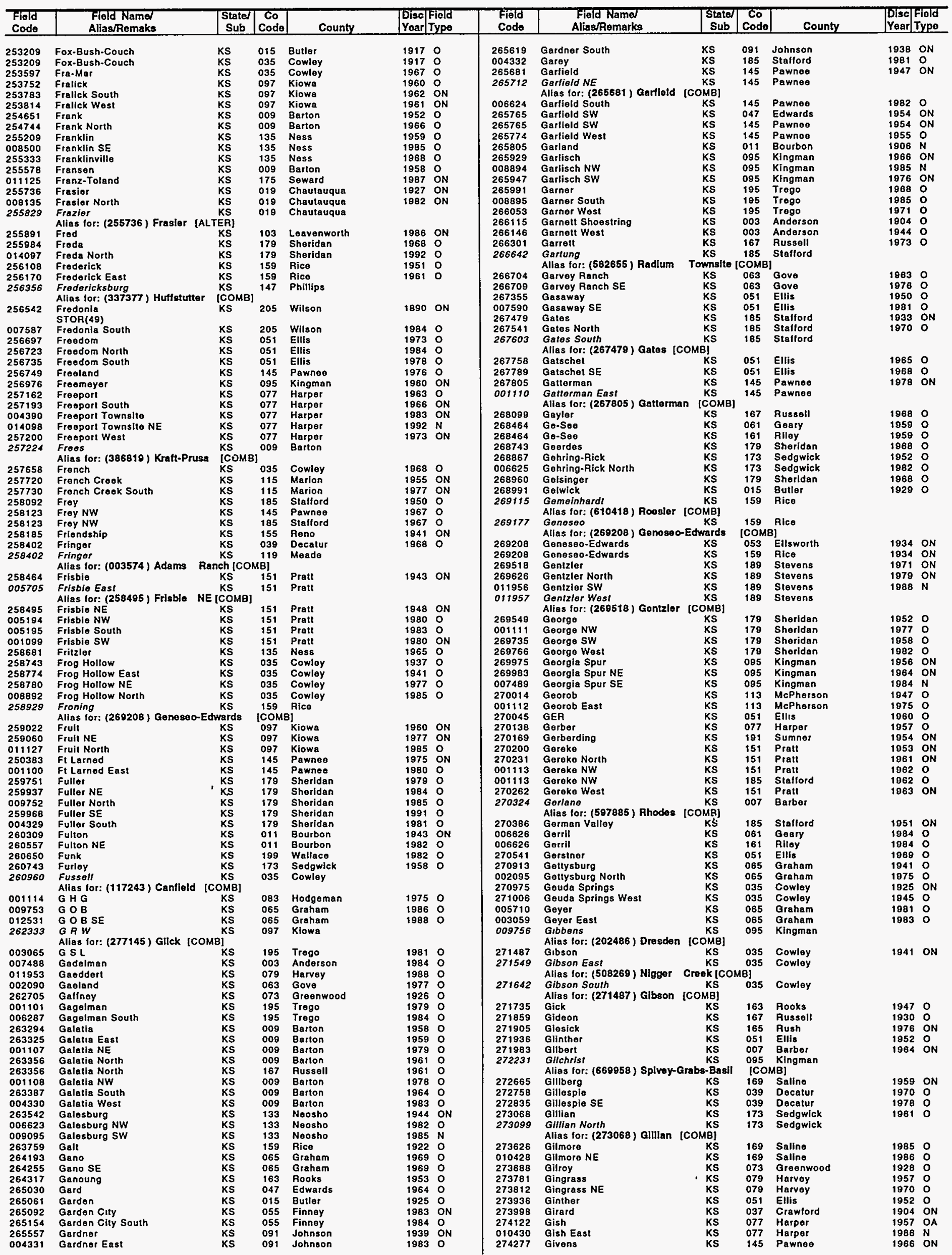


KANSAS

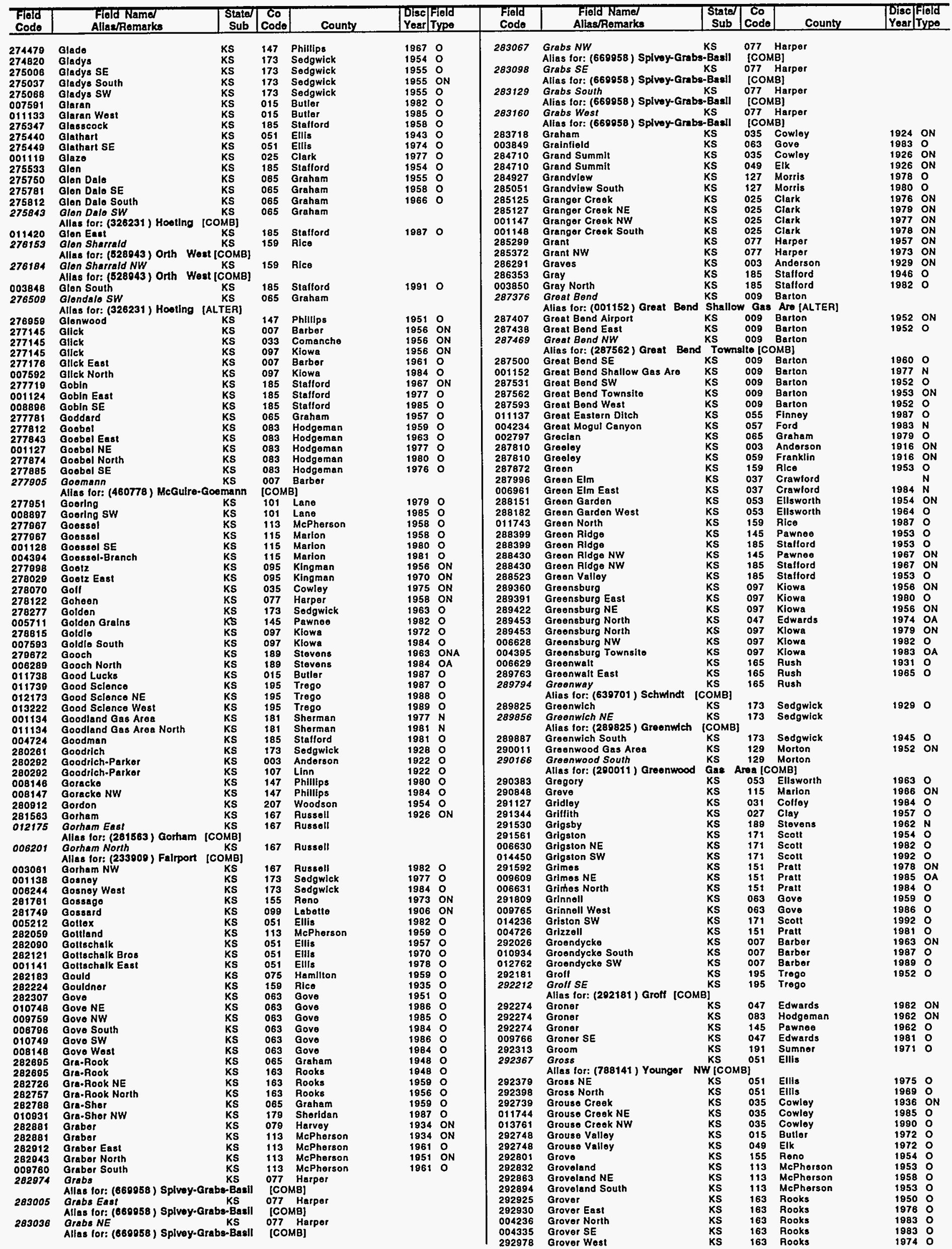


KANSAS

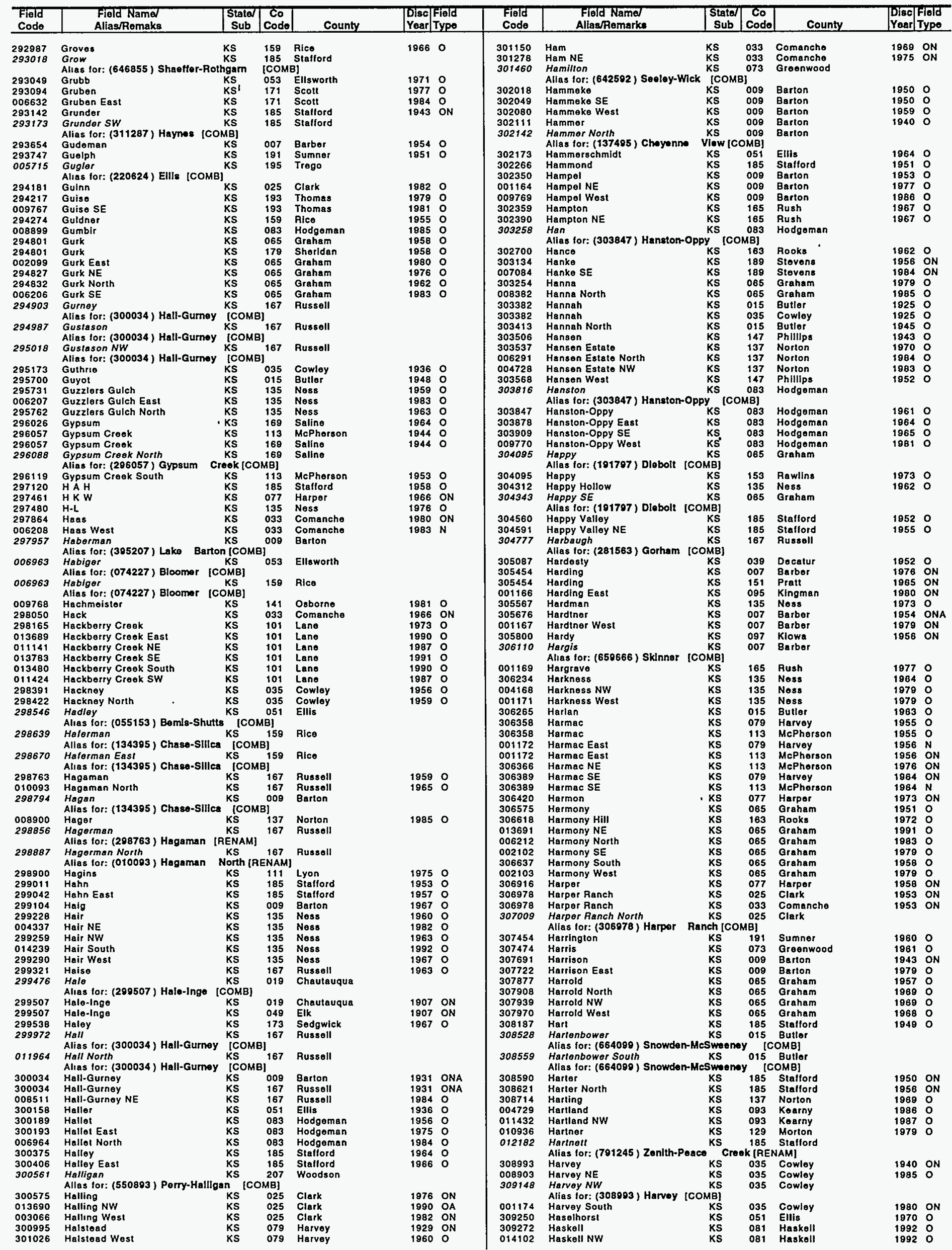


KANSAS

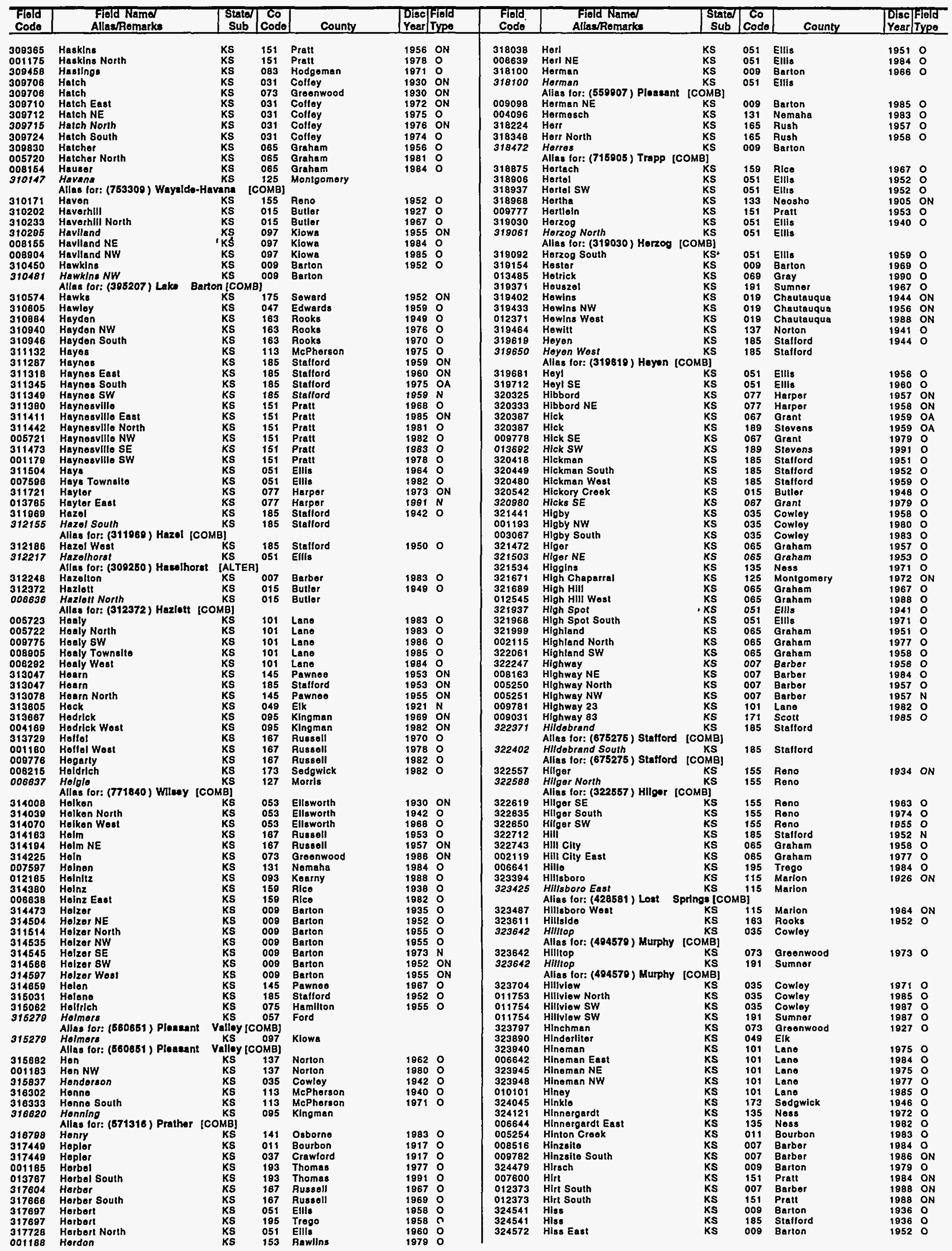


KANSAS

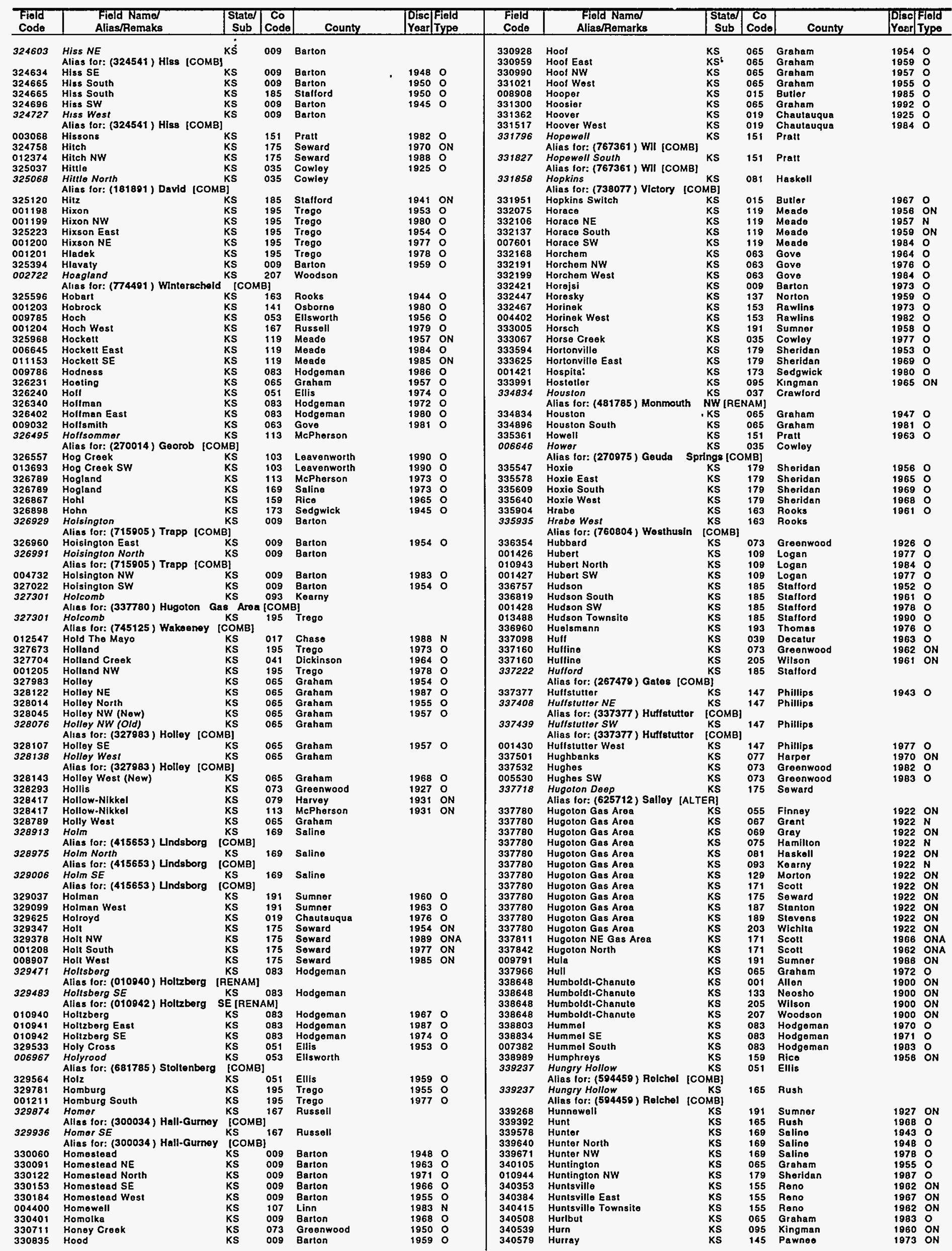


KANSAS

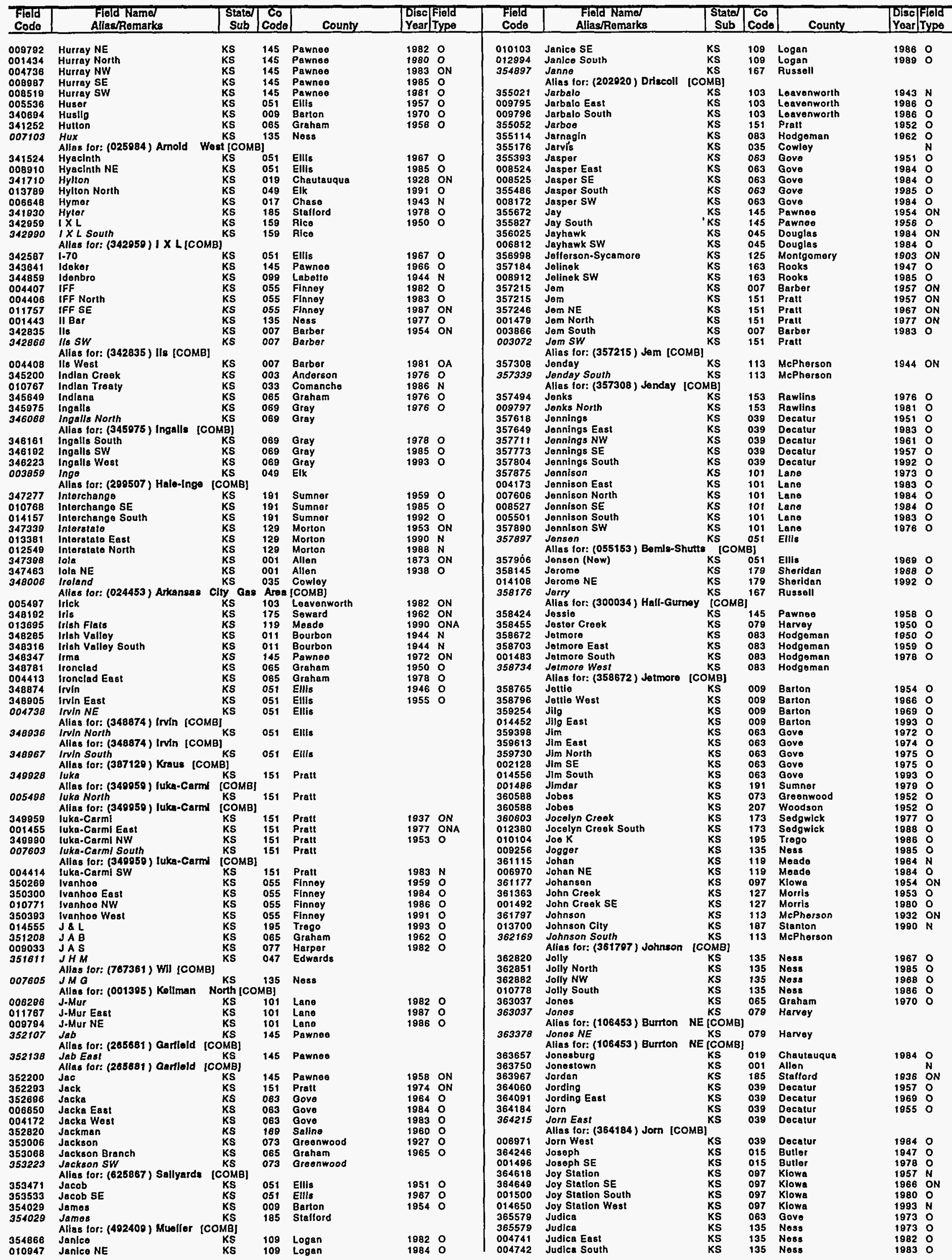


KANSAS

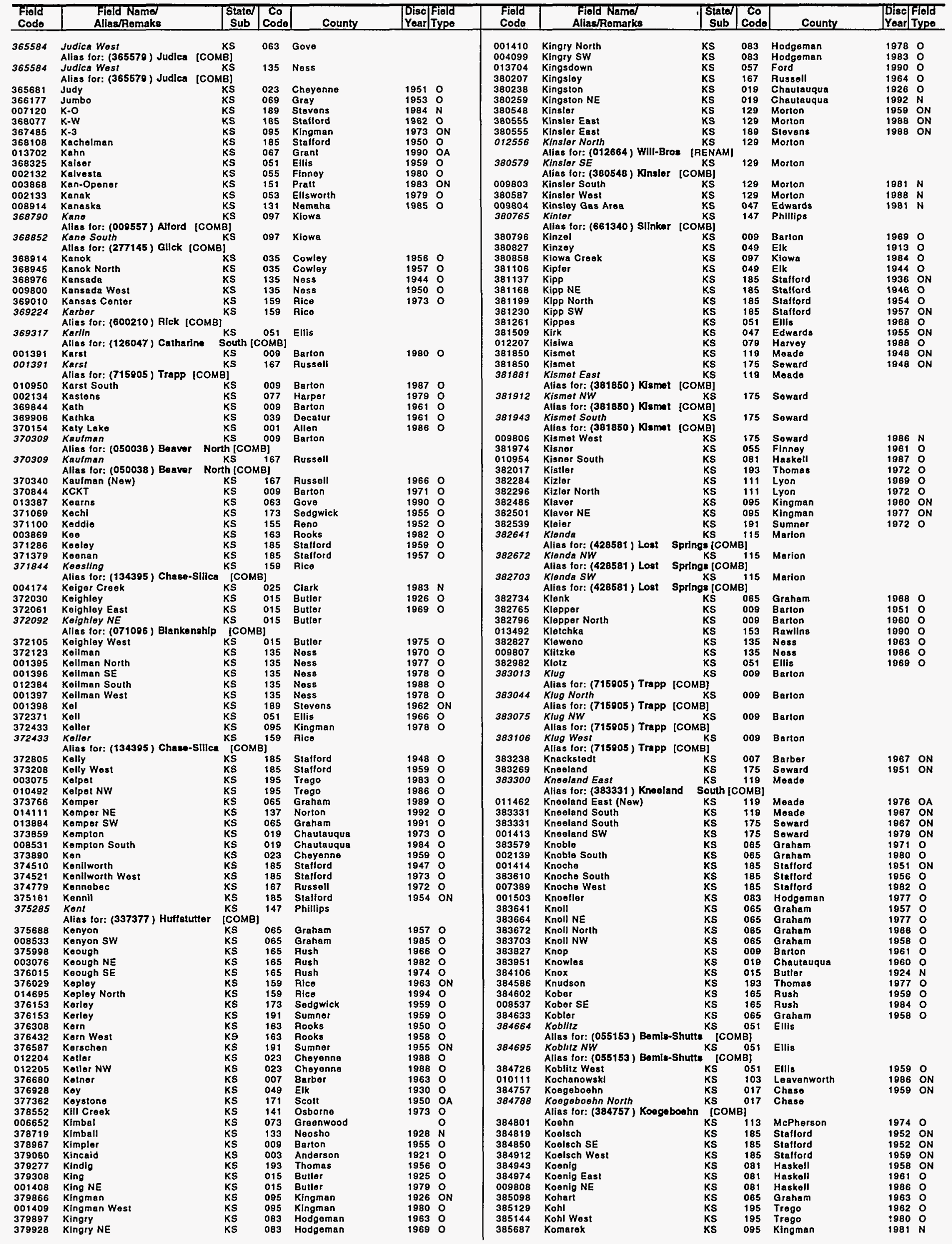


KANSAS

\begin{tabular}{|c|c|c|c|c|c|}
\hline $\begin{array}{l}\text { Flold } \\
\text { Code }\end{array}$ & $\begin{array}{l}\text { Field Named } \\
\text { Allas/Aemarks }\end{array}$ & $\begin{array}{c}\text { Statol } \\
\text { Sub }\end{array}$ & $\begin{array}{c}\text { Co } \\
\text { Code }\end{array}$ & County & | Dise \\
\hline $\begin{array}{l}385718 \\
385935 \\
007400 \\
004100 \\
005511\end{array}$ & $\begin{array}{l}\text { Kompus } \\
\text { Konda } \\
\text { Konda North } \\
\text { Konda SE } \\
\text { Konda Wost }\end{array}$ & $\begin{array}{l}\text { KS } \\
\text { KS } \\
\text { KS } \\
\text { KS } \\
\text { KS }\end{array}$ & $\begin{array}{l}153 \\
057 \\
057 \\
057 \\
057\end{array}$ & $\begin{array}{l}\text { Rawlins } \\
\text { Ford } \\
\text { Ford } \\
\text { Ford } \\
\text { Ford }\end{array}$ & $\begin{array}{l}1959 \\
1956 \\
1984 \\
1982 \\
1983\end{array}$ \\
\hline 007491 & Konecny & KS & 000 & Barton & 1982 \\
\hline 001505 & Konold & KS & 151 & Pratt & 1979 \\
\hline 006821 & Konold East & KS & 151 & Pratt & 1984 \\
\hline $\begin{array}{l}386245 \\
386276\end{array}$ & $\begin{array}{l}\text { Koopman } \\
\text { Koolz }\end{array}$ & $\begin{array}{l}\text { KS } \\
\text { KS }\end{array}$ & $\begin{array}{l}009 \\
053\end{array}$ & $\begin{array}{l}\text { Barton } \\
\text { Ellsworth }\end{array}$ & $\begin{array}{l}1953 \\
1965\end{array}$ \\
\hline 014032 & Kopriva & KS & 153 & Rawlins & 1991 \\
\hline $\begin{array}{l}388431 \\
388462\end{array}$ & $\begin{array}{l}\text { Kostor } \\
\text { Kostnar }\end{array}$ & $\begin{array}{l}\text { KS } \\
\text { KS }\end{array}$ & $\begin{array}{l}179 \\
095\end{array}$ & $\begin{array}{l}\text { Sheridan } \\
\text { Kingman }\end{array}$ & $\begin{array}{l}1968 \\
1960\end{array}$ \\
\hline 386471 & Kostner NW & KS & 095 & Kingman & 1974 \\
\hline 386524 & Kowalsky & KS & 009 & Barton & 1941 \\
\hline 386524 & Kowalsky & KS & 185 & Stafford & 1941 \\
\hline 386555 & $\begin{array}{l}\text { Kowalsky NW } \\
\text { Alias for: (386524) Kowalsky }\end{array}$ & $\begin{array}{l}\text { KS } \\
\text { [COMB] }\end{array}$ & 000 & Barton & \\
\hline $\begin{array}{l}386586 \\
386819\end{array}$ & $\begin{array}{l}\text { Kowatsky SW } \\
\text { Kraft-Prusa }\end{array}$ & $\begin{array}{l}\text { KS } \\
\text { KS }\end{array}$ & $\begin{array}{l}185 \\
009\end{array}$ & $\begin{array}{l}\text { Staflord } \\
\text { Barton }\end{array}$ & $\begin{array}{l}1950 \\
1937\end{array}$ \\
\hline 386819 & Kraft-Prusa & KS & 053 & Ellsworth & 1937 \\
\hline $\begin{array}{l}386818 \\
986850\end{array}$ & $\begin{array}{l}\text { Kraft-Prusa } \\
\text { Krall-Prusa East }\end{array}$ & KS & $\begin{array}{l}167 \\
053\end{array}$ & $\begin{array}{l}\text { Russell } \\
\text { Ellaworth }\end{array}$ & 1937 \\
\hline 388881 & Kraft-Prusa NE & KS & 009 & Barton & 1941 \\
\hline 014353 & $\begin{array}{l}\text { Kraft-Prusa NE } \\
\text { Kraft-Prusa SE } \\
\text { Allas for: }(386819) \text { Kraft-Prusa }\end{array}$ & $\begin{array}{l}\text { KS } \\
\text { [CоMB] }\end{array}$ & $\begin{array}{l}053 \\
009\end{array}$ & $\begin{array}{l}\text { Ellsworth } \\
\text { Barton }\end{array}$ & \\
\hline & Kramer-Stern & KS & 015 & Butler & 1926 \\
\hline $\begin{array}{l}386974 \\
387005\end{array}$ & $\begin{array}{l}\text { Kramor-Stern South } \\
\text { Kramp }\end{array}$ & $\begin{array}{l}\text { Ks } \\
\text { Ks }\end{array}$ & $\begin{array}{l}015 \\
008\end{array}$ & $\begin{array}{l}\text { Butlar } \\
\text { Barton }\end{array}$ & 1947 \\
\hline & Allas for: $(674686)$ st Peter [CC & & & & \\
\hline $\begin{array}{l}008190 \\
387036\end{array}$ & $\begin{array}{l}\text { Krank } \\
\text { Krankonberg }\end{array}$ & $\begin{array}{l}\text { KS } \\
\text { KS }\end{array}$ & $\begin{array}{l}083 \\
083\end{array}$ & $\begin{array}{l}\text { Hodgeman } \\
\text { Hodgeman }\end{array}$ & 1974 \\
\hline & Allias for: (008190) Krank (REN & NAMI & & & \\
\hline 387036 & Krankenberg & KS & 185 & Stafford & 1956 \\
\hline 001508 & Krantz SW & & 135 & Ness & 1980 \\
\hline $\begin{array}{l}387087 \\
387129\end{array}$ & $\begin{array}{l}\text { Kranz } \\
\text { Kraus }\end{array}$ & $\begin{array}{l}\text { KS } \\
\text { KS }\end{array}$ & $\begin{array}{l}195 \\
051\end{array}$ & $\begin{array}{l}\text { Trago } \\
\text { Ellis }\end{array}$ & $\begin{array}{l}1971 \\
1936\end{array}$ \\
\hline 387133 & Kraus East & KS & 051 & Ellis & 1975 \\
\hline 387160 & Kraus North & KS & 051 & Ellis & \\
\hline & Allas for: (387120) Kraus [COM & $M B]$ & & & \\
\hline 387191 & $\begin{array}{l}\text { Kraus NW } \\
\text { Alias for: (387129) Kraus ICOM }\end{array}$ & ks & 051 & Ellis & \\
\hline 004948 & $\begin{array}{l}\text { Alias for: (387129) Kraus [COM } \\
\text { Kraus Wost }\end{array}$ & & 051 & Ellis & \\
\hline & Allas for: (387129) Kraus (COM & MB] & & & \\
\hline 387242 & Krehblel & KS & 113 & Mapharson & 1973 \\
\hline 387687 & Kroutzer & KS & 051 & Ellis & 1968 \\
\hline 387748 & Krior & KS & 000 & Barton & \\
\hline & Alles for: $(386819)$ Kraft-Prusa & - [СОМв] & & & \\
\hline 387780 & Kriloy & KS & 163 & Rooks & 1943 \\
\hline 387842 & Kroegor & KS & 195 & Trego & 1956 \\
\hline 001509 & Krooger North & KS & 185 & Trogo & 1979 \\
\hline 001510 & Krooger South & ks & 195 & Trago & 1979 \\
\hline 001511 & Kropp & KS & 083 & Hodgeman & 1977 \\
\hline 387035 & $\begin{array}{l}\text { Kruckenberg } \\
\text { Alles for: (530307) Otls-Albert }\end{array}$ & $\begin{array}{l}\text { KS } \\
\text { [СОМв] }\end{array}$ & 008 & Barton & \\
\hline 387986 & Krue & KS & 009 & Barton & 1968 \\
\hline 387097 & Krueger & Ks & 051 & Ellis & 1948 \\
\hline 387007 & Kruegor & KS & 163 & Rooks & 1948 \\
\hline $00980 \theta$ & Kruagor SW & KS & 051 & Ellis & 1981 \\
\hline 388007 & Krug & KS & 167 & Russell & 1972 \\
\hline 380028 & Kruse & KS & 163 & Rooks & 1928 \\
\hline 388058 & Kruse NW & KS & 163 & Rooks & 1951 \\
\hline 014456 & Kuendo & KS & 129 & Morton & 1993 \\
\hline 388493 & Kumberg & ks & 007 & Batber & 1976 \\
\hline 000235 & Kumberg SE & KS & 007 & Barber & 1988 \\
\hline 388571 & Kune & KS & 167 & Russell & 1957 \\
\hline 007300 & Kune North & ks & 167 & Russell & 1982 \\
\hline 007381 & Kuno South & KS & 167 & Russoll & 1981 \\
\hline 388602 & Kune sw & KS & 967 & Russoll & 1968 \\
\hline 001863 & Kunz & KS & 051 & Ellis & 1950 \\
\hline 388605 & Kuppotz & KS & 185 & Trego & 1965 \\
\hline 010113 & Kuska & Ks & 193 & Thomas & 1986 \\
\hline 388818 & Kuske & KS & 173 & Sodgwick & 1929 \\
\hline 388850 & Kuske Norlh & KS & 173 & Sedgwick & \\
\hline & Allas for: (388819) Kuske [COM & DMBI & & & \\
\hline 388881 & Kuske Wost & KS & 173 & Sedgwick & 1969 \\
\hline 388912 & Kutina & Ks & 185 & Trogo & 1855 \\
\hline 001513 & Kutina NW & KS & 185 & Trogo & 1978 \\
\hline 010501 & Kutina South & KS & 195 & Trego & 1986 \\
\hline 008297 & Kutina sw & KS & 195 & Trego & 1984 \\
\hline 388943 & Kutlna West & KS & 195 & Trego & 1965 \\
\hline 389463 & $\begin{array}{l}\text { Kysor } \\
\text { Kytint }\end{array}$ & KS & $\begin{array}{l}180 \\
095\end{array}$ & KIngman & 1964 \\
\hline 007126 & $L F J$ & KS & 035 & Cowley & \\
\hline & Alles for: $(439690)$ Maddlx & lorth I & & & \\
\hline 008838 & L F J East & KS & 035 & Cowloy & 1878 \\
\hline 002677 & LF J NW & $\begin{array}{l}\text { KS } \\
\text { KS }\end{array}$ & 035 & Cowloy & 1976 \\
\hline 003078 & L. S & $\begin{array}{l}\text { KS } \\
\text { KS }\end{array}$ & $\begin{array}{l}101 \\
101\end{array}$ & $\begin{array}{l}\text { Lano } \\
\text { Lane }\end{array}$ & $\begin{array}{l}1881 \\
1984\end{array}$ \\
\hline 008182 & $\begin{array}{l}\text { LS East } \\
\text { LS NE }\end{array}$ & KS & $\begin{array}{l}101 \\
101\end{array}$ & Lane & $\begin{array}{l}1984 \\
1983\end{array}$ \\
\hline 004427 & Ls North & KS & 101 & Lano & 1982 \\
\hline 005583 & L S Wost & ks & 101 & Lano & 1983 \\
\hline 380464 & L T P & Ks & 135 & Noss & 1862 \\
\hline 380470 & L T P North & KS & 135 & Noss & 1976 \\
\hline 380081 & La Cygno-Cadmus & KS & 107 & Linn & \\
\hline & Alies for: (393750) Lacygne-Cad & adimus & ALTEF & & \\
\hline 393316 & Lecorto & KS & 065 & Graham & 1856 \\
\hline 002144 & Lecerte North & KS & 085 & Graham & 1880 \\
\hline 393347 & Lacoy & KS & 167 & Russoll & 1969 \\
\hline 393409 & Lecoy NE & KS & 167 & Russell & 1881 \\
\hline 383440 & Lacoy NW & Ks & 167 & Russell & 1984 \\
\hline 393471 & Lacoy SE & KS & 167 & Russell & 1884 \\
\hline $\begin{array}{l}390980 \\
001527\end{array}$ & Lacrosso & $\begin{array}{l}\text { KS } \\
\text { KS }\end{array}$ & $\begin{array}{l}165 \\
165\end{array}$ & $\begin{array}{l}\text { Rush } \\
\text { Rush }\end{array}$ & $\begin{array}{l}1968 \\
1980\end{array}$ \\
\hline 012210 & Lecrosso NW & KS & 165 & Rush & 1988 \\
\hline 393750 & Lacygna-Cadmus & KS & 107 & Linn & 1936 \\
\hline 383936 & Ladore & KS & 133 & Noosho & 1944 \\
\hline $\begin{array}{l}010957 \\
393907\end{array}$ & $\begin{array}{l}\text { Ladore NE } \\
\text { Ladore North }\end{array}$ & $\begin{array}{l}\text { KS } \\
\text { KS }\end{array}$ & $\begin{array}{l}133 \\
133\end{array}$ & $\begin{array}{l}\text { Noosho } \\
\text { Noosho }\end{array}$ & $\begin{array}{l}1987 \\
1944\end{array}$ \\
\hline $\begin{array}{l}383987 \\
007129\end{array}$ & $\begin{array}{l}\text { Ledore North } \\
\text { Ledore South }\end{array}$ & Ks & 098 & $\begin{array}{l}\text { Noosho } \\
\text { Labotte }\end{array}$ & $\begin{array}{l}1984 \\
1984\end{array}$ \\
\hline 008810 & Ladore sw & KS & 099 & Labolte & 1986 \\
\hline
\end{tabular}


KANSAS

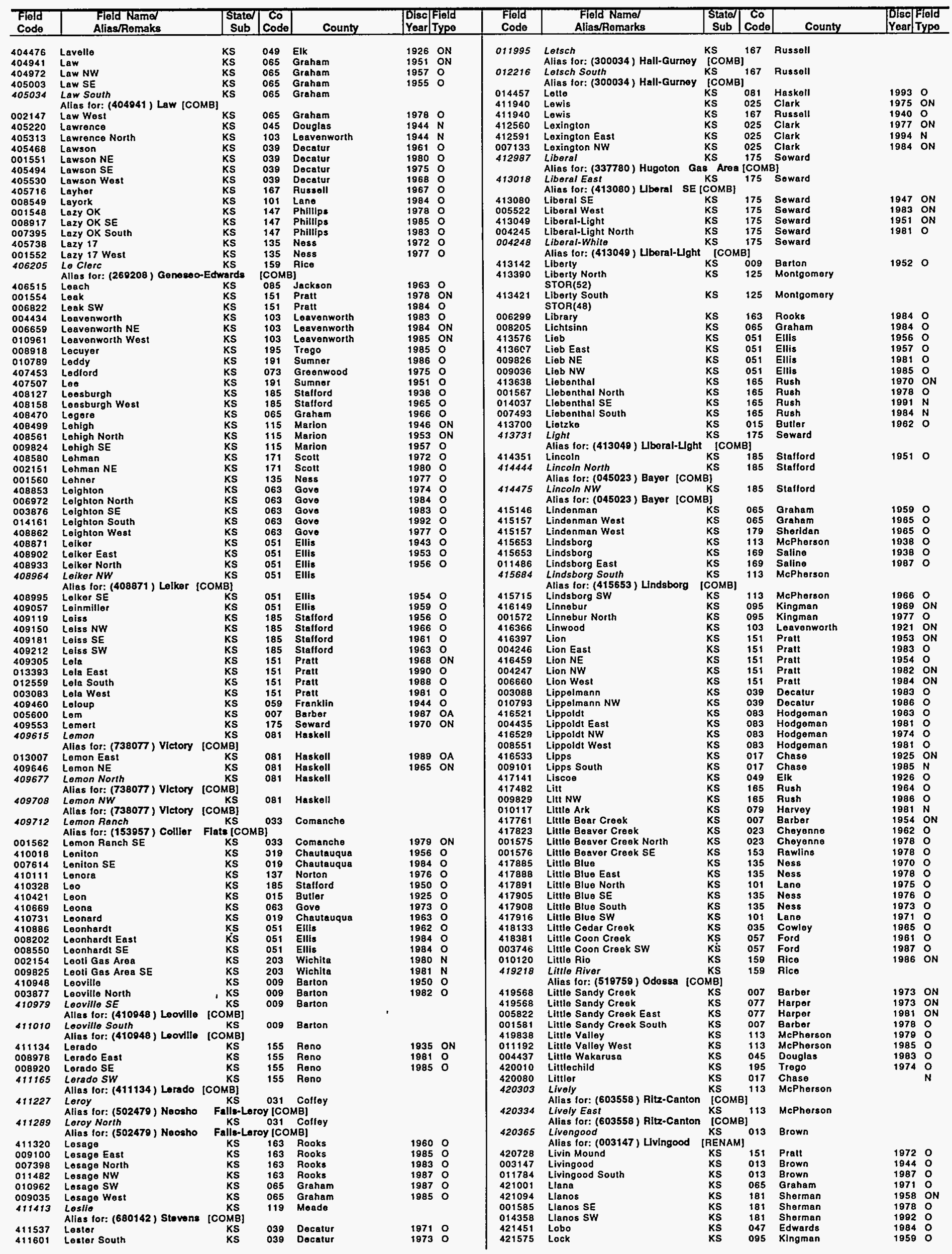


KANSAS

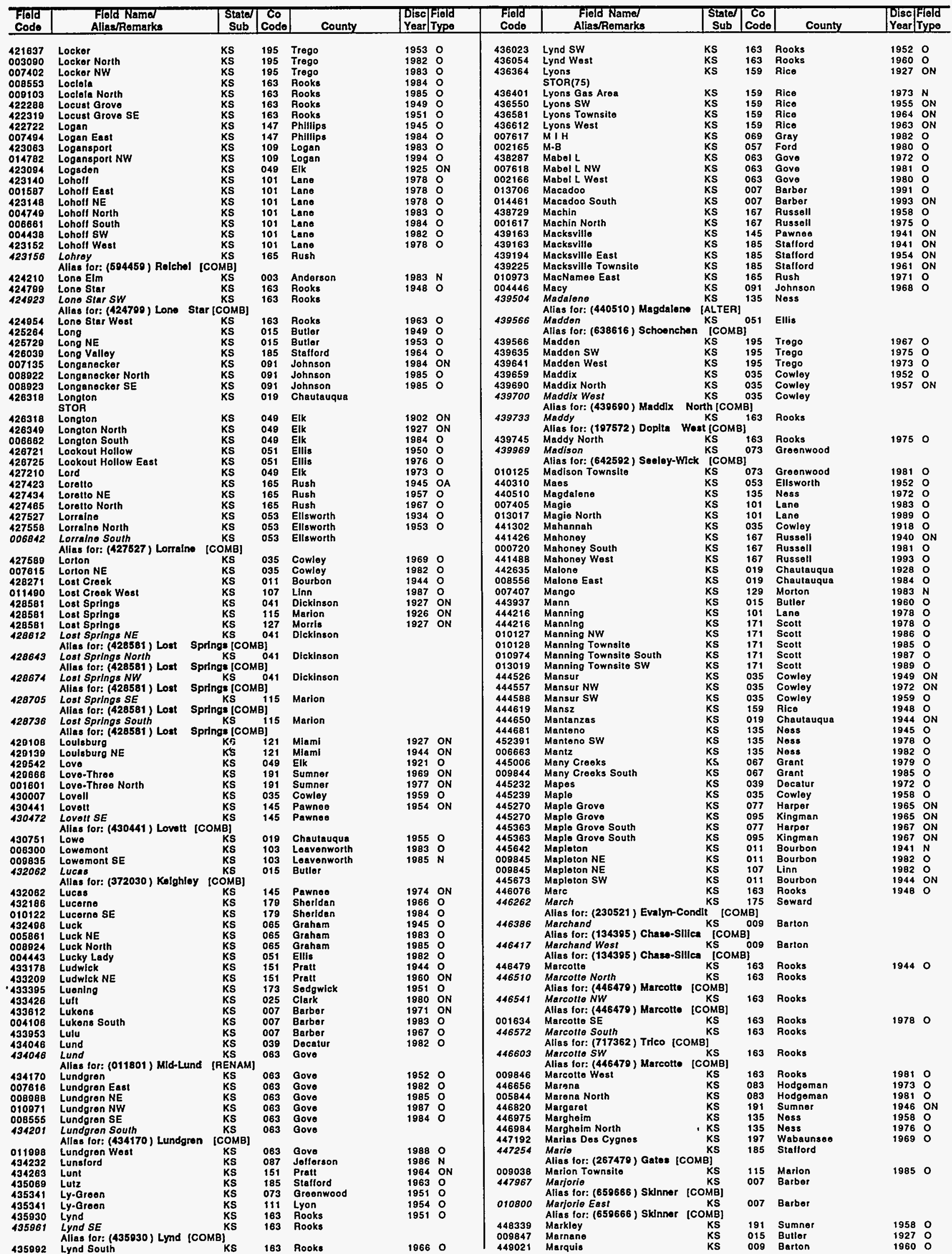


KANSAS

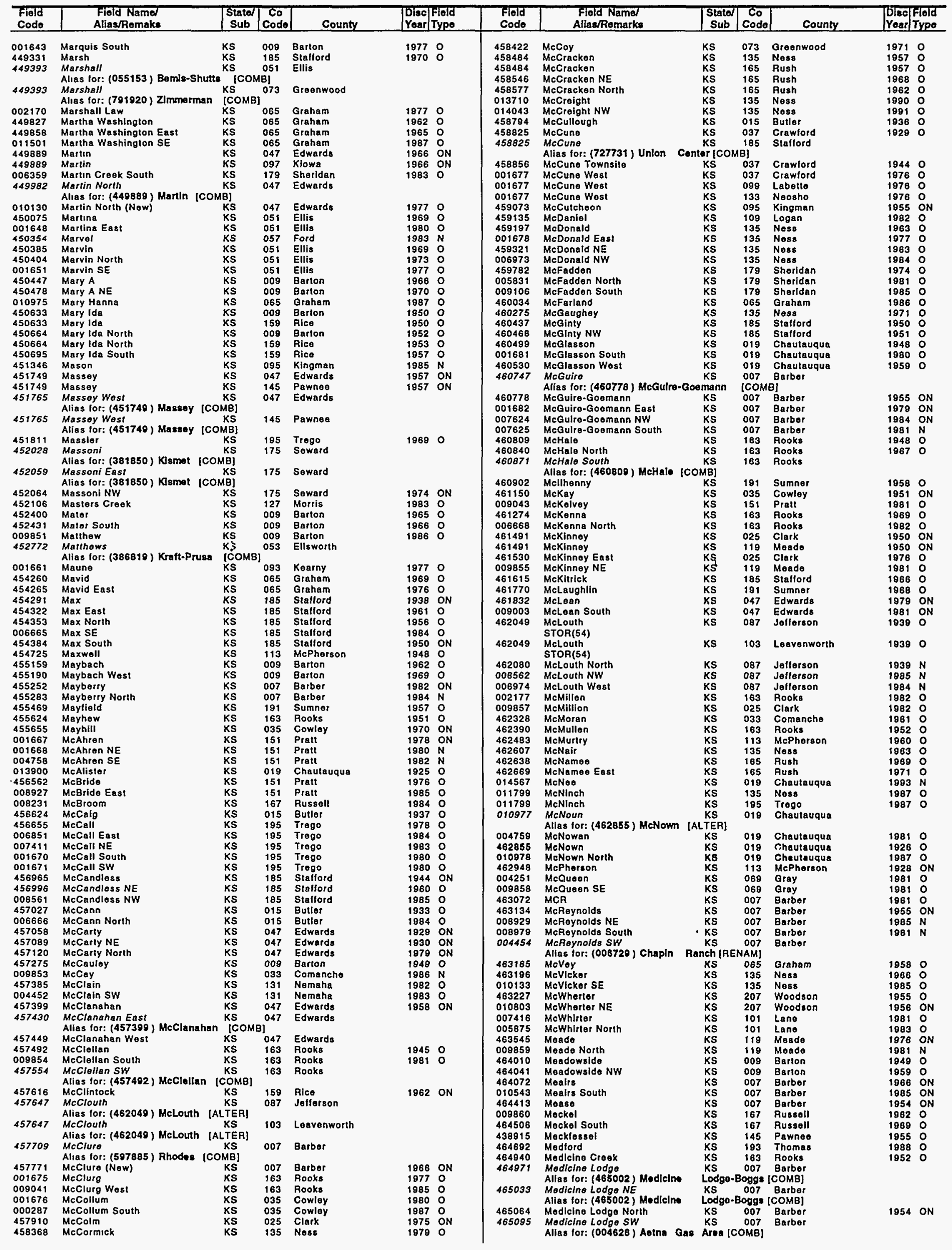


KANSAS

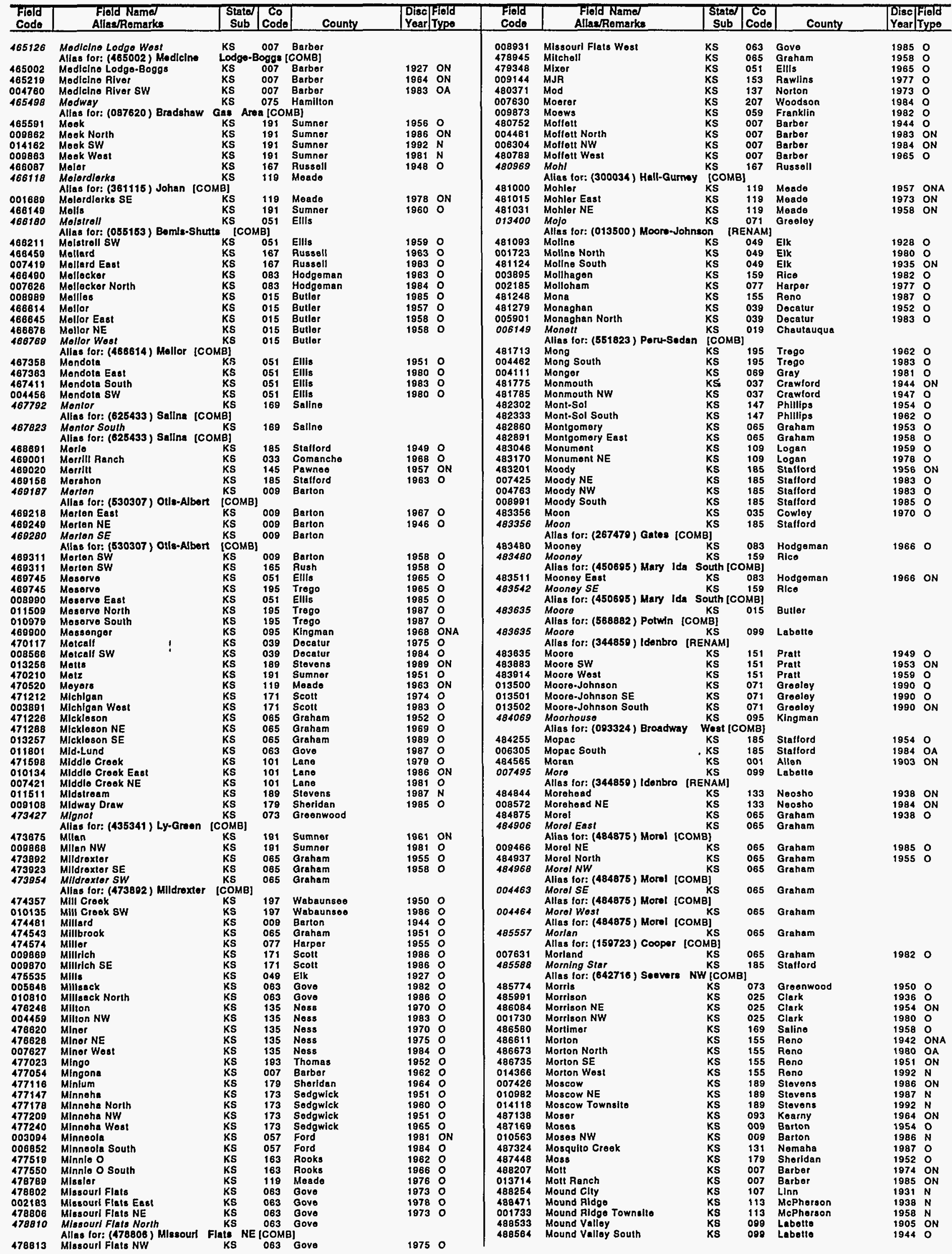


KANSAS

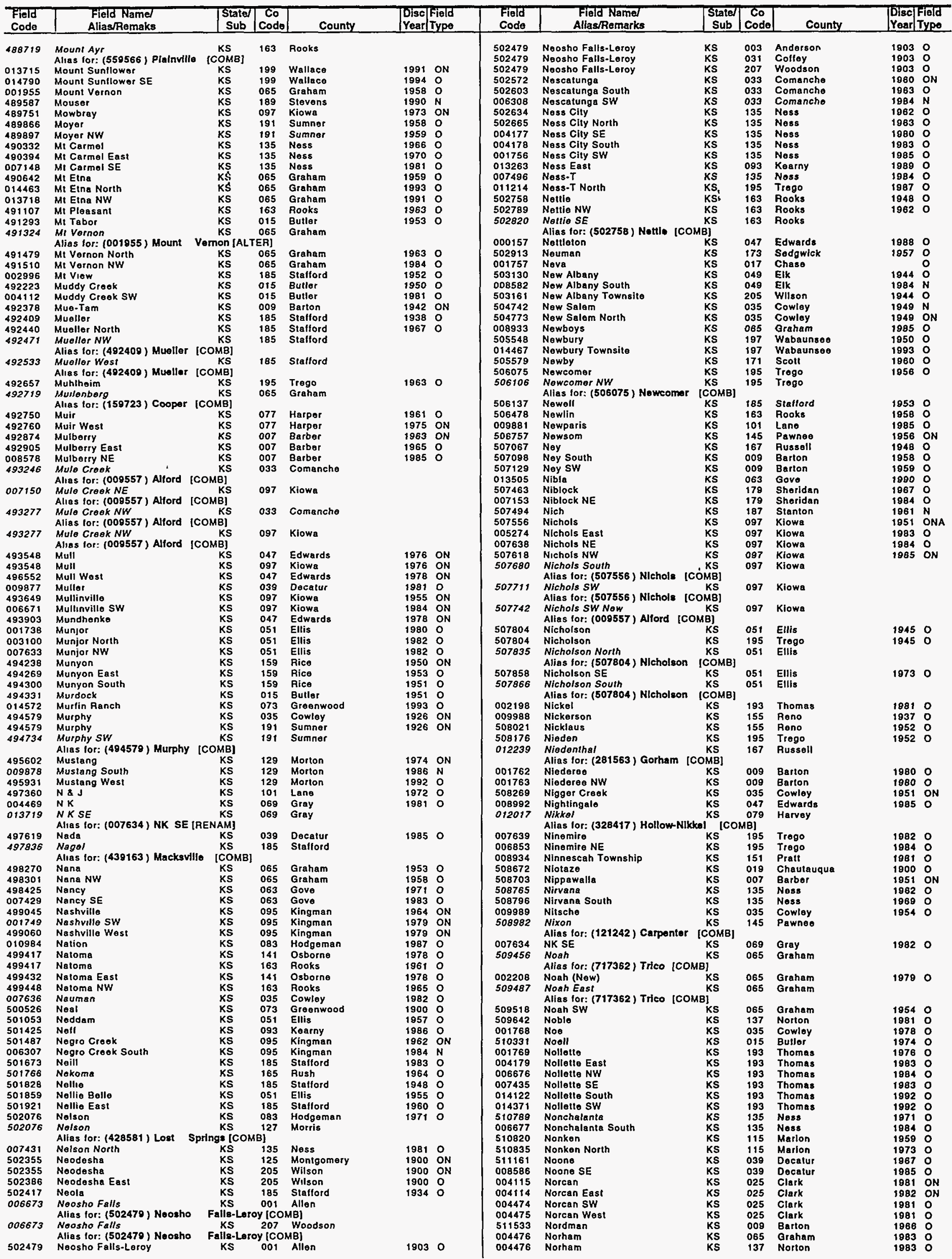


KANSAS

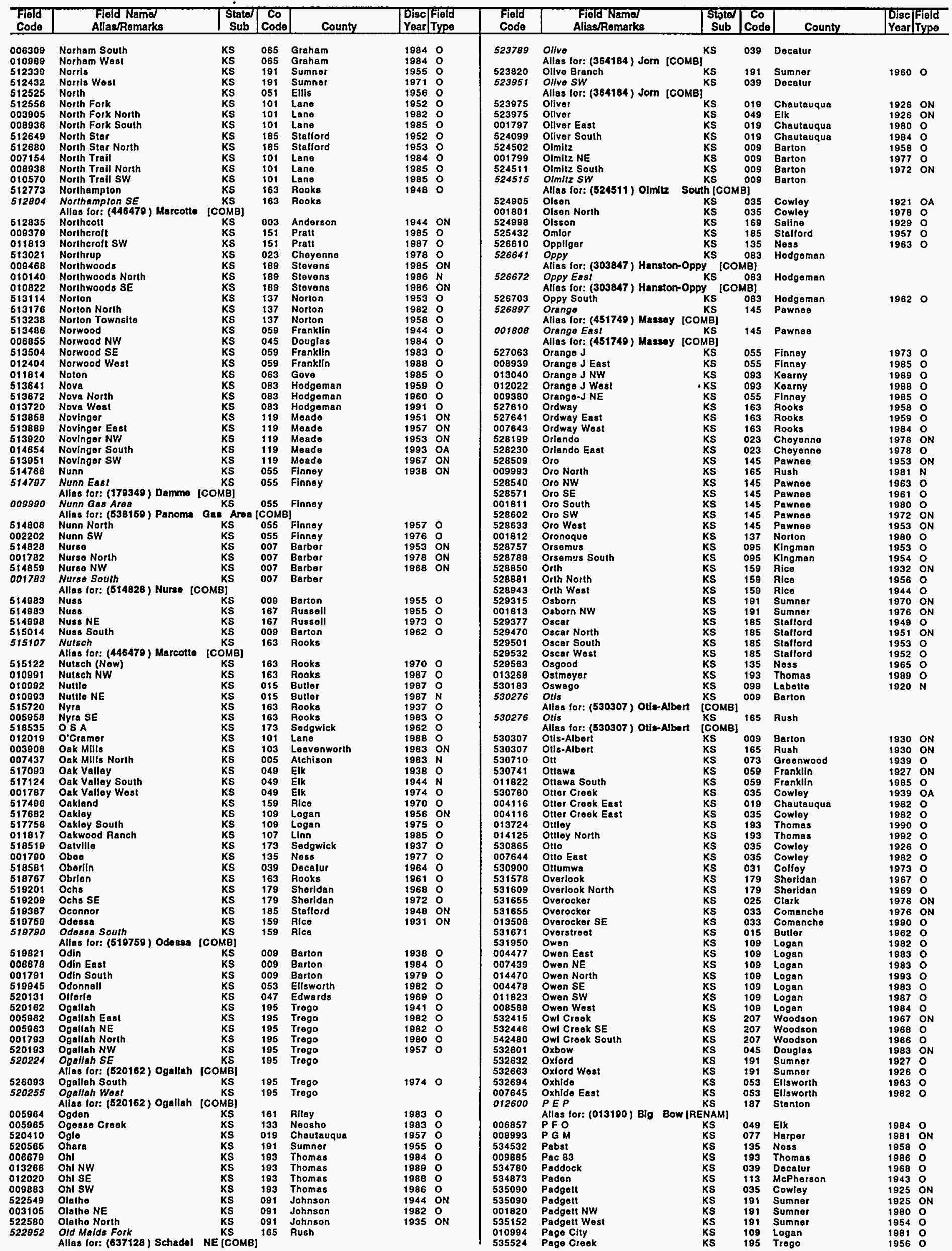


KANSAS

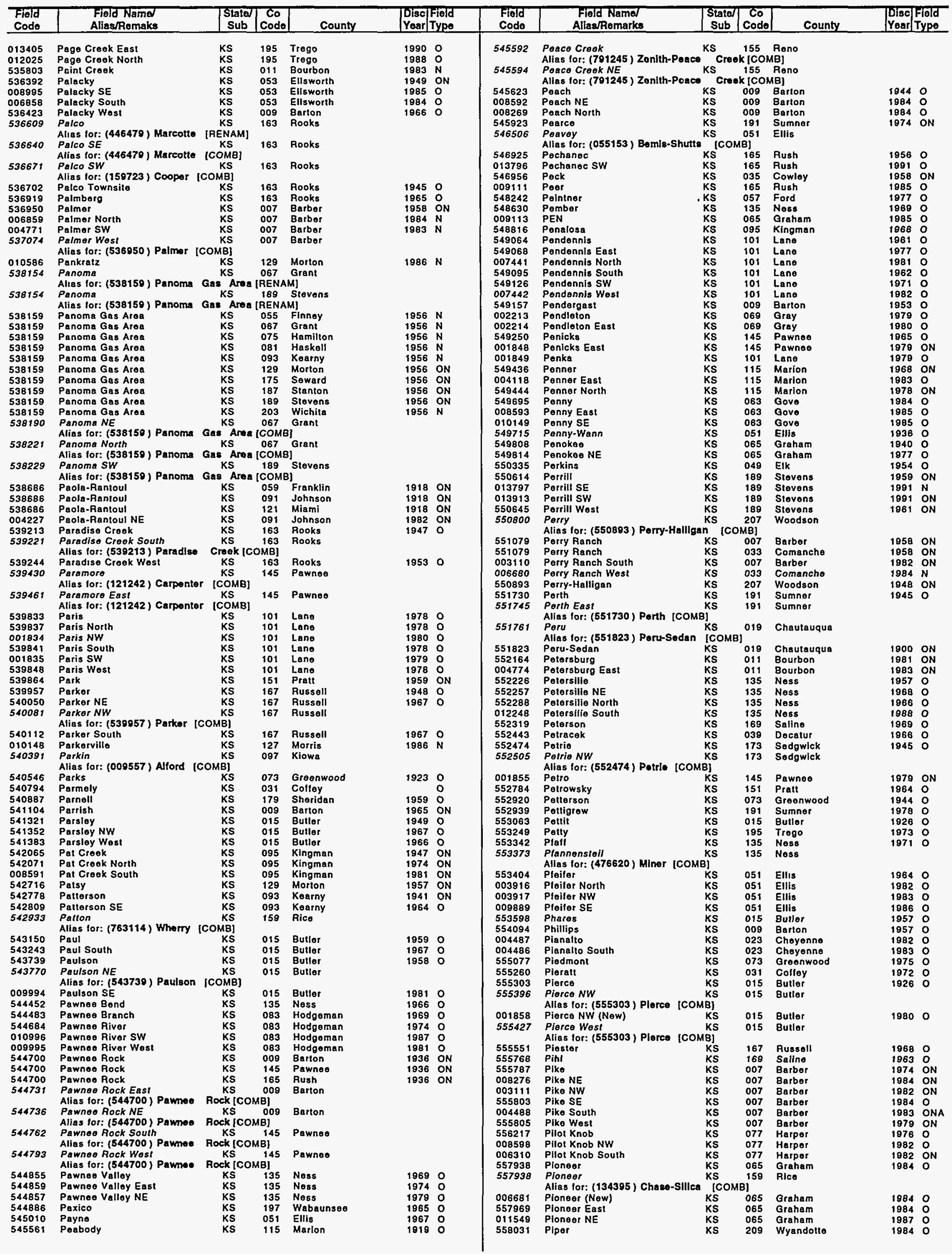


KANSAS

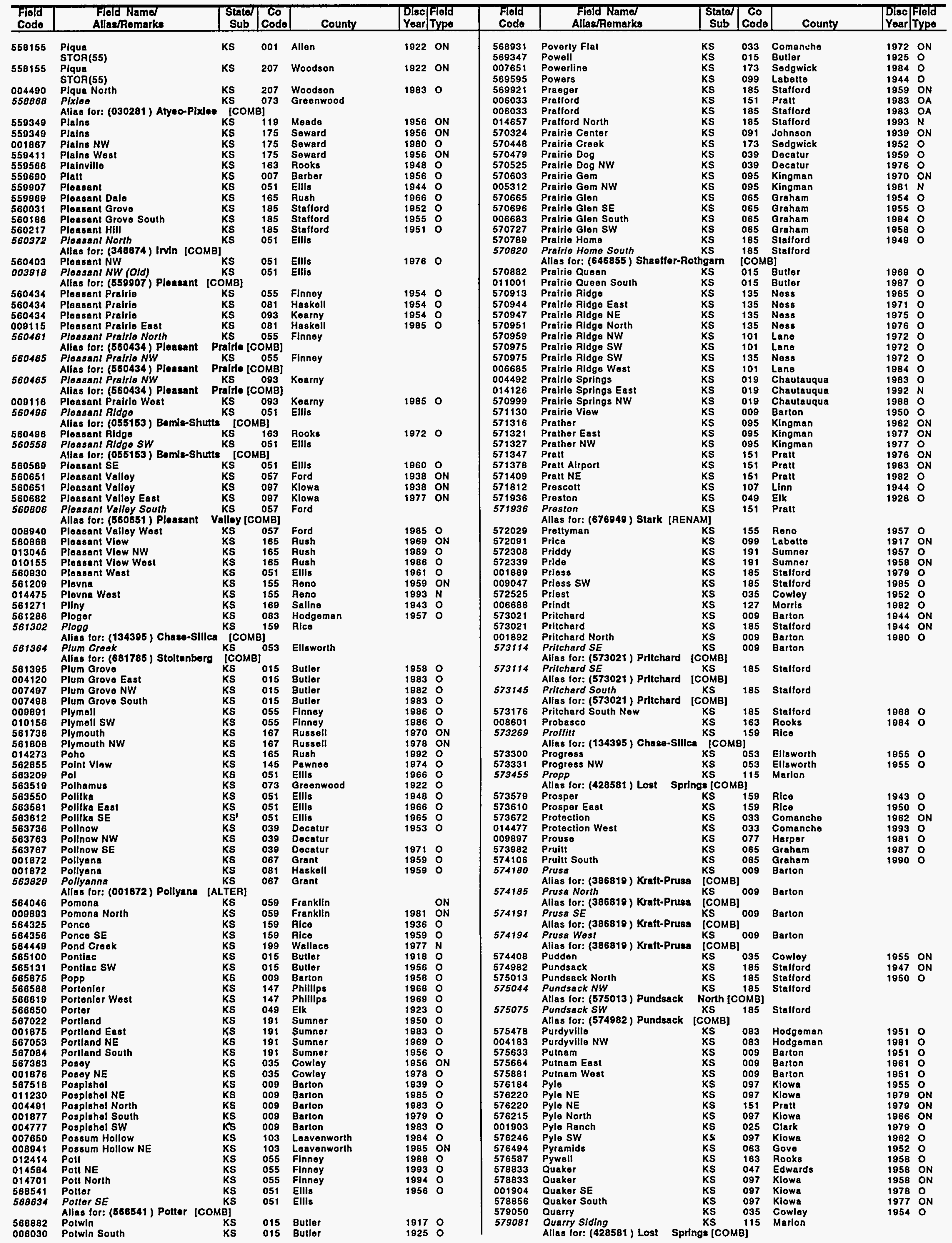




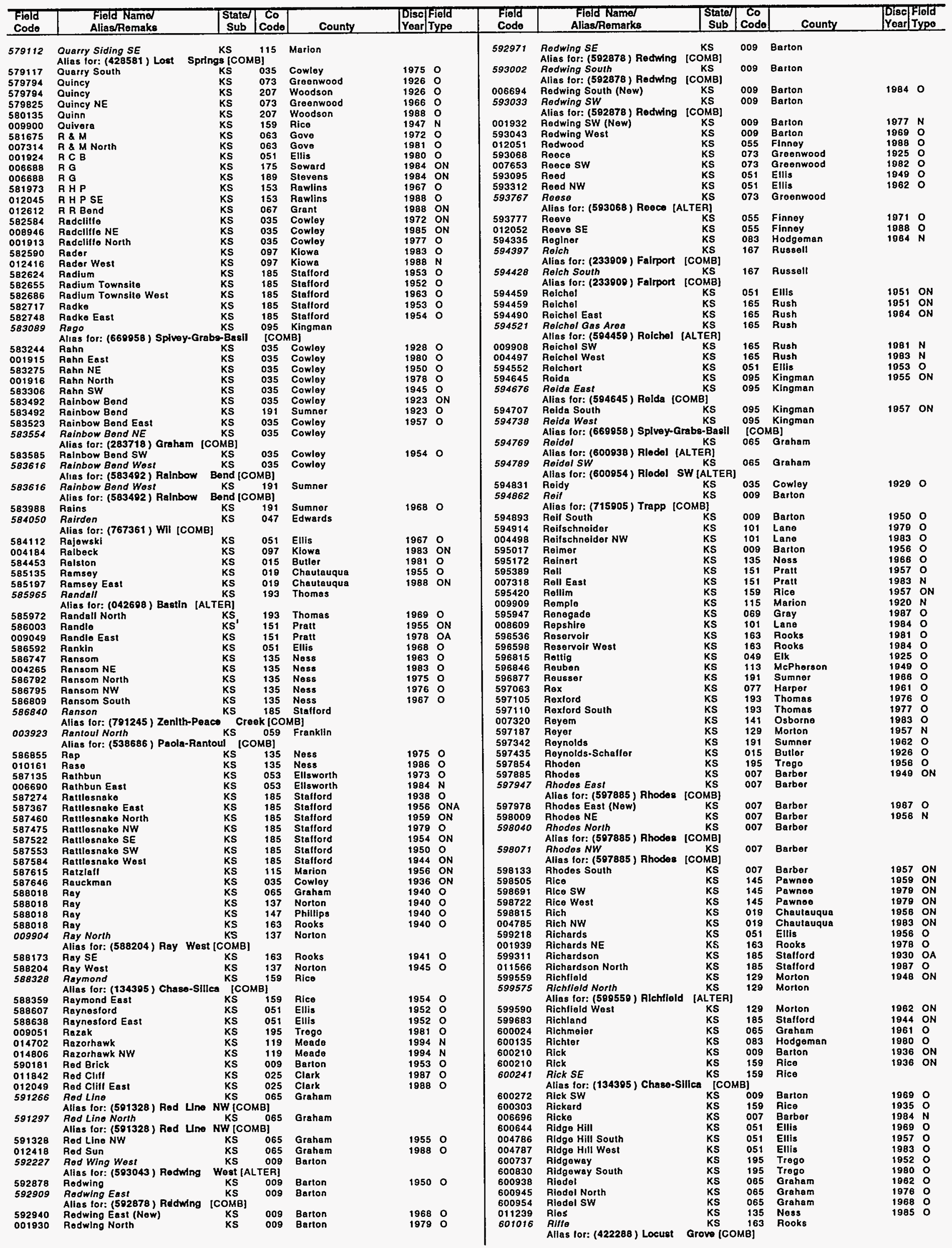


KANSAS

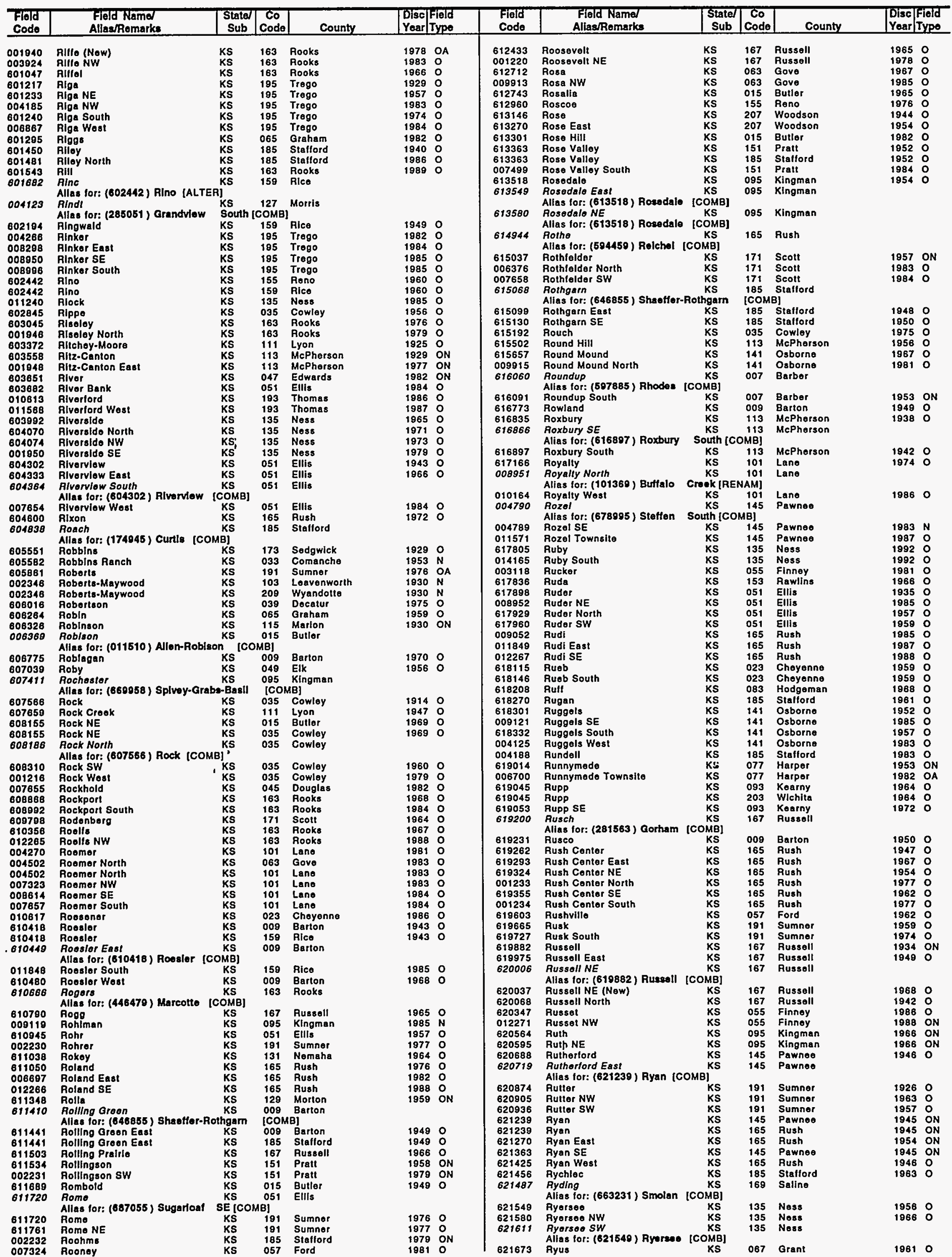


KANSAS

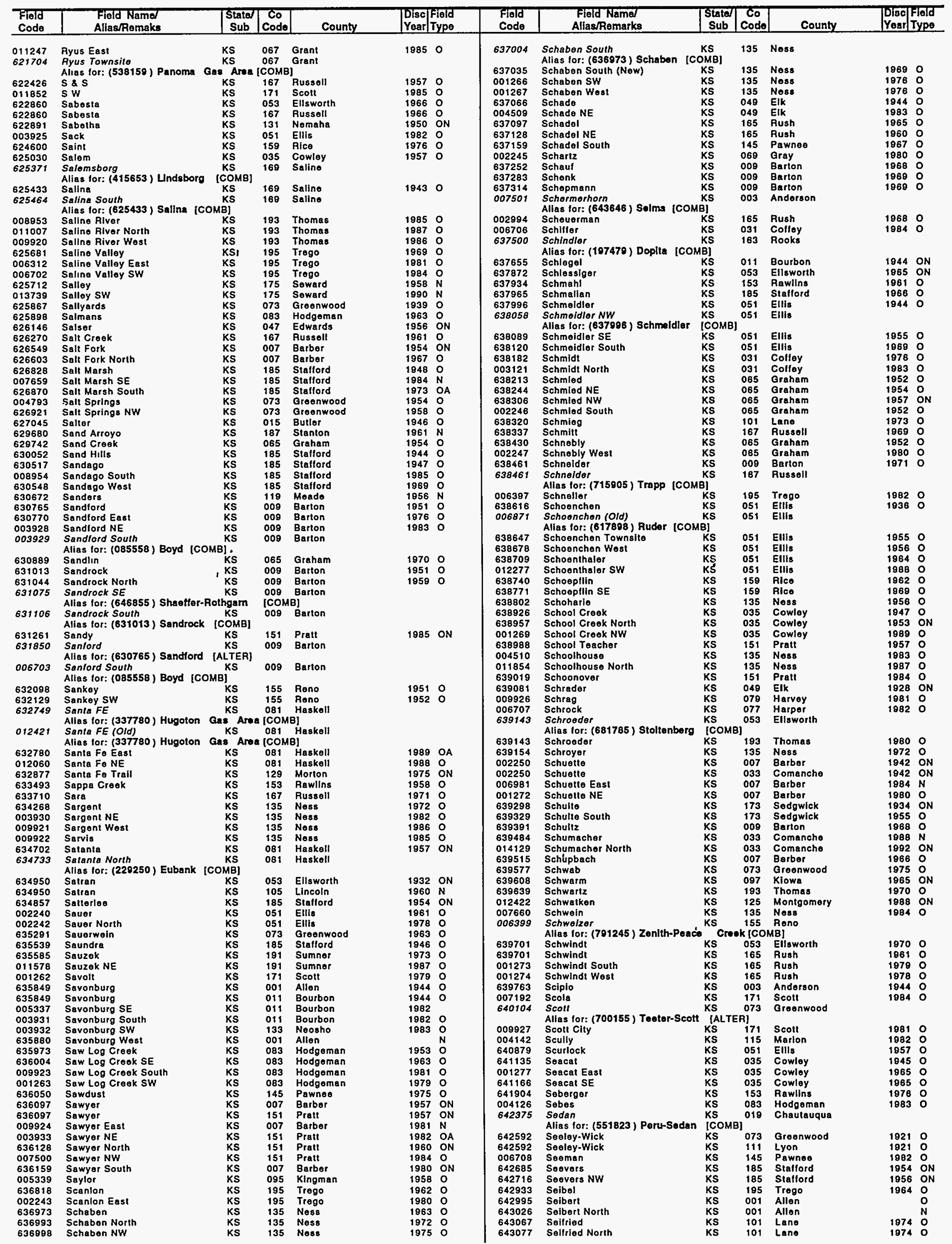


KANSAS

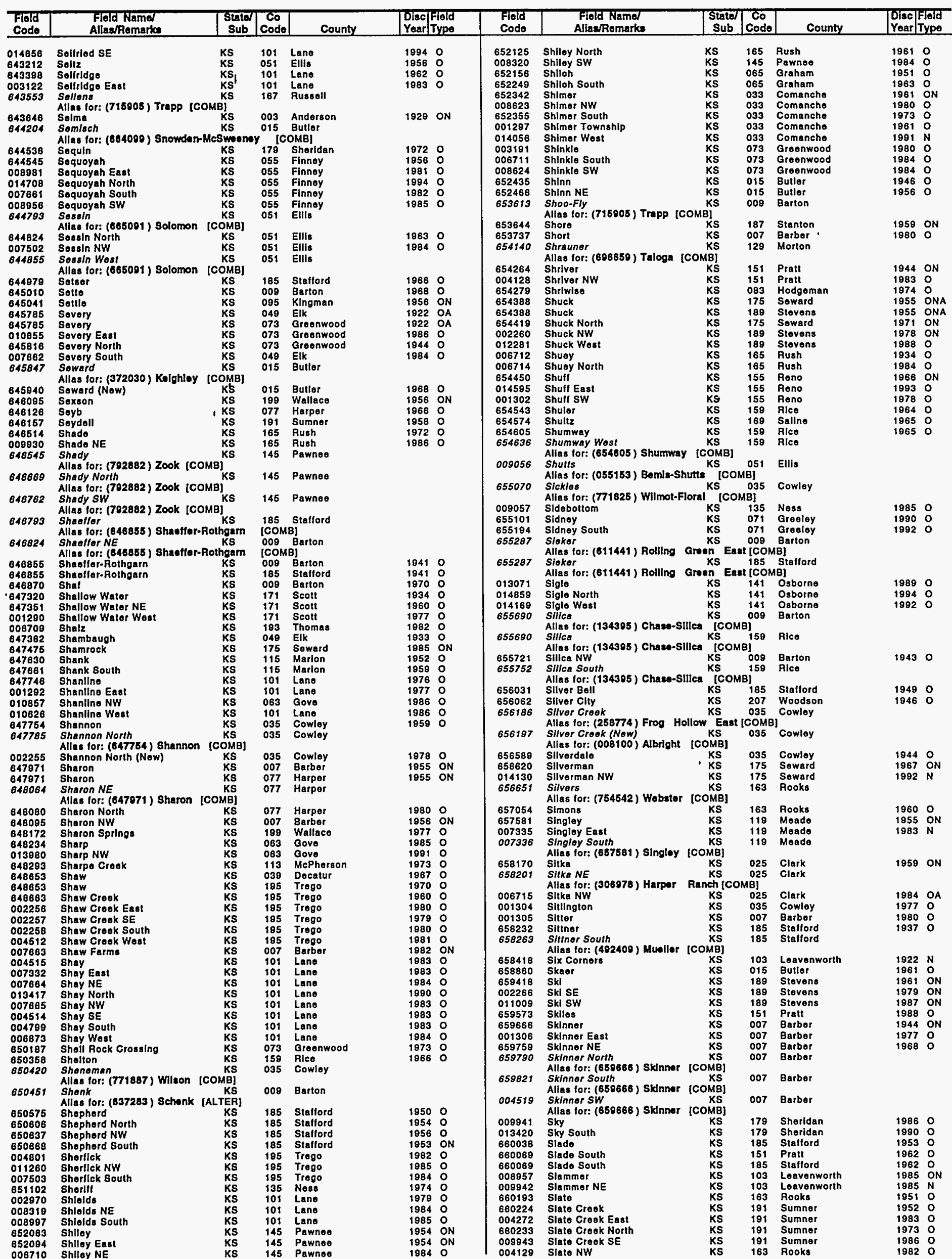


KANSAS

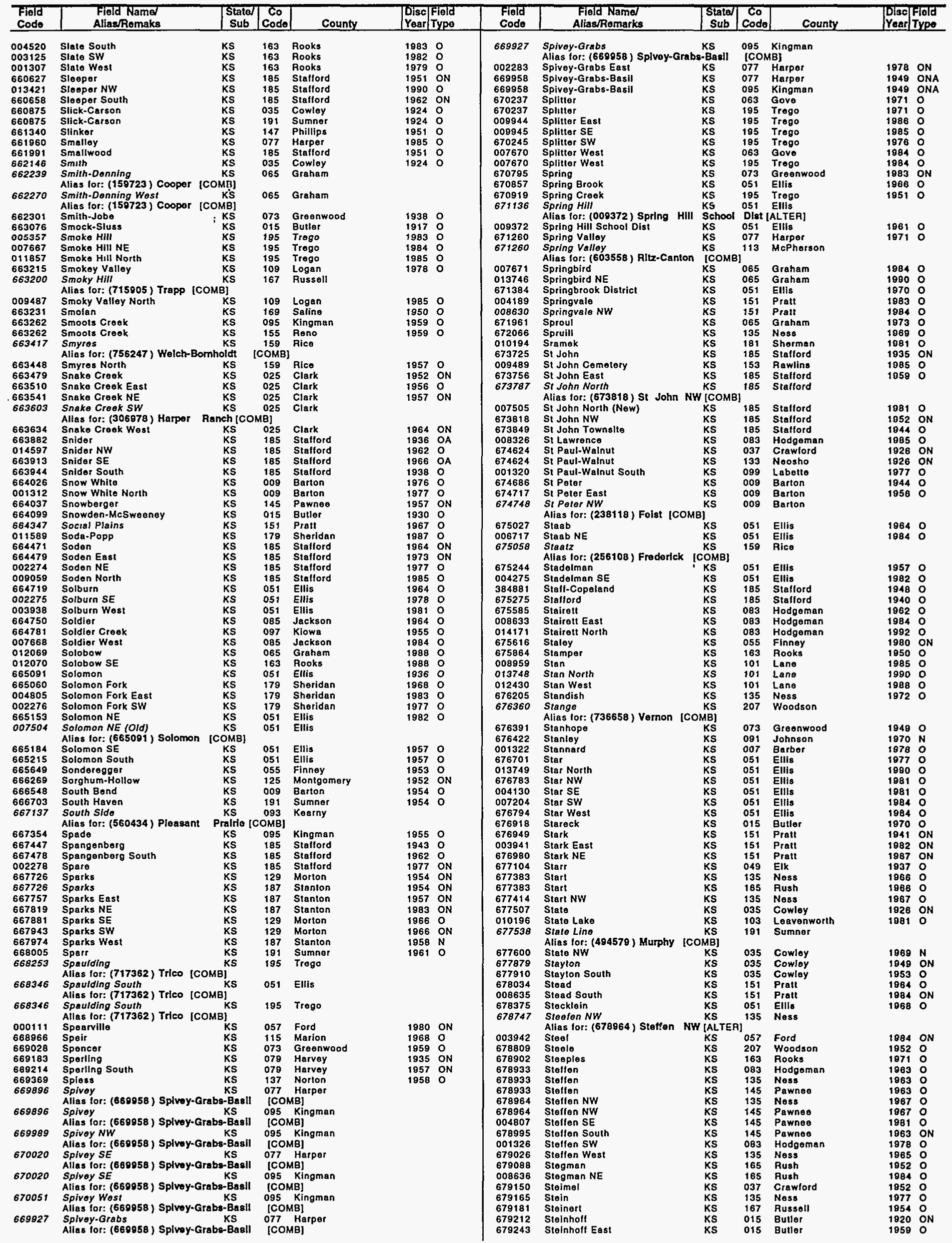


KANSAS

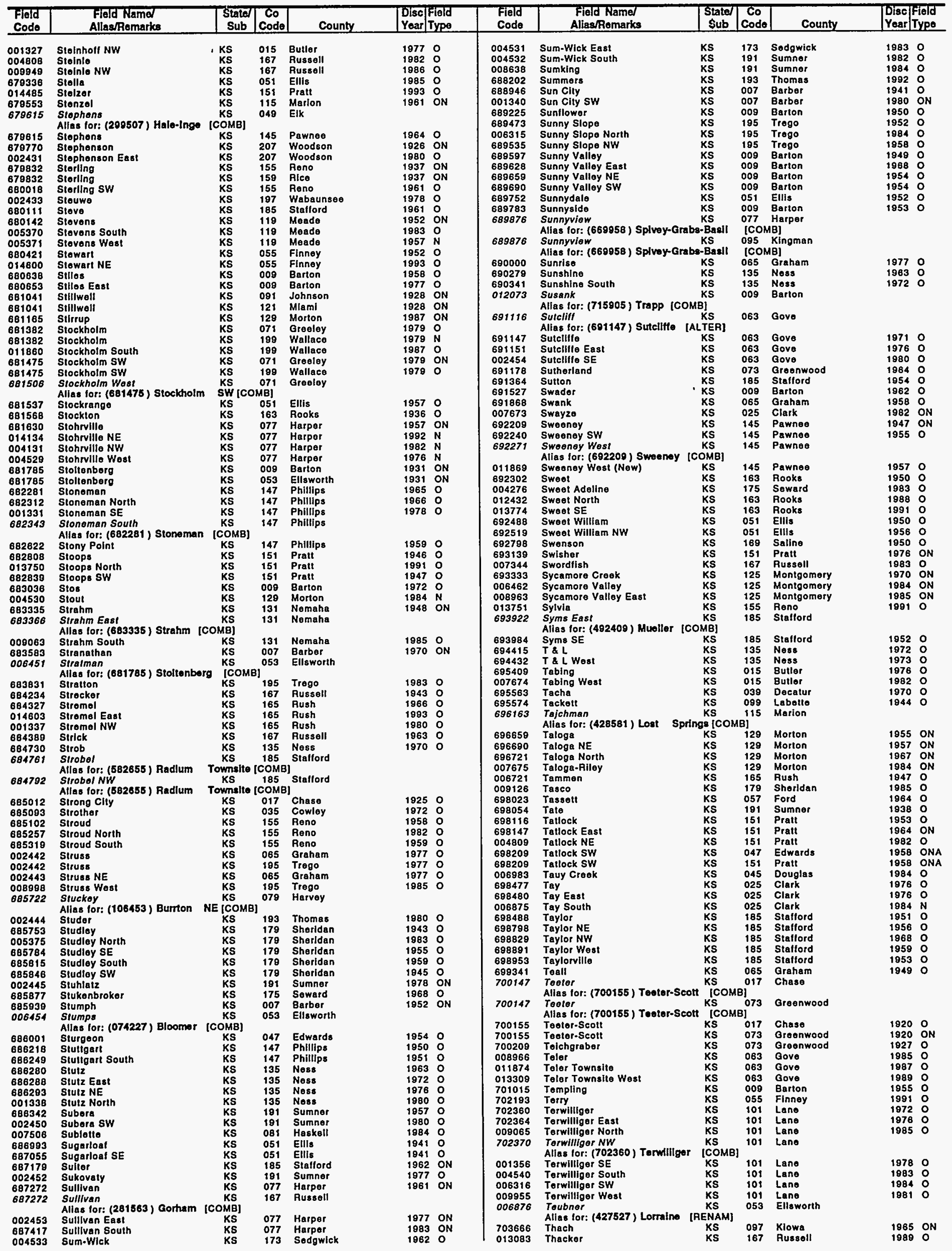


KANSAS

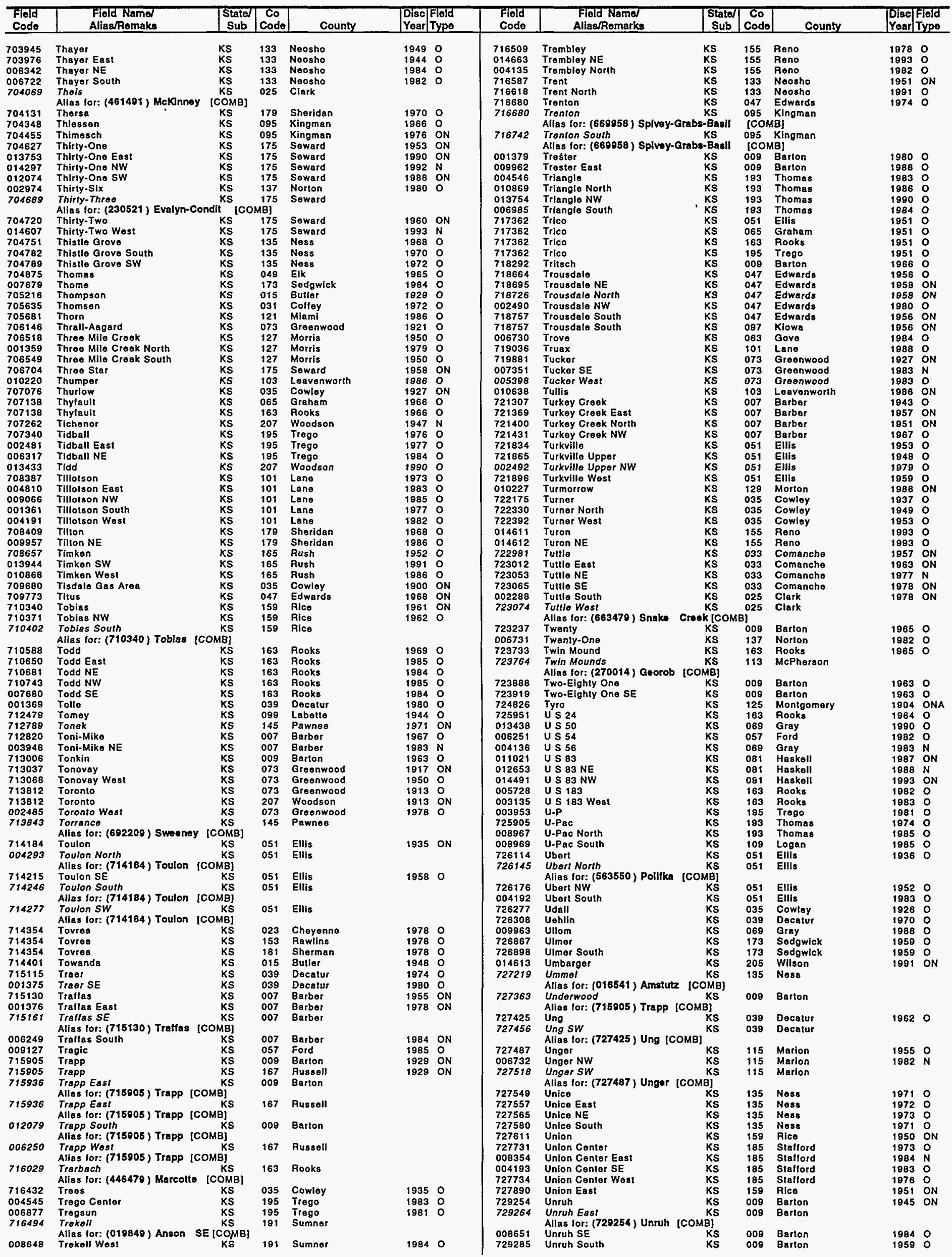


KANSAS

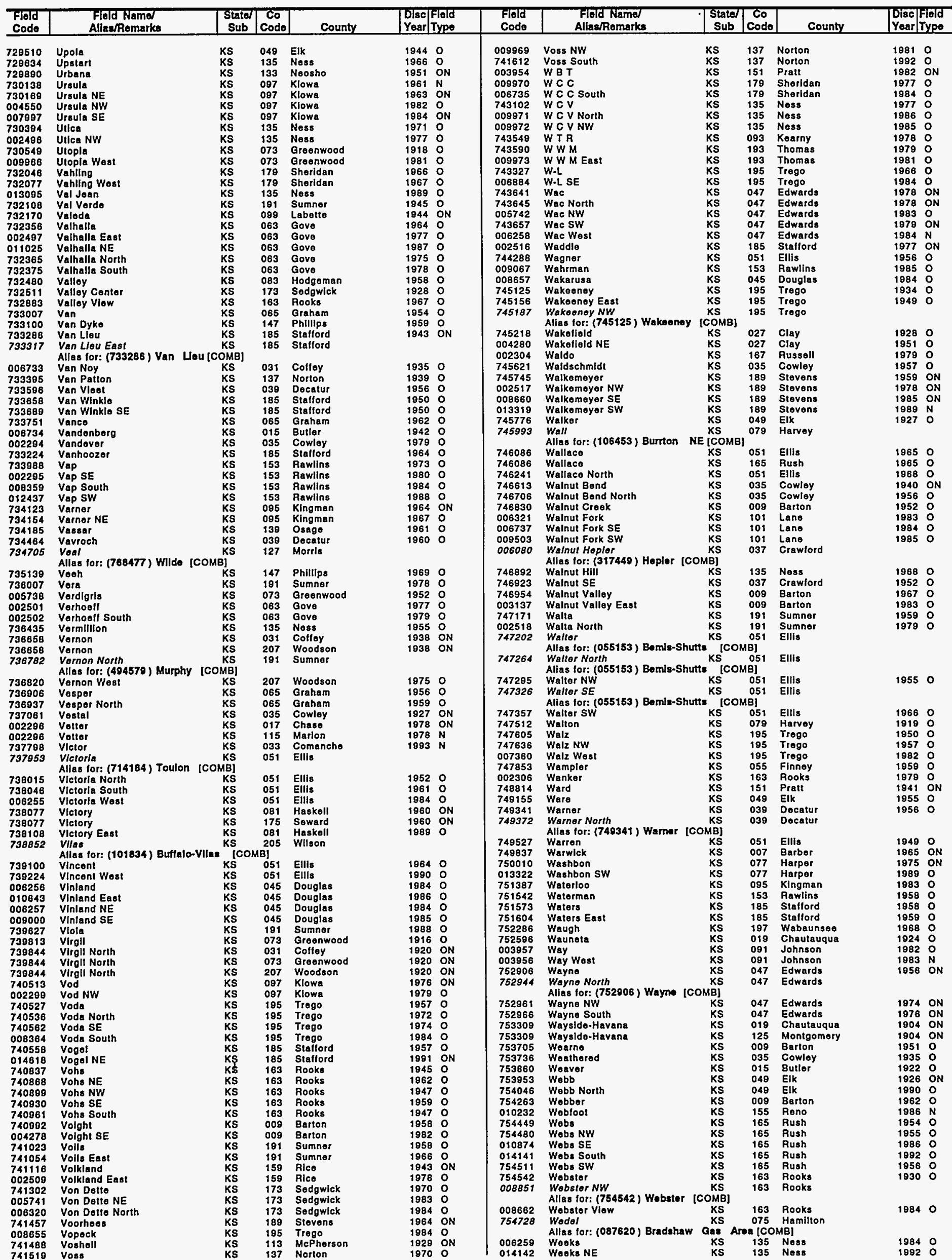


KANSAS

\begin{tabular}{|c|}
\hline $\begin{array}{l}\text { Fiod } \\
\text { Codo } \\
\end{array}$ \\
\hline $\begin{array}{l}009976 \\
014830 \\
010646 \\
755224 \\
014621 \\
006739\end{array}$ \\
\hline $\begin{array}{l}755317 \\
755379\end{array}$ \\
\hline \\
\hline 004560 \\
\hline 755999 \\
\hline $\begin{array}{l}007363 \\
007364\end{array}$ \\
\hline 756092 \\
\hline 756123 \\
\hline 756123 \\
\hline 756185 \\
\hline 756216 \\
\hline $\begin{array}{l}756278 \\
756340\end{array}$ \\
\hline \\
\hline 756433 \\
\hline 756247 \\
\hline 756247 \\
\hline 756309 \\
\hline 002311 \\
\hline 756331 \\
\hline 756331 \\
\hline 756505 \\
\hline \\
\hline 756526 \\
\hline 756557 \\
\hline 757238 \\
\hline 005757 \\
\hline 757270 \\
\hline 757301 \\
\hline 002529 \\
\hline 757332 \\
\hline 013324 \\
\hline 757456 \\
\hline 002313 \\
\hline 002314 \\
\hline 757642 \\
\hline 757642 \\
\hline 757647 \\
\hline 757647 \\
\hline 757651 \\
\hline \\
\hline 014402 \\
\hline 757983 \\
\hline 758003 \\
\hline 004282 \\
\hline 758355 \\
\hline 014862 \\
\hline $\begin{array}{l}758386 \\
758603\end{array}$ \\
\hline $\begin{array}{l}758603 \\
758851\end{array}$ \\
\hline 758866 \\
\hline 758882 \\
\hline 758975 \\
\hline 759006 \\
\hline 002531 \\
\hline 759285 \\
\hline 759378 \\
\hline 759409 \\
\hline 759502 \\
\hline 009977 \\
\hline 759626 \\
\hline 760308 \\
\hline 760502 \\
\hline 760804 \\
\hline 760835 \\
\hline 760846 \\
\hline 004563 \\
\hline 006986 \\
\hline 009978 \\
\hline 760897 \\
\hline \\
\hline 761346 \\
\hline 761362 \\
\hline 761373 \\
\hline 761694 \\
\hline 762308 \\
\hline 762331 \\
\hline 762401 \\
\hline 762432 \\
\hline 762463 \\
\hline 005760 \\
\hline 762484 \\
\hline 762525 \\
\hline \\
\hline 762556 \\
\hline 762587 \\
\hline 762742 \\
\hline 762897 \\
\hline 762959 \\
\hline 762990 \\
\hline \\
\hline \\
\hline 763052 \\
\hline 763083 \\
\hline 763114 \\
\hline 763176 \\
\hline \\
\hline 008971 \\
\hline 008504 \\
\hline
\end{tabular}


KENTUCKY

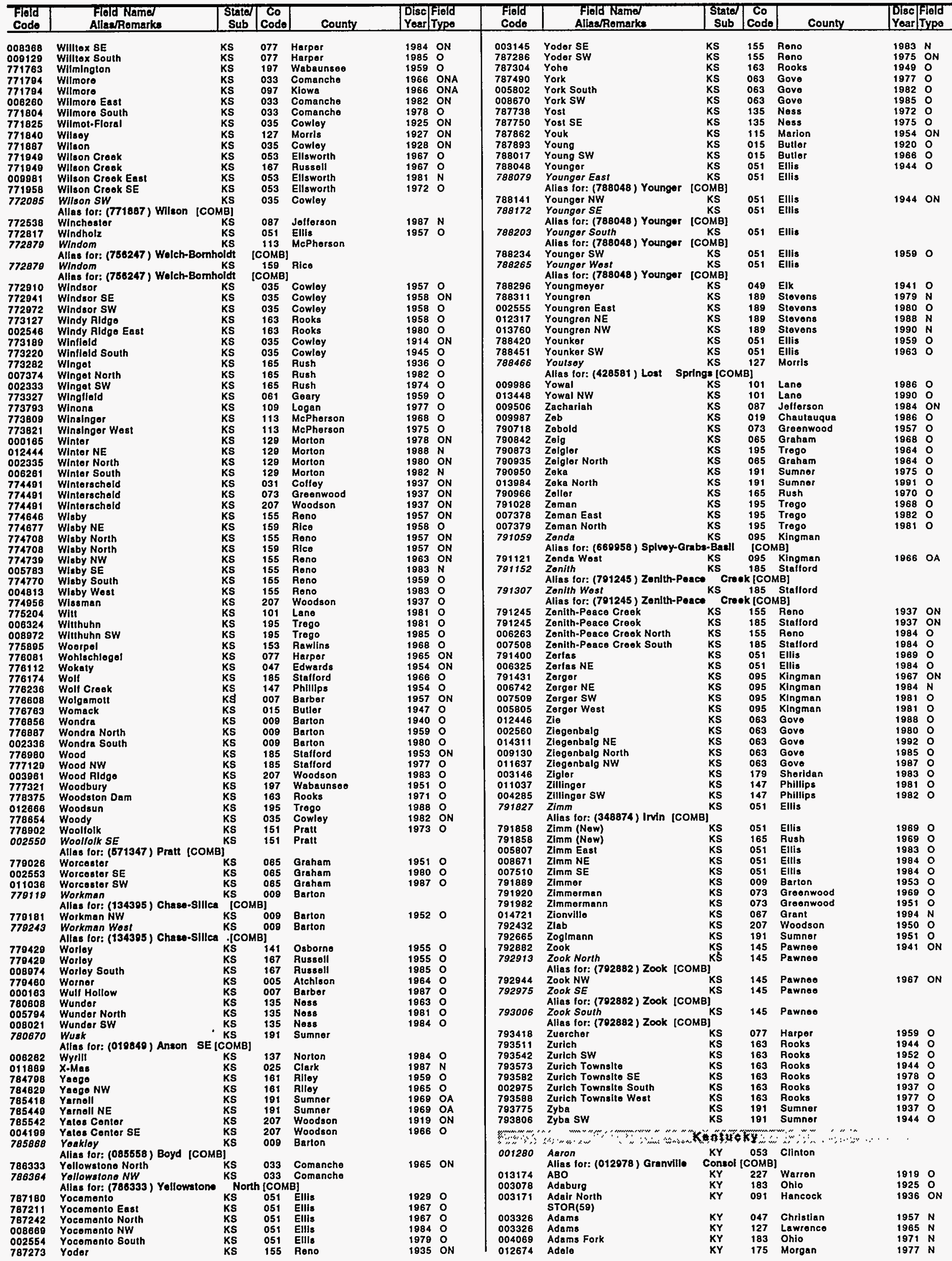




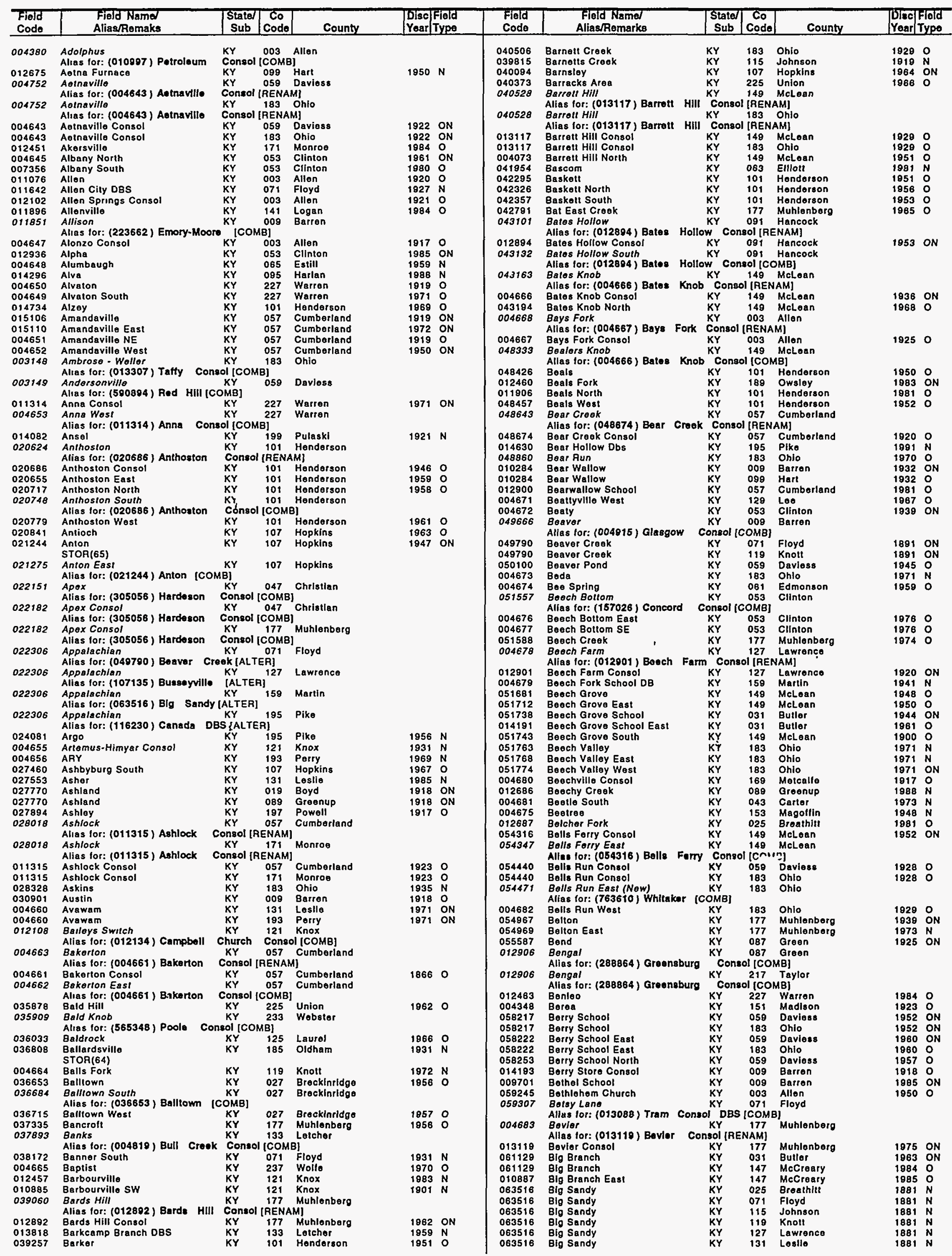


KENTUCKY

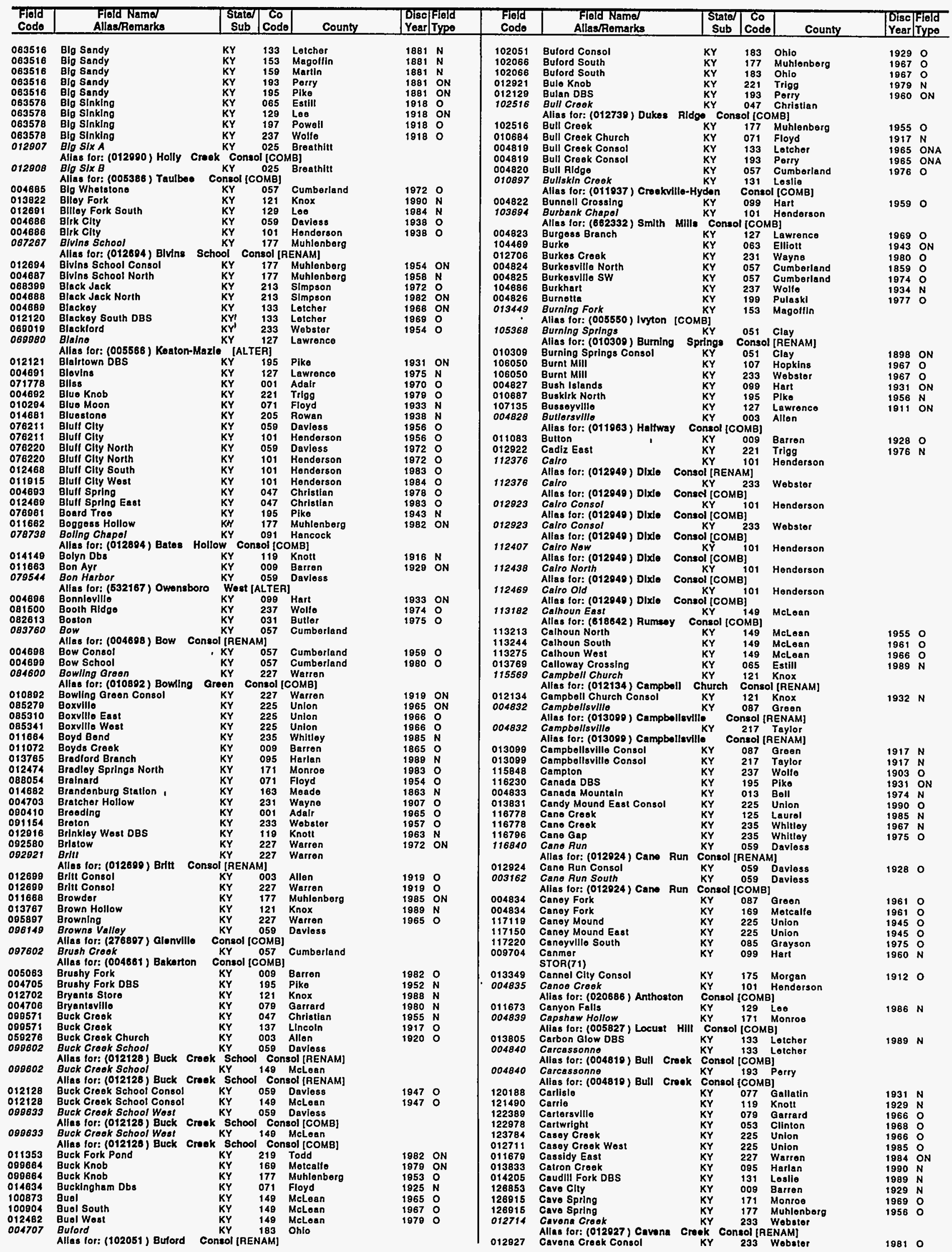




\begin{tabular}{|c|c|c|c|c|c|c|}
\hline $\begin{array}{l}\text { N } \\
\mathbf{O}\end{array}$ & 2806 & 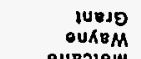 & $\begin{array}{l}180 \\
182\end{array}$ & $\begin{array}{l}\text { NXX } \\
\text { Ax }\end{array}$ & $\begin{array}{l}\text { OBpIY } K_{10} \\
\text { MO॥IOH } K_{1} \text { O }\end{array}$ & $\begin{array}{l}\angle S \angle \varepsilon 0 Z \\
\angle 90200\end{array}$ \\
\hline 0 & & & & Ax & Yjos Rug & EE9ع0Z \\
\hline & SOB! & noux & 611 & 새 & Y0015 kno & $20980 z$ \\
\hline NO & 2861 & Uosucupg & 180 & ix & YHON Bu!lds Bu!dd!IO & $8 \varepsilon \angle 210$ \\
\hline 0 & $\$ 2 B i$ & 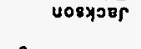 & $\begin{array}{l}601 \\
\text { [gพัอง] }\end{array}$ & 1) 10: & 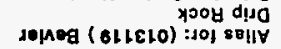 & 288700 \\
\hline & & $\begin{array}{l}\text { 6roquelynw } \\
\text { [вwoj] for }\end{array}$ & $\begin{array}{lll}2<1 \\
\text { TuOS }\end{array}$ & $M^{A x}$ tupejd & 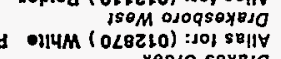 & 090202 \\
\hline & & sulydoH & & & 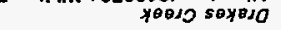 & 980500 \\
\hline 0 & 8881 & volit & 800 & $A x$ & 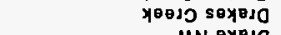 & 980700 \\
\hline $\begin{array}{l}0 \\
0\end{array}$ & $\begin{array}{l}\varepsilon 861 \\
6161\end{array}$ & 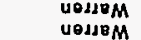 & $\angle 22$ & Ax & MN вx日oso & S0L110 \\
\hline 0 & $8 \times 61$ & vesseg & $\begin{array}{l}206 \\
600\end{array}$ & $\begin{array}{l}\lambda X \\
\Lambda X\end{array}$ & yosnyo sonog & $\begin{array}{l}250202 \\
188700\end{array}$ \\
\hline NO & 2281 & Ksourobjuow & ELI Igwo & 소 & 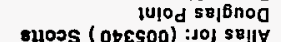 & 206110 \\
\hline & & $\begin{array}{l}\text { purjuguno } \\
\text { [8woj] } 10\end{array}$ & $\begin{array}{l}\angle 90 \\
\text { osuog }\end{array}$ & 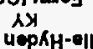 & 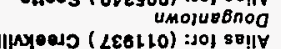 & $088+00$ \\
\hline & & АВ। & ISO & $\Delta x$ & Yoeso elqnoo & 512661 \\
\hline $\mathbf{N}$ & 6861 & pkoly & $1<0$ & xix & SgO Y0U8J K100 & $\angle L \angle E I O$ \\
\hline $\mathbf{N}$ & Ez6l & phois & $1<0$ & $\lambda X$ & $\begin{array}{r}40 \$ 100 \\
\text { (29) yo15 }\end{array}$ & SSHzLO \\
\hline No & 0006 & opeow & 891 & $\Delta x$ & uny 000 & 896761 \\
\hline $\mathbf{N}$ & or6l & noux & 611 & XY & 590100405 uosq00 & $2 \$ 6210$ \\
\hline & & $\begin{array}{l}1018 q 9 M \\
\text { IWYN }\end{array}$ & 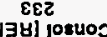 & AX & 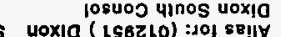 & 196210 \\
\hline & & J018q0 M & & $A x^{4}$ & 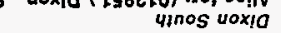 & $090+61$ \\
\hline o & $8+61$ & $10189 \circ M$ & $\sum_{\text {INYNFE }}^{\varepsilon \varepsilon}$ & 빌 & 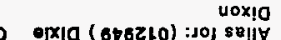 & $866 \varepsilon 6 \mathrm{l}$ \\
\hline & & $1018 q 0 M$ & $\operatorname{cez}$ & AX & josuOJ isom o!xia & ossz10 \\
\hline & & vojun & $\begin{array}{l}\text { IWYNGE } \\
\text { SZZ } \\
\text { [NYNGE }\end{array}$ & $\begin{array}{l}\text { 18] josuos } \\
\text { ix } \\
{[y] \text { [osuog }}\end{array}$ & 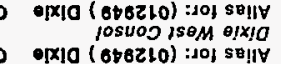 & 056210 \\
\hline & & Uоsıорuон & 101 & & josuOJ isoM olxia & os6z10 \\
\hline & & Jo1sqoM & $\lim _{\operatorname{cez}}$ & $\begin{array}{l}{[y][08400} \\
\wedge \times 1\end{array}$ & 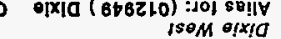 & $9 \varepsilon 6 \varepsilon 6 !$ \\
\hline & & & INVNGE & [) josuog & 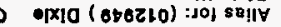 & \\
\hline & & volun & $\begin{array}{l}\text { SZZ } \\
\text { IWYNBK }\end{array}$ & ity & 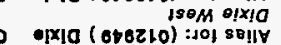 & $9 \varepsilon 6 \varepsilon 6 !$ \\
\hline & & оsсөрuөH & 101 & $\Delta x$ & jsom elxia & $9 \varepsilon 6 \varepsilon 6 t$ \\
\hline & $\varepsilon s 6 !$ & UOssepuer & 101 & $\lambda x$ & บน & $b \angle 8 E 61$ \\
\hline 0 & Et6l & Jo18q日 M & eEz & AX & losuoj จ!x!O & 676210 \\
\hline 0 & $\varepsilon r 6 !$ & vọun & s2z & $\lambda$ त्र & josuog oixic & $6+6210$ \\
\hline & Et6l & UOSsepueH & 101 & $\Lambda$ AX & $\begin{array}{r}\text { losuoj o!x!d } \\
\text { (Is)yoIs }\end{array}$ & 676210 \\
\hline No & StBi & vossopuoH & 101 & $\lambda x$ & o|x!0 & $218 \varepsilon 6 !$ \\
\hline O & $\begin{array}{l}1861 \\
606 !\end{array}$ & $\begin{array}{r}\text { xout } \\
\text { uEBjow }\end{array}$ & $\begin{array}{l}l z ! \\
s<i\end{array}$ & $\hat{\mu x}$ & $\begin{array}{r}\text { souidds uEmys!o } \\
\text { snBuig }\end{array}$ & $6<8+00$ \\
\hline$\ddot{o}$ & $6 \angle B i$ & บoII & 800 & $\lambda$ XX & YIION XO0Jכ נIMOIH!O & $\angle \angle 8 \nabla 00$ \\
\hline o & 0281 & Uoilv & $\varepsilon 00$ & Ax & 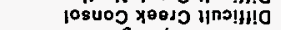 & $8 \angle 8 \nabla 00$ \\
\hline No & $016 i$ & บ0607 & $1 \neq 1$ & הत्र & soullds puowrio & $9+1161$ \\
\hline o & $\angle L 6 I$ & $10189 \theta M$ & $\varepsilon \varepsilon z$ & $\Delta x$ & 4!JON PUOWE! & $s \angle 8+00$ \\
\hline & 6761 & UOSsepUeH & tot & Ax & purisi puowe! & 166061 \\
\hline & & บอุบ!ก & $\begin{array}{l}\text { Leso } \\
\text { Est }\end{array}$ & Aर्र & 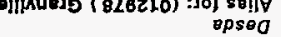 & $p<\theta>00$ \\
\hline $\mathbf{N}$ & 6961 & 1040107 & $\varepsilon \varepsilon t$ & AX & S80 $18100 \mathrm{w}$ & $\angle \$ 6210$ \\
\hline 0 & 2561 & 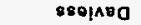 & 690 & $A \times$ & 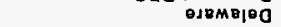 & $\angle E 698$ \\
\hline $\mathbf{N}$ & $8 \angle B I$ & 1040107 & EEi & $A \times$ & xо013 poj80j00 & $\varepsilon \angle 8+00$ \\
\hline o & 9861 & $101890 M$ & $\varepsilon \varepsilon z$ & $A \times$ & X00dכ נ000 & $2+5981$ \\
\hline & & a!̣ะs & 181 & AX & deg dero & $\angle r e E 10$ \\
\hline & & uolu!̣ı & $\begin{array}{l}\text { I8WOOJ } 108 \\
\varepsilon S 0\end{array}$ & $2 \times$ & 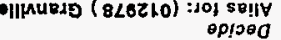 & $\$ 8678 t$ \\
\hline & & & โอพоว & ग) 10040s & 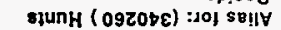 & \\
\hline & & quelunw & $\angle L L$ & $i x$ & puouszed & $\varepsilon \varepsilon \varepsilon+8 t$ \\
\hline & & ọ̣o & 881 & 사 & 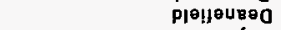 & $0 \rightarrow 2 \forall 81$ \\
\hline $\begin{array}{l}\text { O } \\
\text { N }\end{array}$ & $\begin{array}{l}t S 61 \\
\$ 8061\end{array}$ & $\begin{array}{r}\text { olloM } \\
\text { Bsequejuny }\end{array}$ & LEZ & $\hat{\lambda x y}$ & $\begin{array}{r}\text { oroqskea } \\
\text { souljds uosmea }\end{array}$ & $\begin{array}{l}\text { s十6210 } \\
869110\end{array}$ \\
\hline$\ddot{o}$ & SSEI & 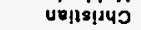 & $\angle 00$ & $\lambda \times$ & yinos UOSMra & 298281 \\
\hline $\mathrm{N}$ & $986 \mathrm{l}$ & u!nsew & BSI & $A x$ & 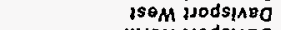 & $80<010$ \\
\hline$\ddot{N}$ & ItBi & บนมอพ & 6St & Ax & YLION JOds!ABa & $2 \angle 8700$ \\
\hline$N$ & $066 \mathfrak{t}$ & nosuyor & sli & 새 & $\begin{array}{r}\text { पLON jodsinga } \\
\text { rouHen }\end{array}$ & $2 \angle 8700$ \\
\hline & & $210 \mathrm{H}$ & 660 & $\Delta \times$ & $\begin{array}{r}\text { pueg s!Aषg } \\
\text { put }\end{array}$ & zezz8t \\
\hline$N$ & 0 S6! & 잉 & 881 & $\lambda \mathbb{X}$ & ISE马 UOSP!ABg & $\angle<028 !$ \\
\hline NO & 2261 & 0!40 & 881 & 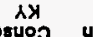 & & $90 z \varepsilon 10$ \\
\hline & & 잉 & EQt & Aत्र & 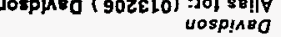 & stozet \\
\hline & & lвพOS & Ol josuos & Ueedo & Bu|pmog $(2680(0): 20,80 ! \mid \forall$ & \\
\hline & & บQNBM & $\angle Z 2$ & $A x$ & GN HOdUQABO & $\$ \angle 9181$ \\
\hline & $8 E 61$ & 0140 & EBi & $\hat{i x}$ & MOIIOH XYBOO & 159081 \\
\hline $\mathrm{N}$ & 5861 & dnuoeso & 680 & Ax & 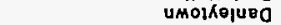 & $\angle 69110$ \\
\hline No & 1961 & Q0UQנMEา & $\angle Z I$ & $\hat{\Lambda} \hat{x}$ & 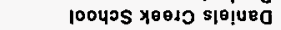 & $18 E 110$ \\
\hline 0 & 9561 & 0!̣० & E8! & $\lambda y$ & 480 & $9996 \angle 1$ \\
\hline 0 & $\varepsilon \angle 6 i$ & Jеру & 100 & $\lambda \times$ & x०20 vojurga & osizio \\
\hline N & $\angle S B$ & sulydoh & LOS & $A x$ & voljea & टहเ $6 \angle \ell$ \\
\hline $\mathbb{N}^{\mathrm{N}}$ & $0<6 i$ & Nued & E6! & $\hat{\lambda x}$ & Көјво & $1<8000$ \\
\hline $\mathbf{N}$ & $0<61$ & 011807 & IEิ & $\lambda \ddot{x}$ & Көןв & $1 \angle 8+00$ \\
\hline 0 & zS6I & UOssopuor & Bot & AX्र & pueg ssejdKo & S6E9<L \\
\hline VNO & $6 \angle 61$ & 0!ाsoา & IEL & AXX & u! $4 \operatorname{sing}$ & $0<8700$ \\
\hline $\mathrm{N}$ & $6+61$ & oxld & SBL & $\hat{\lambda x}$ & S80 MBYsno & $\varepsilon \ngtr 8 \varepsilon 10$ \\
\hline$\ddot{o}$ & 6061 & 10s18puOH & $10 i$ & $\Delta \lambda$ & $180 \mathrm{M}$ 日]!!Asping & seg十 $\angle 1$ \\
\hline 0 & 1961 & $880 ! \wedge 80$ & 690 & $\hat{\lambda x}$ & पHON OIIIASPInO & $880+00$ \\
\hline 0 & troi & ssojara & 690 & $A x$ & ellinspung & $\forall 09 \forall \angle L$ \\
\hline NO & $8 \angle 61$ & 1108 & Elo & Ax & 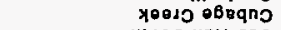 & 698000 \\
\hline 0 & $2 \varepsilon 6 i$ & II8H & 660 & גX्र & yinos uny qno & 898000 \\
\hline $\mathbf{N}$ & 8861 & 111493 & \$90 & $\alpha X$ & 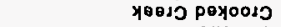 & $56 \varepsilon 1<1$ \\
\hline 0 & PSOI & solung & 180 & $A X$ & llөмшоло & $602 t<t$ \\
\hline 0 & 5861 & 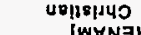 & Lto & AX्र & yinos vonjodo & Z\$8E10 \\
\hline & & 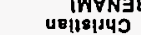 & 8] 1 ton & QH & 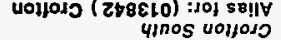 & $\langle t|l| l t$ \\
\hline & & & & & yols & \\
\hline & & 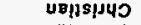 & $\angle P O$ & $A X$ & 1589 4013013 & $12 \angle 600$ \\
\hline $\mathbf{N}$ & 8281 & 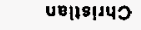 & $\angle 70$ & 서 & 401ן013 & $9111<1$ \\
\hline & & & [ดพัดว] & ostuog & uopeyirg $(199600)$ :10) sa!| & \\
\hline & & puE|10quno & $\angle S O$ & $A X$ & yooso snooso & $\varepsilon 20 \mathrm{l}\langle t$ \\
\hline & & lewo & opl [osuos & O Eply & 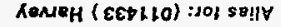 & \\
\hline & & & 100 & AX & y0010 sno010 & $\varepsilon 201<t$ \\
\hline & & $\| \operatorname{lnsen} y$ & $\angle 02$ & $\Lambda x$ & snooso & 269110 \\
\hline $\mathbf{N}$ & 2581 & juedo & 180 & $A x$ & MN UepueIIIJ & DELELO \\
\hline 0 & 1861 & $\| \cos n y$ & 202 & $A X$ & & $P 98500$ \\
\hline 0 & 1861 & $8 \mid s \theta q u n{ }^{2}$ & $\angle S O$ & AX & ISOM OdOQS10010 & 798700 \\
\hline 0 & 1981 & $\| \operatorname{\theta essn} y$ & $\angle O Z$ & $\Delta \times$ & josuoj osoqsjoeso & $08 \varepsilon 69 !$ \\
\hline $\operatorname{ad} K_{1}$ & $\left|\begin{array}{ll}280 \lambda \\
\operatorname{seg}\end{array}\right|$ & nos & \begin{tabular}{l|l} 
कpop & qn \\
०2 & pis
\end{tabular} & $\begin{array}{l}\text { ns } \\
\text { ins }\end{array}$ & $\begin{array}{l}\text { कyrueu/se!ly } \\
\text { pund pjoly }\end{array}$ & $\begin{array}{l}\text { өpos } \\
\text { pløit }\end{array}$ \\
\hline
\end{tabular}


KENTUCKY

\begin{tabular}{|c|c|c|c|}
\hline $\begin{array}{l}\text { Fleld } \\
\text { Codo }\end{array}$ & $\begin{array}{l}\text { Fild Name } \\
\text { Allaa/Remarks }\end{array}$ & $\begin{array}{l}\text { Statd } \\
\text { Sub }\end{array}$ & $\begin{array}{c}\text { Co } \\
\text { Codo }\end{array}$ \\
\hline 004884 & Dubre & KY & 057 \\
\hline 011706 & Dubre SE & KY & 057 \\
\hline 012955 & Duck Fork & KY & 129 \\
\hline 205199 & Duke & SY & 003 \\
\hline 205261 & $\begin{array}{l}\text { Allas lor: (005387) Taylor Se } \\
\text { Dukes fidge }\end{array}$ & School Co & onsol ICC \\
\hline 200200 & $\begin{array}{l}\text { Oukes fidge } \\
\text { Allas for: (012739) Dukez RId }\end{array}$ & Fldge $\cdot$ Cons & $\begin{array}{l}047 \\
\text { nOOL IREN }\end{array}$ \\
\hline 012739 & Dukes Ridge Consol & KY & 047 \\
\hline $\begin{array}{l}2052 \theta 2 \\
2052 \theta 2\end{array}$ & $\begin{array}{l}\text { Dukes School } \\
\text { Dukes School }\end{array}$ & $\begin{array}{l}\mathrm{KY} \\
\mathrm{KY}\end{array}$ & $\begin{array}{l}027 \\
091\end{array}$ \\
\hline 012740 & Duncen Rildge & KY & 141 \\
\hline 004885 & Duncan Valley & KY & 231 \\
\hline 090910 & Duncan Valley South & KY & 231 \\
\hline 206160 & Dundeo & KY & 183 \\
\hline 004087 & Dundoo South & $k Y$ & 183 \\
\hline 206842 & Dunmor & KY & 177 \\
\hline $\begin{array}{l}012158 \\
200330\end{array}$ & Dunmor Wost & $\underset{K Y}{K Y}$ & 177 \\
\hline $\begin{array}{l}208330 \\
208307\end{array}$ & $\begin{array}{l}\text { Duteh Grook } \\
\text { Dyson Grook }\end{array}$ & $\begin{array}{l}\mathrm{Kr} \\
\mathrm{KY}\end{array}$ & $\begin{array}{l}057 \\
225\end{array}$ \\
\hline $\begin{array}{l}004886 \\
210463\end{array}$ & $\begin{array}{l}\text { Eagan North } \\
\text { Eaglo Creok }\end{array}$ & $\begin{array}{l}K Y \\
K Y\end{array}$ & $\begin{array}{l}235 \\
077\end{array}$ \\
\hline & STOR(63) & & \\
\hline 290463 & $\begin{array}{l}\text { Eagle Crook } \\
\text { STOR(63) }\end{array}$ & KY & 081 \\
\hline 210483 & $\begin{array}{l}\text { Eaglo Crook } \\
\text { STOR(63) }\end{array}$ & $\mathrm{kr}$ & 187 \\
\hline 211083 & Earro Crook & KY & 107 \\
\hline 011843 & Earlo Crook East & KY & 107 \\
\hline 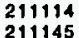 & $\begin{array}{l}\text { Eastilington } \\
\text { Earflington Wost }\end{array}$ & $\underset{K Y}{K Y}$ & 107 \\
\hline 211145 & $\begin{array}{l}\text { Earylington Wost } \\
\text { East Fork }\end{array}$ & & 107 \\
\hline 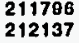 & $\begin{array}{l}\text { East Fork } \\
\text { Eastorn }\end{array}$ & $\begin{array}{l}\text { KY } \\
\text { KY }\end{array}$ & $\begin{array}{l}169 \\
025\end{array}$ \\
\hline 212137 & Eastorn & & 071 \\
\hline & Alias for: $(049790)$ Basver $\mathrm{Cr}$ & Iok IALT & TER] \\
\hline 212137 & $\begin{array}{l}\text { Eastern } \\
\text { Allas for: (107135) Busseyvilte }\end{array}$ & 1. ${ }^{K Y}$ & ${ }^{127}$ \\
\hline 212137 & 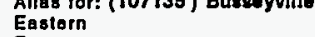 & KY & ${ }^{19]}$ \\
\hline 212261 & Easton & KY & 091 \\
\hline 212261 & Easton & KY & 183 \\
\hline 211920 & Eastiviow & KY & 183 \\
\hline 212323 & $\begin{array}{l}\text { Eastwood Forry } \\
\text { Allas }\end{array}$ & $\begin{array}{c}\mathrm{KY} \\
\text { Consol ICO }\end{array}$ & 233 \\
\hline 012744 & Eatln Fork & KY 10 & 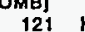 \\
\hline 012744 & Eatin Fook & KY & 235 \\
\hline 212540 & Ebonezer Church & KY & 169 \\
\hline 212695 & Echo & KY & 168 \\
\hline 007863 & Echo North & KY & 168 \\
\hline 213408 & Edon & KY & 159 \\
\hline 214710 & $\begin{array}{l}\text { Edmonton } \\
\text { Aligs }\end{array}$ & KY & 169 \\
\hline 012957 & $\begin{array}{l}\text { Alias for: }(012957) \text { Edmonton } \\
\text { Edmonton Consol }\end{array}$ & $\begin{array}{l}\text { Consol } \\
\mathrm{KY}\end{array}$ & $\begin{array}{l}\text { [RENAM } \\
\text { 169 }\end{array}$ \\
\hline 004890 & Edmonton North & KY & 169 \\
\hline & Allas for: (214741) Edmonton & n North & Consol ! \\
\hline $\begin{array}{l}214741 \\
214772\end{array}$ & $\begin{array}{l}\text { Edmonton North Consol } \\
\text { Edmonton South }\end{array}$ & $\begin{array}{l}\text { KY } \\
\text { KY }\end{array}$ & $\begin{array}{l}169 \\
169\end{array}$ \\
\hline & Allas for: (012957) Edmonton & n Consol & I (сомв] \\
\hline $\begin{array}{l}215521 \\
009731\end{array}$ & $\begin{array}{l}\text { Edsol } \\
\text { Ekron }\end{array}$ & $\begin{array}{l}\text { KY } \\
\text { KY }\end{array}$ & $\begin{array}{l}083 \\
163\end{array}$ \\
\hline & Elam Flats & KY & \\
\hline & Allas for: $(012878)$ Zlon North & ith Consol & I ICOMB] \\
\hline 217896 & & $K Y$ & 059 \\
\hline 217896 & & KY & 149 \\
\hline 217027 & Elba North & KY & 059 \\
\hline 217927 & Elba North & KY & $149 \quad 1$ \\
\hline 217058 & Elba South & KY & $149 !$ \\
\hline 217809 & Elba Wast & $K Y$ & 149 \\
\hline & $\begin{array}{l}\text { Allas for: (1013050) Hangers } \\
\text { Elbow Bond }\end{array}$ & $\underset{K Y}{\text { Landing }}$ & $\begin{array}{l}\text { Consol } \\
171\end{array}$ \\
\hline 013475 & Elcomb & $\widehat{K Y}$ & 095 \\
\hline 012748 & Ellisha Braneh & KY & 147 \\
\hline 219188 & Elk Croek & KY & 107 \\
\hline 218260 & Elk Croek East & KY & 107 \\
\hline 010385 & Elkatawa & KY & 025 \\
\hline 011710 & Elkhoin Clty BS & KY & $185 F$ \\
\hline 011711 & Elkhorn Clty BS East & KY & $195 \mathrm{~F}-\mathrm{C}(\mathrm{C}-\mathrm{C}$ \\
\hline 219873 & Elkton & KY & 2197 \\
\hline 012749 & & $\underset{K Y}{K Y}$ & 0019 \\
\hline $\begin{array}{l}004882 \\
222081\end{array}$ & $\begin{array}{l}\text { Ellls Fork } \\
\text { Elna }\end{array}$ & $\begin{array}{l}\text { KY } \\
\text { KY }\end{array}$ & $\begin{array}{ll}207 & 5 \\
115 & \mathrm{~J}\end{array}$ \\
\hline 222081 & Elna & KY & 175 \\
\hline 222577 & Elrod & KY & $199 \mathrm{~F}$ \\
\hline 004893 & Embarton & KY & 171 \\
\hline 013853 & Emlly Croak DBS & KY & $159 \quad 1$ \\
\hline 223662 & Emory-Moore & KY & 0098 \\
\hline 221313 & Emplio & KY & 047 \\
\hline & $\begin{array}{l}\text { Erllino } \\
\text { Allas for: (013039) Opalda }\end{array}$ & $\begin{array}{l}\text { KY } \\
\text { neolico }\end{array}$ & OMB \\
\hline & 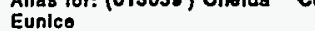 & KY & 001 \\
\hline & Eutorpo & KY & 059 \\
\hline 230211 & Eutorpo & KY & 101 \\
\hline 230242 & Euterpe North & $K Y$ & $101 i$ \\
\hline & Allas for: (230211) Euterpe (C & [COMB] & \\
\hline 231234 & Everatt School & $\mathrm{KY}$ & $177 \mathrm{~N}-2-3-1$ \\
\hline 004895 & Exlo & $\mathrm{KY}_{\mathrm{KY}}$ & 087 \\
\hline 012960 & $\begin{array}{l}\text { Exio South } \\
\text { Allas for: (584877) Pickett }\end{array}$ & $\underset{\text { onsol IC }}{K Y}$ & 087 \\
\hline & $\begin{array}{l}\text { Alas for: (584677) Pickott } \\
\text { Falrplay }\end{array}$ & $\begin{array}{l}\text { Oneol IC } \\
\text { KY }\end{array}$ & \\
\hline 233940 & Falrviow & KY & 235 \\
\hline $\begin{array}{l}00486 \\
004896\end{array}$ & $\begin{array}{l}\text { Falrrvlow Church } \\
\text { Falrylaw Church }\end{array}$ & KY & 008 \\
\hline 146 & $\begin{array}{l}\text { Farlivew Church } \\
\text { Falth North }\end{array}$ & ${ }_{K Y}^{K Y}$ & $\begin{array}{l}061 \\
149\end{array}$ \\
\hline & alls of & KY & 027 \\
\hline & Falls of Ros & KY & 028 \\
\hline 004897 & Fsllsburg & KY & $127 \mathrm{C}$ \\
\hline & Ilas lor: (014094) & Consol I & [RENAM] \\
\hline & Fallsburg Coneol & $\underset{K Y}{K Y}$ & $127 i$ \\
\hline 235459 & $\begin{array}{l}\text { Fannin } \\
\text { Fannak }\end{array}$ & $K_{K Y}^{K Y}$ & $063 E$ \\
\hline 004898 & Fannys Creak & $\underset{k Y}{K Y}$ & $053 c$ \\
\hline $\begin{array}{l}004258 \\
0.12522\end{array}$ & $\begin{array}{l}\text { annys Creok } \\
\text { arler DBS }\end{array}$ & $\begin{array}{l}\mathrm{KY} \\
\mathrm{KY}\end{array}$ & $\begin{array}{ll}057 & C \\
193 & P\end{array}$ \\
\hline & wing & KY & $159 \mathrm{i}$ \\
\hline & hureh $\mathrm{v}$ & KY & 159 \\
\hline
\end{tabular}




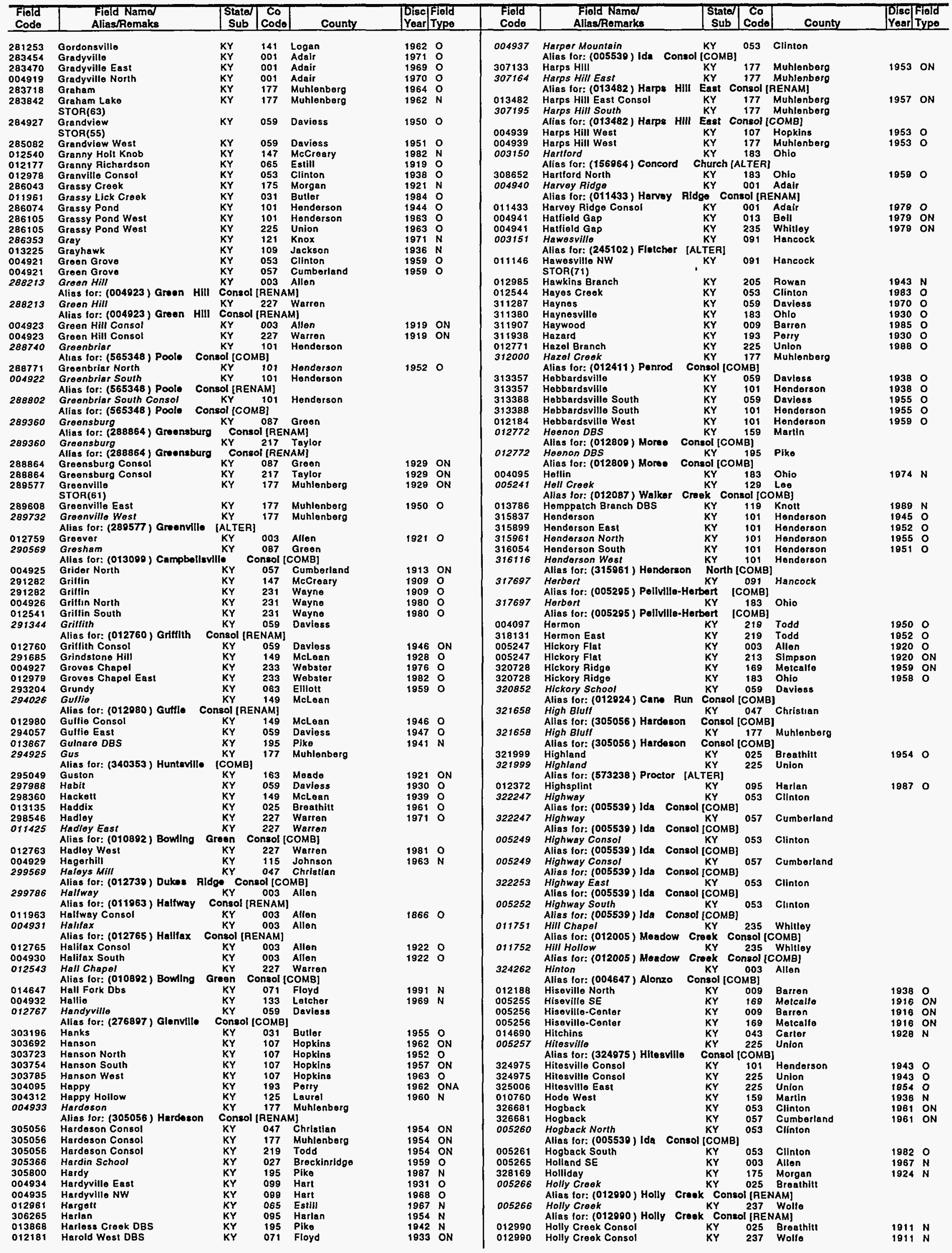


KENTUCKY

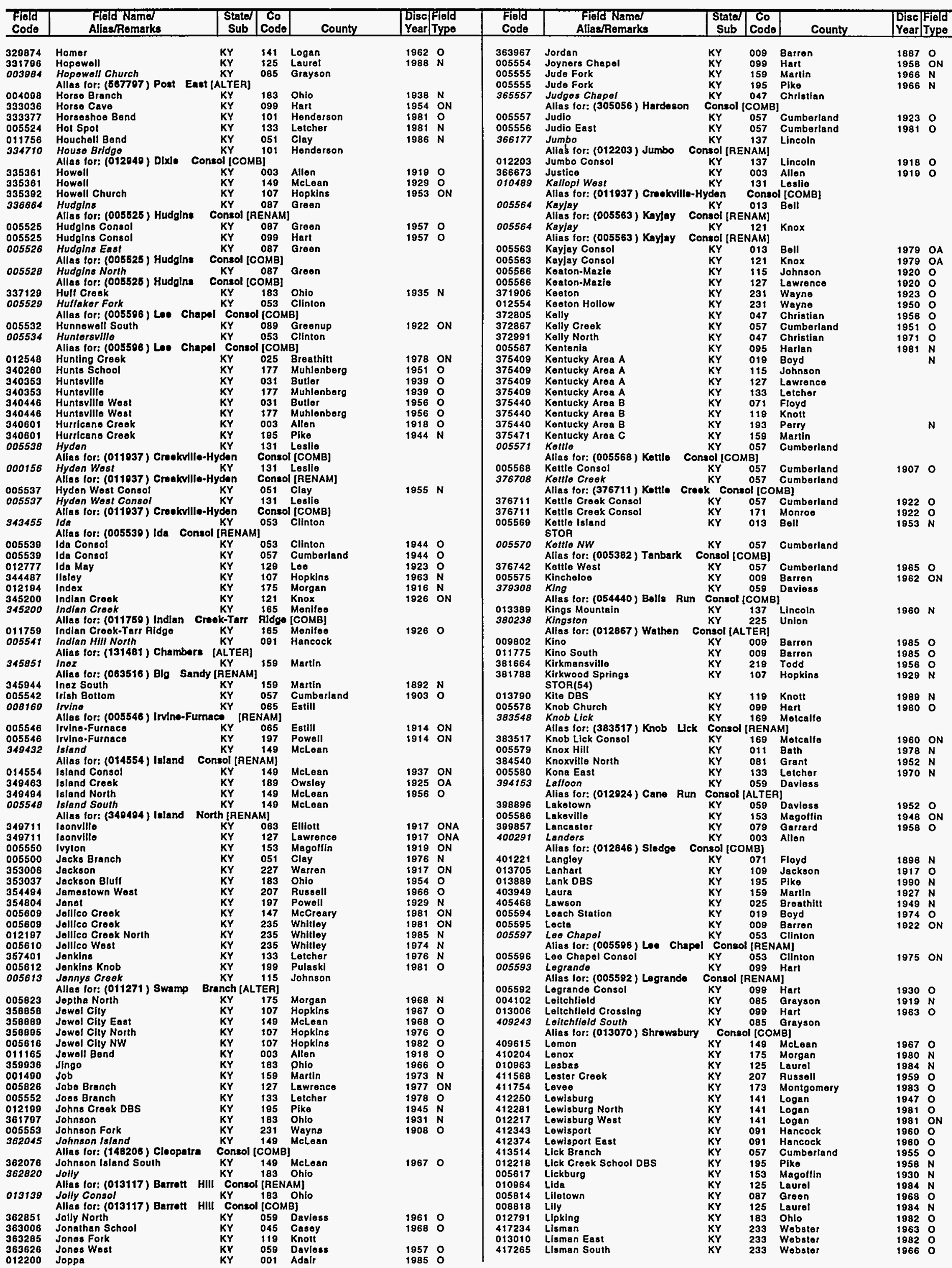




\begin{tabular}{|c|}
\hline $\begin{array}{l}\text { Field } \\
\text { Code }\end{array}$ \\
\hline 005817 \\
\hline 005818 \\
\hline $\begin{array}{l}418474 \\
013890\end{array}$ \\
\hline $\begin{array}{l}013890 \\
0058819\end{array}$ \\
\hline \\
\hline 012792 \\
\hline 012793 \\
\hline 005821 \\
\hline 004103 \\
\hline 419776 \\
\hline $\begin{array}{l}419807 \\
419931\end{array}$ \\
\hline$\$ 18993$ \\
\hline 418993 \\
\hline 005825 \\
\hline $\begin{array}{l}013011 \\
420148\end{array}$ \\
\hline 420427 \\
\hline 014255 \\
\hline 014255 \\
\hline 420458 \\
\hline 420458 \\
\hline 420520 \\
\hline 420582 \\
\hline 004104 \\
\hline 420675 \\
\hline 420675 \\
\hline 420706 \\
\hline 420799 \\
\hline \\
\hline 005827 \\
\hline 014257 \\
\hline 013792 \\
\hline 423063 \\
\hline 005621 \\
\hline 424799 \\
\hline \\
\hline 424830 \\
\hline 424830 \\
\hline 424830 \\
\hline 124861 \\
\hline \\
\hline$\$ 24892$ \\
\hline 424954 \\
\hline 425326 \\
\hline 425419 \\
\hline \\
\hline 004105 \\
\hline 011790 \\
\hline 012226 \\
\hline 004440 \\
\hline 012567 \\
\hline 429046 \\
\hline 012794 \\
\hline 012794 \\
\hline 432899 \\
\hline 011792 \\
\hline 011792 \\
\hline 435100 \\
\hline \\
\hline 080794 \\
\hline 010785 \\
\hline 436333 \\
\hline 438543 \\
\hline 438543 \\
\hline 438543 \\
\hline 438574 \\
\hline 438605 \\
\hline 438636 \\
\hline 005838 \\
\hline 005838 \\
\hline 440051 \\
\hline$\$ 40155$ \\
\hline 440481 \\
\hline 440775 \\
\hline \\
\hline 440775 \\
\hline 440775 \\
\hline 443038 \\
\hline 443255 \\
\hline 443875 \\
\hline 005843 \\
\hline 445340 \\
\hline 013708 \\
\hline \\
\hline \\
\hline 013896 \\
\hline 013020 \\
\hline 449579 \\
\hline 013022 \\
\hline 448765 \\
\hline 449951 \\
\hline 006360 \\
\hline 004108 \\
\hline 004107 \\
\hline 004107 \\
\hline 451408 \\
\hline 006363 \\
\hline 454725 \\
\hline
\end{tabular}


KENTUCKY

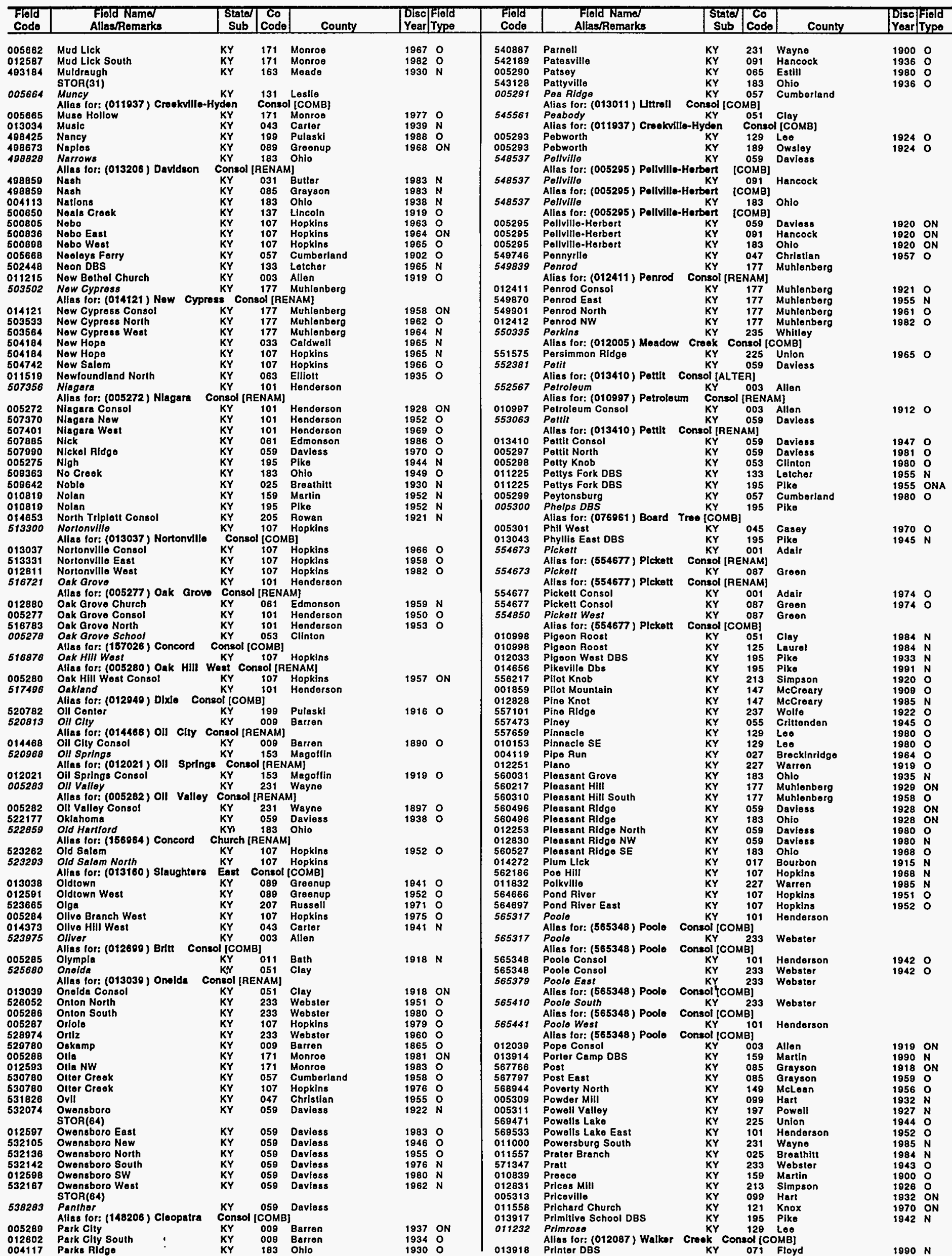




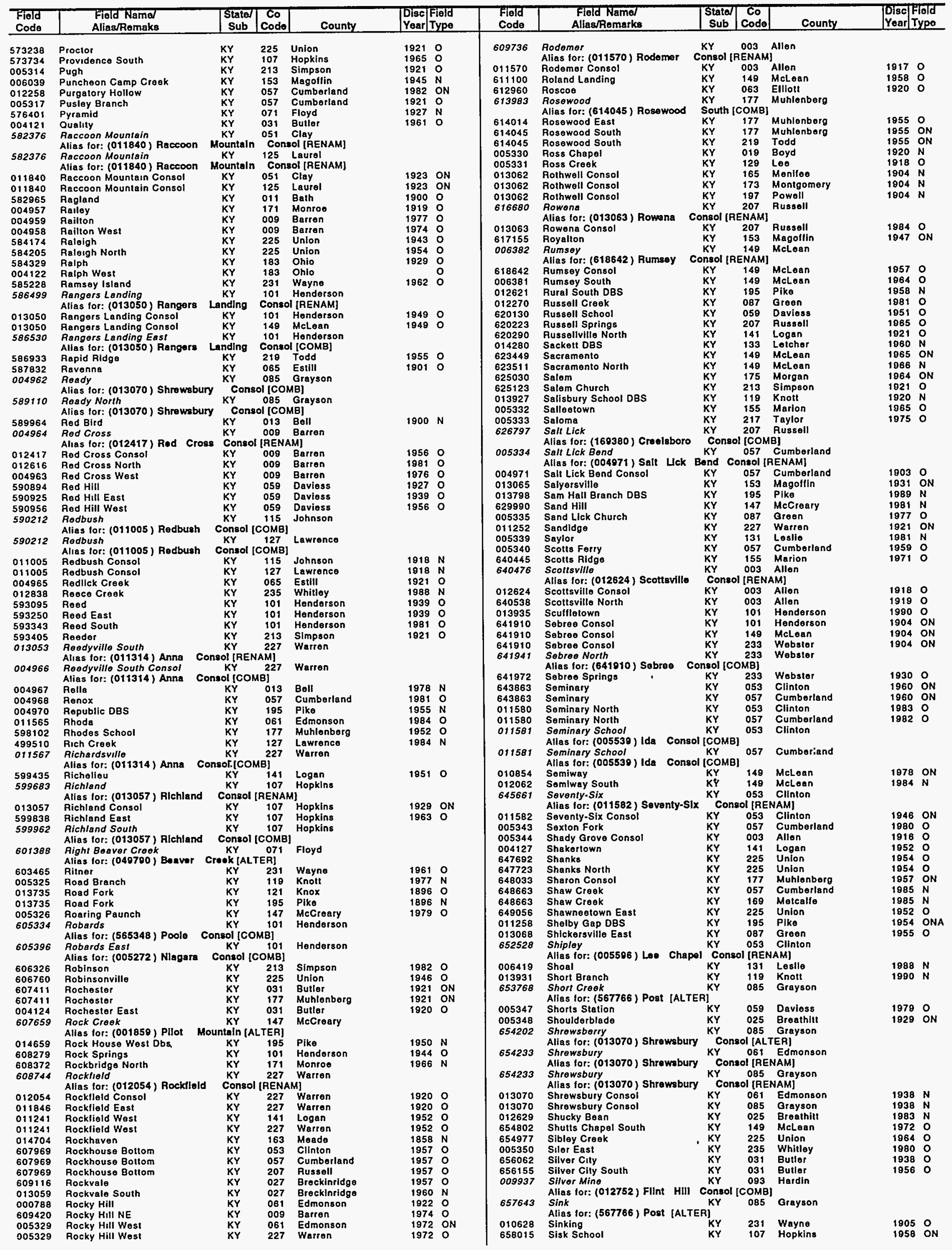


$\begin{array}{ll}0 & \\ \text { N } & 861 \\ \text { N } & 6161\end{array}$

N 886

O 906

N 2561

N 0661

O 8861

N $\angle 16 !$

$\begin{aligned} \text { NO } & 1261 \\ \text { NO } & 8961\end{aligned}$

NO 1861

$\forall$ NO 9261

N 2961

NO $\vdash \varepsilon 6 เ$

N 2261

- 1861

$\begin{array}{ll}0 & 996 ! \\ N & 0861\end{array}$

O LLGL

아 $\$ 861$

NO 9961

NO 1281

$\begin{array}{ll}0 & 9981 \\ N & S+61\end{array}$

N Ot61

O OS6!

$N \$<61$

- 6E6!

N 1561

NO
O 9261

9261

O 1961

NO 9861

NO $6|6|$

O 9861

O 9981

( 2861

- 8161

NO 8161

- 2961

N 9961

- 6961

- 696

$\begin{array}{lll}0 & \$ 61 \\ 0 & 8261\end{array}$

- 9261

(n)

O 696!

- 6161

No

O 2261

o lo6i

N 8L6!

NO EBE!

NO
N 9861
S

NO $\$ 861$

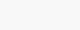

- $\$ 961$

0
0 $\$ 961$

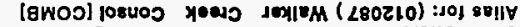

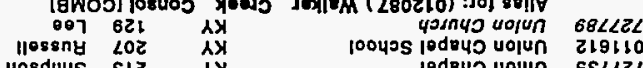
vosduIS ELC AXX jedeus volun SELLCL

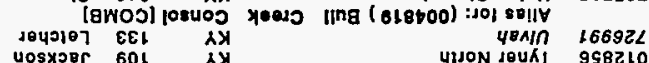
UOSXOP 601 AX vosyor 601 AX

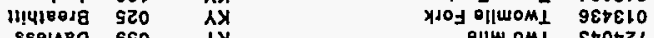

$880 ! \wedge 80$ 6SO $\quad$ SX

UEן1sE 560 AX

$\begin{array}{lll}\text { OUAOM } & \text { IEL } & A X \\ A X & \end{array}$

र́18MO 681

BrequolunW LLI AXY

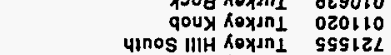

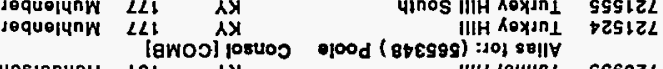
L 101 사

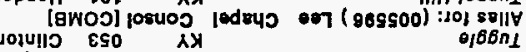

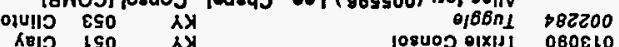

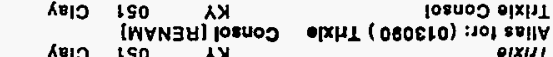

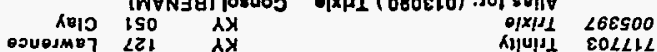

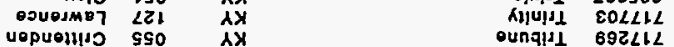

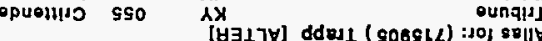

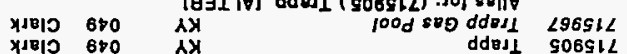
XIEIO 670 A

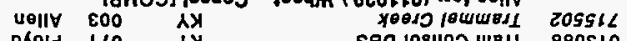

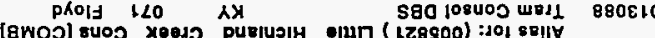

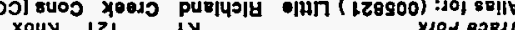

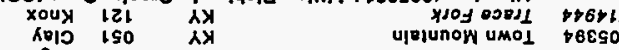

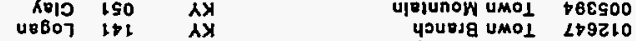

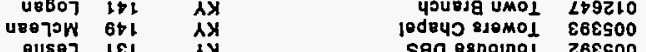

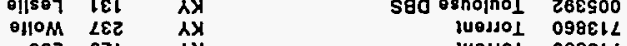

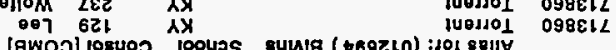

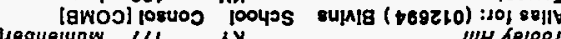

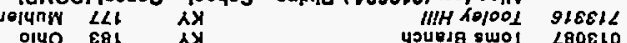
OI4O E8I AX

DosuoW HL $\mathrm{AX}$

oxId sel AX

Oxld 561 AXX

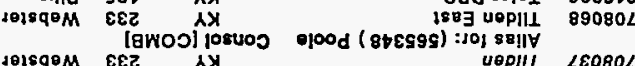
मө1sq०M EEZ AY

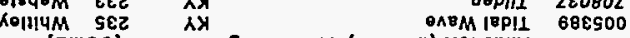

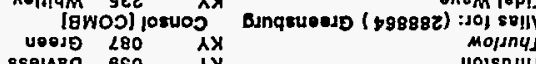
MolsnyL $9<0<0<$

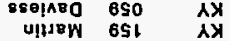
pKoIJ 120 AXX

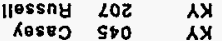

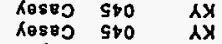

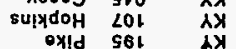

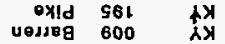

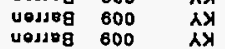

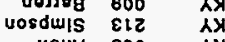

uөाY 800 Aर्र

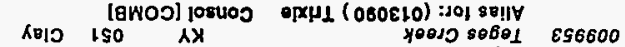

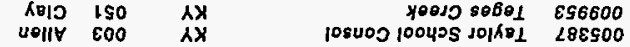

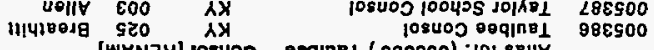

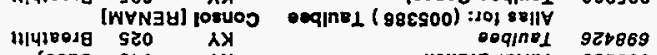

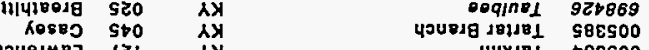

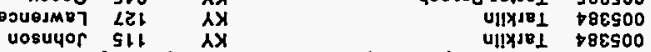

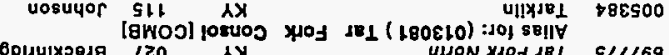

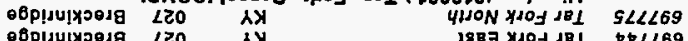

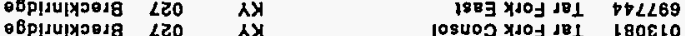

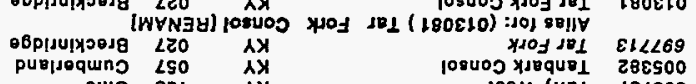

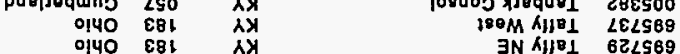

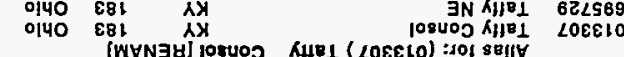

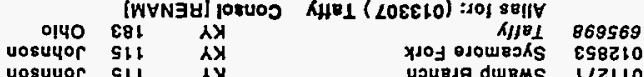
uosuyor S11 $A$ AX

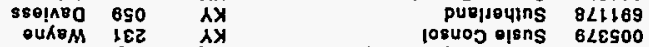

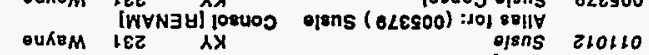

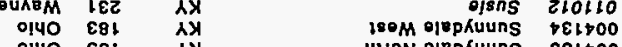

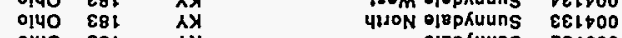

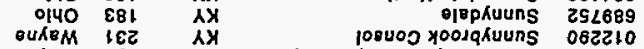

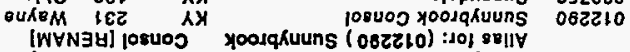

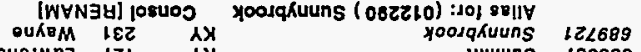

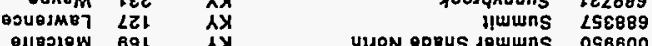

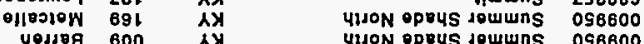

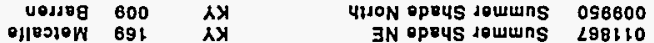

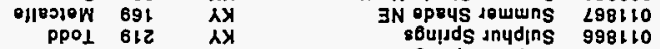

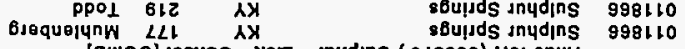

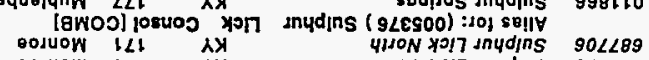

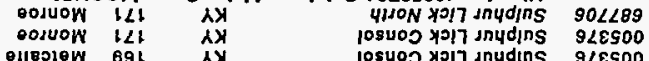

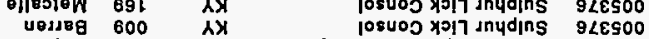

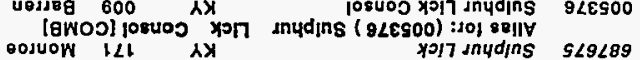

O 9061
No 8961

$\begin{array}{ll}0 & \$ 661 \\ 0 & \$ 661\end{array}$

- 1661

$\begin{array}{ll}N & 2 \angle B ! \\ O & 086 !\end{array}$

$\begin{array}{ll}\text { O } & 0861 \\ N & 2 \angle 61\end{array}$

N 6861

$\begin{array}{ll}\text { O } & 6161 \\ \text { O } & 0 \angle 6 !\end{array}$

NO 2961

$N$ 256!
$N$
$286 !$

O 6081

O E06

N $0<B i$

O 6881

$\begin{array}{ll}0 & 1261 \\ 0 & 2961 \\ 0 & 9161\end{array}$

o ع96।

$0 \$ 781$

O 5961

O Est।

$\begin{array}{ll}\text { O } & 6261 \\ \text { O } & 6261\end{array}$

$\begin{array}{ll}0 & 6261 \\ 0 & 0861\end{array}$

O 8961

0
0 2961

No 9961

NO $996 !$

$\begin{array}{rl}\text { NO } & 956 ! \\ O & 676 !\end{array}$

O 1461

$\begin{array}{ll}O & 8861 \\ N & 066 !\end{array}$

$\mathrm{N}$ S86!

o L961

N 1861

$\begin{array}{ll}N & 1861 \\ 0 & 8161 \\ 0 & 1561 \\ 0 & 2561\end{array}$

$\begin{array}{ll}0 & \text { ZS61 } \\ \text { O } & \text { IS6I }\end{array}$

$\begin{array}{ll}0 & \lfloor 96 ! \\ 0 & \$ 56 !\end{array}$

$\begin{array}{lll}0 & \$ 56 ! \\ 0 & 2 \$ 6 !\end{array}$

$\begin{array}{ll}0 & 276 ! \\ 0 & 2761\end{array}$

N 0861

$\begin{array}{ll}\text { O } & \varepsilon 06 ! \\ \text { O } & \varepsilon 86 !\end{array}$

- 6561

o $\$ 961$

$\begin{array}{ll}0 & 2681 \\ N & 8961\end{array}$

$\begin{array}{ll}0 & 8161 \\ 0 & 8161\end{array}$

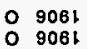

\section{- 9961}

$\begin{array}{ll}\text { O } & \angle \$ 6 ! \\ \text { O } & \angle 96 !\end{array}$

$\begin{array}{ll}\text { O } & 296 ! \\ N & \varepsilon 96 !\end{array}$

$\begin{array}{ll}\text { NO } & 8961 \\ \text { NO } & 8961\end{array}$

$\begin{array}{ll}0 & L \forall 8 \mid \\ 0 & L \forall 61\end{array}$

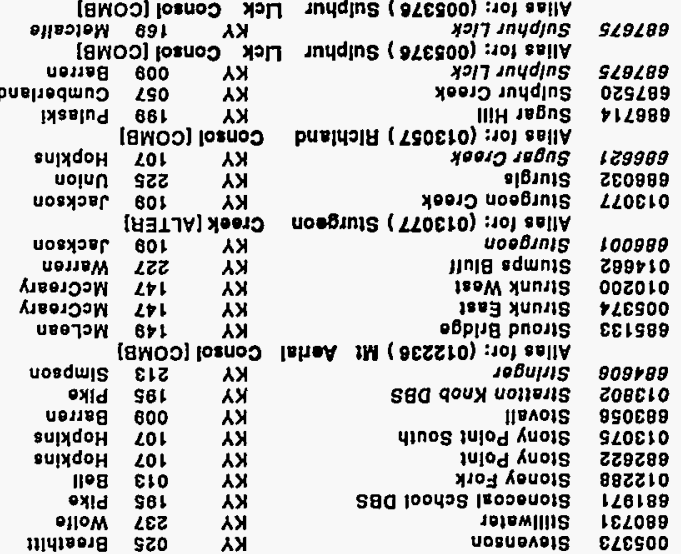

M14180J8 520 AX

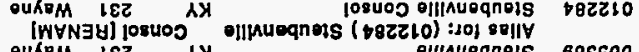

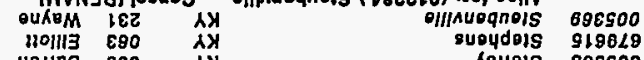

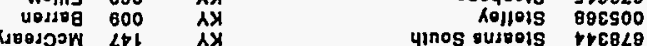

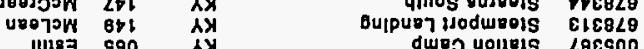

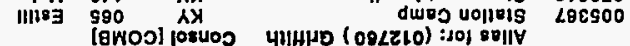

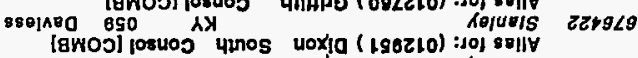
$101999 M$ EEZ $A X$ UIJON OdOLURIS OOr $9 \angle 9$

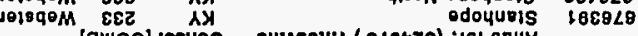

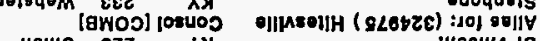
uojun 922 사 juosulA is rearls

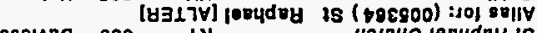

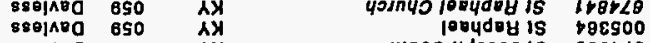
880jAв 6SO AX BselazO 6SO AX ULJON Ydosor IS SCOVL9

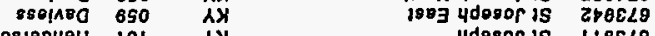
บosiopueH 201 새

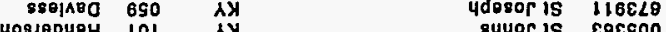

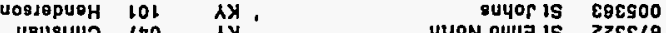

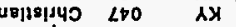

$\begin{array}{lll}\text { sulydOH } & \text { LOL } & \text { AX } \\ \text { sulydOH } & \text { LOL } & \text { AX }\end{array}$ ULON OWIJ is ZZEELO

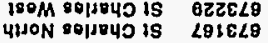
sulydOH $\angle O H$ BY HOIS

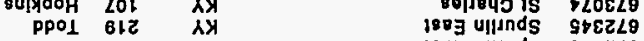

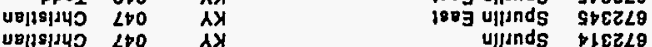

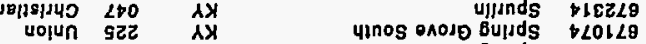

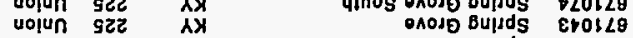
uossepueh 101 AX HOIII 890 AX

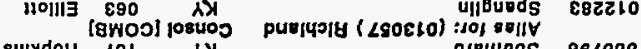

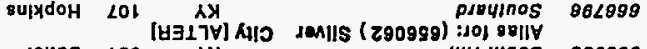

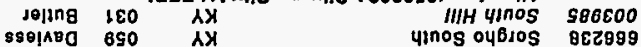

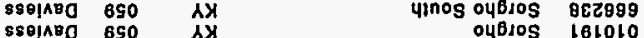

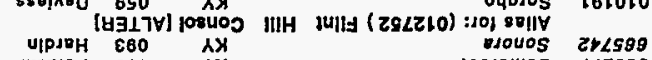

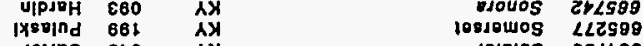

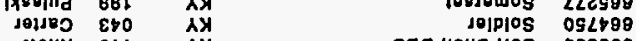
HOU 611 AX्र $\quad$ SEA IIOUS 1105 BSES00

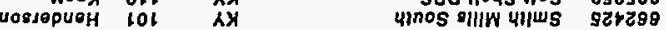

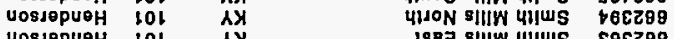

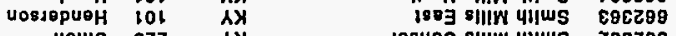

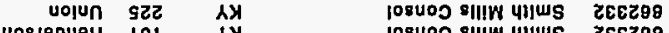

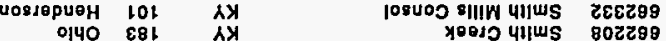
pupjoqung 290 AX ppol 612 $A$ AX uojun $S Z 2$ AX

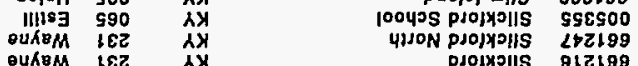

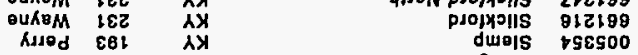

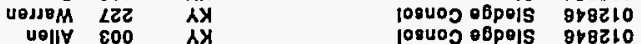

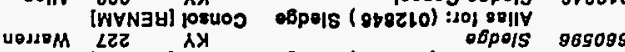
[WVNGH] josuos eopess (968Z10): UOIY 800 AH

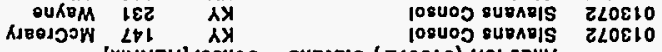
OUKBM IWVNGH] lOSUOS SUDADIS (ZLOELO) :JOJ ODIJ SUDAVIS ESESOO AWROJOOW LTH

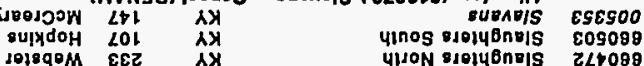

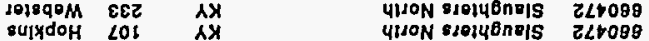
sUIydOH $\angle O L$ XY

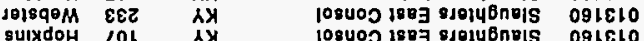

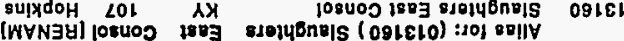

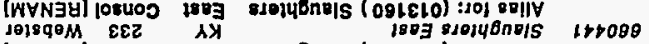

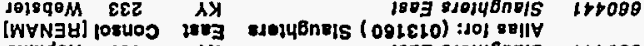

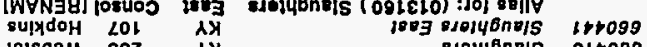

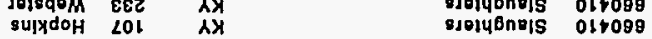

$810146 \mathrm{nV} / \mathrm{s} \quad 017098$ 


\section{KENTUCKY}

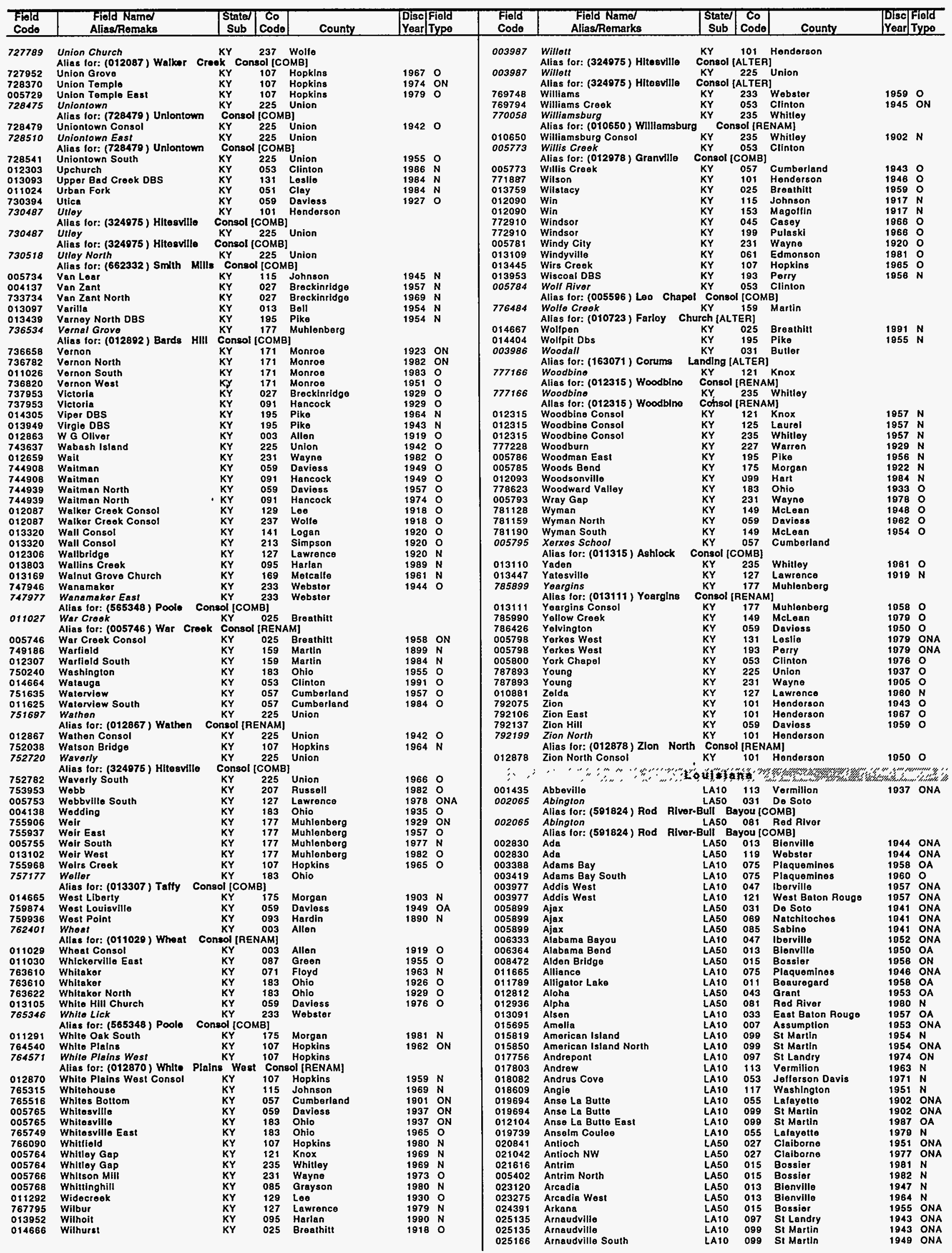


LOUISIANA

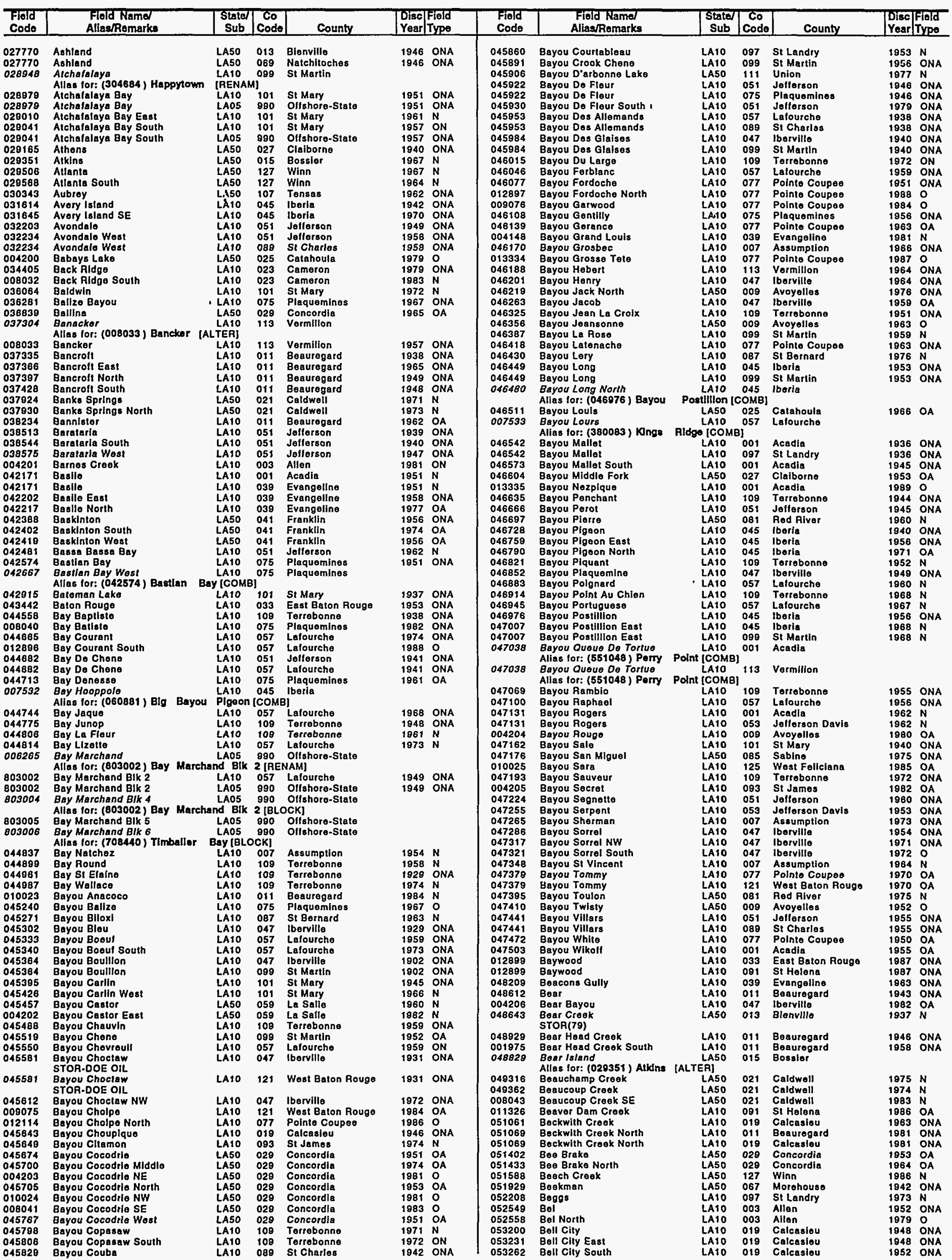


LOUISIANA

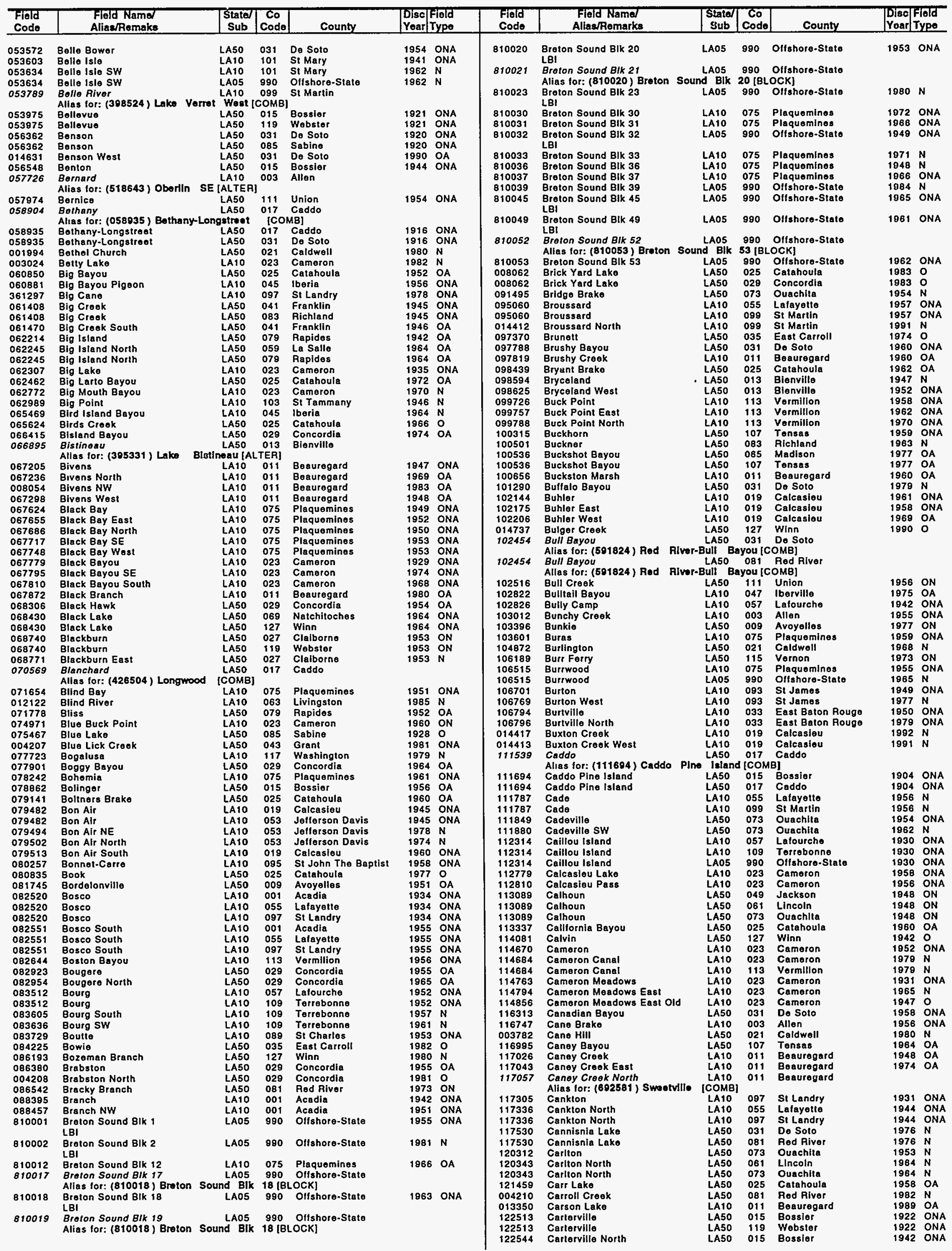


LOUISIANA

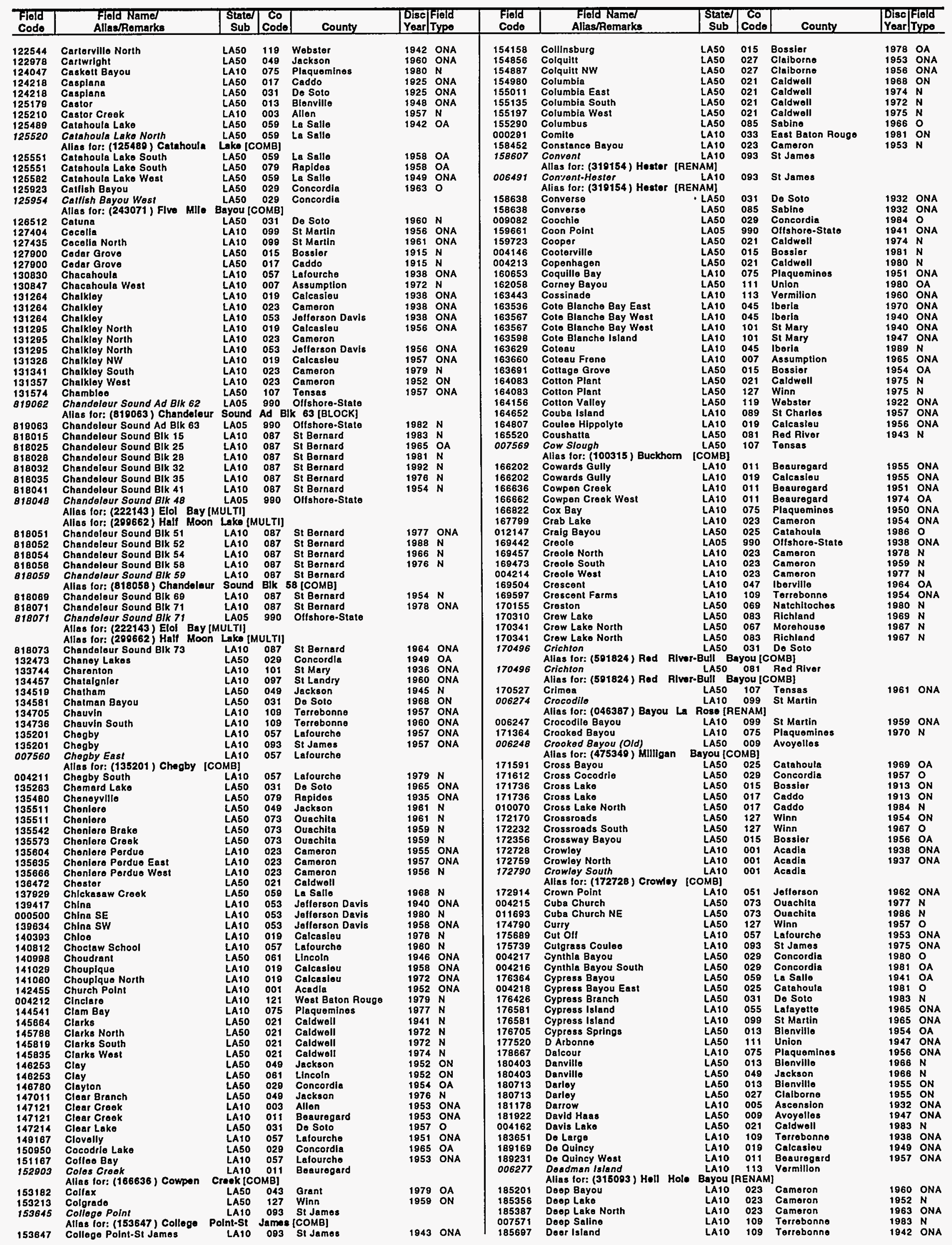




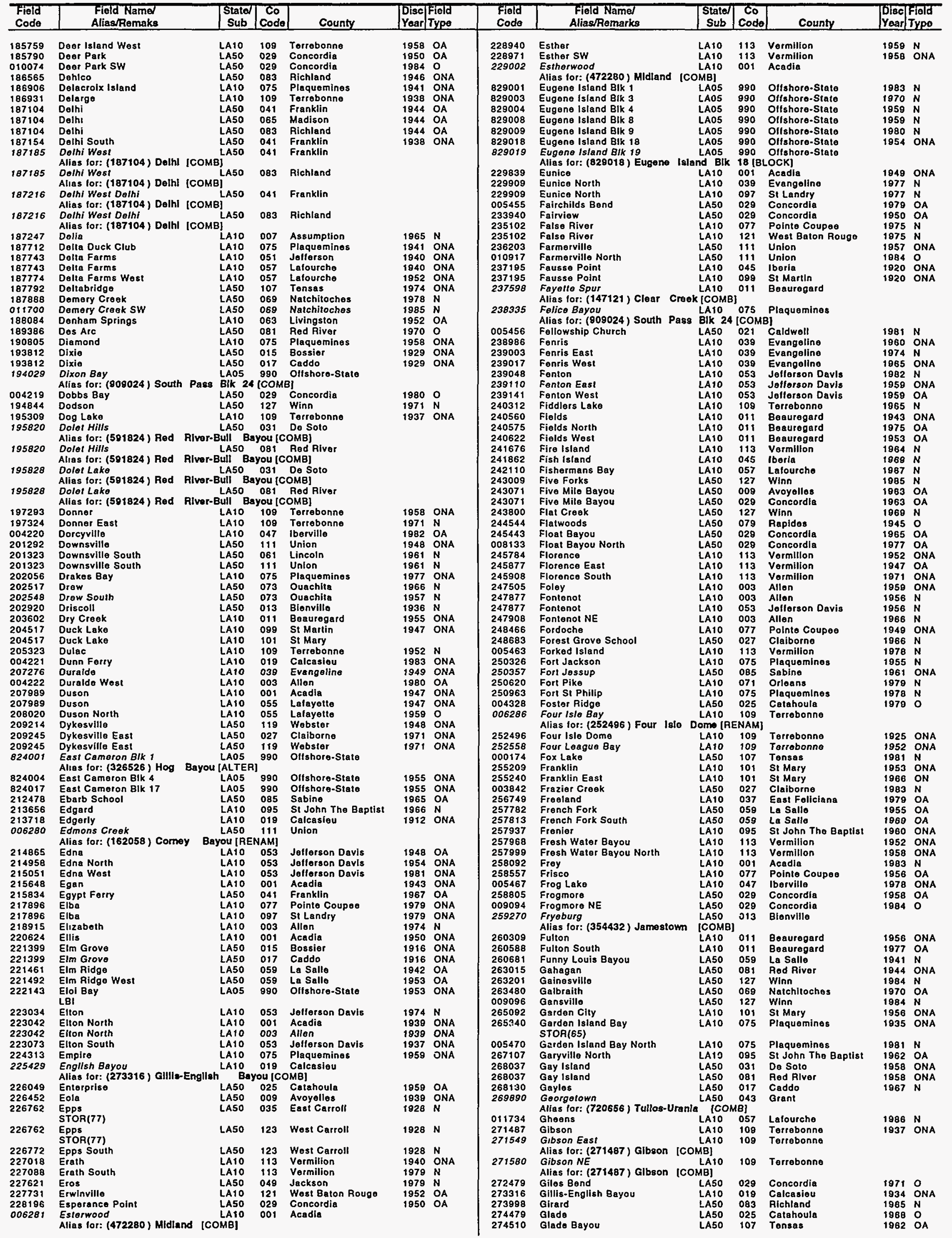


LOUISIANA

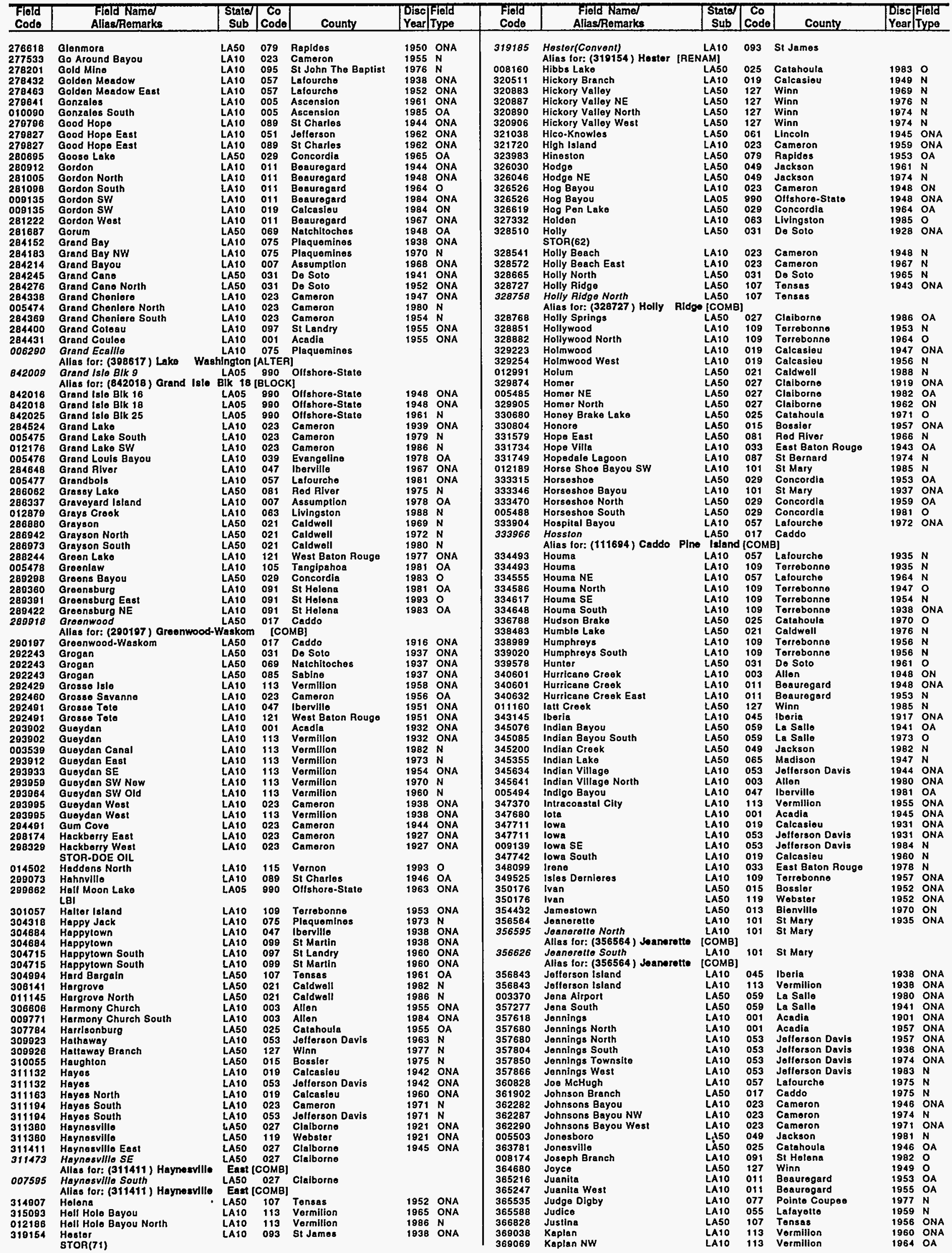


LOUISIANA

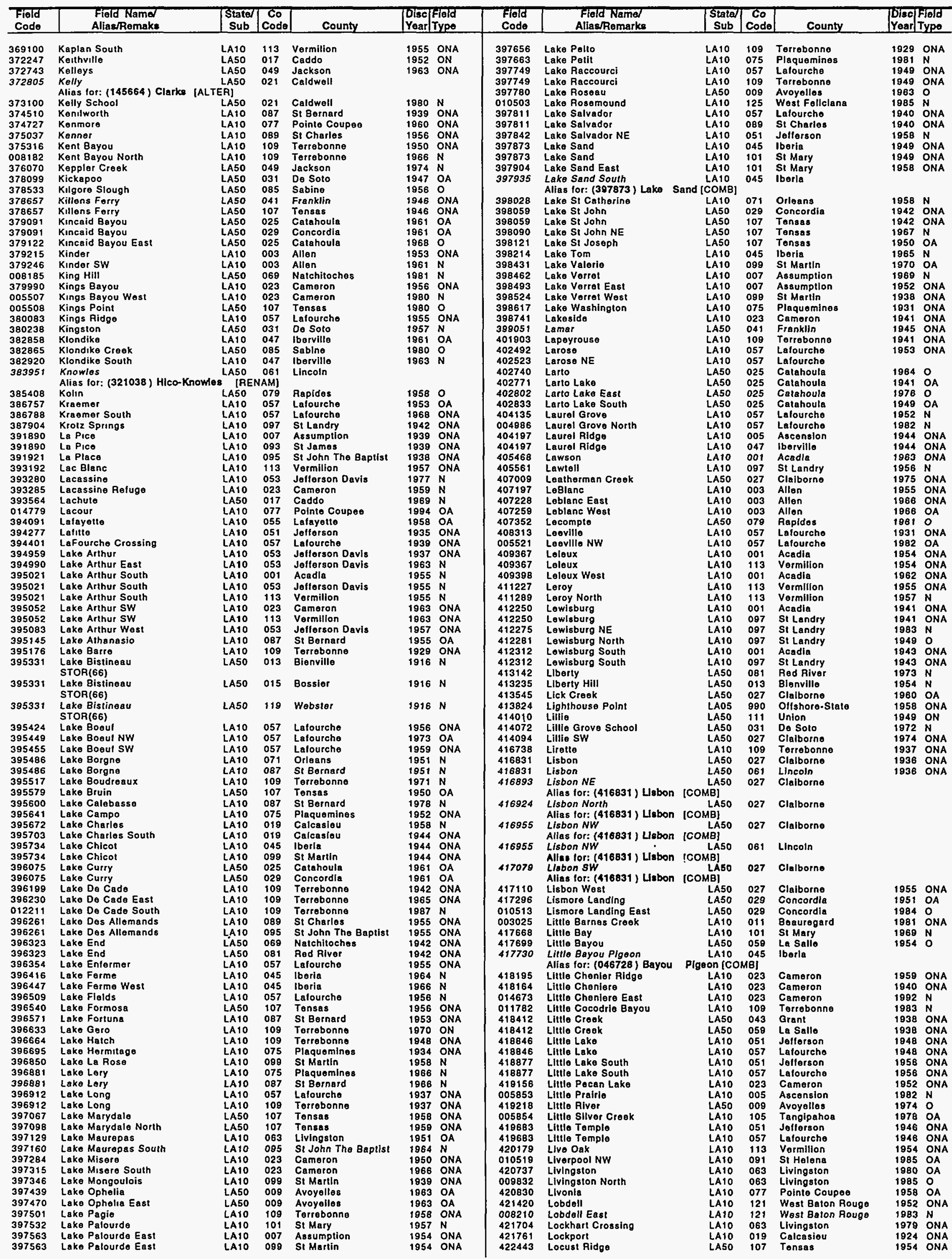


LOUISIANA

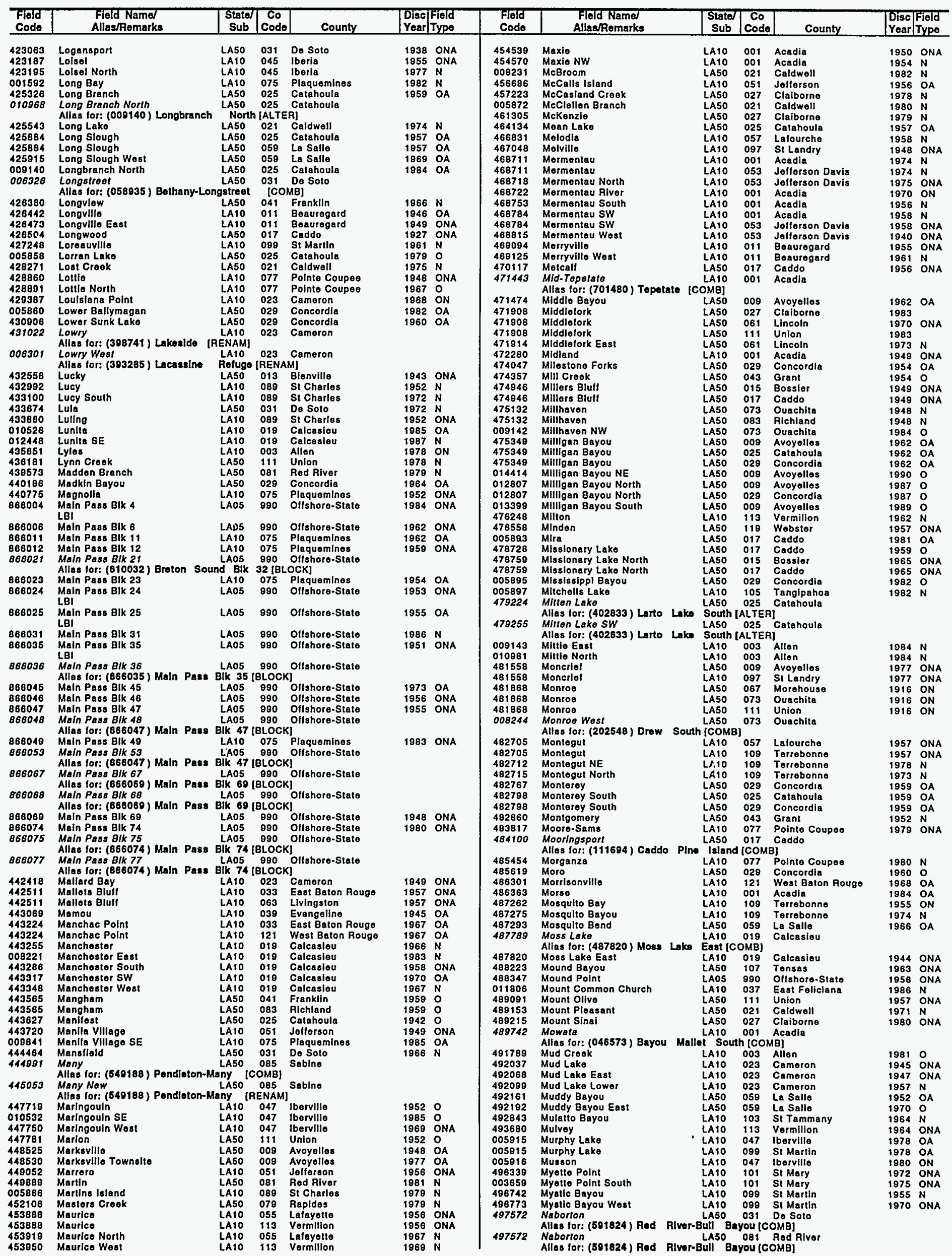


LOUISIANA

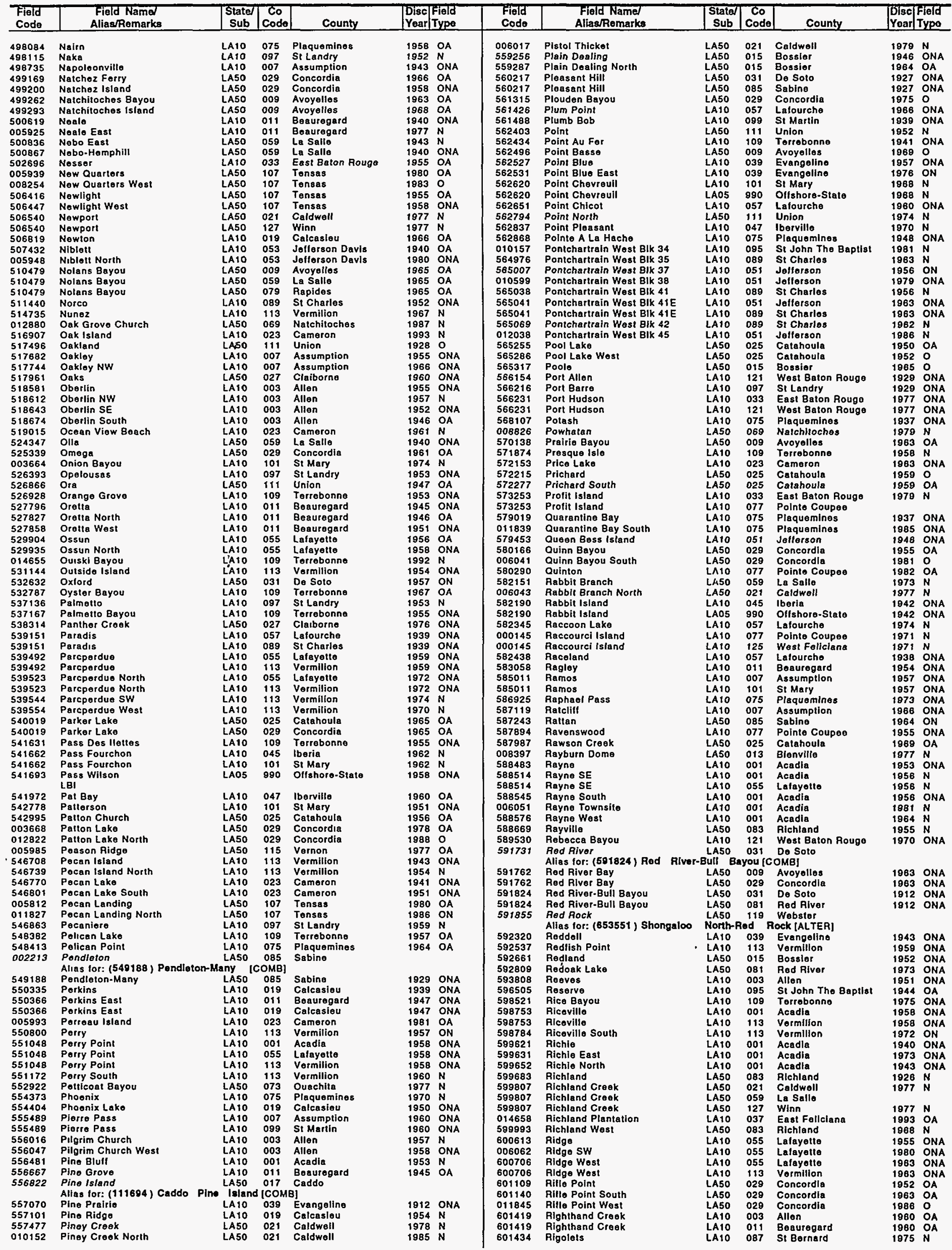


LOUISIANA

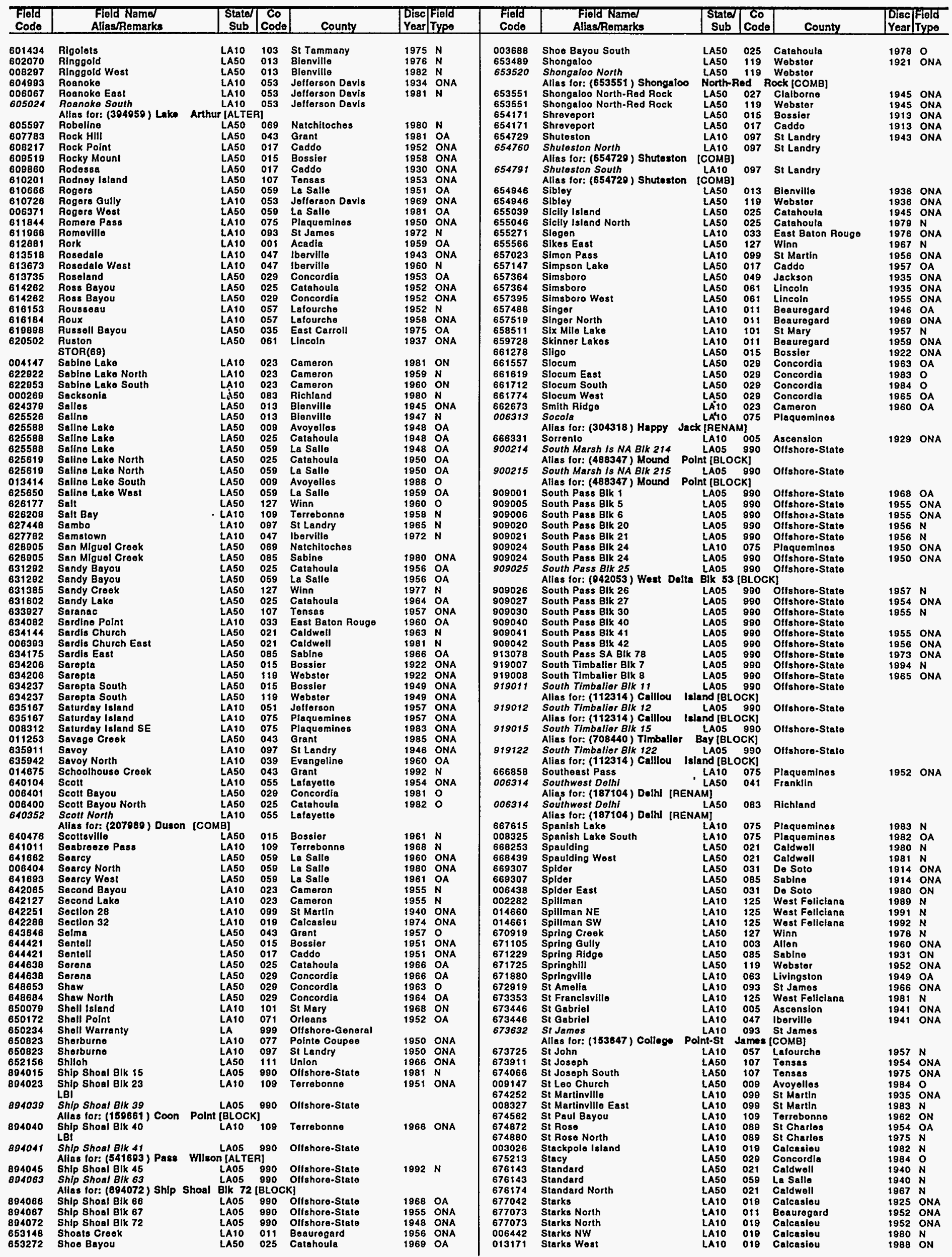




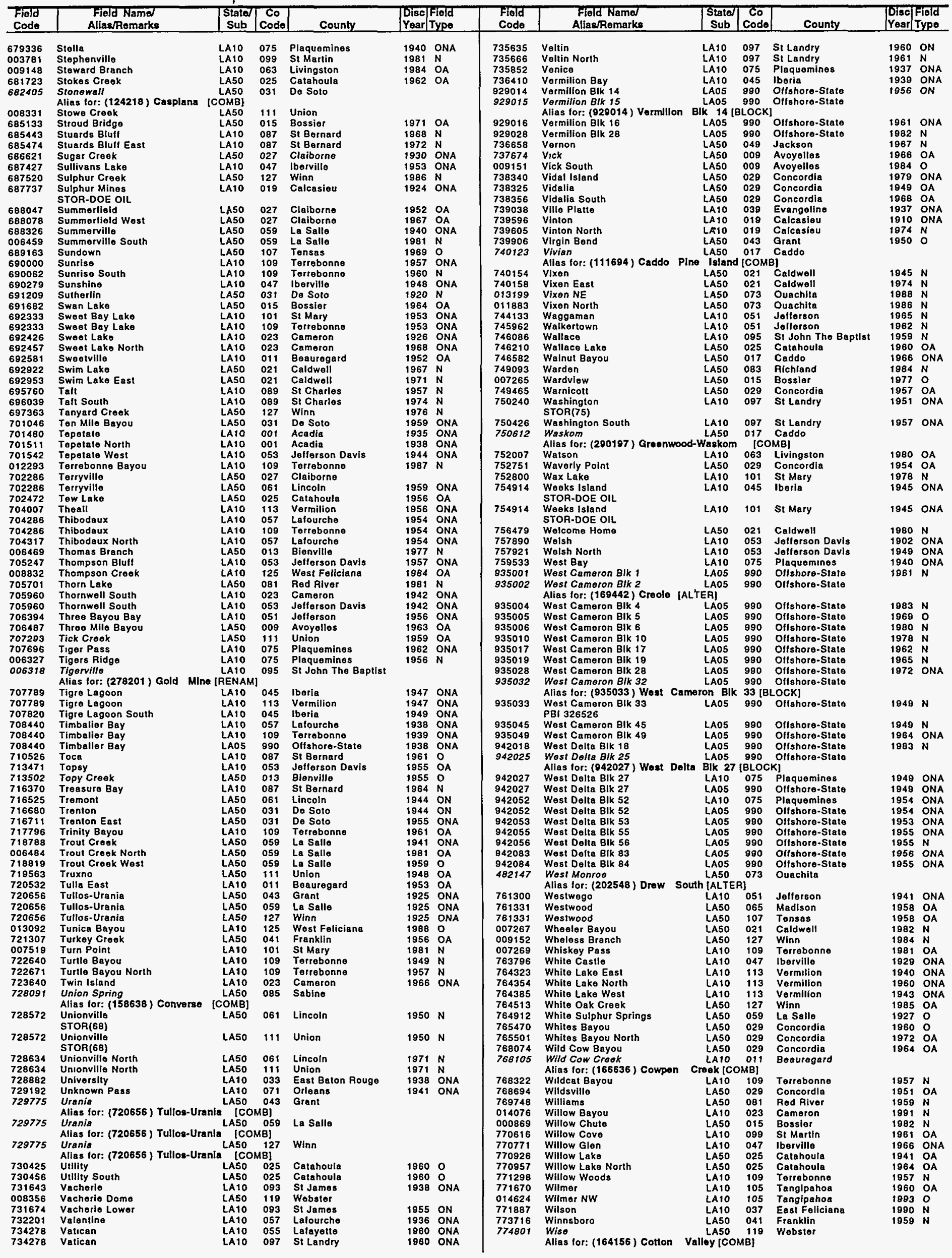


MICHIGAN

\begin{tabular}{|c|}
\hline $\begin{array}{l}\text { Field } \\
\text { Code } \\
\end{array}$ \\
\hline 774925 \\
\hline 776329 \\
\hline 777150 \\
\hline 777600 \\
\hline 777662 \\
\hline 780189 \\
\hline 780911 \\
\hline 007273 \\
\hline 781369 \\
\hline 791375 \\
\hline 791375 \\
\hline (20780 \\
\hline 793674 \\
\hline 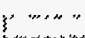 \\
\hline 002210 \\
\hline $00<<10$ \\
\hline 489401 \\
\hline 501504 \\
\hline 549498 \\
\hline$\xi$ \\
\hline 003108 \\
\hline 003326 \\
\hline $\begin{array}{l}003326 \\
003512\end{array}$ \\
\hline $\begin{array}{l}003512 \\
004143\end{array}$ \\
\hline $\begin{array}{l}001263 \\
012673\end{array}$ \\
\hline 003729 \\
\hline 004008 \\
\hline \\
\hline $\begin{array}{l}003361 \\
003362\end{array}$ \\
\hline 006200 \\
\hline 006240 \\
\hline $\begin{array}{l}0067386 \\
0007728\end{array}$ \\
\hline 007045 \\
\hline \\
\hline 007914 \\
\hline 007914 \\
\hline 007914 \\
\hline 009960 \\
\hline 010083 \\
\hline $\begin{array}{l}002761 \\
002762\end{array}$ \\
\hline $\begin{array}{l}002782 \\
002783\end{array}$ \\
\hline 012323 \\
\hline $\begin{array}{l}014728 \\
002764\end{array}$ \\
\hline 013955 \\
\hline 013956 \\
\hline 013331 \\
\hline 012920 \\
\hline 012998 \\
\hline $\begin{array}{l}009514 \\
021818\end{array}$ \\
\hline 021616 \\
\hline 021616 \\
\hline 021616 \\
\hline 021616 \\
\hline 021616 \\
\hline 021616 \\
\hline 021616 \\
\hline 021616 \\
\hline 021616 \\
\hline $\begin{array}{l}021616 \\
023027\end{array}$ \\
\hline 007702 \\
\hline \\
\hline \\
\hline 007703 \\
\hline 028142 \\
\hline 028173 \\
\hline 029506 \\
\hline $\begin{array}{l}030312 \\
03077\end{array}$ \\
\hline \\
\hline \\
\hline 002766 \\
\hline 002767 \\
\hline 002769 \\
\hline 030801 \\
\hline \\
\hline $\begin{array}{l}008515 \\
007706\end{array}$ \\
\hline 035010 \\
\hline \\
\hline 012681 \\
\hline \\
\hline 01131 \\
\hline 002770 \\
\hline 007708 \\
\hline 002771 \\
\hline 002772 \\
\hline $\begin{array}{l}002773 \\
00274\end{array}$ \\
\hline $\begin{array}{l}0027744 \\
002775\end{array}$ \\
\hline 002776 \\
\hline 037676 \\
\hline 40 \\
\hline 37 \\
\hline \\
\hline \\
\hline \\
\hline \\
\hline \\
\hline 002778 \\
\hline 002779 \\
\hline
\end{tabular}


MICHIGAN

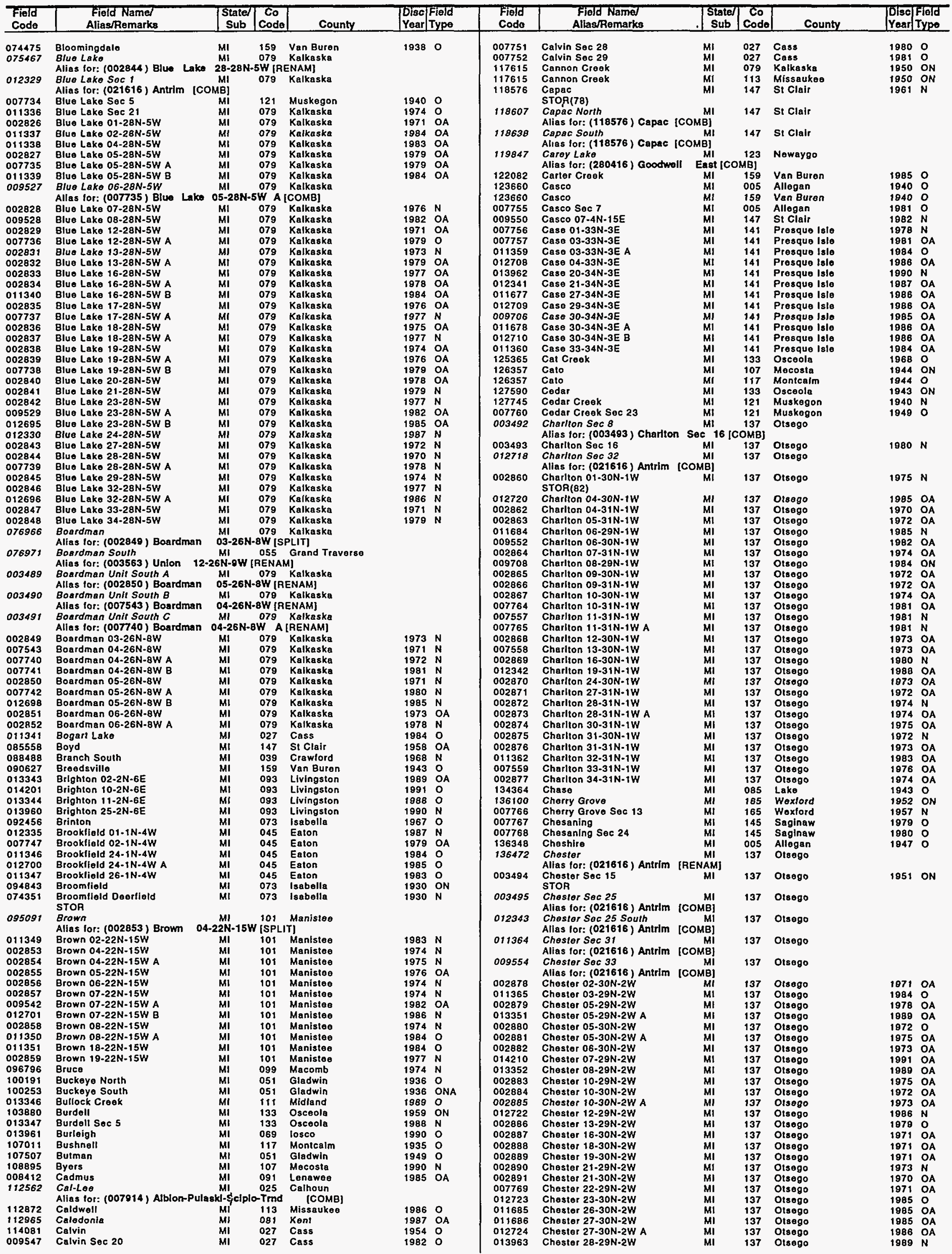


MICHIGAN

\begin{tabular}{|c|c|c|c|c|c|c|c|c|c|c|c|c|c|}
\hline $\begin{array}{l}\text { Flold } \\
\text { Codo }\end{array}$ & $\begin{array}{l}\text { Field Name/ } \\
\text { Alias/Aemarks }\end{array}$ & $\begin{array}{c}\text { Statel } \\
\text { Sub }\end{array}$ & $\begin{array}{c}\text { Co } \\
\text { Code }\end{array}$ & County & \begin{tabular}{|l|l|} 
Disc & \\
Year & \\
\end{tabular} & $\begin{array}{l}\text { Field } \\
\text { Type }\end{array}$ & $\begin{array}{l}\text { Field } \\
\text { Code }\end{array}$ & $\begin{array}{l}\text { Field Name/ } \\
\text { Alias/Remarks }\end{array}$ & \begin{tabular}{|c|} 
Stated \\
Sub
\end{tabular} & \begin{tabular}{c|} 
Co \\
Code \\
\end{tabular} & County & $\left.\mid \begin{array}{l}\text { Dise } \\
\text { Year }\end{array}\right]$ & $\begin{array}{l}\text { Fiteld } \\
\text { Typo }\end{array}$ \\
\hline $\begin{array}{l}002893 \\
002893 \\
011687 \\
138782 \\
139418 \\
139479 \\
007771 \\
009558 \\
009558\end{array}$ & $\begin{array}{l}\text { Chester } 30-30 N-2 W \\
\text { Chaster } 34-30 N-2 W \\
\text { Choster } 35-30 N-2 W \\
\text { Chosterfiold } \\
\text { China Bollo } \\
\text { Chlna East } \\
\text { China East Sac } 13 \\
\text { China Soc } 12 \\
\text { Ching Sac } 31 \\
\text { Allas for: }(164590) \text { Cottrelluille }\end{array}$ & $\begin{array}{l}\text { MI } \\
\text { MI } \\
M I \\
M I \\
M I \\
M I \\
M I \\
M I \\
M I \\
\text { [COMB] }\end{array}$ & $\begin{array}{l}137 \\
137 \\
137 \\
099 \\
147 \\
147 \\
147 \\
147 \\
147\end{array}$ & $\begin{array}{l}\text { Otsego } \\
\text { Otsego } \\
\text { Otsego } \\
\text { Macomb } \\
\text { St Clair } \\
\text { St Clair } \\
\text { St Clair } \\
\text { St Clair } \\
\text { St Clair }\end{array}$ & $\begin{array}{l}1973 \\
1978 \\
1986 \\
1962 \\
1963 \\
1969 \\
1979 \\
1962\end{array}$ & $\begin{array}{l}\text { OA } \\
O A \\
O A \\
O A \\
\text { OA } \\
N \\
N \\
N \\
O A\end{array}$ & $\begin{array}{l}152531 \\
153182 \\
153182 \\
011373 \\
013357 \\
012729 \\
007564 \\
012344 \\
007565 \\
153988\end{array}$ & $\begin{array}{l}\text { Cole Lake } \\
\text { Colfax } \\
\text { Colfax } \\
\text { Colfax Sec } 35 \\
\text { Collax } 14-25 \mathrm{~N}-13 \mathrm{~W} \\
\text { Colfax } 24-25 \mathrm{~N}-13 \mathrm{~W} \\
\text { Colfax } 25-25 \mathrm{~N}-13 \mathrm{~W} \\
\text { Colfax } 31-25 \mathrm{~N}-13 \mathrm{~W} \text { A } \\
\text { Collax } 36-25 \mathrm{~N}-13 \mathrm{~W} \\
\text { Collin }\end{array}$ & $\begin{array}{l}M I \\
M I \\
M I \\
M I \\
M I \\
M I \\
M I \\
M I \\
M I \\
M I\end{array}$ & $\begin{array}{l}123 \\
107 \\
127 \\
127 \\
019 \\
019 \\
019 \\
019 \\
019 \\
147\end{array}$ & $\begin{array}{l}\text { Nowaygo } \\
\text { Mecosta } \\
\text { Ocoana } \\
\text { Ocoana } \\
\text { Benzle } \\
\text { Benzlo } \\
\text { Benzle } \\
\text { Benzle } \\
\text { Benzio } \\
\text { St Clair }\end{array}$ & $\begin{array}{l}1968 \\
1945 \\
1952 \\
1952 \\
1989 \\
1985 \\
1981 \\
1988 \\
1976 \\
1968\end{array}$ & $\begin{array}{l}0 \\
O N \\
O \\
O \\
O \\
O \\
N \\
O A \\
O \\
O A \\
O A \\
O N\end{array}$ \\
\hline 139603 & China South & Mi & 147 & St Clate & 1961 & $\mathbf{N}$ & & STOR(81) & & & & & \\
\hline $\begin{array}{l}0077772 \\
013354\end{array}$ & $\begin{array}{l}\text { Chlppowa Soc } 10 \\
\text { Church Lake }\end{array}$ & $\begin{array}{l}\text { MI } \\
\text { MI }\end{array}$ & $\begin{array}{l}073 \\
135\end{array}$ & $\begin{array}{l}\text { laabolla } \\
\text { Oscoda }\end{array}$ & $\begin{array}{l}1861 \\
1988\end{array}$ & $\stackrel{0}{\mathrm{o}}$ & 155290 & $\begin{array}{l}\text { Columbus } \\
\text { STOR(72) }\end{array}$ & MI & 1478 & St Clair & 1964 & $\mathbf{N}$ \\
\hline 144880 & Claro Clity & $M !$ & 035 & Clara & 1837 & ON & 155352 & Columbus North & MI & 147 & St Clair & 1808 & $O A$ \\
\hline $\begin{array}{l}144889 \\
008710\end{array}$ & $\begin{array}{l}\text { Claro Clty } \\
\text { Clarence } 19-1 \mathrm{~S}-4 \mathrm{~W}\end{array}$ & $\begin{array}{l}\text { MI } \\
\text { MI }\end{array}$ & $\begin{array}{l}073 \\
025\end{array}$ & $\begin{array}{l}\text { Isabolla } \\
\text { Calhoun }\end{array}$ & $\begin{array}{l}1937 \\
1971\end{array}$ & $\mathrm{ON}^{N}$ & $\begin{array}{l}007794 \\
007797\end{array}$ & $\begin{array}{l}\text { Columbus Soc } 2 \\
\text { Columbus Sec } 3\end{array}$ & MI & $\begin{array}{l}147 \\
147\end{array}$ & $\begin{array}{l}\text { St Clair } \\
\text { St Clair }\end{array}$ & $\begin{array}{l}1971 \\
1968\end{array}$ & $\begin{array}{l}O A \\
O A\end{array}$ \\
\hline $\begin{array}{l}007774 \\
007775 \\
000550 \\
007776 \\
007777 \\
009580 \\
009561\end{array}$ & 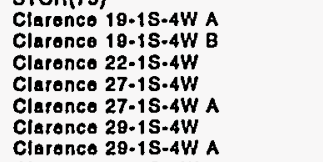 & $\begin{array}{l}M I \\
M I \\
M I \\
M I \\
M I \\
M I \\
M I\end{array}$ & $\begin{array}{l}025 \\
025 \\
025 \\
025 \\
025 \\
025 \\
025\end{array}$ & $\begin{array}{l}\text { Calhoun } \\
\text { Calhoun } \\
\text { Calhoun } \\
\text { Calhoun } \\
\text { Galhoun } \\
\text { Calhoun } \\
\text { Calhoun }\end{array}$ & $\begin{array}{l}1977 \\
1978 \\
1982 \\
1979 \\
1981 \\
1982 \\
1982\end{array}$ & $\begin{array}{l}N \\
N \\
N \\
O A \\
O A \\
N \\
N\end{array}$ & $\begin{array}{l}012730 \\
007795 \\
007796 \\
007798 \\
009570 \\
155538\end{array}$ & $\begin{array}{l}\text { Columbus Sec } 4 \text { A } \\
\text { Columbus Sec } 20 \\
\text { Columbus Sec } 23 \\
\text { Columbus Sec } 32 \\
\text { Columbus Sec } 35 \\
\text { Columbus West } \\
\text { STOR(73) }\end{array}$ & $\begin{array}{l}M I \\
M I \\
M I \\
M I \\
M I \\
M I\end{array}$ & $\begin{array}{l}147 \\
147 \\
147 \\
147 \\
147 \\
147\end{array}$ & $\begin{array}{l}\text { St Clair } \\
\text { St Clair } \\
\text { St Clair } \\
\text { St Clair } \\
\text { St Clair } \\
\text { St Clair }\end{array}$ & $\begin{array}{l}1886 \\
1872 \\
1965 \\
1970 \\
1982 \\
1967\end{array}$ & $\begin{array}{l}\mathbf{N} \\
\mathbf{N} \\
\mathbf{N} \\
\mathbf{N} \\
\mathbf{N} \\
\mathbf{N}\end{array}$ \\
\hline 009562 & Clarance 32-1S-4W & $M I$ & 025 & Calhoun & 1982 & OA & 156530 & Comstock See 5 & M! & 077 & Kalamazoo & 1949 & 0 \\
\hline 007778 & Clarence $33.1 \mathrm{~S} .4 \mathrm{~W}$ & MI & 025 & Galhoun & 1981 & $\mathrm{~N}$ & 156902 & Concord & Mi & 075 & Jackson & 1953 & 0 \\
\hline 007779 & Clarence 34-1S-4W & $M I$ & 025 & Calhoun & 1981 & OA & 013358 & Conners Marsh & $M i$ & 039 & Crawlord & 1989 & $\mathbf{N}$ \\
\hline 007780 & Clarence $35.15-4 \mathrm{~W}$ & $M I$ & 025 & Calhoun & 1981 & $O A$ & 007799 & Convis 08-1S-6W & MI & 025 & Calhoun & 1975 & OA \\
\hline 009563 & Claybanks 02-13N-18W & MI & 127 & Oceana & 1982 & $O A$ & 007800 & Convis 08-1S-6W A & MI & 025 & Calhoun & 1975 & OA \\
\hline 011368 & Claybanks $02-13 \mathrm{~N}-18 \mathrm{BW}$ & MI & 127 & Oceana & 1984 & $\mathrm{~N}$ & 011691 & Convis 08-1S-6W B & MI & 025 & Calhoun & 1986 & $O A$ \\
\hline 007782 & Claybanks $11-13 \mathrm{~N}-18 \mathrm{~W}$ & MI & 127 & Oceana & 1980 & N & 007801 & Convis 09-1S-6W & $M 1$ & 025 & Calhoun & 1981 & 0 \\
\hline $\begin{array}{l}146780 \\
146780\end{array}$ & $\begin{array}{l}\text { Clayton } \\
\text { Clayton }\end{array}$ & $\begin{array}{l}M I \\
M I\end{array}$ & $\begin{array}{l}011 \\
129\end{array}$ & $\begin{array}{l}\text { Arenac } \\
\text { Ogemaw }\end{array}$ & $\begin{array}{l}1935 \\
1935\end{array}$ & $\begin{array}{l}\text { ON } \\
\text { ON }\end{array}$ & $\begin{array}{l}009571 \\
007802\end{array}$ & $\begin{array}{l}\text { Convis } 12-1 \mathrm{~S}-6 \mathrm{~W} \\
\text { Convis } 16-1 \mathrm{~S}-6 \mathrm{~W}\end{array}$ & $\begin{array}{l}M ! \\
M !\end{array}$ & $\begin{array}{l}025 \\
025\end{array}$ & $\begin{array}{l}\text { Calhoun } \\
\text { Calhoun }\end{array}$ & $\begin{array}{l}1982 \\
1981\end{array}$ & $\begin{array}{l}N \\
0\end{array}$ \\
\hline 147214 & Clear Lake & MI & $\begin{array}{l}159 \\
101\end{array}$ & Van Buren & 1950 & 0 & $\begin{array}{l}007803 \\
009572\end{array}$ & $\begin{array}{l}\text { Convis } 16-15 \cdot 6 W \text { A } \\
\text { Convis } 17-1 S-6 W\end{array}$ & $M i$ & 025 & Calhoun & 1981 & 0 \\
\hline 148185 & Alias for: $(002895)$ Cleon $11-8$ & $4 N-13 W$ & ISPLIT & & & & 012731 & Convis $17.15-6$ W A & $M i$ & 025 & Calhoun & $\begin{array}{l}1882 \\
1985\end{array}$ & o \\
\hline 002895 & $\begin{array}{l}\text { Cleon } 11-24 \mathrm{~N}-13 \mathrm{~W} \\
\text { Cloon } 12-24 \mathrm{~N}-13 \mathrm{~W}\end{array}$ & $\begin{array}{l}M I \\
M I\end{array}$ & 101 & Manistee & 1973 & $O A$ & 007804 & Convis $18.15 .6 \mathrm{~W}$ & Mi & 025 & Calhoun & 1975 & OA \\
\hline $\begin{array}{l}002896 \\
002897\end{array}$ & $\begin{array}{l}\text { Cloon 12-24N-13W } \\
\text { Cloon 12.24N-13W A }\end{array}$ & $\begin{array}{l}M I \\
M I\end{array}$ & $\begin{array}{l}101 \\
101\end{array}$ & $\begin{array}{l}\text { Manistee } \\
\text { Manistee }\end{array}$ & $\begin{array}{l}1974 \\
1975\end{array}$ & $\begin{array}{l}O A \\
O A\end{array}$ & $\begin{array}{l}007805 \\
012345\end{array}$ & $\begin{array}{l}\text { Convis } 19-1 \mathrm{~S}-6 \mathrm{~W} \\
\text { Convis } 19-1 \mathrm{~S}-6 \mathrm{~W} \text { A }\end{array}$ & $\begin{array}{l}\text { MI } \\
\text { MI }\end{array}$ & $\begin{array}{l}025 \\
025\end{array}$ & $\begin{array}{l}\text { Galhoun } \\
\text { Calhoun }\end{array}$ & $\begin{array}{l}1976 \\
1988\end{array}$ & $\stackrel{O A}{A}_{0}$ \\
\hline 007562 & Cloon 13-24N-13W & MI & 101 & Manisteo & 1977 & $O A$ & 006750 & Convis 20-1S-6W & $M I$ & 025 & Calhoun & 1981 & o \\
\hline 002898 & Cloon 14-24N-13W & $M I$ & 101 & Manistoe & 1973 & $O A$ & 006752 & $\begin{array}{l}\text { Convis } 20-15-6 W \\
\text { Convis } 21-15-6 W\end{array}$ & MI & 025 & $\begin{array}{l}\text { Calnoun } \\
\text { Galhoun }\end{array}$ & $\begin{array}{l}1981 \\
1980\end{array}$ & $\stackrel{0}{0}$ \\
\hline $\begin{array}{l}002899 \\
002800\end{array}$ & $\begin{array}{l}\text { Cloon 14-24N-13W A } \\
\text { Cloon 15-24N-13W }\end{array}$ & $\begin{array}{l}M I \\
M I\end{array}$ & $\begin{array}{l}101 \\
101\end{array}$ & $\begin{array}{l}\text { Manisteo } \\
\text { Manisteo }\end{array}$ & $\begin{array}{l}1976 \\
1974\end{array}$ & $\mathrm{OA}_{\mathrm{N}}$ & $\begin{array}{l}006753 \\
006754\end{array}$ & $\begin{array}{l}\text { Convis } 21-1 S-6 W \text { A } \\
\text { Convis } 22-1 \mathrm{~S}-6 \mathrm{~W}\end{array}$ & $\underset{M I}{M I}$ & 025 & $\begin{array}{l}\text { Calhoun } \\
\text { Calhoun }\end{array}$ & $\begin{array}{l}1981 \\
1981\end{array}$ & $\begin{array}{l}0 \\
0\end{array}$ \\
\hline 002001 & Cloon 16-24N-13W & MI & 101 & Manistee & 1980 & $\mathbf{N}$ & 009573 & Convis 23-15-6W & MI & 025 & Calhoun & 1982 & OA \\
\hline 002802 & Cloon 17-24N-13W & MI & 101 & Manisteo & 1978 & $\mathbf{N}$ & 009574 & Convis 23-1S-6W A & MI & 025 & Calhoun & 1982 & $N$ \\
\hline 002903 & Cloon $19.24 \mathrm{~N} \cdot 13 \mathrm{~W}$ & MI & 101 & Manisteo & 1976 & $\mathbf{N}$ & 006755 & Convis 25-1S-6W & MI & 025 & Calhoun & 1975 & $\circ$ \\
\hline $\begin{array}{l}002804 \\
002805\end{array}$ & Cleon 19-24N-13W A & $M !$ & 101 & Manistee & 1977 & $O A$ & 006756 & Convis 26-15-6W & MI & 025 & Calhoun & 1978 & 0 \\
\hline $\begin{array}{l}002905 \\
002808\end{array}$ & $\begin{array}{l}\text { Cloon 19-24N-13W B } \\
\text { Cloon } 20.24 \mathrm{~N}-13 W\end{array}$ & $\begin{array}{l}M ! \\
M I\end{array}$ & $\begin{array}{l}101 \\
101\end{array}$ & $\begin{array}{l}\text { Manisteo } \\
\text { Manisteo }\end{array}$ & $\begin{array}{l}1980 \\
1974\end{array}$ & $\mathrm{OA}_{\mathbf{N}}$ & $\begin{array}{l}006757 \\
007568\end{array}$ & $\begin{array}{l}\text { Convis } 29-15-6 W \\
\text { Convis } 30-15-6 W\end{array}$ & MI & $\begin{array}{l}025 \\
025\end{array}$ & $\begin{array}{l}\text { Calhoun } \\
\text { Galhoun }\end{array}$ & $\begin{array}{l}1980 \\
1875\end{array}$ & $\begin{array}{l}\circ \\
\mathrm{OA}\end{array}$ \\
\hline 000564 & Cleon $20-24 \mathrm{~N}-13 \mathrm{~W} \mathrm{~A}$ & MI & 101 & Manlstee & 1979 & $\mathbf{N}$ & 006758 & Convis 30-15.6W A & MI & 025 & Calhoun & 1981 & 0 \\
\hline $\begin{array}{l}002907 \\
002908\end{array}$ & $\begin{array}{l}\text { Cloon 22-24N-13W } \\
\text { Cloon 23-24N-13W }\end{array}$ & $\begin{array}{l}M ! \\
M !\end{array}$ & $\begin{array}{l}101 \\
101\end{array}$ & $\begin{array}{l}\text { Manistoo } \\
\text { Manisteo }\end{array}$ & $\begin{array}{l}1975 \\
1980\end{array}$ & $\begin{array}{l}N \\
O A\end{array}$ & $\begin{array}{l}009575 \\
159475\end{array}$ & $\begin{array}{l}\text { Convis 32-1S-6W } \\
\text { Coon Creok }\end{array}$ & MI & 025 & $\begin{array}{l}\text { Calhoun } \\
\text { Macomb }\end{array}$ & $\begin{array}{l}1982 \\
1963\end{array}$ & $\stackrel{\circ}{\mathrm{O}}$ \\
\hline 002809 & Cleon 24-24N-13W & Mi & 101 & Manlatoo & 1977 & N & 159723 & Cooper & MI & 077 & Kalamazoo & 1979 & o \\
\hline 009585 & Cloon $25.24 \mathrm{~N} \cdot 13 \mathrm{~W}$ & MI & 101 & Menisteo & 1882 & 0 & 160157 & Coopersville & Mi & 139 & Oltawa & 1939 & $\mathbf{N}$ \\
\hline 002910 & Cloon $26-24 N-13 W$ & $M i$ & 101 & Manisteo & 1976 & $\mathrm{~N}$ & 009386 & Corwith $12-32 \mathrm{~N}-1 \mathrm{~W}$ & MI & 137 & Otsego & 1982 & $\mathbf{N}$ \\
\hline 002811 & cloon $26-24 \mathrm{~N}-13 \mathrm{~W}$ A & $M t$ & 101 & Manistoe & 1979 & $\mathrm{~N}$ & 009387 & Corwith $14-32 N-1$ W & $M I$ & 137 & Otsego & 1982 & $\mathbf{N}$ \\
\hline 000568 & Cloon 26-24N-13W B & MI & 101 & Manisteo & 1982 & $O A$ & 012346 & Corwith $22.32 \mathrm{~N}-1 \mathrm{~W}$ & MI & 137 & Otsego & 1988 & 0 \\
\hline 002812 & Cloon $27-24 \mathrm{~N}-13 \mathrm{~W}$ & $M !$ & 101 & Manisteo & 1976 & $\mathbf{N}$ & 009388 & Corwith 23-32N-1 W & MI & 137 & Otsogo & 1982 & $\mathbf{N}$ \\
\hline 002813 & Cloon $27.24 \mathrm{~N}-13 \mathrm{~W}$ A & $M I$ & 101 & Manistee & 1977 & $\ddot{N}$ & 009389 & Corwith $31-32 \mathrm{~N}-1 \mathrm{~W}$ & Mi & 137 & Otsago & 1982 & $\ddot{O A}_{A}$ \\
\hline 002914 & Cloon $28-24 N-13 W$ & Mi & 101 & Manistao & & & 164580 & Cottrallville & MI & 147 & St Clair & 1958 & ON \\
\hline 002815 & Alias for: $(002915)$ Cleon & & $10 \mathrm{MB}$ & & & & 006760 & Cottrollville 05-3N-16E & MI & 147 & St Clair & 1881 & $\mathbf{N}$ \\
\hline $\begin{array}{l}002915 \\
002916\end{array}$ & $\begin{array}{l}\text { Cloon 29-24N-13W } \\
\text { Cloon } 29-24 \mathrm{~N}-13 \mathrm{~W} \text { A }\end{array}$ & $\begin{array}{l}M I \\
M I\end{array}$ & $\begin{array}{l}101 \\
101\end{array}$ & $\begin{array}{l}\text { Manisteo } \\
\text { Manistee }\end{array}$ & $\begin{array}{l}1978 \\
1979\end{array}$ & $\stackrel{N}{N}$ & 168233 & $\begin{array}{l}\text { Cranberry Lake } \\
\text { STOR(48) }\end{array}$ & MI & 035 & Clare & 1943 & ON \\
\hline 012726 & $\begin{array}{l}\text { Cloon } 30 \cdot 24 \mathrm{~N}-13 \mathrm{~W} \\
\text { Cloon } 31 \cdot 24 \mathrm{~N}-13 \mathrm{~W}\end{array}$ & $M I$ & $\begin{array}{l}101 \\
101\end{array}$ & $\begin{array}{l}\text { Manisteo } \\
\text { Manisteo }\end{array}$ & $\begin{array}{l}1986 \\
1977\end{array}$ & $\stackrel{N}{O A}$ & 168233 & $\begin{array}{l}\text { Cranberry Lake } \\
\text { STOR(48) }\end{array}$ & MI & 113 & Missaukeo & 1943 & ON \\
\hline 002918 & Cloon $32-24 \mathrm{~N}-13 \mathrm{~W}$ & MI & 101 & Menisteo & 1977 & $\mathrm{~N}$ & 168264 & Cranberry Lake East & M! & 035 & Clare & 1963 & OA \\
\hline 002918 & Cloon $32-24 \mathrm{~N}-13 \mathrm{~W} / \mathrm{A}$ & $M I$ & 101 & Manisteo & 1978 & oA & 013360 & Cranberry Lako North & MI & 113 & Missaukeo & 1989 & N \\
\hline 002920 & Cloon $32.24 \mathrm{~N}-13 \mathrm{~W} \mathrm{~B}$ & MI & 101 & Manisteo & 1980 & $O A$ & 171426 & Crooked Lake & M1 & 005 & Allegan & $\$ 949$ & $\ddot{o}$ \\
\hline 148733 & Clinton & $M I$ & 169 & Weshtenaw & 1953 & 0 & 172387 & Croton & MI & 123 & Nowaygo & t851 & $\mathrm{N}$ \\
\hline $\begin{array}{l}151322 \\
152035\end{array}$ & $\begin{array}{l}\text { Colloe Lake } \\
\text { Cold Sprlngs }\end{array}$ & $M !$ & $\begin{array}{l}159 \\
079\end{array}$ & $\begin{array}{l}\text { Van Buren } \\
\text { Kalkaska }\end{array}$ & 1946 & 0 & 173193 & STOR(57) & MI & 017 & & 1850 & 0 \\
\hline & Allas for: (002928) Cold & $g=21-28$ & : 8 N-6W & $\checkmark$ [RENAM] & & & 173441 & $\begin{array}{l}\text { Crump } \\
\text { Crystal }\end{array}$ & MI & $\begin{array}{l}017 \\
117\end{array}$ & $\begin{array}{l}\text { Bay } \\
\text { Montcalm }\end{array}$ & $\begin{array}{l}1850 \\
1935\end{array}$ & 0 \\
\hline $\begin{array}{l}002821 \\
007784\end{array}$ & $\begin{array}{l}\text { Cold Springs } 01-28 N-6 W \\
\text { Cold Springs } 01-28 N-6 \text { W A }\end{array}$ & MI & $\begin{array}{l}079 \\
079\end{array}$ & $\begin{array}{l}\text { Kalkaska } \\
\text { Kalkaska }\end{array}$ & $\begin{array}{l}1973 \\
1976\end{array}$ & $\underset{N}{N}$ & $\begin{array}{l}173596 \\
006761\end{array}$ & $\begin{array}{l}\text { Crystal Vallay } \\
\text { Crystal Valley Sec } 19\end{array}$ & $\begin{array}{l}\text { MI } \\
M I\end{array}$ & $\begin{array}{l}127 \\
127\end{array}$ & $\begin{array}{l}\text { Ocoana } \\
\text { Oceane }\end{array}$ & $\begin{array}{l}1845 \\
1971\end{array}$ & ON \\
\hline 000567 & Cold Springs $10-28 \mathrm{~N}-6 \mathrm{~W}$ & $M I$ & 078 & Kalkaska & 1982 & $O A$ & 173658 & Crystel Velloy South & MI & 127 & Ocoana & 1969 & 0 \\
\hline 002922 & Gold Springs $12-28 \mathrm{~N}-6 \mathrm{~W}$ & MI & 078 & Kalkaska & 1973 & ON & $\begin{array}{l}174758 \\
175410\end{array}$ & Currio & Mt & 073 & Isebella & 1836 & 0 \\
\hline 009588 & Cold Springs 13-28N-6W & MI & 079 & Kalkaska & 1982 & OA & & Alias for: $(021616)$ Antrim & [COMB] & & & & \\
\hline 002924 & Cold Springs $14.28 \mathrm{~N}-6 \mathrm{~W}$ & $M I$ & 079 & Kalkaska & 1976 & $O A$ & 178946 & Dallas & MI & 037 & Cllnton & 1942 & 0 \\
\hline 011368 & Cold Springs $16.28 \mathrm{~N}-6 \mathrm{~W}$ & $M I$ & 079 & Kalkaska & 1984 & $O A$ & 179132 & Dalton & MI & 121 & Muskegon & 1940 & 0 \\
\hline 002825 & Cold Springs $18.28 \mathrm{~N}-6 \mathrm{~W}$ & MI & 079 & Kalkaska & 1974 & $O A$ & 182883 & Day & MI & 117 & Montcalm & 1934 & ON \\
\hline 002026 & Cold Springs $19.28 \mathrm{~N} \cdot 6 \mathrm{~W}$ & $M I$ & 079 & Kalkaska & 1971 & $O A$ & 006762 & Day Sec 13 & MI & 117 & Montcalm & 1971 & 0 \\
\hline 002027 & Cold Springs 20-28N-6W & $M I$ & 079 & Kaikaska & 1971 & $\hat{O A}$ & 011382 & Dayton Sec 16 & MI & 123 & Nowaygo & 1957 & 0 \\
\hline 007785 & Cold Springs $20.28 \mathrm{~N}-6 \mathrm{~W}$ A & Mi & 079 & Kalkaska & 1981 & $N$ & 011383 & Decatur Sec 4 & $M I$ & 159 & Van Buren & 1942 & 0 \\
\hline 002928 & Cold Springs 21-28N-6W & MI & 079 & Kalkaska & 1970 & OA & 185418 & Deep River & MI & 011 & Arenac & 1936 & ON \\
\hline 007786 & Cold Springs 21-28N-6W A & MI & 079 & Kalkaska & 1978 & 0 & 185914 & Deortield & MI & 107 & Mecosta & 1956 & 0 \\
\hline 007563 & Cold Springs 22-28N-6W & MI & 079 & Kalkaska & 1976 & OA & 185914 & Dooptiald & MI & 115 & Monroo & 1920 & 0 \\
\hline 007787 & Cold Springs 22-2BN-6W A & MI & 079 & Kalkaska & 1978 & $\mathrm{OA}$ & 187898 & Demings Lake & MI & 091 & Lonawoo & 1988 & $\mathrm{~N}$ \\
\hline 002029 & Cold SprIngs 23-28N-6W & MI & 079 & Kalkaske & 1974 & OA & 188332 & Dennison & MI & 139 & Ottawa & 1963 & 0 \\
\hline 002030 & Cold Springs $24-28 N-6 W$ & Mu & 079 & Kalkaske & 1974 & $\mathbf{N}$ & 188611 & Denton Creek & MI & 143 & Roscommon & 1882 & OA \\
\hline 002031 & $\begin{array}{l}\text { Cold Springs } 24-28 \mathrm{~N}-6 \mathrm{~W} \text { A } \\
\text { Cold Spilngs } 25.2 \mathrm{BN}-6 \mathrm{~W}\end{array}$ & $M \prime$ & $\begin{array}{l}079 \\
079\end{array}$ & $\begin{array}{l}\text { Kalkaska } \\
\text { Kalkaska }\end{array}$ & $\begin{array}{l}1979 \\
1971\end{array}$ & $\mathrm{OA}_{\mathrm{N}}$ & $\begin{array}{l}190898 \\
191146\end{array}$ & $\begin{array}{l}\text { Diamond Crystal Salt } \\
\text { Oiamond Springs }\end{array}$ & $\begin{array}{l}M I \\
M I\end{array}$ & $\begin{array}{l}147 \\
005\end{array}$ & $\begin{array}{l}\text { St Clair } \\
\text { Allogan }\end{array}$ & $\begin{array}{l}1927 \\
1938\end{array}$ & $\begin{array}{l}N \\
0\end{array}$ \\
\hline $\begin{array}{l}002833 \\
002833\end{array}$ & Cold Springs 25-2BN-6W A & MI & 079 & Kalkaska & 1972 & OA & $\begin{array}{l}181746 \\
191935\end{array}$ & $\begin{array}{l}\text { Ulamond Springs } \\
\text { Dighton }\end{array}$ & MI & 133 & $\begin{array}{l}\text { Aliogan } \\
\text { Osceola }\end{array}$ & $\begin{array}{l}1938 \\
1991\end{array}$ & $\mathbf{N}$ \\
\hline 002834 & Cold Springs $28.28 \mathrm{~N}-6 \mathrm{~W}$ & MI & 079 & Kalkaska & 1975 & OA & 198285 & Dort & MI & 005 & Allogan & 1938 & $\mathrm{ON}$ \\
\hline 002935 & Cold Springs 28-28N-6W A & MI & $079^{\circ}$ & Kalkaska & & & 006764 & Dorr Sec 17 & MI & 005 & Allegan & $\$ 951$ & $\mathbf{N}$ \\
\hline 002038 & $\begin{array}{l}\text { Alias for: (007788) Cold } \mathbf{S} \\
\text { Cold Springs } 29.28 \mathrm{~N} .6 \mathrm{~W}\end{array}$ & $g_{M I}^{33-}$ & $\begin{array}{l}28 N-6 W \\
079\end{array}$ & $\begin{array}{l}\text { V [RENAM] } \\
\text { Kalkaska }\end{array}$ & 1976 & OA & $\begin{array}{l}006765 \\
200207\end{array}$ & $\begin{array}{l}\text { Dorr Sec } 21 \\
\text { Douglass }\end{array}$ & $M I$ & 005 & Allogan & 1940 & \\
\hline $\begin{array}{l}002838 \\
011370\end{array}$ & $\begin{array}{l}\text { Cold Springs 29.28N-6W } \\
\text { Cold Springs } 29 \cdot 28 \mathrm{~N}-6 \mathrm{~W} \text { A }\end{array}$ & MI & $\begin{array}{l}079 \\
079\end{array}$ & $\begin{array}{l}\text { Kalkaska } \\
\text { Kalkaska }\end{array}$ & $\begin{array}{l}1976 \\
1978\end{array}$ & OA & $\begin{array}{l}200207 \\
006766\end{array}$ & $\begin{array}{l}\text { Douglass } \\
\text { Douglass Soc } 3\end{array}$ & MI & 117 & Montcalm & 1943 & ON \\
\hline 011371 & $\begin{array}{l}\text { Cold Springs 29-28N-6W A } \\
\text { Cold Springs 29-28N-6W B }\end{array}$ & $M I$ & 079 & $\begin{array}{l}\text { Kalkaska } \\
\text { Kalkaska }\end{array}$ & 1983 & OA & $\begin{array}{l}006766 \\
200672\end{array}$ & $\begin{array}{l}\text { Douglass Sac } 3 \\
\text { Dover }\end{array}$ & MI & $\begin{array}{l}117 \\
137\end{array}$ & $\begin{array}{l}\text { Montcalm } \\
\text { Otsogo }\end{array}$ & & \\
\hline 002037 & Cold Springs 30-28N-6W & MI & 079 & Kalkaska & 1974 & $O A$ & & Alias for: 10029 & & [SPLIT] & & & \\
\hline 012728 & Cold Springs $30.28 \mathrm{~N}-6 \mathrm{~W}$ A & MI & 078 & Kalkaska & 1986 & $O A$ & 003496 & Dover Sac 13 & Mi & 137 & Otsego & & \\
\hline 009713 & Cold Springs $31-28 \mathrm{~N}-6 \mathrm{~W}$ & MI & 079 & Kalkaska & 1974 & ON & & Allas for: (021616) Antrim & [Сомв] & & & & \\
\hline & STOR(BO) & & & & & & 002940 & Dover $12-31 \mathrm{~N}-2 \mathrm{~W}$ & Mi & 137 & Otsego & 1873 & OA \\
\hline 002838 & Cold Springs $32.28 \mathrm{~N}-6 \mathrm{~W}$ & MI & 079 & Kalkaska & 1977 & $O A$ & 012351 & Dover $15-31 \mathrm{~N}-2 \mathrm{~W}$ & Mi & 137 & Otsogo & 1987 & 0 \\
\hline 007788 & Cold Springs $33-28 \mathrm{~N}-6 \mathrm{~W}$ & MI & 079 & Kalkaska & 1977 & 0 & 002941 & Dover $21-31 N-2 W$ & M! & 137 & Otsego: & 1975 & OA \\
\hline 007789 & Cold Springs 35-28N-6W & MI & 078 & Kalkaska & 1978 & $\mathbf{N}$ & 002942 & Dover $22-31 N-2 W$ & MI & 137 & Otsego & 1975 & $O A$ \\
\hline 011372 & Cold Springs 35.28N.6W A & $M I$ & 079 & Kalkaska & 1984 & $\mathbf{N}$ & 002843 & Dover 22-31N-2W A & M! & 137 & Olsogo & 1977 & $O A$ \\
\hline 002939 & Cold Springs 36-2BN-6W & MI & 079 & Kalkaska & 1978 & $\mathbf{N}$ & 009586 & Dover $22.31 \mathrm{~N}-2 \mathrm{~W}$ B & Mi & 137 & Otsego & 1982 & 0 \\
\hline 152252 & Coldwator & $\mathrm{MI}$ & 073 & losbella & 1945 & ON & 012735 & Dover $25.31 \mathrm{~N}-2 \mathrm{~W}$ & $M !$ & 137 & Otsogo & 1986 & OA \\
\hline 152283 & $\begin{array}{l}\text { STOR(56) } \\
\text { Coldwater South }\end{array}$ & MI & 073 & Isabolla & 1951 & 0 & $\begin{array}{l}002944 \\
002945\end{array}$ & $\begin{array}{l}\text { Dover } 27-31 N-2 W \\
\text { Dover } 33-31 N-2 W\end{array}$ & $\begin{array}{l}M ! \\
M !\end{array}$ & $\begin{array}{l}137 \\
137\end{array}$ & $\begin{array}{l}\text { Otsego } \\
\text { Otsego }\end{array}$ & $\begin{array}{l}1975 \\
1974\end{array}$ & $\begin{array}{l}0 \\
O A\end{array}$ \\
\hline
\end{tabular}




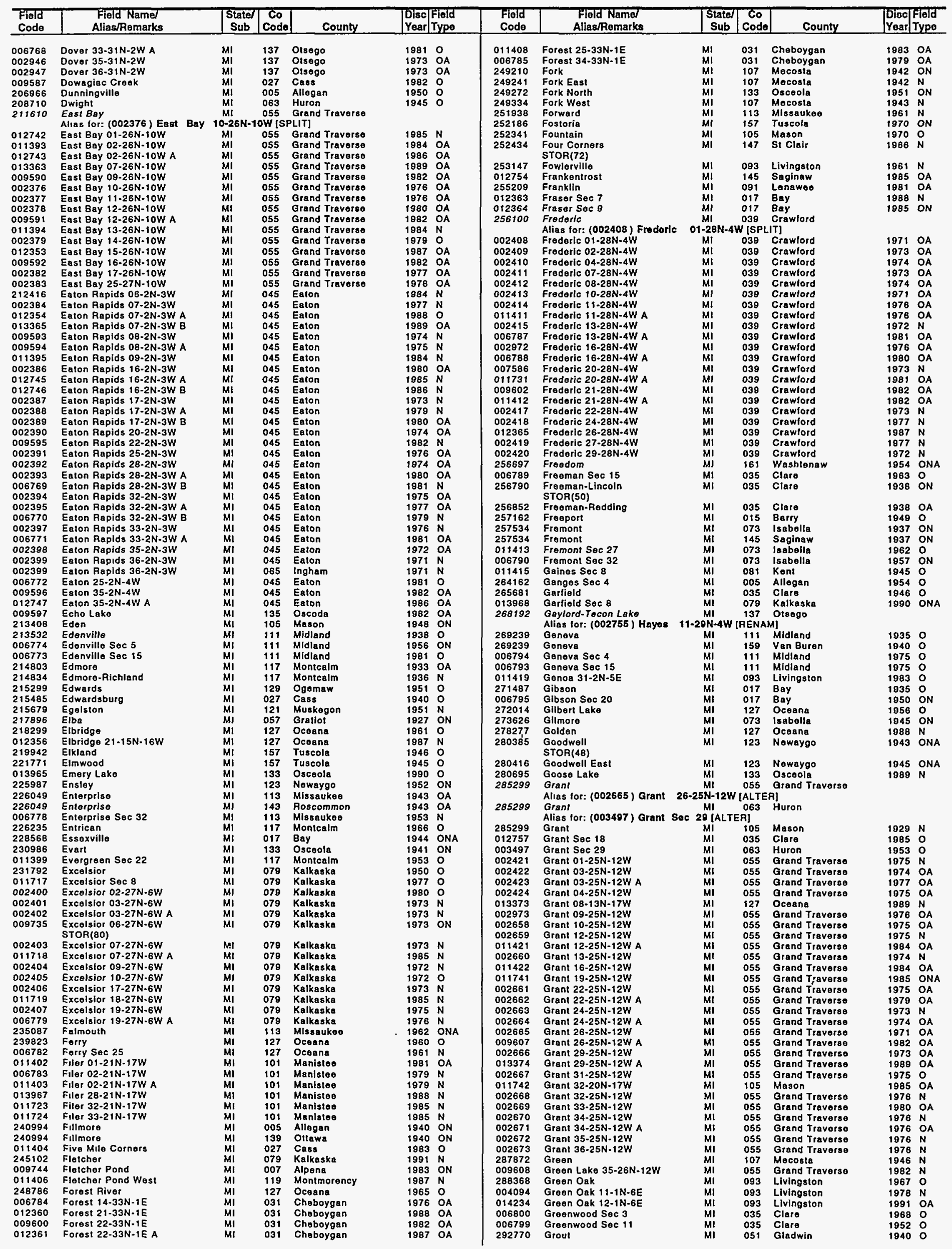


MICHIGAN

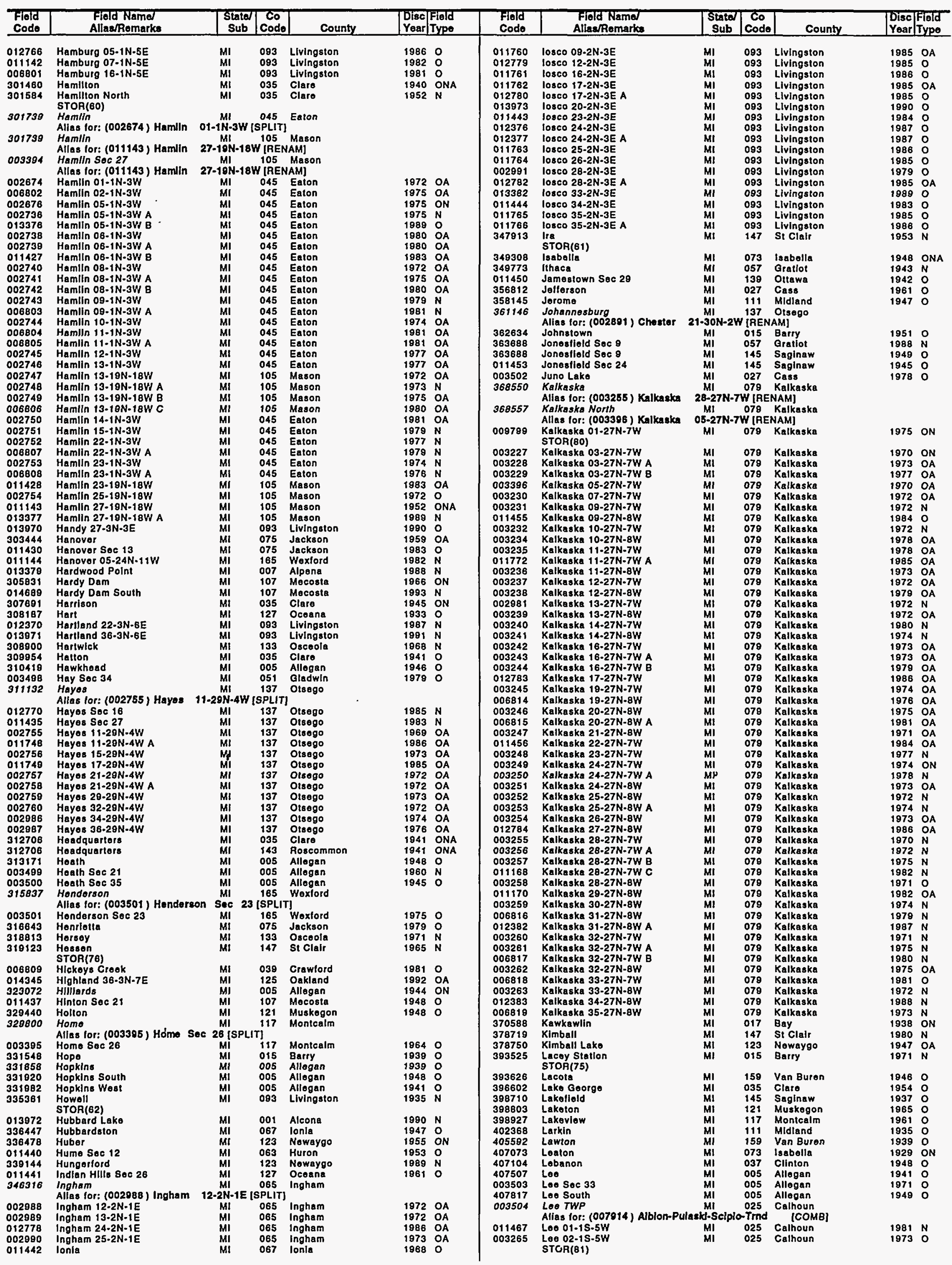


MICHIGAN

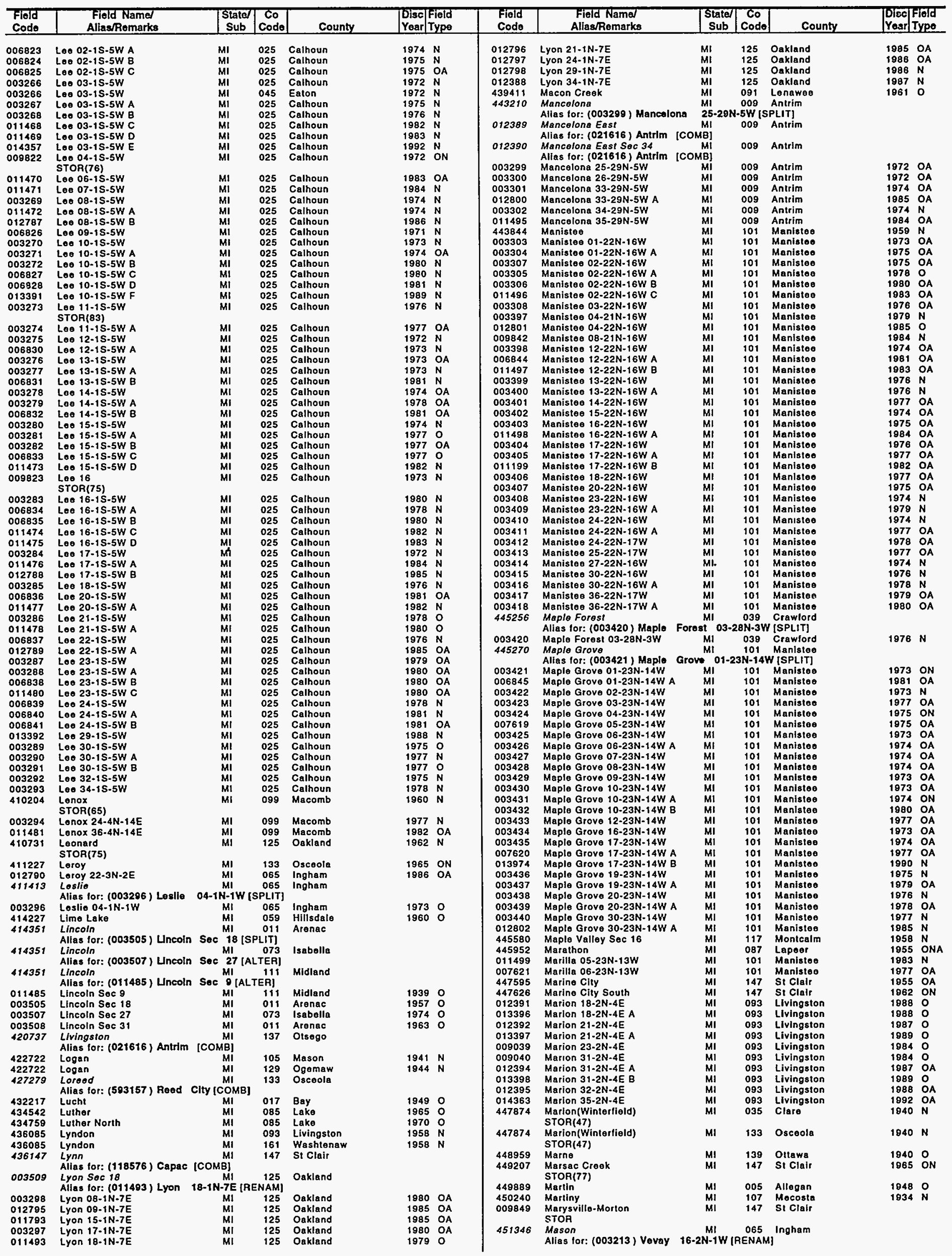


MICHIGAN

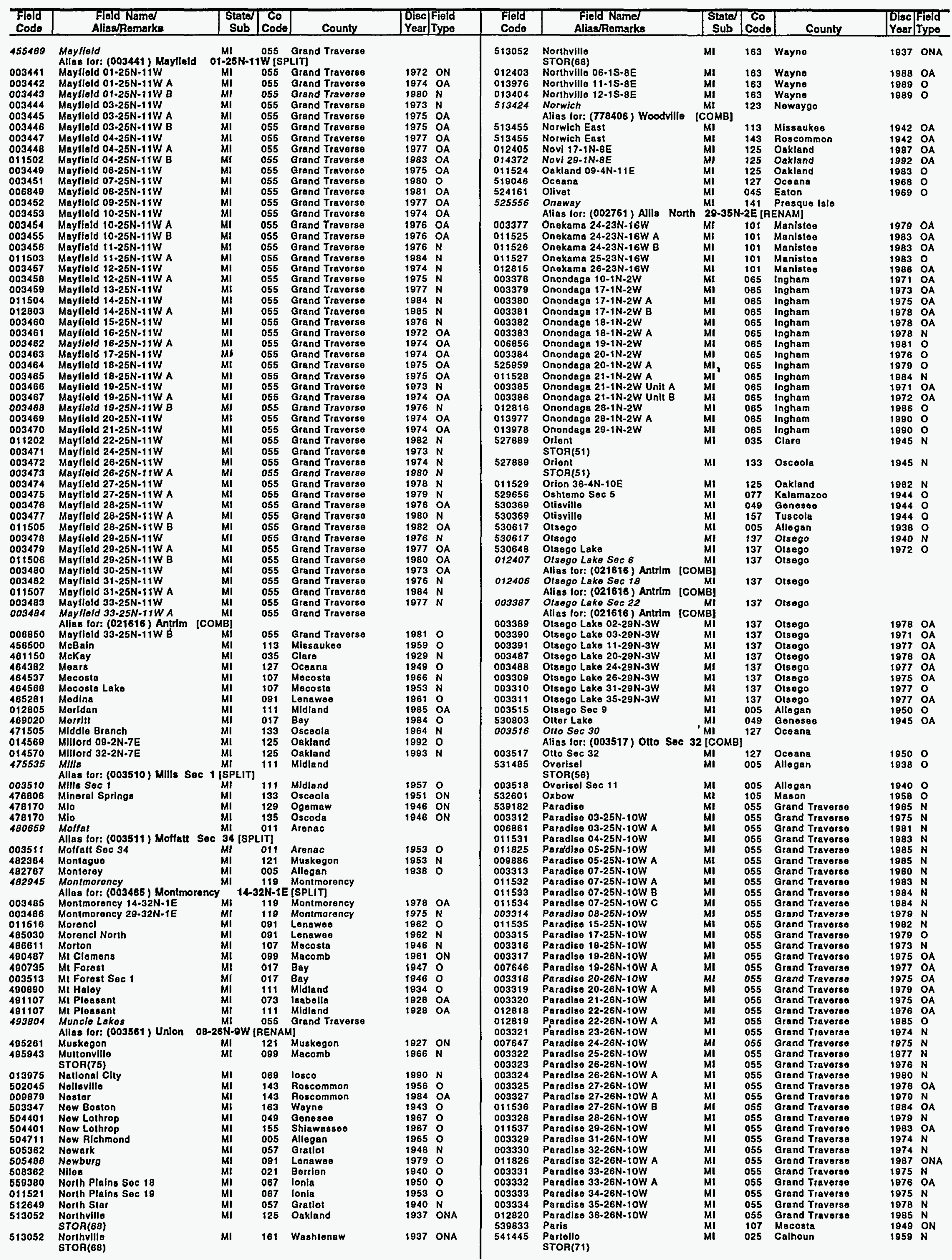




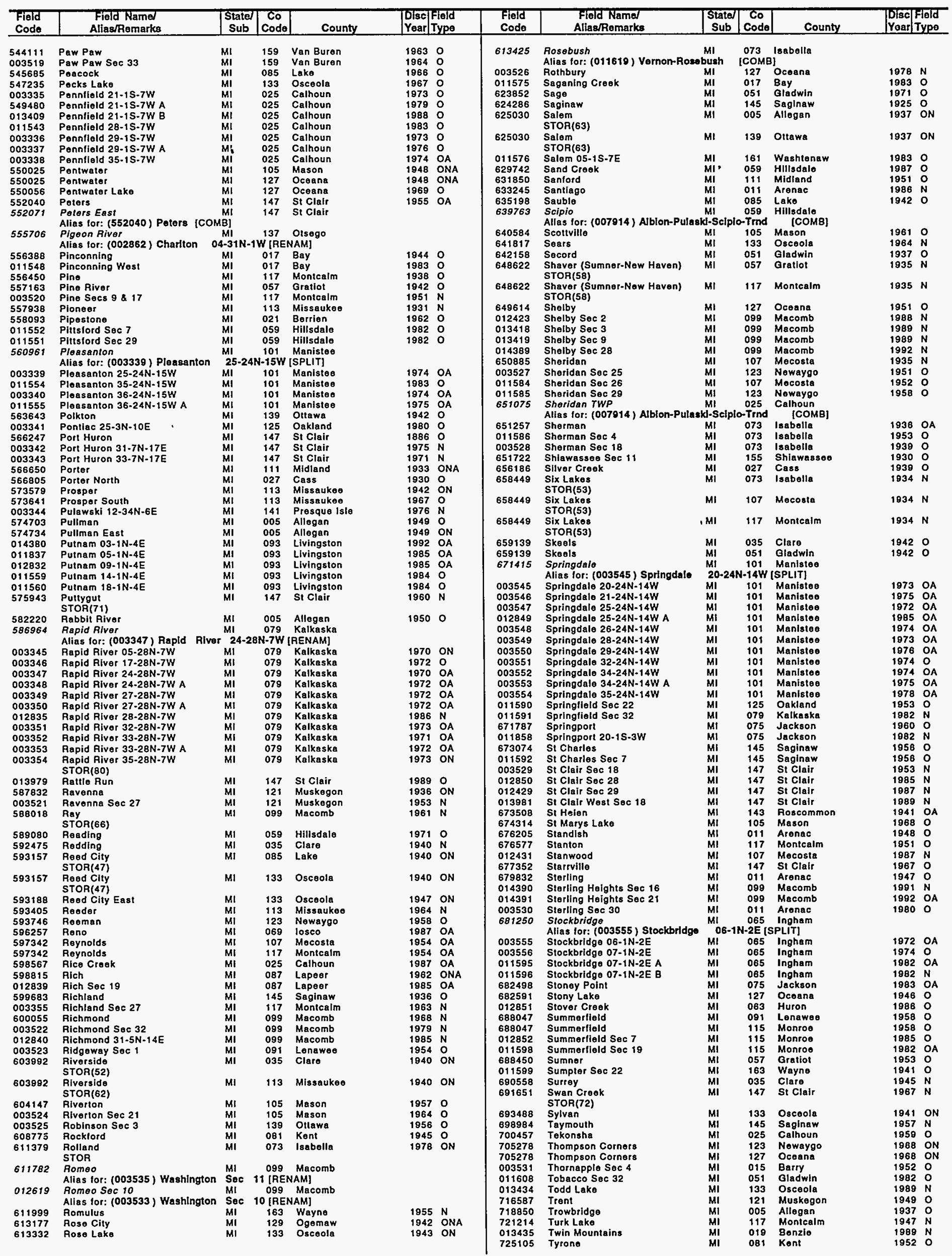


MISSISSIPPI

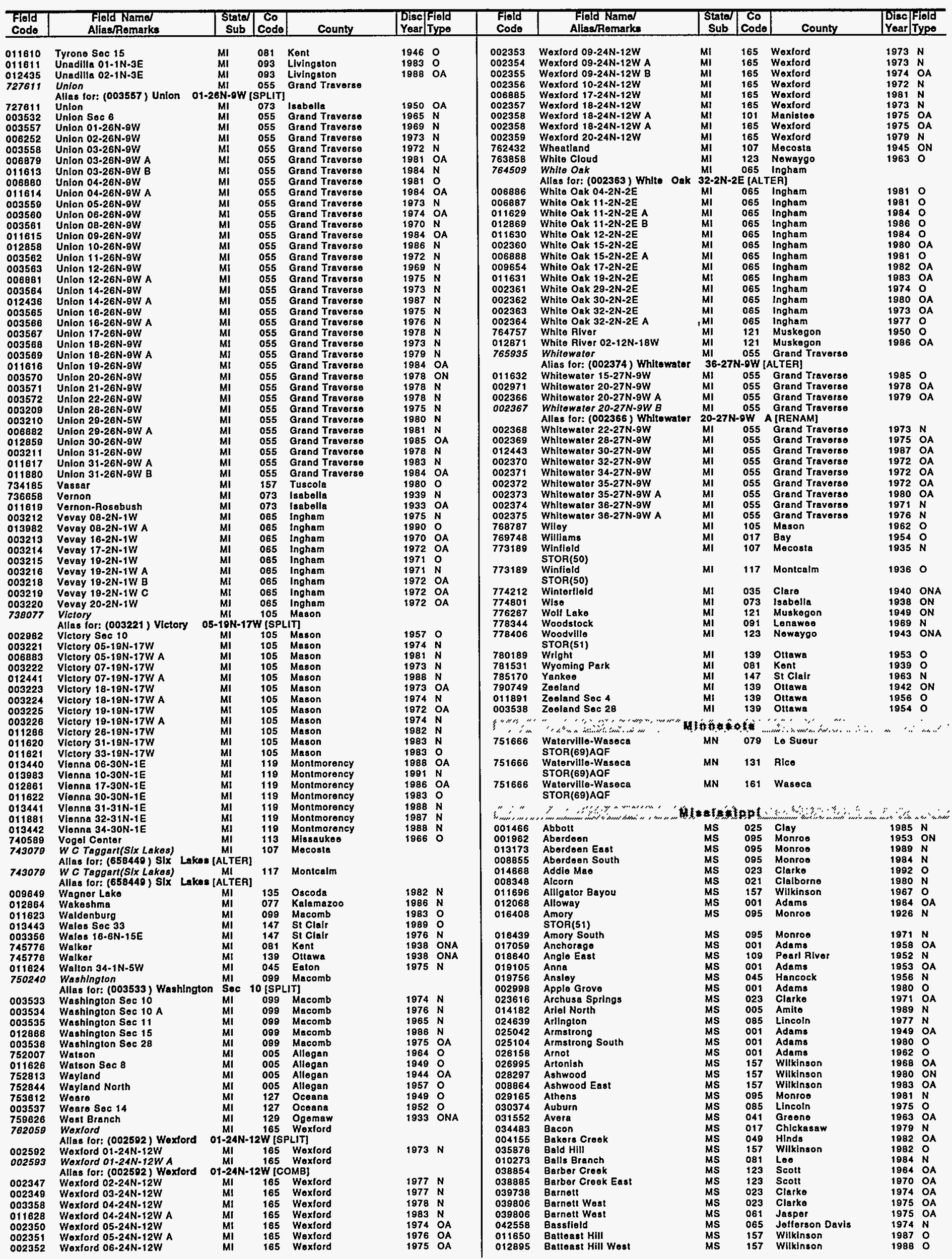


MISSISSIPPI

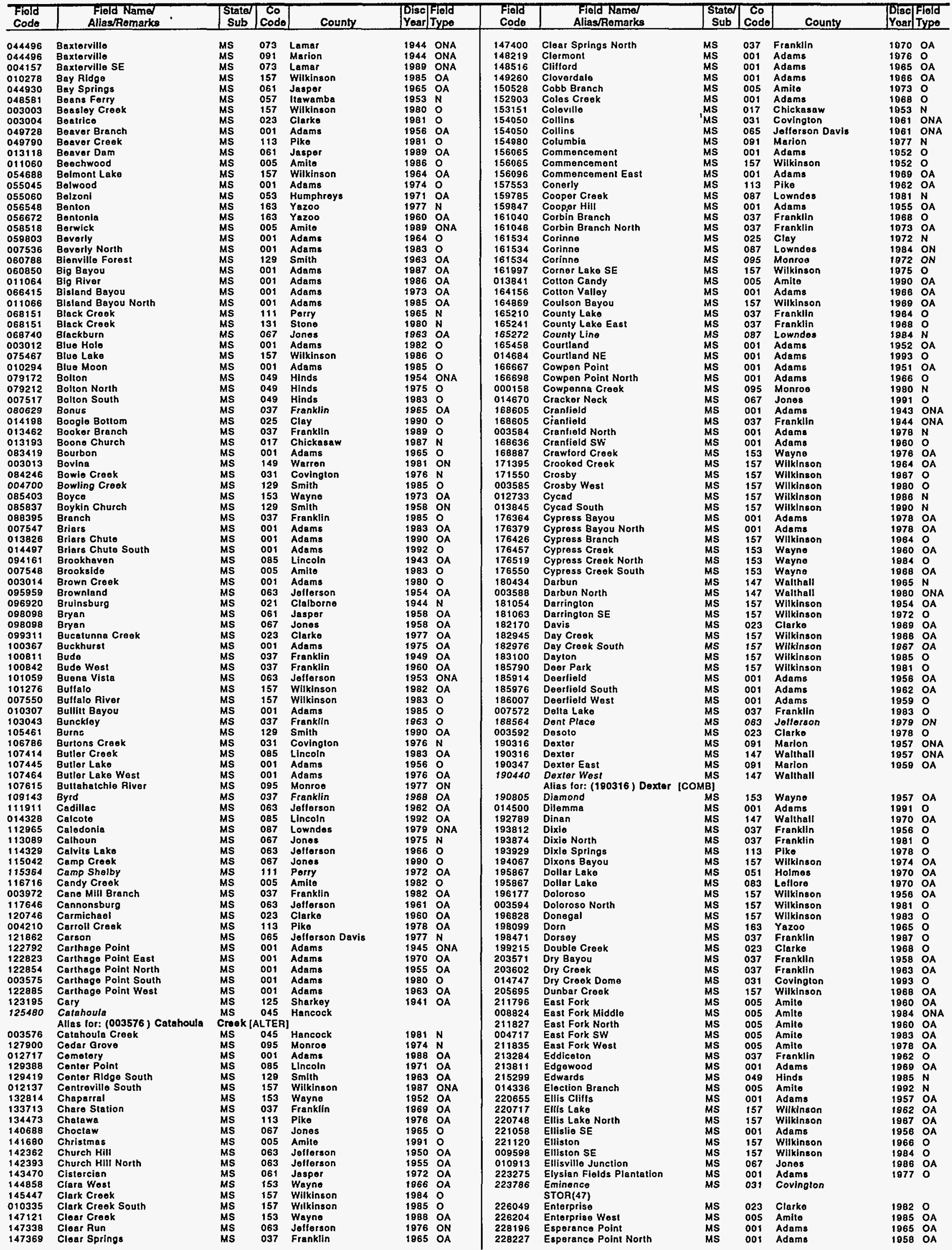


MISSISSIPPI

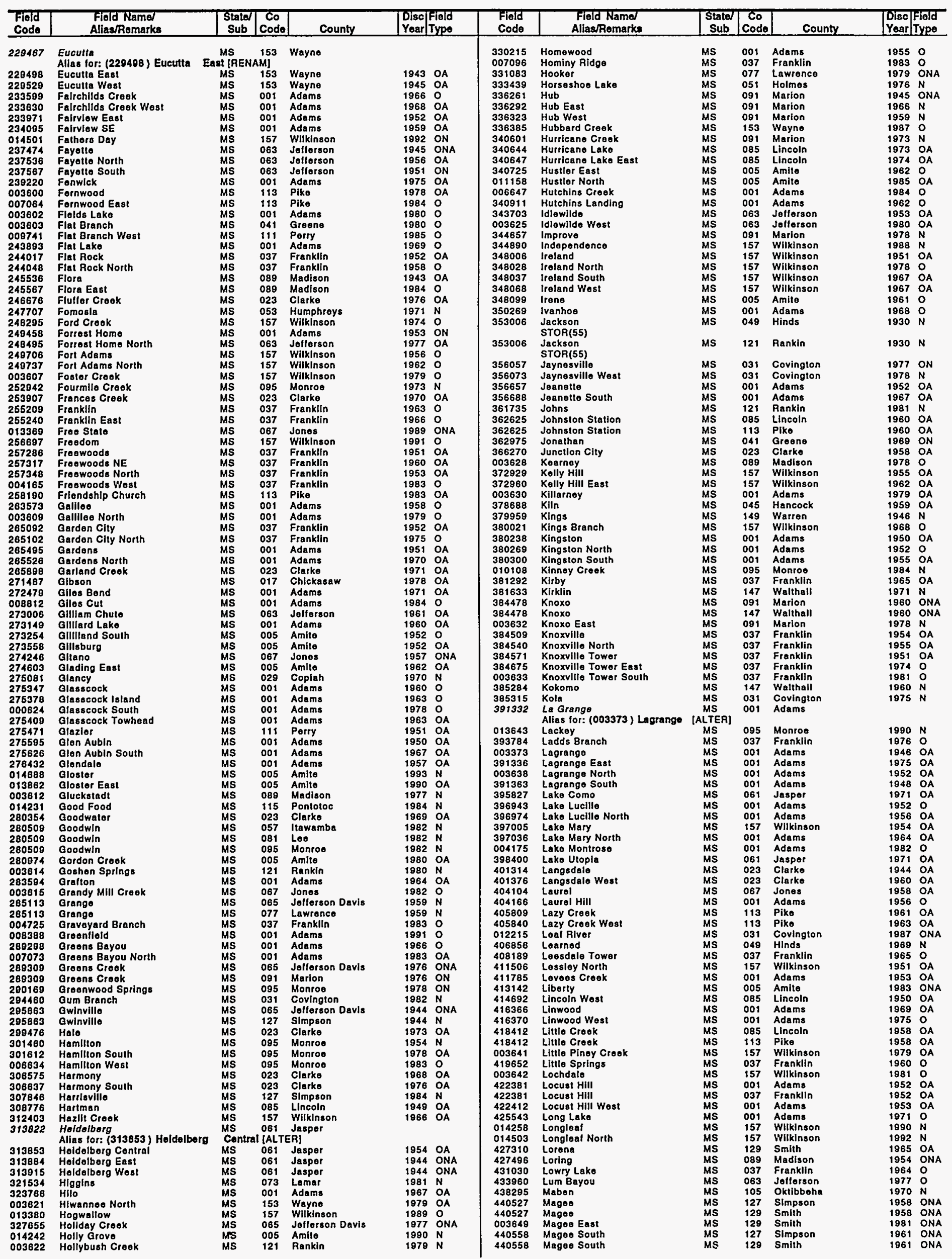




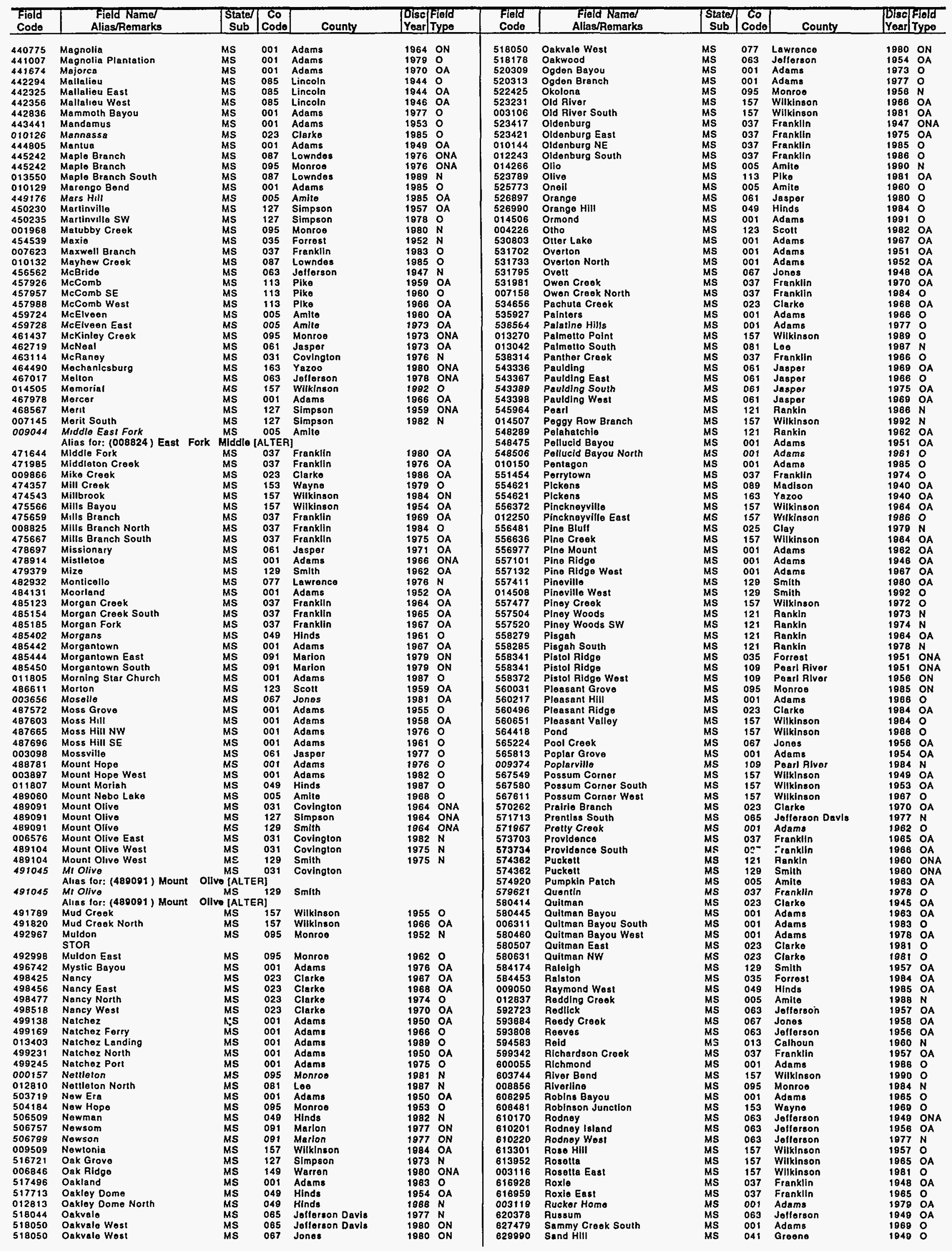


MISSOURI

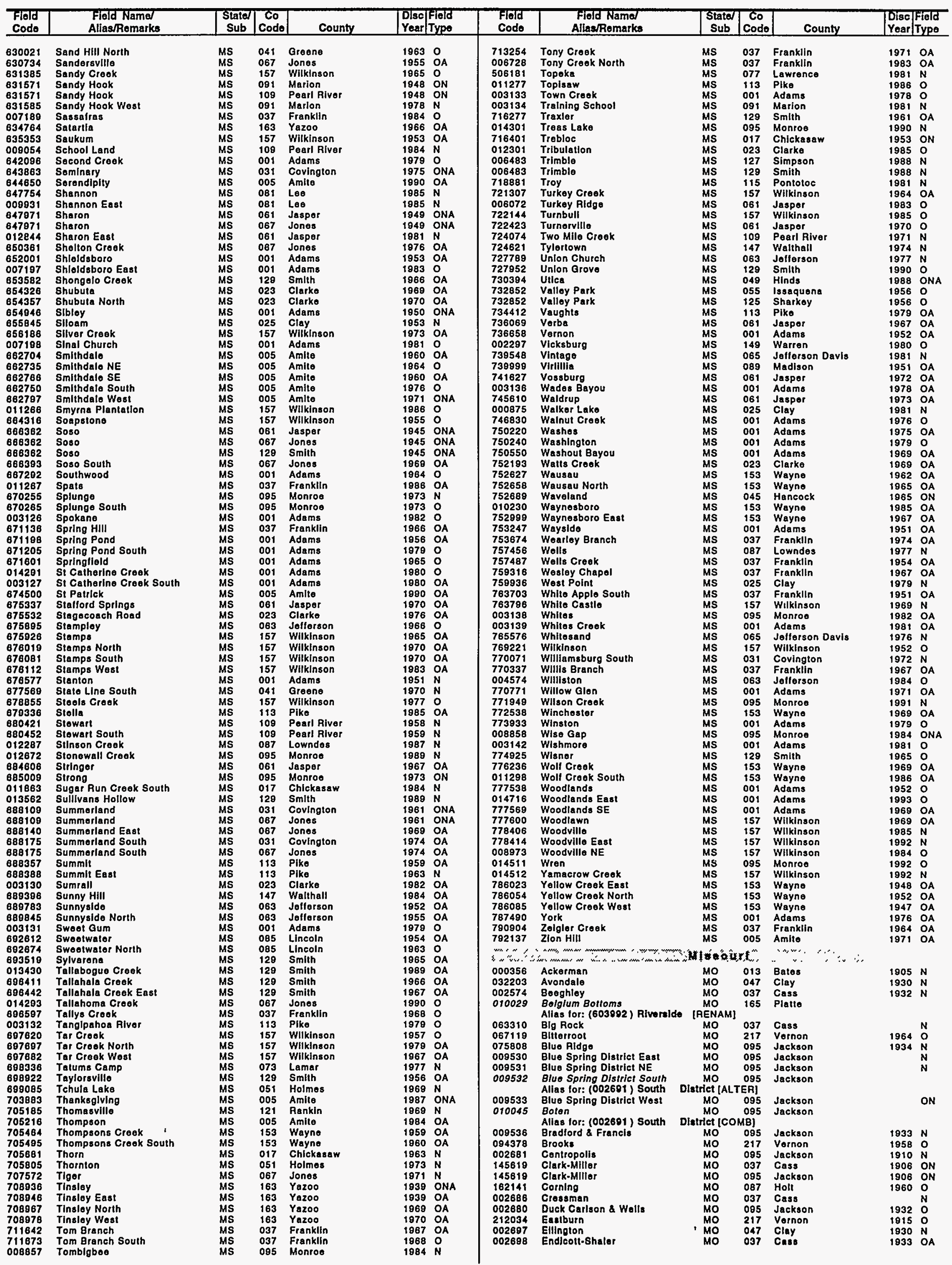




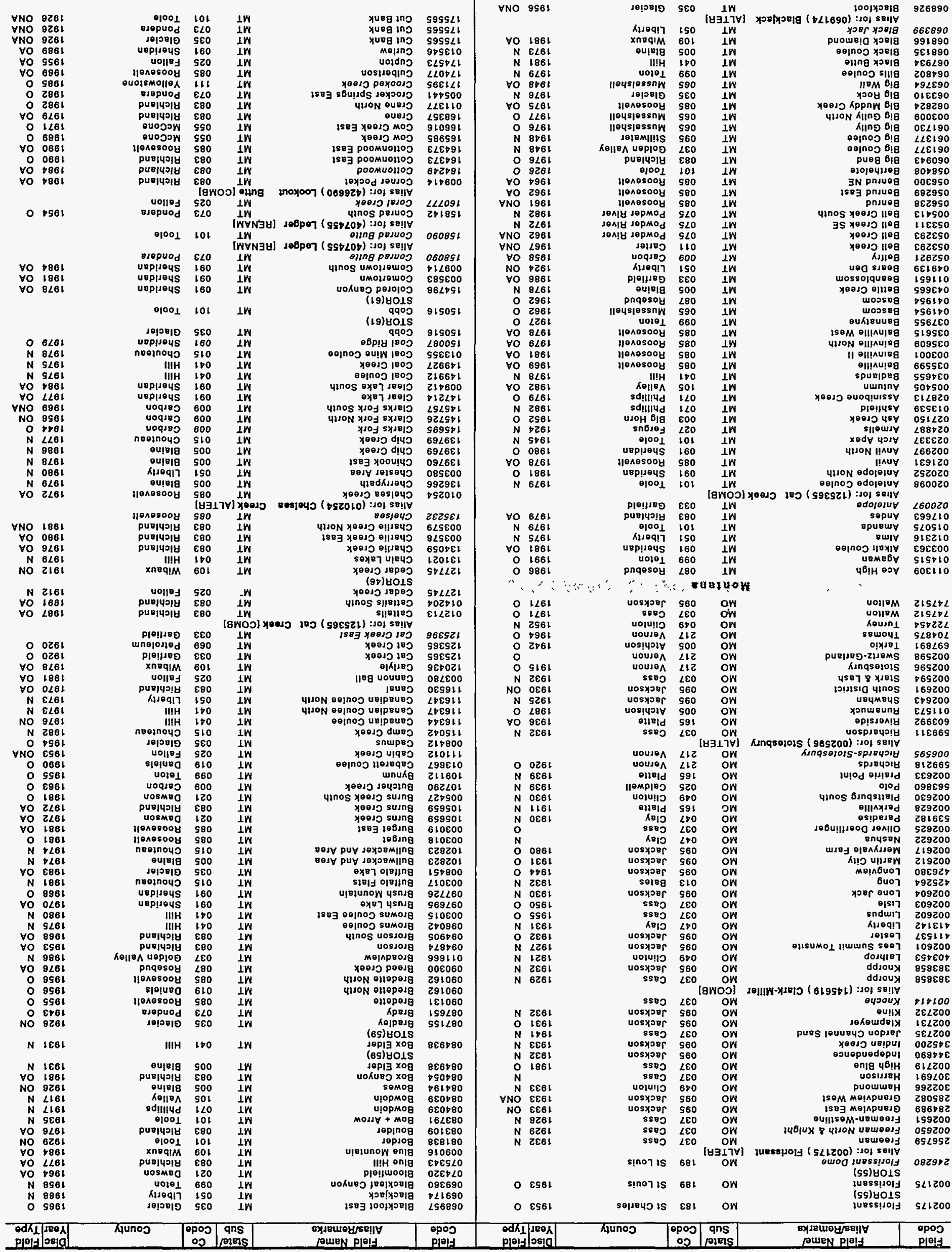


MONTANA

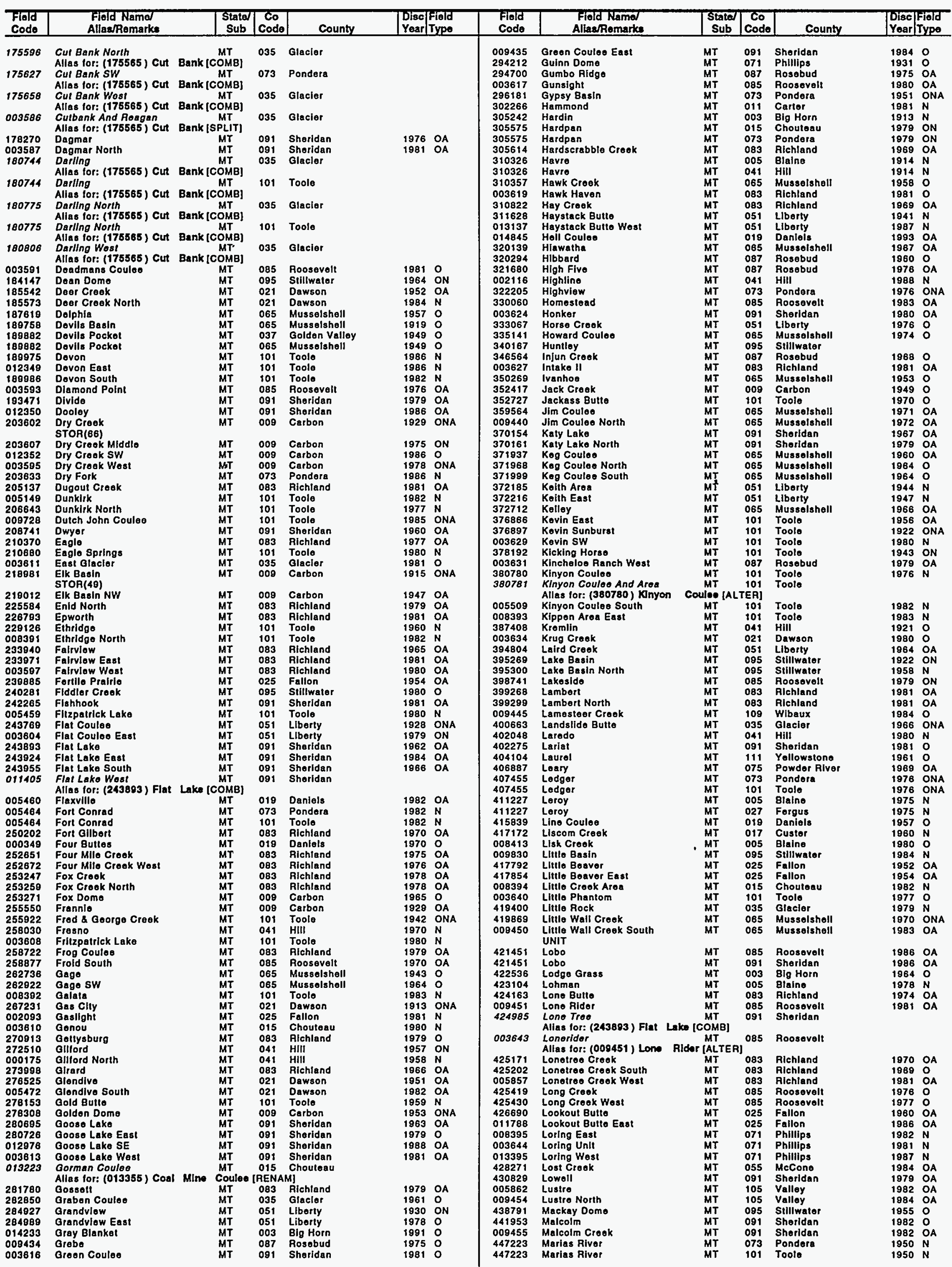

Energy Information Administration/Oil and Gas Field Code Master List 1994 
MONTANA

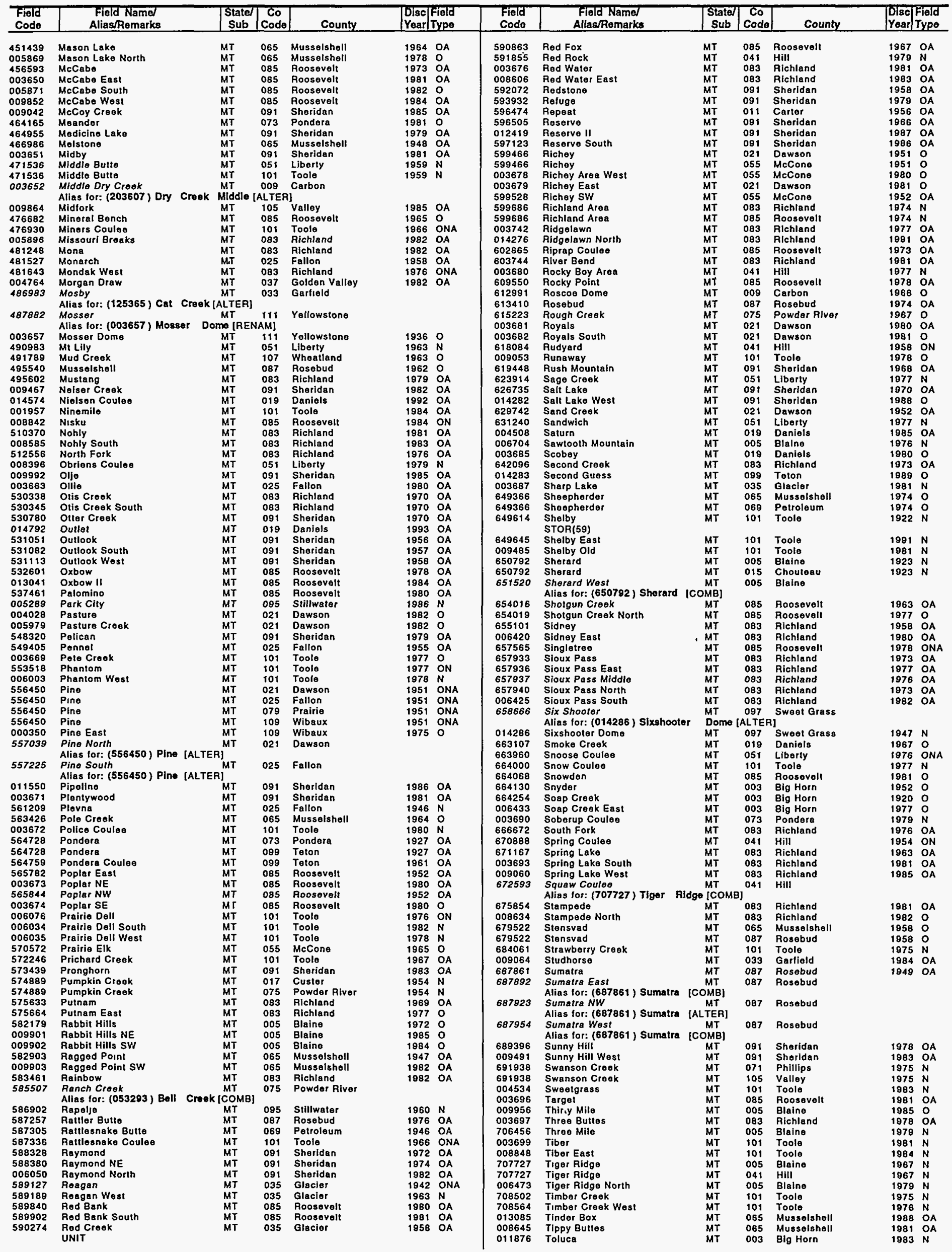


NEBRASKA

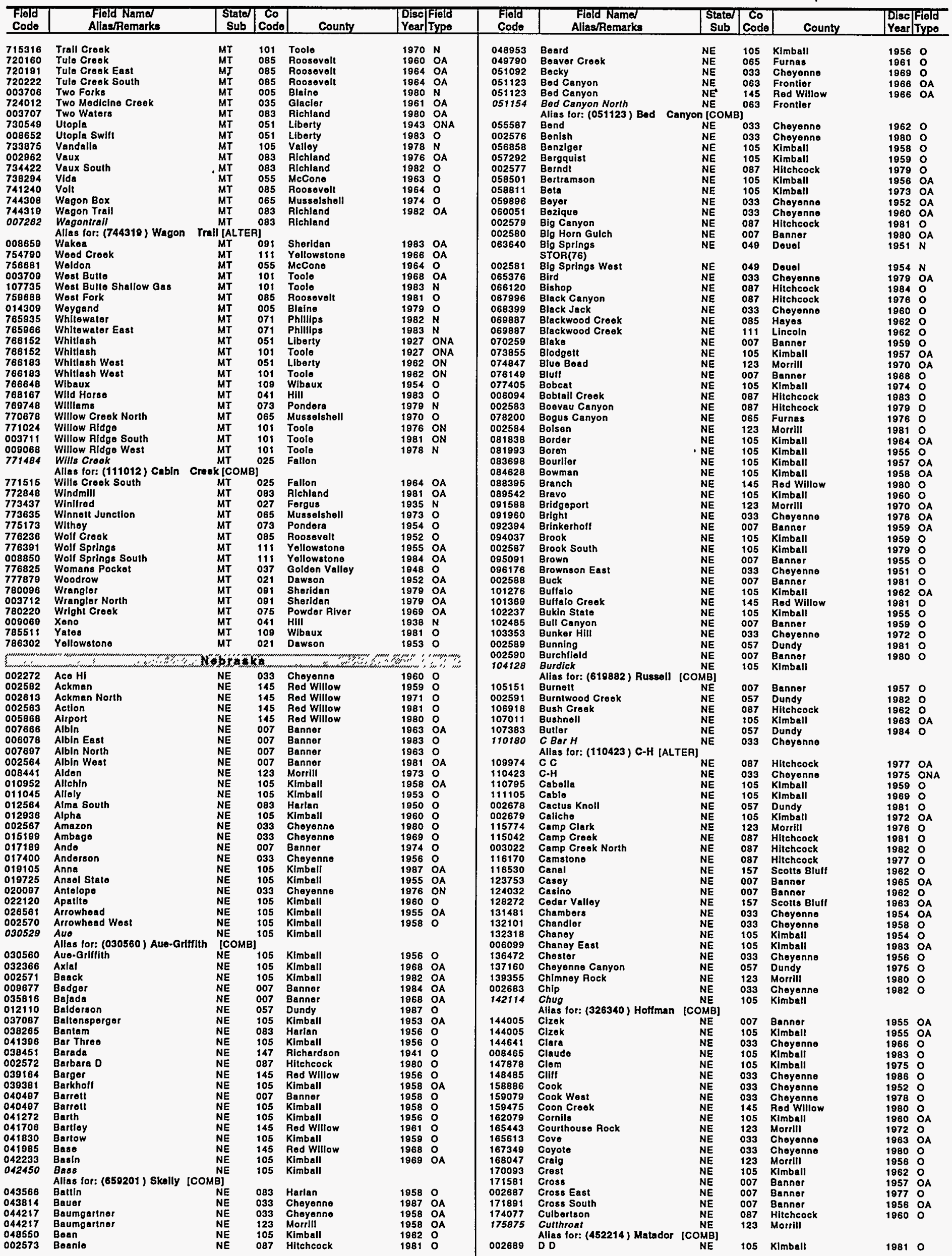


NEBRASKA

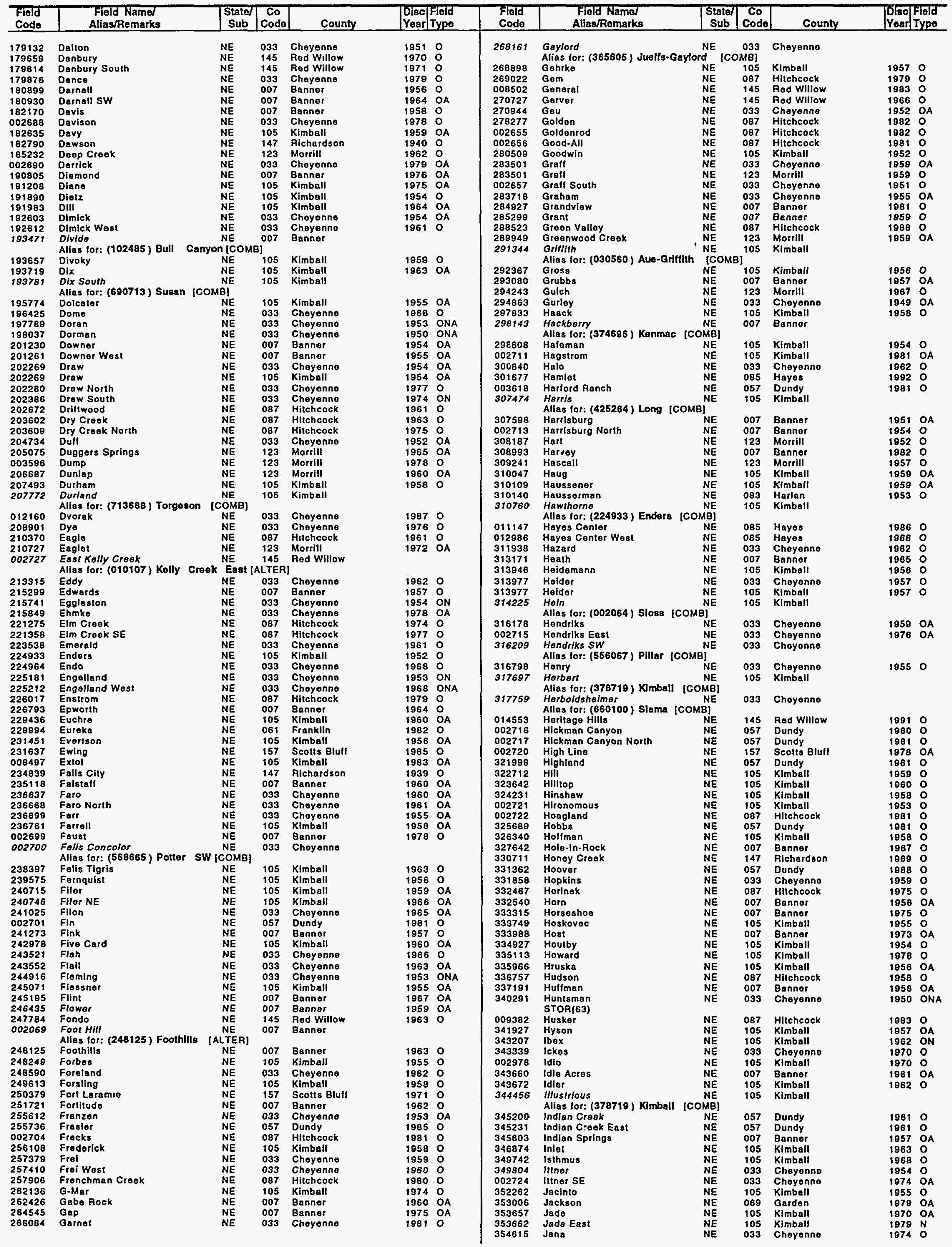


NEBRASKA

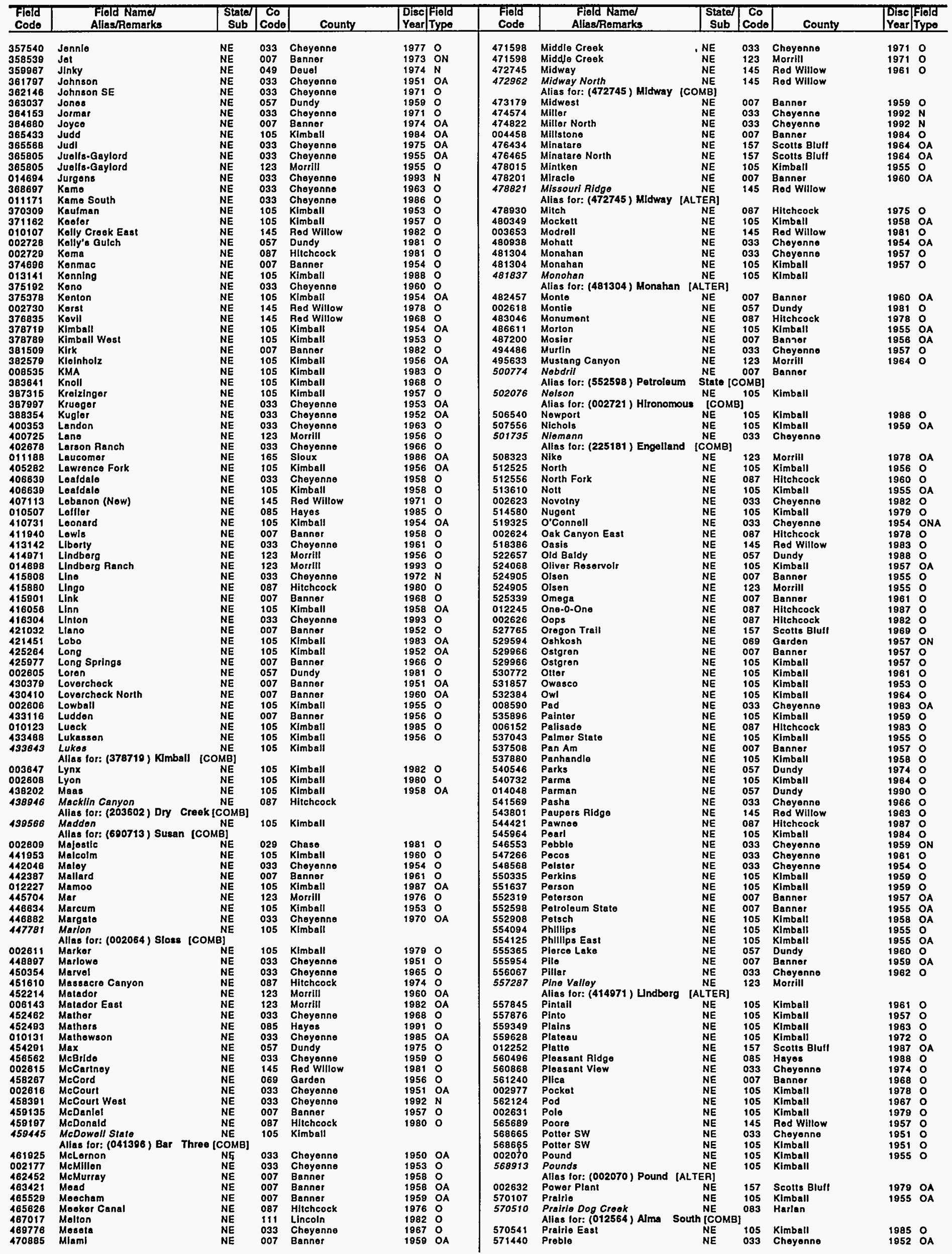

Energy Intormation Administration/OIl and Gas Field Code Master List 1994 
NEBRASKA

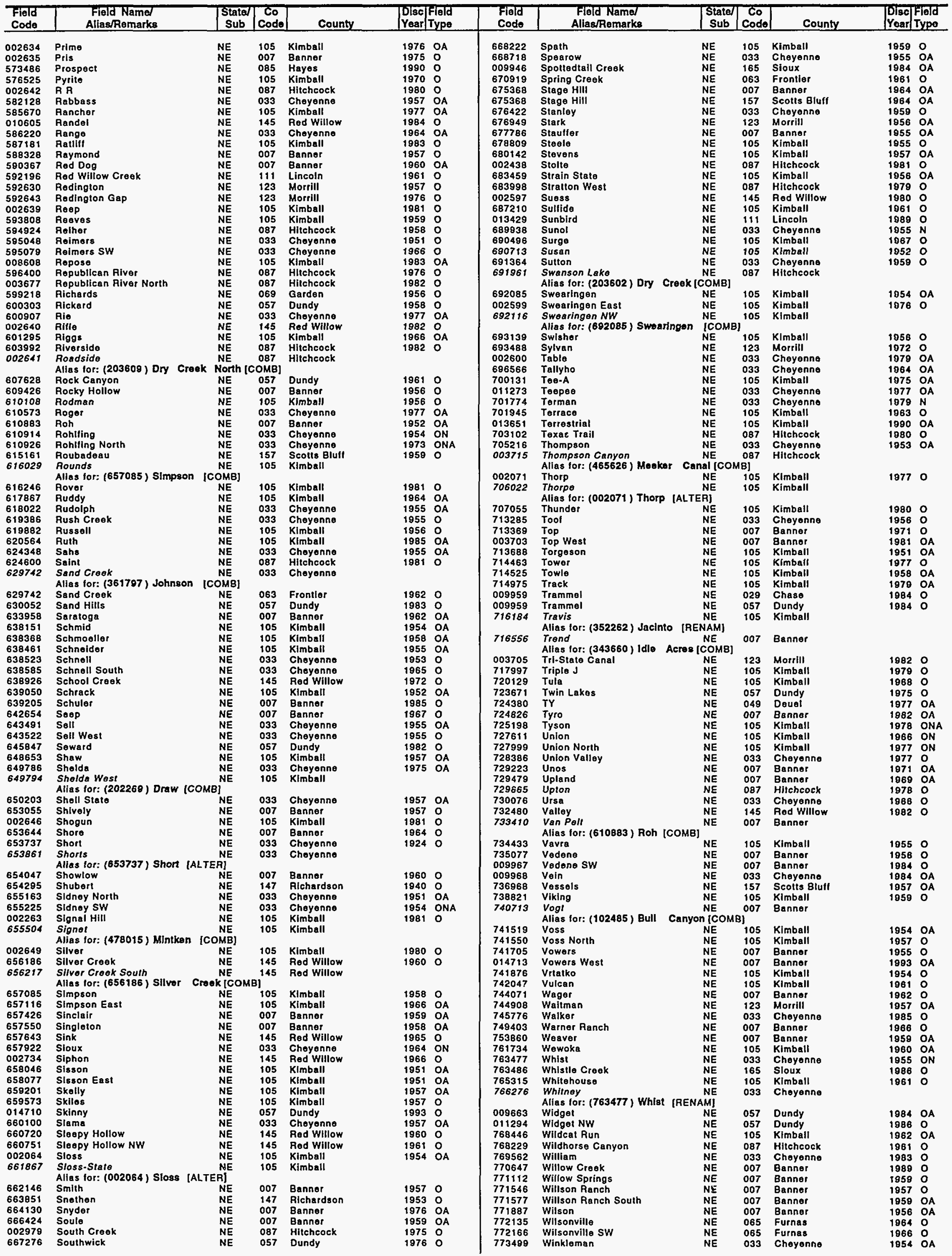


NEW MEXICO

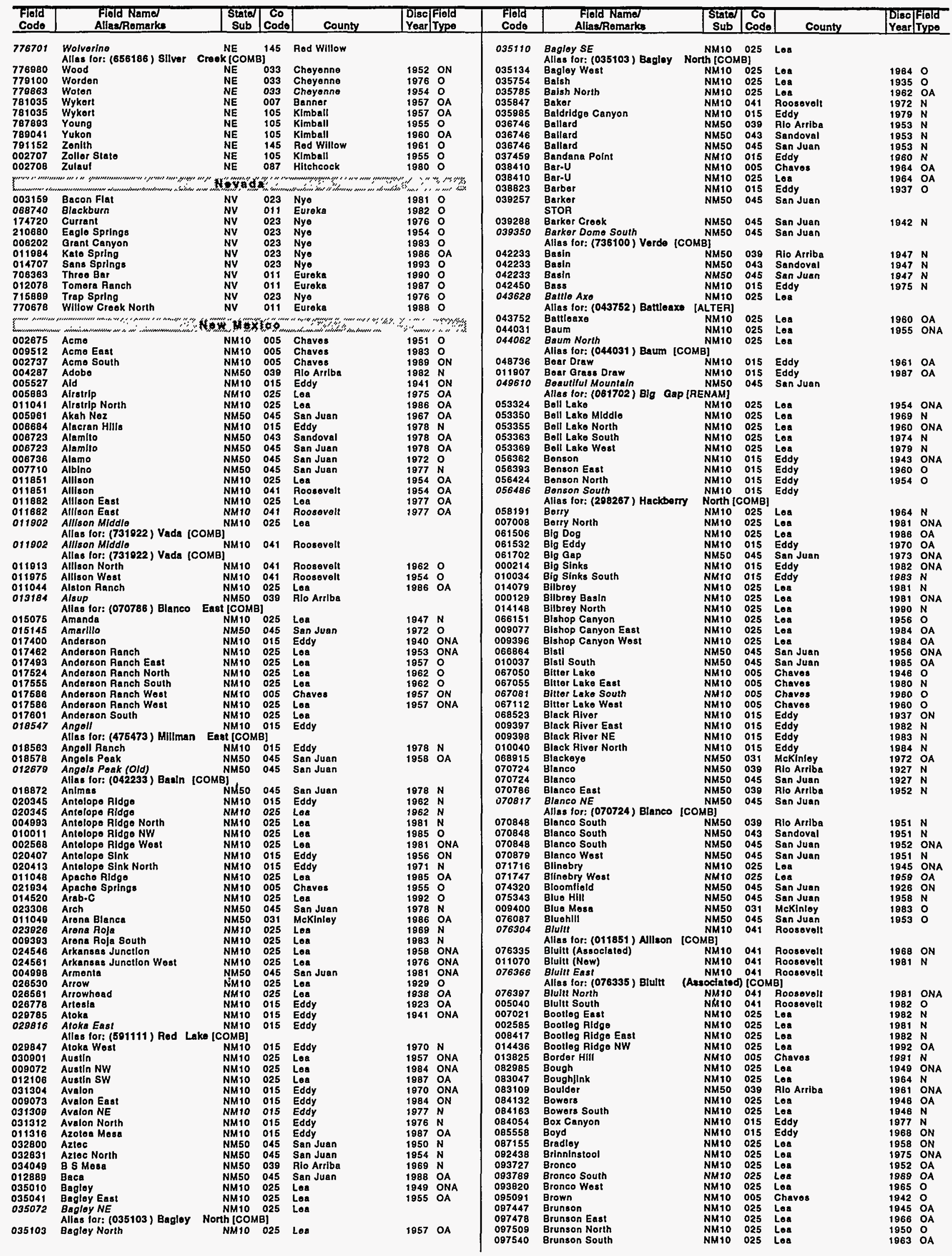


NEW MEXICO

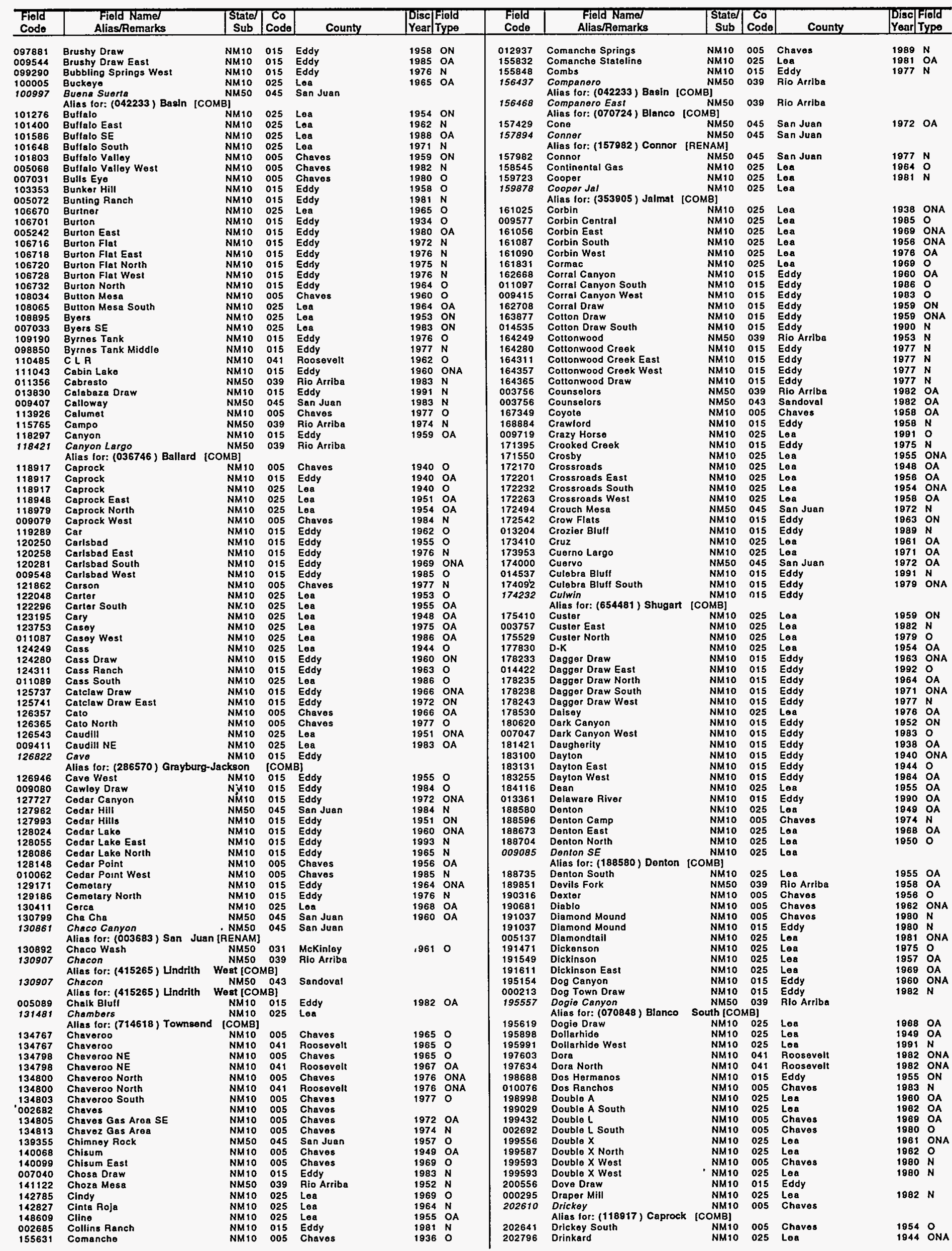


NEW MEXICO

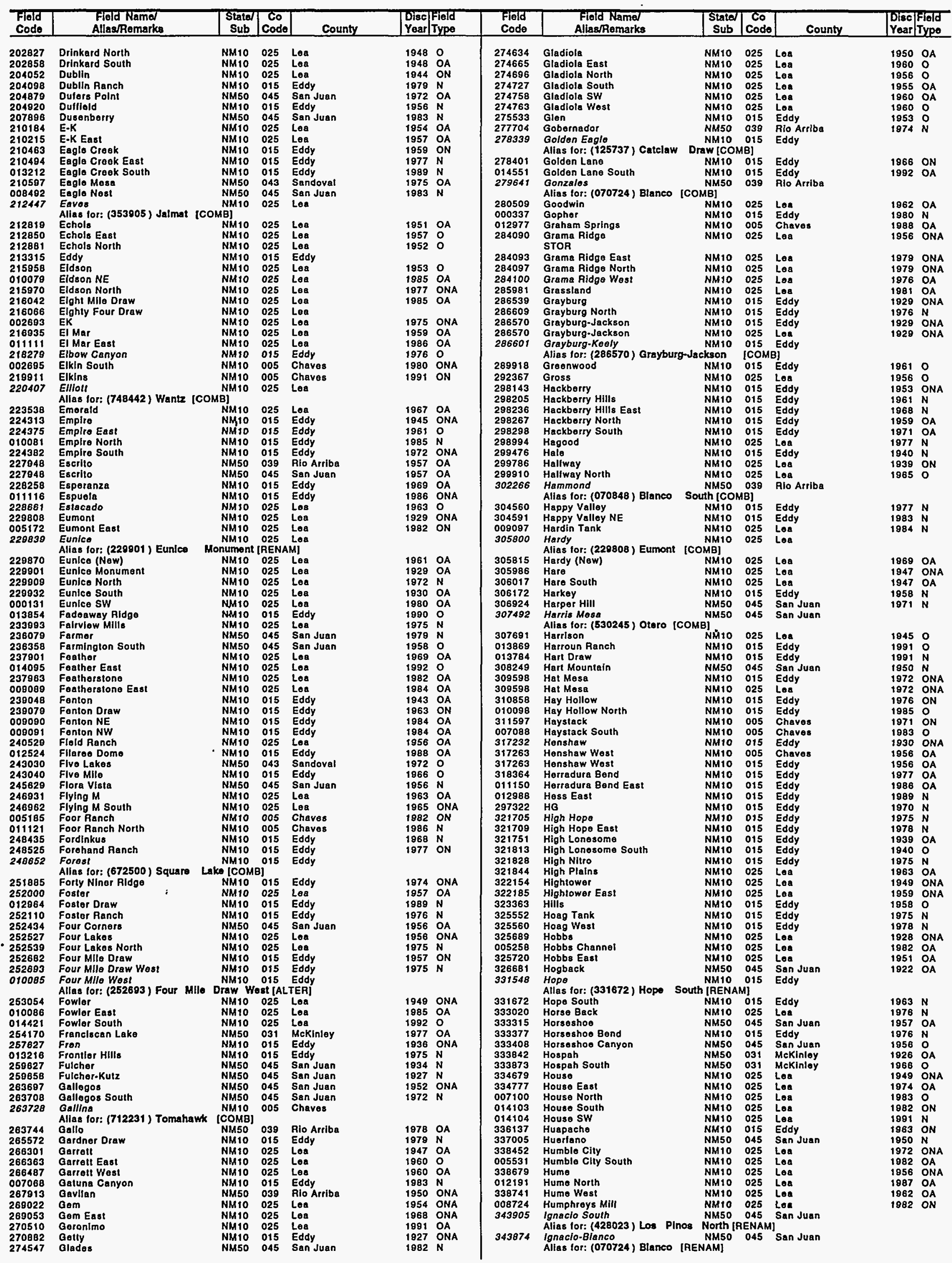


NEW MEXICO

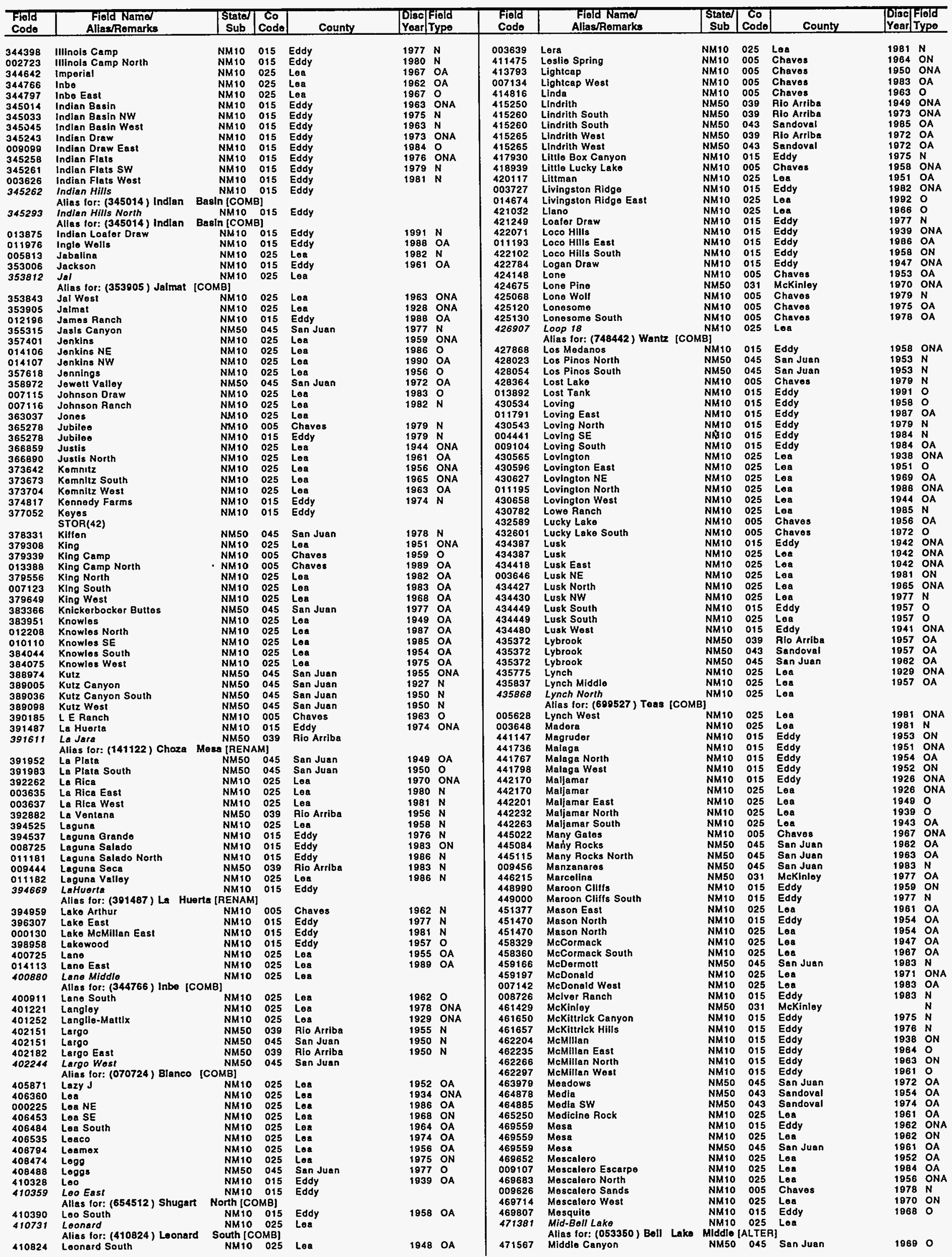


NEW MEXICO

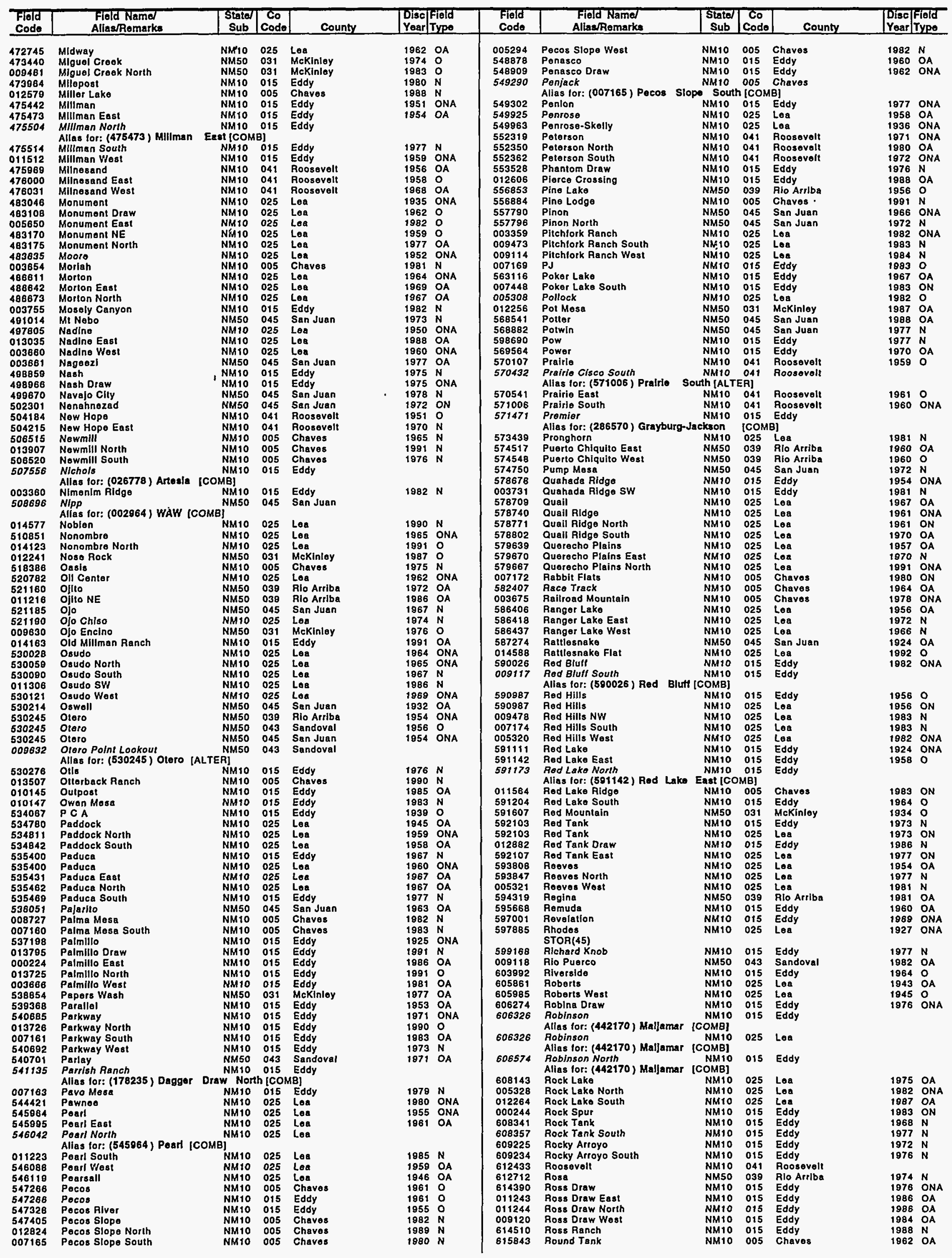


NEW MEXICO

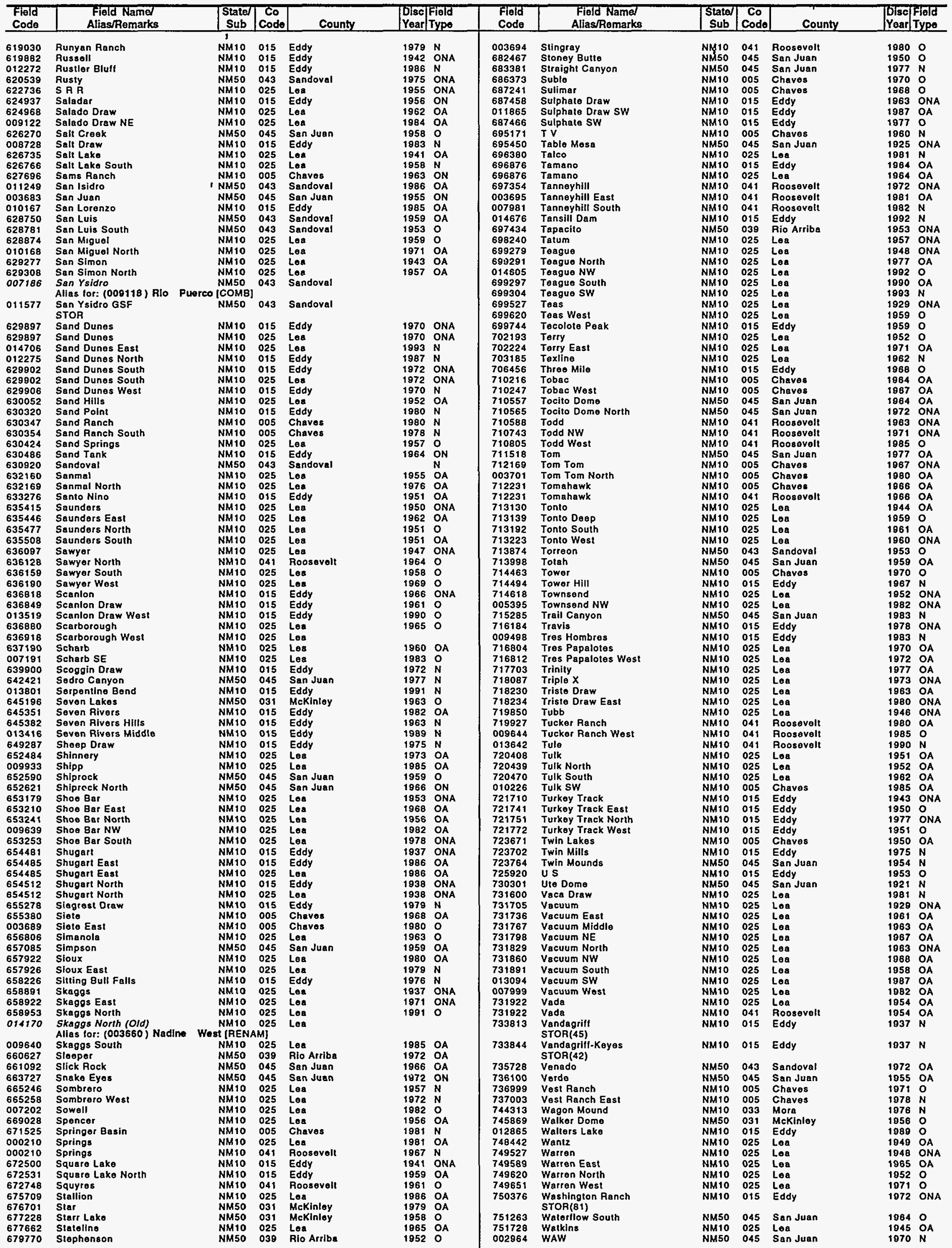


NEW YORK

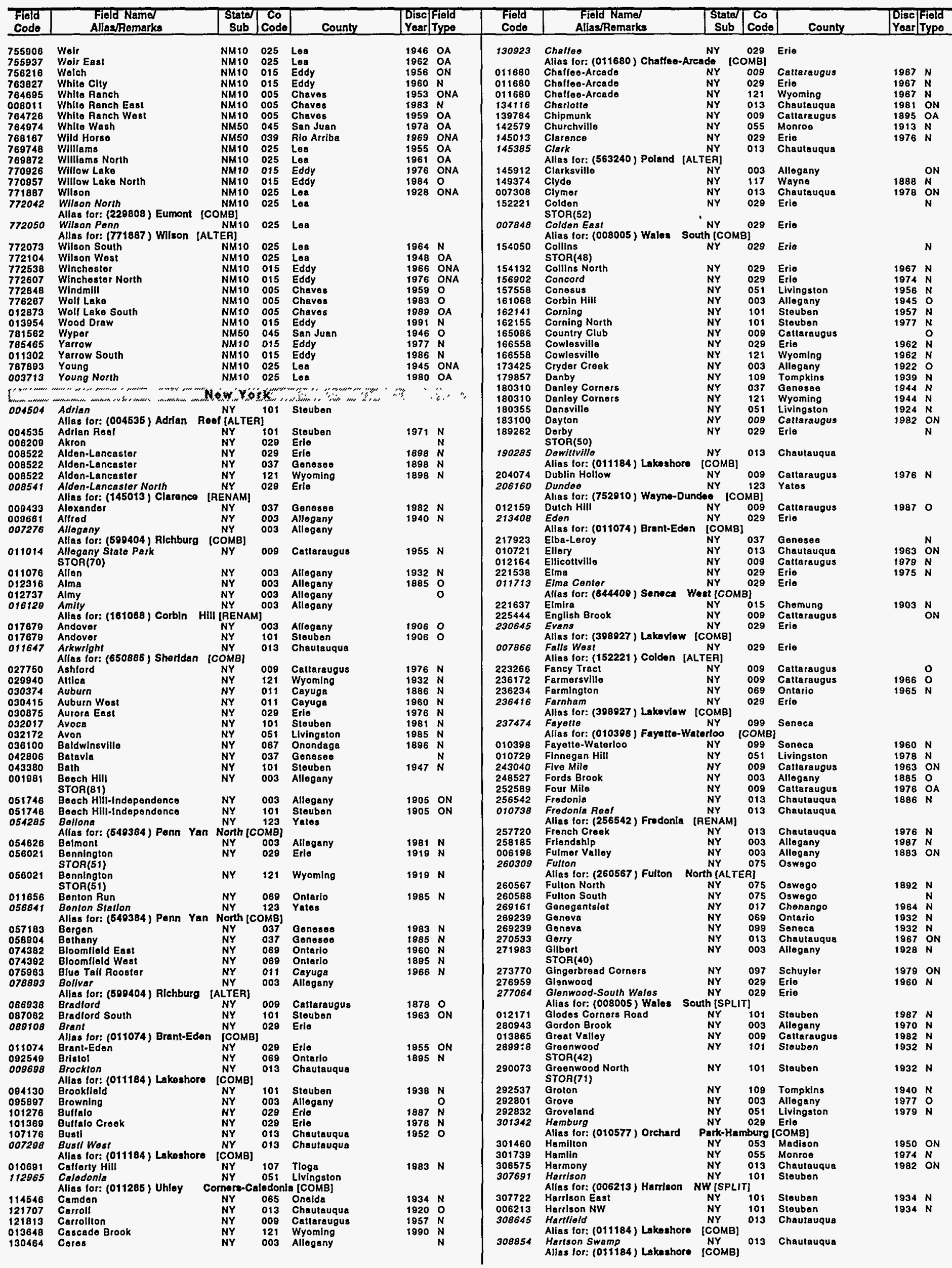




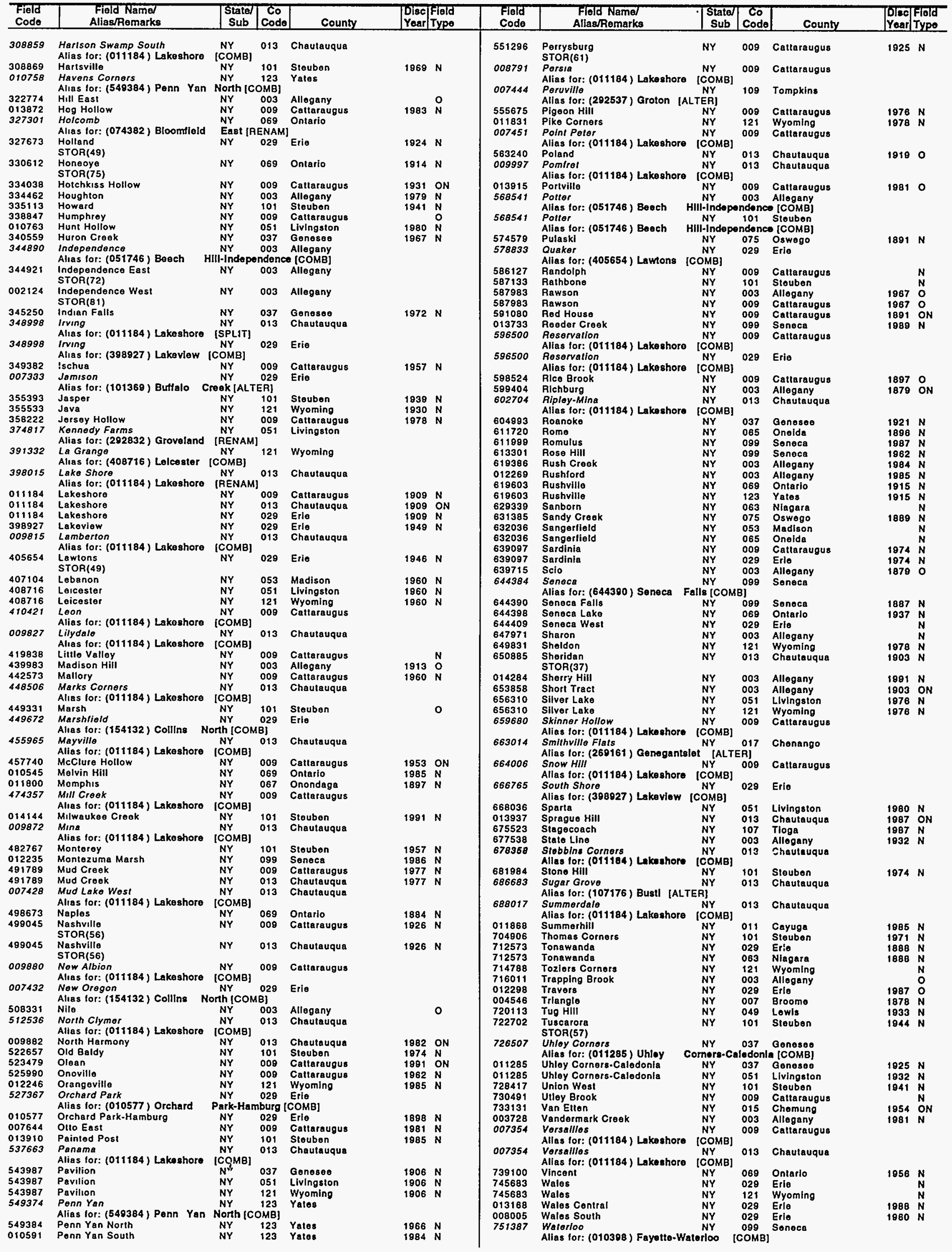


NORTH DAKOTA

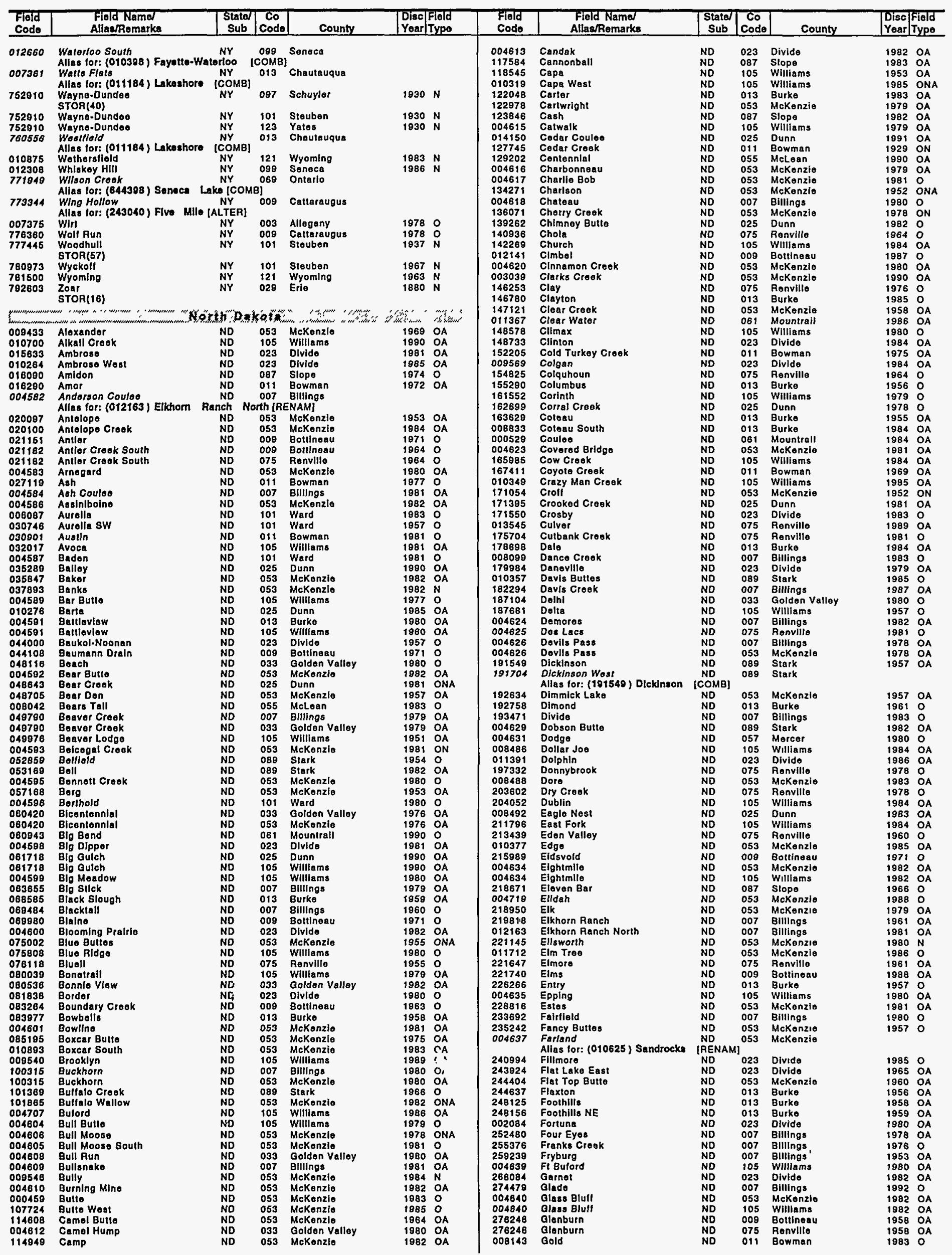


NORTH DAKOTA

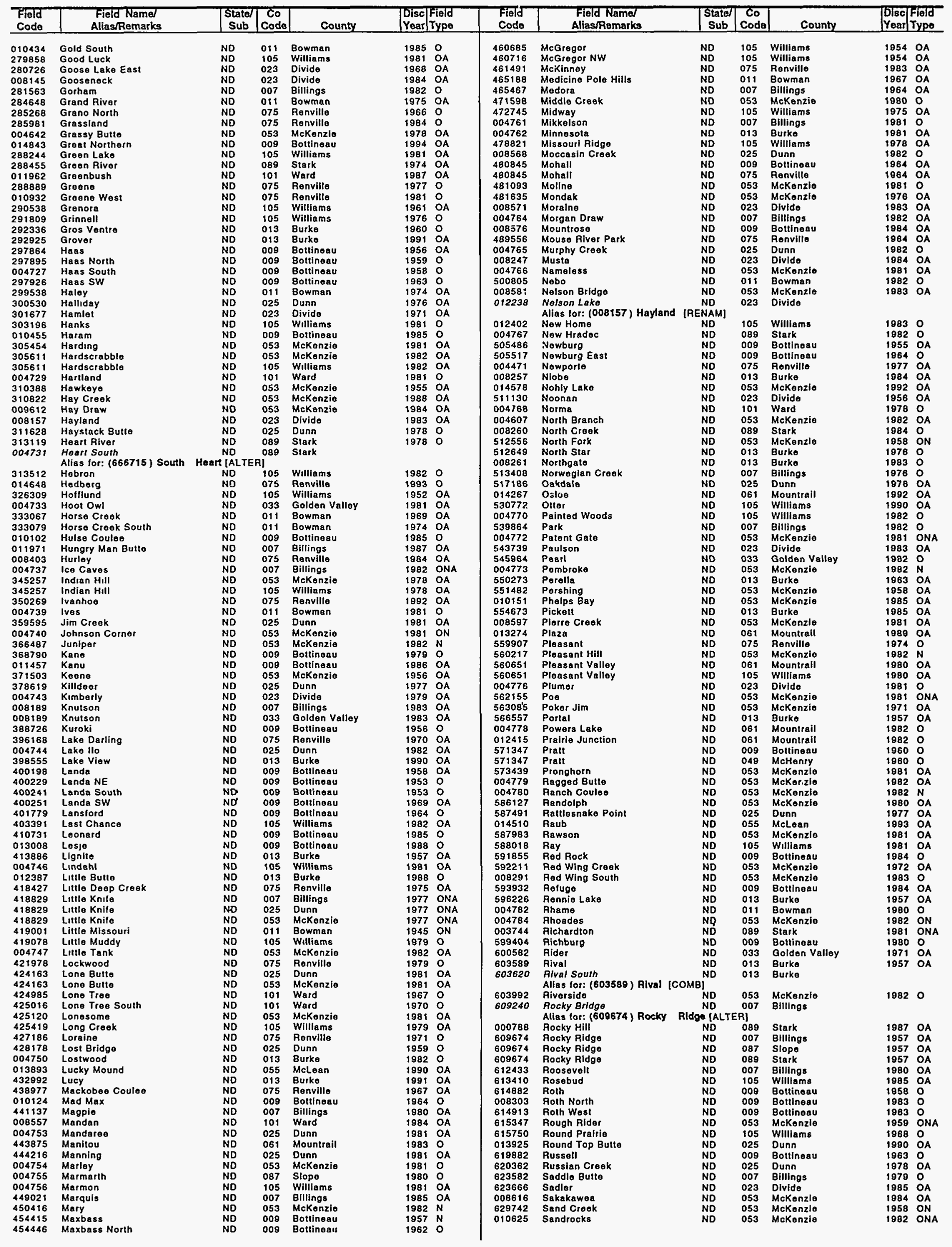




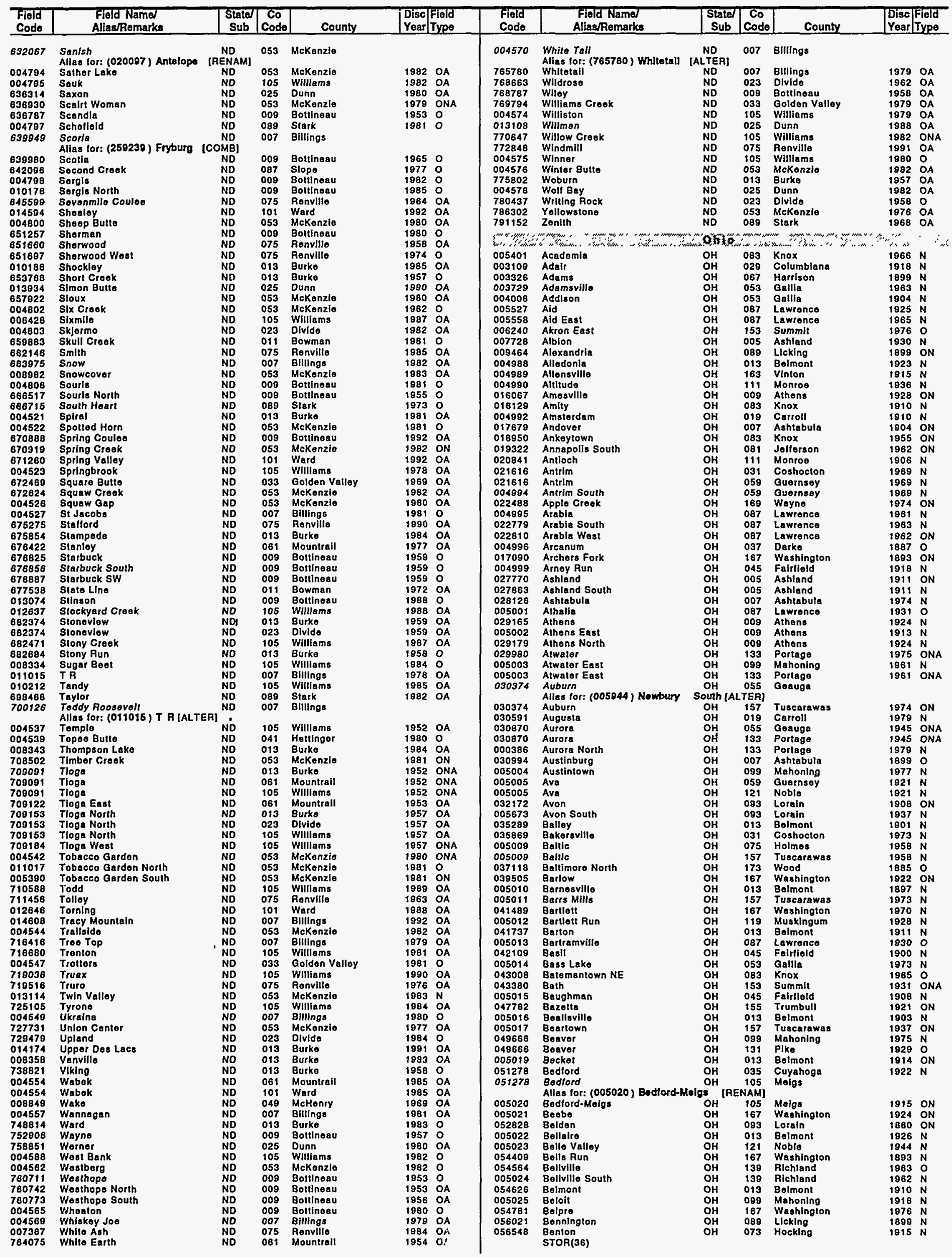


$\mathrm{OHIO}$

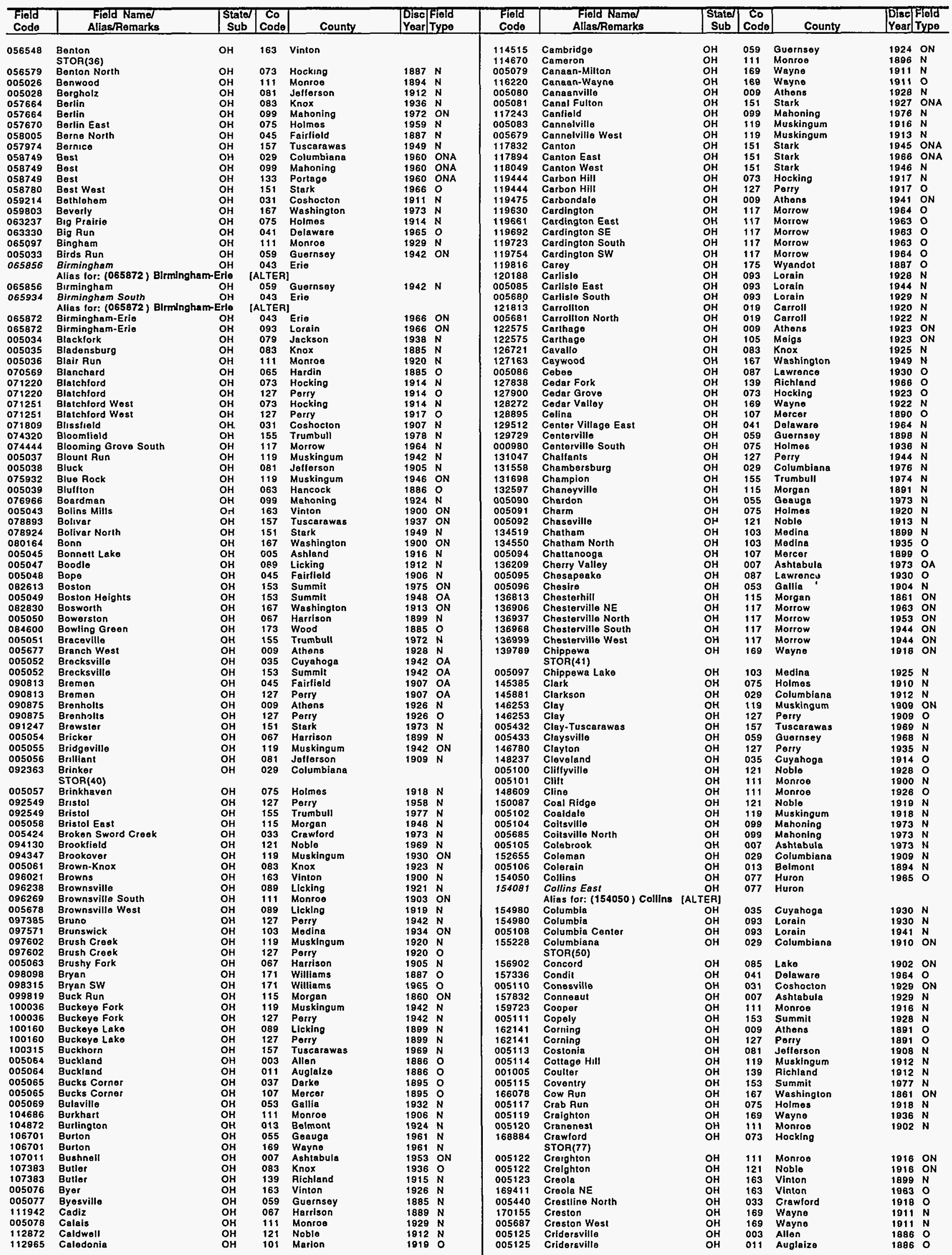


OHIO

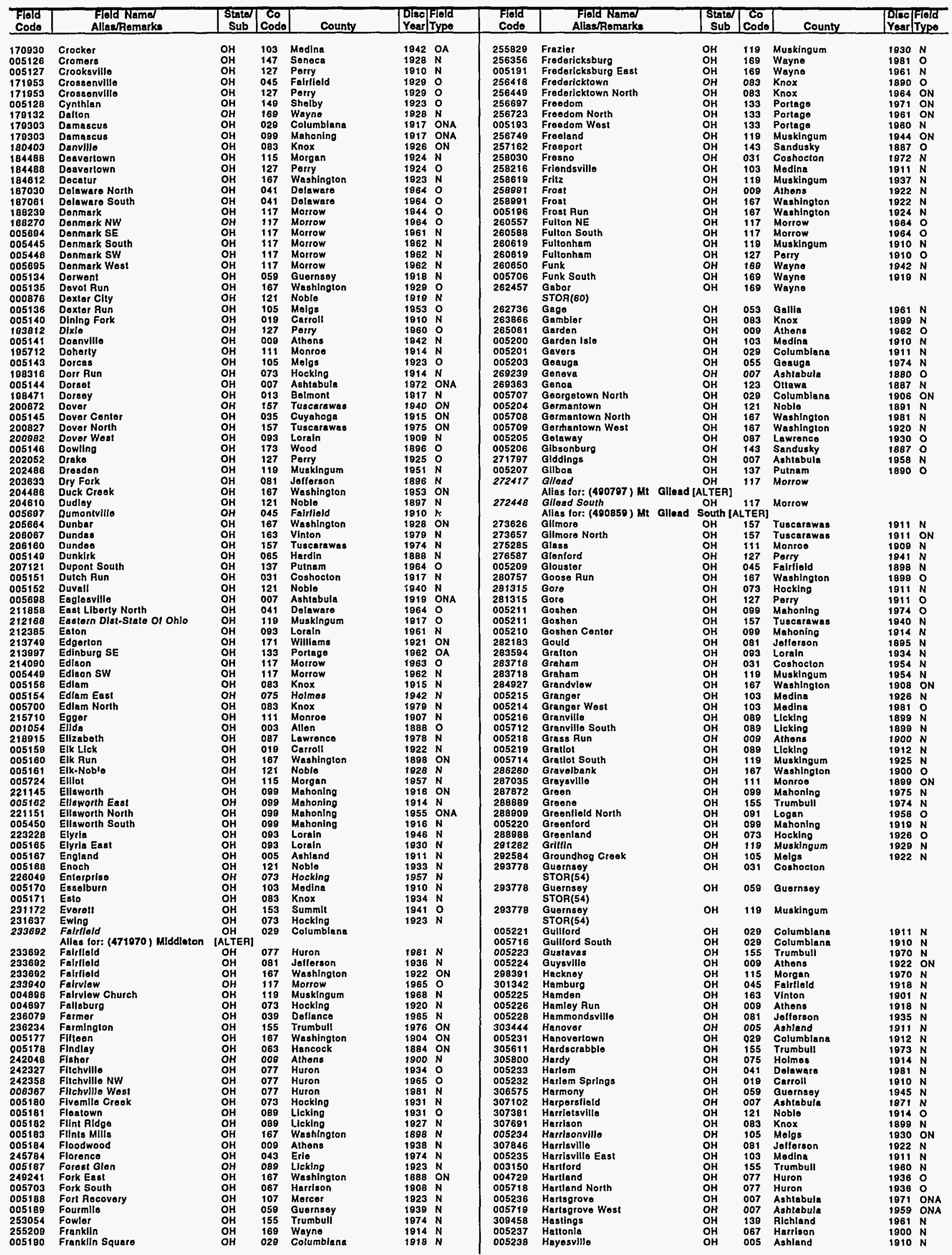




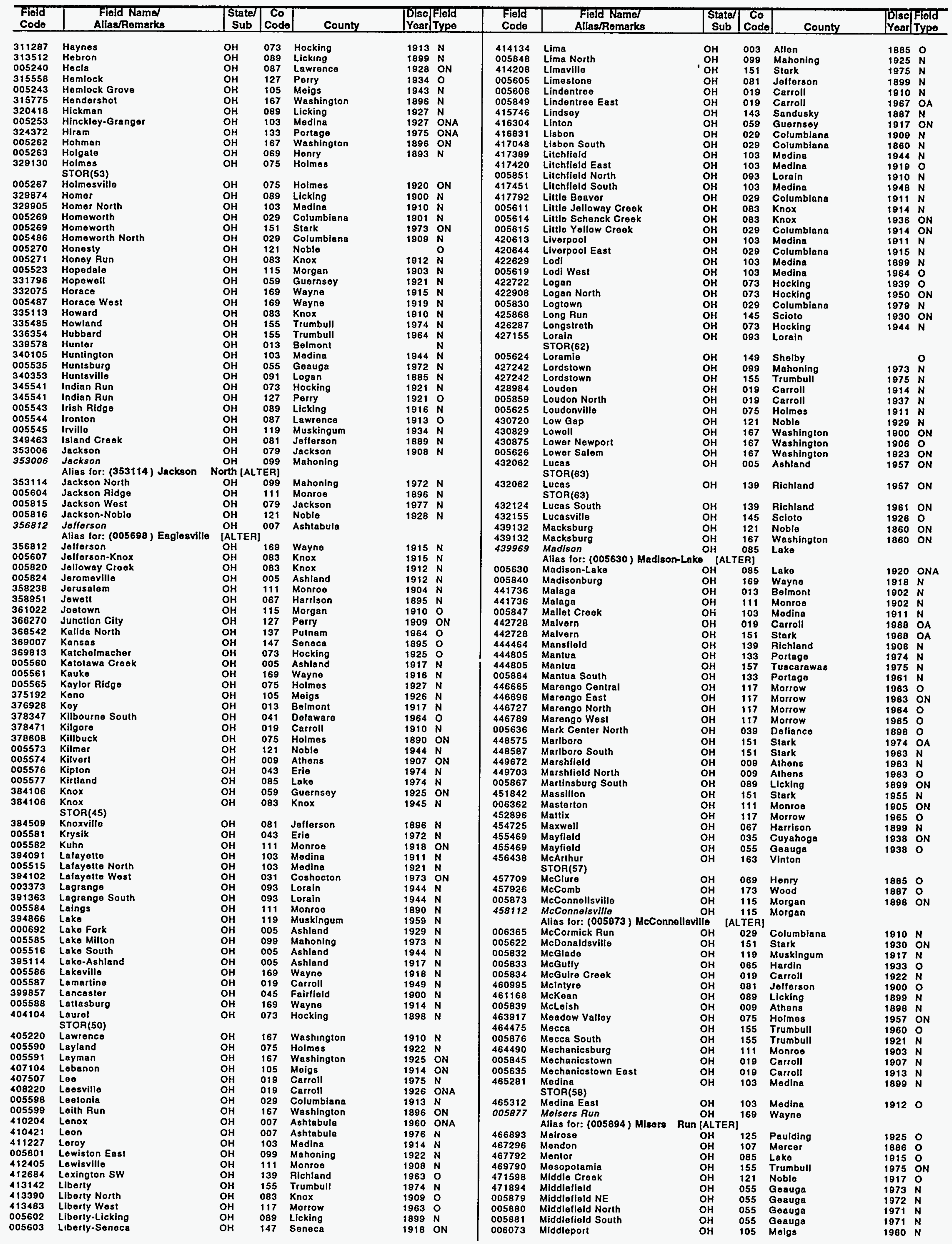


OHIO

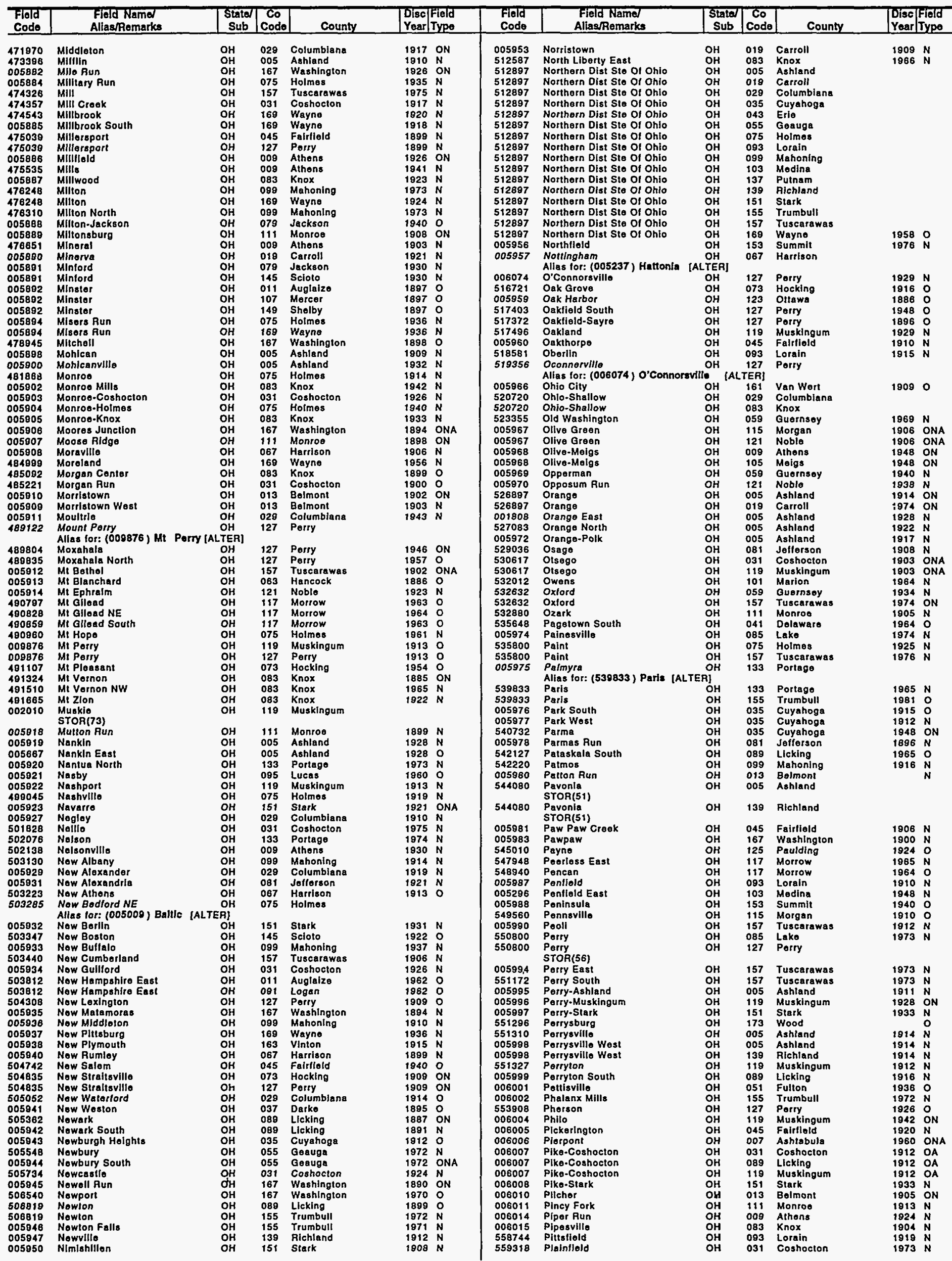

Energy Information Administration/Oil and Gas Field Code Master List 1994 


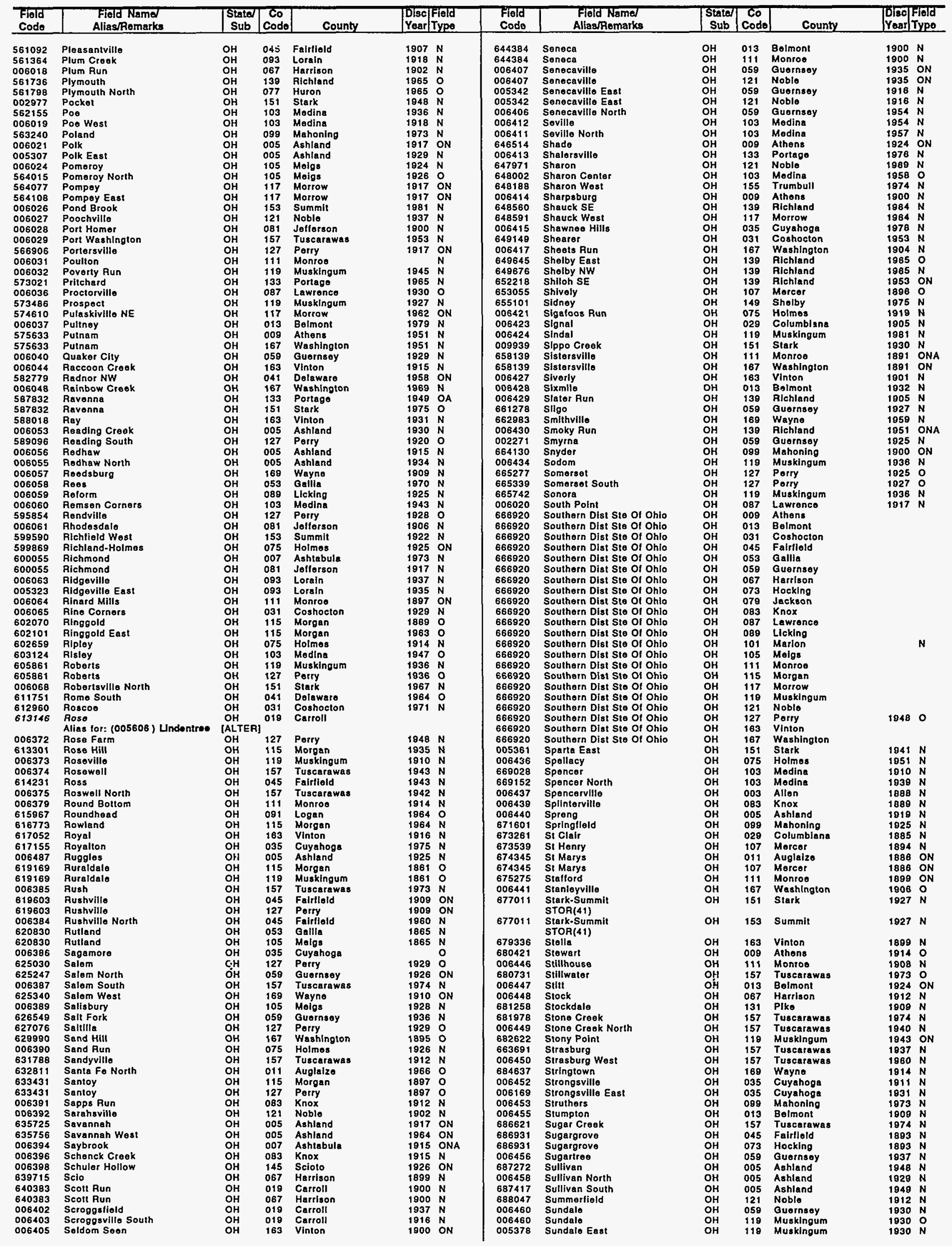


OKLAHOMA

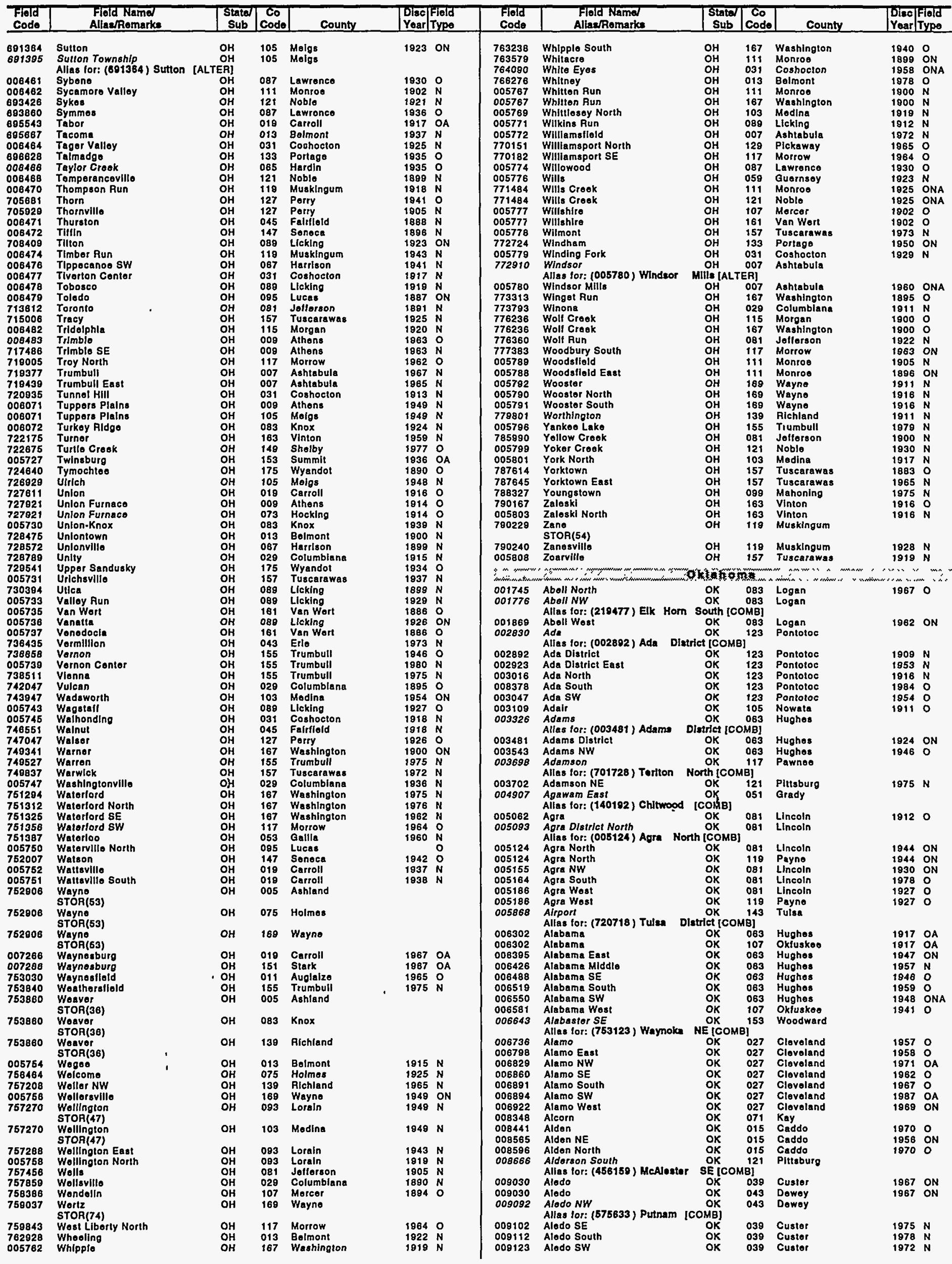


OKLAHOMA

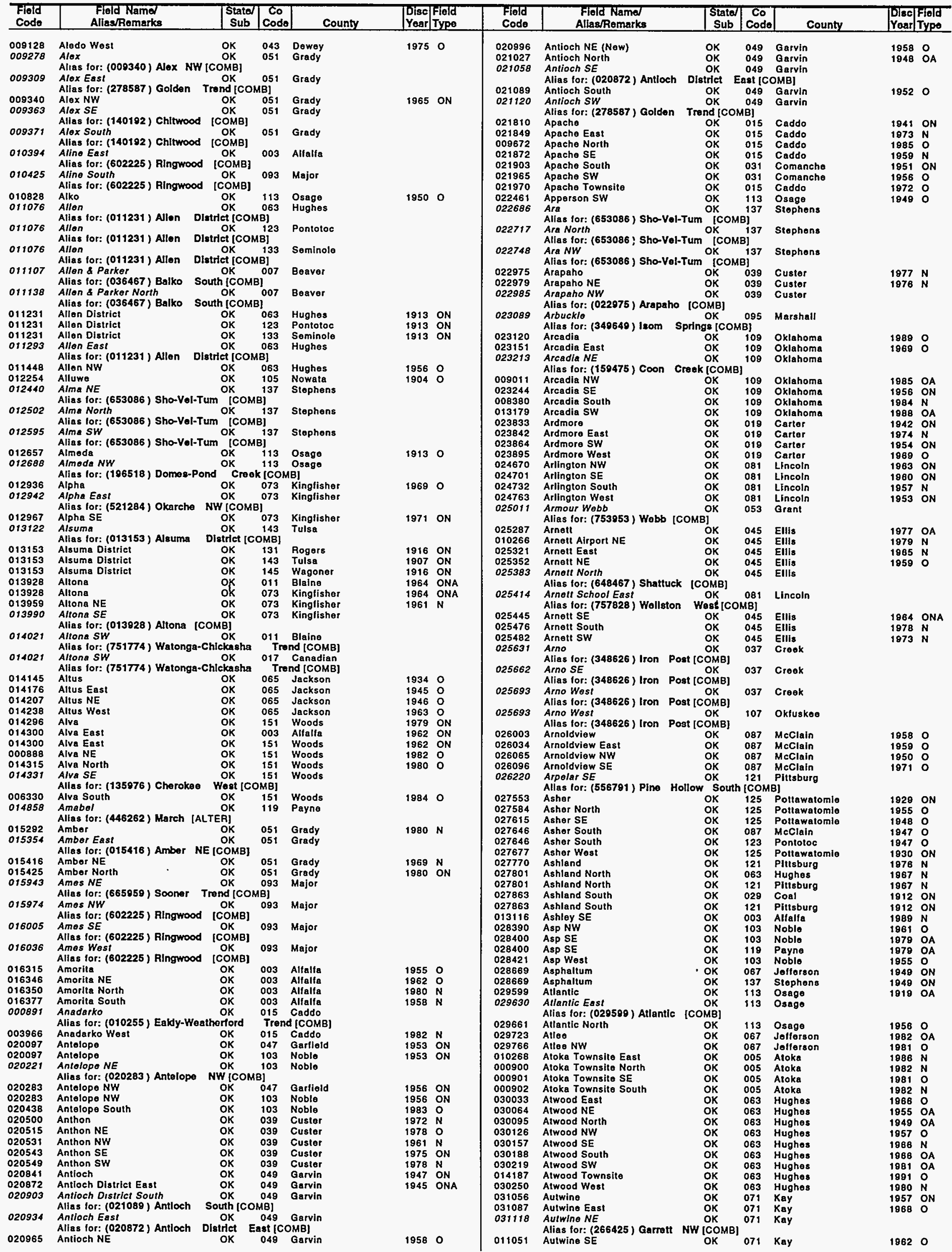


OKLAHOMA

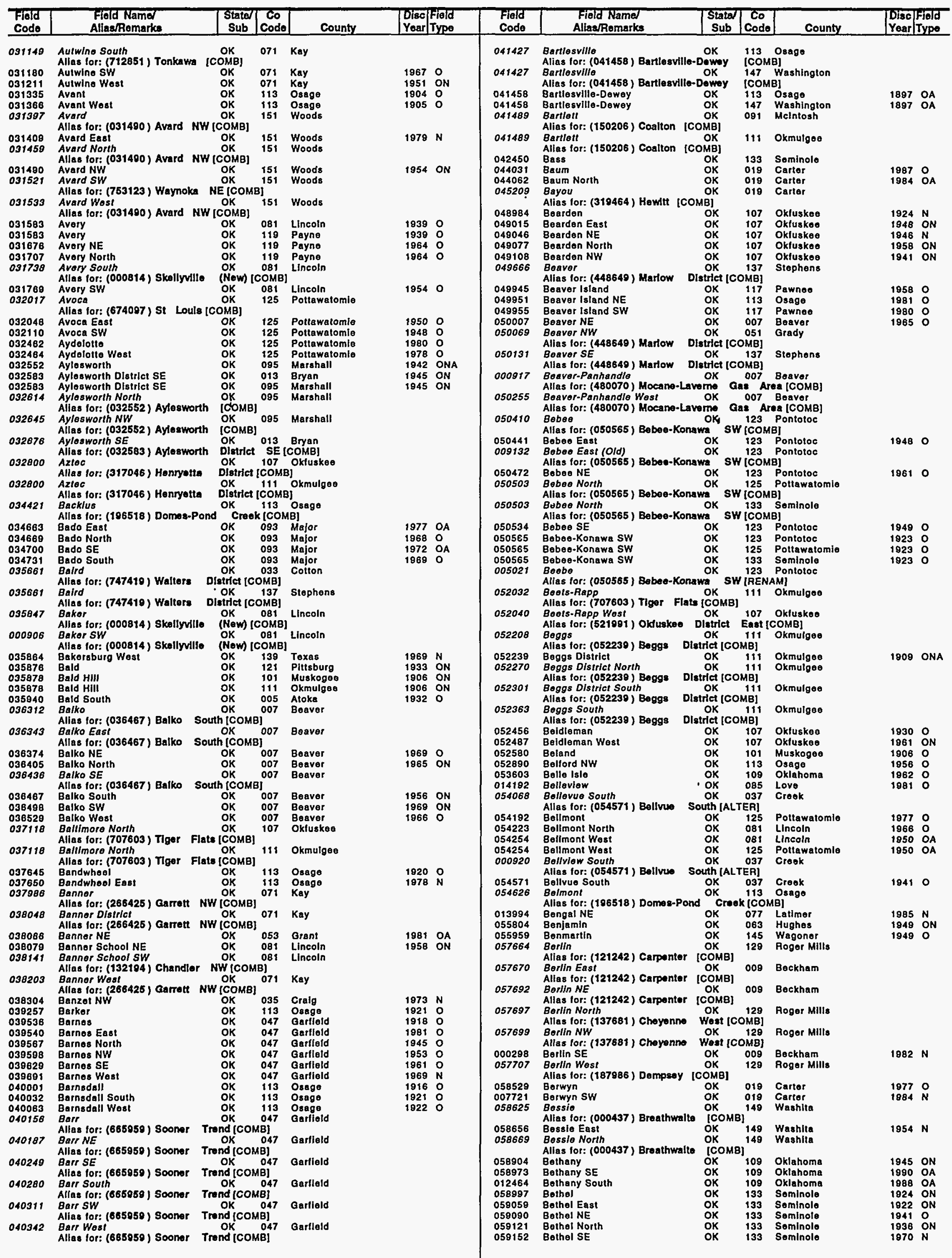


OKLAHOMA

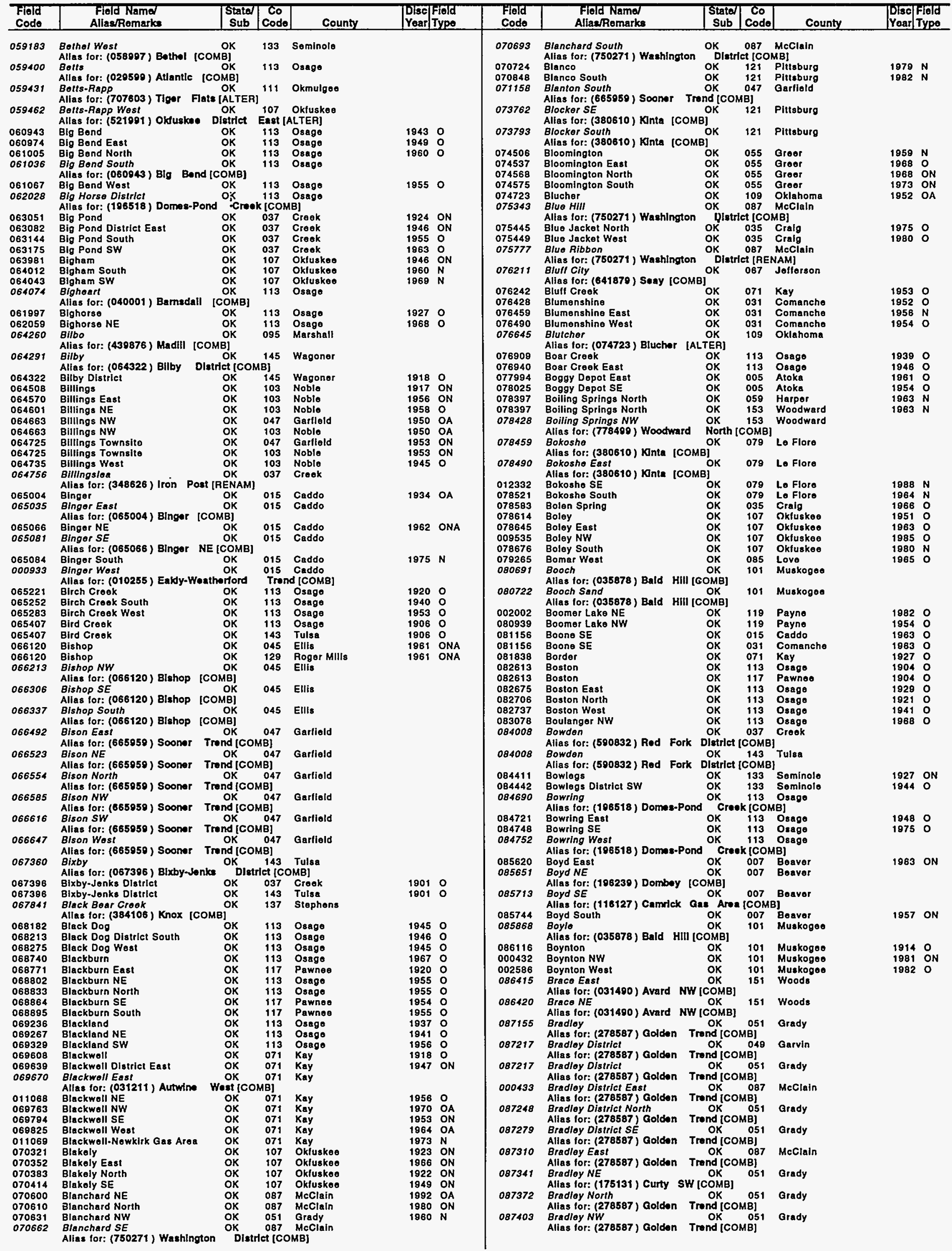


OKLAHOMA

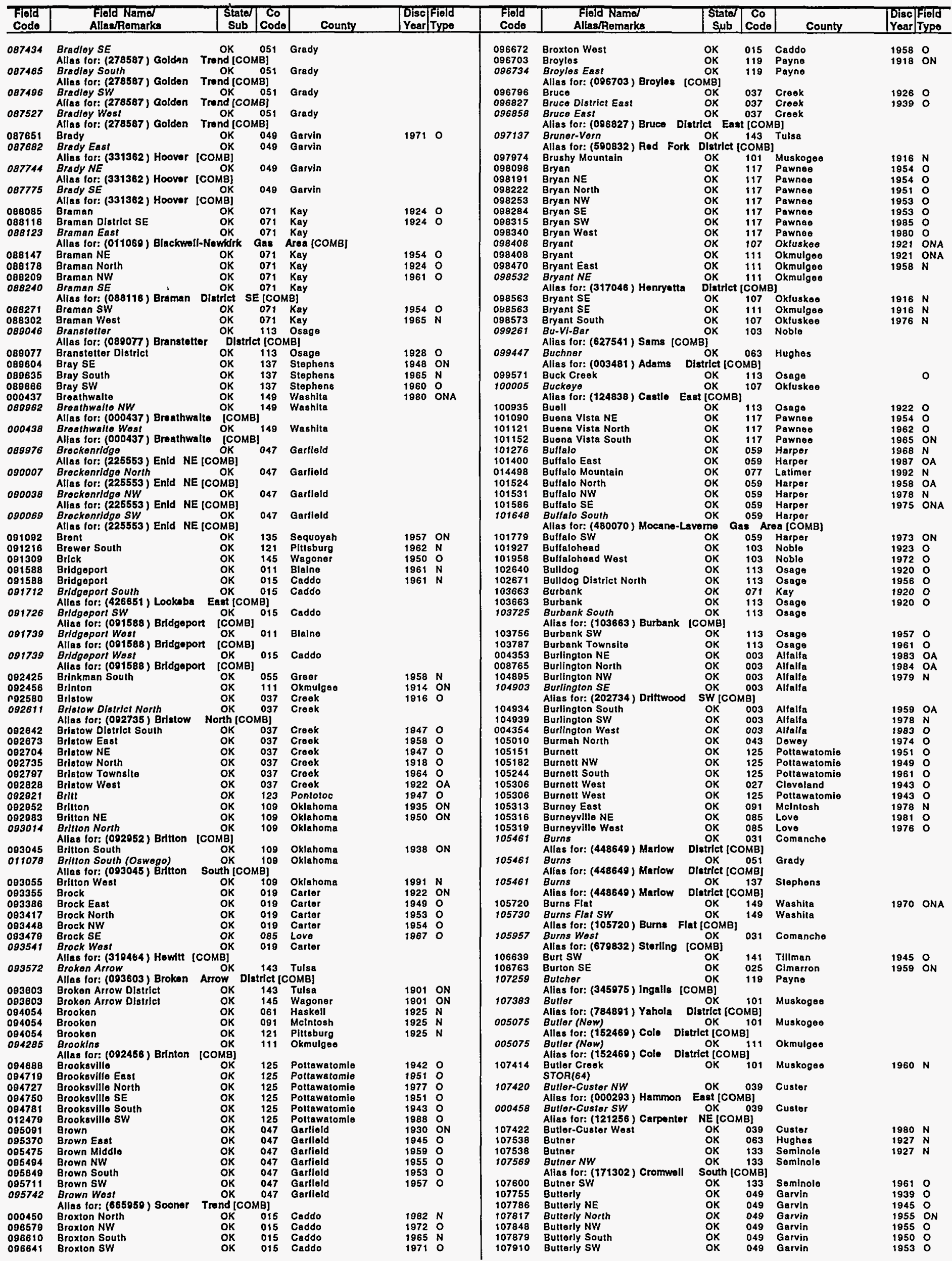


OKLAHOMA

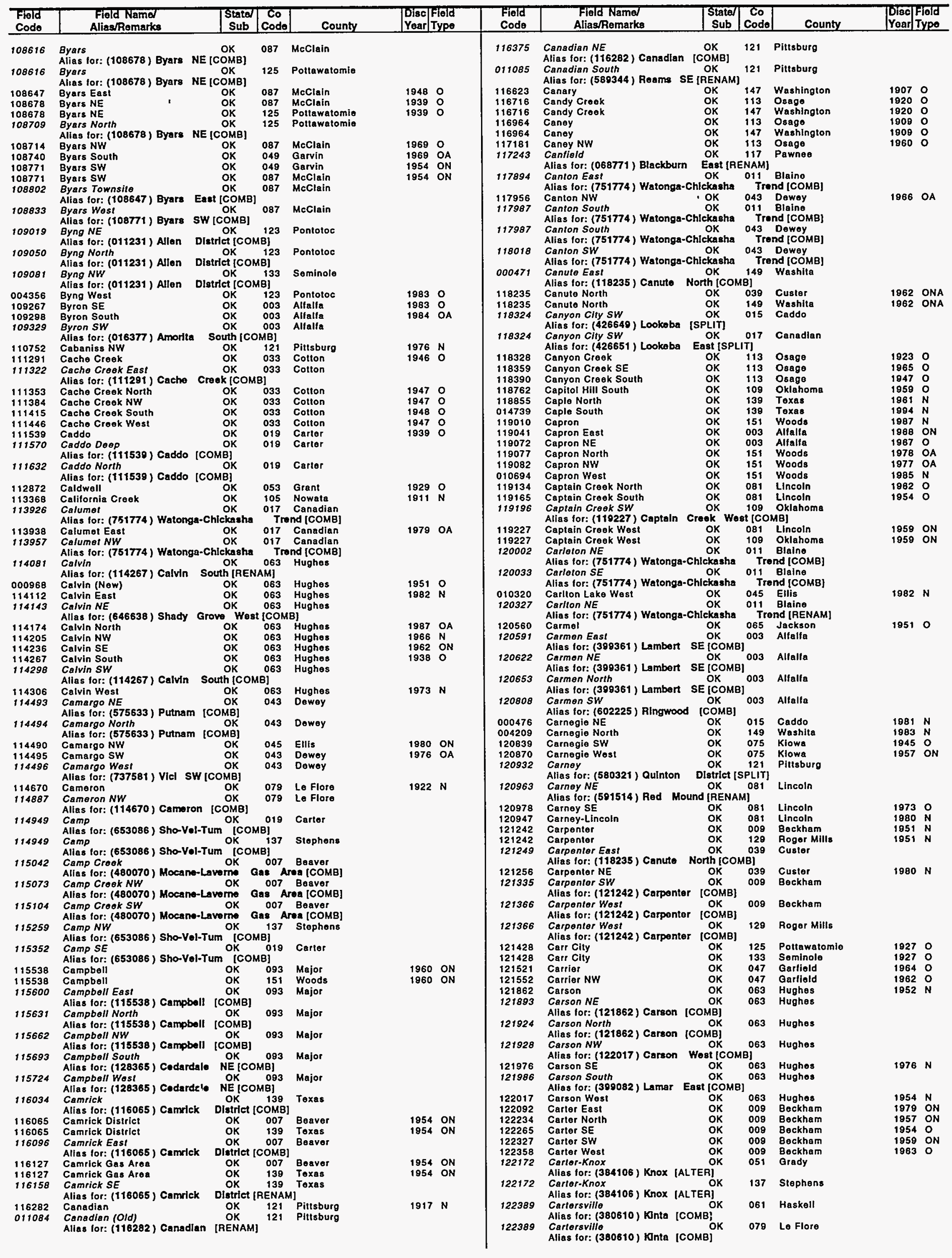


OKLAHOMA

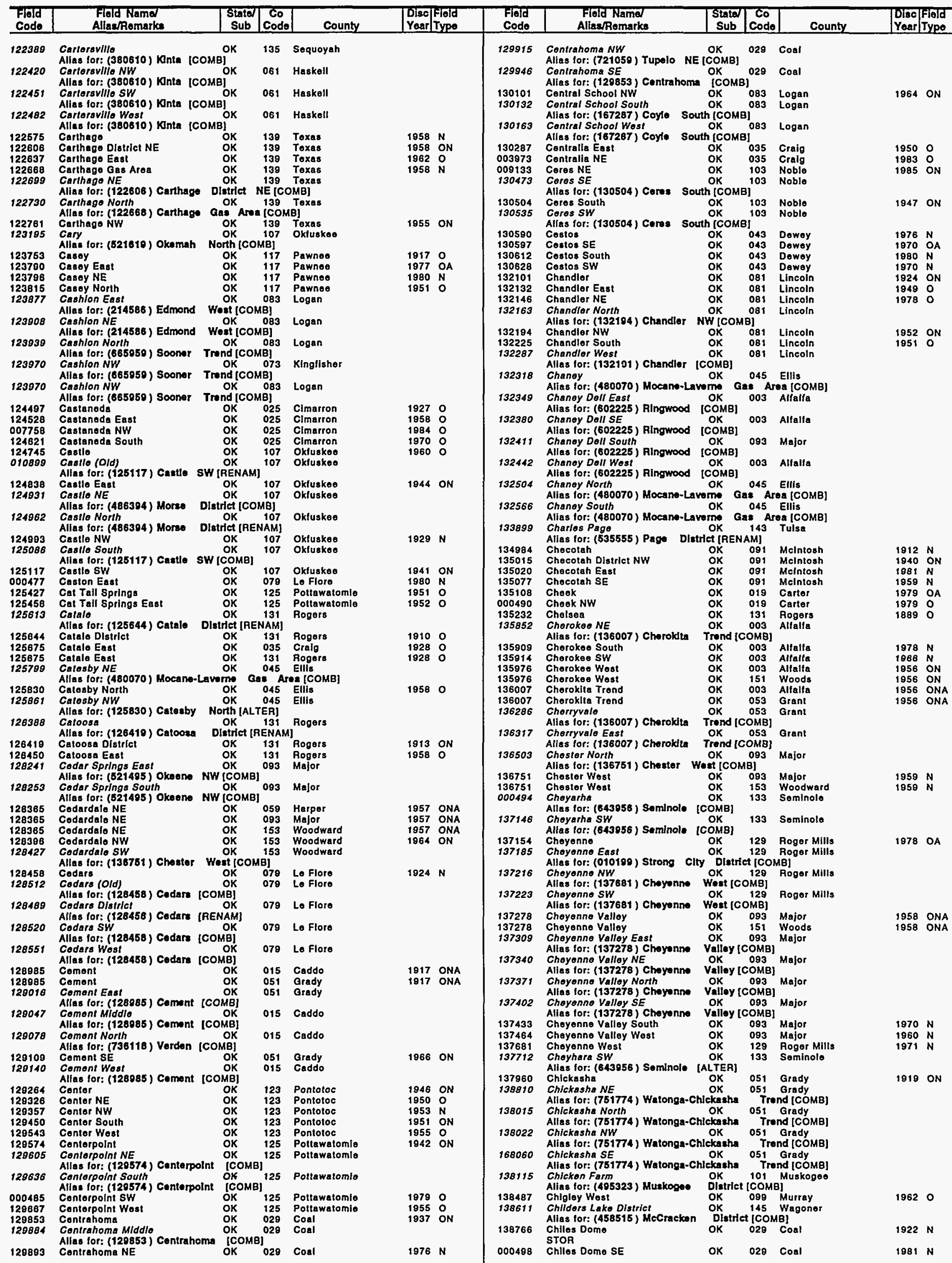


OKLAHOMA

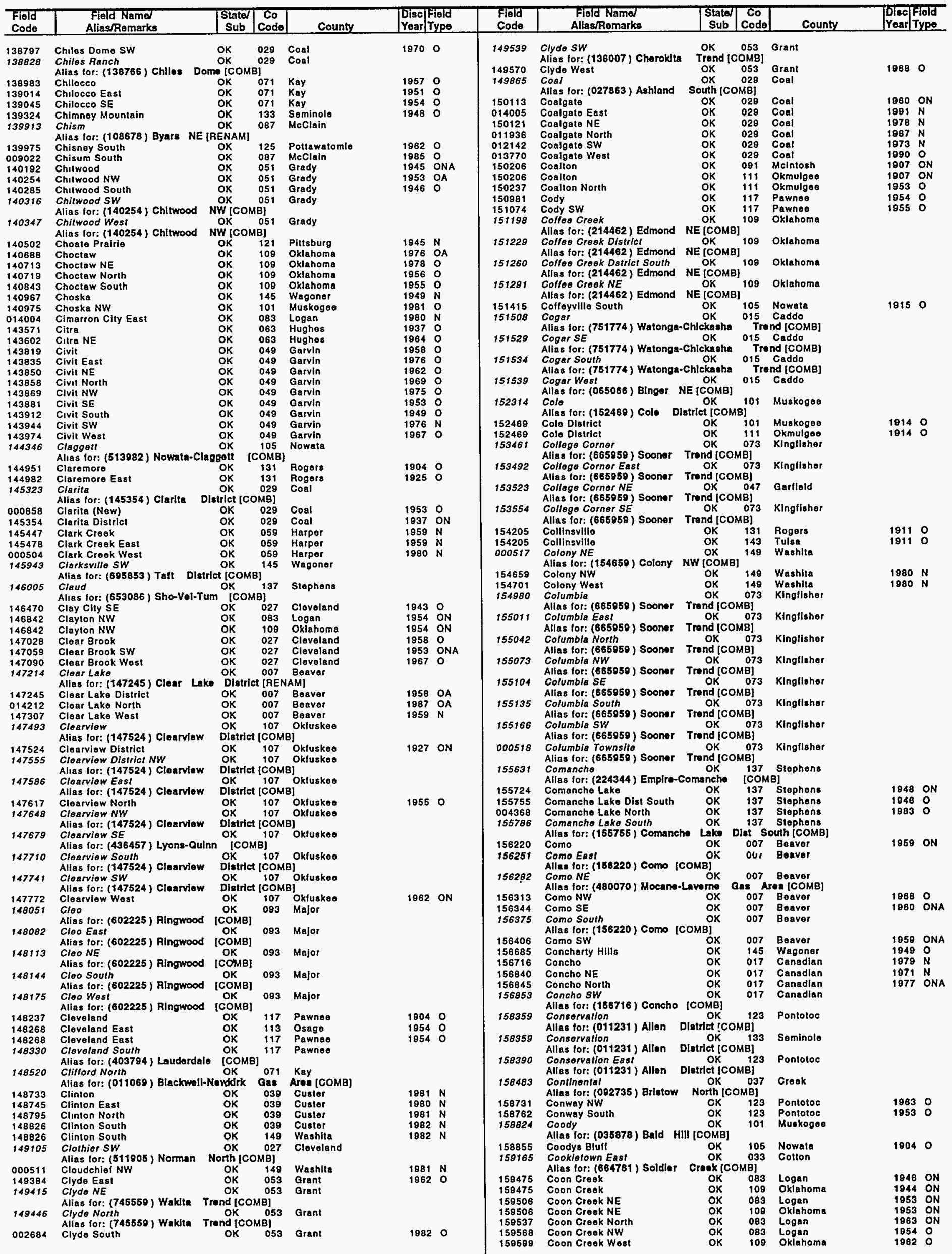


OKLAHOMA

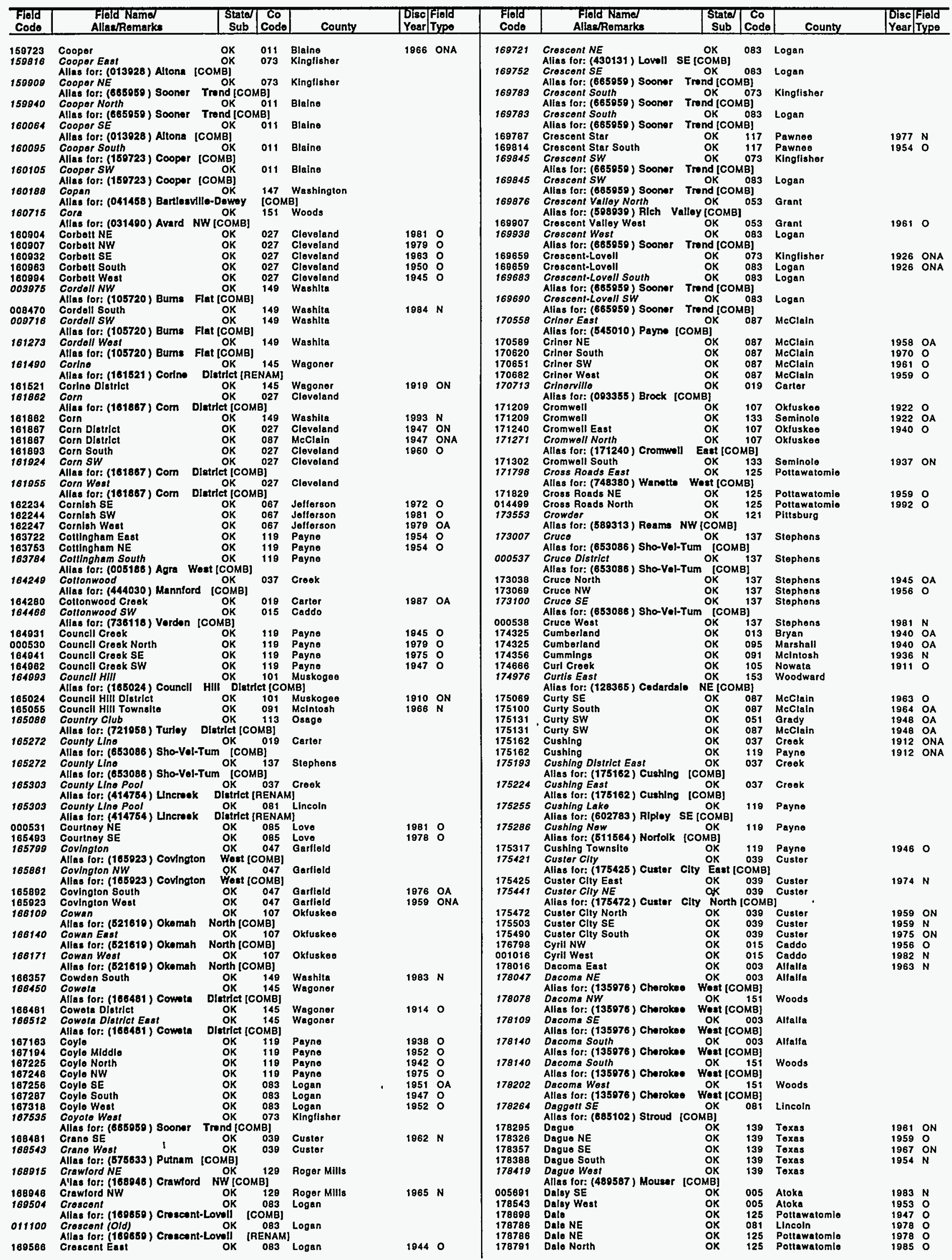


OKLAHOMA

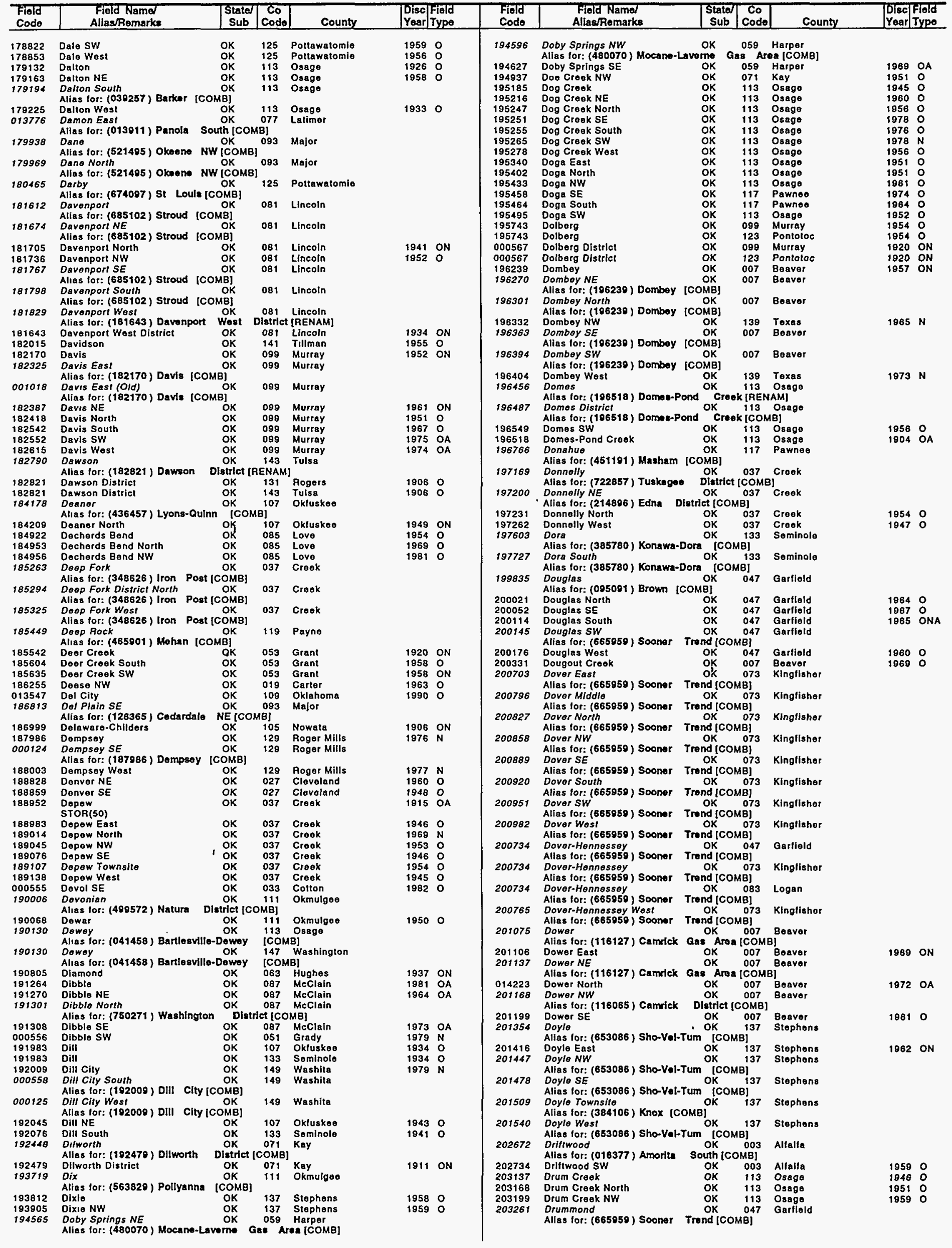


OKLAHOMA

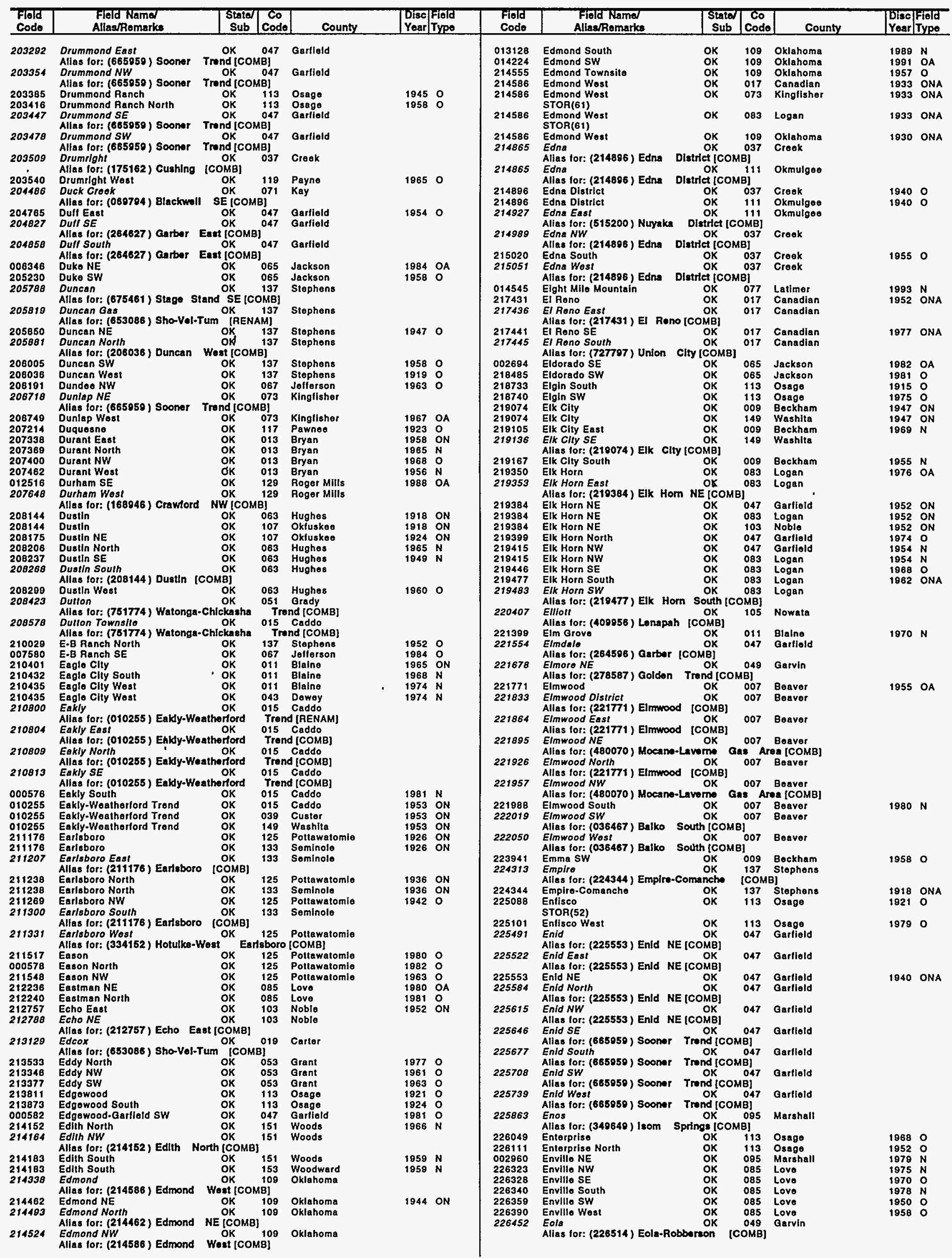


OKLAHOMA

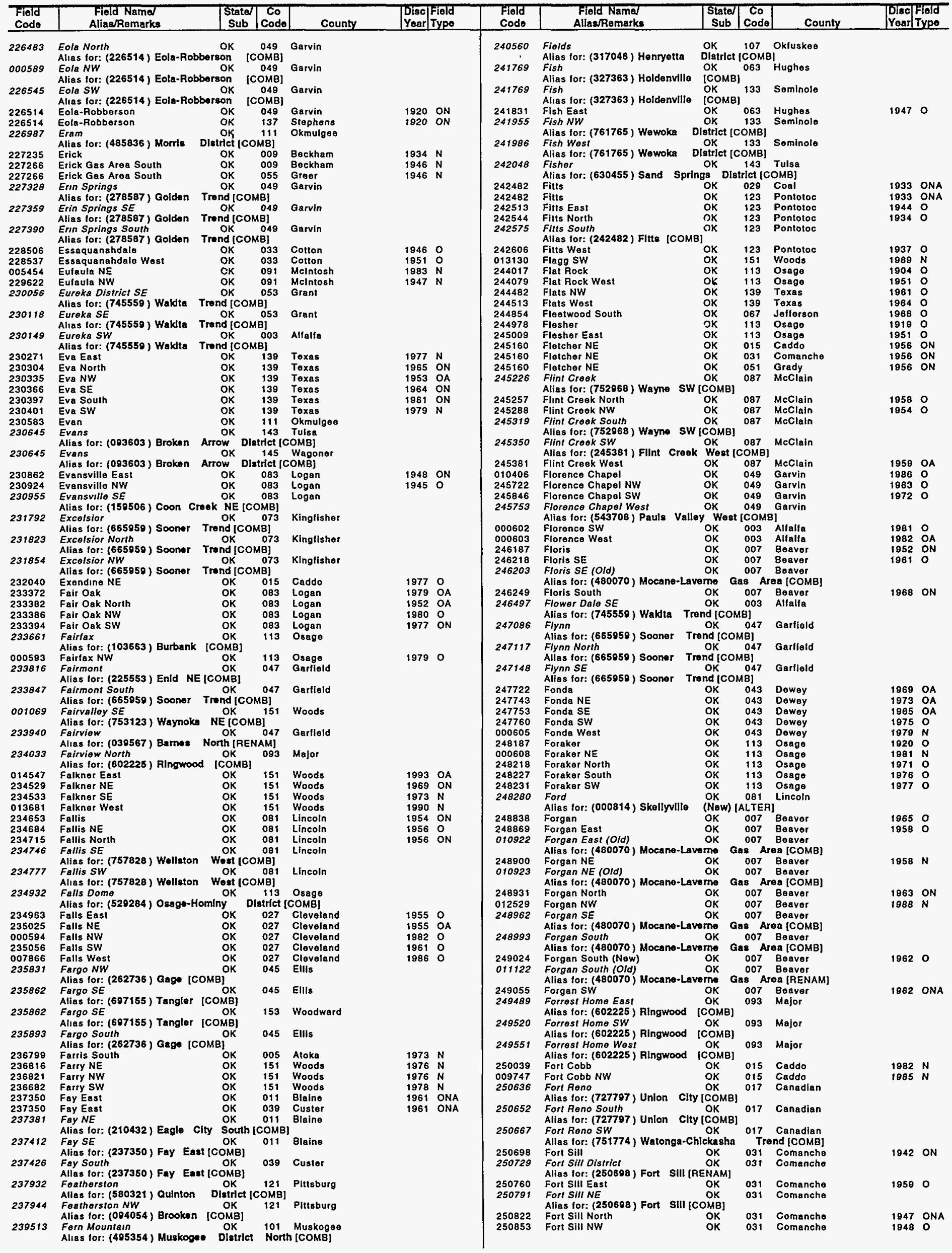


OKLAHOMA

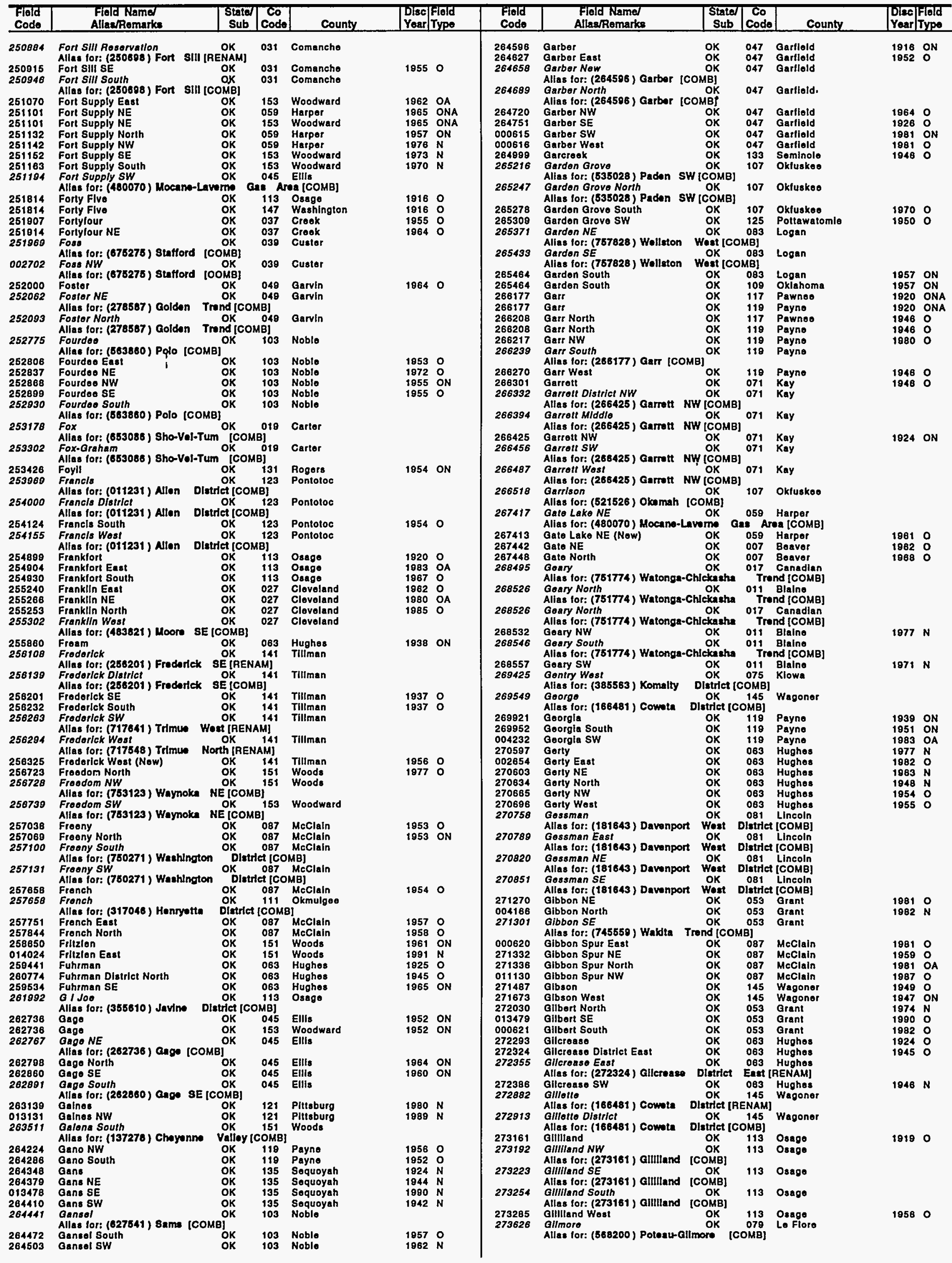




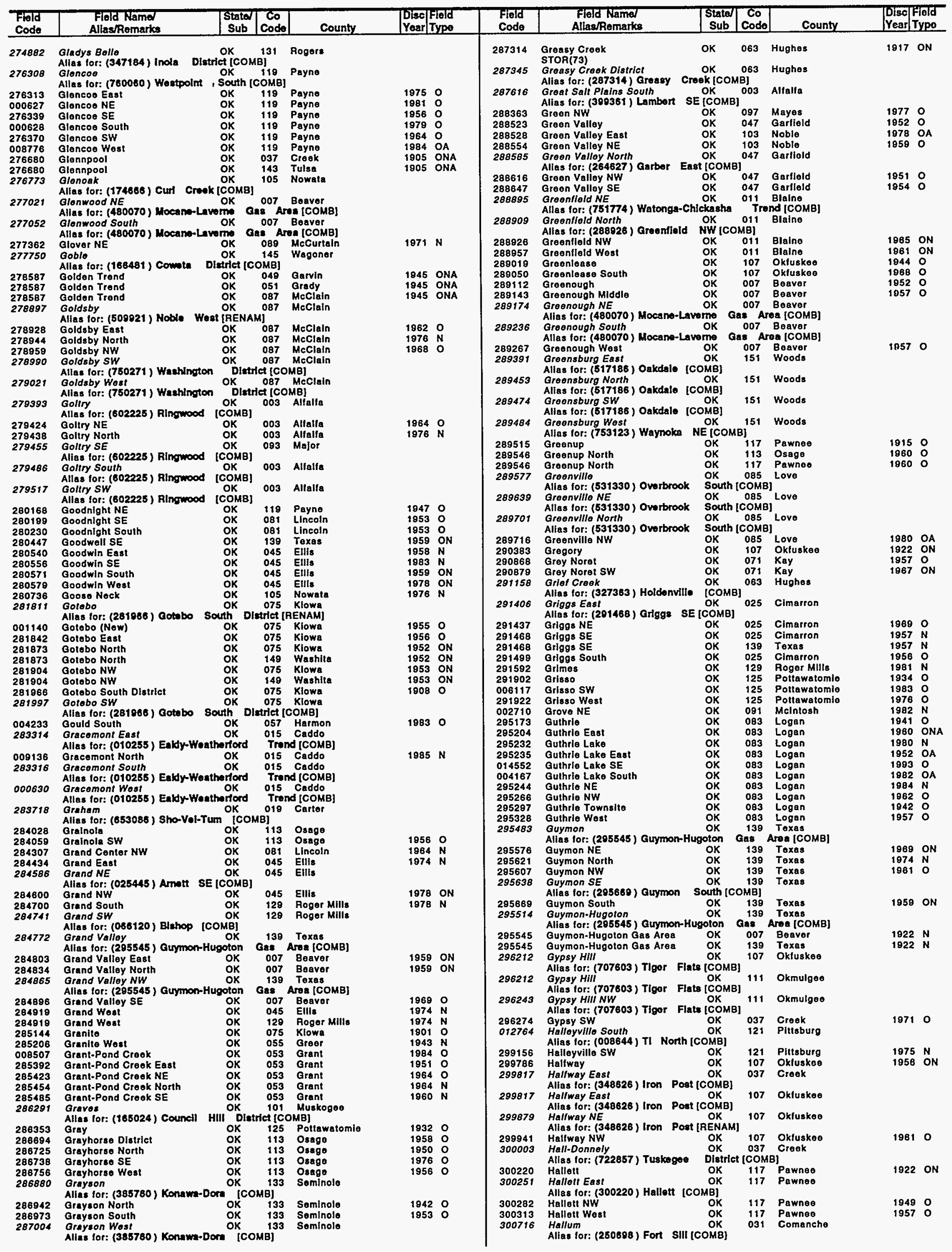


OKLAHOMA

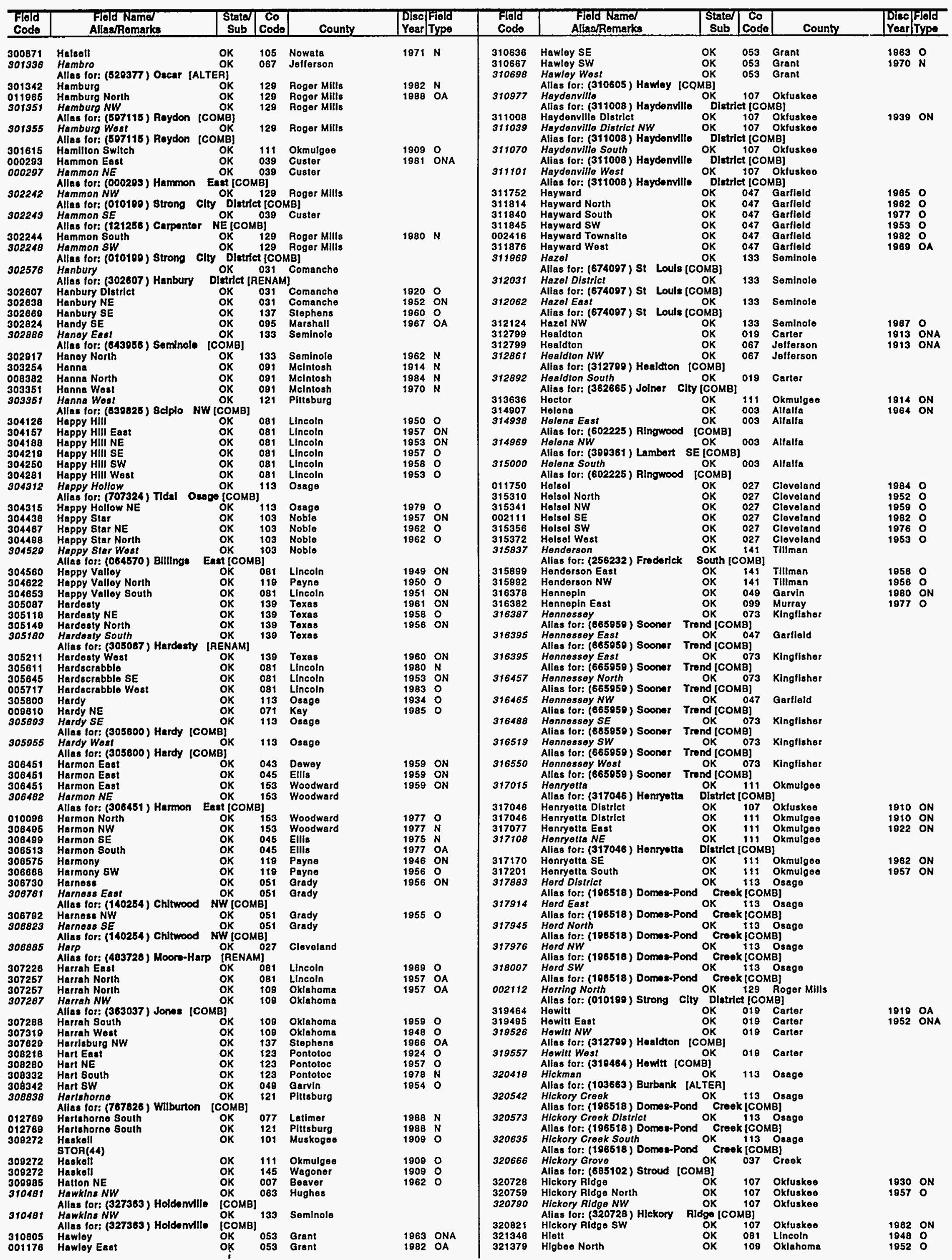




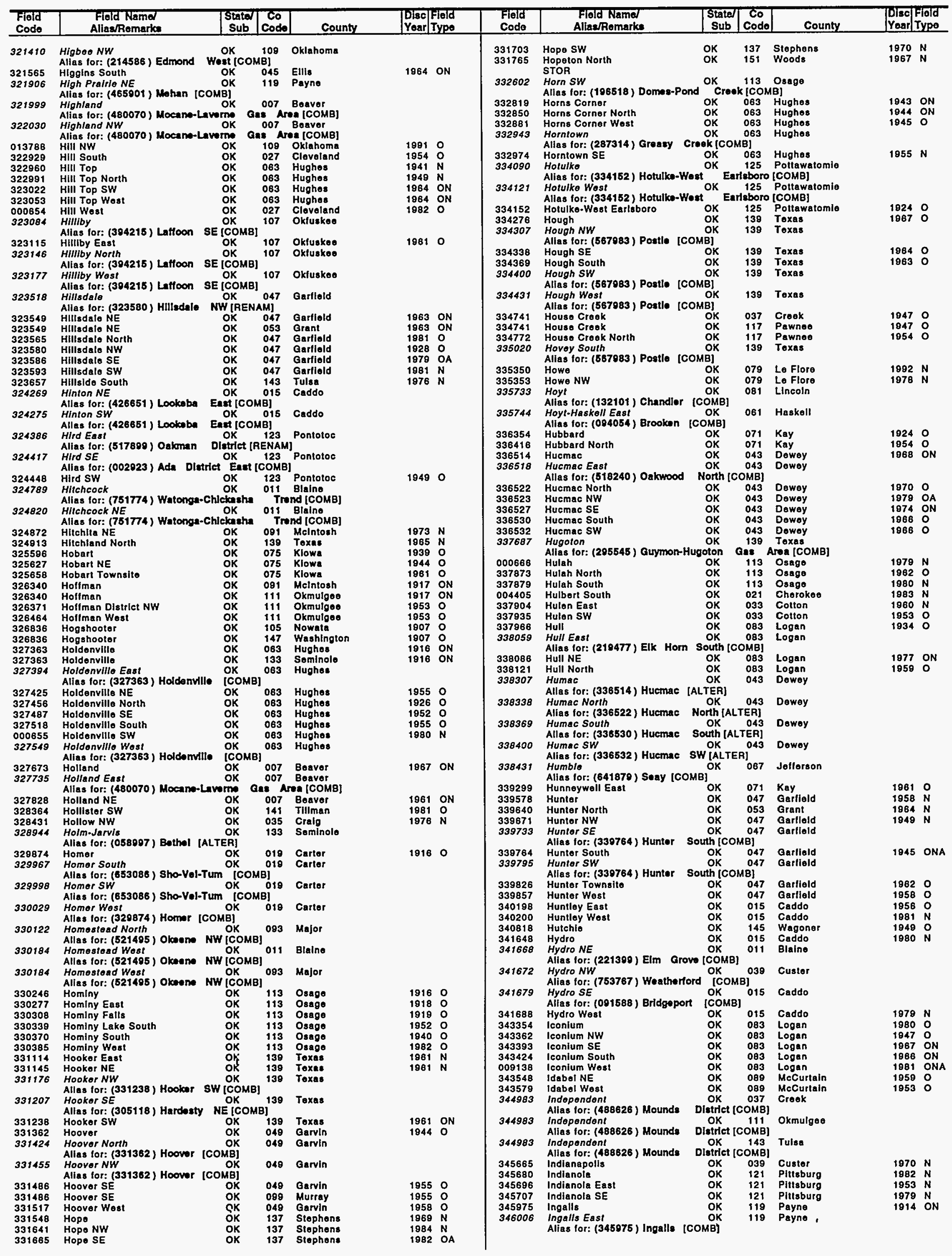


OKLAHOMA

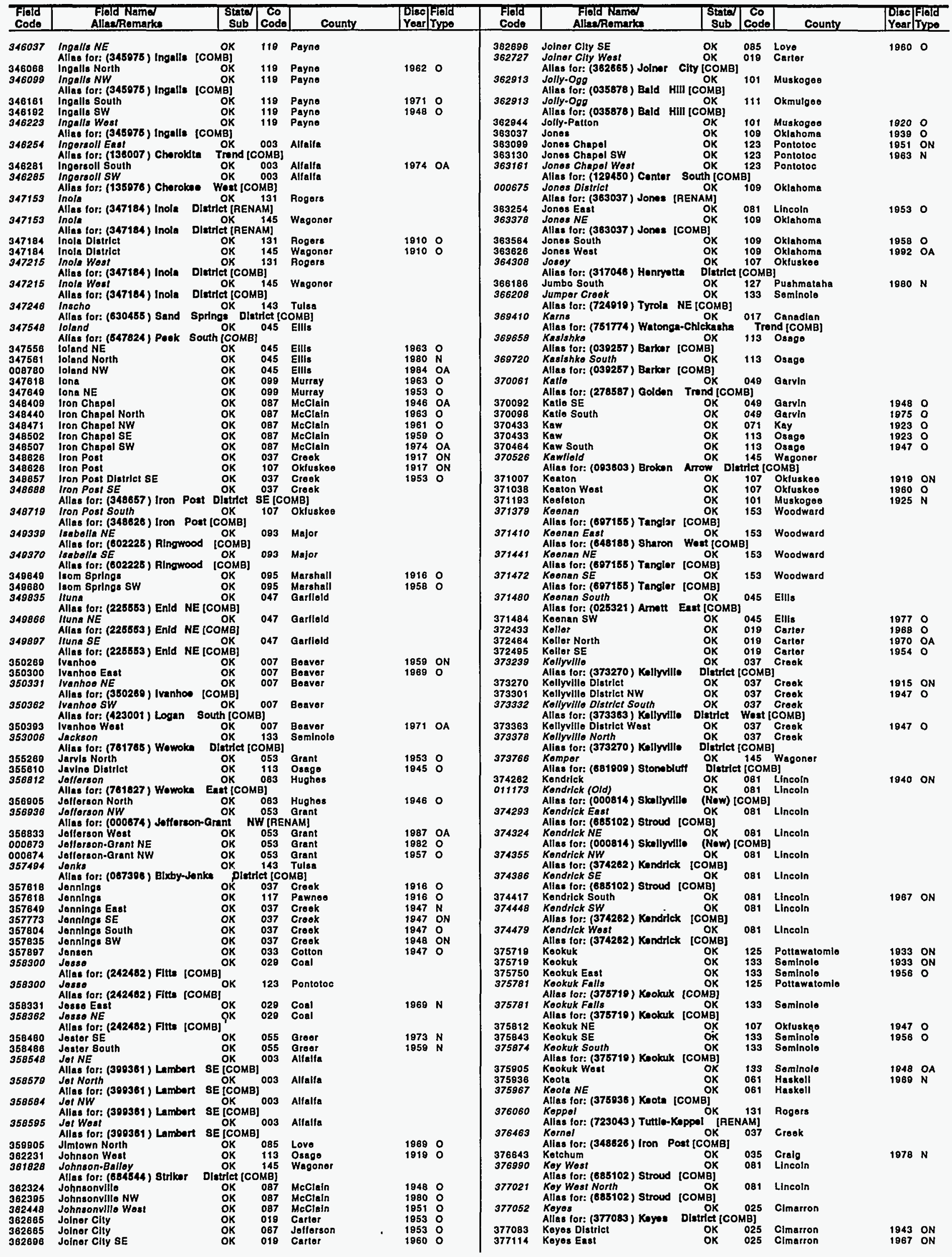




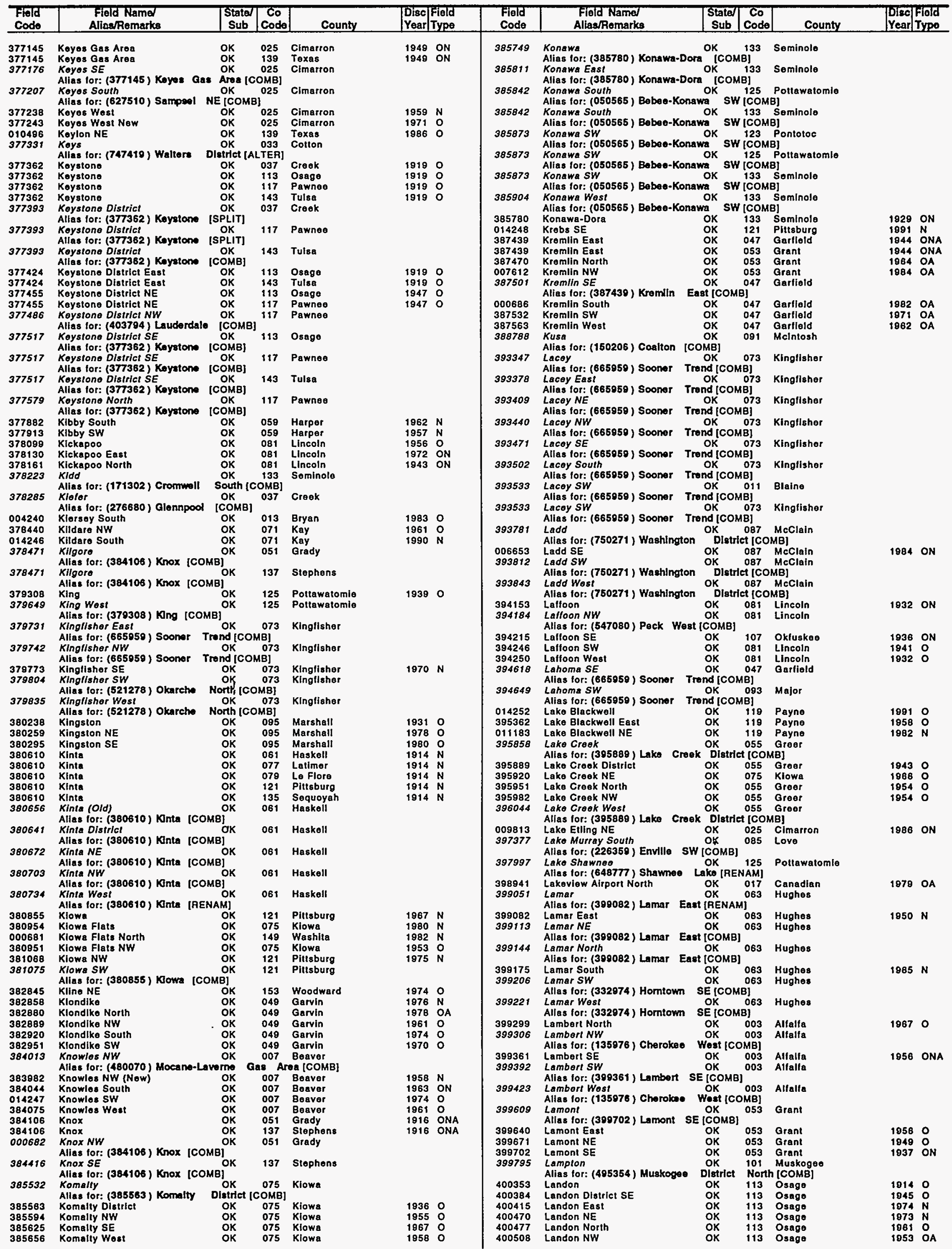


OKLAHOMA

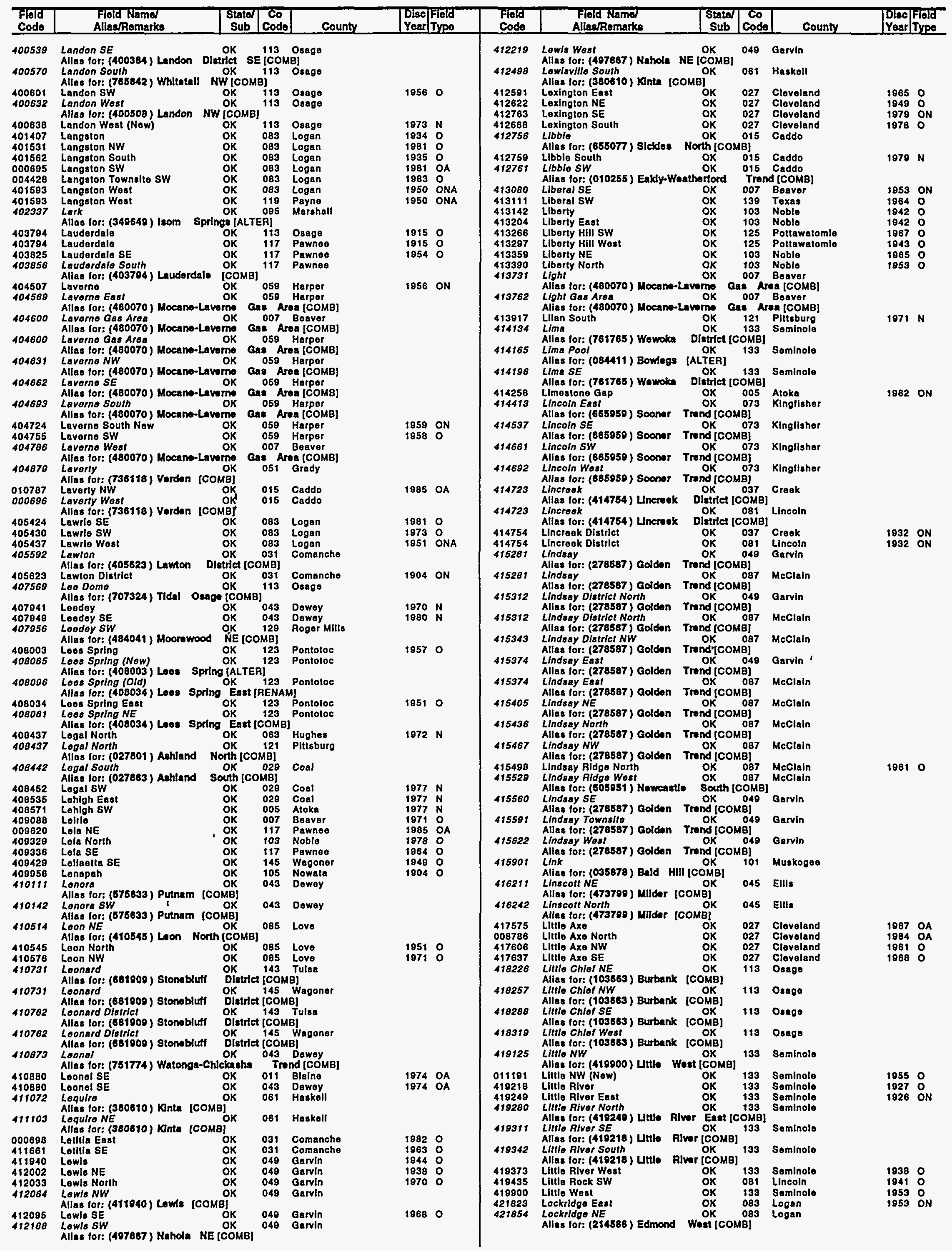




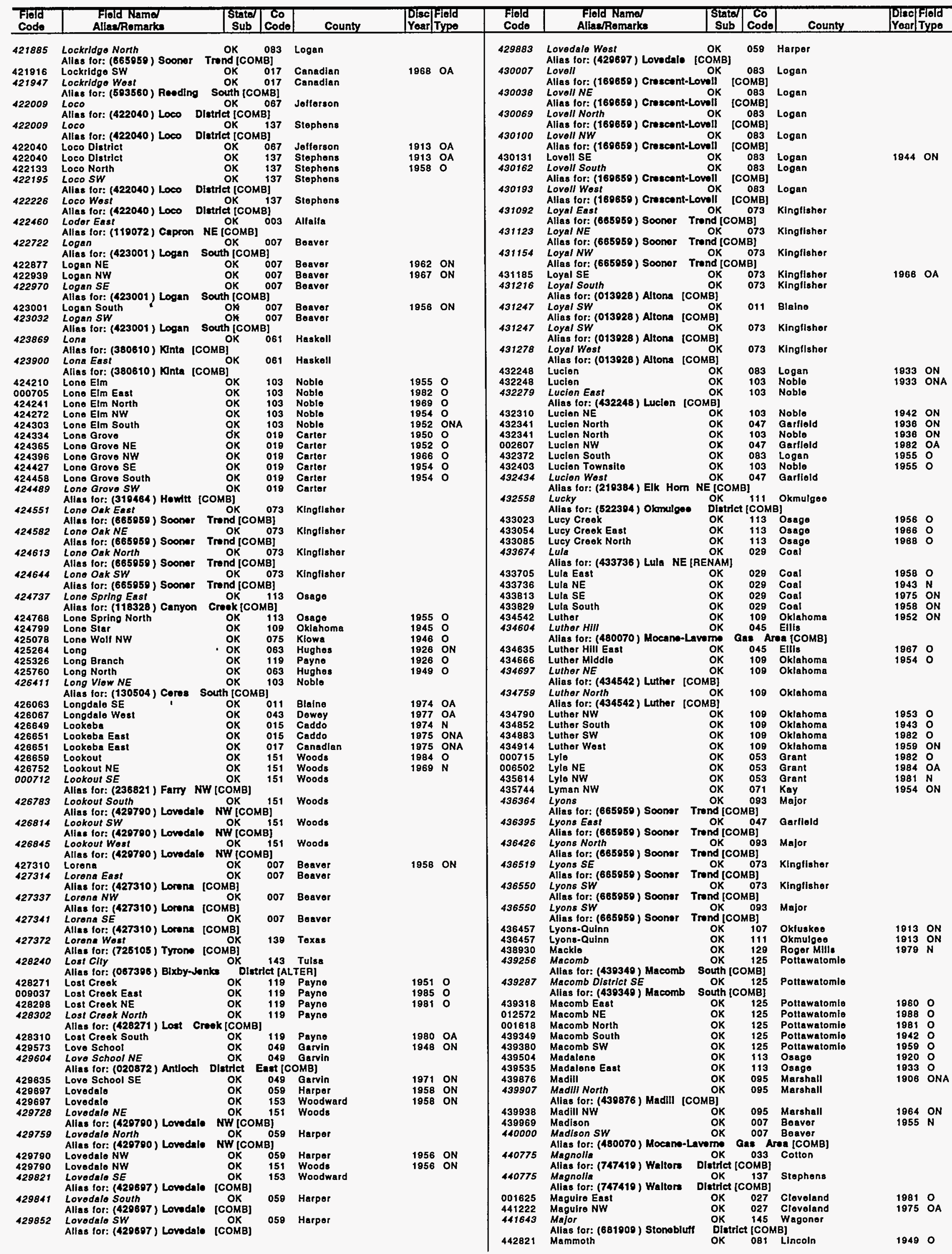


OKLAHOMA

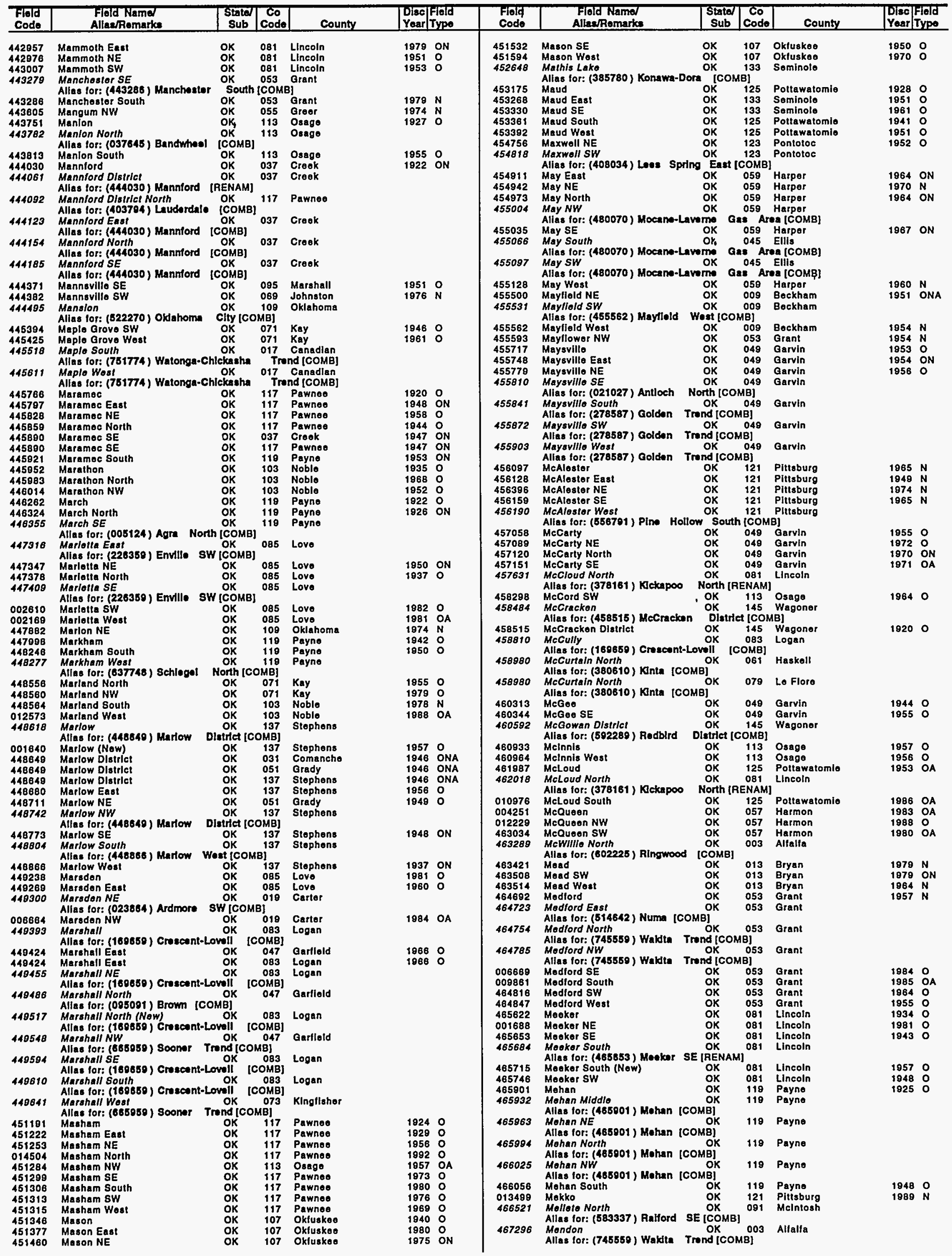




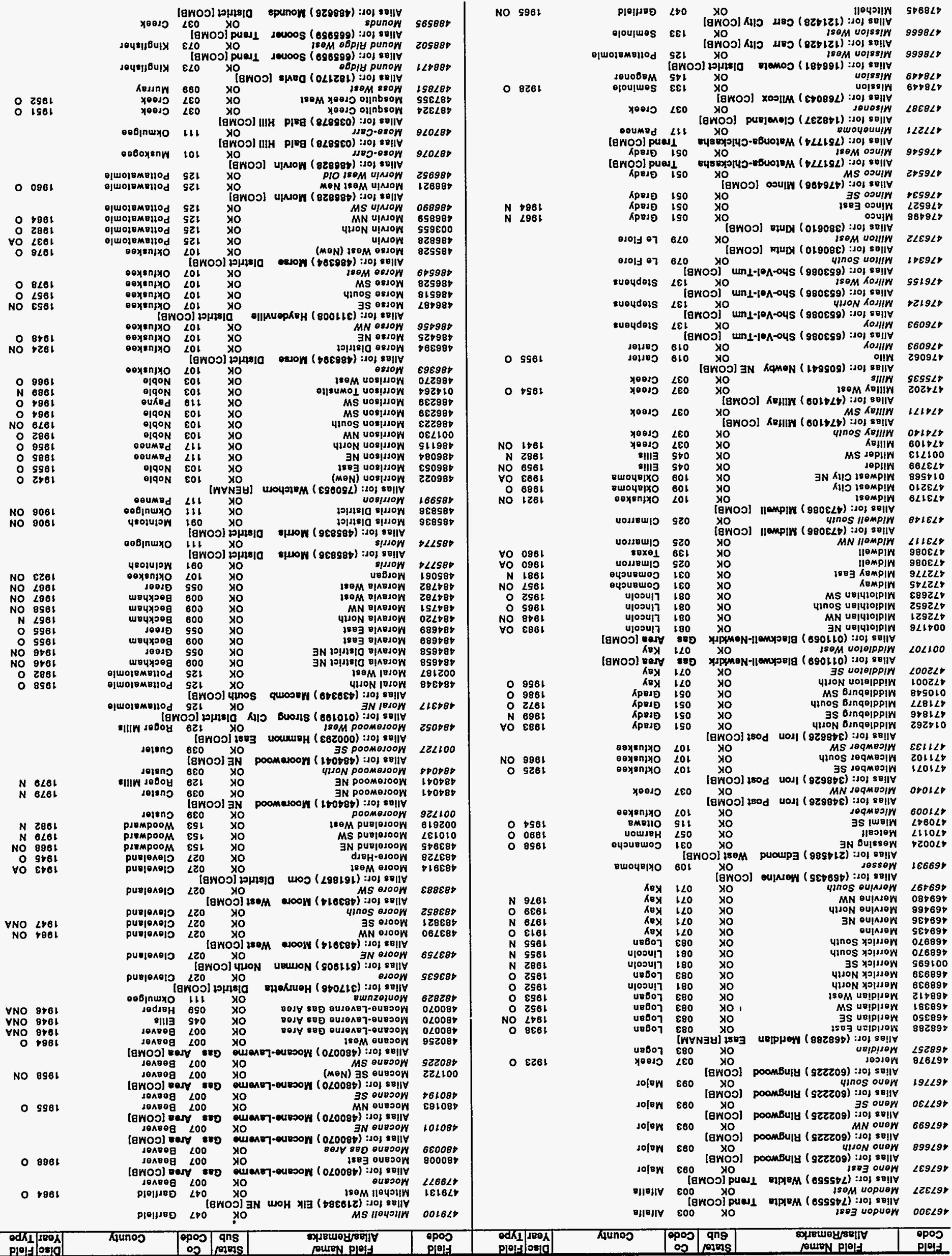


NO 626

No 916 N $Z 861$

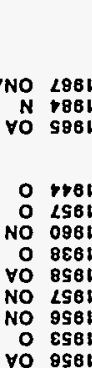

NO 628

No 898

O 658 (

098

0698

NO SOB:

$\begin{array}{ll}\text { O } & 1981 \\ 0 & \varepsilon 96 t\end{array}$

No $9 \varsigma 6$

NO ES6

$\forall 0$ 296!

$\begin{array}{ll}0 & 8181 \\ 0 & 1961\end{array}$

$\begin{array}{ll}0 & 1981 \\ 0 & 0961\end{array}$

0
0

$0+\varepsilon \theta$

$\forall O$
Y 9865

$0 \quad 9861$
$\forall 0 \quad 1 \angle 61$

N $9 \angle 8$ ! N $6 \angle 6 !$
NO $8<6 !$

O 6761

ㅇ 1961

$\begin{array}{rl}\forall 0 & 8561 \\ 0 & \$ S 61\end{array}$

O $5 s 8$

N $\angle 861$

$\begin{array}{ll}N & 2861 \\ \text { O } & \angle 161\end{array}$

$\forall \mathrm{O}$ s86

O $8 \angle 6$

$\begin{array}{ll}0 \\ 0 & 2661\end{array}$

N 6861

\begin{abstract}
solang 680

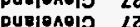

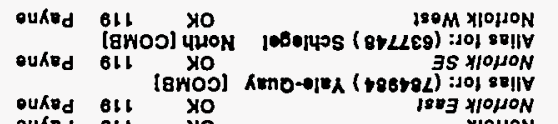

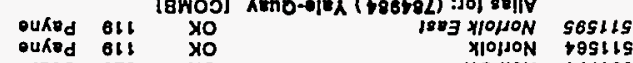

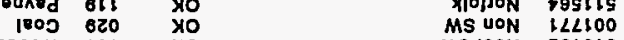
$900 M$ isi X्रO MS $100 \mathrm{~N}$

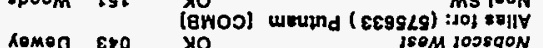

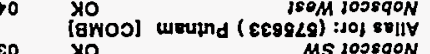
Lo1015 MS rosegON 9LOOLIS 10meO

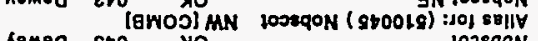

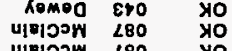

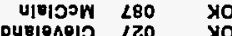

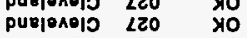

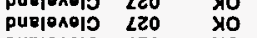

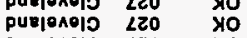
purjonojo 250 T्रO

$$
\begin{aligned}
& \text { Y्र० } \\
& \text { YXO } \\
& \hline 0
\end{aligned}
$$

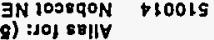
jossqON SL660S

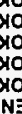

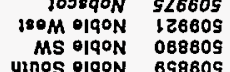
$\begin{aligned} \text { पINOS DIQDN } & 658605 \\ \text { JS OIQON } & 828605\end{aligned}$ $\begin{aligned} \text { IS OIQON } & 828605 \\ \text { MN OIQON } & \text { LEL60S }\end{aligned}$ $\begin{array}{rr}M N \text { OIवON } & \text { LBL6OS } \\ \text { YION OIQON } & 99 \angle 60 S\end{array}$ $\exists N$ OIOON $\$ 0 \angle 60 S$ (MON) OIQON SELBOS

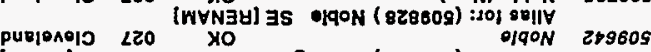
(EพOO) pued

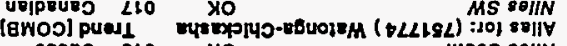
(8w0ol pue!

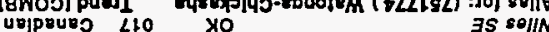

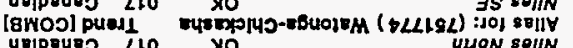
up|paues L10 Xo

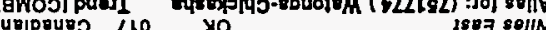
$80 r 805$

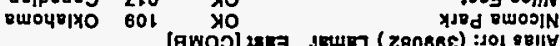
668805

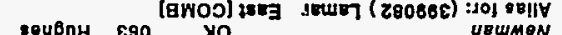

AOX 690 त्रO

$180 \mathrm{M}$ XIYMMON SBE9OS

AEX 1200 X्र०

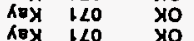

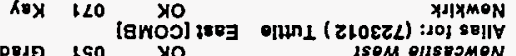

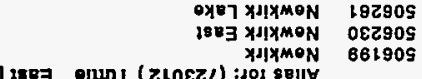

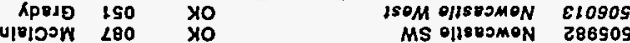

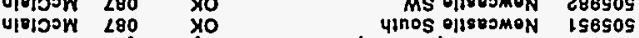

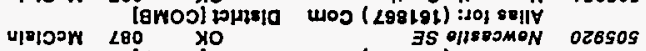

[EmoJ] panaja unos ( L9819L) :

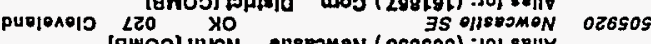

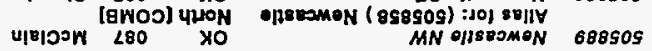
पाए100W 280 त्रO

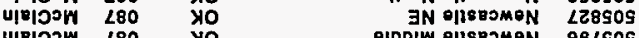

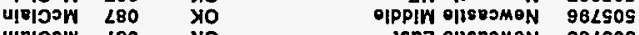

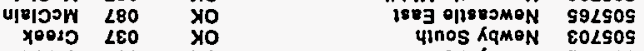
Y0015 LEO त्रo

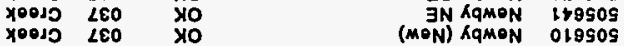

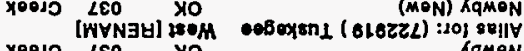
Yoojo 2 OO ro

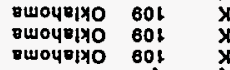
x० $180 M$ IIIOMON 6LSSOS

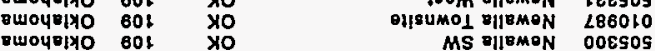

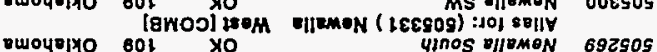

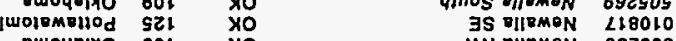

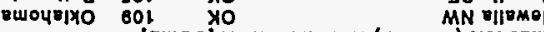

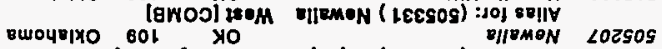

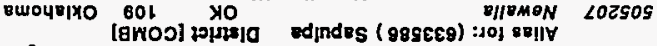

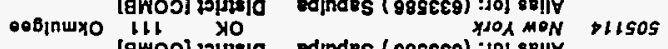

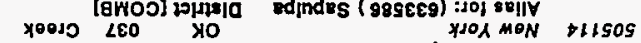

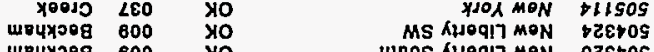
шह४

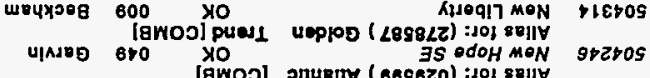
-6eso El/ X्र०

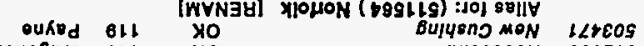

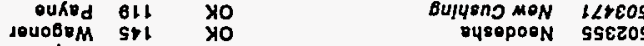

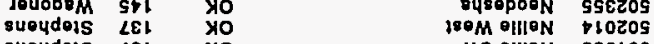
suoudols $\angle E L$ Y्रO MS PIIION E8BLOS

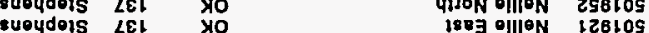

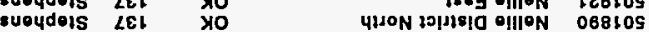
ISTOO un

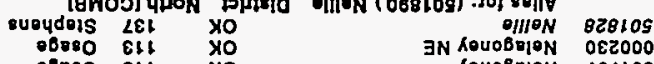
OBV8O ELI X XO ROUOBVION L6LLOS

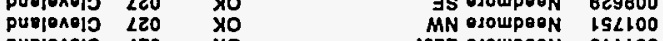

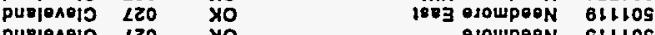

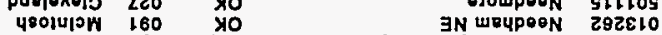

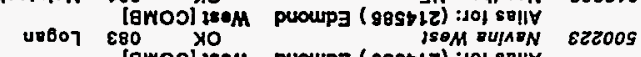

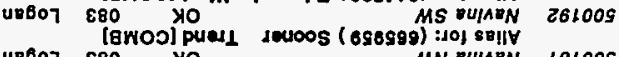

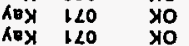

$\begin{array}{lll}x \in X & 1<0 \\ x \in X & 1<0\end{array}$

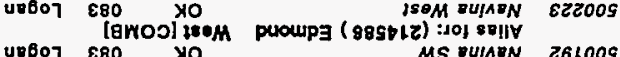

10101

(acos

- 8E6!

- 2981

O 8Z81

NO $\begin{array}{r}0 \\ \text { N }\end{array}$

No 8961

$\forall 0 \$ 86 !$

$\forall 0$ $9<61$

N 2981

$\begin{array}{lll}N & 2961 \\ O & 1961\end{array}$

$\begin{array}{ll}\text { O } & 1961 \\ \mathrm{O} & 6861\end{array}$

YNO 088 เ

O S581

$\begin{array}{ll}0 & 9161 \\ N & 0881\end{array}$

N
$\forall O 88$ ' $9 \angle 6 !$

No E96!

No $896 !$

N 286 b

$\forall O$ LL6!

$\forall N O$ ZL6!

$\mathrm{N} \mathrm{OL6b}$

NO 9561

NO 9561

NO 9561

NO $1 \angle 6 !$

O 0581

NO 2961

N E86!

$\begin{array}{rr}\text { O } & 9061 \\ \text { NO } & \$ 681\end{array}$

N 0861

- SS6!

O 896

o 8561

$\begin{array}{rl}\forall O & L<8 ! \\ 0 & 6961\end{array}$

VO $6 \angle 61$

$\begin{array}{ll}0 & 2961 \\ 0 & 6961\end{array}$

o SG6

0 sक61

0 8\$8।

No \$961

O 5961

NO 2961

N 1981

N 9L6!

$N$
$N$ 8961

$N>961$

$\begin{array}{cc}N O & 19 B 1 \\ N & 08 B 1\end{array}$

- $6 \$ 6 t$

No 1581

NO 806!

NO 8081

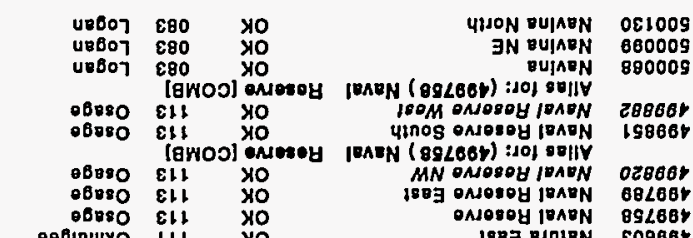

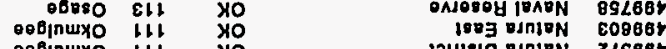

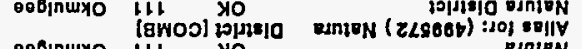

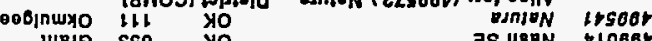

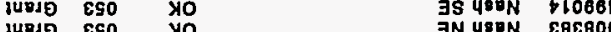

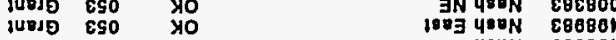$$
\text { jüg ESO Y्रO }
$$$$
\text { AEY } 1 \angle 0 \text { Y्रO UIJON UJPJON LOLBOH }
$$

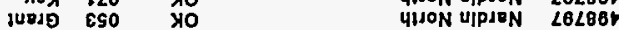

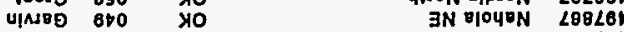

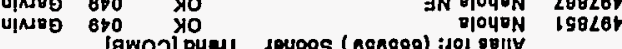

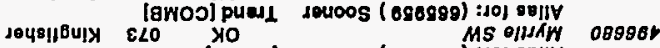

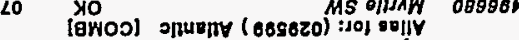$$
\text { OBвsO Elt Y्र० }
$$$$
\text { oB890 EL X Y X }
$$

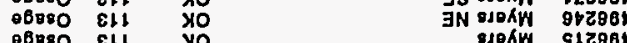

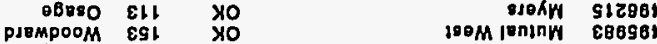

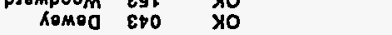

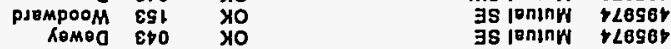$$
\text { PJемроOM ESI Y्रO }
$$$$
\text { PJEMPOOM ESI Y्रO }
$$

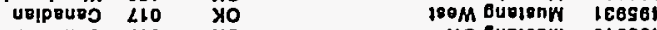

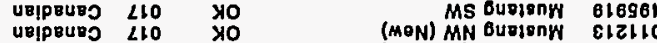$$
\text { [WVNBU] 4LON BUmBnm ( }
$$

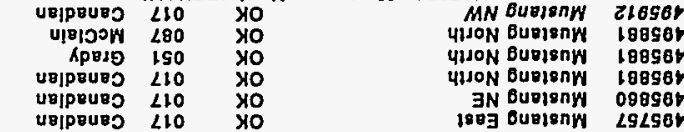

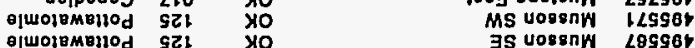

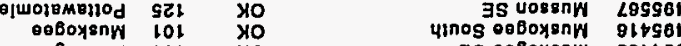

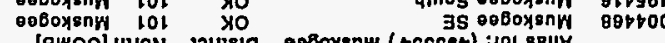

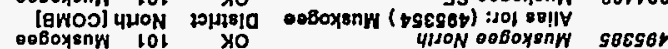

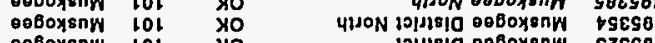$$
\text { 00Boग्रsn } 101 \text { Y. }
$$

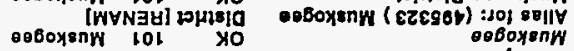

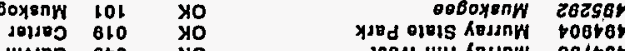

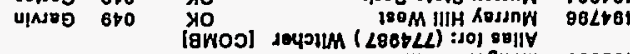$$
\text { вwо4в:10 } 601 \text { Y्र० }
$$

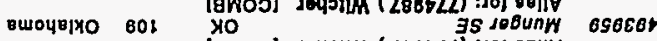

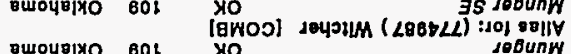

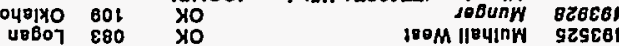$$
\text { uBBo7 } 880 \text { X0 }
$$$$
\text { uB607 } 880 \text { XO }
$$

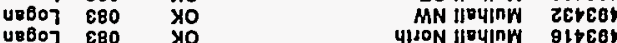

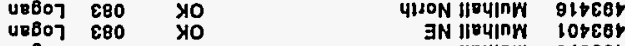$$
\text { U8B०7 } 880 \text { X्र० }
$$

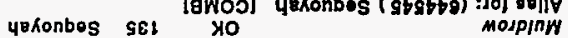

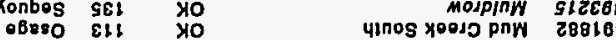

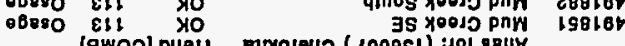

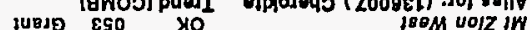$$
\text { IU8נo हSO X्र० }
$$

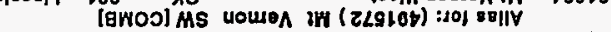

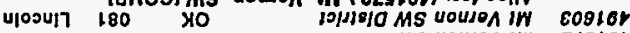

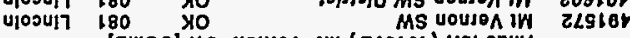

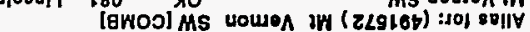

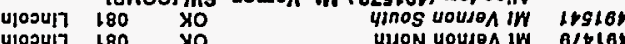$$
\text { Ulo⿰u口7 } 180 \text { X्रO }
$$$$
\text { Uloouin } 180 \text { X्रo } 3 N \text { vouson iN }
$$

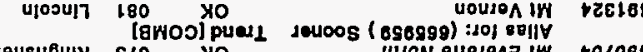

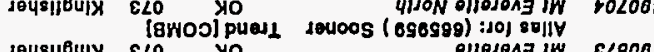

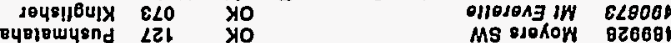

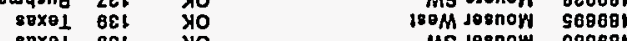

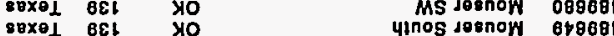$$
\text { soxe1 6E1 }
$$

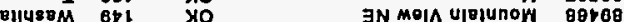

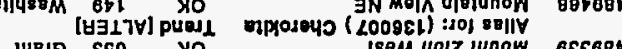$$
\text { IUEJO ESO YXO JSOM UOIZ IUNOH OEE68\% }
$$

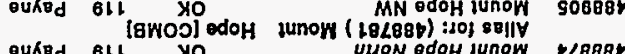

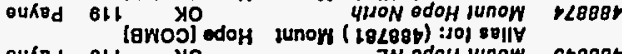$$
\text { oukid 611 Y्रO }
$$

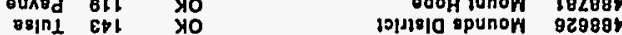

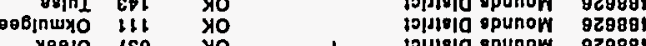$$
\text { yo010 L80 Y्र० }
$$

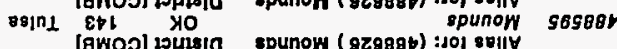

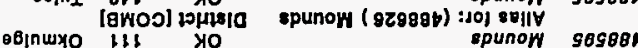

UDBO7 EBO YO MN BU/AEN 191005 
OKLAHOMA

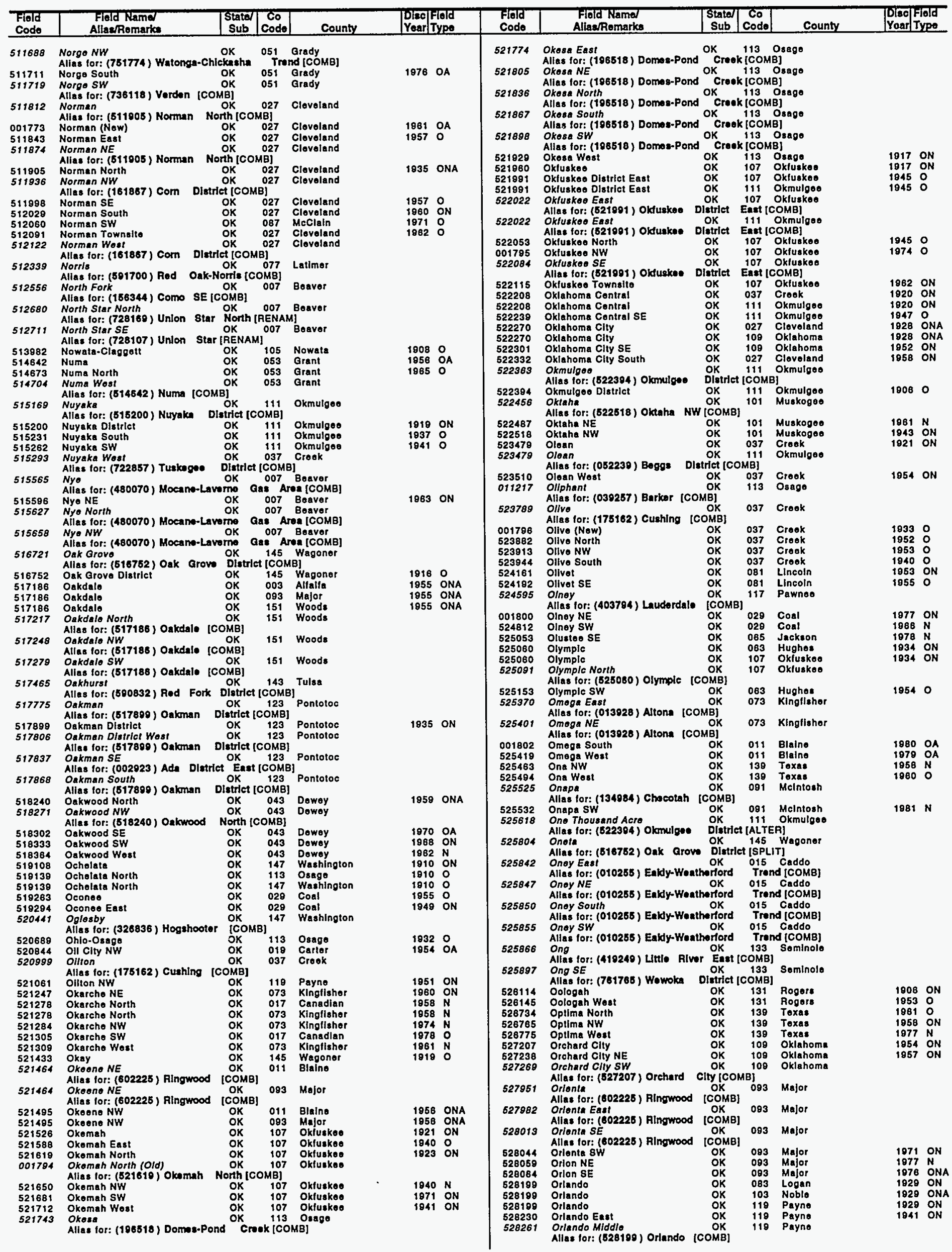


OKLAHOMA

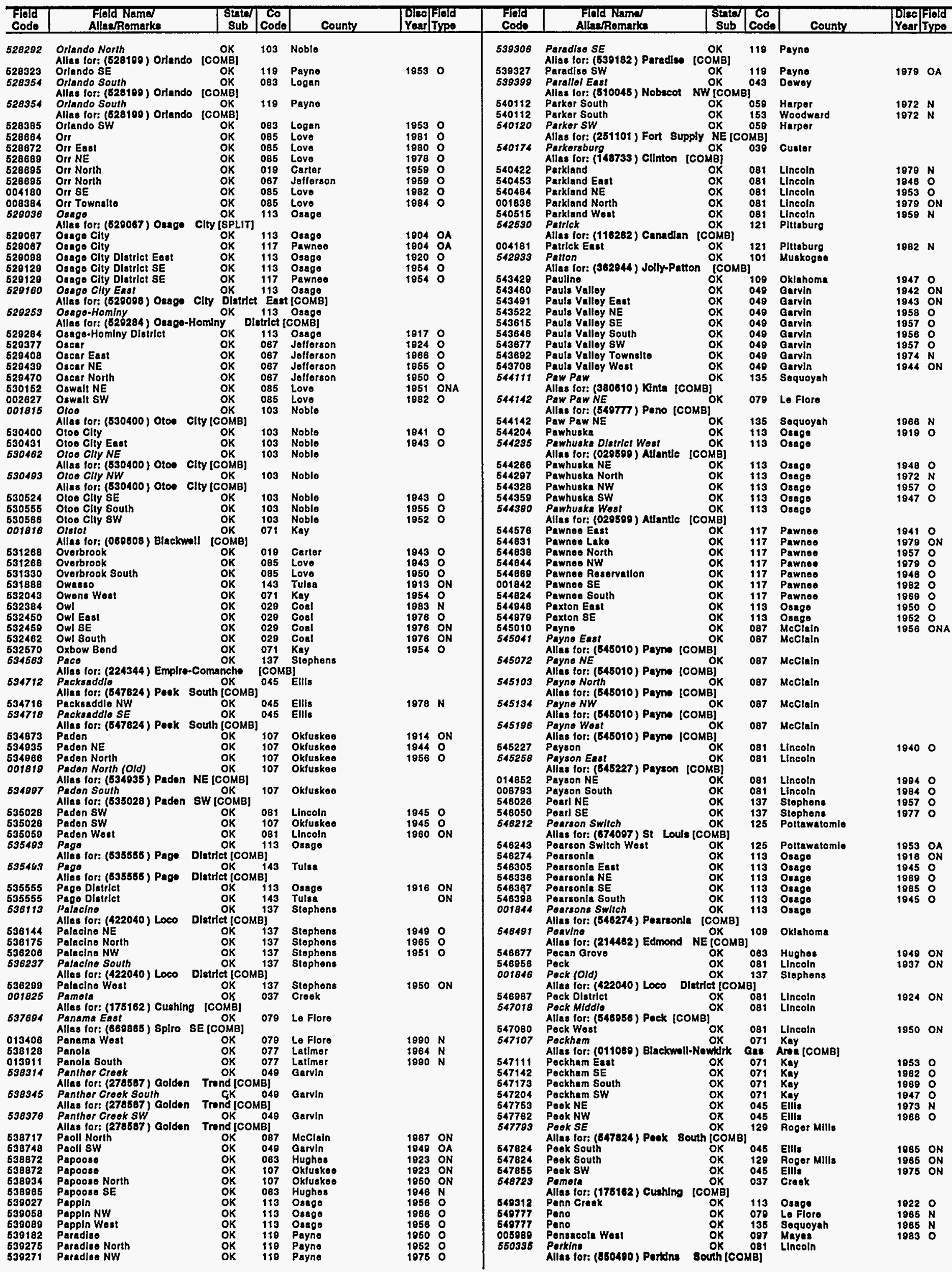


OKLAHOMA

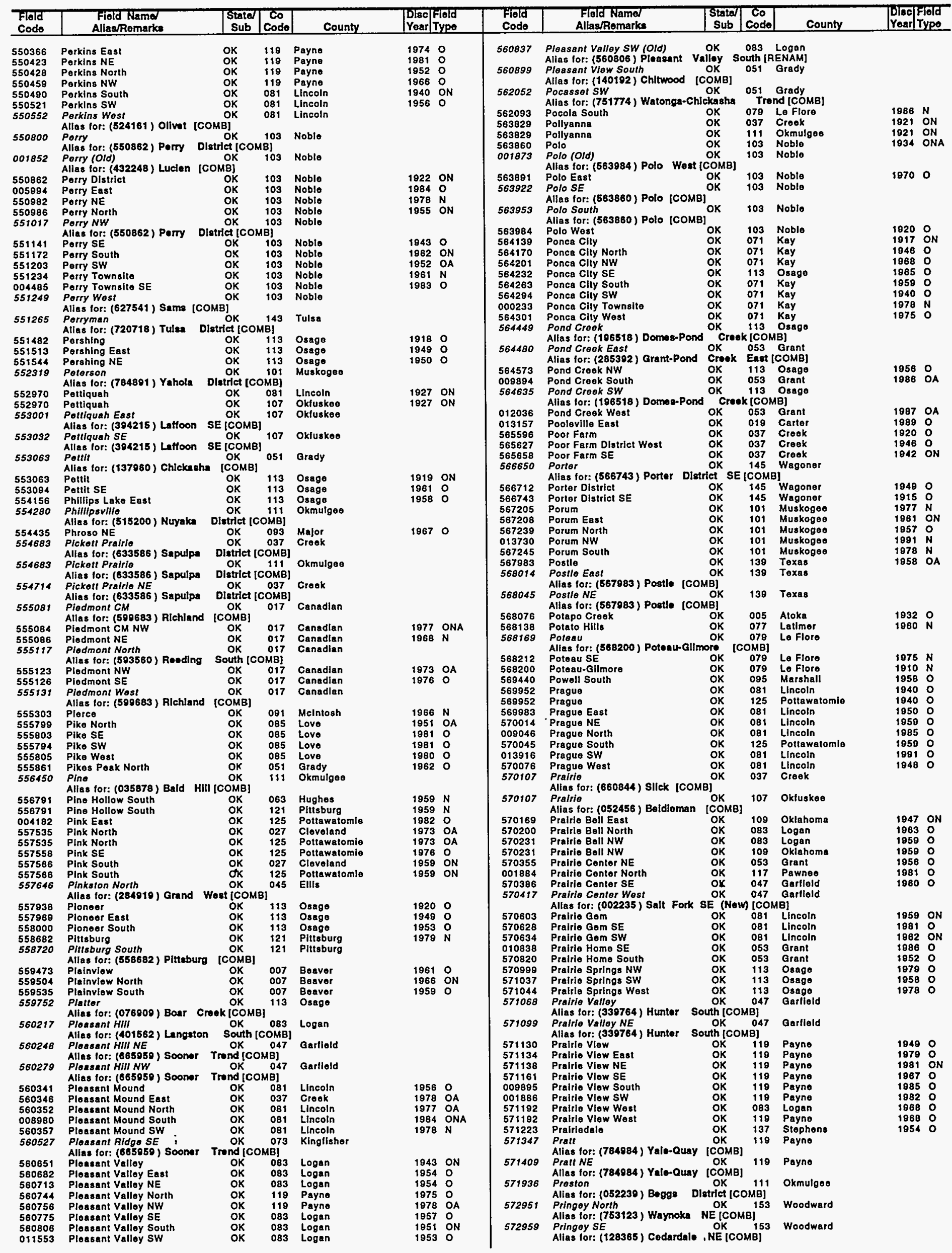


OKLAHOMA

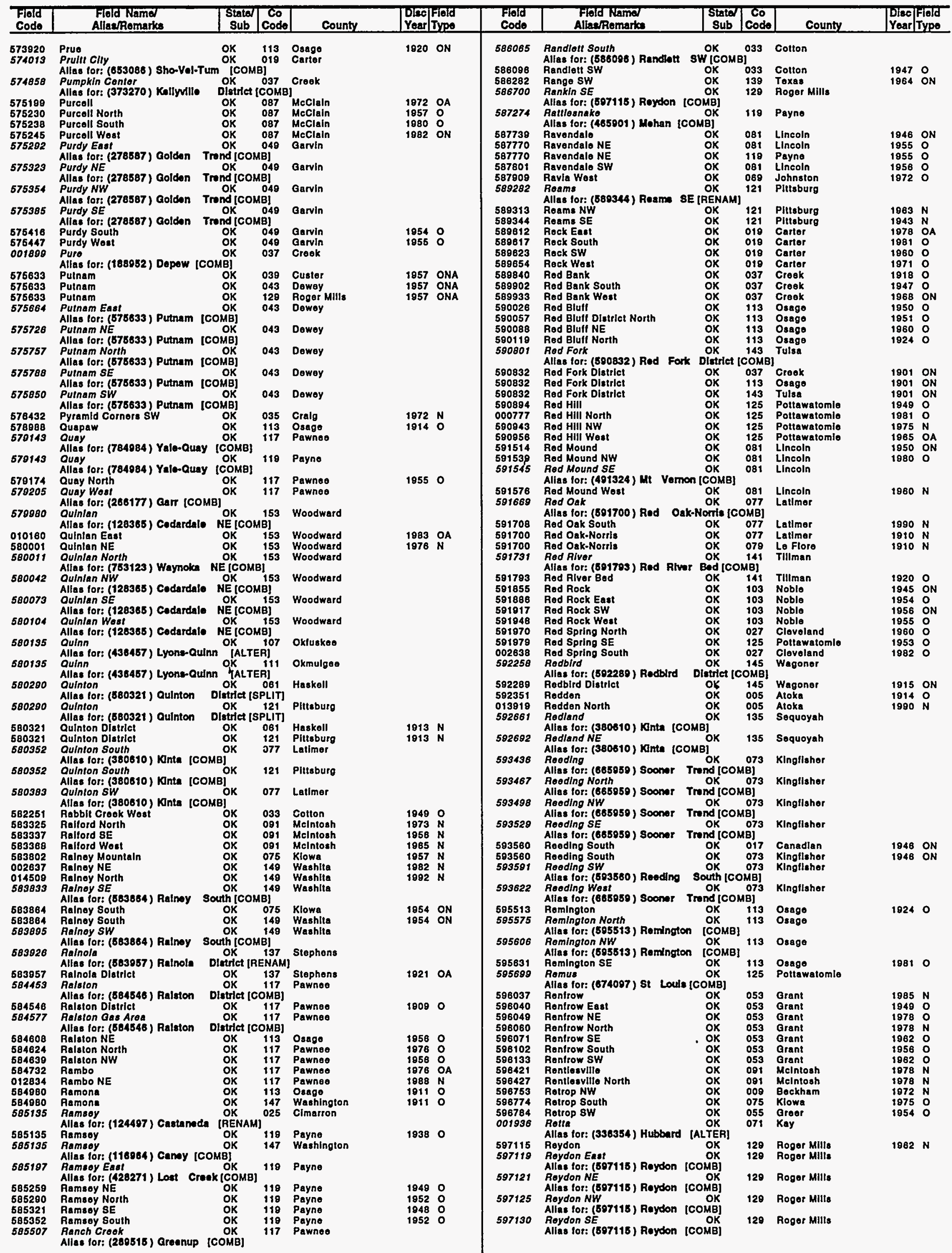




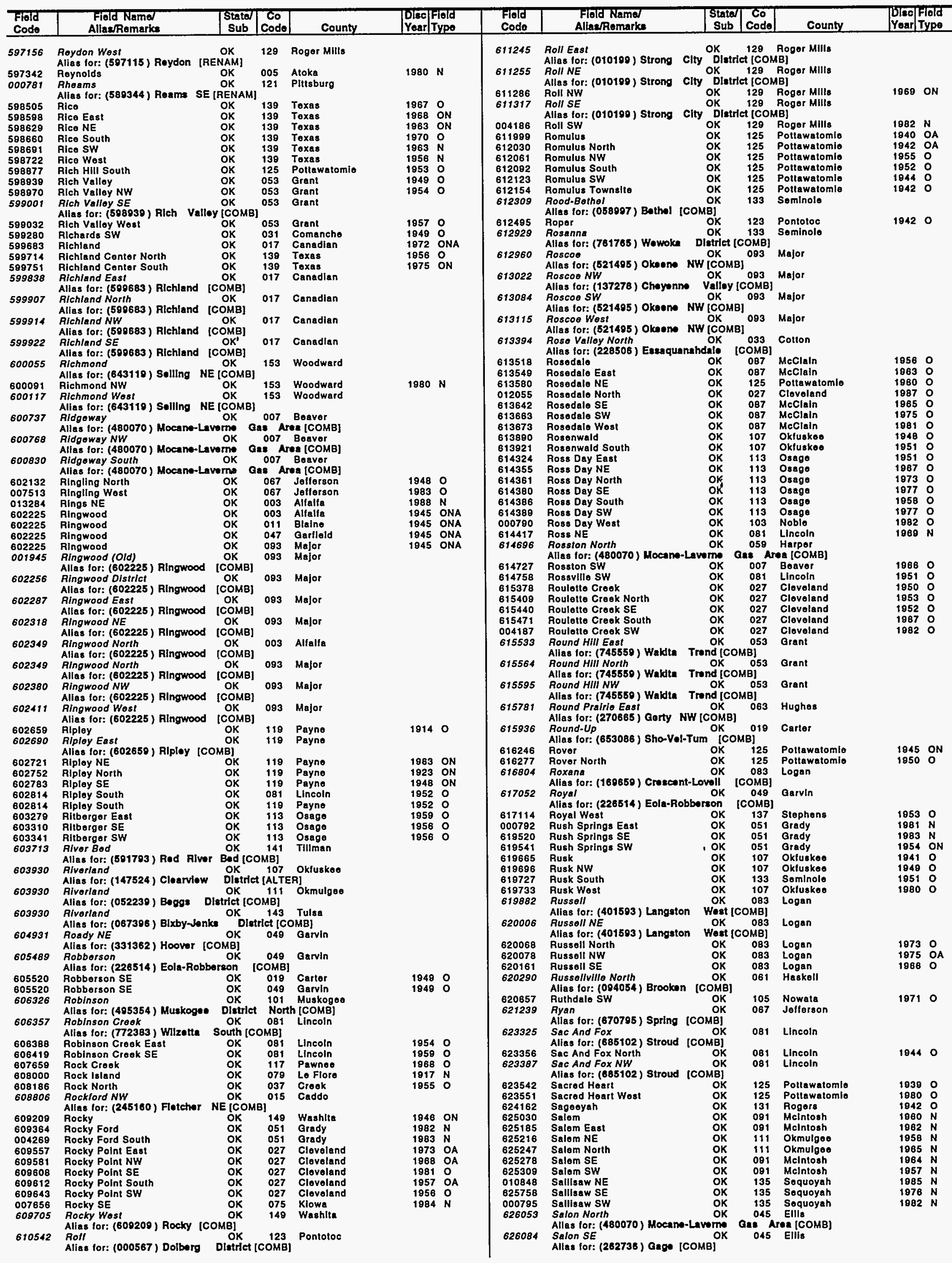


OKLAHOMA

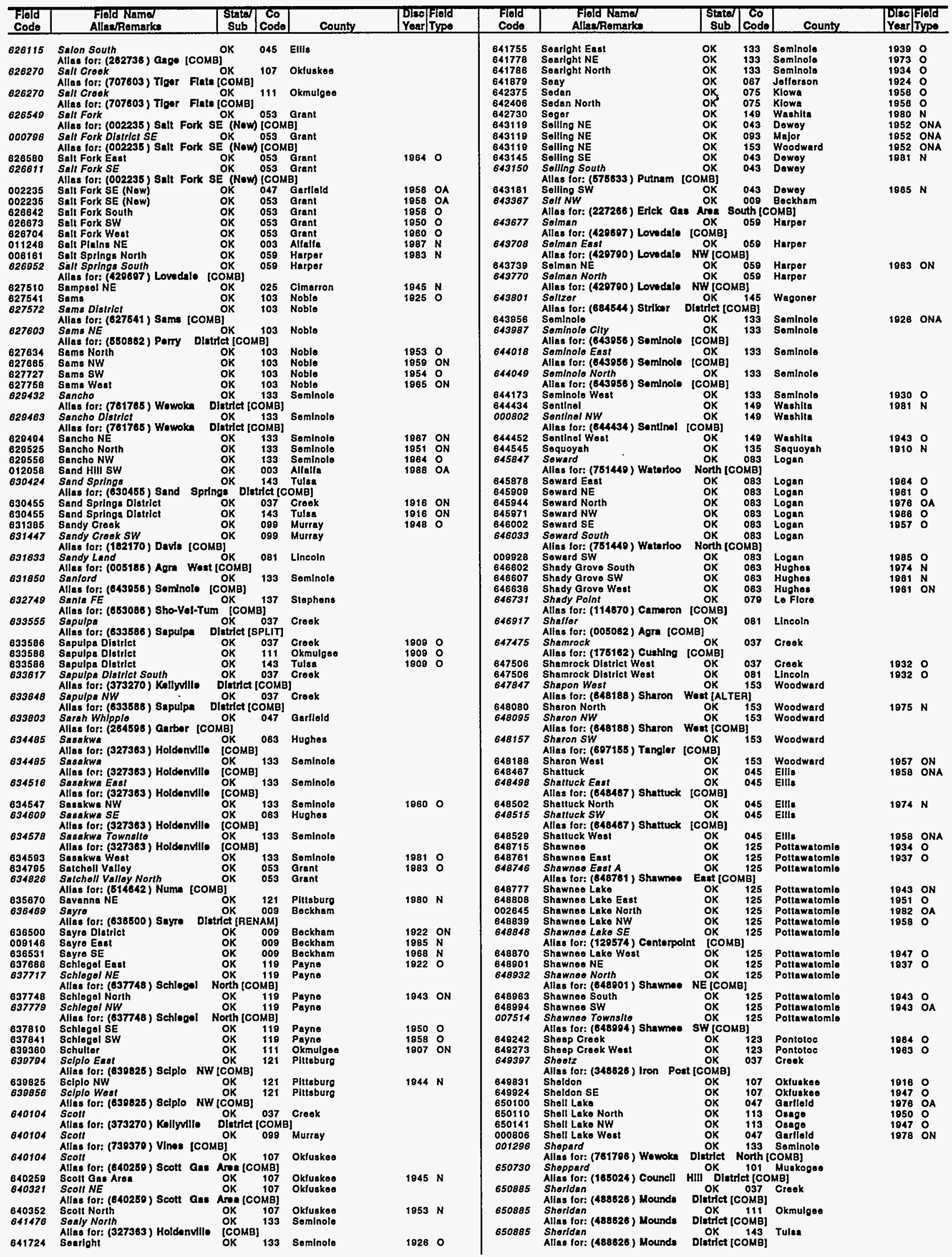


OKLAHOMA

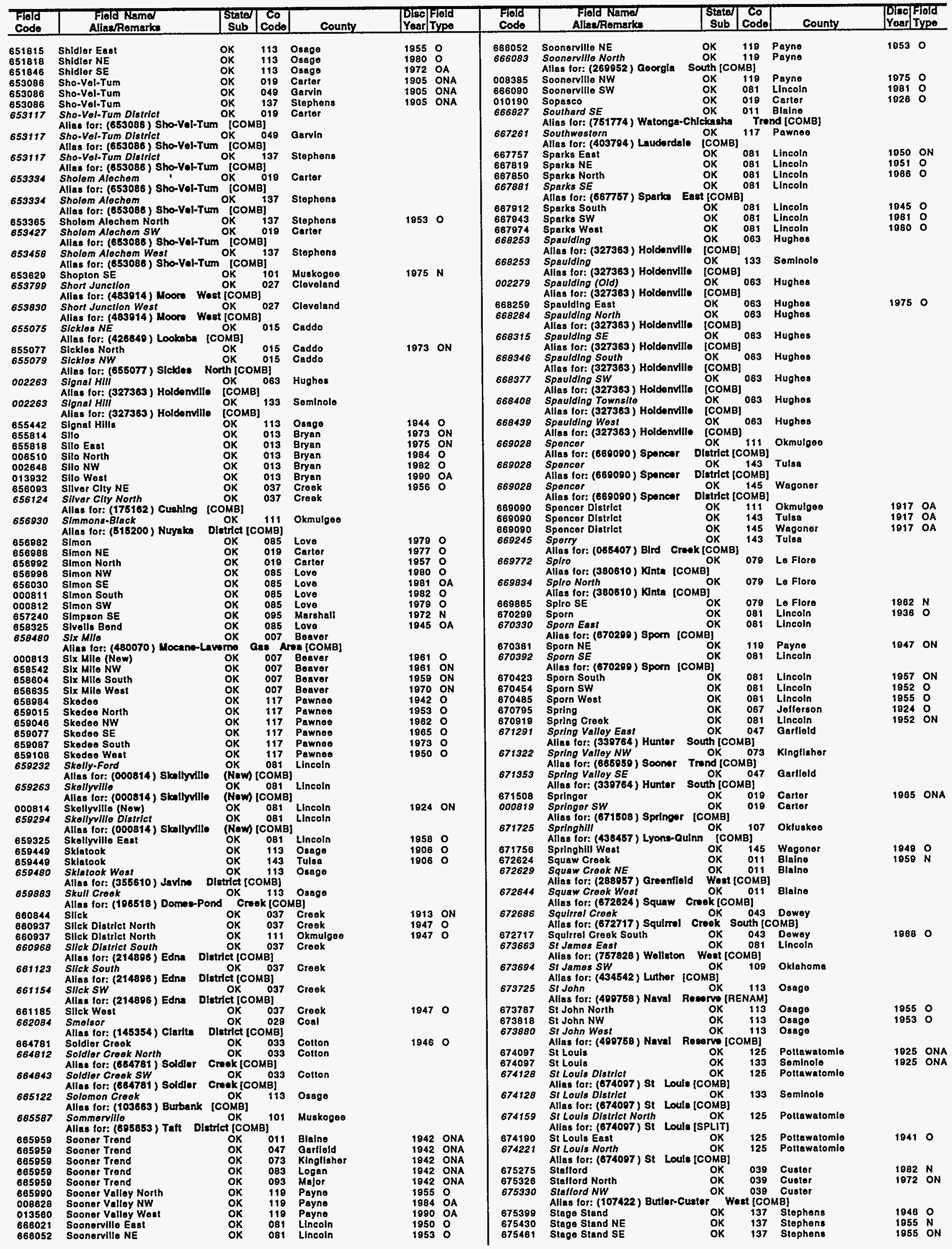


OKLAHOMA

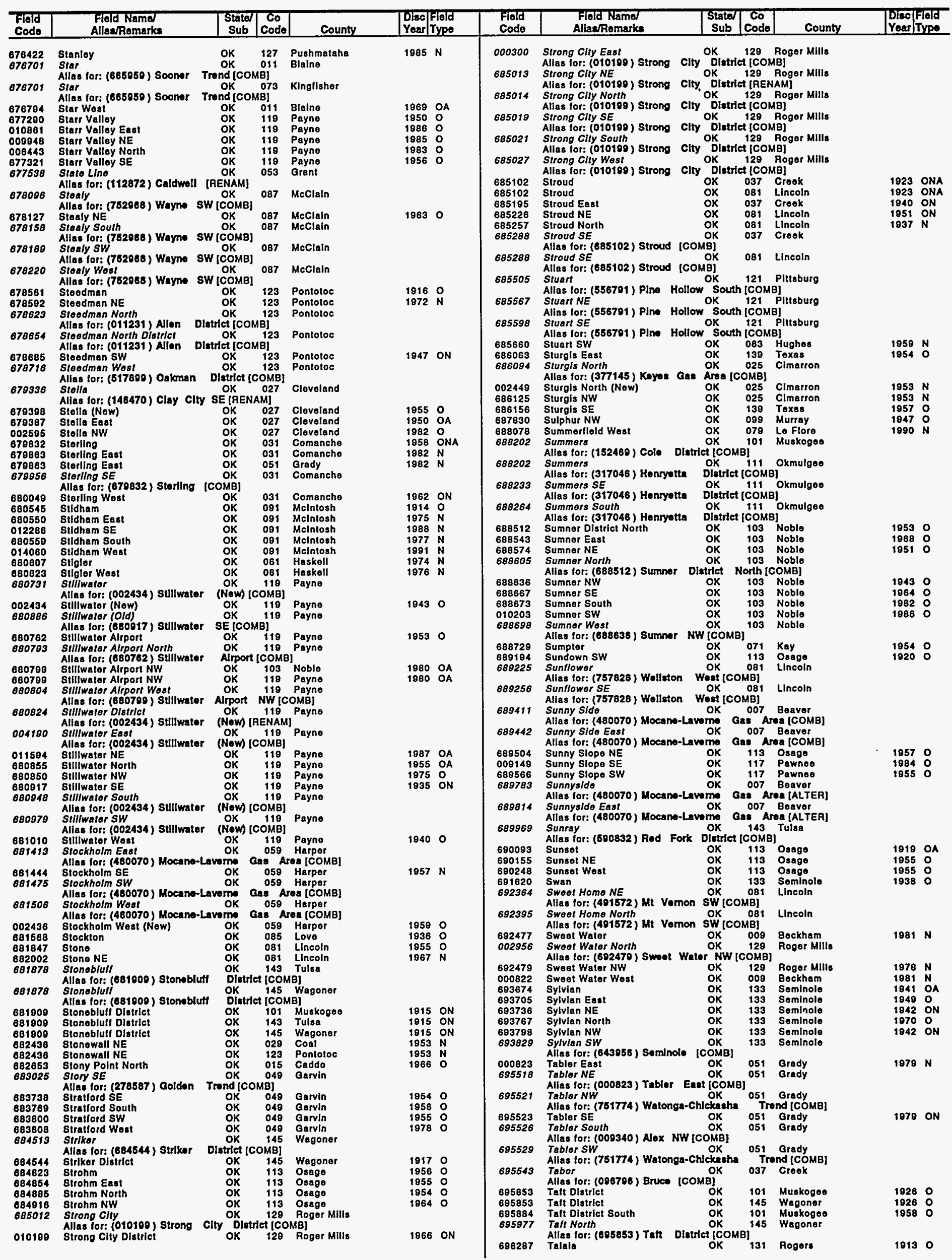


OKLAHOMA

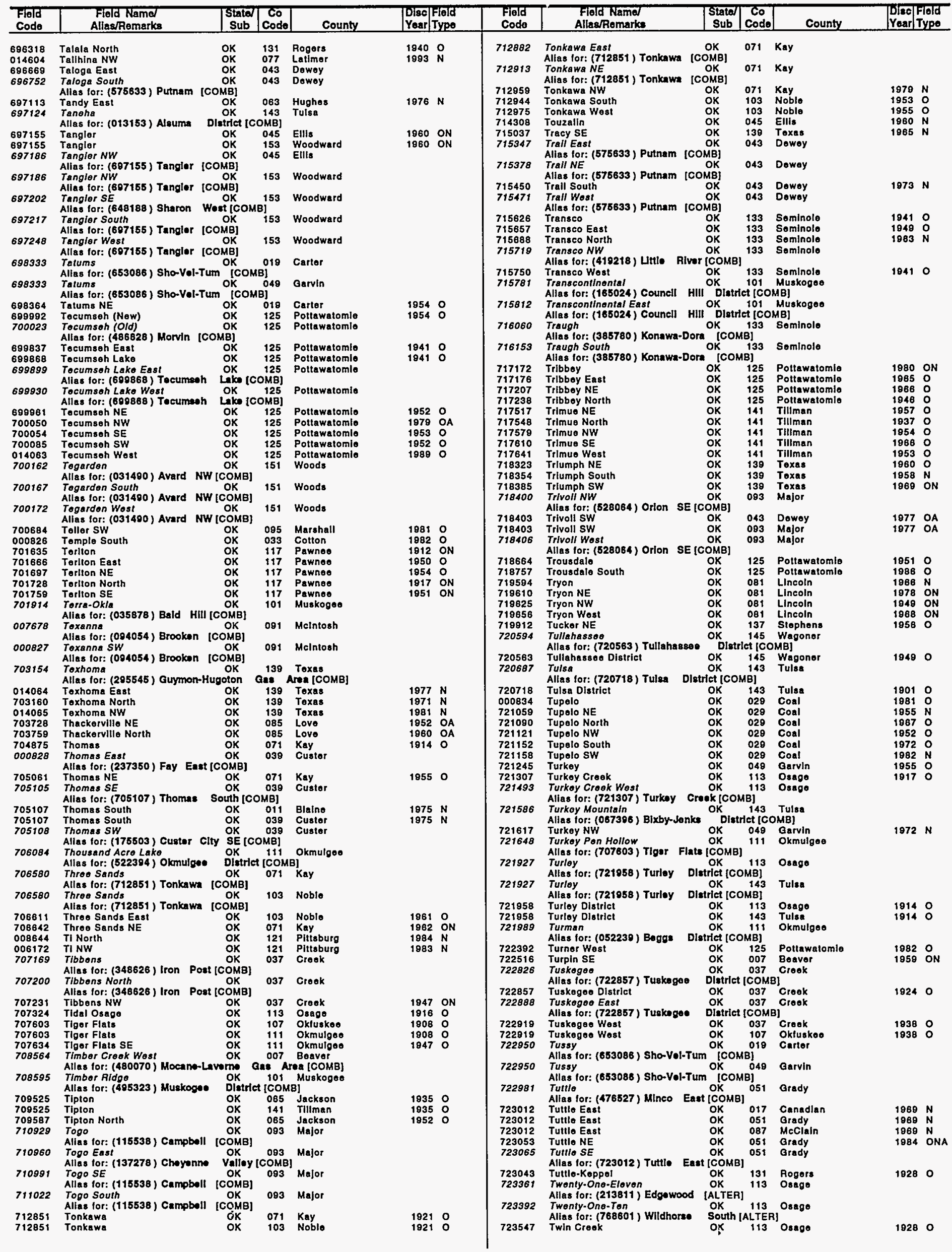




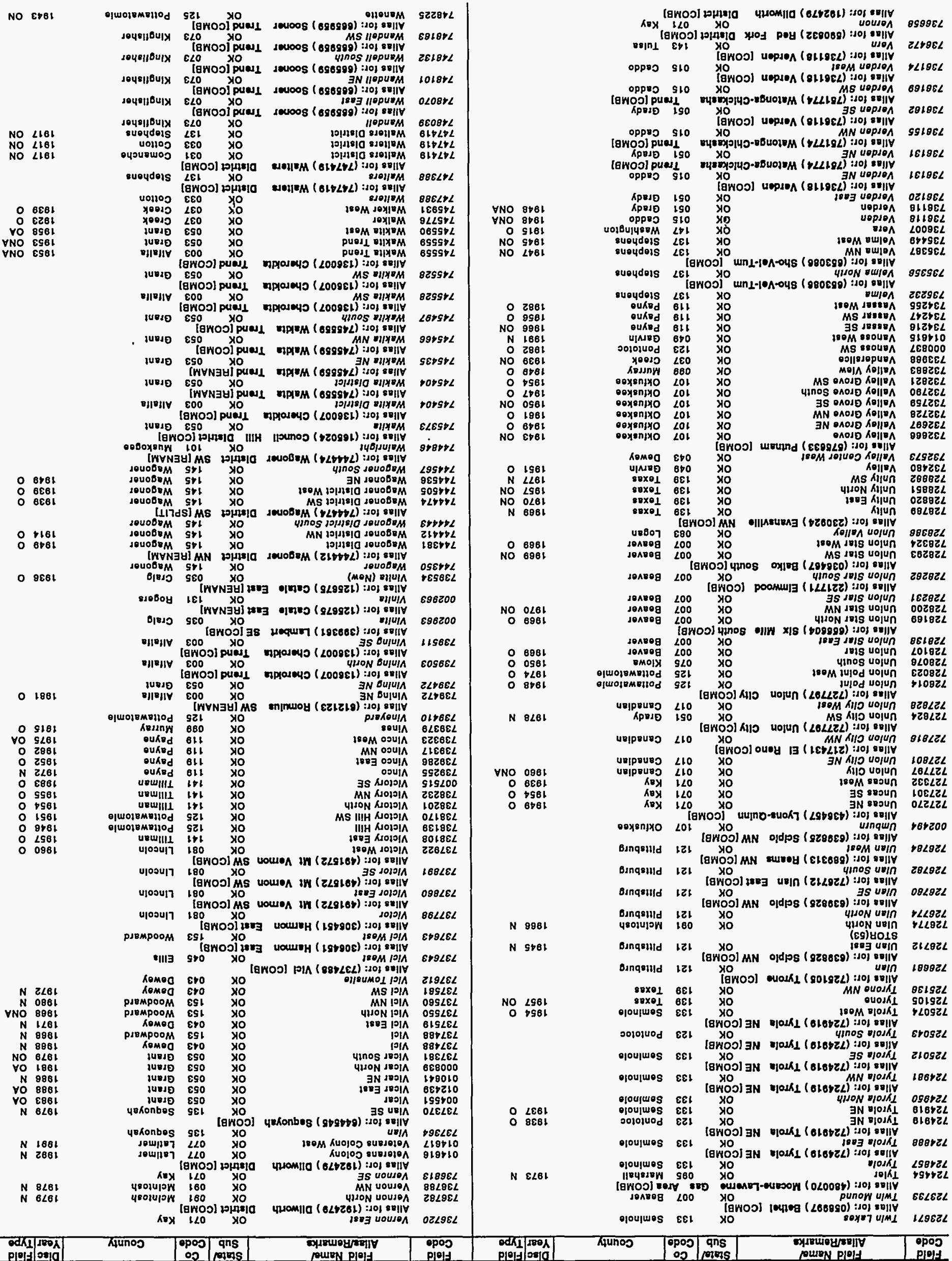


OKLAHOMA

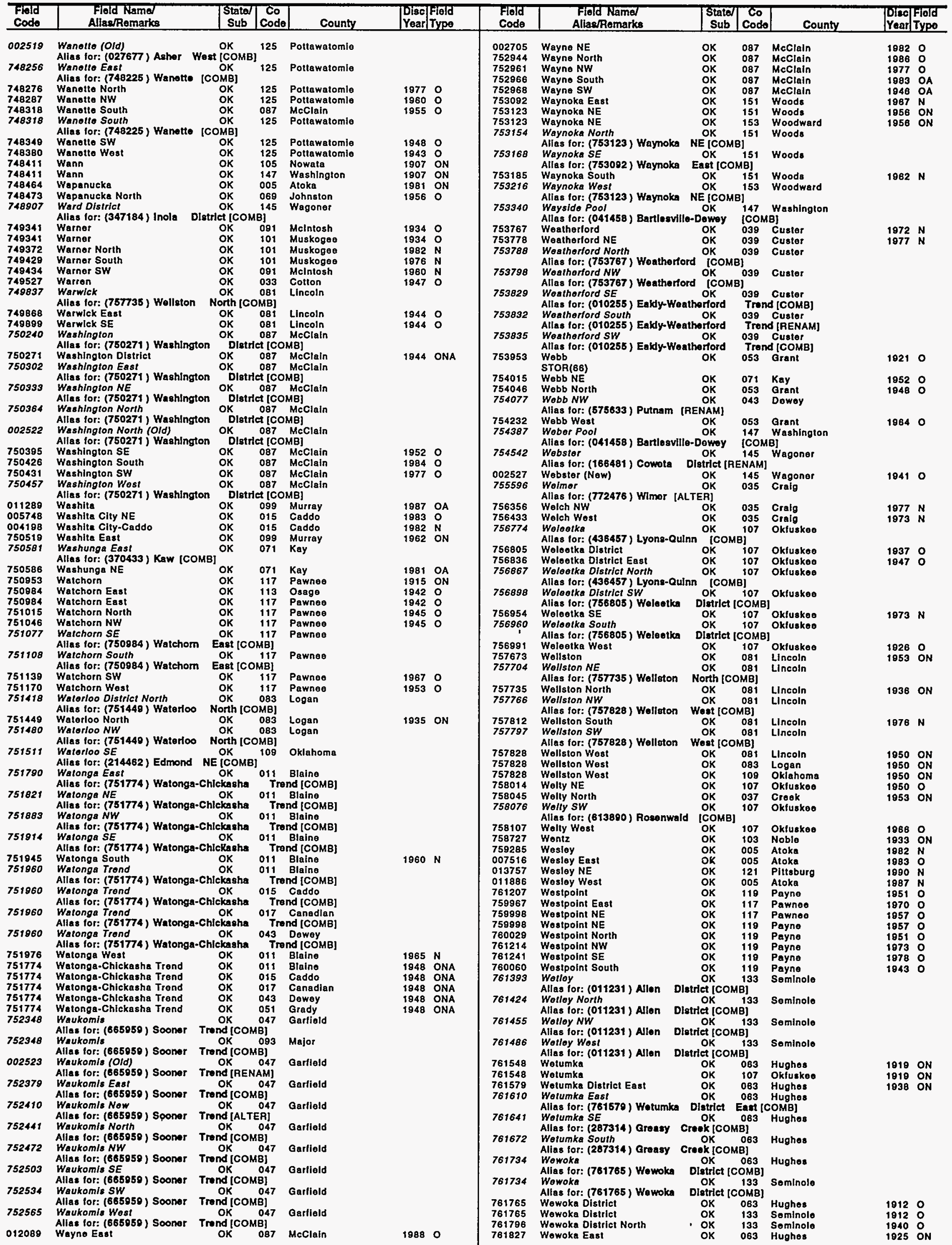




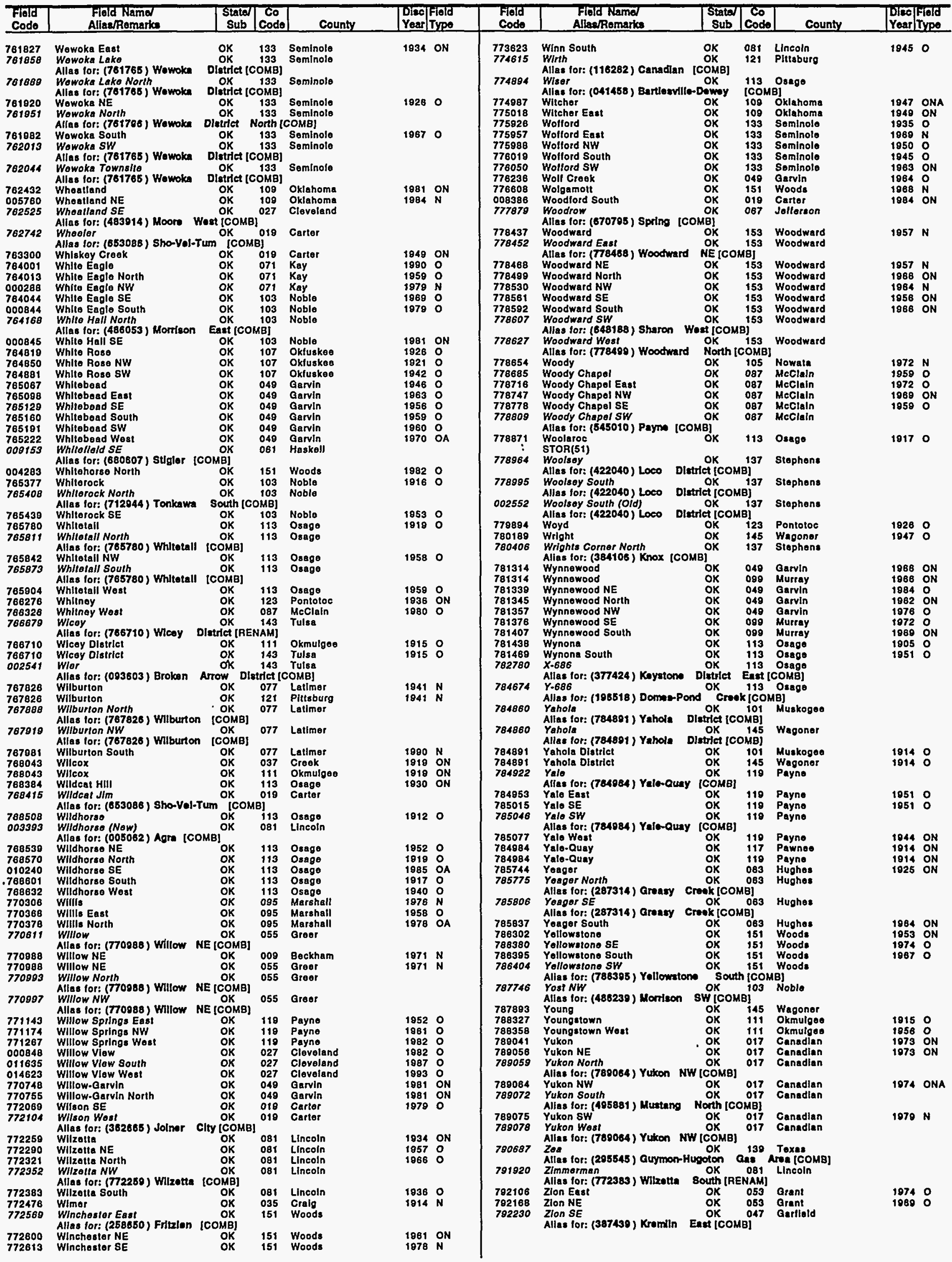




\section{OREGON}

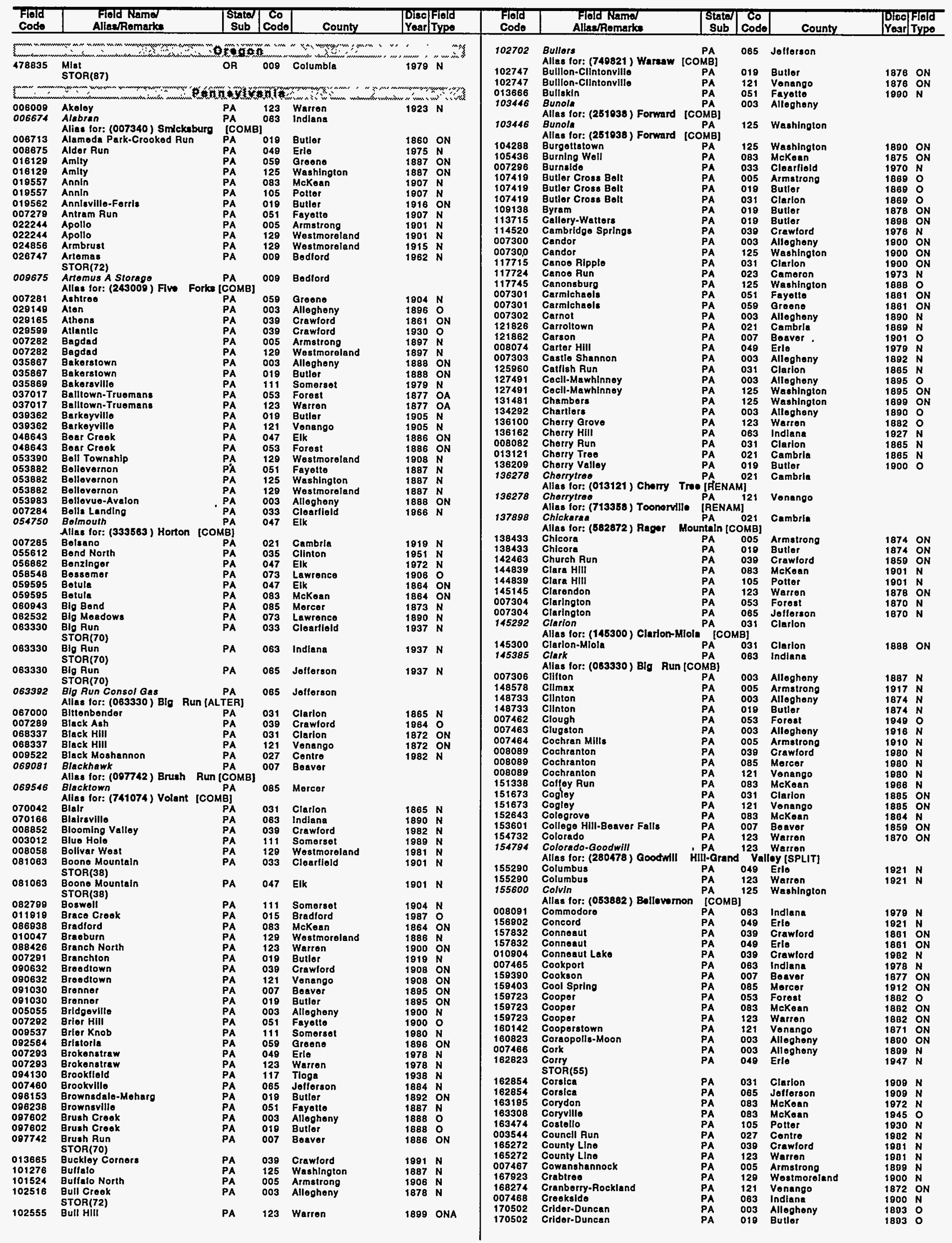


PENNSYLVANIA

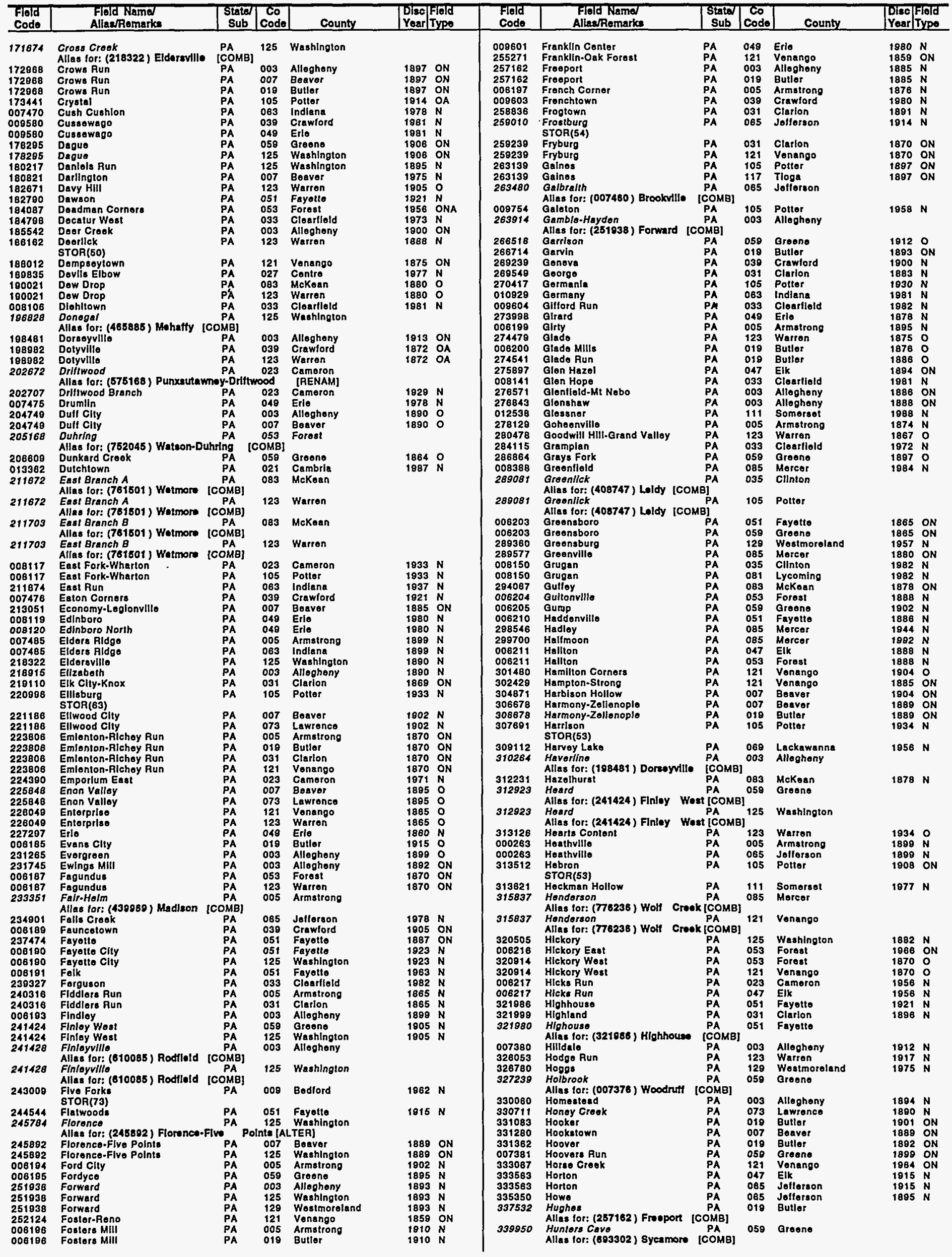


PENNSYLVANIA

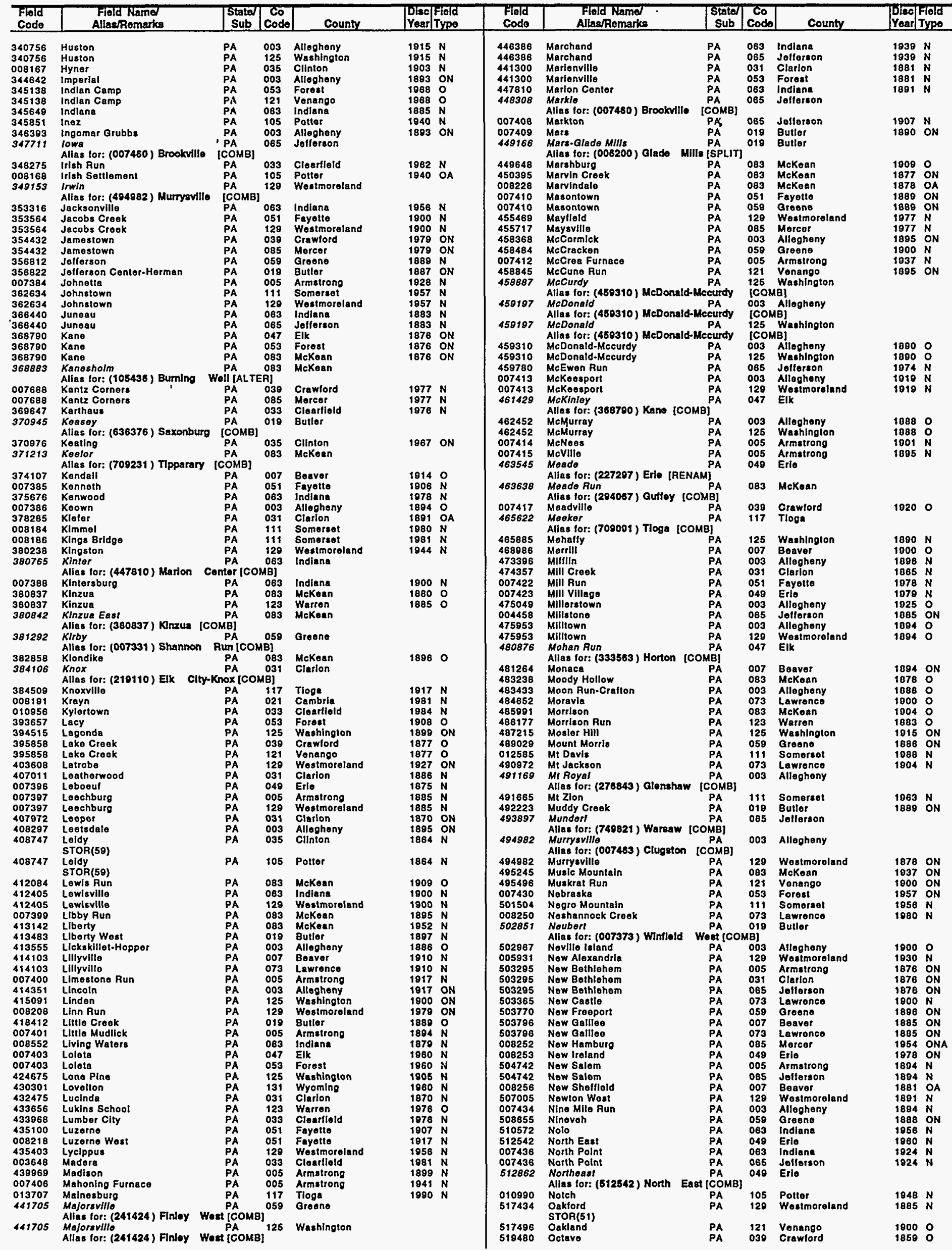


PENNSYLVANIA

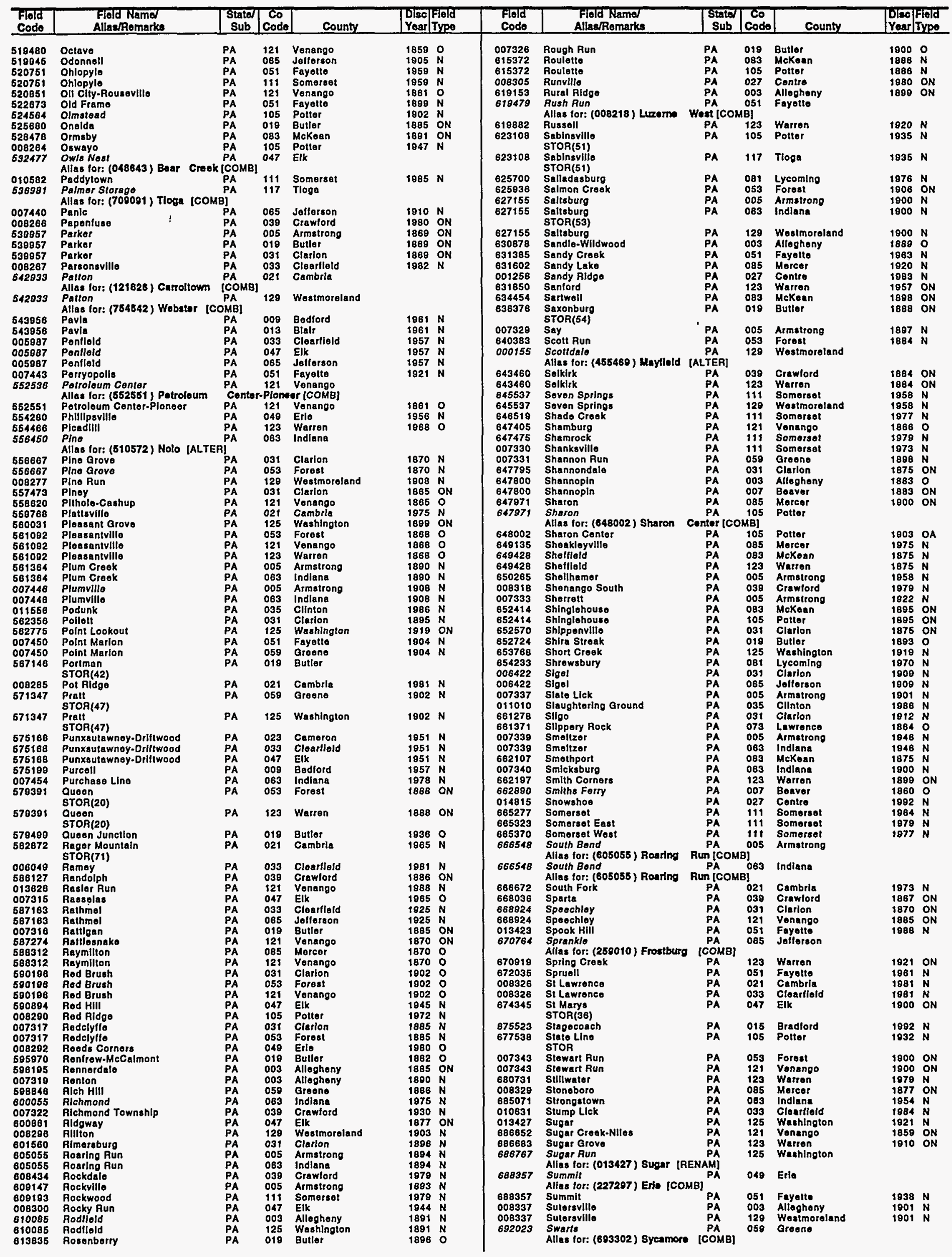




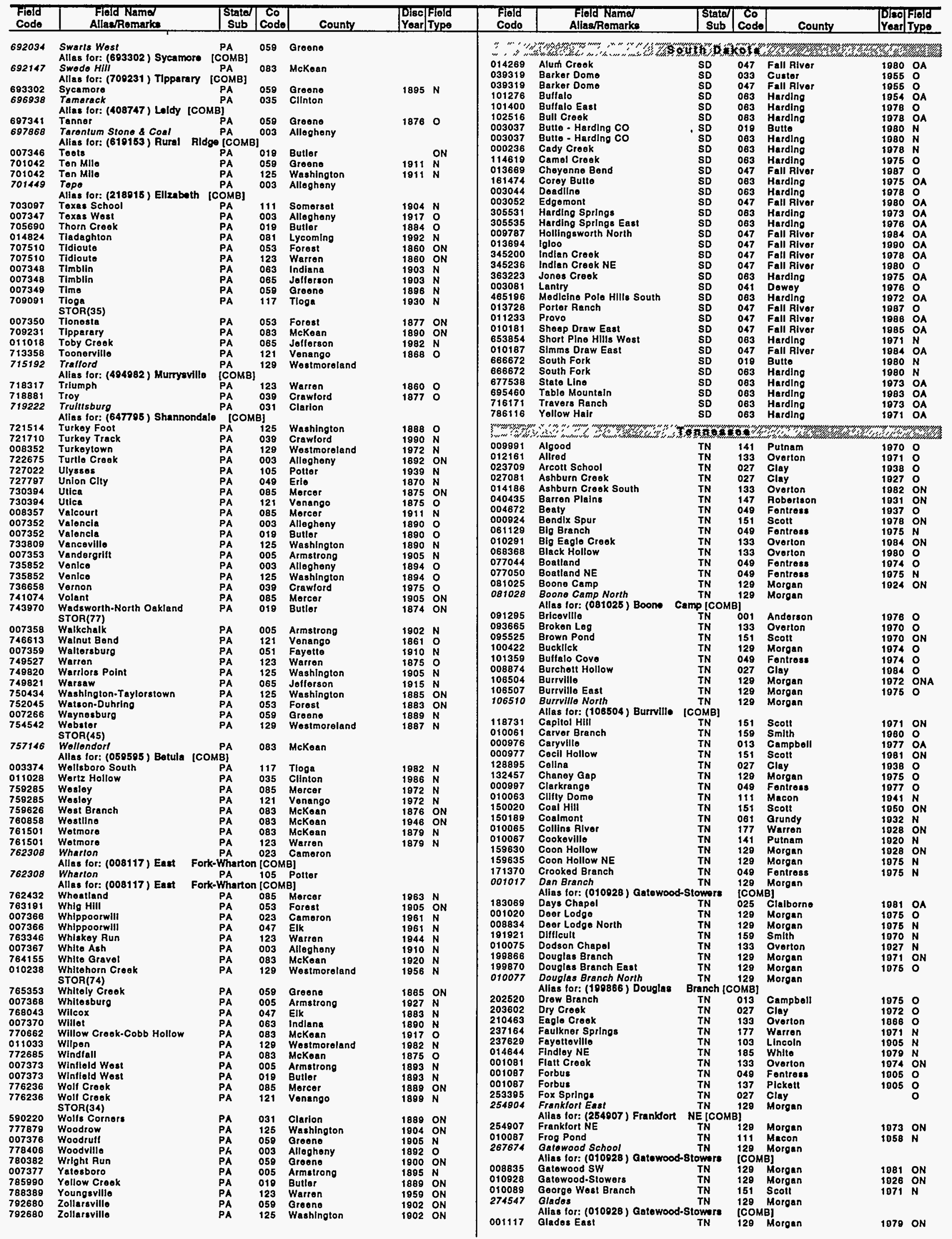


TEXAS

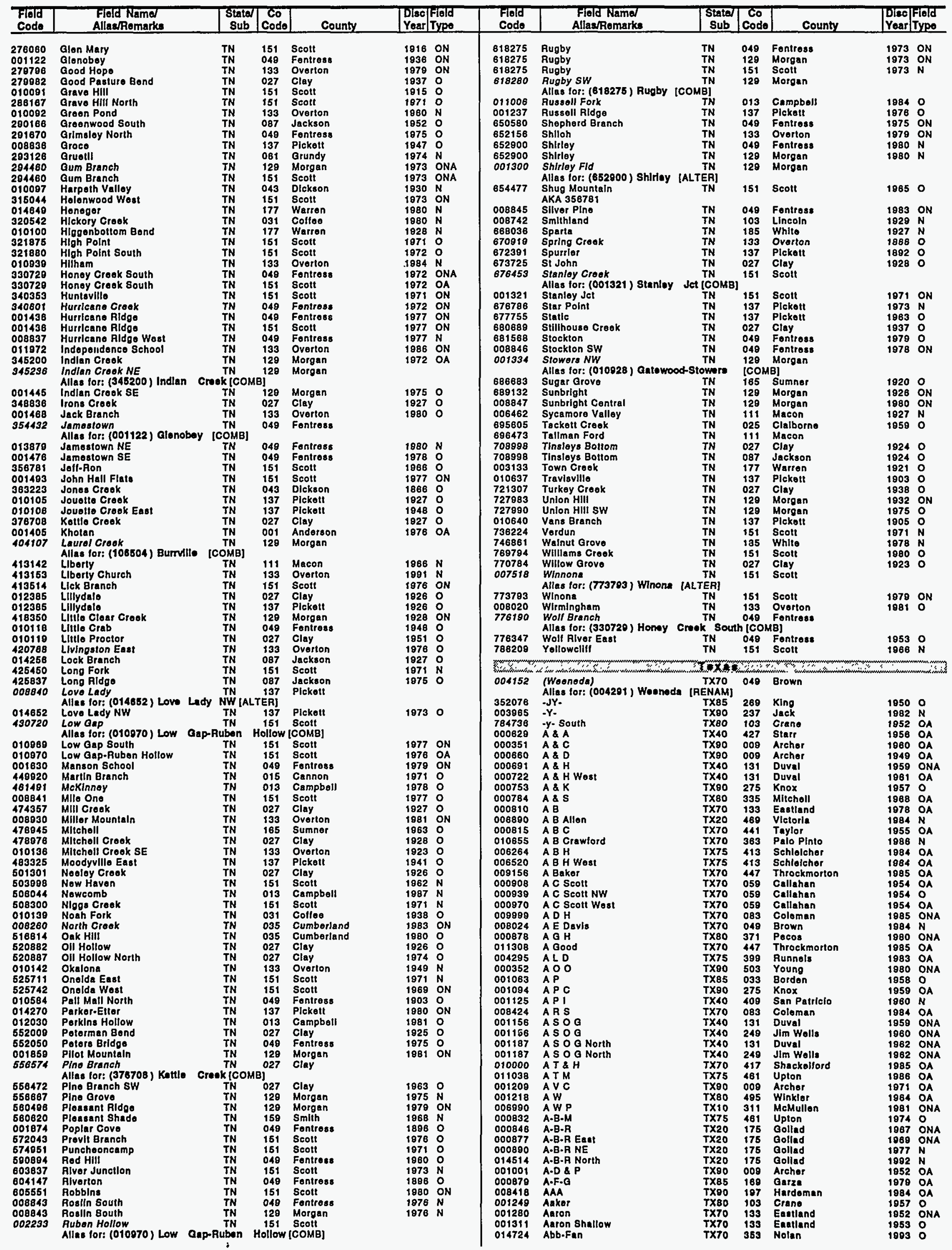


TEXAS

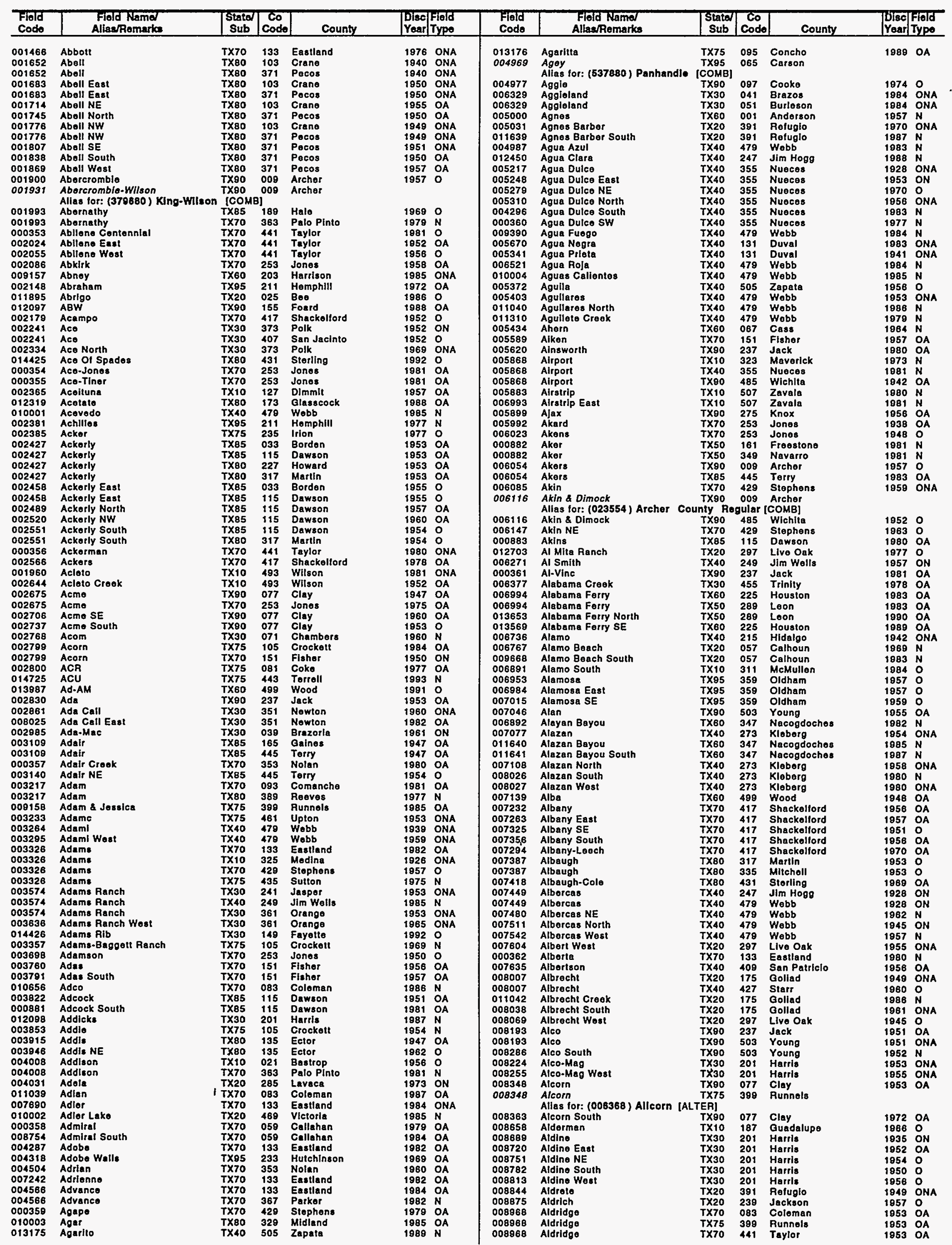


TEXAS

\begin{tabular}{|c|c|c|c|}
\hline $\begin{array}{l}\text { Frold } \\
\text { Code }\end{array}$ & $\begin{array}{l}\text { Frold Nama } \\
\text { AllaarRemarks }\end{array}$ & $\begin{array}{c}\text { State } \\
\text { Sub }\end{array}$ & $\begin{array}{l}c 0 \\
\text { Codo }\end{array}$ \\
\hline 008989 & Aldwell & Tho & 383 \\
\hline 009020 & $\begin{array}{l}\text { Allas for: }(670800) \text { Spraborry } \\
\text { Aldwoll Ranch }\end{array}$ & Trond & Arealcc \\
\hline $\begin{array}{l}009020 \\
009102\end{array}$ & $\begin{array}{l}\text { Aldwoll Ranch } \\
\text { Alodo SE }\end{array}$ & $\begin{array}{l}\text { TX75 } \\
\text { TX70 }\end{array}$ & $\begin{array}{l}435 \\
367\end{array}$ \\
\hline 009112 & Alodo South & TX70 & 367 \\
\hline 000363 & Alejandrenas & $T \times 40$ & 505 \\
\hline 008278 & Alox & $T \times 85$ & 445 \\
\hline $\begin{array}{l}000433 \\
000433\end{array}$ & $\begin{array}{l}\text { Aloxandor } \\
\text { Aloxandor }\end{array}$ & $\begin{array}{l}\text { TX95 } \\
\text { TX75 }\end{array}$ & $\begin{array}{l}211 \\
399\end{array}$ \\
\hline & & TX70 & 433 \\
\hline $009 \times 33$ & Aloxandor & TX85 & 445 \\
\hline 000384 & Alexender South & $\begin{array}{r}T \times 70 \\
T \times 70\end{array}$ & 433 \\
\hline $\begin{array}{l}009464 \\
009881\end{array}$ & $\begin{array}{l}\text { Alexandrla } \\
\text { Allrod }\end{array}$ & $\begin{array}{l}T \times 70 \\
T \times 40\end{array}$ & $\begin{array}{l}417 \\
249\end{array}$ \\
\hline 009712 & Allred Doep & $7 \times 40$ & 249 \\
\hline $\begin{array}{l}009160 \\
000774\end{array}$ & $\begin{array}{l}\text { Allred North } \\
\text { Allred Wost }\end{array}$ & $\begin{array}{l}7 \times 40 \\
7 \times 40\end{array}$ & $\begin{array}{l}249 \\
249\end{array}$ \\
\hline $\begin{array}{l}000774 \\
009743\end{array}-1$ & Allrod.Donnoll & $\begin{array}{l}7 \times 40 \\
T \times 70\end{array}$ & $\begin{array}{l}249 \\
429\end{array}$ \\
\hline 006743 & Aloeny & TX20 & 285 \\
\hline $\begin{array}{l}009836 \\
009877\end{array}-37$ & $\begin{array}{l}\text { Algoinon } \\
\text { Algoa }\end{array}$ & $\underset{T \times 30}{T \times 75}$ & $\begin{array}{l}309 \\
187\end{array}$ \\
\hline 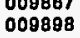 & Algoa Nornh & TX30 & 187 \\
\hline 005871 & Algoe NW & $T_{T \times 30}^{T \times 30}$ & 187 \\
\hline $\begin{array}{l}009929 \\
008672\end{array}-3$ & $\begin{array}{l}\text { Algoa Orehard } \\
\text { Algoa South }\end{array}$ & $\begin{array}{l}T \times 30 \\
T \times 30\end{array}$ & $\begin{array}{l}187 \\
038\end{array}$ \\
\hline 008672 & Aloos South & $\begin{array}{l}T \times 30 \\
T \times 30\end{array}$ & 187 \\
\hline .009934 & $\begin{array}{l}\text { Algos Why } \\
\text { Algoe Wost }\end{array}$ & $\begin{array}{l}T \times 30 \\
T \times 30\end{array}$ & $\begin{array}{l}039 \\
039\end{array}$ \\
\hline $\begin{array}{l}009838 \\
010022\end{array}$ & $\begin{array}{l}\text { Alloos Woos } \\
\text { Allco }\end{array}$ & $7 \times 40$ & $\begin{array}{l}038 \\
248\end{array}$ \\
\hline $\begin{array}{l}010053 \\
010084\end{array}$ & $\begin{array}{l}\text { Allco Ann } \\
\text { Allico Doop }\end{array}$ & $\begin{array}{l}T \times 70 \\
T \times 40\end{array}$ & $\begin{array}{l}133 \\
249\end{array}$ \\
\hline $\begin{array}{l}010084 \\
002978\end{array}$ & $\begin{array}{l}\text { Allce Deop } \\
\text { Alleo Doop SW }\end{array}$ & $\underset{T \times 40}{T \times 40}$ & $\begin{array}{l}248 \\
249\end{array}$ \\
\hline 000385 & Allco East & $T \times 40$ & 249 \\
\hline $\begin{array}{l}010115 \\
010148\end{array}$ & $\begin{array}{l}\text { Alleo North } \\
\text { Alleo SE }\end{array}$ & $\begin{array}{l}T \times 40 \\
T \times 40\end{array}$ & $\begin{array}{r}249 \\
249\end{array}$ \\
\hline $\begin{array}{l}010186 \\
010177\end{array}$ & $\begin{array}{l}\text { Alleo So } \\
\text { Alleo South }\end{array}$ & $\begin{array}{l}T \times 40 \\
T \times 40\end{array}$ & $\begin{array}{l}249 \\
249\end{array}$ \\
\hline 010208 & Alice West & $T \times 40$ & 248 \\
\hline 010298 & Allcoanno & $T \times 70$ & 417 \\
\hline 010270 & Allas for: (646494) Shackolfore & d NEIC & OMB] \\
\hline 010270 & $\begin{array}{l}\text { Allcoanne South } \\
\text { Allas for: (646404) Shackolfore }\end{array}$ & TX70 & $\begin{array}{l}417 \\
\text { OMBI }\end{array}$ \\
\hline 010301 & Allot & TX30 & 201 \\
\hline 010332 & Aliof South & $T \times 30$ & 201 \\
\hline 012678 & Aljonda - Blp & TX85 & 169 \\
\hline 010700 & Alkall Crook & $T \times 70$ & 151 \\
\hline 010702 & Alkali Crook SW & $T \times 70$ & 151 \\
\hline 010736 & Alkok & $T \times 20$ & 175 \\
\hline 090735 & Alkok & $T \times 20$ & 381 \\
\hline 010786 & Alkok East & $T \times 20$ & 175 \\
\hline $\begin{array}{l}010707 \\
010797\end{array}-3$ & $\begin{array}{l}\text { Alkok North } \\
\text { Alkok Nort }\end{array}$ & $\begin{array}{l}T \times 20 \\
T \times 20\end{array}$ & $\begin{array}{l}175 \\
381\end{array}$ \\
\hline $\begin{array}{l}010797 \\
008420\end{array}-100$ & $\begin{array}{l}\text { Alkok North } \\
\text { Alkok Wost }\end{array}$ & $\begin{array}{l}T \times 20 \\
T \times 20\end{array}$ & $\begin{array}{l}381 \\
175\end{array}$ \\
\hline 010858 & Allar & TXOO & 503 \\
\hline 006998 & Allar-Buzz & $T \times 75$ & 081 \\
\hline 010800 & Allar-Morks & $T \times 80$ & 389 \\
\hline & Allas for: (373921) Ken Regan & $n$ (сомв & \\
\hline 010921 & Allar-Warron & TX80 & 503 \\
\hline 010857 & Allard 8mith & $\underset{T \times 90}{T \times 75}$ & 237 \\
\hline $\begin{array}{l}006388 \\
011078\end{array}$ & $\begin{array}{l}\text { Allcoern } \\
\text { Allon }\end{array}$ & $\begin{array}{l}T \times 75 \\
7 \times 70\end{array}$ & 399 \\
\hline $\begin{array}{l}011078 \\
011078\end{array}$ & $\begin{array}{l}\text { Allon } \\
\text { Allon }\end{array}$ & $\begin{array}{l}T \times 70 \\
T \times 40\end{array}$ & $\begin{array}{l}133 \\
247\end{array}$ \\
\hline 011076 & Allon & $T \times 75$ & 308 \\
\hline 011078 & Allen & $T \times 70$ & 417 \\
\hline 011078 & Allon & $T \times 40$ & 427 \\
\hline & Allas for: (014362) Alvarez (C) & :OMB] & \\
\hline $\begin{array}{l}011078 \\
011078\end{array}-30$ & $\begin{array}{l}\text { Allon } \\
\text { Allon }\end{array}$ & $\begin{array}{l}T \times 75 \\
T \times 30\end{array}$ & $\begin{array}{l}451 \\
481\end{array}$ \\
\hline $\begin{array}{l}011076 \\
011180\end{array}$ & $\begin{array}{l}\text { Allon \& Altehlo } \\
\text { Allon a }\end{array}$ & $\begin{array}{l}T \times 30 \\
T \times 70\end{array}$ & $\begin{array}{l}481 \\
363\end{array}$ \\
\hline 011200 & Allan 8 Smlth & TX85 & 233 \\
\hline 000885 & Allon Androw & $T \times 80$ & 431 \\
\hline 011282 & Allon Domo & $\begin{array}{l}\operatorname{Tx} 30 \\
-T y 75\end{array}$ & 039 \\
\hline 000336 & Allen HIII & TX75 & 451 \\
\hline 011417 & $\begin{array}{l}\text { Allon North } \\
\text { Allon Banch }\end{array}$ & $\begin{array}{l}T \times 40 \\
T \times 30\end{array}-1-100$ & 247 \\
\hline 007603 & $\begin{array}{l}\text { Allon Ranch } \\
\text { Allon Ganch }\end{array}$ & $\begin{array}{c}T \times 30 \\
T \times 20\end{array}-1-100$ & $\begin{array}{r}089 \\
285\end{array}$ \\
\hline $\begin{array}{l}007693 \\
011324\end{array}$ & $\begin{array}{l}\text { Allon fanch } \\
\text { Allon-Hollday }\end{array}$ & $\begin{array}{l}T \times 20 \\
T \times 85\end{array}$ & $\begin{array}{l}285 \\
415\end{array}$ \\
\hline 011470 & Allon-Parker & Tะ95 & 285 \\
\hline 011479 & Allon-Parkor & TXं95 & 357 \\
\hline 000366 & $\begin{array}{l}\text { Allon-Six } \\
\text { Allopiown }\end{array}$ & $T \times 90$ & 237 \\
\hline $\begin{array}{l}011634 \\
011634\end{array}-3$ & $\begin{array}{l}\text { Allontown } \\
\text { Allontown }\end{array}$ & & 005 \\
\hline $\begin{array}{l}011634 \\
009870\end{array}-30$ & $\begin{array}{l}\text { Allontown } \\
\text { Allio }\end{array}$ & $\cdot \begin{array}{l}7 \times 60 \\
7 \times 75\end{array}$ & $\begin{array}{l}087 \\
435\end{array}$ \\
\hline $\begin{array}{l}009670 \\
012877\end{array}-30$ & $\begin{array}{l}\text { Alllo } \\
\text { Allogator }\end{array}$ & $\begin{array}{l}T \times 75 \\
T \times 30 \\
T \times 30\end{array}$ & $\begin{array}{l}435 \\
477\end{array}$ \\
\hline $\begin{array}{l}012677 \\
01168\end{array}$ & & $\begin{array}{l}7 \times 30 \\
T \times 30\end{array}$ & $\begin{array}{l}477 \\
071\end{array}$ \\
\hline $\begin{array}{l}011688 \\
011727\end{array}$ & $\begin{array}{l}\text { Allloator Bayou } \\
\text { Allgator Bayou East }\end{array}$ & $\begin{array}{l}1 \times 30 \\
1 \times 30\end{array}$ & 071 \\
\hline $\begin{array}{l}003788 \\
0.726\end{array}$ & Alllgator Bayou SW & $T \times 30$ & 071 \\
\hline 011758 & Alllgator Bayou Wost & $\begin{array}{l}7 \times 30 \\
7 \times 50\end{array}$ & 071 \\
\hline 008421 & Alllogator Crook & $T \times 50$ & 349 \\
\hline 011789 & Alllgator Lako & $7 \times 30$ & 351 \\
\hline 000367 & Allloator Lako North & $\begin{array}{r}T \times 30 \\
T \times 30\end{array}$ & 039 \\
\hline 011820 & $\begin{array}{l}\text { Alllgator Polnt } \\
\text { Alligator Slough }\end{array}$ & $\begin{array}{l}T \times 30 \\
T \times 10\end{array}$ & \\
\hline $\begin{array}{l}001984 \\
011851\end{array}$ & $\begin{array}{l}\text { Alligator slough } \\
\text { Allison }\end{array}$ & $\underset{T \times 10}{T \times 10}$ & $\begin{array}{l}507 \\
429\end{array}$ \\
\hline 011851 & Allison & $7 \times 75$ & 143 \\
\hline 011851 & Allison & $T \times 90$ & 485 \\
\hline 011024 & Alilion Parks & TX85 & 211 \\
\hline 011821 & Allleon Parks & TX95 & 483 \\
\hline $\begin{array}{l}007243 \\
011927\end{array}$ & $\begin{array}{l}\text { Allloon Parke SE } \\
\text { Allison Ranch }\end{array}$ & $\begin{array}{l}T \times 95 \\
T \times 80\end{array}$ & 483 \\
\hline & $\begin{array}{l}\text { Allison Ranch } \\
\text { Allson South }\end{array}$ & $\begin{array}{l}T \times 80 \\
T \times 75 \\
T \times 75\end{array}$ & $\begin{array}{l}371 \\
443\end{array}$ \\
\hline 000314 & Allieon-Briti & TX95 & 483 \\
\hline 011892 & Allison-Holt & TX95 & 483 \\
\hline 012008 & Allmyors & $\begin{array}{l}7 \times 90 \\
T \times 90\end{array}$ & 503 \\
\hline 012161 & Allrod & $T \times 90$ & 197 \\
\hline 012182 & Allrod-Peory & TX90 & 009 \\
\hline 012285 & Allyaises & $\begin{array}{r}T \times 90 \\
T \times 70\end{array}$ & 237 \\
\hline 007244 & Allyn & $T \times 70$ & 441 \\
\hline 011643 & Alm & $T \times 20$ & 285 \\
\hline 012318 & Alma & $7 \times 80$ & 337 \\
\hline $\begin{array}{l}01237 \\
010008\end{array}$ & Alma Boll & $\begin{array}{l}T \times 20 \\
T \times 70\end{array}$ & 297 \\
\hline $\begin{array}{l}010858 \\
01008\end{array}$ & $\begin{array}{l}\text { Alma Johnson } \\
\text { Alma Jum }\end{array}$ & $7 \times 70$ & 083 \\
\hline
\end{tabular}




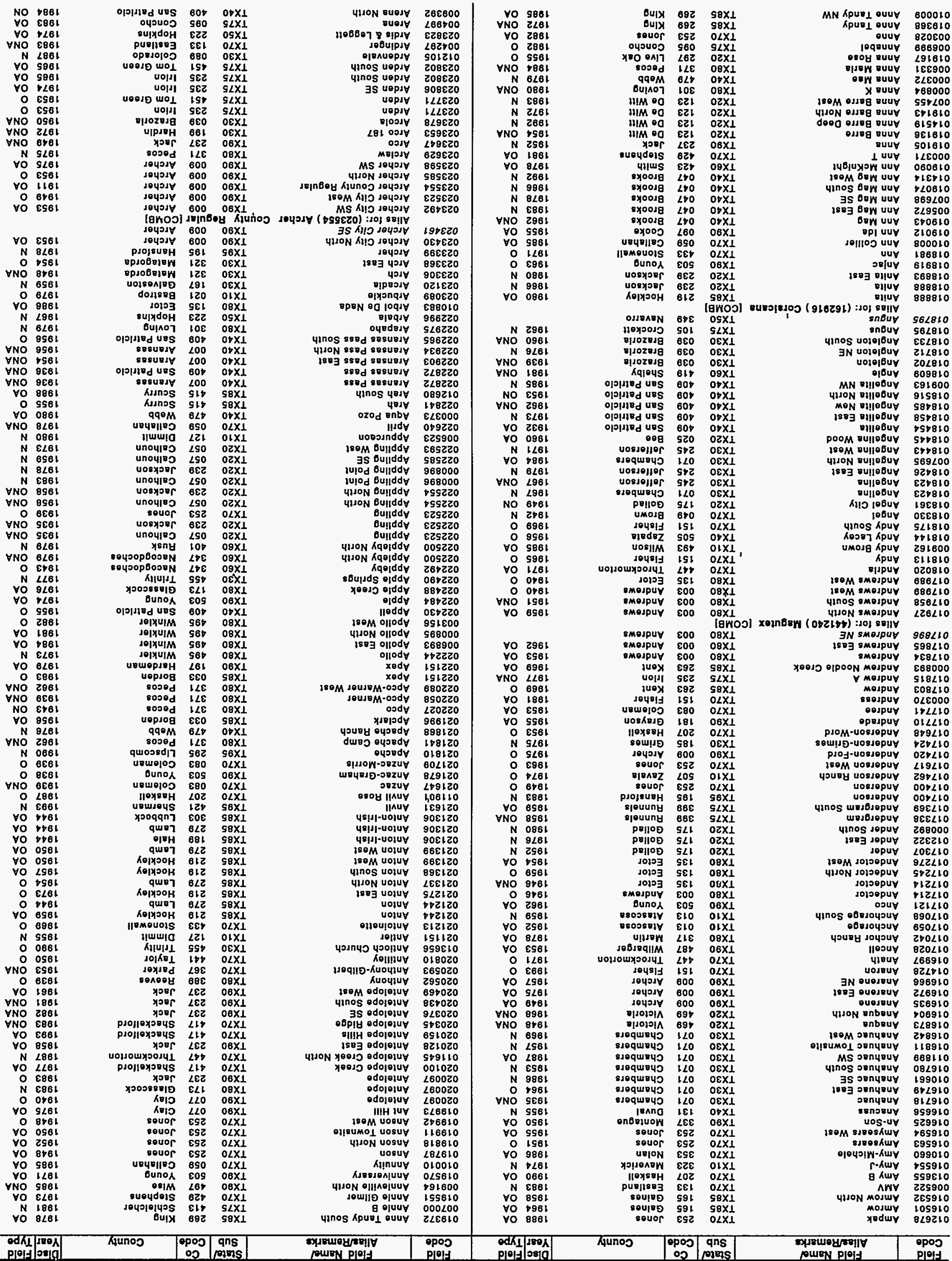


TEXAS

\begin{tabular}{|c|c|c|c|c|c|c|c|c|c|c|c|c|c|}
\hline $\begin{array}{l}\text { Flotd } \\
\text { Codo } \\
\end{array}$ & $\begin{array}{l}\text { Fild Nama } \\
\text { Allas/Remarks }\end{array}$ & $\begin{array}{l}\text { Stated } \\
\text { Sub }\end{array}$ & $\begin{array}{c}\text { Co } \\
\text { Code }\end{array}$ & County & {$\left[\begin{array}{l}\text { bisc } \\
\text { Year }\end{array}\right]$} & $\begin{array}{l}\text { Fierd } \\
\text { Typo }\end{array}$ & $\begin{array}{l}\text { Fiteld } \\
\text { Code }\end{array}$ & $\begin{array}{l}\text { Fleld Name/ } \\
\text { Allas/Romarks }\end{array}$ & $\begin{array}{c}\text { Stated } \\
\text { Sub }\end{array}$ & $\begin{array}{c}\text { Co } \\
\text { Codo }\end{array}$ & County & \begin{tabular}{|l|l} 
Disec & T \\
Yoar & T \\
\end{tabular} & $\begin{array}{l}\text { Fied } \\
\text { Typo }\end{array}$ \\
\hline $\begin{array}{l}023857 \\
000897 \\
023888 \\
023888 \\
024018 \\
024068 \\
0240081 \\
024081 \\
024143 \\
024143 \\
024174 \\
024174 \\
024205 \\
024238 \\
000374 \\
024422\end{array}$ & $\begin{array}{l}\text { Aronas } \\
\text { Aronosa Creok } \\
\text { Aronoeo } \\
\text { Arenoso } \\
\text { Arsnoso West } \\
\text { Argonta } \\
\text { Argo } \\
\text { Arlek } \\
\text { Arlek } \\
\text { Arles } \\
\text { Arlos } \\
\text { Arles East } \\
\text { Arles Wost } \\
\text { Ark-La-Tox } \\
\text { Arkansas Clty }\end{array}$ & $\begin{array}{l}T \times 80 \\
T \times 20 \\
T \times 80 \\
T \times 80 \\
T \times 80 \\
T \times 20 \\
T \times 80 \\
T \times 85 \\
T \times 85 \\
T X T 0 \\
T \times 90 \\
T X 90 \\
T X 90 \\
T X 80 \\
T X 40\end{array}$ & $\begin{array}{l}475 \\
469 \\
135 \\
495 \\
405 \\
297 \\
003 \\
153 \\
189 \\
059 \\
337 \\
337 \\
337 \\
077 \\
427\end{array}$ & $\begin{array}{l}\text { Ward } \\
\text { Victoria } \\
\text { Ector } \\
\text { Winkler } \\
\text { Winkler } \\
\text { Live Oak } \\
\text { Andrews } \\
\text { Floyd } \\
\text { Halo } \\
\text { Callahan } \\
\text { Montague } \\
\text { Montague } \\
\text { Montegue } \\
\text { Clay } \\
\text { Start }\end{array}$ & $\begin{array}{l}1970 \\
1979 \\
1965 \\
1965 \\
1967 \\
1978 \\
1956 \\
1948 \\
1948 \\
1984 \\
1953 \\
1960 \\
1957 \\
1981 \\
1956\end{array}$ & $\begin{array}{l}O A \\
N \\
O A \\
O A \\
O A \\
O A \\
O A \\
O \\
O N A \\
O A \\
N \\
O A \\
O \\
O A \\
O A \\
O A \\
O N A\end{array}$ & $\begin{array}{l}028607 \\
028731 \\
028855 \\
000383 \\
000899 \\
028886 \\
028917 \\
000859 \\
028103 \\
029134 \\
029165 \\
029179 \\
029289 \\
029320\end{array}$ & $\begin{array}{l}\text { Aspermont Wost } \\
\text { Assumption } \\
\text { Assumption South } \\
\text { Astin } \\
\text { Atak } \\
\text { Atapco } \\
\text { Atapco North } \\
\text { Atcherson } \\
\text { Atcheson } \\
\text { Atchley } \\
\text { Athens } \\
\text { Athens North } \\
\text { Athey } \\
\text { Athey East } \\
\text { Alies for: (020289) Athey (C }\end{array}$ & $\begin{array}{l}T \times 70 \\
T \times 40 \\
T \times 40 \\
T \times 70 \\
T \times 80 \\
T \times 80 \\
T \times 80 \\
T \times 80 \\
T \times 90 \\
T \times 70 \\
T \times 80 \\
T \times 50 \\
T \times 80 \\
T \times 80 \\
T\end{array}$ & $\begin{array}{l}433 \\
247 \\
247 \\
083 \\
371 \\
103 \\
103 \\
097 \\
097 \\
083 \\
389 \\
213 \\
371 \\
371\end{array}$ & $\begin{array}{l}\text { Stonowall } \\
\text { JIm Hogg } \\
\text { JIm Hogg } \\
\text { Coleman } \\
\text { Pocos } \\
\text { Crano } \\
\text { Crane } \\
\text { Cooke } \\
\text { Cooke } \\
\text { Coleman } \\
\text { Reoves } \\
\text { Honderson } \\
\text { Pocos } \\
\text { Pocos }\end{array}$ & $\begin{array}{l}1958 \\
1974 \\
1981 \\
1881 \\
1979 \\
1958 \\
1961 \\
1977 \\
1947 \\
1953 \\
1972 \\
1975 \\
1985\end{array}$ & $\begin{array}{l}O A \\
N \\
N \\
O N \\
O A \\
O A \\
O A \\
O A \\
O A \\
O \\
O A \\
N \\
N \\
O N A \\
O N A\end{array}$ \\
\hline 024458 & Askansas Clty North & $7 \times 40$ & 427 & Starr & 1978 & $\mathbf{N}$ & 008394 & Athey East (Naw) & $T \times 80$ & 371 & Pecos & 1984 & 0 \\
\hline 024577 & Aflodgo & $7 \times 75$ & 081 & Coke & 1948 & $O A$ & 029351 & Atkins & $T \times 70$ & 428 & Stephens & 1950 & 0 \\
\hline $\begin{array}{l}024577 \\
024577\end{array}$ & $\begin{array}{l}\text { Arlodgo } \\
\text { Arlodgo }\end{array}$ & $\begin{array}{l}T \times 70 \\
T \times 70\end{array}$ & $\begin{array}{l}133 \\
353\end{array}$ & $\begin{array}{l}\text { Eastiand } \\
\text { Nolan }\end{array}$ & $\begin{array}{l}1979 \\
1848\end{array}$ & $\begin{array}{l}\text { ONA } \\
O A\end{array}$ & $\begin{array}{l}029356 \\
029382\end{array}$ & $\begin{array}{l}\text { Atkins-Fleming } \\
\text { Atkinson }\end{array}$ & TX85 & $\begin{array}{l}263 \\
255\end{array}$ & Kent & 1972 & OA \\
\hline 024608 & Arleno & $T \times 85$ & 169 & Garza & 1958 & $O A$ & 029382 & Alkinson & $T \times 20$ & 297 & Llve Oak & 1969 & $\mathbf{N}$ \\
\hline $\begin{array}{l}024608 \\
024608\end{array}$ & $\begin{array}{l}\text { Arlono } \\
\text { Arlone }\end{array}$ & $\begin{array}{l}T \times 75 \\
T \times 70\end{array}$ & $\begin{array}{l}413 \\
417\end{array}$ & $\begin{array}{l}\text { Schlelcher } \\
\text { Shackelford }\end{array}$ & $\begin{array}{l}1983 \\
1979\end{array}$ & $\underset{N}{N}$ & $\begin{array}{l}029382 \\
000384\end{array}$ & $\begin{array}{l}\text { Atkinson } \\
\text { Atkinson Island }\end{array}$ & $\begin{array}{l}T \times 75 \\
T \times 30\end{array}$ & $\begin{array}{l}451 \\
071\end{array}$ & $\begin{array}{l}\text { Tom Green } \\
\text { Chambers }\end{array}$ & $\begin{array}{l}1052 \\
1980\end{array}$ & $\begin{array}{l}\ddot{0} \\
0 A\end{array}$ \\
\hline $\begin{array}{l}000375 \\
024639\end{array}$ & $\begin{array}{l}\text { Arlono North } \\
\text { Arlington }\end{array}$ & $\begin{array}{l}7 \times 70 \\
7 \times 80\end{array}$ & $\begin{array}{l}417 \\
503\end{array}$ & $\begin{array}{l}\text { Shackolford } \\
\text { Young }\end{array}$ & $\begin{array}{l}1979 \\
1962\end{array}$ & $\stackrel{O A}{O A}$ & $\begin{array}{l}029413 \\
029444\end{array}$ & $\begin{array}{l}\text { Atkinson NE } \\
\text { Atkinson NW }\end{array}$ & $\begin{array}{l}T \times 75 \\
T \times 10\end{array}$ & $\begin{array}{l}451 \\
013\end{array}$ & $\begin{array}{l}\text { Tom Groen } \\
\text { Atascosa }\end{array}$ & $\begin{array}{l}1959 \\
1980\end{array}$ & $\mathrm{OA}_{N}$ \\
\hline 003788 & $\begin{array}{l}\text { Arllo } \\
\text { Allas for: }(003828) \text { Dowey }\end{array}$ & $\begin{array}{l}\text { TX80 } \\
\text { Lake South }\end{array}$ & $\begin{array}{l}173 \\
1 \mathrm{COMB}\end{array}$ & B] & & & $\begin{array}{l}029444 \\
029475\end{array}$ & $\begin{array}{l}\text { Atkjnson NW } \\
\text { AtkInson West }\end{array}$ & $\begin{array}{l}T \times 20 \\
T \times 20\end{array}$ & $\begin{array}{l}297 \\
297\end{array}$ & $\begin{array}{l}\text { Livo Oak } \\
\text { Live Oak }\end{array}$ & $\begin{array}{l}1972 \\
1978\end{array}$ & $\stackrel{N}{N}$ \\
\hline 024818 & Armor & $T \times 80$ & 103 & Crane & 1955 & $O A$ & 028475 & Atkinson West & $T \times 75$ & 451 & Tom Groon & 1985 & $O A$ \\
\hline $\begin{array}{l}024980 \\
000280\end{array}$ & $\begin{array}{l}\text { Armour } \\
\text { Armray }\end{array}$ & $\begin{array}{l}T \times 30 \\
T \times 70\end{array}$ & $\begin{array}{l}321 \\
143\end{array}$ & $\begin{array}{l}\text { Matagorda } \\
\text { Erath }\end{array}$ & $\begin{array}{l}1938 \\
1979\end{array}$ & $\stackrel{O}{N}$ & $\begin{array}{l}029568 \\
028723\end{array}$ & $\begin{array}{l}\text { Allanta South } \\
\text { Atles }\end{array}$ & $\begin{array}{l}T \times 60 \\
T \times 40\end{array}$ & $\begin{array}{l}087 \\
131\end{array}$ & $\begin{array}{l}\text { Cass } \\
\text { Duval }\end{array}$ & $\begin{array}{l}1982 \\
1950\end{array}$ & $\begin{array}{l}\text { N } \\
\text { ONA }\end{array}$ \\
\hline 025042 & Armstiong & TXB5 & 101 & Cottle & 1982 & $\mathrm{~N}$ & 029723 & Atloo & $T \times 10$ & 283 & La Sallo & 1983 & $\mathrm{~N}$ \\
\hline $\begin{array}{l}025042 \\
000376\end{array}$ & $\begin{array}{l}\text { Armstrong } \\
\text { Armstrong NW }\end{array}$ & $\begin{array}{l}T \times 40 \\
7 \times 40\end{array}$ & $\begin{array}{l}247 \\
247\end{array}$ & Jim Hogg & $\begin{array}{l}1943 \\
1979\end{array}$ & ONA & $\begin{array}{l}029754 \\
008427\end{array}$ & $\begin{array}{l}\text { Atloo NE } \\
\text { Atoll }\end{array}$ & $\begin{array}{l}T \times 40 \\
\text { TX90 }\end{array}$ & $\begin{array}{l}131 \\
503\end{array}$ & $\begin{array}{l}\text { Duvel } \\
\text { Young }\end{array}$ & $\begin{array}{l}1963 \\
1984\end{array}$ & $\stackrel{N}{N}$ \\
\hline 025104 & Armstrong South & $T \times 40$ & 247 & Jim Hogg & 1948 & ON & 029878 & Atrice & $T \times 75$ & 451 & Tom Groen & 1954 & ONA \\
\hline $\begin{array}{l}025118 \\
025187\end{array}$ & $\begin{array}{l}\text { Armstrong West } \\
\text { Arneckevillo }\end{array}$ & $\begin{array}{l}T \times 40 \\
T \times 20\end{array}$ & $\begin{array}{l}247 \\
123\end{array}$ & $\begin{array}{l}\text { JIm Hogo } \\
\text { De witt }\end{array}$ & $\begin{array}{l}1973 \\
1950\end{array}$ & N & 029971 & $\begin{array}{l}\text { Alloyac } \\
\text { Altoyec }\end{array}$ & $\begin{array}{l}T \times 60 \\
T \times 60\end{array}$ & 347 & Nacogdoches & 1885 & $\mathrm{OA}_{\mathrm{N}}$ \\
\hline 025228 & $\begin{array}{l}\text { Arneckavillo } \\
\text { Arneckovillo East }\end{array}$ & $7 \times 20$ & $\begin{array}{l}123 \\
123\end{array}$ & De WItt & $\begin{array}{l}1950 \\
1964\end{array}$ & $\mathrm{ONA}^{\mathrm{NA}}$ & 010663 & Attoyac West & $\begin{array}{l}1 \times 60 \\
T \times 60\end{array}$ & $\begin{array}{l}401 \\
401\end{array}$ & $\begin{array}{l}\text { Rusk } \\
\text { Rusk }\end{array}$ & $\begin{array}{l}1958 \\
1985\end{array}$ & $\underset{\mathbf{N}}{\mathbf{N}}$ \\
\hline 001873 & Arnockaville NW & $T \times 20$ & 123 & De Witt & 1982 & $\mathbf{N}$ & 011803 & Atwell & $T \times 70$ & 059 & Callahan & 1987 & OA \\
\hline $\begin{array}{l}000898 \\
025258\end{array}$ & $\begin{array}{l}\text { Arneckovillo SE } \\
\text { Arneckovilio South }\end{array}$ & $\begin{array}{l}T \times 20 \\
T \times 20\end{array}$ & $\begin{array}{l}123 \\
123\end{array}$ & $\begin{array}{l}\text { De Witt } \\
\text { De Witt }\end{array}$ & $\begin{array}{l}1981 \\
1953\end{array}$ & $\stackrel{N}{N}$ ONA & $\begin{array}{l}030002 \\
030219\end{array}$ & $\begin{array}{l}\text { Atwood } \\
\text { Atwood SW }\end{array}$ & $\begin{array}{l}T \times 70 \\
T \times 70\end{array}$ & $\begin{array}{l}441 \\
441\end{array}$ & $\begin{array}{l}\text { Taylor } \\
\text { Taylor }\end{array}$ & $\begin{array}{l}1949 \\
1856\end{array}$ & $\begin{array}{l}0 \\
0\end{array}$ \\
\hline 025200 & Arnott Aroa & TXso & 293 & Limestone & 1961 & 0 & 030250 & Atwood West & Tx70 & 441 & Taylor & 1953 & OA \\
\hline 025538 & Arnim & $T \times 30$ & 149 & Fayotte & 1945 & ONA & 030374 & Auburn & TX70 & 428 & Stéphens & 1984 & $O A$ \\
\hline 025569 & Arnim Eest & $T \times 30$ & 148 & Fayotte & 1950 & ONA & 000385 & Audabon & TX90 & 497 & Wlse & 1981 & OA \\
\hline $\begin{array}{l}025800 \\
025631\end{array}$ & $\begin{array}{l}\text { Arnn } \\
\text { Arno }\end{array}$ & $\begin{array}{l}\text { TX70 } \\
\text { TX80 }\end{array}$ & $\begin{array}{l}143 \\
389\end{array}$ & $\begin{array}{l}\text { Erath } \\
\text { Reoves }\end{array}$ & $\begin{array}{l}1948 \\
1956\end{array}$ & $\stackrel{0}{\text { ON }}$ & $\begin{array}{l}030436 \\
030467\end{array}$ & $\begin{array}{l}\text { Audas-Grahem } \\
\text { Audra }\end{array}$ & $\begin{array}{l}\text { TX70 } \\
T \times 70\end{array}$ & $\begin{array}{l}44 t \\
441\end{array}$ & $\begin{array}{l}\text { Taytor } \\
\text { Taylor }\end{array}$ & $\begin{array}{l}1855 \\
1957\end{array}$ & $\begin{array}{l}O A \\
0\end{array}$ \\
\hline 025644 & Aino North & rX80 & 389 & Reeves & 1978 & $O A$ & 030498 & Audra South & $T \times 70$ & 441 & Taylor & 1958 & 0 \\
\hline 025724 & Arnold & TX90 & 337 & Montague & 1955 & 0 & 007246 & Audroy & $T \times 70$ & 133 & Eastland & 1802 & ONA \\
\hline 025724 & Arnold & TX20 & 489 & Victoria & 1978 & N & 013332 & Augustine & $\begin{array}{l}T \times 70 \\
T Y 70\end{array}$ & 133 & Eastland & 1989 & $O A$ \\
\hline $\begin{array}{l}025731 \\
025755\end{array}$ & $\begin{array}{l}\text { Arnold Bruner } \\
\text { Arnold-Davld }\end{array}$ & $\begin{array}{l}T \times 90 \\
T \times 40\end{array}$ & $\begin{array}{l}\mathbf{3 3 7} \\
\mathbf{2 7 3}\end{array}$ & $\begin{array}{l}\text { Montague } \\
\text { Kleberg }\end{array}$ & $\begin{array}{l}1973 \\
1980\end{array}$ & OA & $\begin{array}{l}030653 \\
030684\end{array}$ & $\begin{array}{l}\text { Auld } \\
\text { Auld-Shipmen }\end{array}$ & $\begin{array}{l}T \times 70 \\
T \times 10\end{array}$ & 353 & Nolan & 1057 & $\stackrel{0}{N}$ \\
\hline 025755 & Arnold-David & $T \times 40$ & 355 & Nueces & 1960 & ONA & 030715 & $\begin{array}{l}\text { Auld-Shipman } \\
\text { Aunt Em }\end{array}$ & TX70 & $\begin{array}{l}283 \\
429\end{array}$ & $\begin{array}{l}\text { La Salte } \\
\text { Stephons }\end{array}$ & $\begin{array}{l}1962 \\
1851\end{array}$ & OA \\
\hline 025786 & Arnold-David North & $T \times 40$ & 355 & Nusces & 1961 & ONA & 008087 & Auralia & $T \times 90$ & 275 & Knox & 1883 & $O A$ \\
\hline 025817 & Arnold-Mullo & $7 \times 40$ & 131 & Duval & 1970 & $O A$ & 030870 & Aurore & $T \times 40$ & 247 & Jim Hogg & 1952 & $\mathbf{N}$ \\
\hline 025941 & Arnold-Woldon & $T \times 10$ & 311 & McMullen & 1962 & $O A$ & 030875 & Aurora East & $T \times 40$ & 247 & Jim Hogg & 1971 & $\mathbf{N}$ \\
\hline 025972 & Arnold-Weldon South & TX10 & 311 & McMulien & 1963 & 0 & 000386 & Aurora North & $T \times 40$ & 247 & Jim Hogg & 1979 & $\mathbf{N}$ \\
\hline 026180 & $\begin{array}{l}\text { ARP } \\
\text { Arrlbs }\end{array}$ & $\begin{array}{l}T \times 60 \\
T \times 40\end{array}$ & $\begin{array}{l}423 \\
249\end{array}$ & $\begin{array}{l}\text { Smith } \\
\text { JIm Wolls }\end{array}$ & $\begin{array}{l}1954 \\
1057\end{array}$ & $\begin{array}{l}0 \\
0\end{array}$ & 000903 & $\begin{array}{l}\text { Ausa } \\
\text { Austox }\end{array}$ & $\begin{array}{l}\text { TX85 } \\
T \times 70\end{array}$ & 033 & Bordon & 1980 & $O A$ \\
\hline $\begin{array}{l}028344 \\
026375\end{array}$ & $\begin{array}{l}\text { Arrlba } \\
\text { Arrlba LIbra }\end{array}$ & $\begin{array}{l}1 \times 40 \\
T \times 70\end{array}$ & $\begin{array}{l}249 \\
353\end{array}$ & $\begin{array}{l}\text { Jlm Wolls } \\
\text { Nolan }\end{array}$ & $\begin{array}{l}1957 \\
1971\end{array}$ & ONA & $\begin{array}{l}007247 \\
030901\end{array}$ & $\begin{array}{l}\text { Austex } \\
\text { Austin }\end{array}$ & $\begin{array}{l}T \times 70 \\
T \times 90\end{array}$ & $\begin{array}{l}363 \\
503\end{array}$ & Palo Pinto & 1981 & ON \\
\hline $\begin{array}{l}026375 \\
026389\end{array}$ & Arrington & TX95 & 233 & $\begin{array}{l}\text { Nolan } \\
\text { Hutchinson }\end{array}$ & 1985 & ONA & 011050 & $\begin{array}{l}\text { Austin } \\
\text { Austin Bayou }\end{array}$ & $\begin{array}{l}\text { TX90 } \\
T \times 30\end{array}$ & 503 & Young & 1953 & OA \\
\hline $\begin{array}{l}026389 \\
026389\end{array}$ & Arrington & TX30 & $48 t$ & $\begin{array}{l}\text { Hutchinson } \\
\text { Wharton }\end{array}$ & 1977 & $O A$ & 030906 & $\begin{array}{l}\text { Austin Bayou } \\
\text { Austin Colloge }\end{array}$ & $\begin{array}{l}T \times 30 \\
T \times 30\end{array}$ & $\begin{array}{l}039 \\
039\end{array}$ & Brazoria & $\begin{array}{l}1985 \\
1976\end{array}$ & $\underset{N}{N}$ \\
\hline 012455 & Arrington West & $T \times 95$ & 233 & Hutehinson & 1888 & $O A$ & 030963 & Austin Pierce & $7 \times 10$ & 177 & Gonzales & 1901 & $O A$ \\
\hline 026406 & Arrlota & $T \times 30$ & 189 & Herdln & 1932 & ONA & 030932 & Austin-Brixay & $T \times 90$ & 077 & Clay & 1989 & $O A$ \\
\hline 026437 & Arrlola South & $T \times 30$ & 199 & Hardin & 1986 & ONA & 007248 & Austln-Feilf & $T \times 70$ & 253 & Jones & 1983 & $O A$ \\
\hline 026468 & Arron & $T \times 90$ & 237 & Jack & 1958 & $\circ$ & 031025 & Austonlo & $T \times 60$ & 225 & Houston & 1965 & 0 \\
\hline $\begin{array}{l}026561 \\
026561\end{array}$ & $\begin{array}{l}\text { Arrowhead } \\
\text { Arrowhead }\end{array}$ & $\begin{array}{l}T \times 90 \\
T \times 90\end{array}$ & $\begin{array}{l}000 \\
077\end{array}$ & $\begin{array}{l}\text { Archor } \\
\text { Clay }\end{array}$ & $\begin{array}{l}1880 \\
1880\end{array}$ & $\begin{array}{l}O A \\
O A\end{array}$ & $\begin{array}{l}010014 \\
031033\end{array}$ & $\begin{array}{l}\text { Austonio NE } \\
\text { Austwell }\end{array}$ & $\begin{array}{l}T \times 60 \\
T \times 20\end{array}$ & $\begin{array}{l}225 \\
391\end{array}$ & $\begin{array}{l}\text { Houston } \\
\text { Refugio }\end{array}$ & $\begin{array}{l}1985 \\
1975\end{array}$ & $\begin{array}{l}O \\
\text { ONA }\end{array}$ \\
\hline 026561 & Arrowhead & $7 \times 40$ & 215 & Hidalgo & 1948 & ONA & 010664 & Austwell SW & $T \times 20$ & 391 & Refugio & 1986 & $O A$ \\
\hline 026581 & Arrowhead & $T \times 40$ & 427 & Starr & 1948 & $O A$ & 031043 & Austwell West & TX20 & 391 & Rofugio & 1977 & N \\
\hline 000294 & Arrowhend Ranch & $T \times 40$ & 215 & Hidalgo & 1881 & $\mathbf{N}$ & 006524 & Auta & $T \times 80$ & 371 & Pecos & 1984 & ONA \\
\hline 028592 & Arroyo & $T \times 20$ & 469 & Victoria & 1970 & $\mathbf{N}$ & 009385 & Auzia & TX10 & 507 & Zavala & 1984 & $\mathbf{N}$ \\
\hline $\begin{array}{l}026623 \\
006218\end{array}$ & $\begin{array}{l}\text { Arroyo Colorado } \\
\text { Arroyo Colozado North }\end{array}$ & $\begin{array}{l}T \times 40 \\
T \times 40\end{array}$ & $\begin{array}{l}489 \\
489\end{array}$ & $\begin{array}{l}\text { Willacy } \\
\text { Willacy }\end{array}$ & $\begin{array}{l}1870 \\
1881\end{array}$ & $\stackrel{N}{N}$ & $\begin{array}{l}031273 \\
013814\end{array}$ & Ava Loo & $\begin{array}{l}T \times 85 \\
T \times 50\end{array}$ & 219 & Hockley & 1968 & $\mathrm{O}$ \\
\hline $\begin{array}{l}006218 \\
026654\end{array}$ & $\begin{array}{l}\text { Arroyo Colorado North } \\
\text { Arroyo Garza }\end{array}$ & $\begin{array}{l}T \times 40 \\
T \times 40\end{array}$ & $\begin{array}{l}489 \\
505\end{array}$ & $\begin{array}{l}\text { Willacy } \\
\text { Zapata }\end{array}$ & $\begin{array}{l}1981 \\
1968\end{array}$ & $\begin{array}{l}\mathbf{N} \\
\mathbf{N}\end{array}$ & $\begin{array}{l}013814 \\
001977\end{array}$ & $\begin{array}{l}\text { Aventura Ranch } \\
\text { Averitt }\end{array}$ & $\begin{array}{l}\text { TX50 } \\
7 \times 80\end{array}$ & $\begin{array}{l}467 \\
135\end{array}$ & $\begin{array}{l}\text { Van Zandt } \\
\text { Ector }\end{array}$ & $\begin{array}{l}1980 \\
1982\end{array}$ & $\begin{array}{c}N \\
0\end{array}$ \\
\hline 026885 & Arroyo Grando & $T \times 40$ & 427 & Starr & 1976 & $O A$ & 010015 & Avery Kibbe & $T \times 40$ & 248 & Jim Walls & 1885 & $\mathbf{N}$ \\
\hline 010012 & Arroyo Los Olmos & $T \times 40$ & 427 & Starr & 1985 & ONA & 031800 & Aviators & $T \times 40$ & 479 & Wabb & 1022 & ONA \\
\hline 026688 & $\begin{array}{l}\text { Arroyo Nogro } \\
\text { Allas for: }(546119) \text { Pearsall }\end{array}$ & $\begin{array}{c}T \times 10 \\
\text { [CoMB] }\end{array}$ & 507 & Zavala & & & 031831 & $\begin{array}{l}\text { Avlators North } \\
\text { Alles for: }(031800) \text { Avlators }\end{array}$ & $\begin{array}{l}\mathrm{TX} 40 \\
{[\text { [сомв] }}\end{array}$ & 479 & Webb & & \\
\hline 008423 & $\begin{array}{l}\text { Arroyo Rojo } \\
\text { Artbow }\end{array}$ & $\begin{array}{r}T \times 20 \\
T \times 85\end{array}$ & 239 & Jackson & 1984 & $N$ & 013182 & Avlators North (Now) & $T \times 40$ & 479 & Wobb & 1981 & $\mathbf{N}$ \\
\hline & Artbow & $7 \times 85$ & $16 \theta$ & Garza & 1977 & OA & 031845 & Aviators South & $T \times 40$ & 470 & Wobb & 1076 & $\mathbf{N}$ \\
\hline 000370 & Artaburn & $T \times 70$ & 363 & Pato Pinto & 1978 & $\mathbf{N}$ & 031845 & Avlators South & $T \times 40$ & 505 & Zapata & 1876 & $\mathbf{N}$ \\
\hline 000378 & Antoburn & $T \times 70$ & 387 & Parkar & 1978 & $N$ & 031821 & Avinger & $T \times 60$ & 087 & Cass & 1874 & ONA \\
\hline 028809 & Artosla Wolts Wost & TX10 & 283 & La Sallo & 1867 & $\mathbf{N}$ & 031821 & Avinger & $T \times 60$ & 315 & Marion & 1974 & ONA \\
\hline 026845 & Artosian Croek & TX20 & 391 & Rofuglo & 1980 & $\mathbf{N}$ & 031882 & Avis & $T \times 90$ & 237 & Jack & 1955 & ONA \\
\hline 026002 & Arthur & TX90 & 009 & Archer & 1986 & 0 & 031893 & Avis East & $T \times 90$ & 237 & Jack & 1852 & $O A$ \\
\hline 026802 & Arthur & TX85 & 033 & Bordon & 1854 & 0 & 031924 & Avls North & $T \times 80$ & 237 & Jack & 1953 & ONA \\
\hline 026833 & Arthur Harvey & TX30 & 477 & Washington & 1952 & $O A$ & 031955 & Avls South & TX90 & 237 & Jack & 1954 & $O A$ \\
\hline 027057 & Arvanna & TX85 & 115 & Dawson & 1953 & 0 & 031986 & Avis West & TX90 & 237 & Jack & 1953 & 0 \\
\hline 013813 & Arvanna North & TX85 & 115 & Dawson & 1991 & $O A$ & 014080 & Avo Grando & TX30 & 481 & Wharton & 1991 & $\mathbf{N}$ \\
\hline 000381 & Ascot & TX70 & 083 & Comanche & 1972 & $N$ & 032017 & Avoca & $T \times 70$ & 253 & Jones & 1937 & OA \\
\hline 0271119 & Ash & $7 \times 75$ & 399 & Runnels & 1968 & $O A$ & 032078 & Avaca North & $T \times 70$ & 253 & Jones & 1840 & $O A$ \\
\hline 027336 & Ash North & $T \times 50$ & 467 & Van Zandt & 1958 & $\mathbf{N}$ & 032105 & Avoca NW & $T \times 70$ & 253 & Jonos & 1973 & 0 \\
\hline 027387 & Aahburn & $\begin{array}{l}T \times 70 \\
x y 0\end{array}$ & 049 & Brown & $\begin{array}{l}1983 \\
1860\end{array}$ & $\stackrel{\circ}{\circ}$ & 032141 & Avoca Wast & $T \times 70$ & 253 & Jones & 1841 & 0 \\
\hline 027398 & Ashby & $T \times 30$ & 321 & Matagorda & 1860 & ONA & 032203 & Avondale & TX50 & 439 & Tarrant & 1993 & $\mathbf{N}$ \\
\hline 003157 & Ashby East & $\begin{array}{l}T \times 30 \\
T \times 50\end{array}$ & $\begin{array}{l}321 \\
231\end{array}$ & $\begin{array}{l}\text { Matagorda } \\
\text { Hur.: }\end{array}$ & $\begin{array}{l}1983 \\
1952\end{array}$ & $\begin{array}{c}N \\
0\end{array}$ & 007001 & Awalt & $T \times 90$ & 237 & Jack & 1982 & $O A$ \\
\hline 027420 & Ashby-Remsey & TX50 & 231 & $\begin{array}{l}\text { Hur:: } \\
\text { Wise }\end{array}$ & $\begin{array}{l}1952 \\
1958\end{array}$ & 0 & 000387 & Awtry & $T \times 90$ & 008 & Archer & 1877 & $O A$ \\
\hline 027491 & Asho & $T \times 90$ & 497 & Wise & 1958 & ON & 032335 & Axalson & $T \times 70$ & 441 & Taylor & 1852 & 0 \\
\hline 027522 & Asho Ranch & TX90 & 497 & Wiso & 1863 & o & 032458 & Aycock & $T \times 85$ & 188 & & 1955 & $O A$ \\
\hline 027708 & Asherton & $\begin{array}{l}T \times 10 \\
T Y 10\end{array}$ & 127 & Dimmit & 1955 & ONA & 032552 & Aylesworth & TX80 & 389 & Reevos & 1953 & ONA \\
\hline 027739 & Ashorton East & $T \times 10$ & 127 & $\begin{array}{l}\text { Dimmit } \\
\text { Lavaca }\end{array}$ & $\begin{array}{l}1968 \\
1982\end{array}$ & ONA & 032707 & Azalea & TX80 & 103 & Crane & 1958 & $\begin{array}{l}\text { ONA } \\
\text { ONA }\end{array}$ \\
\hline 027750 & Ashford & $T \times 20$ & 285 & $\begin{array}{l}\text { Lavaca } \\
\text { Jones }\end{array}$ & 1882 & ONA & 032707 & Azalea & TX80 & 329 & Midland & 1950 & ONA \\
\hline 027894 & Aahloy & $T \times 70$ & 253 & Jones & 1960 & 0 & 011052 & Azaloa East & TX80 & 329 & Midland & 1985 & ONA \\
\hline 027804 & Aahioy & $T \times 20$ & 285 & Laveca & 1889 & $N$ & 032769 & Azaloa South & TX80 & 329 & & 1857 & $\mathbf{N}$ \\
\hline 004585 & Ashloy Mlzer & $\begin{array}{l}T \times 50 \\
T \times 70\end{array}$ & 213 & $\begin{array}{l}\text { Henderson } \\
\text { Stonewall }\end{array}$ & $\begin{array}{l}1983 \\
1953\end{array}$ & $O A$ & $\begin{array}{l}000904 \\
032738\end{array}$ & Azales Woat & TX80 & 328 & Midland & 1981 & $O A$ \\
\hline $\begin{array}{l}028019 \\
000382\end{array}$ & ro & $\begin{array}{l}\text { TX70 } \\
\text { TX90 }\end{array}$ & $\begin{array}{l}433 \\
503\end{array}$ & $\begin{array}{l}\text { Stonowall } \\
\text { Young }\end{array}$ & $\begin{array}{l}1953 \\
t 880\end{array}$ & $\begin{array}{l}O A \\
O A\end{array}$ & $\begin{array}{l}032738 \\
007249\end{array}$ & $\begin{array}{l}\text { Azslos-Halo } \\
\text { Azlo South }\end{array}$ & $\begin{array}{l}T \times 80 \\
T \times 70\end{array}$ & 328 & Midland & 1883 & $\stackrel{\circ}{O N A}^{\circ}$ \\
\hline & $\begin{array}{l}\text { Ashtale } \\
\text { Ashton }\end{array}$ & TX75 & $\begin{array}{l}503 \\
398\end{array}$ & $\begin{array}{l}\text { Young } \\
\text { Runnols }\end{array}$ & 1951 & $O A$ & $\begin{array}{l}007248 \\
032800\end{array}$ & $\begin{array}{l}\text { Azlo South } \\
\text { Aztec }\end{array}$ & $\begin{array}{r}1 \times 70 \\
\times \times 85\end{array}$ & $\begin{array}{l}387 \\
501\end{array}$ & & & $\begin{array}{l}\text { ONA } \\
\text { OA }\end{array}$ \\
\hline $\begin{array}{l}028142 \\
028287\end{array}$ & $\begin{array}{l}\text { Ashton } \\
\text { Aehwood }\end{array}$ & $T \times 30$ & 321 & Matagorda & 1944 & $\mathbf{N}$ & 033522 & $\begin{array}{l}\text { Aztec } \\
B \& B\end{array}$ & $\begin{array}{l}1 \times 85 \\
T \times 75\end{array}$ & $\begin{array}{l}501 \\
399\end{array}$ & $\begin{array}{l}\text { Yoakum } \\
\text { Runnels }\end{array}$ & $\begin{array}{l}1989 \\
1972\end{array}$ & $\begin{array}{l}O A \\
O A\end{array}$ \\
\hline 012887 & Askew & $T \times 90$ & 237 & Jack & 1989 & $O A$ & 033522 & $B \& B$ & TX95 & 483 & Wheoler & 1982 & ONA \\
\hline 009165 & Asog & $T \times 40$ & 131 & Duval & 1885 & $\mathbf{N}$ & 011648 & B \& D & TX80 & 503 & You & 1887 & $\mathbf{N}$ \\
\hline 028483 & Aspormont & $r \times 70$ & 433 & Stonewall & 1940 & $\circ$ & 033615 & $\mathrm{BAH}$ & $7 \times 75$ & 105 & Crockelt & 1958 & $O A$ \\
\hline 028514 & Aspermont Eest & TX70 & 433 & Stonowall & 1959 & $\circ$ & 013183 & 882 & IX70 & 048 & Brown & 1989 & 0 \\
\hline & & $T \times 70$ & 433 & Stonowall & 1951 & $O A$ & 013183 & B \& L & TX70 & 429 & Stephens & 1982 & OA \\
\hline 02 & As & $T \times 70$ & 33 & tonowall & 1850 & & 033553 & $M$ & $\times 90$ & 337 & Aontague & 1953 & OA \\
\hline
\end{tabular}


TEXAS

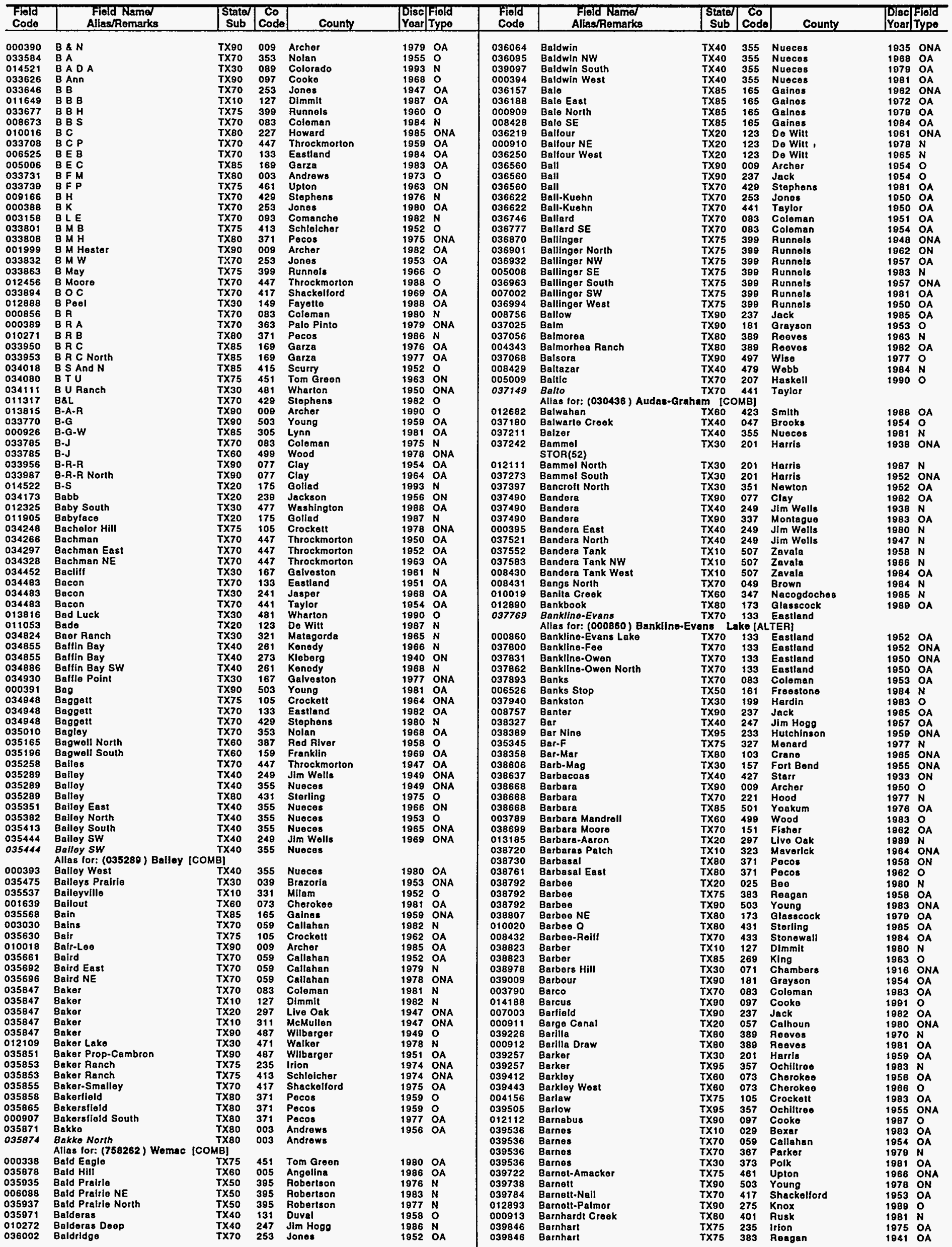


TEXAS

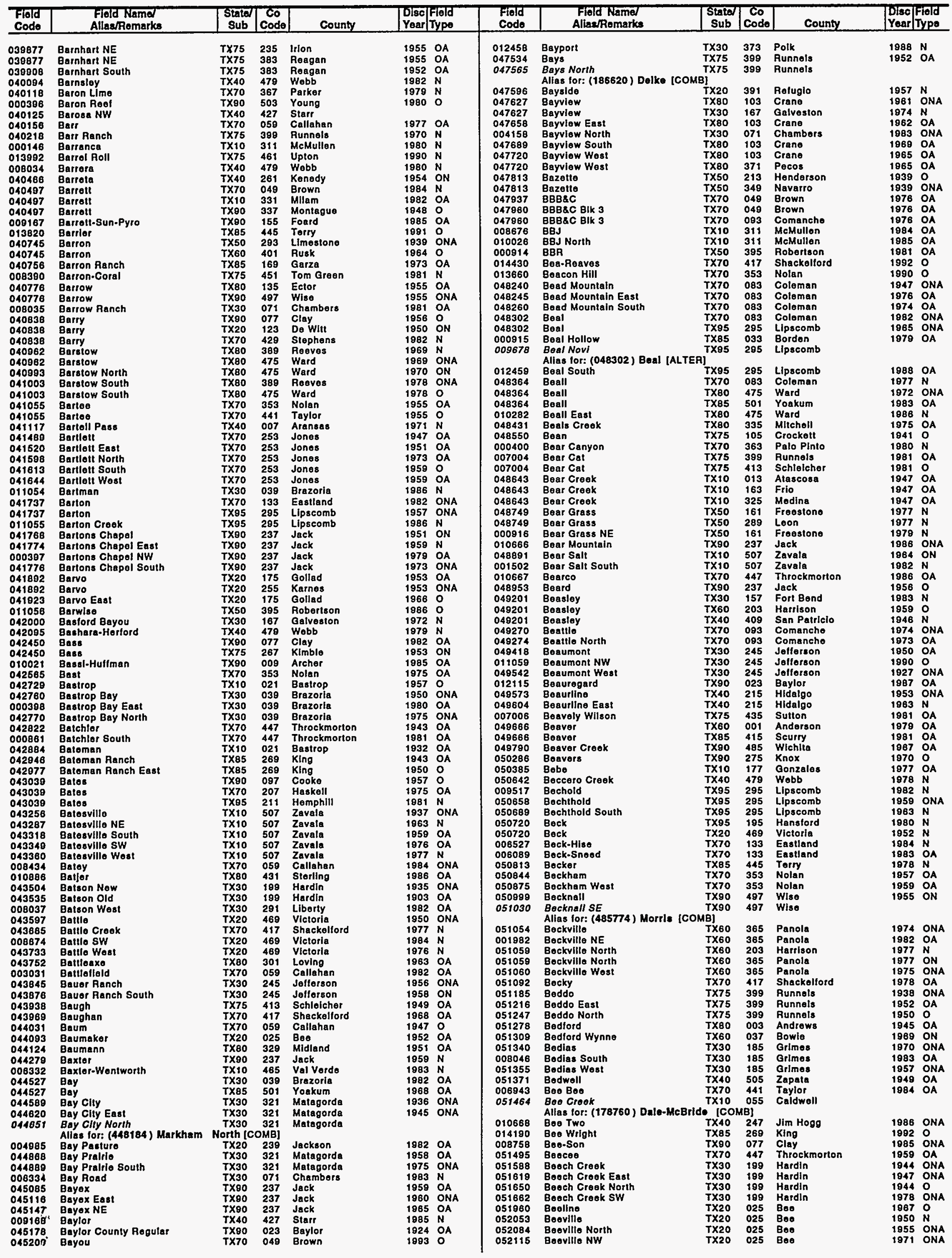


TEXAS

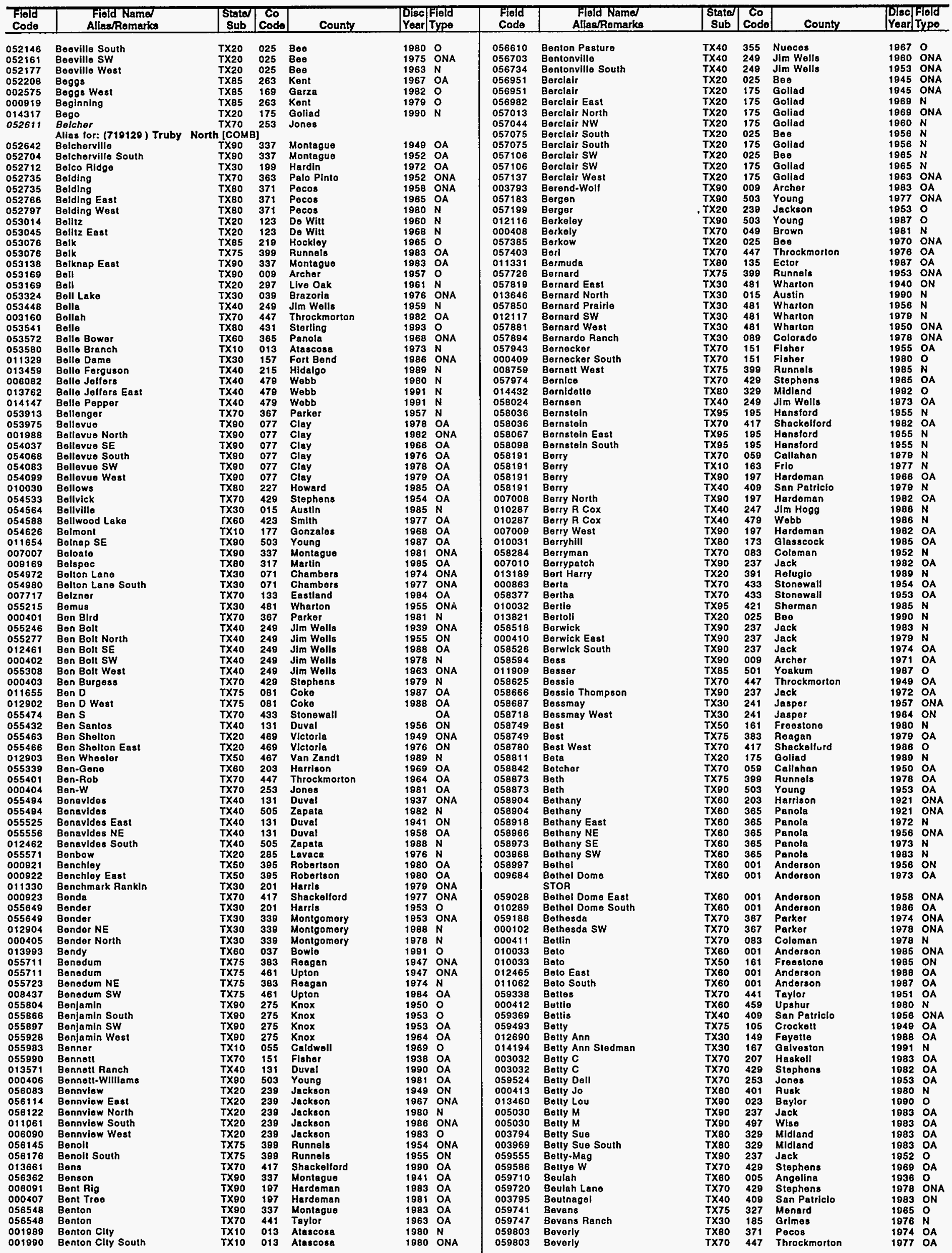


TEXAS

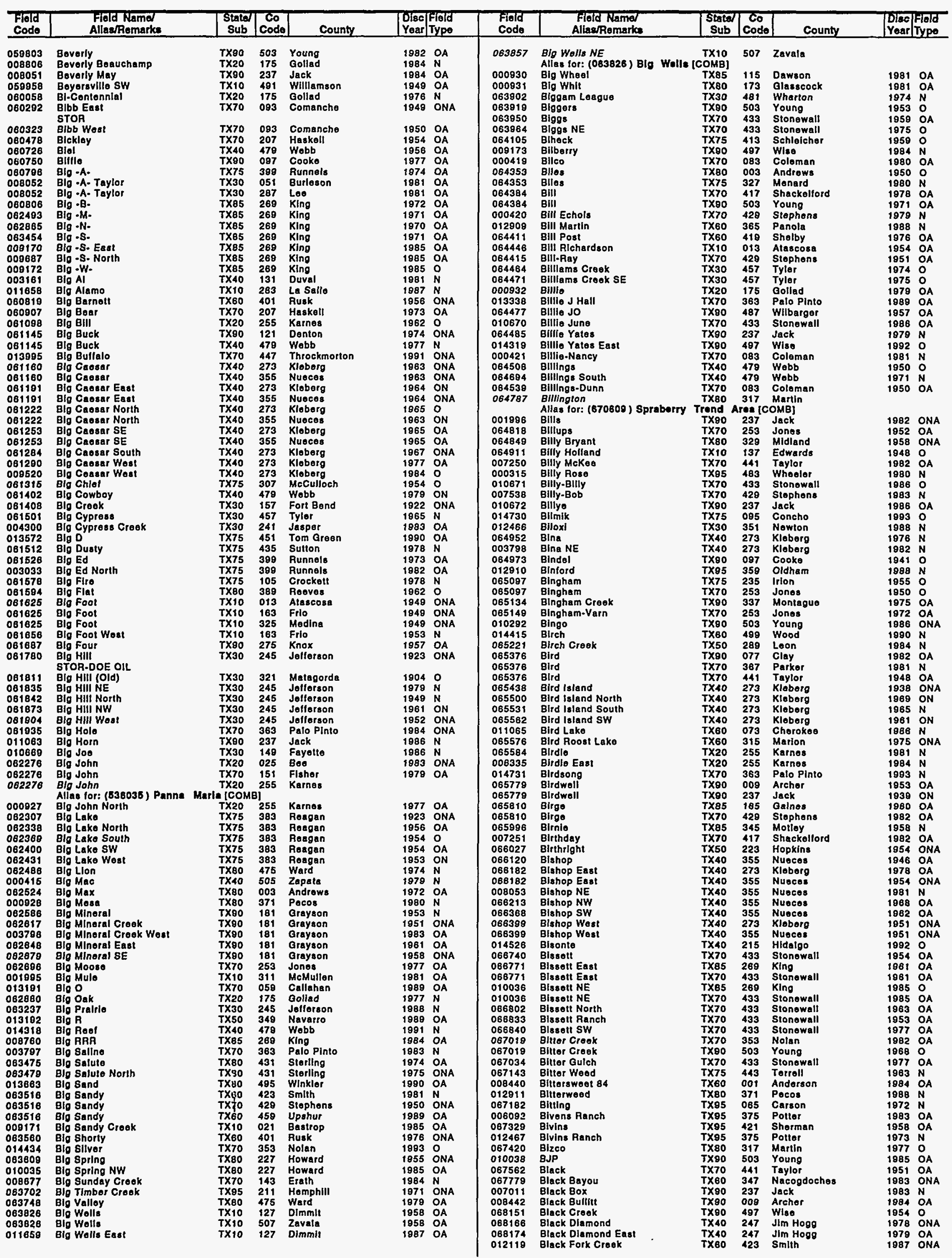




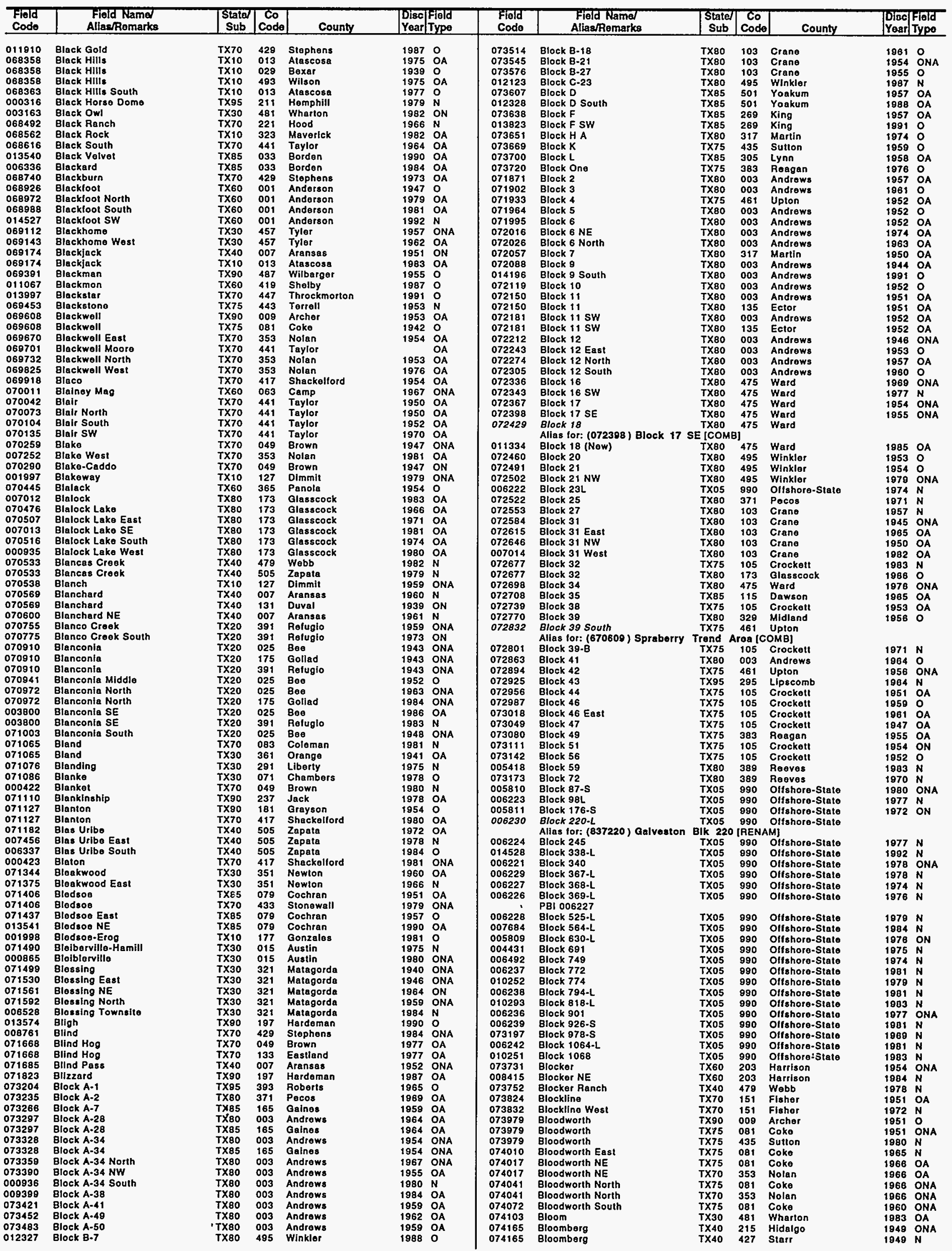


TEXAS

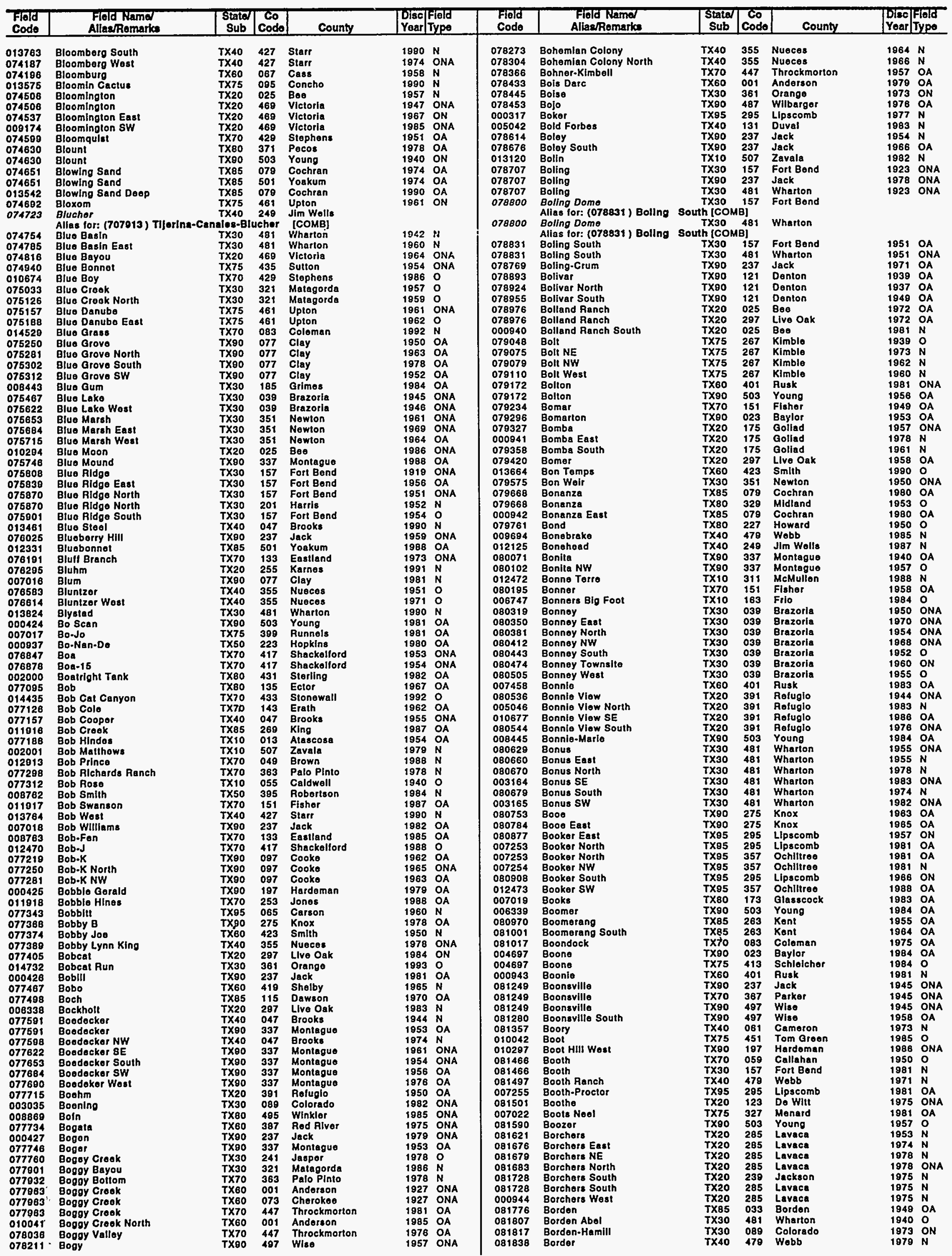


TEXAS

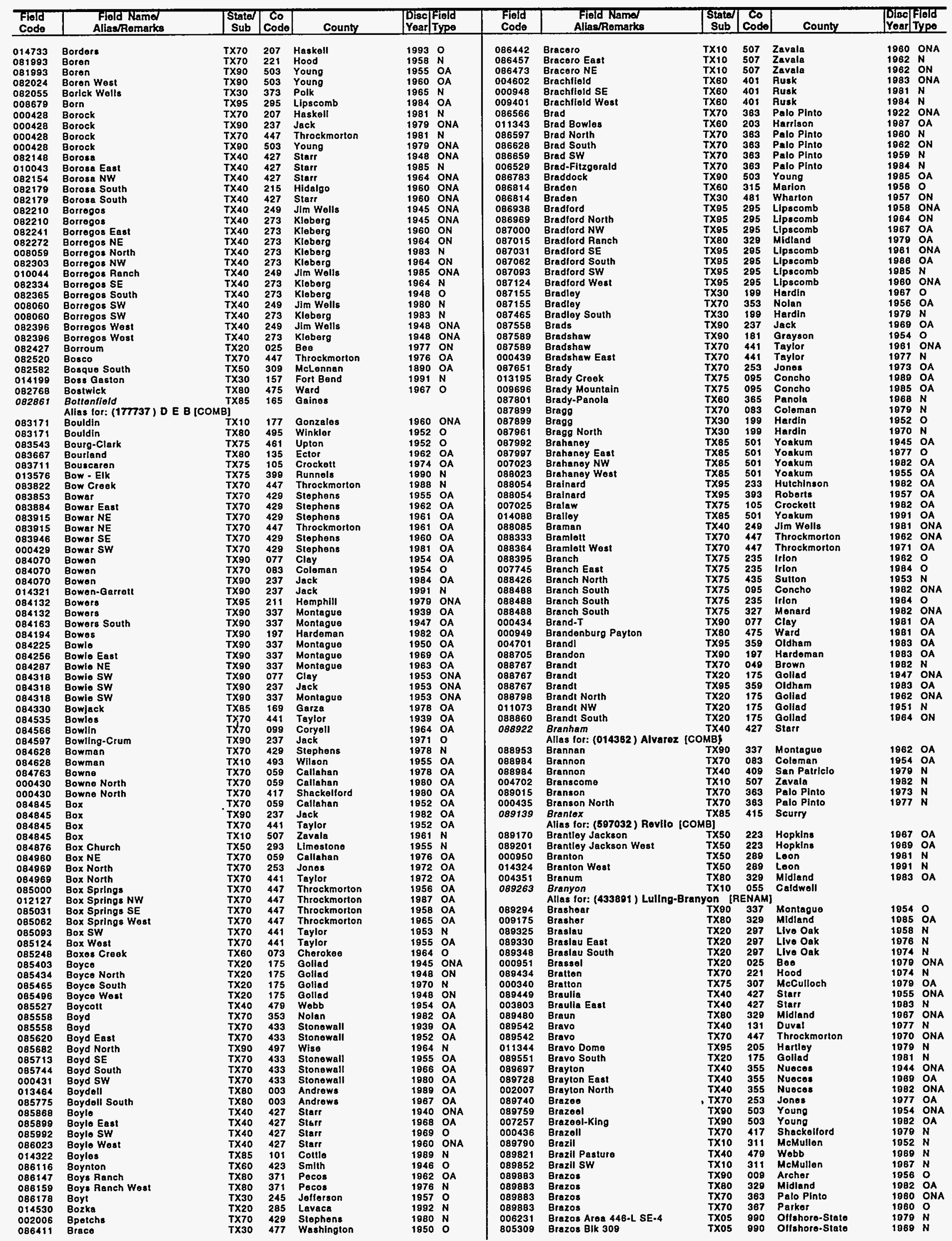


TEXAS

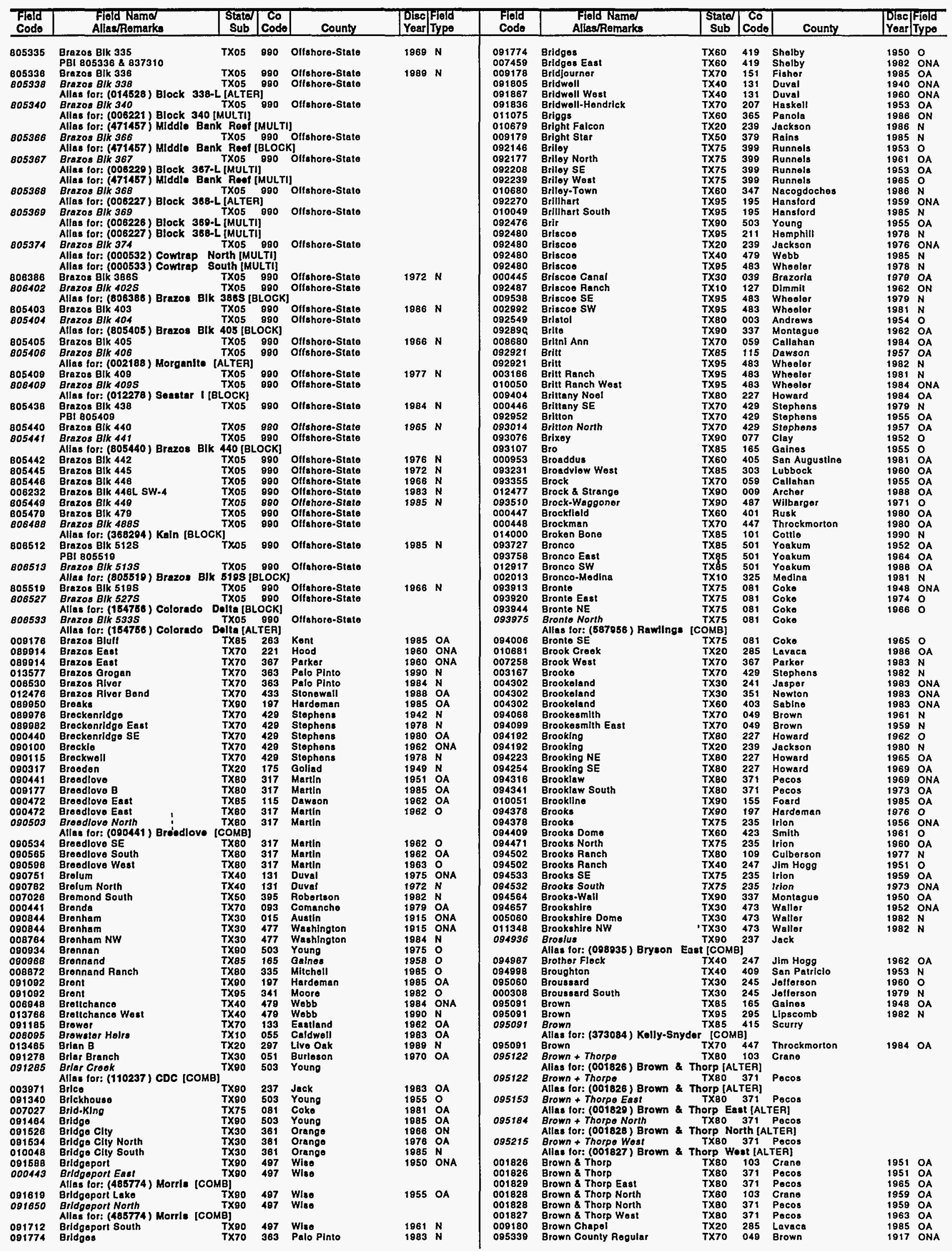


$\begin{array}{rl}\forall O & 898 \\ \forall O & 058 \\ \forall N O & 158 \\ \forall O & \text { จSB } \\ N & 6 L 6\end{array}$

O 9981

YNO 0261

Yo 0981

Yo 9881

YNo Es8:

N 5985

YNO $\checkmark S B 1$

N 8*81

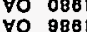

$\begin{array}{rl}\forall & 9881 \\ N & 1888\end{array}$

YNO $99 B 1$

$\forall$ O 886 !

YNO E9B!

VNO 8461

N

N $S \angle B 1$

No

YN $\quad$ BL81

Yo ESBI

YNO 2861

N 1881

Yo 2861

N 5868

o 2881

$\begin{array}{rl}N & 2761 \\ \forall O & 0581\end{array}$



Yo 8881

Y 2881

o Es6

Yo z96:

$\forall N O$ B961

No 19861

Vo 25801

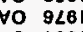

o 1581

o $\angle 261$

N 1881

VNO 9881

N $2 \angle 81$

YNO 6961

YNO 6961

$\begin{array}{lll}\forall O & 2561 \\ \forall O & 2961\end{array}$

$\forall 0$ 8261

$\forall O$
$\forall N O$ L 2961
OS6I

Y 0861

$\forall N O$ EE6

$N$ 28B

N $\mathrm{N}$ ช881

vo $\$ 96$

VNO 2561

vo 1861

$\forall N O$ SLQ

NO $026 !$

$\forall \mathrm{V} \varepsilon \varepsilon 61$

$\begin{array}{ll}\forall 0 & \varepsilon \varepsilon 61 \\ \forall 0 & 8+81\end{array}$

Yo 0681

7NO 6761

$\forall$ YNO 698

$\forall$ ҰO 2861

NO 9861

$\forall$ YO 8 ㄴ6

YNO SLBI

$\forall 0$ BS61

O 0861

N 8LBL

N 586

YNO 9961

vo 596

$N$
$Y 086$

Yo 086

NO 928

$N$ s981

N 2981

$\forall N O$ OS6!
NO $2 E 6 !$

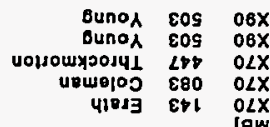

AEIO $2 \angle 0$ OEXI

BBE $s \angle X \perp$

lodser 5 stzer oex

you $2 E Z$ OEXI

soutug s91 s8X1

Bunoर EOS 0BX

Bunoᄉ EOS 06X1

Bunoג 005 o6x

bunol 809 06X1

O4Du०D 960 SLXI

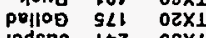

sodsor 1tz OEXI

jodsor ltz $0 E x 1$

zodsor tiv oEXI

jodsor ltz oex

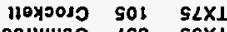

W曰I140 LSE S6X1

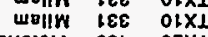

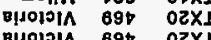

Buno人 EOS 06X1

Buno人 EOS $06 \times 1$

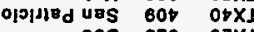

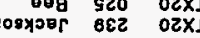

गouuny $662 \quad 5 \angle X 1$

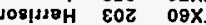

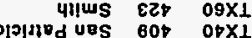

souor Egz OLX

บop

201א政 It $02 X 1$

\|өчगा| $58 \varepsilon$ 08XI

JO|AB it $0 \angle X 1$

-DगIIYOO LSE S6X

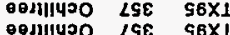

-

0.11490 258 S8X

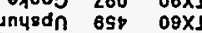

JOEJeqIIM LBV 08X

$\begin{array}{lll}\text { voly } & 692 & 58 \times 1 \\ \text { vold } & 195 & 52 \times 1\end{array}$

xoon $\angle E z$ SEX

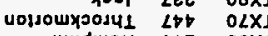

1114dw日 112 sex

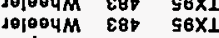

11000 112 $56 \times$

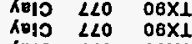

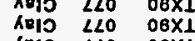

$\begin{array}{lll}0 \times 105 & 180 & 52 X 1 \\ \text { voo7 } & 682 & 0 S X 1\end{array}$

บ007 882 OSX

4007 682 os 051

tolर $\theta_{1}$ itt $02 x$



sunos sit s8X

0y00 $180 \quad 92 \times 1$

souun

voo 782 OSX

92180 691 58X

$990 M$ 6Lt asX

IeAno ist orx

IEAng IEL OPXI

sourax SSE OZX

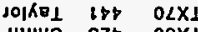

4yus $8 z t$ 09X

$2<0 \quad 08 \times 1$

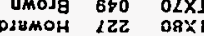

xou $9 \angle 2$ 06X

48 $34000 \quad 620 \quad 58 \times$

JOXJ10d $\angle 98 \quad 0<x_{1}$

18xpojo sol sLx

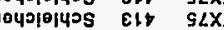

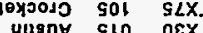

of

口p

вp

epJofelaW LZE OEXI

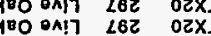

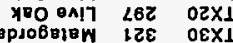

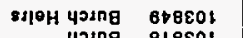
yo2ng 8t8عot

Bqing zE9EO

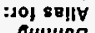

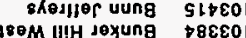

IIIH dexung $88 \varepsilon \varepsilon 0$

uexung zzerol

RouryeW-soBung 298200

MS reBung o9z201

MN J8Bung 86L80

o6pisg sobung orteot

robung 9etrot

getzot

อxจา Xכpung 296000

3S Bung 18620

MN Bung 056zo

4jJON Bung 616201

\begin{tabular}{ll}
3 8ung & 890800 \\
Bung & 888200 \\
\hline
\end{tabular}

จ18g-dung $z \angle 8 z 0$ L

48wing 088200

118201

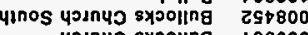

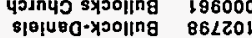

y00ing $s 6 \angle 201$

yoejo өpl4

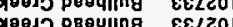

delng $\varepsilon \varepsilon<z 0$

Bopling 096000

41nos p1811ng 609201

psen|⿰氵 908010

pselng 8Lszol

pגeing 8LSzOL

1803 x०0.0 IIng $0 \angle 0500$

xө013 ing 915z0

ye825 Ing 9iszo

IIng $\varepsilon z \not z 00$

जs دO1ng $26 \varepsilon z 01$

1S89 Jejng $628 \angle 00$

มำ

a!ng 992201

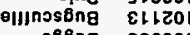

\begin{tabular}{cc} 
8B8ng & 856000 \\
Bng & 280200 \\
\hline
\end{tabular}

บoyn $080<00$

wnyng OEOLOO

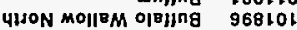

MollBM 이배 $\angle \angle 810$

MO|lBM 이뱅 S9810

unnos abujods olvung z2L10

3S erupds o뱅 $1>\angle 10$

4h

Bujds oleung $6 \angle 910$

Bujds olघyna BZ0L0O

4b910

JS 이밸 98910

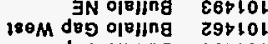

deg o|베

1003 이료 0008

xood5 이료 69810

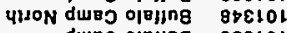

durg 이교 $8 \varepsilon \varepsilon 10$

이밸 990500

이료 9L210

ouens oueng 8LSE10

BIs! घueng 65010

ollons zueng
eprons bueng

دol40ng 880110

sures png
ag png
png

png 289001

र०ก: $2 \angle 900$

:

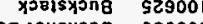

$1049 \times 3 n g$ 996000

(O)

43nos Ujouxगng 105001

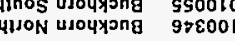

w10uxong sirool

usouxong ste001

SOM OXOXon 157000

Unos orexong Eszool

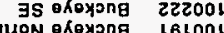

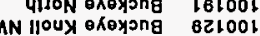

Ba 1100 प्र oxeyong 86000

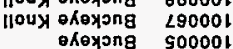

$\forall 0$ 0561

N $S \angle 61$

Yo L L61

$N \quad \nabla<61$

N 5861

$\forall N O$ YNO 296

$\forall$ Y $\mathbf{9 8 6 1}$

vo 8266

Yo 2568

$\forall 0$ 8ㅏ

$\forall$ Y 286

No 2961

$\forall 0$ 1961

Vo 0561

Vo 9561

$\begin{array}{rl}\forall O & 8561 \\ 7 \text { NO } & 5181\end{array}$

$\forall$ VNO $\angle>61$

o 6561

Y 8961

Yo 2961

$\forall \bigcirc 896$

Yo 9561

$\forall \mathrm{Y} 886$

$\begin{array}{ll}\forall 0 & 5961 \\ \forall & 5<61\end{array}$

$\forall 0$ 9v6

$\forall$ YNO 0961

$\forall 0$
$\forall 0$

o Bro:

Yo 8861

$\forall$ YO $626 \mathrm{t}$

Yo 9S61

vo 0961

vo 2861

$N$
$N$

N 6561

$\forall O$ L $\angle 261$

$\begin{array}{cc}N & 1+6 ! \\ N & 1 \rightarrow 6 !\end{array}$

$N$ ib6t

NO 0861

$\forall{ }_{N} 2261$

$\forall N O$ YTGL

YNO 1261

YNO $2: 261$

YNO 0961

$\begin{array}{cc}N & 6261 \\ 0 & 2861\end{array}$

YNO 9281

$N$
$N$
$N$

Yo 1864

$\forall 0$ \& 886

O OSB!

$\begin{array}{ll}N & \$ 186 \\ N & 68 B t \\ N & \end{array}$ 
TEXAS

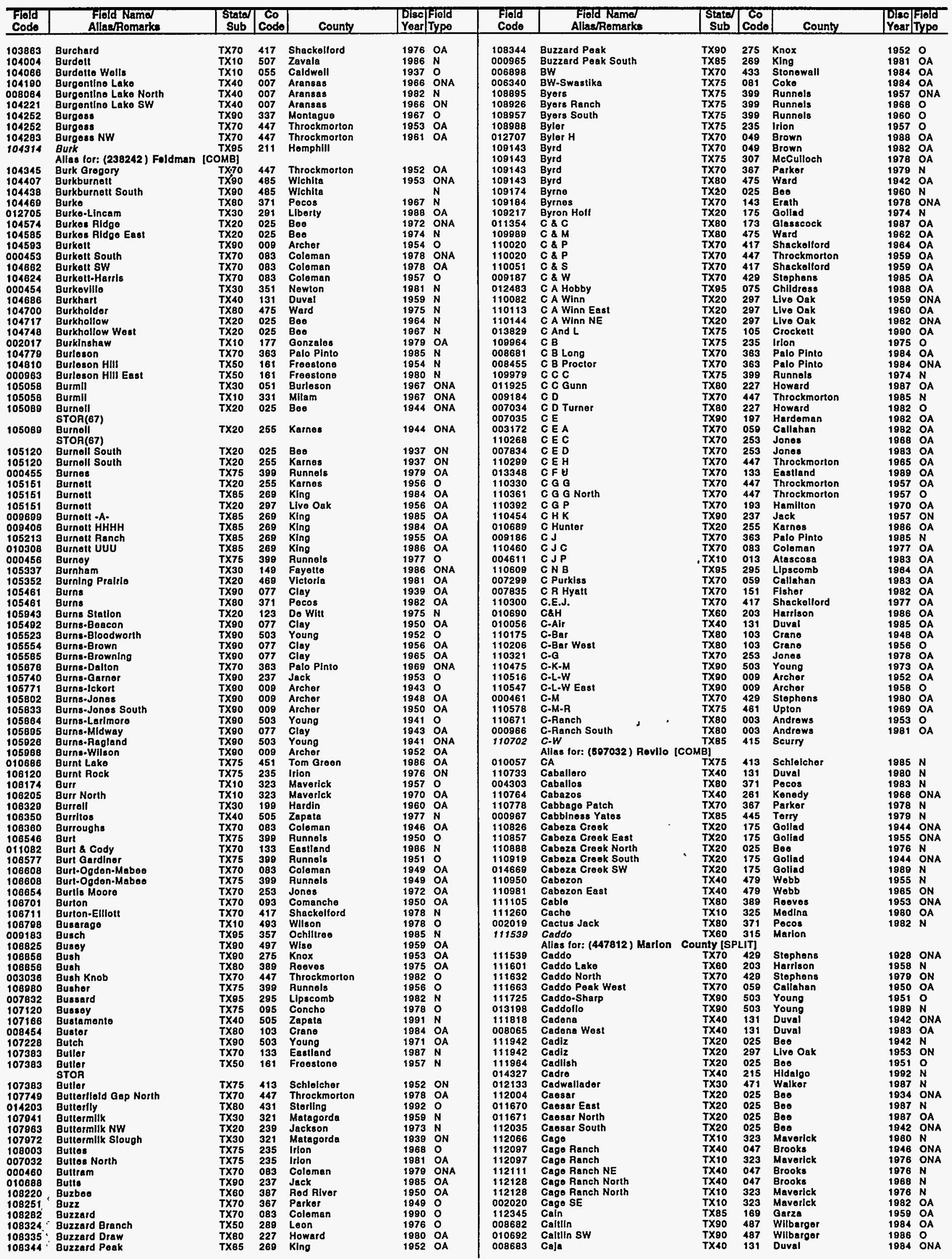


TEXAS

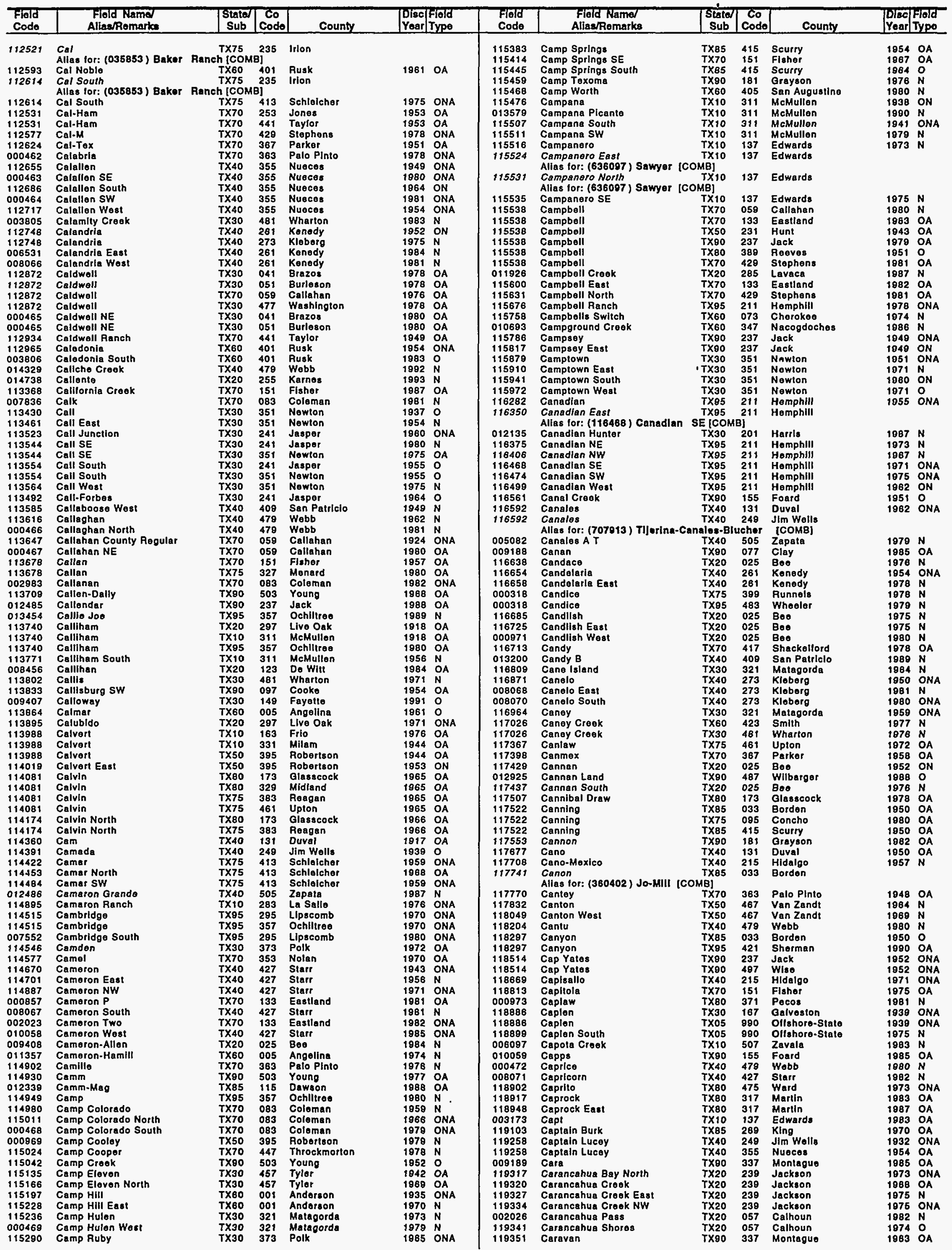


TEXAS

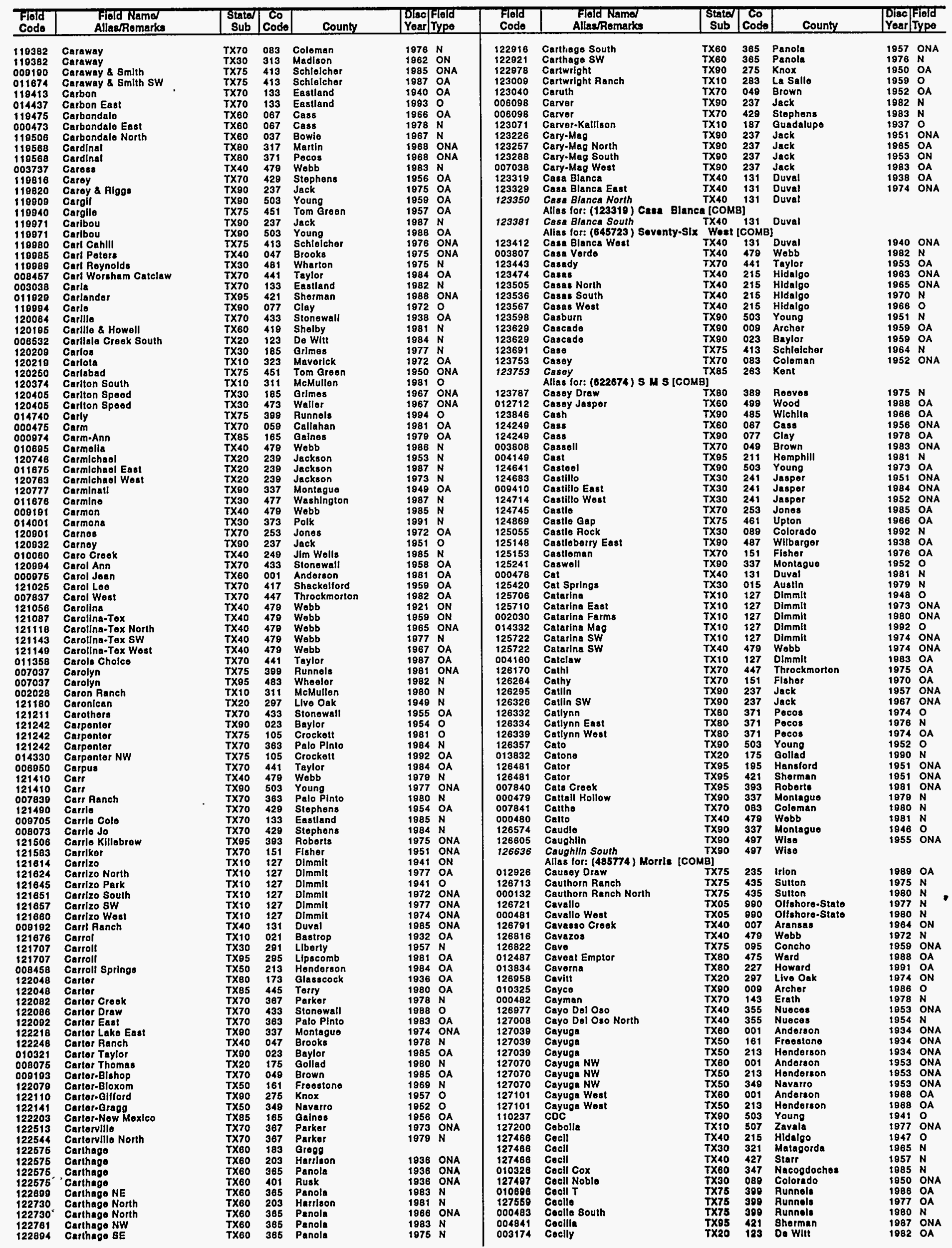


TEXAS

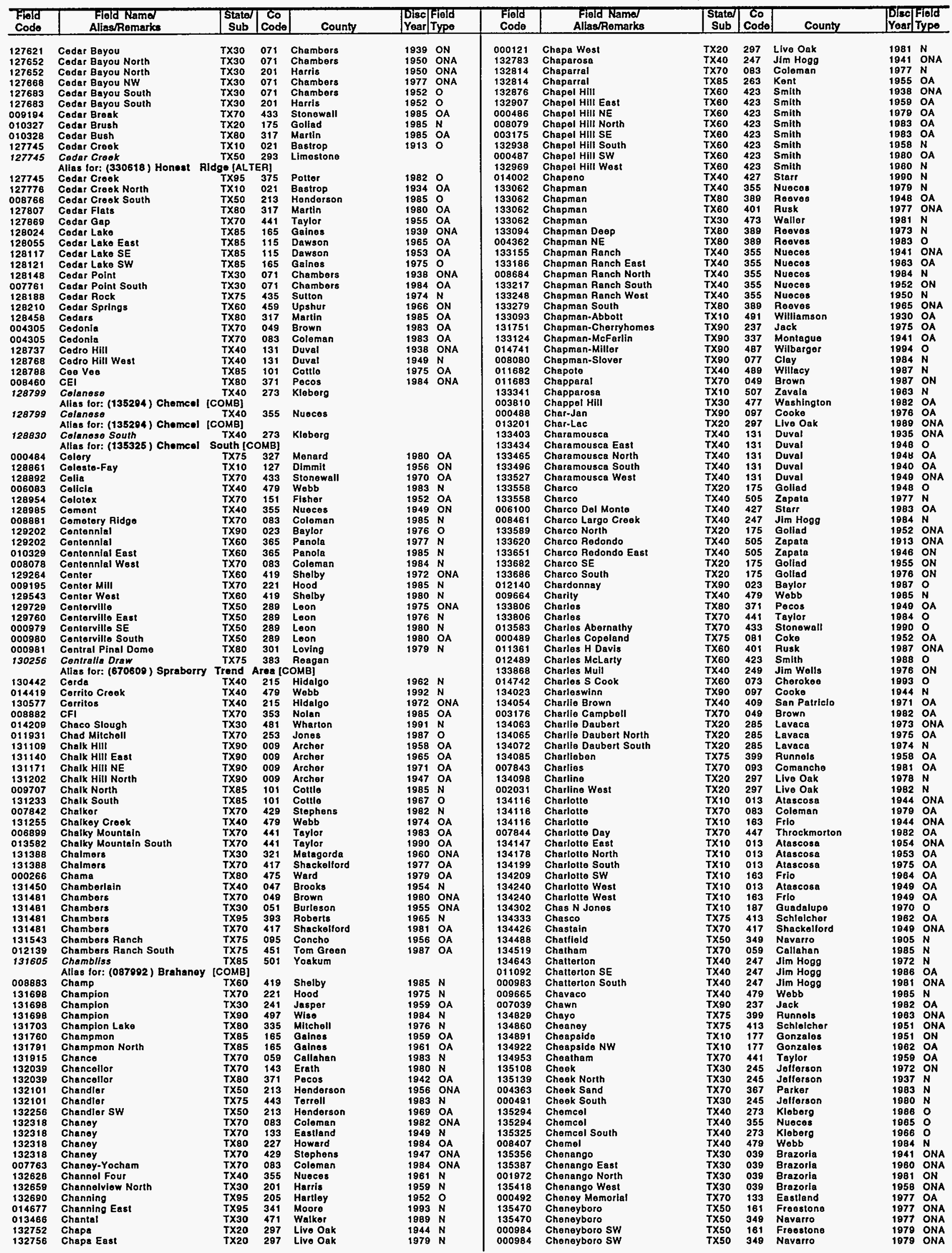


TEXAS

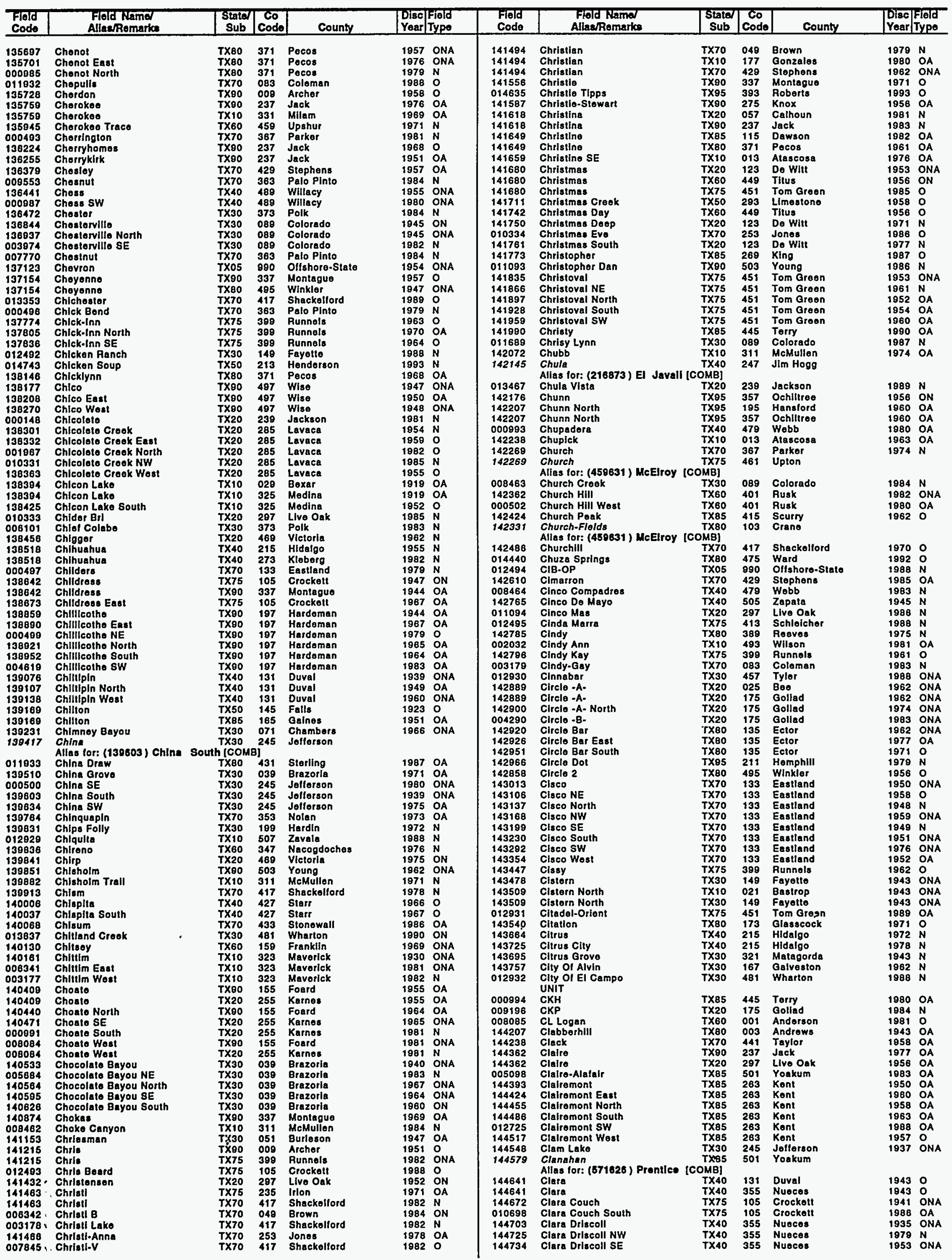


TEXAS

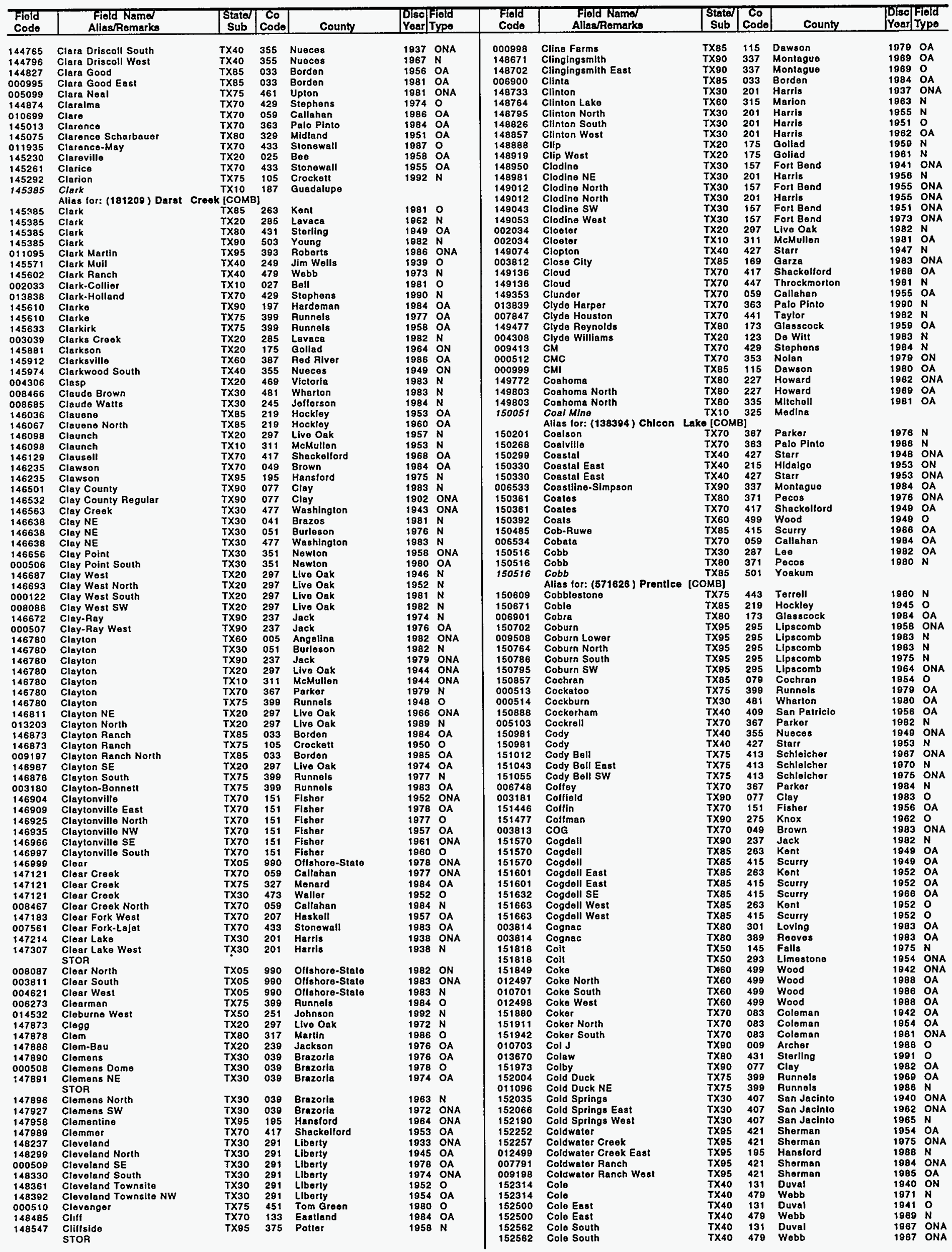


TEXAS

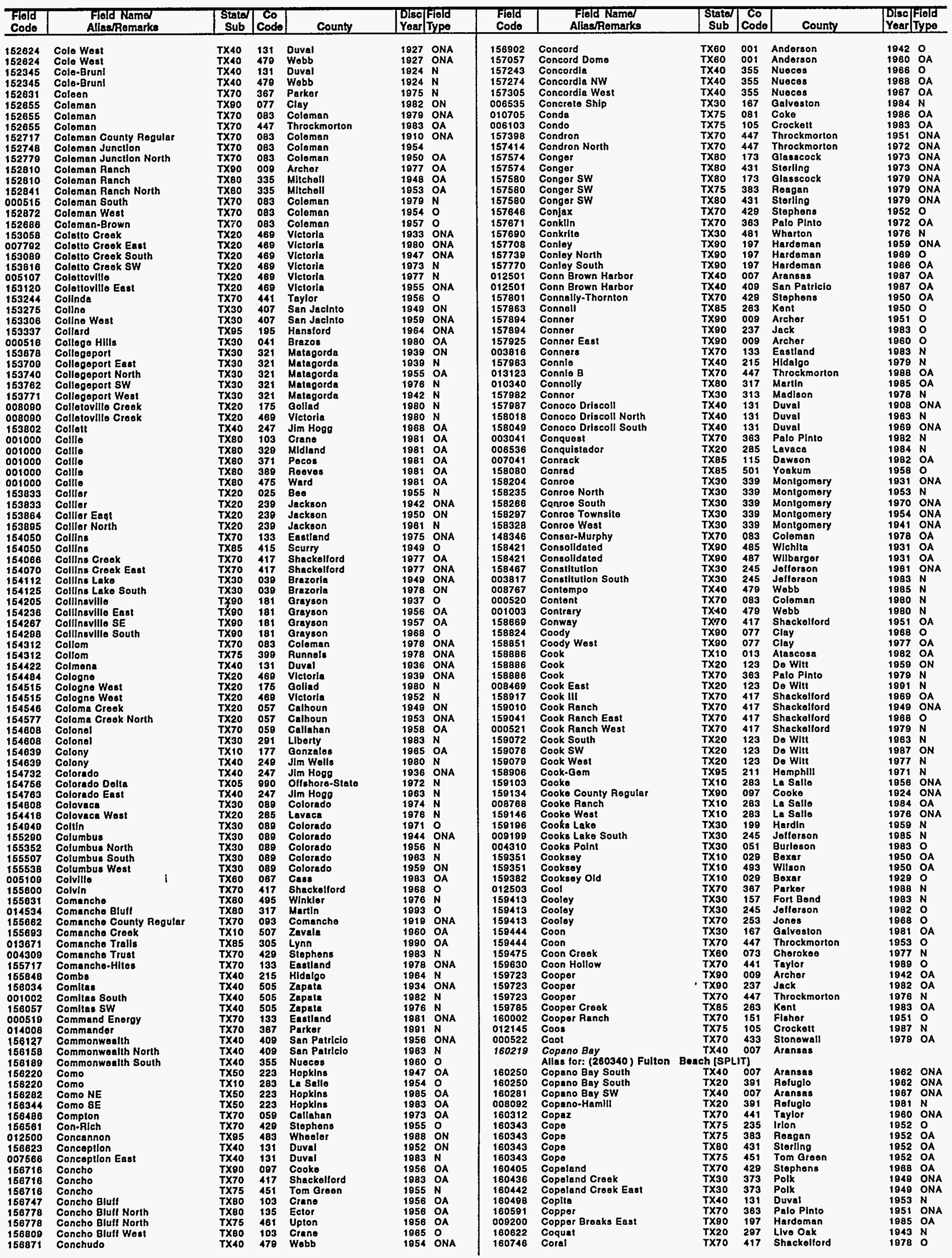

Energy Information Administration/Oil and Gas Field Code Master List 1994 
TEXAS

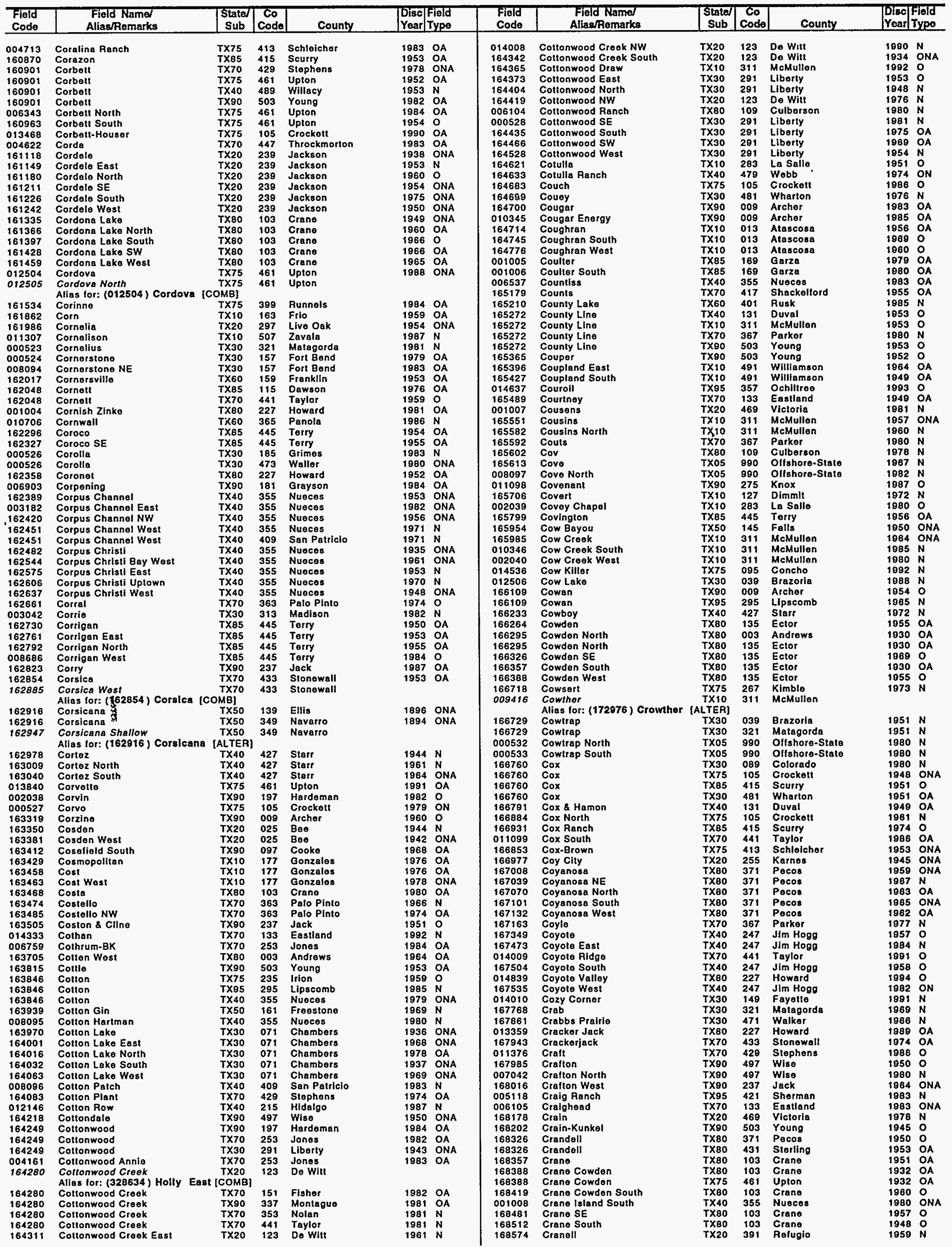


TEXAS

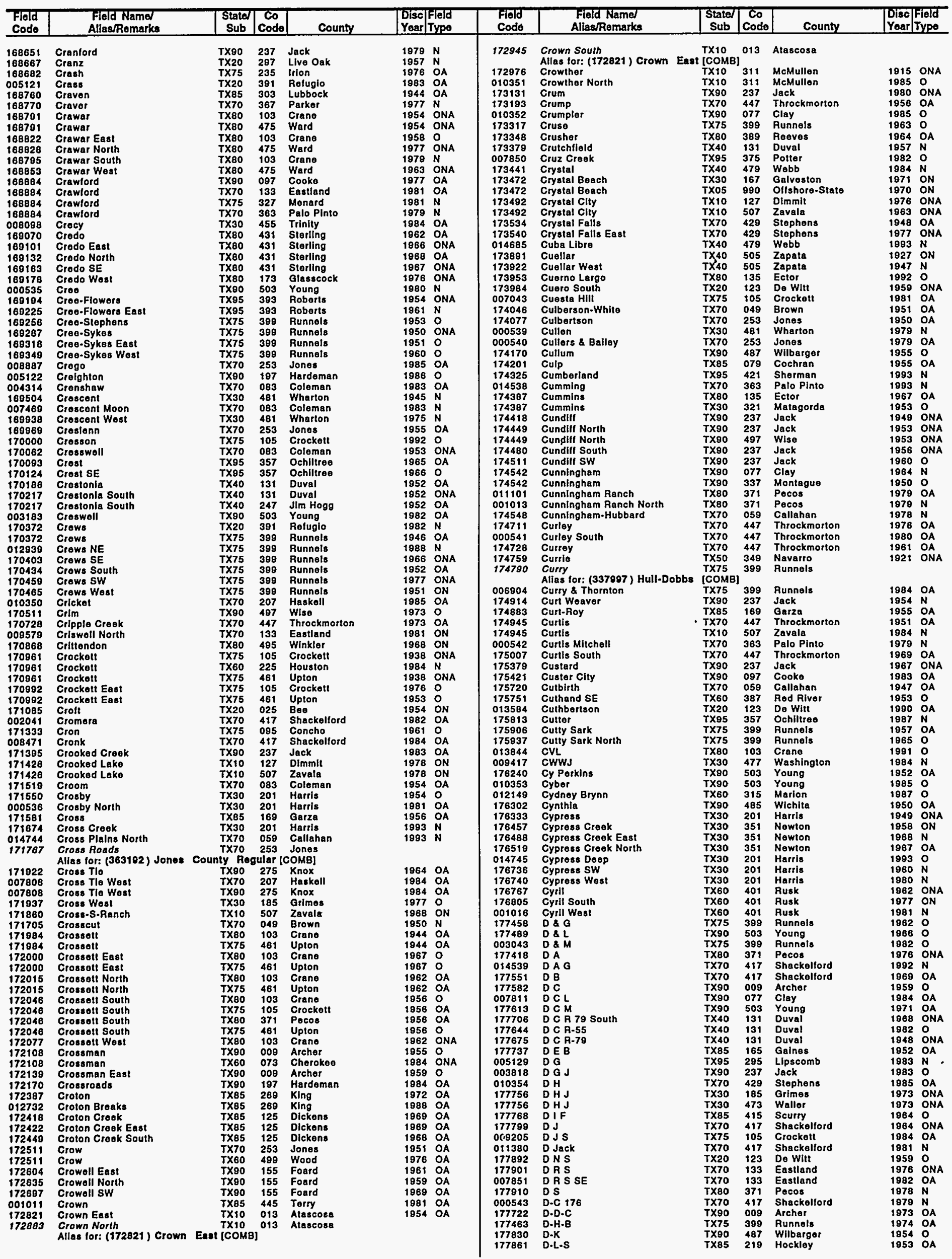


TEXAS

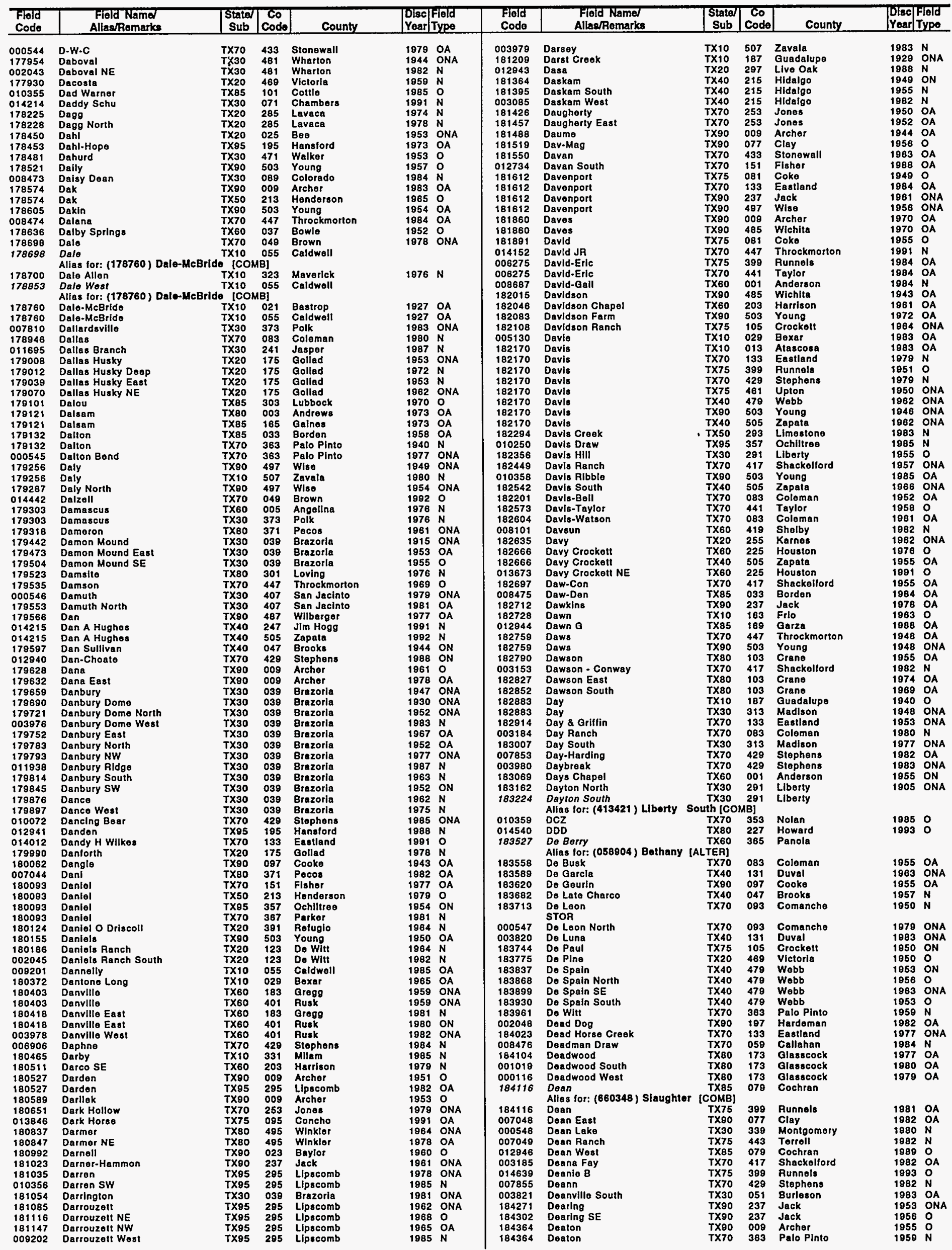




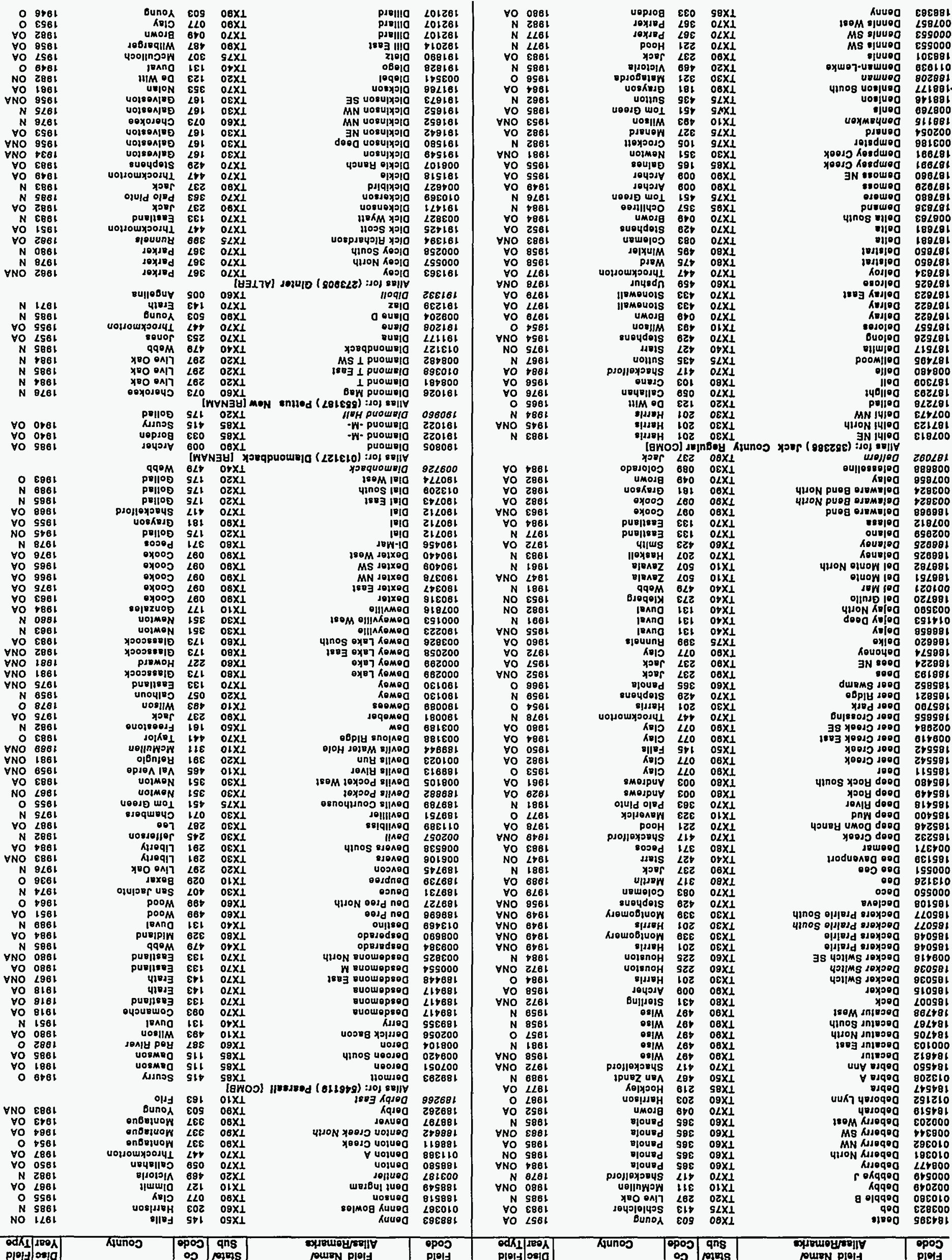


TEXAS

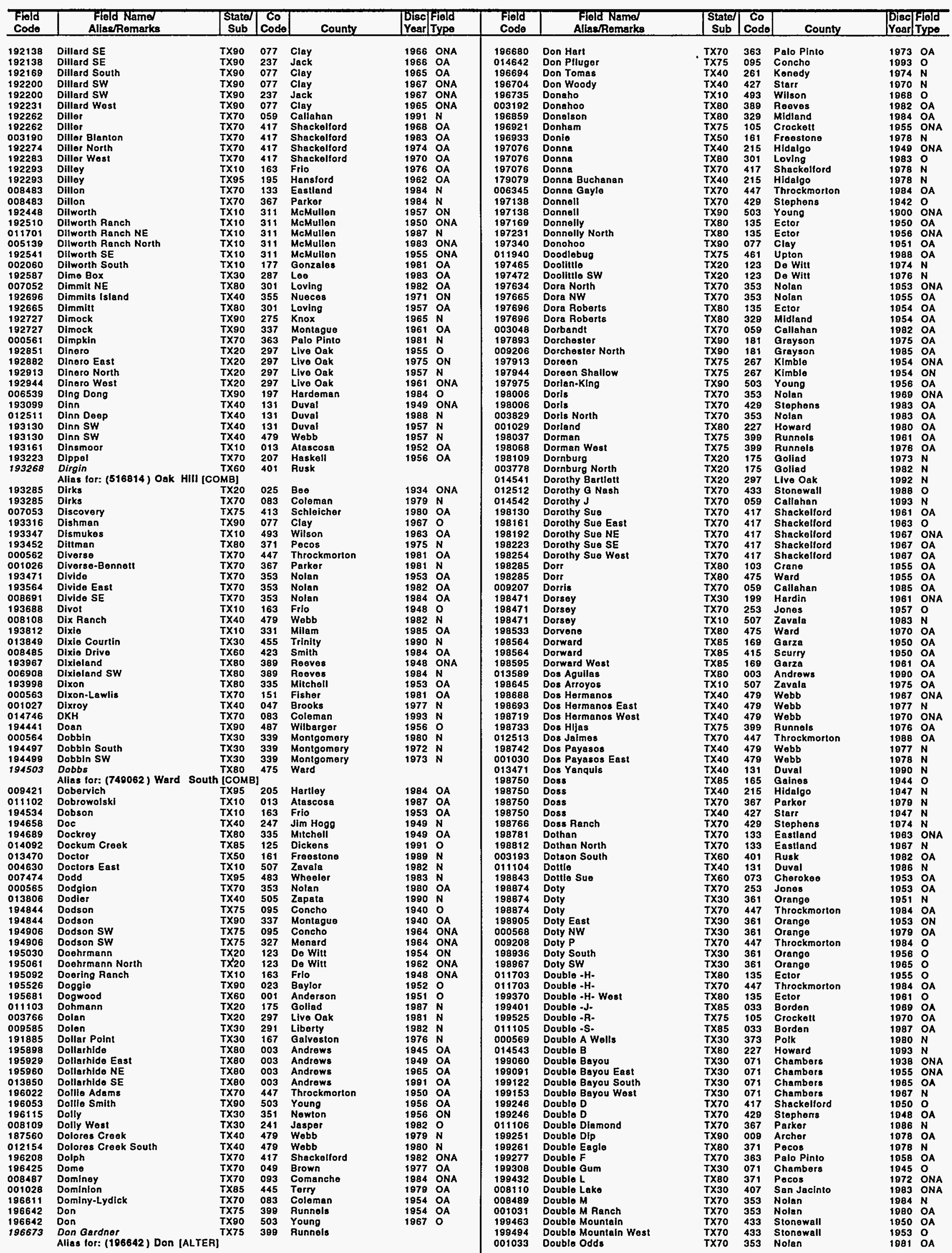


TEXAS

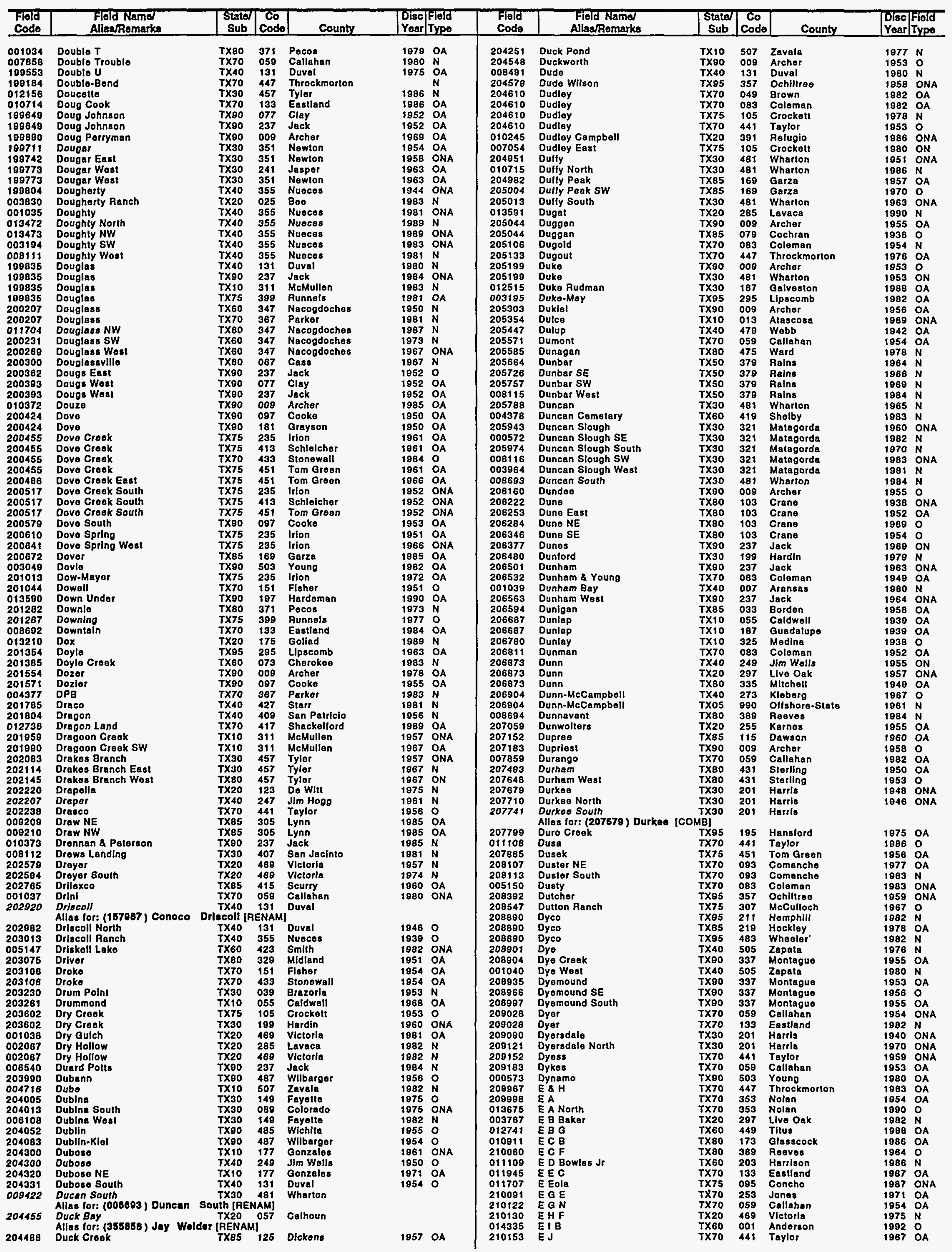




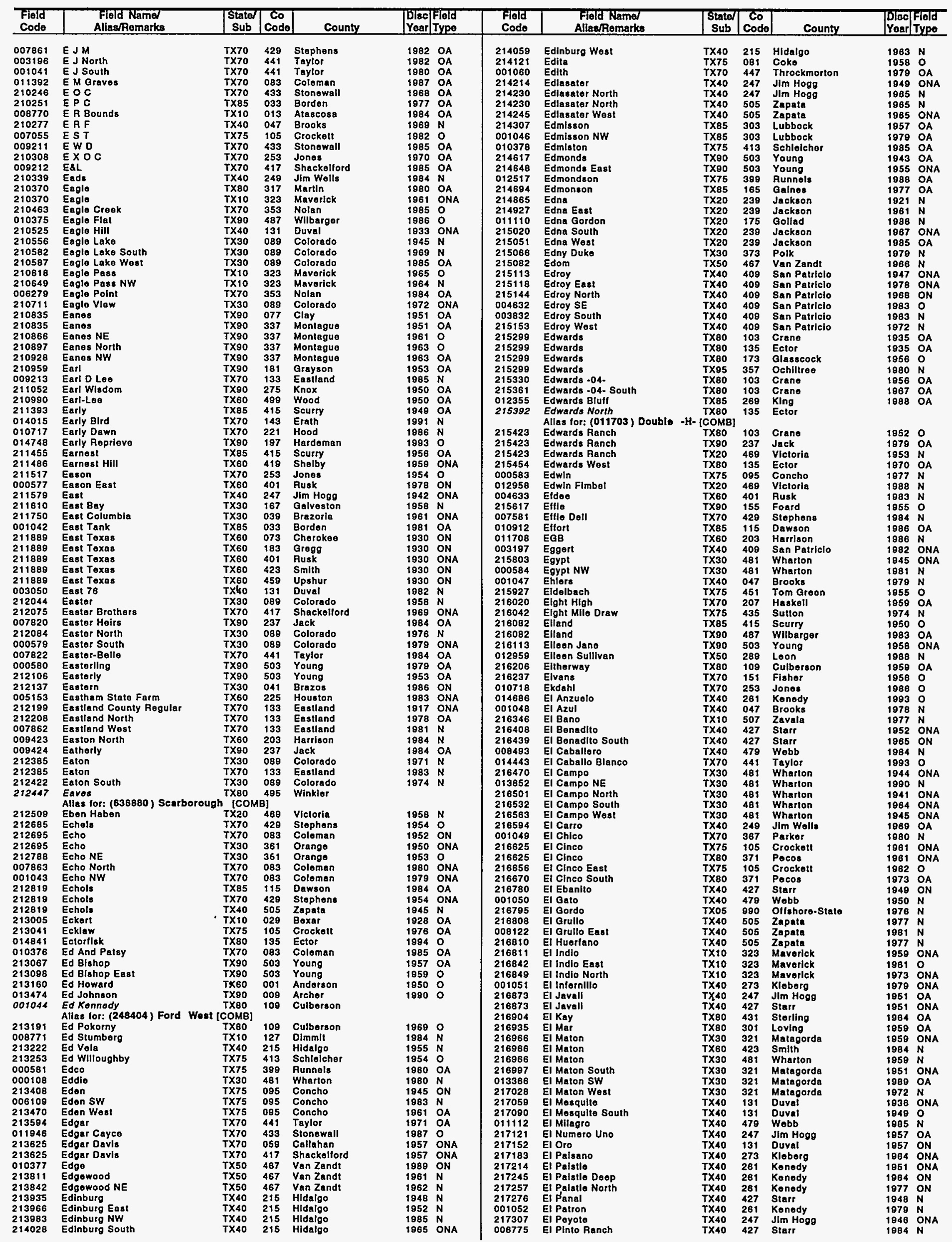


TEXAS

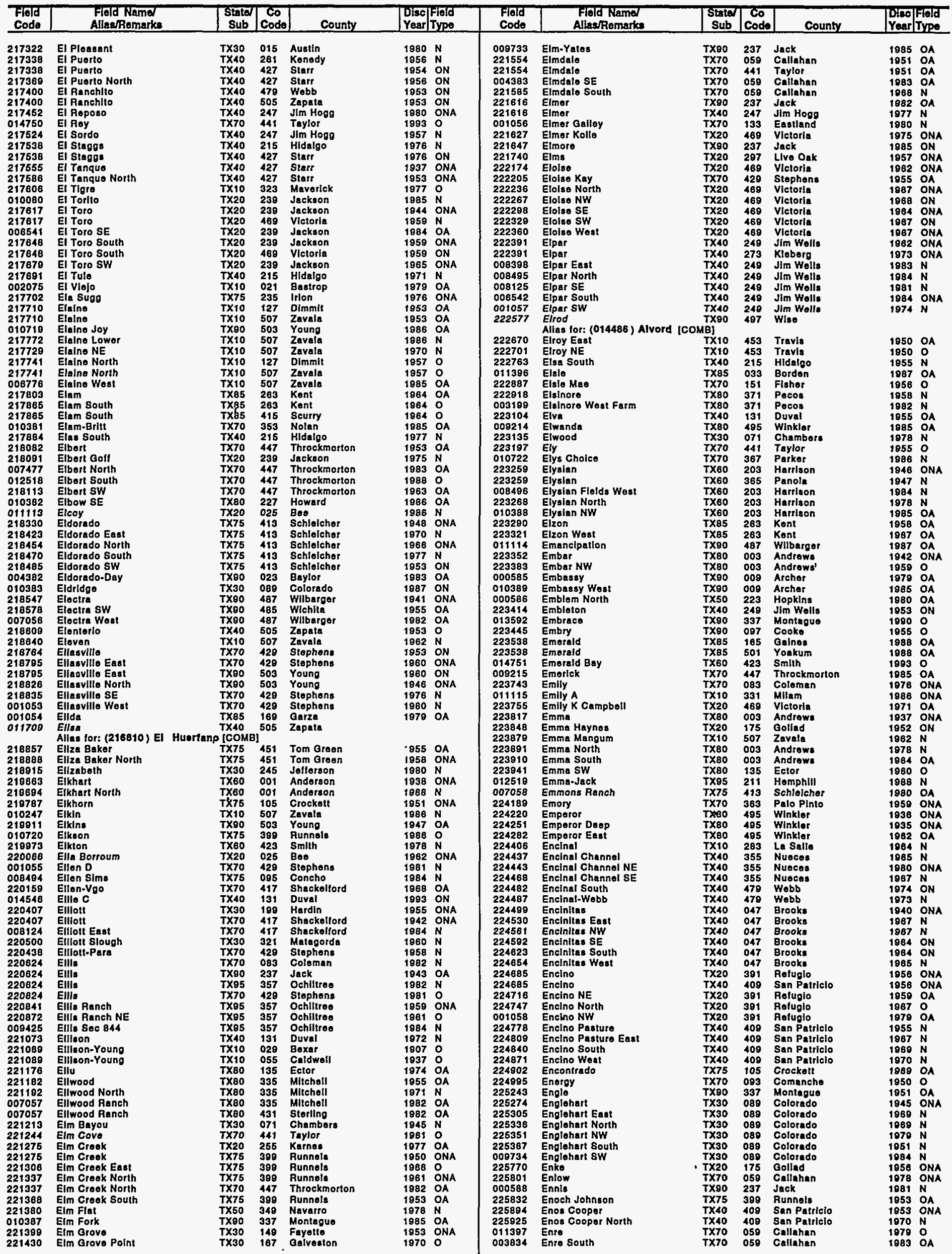


TEXAS

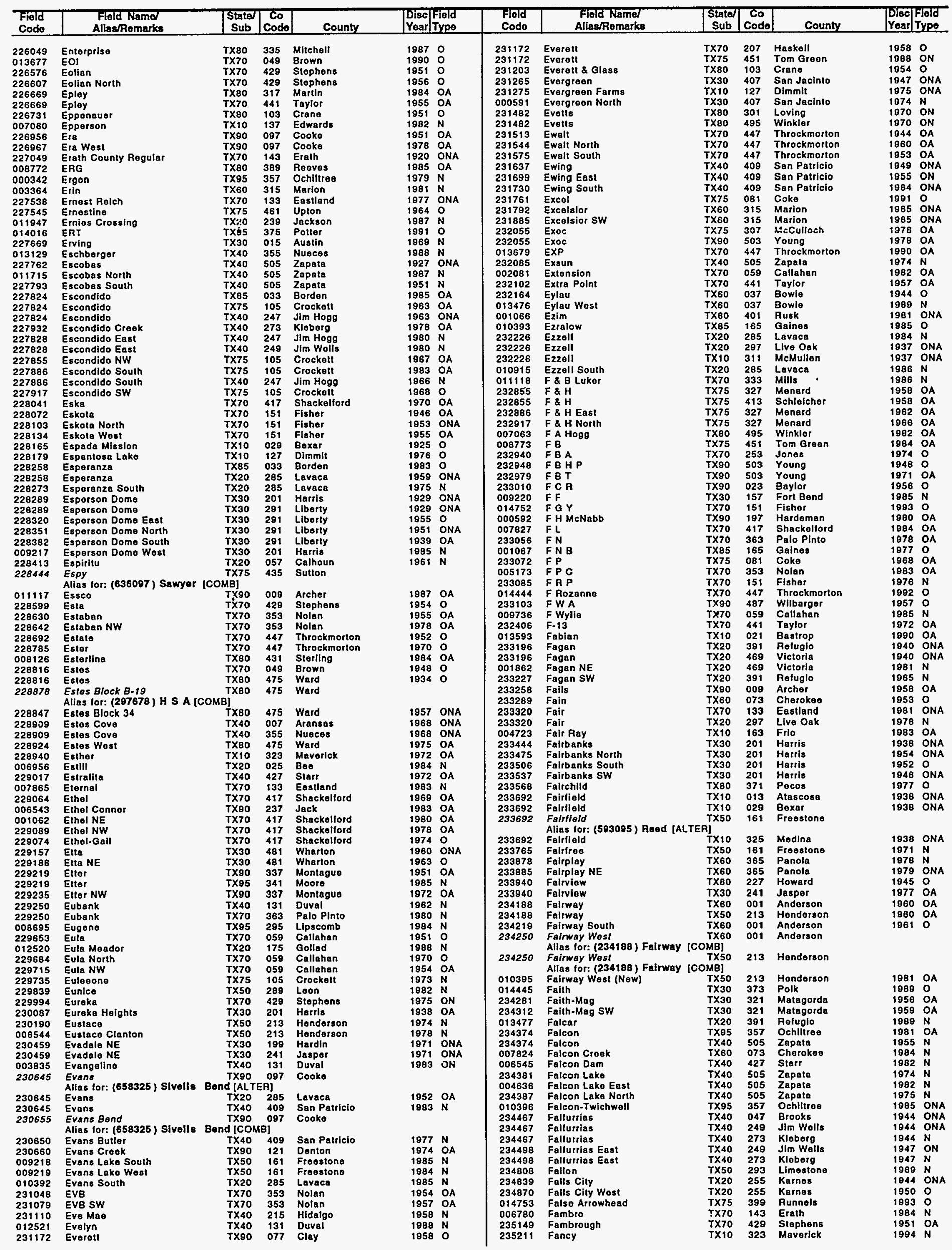


TEXAS

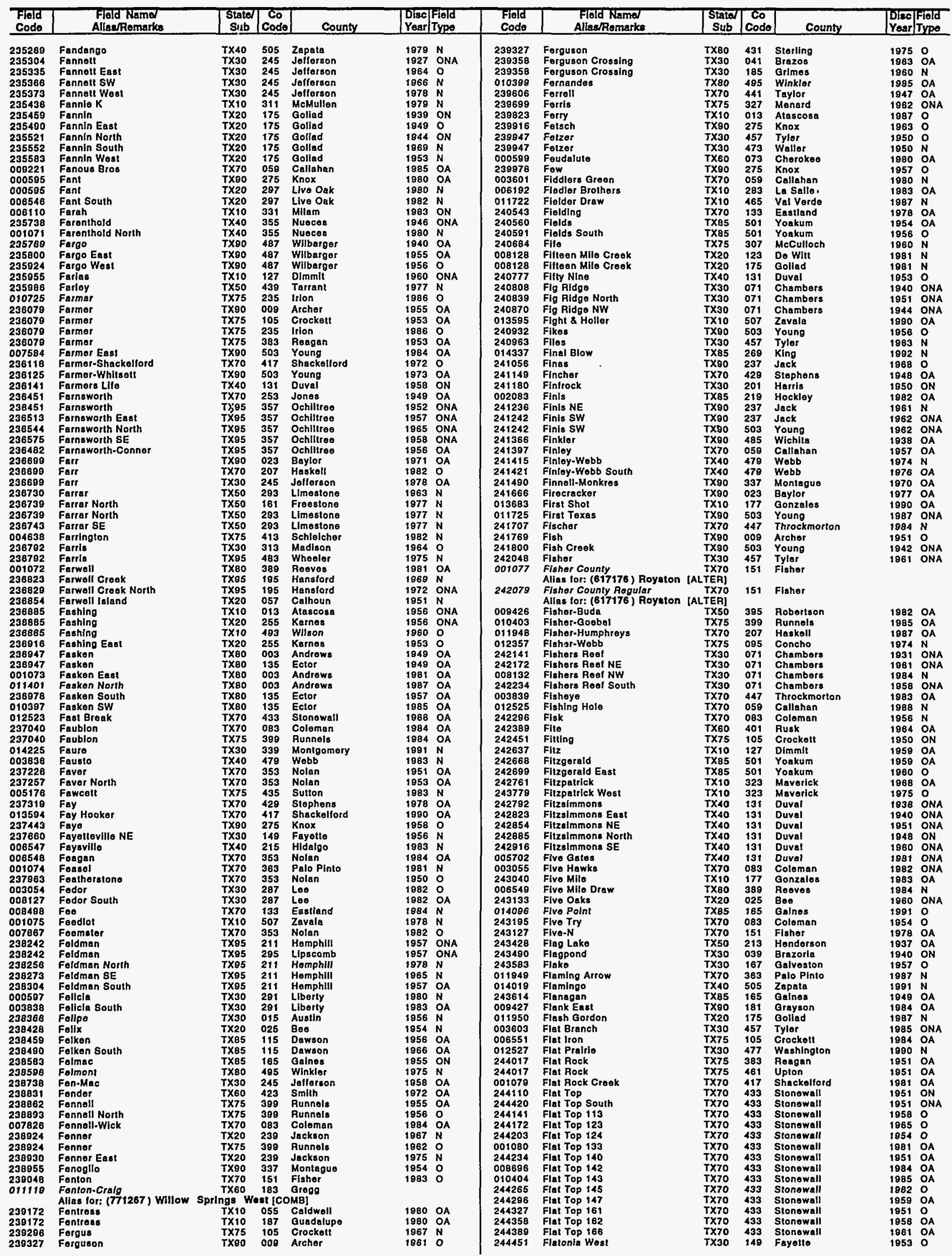


TEXAS

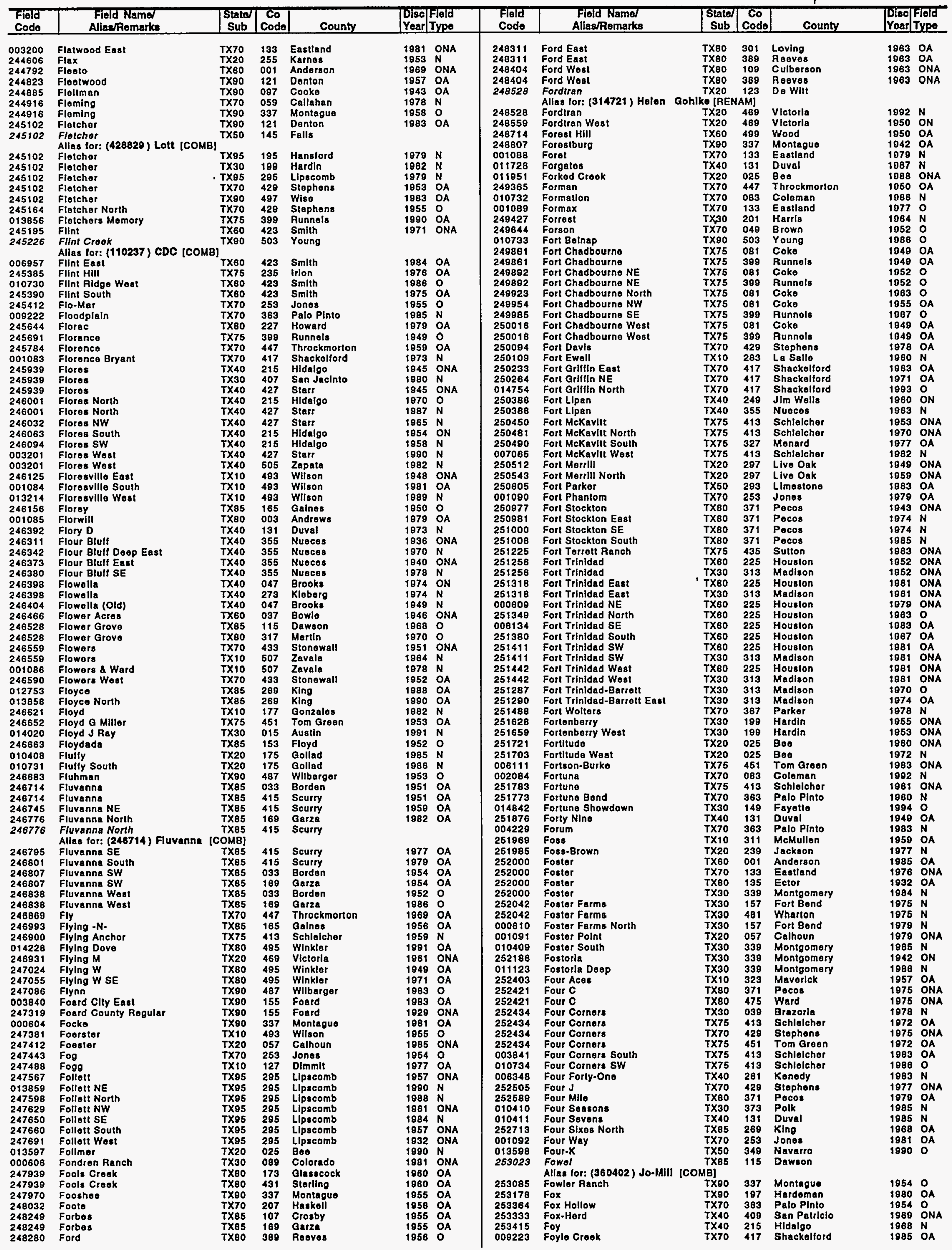


TEXAS

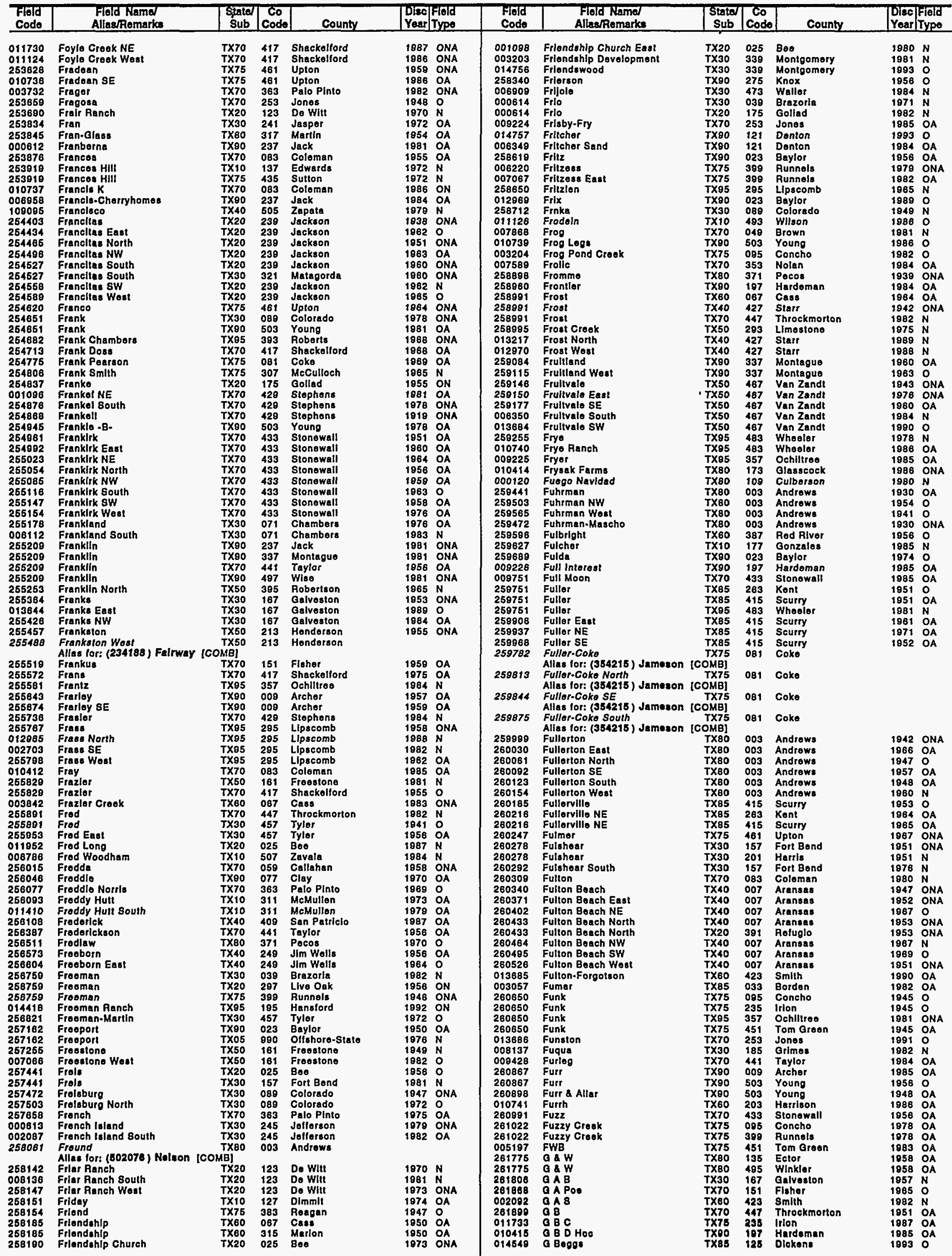


TEXAS

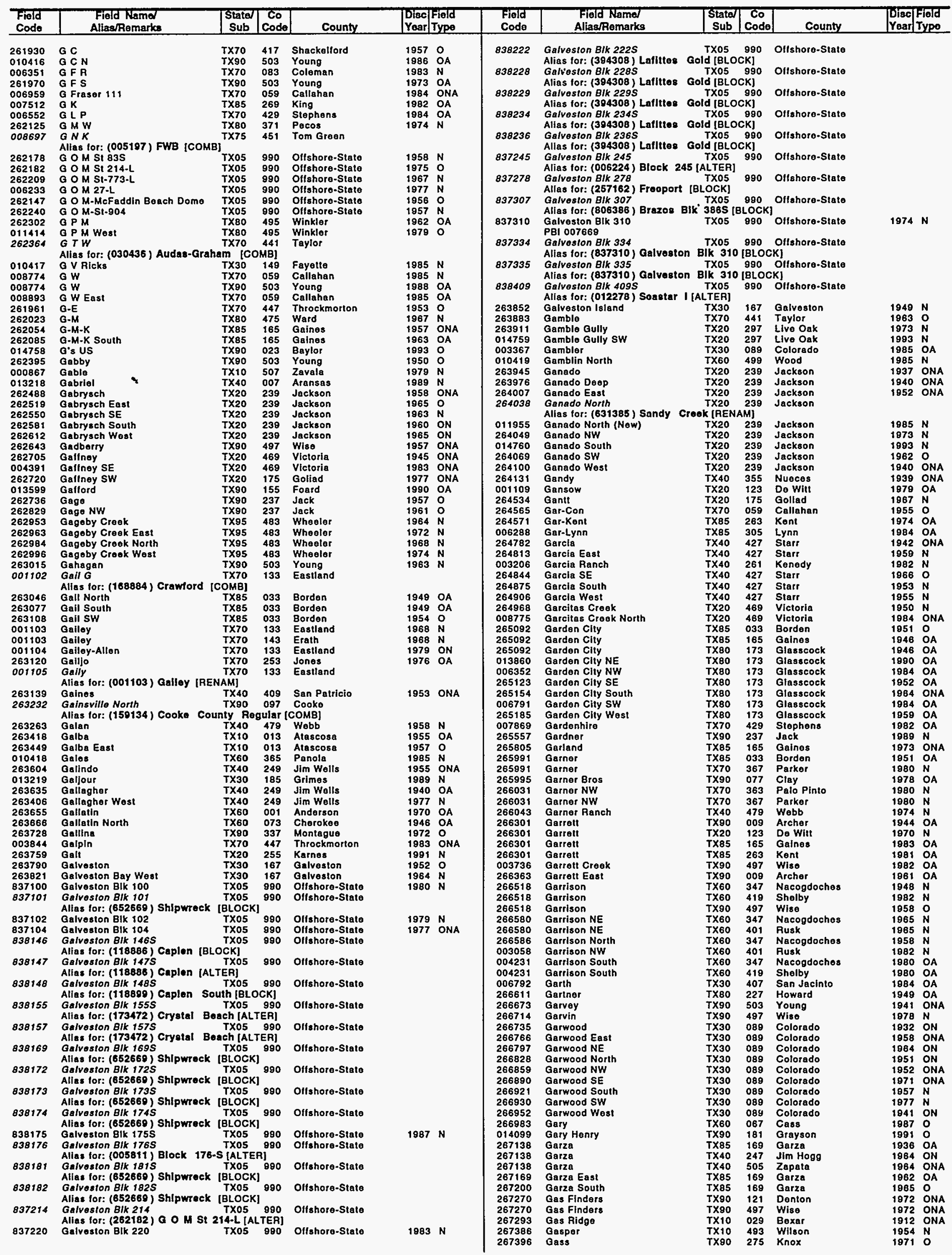


TEXAS

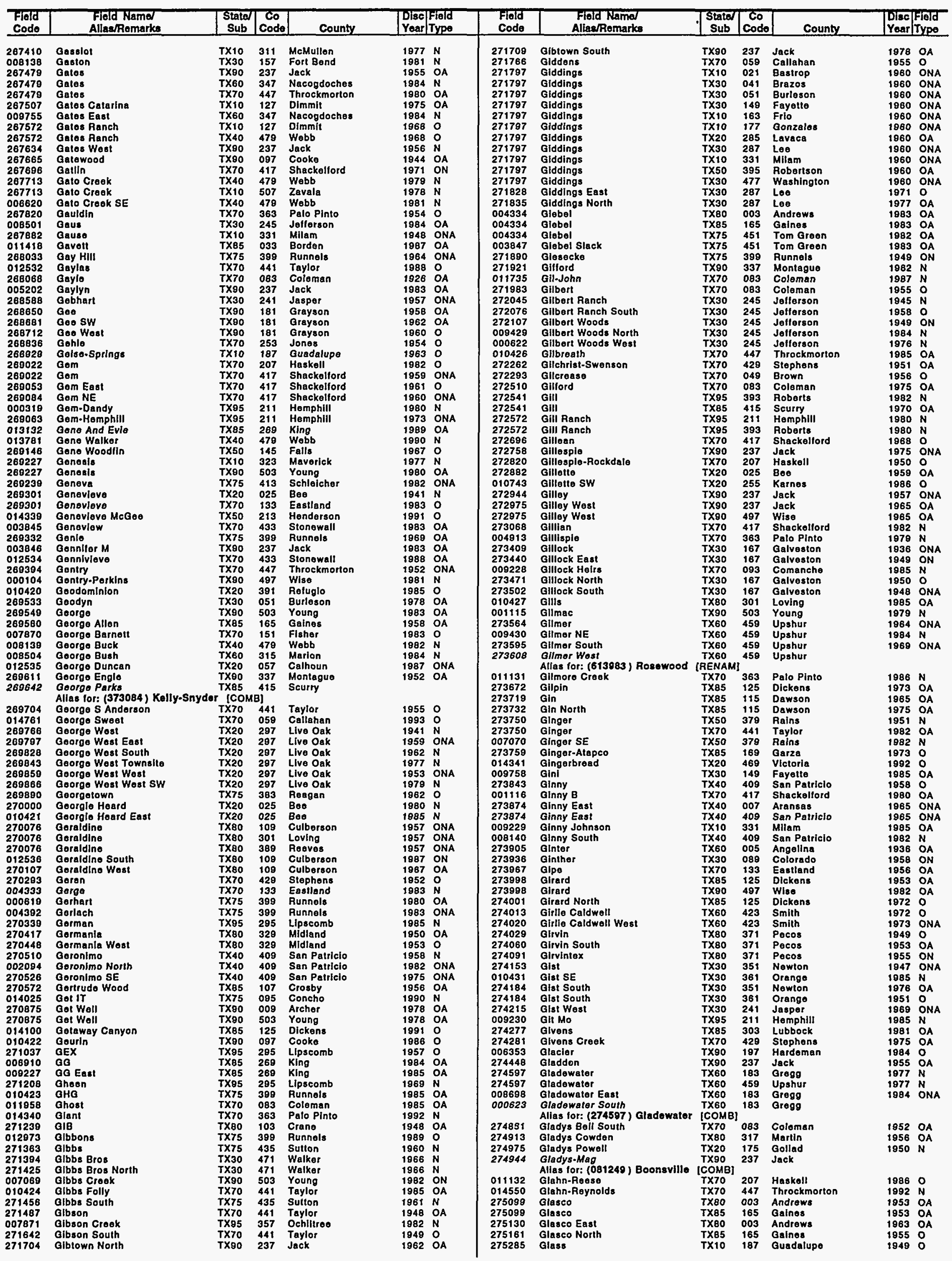


TEXAS

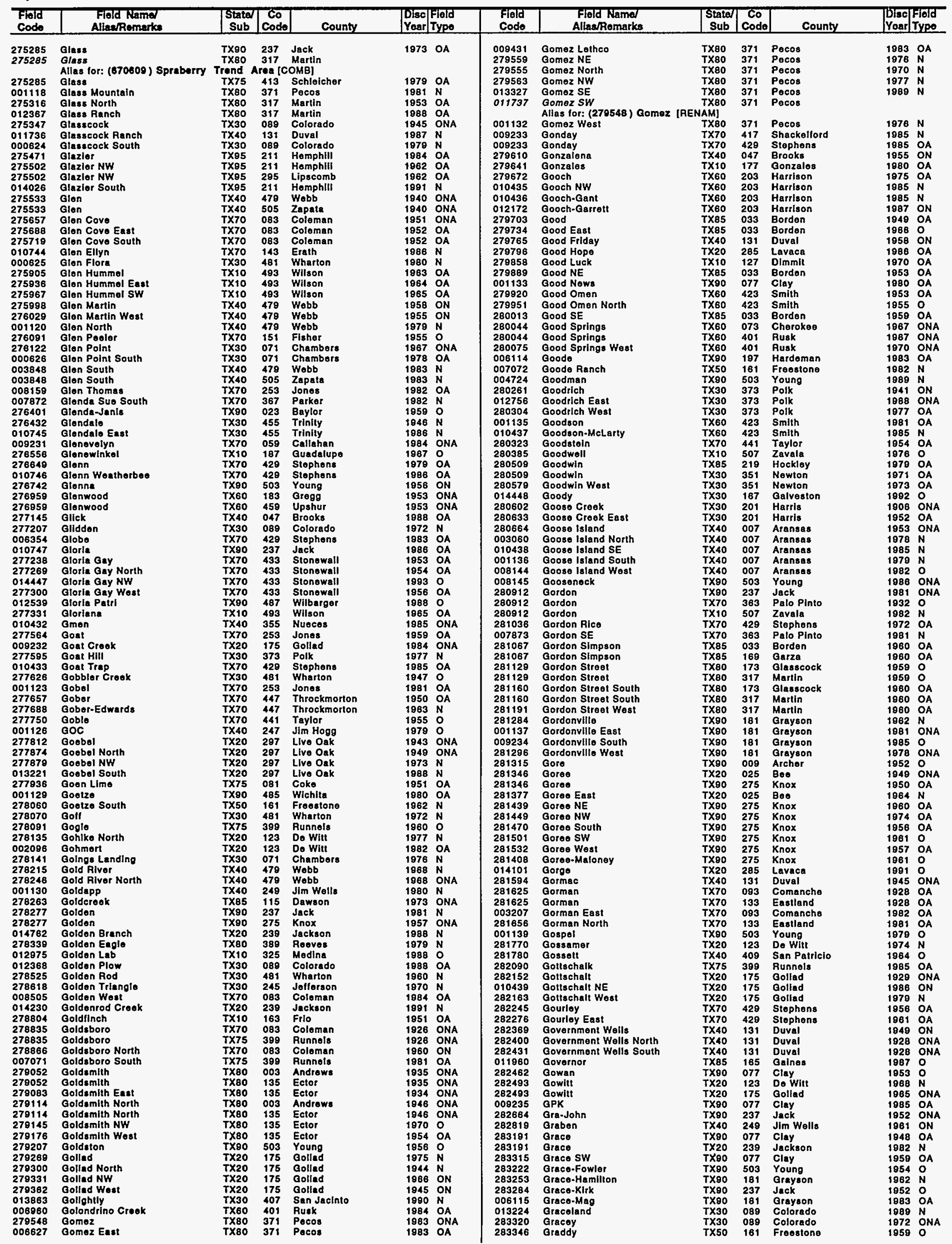


TEXAS

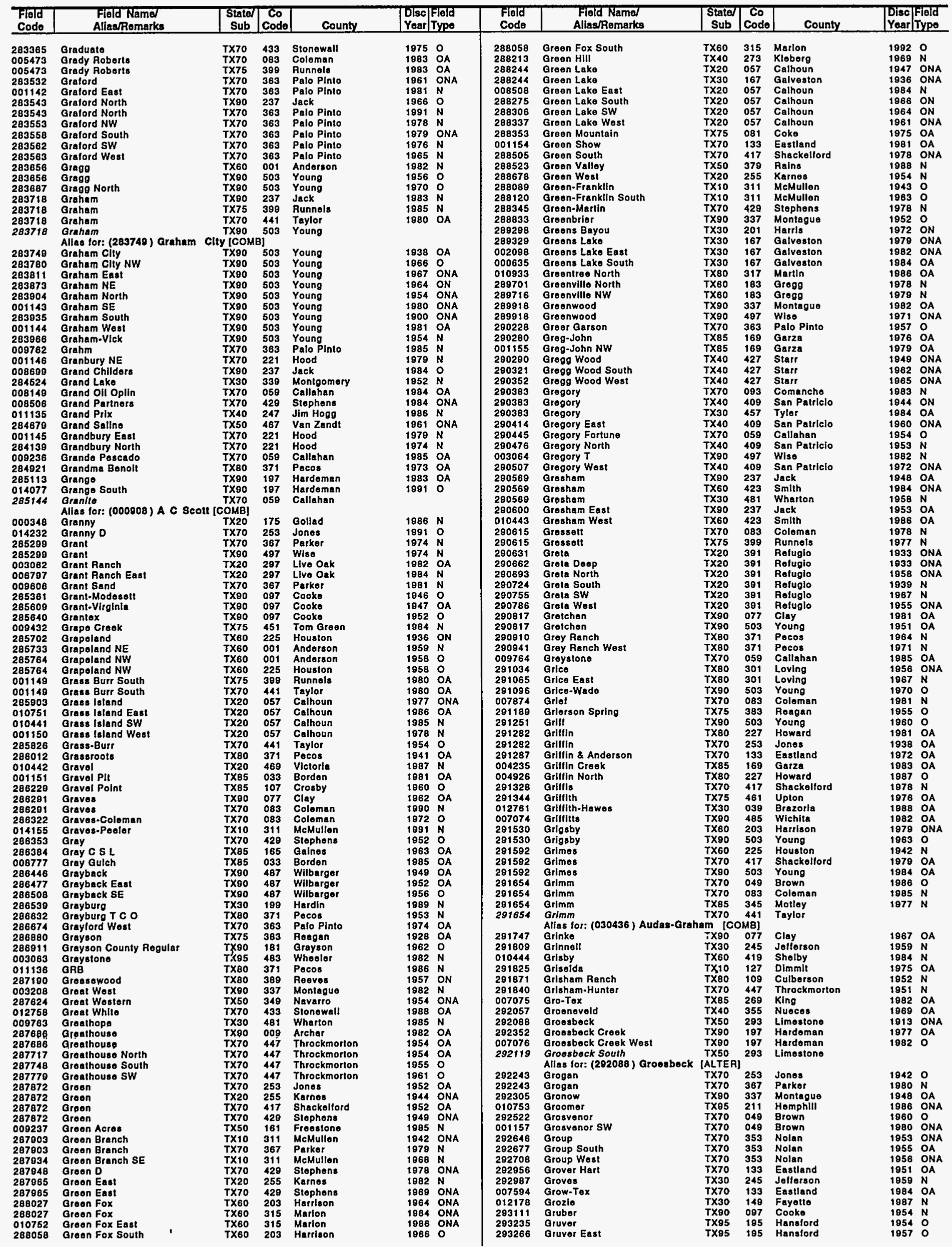


TEXAS

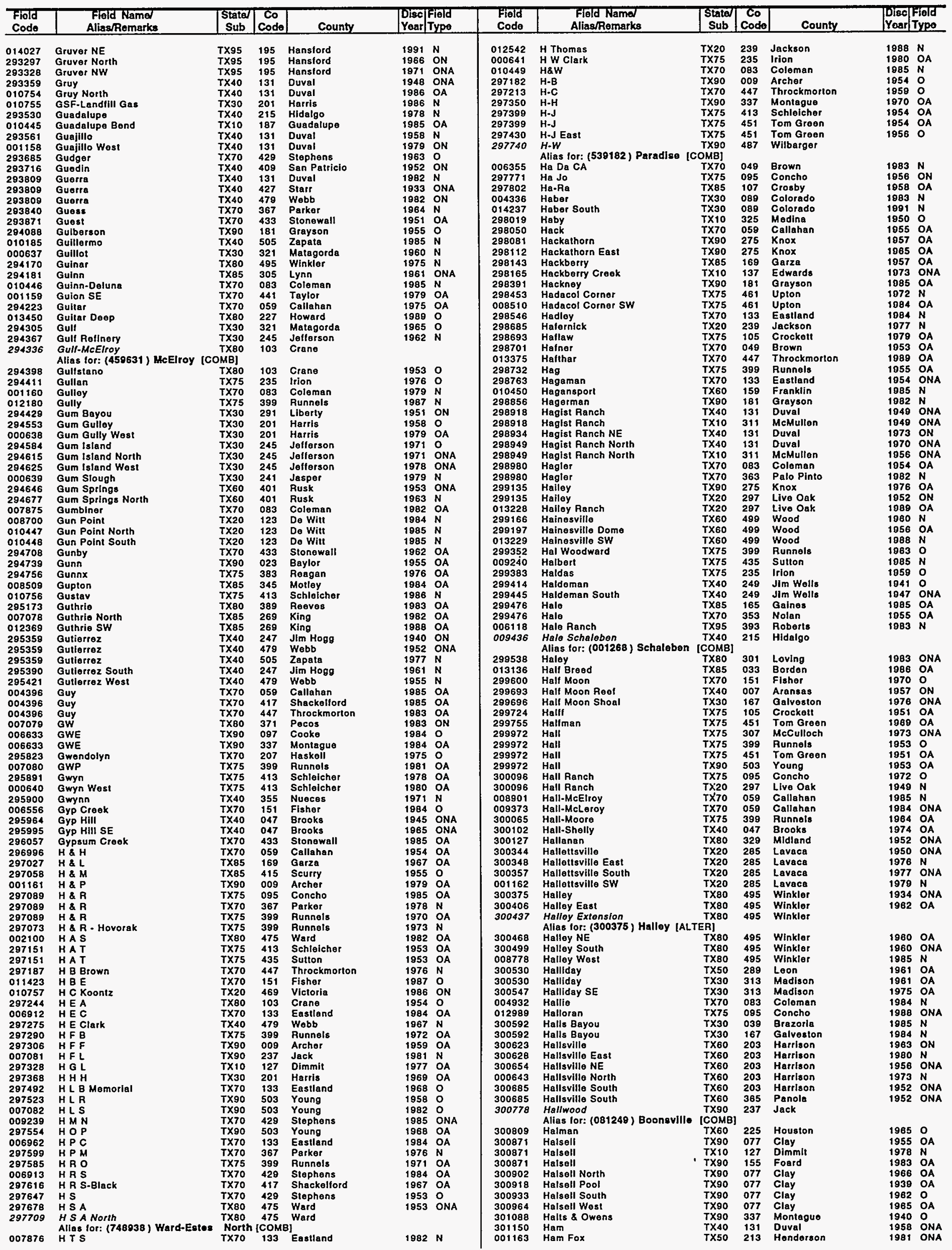


TEXAS

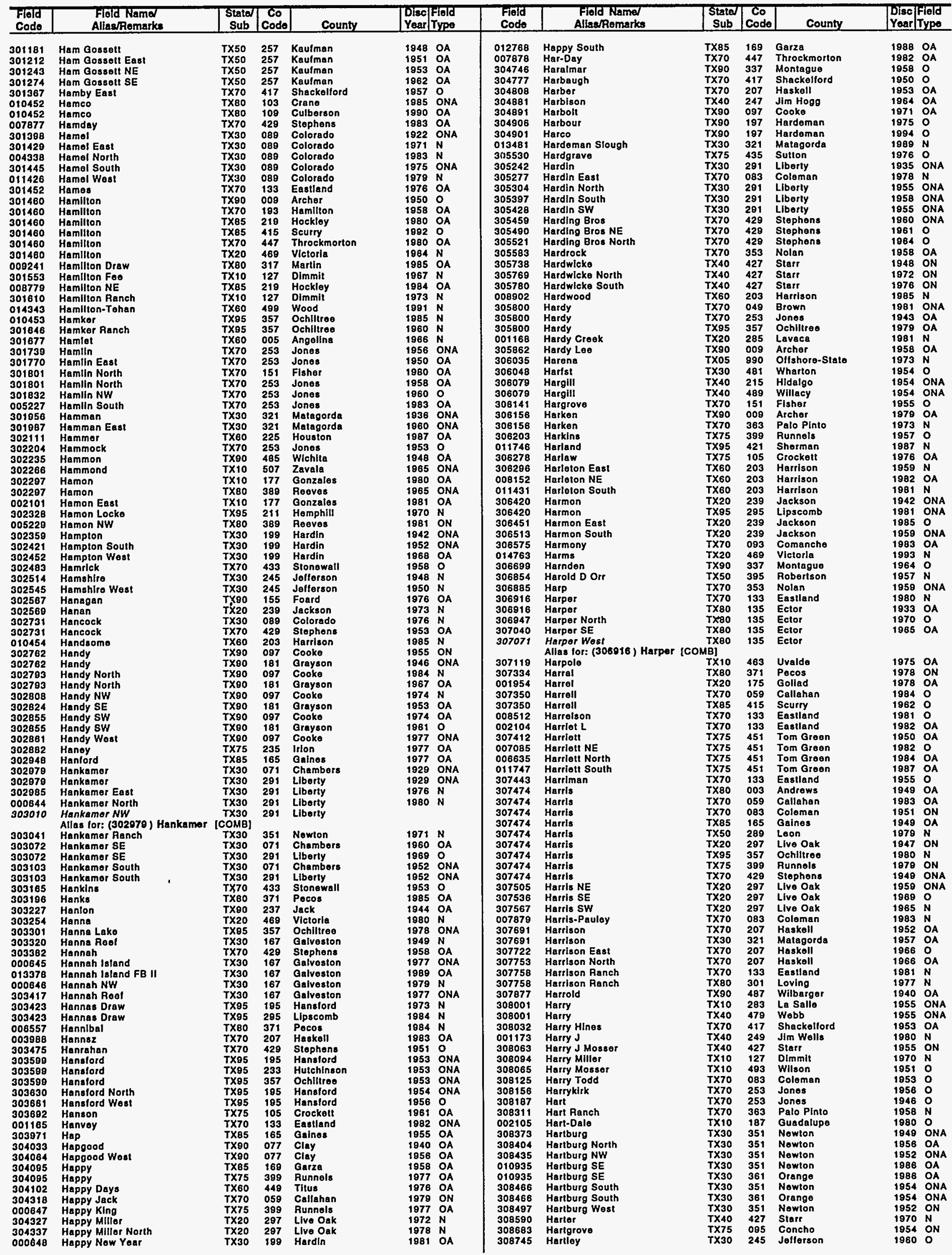


TEXAS

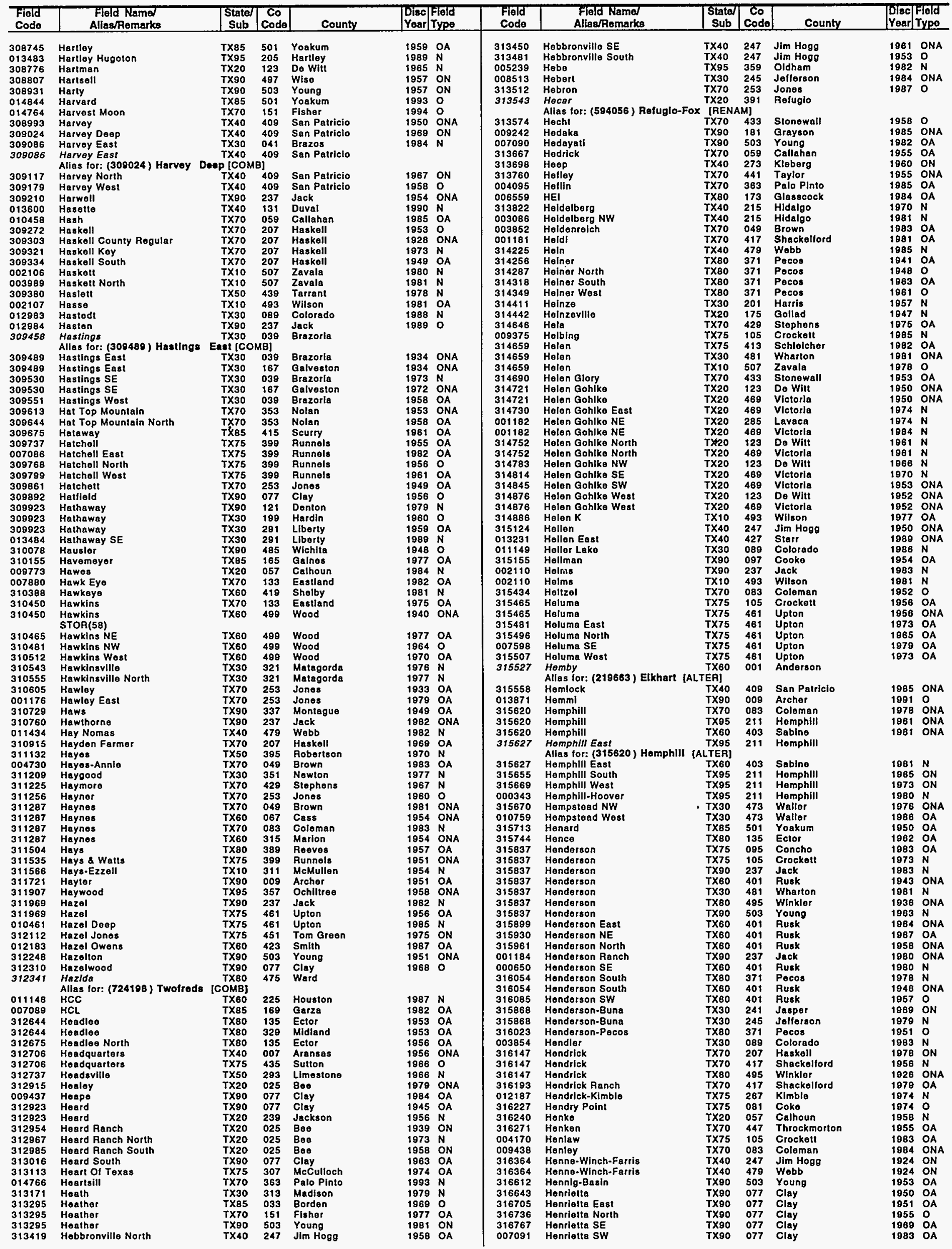


TEXAS

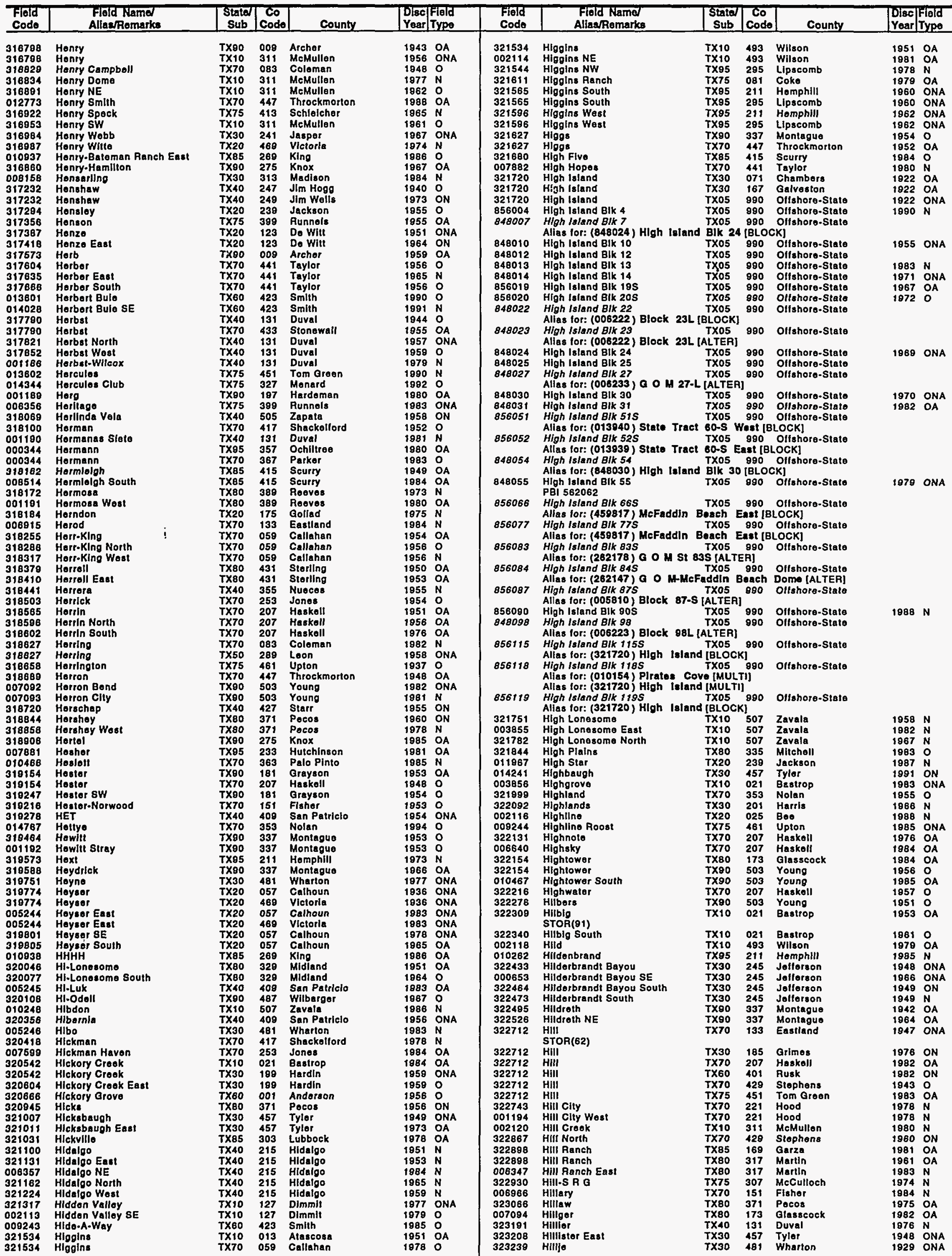


TEXAS

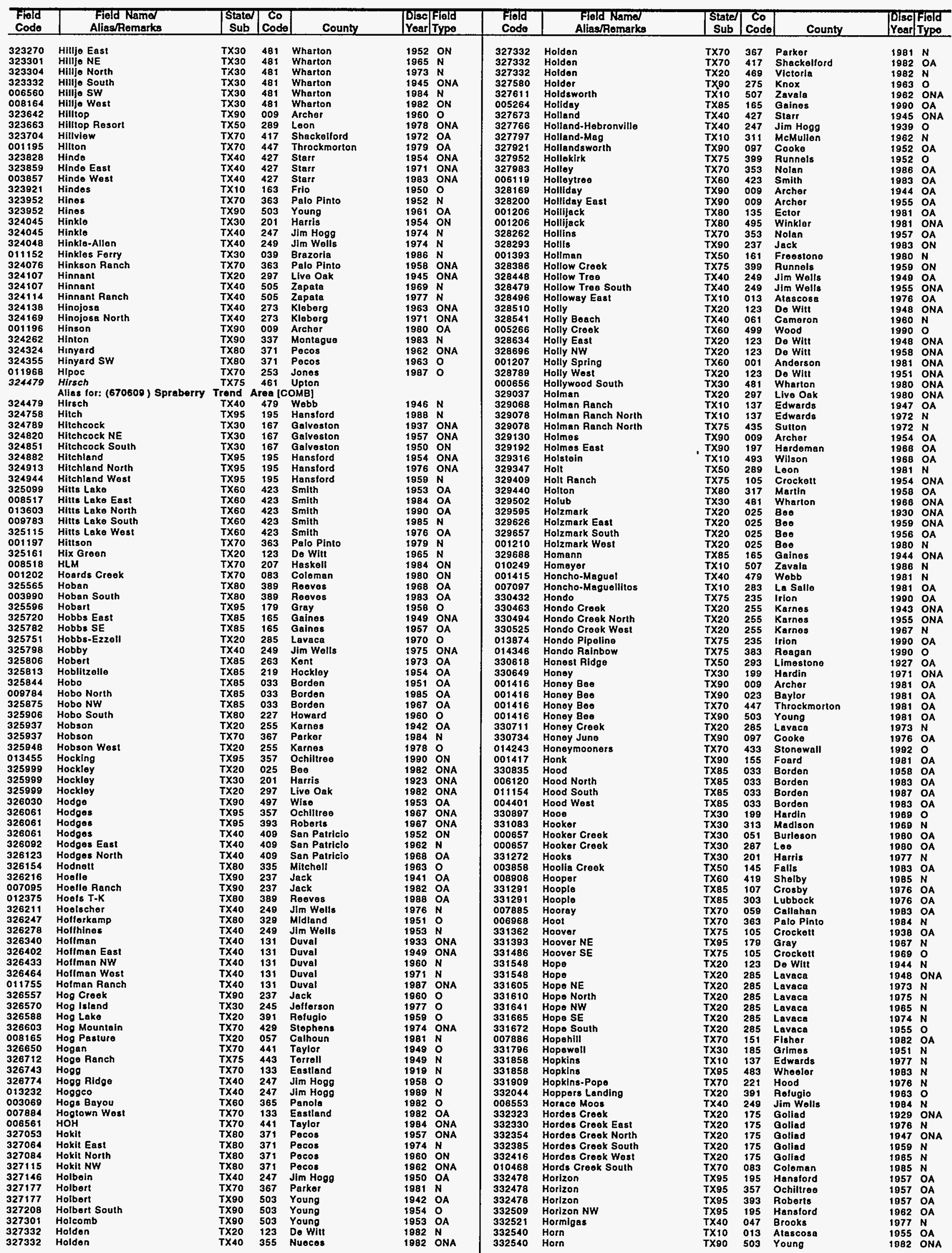


TEXAS

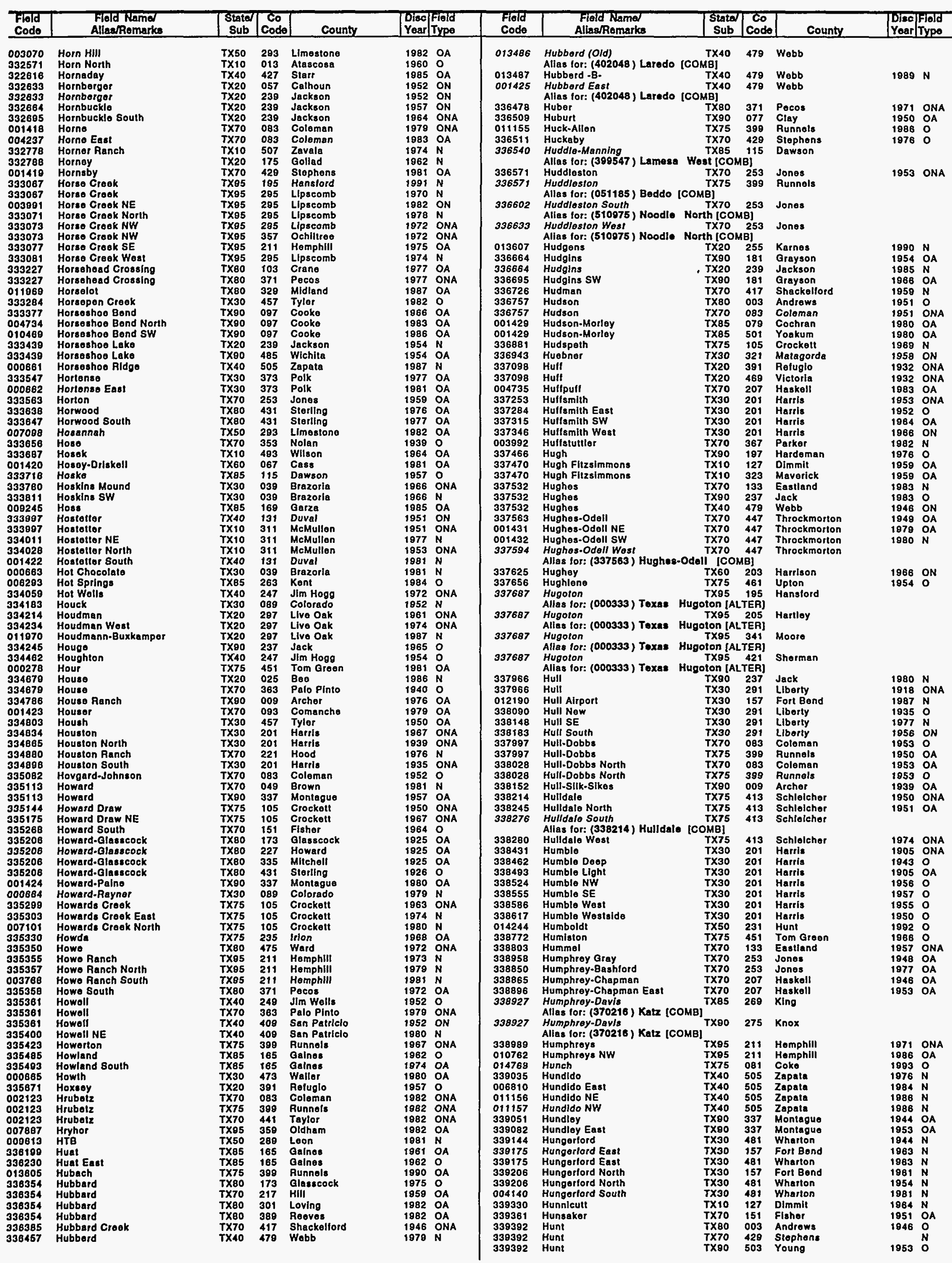




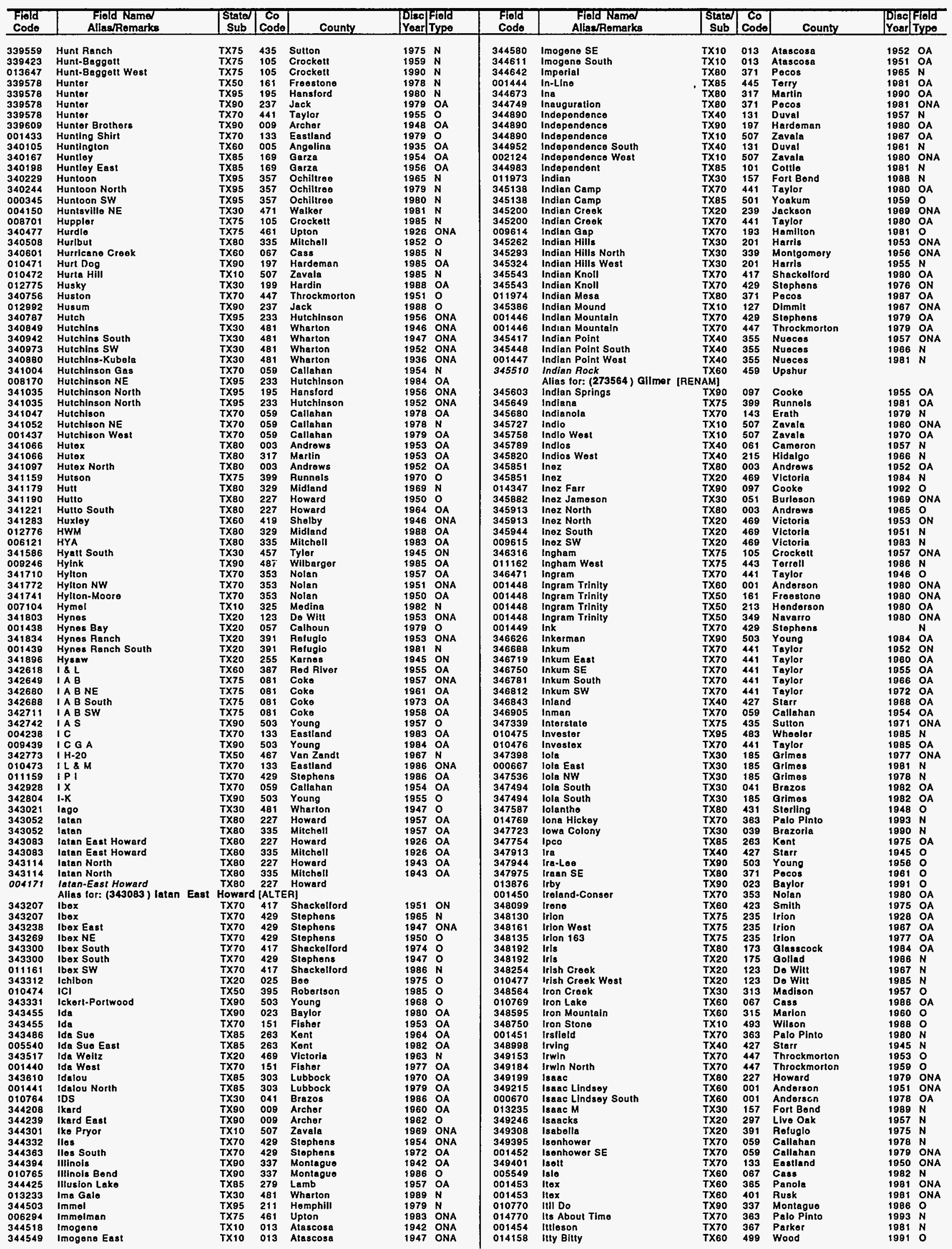


TEXAS

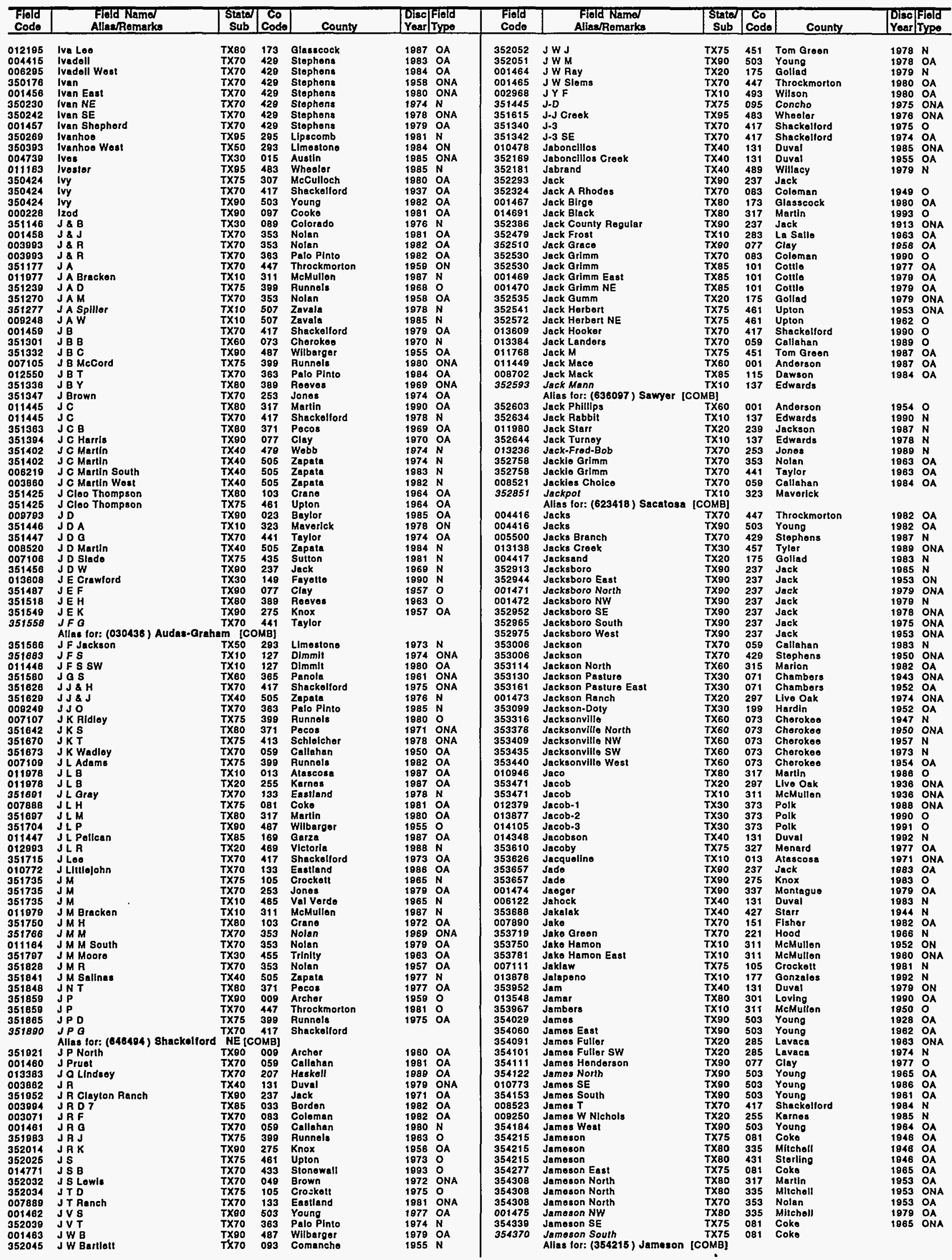


TEXAS

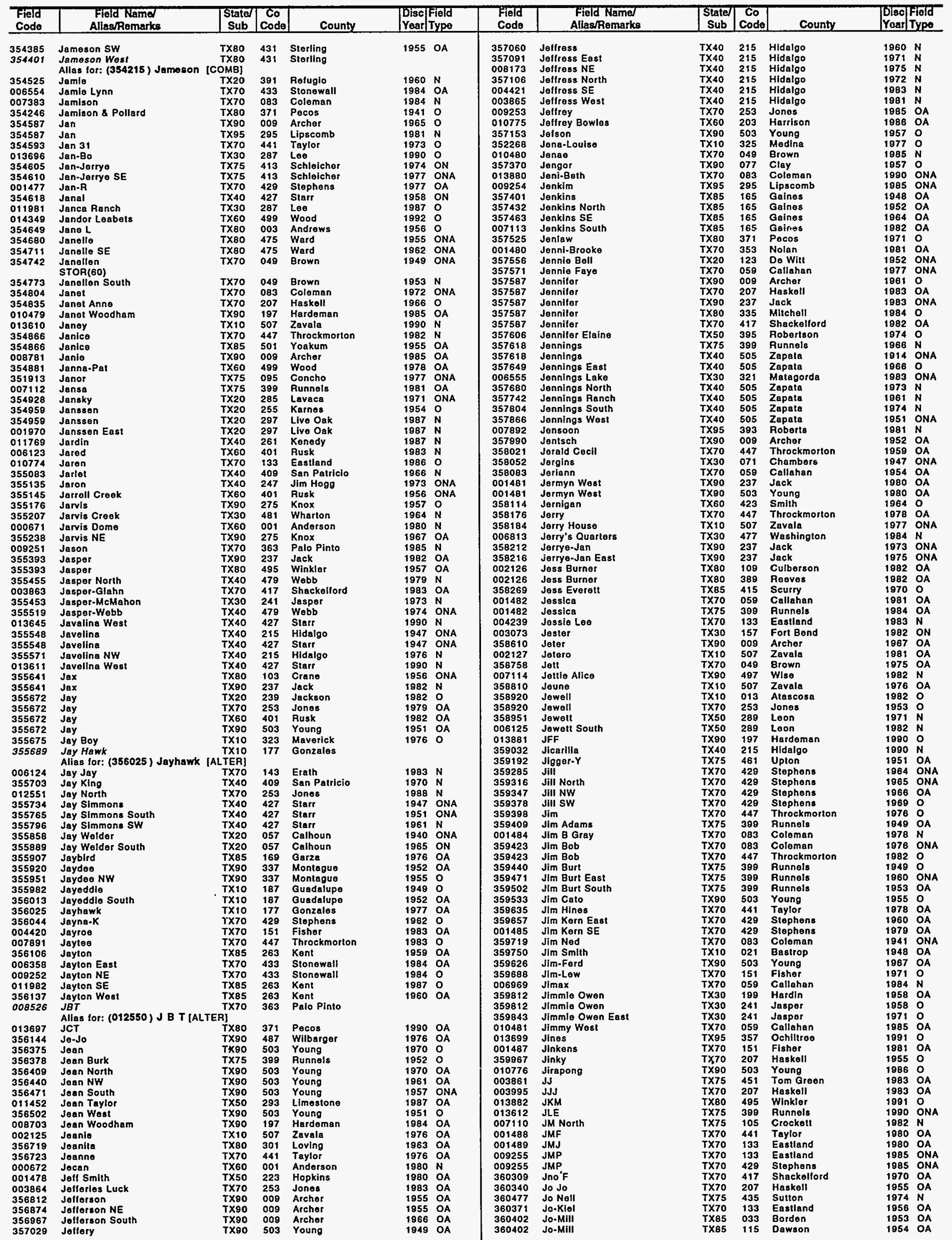


TEXAS

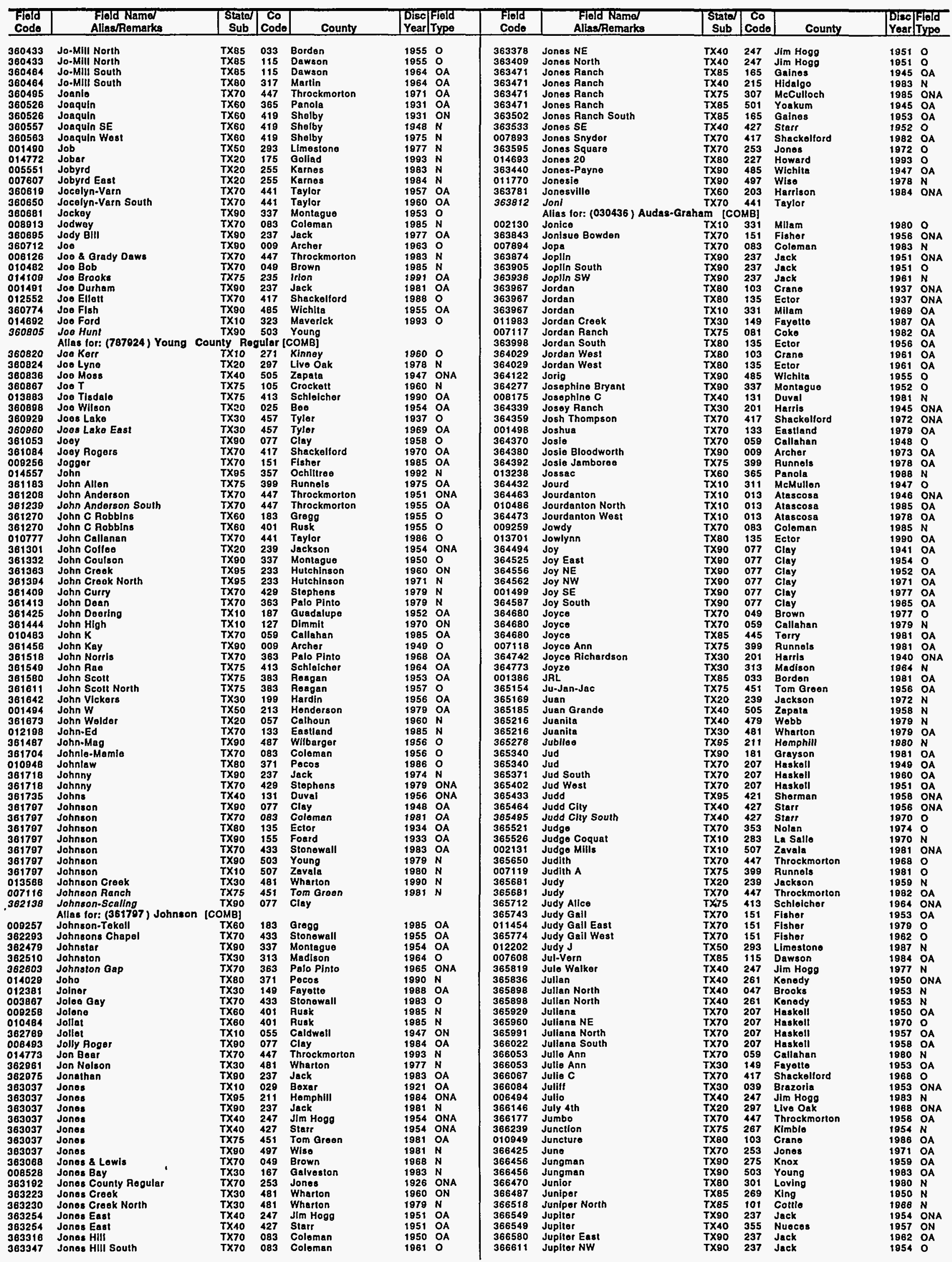


TEXAS

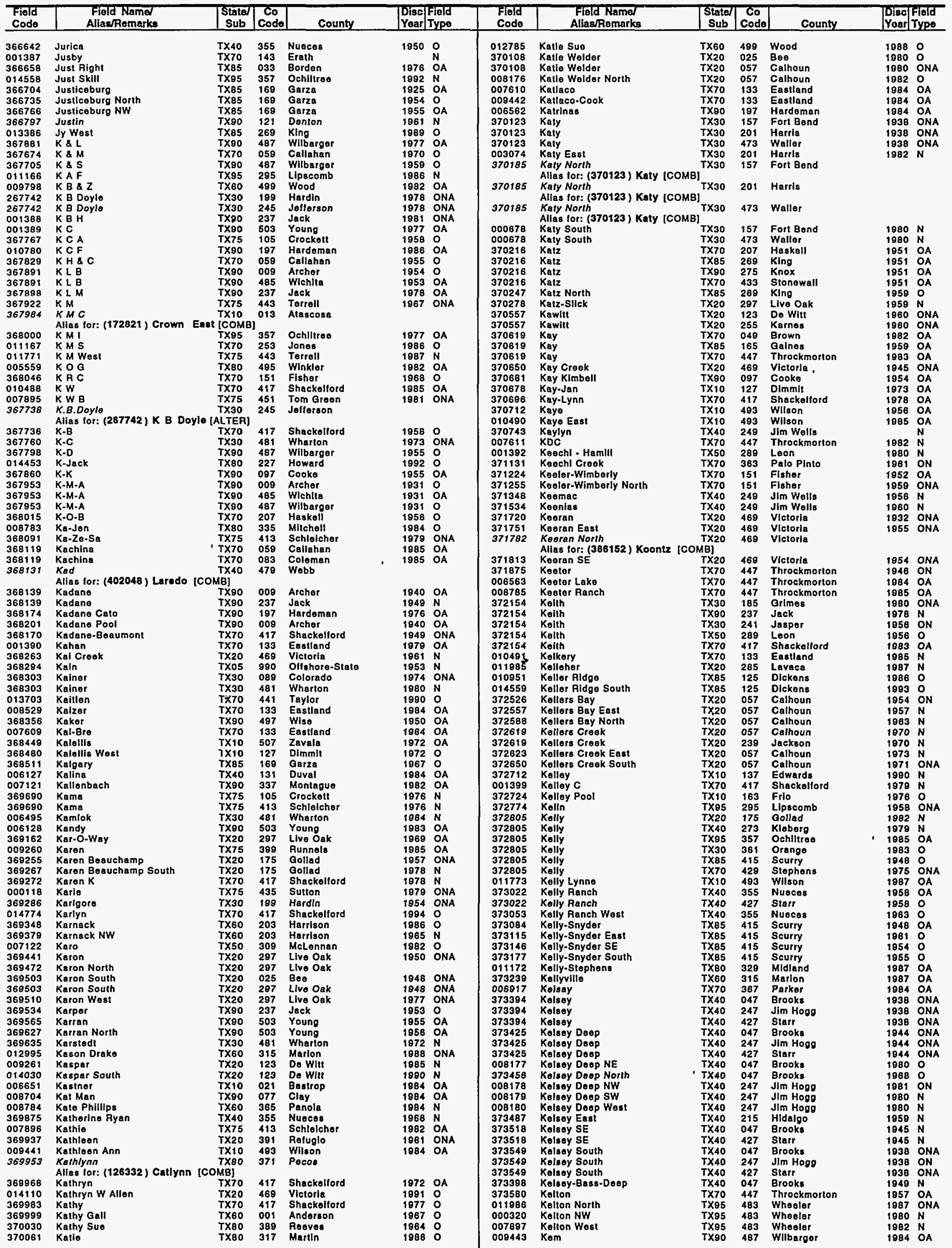


TEXAS

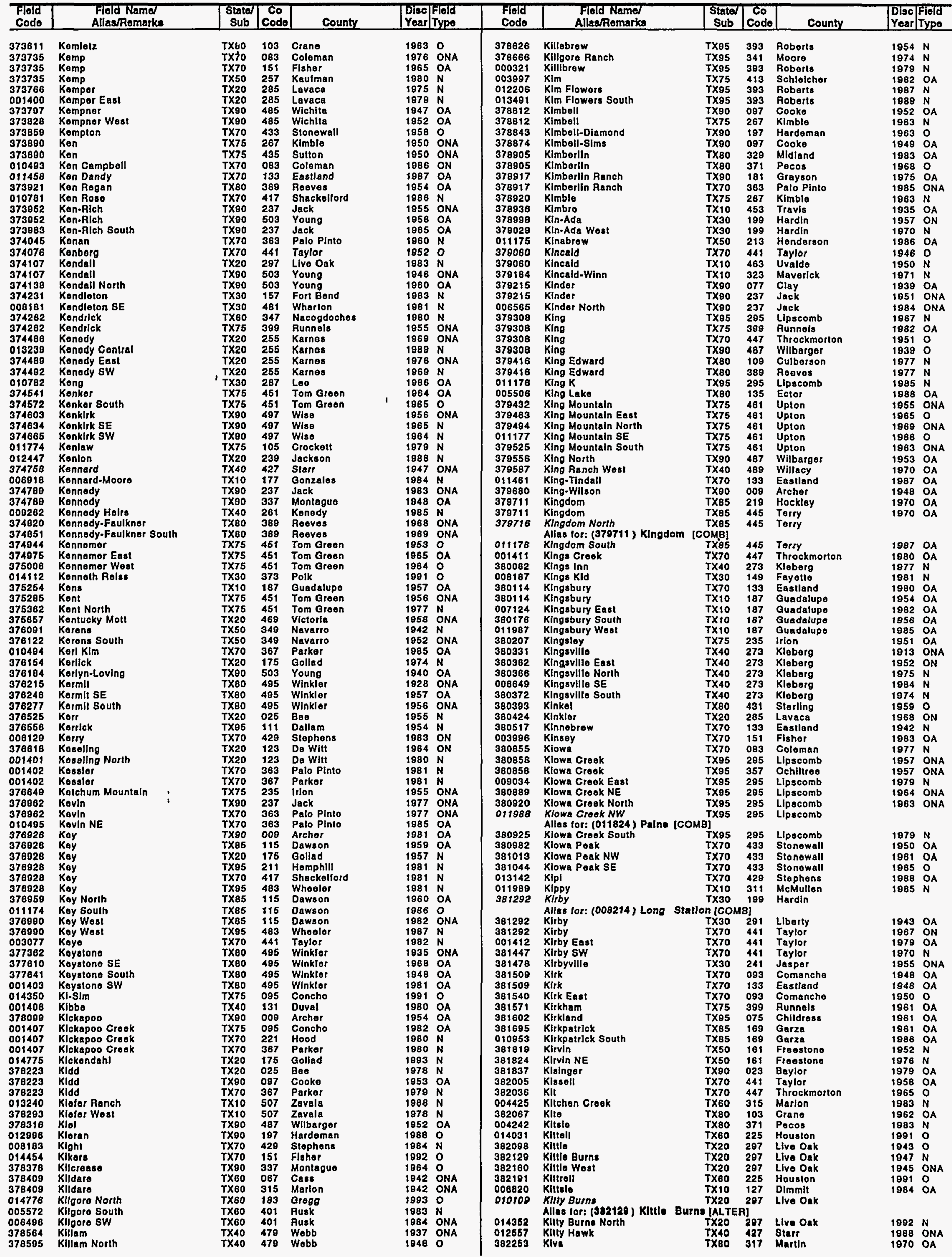


TEXAS

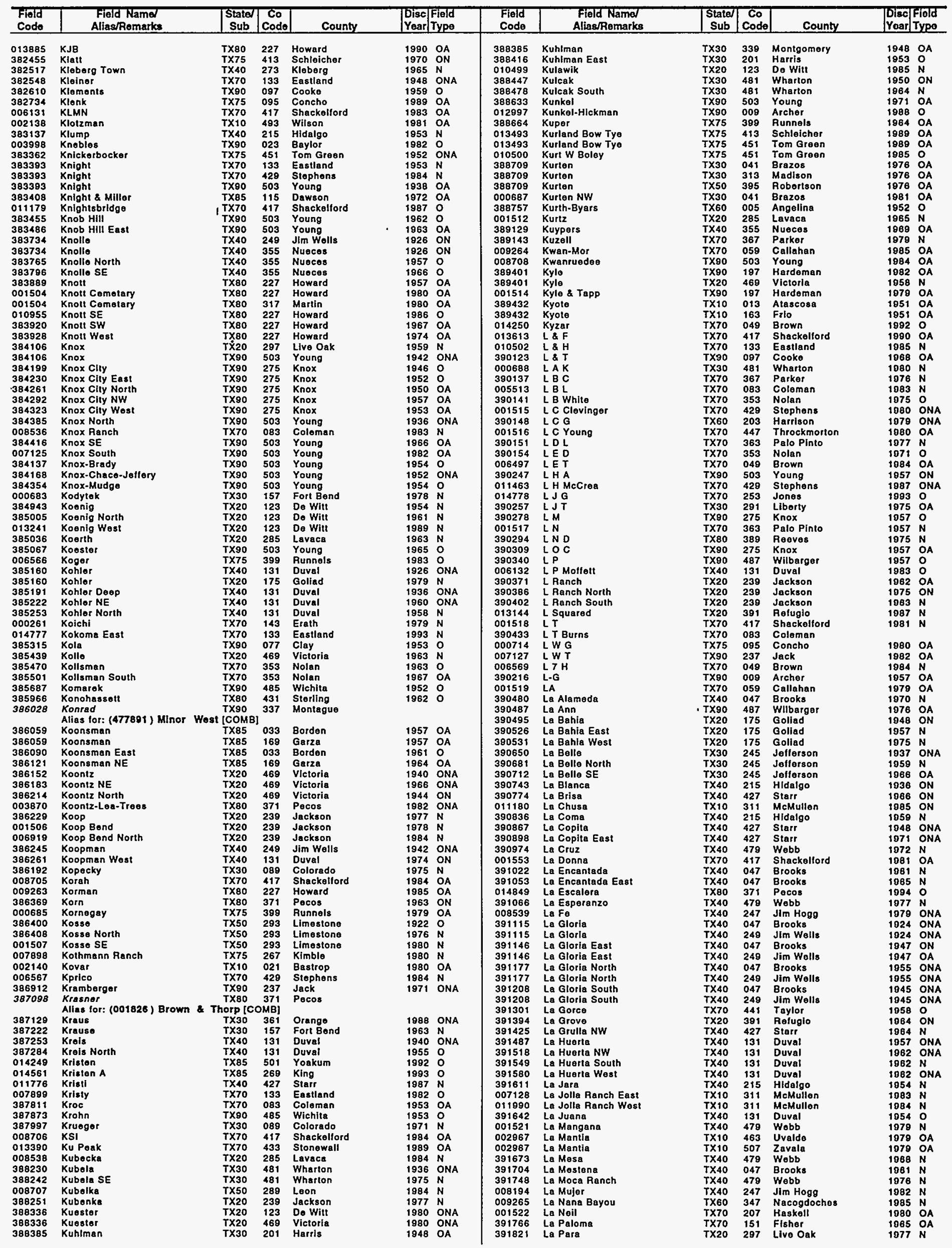


TEXAS

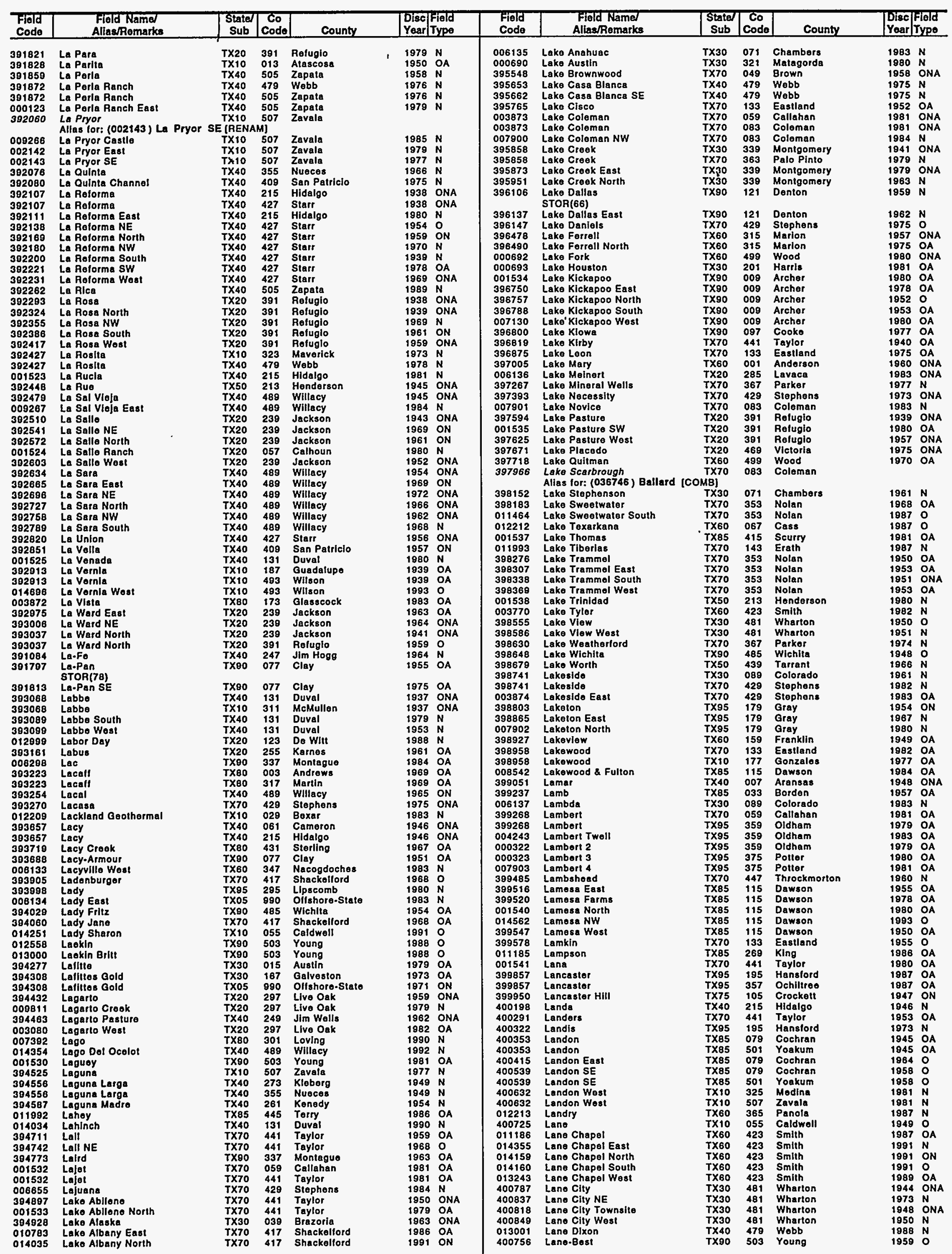


TEXAS

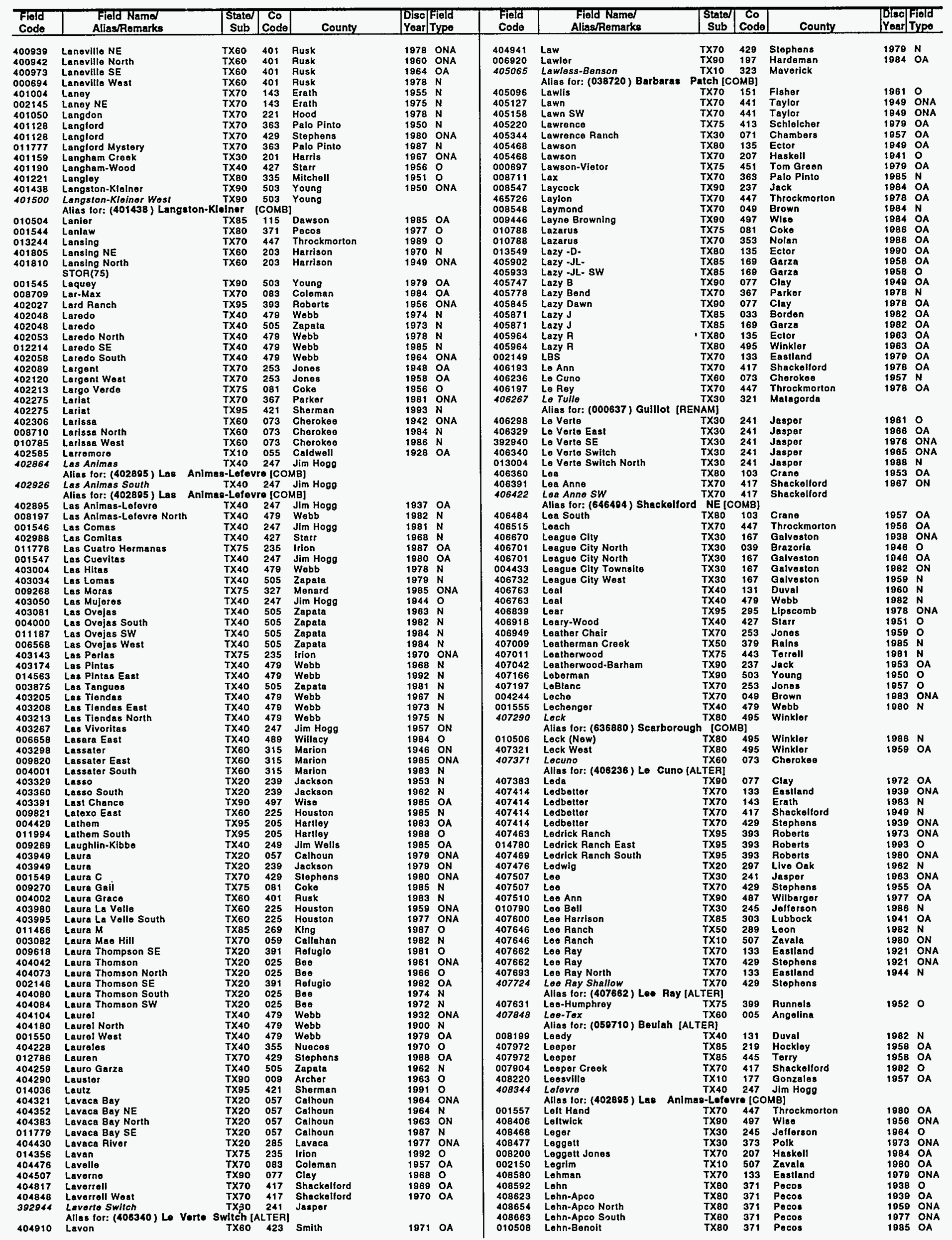


TEXAS

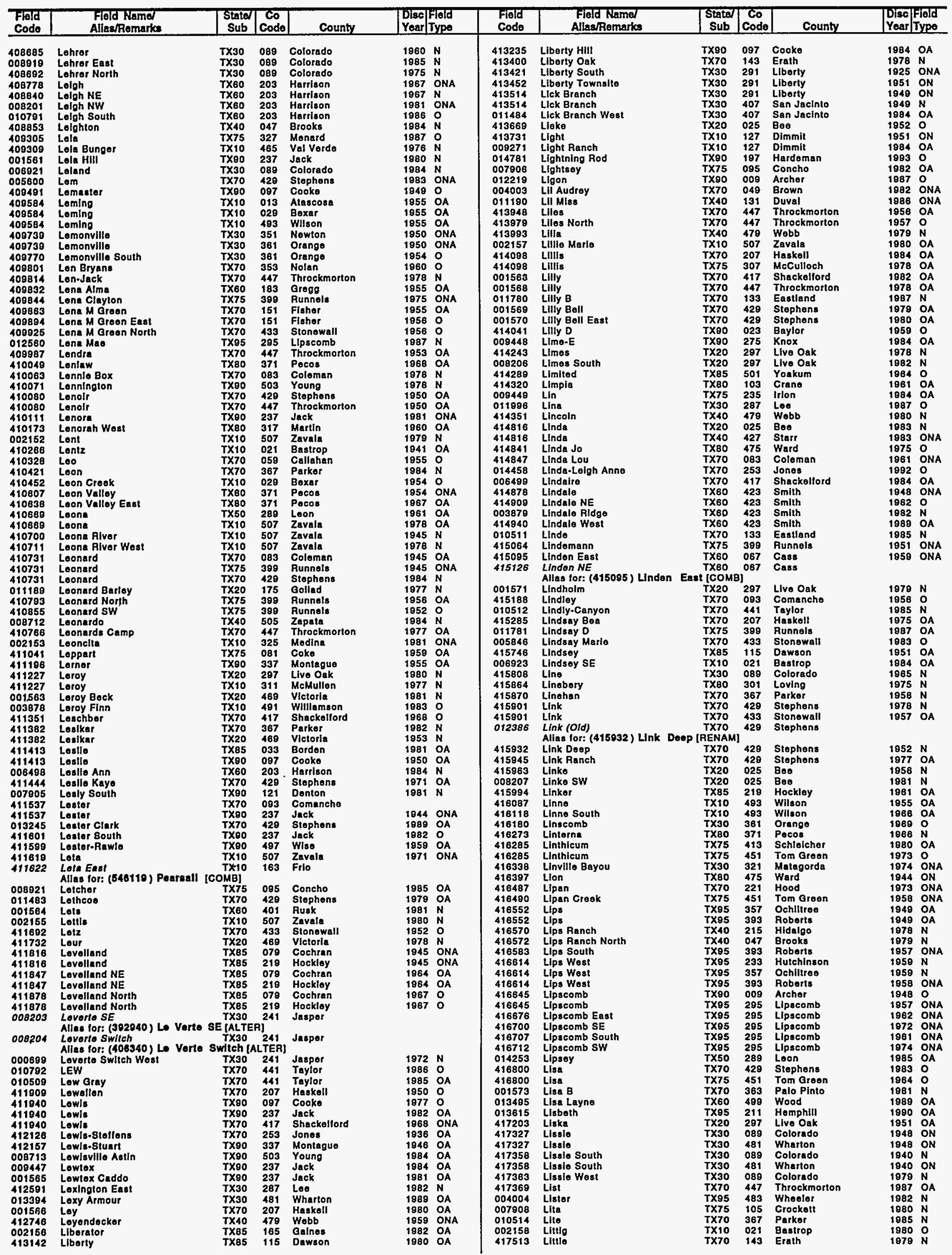


TEXAS

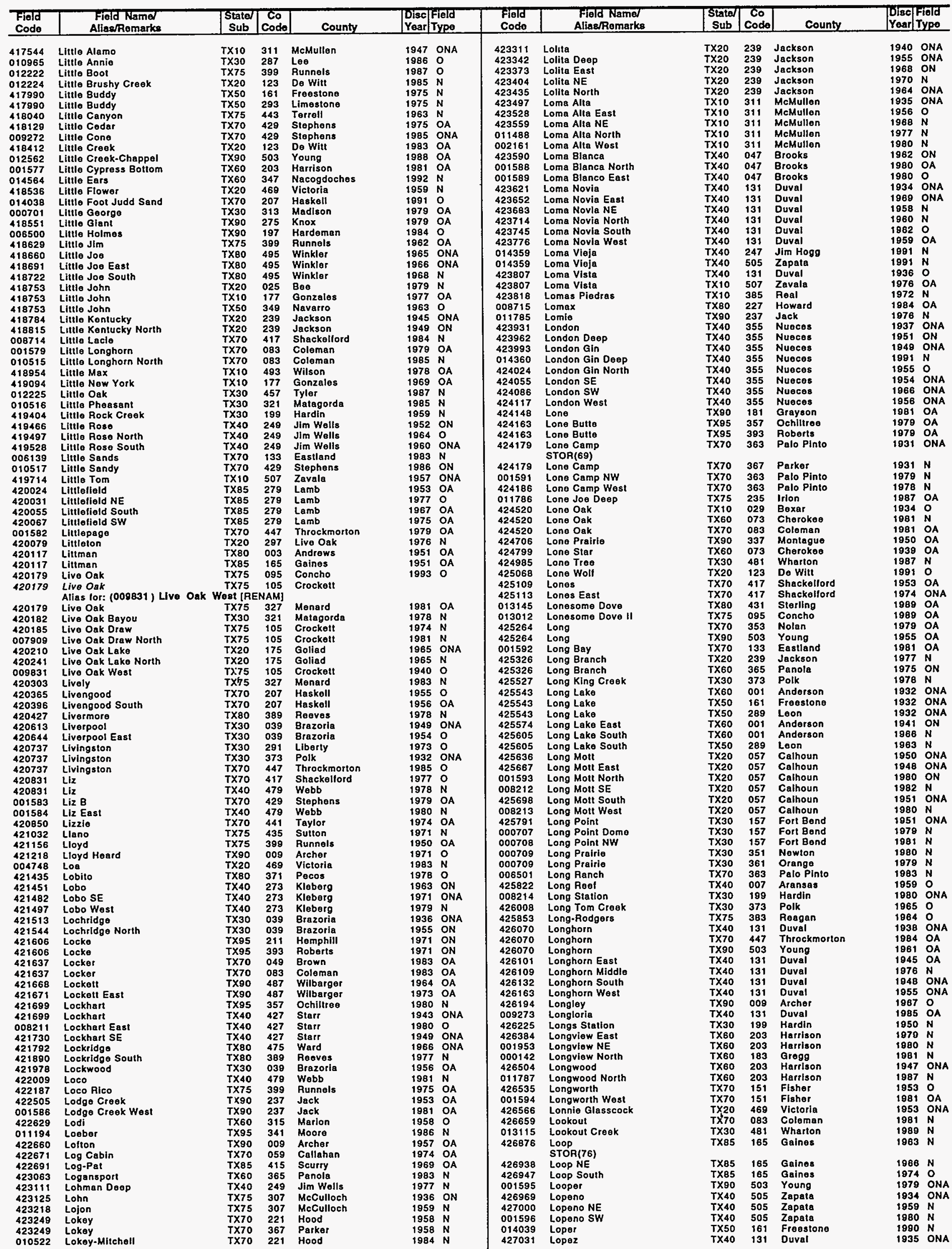


TEXAS

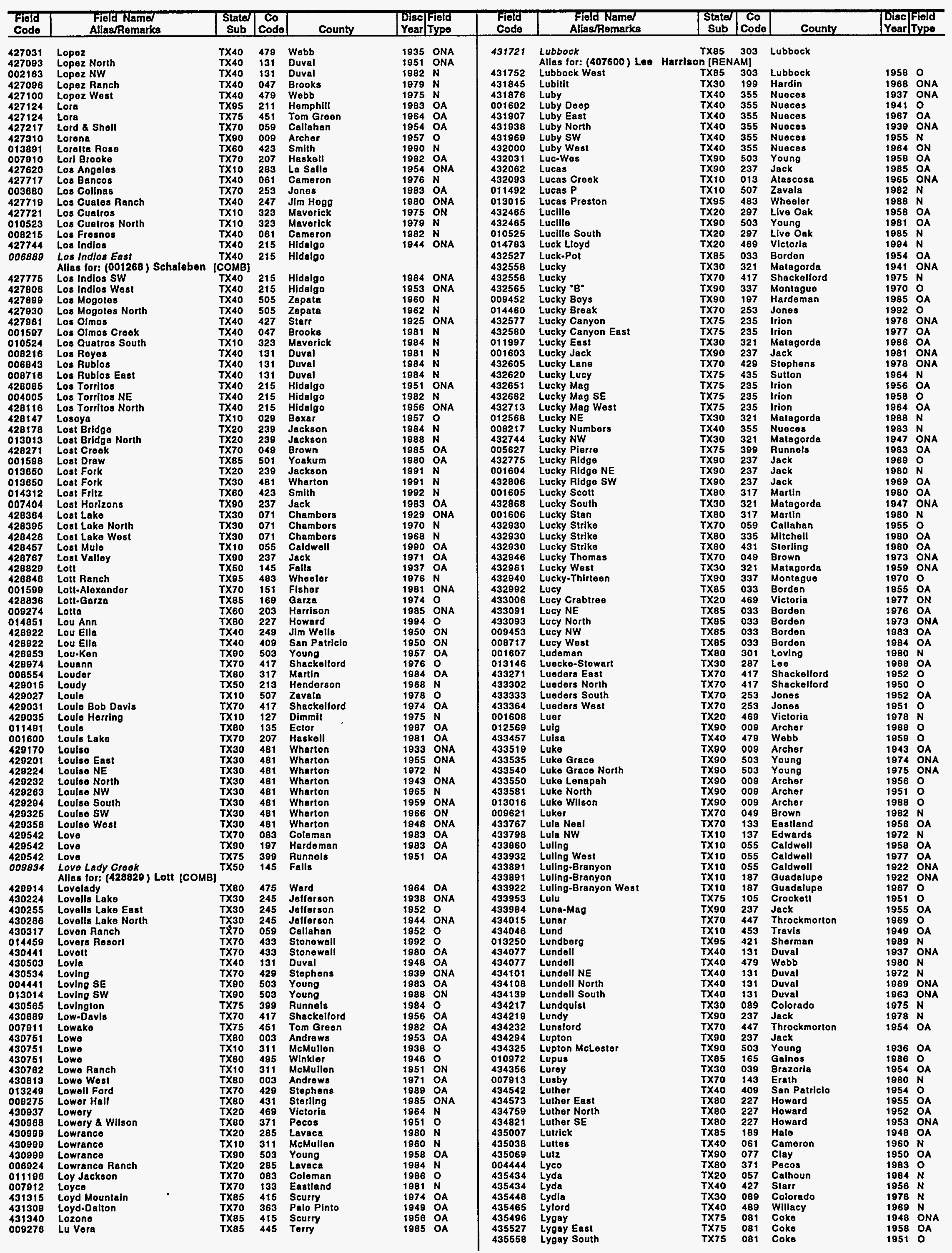


TEXAS

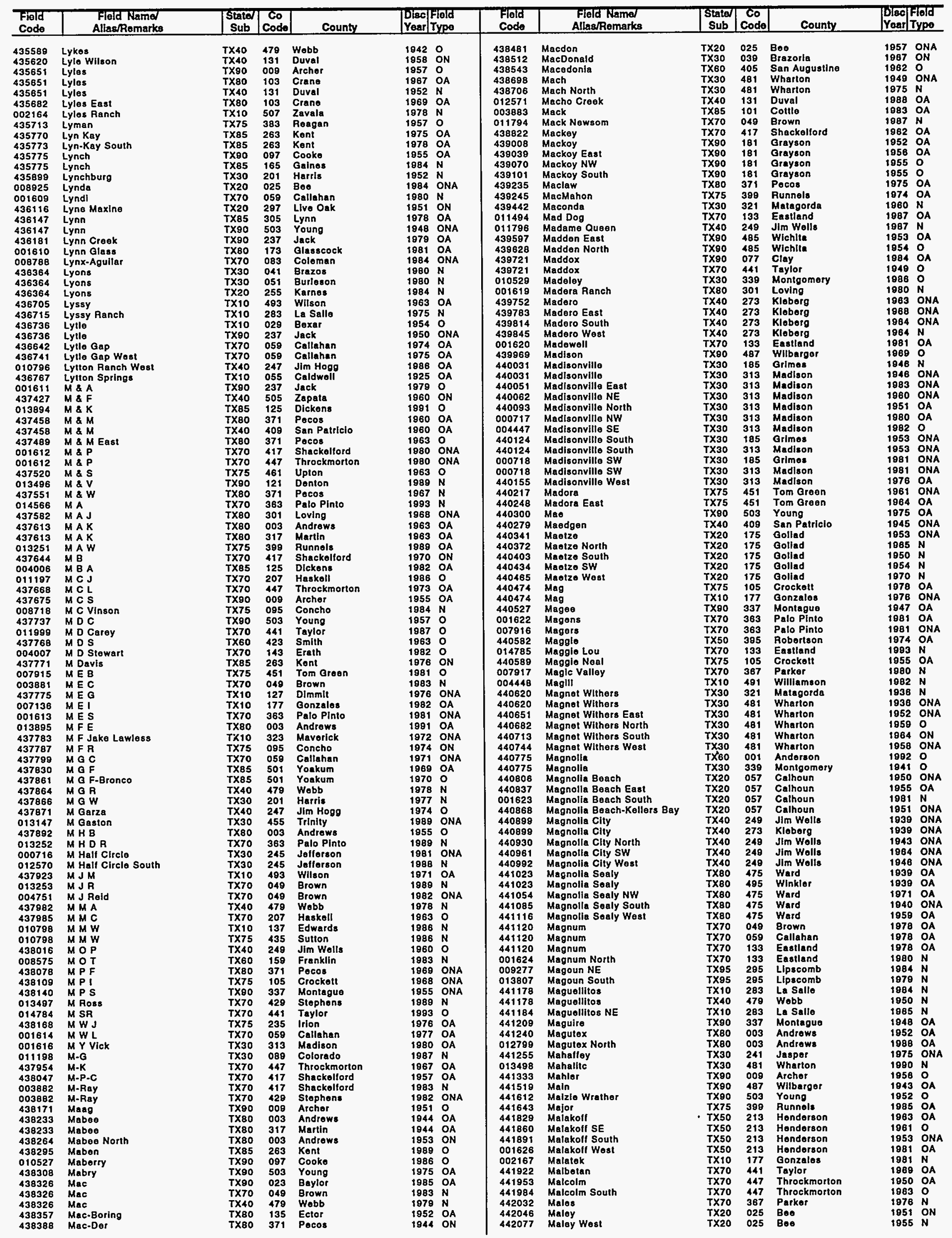


TEXAS

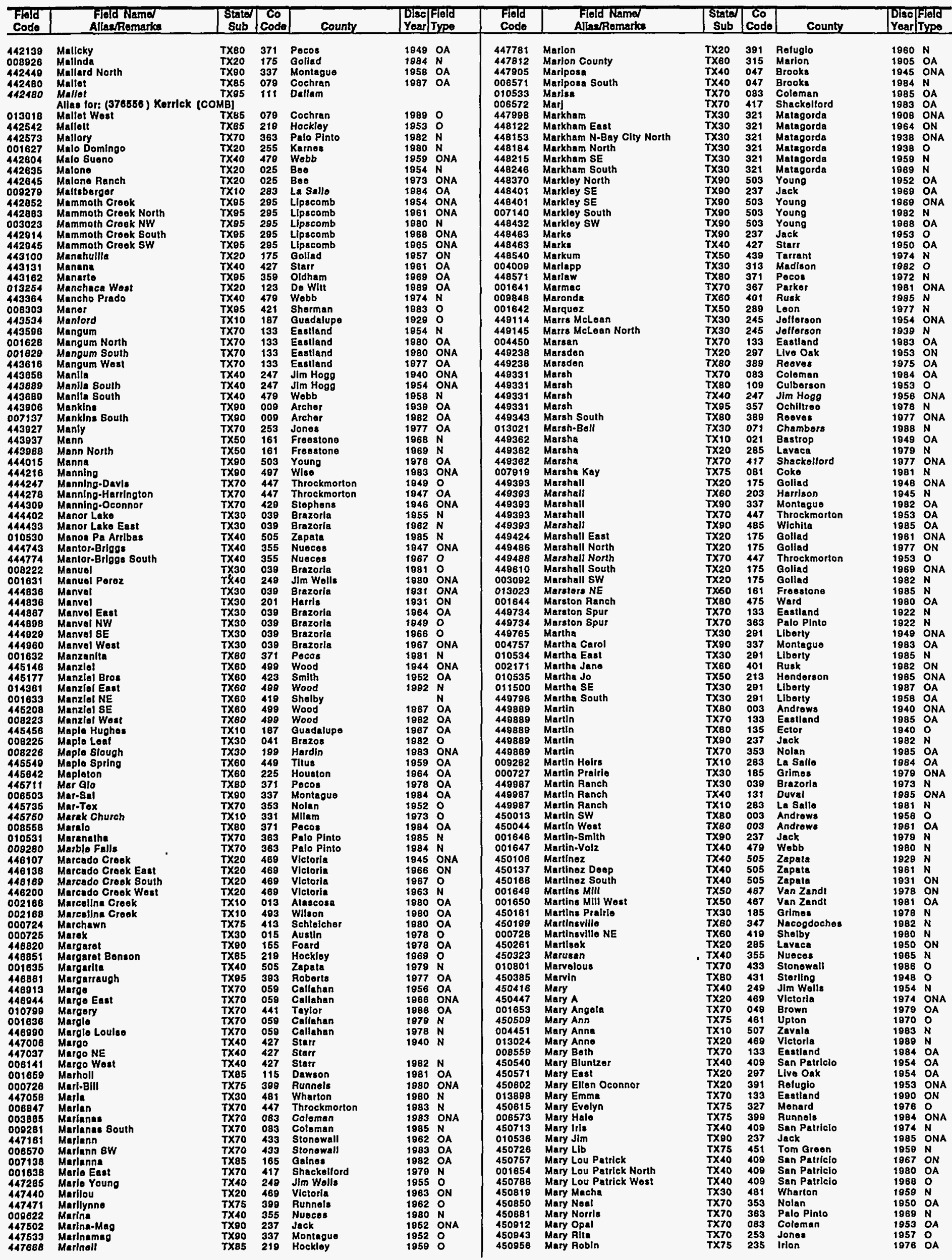

Energy Information Administration/Oil and Gas Field Code Master List 1994 


\section{TEXAS}

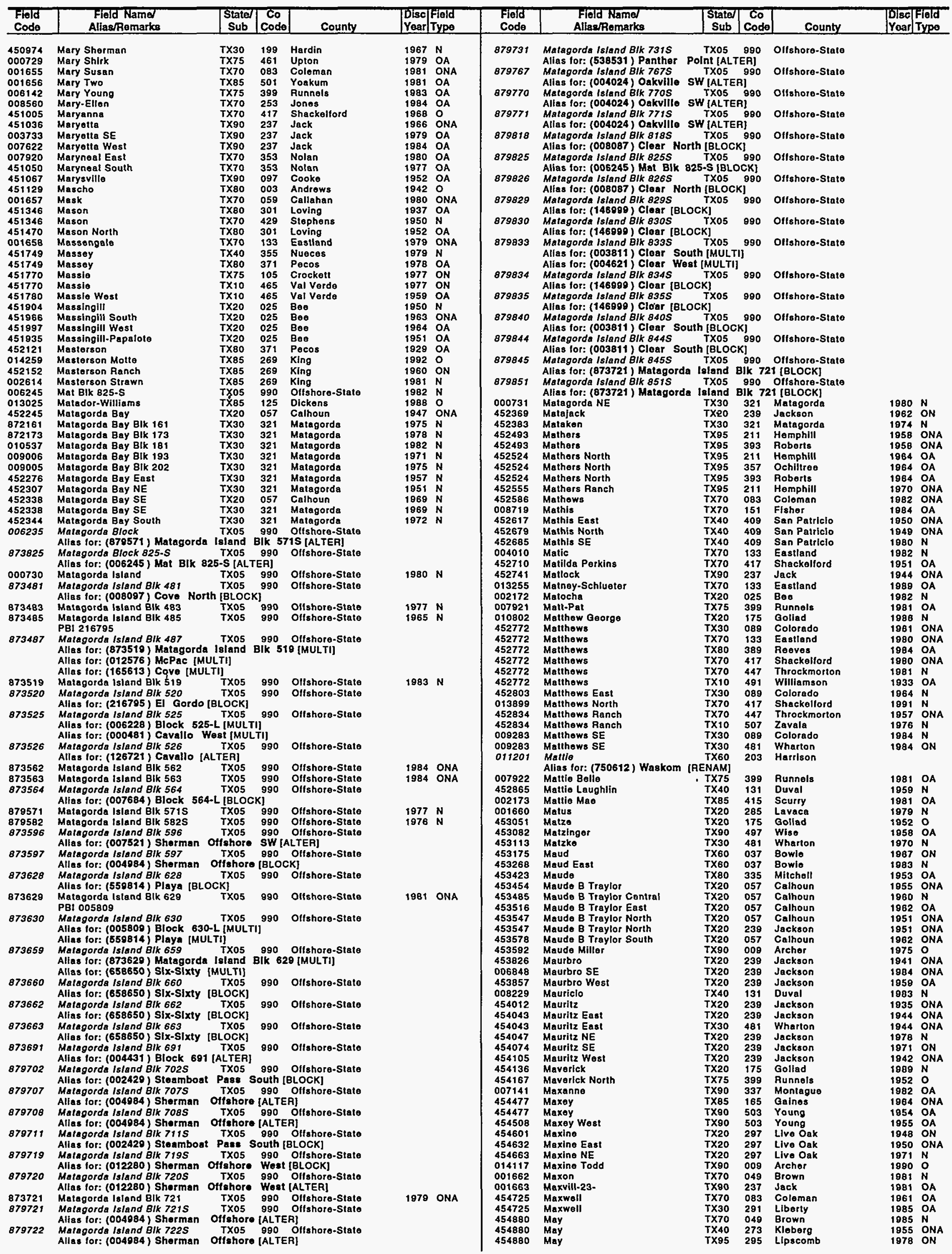


TEXAS

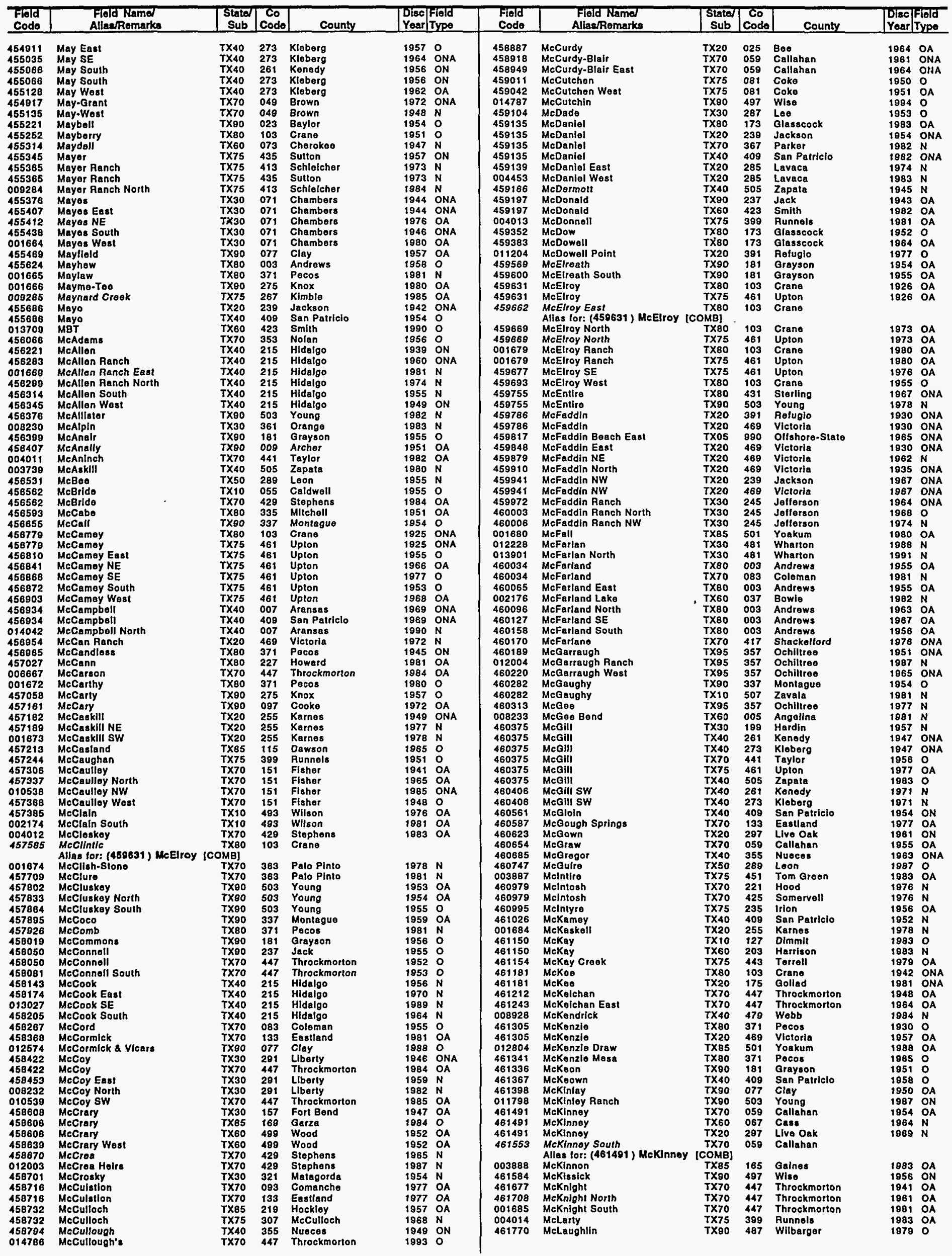


TEXAS

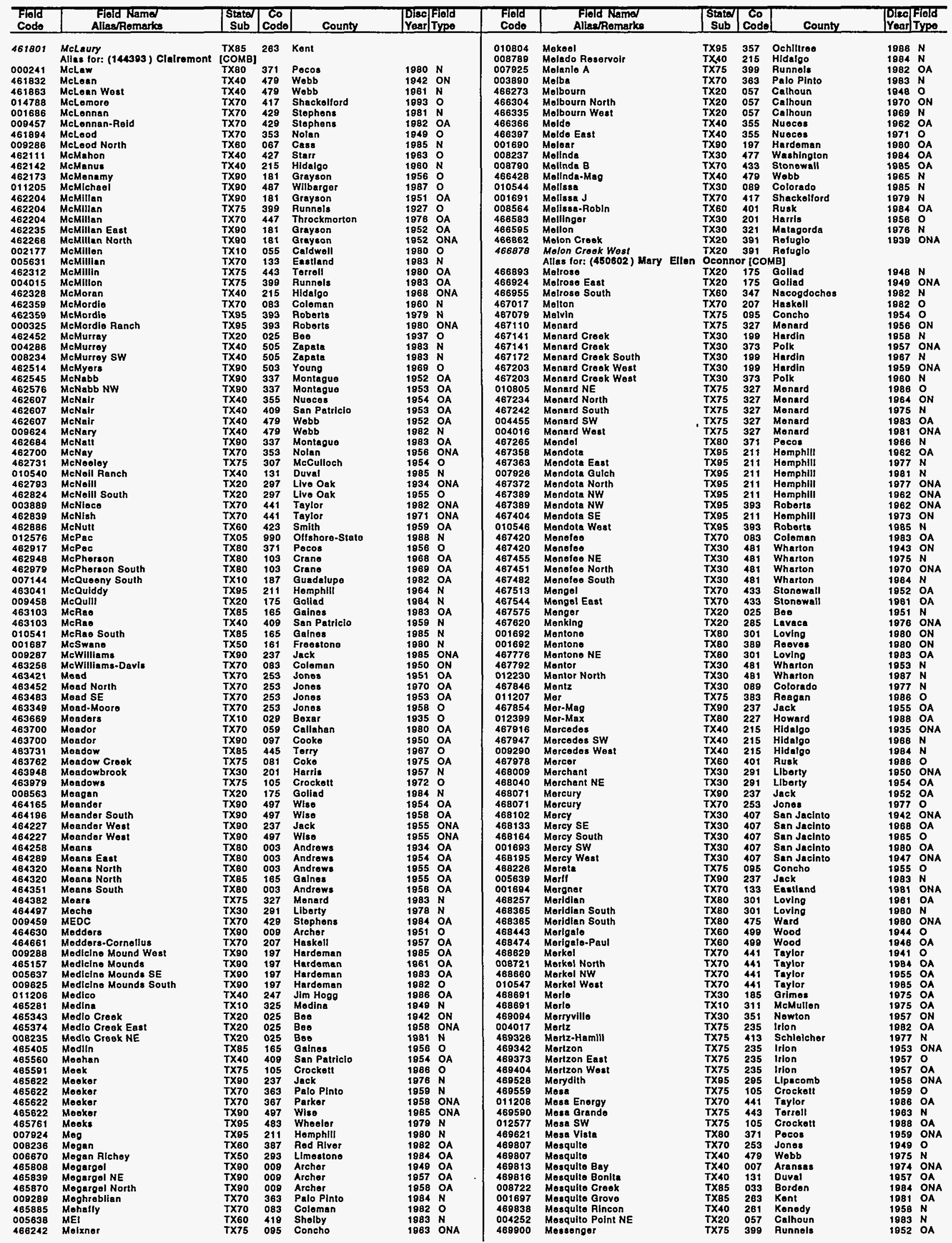


TEXAS

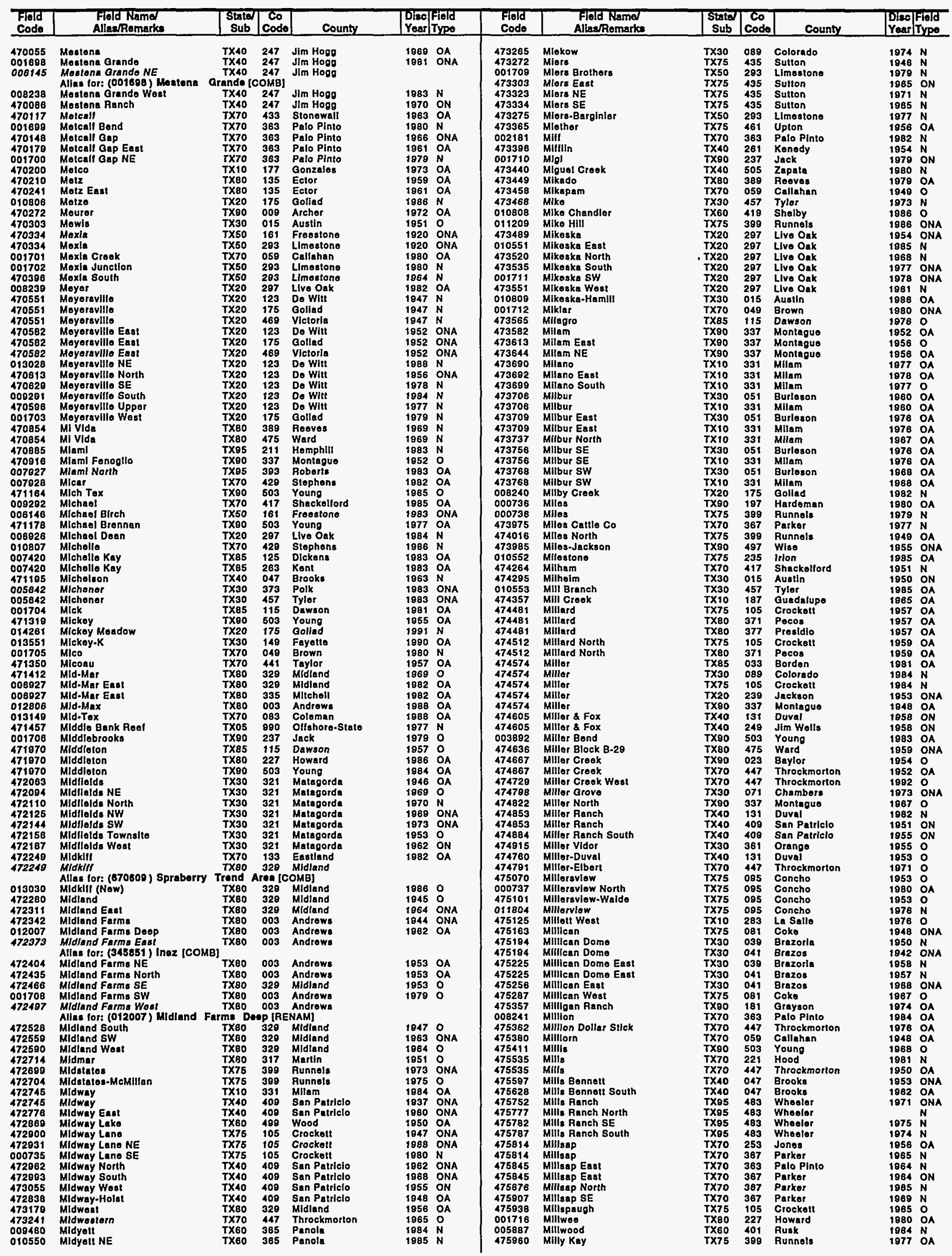


TEXAS

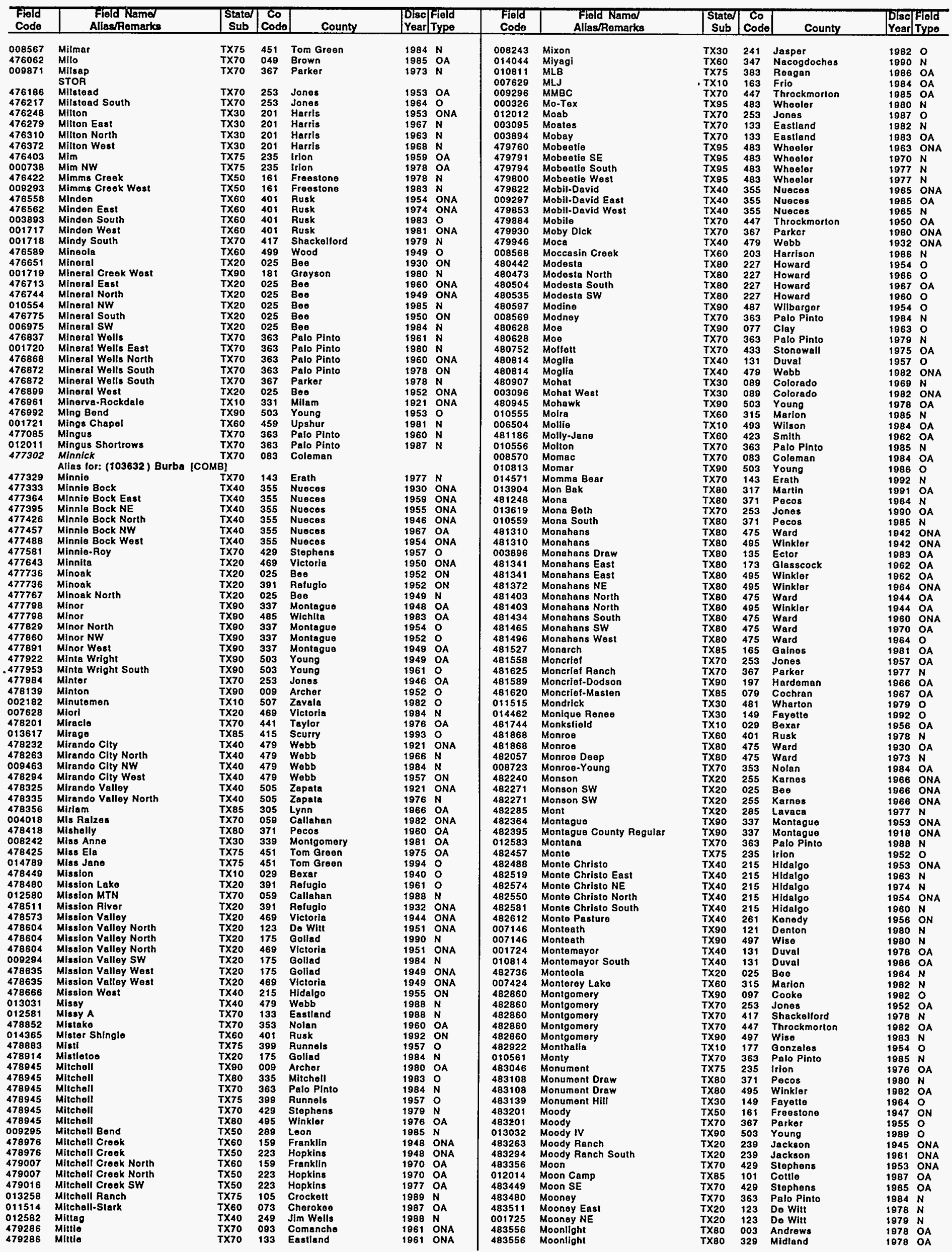


TEXAS

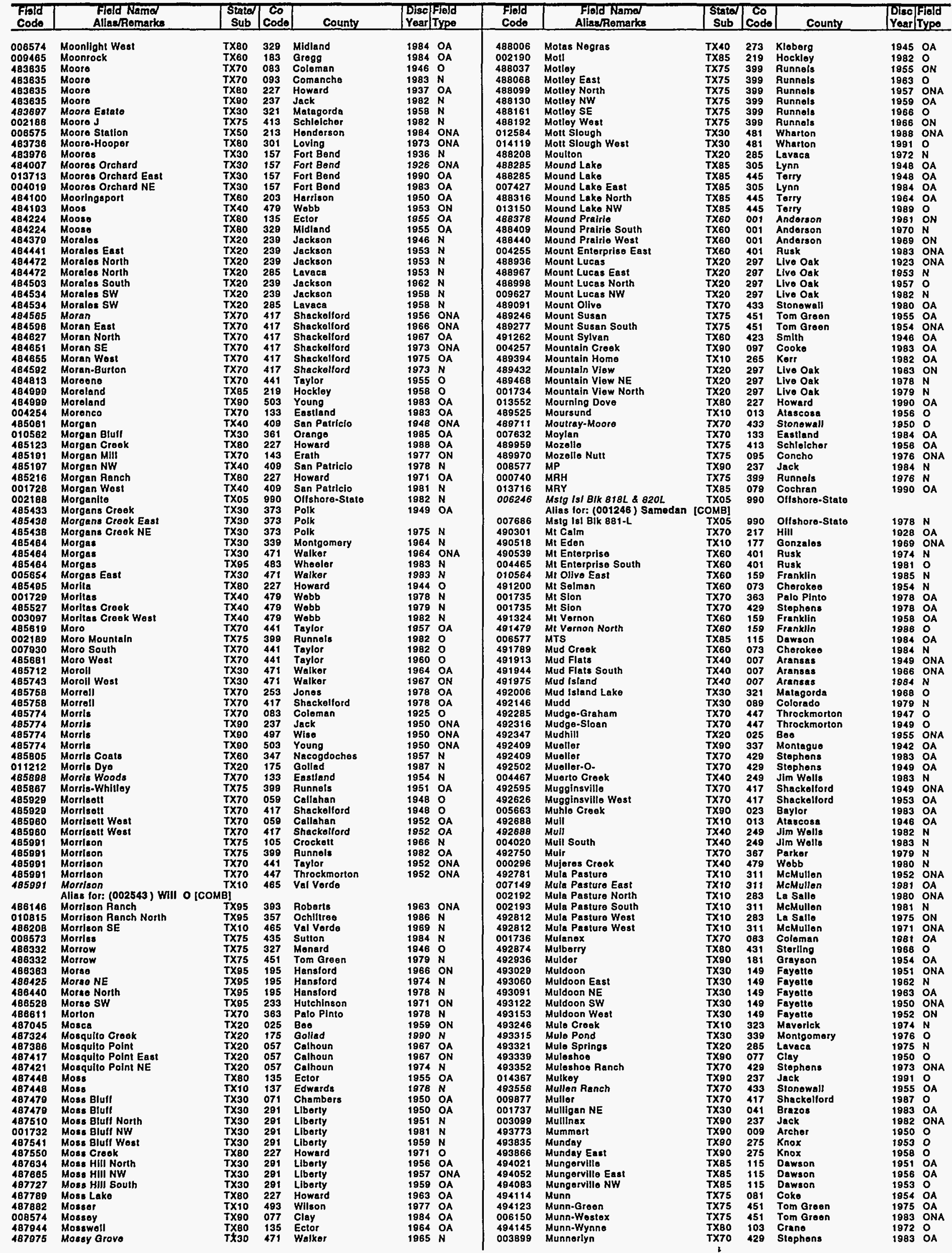




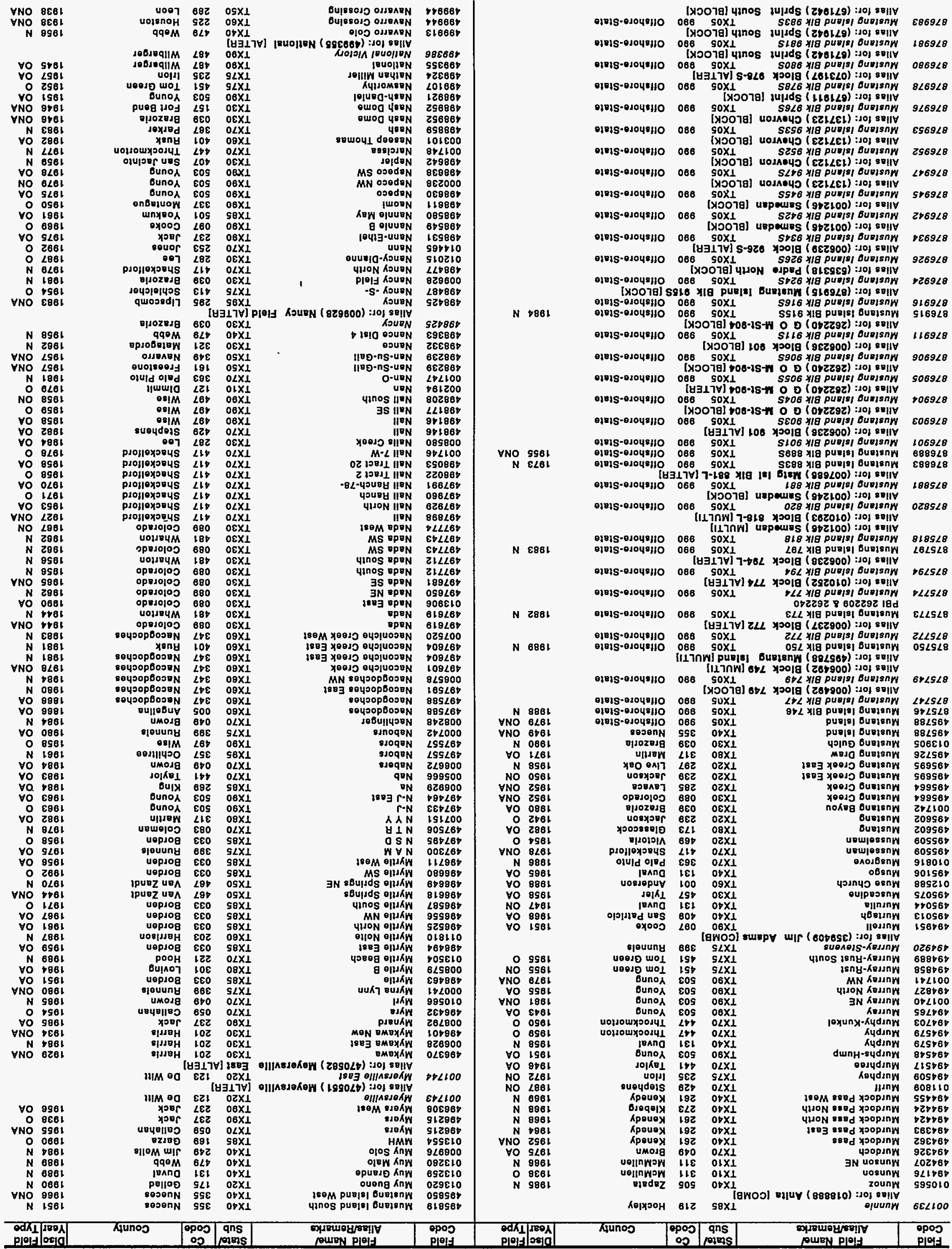




\begin{tabular}{|c|}
\hline 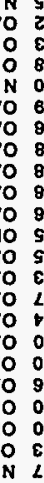 \\
\hline
\end{tabular}

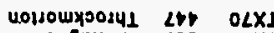

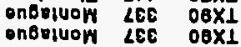

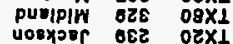

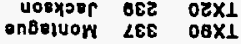
xoux sLz $06 \times 1$ $\begin{array}{rll}007 & 282 & \text { OEXI } \\ \text { uosejung } & 150 & \text { OEXI }\end{array}$ $\begin{array}{rrrr}\text { uosoping } & 150 & 0 E X I \\ 007 & L 82 & 0 E X I\end{array}$ \begin{tabular}{ccc} 
vosoping \\
ISO & $0 \times X_{1}$ \\
\hline 002 & $0 \times 1$
\end{tabular} $\begin{array}{ccc}007 \\ \text { voseing } \\ 150 & 0 \times X 1 \\ \text { OEXI }\end{array}$

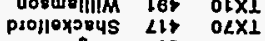

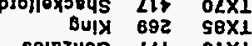

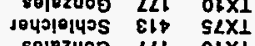

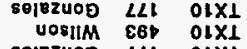
solezuog LLL OLXI

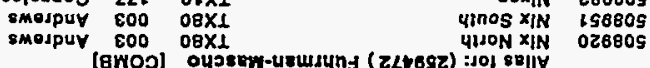
smojpur 800 08X1 00נ11400 LSE $58 \times 1$ voprew SIE OxO0 180 sLXI

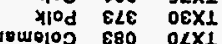
sasues 200 orXi ensuedy 200 orx SOAOOY 682 OOXXI

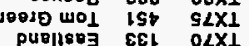
puriles EEt $0 \angle X I$ purpses $\varepsilon \varepsilon 1$ 0LXI ADellim 685 orxi

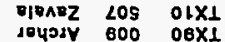

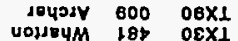
UoगयपM 18\$ OEX1

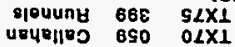

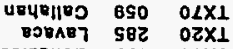
$\begin{array}{lll}\text { 8018zuog } & \angle L 1 & 0 L X 1 \\ \text { 8peutung } & 668 & 5 \angle X 1\end{array}$

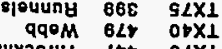

Yo 6261

vo $8<8 !$

YNO $\angle 261$

O EBst

YNO 8861

$\checkmark N O$ Or61

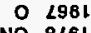

YNO 8261

N 8861

$\forall N O$ LS61

N $0<61$

N 1261

을

YO ES61

vo 8561

YNO $\begin{aligned} \text { YL6! } \\ \text { ES6! }\end{aligned}$

NO 8961

YNO 8961

O $\$ 961$

Yo $\$ 561$

$\forall$ YNO ISBI

N 8801

N SL6i

Yo 296 !

$\forall O$ isei

vo 6961

Yo 6961

No 1961

N 1801

$\begin{array}{lll}\text { YNO } & 5981 \\ \text { N } 1886 !\end{array}$

$\begin{array}{lll}N & 188 ! \\ \text { O } & 858 !\end{array}$

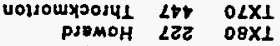

promoH LEz ISE $08 \times 1$

obiepir siz oxxit

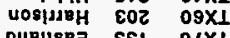

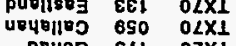

pणI00 SLL O2X1

UOIMON ISE 0 EXX

Q98M BLt $0+\times 1$

บจw0100 880 0LXI

oexojoyo $8 \angle 0$ 09X1

이려 891 ol $\times 1$

duro 890 09x।

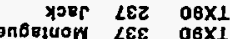

xour $2 E 2$ oxx

xin $2<0$ 08x1

xijo $2 \angle 0$ o8x

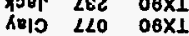

oburdo loE OEXI

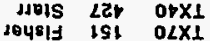

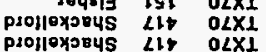

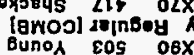

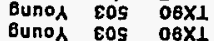

$\begin{array}{lll}\text { Bunos } & \text { cos } & \text { obXI } \\ \text { Bunos } & \text { Eog } & \text { OBXI }\end{array}$

$\begin{array}{lll}\text { bunne } & \text { EOs } & 08 \times 1 \\ \text { Bunod } & \text { Eos } & \text { OBXI }\end{array}$

Bunoᄉ EOS OBXI

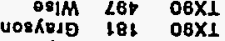

$\begin{array}{ccc}\text { vivdez } & \text { SOS } & \text { orXI } \\ \text { XDIO } & \angle L O & \text { OBX1 }\end{array}$

YNO $\angle Y B L$

Yo ع861

N $2 \angle 6 !$

ZNO 1.8B!

$\forall N O$ ILB!

N $6<0 !$

St81

$\begin{array}{ll}N & 1 \\ N & 0<81 \\ N & 1\end{array}$

N
N
1

N 6 ret

Yo SSB1

VNO 6SGI

N 2861

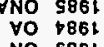

XDI L $\angle 20$ O8XI

$\begin{array}{lll}x \in 19 & \angle \angle 0 & 08 \times 1 \\ x \geqslant 04 & \angle 8 Z & 08 \times 1\end{array}$

20|k8 220 08X1

JE्राएM $1 \angle b \quad 0 E X 1$

ulsenY sio oEXI

ufleny sio oexl

uluy

บ01נv4M 18p OEX1

vouseyM los oEX1

81804 L8 oEx

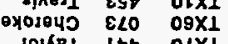

solka 1 it $0<1$

OlBnioy IBE $02 \times 1$

$\begin{array}{lll}x=n y & 10 \% & 09 \times 1 \\ 990 M & 6<5 & 0 \times X 1\end{array}$

WIN MON OBBtOS

UODPI AN 268709

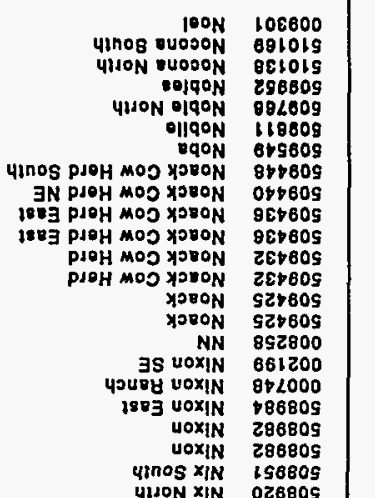

YNo $\begin{aligned} \text { Z } \\ \text { \& }\end{aligned}$

YNO $898 !$

YNO $8 \angle B L$

$\forall N O$ †ESI

YNO $\$ E B !$

YNO $\mathrm{SSBL}$

VNO 1581

YO $\angle 261$

Yo 8861
YNO 2581

IEO K A

(29) YOLS
A10 YJON MON grisos

Oกヨ 9300 1 MON EOBEOO

$180 \Omega$ MON 708800

MS UID MON O00SOS

MS WIn MeN O0OSOS

$S$ win MON $89 \angle 100$

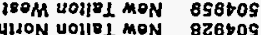

$18 B \exists$ UOIIEI MON BOBHOS

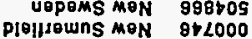

UEAOS MEN POBrog

pUQB JOAJY MON $88 B 600$

joodsold MON
285900
ouens OlघW MON
- 1863

Yo 2881

ro $\begin{array}{r}+961 \\ \text { O }\end{array}$

Yo 8986 เ

Yo 6581

Yo 2881

$N$
$\forall \angle 61$
VNO $2 \angle 6 !$

0 L 151

Yo 1981

yo 1961

Yo 2961

Yo 7881

$\forall 0$ \$SBL

N E8B!

응 2765

N 88661

N 2861

Yo 2581

Yo 1581

Yo 2968

: 5461

- EsBt

N 28061

vo $\$ 261$

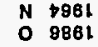

NO 8886

No 8281

o $988 t$

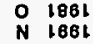

Yo 0286

YO $0<61$

N 8966

Y 9988 .

Yo 1886

$\forall 0$ EsB

N $8<81$

Yo 928!

Yo 1881

vo 2988 .

Yo 9981

YO 1884

YO 2889

YNO $998 \mathrm{~B}$

NO 3981

NO 8581

N 9881

YNO $1+81$

vo 8286

Yo 1881

vo 2283

vo 2581

Yo 6861

Yo 6981

Yo 8961

yo 8981

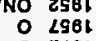

- $2 \angle 61$

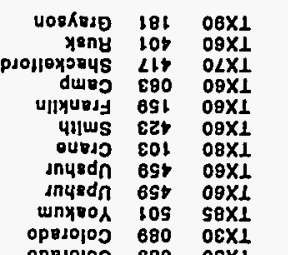

$\begin{array}{lll}680 & 0 E \times 1 \\ \text { opघJolog } & 880 & \text { OEX1 } \\ 0\end{array}$

01गम18 UES 60\% OPX1

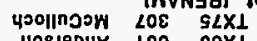

spoutung BBE SLXI

SO섬 $1 * 8$ OLXI

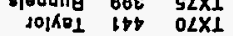

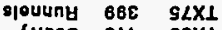

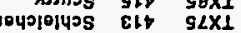

pooM 8Bt 09x1

1

8Bop wir Liz orXI

ollox日 $6+1$ oEXI

sasuesy 200 orX1

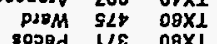

DiBazz 205 OIXI

OSTRAN GTE OSX1

UEION ESE OLXI

HOYSगW SEE OEXI

U日|ON ESE OLXI

UEION ESE $0 \angle X 1$

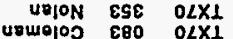

seวonN 998 O†X1

$\begin{array}{lll}\text { sulydoH } & \varepsilon z z & 0 \text { oxI } \\ \text { uilsny } & \text { sio } & 0 \varepsilon \times 1\end{array}$

uाเent sL

$\begin{array}{lll}\text { UjpiEH } & 681 & 08 \times 1 \\ \text { eMejpuY } & \text { E00 } & \text { O8XI }\end{array}$

$\begin{array}{lll}\text { smespuY } & 800 & 08 \times 1 \\ \text { suoudors } & 62 b & 0<\times 1\end{array}$

บолу⿰чM 18\$ OEXL

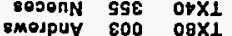

gpounny $8 B 2$ sLXL

UBION ESE OLXI

10481 19: $02 \times 1$

voluoa lit o6X1

souor Egz $0 \angle X_{1}$

sevor est 0Lx1

souor ESE OLXI

OU0180uा $\angle E 2$ OBXI

pdoj|0x284S LLt $0 \angle X I$

20481 19L $02 \times 1$

JuAna $1 E t$ orXI

jeana $1 E 1$ orXI

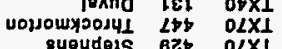

suoudars BZt $0<x 1$

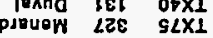

pueg म0ड 291 0EX1

pueg 120 L 291 OEXI

PUeg 100 LSL $08 \times 1$

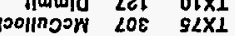

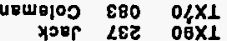

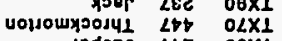

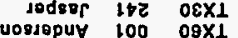

$\begin{array}{lll}\text { บossopuy } & 100 & 09 \times 1 \\ 00 \times 01040 & \varepsilon \angle 0 & 09 \times 1\end{array}$

oөx01040 $\varepsilon \angle 0$ 09X1

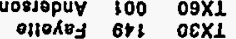

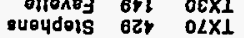

8900 280 09X1

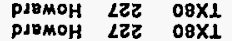

Xyogr $\angle E z$ O8X1

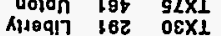

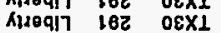

$\circ>881$

YNO 5981

N S981

N 6281

Yo 2881

N 2681

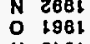

N S\$8t

No 2984

Uold84M L85 OEXL

05X1

घ8шерлв 600 08X1

PJPMOH L2Z OBXI

punod Eos obxi

8)

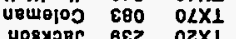

ouossowा ह62 OSXI

$09 \times 1$
I 91717
$05 \times 1$

$\begin{array}{rll}\text { voo7 } & 682 & 05 \times 1 \\ \text { บ08snoH } & \text { SEZ } & \text { O9X1 }\end{array}$

Yo
yo
$2886 !$

BOF MON Z9LLOO

OPUO7 MON 6Ectog

Inv OdOH MON gizrog

odoh moN tolros 
TEXAS

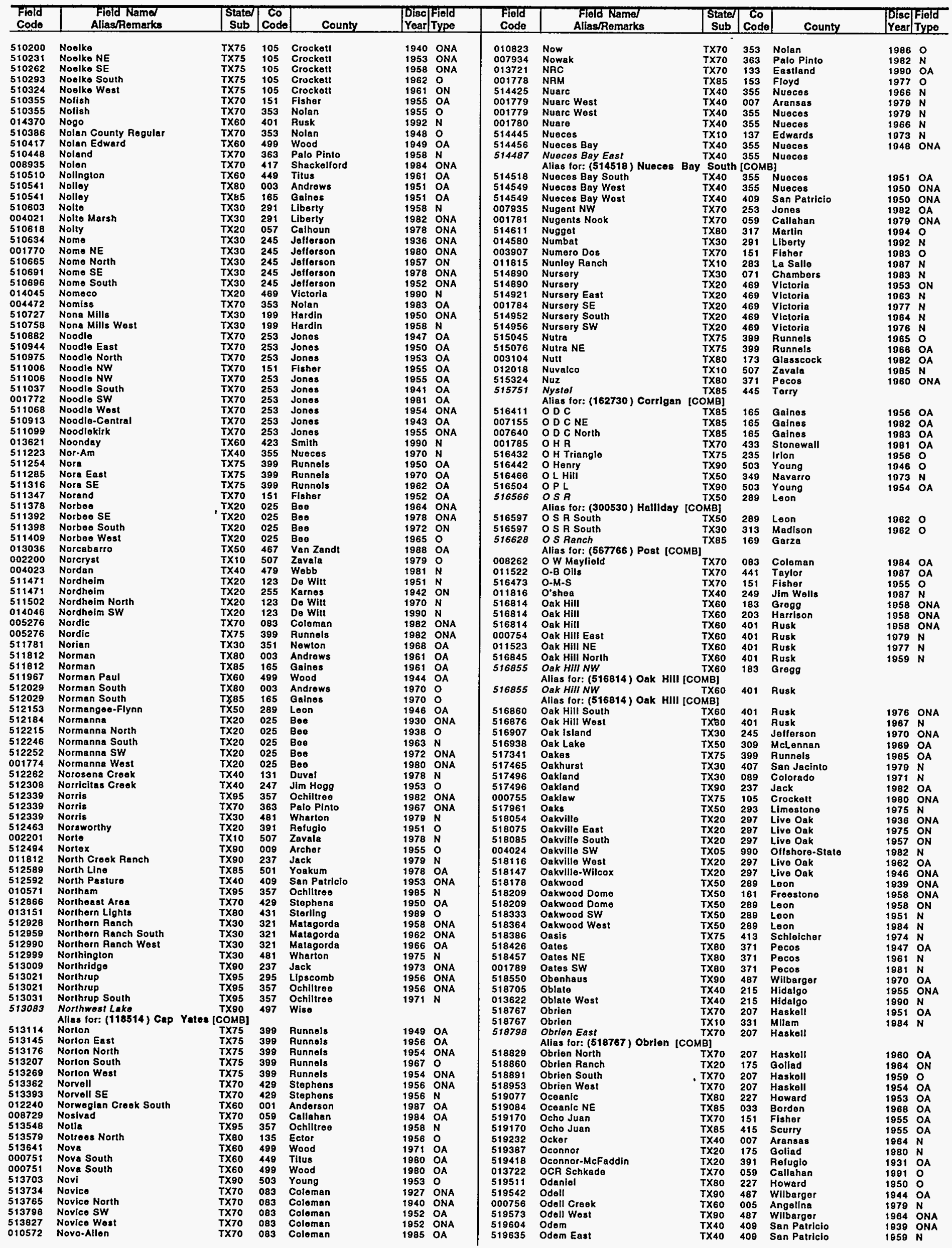


TEXAS

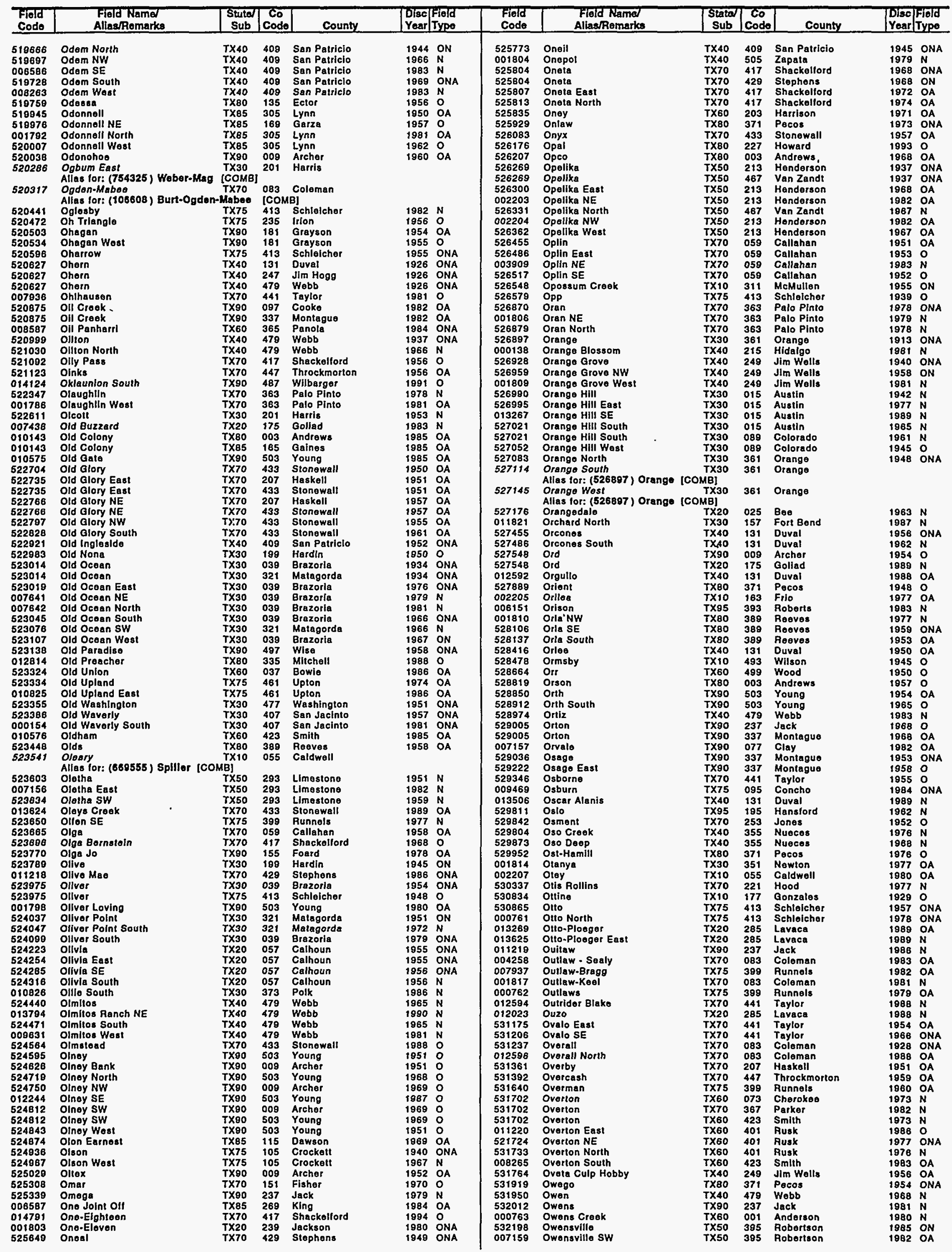


TEXAS

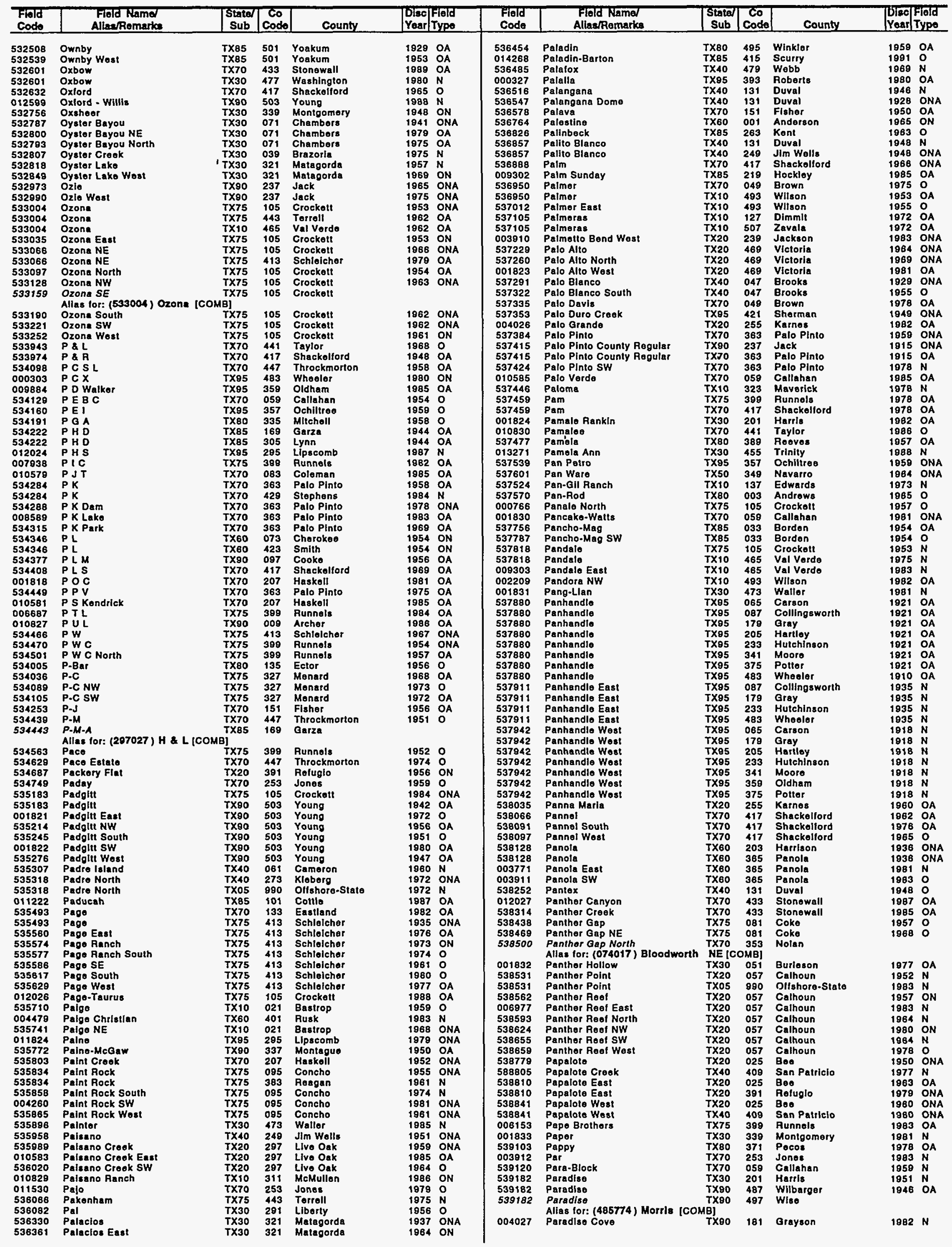


TEXAS

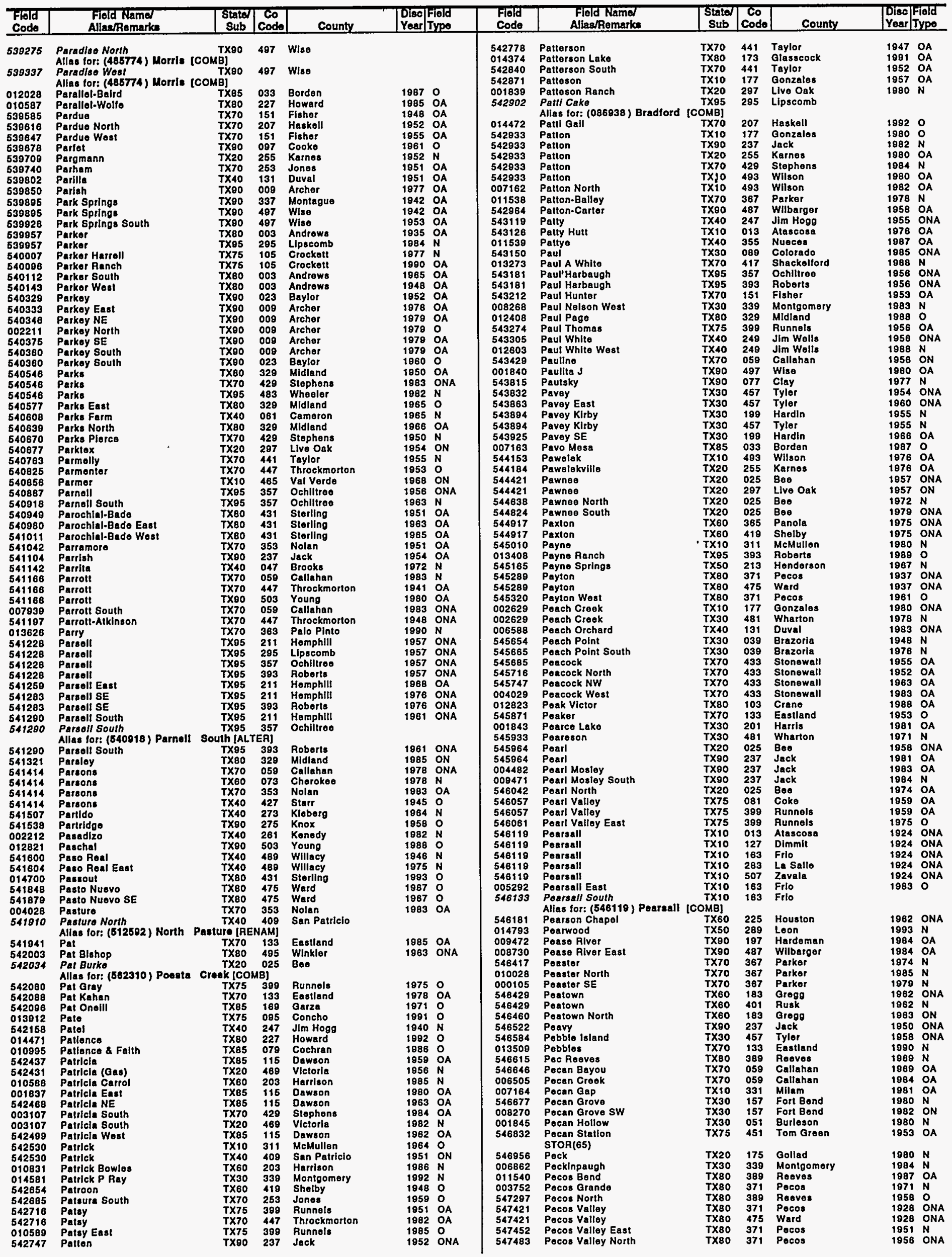


TEXAS

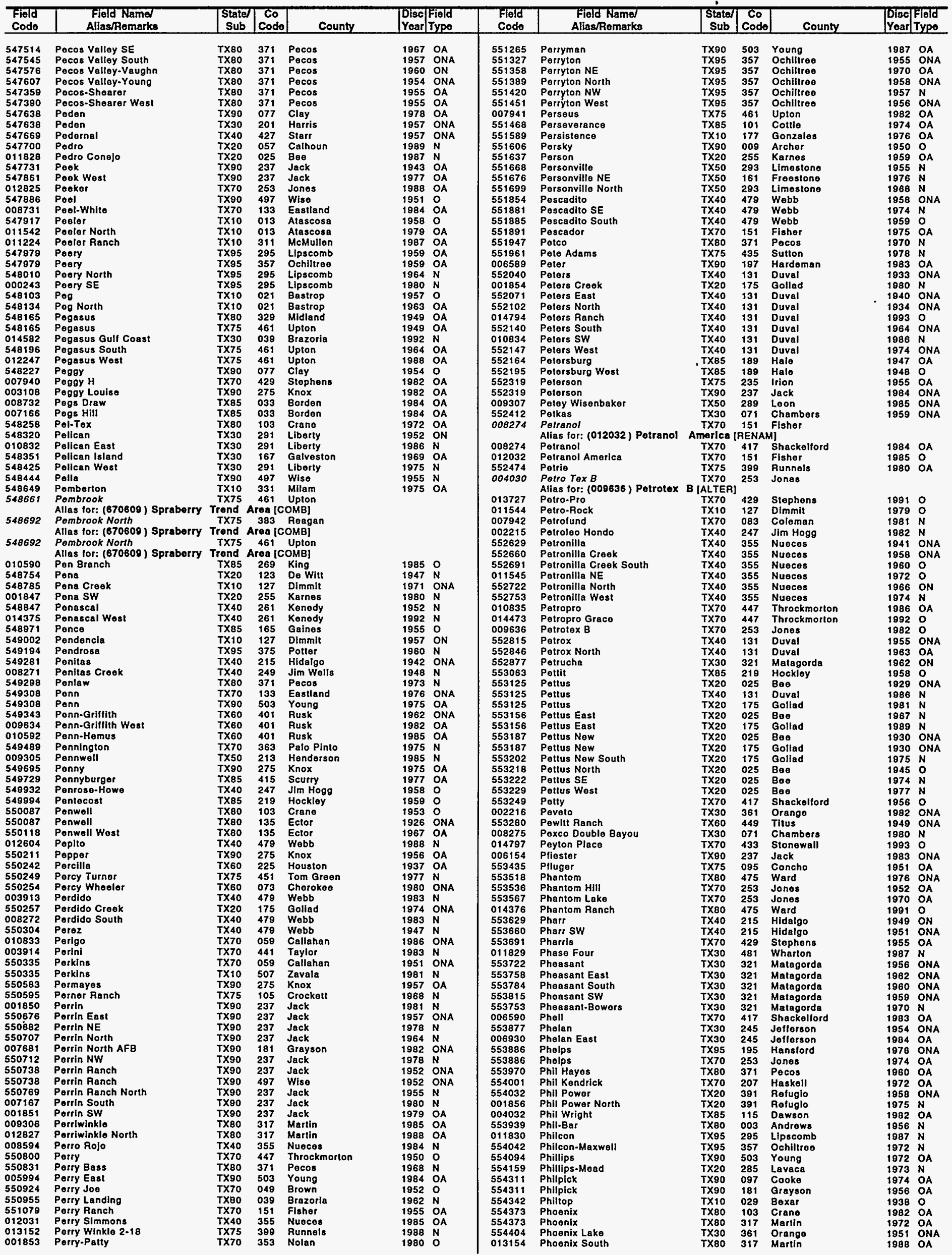


TEXAS

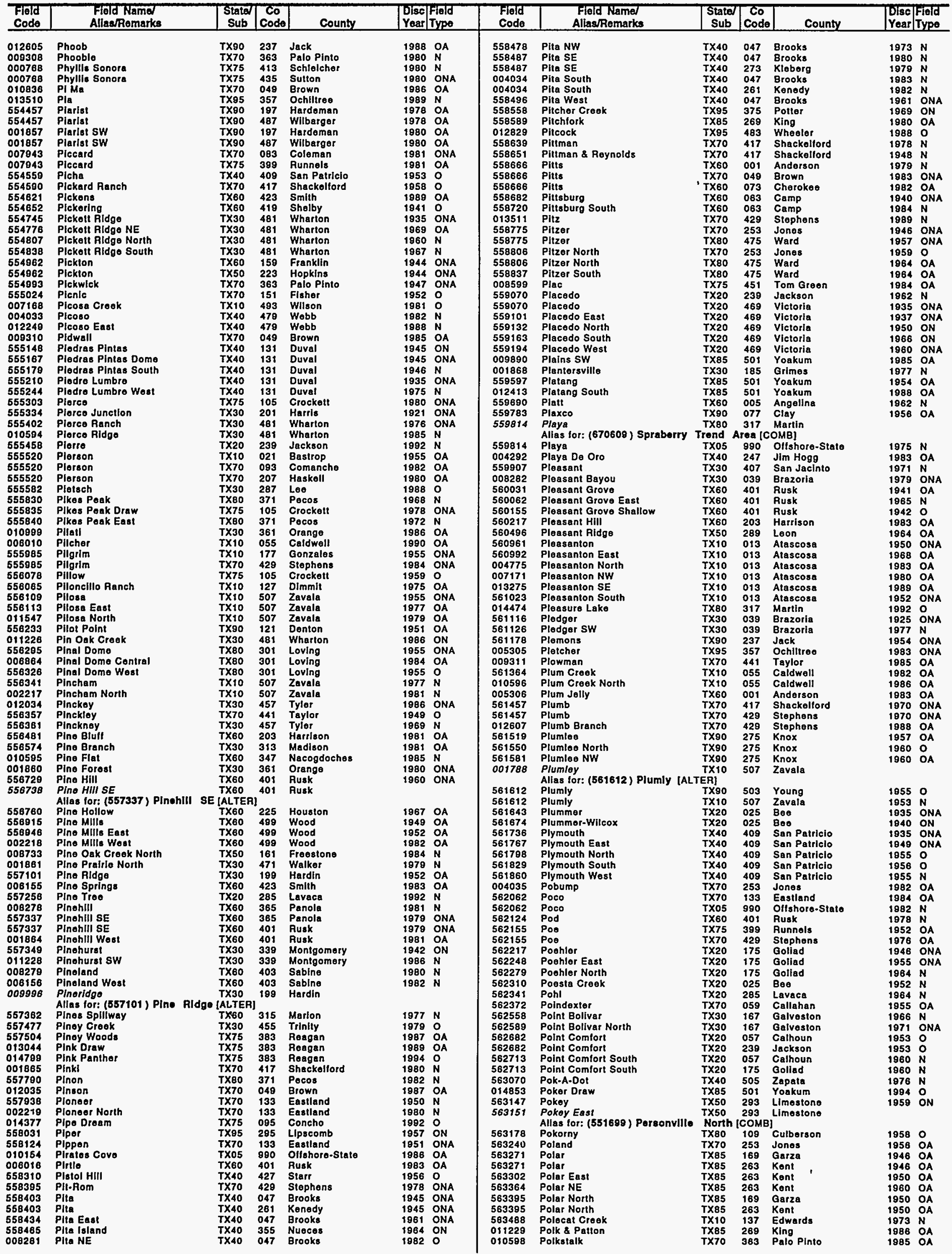


TEXAS

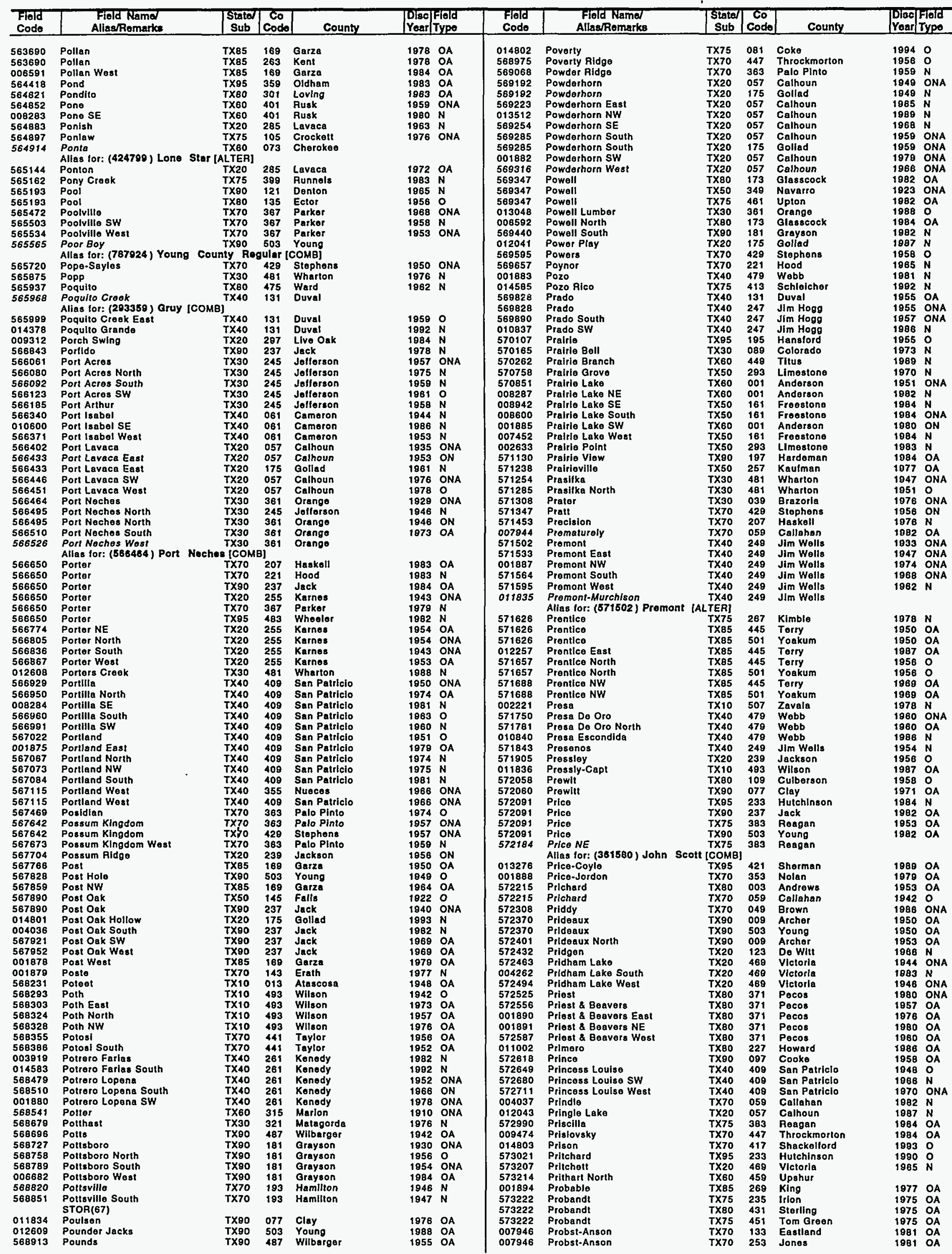


TEXAS

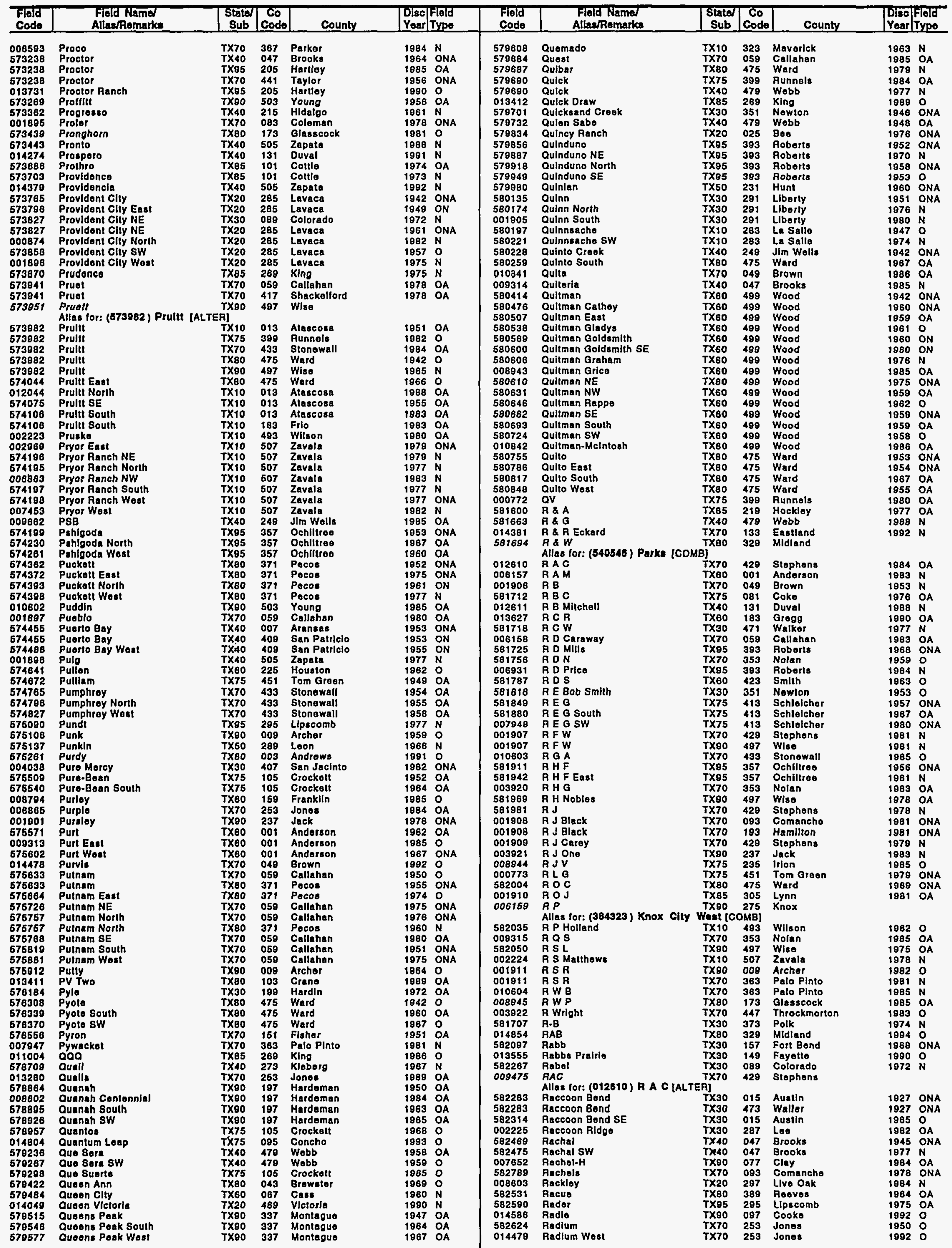




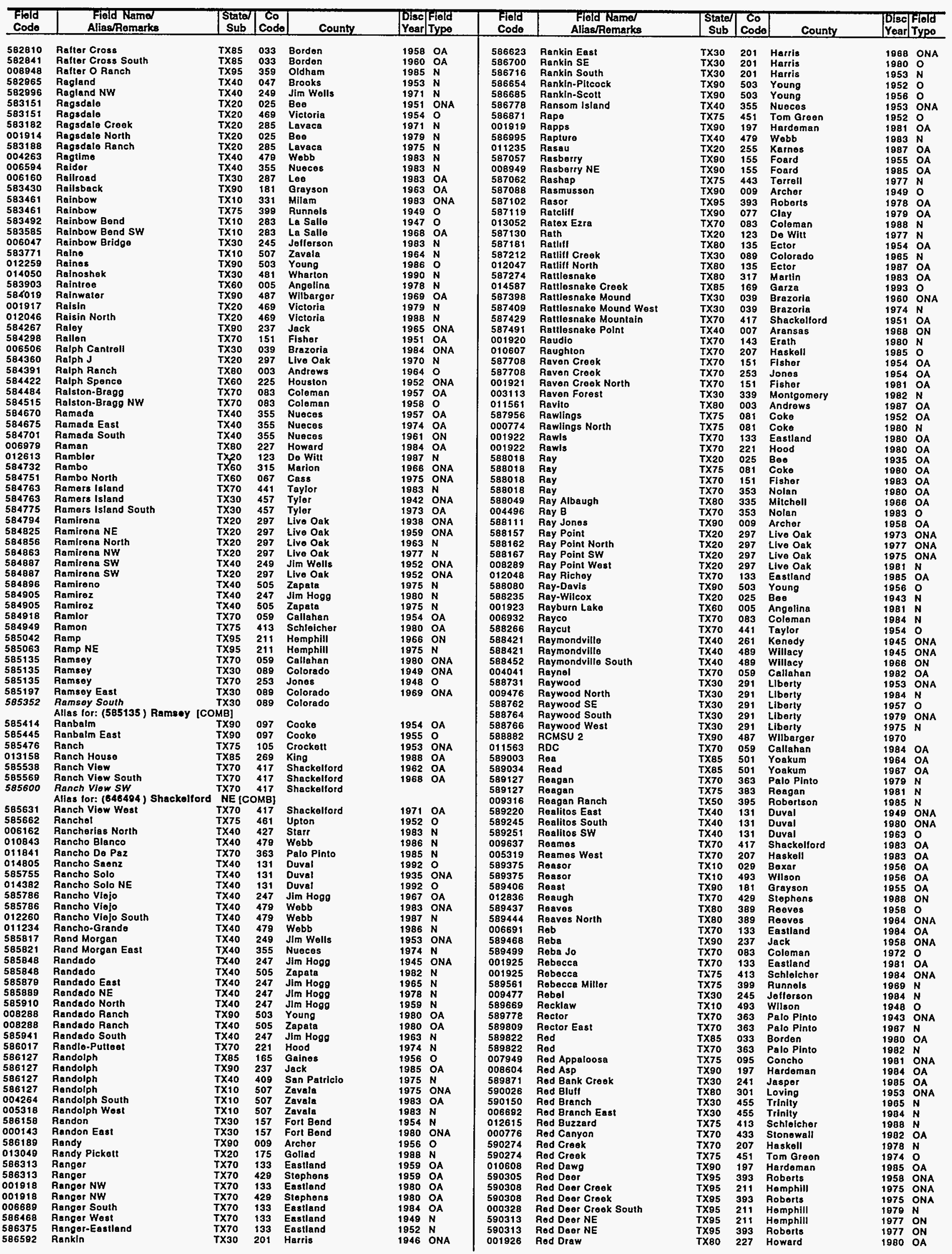


TEXAS

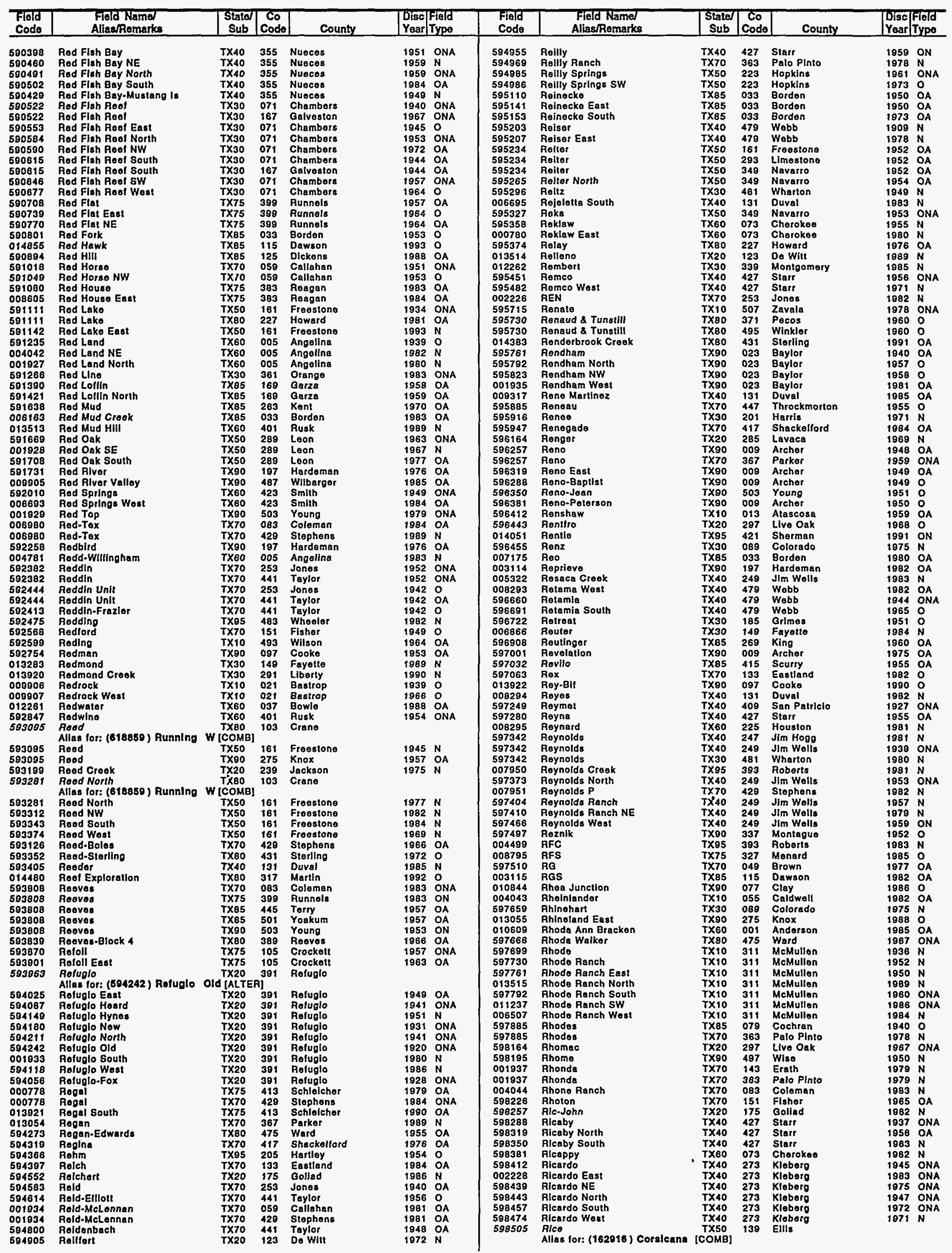


TEXAS

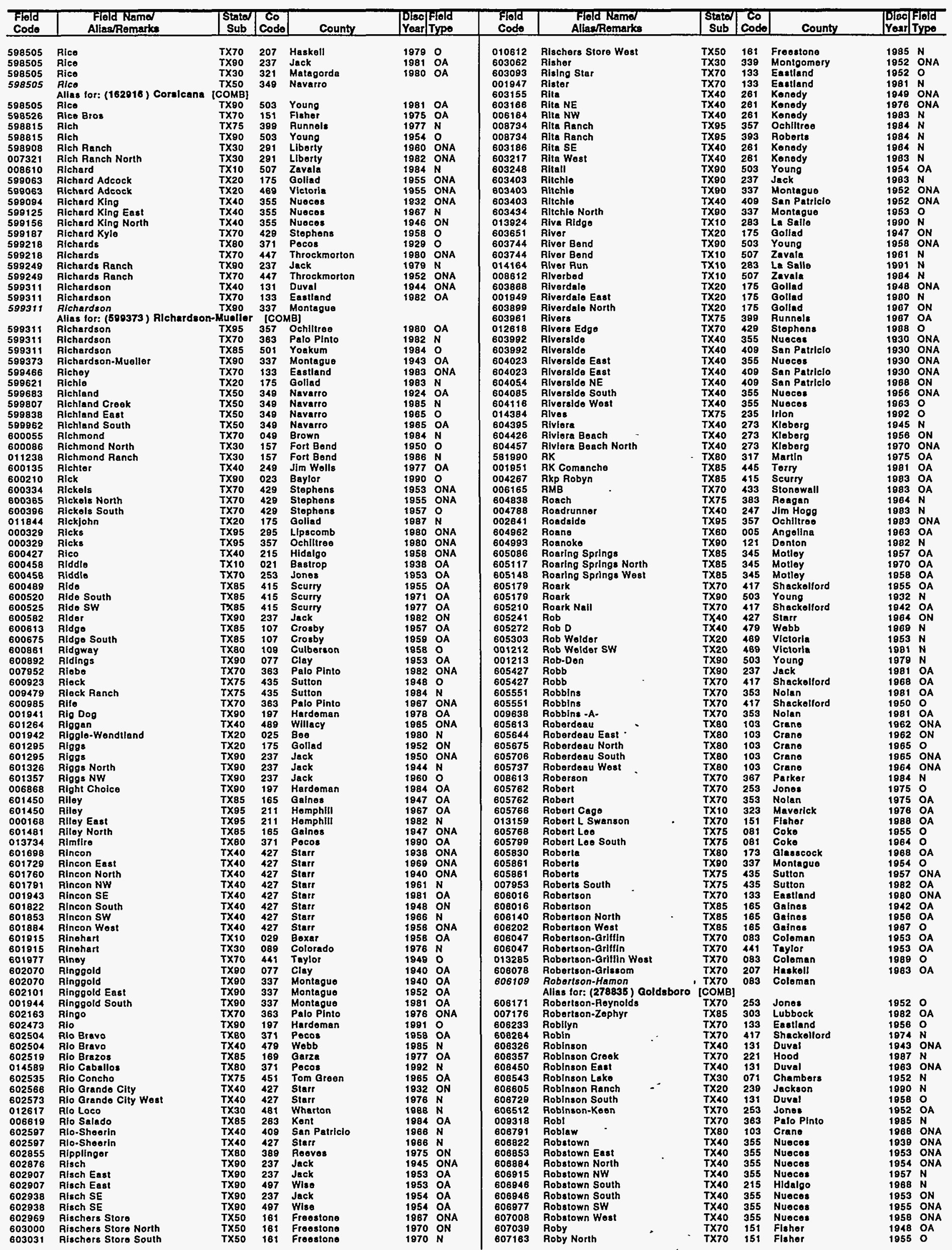


TEXAS

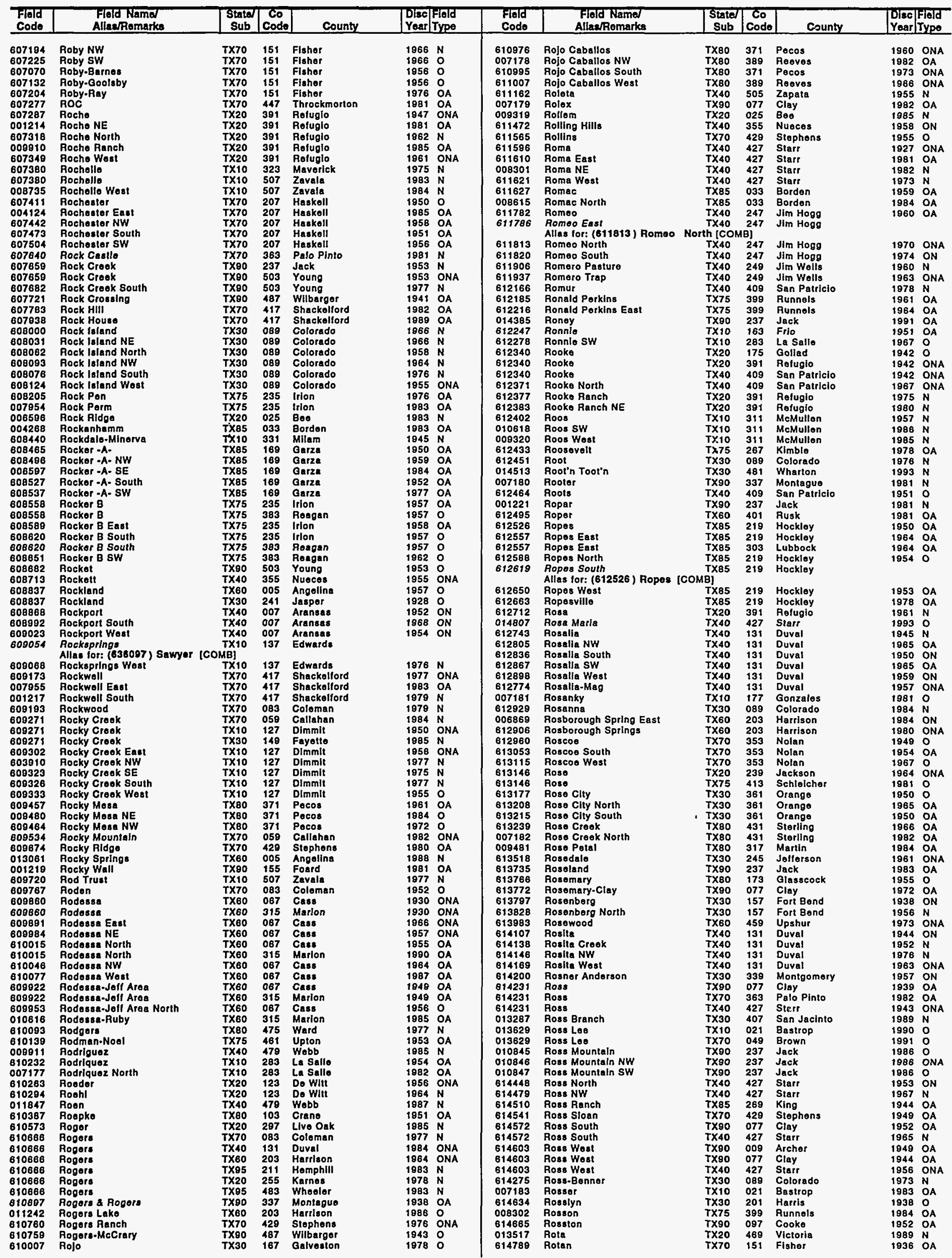


TEXAS

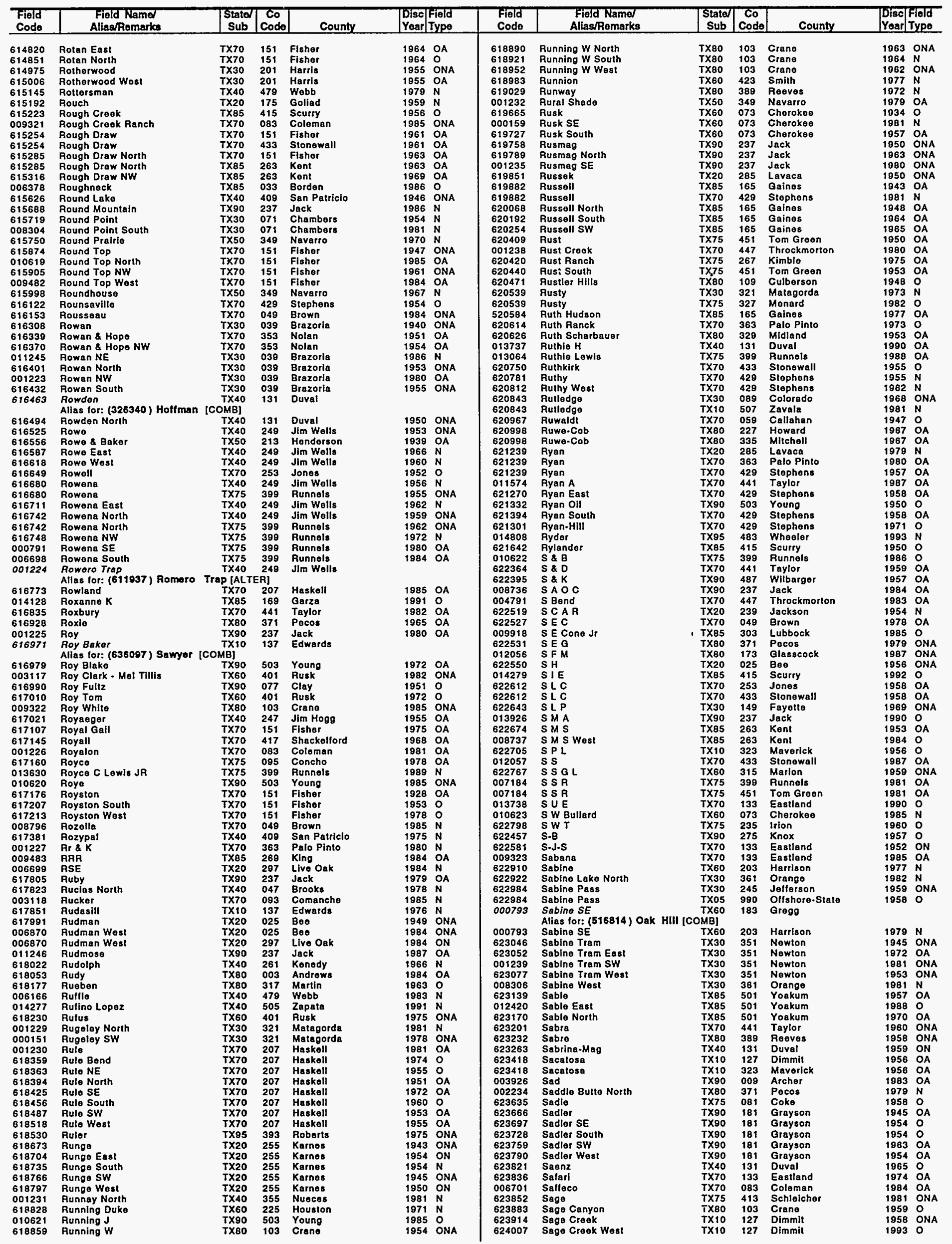


TEXAS

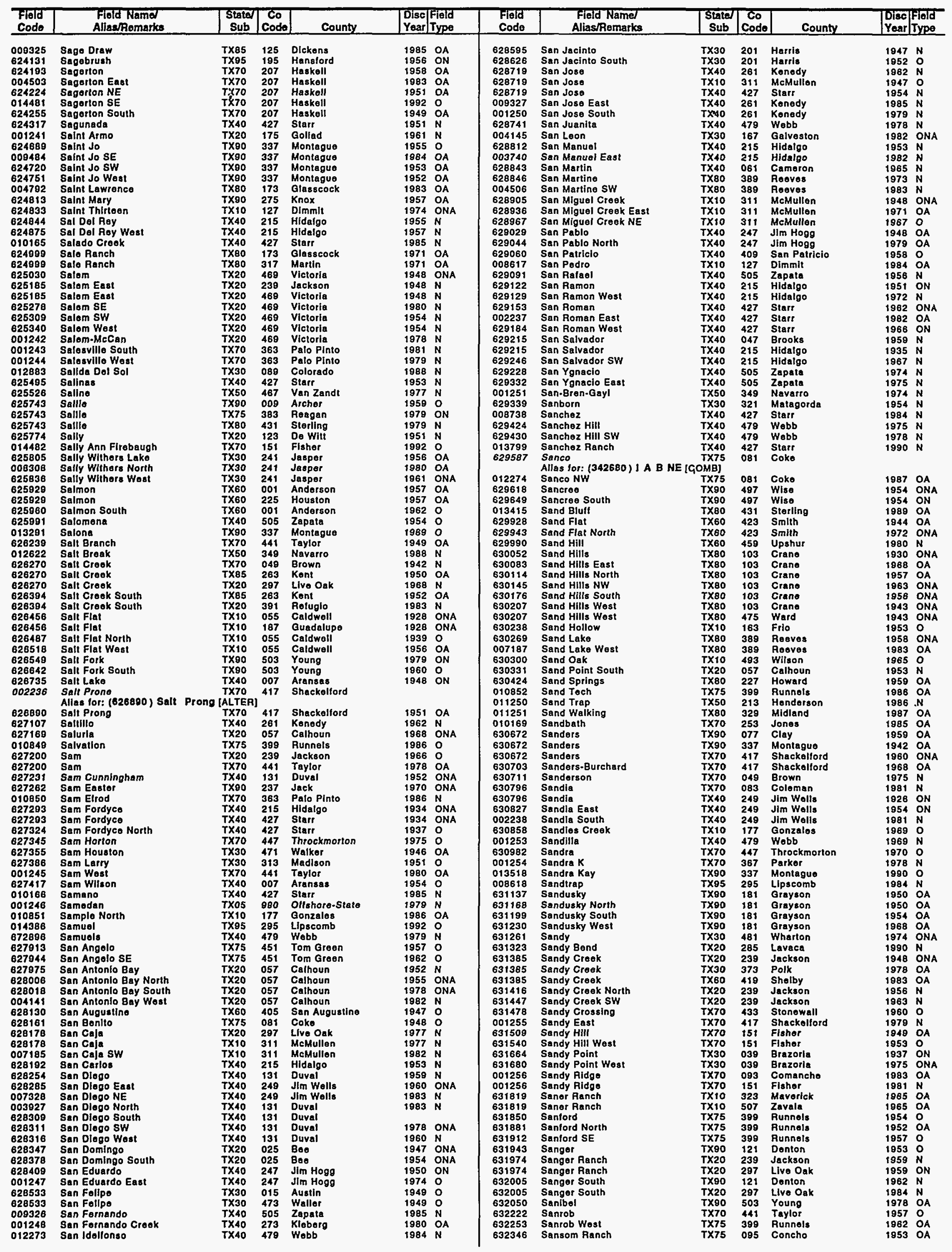


TEXAS

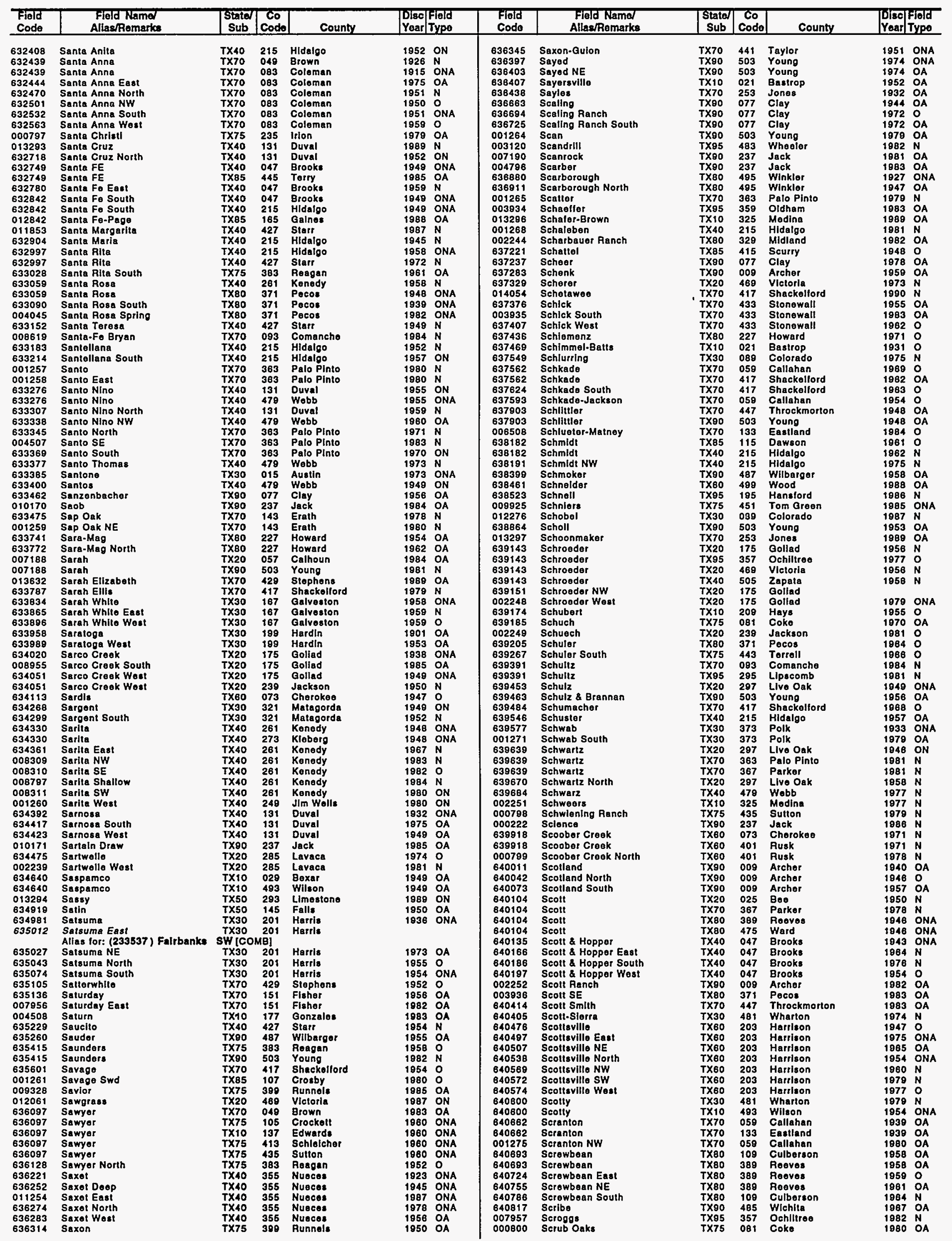


TEXAS

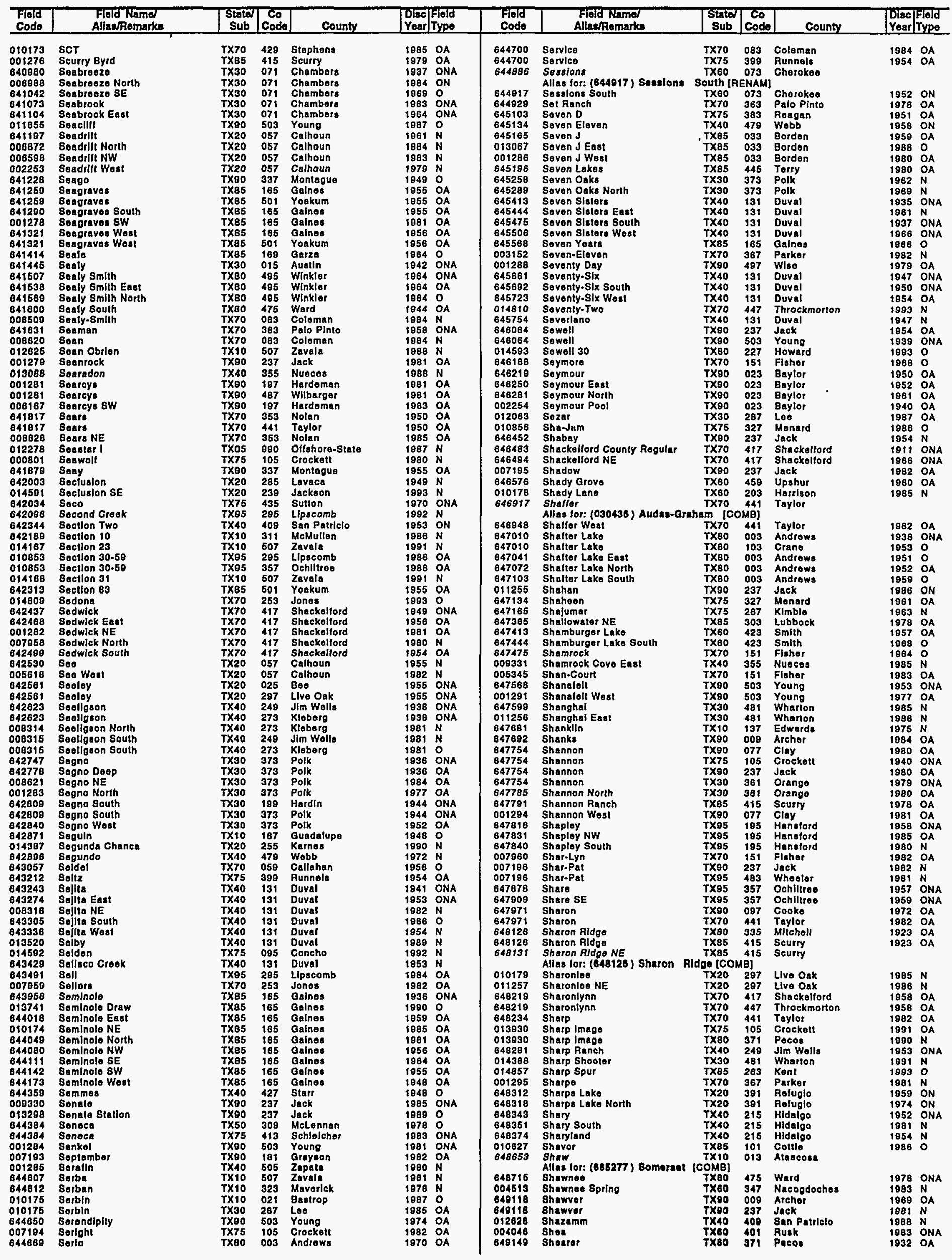


TEXAS

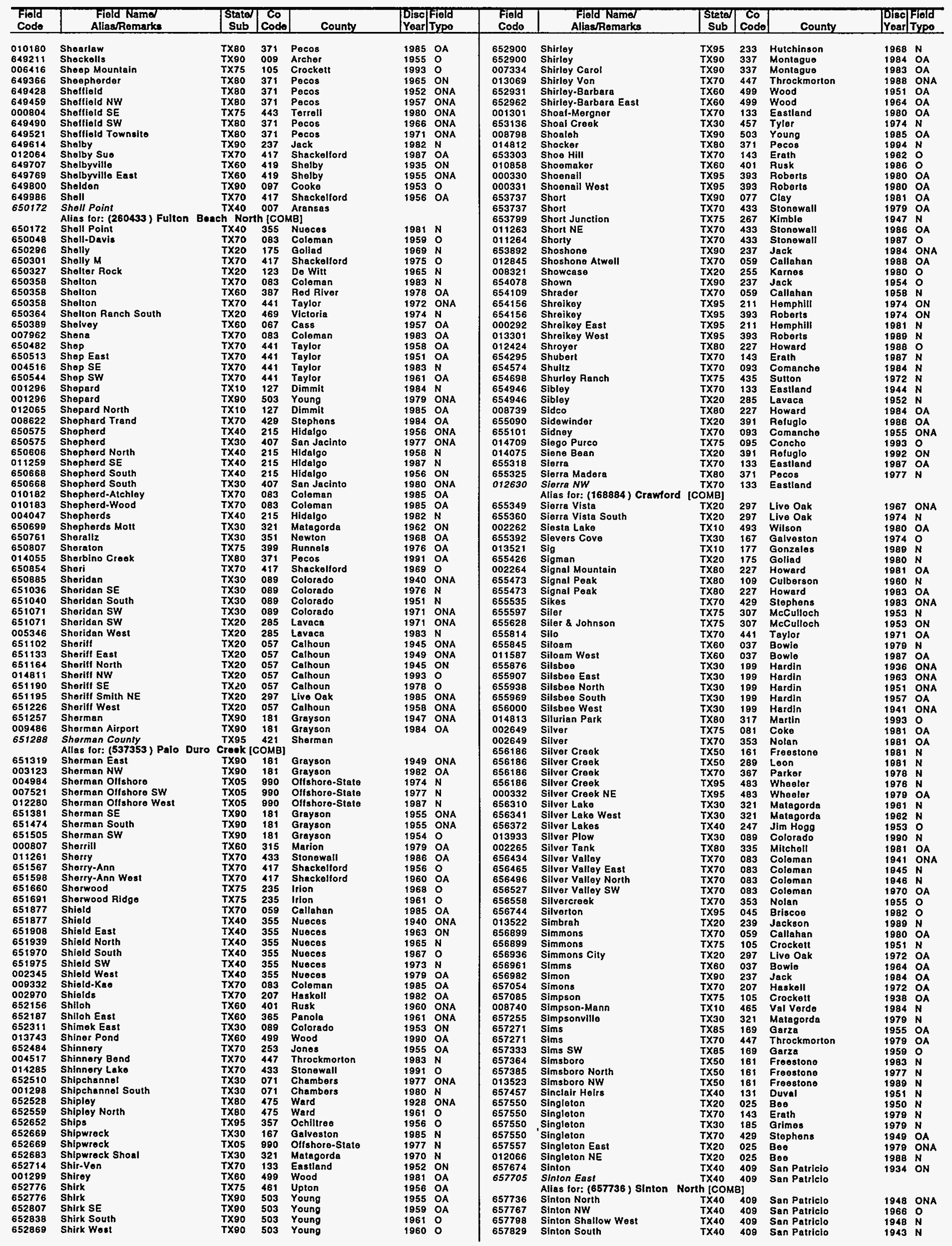


TEXAS

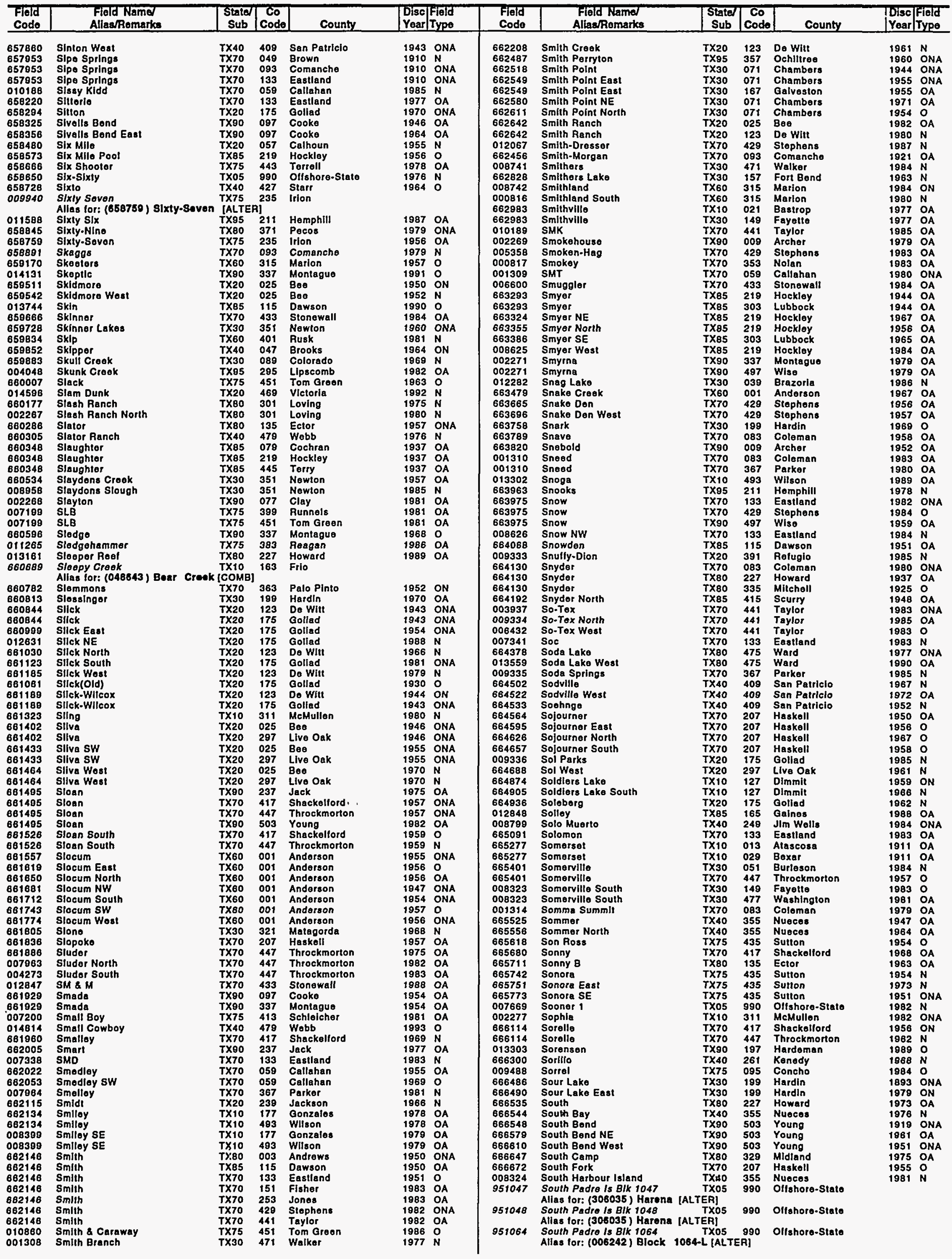


TEXAS

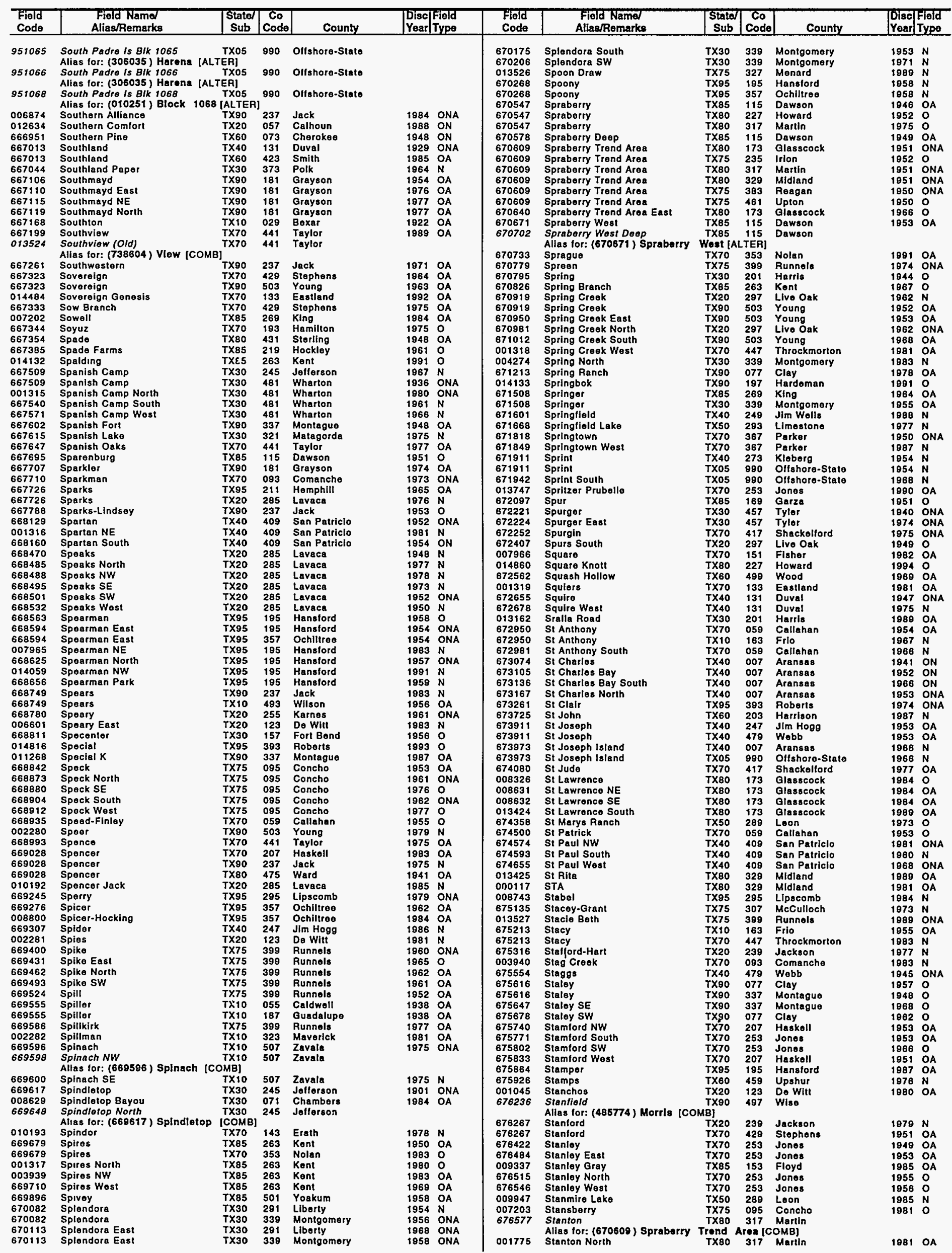


TEXAS

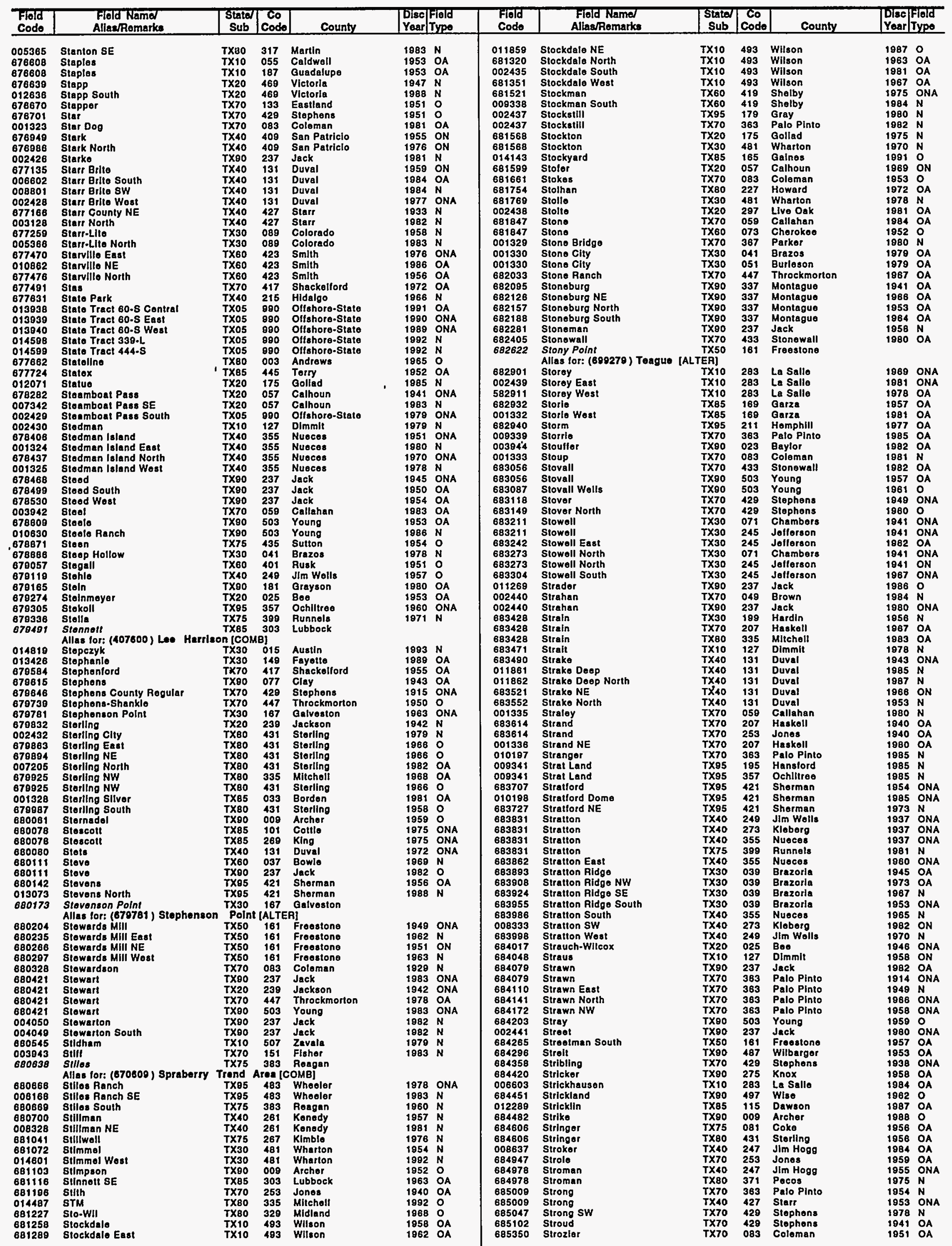


TEXAS

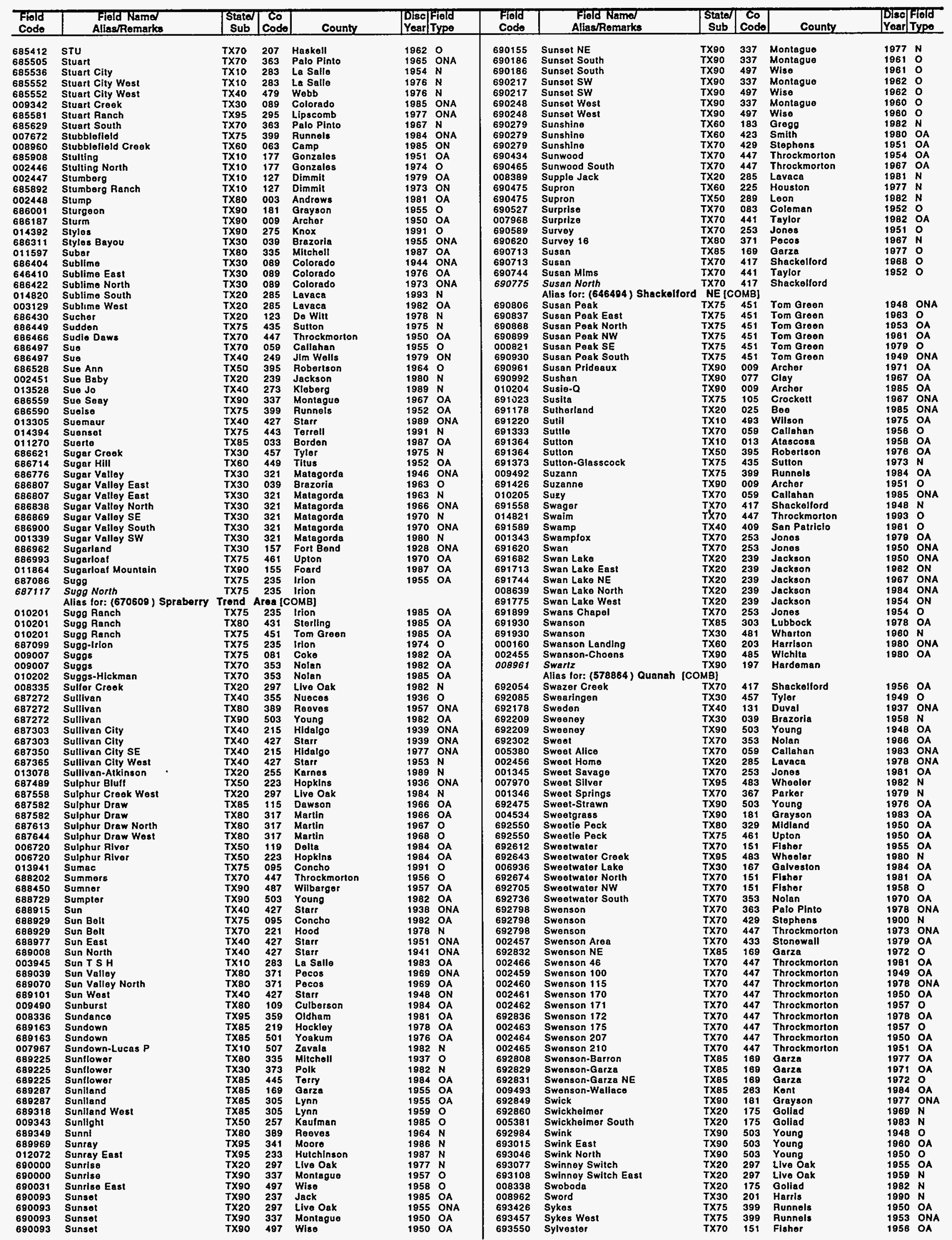


TEXAS

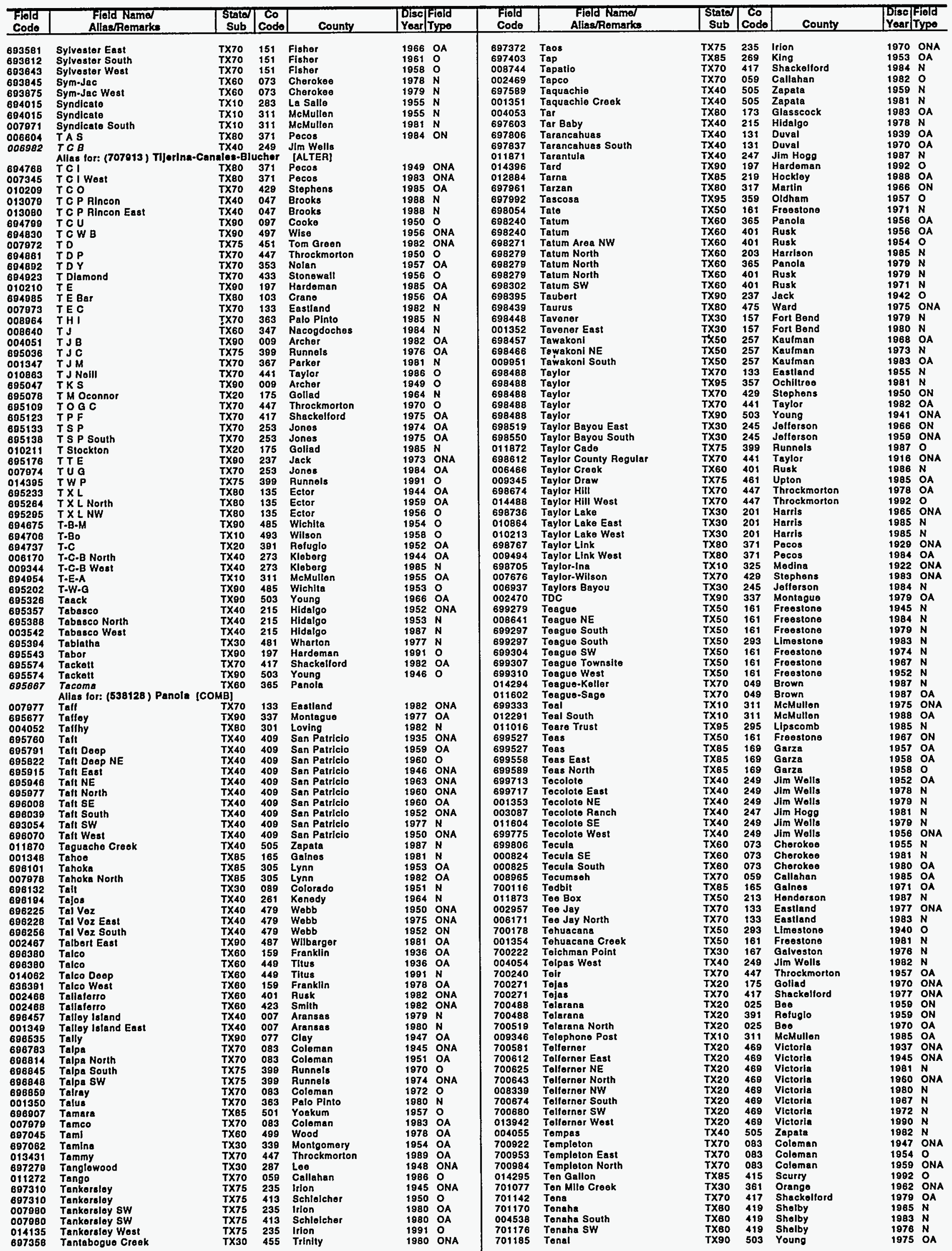


TEXAS

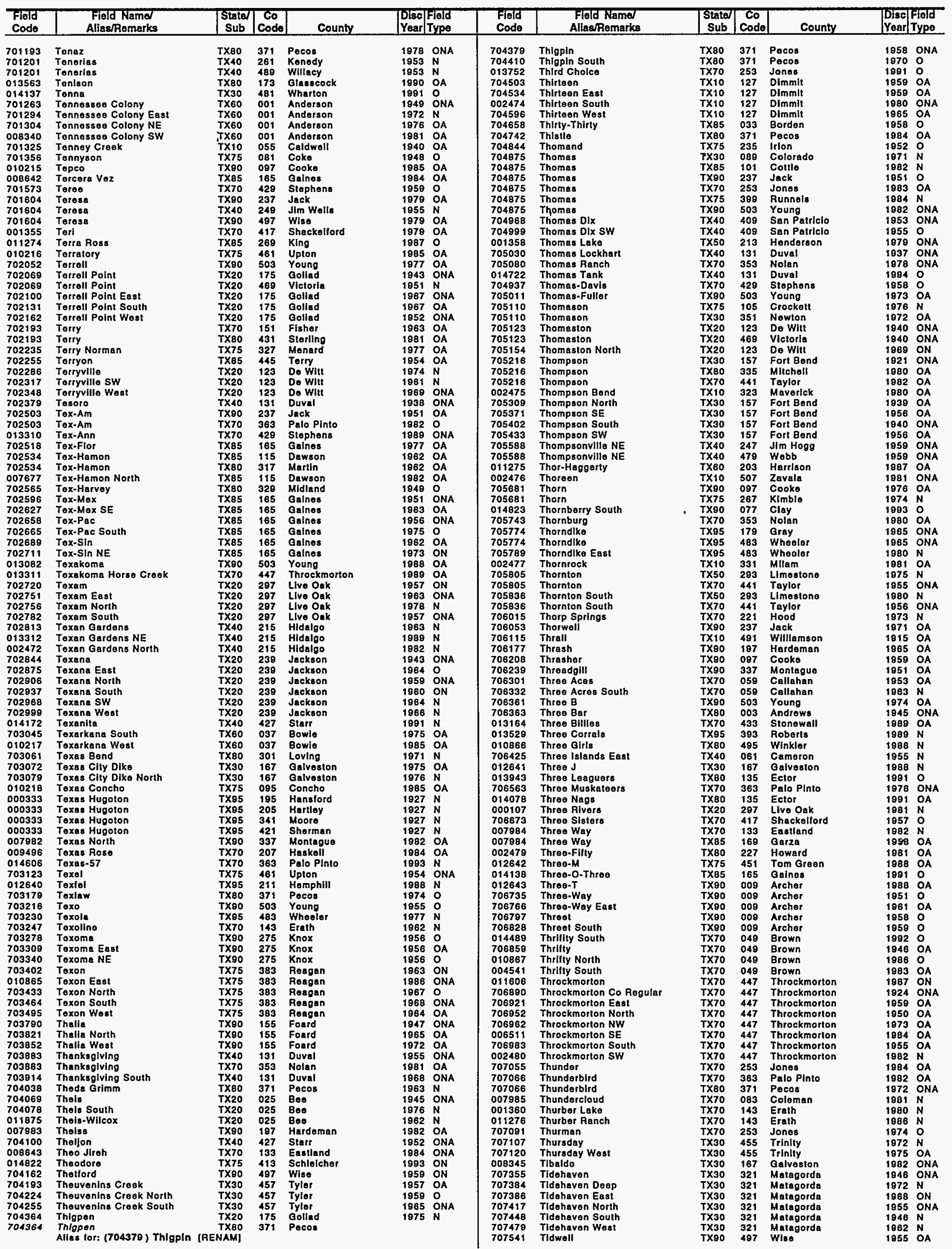


TEXAS

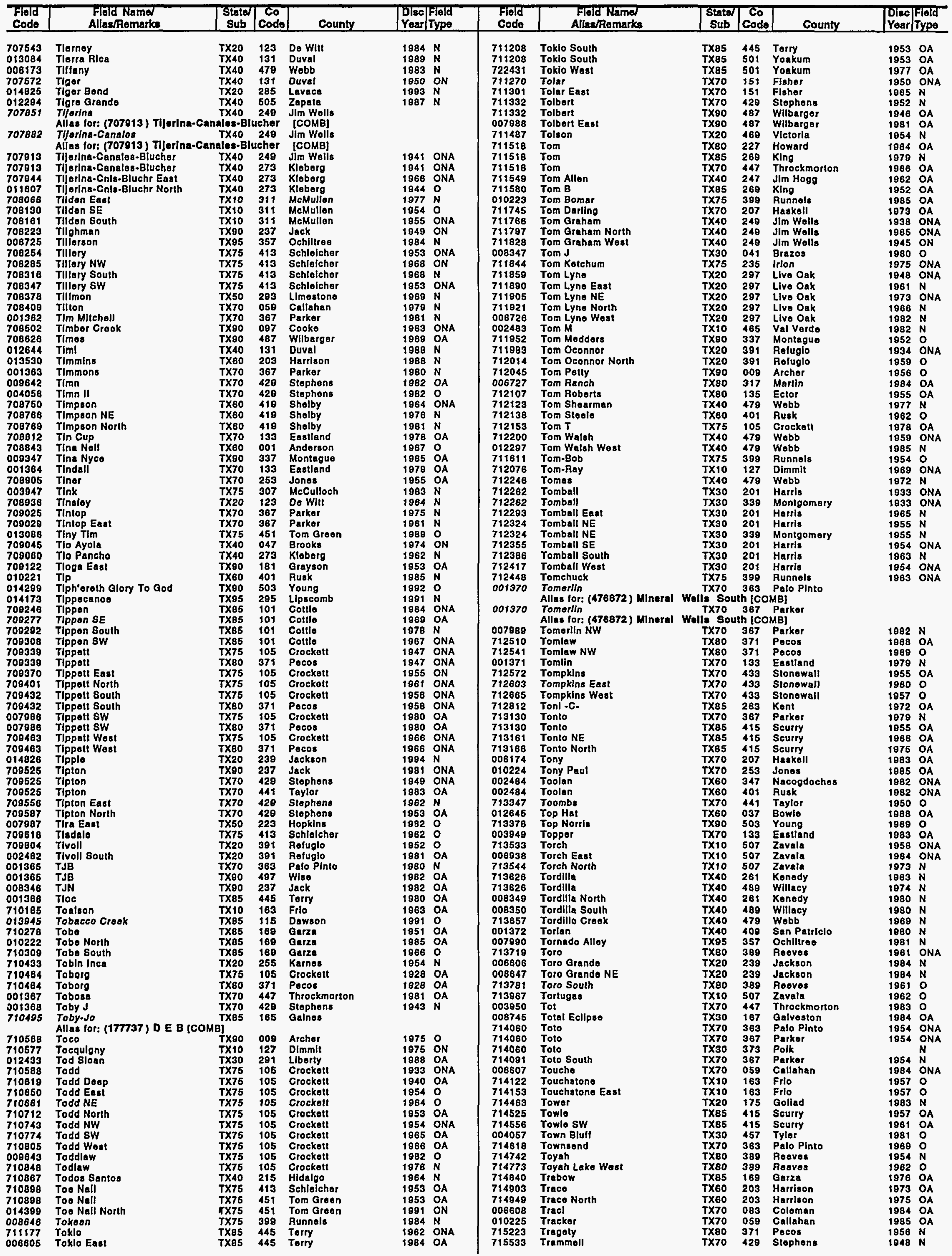


TEXAS

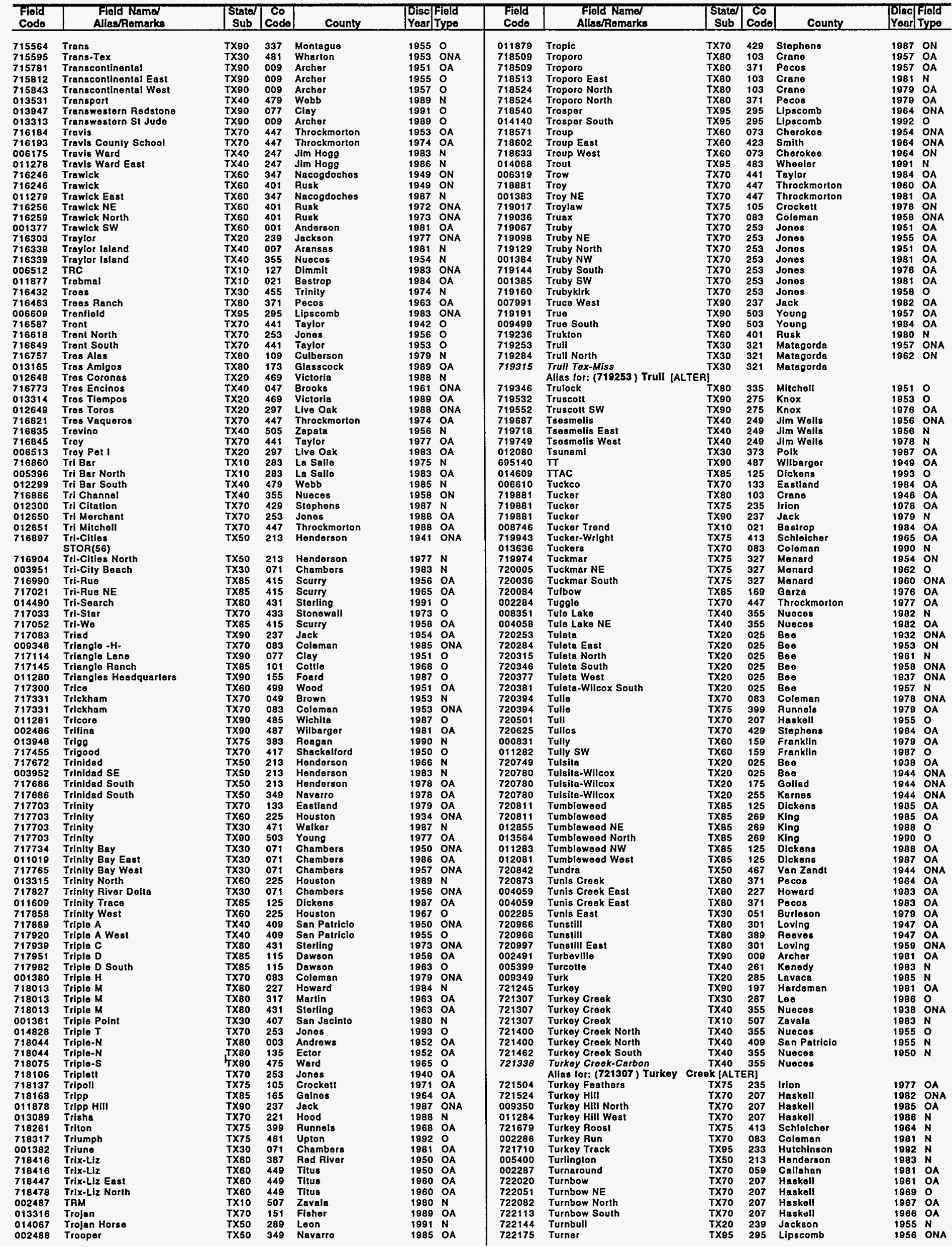


TEXAS

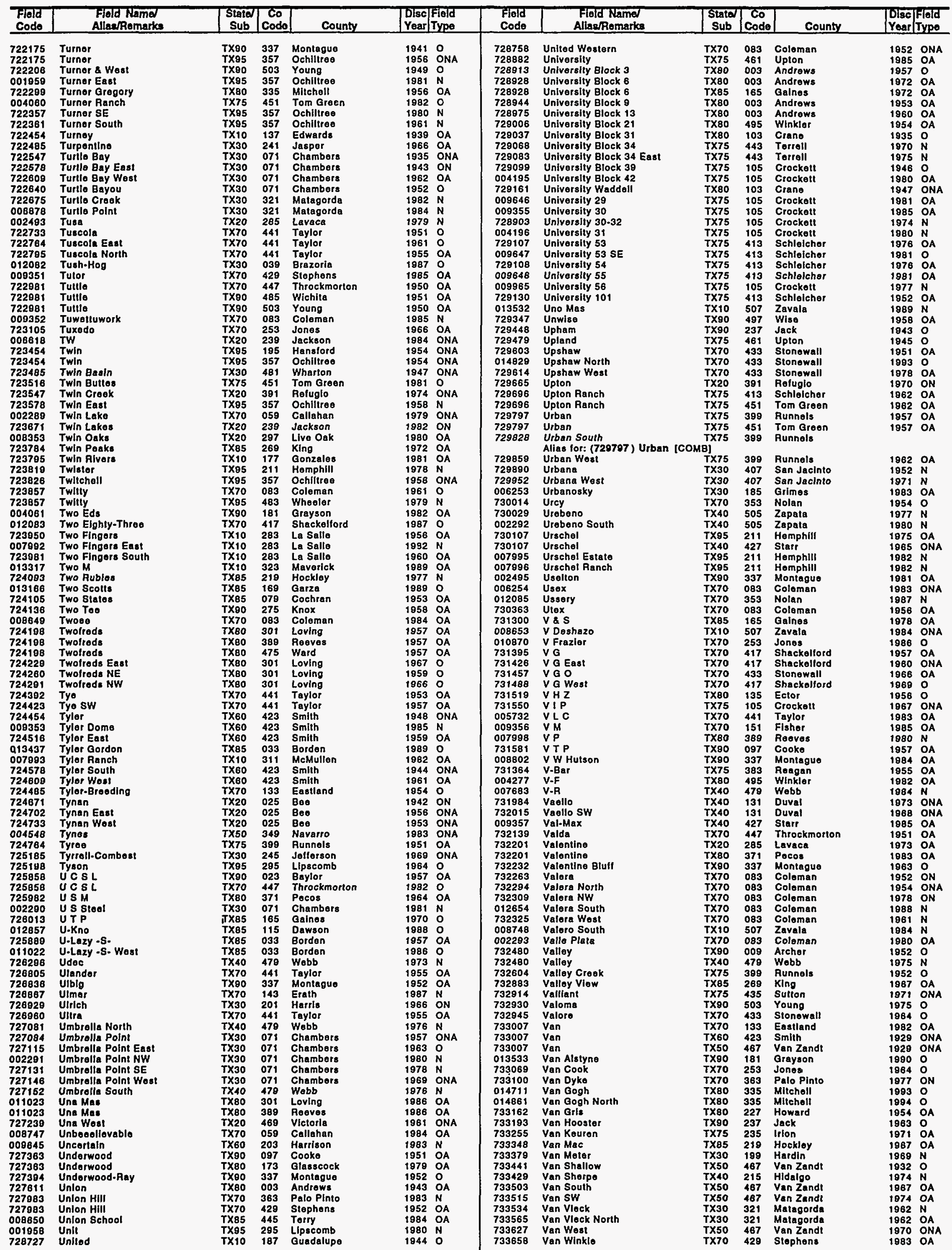


TEXAS

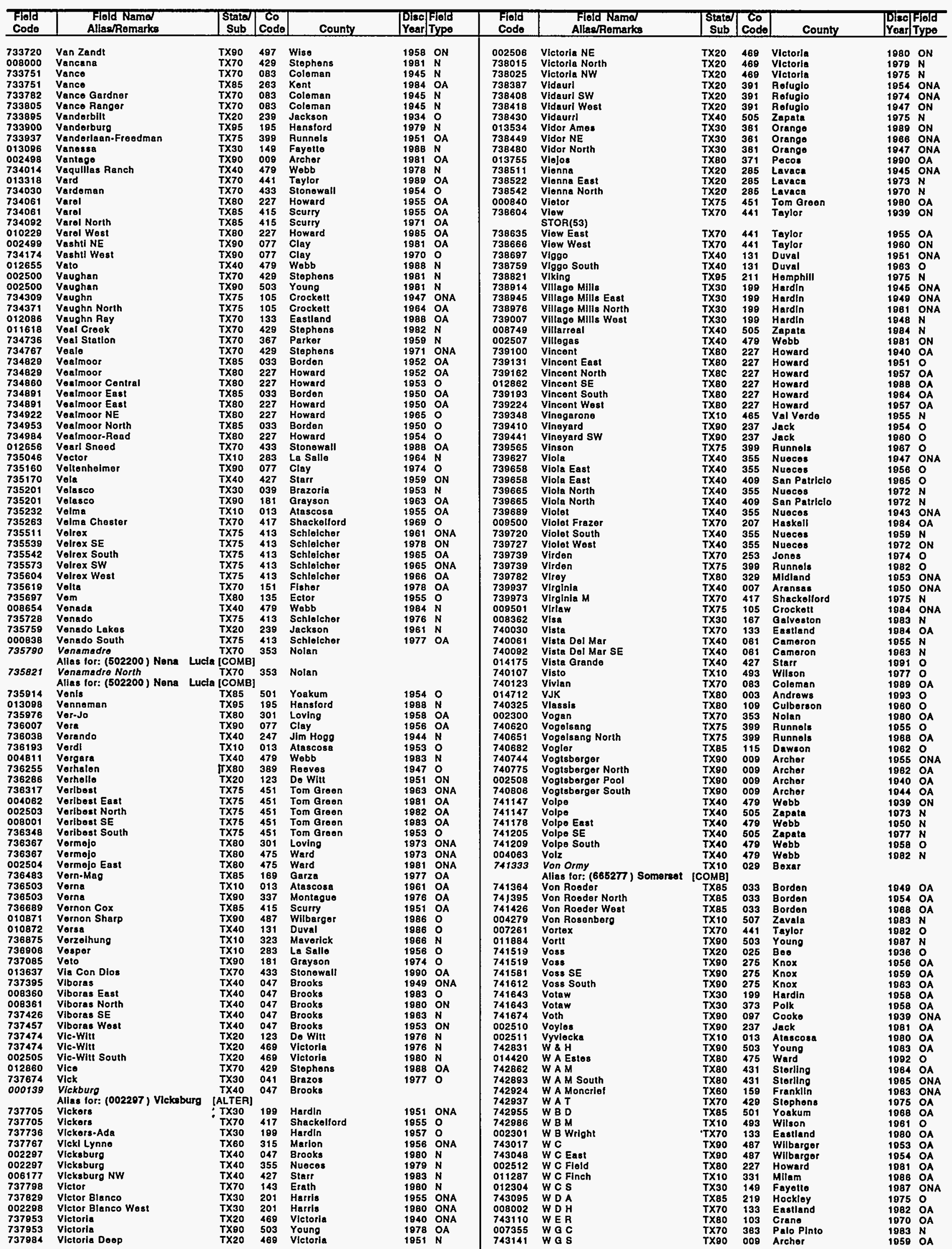


TEXAS

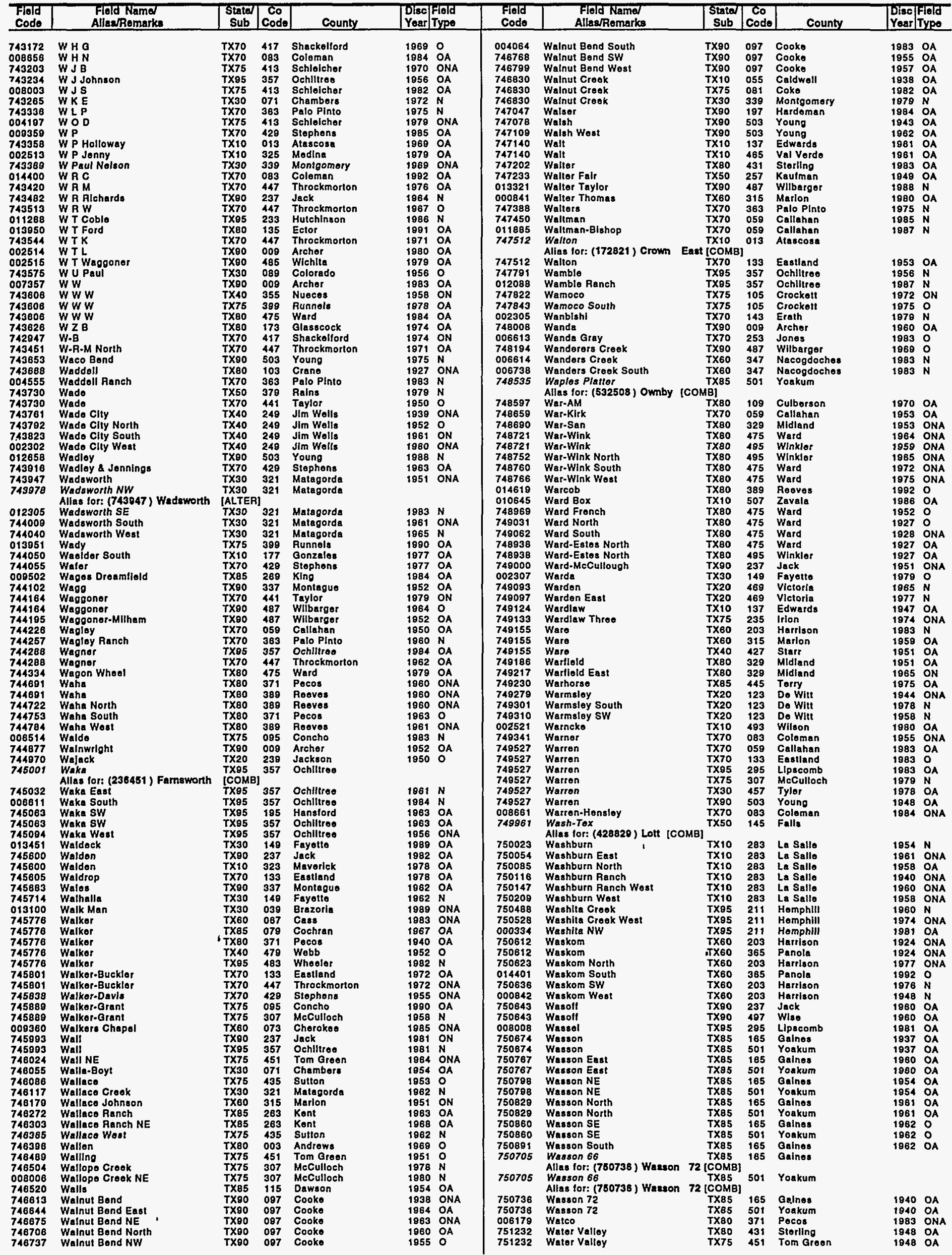


TEXAS

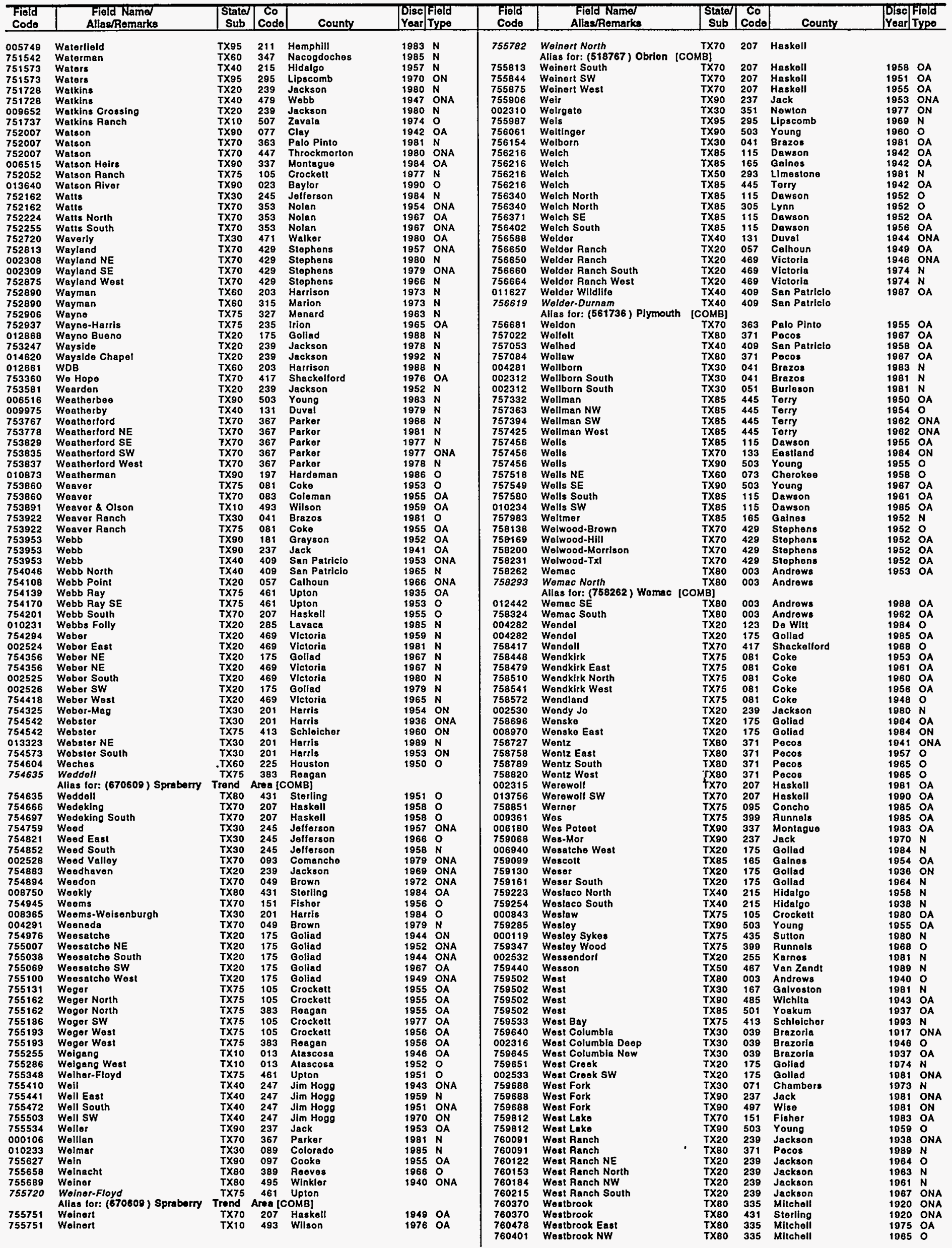


TEXAS

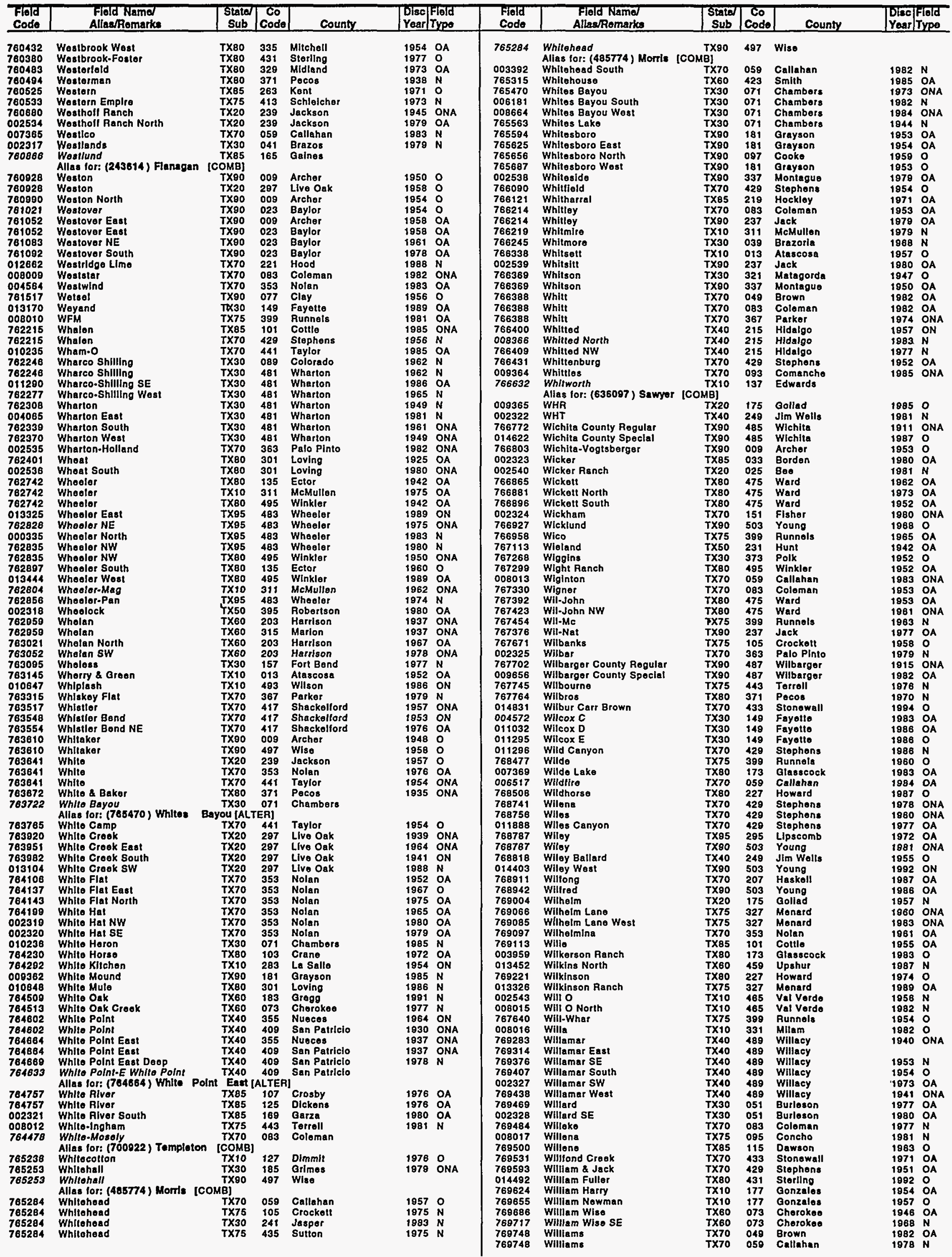


TEXAS

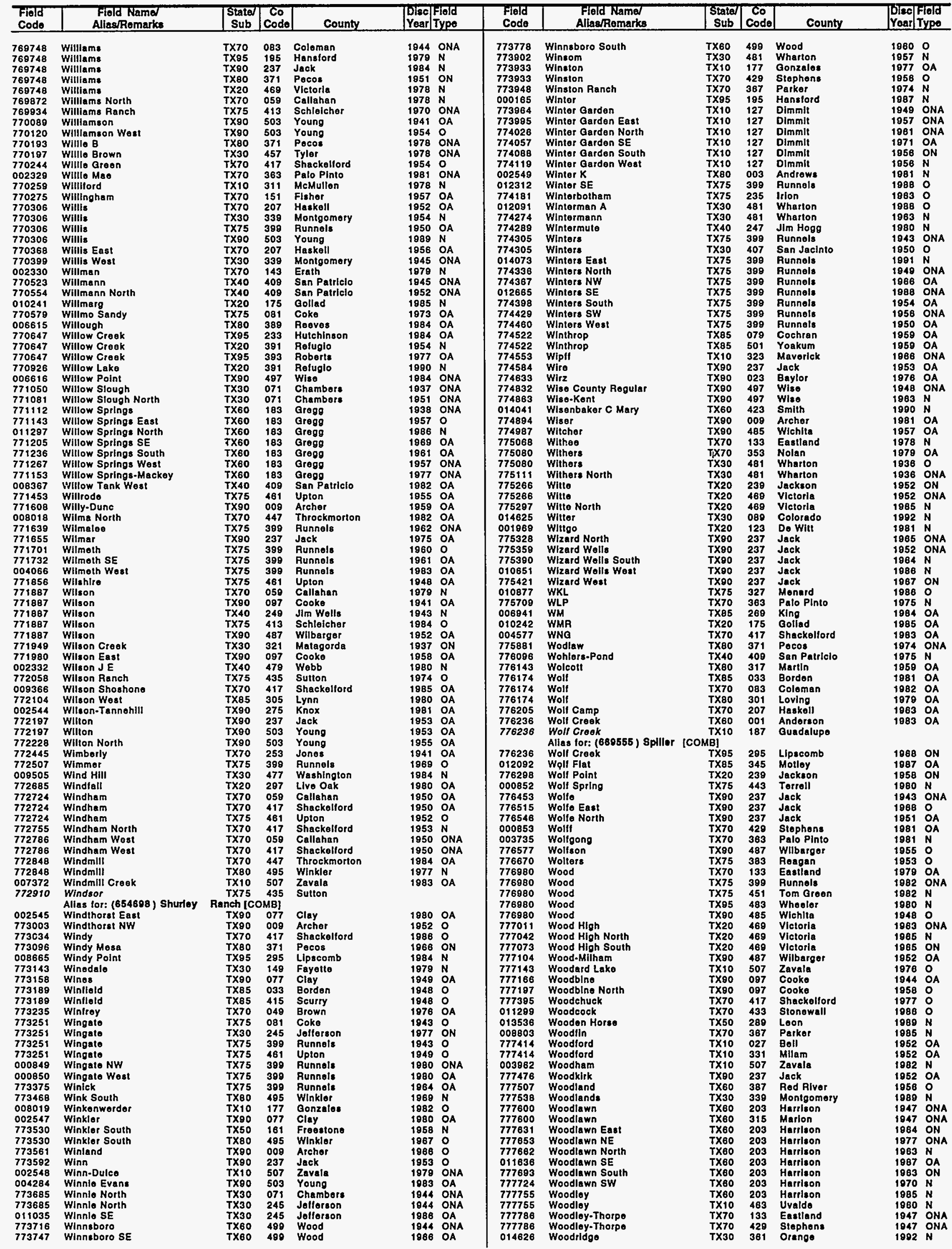


TEXAS

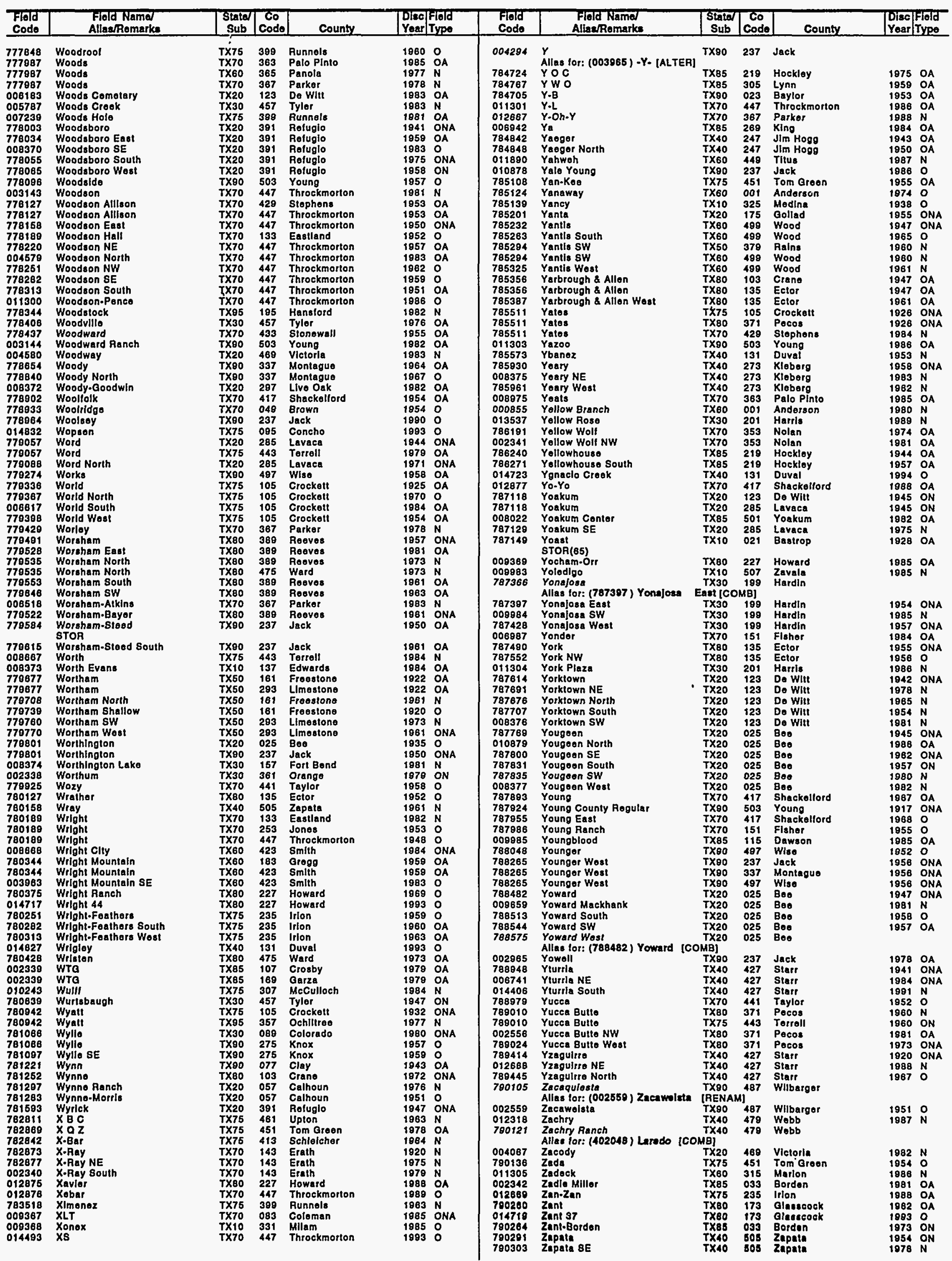


TEXAS

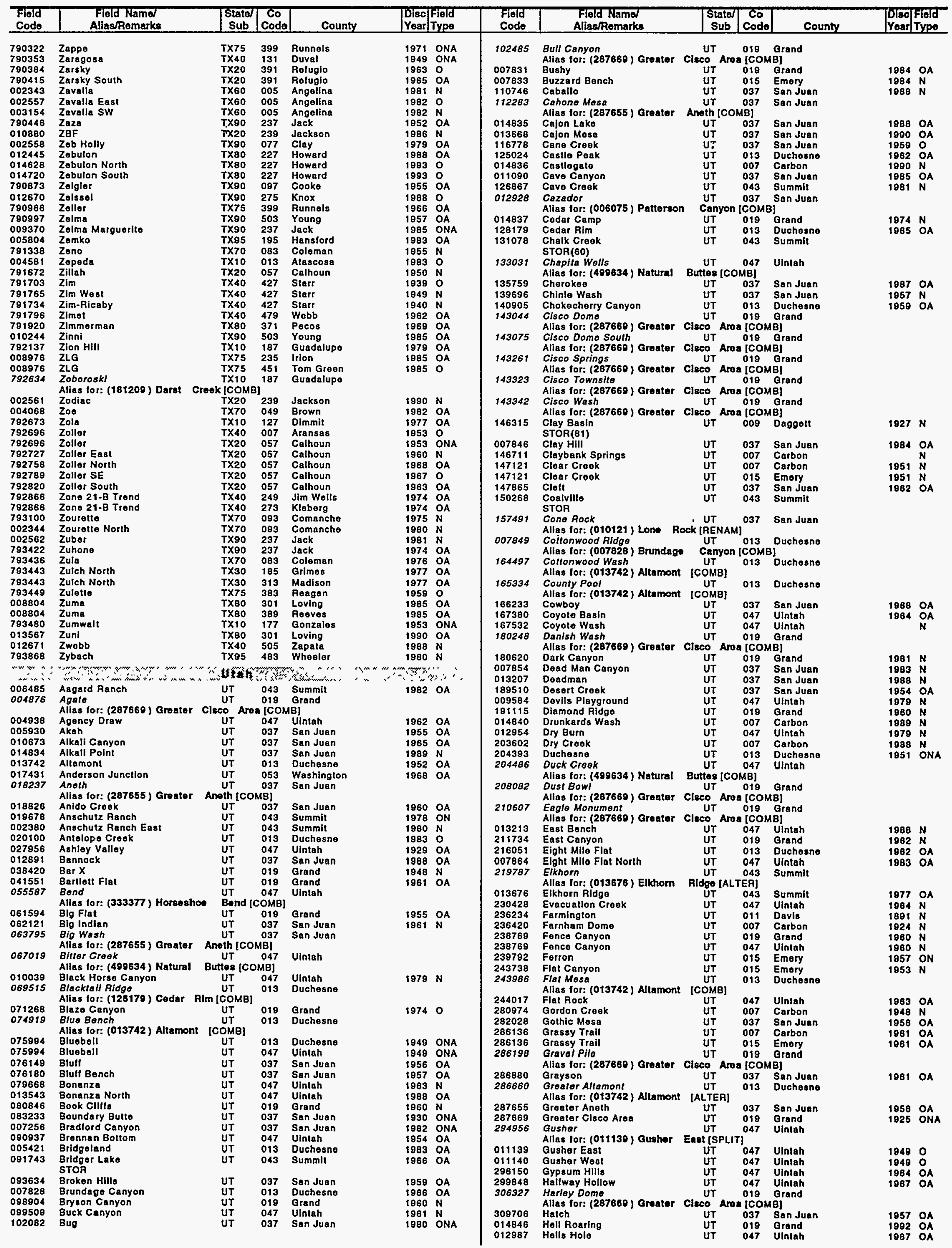


WEST VIRGINIA

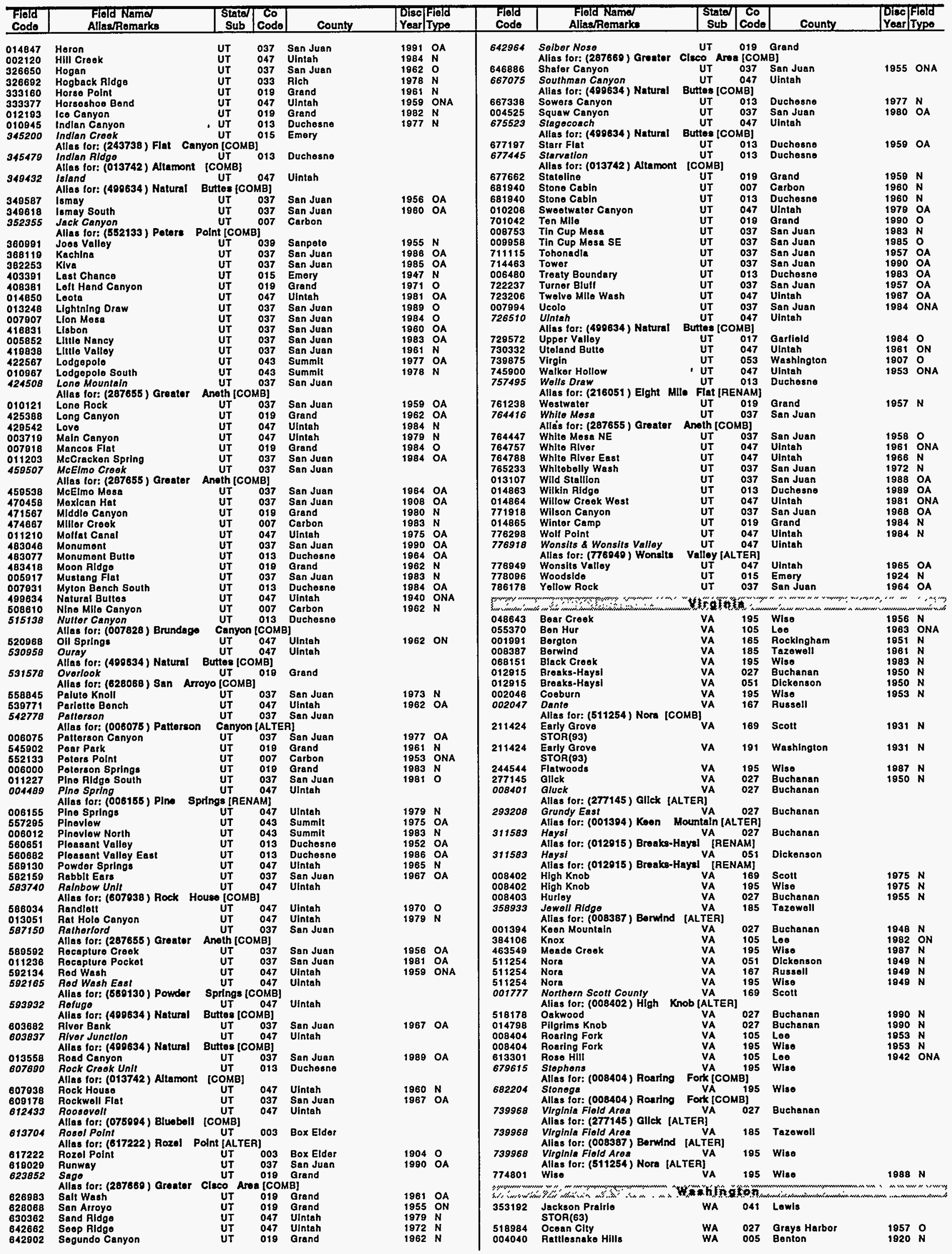




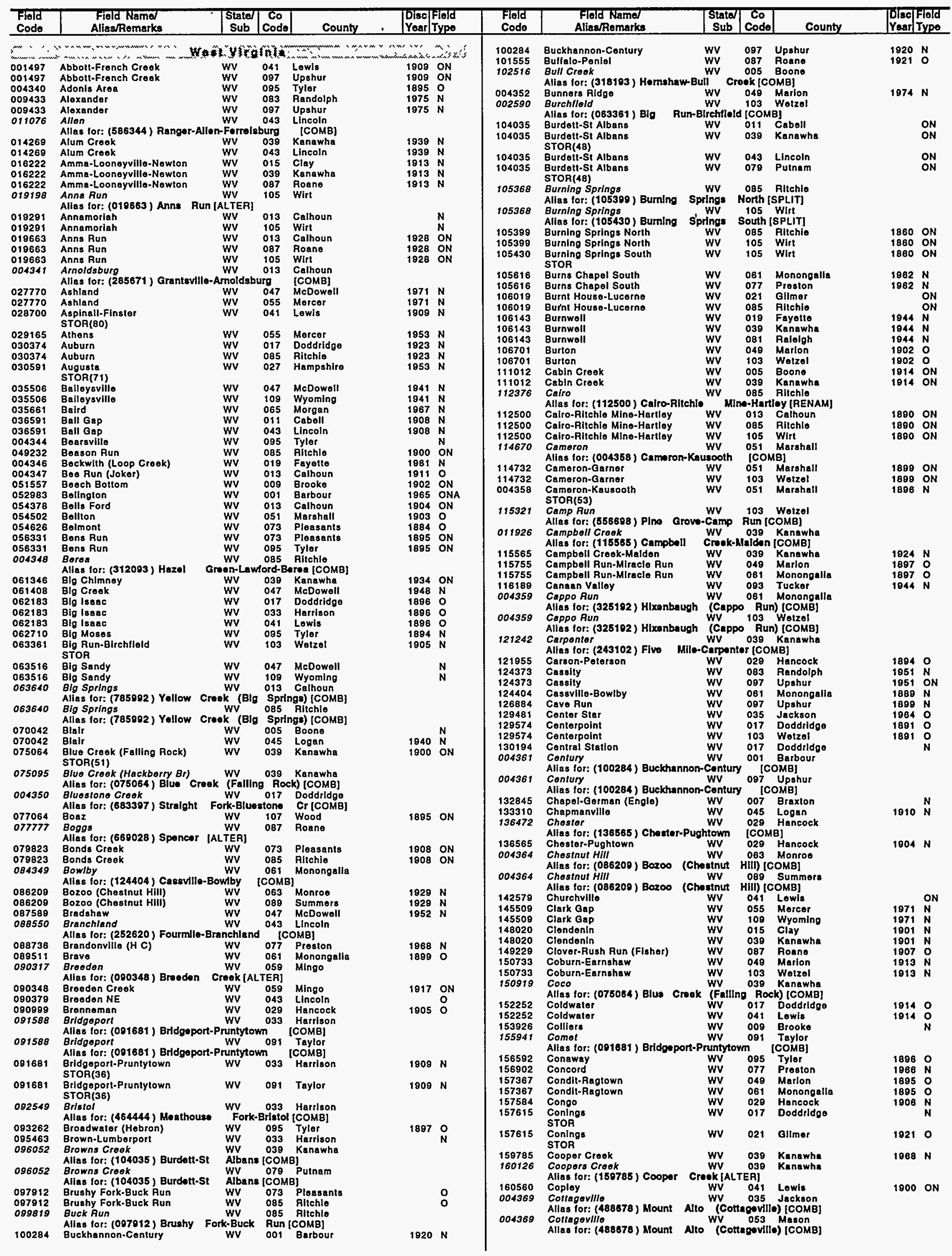


WEST VIRGINIA

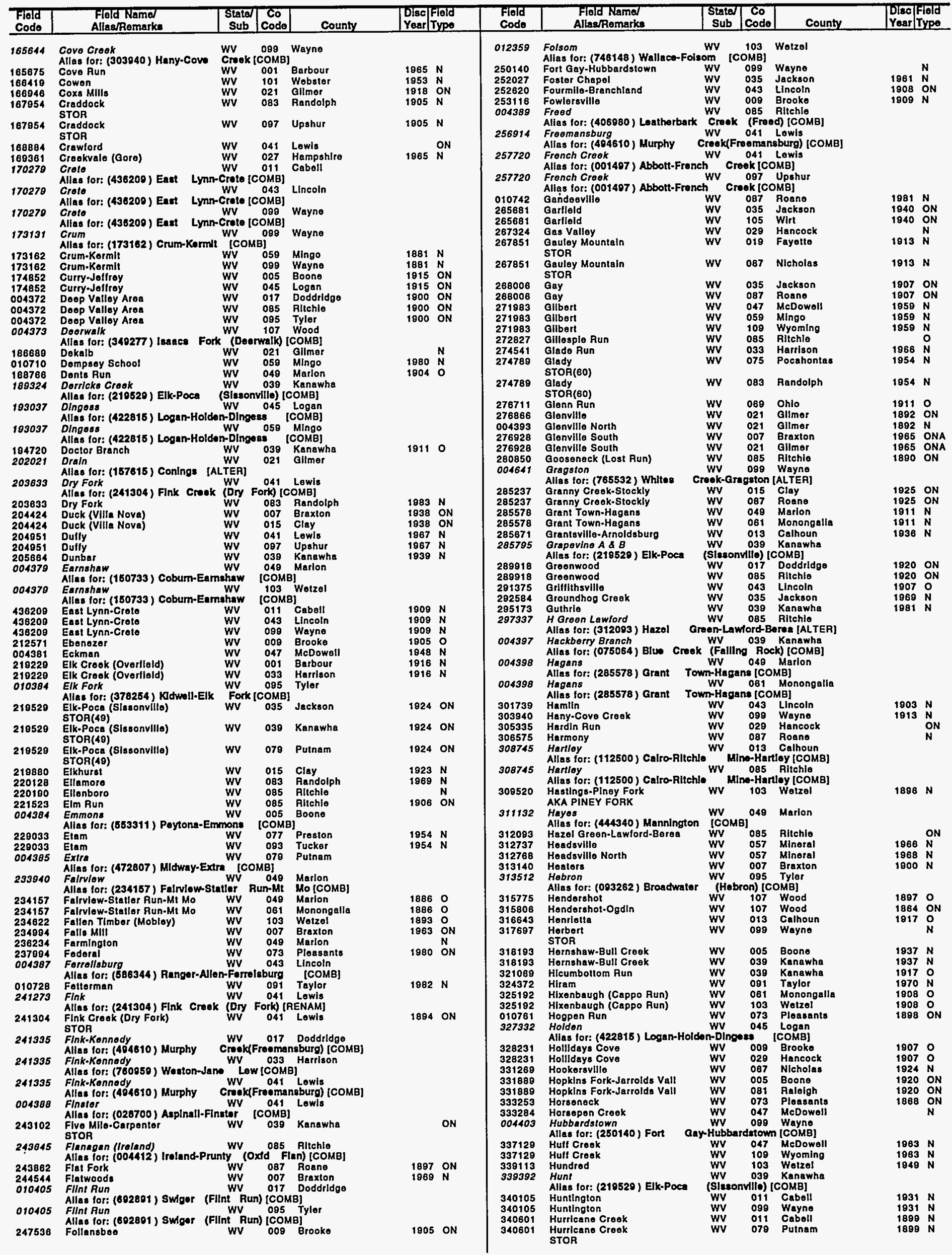




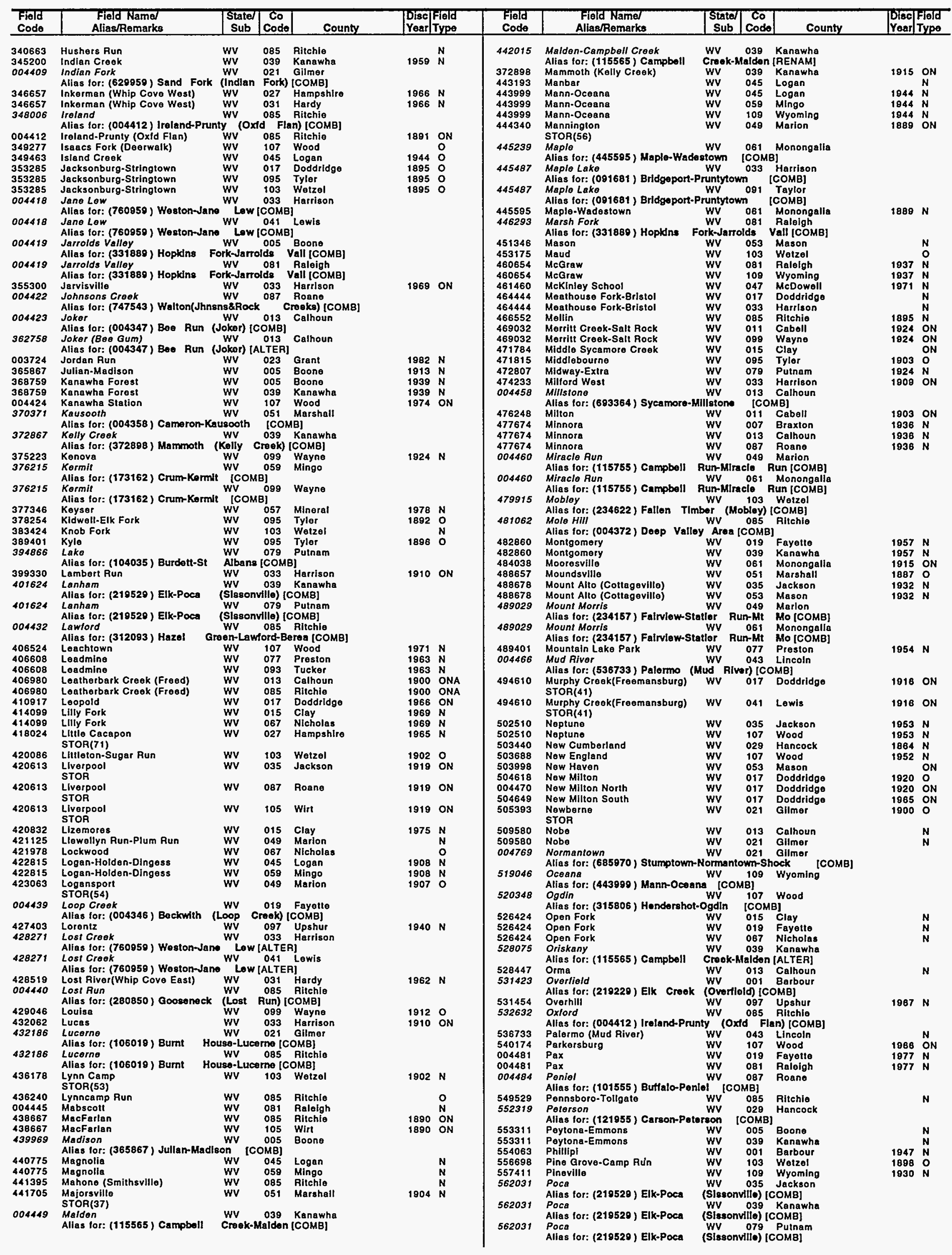


WEST VIRGINIA

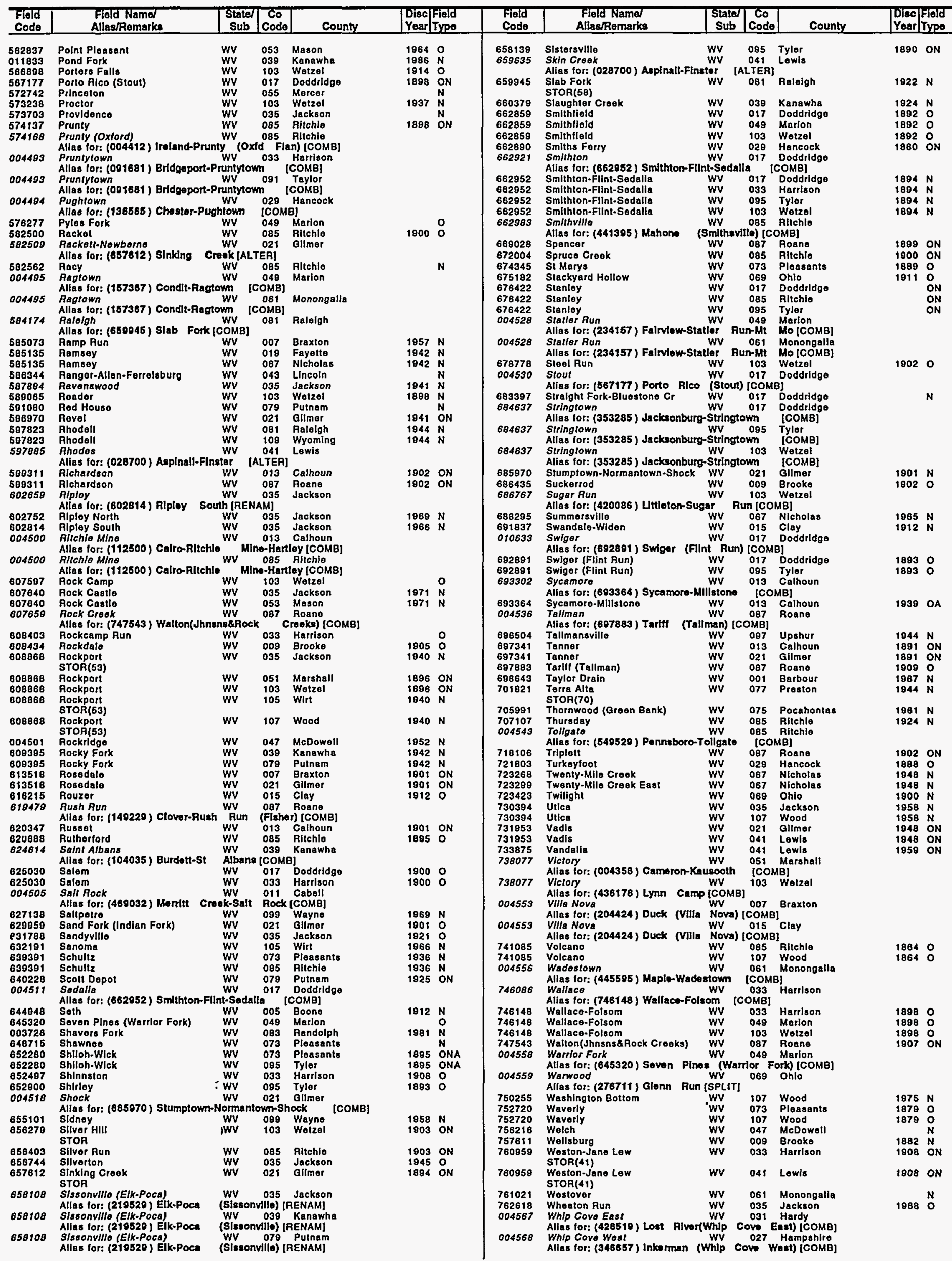




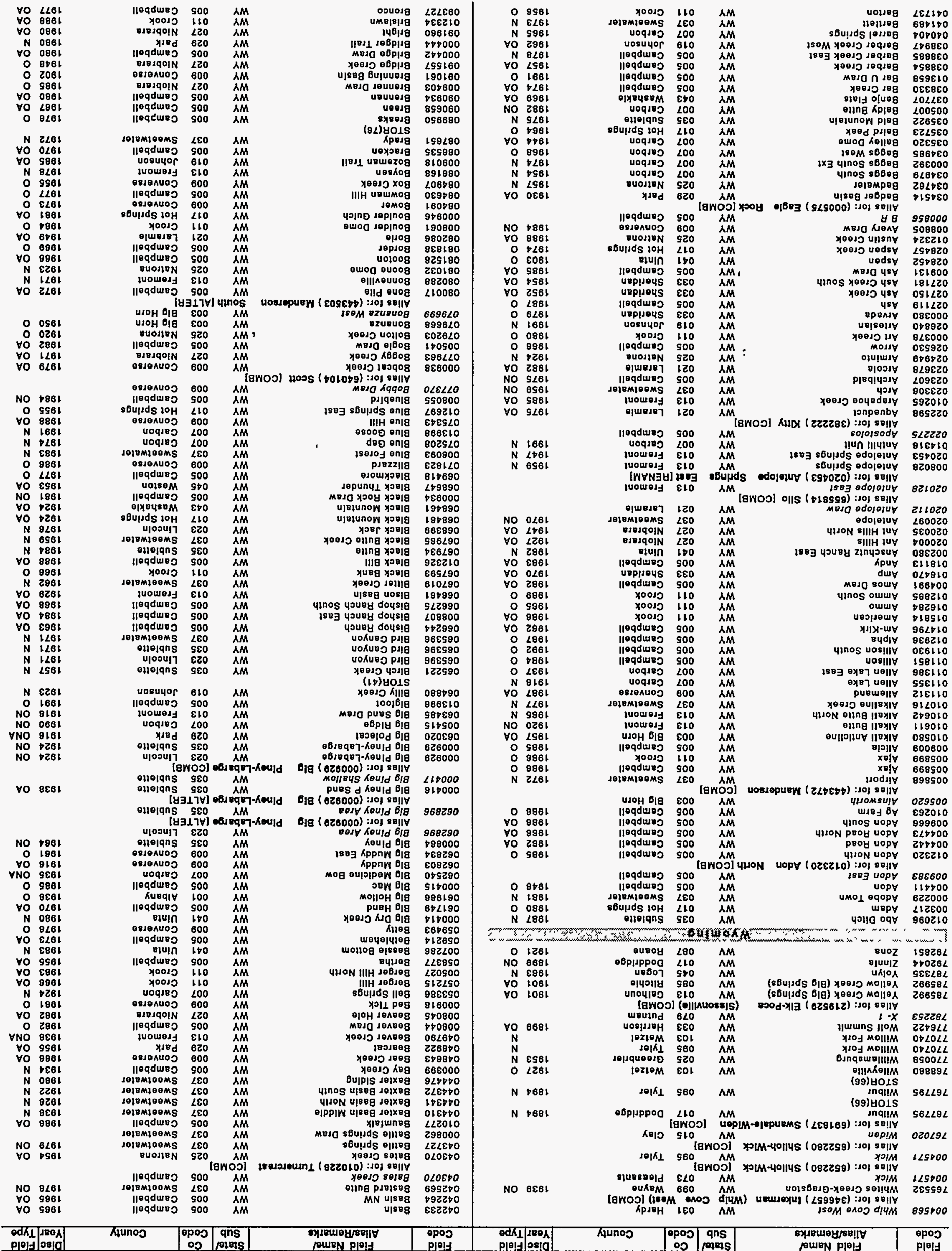


WYOMING

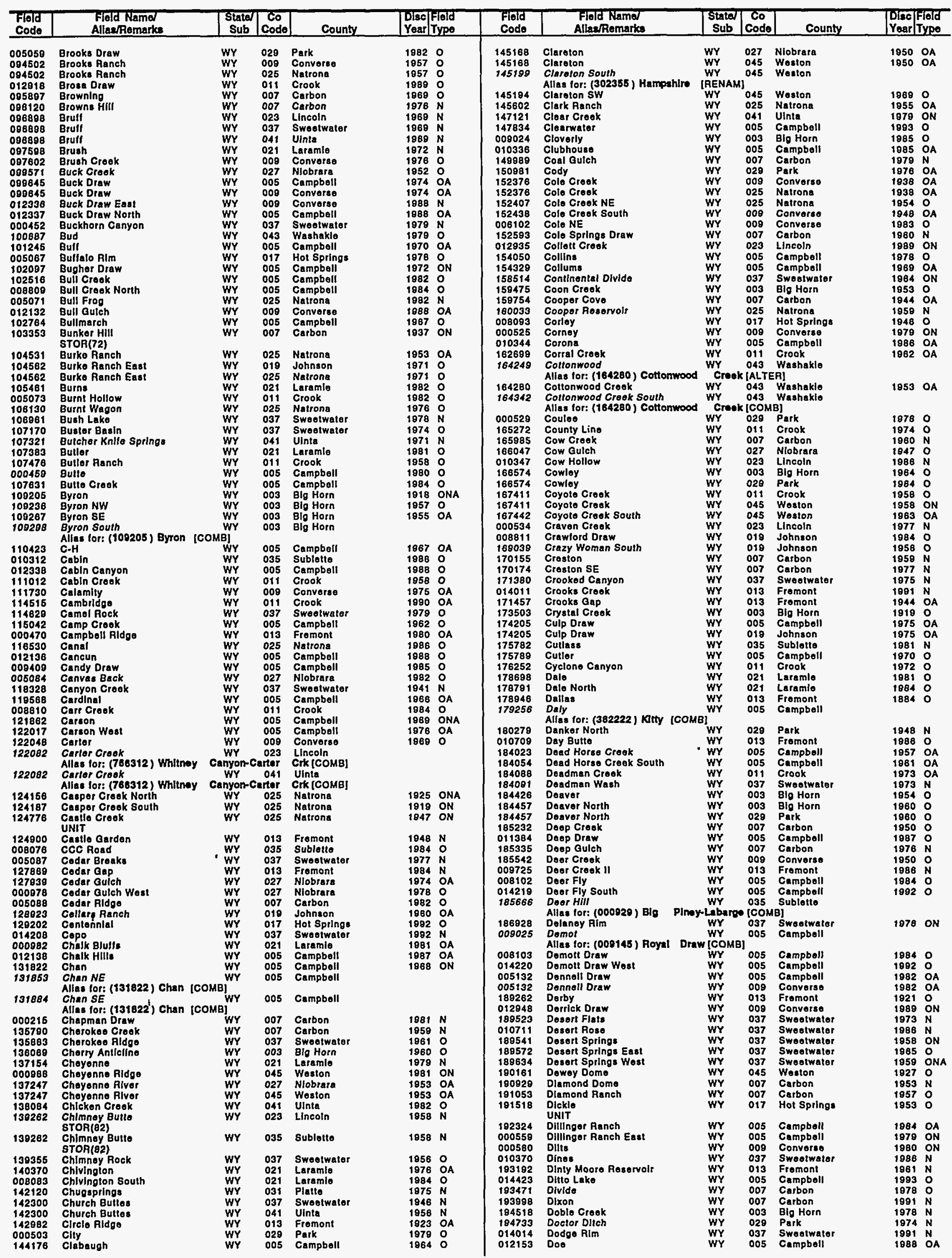




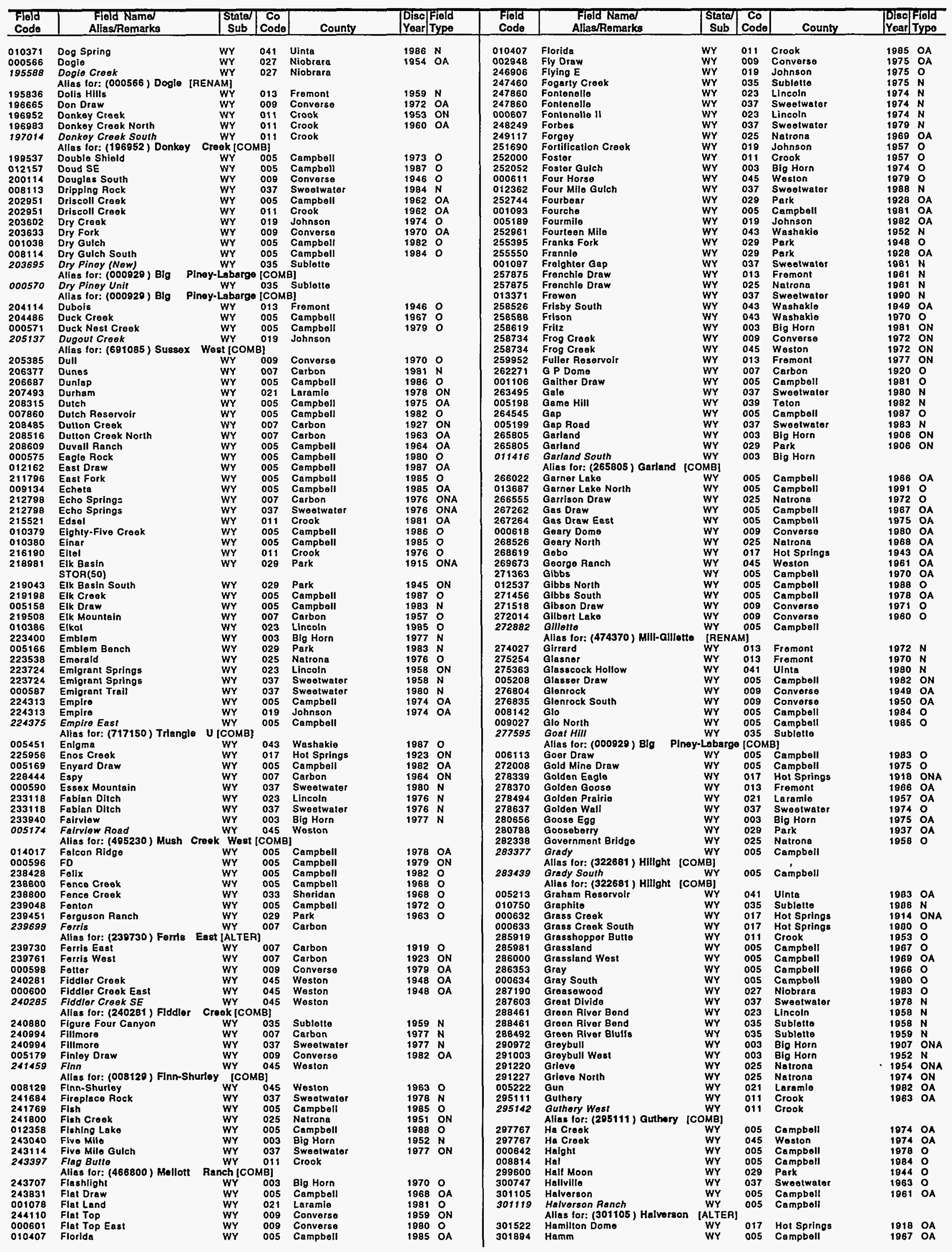


WYOMING

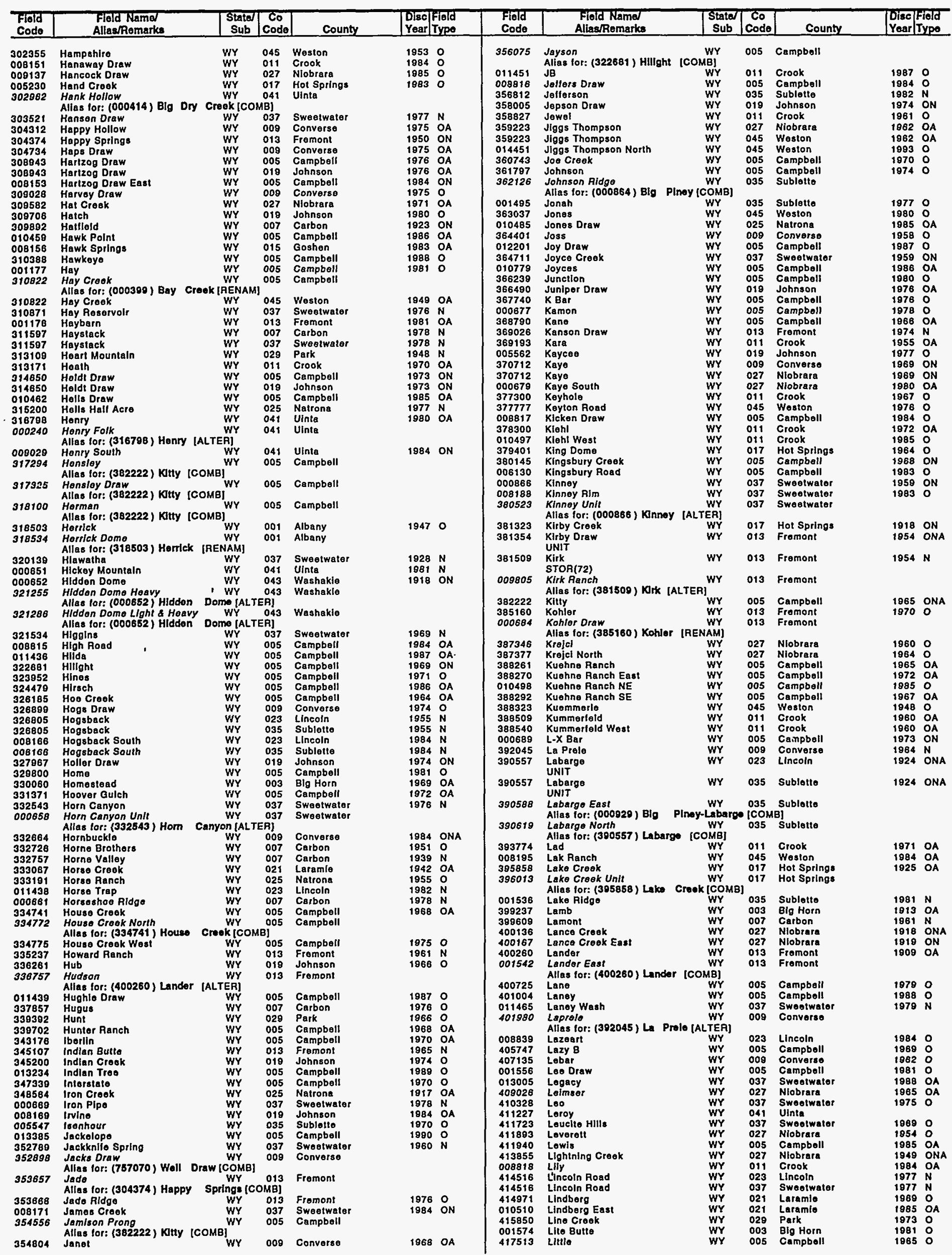




\begin{tabular}{|c|c|}
\hline $\begin{array}{l}\text { Frold } \\
\text { Code }\end{array}$ & $\begin{array}{l}\text { Fivid Nama } \\
\text { Alias/Remarks }\end{array}$ \\
\hline 417947 & $\begin{array}{l}\text { Lithe Buck Creak } \\
\text { Allas for: }(400136) \text { Lance C }\end{array}$ \\
\hline 417978 & Littlo Buck Creek (Now) \\
\hline$\$ 18000$ & Littlo Buffalo Besin \\
\hline 418000 & Littlo Buffalo Basin \\
\hline 000700 & Littlo Bughar Draw \\
\hline 418443 & Littlo Dome \\
\hline 498567 & Litile Grass Creok \\
\hline 418908 & Little Laramlo \\
\hline 418970 & Little Medleine Bow \\
\hline $\begin{array}{l}419001 \\
419032\end{array}$ & $\begin{array}{l}\text { Littlo Missouri } \\
\text { Litlle Mitchell Croek }\end{array}$ \\
\hline $\begin{array}{l}419032 \\
419063\end{array}$ & $\begin{array}{l}\text { Little Mitchell Craok } \\
\text { Little Mo }\end{array}$ \\
\hline 006138 & Little Monument \\
\hline 006138 & Littlo Monument \\
\hline 419187 & Littlo Polocat \\
\hline 001580 & Littlo Powder \\
\hline 419559 & Littlo Sand Draw \\
\hline 418621 & Littlo Snake \\
\hline 000702 & Littlo Thunder \\
\hline $\begin{array}{l}419962 \\
006140\end{array}$ & $\begin{array}{l}\text { Littlo Worm Creok } \\
\text { Loblolly }\end{array}$ \\
\hline 000703 & Lodgepolo Croek \\
\hline 422784 & Logan Draw \\
\hline 008818 & Lone Coder \\
\hline 425171 & Lonetreo Creek \\
\hline 425371 & Long Butte \\
\hline 425371 & Long Butte \\
\hline 425419 & Long Crook \\
\hline & Alias for: (000711) Longs $c$ \\
\hline 425481 & Long Groon \\
\hline 425512 & $\begin{array}{l}\text { Long island } \\
\text { Alias for: }(000929) \text { Big PIn }\end{array}$ \\
\hline 000708 & Long island Unit \\
\hline 000710 & $\begin{array}{l}\text { Allas for: }(000929) \text { ilg PIn } \\
\text { Long Treo }\end{array}$ \\
\hline 011489 & Long Troo North \\
\hline 000711 & Longs Grook \\
\hline 426876 & Loop \\
\hline 428209 & Lost Cabin \\
\hline 428271 & Lost Crook \\
\hline 428271 & Lost Crook \\
\hline 000713 & Lost Creok Basin \\
\hline 428550 & Lost Soldier \\
\hline 428550 & Lost Soldier \\
\hline 428581 & Lost Springs \\
\hline 428767 & Lost Valley \\
\hline $\begin{array}{l}430007 \\
430010\end{array}$ & $\begin{array}{l}\text { Lovall } \\
\text { Lovell Draw }\end{array}$ \\
\hline 431061 & Lox \\
\hline 000141 & Luckey Dltch \\
\hline 008820 & Lucky Lindy \\
\hline $\begin{array}{l}436643 \\
437706\end{array}$ & $\begin{array}{l}\text { Lysitio } \\
M-D\end{array}$ \\
\hline $\begin{array}{l}437706 \\
437706\end{array}$ & $\begin{array}{l}M-D \\
M-D\end{array}$ \\
\hline 008219 & $\begin{array}{l}M-D \\
M-D \text { North }\end{array}$ \\
\hline $\begin{array}{l}008219 \\
438326\end{array}$ & $\begin{array}{l}\text { M-D North } \\
\text { Mac }\end{array}$ \\
\hline 438568 & $\begin{array}{l}\text { Mac } \\
\text { Maddon }\end{array}$ \\
\hline 439580 & Maddon Doep \\
\hline & Allas for: (439566) Madden \\
\hline 008220 & Mader Draw \\
\hline 441426 & $\begin{array}{l}\text { Mahoney } \\
\text { Alies for: }(000718) \text { Mahonay }\end{array}$ \\
\hline 000718 & $\begin{array}{l}\text { Alias for: }(000719) \text { Mahonay } \\
\text { Mahonay Domo }\end{array}$ \\
\hline 005632 & Mahonoy Dome East \\
\hline 441443 & Mahoney Draw \\
\hline 441457 & $\begin{array}{l}\text { Mahoney East } \\
\text { Altas tor: }(005632) \text { Mahoney }\end{array}$ \\
\hline 000720 & Mahonoy South \\
\hline 000721 & Maki Crook \\
\hline 442387 & Mallard \\
\hline 008821 & Malmquist \\
\hline 008822 & Mammoth Hollow \\
\hline$\$ 43472$ & Manderson \\
\hline 143503 & Manderson Sauth \\
\hline 444216 & Manning \\
\hline 445232 & Mapos \\
\hline 447176 & Marianno \\
\hline 008227 & Marnio \\
\hline $\begin{array}{l}012396 \\
448393\end{array}$ & $\begin{array}{l}\text { Marnie Sauth } \\
\text { Marshalf }\end{array}$ \\
\hline 450000 & Martin Spring \\
\hline 006361 & Mary Draw \\
\hline $\begin{array}{l}006361 \\
012387\end{array}$ & Mary Draw \\
\hline $\begin{array}{l}012397 \\
451346\end{array}$ & $\begin{array}{l}\text { Marys Reservoir } \\
\text { Mason }\end{array}$ \\
\hline 451625 & Messacre Hills \\
\hline 452121 & Mastorson \\
\hline 452183 & Mat \\
\hline 452493 & Mathors \\
\hline 454198 & Mavorick SprIngs \\
\hline 454228 & Mavarick Springs SE \\
\hline 454880 & May \\
\hline 455469 & $\begin{array}{l}\text { Mayfield } \\
\text { Alias for: (000632) Grass }\end{array}$ \\
\hline & \\
\hline 008823 & Maysdorl South \\
\hline 006144 & MeCroory \\
\hline $\begin{array}{l}458783 \\
459259\end{array}$ & $\begin{array}{l}\text { MeCulloch Poak } \\
\text { MeDonald Draw }\end{array}$ \\
\hline & Alias for: $(000029)$ Blg \\
\hline 000733 & MePhorson Springs \\
\hline 463762 & $\begin{array}{l}\text { Mosdow Crook } \\
\text { UNIT }\end{array}$ \\
\hline 463793 & $\begin{array}{l}\text { UNIT } \\
\text { Moadow Croek East }\end{array}$ \\
\hline 463824 & \\
\hline 463855 & Moadow Crook SE \\
\hline & Alias for: (463793) Moadow \\
\hline $\begin{array}{l}463886 \\
464900\end{array}$ & $\begin{array}{l}\text { Moadow Crook West } \\
\text { Medicine Bow }\end{array}$ \\
\hline 464809 & $\begin{array}{l}\text { Allas for: }(464809) \text { Medicine } \\
\text { Madielne Bow South }\end{array}$ \\
\hline
\end{tabular}


WYOMING

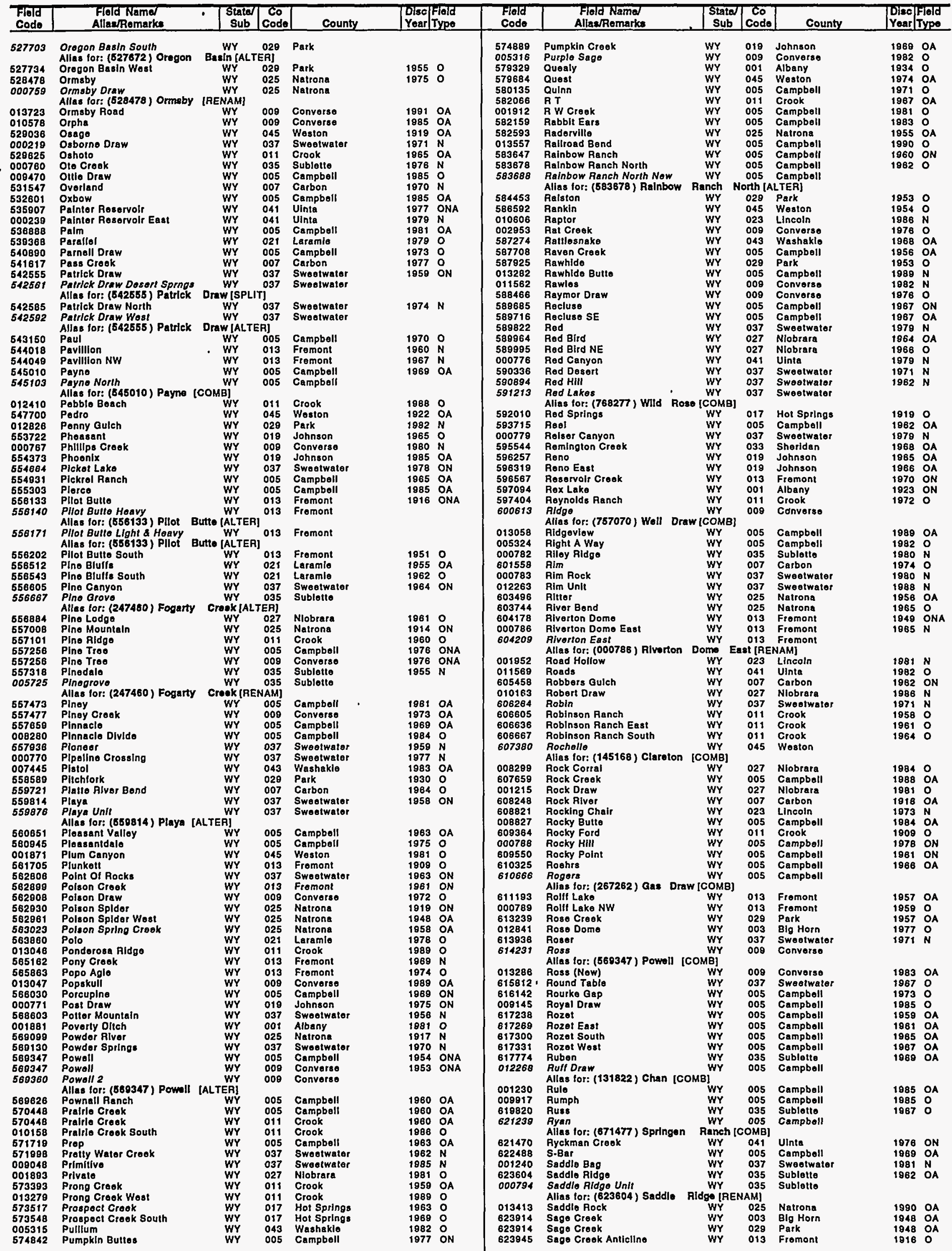




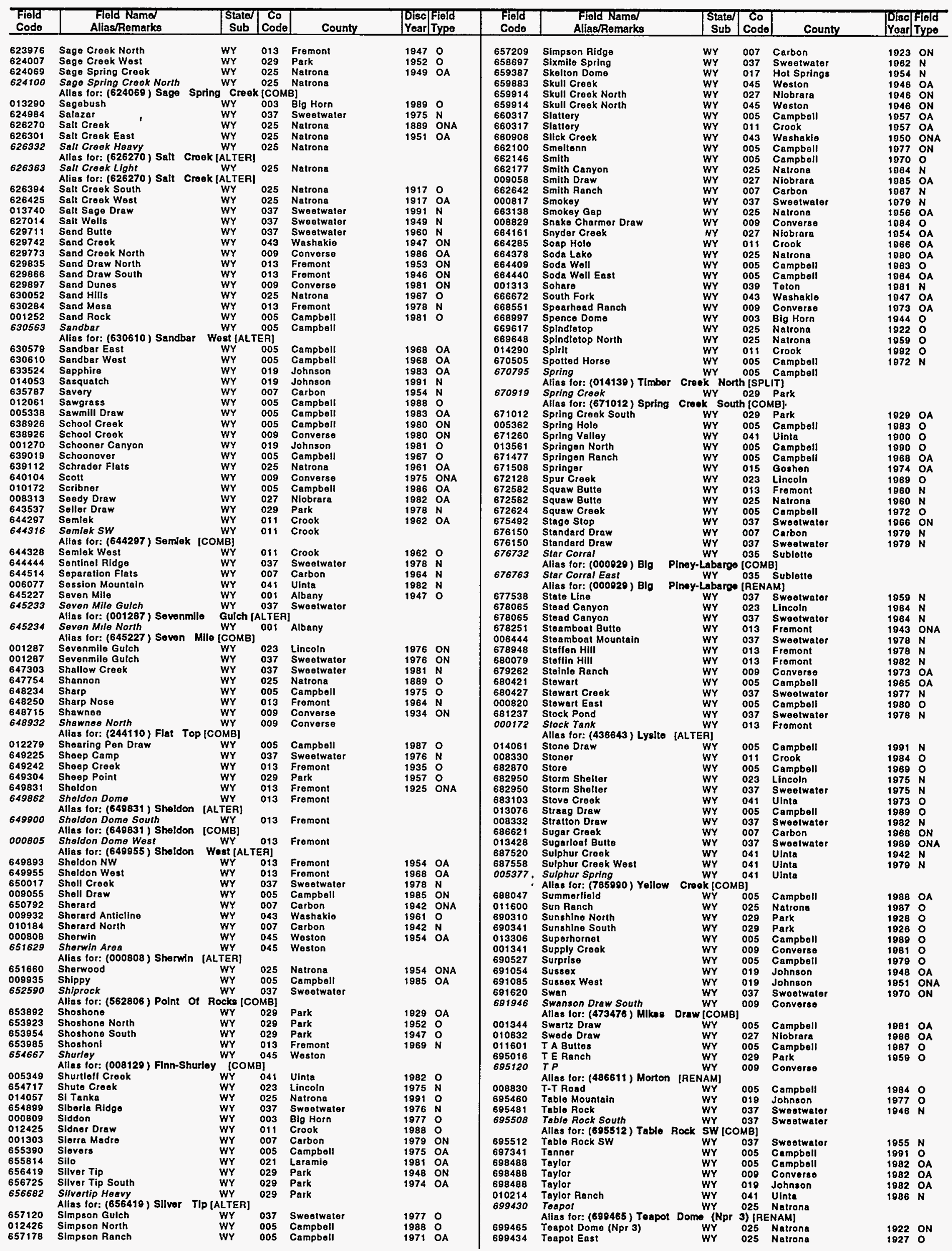


F.O.- CALIFORNIA

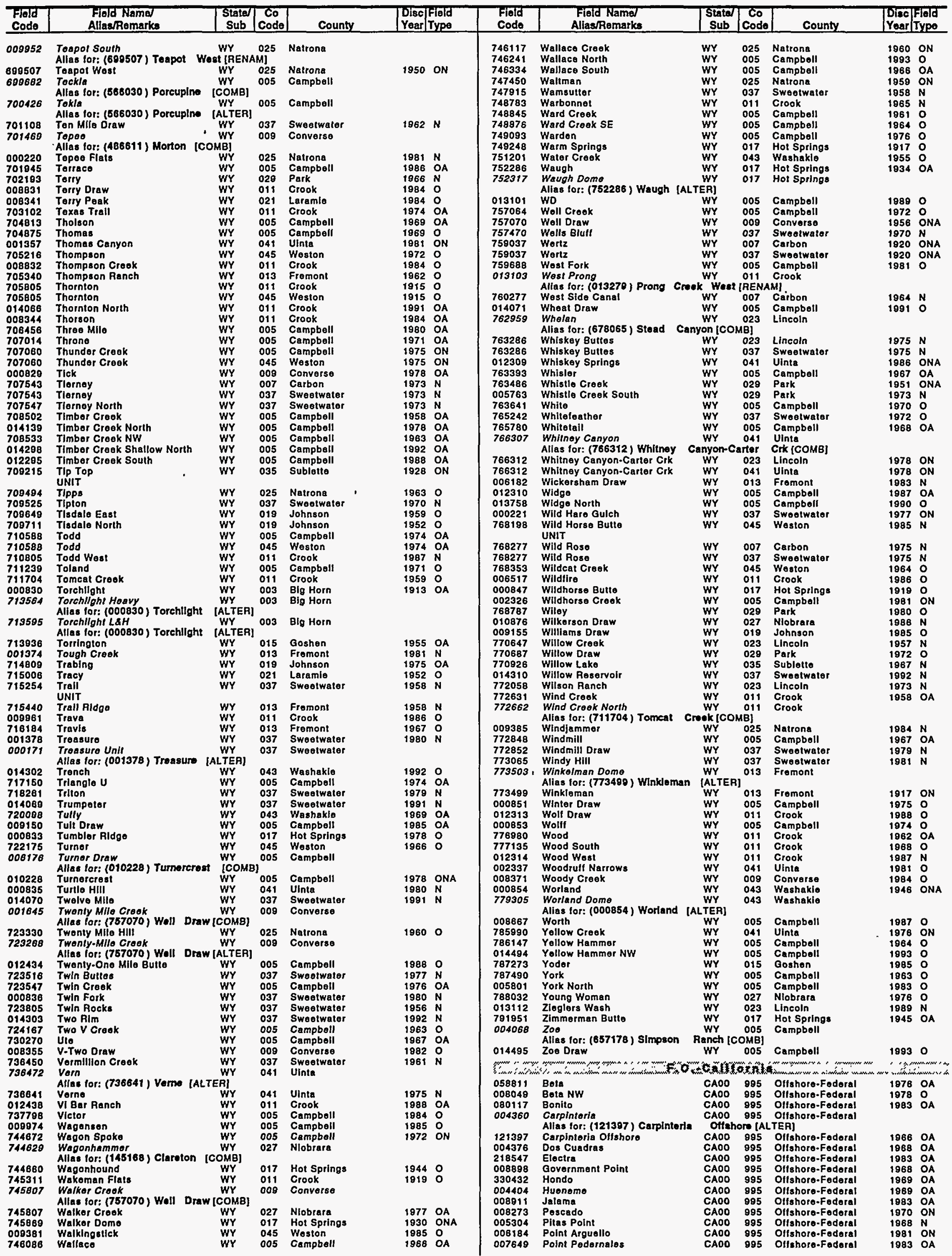




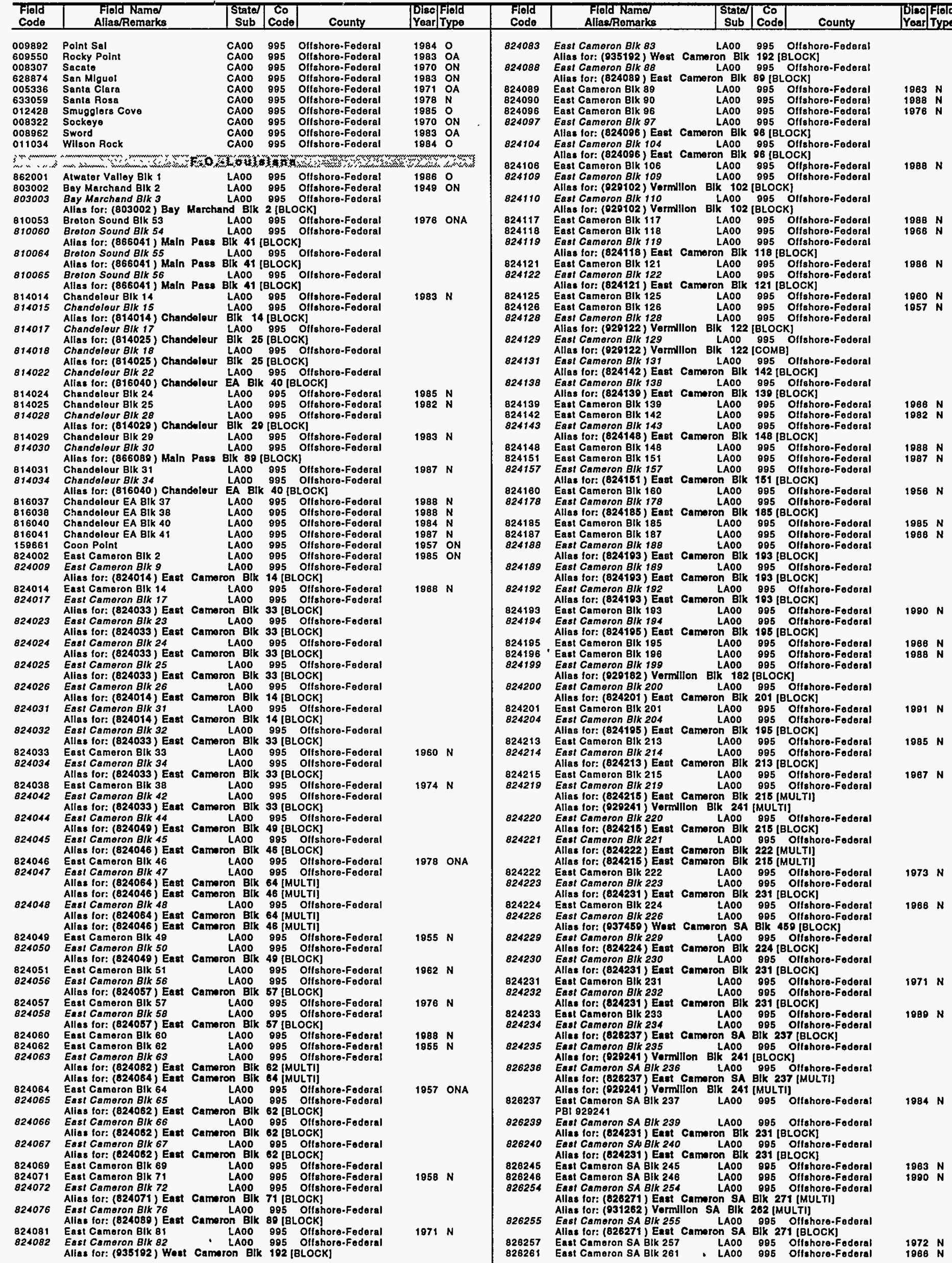




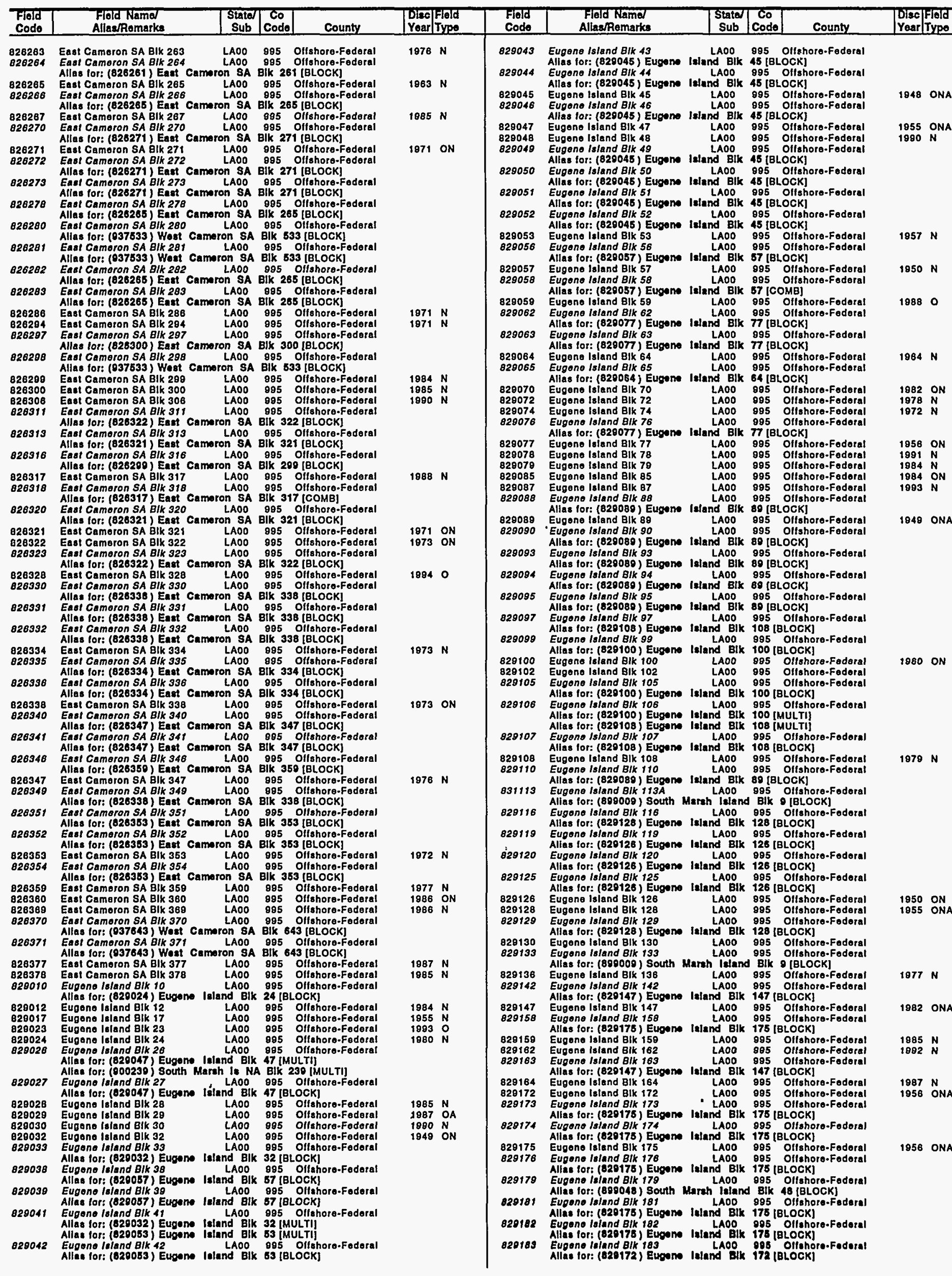




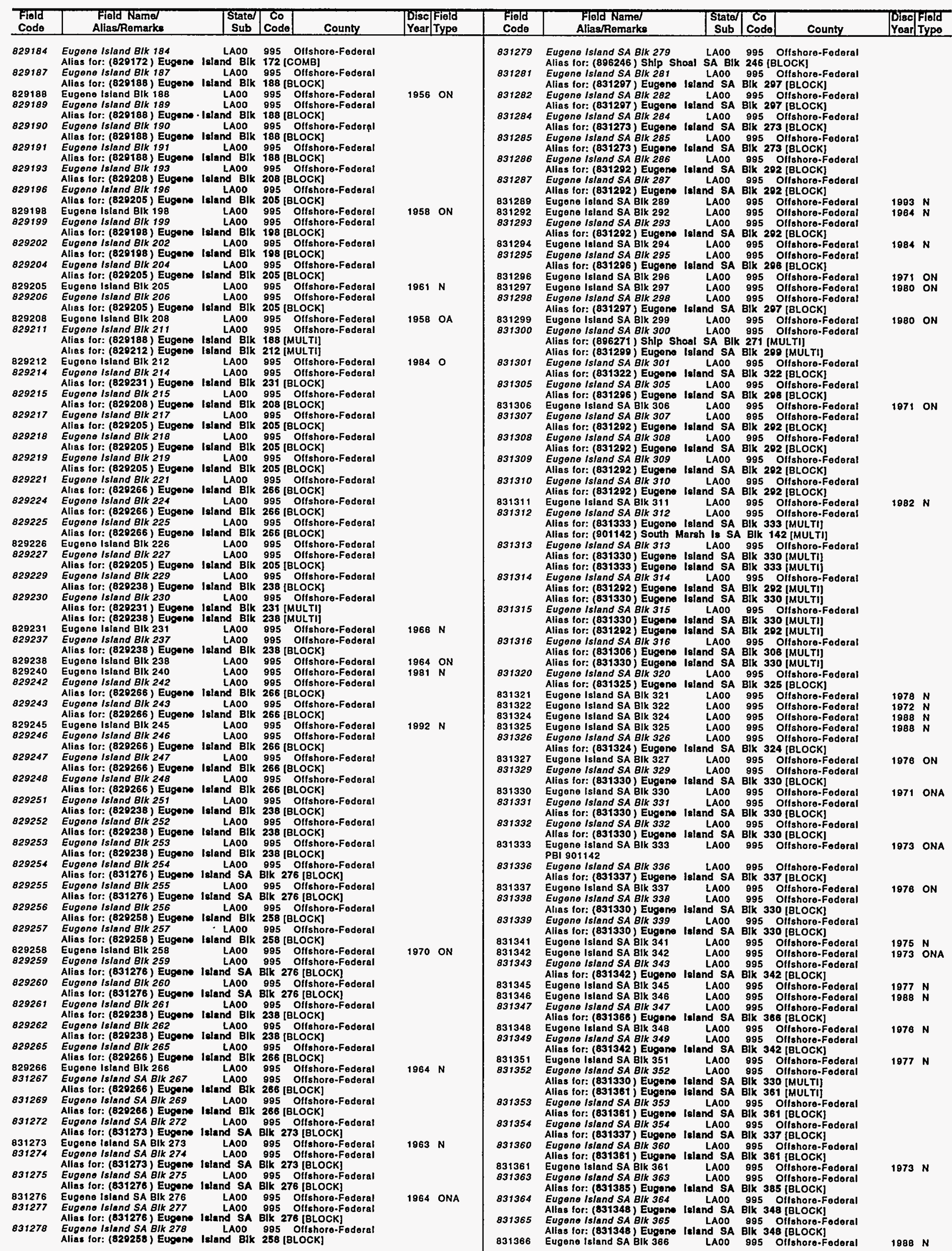




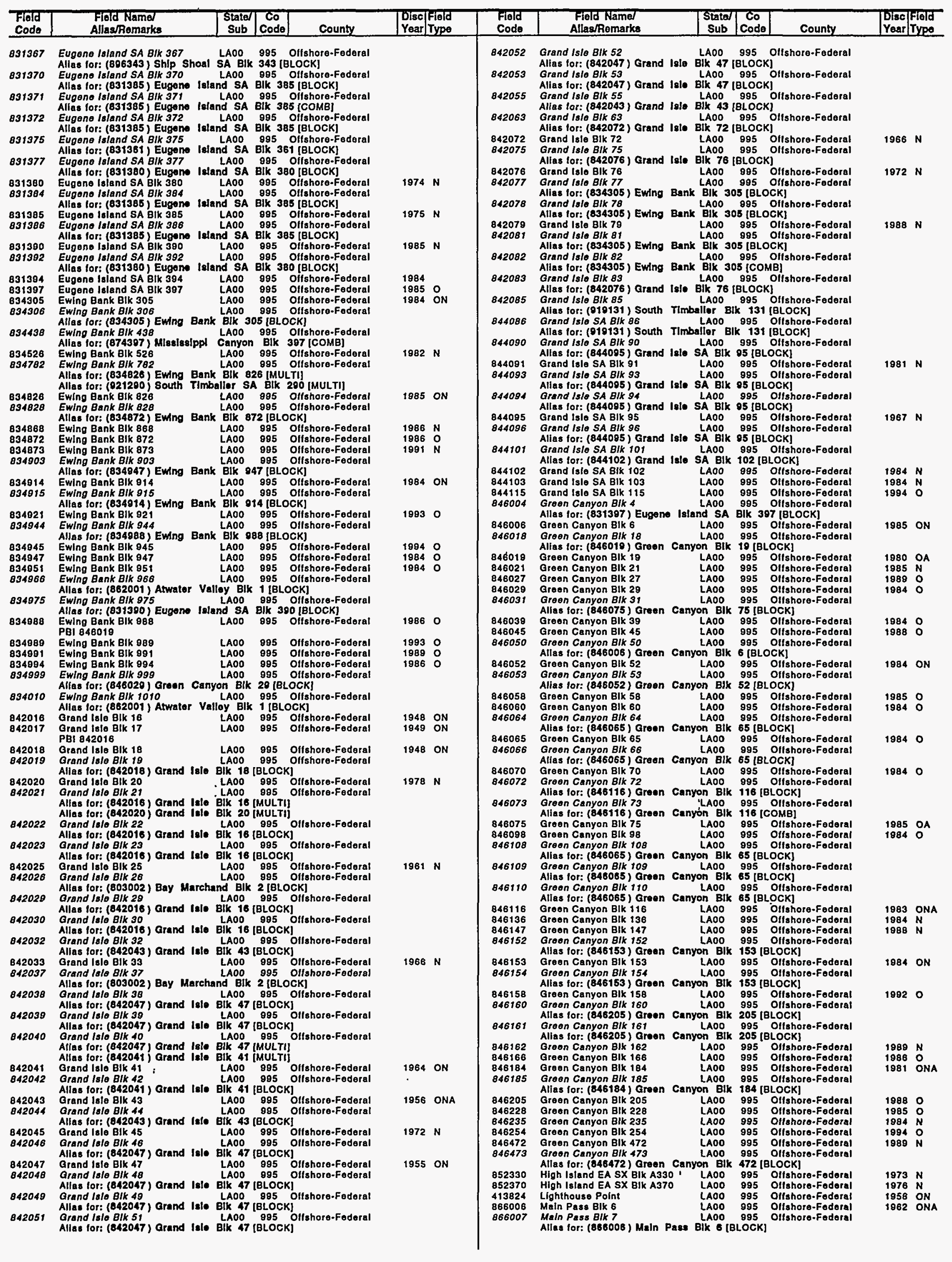




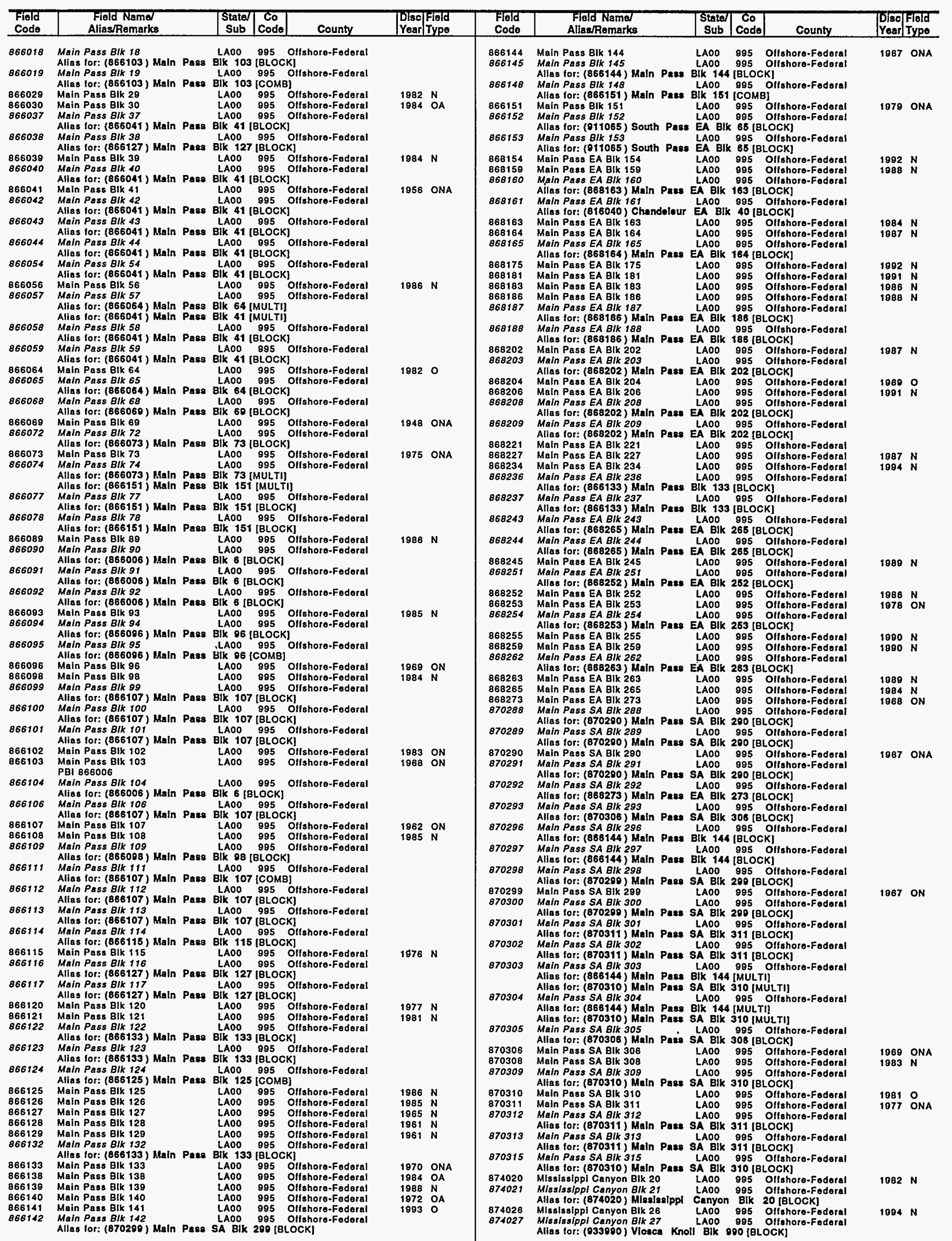


F.O.-LOUISIANA

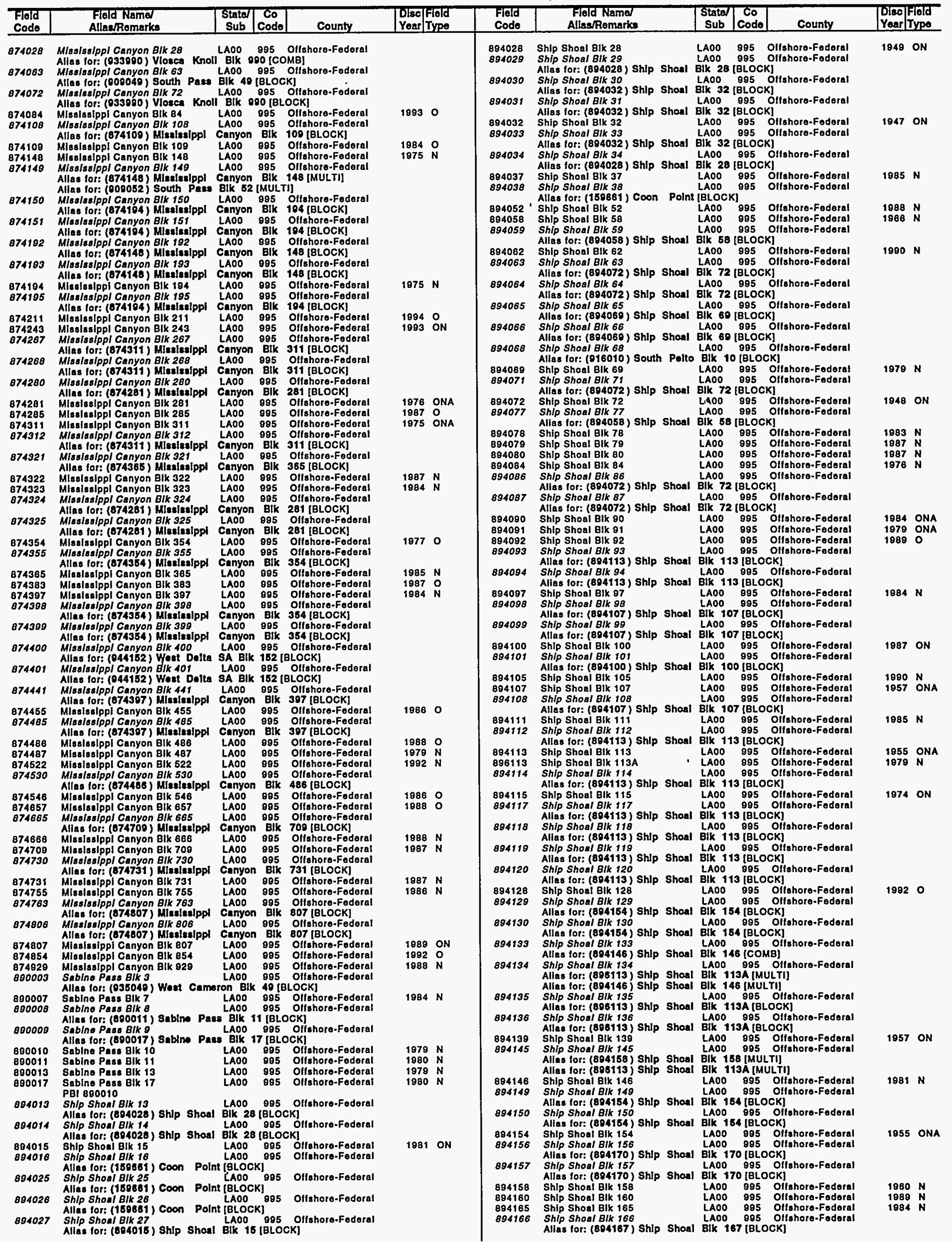




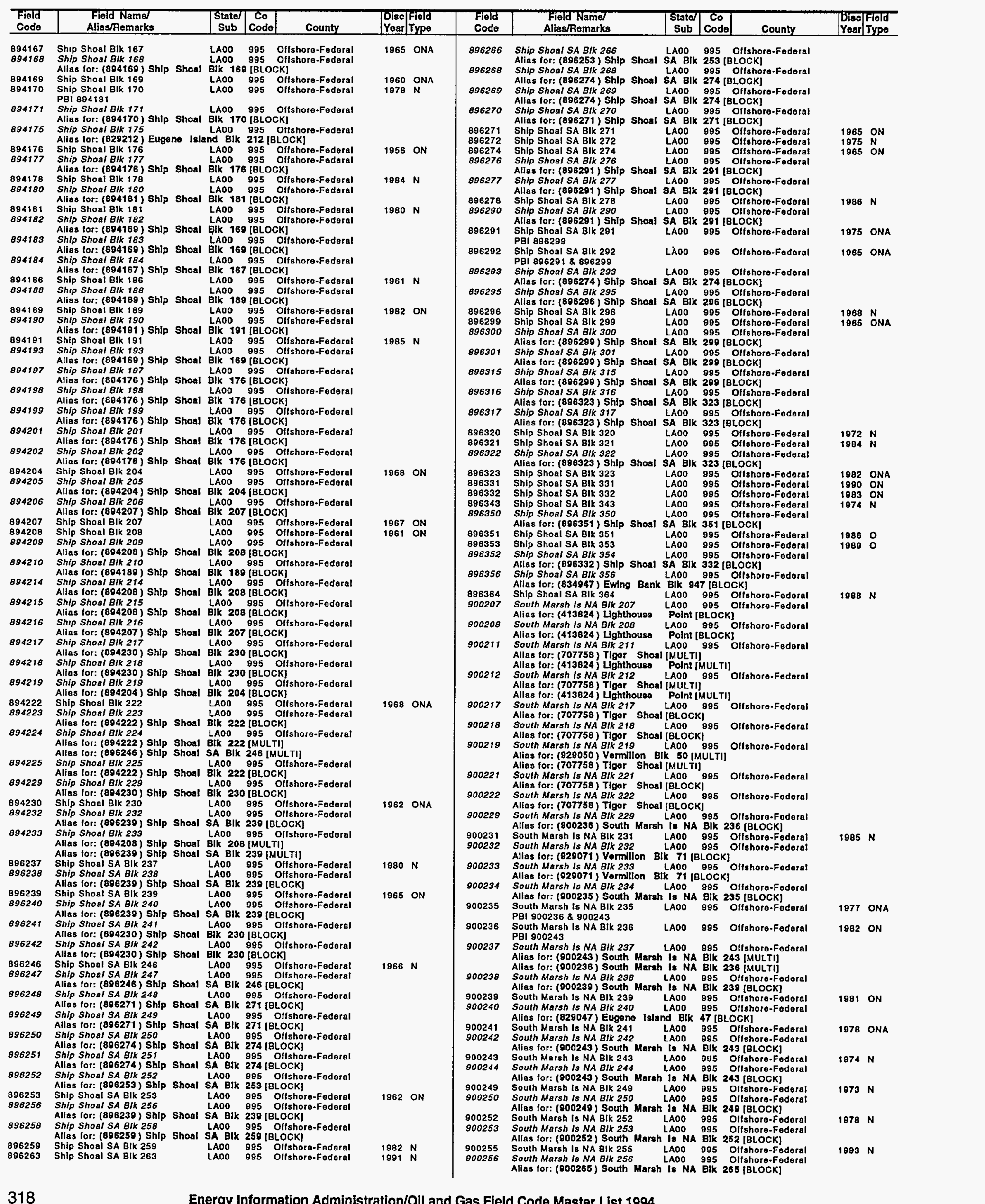




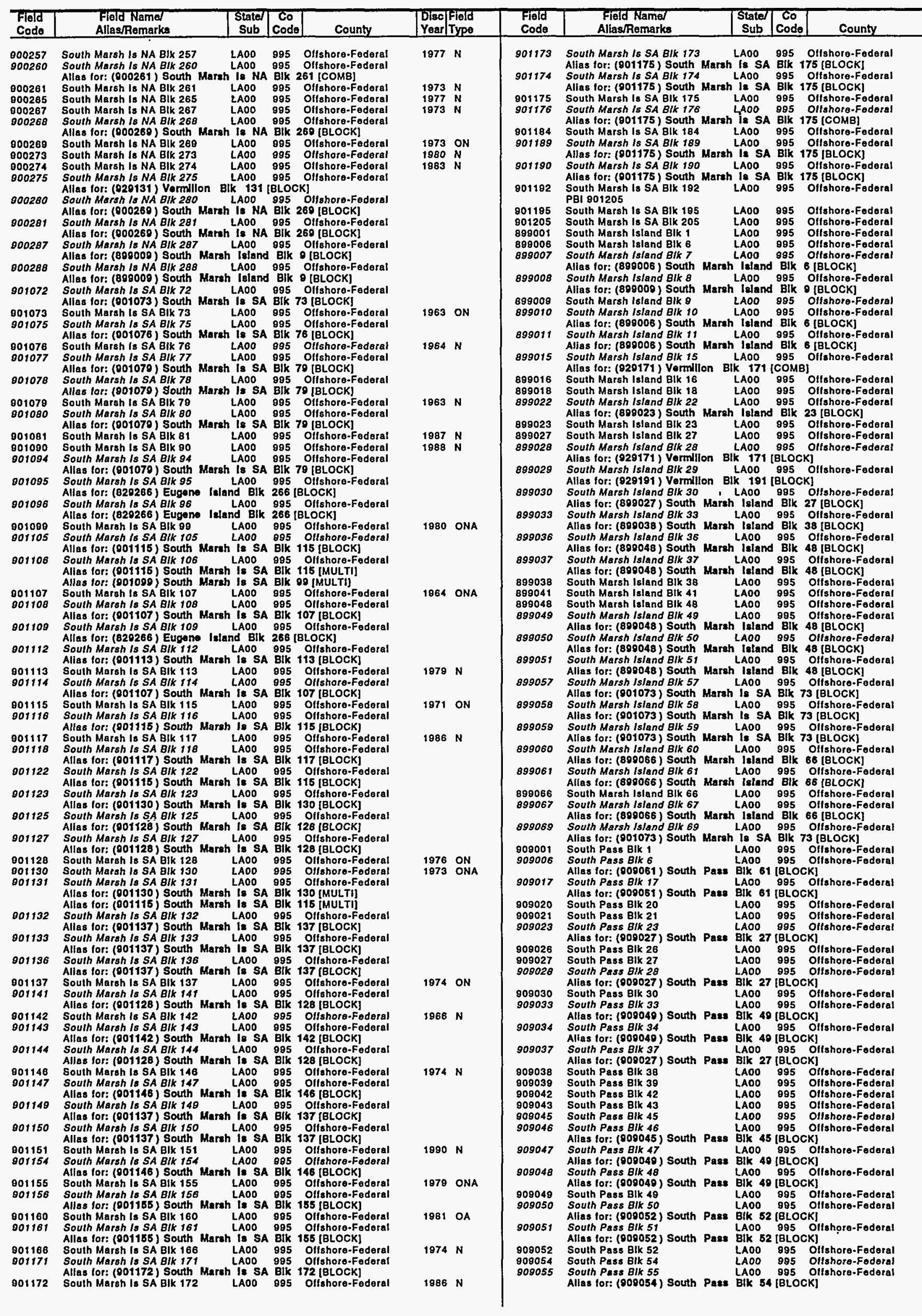

1973 ON

$1974 N$

$1902 \mathrm{~N}$

$1992 \mathrm{ON}$
$1985 \mathrm{~N}$

1982 ONA

1985 ON

$1982 \mathrm{ON}$

$1960 \%$

1963 ON

1961 ON

1983 ON

1968 OA

$1956 \mathrm{~N}$

$1957 \mathrm{~N}$

1854 ONA

$1955 \mathrm{~N}$

1962 ON

$1858 \mathrm{~N}$

1989 ONA
1981 N

1874 ONA

1968 ONA
1969 ON 
$\begin{array}{ll}N \quad 188 \\ \\ N & 188 \\ N & 888 \\ N & 886 \\ N & 986\end{array}$

N $\mathbf{9 8 6 1}$

N 2961

YNO 8861

N sort

N 8L61

$N$ SL84

$0<61$

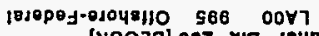

902 길 20$][8 q u ! \perp$ 4InOS

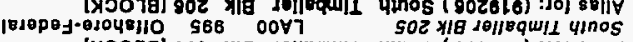

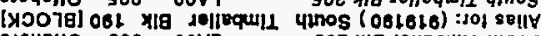

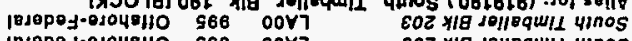

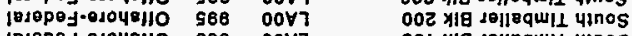

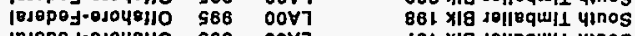

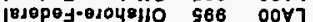

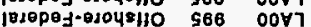

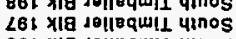

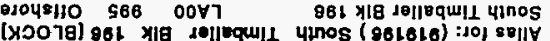

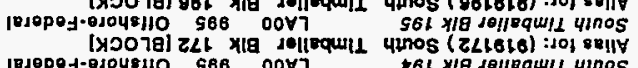

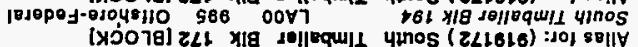

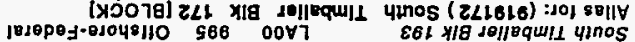

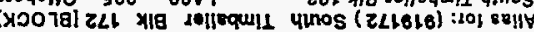

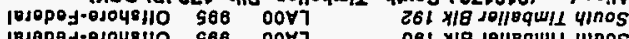

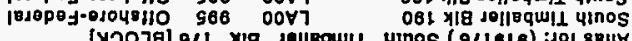

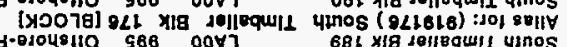

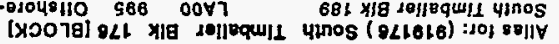

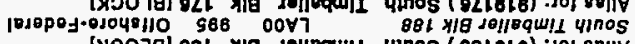

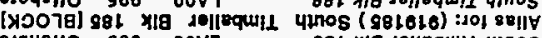

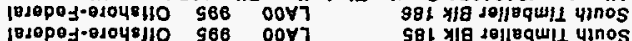

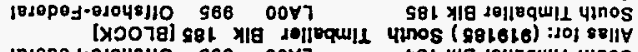

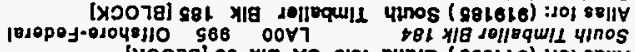

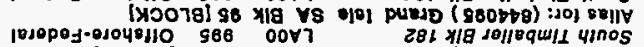

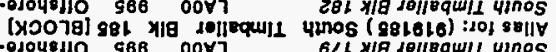

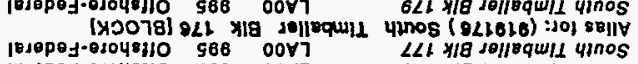

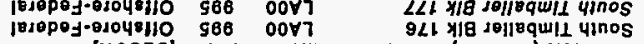

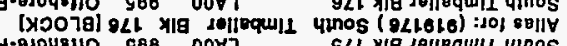

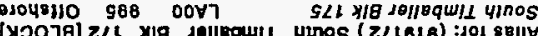

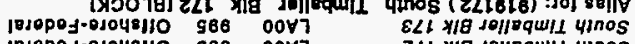

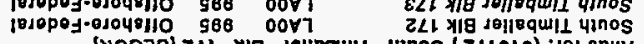

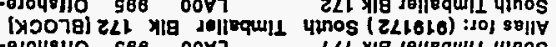

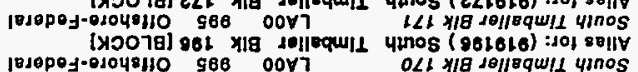

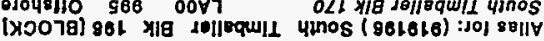
101000-

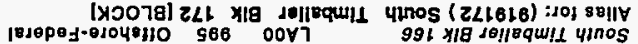

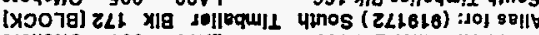

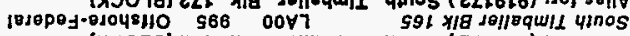

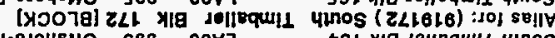
(8jepoj-01045110 568 00V7

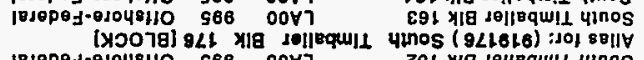

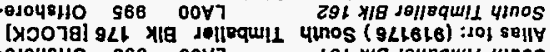

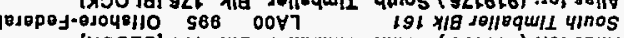

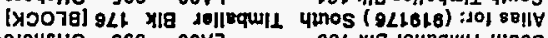

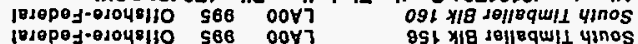

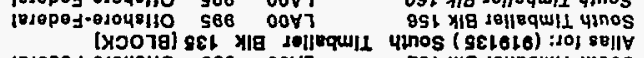

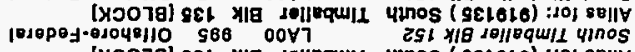

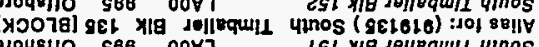

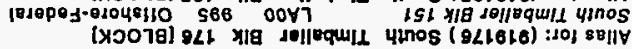

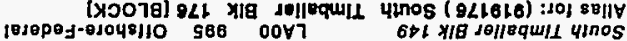

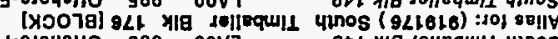

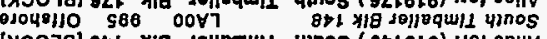

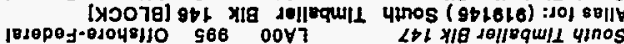

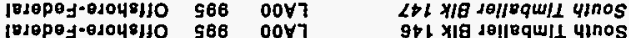

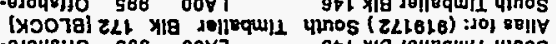

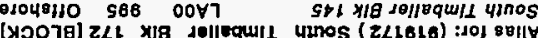

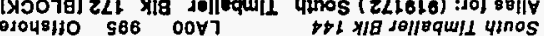

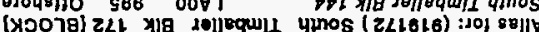

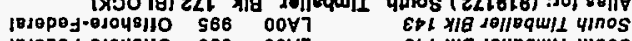

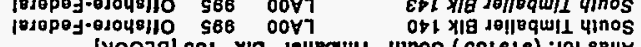

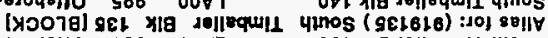
|ejopej-01049110 $\$ 86$ oOV7 981 XIG |вג0p0J

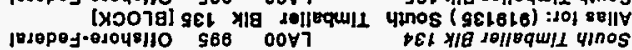

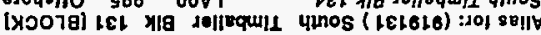
(X)

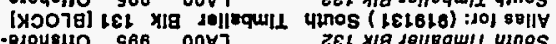

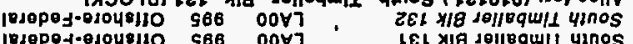

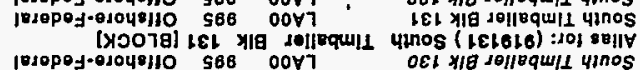

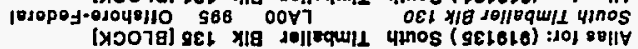

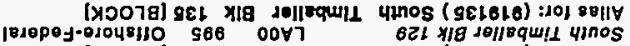

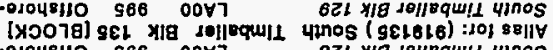

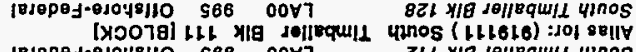

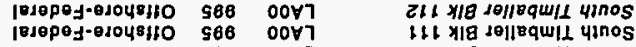

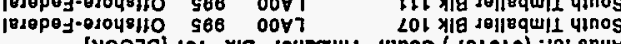

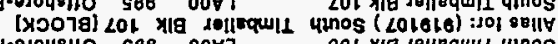

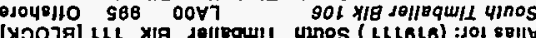

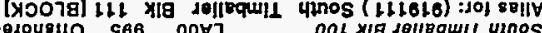

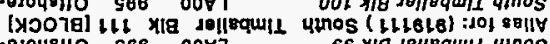
-0.049110 966 00\%7

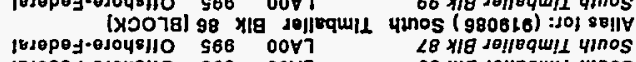
181000-01049110 566 00Y7 $\begin{array}{rl}\forall N O & 9961 \\ N & \forall 861 \\ N & 9861 \\ N & 8861\end{array}$

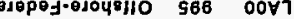

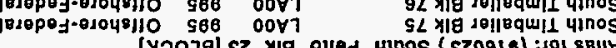

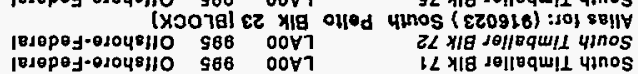

SLLGLG

981616 98 xis de\|lहquil uाnos $\angle L$ xig Le|lBqwil yinos $2 \angle 065$ s02616

802616

002616

861616

961816

561616

$\$ 61616$

861616

261616
061616

681616

881616

981616

S81616

$\$ 81616$

281616

621616

$\angle 21616$

21616

121616

$0<1616$

691616

991616

S91616

p 91616

291616

191616

091616

951616

เ51610

$6 \$ 1616$

$B \geqslant 1616$

161616

s\$1616

$\$ \$ 1616$

E\$1616

EI6!6

Eะ1616

zE1616

oE:616

621616

821616

211616

LO1616

801616

001616

660616

80616

$9 \angle 0618$

$z<0616$
N $6 \angle 61$

NO ss8t

- OgBt

$\forall N O \quad \geqslant \angle 61$
$\forall N O \quad \$ \angle 61$

NO $6+61$

N $6 \angle 6$ t

- 2966

YNO 2961

YNo

O $8 \angle 8 b$

No $\theta \angle 6$

No $S \angle \theta \mid$

8861

$\begin{array}{rl}N & 286 ! \\ \forall O & 186 !\end{array}$

$\forall$ NO 696

N 9861

NO $\varepsilon<6$

$\forall$ NO $\$ 801$

N 066 t

opos
plof

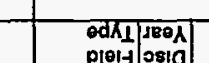

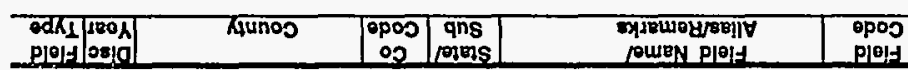

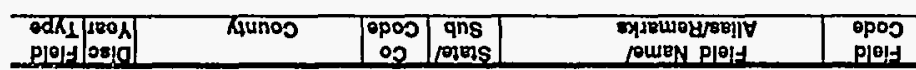

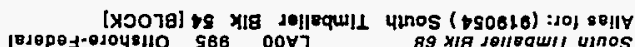

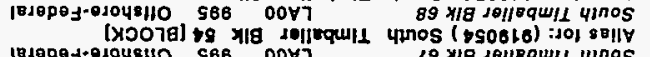

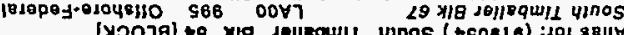

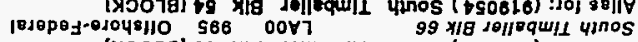

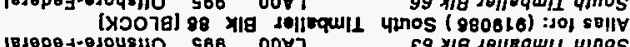

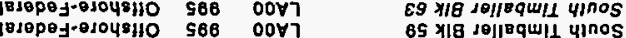
100 566 007า 65 ×18

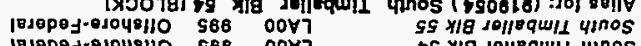

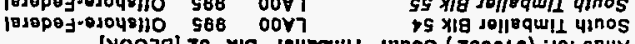

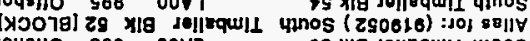
(818pej-01048110 568 00V7

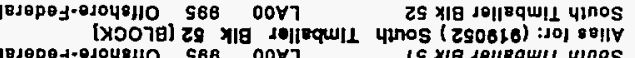

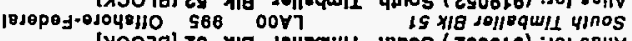

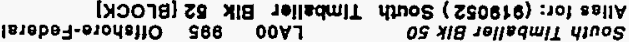

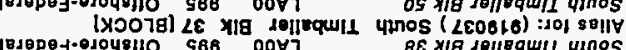

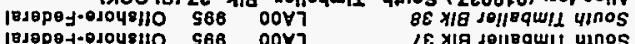

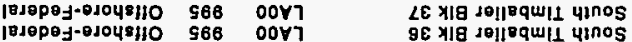

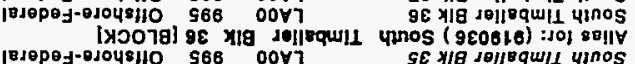

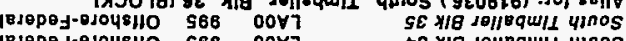

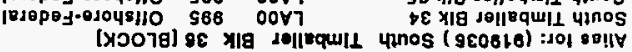

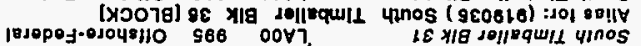

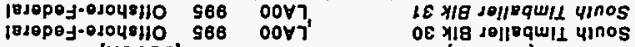

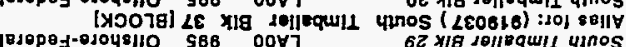

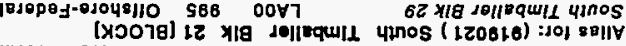

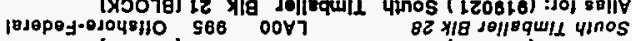

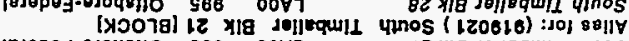

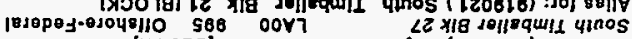

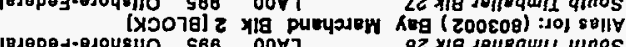

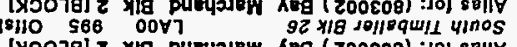

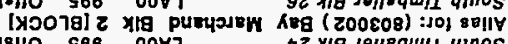

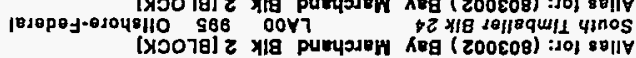

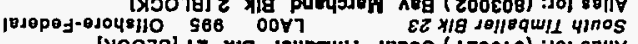

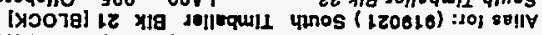

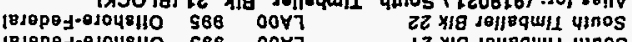

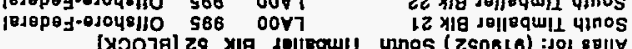

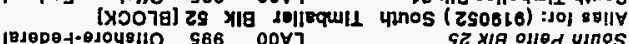

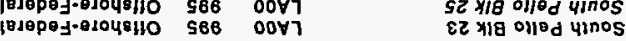

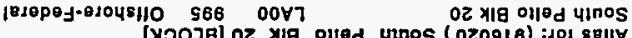

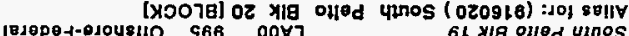

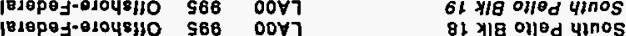

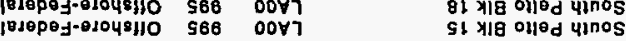

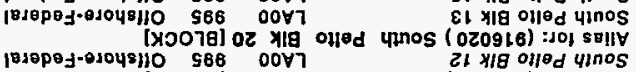

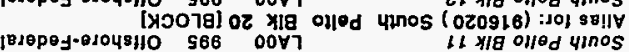

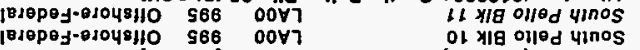

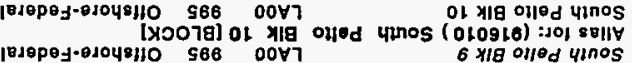

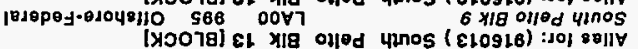

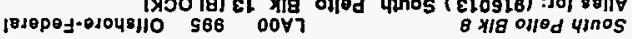

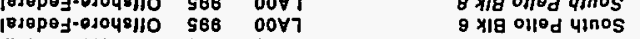

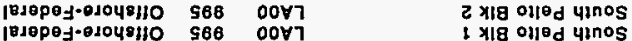

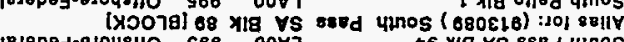
(40 $\$ 18$ VS ss0d 41 nos

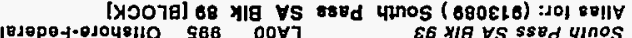

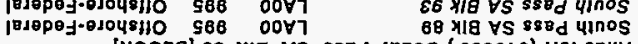

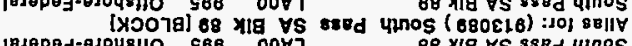

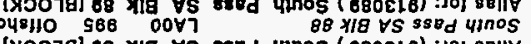

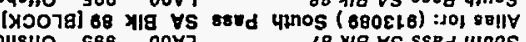

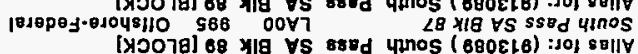

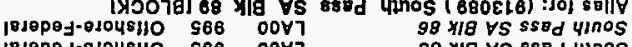
1010p0J-0104s110 568 00V7

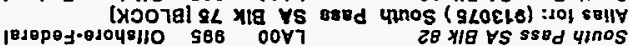

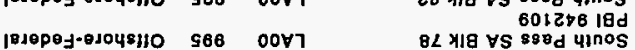

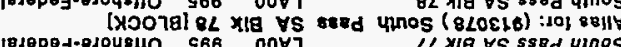

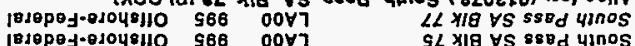

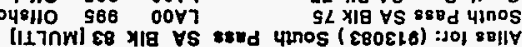

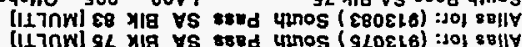

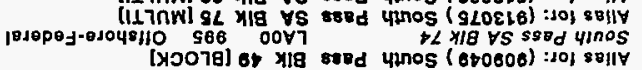

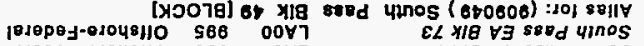

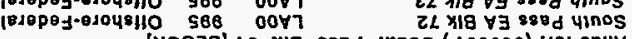

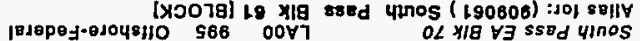

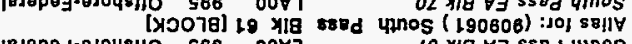
$297 / 8 \forall \exists$ S58d 4INOS

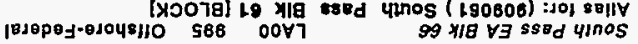

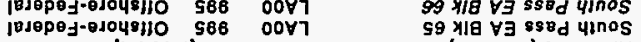
(X0078) 99 y,

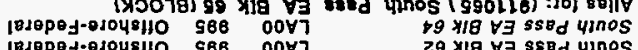

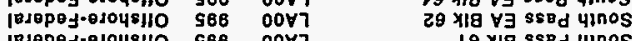
1010p0]-01049110 568 OOV7

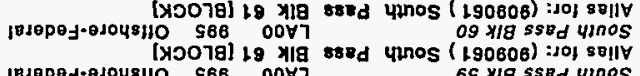

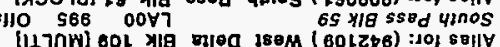

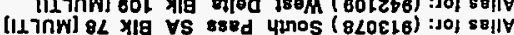

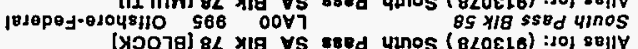

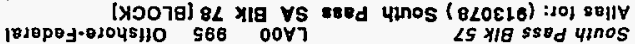

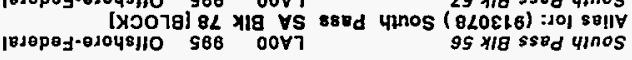

890616

$\angle 90616$ 990616

890616

650616

$\checkmark 50616$ 850616
250016

150616

050616

$8 \varepsilon 0616$ $\angle C 0818$
920616 580616
$\checkmark 60816$ 


\section{F.O.-LOUISIANA}

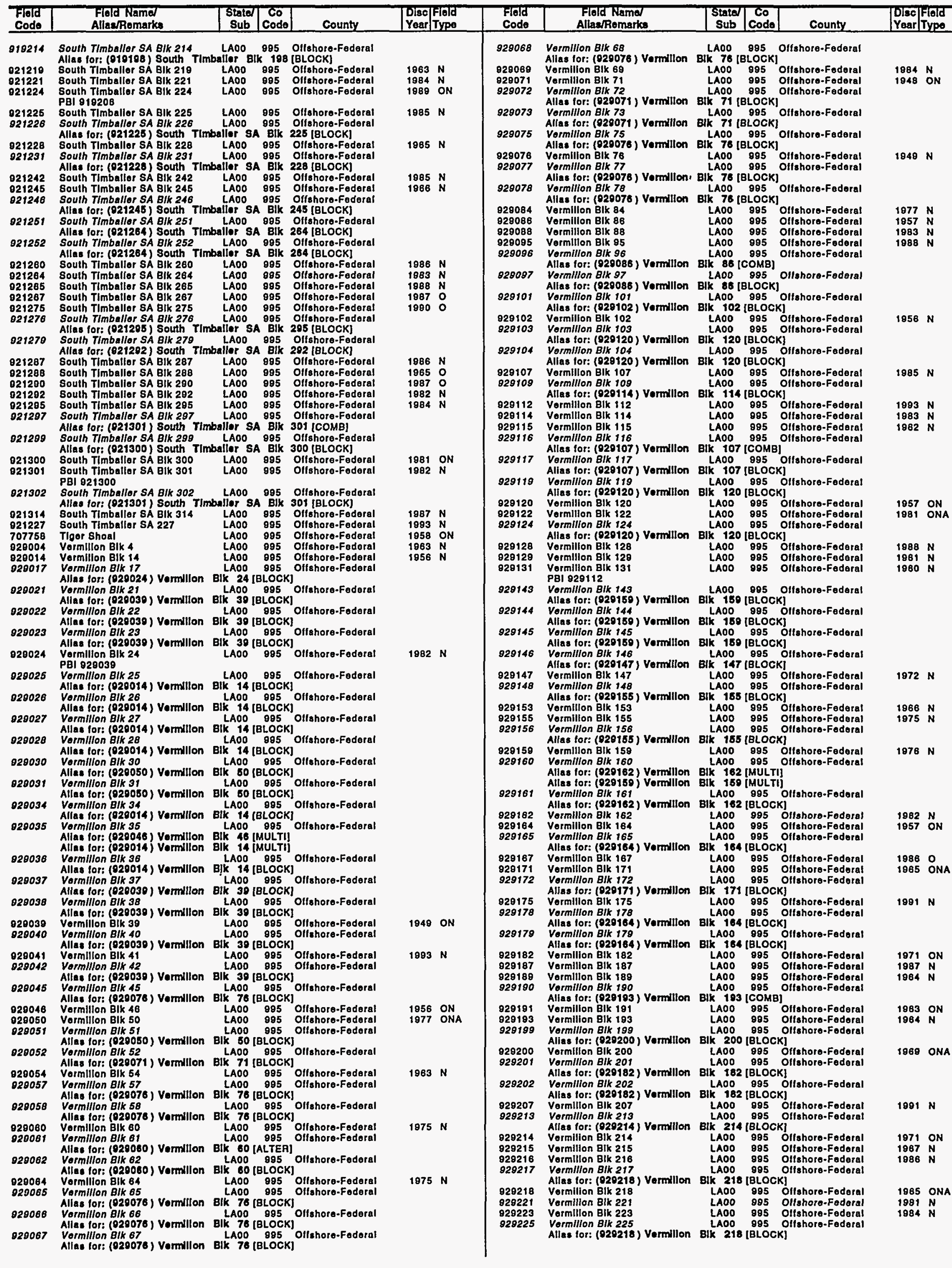




\section{F.O.-LOUISIANA}

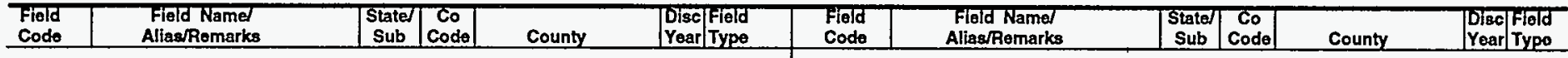

929226 Vormillon Blk 226 LAOO 995 Olfshora-Federal

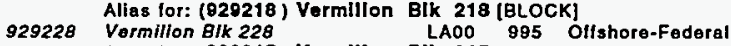

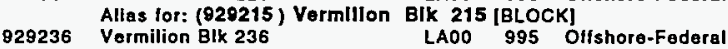
$\begin{array}{lll}929236 & \text { Vermilion Blk } 236 & \text { LAOO } 995 \text { Orshore-Foderal } \\ 929237 & \text { Vormilion BIK } 237 & \text { LAOO } 995 \text { Offshore-Federal }\end{array}$ 929237 Vormilion Blk 237 LAOC 995 Offshore-Federal 929241 Vermilion Blk 241 LA00 995 Offshore-Federal 929242 Vormillon BIK 242 LAOO 995 Olishore-Federal

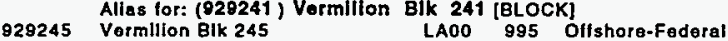
929246 Vormilion BIK 246 LA00 995 Ollshore-Foderal 929247 Alias for: (929245) Vermllton Blk 245 (BLOCK) 929247 Vormilion Blk 247
Alias for: (931255) Vermillon SA Blk 255 [BLOCK] 929248 Vermillon BIK 248 LAOO 995 Ollshore-Federal

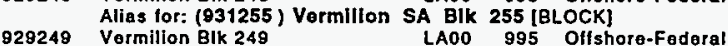
PBI 931250

929250 Vermilion Blk $250 \quad$ LA0O 995 Olfshore-Federal $\begin{array}{llll}929250 & \text { Vermilion Blk 250 } & \text { LA00 } 995 & \text { Olfshore-Fodaral } \\ 929251 & \text { Vormilion BIK 251 } & \text { LAOO } 995 \text { Olfshore-Foderal }\end{array}$ Allas tor: (029250) Vormllion Blk 250 [BLOCK] Vormillon SA BIK 252 LAOO 995 Ollshore-Federal Alias for: (931273) Vermllion SA BIK 273 [MULTI] Alias for: (929250) Vermllion BIK 250 [MULTI]

931253 Vormillon SA BIK 253 LAOO 995 Olfshore-Federal Alias for: (929250) Vermllion Blk 250 [MULTI]

931255 Alias for: (931273) Vermillon SA Blk 273 [MULTI]

931255
931256 Alias for: (931255) Vermillon SA BIk 255 (BLOCK]

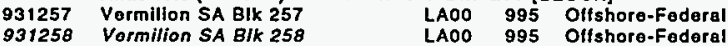
Alias for: (931265) Vermillon SA BIk 265 [MULTI]

931260 Vermilion SA B/K 260 ) Vermllion Blk 245 [MULTI] Alias for: (931262) Vermillon SA BIk 262 [8LOCK]
[BLOCK

931261 Vormillon SA BIK 261 LA0O 995 Olfshore-Federal Alias for: (931262) Vermilion SA BIK 262 (BLOCK]

931262 Vormilion SA BlK 262 LAOO 995 Otfshore-Foderal 031264 Vermillon SA BIK 264 LAOO 995 Olfshore-Federa 931265 Allas for: (031265) Vermllion SA BIK 265 [BLOCK]

Varmilion SA Blk 265 LAOO 995 Olfshora-Foderal

$\begin{array}{llll}931266 & \text { Vermilion SA BIk 266 } & \text { LAOO } 995 & \text { Olfshore-Federal } \\ 931267 & \text { Vermillon SA BlK 267 } & \text { LA00 } 995 \text { Offshoro-Federal }\end{array}$ Vermillon SA BIK 267 LAOO 995 Olfshore-Federal Alias for: (931255) Vermillon SA Blk 255 [BLOCK]

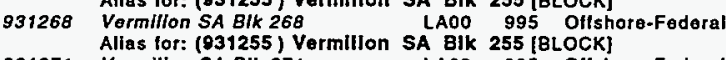
931271 Vermilion SA BIK 271 . LAOO 995 Olishore-Federal
Alias for: (931273) VermIlion SA BIk 273 [COMB]

931273 Vermilion SA Blk 273 LAOO 995 Ollshore-Federal

931274 Vormilion SA BIK 274 LAOO 995 Olfshore-Federal Alias for: (931288) Vormilion SA BIK 288 [BLOCK]

931275 Vermilion SA Blk 275 LAOO 995 Offshore-Federal

931276 Vormilion SA BIK 276 LAOO 995 OfIshoro.Foderal

931277 Alias for: (931287) Vermillon SA Blk 287 [BLOCK] 931277 Vermilion SA BIK 277 Varllion SA BIK 287 [BLOCK]
Alias tor: (931287) Vormillon

931281 Vormillion SA Blk 281 LAOO 995 Olfshore-Foderal

931282 Vormilion SA Blk 282 LAOO 995 Ollshore-Foderal Alles for: (826271) East Cameron SA Blk 271 [BLOCK]

931284 Vormilion SA BlK 284 LAOD 995 Offshore-Federal

$\begin{array}{llll}931287 & \text { Vetmillion SA Blk 287 } & \text { LAOO } 995 & \text { Offshore-Foderal } \\ 931288 & \text { Vermilion SA Blk 28B } & \text { LAOO } 995 \text { Offshore-Foderal }\end{array}$

931289 Vormilion SA BIK 289 LAOO 995 Ofishore-Federal Vormilion $S A$ Blk 289
Alias for: (931288) Vermilion SA BIK 288 [BLOCK]

931292 Vermilion SA BIk 292 LAOO 995 Olishore.Federal

931296 Vermillon SA Blk 296 , LAOO 995 Offshore-Federal 931302 Vormilion SA BIk 302
Alias for: $(931320)$ Vormillion SA BIk 320 [BLOCK]
931310 Vermillon SA BIk 310
LAOO 995 Offshore-Federal

931310 Vermillon SA Blk 310

$\begin{array}{lll}\text { LAOO } & 995 & \text { Offshore-Federal } \\ \text { LAOO } & 895 & \text { Offshore-Federal }\end{array}$

PBI 931310 Barmion SA BIK 314

LA00 995 Ottshore-Federat

931314 Alias for: (931315) Vermillon SA Blk 315 [BLOCK]
931315 Vermilion SA Elk 315
LAOO 995 Oftshore-Federal $\begin{array}{llll}\text { Vermilion SA Elk } 315 & \text { LAOO } & 995 & \text { Offshore-Foderal } \\ \text { Vermilion SA BIk } 318 & \text { LAOO } & 995 & \text { Olfshore-Foderat }\end{array}$ $\begin{array}{llll}\text { Vermilion SA BIK } 318 & \text { LAOO } & 995 & \text { Olfshore-Faderat } \\ \text { Vermilion SA BIK } 320 & \text { LAOO } & 995 & \text { Offshore-Federal }\end{array}$ $\begin{array}{lll}\text { Vermilion SA BIK } 320 & \text { LAOO } 995 & \text { Offshore-Foderal } \\ \text { Vermillion SA BIK } 321 & \text { LAOO } 995 \text { Offshore-Federal }\end{array}$ Alias for: (931320) Vermillon SA BJk 320 (BLOCKI Alias for: (931320) Vermillon SA BIk 320 [BLOCK]
Vermilion SA BIK 325
LAOO 995 Offshoro-Foderal Vormilion SA BIK 325
Alias for: (931320) Vormillon SA B1k 320 [BLOCK] Vermilion SA BIK 326 LAOO 995 Offshore-Faderal Alias for: $(931320)$ Vormillon SA BIK 320 [BLOCK]

931328 Vermillon SA Blk 328 LAOO 995 Offshore-Federal

931329 Vermilion SA BIK 329 LAOO 995 Offshore-Federal

931330 Vermilion SA BIK 330 LAOO 995 Oftshore-Foderal Alies for: (931331) Vormilion SA BIk 331 iBLOCK)

931331 Vormilion SA BIk 331 LAOO 995 Olfshore-Faderal

931332 Vormilion SA Blk 332

Allas for: (931329) Vormillon SA BIK 329 (BLOCK)

LAOO 995 Offshore-Foderal Alias for: (931340) Varmllion SA BIK 340 (BLOCK]

931340 Vermilion SA BIK 340 LAOO 995 Offshore.federal

931342 Vermillon SA BIK 342 LAOO 995 Olishore-Foderal

931348 Vermilion SA Blk 348 LAOO 995 Olfshore-Federal

LAOO 995 Olfshor

Alias for: (931350) Vermillon SA Blk 350 [BLOCK]

$\begin{array}{llll}931359 & \text { Vormilion SA Blk } 359 & \text { LAOO } 995 & \text { Offshore-Federal } \\ 931362 & \text { Vormllion SA Blk } 362 & \text { LAOO } 995 \text { Olfshore-Foderat }\end{array}$ Alias for: (931370) Vermillon SA BIK 370 [BLOCK]

931369 Vermilion SA BIK 369 LADO 995 Oifshore-Federal Vermilion SA BIk $370 \quad$ LA00 995 Olishora-Federal PBI 931369

931371 Vermilion SA BIk 371 LA00 995 Olfshore-Federal 931372 Vormllion SA Blk 372 LAOO 995 Olfshore-Federal

Alias for: (931370) Vormillon SA Blk 370 [BLOCK]

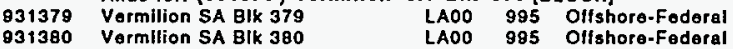

931381 Vormilion SA BIK 381

Vormilion SA Blk 381 LAOO 995 Olfshoro-Fodera

931386 Vermilion $S A$ B 13386 ) Vermllion SA Blk 380 [BLOCK] Vermilion SA Blk 386
Alias for: (931360) Vermlllon SA BIK 369 [BLOCK]

$1971 \mathrm{~N} \quad 931392$ Vermilion SA Blk 392 LAOO 995 Ofishoro-Fodera

\begin{tabular}{l|ll}
931395 & $\begin{array}{l}\text { Vermilion SA Blk 395 } \\
\text { Alias for: (931412) Vermillon SA BAO } 995 \text { Oflk } 412 \text { [BLOCK] }\end{array}$ \\
$975 \mathrm{~N}$ & 931397 & Vermilion SA B1k 397
\end{tabular}

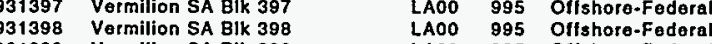

31399 Vermilion SA Blk 399 LAOO 995 Olfshoro-Fodera

1962 ON Alias for: (931412) Vermillion SA BIK 412 [BLOCK]

LAOO 995 Ottshoro.Foderal

931407 Vermilion SA BIK 407 LAOO 995 Olfshore-Federa

931412 Vermilion SA BIK 412

933022 Viosca Knoll Blk 22

LAOO 995 Offshore-Foderal

PBI:886990

$1989 N$

933024 Viosca Knoll Blk 24

933027 Viosca Knoll Blk 27

933031 Viosca Knoll Blk 31

933032 Viosca Knoll Blk 32

933068 Viosca Knoll Blk 68

933069 Vlosca Knoll Blk 69

Viosca Knoll Blk 74

933076
93117
Viosca Knoll Blk 117

Viosca Knoll Blk 118

1965 ON

Alias for: (033074)

933124 ' Viosca Knoll Blk 124

933161

$1989 \mathrm{~N}$

Vlosca Knoll B/k 203

Alias for: (933204) V

933204 Viosca Knoll B1k 204

Viosea Knoll BI1 213

933252 Viosca Knoll BIK 252

933256 Viosca Knoll Blk 256

$1975 \mathrm{~N}$

Viosca Knoll Blk 294

933694 Alias for: (888259) M

1970 ON

$1992 \mathrm{~N}$

933698

Vlasca Knoll Blk 694

Viosca Knoll Blk 698

Alias for: (868255)

933780 Viosca Knoll Blk 780

LAOO 995 Offshore-Foderal

LAOO 995 Oftshoro-Foderal

LAOO 995 Ofishoro-Federal

LAOO 995 Ofishore-Federa

LAOD 995 Offshore-Federal

[A Blk 74 [BLOCK]

LAOO 995 Offshore-Foderal

LAOD 995 Ofishore-Foderal LAOO 995 OfIshoro-Foderal

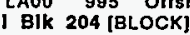

LAOO 995 Ofishore-Fodera LAO0 995 Offshore-Federa LA00 995 Oflshore-Fodera LAOO 995 OfIshore.Federa LAOO 995 OfIshore-Federa 995 Oltshore-Fodera LAO0 995 Offishore-Fodoral

933783 Viosca Knoll Blk 783 LAOO 995 Olishore-Federal LAOD 995 Oflshore-Federal

$1964 N$

033814 Viosca Knoll B/k 814 Alias for: (870308) M

LAOO 995 Ofishoro.Foderal

A BIK 259 [BLOCK]

A Blk 255 [BLOCK]

$1990 N$

933817

Vlosca Knoll Blk 817

SA BIk 308 [BLOCK]

LAOO 995 Offshore-Fadoral
LAOK 308 [BLOCK] $\begin{array}{lll}\text { Viosea Knoll Blk } 826 & \text { LAOO } & 995 \text { Offshore-Fedoral }\end{array}$ LAlias for: (933825) Vlosea Knoll BlK 825 [BLOCK]
[BL Offshor

933862 Viosca Knoll B1k 862 LAOO 995 Olfshore-Federa

Viosca Knoll BJk 864 LAOO 995 Olfshore-Federa

933869 Viosca Knoll BlK 869 LA00 995 Olfshore-Federa

933873 Alias for: (933825) Vlosca Knoll Blk 825 (BLOCK)

LAOOO 995 Offshoro-Fodera Viosca Knall BIK B99
Alias for: (911062) South Pass EA BA BIK 62 [BLOCK]

$1989 \mathrm{~N}$

$\begin{array}{ll}1976 & N \\ 1964 & N\end{array}$

933900 Viosca Knoll BIK 900 LA00 995 Ofishore-Fodora
Alias for: (911062) South Pass EA BIK 62 [BLOCK]

933912 Viosce Knoll BIK 912 LA00 995 Olfshore-Federal

933915 Alias for: (833956) Vlosca Knoll Blk 956 [BLOCK]

LAOO 995 Oifshore-Federa

$\begin{array}{llll}933956 & \text { Viosca Knoll Blk 956 } & \text { LAOO } 995 & \text { Offshore-Foderal } \\ \text { 933957 Viosce Knoll BIK 957 } & \text { LAOO } 995 \text { Offshore-Federal }\end{array}$

$1993 N$

$1977 \mathrm{~N}$ Alias tor: (933956) Vlos

1966 ON

$1980 \mathrm{OA}$

$1982 \mathrm{~N}$

933989 Viosca Knoll BIK 989 LAOD 995 Olfshora-Fodora

933990 Alias for: (933990) Vlosea Knoll Blk 990 [BLOCK]

LAOO 995 Olshore-Federal

$\begin{array}{lllll}\mathbf{9 3 5 0 1 6} & \text { West Cameron Blk 16 } & \text { LAOO } & 995 & \text { Olfshore-Federal } \\ \mathbf{9 3 5 0 1 7} & \text { West Cameron Blk 17 } & \text { LAOO } & \mathbf{9 9 5} & \text { Olishore-Federal }\end{array}$

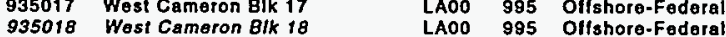

Wast Cameron Blk 18
Alias for: (935017) Wost Cameron BIK 17 [BLOCK]

$1972 \mathrm{~N}$

935020 Wost Cameron Blk 20 LAOO 995 Olishore-Fedoral

935021 Alias for: (935045) West Cameron Bik 45 [BLOCK] Alias tor: (935045) Wost Cameron Blk 45 [BLLCKK]
West Cameron BIK 22

West Cameron BIK 22 LAOO 995 Olfshore-Foderal

935028 Alias for: (035O45) Wost CamarOA BNk 45 [BLOCK]

$\begin{array}{lll}\text { West Cameron Blk 28 } & \text { LA00 } 995 \text { Olfshore-Federal } \\ \text { West Cameron Blk 33 } & \text { LA00 } 995 \text { Offshore-Foderal }\end{array}$

1976

LA00 995 OffBhore-Fodera West Cameron Blk 34
Atias tor: (935066) West Cameron BIk 66 [BLOCK]

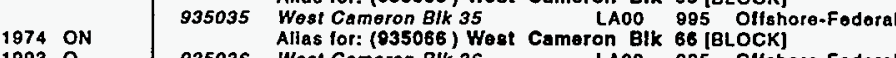

1993 O

Allas for: (935086) Weat Cameron Bik 66 [BLOCK]

West Cameron Blk 36 LAO 995 OIfsh
Alias lor: (935066) Wost Cameron Blk 66 [BLOCK]

935040 Wast Cameron B1k 40

935041 Wost Cameron BIk 41 LAOO 995 Olfshoro-Federal

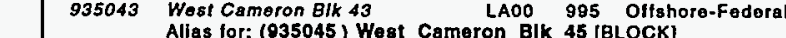

$1987 \mathrm{~N}$

935044

Alias for: (935045) Wost Cameron BIk 45 [BLOCK]
[B5045 $\begin{array}{lllll}935045 & \text { West Cameron Blk 45 } & \text { LAO0 } 995 & \text { Ollshore-Federal } \\ 935047 & \text { Wost Cameron Blk } 47 & \text { LAOO } 995 \text { Ollshora-Federal }\end{array}$

$1988 N$

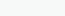

1975 ON
1975 ON

19930

LAOO 995 Ollshore-Federal

935049 Alias for: (935017) Wast Cameron BlK 17 [BLOCK]

W35053 Wast Cameron Blk 49 LA00 995 Offshoro-Foderal

Alias for: (935040) West Cameron Blk 49 (BLOCK)

Wast Cameron Blk 55 LA00 995 Offshore.Foderal

Alies for: (935045) Wost Camoron BIK 95 [BLOCK]
[BLOCK

$1987 \mathrm{~N}$

935057 West Csmeron Blk 57 Cameron Blk 45 [BLOCK]
Alias for: (935045) Wast Cameron BIK 45 [BLOCK]

$1982 \mathrm{~N}$

$1977 \mathrm{~N}$
$1993 \mathrm{~N}$

$1980 \mathrm{~N}$

$1990 \mathrm{~N}$

$1988 \mathrm{~N}$

$1980 \mathrm{~N}$

$1987 \mathrm{~N}$

$1990 \mathrm{~N}$

$1892 \mathrm{O}$

$1889 \mathrm{~N}$

$1989 \mathrm{~N}$

$1889 \mathrm{~N}$

$1985 \mathrm{~N}$

$1988 \mathrm{~N}$

$1994 N$

$1988 \mathrm{~N}$

$1985 \mathrm{~N}$

$1893 \mathrm{~N}$

1988 ON

$1985 \mathrm{O}$

19760

$1988 \mathrm{~N}$

$1993 \circ$

$198 B \mathrm{~N}$

19810 


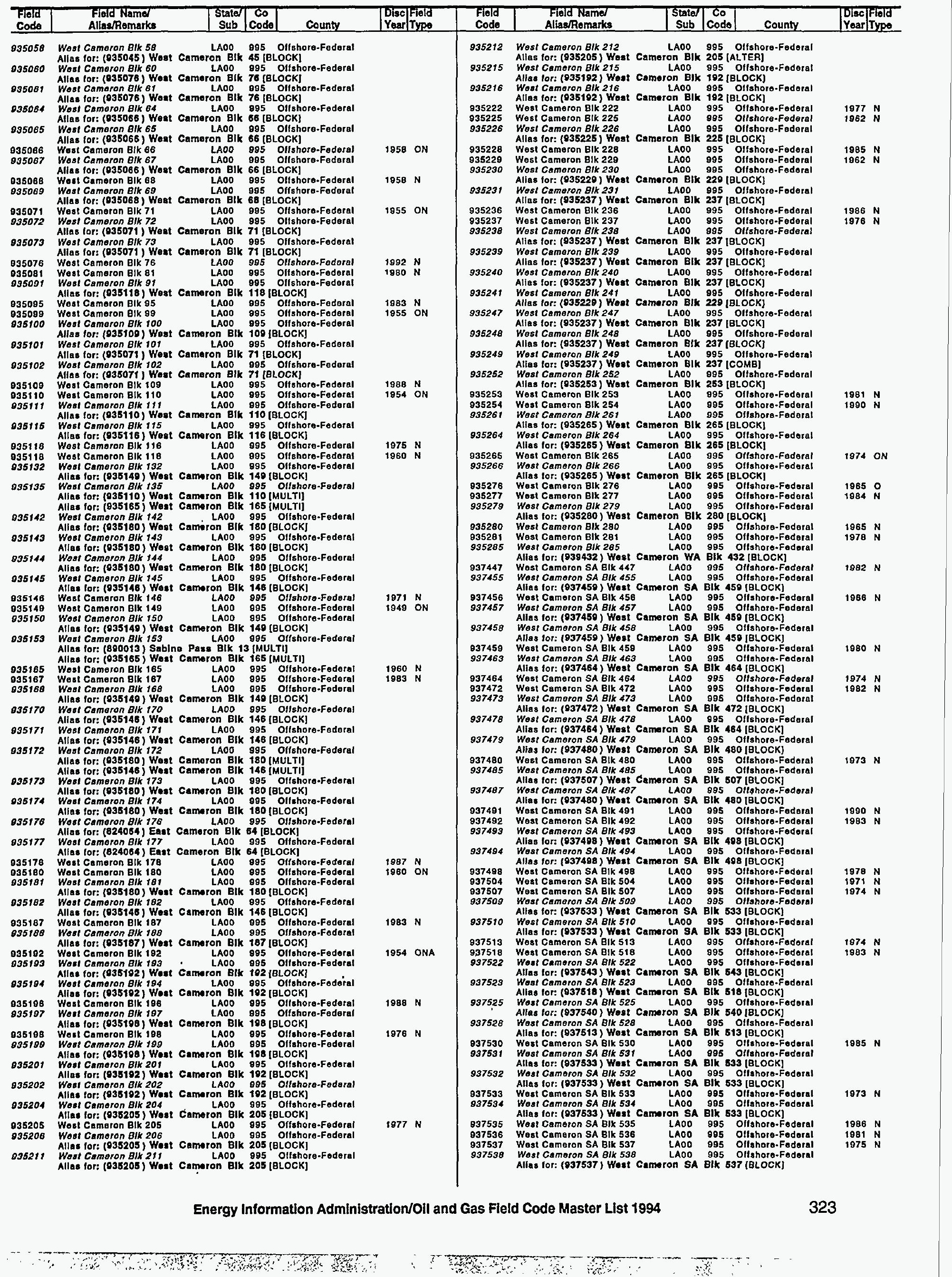




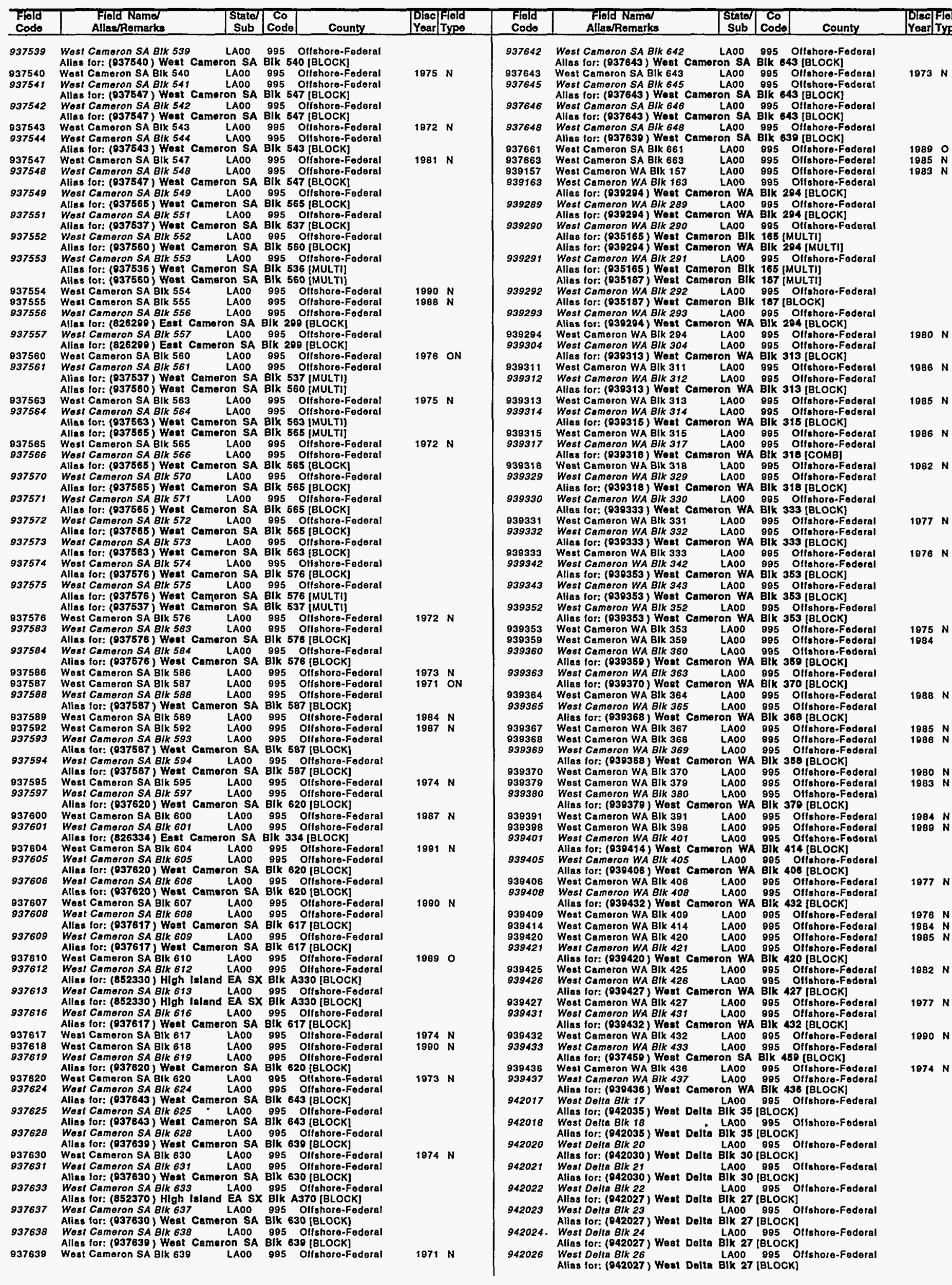




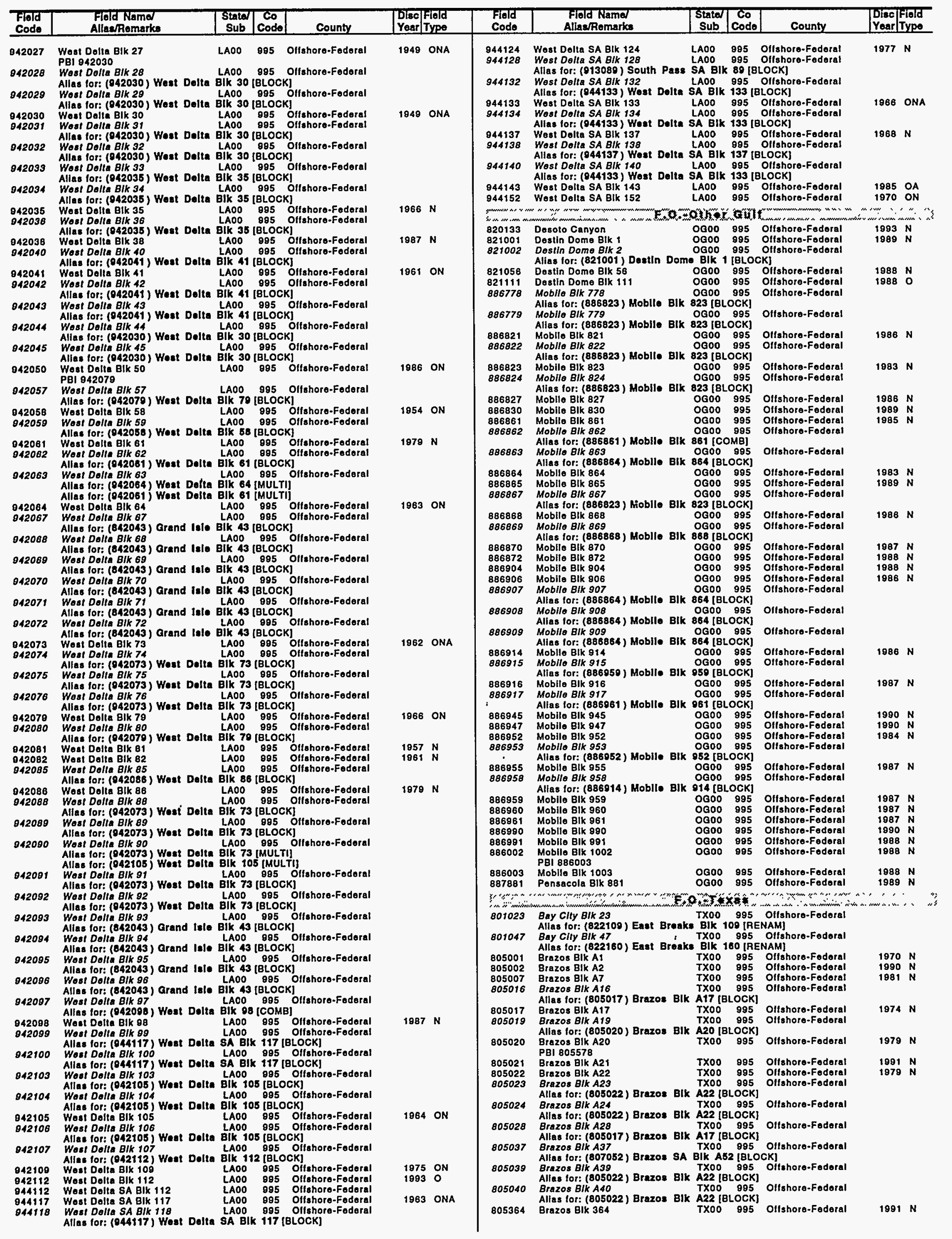




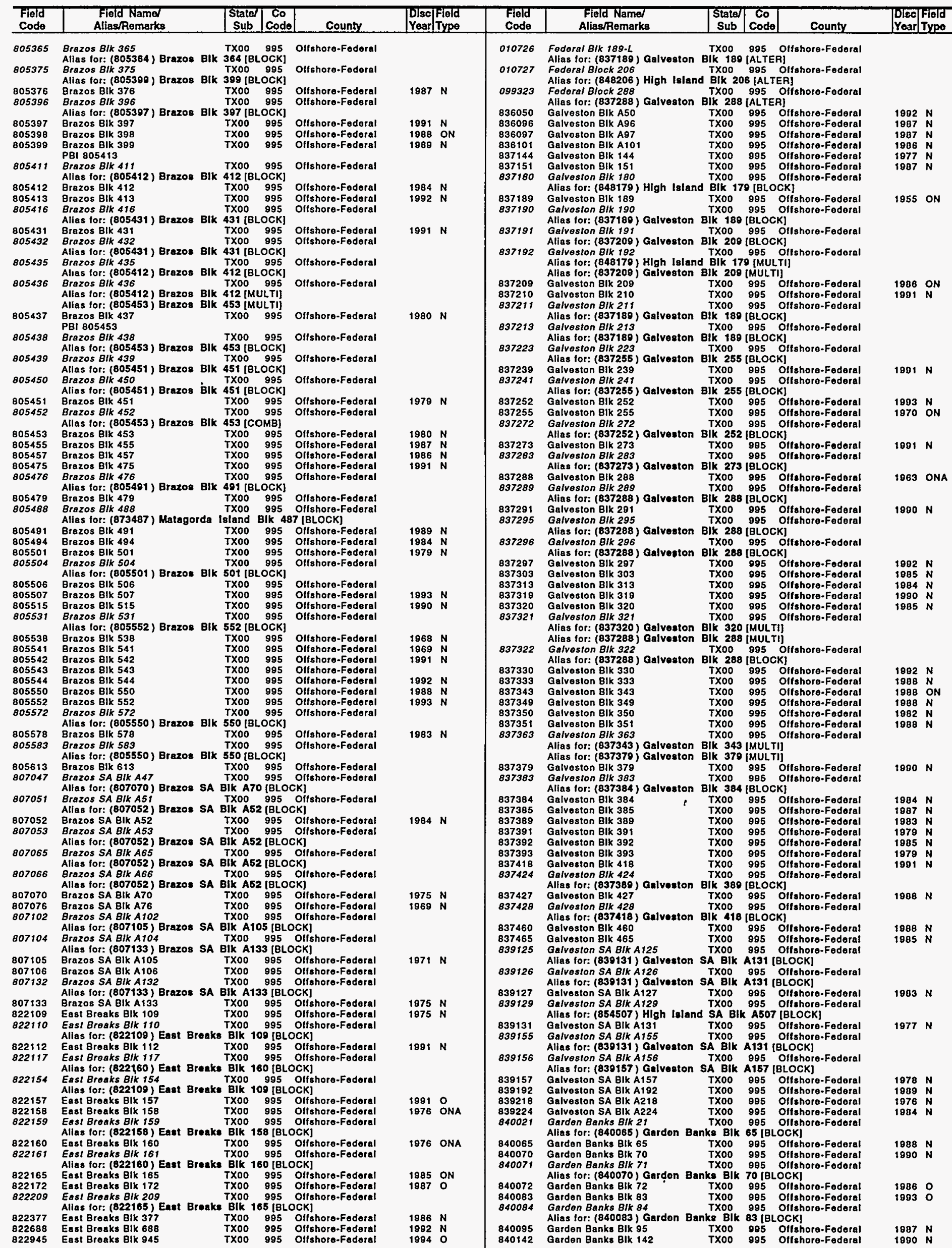




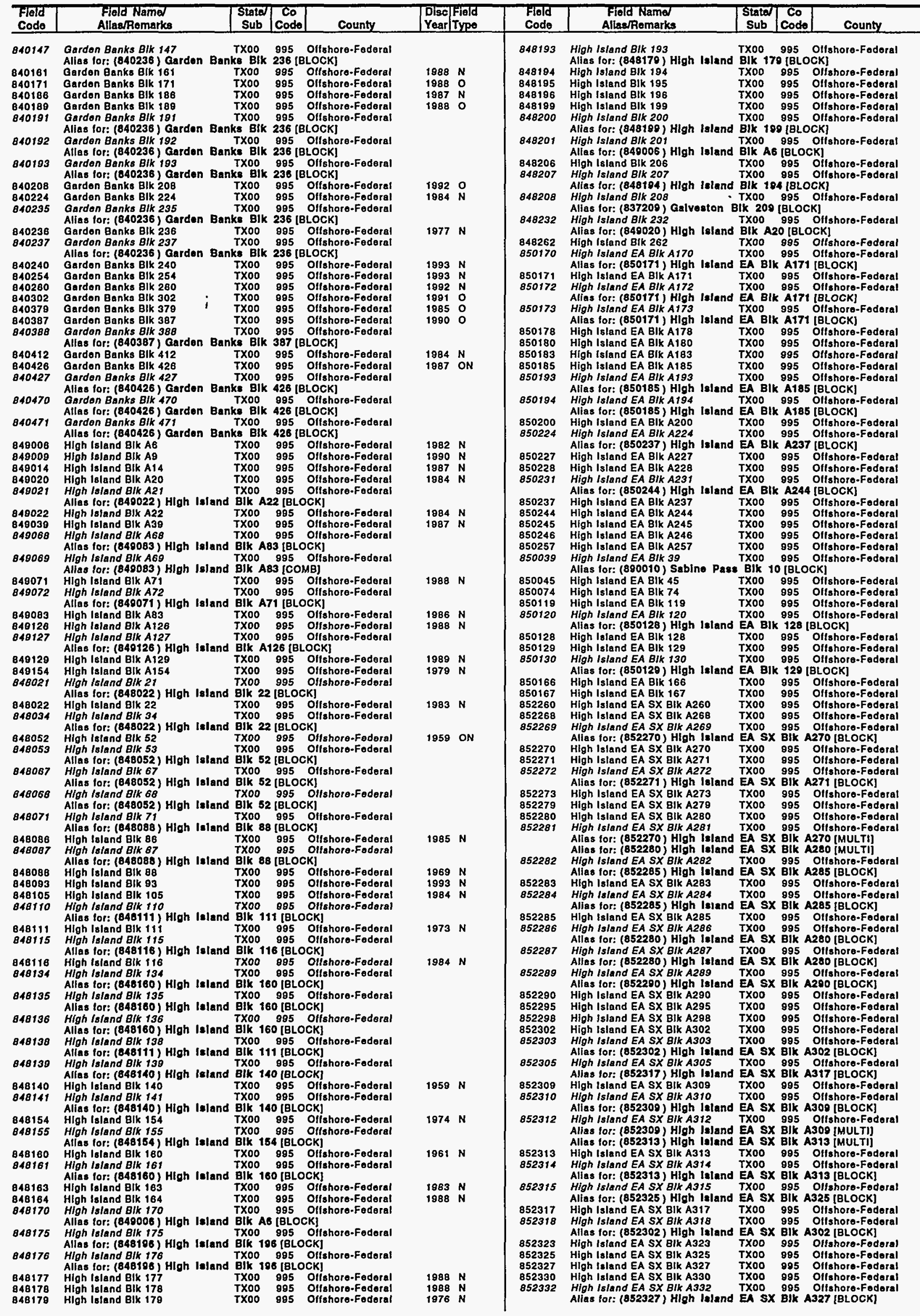
\begin{tabular}{|l|l|l|}
\hline Disc & Field \\
Year & Type \\
\hline
\end{tabular}

$1984 \mathrm{~N}$

$1980 \mathrm{~N}$

$1985 \mathrm{~N}$
$1980 \mathrm{~N}$

1968 ONA

$1890 \mathrm{~N}$

$1987 \mathrm{~N}$

$1986 \mathrm{~N}$

$1987 \mathrm{~N}$

$1984 N$

$1990 N$

$1988 \mathrm{~N}$

$1983 \mathrm{~N}$

$1891 \mathrm{~N}$
$1885 \mathrm{~N}$

19880

$1985 N$
$1983 N$

$1987 \mathrm{~N}$

$1968 \mathrm{~N}$

$1984 N$

$1987 N$
$1889 N$

1889
1988

$1975 \mathrm{~N}$

$1973 \mathrm{~N}$

$1974 N$

$1973 \mathrm{~N}$

$1978 N$

$1977 \mathrm{~N}$

$1980 \mathrm{~N}$

$1974 N$
$1975 N$

1974 N

$1976 \mathrm{~N}$

$1974 N$

$1974 \mathrm{~N}$

$1974 N$

$1973 \mathrm{~N}$ 


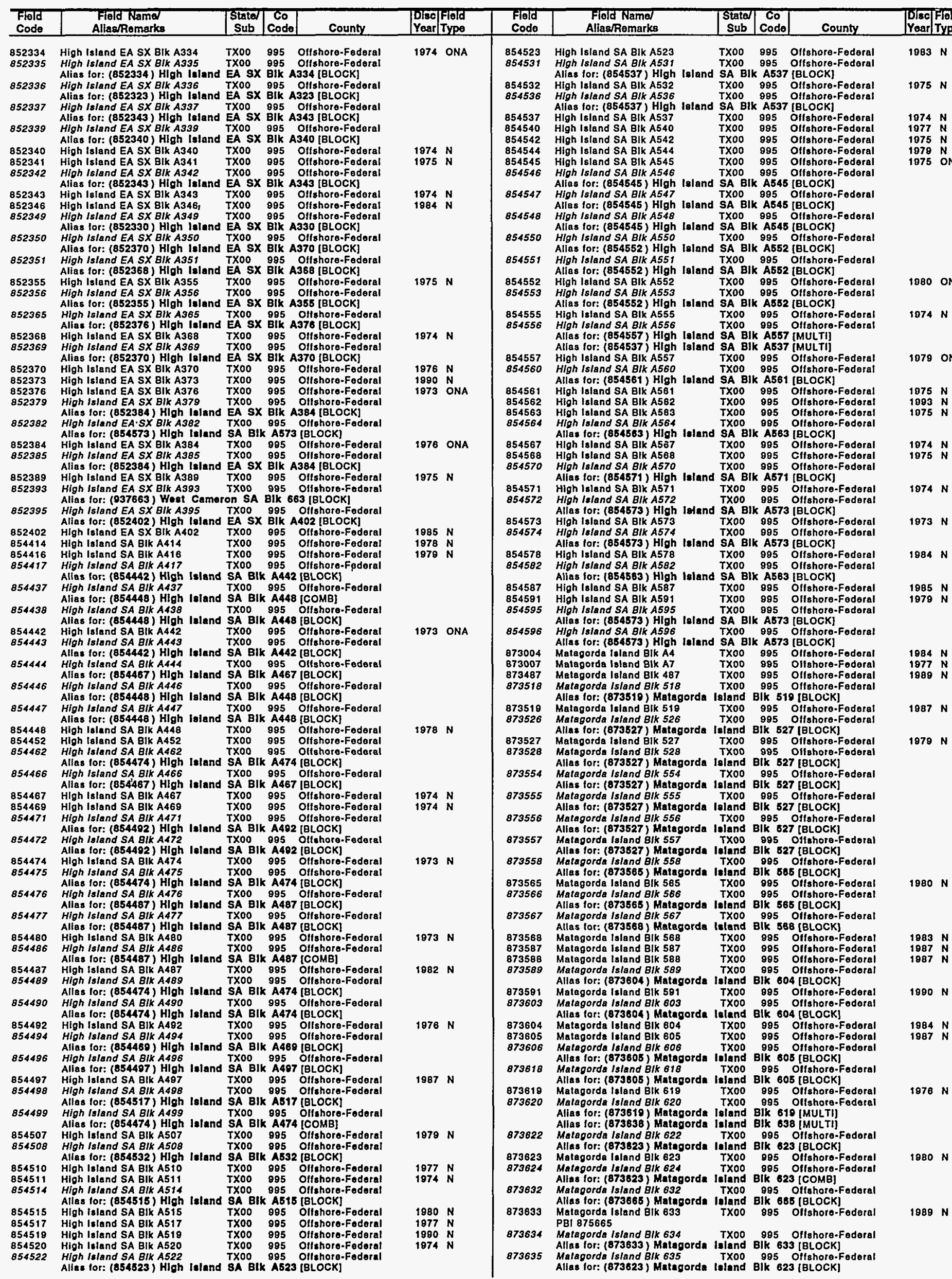


F.O.-TEXAS

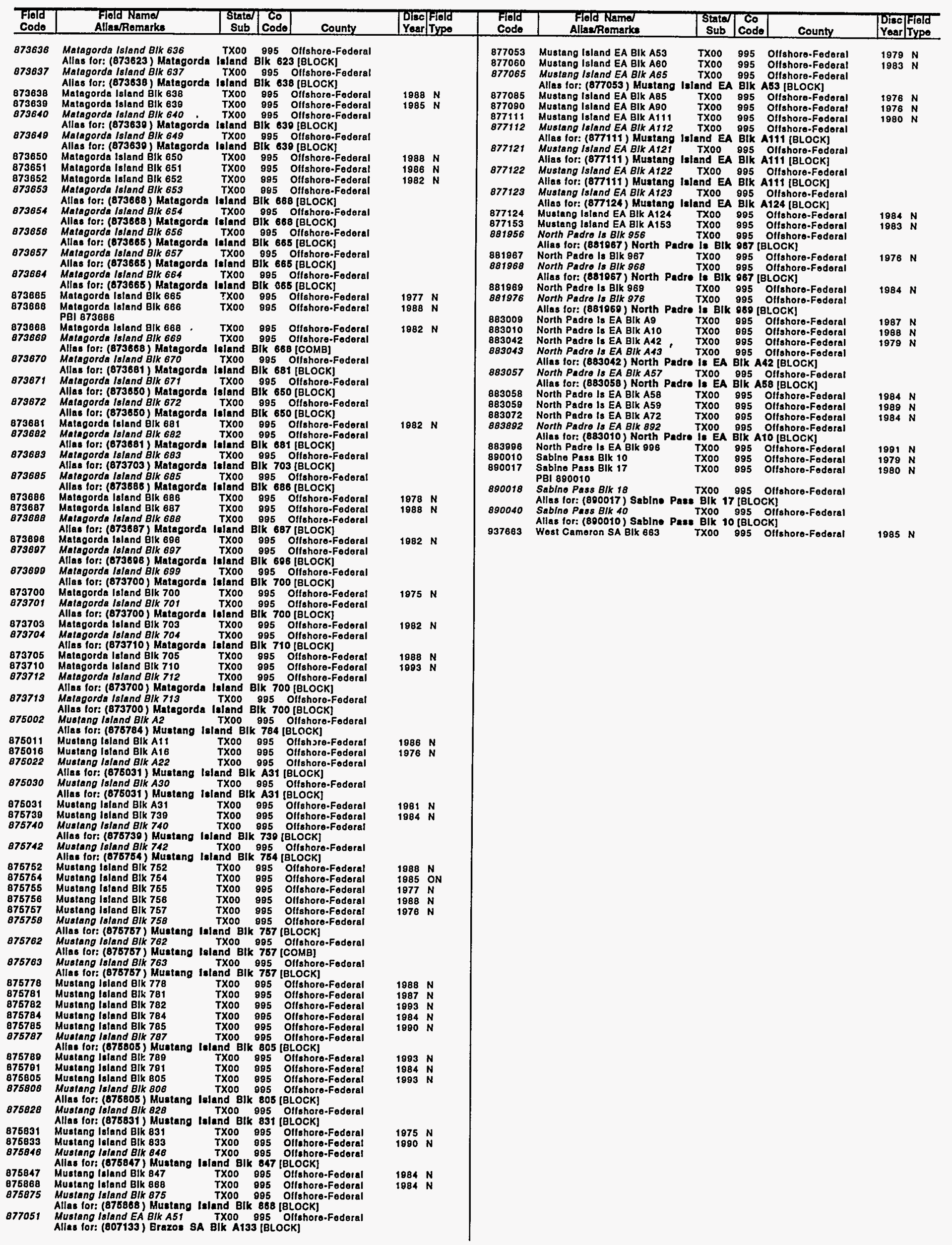





\section{Oil and Gas \\ Field Code Index}




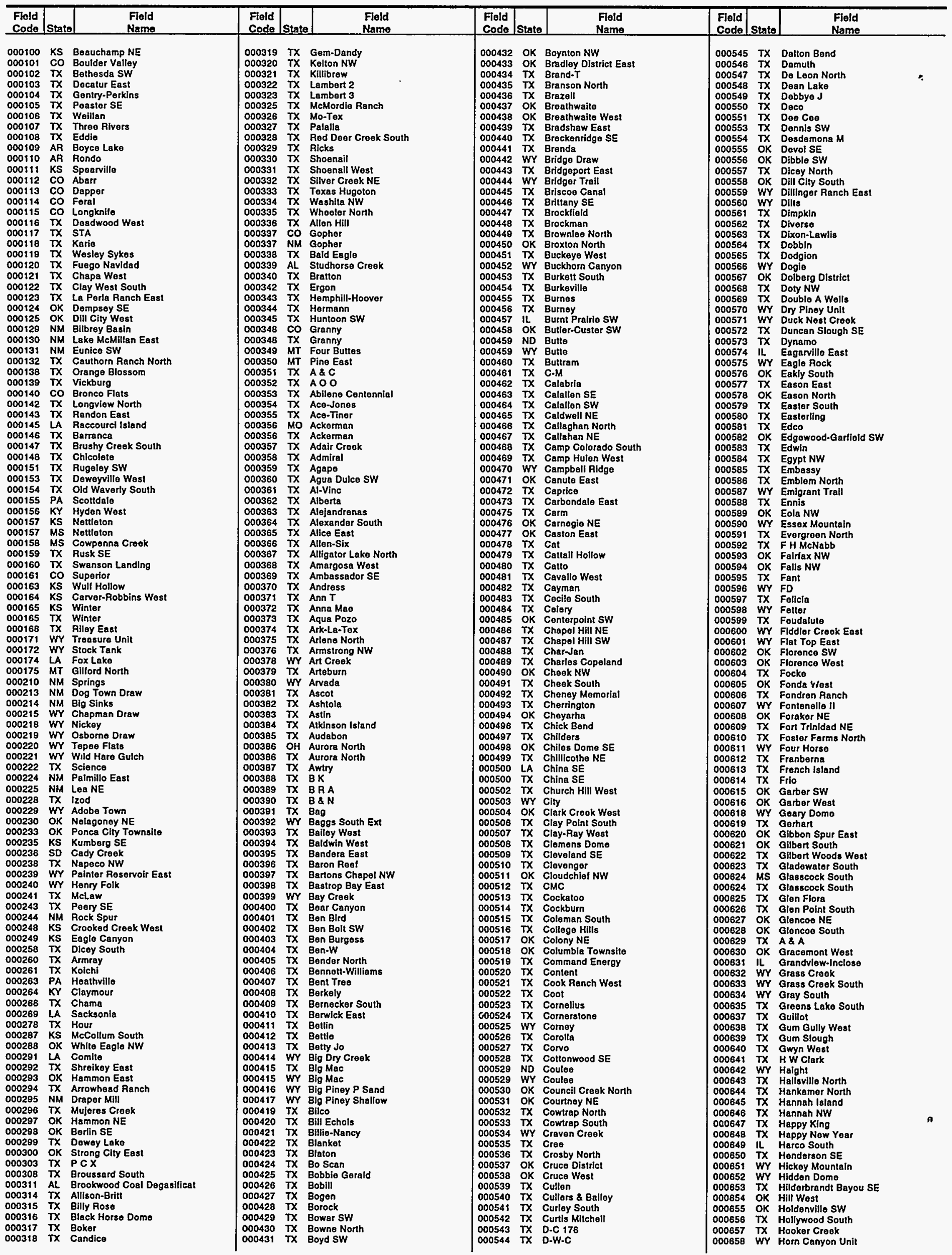




\begin{tabular}{|c|c|c|c|c|c|c|c|c|c|c|c|}
\hline \begin{tabular}{l|} 
Flold \\
Code
\end{tabular} & Stato & $\begin{array}{l}\text { Fiold } \\
\text { Name }\end{array}$ & $\begin{array}{l}\text { Fiold } \\
\text { Code }\end{array}$ & Stato & $\begin{array}{l}\text { Flold } \\
\text { Name }\end{array}$ & $\begin{array}{l}\text { Fiold } \\
\text { Code }\end{array}$ & Stato & $\begin{array}{l}\text { Fiold } \\
\text { Name }\end{array}$ & $\begin{array}{l}\text { Fiold } \\
\text { Code }\end{array}$ & State & $\begin{array}{l}\text { Field } \\
\text { Name }\end{array}$ \\
\hline 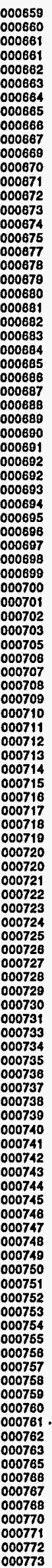 & 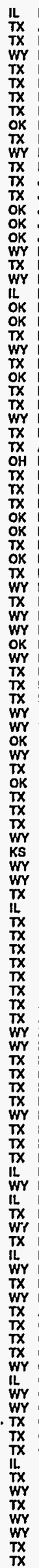 & 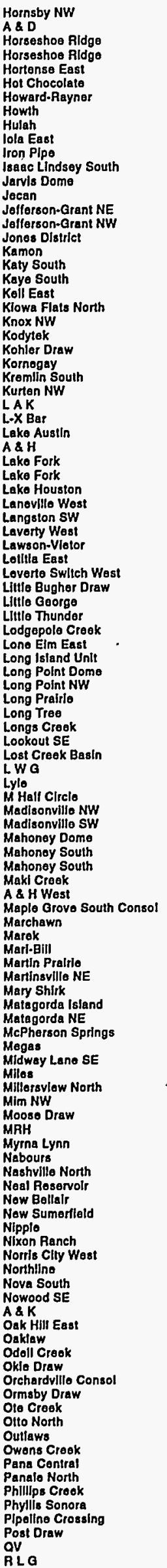 & 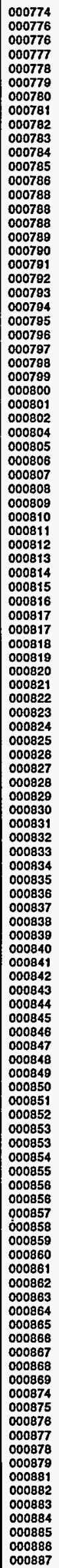 & 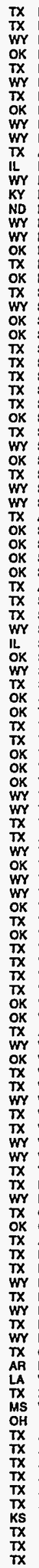 & 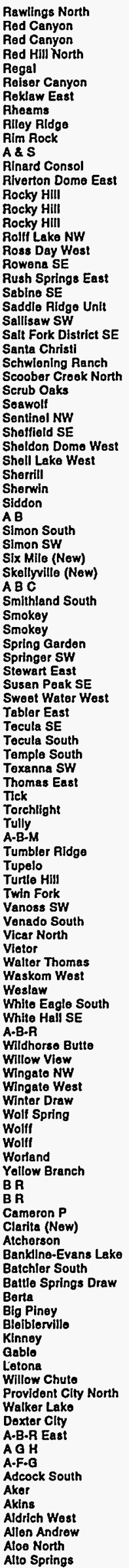 & 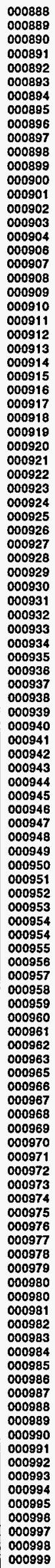 & 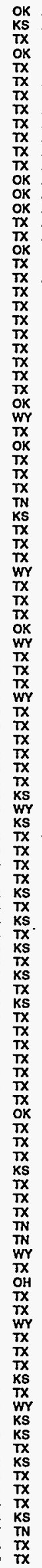 & 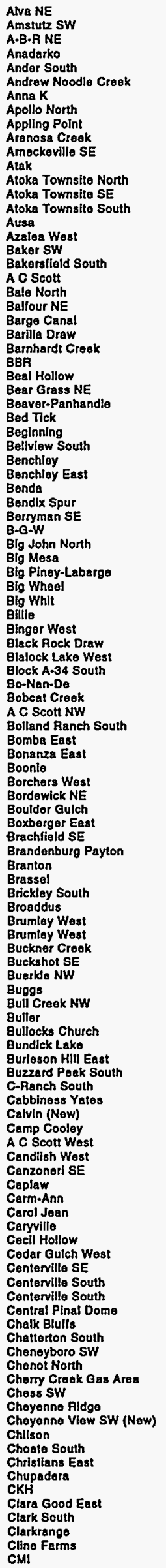 & 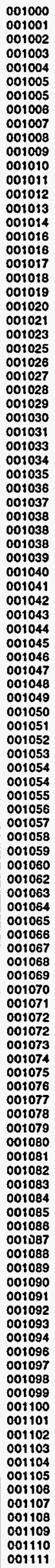 & 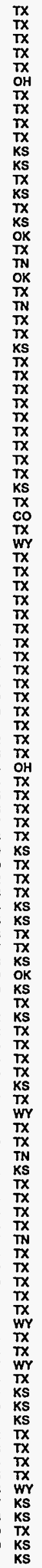 & 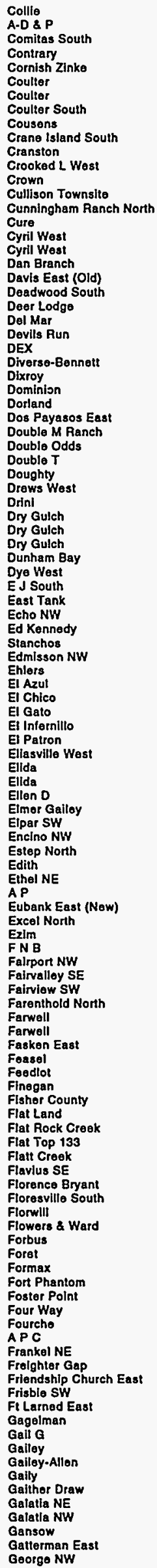 \\
\hline
\end{tabular}




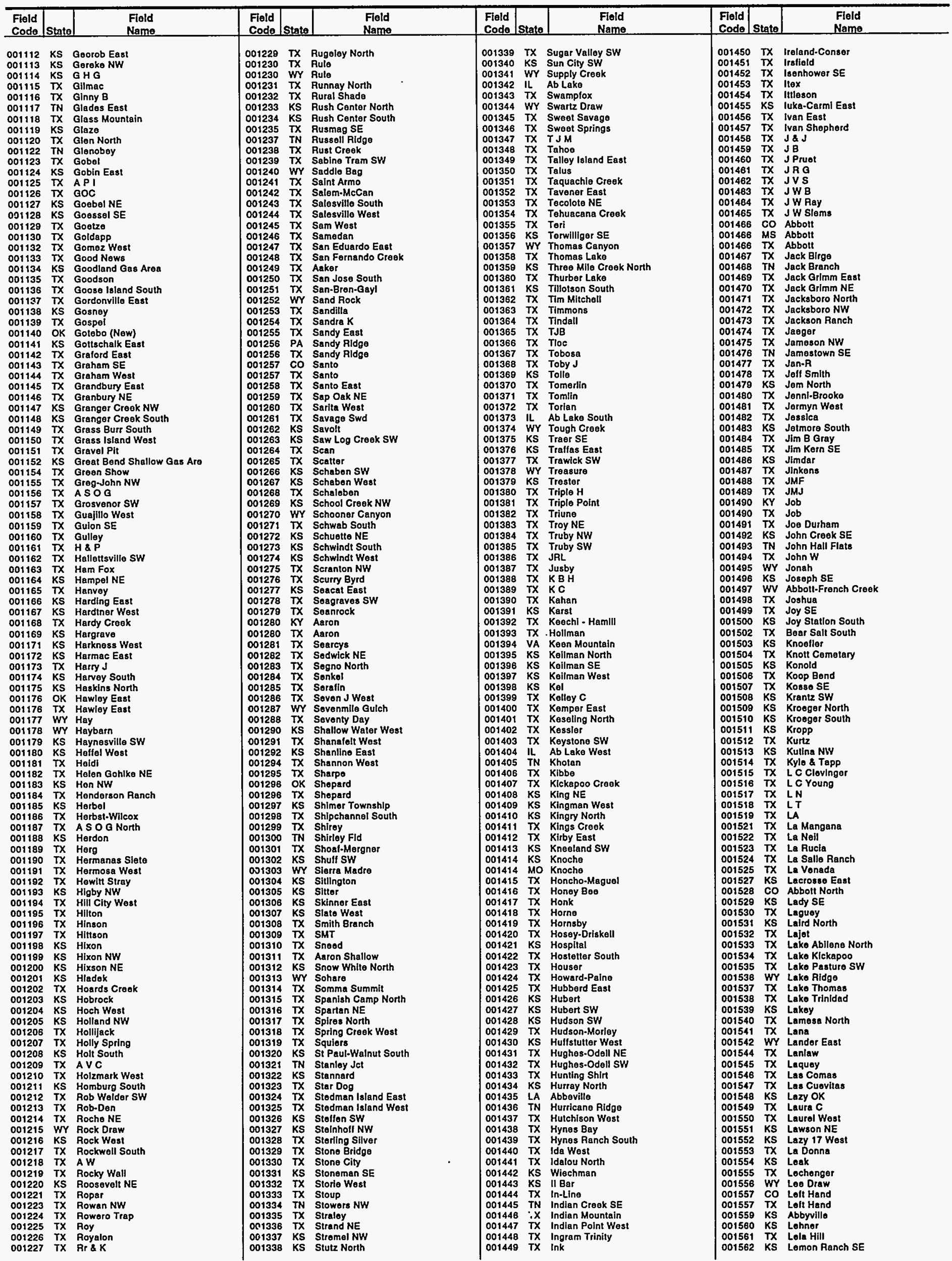




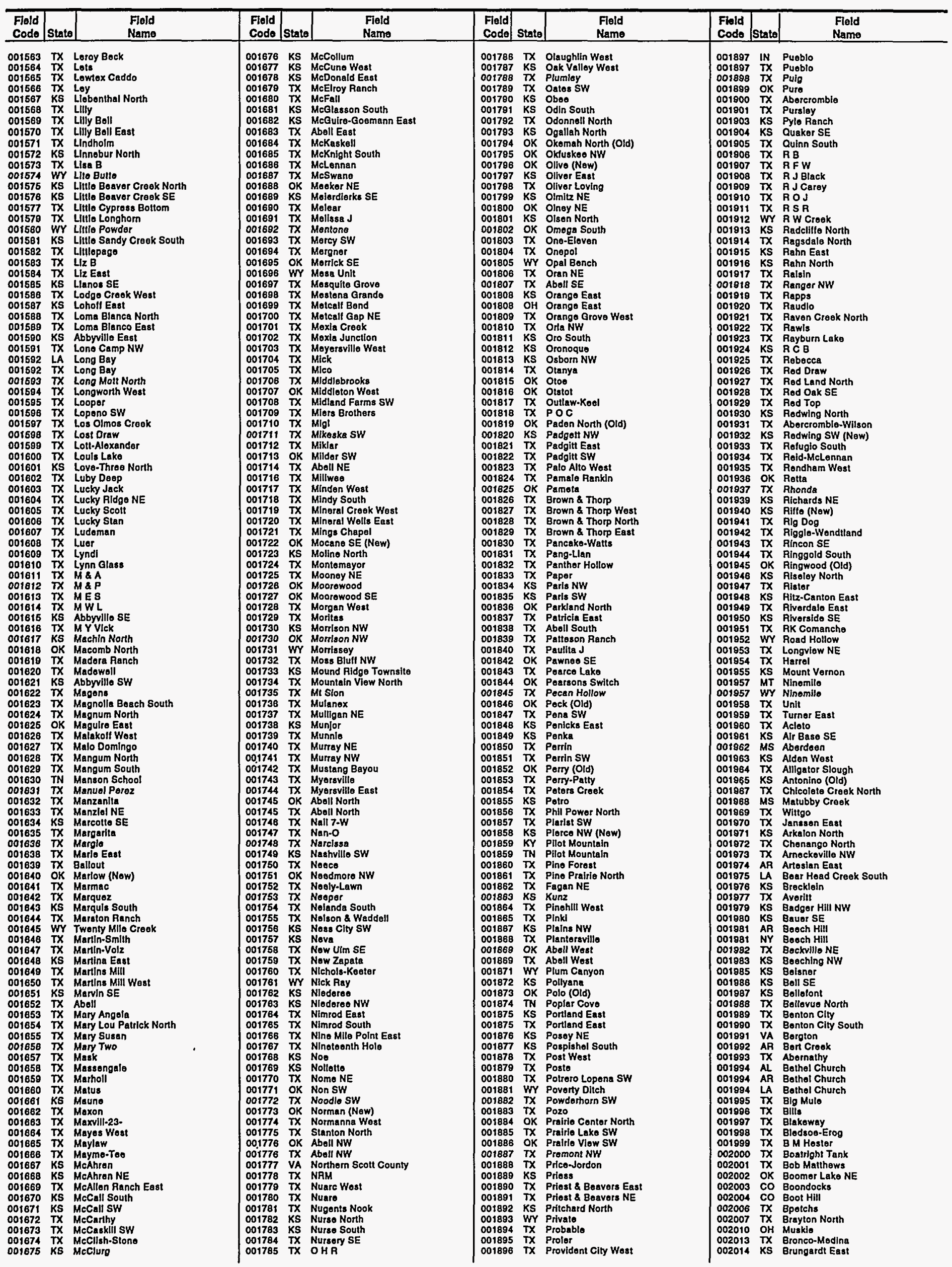




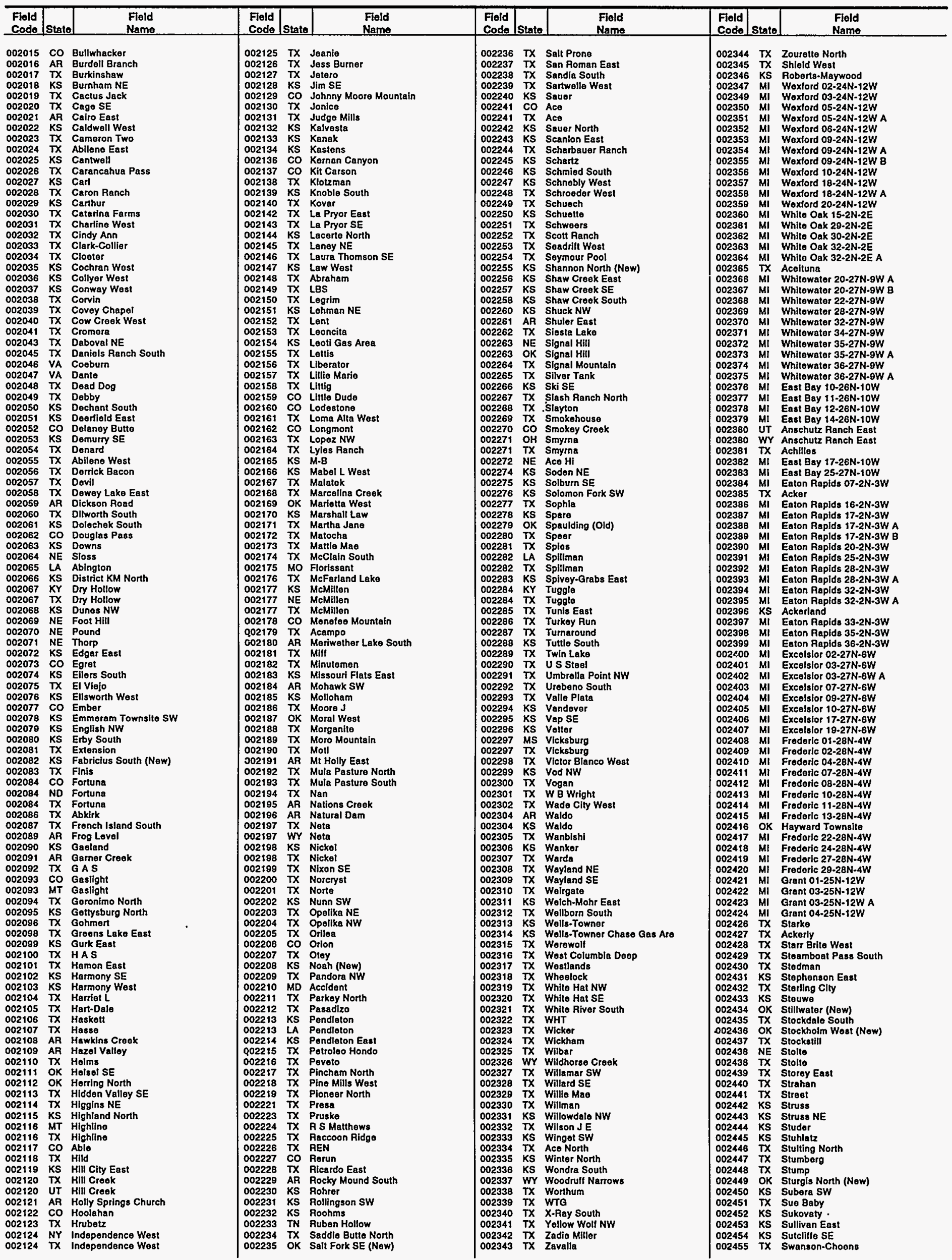




\begin{tabular}{|c|c|c|c|c|c|c|c|c|c|c|c|}
\hline $\begin{array}{l}\text { Frold } \\
\text { Code }\end{array}$ & State & $\begin{array}{l}\text { Floid } \\
\text { Name } \\
\end{array}$ & $\begin{array}{l}\text { Fiold } \\
\text { Codo }\end{array}$ & State & $\begin{array}{l}\text { Field } \\
\text { Name } \\
\end{array}$ & $\begin{array}{l}\text { Fiold } \\
\text { Codo }\end{array}$ & Stato & $\begin{array}{l}\text { Field } \\
\text { Namo } \\
\end{array}$ & $\begin{array}{l}\text { Flold } \\
\text { Codo } \\
\end{array}$ & State & $\begin{array}{l}\text { Fiold } \\
\text { Nemo }\end{array}$ \\
\hline 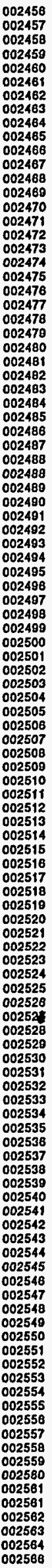 & 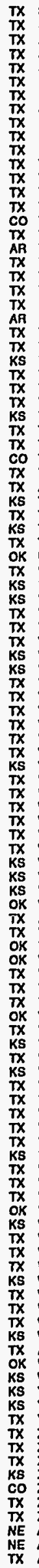 & 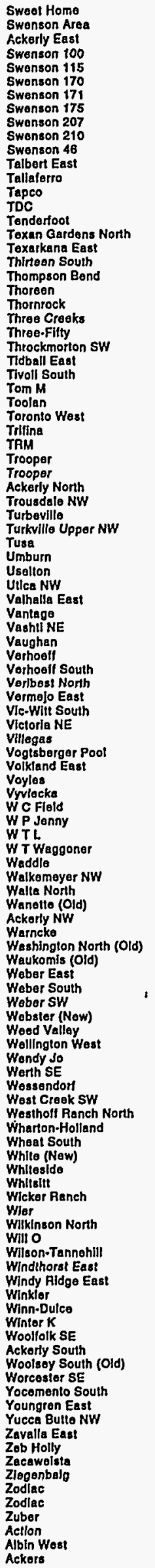 & 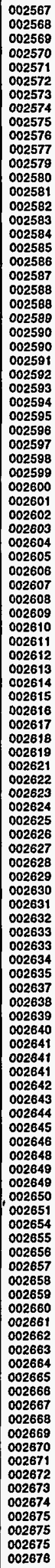 & 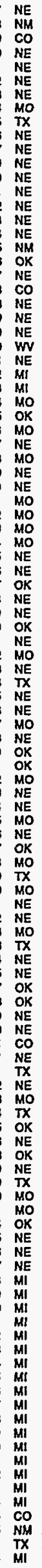 & 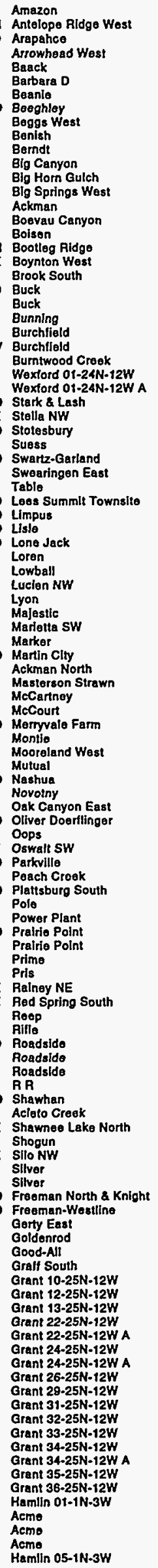 & 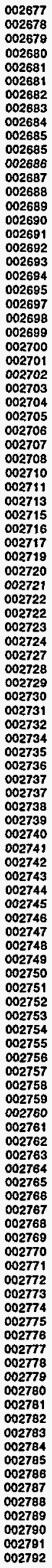 & 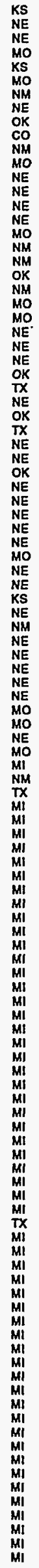 & 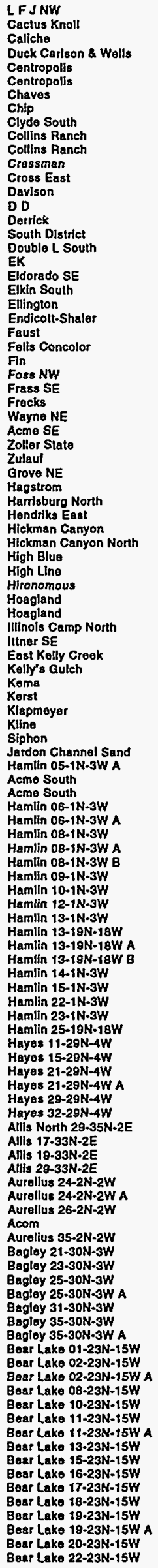 & 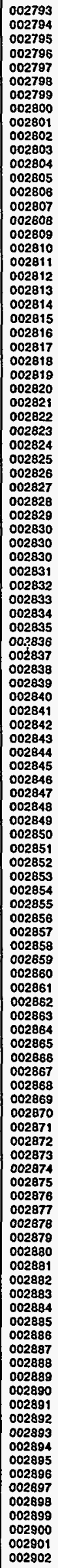 & 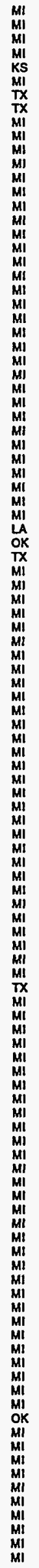 & 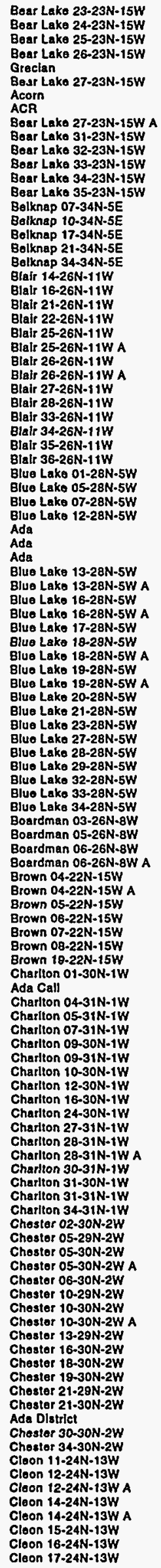 \\
\hline
\end{tabular}




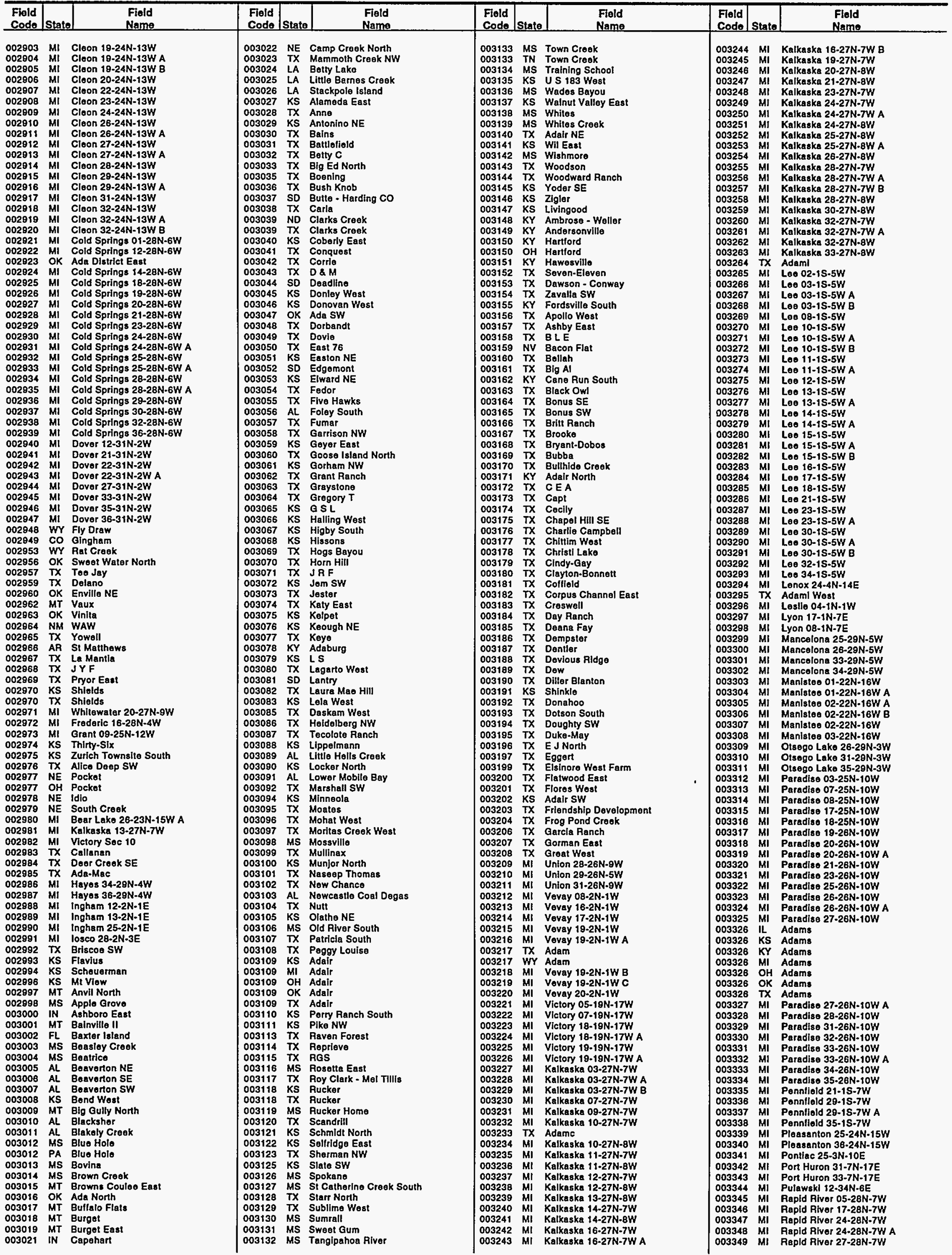




\begin{tabular}{|c|c|c|c|c|c|c|c|c|c|c|c|c|}
\hline $\begin{array}{l}\text { Fleld } \\
\text { Code }\end{array}$ & State & $\begin{array}{l}\text { Fiold } \\
\text { Name }\end{array}$ & & $\begin{array}{l}\text { Field } \\
\text { Code }\end{array}$ & State & $\begin{array}{l}\text { Field } \\
\text { Name }\end{array}$ & $\begin{array}{l}\text { Field } \\
\text { Codo }\end{array}$ & State & $\begin{array}{l}\text { Field } \\
\text { Name }\end{array}$ & \begin{tabular}{|l|} 
Fiold \\
Codo \\
\end{tabular} & State & $\begin{array}{l}\text { Fiold } \\
\text { Name } \\
\end{array}$ \\
\hline 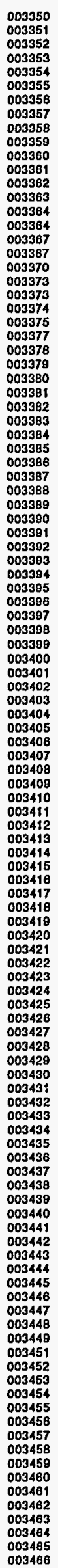 & 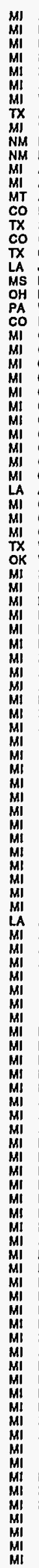 & 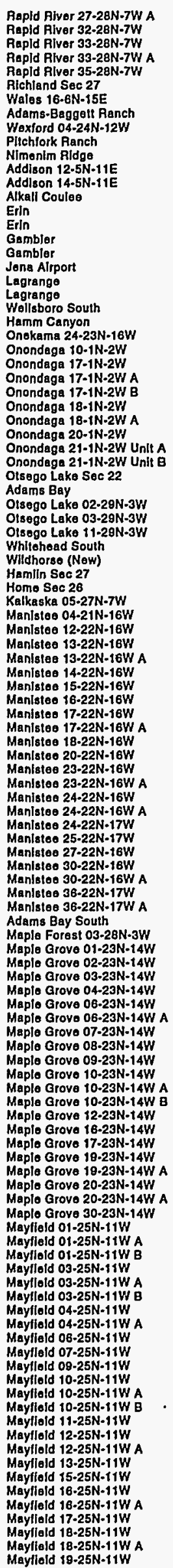 & . & 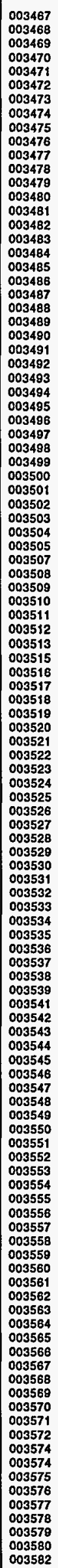 & 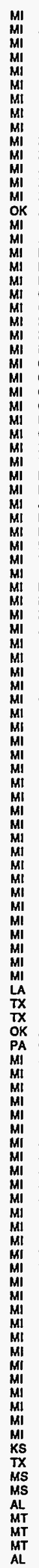 & 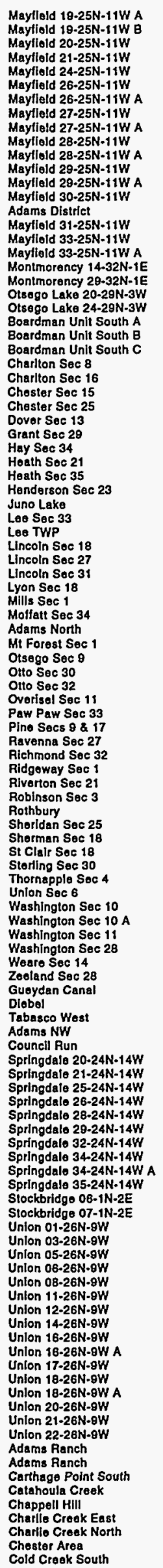 & 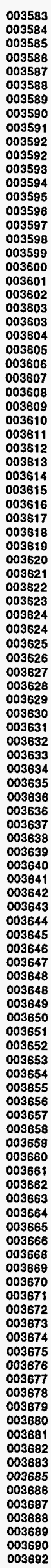 & 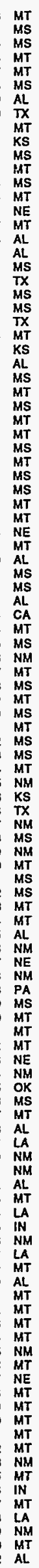 & 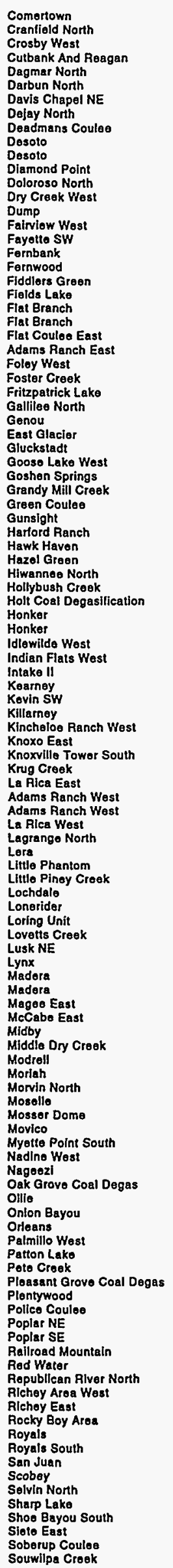 & 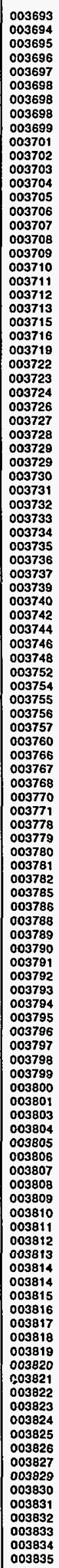 & 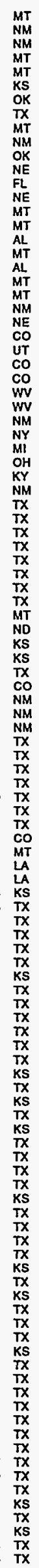 & 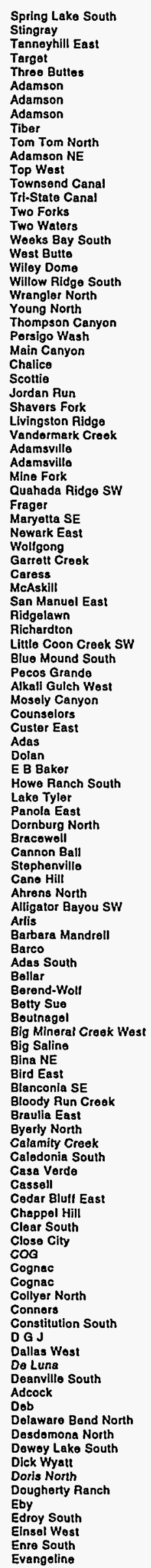 \\
\hline
\end{tabular}




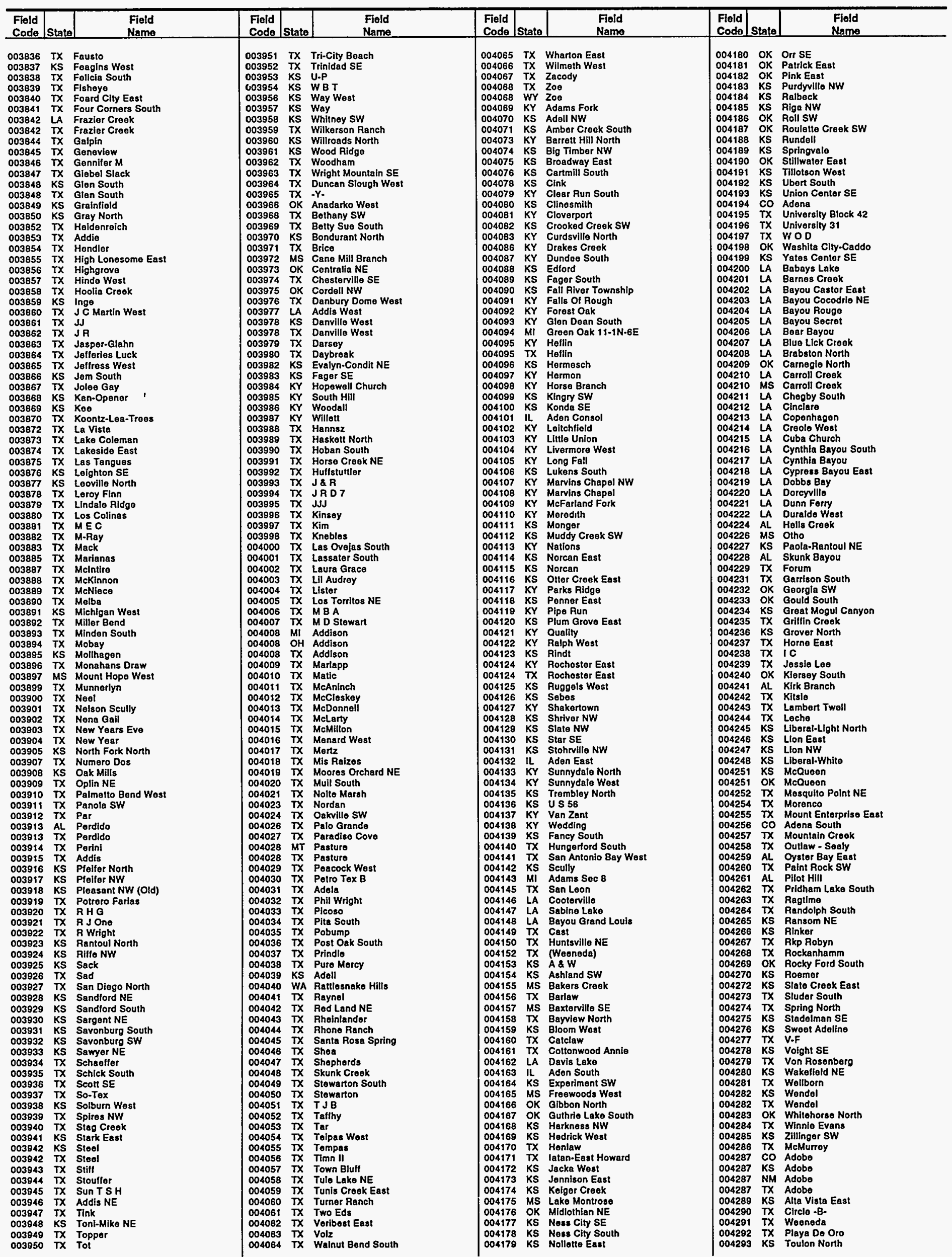


004736

\begin{tabular}{|c|c|c|c|c|c|c|c|c|c|c|c|}
\hline $\begin{array}{l}\text { Flold } \\
\text { Code }\end{array}$ & State & $\begin{array}{l}\text { Flold } \\
\text { Name }\end{array}$ & $\begin{array}{l}\text { Flold } \\
\text { Codo }\end{array}$ & State & $\begin{array}{l}\text { Fiold } \\
\text { Namo }\end{array}$ & \begin{tabular}{l|} 
Flold \\
Code
\end{tabular} & State & $\begin{array}{c}\text { Feld } \\
\text { Name } \\
\end{array}$ & $\begin{array}{l}\text { Flold } \\
\text { Code }\end{array}$ & State & $\begin{array}{l}\text { Fleid } \\
\text { Namo }\end{array}$ \\
\hline 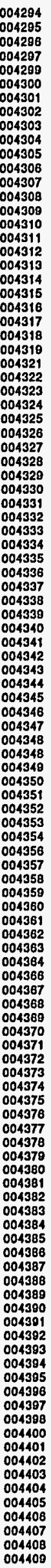 & 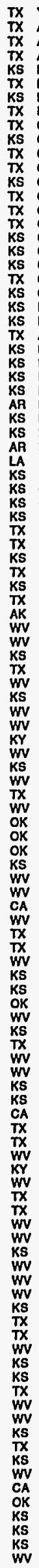 & 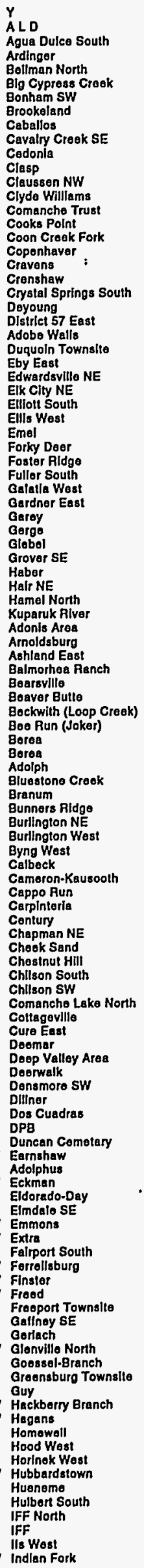 & 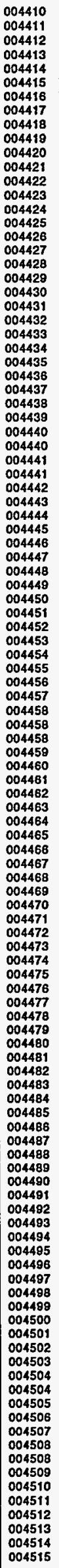 & 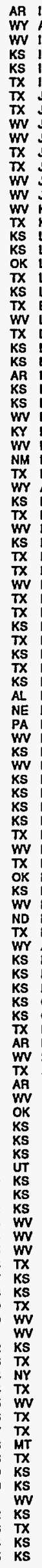 & 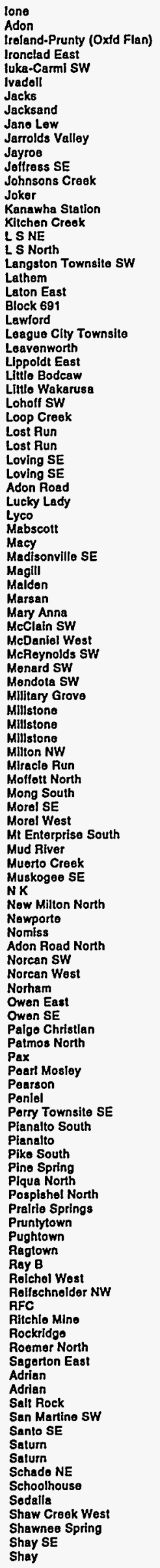 & 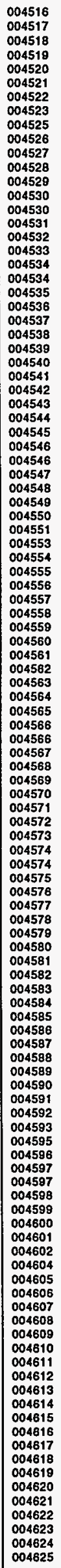 & 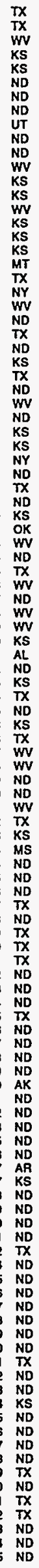 & 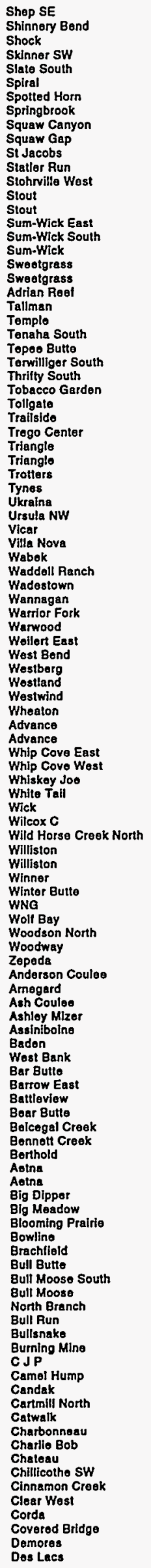 & 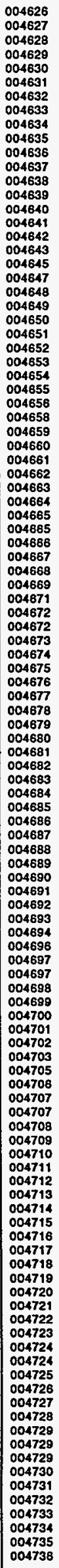 & 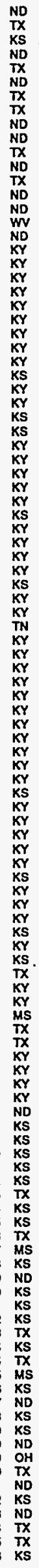 & 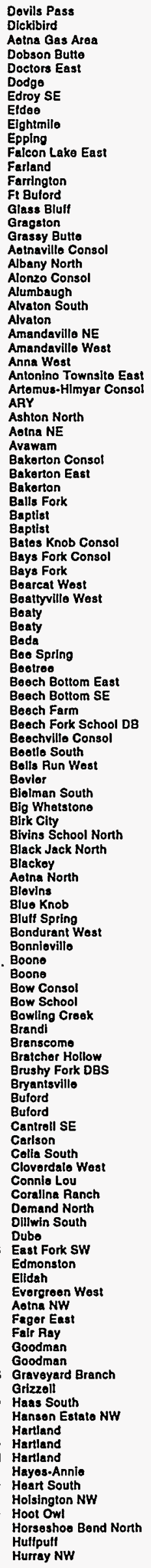 \\
\hline
\end{tabular}




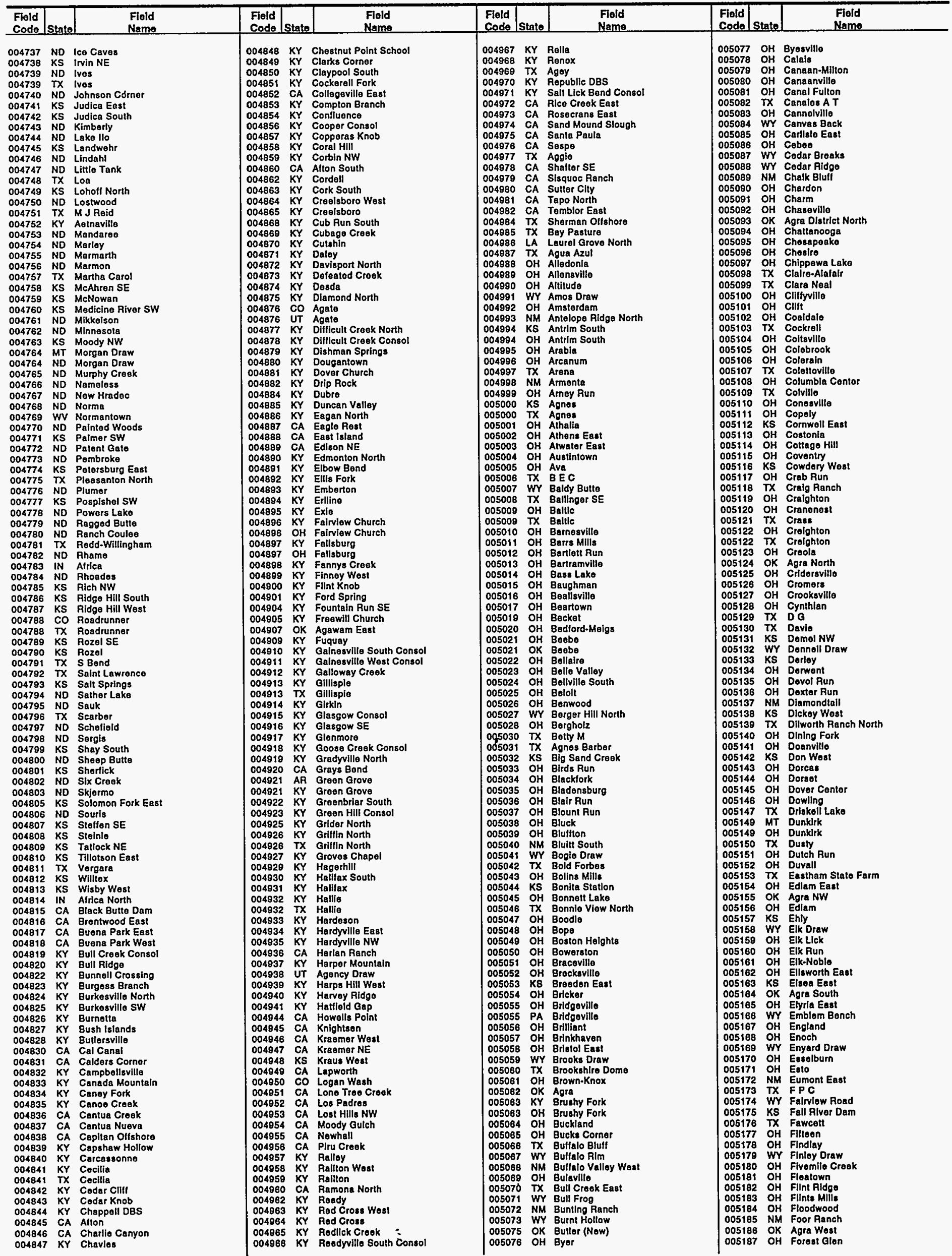




\begin{tabular}{|c|c|c|c|c|c|c|c|c|c|c|c|}
\hline $\begin{array}{l}\text { Fiold } \\
\text { Code }\end{array}$ & State & $\begin{array}{l}\text { Field } \\
\text { Name }\end{array}$ & $\begin{array}{l}\text { Fiold } \\
\text { Code }\end{array}$ & State & $\begin{array}{l}\text { Fleld } \\
\text { Name } \\
\end{array}$ & $\begin{array}{l}\text { Field } \\
\text { Coda } \\
\end{array}$ & Stato & $\begin{array}{l}\text { Field } \\
\text { Namo } \\
\end{array}$ & $\begin{array}{l}\text { Fiold } \\
\text { Codo }\end{array}$ & Stato & $\begin{array}{l}\text { Fleld } \\
\text { Name }\end{array}$ \\
\hline 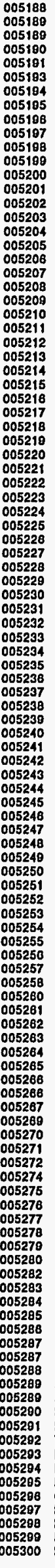 & 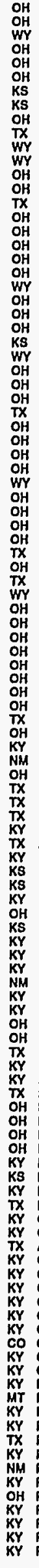 & 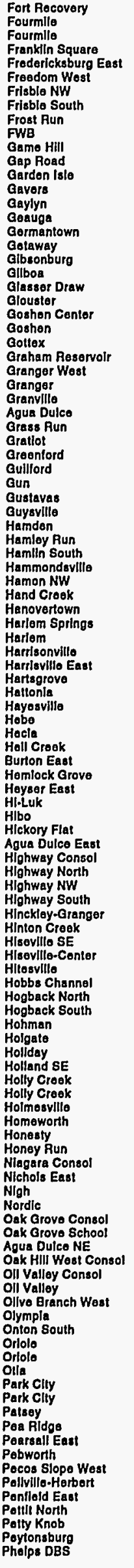 & 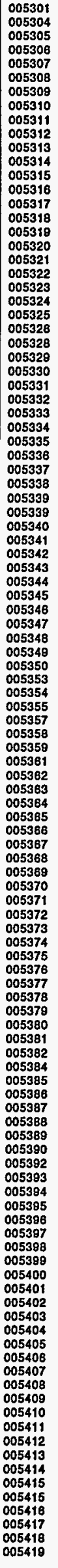 & 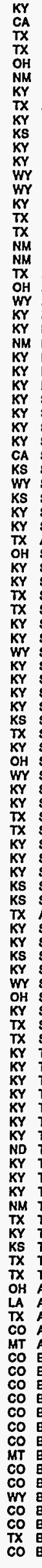 & 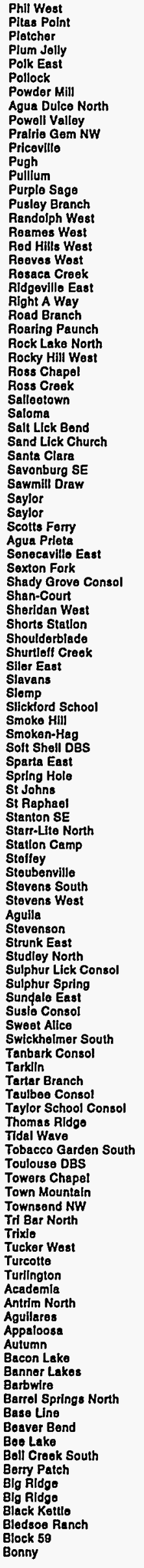 & 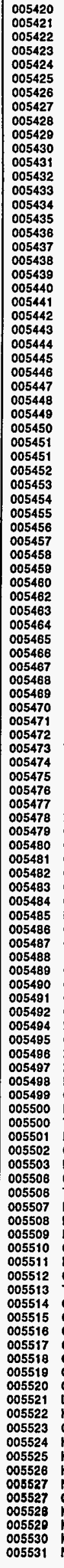 & 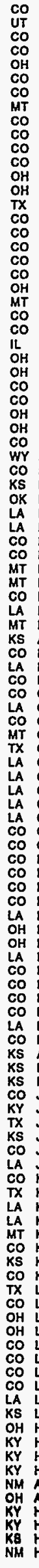 & 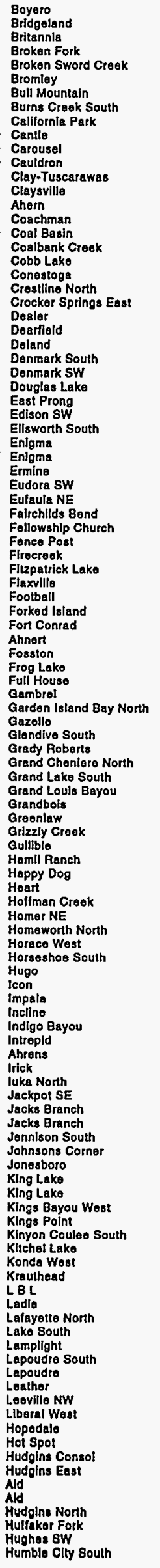 & 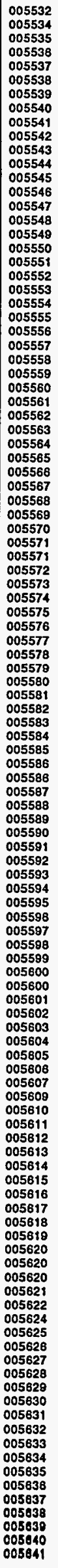 & 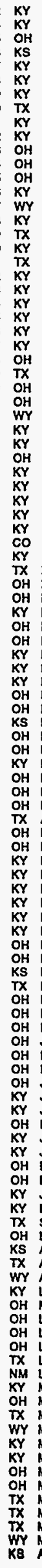 & 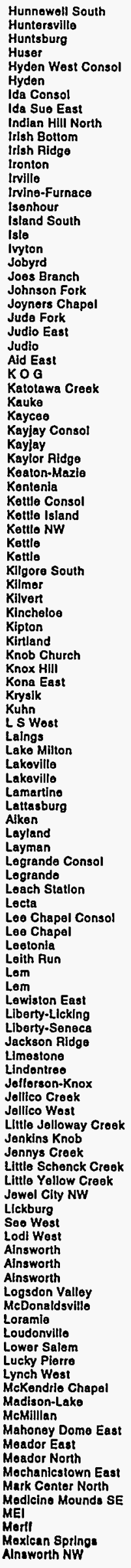 \\
\hline
\end{tabular}




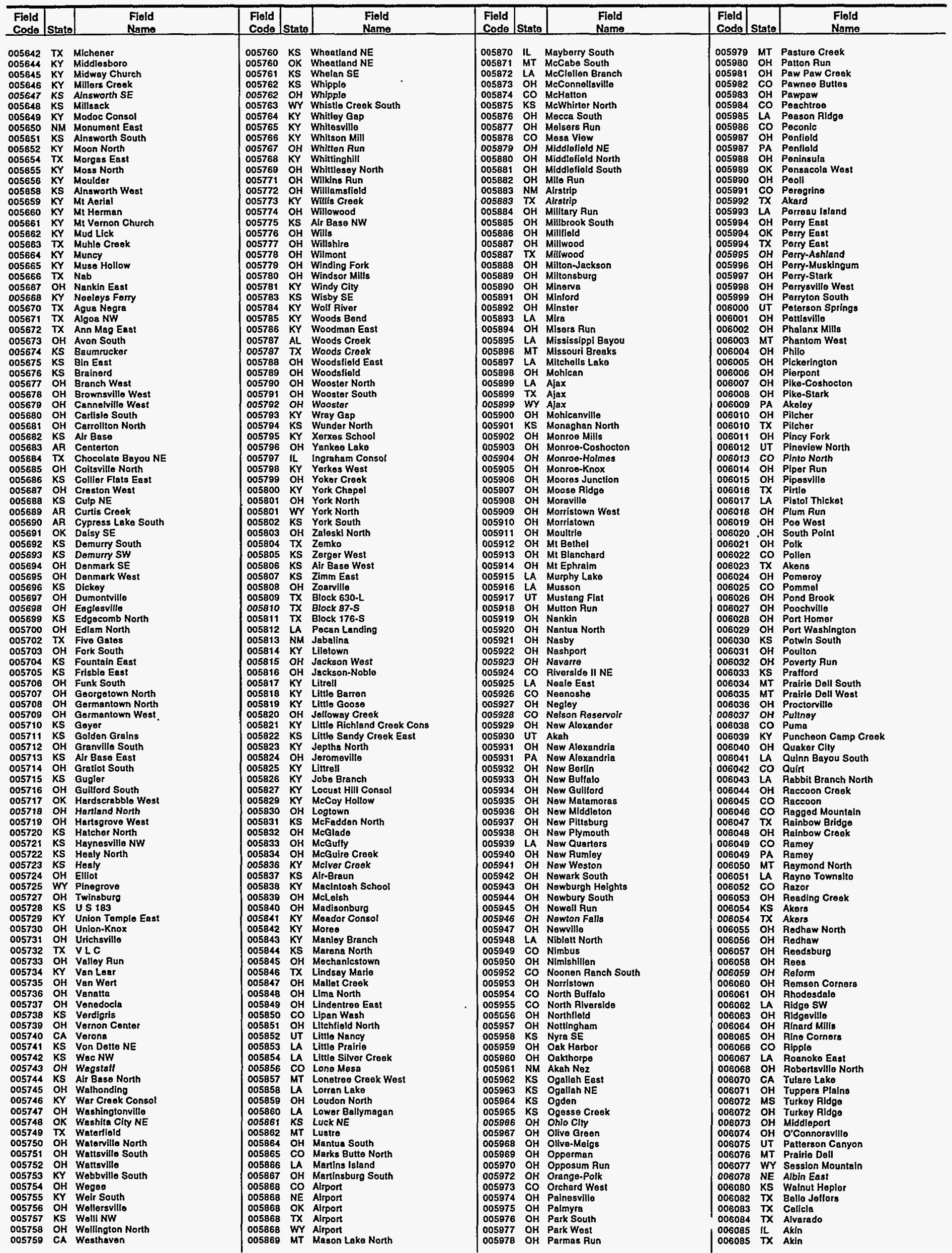




\begin{tabular}{|c|c|c|c|c|c|c|c|c|c|c|c|}
\hline $\begin{array}{l}\text { Flold } \\
\text { Code }\end{array}$ & State & $\begin{array}{l}\text { Fiald } \\
\text { Name }\end{array}$ & $\begin{array}{l}\text { Fiold } \\
\text { Code }\end{array}$ & State & $\begin{array}{l}\text { Field } \\
\text { Name }\end{array}$ & $\begin{array}{l}\text { Fiold } \\
\text { Code }\end{array}$ & State & $\begin{array}{l}\text { Fiold } \\
\text { Name }\end{array}$ & $\begin{array}{l}\text { Fleld } \\
\text { Code } \\
\end{array}$ & State & $\begin{array}{l}\text { Fiold } \\
\text { Name }\end{array}$ \\
\hline 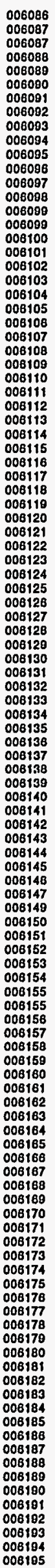 & 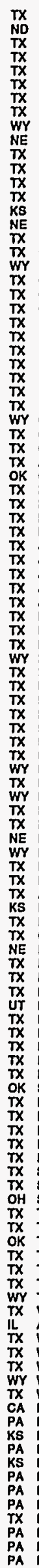 & 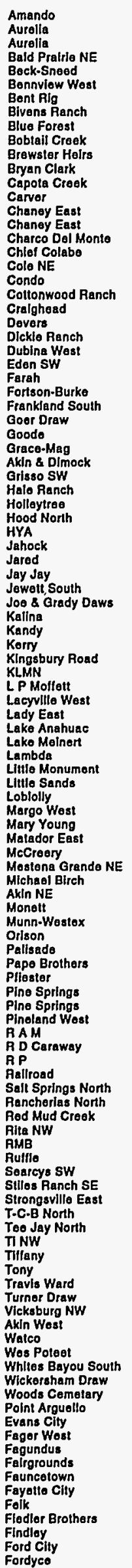 & 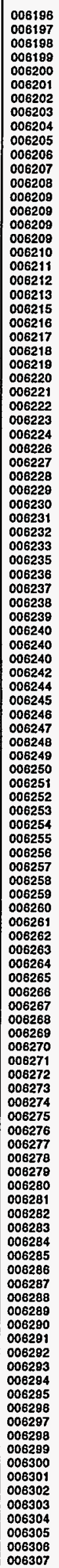 & 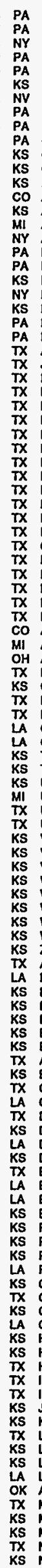 & 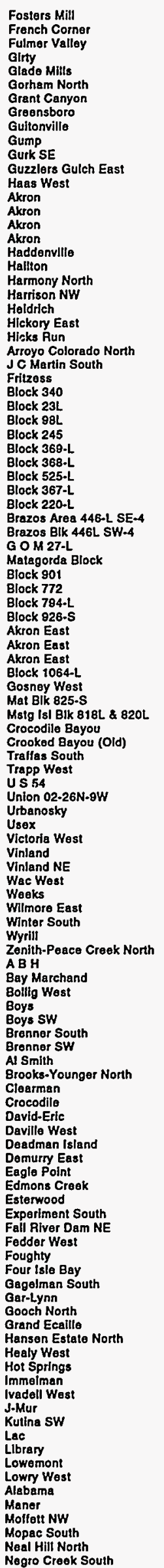 & 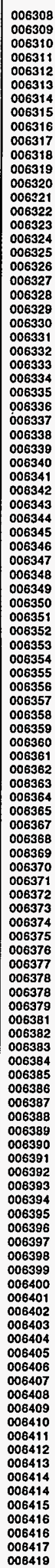 & 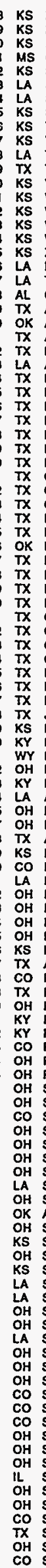 & 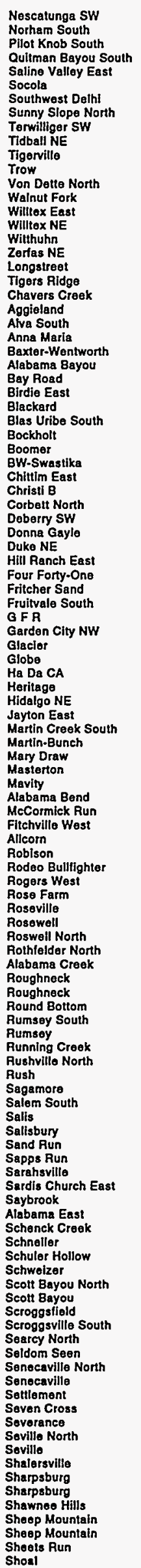 & 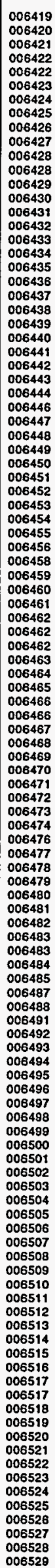 & 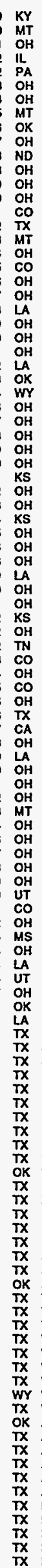 & 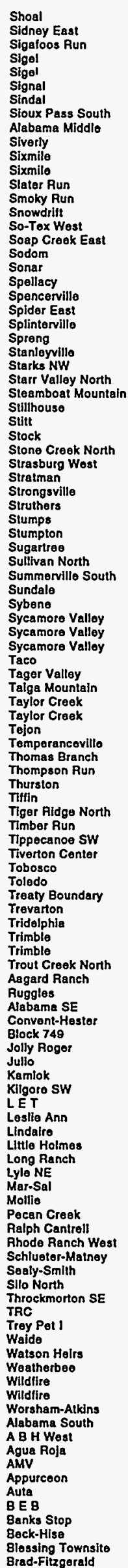 \\
\hline
\end{tabular}




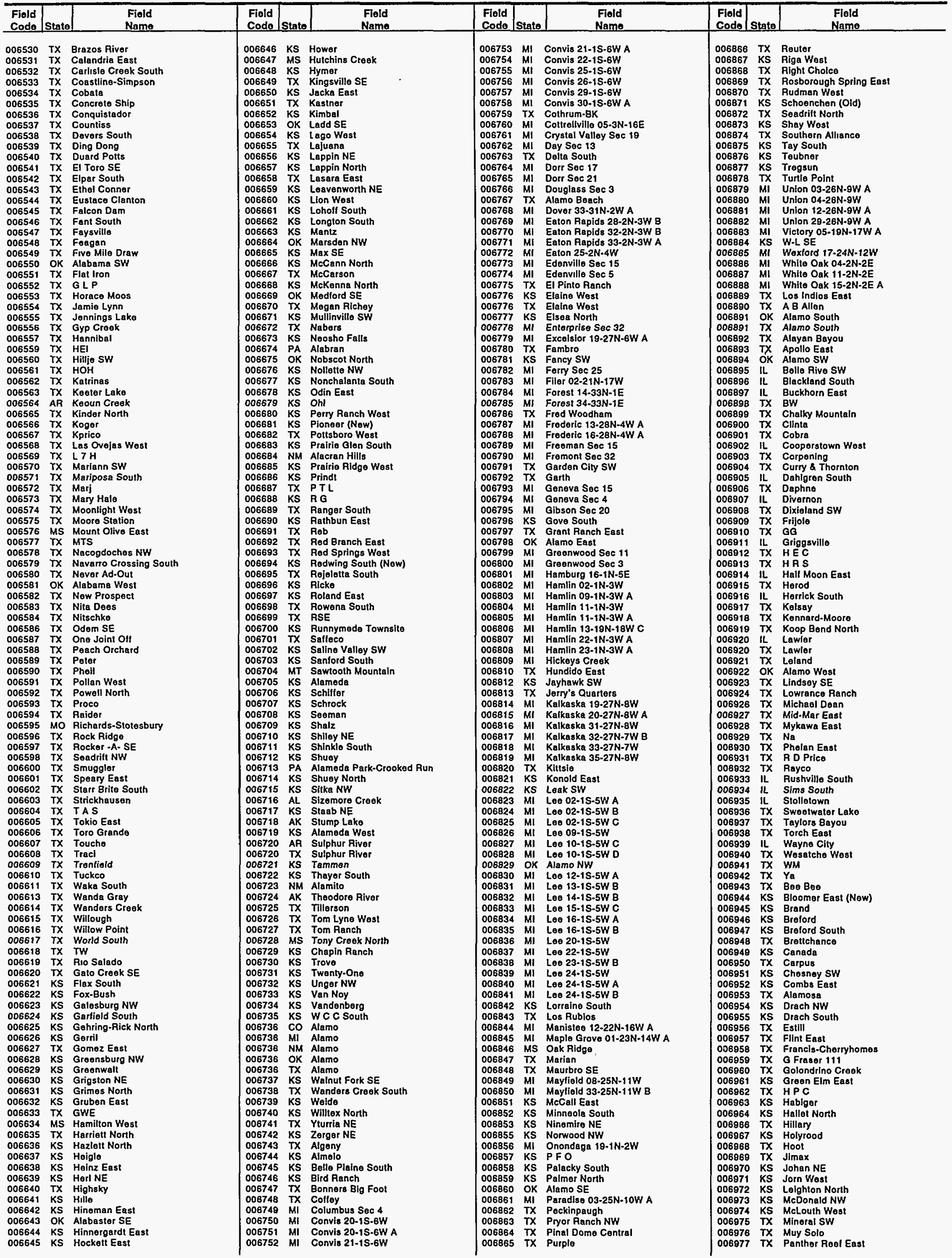




\begin{tabular}{|c|c|c|c|c|c|c|c|c|c|c|c|}
\hline $\begin{array}{l}\text { Flold } \\
\text { Codo }\end{array}$ & Stato & $\begin{array}{l}\text { Fiold } \\
\text { Namo }\end{array}$ & $\begin{array}{l}\text { Fleid } \\
\text { Code }\end{array}$ & State & $\begin{array}{l}\text { Fiold } \\
\text { Namo }\end{array}$ & $\begin{array}{l}\text { Field } \\
\text { Code }\end{array}$ & State & $\begin{array}{l}\text { Fleld } \\
\text { Name }\end{array}$ & $\begin{array}{l}\text { Fleld } \\
\text { Code } \\
\end{array}$ & State & $\begin{array}{l}\text { Field } \\
\text { Name }\end{array}$ \\
\hline 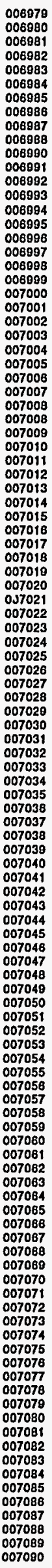 & 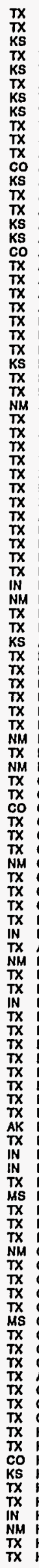 & 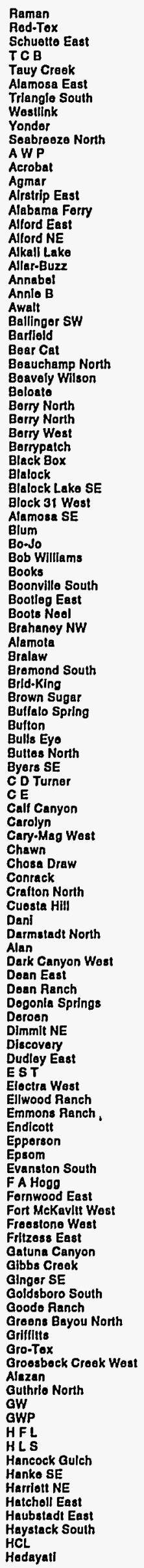 & 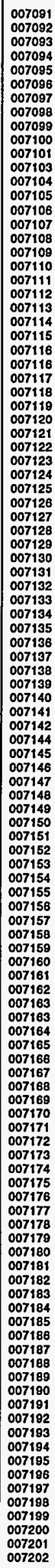 & 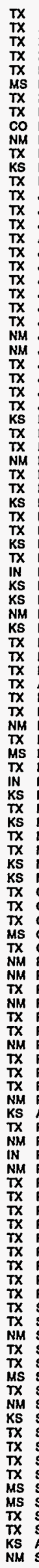 & 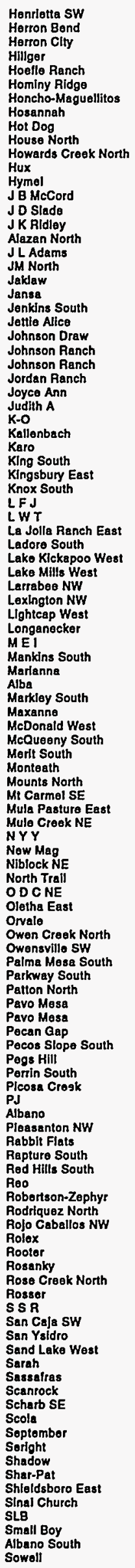 & 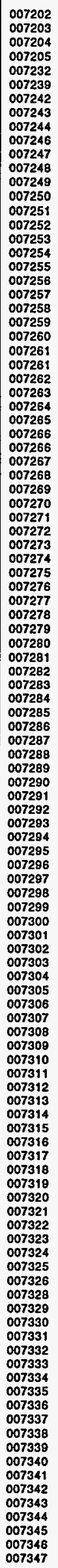 & 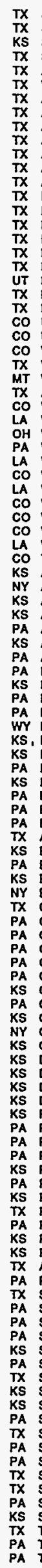 & 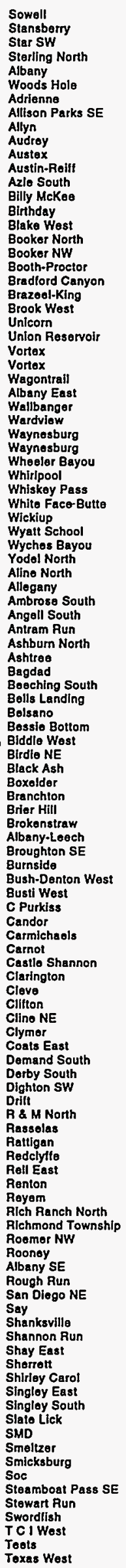 & 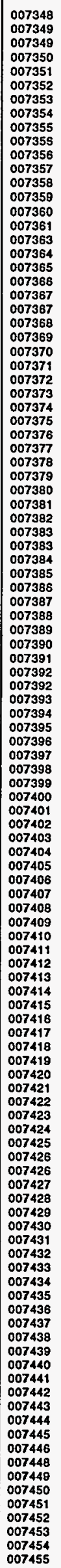 & 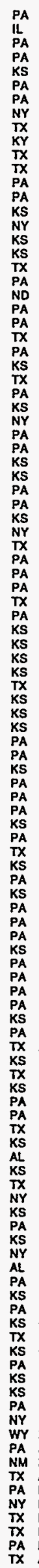 & 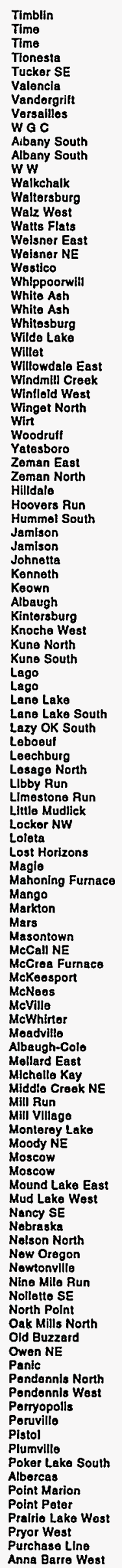 \\
\hline
\end{tabular}




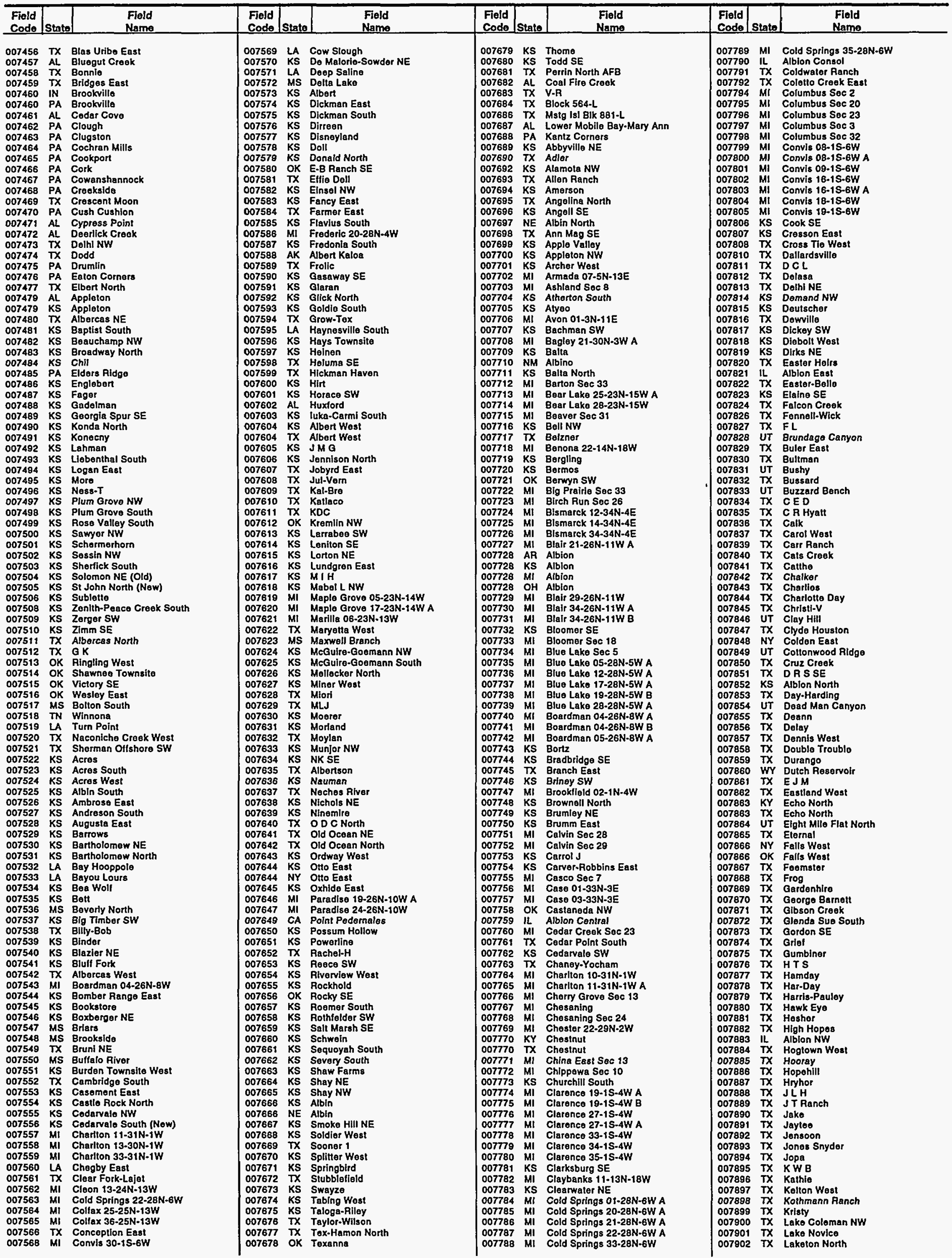




\begin{tabular}{|c|c|c|c|c|c|c|c|c|c|c|c|}
\hline $\begin{array}{l}\text { Flold } \\
\text { Codo }\end{array}$ & State & $\begin{array}{l}\text { Flold } \\
\text { Name }\end{array}$ & $\begin{array}{l}\text { Fiold } \\
\text { Code }\end{array}$ & State & $\begin{array}{l}\text { Fiold } \\
\text { Name } \\
\end{array}$ & $\begin{array}{l}\text { Fiold } \\
\text { Codo }\end{array}$ & State & $\begin{array}{l}\text { Field } \\
\text { Name } \\
\end{array}$ & \begin{tabular}{l|} 
Fiold \\
Code \\
\end{tabular} & State & $\begin{array}{l}\text { Field } \\
\text { Name } \\
\end{array}$ \\
\hline 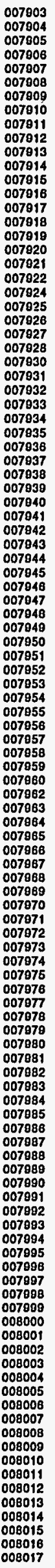 & 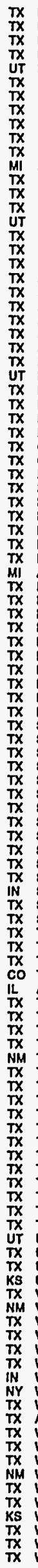 & 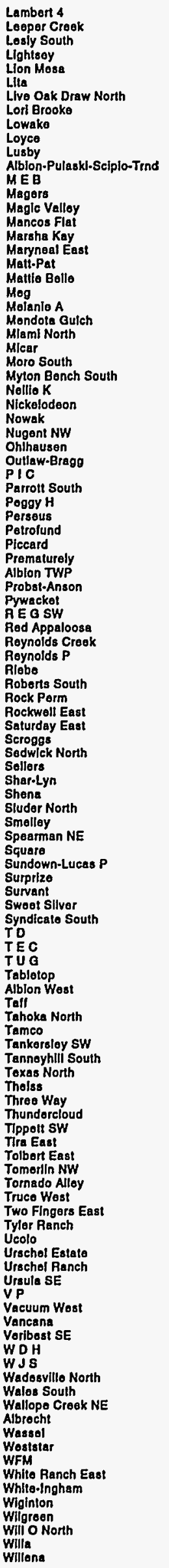 & 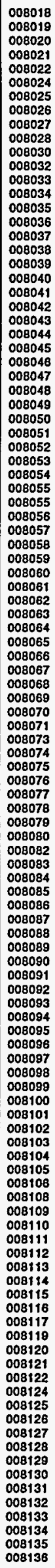 & 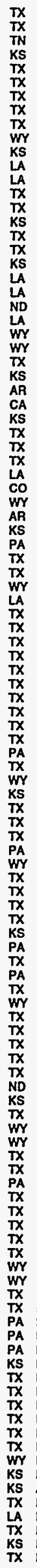 & 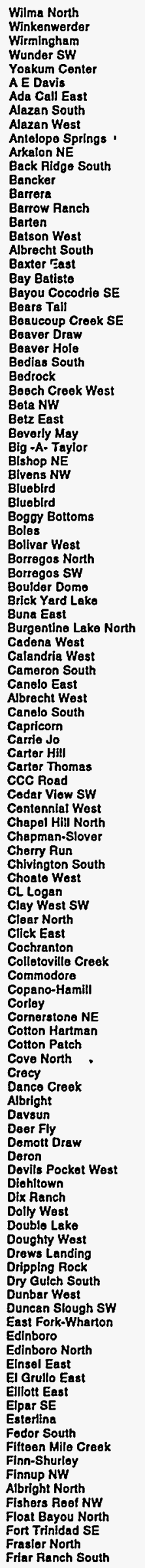 & 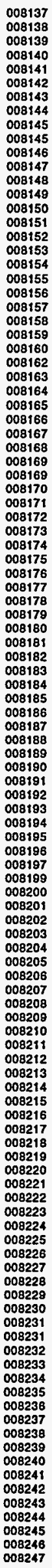 & 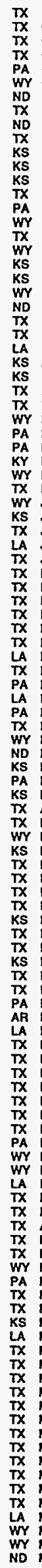 & 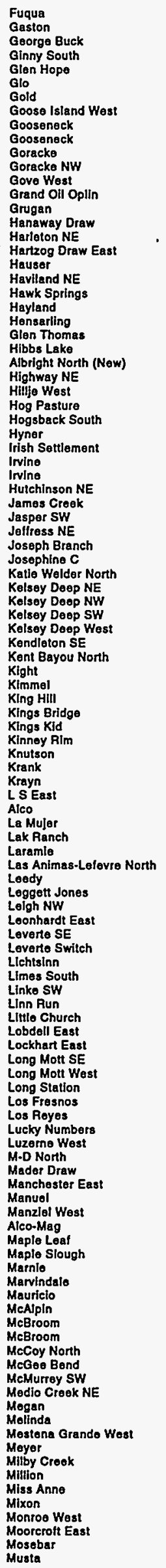 & 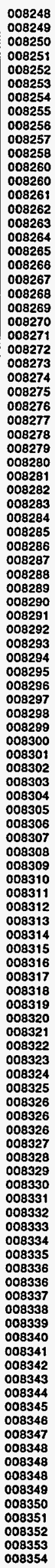 & 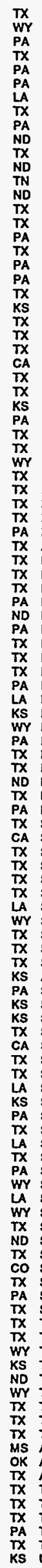 & 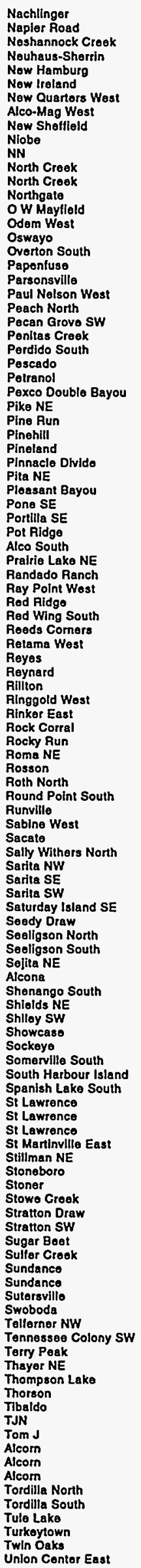 \\
\hline
\end{tabular}




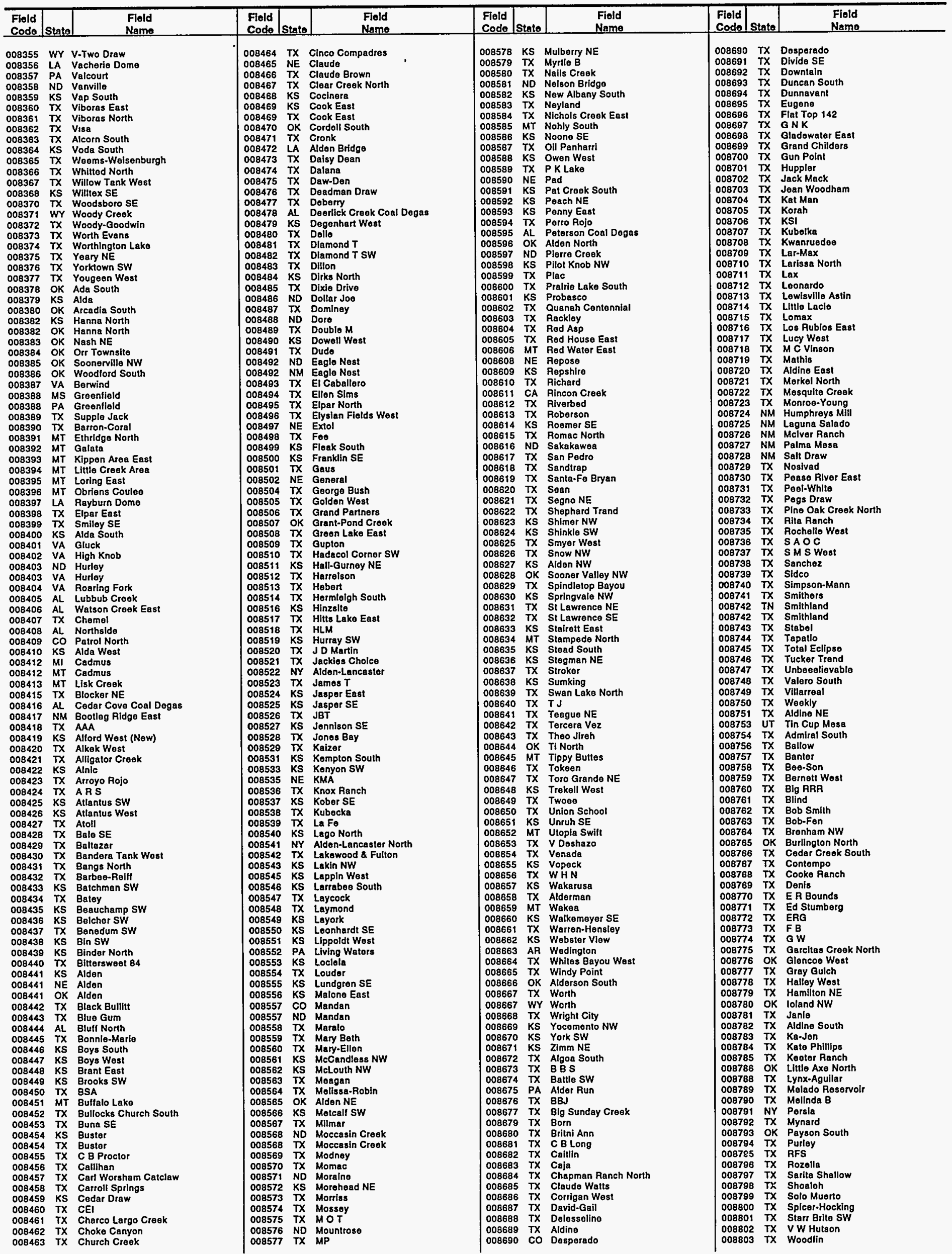




\begin{tabular}{|c|c|c|c|c|c|c|c|c|c|c|c|}
\hline $\begin{array}{l}\text { Flold } \\
\text { Codo }\end{array}$ & State & $\begin{array}{l}\text { Flold } \\
\text { Name }\end{array}$ & $\begin{array}{l}\text { Fleld } \\
\text { Codo }\end{array}$ & State & $\begin{array}{l}\text { Field } \\
\text { Name }\end{array}$ & $\begin{array}{l}\text { Fiold } \\
\text { Codo }\end{array}$ & State & $\begin{array}{l}\text { Fiold } \\
\text { Name }\end{array}$ & \begin{tabular}{l|} 
Fiold \\
Code \\
\end{tabular} & State & $\begin{array}{l}\text { Fiold } \\
\text { Name }\end{array}$ \\
\hline 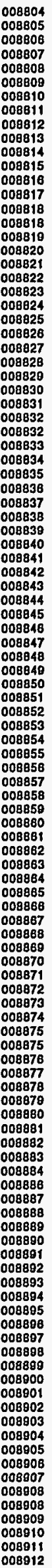 & 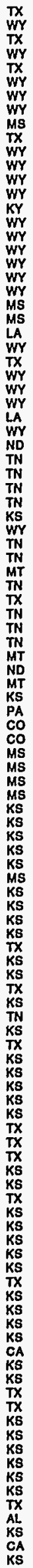 & 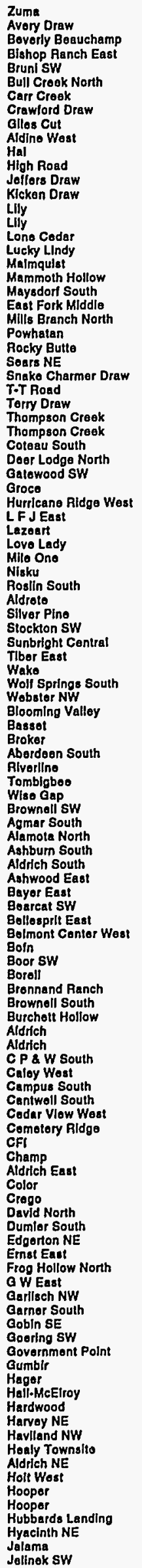 & 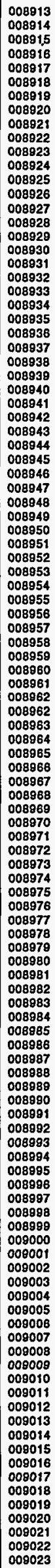 & 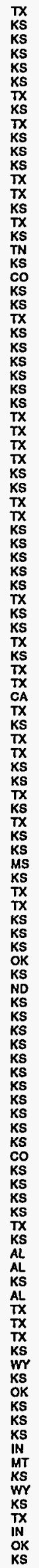 & 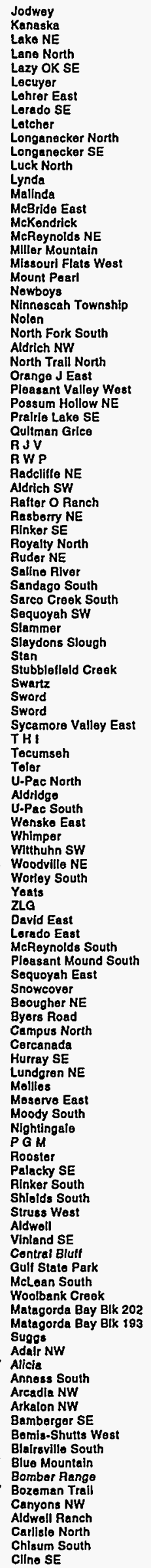 & 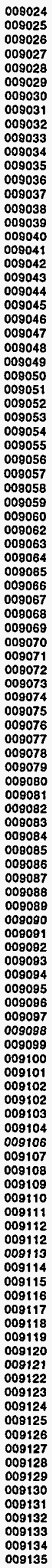 & 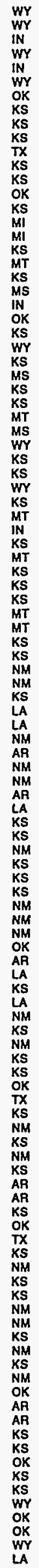 & 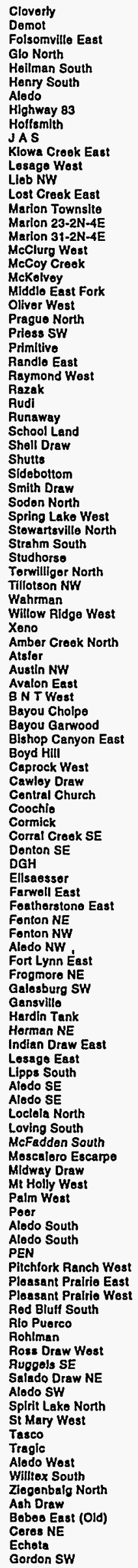 & 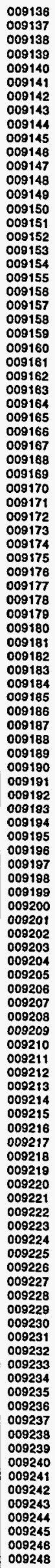 & 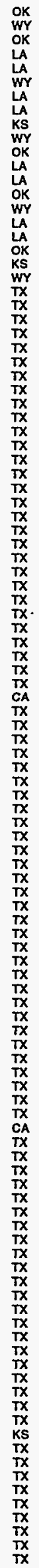 & 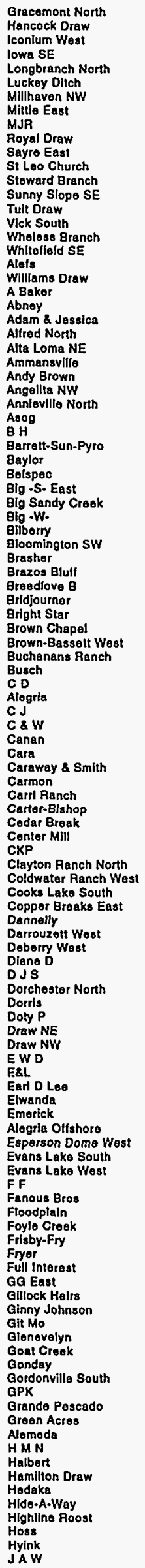 \\
\hline
\end{tabular}




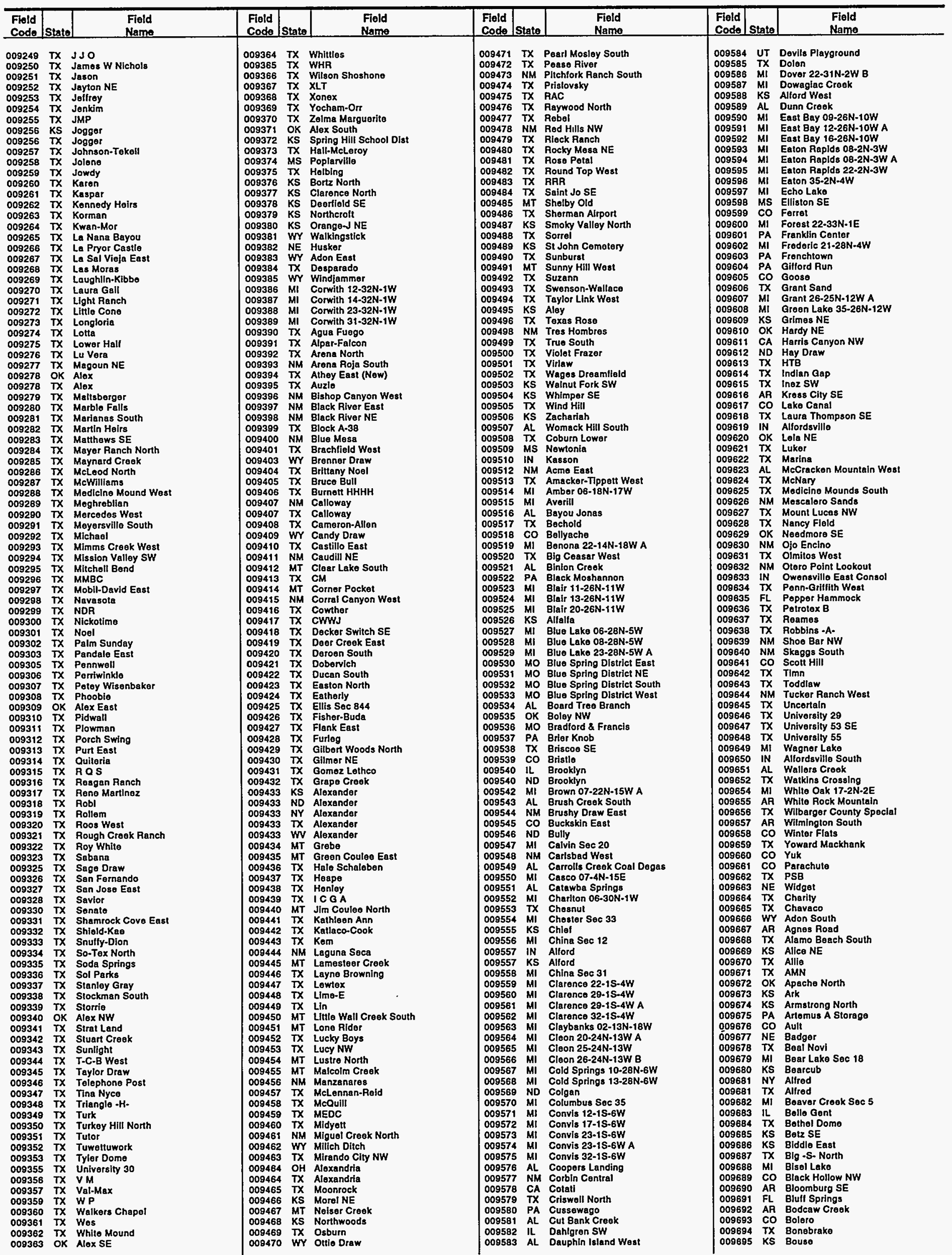




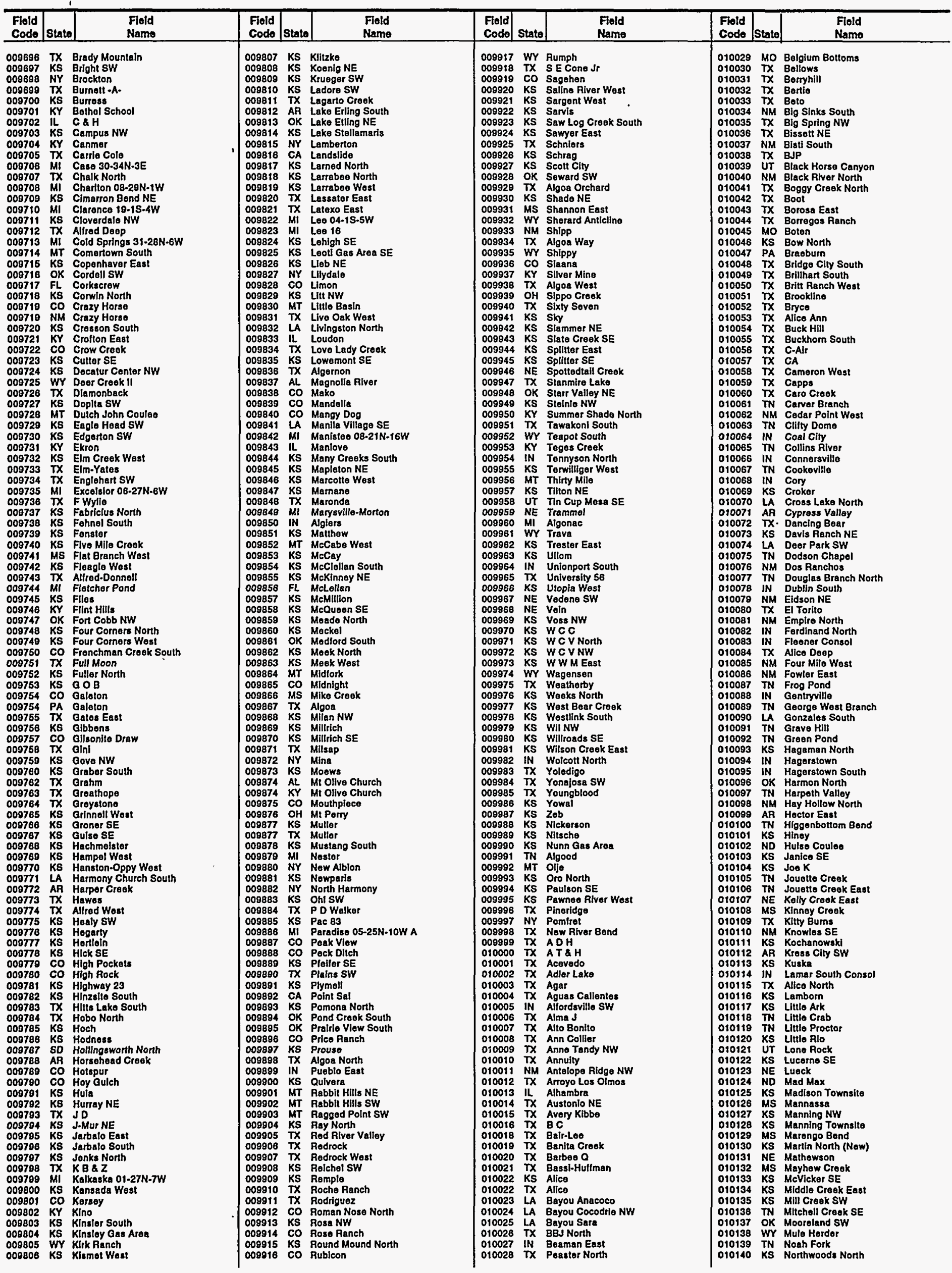

Energy Information Administration/Oil and Gas Field Code Master List 1994 


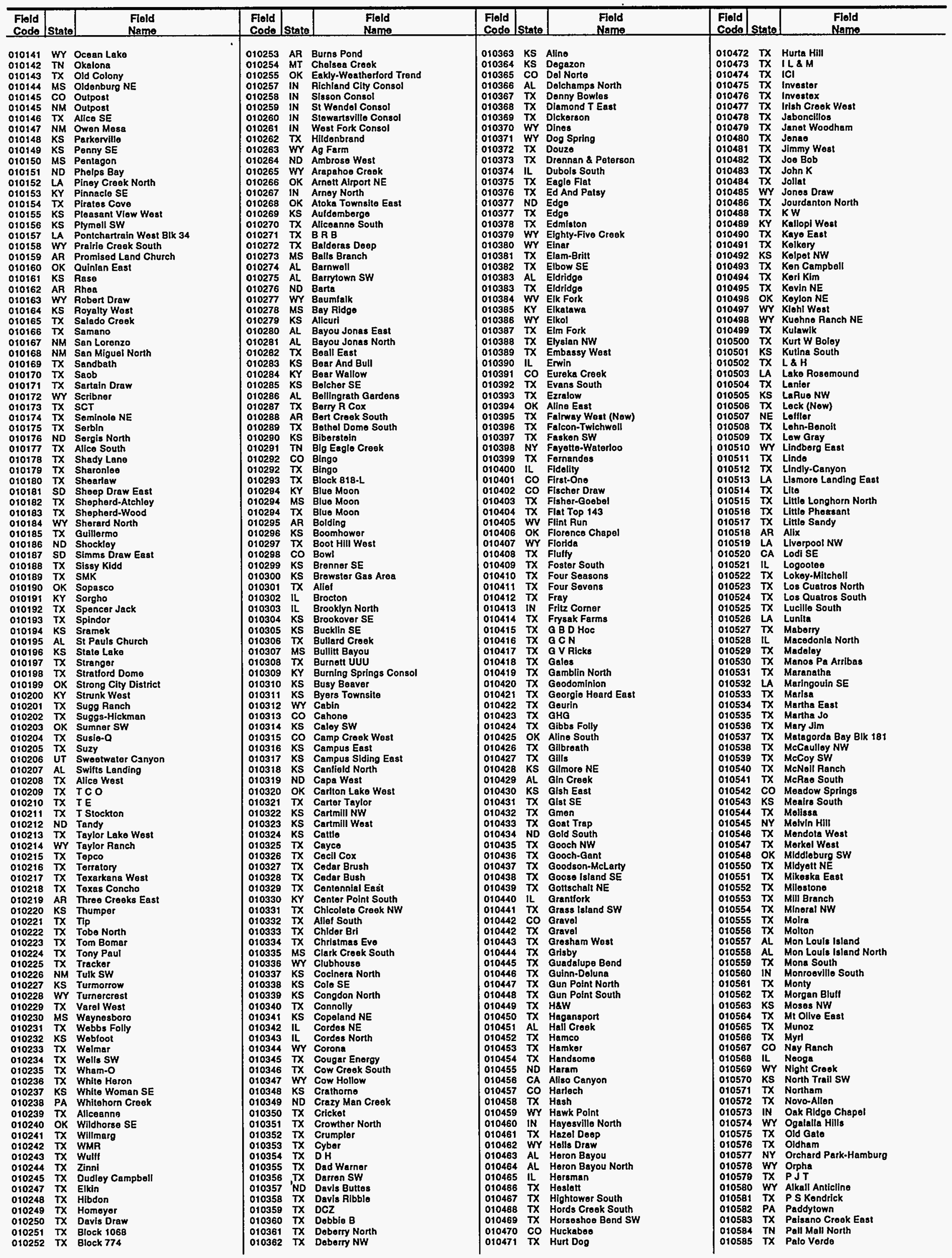




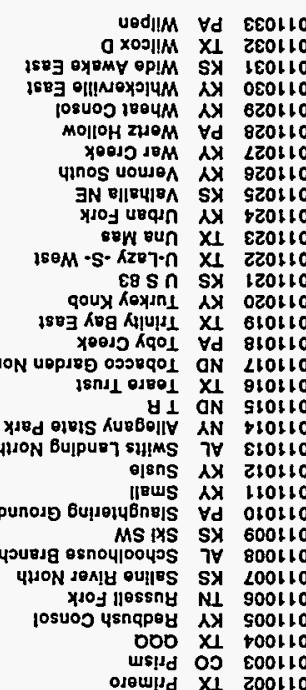

unnos veono oluted sx yanos 6angsiomod

Ine!ld $X_{1} 668010$ osuoo wnejonod ix 206010 MS LOA OQUMB $4 ! 1058$ oovolise $X I$ S66010

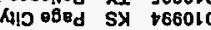
$\exists N$ OIMnN $S Y$ E 860010 OMUN SY 266010

$M N$ yอsinN $S Y$ YDION $\forall d$ O66010 Jogin NI 886010

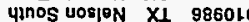
OIBABN OO SBGOLO JOLEN SX \$860LO uв6014 IN 886010 3N MOssow $S X$ 286010 पLSON DIया $\forall 7 \quad 186010$ yinos Y28d II!W NI 086010 पINOS ONOSOW SX 6LBOLO UMONDW SY 8L60LO 4!nOS pnoาow XX घUUEH NBW $S Y$ SLGOL

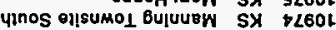
NDEW SX ELBOLIO (1) 226010 MN UOJPUn? $S \times 1$ L60lO 4

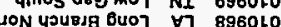
4in png pre olul 05996010 $1977 \cdot x_{1}$ s96010 (20987 $\lambda x$ t96010 MS obase $S X$ Z 296010

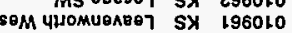
MS U[dd日7 SX 096010 MS UPbut $S X$ 6S60L0 IS Uहurye $5 \times$ 856010 GN EIOp87 SX LS60LO जि IS I10uX XI SS6010

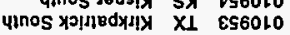
UIYणाY $3 N$ 256010

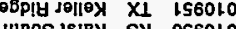

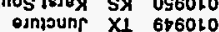
Mejuyor $X_{1}$ 8r6020 כN oover XI 9p6010 UORUEO UE!puI in st60s MN WOB 4

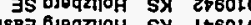
Geqziा พвบIIH N1 6860เ0 HHHH XI 886010 $188 \exists$ yวuв ив

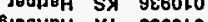
تs 4hON Isem 040010 ON 280010 $M N$ LOYS-810 SX IE6010 KuBuseo $\forall d$ 6z6010 त्रoojo doD NH LZ6010 4I0 0эร1s 74926010 JS $\times 0$ S SX 92601 (PIO) $\exists$ N UBB (pIO) iseg UDB1OJ XO X26010

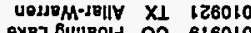

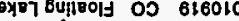

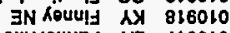
YHON OIINSOUsey $\forall 7$ L16010

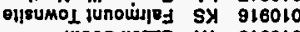
4inos llozz $X_{1} \mathbf{s 1 6 0 1 0}$ MS UPUOJ-URिA SX \$16010 uopJune OIIASIlla SW E16010 $\begin{array}{lll}\text { म0月3 } & X \perp & 216010 \\ 823 & X 1 & 116010\end{array}$ unos koljes uesuna $\lambda$ OX تs Hua sर्र 600010

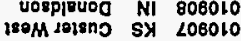

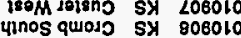
4RON Bu[PU:7 810d005 $7 \forall$ s08010 exp7 invouvos $\forall d$ rocolo

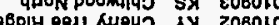
Apurus sy 100010 uinos 11040 Sx 006010 (PIO) өा880 xo 668010 Y0010 unos jung $7 \forall 868010$

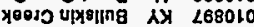
4มON Jejung Sर्र 868010 MS Plopumos $X_{\perp}$ S68010 unos jBoxog oN c68010 josuon U日esg Bullmog AX Z68010 woog of te8010 sxग8W-jEll X1 068010 MS se91日 SX 888010

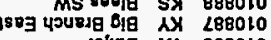
MS OIInnoque พ7 8 SX +88010 loqy $X 1$. Jisey sx z88010 eplez ix 188010 $\exists 87$ Xर 188010

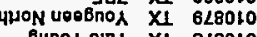
Bunos $018 \lambda X_{1} \quad 828010$

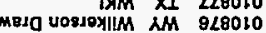
PIPISLOYIOM AN SLBOLO

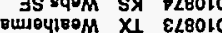
osson $X 112 \angle 8010$ digus UOUsoA $X_{1}$ 1L8010 sẹzeds $A X_{1}$ 0L8010 पMON QIBUBIAI $S X$ 698010

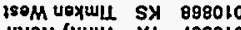
4RON HI] $_{41} X_{1} \quad$ 298010 शมอ 0014L XI 998010 $180 \exists$ 0x107 IIIPN $\mathbf{C}_{1} \mathbf{X}_{1} \quad 898010$ FN OlInN⿴囗十 $X_{1}$ 298010

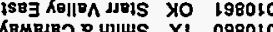
KBMBIES 8 4यLS $X_{1} 098010$ $\begin{array}{rll}\text { JiJl } & X_{1} & 658010 \\ X_{1} & 858010\end{array}$

MN Ou|juruS $S X \quad L 58010$ WET-ESS XI 958010

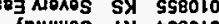

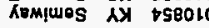
6S-0E UOाPOS $X_{\perp}$ ES8010 y001 pues $X_{1}$ Z58010 4HON ODduES $X I$ IS8010 podj weS XI 058010 UO|HEAIES XI 6r80L 3N MESIIIBS XO 8†BOLL

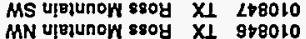

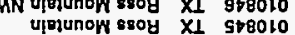
uppunow ssoy $X_{1}$ st8olo

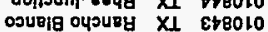

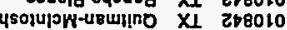

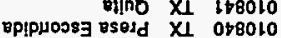
esoesd $x \times$ 688010

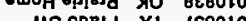
MS op Edd $X_{1} \quad \angle 88010$ BWId XI 988010 osdodiod $X_{1}$ se8010 o8jued $x_{1}$ EE80t0

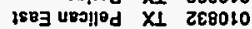

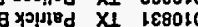
थिpएwed $X_{1}$ 0ह8010 oxiy Xo 8z8010 ind $X \perp$ LZ8010

4เnos $\theta 110 x_{1} \quad 9 z 8050$ 8 puridn plo $x_{1}$ sz8010 $\begin{array}{ccc}10040 \\ \text { MON } X I & \text { EZBOLIO }\end{array}$ JS spoom4nON SY ZZBOL IIND ISOMYHON TV 128010 InO IEIUOO YHON TY OZBOL Xo0jo DIN OUIN XI 818010 जS DाfPMON X्रO L180LO

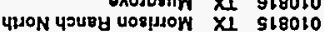

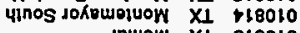
as Квg Ө!!qOW EרW XI 118010

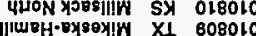

seppueyo oxis XI 808010

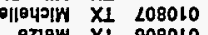
9N Prouow $X 1$ s08010 pooxow $X I$ rosolo IN 10204MOW SX E08010

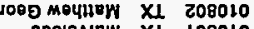
Onojorar XI 108010 089 op Nodsw $X \perp$ 68L010 $M W W X 1882010$

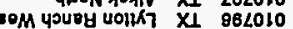
1004อS X188 UURT NN S8LO10

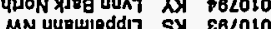
43nos 439797.262010 $1108007 \times 1 \quad 062010$ Appo7 $5 x$ 68LO10 Snser $X 1$ 88LO10

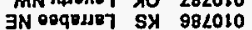

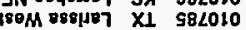

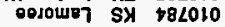

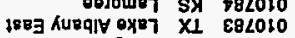
Buey $X_{1} \quad 28 \angle 010$

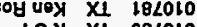
zD mnos fllor sy 8<Loto gyelpo Buodujir X1 $0<2010$

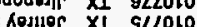
ueate $x_{1}$ vLLO10

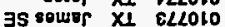

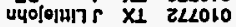

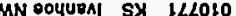
oO IIII $x_{1} \quad 0<2010$ อxष7 प04 $\times 1$ 69<010

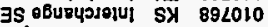

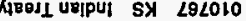
1809 X0XIV XI $99 \angle 0.0$ XIIIII $X \perp$ s9LOLIO

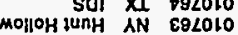
MN BKouydwn XI 292010 ny yedBoH $A M$ 19L010 

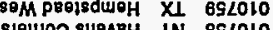
Zuoox $O H$ XI $X 91010$

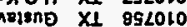

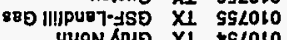
4म

$1883 \times 0+400010 \times 1 \quad 29 \angle 010$

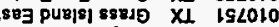

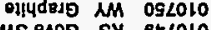
MN QAOD SX $87 \angle 010$ E1010 XI $\angle$ L LO10

政

1883 OाP UताI U019 $x_{1}$ trLO10 MS OHOll19 $X_{1}$ ErLO10 yมn $x_{1}$ it<2010

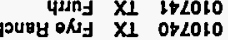
8807 Bos $X_{1}$ BELO10 jo84 घiU0pest AN 8ELO10

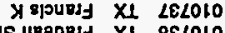

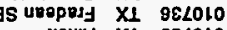

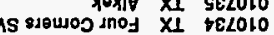
deujog rog $x_{1} \quad \varepsilon E \angle O 10$ Uo: 4inos Ajn $X 1$ iezolo Mobply yuly $x_{1}$ oezo10 usbauly XN Bzzolo 90ट $\times 0018009$ $7-681$ ×19 propes XI $82 \angle 010$ sOM

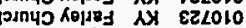

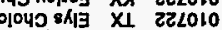

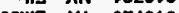

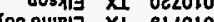
$\begin{array}{llll} & 1480 \times 3 \\ x 1 & 812010\end{array}$

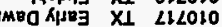

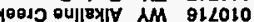
4roN Kiyno $x 1$ s1<010 Xoos Bnog $X_{1}$ th $\angle 010$

(10;1)

$\exists S \mu 01100$ 7ళ $21<010$ osoy ueseo is t1LO10 poous fosduse MM Ot 2010 oung k80 is 602010 MN

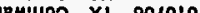
15ugo X1 4כing $\begin{array}{rlll}105 & X 1 & 802010\end{array}$ yinos oxos $x_{1} \quad 102010$ (0030 $\| 8 \times 1 y \quad X_{1} \quad 002010$

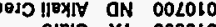

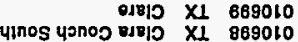

ก⿻40 SX 269010 $11095 \times 1969010$ som uorda $x 1$ s68010

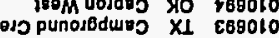
MS Uנग100 $x_{\perp} \quad 269010$ MIH AHOJjED AN 169010

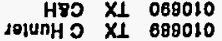
sing $X 1889010$

LHON XJjYSE $A Y 1 \quad \angle 89010$ xp 7 juing $X \pm 1999010$

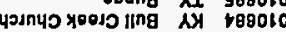
seos png $X 1$ c89010 $x_{1}$ umo1-1019 $x_{1}$

(1)

189380040909 35 Moin oluvog $x / 140010$ pjexouog oD $0<0010$ Avg unovos uog $7 y$ SL9010 บokUBD IIPYIY in EL9010 oxitir XI zL8010 Alnd $x_{1}$

cor $618 x_{1} \quad 099010$

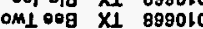

021809 X1 899010 u티 р४ә8 M0148E $7 Y$ S99010 MS IIOM!EnY XI t90010 


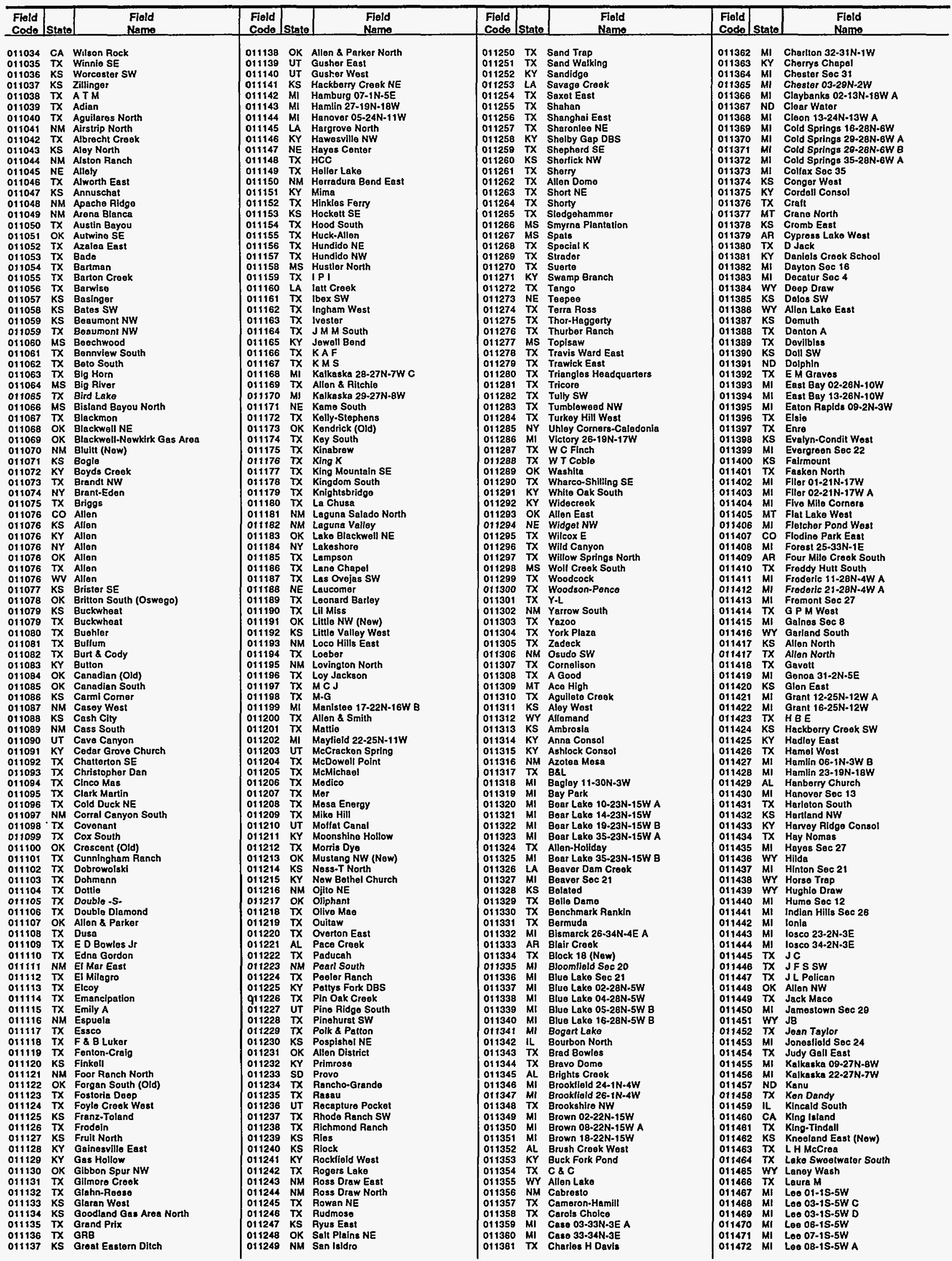




\begin{tabular}{|c|c|c|c|c|c|c|c|c|c|c|c|}
\hline $\begin{array}{l}\text { Fiald } \\
\text { Code }\end{array}$ & Stato & $\begin{array}{l}\text { Fiold } \\
\text { Namo }\end{array}$ & $\begin{array}{l}\text { Field } \\
\text { Code }\end{array}$ & State & $\begin{array}{l}\text { Field } \\
\text { Name }\end{array}$ & $\begin{array}{l}\text { Field } \\
\text { Code }\end{array}$ & State & $\begin{array}{l}\text { Fiold } \\
\text { Name }\end{array}$ & \begin{tabular}{|l|} 
Fiold \\
Codo \\
\end{tabular} & Stato & $\begin{array}{l}\text { Fiold } \\
\text { Name }\end{array}$ \\
\hline 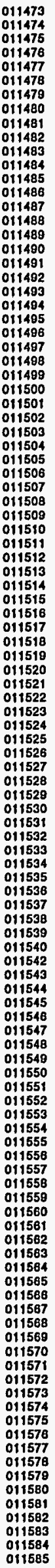 & 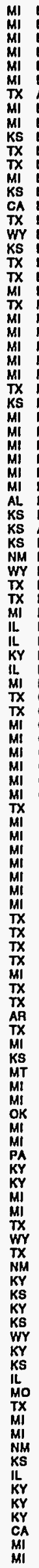 & 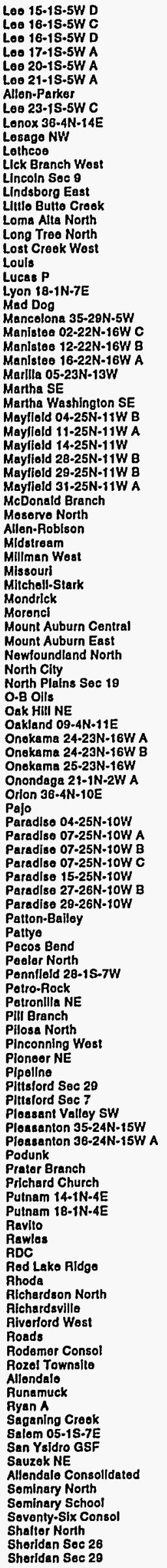 & 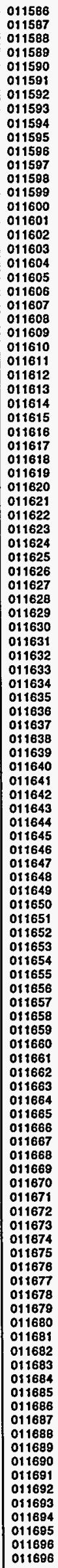 & 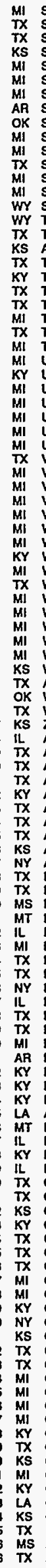 & 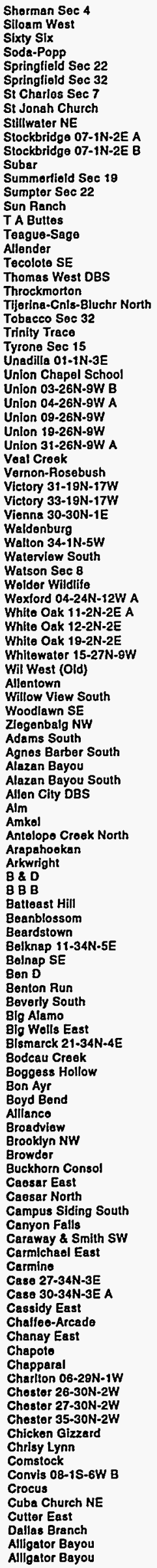 & 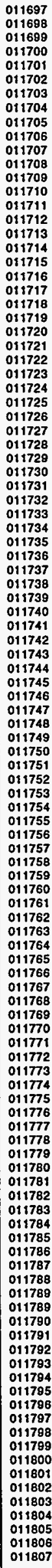 & 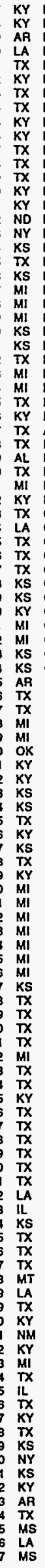 & 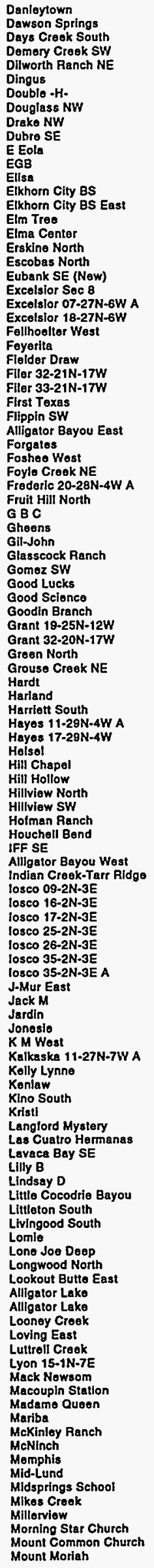 & 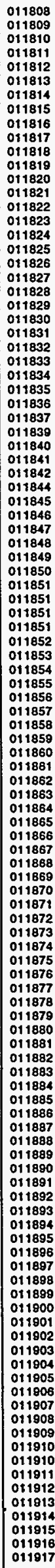 & 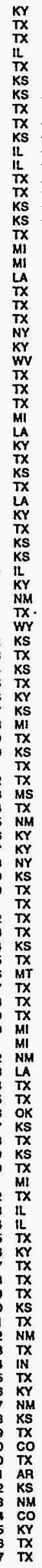 & 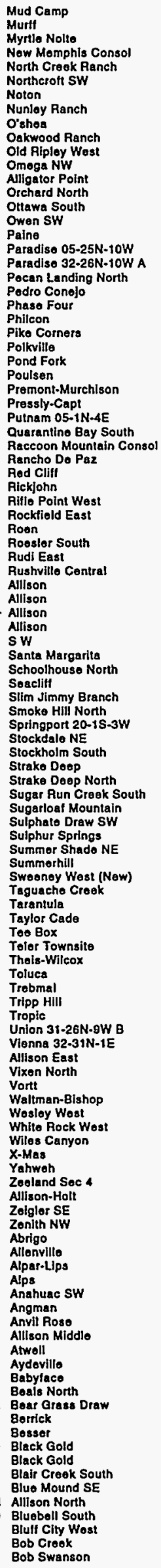 \\
\hline
\end{tabular}




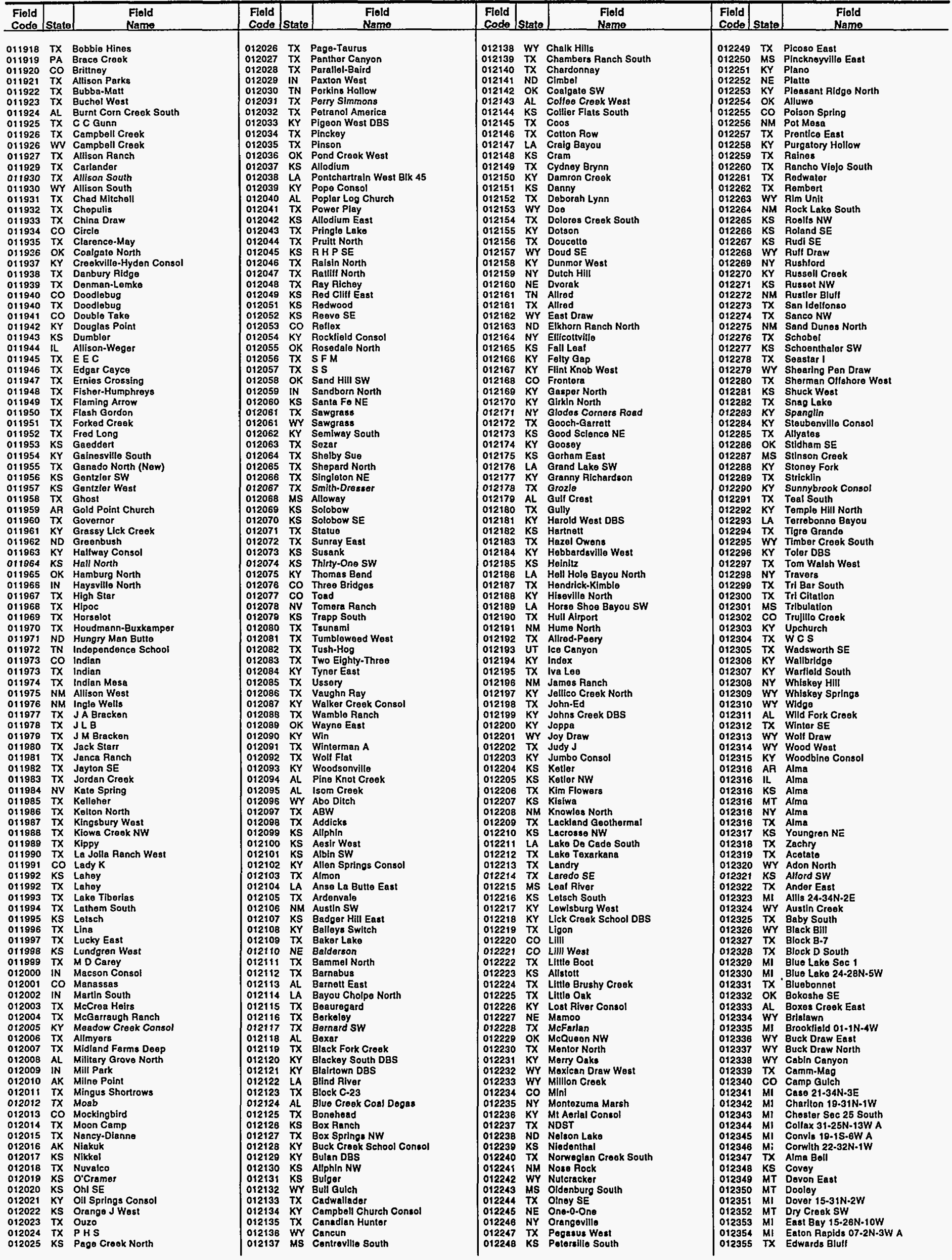




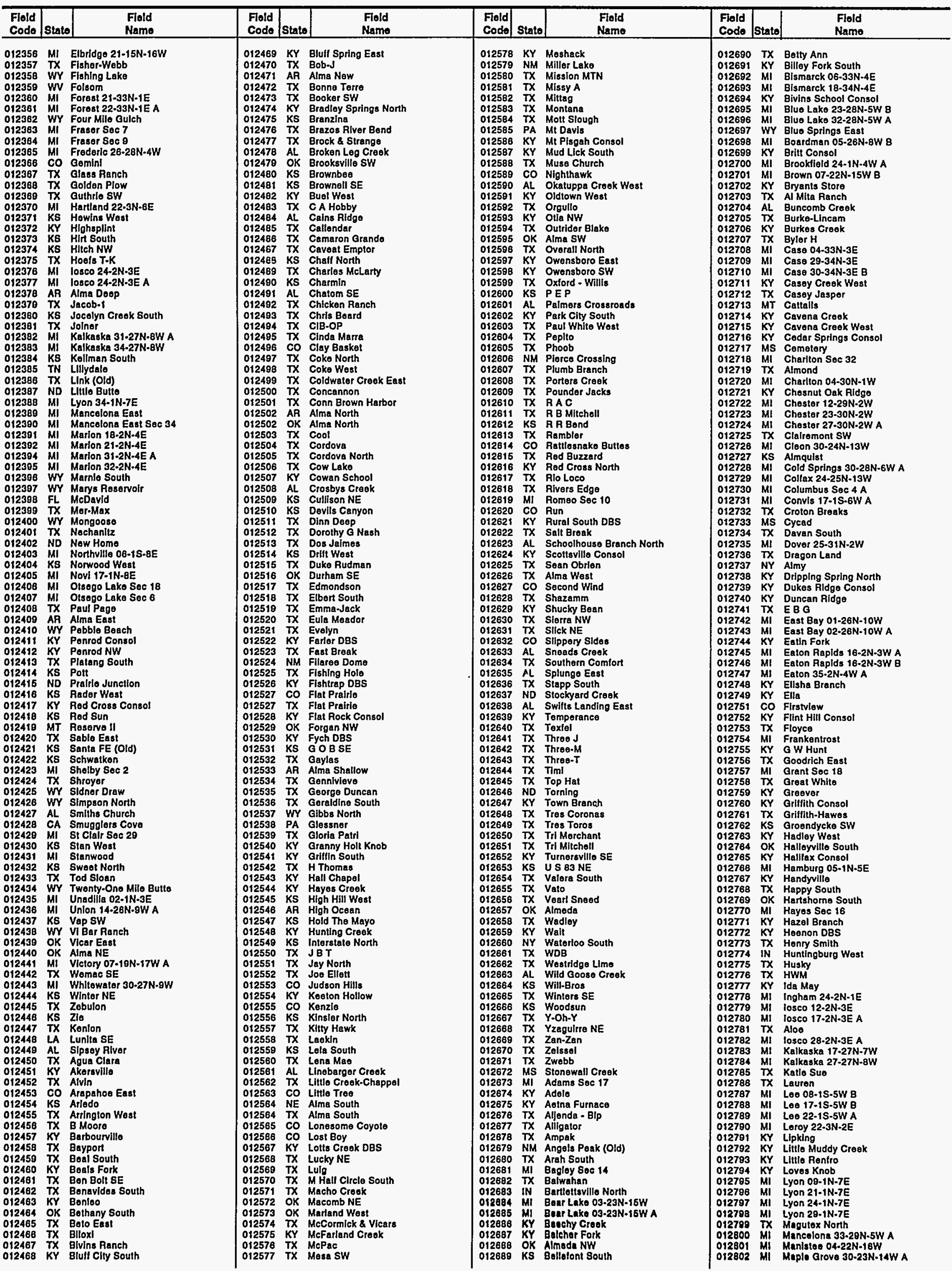




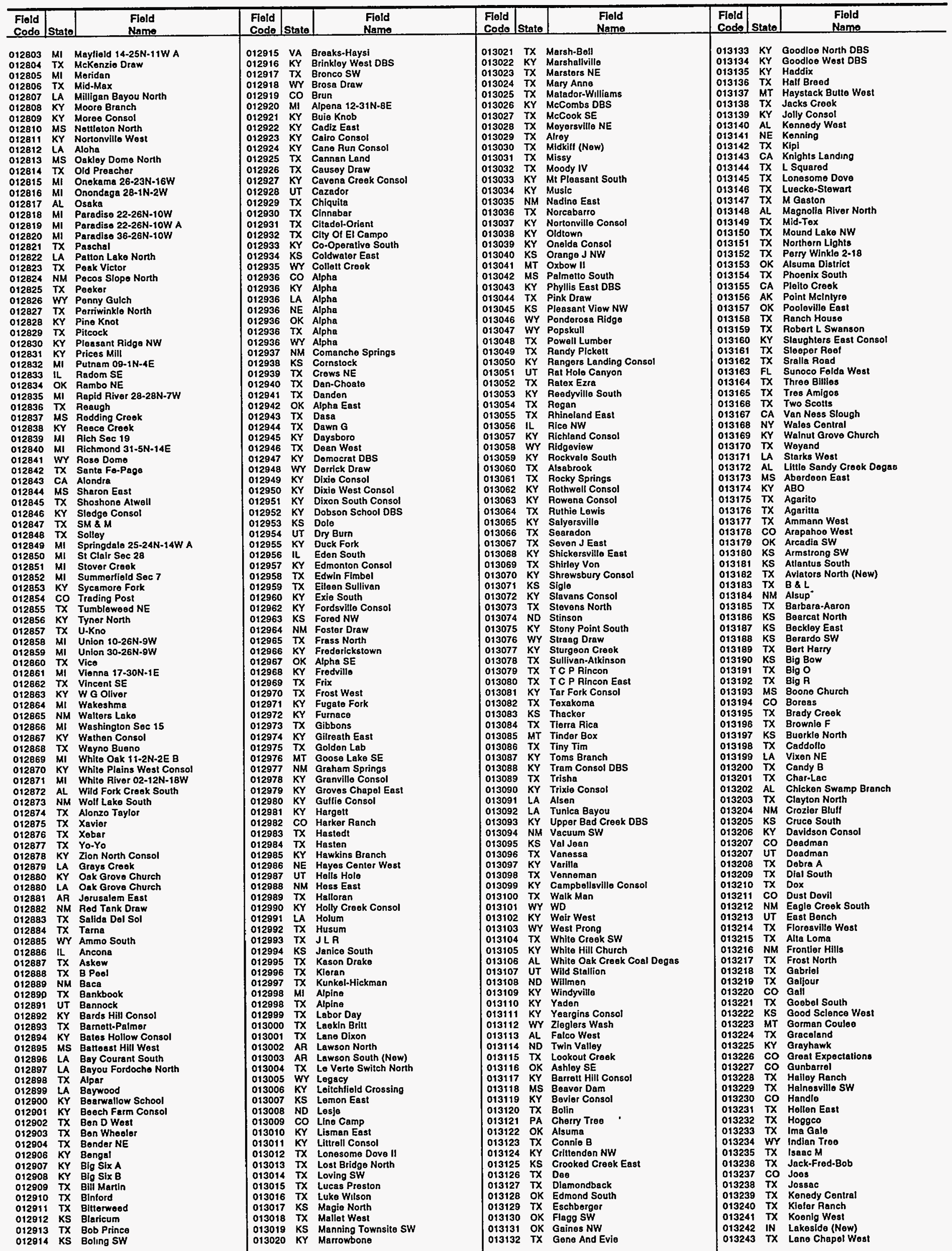




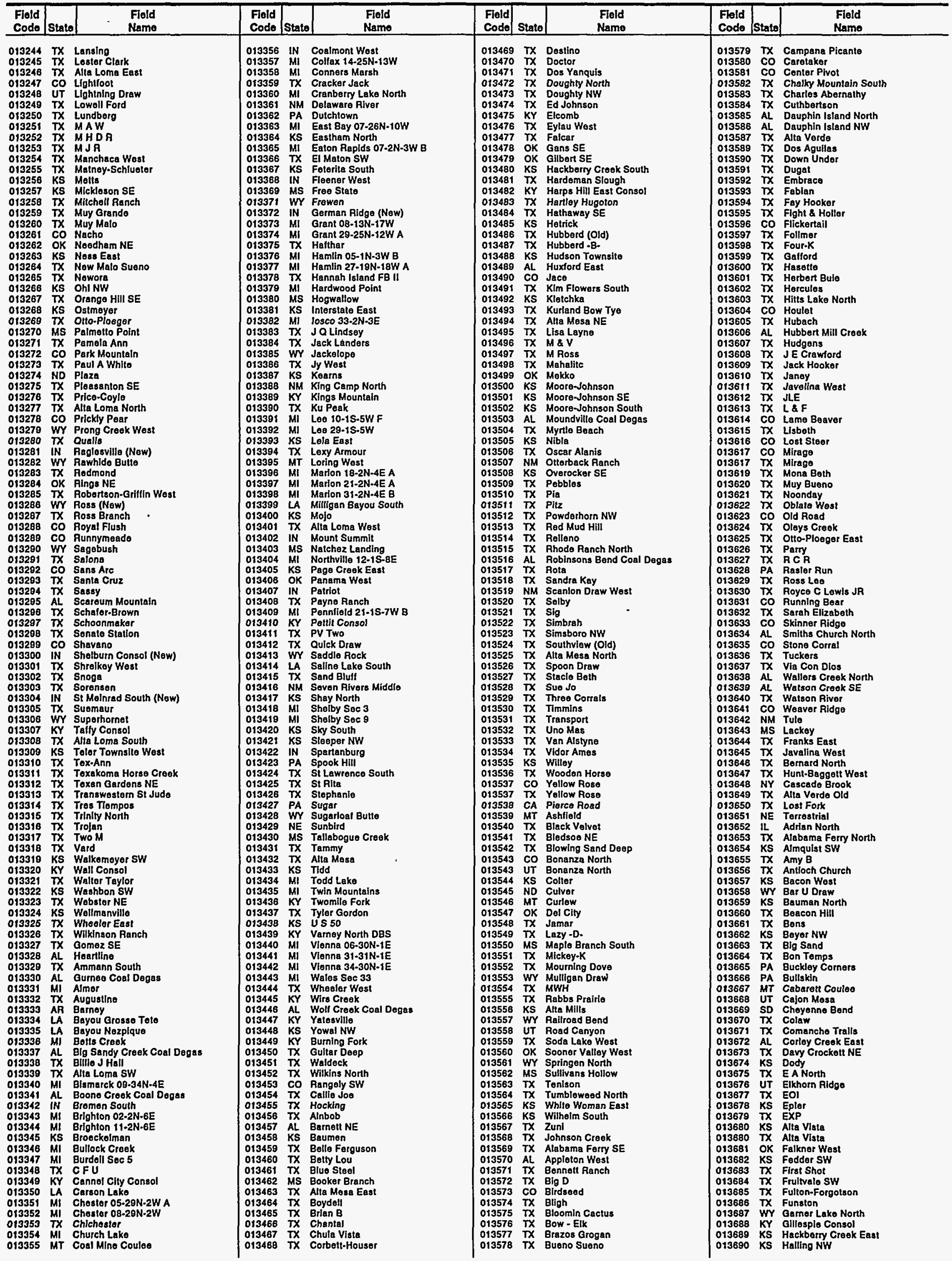




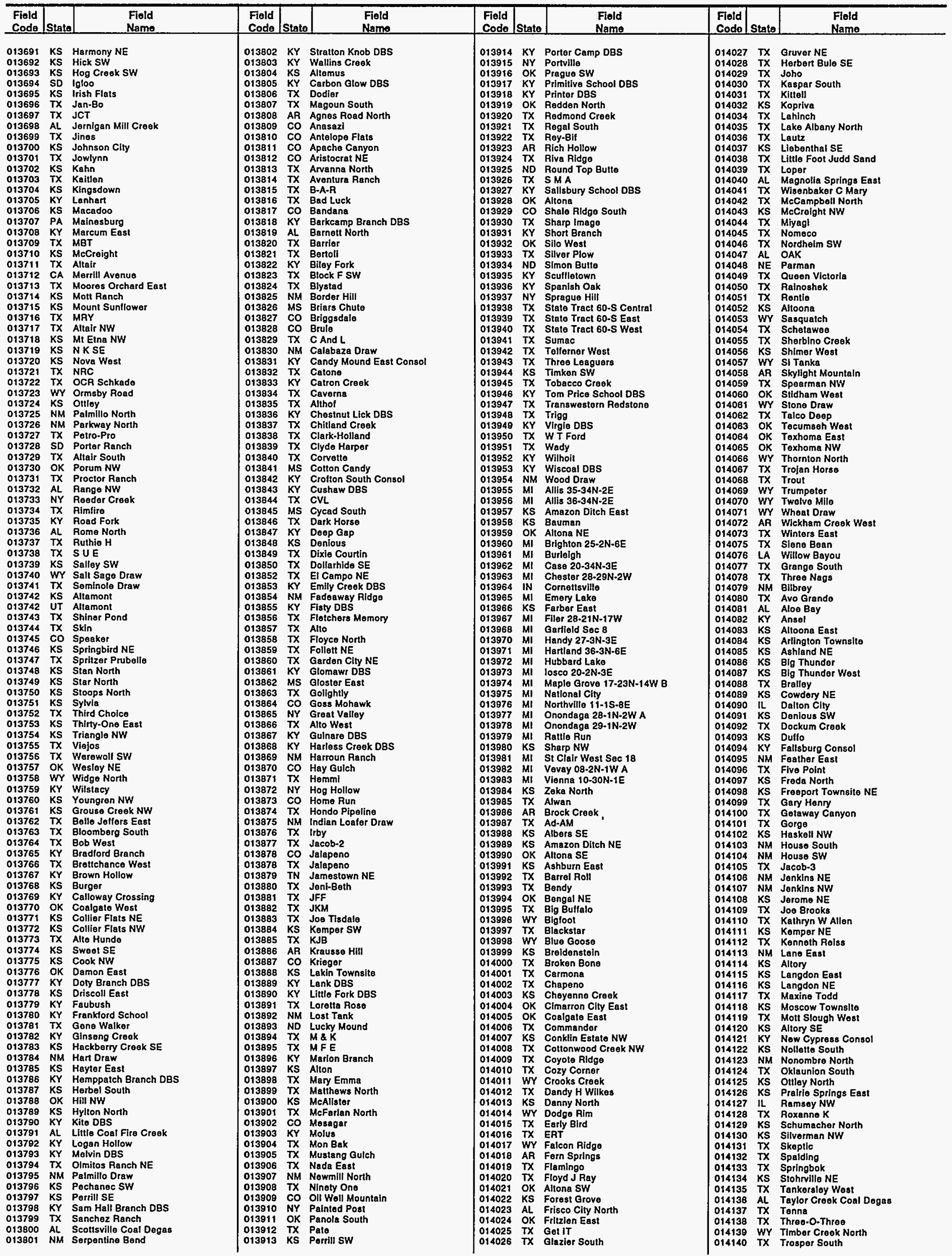


014592

\begin{tabular}{|c|c|c|c|c|c|c|c|c|c|c|c|}
\hline $\begin{array}{l}\text { Fleld } \\
\text { Codo }\end{array}$ & Stato & $\begin{array}{l}\text { Flold } \\
\text { Namo } \\
\end{array}$ & $\begin{array}{l}\text { Flold } \\
\text { Code } \\
\end{array}$ & Stato & $\begin{array}{l}\text { Fold } \\
\text { Name } \\
\end{array}$ & $\begin{array}{l}\text { Fiold } \\
\text { Code }\end{array}$ & Stalo & $\begin{array}{l}\text { Fiold } \\
\text { Name } \\
\end{array}$ & $\begin{array}{l}\text { Fiold } \\
\text { Code }\end{array}$ & State & $\begin{array}{l}\text { Fiold } \\
\text { Namo }\end{array}$ \\
\hline 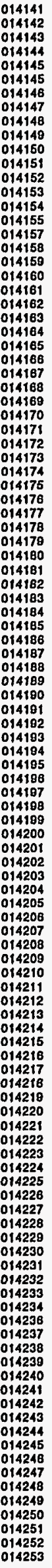 & 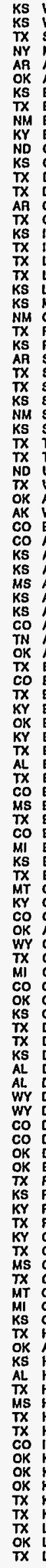 & 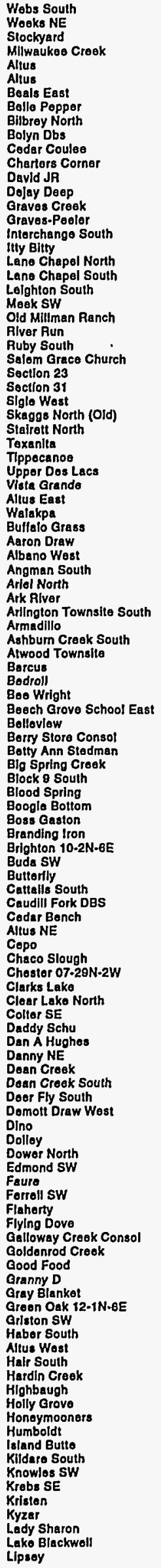 & 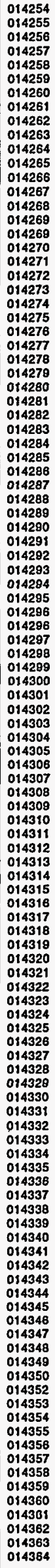 & 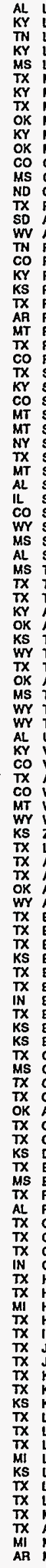 & 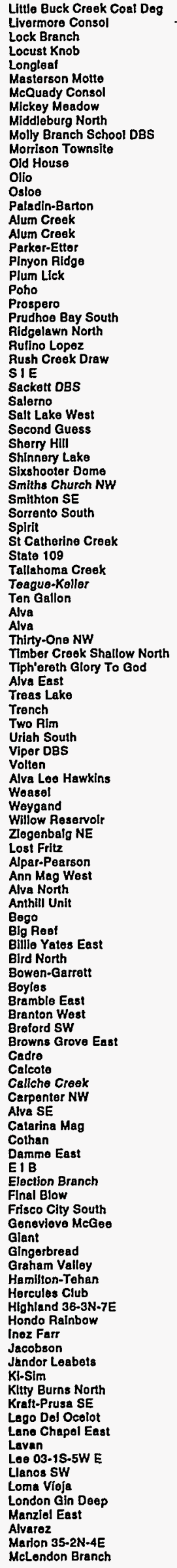 & 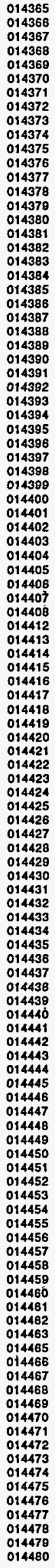 & 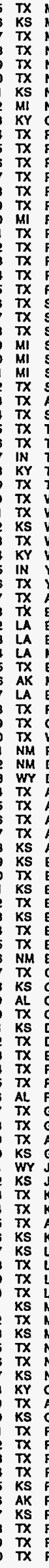 & 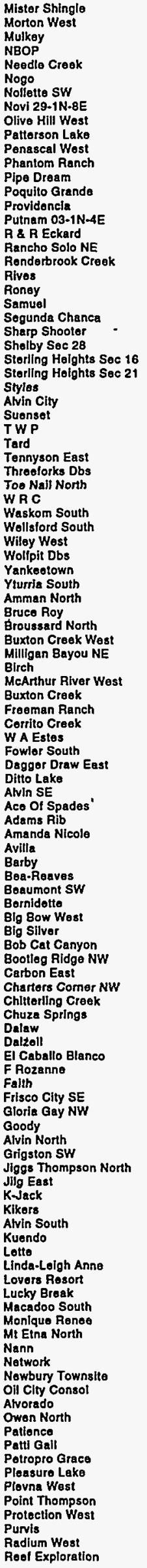 & 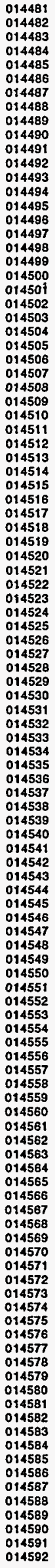 & 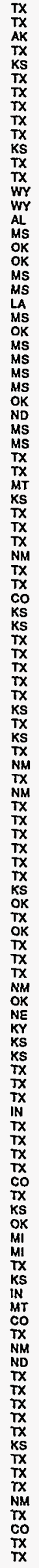 & 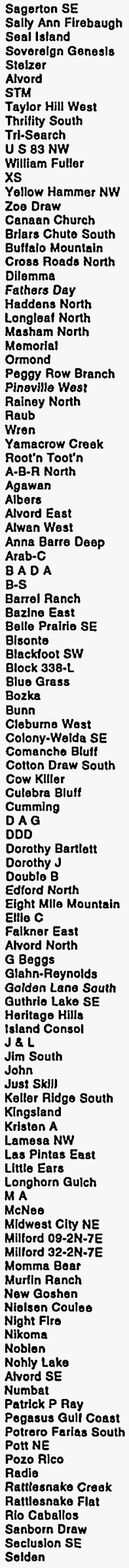 \\
\hline
\end{tabular}


014593

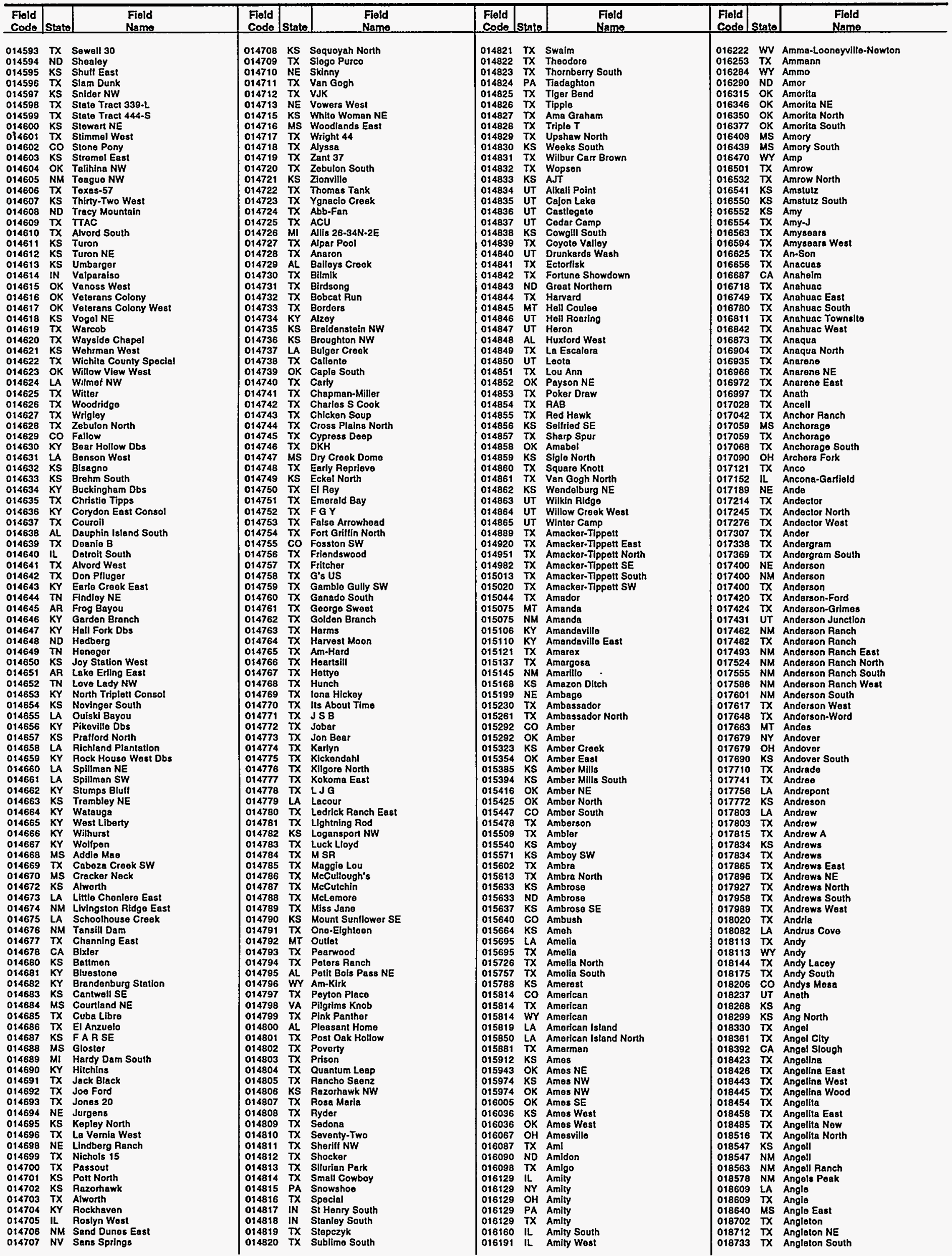




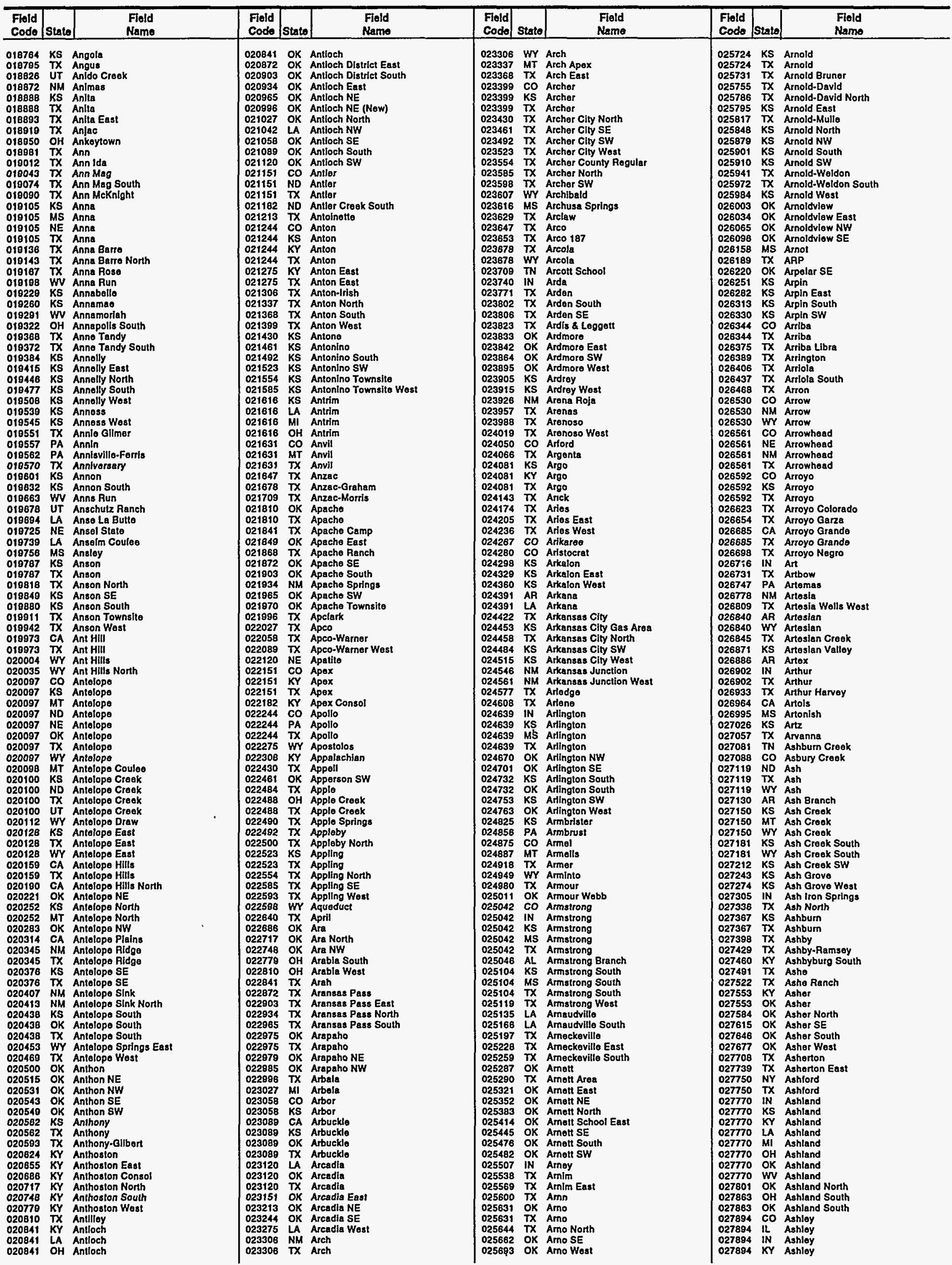




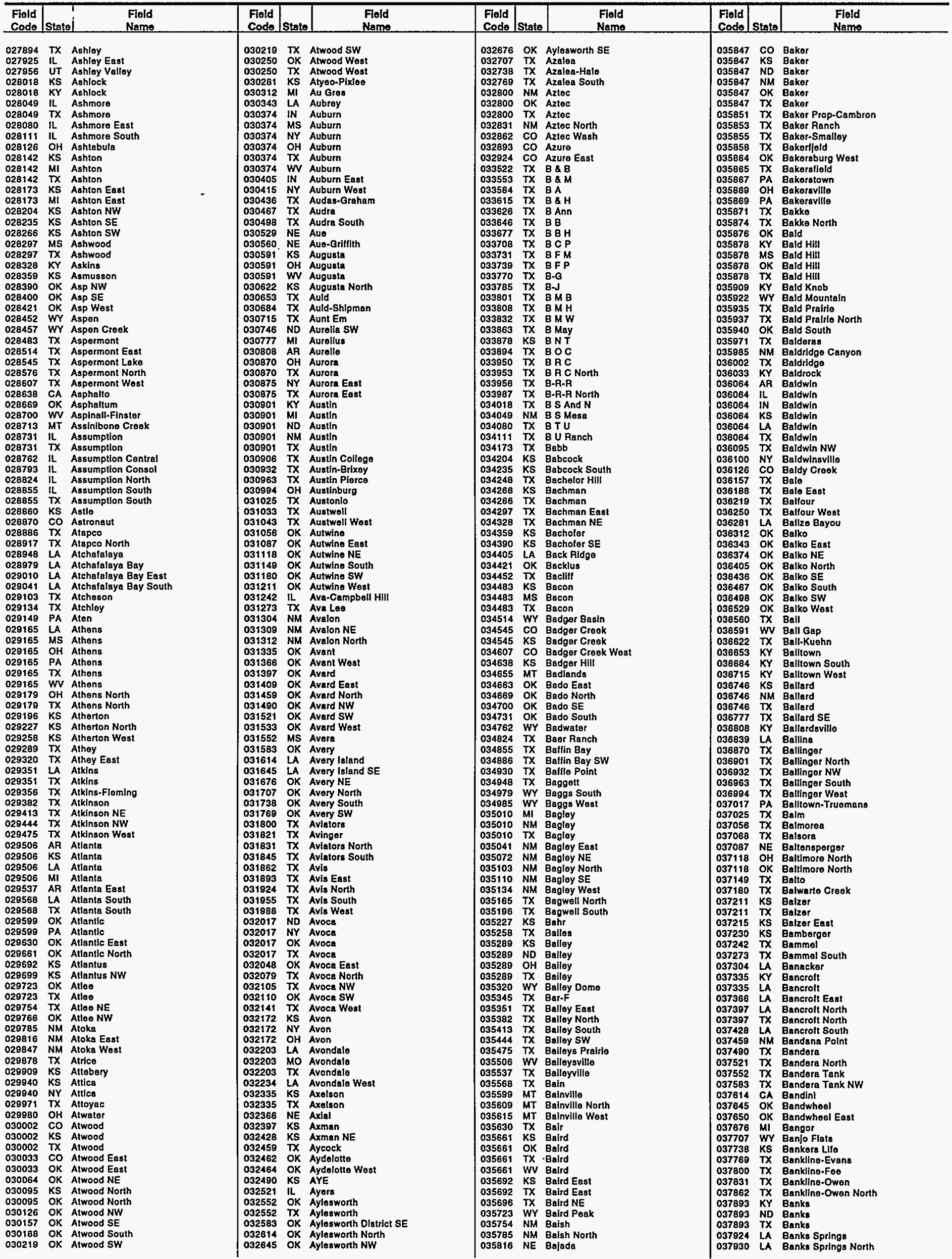


048457

\begin{tabular}{|c|c|c|c|c|c|c|c|c|c|c|c|}
\hline $\begin{array}{l}\text { Fiold } \\
\text { Codo }\end{array}$ & State & $\begin{array}{l}\text { Fiold } \\
\text { Namo } \\
\end{array}$ & $\begin{array}{l}\text { Flold } \\
\text { Code } \\
\end{array}$ & Stato & $\begin{array}{l}\text { Fiold } \\
\text { Name } \\
\end{array}$ & $\begin{array}{l}\text { Fleld } \\
\text { Codo }\end{array}$ & State & $\begin{array}{l}\text { Field } \\
\text { Name } \\
\end{array}$ & $\begin{array}{l}\text { Flold } \\
\text { Code }\end{array}$ & State & $\begin{array}{l}\text { Fleld } \\
\text { Name } \\
\end{array}$ \\
\hline 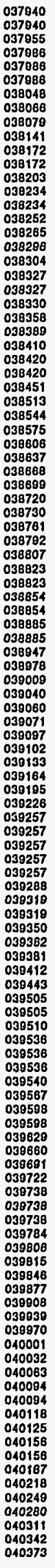 & 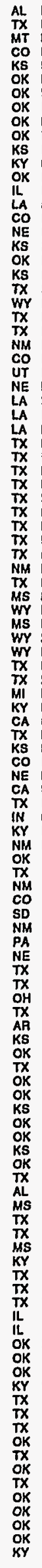 & 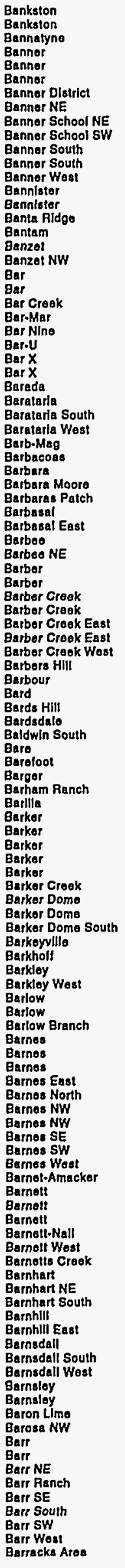 & 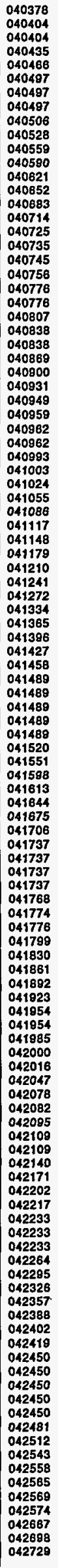 & 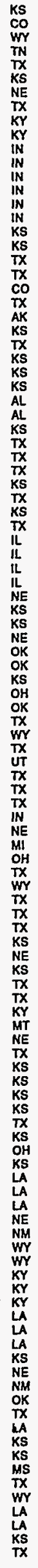 & 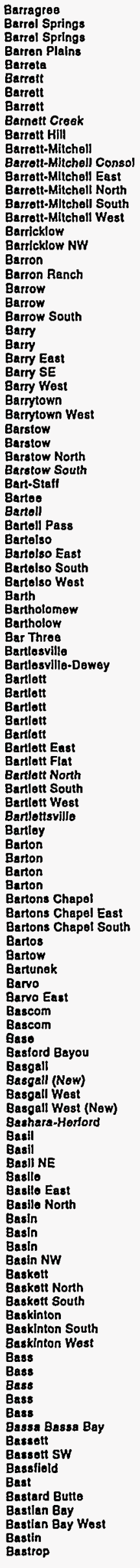 & 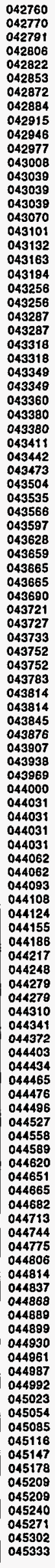 & 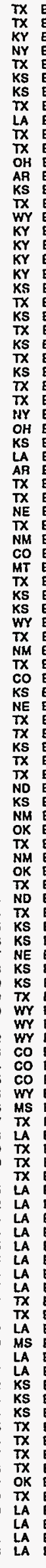 & 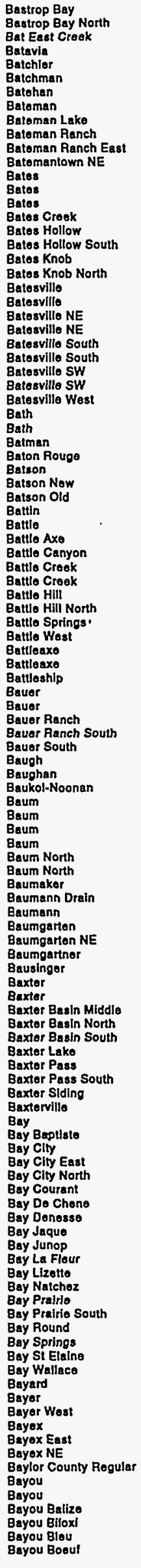 & 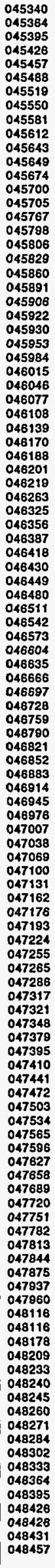 & 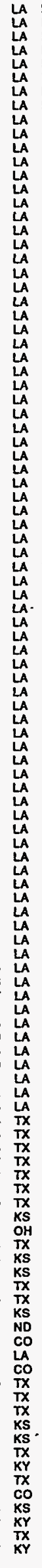 & 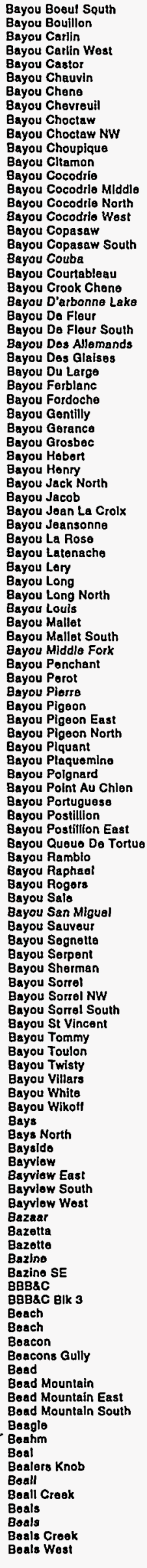 \\
\hline
\end{tabular}

Energy Information Administration/Oll and Gas Field Code Master List 1994 


\begin{tabular}{|c|c|c|c|c|c|c|c|c|c|c|c|}
\hline $\begin{array}{l}\text { Fleld } \\
\text { Codo }\end{array}$ & State & $\begin{array}{l}\text { Field } \\
\text { Name }\end{array}$ & $\begin{array}{l}\text { Fiold } \\
\text { Code }\end{array}$ & State & $\begin{array}{l}\text { Field } \\
\text { Name }\end{array}$ & $\begin{array}{l}\text { Fiold } \\
\text { Code } \\
\end{array}$ & State & $\begin{array}{l}\text { Fild } \\
\text { Name }\end{array}$ & $\begin{array}{l}\text { Fiold } \\
\text { Code }\end{array}$ & State & $\begin{array}{l}\text { Flold } \\
\text { Name }\end{array}$ \\
\hline 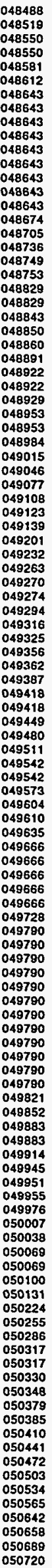 & 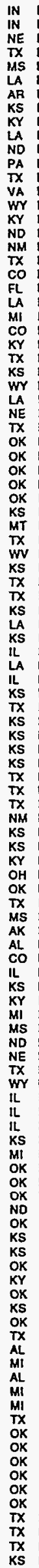 & 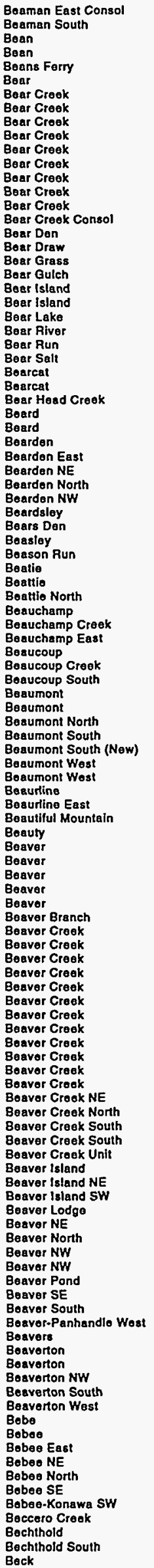 & 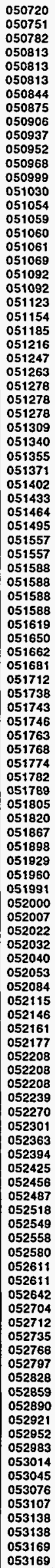 & 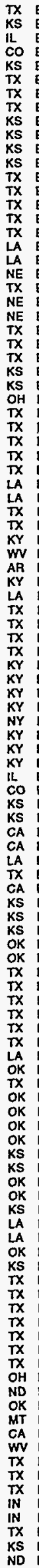 & 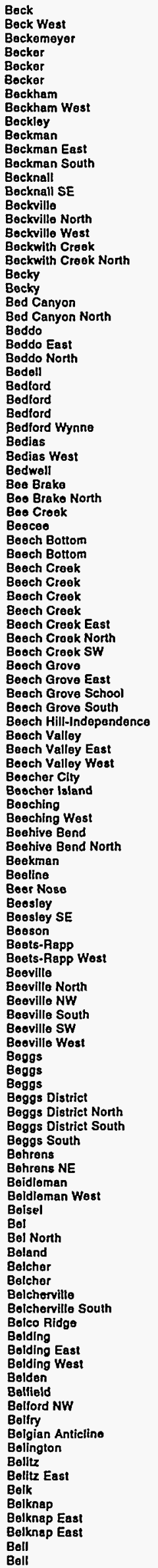 & 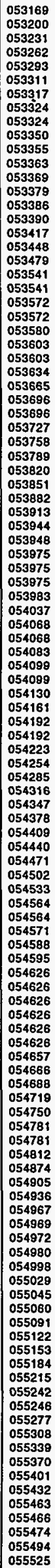 & 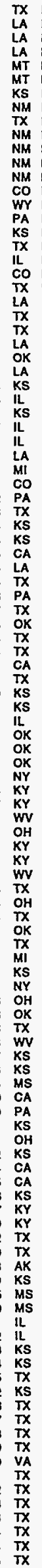 & 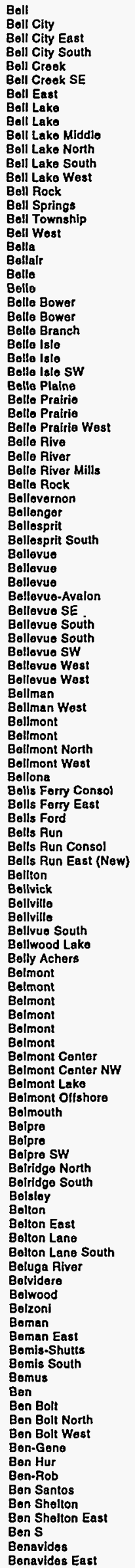 & 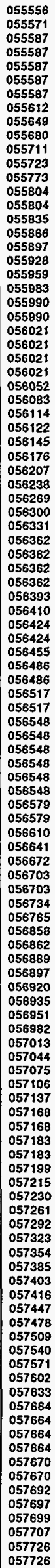 & 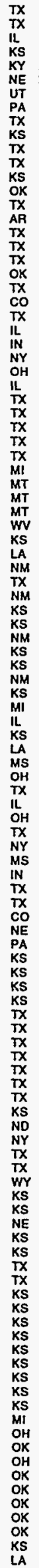 & 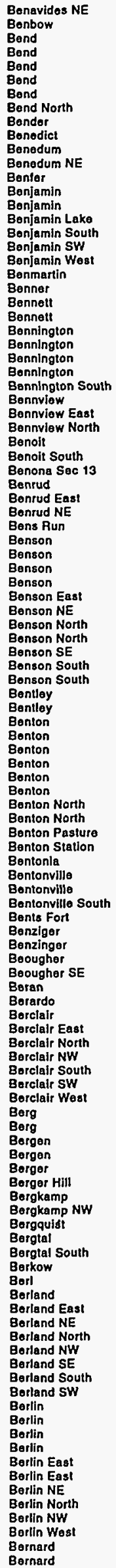 \\
\hline
\end{tabular}




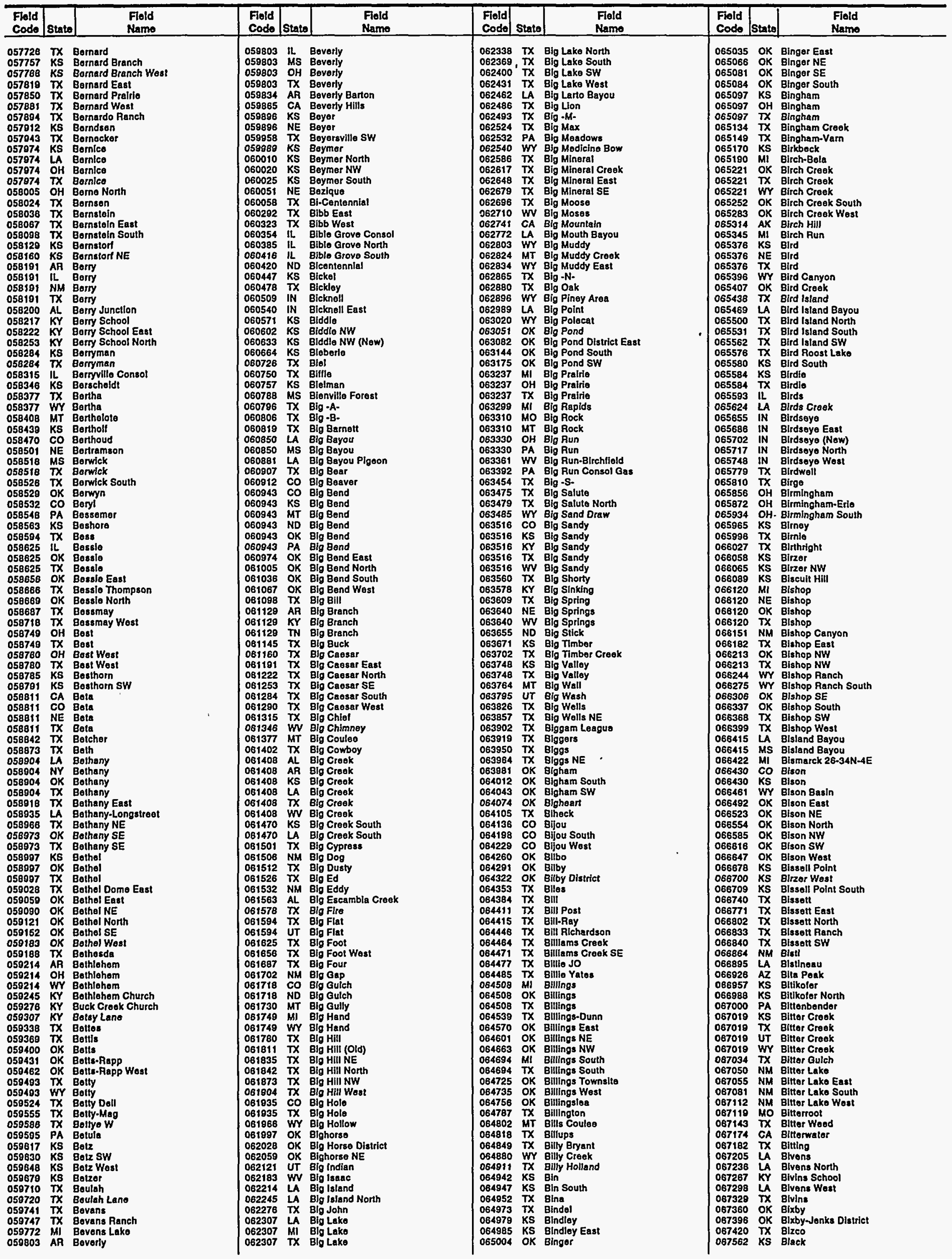




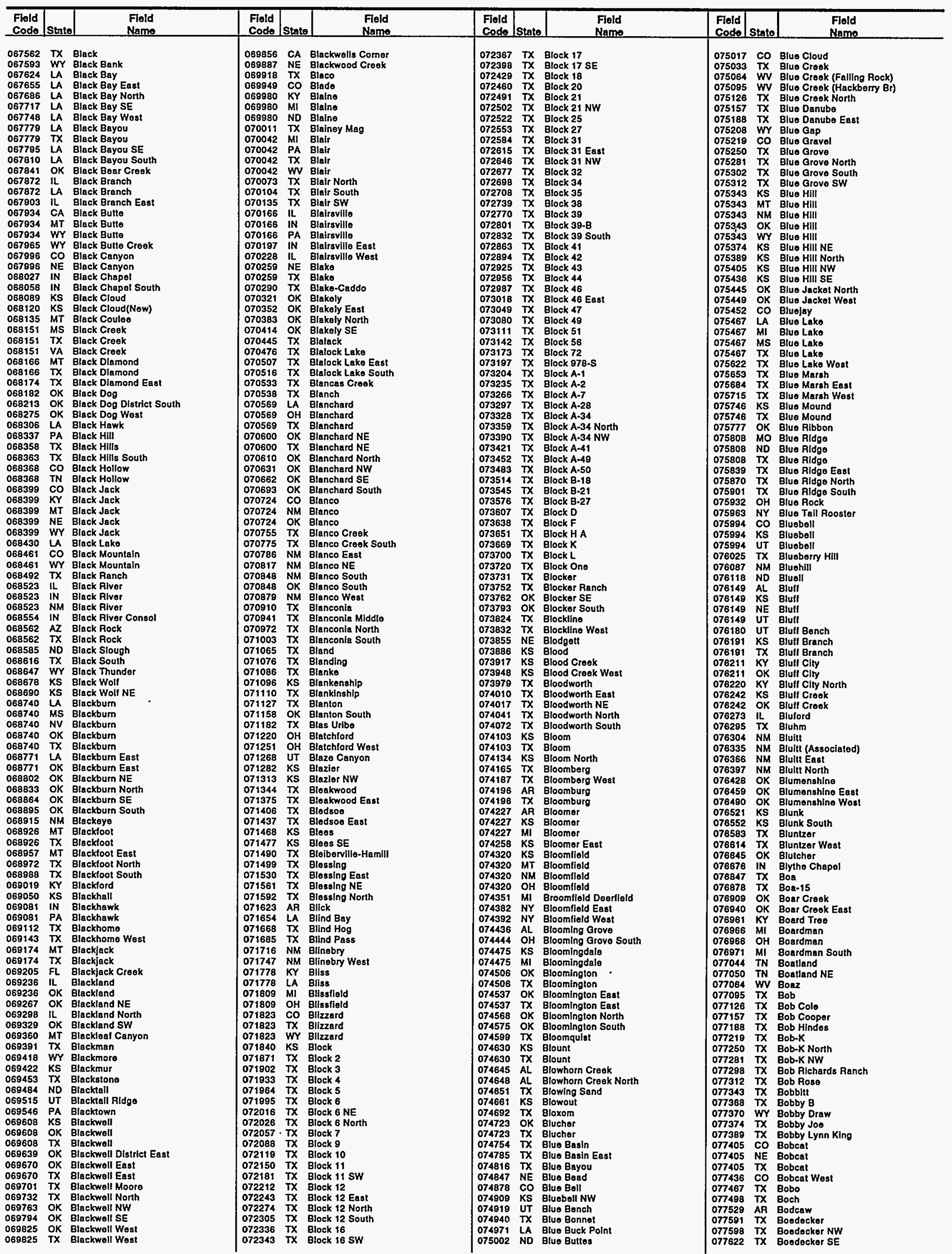


087682

\begin{tabular}{|c|c|c|c|c|c|c|c|c|c|c|c|}
\hline $\begin{array}{l}\text { Fiold } \\
\text { Code }\end{array}$ & Stato & $\begin{array}{l}\text { Flold } \\
\text { Name }\end{array}$ & $\begin{array}{l}\text { Fiold } \\
\text { Codo }\end{array}$ & State & $\begin{array}{l}\text { Field } \\
\text { Name } \\
\end{array}$ & \begin{tabular}{l|} 
Field \\
Codo
\end{tabular} & State & $\begin{array}{c}\text { Field } \\
\text { Name }\end{array}$ & $\begin{array}{l}\text { Fiold } \\
\text { Code }\end{array}$ & State & $\begin{array}{l}\text { Fiold } \\
\text { Namo }\end{array}$ \\
\hline 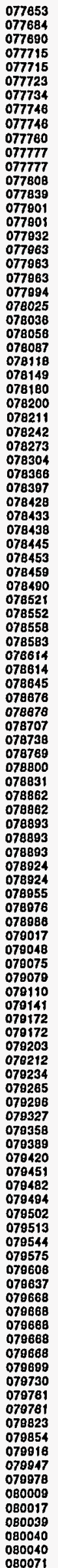 & 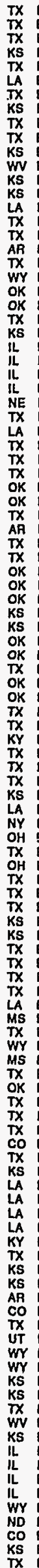 & 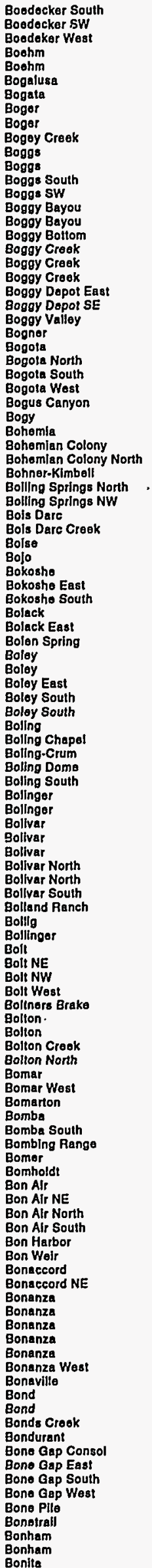 & 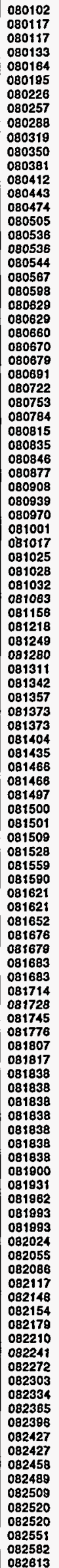 & 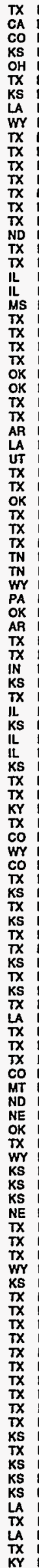 & 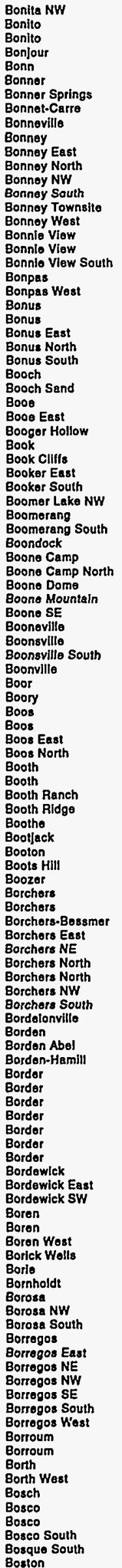 & 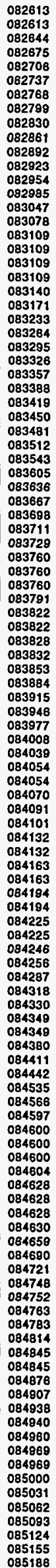 & 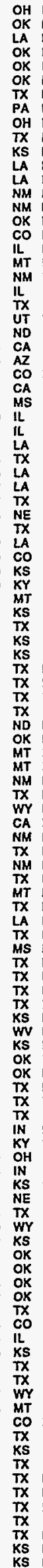 & 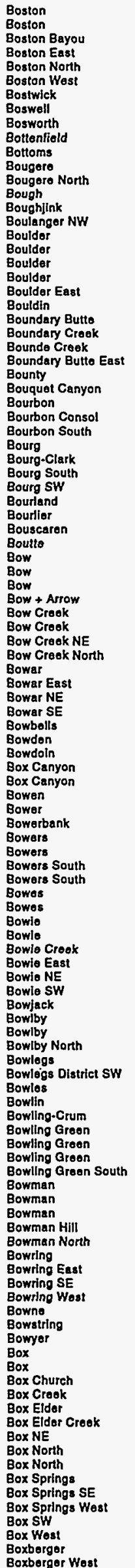 & 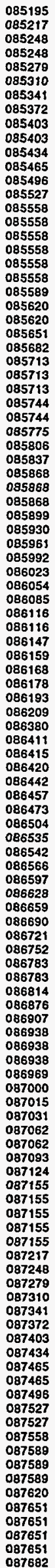 & 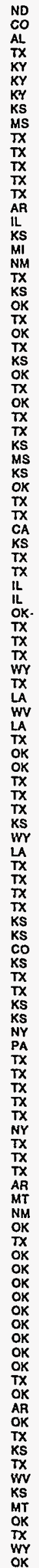 & 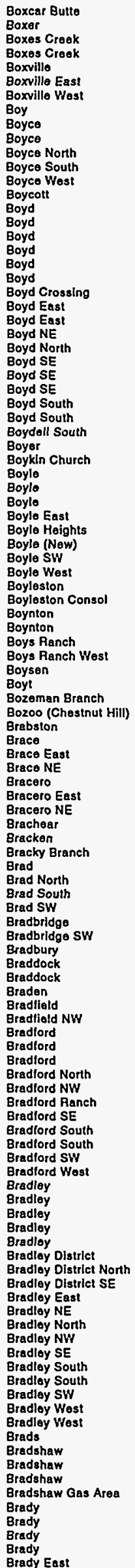 \\
\hline
\end{tabular}




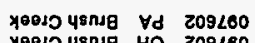
Y0015 पूu HO 209280

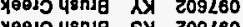

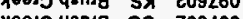

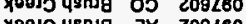

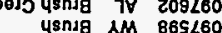

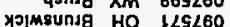
4INOS UOEUnA WIN OOSL6O YOUEY UOBUAS $X I$ EISLEO

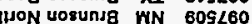
1803 UOSUn OSUNA WN $\angle D T \angle B O$ JN oung $S X$ Sit $\angle 60$ ouns $X \perp \quad s 8 \varepsilon \angle 60$ oung Ho $58 \varepsilon \angle 60$ oung $5 x$ SBEL60 1 as luns $X \perp$ EZEL60

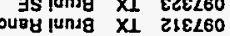
4HON IUNA $X_{1} \quad 262 \angle 80$ 1883 jund $X \perp \quad$ 192L60 Iundg $X_{1}$ OEZL60

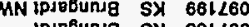

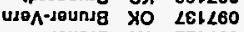
Jound $X 1$ ZZ1 $\angle 60$ unos ebapung $S \times$ SOL 260 OBepuns SX SLOL60 4LON WWRG $S x$ 6SOL60 wwns $S x$ tro<60 $M N$ Koquns $X$ रopuns $X_{1} \quad 286960$ Koperns $s \times 286960$

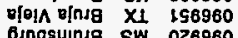
QSU!nja SW 026960

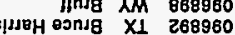
oly-eэnja $X \perp$ t88960 1983 o5ns yo 898960

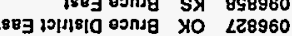
องกง XI $98 \angle 960$

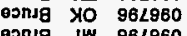
oรng IW $96 \angle 960$

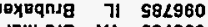
JPW-nJ $X_{1} \quad \$ 92960$ 801रodg प्र० bEL960 80jरodg yo 802960 180M U01X018 YO $Z 29960$ MS volxodg X्रO $\$ \$ 9960$

4nos uopxodg yo 019960

MN uolxosa yo 6LS960 180M poomumoja $X_{1} \quad 865960$ 4.

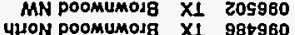
4LON POомUMолg $X_{1} 985960$ роомuмогg $X_{1}$ tZt960

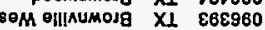

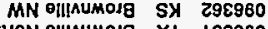
\begin{tabular}{lll} 
Ollnumojg $X \perp$ & $L \varepsilon \varepsilon 980$ \\
\hline &
\end{tabular} DIjAumos $5 \times 008960$

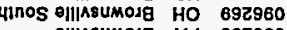
ellinsumodg $\forall d 8 \varepsilon 2960$

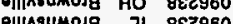
(1) owor vosumas $X_{I} \quad 202960$ 8seuew-oppeumos

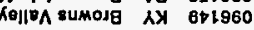
II SUM IIIH sumos XM OZI960 1883 sumas S Xoes sunos 001nоo sumos IN Z50980 sumore HO 120960 oejumos $x 1$ OBBSE0 oojumose $X 1$ o8sse puriumora SW คөppra-bujumoig $X I$ BZ6560 Bujumose $A M \quad \angle 68960$ BuIUMOJ日 $X I$ L68SBO BujuMOLE AN L68S6O BujuMOJ日 AX L68560 Bu!UAOJ $S X \quad$ L68S60

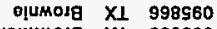
yinos pjojfumore $X_{\perp}$ se8560 3S ploljumore $X 1$ t08S60 I0UMOJ日 XI ELLS60 IlOUMOL $S X$ EL ELSBO 280M UMOJ日 त्र० Z๐ $\angle S 60$ MS UMO s!bInis-uMOLG SX 089S60 4inos umose Yo 679560 49U8Y UMOIE XI 955580

puod UMOse NI SZSS60 MN UMOS日 YY \$6t560 OPPPIW UMOS XO SLtS60 Нөчगुष

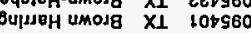
1803 uм०s $x_{0} 0 \angle E S 60$

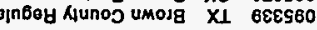
YHON HOSSBg-UMOJ日 $X \perp$ LLZS60
H0888g-umosg XI 972560 190M OdJO4L + UMOJG XI SICS60 4poN ad1041 + umasg $X \perp$ zटIS60 ImOJ日 $\quad X_{1} \quad 160560$ 4M018 X్ 160560 UMOJ日 WN 160560 uMOLg IN 160560 mose IN 160560 unoje $71 \quad 160560$ puesnos $x_{1} 090560$ $\begin{array}{rrr}\text { plessnoig } & \forall 7 & 090560 \\ \text { ujnos uojuชnojg } & 71 & 620560\end{array}$ 4HON UojuBnod s sर E10560

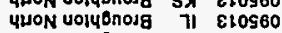
volyBnosg $x \perp \frac{11}{88680}$ volubnos $\quad \mathbf{s} x \quad 866760$ vol4Bnosa 71 866+60 X001] मO41019 XI 296760 snj802 $X_{1} \quad 986 \$ 60$ 4inos vosiole IW $508 p 60$ vossosg IN $t<8 t 60$ pjollwood IN Erar60

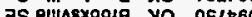
IS olfnsxoodg xo $05<660$ 18Bב 9Hasxoos $\times 0$ 8

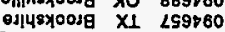
OJnqsy0os $\mathrm{NI} 929760$ 106unOR-sY0019 SX S65t60

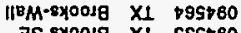
IS sश001 $X_{1}$ EESt80 4InOS *xo01 $X_{1}$ EEst60 4InOS $8 \times 0019$ Sx 2 ESt60

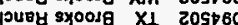
पLON 3 X0018 $X_{1} \quad 1<\$ 760$ Qurod 8x0018 $x_{1}$ 60\%\$60 sXo0دg $X_{1} 8 \angle E P 60$ sx0019 OW 8LE760 Sx00J8 SX 8LCt60 J日AOXOOJ日 HO LVEP6O nos MBjYO019 $X X$ LEEt60 MEIX0018 XI 91E\$60 sulyoos Xo sazr60

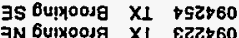

$\exists N$ Guikoosg $X_{\perp} \quad \varepsilon Z Z 760$

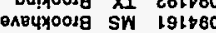

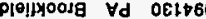

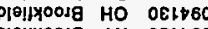
Ple!ly 1803 41\}เ158\%0018 $X_{1} 660 t 60$

$\begin{array}{lll}X 1 & 890760 \\ 409000 & X 1 & 750560\end{array}$ जoxpoug XO $\$ 50 \% 60$ as ęuos $X I \quad 900860$ पHON OIUOJ8 $X I \quad S \angle 6860$

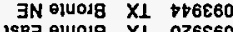
ISB] eluod XI $X 26860$ өyuodg XI E16860 8!ueX-vosuojg $S$ X 288860 تS vosuosg 5 Sर 158660 I80M ovuos $\mathrm{HN}$ OZBE60 I803 05001 $X_{1}$ 8SLE60 0000د $X$ oovoug $X 1$ LZLE60

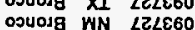

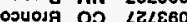
गөмшолg 5 र $989 \varepsilon 60$

Do7 vox NL s95e60 MO8 uexosg oD Lt9860

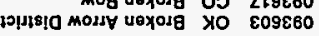
MorH vexoJg xo ZLSE6o 158M x

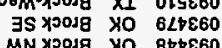
पMON YOOJg XO LL\$E60 1883 Y9018 XO $988 \varepsilon 60$ Y2019 $X_{1}$ SsCE60 Xण0। Y्रO SSEE60 yण0dg SY SSEE60 190M Rөмpвodg SX्र $\forall Z E \varepsilon 60$

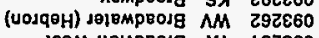
ISOM Mอ!یPвOAg $X_{\perp} \quad 1 \varepsilon z \varepsilon 60$ 4inos ejdd! PBodg NI 69Lع60 oldd!y peos NI $8 \varepsilon 1 \varepsilon 60$ OدB XI LOLE60

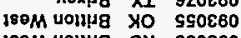

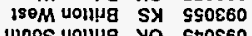
4inos voln!l yo stoe60 MN Uon!ug SX 620860 4นON UOHम $X \perp$ 10860 4HON VOIIIS XO \$10E60

$\exists N$ Uolyयg XO E86260

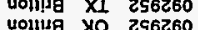
บol!! $S \times$ ZS6260 HII $X 1$ LI 126260 मแ⿰

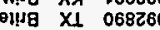

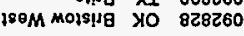

I80M MOIsน" NI 8Z8Z60 0! SUMO1 Molsyg Xo $\angle 6 \angle Z 60$ 4inos Molspla NI 99L260 4LON MOISL8 Xo SELZ60 4LON MOLSHg NI SELZ60 JN M입 Xo $\$ 0 \angle 260$

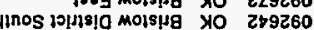
4HON JOHSIO MOISH Y YO 139260 molsyg tyo 085z60 m이냅 $\mathrm{NX} 085260$ morsug ojsid iosug $X 1$ 6\$5260 iojsing HO 6 tSZ60 pis?ug AN $6 \$ S Z 60$ Jolsts $5 \times$ 8tSZ80

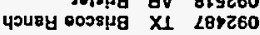
cosspg $X_{1} \quad 08>260$ गयद $X 11920 Z 60$

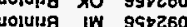
oolsujuup WN $9 \varepsilon \downarrow 260$ 4inos uвuxyula xo szbz60 Hочнхuн $\exists N$ \$6EZ60

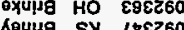

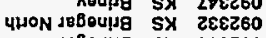
LeBouj! SX 508260 मะपा!! $x_{1}$ 0LZZ60 उS JS Rolpg $X_{1} 802260$

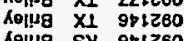
unos MN 1400 SX $\$ 80260$ 4นON 14614 SX ESOZ60 $18031461 \mathrm{~s}$ sX 220260 146148 iM 096160 146149 $\mathrm{BN}$ 096160 150M хорриен-лөмрня $X_{1} \quad 9 \varepsilon 8160$

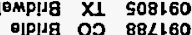
8Bpug $x I \$ 2<160$

Oxष7 JOBpyg in E\<160 ISOM LOdo6pis Y YO 6ELL6O MS uodobply त्रo 9ZL160 uinos HodeBpyg XI ZLLL60 4nos hodeBpy to $21<160$ 14inJd-HodeBppg $M M \quad 189160$ 4นON HOdeBpHg $X_{1}$ OS9160

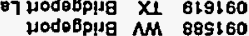
HOdoBpH $A M$ 88S560 Hode6p19 $X_{1}$ 88Sเ60 Hode6pla त्र० 88S160

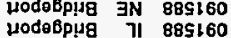
7001כ o8plag is $\angle S S 160$ 4RON A!J e6p!g XI TESI60 A:15 eBpHa $X_{1}$ 9zsL160

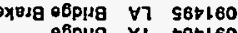

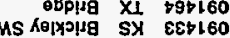
1983

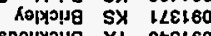
SกOYพगम XI O†Es60

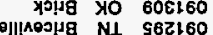

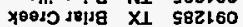

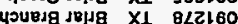
JOISMOSg HO L†ZL6O unos semosg YO 912160 J8Mosa $X_{1} 581180$ 4010J日 ix $\$ 5150$

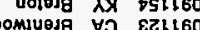
poomives Iบ9נ8 XI 260160

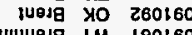

บ1988 Bupuues MM 190160

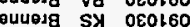
บвшөบuesa $\mathrm{MM} 666060$ puвuvesg XI 896060 wollos usuUes in $2 \varepsilon 6060$

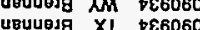

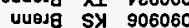
Sy०पणQد日 HO SL8060 шв4यед XI $\$>8060$ แष्पued Sत्र $\$ \$ 8060$ บow日s HO عl8060

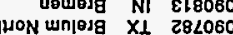

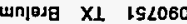

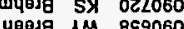

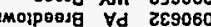
Oा!nspoesg IN L29060 ISOM OAOIPOOSG $X_{1} \quad 965060$ 4inos onop료 $X_{1}$ S9SOBO IS OAOpp8018 $X_{1}$ tes060

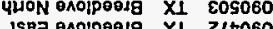

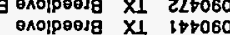
Bu!poesg $A Y$ Ott060 


\begin{tabular}{|c|c|c|c|c|c|c|c|c|c|c|c|}
\hline $\begin{array}{l}\text { Flold } \\
\text { Code }\end{array}$ & State & $\begin{array}{l}\text { Fiold } \\
\text { Name }\end{array}$ & $\begin{array}{l}\text { Fiold } \\
\text { Codo } \\
\end{array}$ & State & $\begin{array}{c}\text { Field } \\
\text { Name } \\
\end{array}$ & $\begin{array}{l}\text { Field } \\
\text { Code }\end{array}$ & Stato & $\begin{array}{c}\text { Field } \\
\text { Name } \\
\end{array}$ & $\begin{array}{l}\text { Fiold } \\
\text { Codo } \\
\end{array}$ & State & $\begin{array}{l}\text { Fiold } \\
\text { Name }\end{array}$ \\
\hline 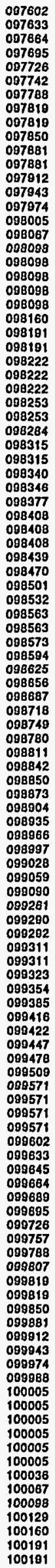 & 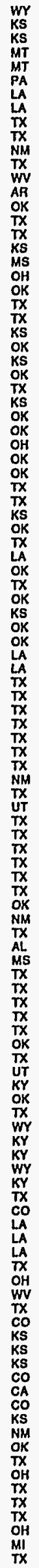 & 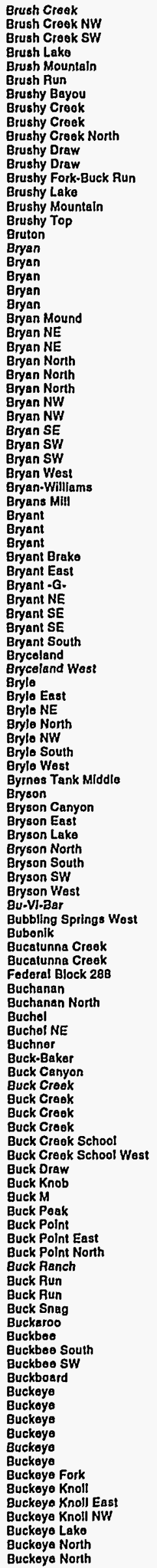 & 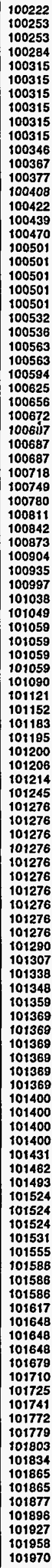 & 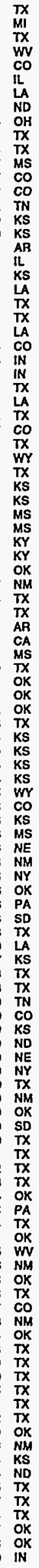 & 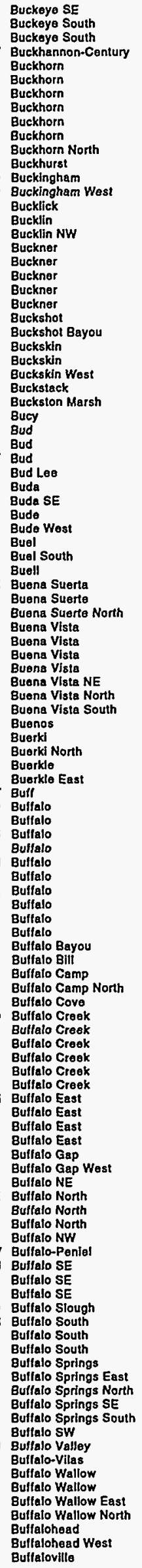 & 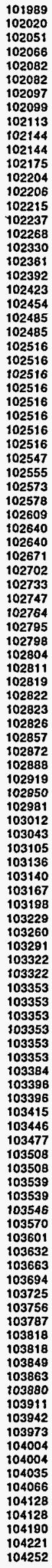 & 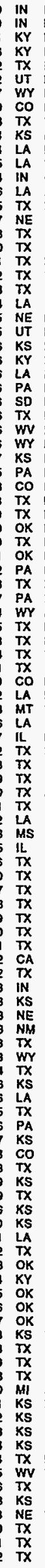 & 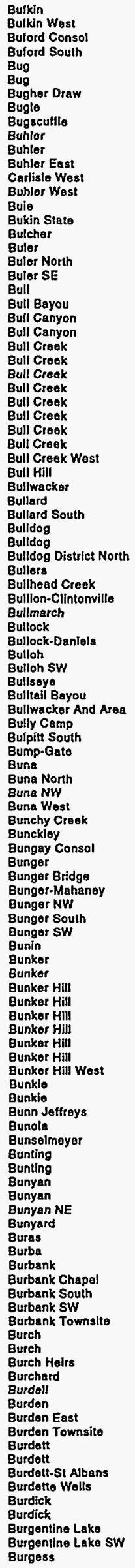 & 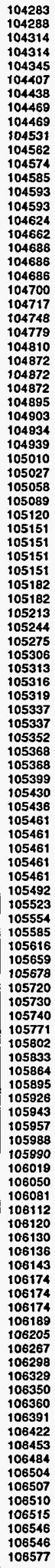 & 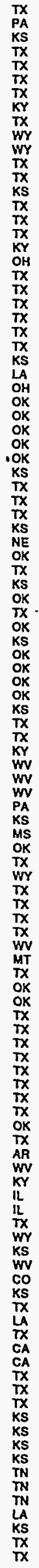 & 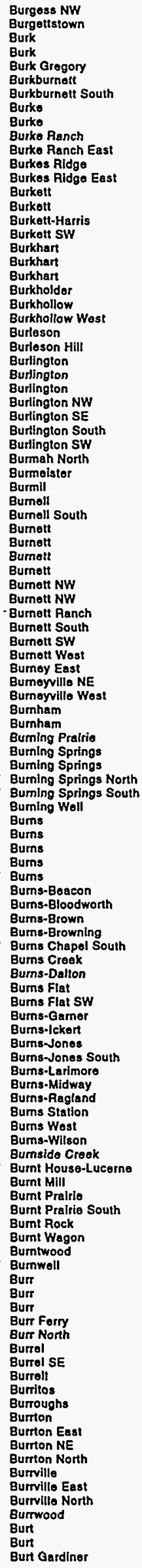 \\
\hline
\end{tabular}

Energy Information Administration/Oil and Gas Field Code Master List 1994 


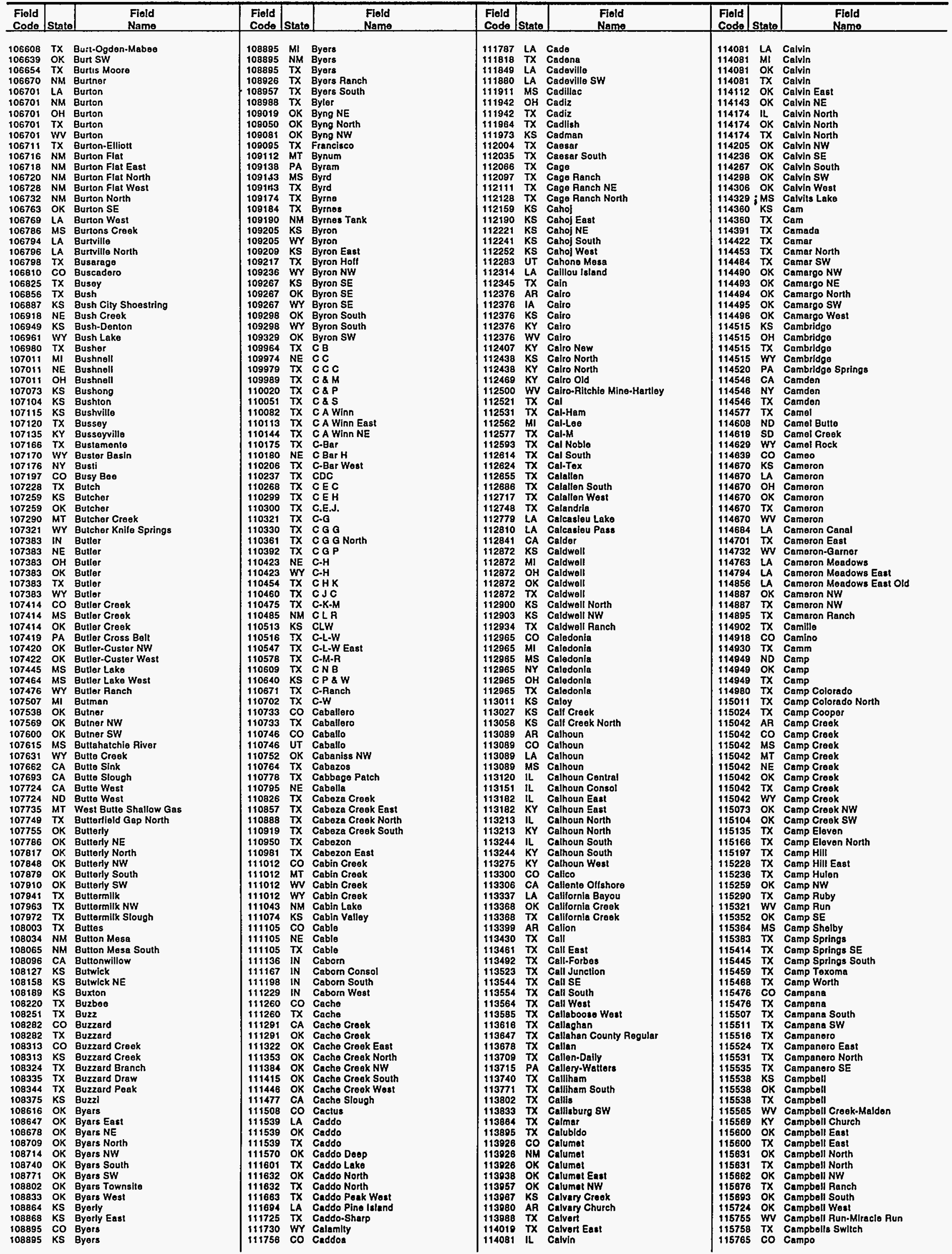




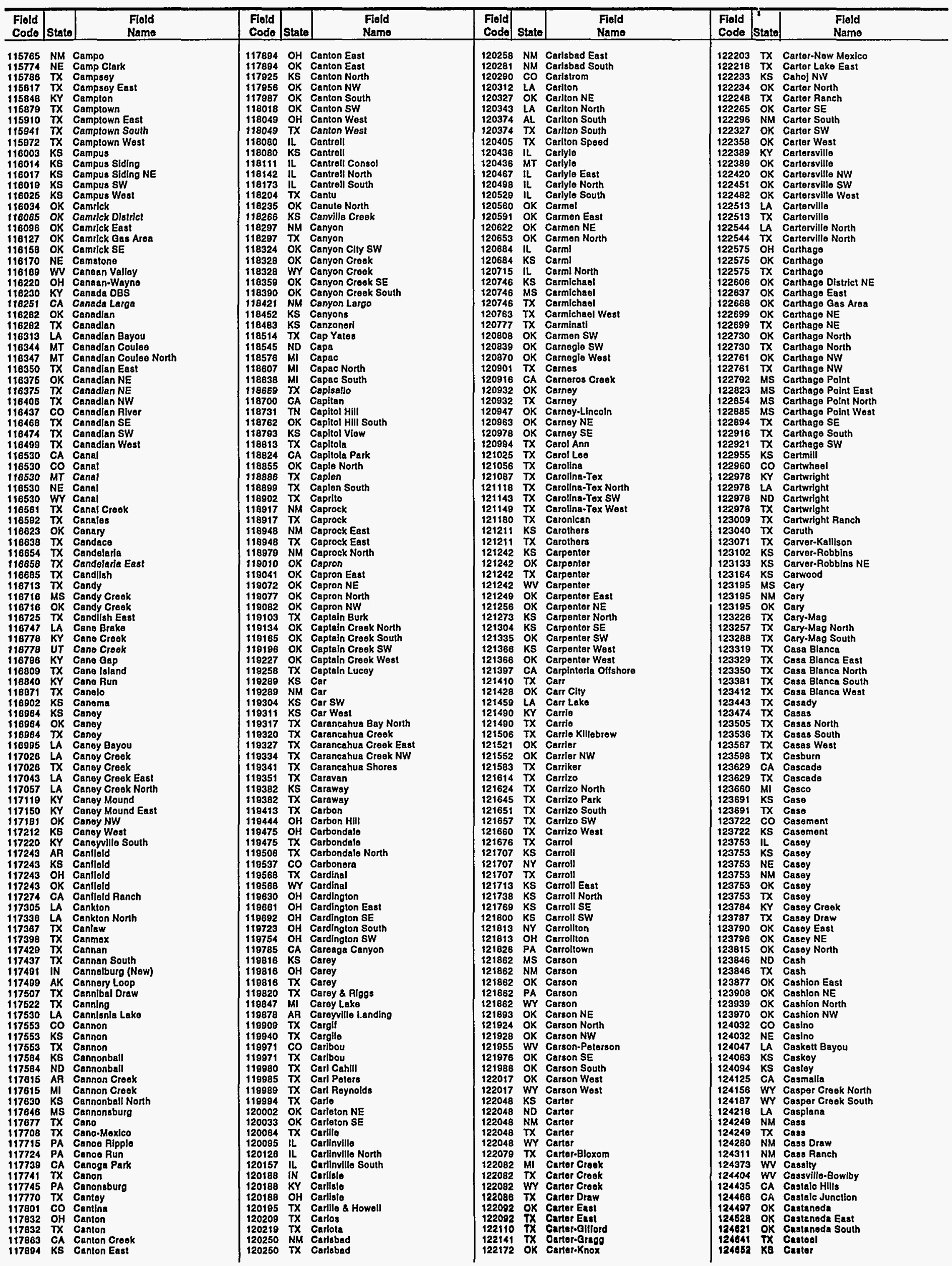




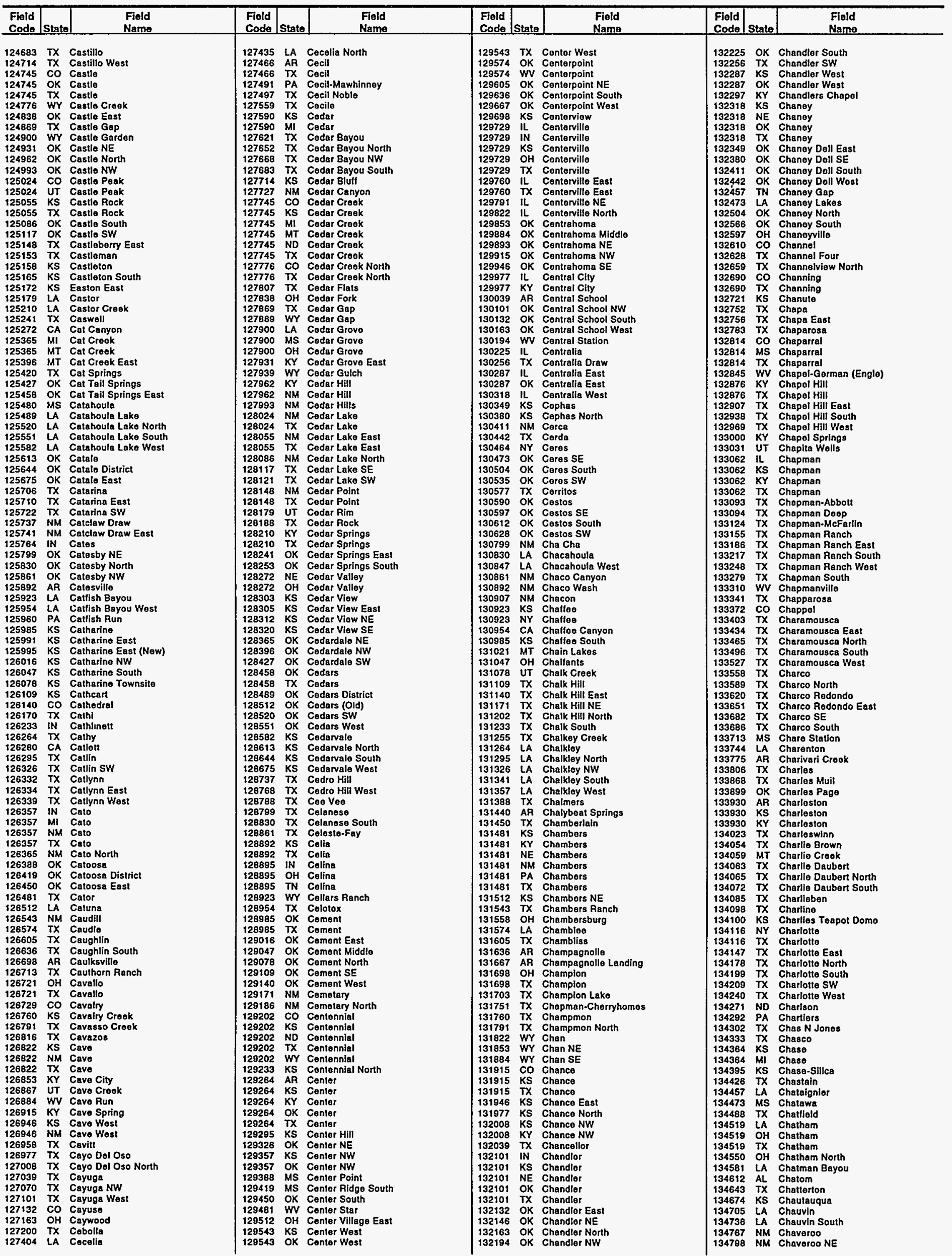




\begin{tabular}{|c|c|c|c|c|c|c|c|c|c|c|c|}
\hline $\begin{array}{l}\text { Flold } \\
\text { Codo }\end{array}$ & State & $\begin{array}{l}\text { Flold } \\
\text { Name }\end{array}$ & $\begin{array}{l}\text { Fiold } \\
\text { Codo }\end{array}$ & Stato & $\begin{array}{l}\text { Fiold } \\
\text { Name }\end{array}$ & $\begin{array}{l}\text { Flold } \\
\text { Codo }\end{array}$ & State & $\begin{array}{l}\text { Field } \\
\text { Namo }\end{array}$ & $\begin{array}{l}\text { Field } \\
\text { Codo }\end{array}$ & State & $\begin{array}{l}\text { Flold } \\
\text { Name }\end{array}$ \\
\hline 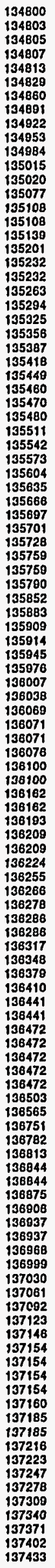 & 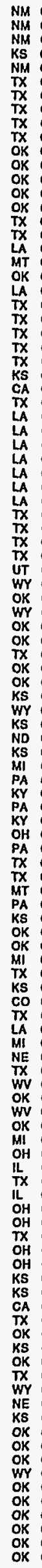 & 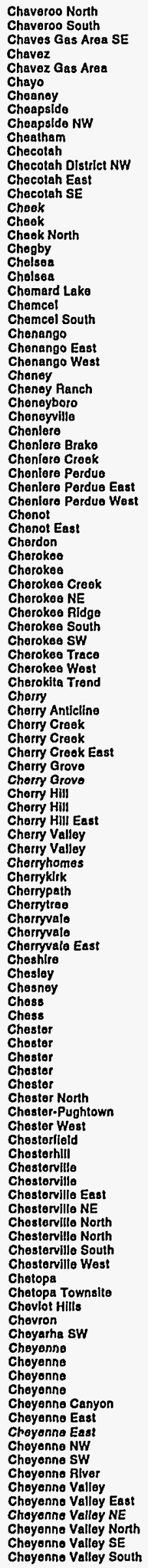 & 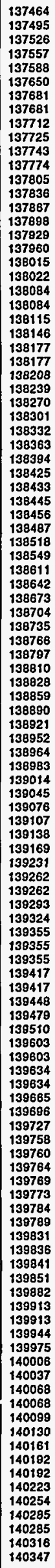 & 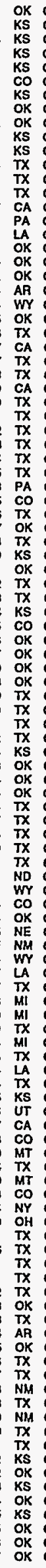 & 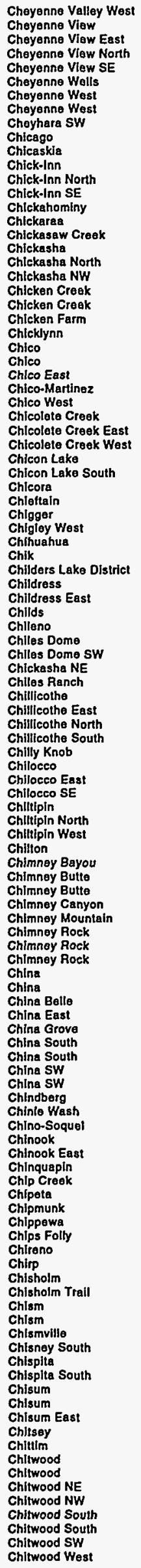 & 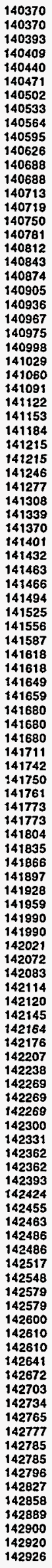 & 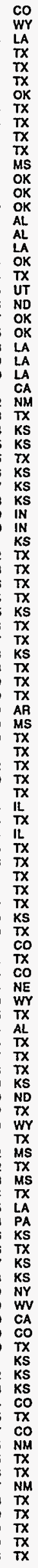 & 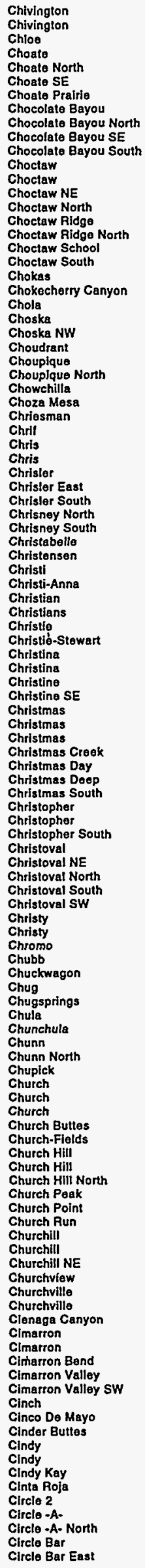 & 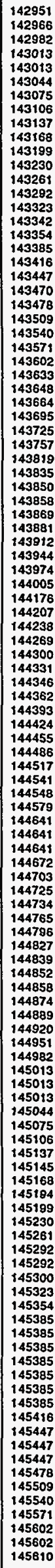 & 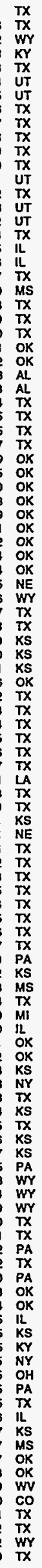 & 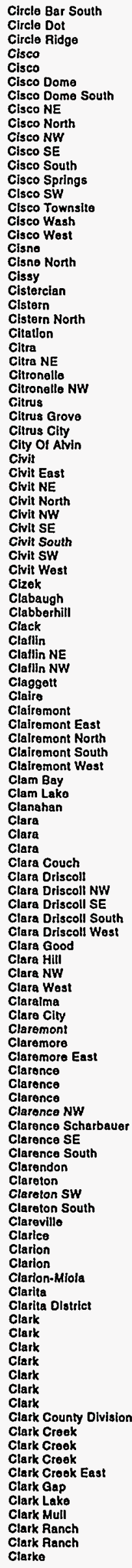 \\
\hline
\end{tabular}




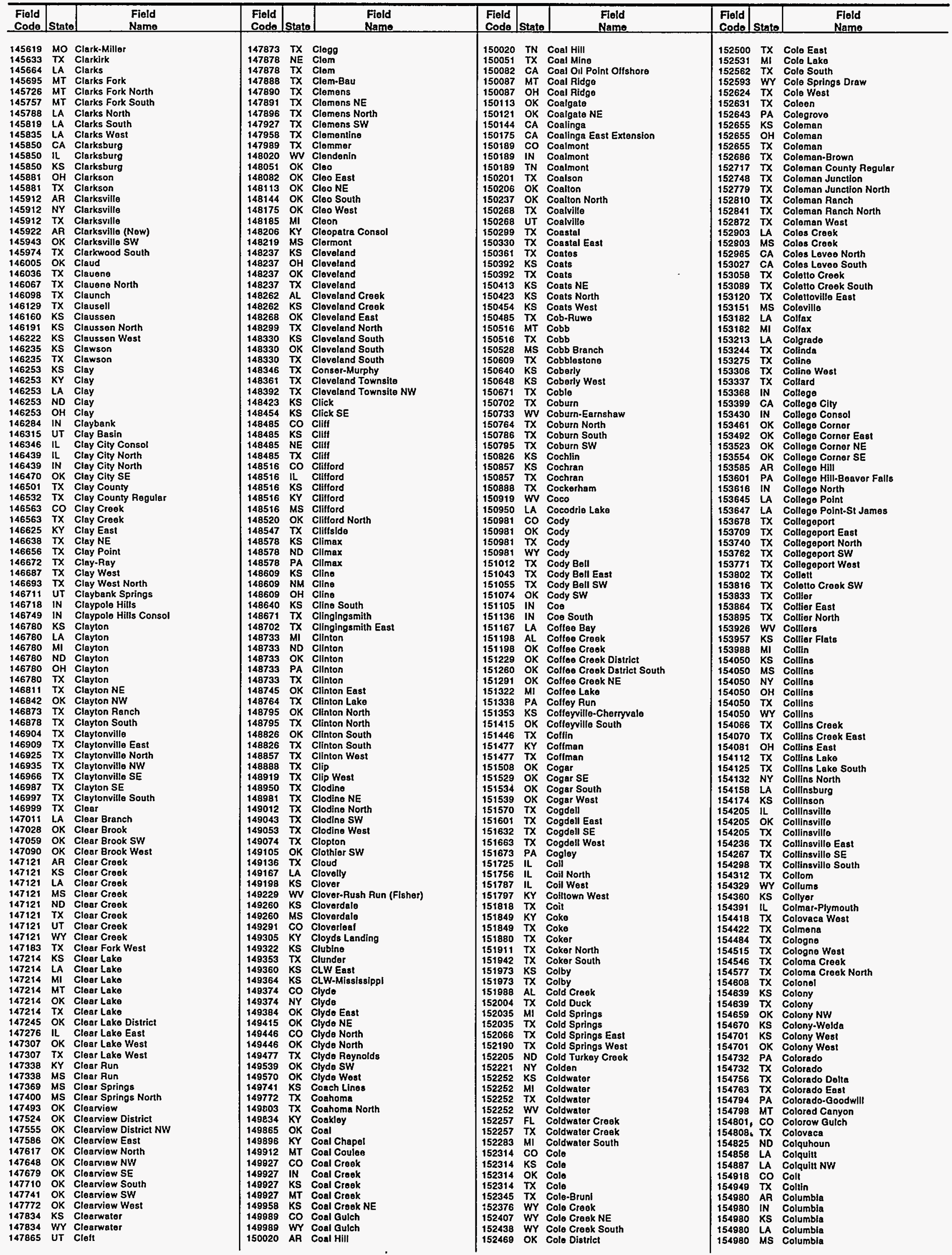




\begin{tabular}{|c|c|c|c|c|c|c|c|c|c|c|c|}
\hline $\begin{array}{l}\text { Fiold } \\
\text { Codo }\end{array}$ & Stato & $\begin{array}{l}\text { Fiold } \\
\text { Name }\end{array}$ & $\begin{array}{l}\text { Field } \\
\text { Code } \\
\end{array}$ & State & $\begin{array}{l}\text { Field } \\
\text { Name } \\
\end{array}$ & $\begin{array}{l}\text { Field } \\
\text { Code }\end{array}$ & State & $\begin{array}{c}\text { Field } \\
\text { Name } \\
\end{array}$ & $\begin{array}{l}\text { Fiold } \\
\text { Code }\end{array}$ & State & $\begin{array}{l}\text { Field } \\
\text { Name } \\
\end{array}$ \\
\hline 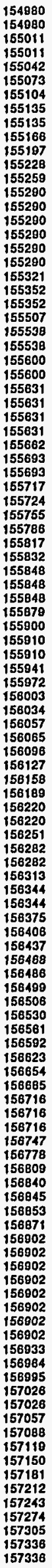 & 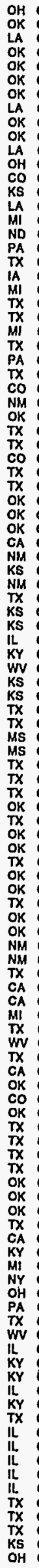 & 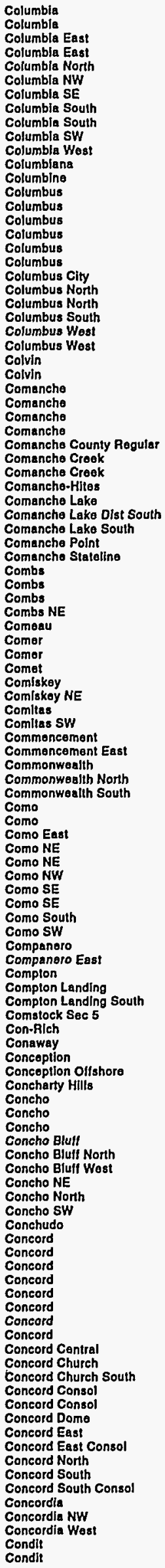 & 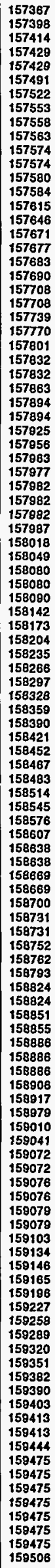 & 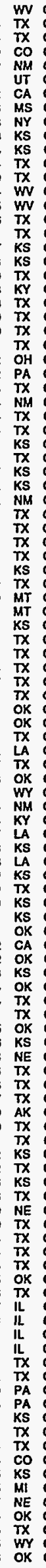 & 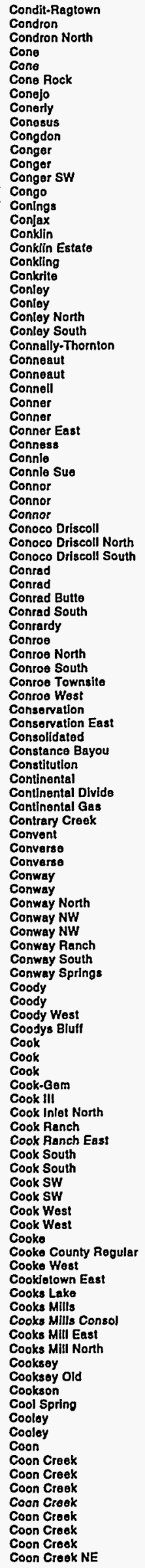 & 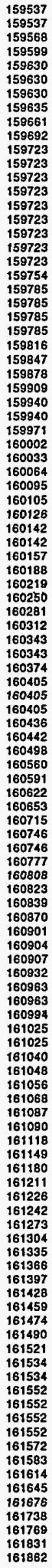 & 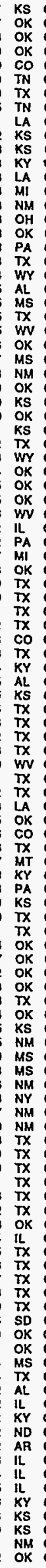 & 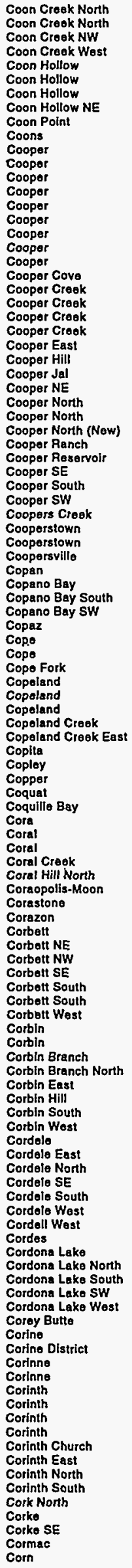 & 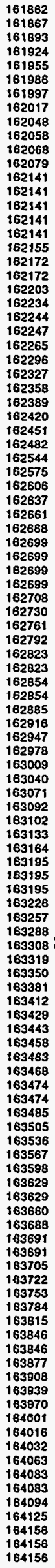 & 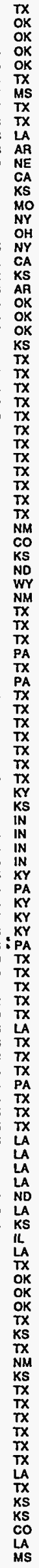 & 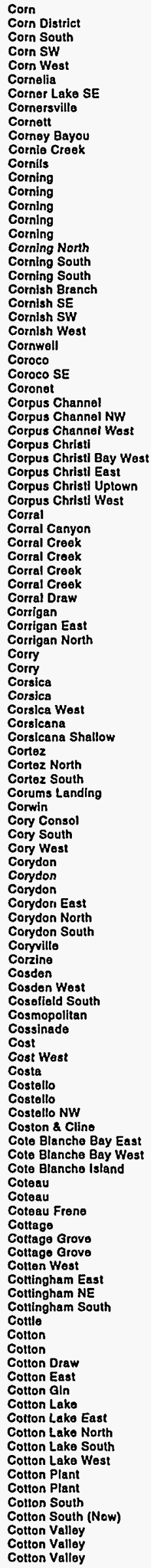 \\
\hline
\end{tabular}




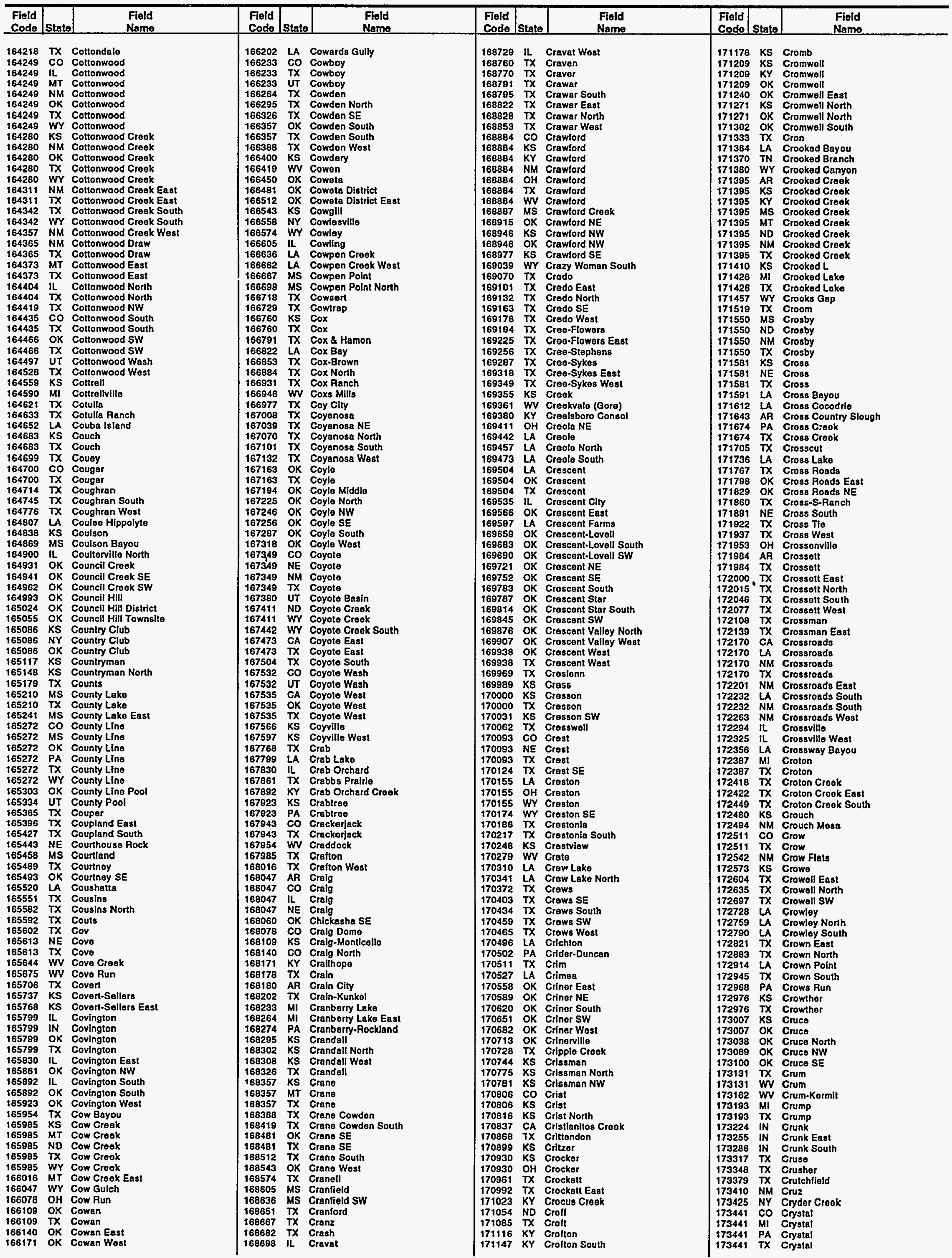




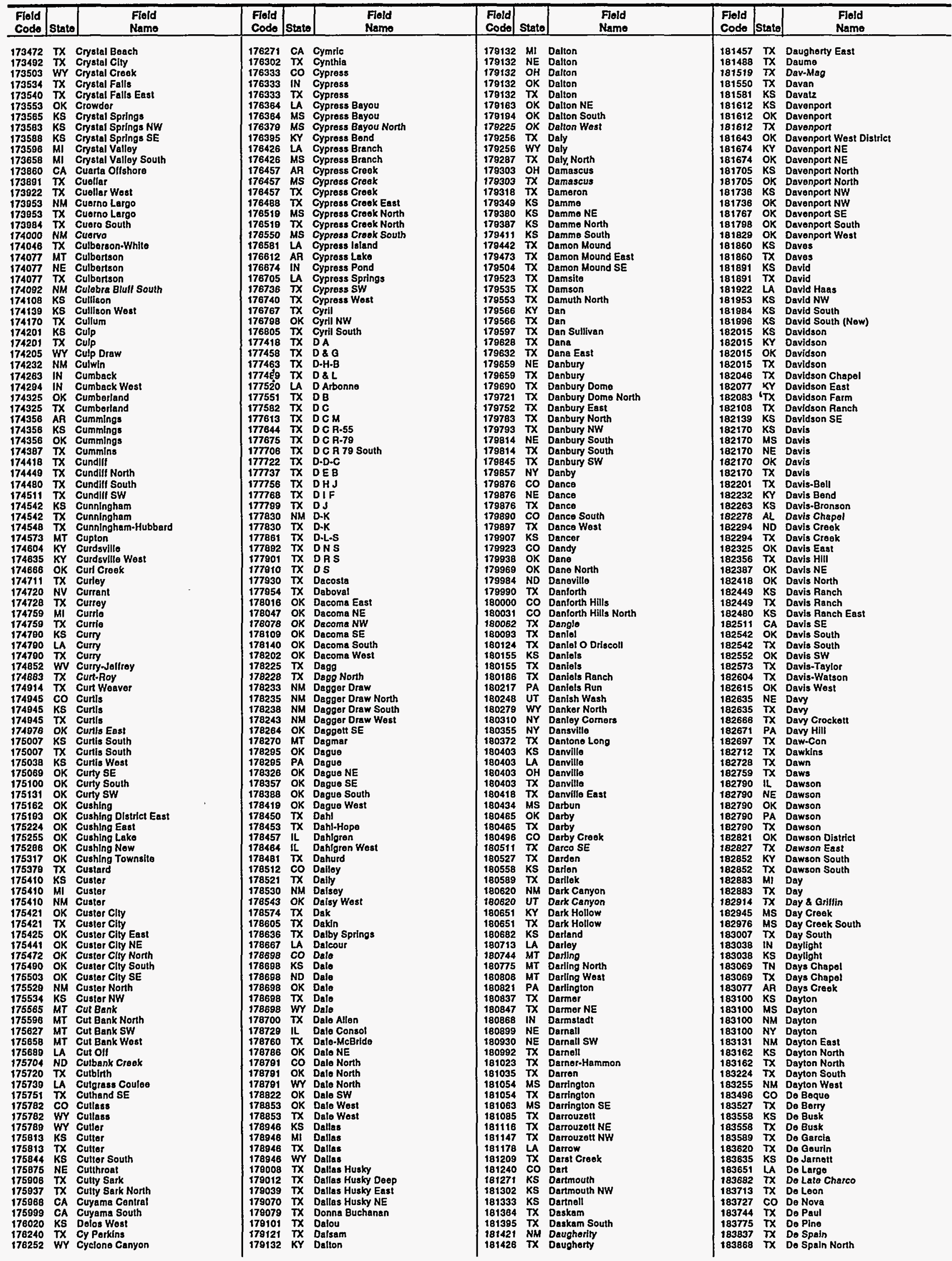




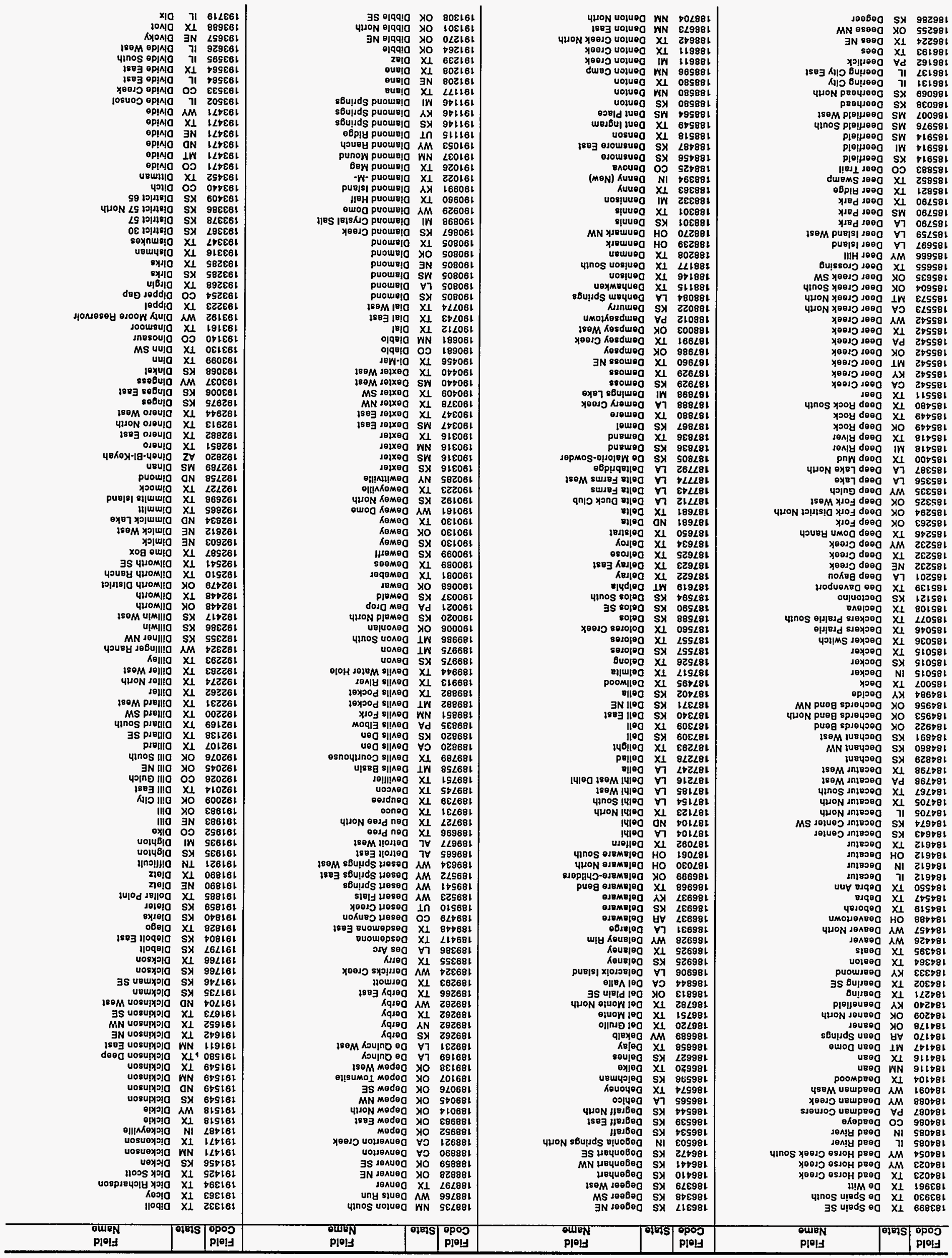




\begin{tabular}{|c|c|c|c|c|c|c|c|c|c|c|c|}
\hline $\begin{array}{l}\text { Fleld } \\
\text { Code }\end{array}$ & Stato & $\begin{array}{l}\text { Fleld } \\
\text { Name }\end{array}$ & $\begin{array}{l}\text { Fiold } \\
\text { Code }\end{array}$ & Stato & $\begin{array}{l}\text { Flold } \\
\text { Name }\end{array}$ & $\begin{array}{l}\text { Fiold } \\
\text { Codo }\end{array}$ & Stale & $\begin{array}{l}\text { Fiold } \\
\text { Name }\end{array}$ & \begin{tabular}{l|} 
Fleld \\
Code \\
\end{tabular} & State & $\begin{array}{c}\text { Field } \\
\text { Name } \\
\end{array}$ \\
\hline 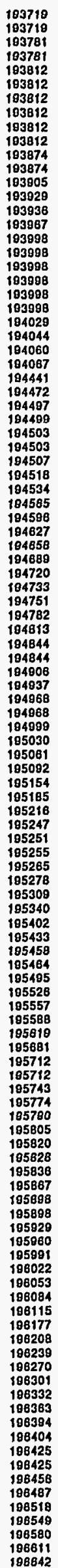 & 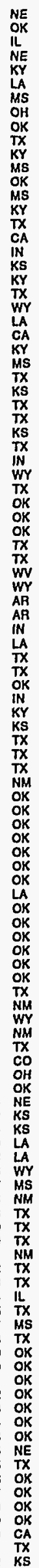 & 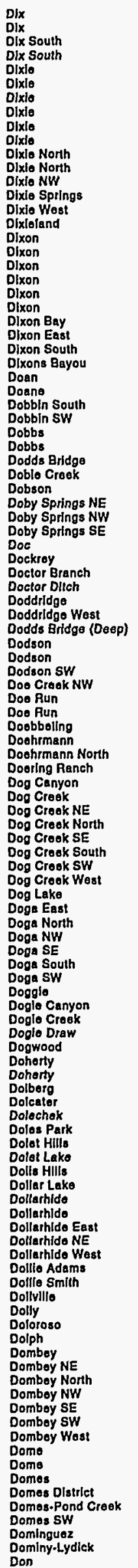 & 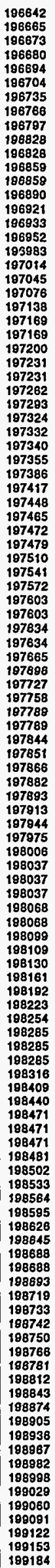 & 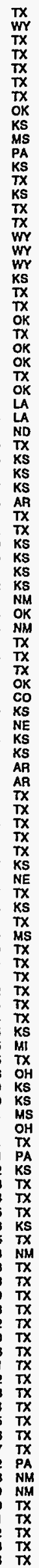 & 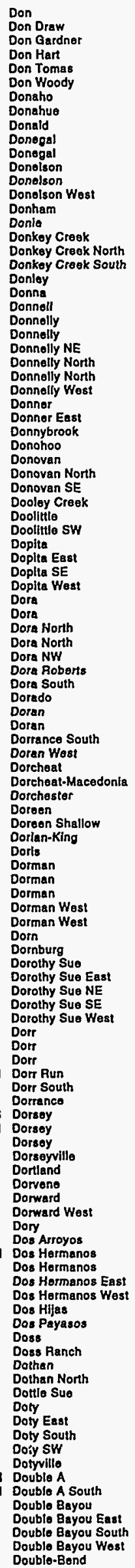 & 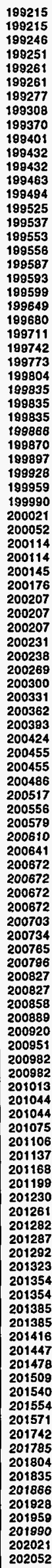 & 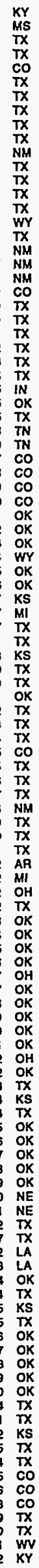 & 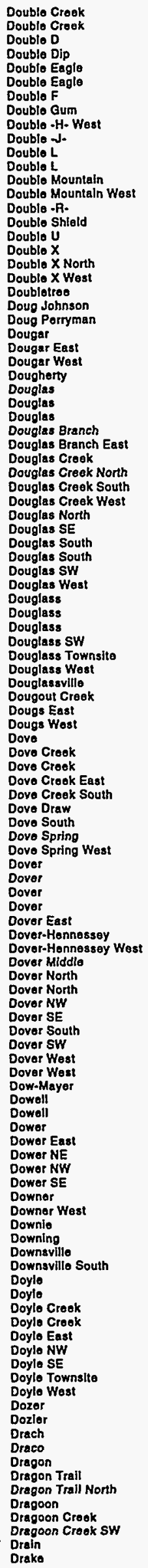 & 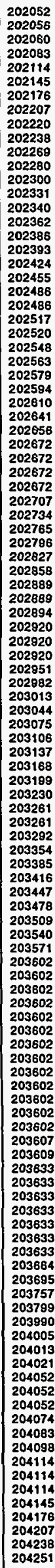 & 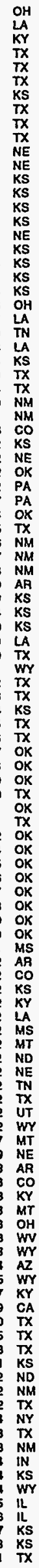 & 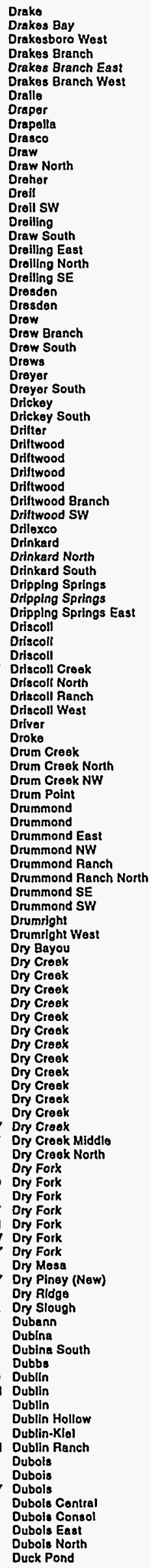 \\
\hline
\end{tabular}

Energy Information Administration/Oil and Gas Field Code Master List 1994 


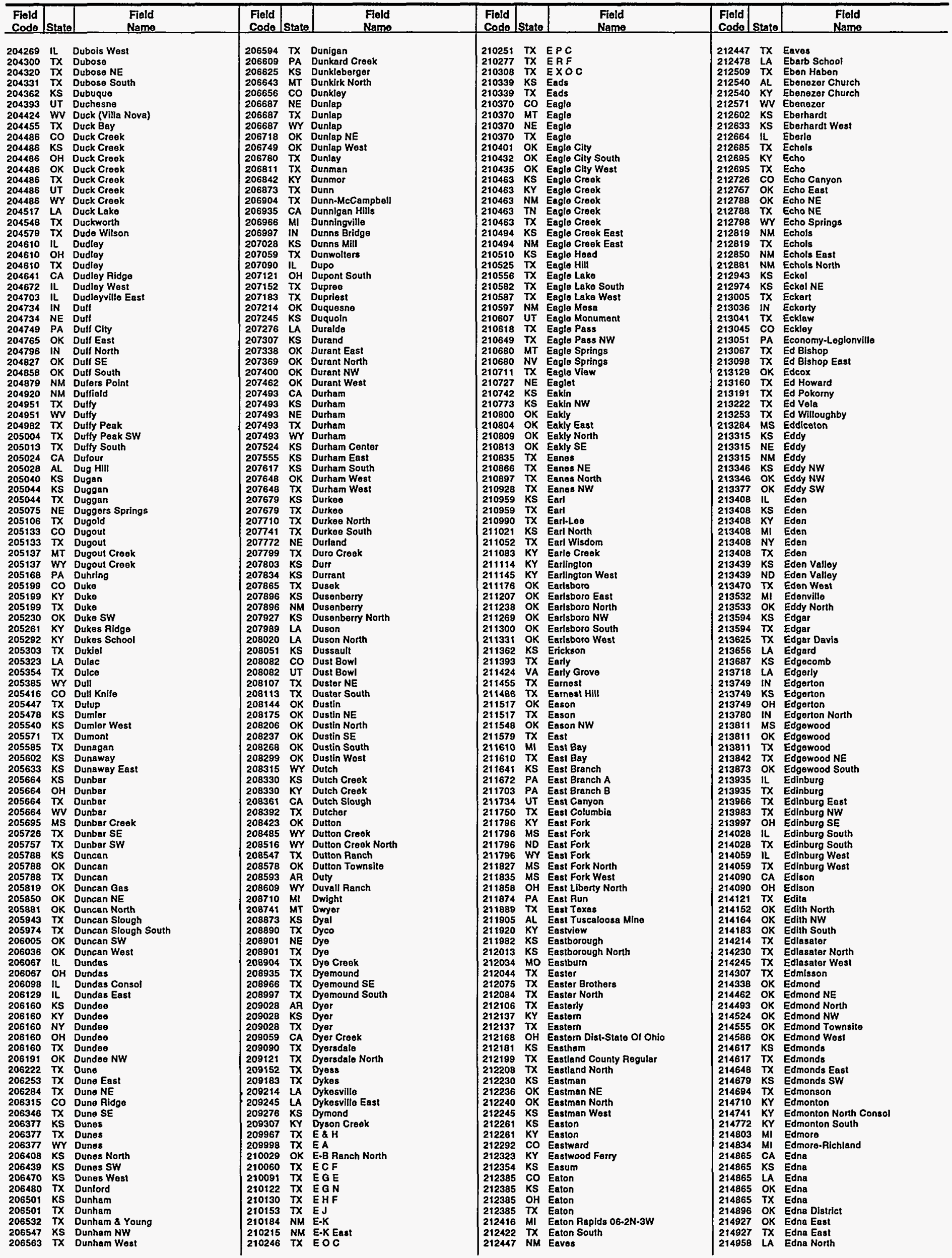




\begin{tabular}{|c|c|c|c|c|c|c|c|c|c|c|c|}
\hline $\begin{array}{l}\text { Flold } \\
\text { Codo }\end{array}$ & State & $\begin{array}{l}\text { Flold } \\
\text { Namo } \\
\end{array}$ & $\begin{array}{l}\text { Fiold } \\
\text { Code }\end{array}$ & Stato & $\begin{array}{l}\text { Field } \\
\text { Name }\end{array}$ & \begin{tabular}{l|l} 
Frold \\
Code
\end{tabular} & Stato & $\begin{array}{l}\text { Fiold } \\
\text { Namo }\end{array}$ & $\begin{array}{l}\text { Fleid } \\
\text { Code }\end{array}$ & State & $\begin{array}{l}\text { Field } \\
\text { Name }\end{array}$ \\
\hline 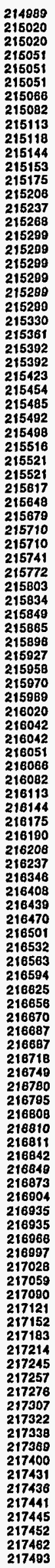 & 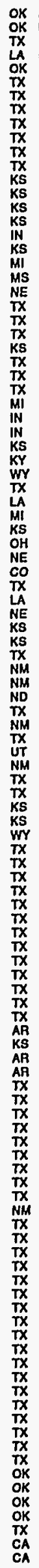 & 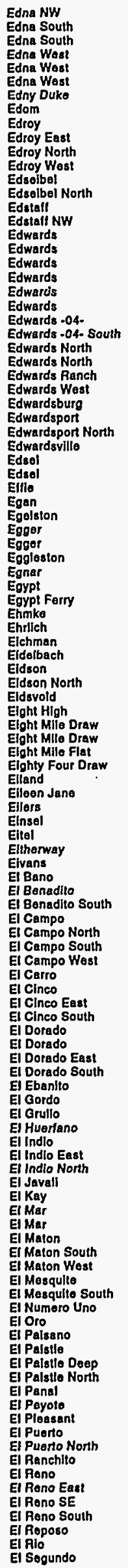 & 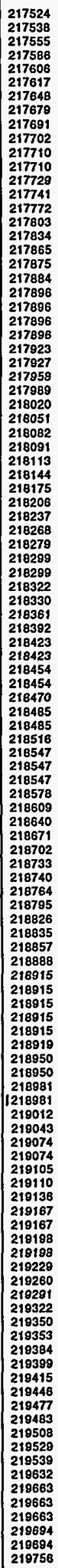 & 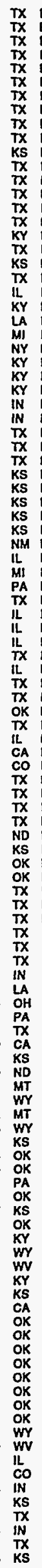 & 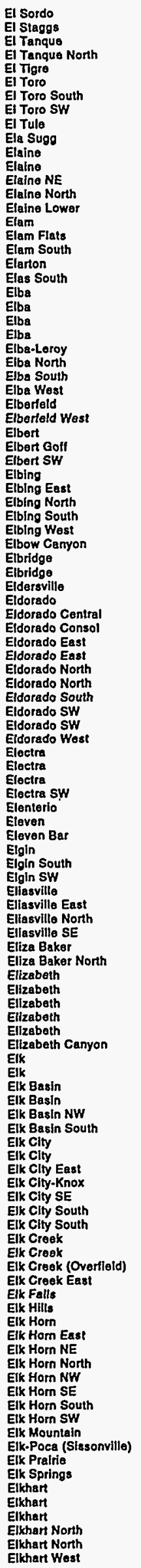 & 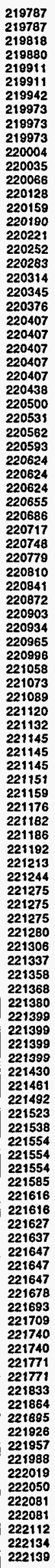 & 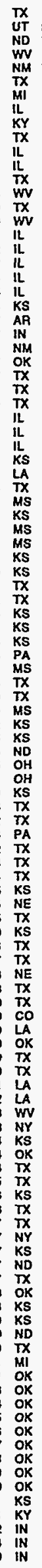 & 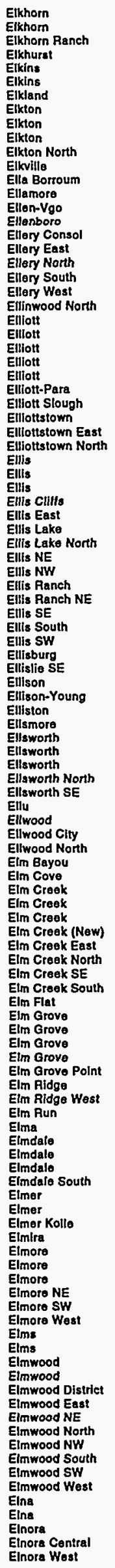 & 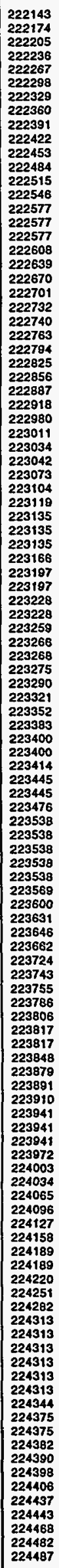 & 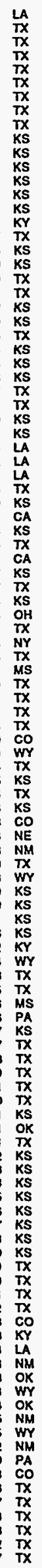 & 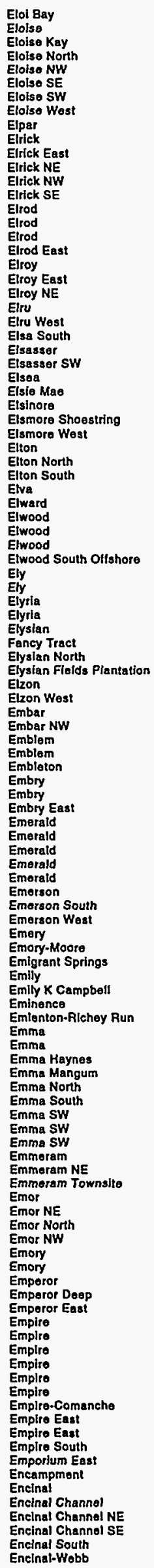 \\
\hline
\end{tabular}

Energy Information Administration/Oil and Gas Fleld Code Master List 1994 


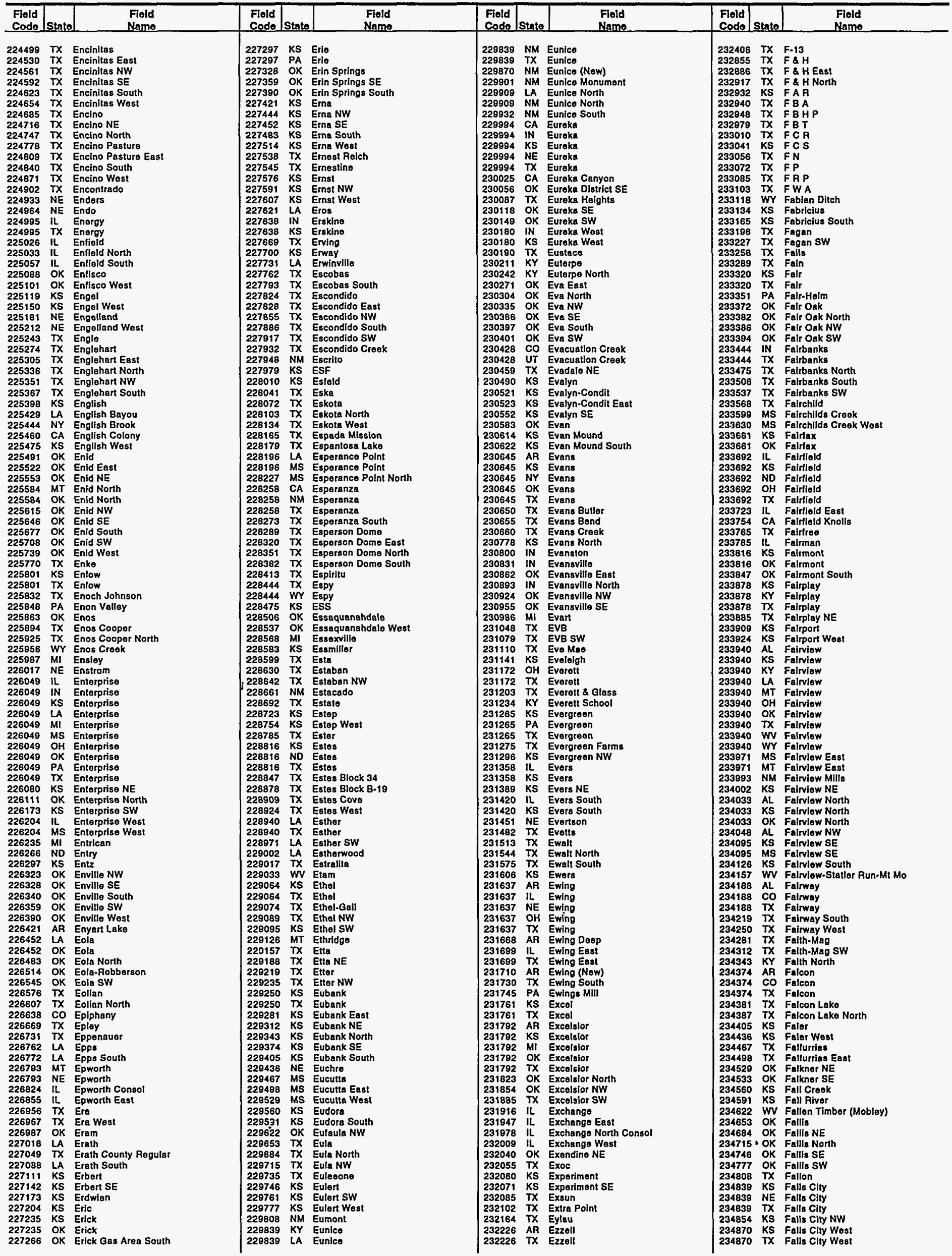




\begin{tabular}{|c|c|c|c|c|c|c|c|c|c|c|c|}
\hline $\begin{array}{l}\text { Flold } \\
\text { Codo }\end{array}$ & State & $\begin{array}{l}\text { Fleld } \\
\text { Name }\end{array}$ & $\begin{array}{l}\text { Field } \\
\text { Code }\end{array}$ & Stato & $\begin{array}{l}\text { Fiold } \\
\text { Name }\end{array}$ & $\begin{array}{l}\text { Field } \\
\text { Code }\end{array}$ & State & $\begin{array}{l}\text { Field } \\
\text { Name }\end{array}$ & \begin{tabular}{l|} 
Fiold \\
Codo
\end{tabular} & State & $\begin{array}{l}\text { Fiold } \\
\text { Namo }\end{array}$ \\
\hline 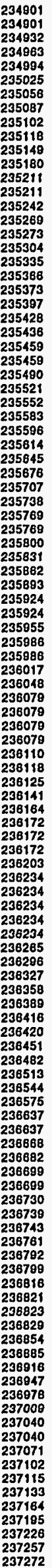 & 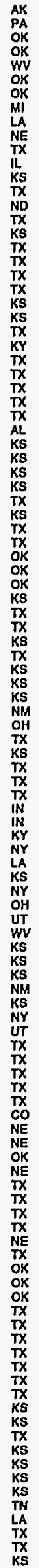 & 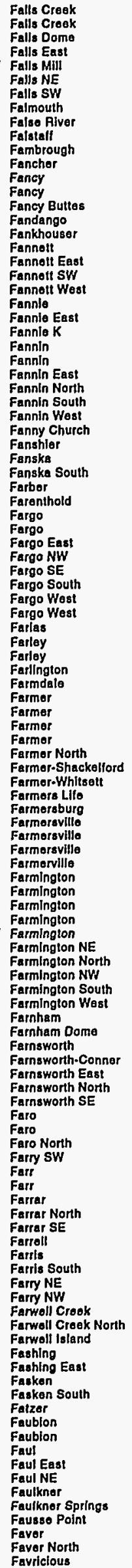 & 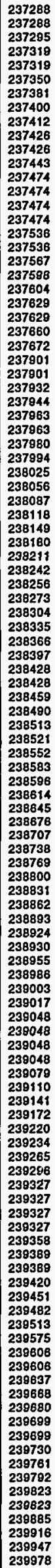 & 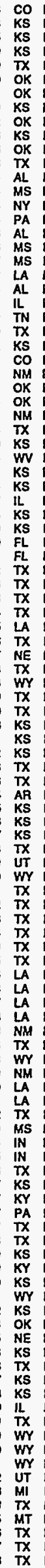 & 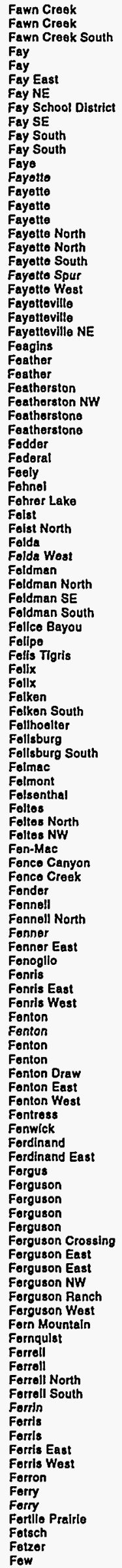 & 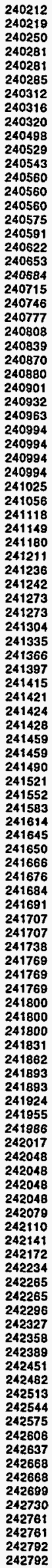 & 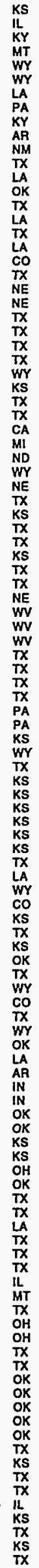 & 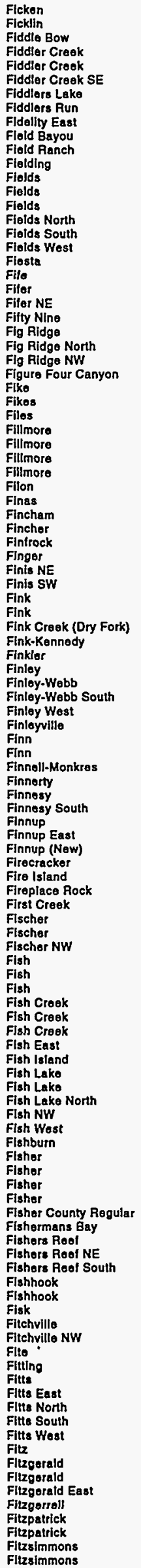 & 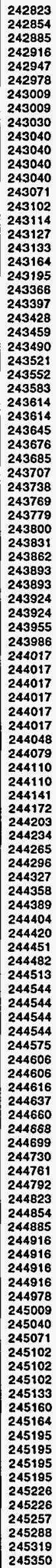 & 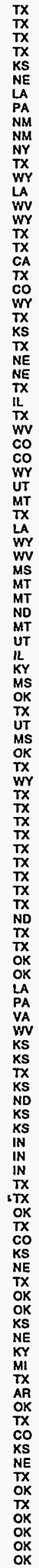 & 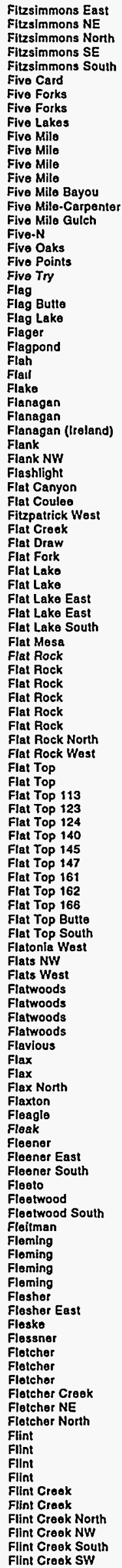 \\
\hline
\end{tabular}




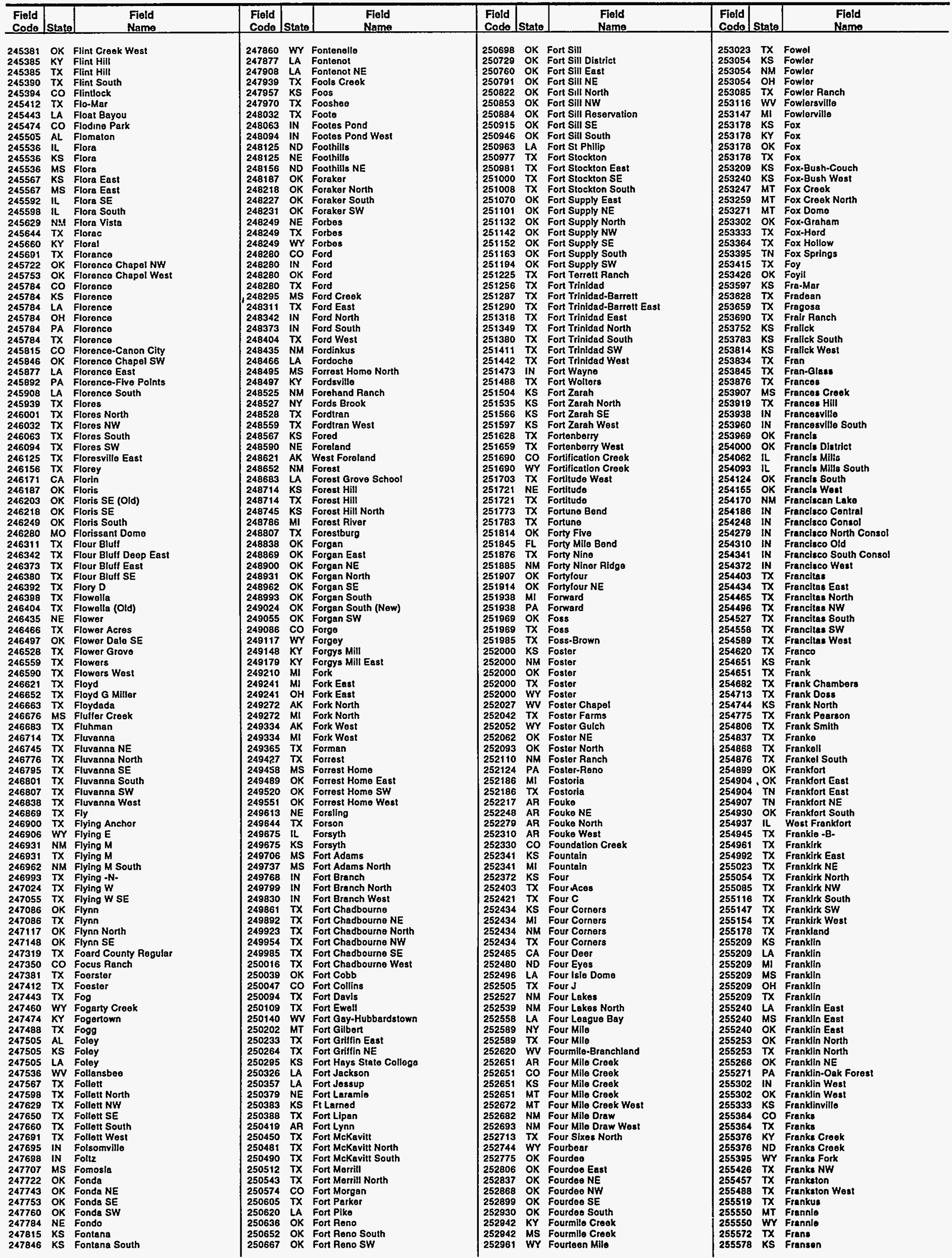




\begin{tabular}{|c|c|c|c|c|c|c|c|c|c|c|c|}
\hline $\begin{array}{l}\text { Fiold } \\
\text { Code }\end{array}$ & State & $\begin{array}{l}\text { Fiold } \\
\text { Name }\end{array}$ & $\begin{array}{l}\text { Flold } \\
\text { Code }\end{array}$ & State & $\begin{array}{l}\text { Field } \\
\text { Name }\end{array}$ & $\begin{array}{l}\text { Field } \\
\text { Codo }\end{array}$ & State & $\begin{array}{l}\text { Field } \\
\text { Name }\end{array}$ & $\begin{array}{l}\text { Fiold } \\
\text { Code }\end{array}$ & State & $\begin{array}{l}\text { Fleld } \\
\text { Name }\end{array}$ \\
\hline 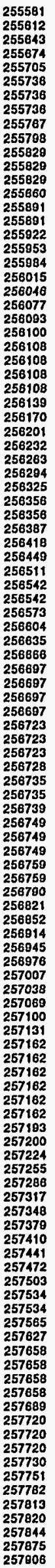 & 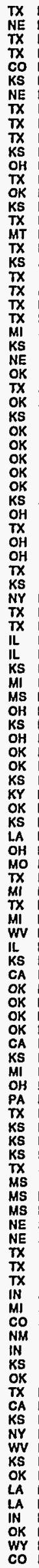 & 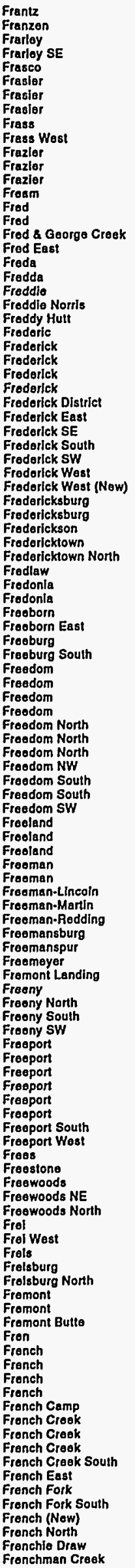 & 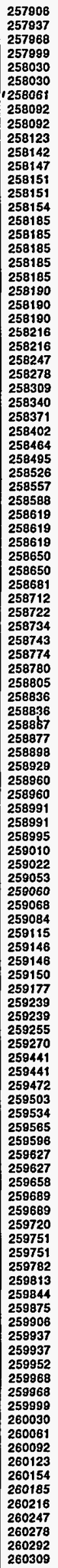 & 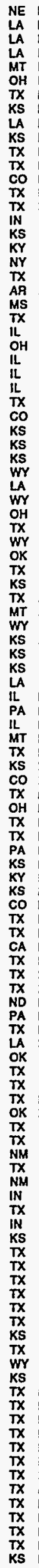 & 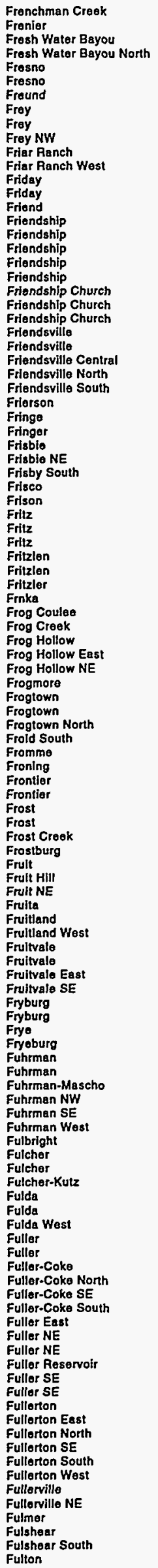 & 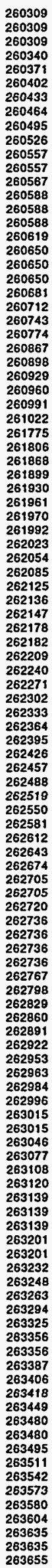 & 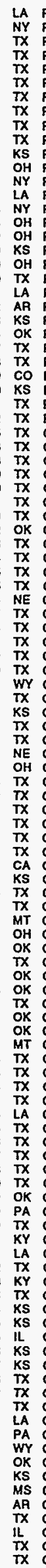 & 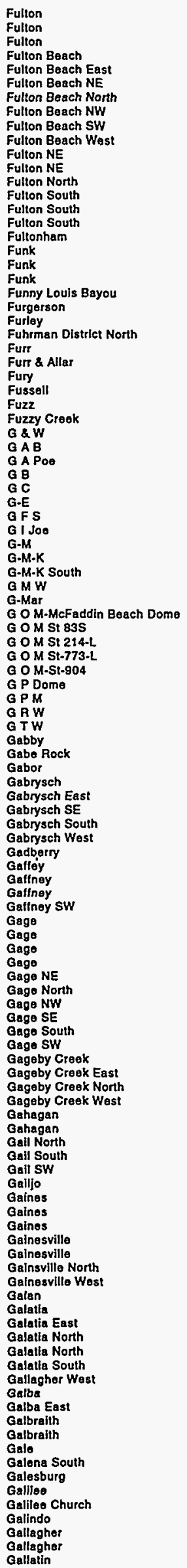 & 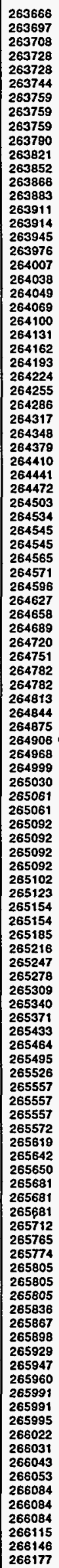 & 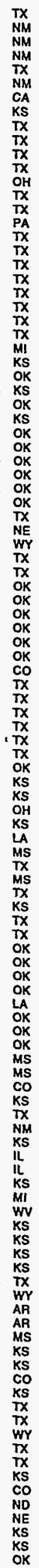 & 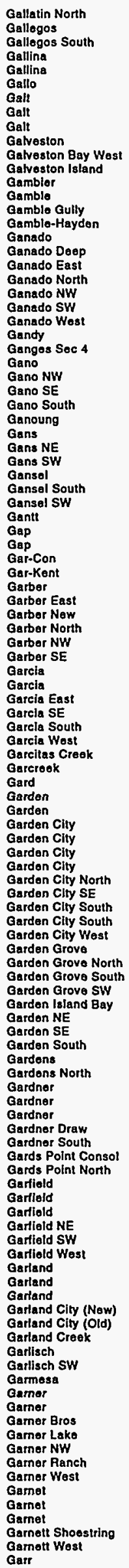 \\
\hline
\end{tabular}




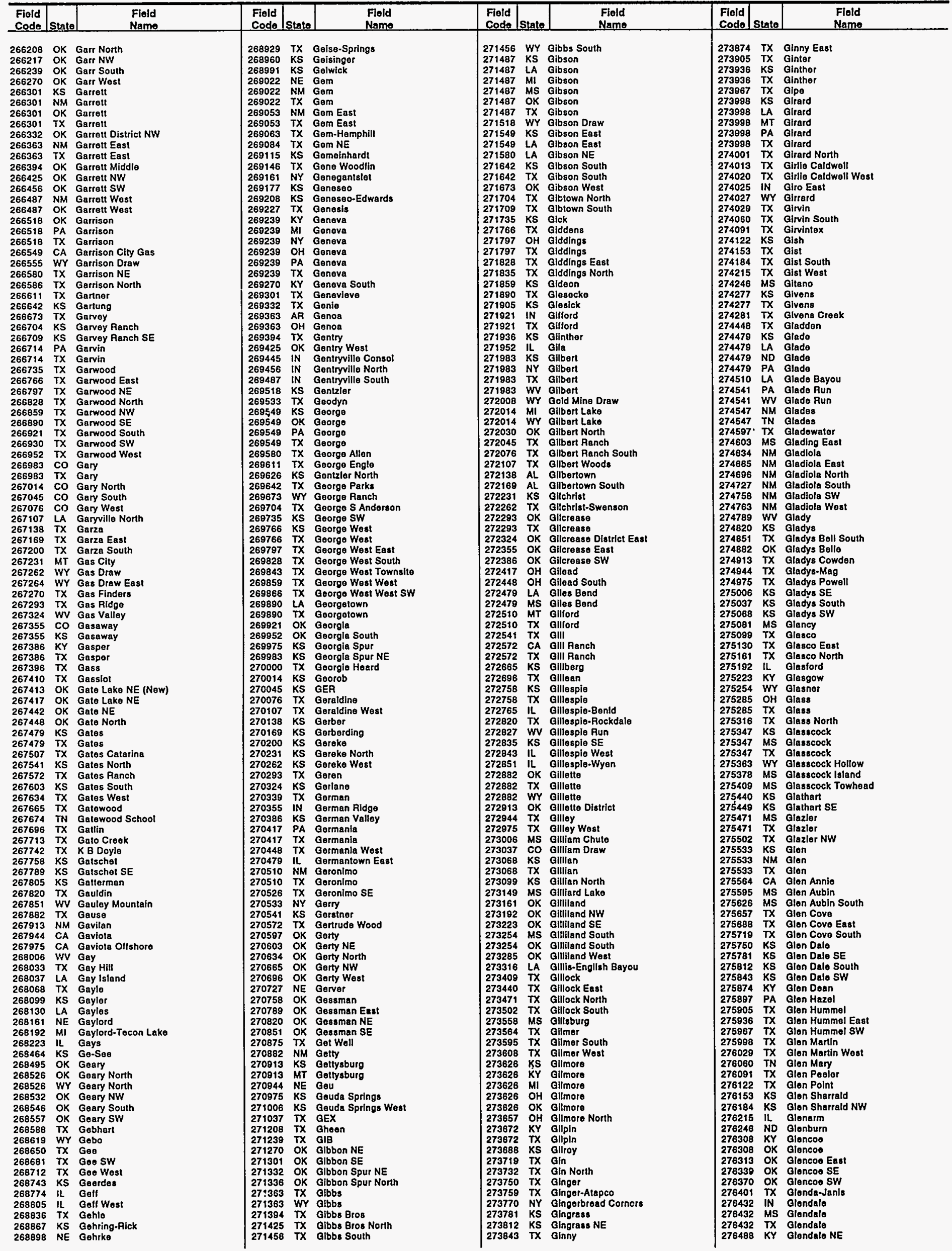




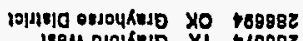

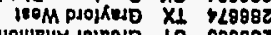

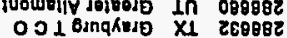

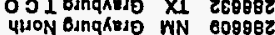

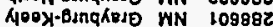

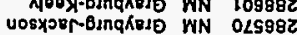
BInqhato X1 BESSBZ Bunque WN 689982

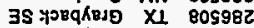

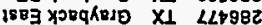

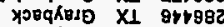

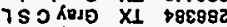
रQנ

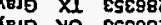
रห19 NX Es8982 XE9 PUP|EI PJORAAEJO V7 LEE98Z

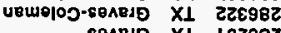
sonerg XI 162982

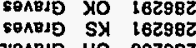

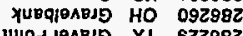
lulod laABIS XI 622998 olld loakg in 861982 ISOM PUOd KSSEIS AX SOI982 puod KsSE19 $A X$ DLO9BZ อXำXssed $\forall 7$ Z9098Z

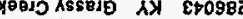

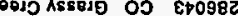
sloojsseja XI z1088 $189 \mathrm{M}$ PUV|88E19 AM 00098Z purissed AM puBisese WN PUB|88E10 ON L8658Z Oling JoddOYSEEIJ AM GLGSBZ

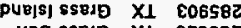
Jung-sserg XI $X_{1} \quad 92898 Z$

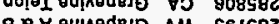
8

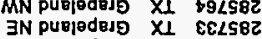

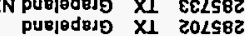

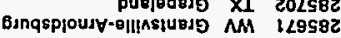

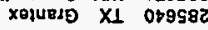

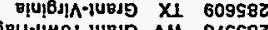
s40. Fs

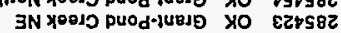

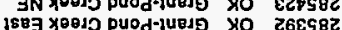

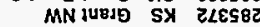

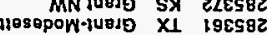
juखd XI 662582 14010 $3 N$ 66Z58Z JuEJ IW 68ZSBZ पHON OUBA ON

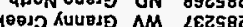

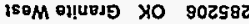

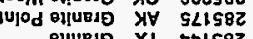

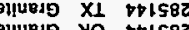

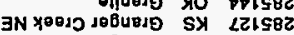
X0015 ๑6uвJ XI Ells82 ๑ธนשנ SW ELISBz IsoM malnpuejg OW z80s8 促

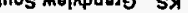

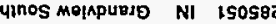

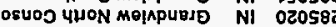

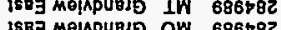

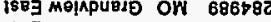
1803 м예술요 NI 686782

|вנ)

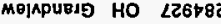

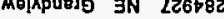
мөाкрuвg IN LZ6r8Z

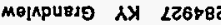

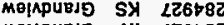
Meinpuвd NI LZ6rgz

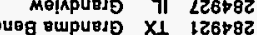

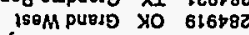
जs Кө|ाष

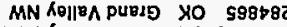

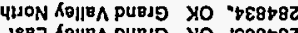

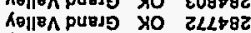
(0) MS PUDJ YO $1+\angle 482$

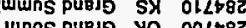
eu!!ES PUBdg XI 6L958Z

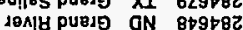

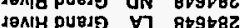

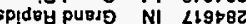
MN PU⿴囗十 YO 009582 asow puesg OJ ssst8z

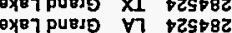

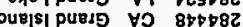

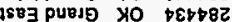
-

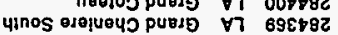

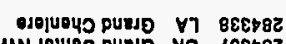
MN JOSUEO PUINO XO LOERB ONe noej putuo v1 sterse MN MN Rog puvo vi thetrge 4 Ddurso os $121+82$ uridwe SOM OBPIY zWES WN 00158Z (4) 803 oBpit pwes WN EBOpB

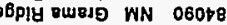
AS घоำाघ 11810 00 82068

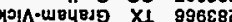
ynos 4นON

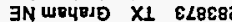
อx日 1803 w8 MN Ail WBपष

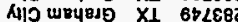
עह4घJפ $X 1 \quad 81 \angle 882$

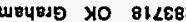
ШЕपणJ⿹ HO $81 \angle 8 B Z$

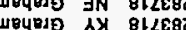

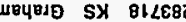
แryणdD NI 81 288 UHON B6EJO XI LB9E8Z BBDJ $X_{1} \quad 859882$ BBVJE UY 959882

4InOS U0ำ บำघ10 HO 769882

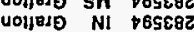

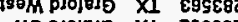
MS pJojerg XI 28s88

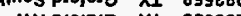
MN PJojug $X \perp$ ESSE8

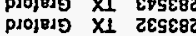
HणJO $3 N$ 10588

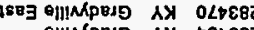

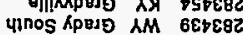
АрЕנ

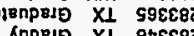

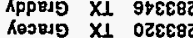

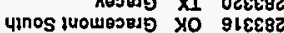
MS O5EIS XI SIEER प องषנפ XI ESzE8 90010 XI $X_{1} \quad 228882$

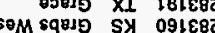
yinos SqBJ $S X$ S?

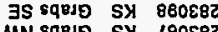
IN sque $S X$ L 283 89810 SX 50088 89010 SY $\$ \angle 8882$

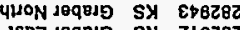

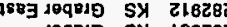

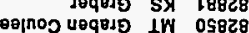
U8qEIS $X_{1} \quad 61828$

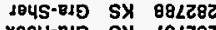

IHON XOOY-8JO SX LSLZBZ

$\exists N$ YOOU-BJ $S X$ xoog-8Jo sर्र s8928 димо0 XI E86z8 UвMOO $X_{1} \quad 295 z 8$

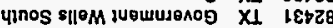

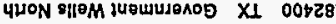

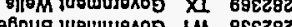
คAOO $\mathrm{SX}$ LOEZ8 1803 KopjnoD XI 9LZZ8Z Xopnog $X_{1}$ Stzz8z pino pinos HO Ealz8 pInoo Sx E8เzв

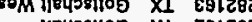

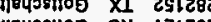

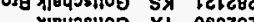

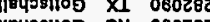
(1) DF ग4100 in $82028 Z$

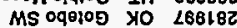

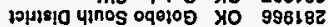
MN Og.105 YO +0818 4HON OQPIOS YO EL818 Hessog $\times 1$ OBLL82 HOSsog iW OBLL82 nOSSOD $71 \quad 08 \angle 283$ (1)

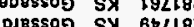
pressog sर पमОN URW100 $X_{1} \quad 999182$

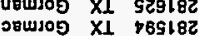

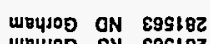

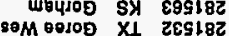
MS 00100 XI LOSLR 4nOS $00100 x_{1} x_{0<182}$ AN 00100 X1 atplaz

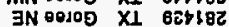

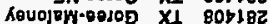
188900100 XI LLE18Z

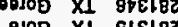

soM बा11 Olliauopjog XI razsoz

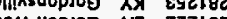
(1) 150M IOOHS Uoptog $x_{1}$ (18)

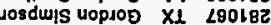

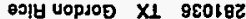
पHON UOPIOS Y1

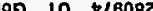

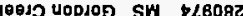

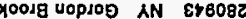
uopiog XI 216082 4ор109 $\forall 7$ zl608z copso st ct608z

(บกy 1807) xр000 CJoqasoog AM $80 \angle 08 Z$ UNY 08000 HO $\angle S \angle 08 Z$

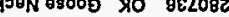

1883 OXVT

3 0X07 08000 IN $92208 \mathrm{C}$ 0xB7 08000 IN 969082 อxp708000 if 589082

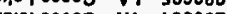
p4818 08000 XI t9908Z 003 85000 MM 959092

150马 $\times 1000$ X00J0 08000 XI $20908 Z$ X005 (190M

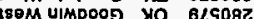
4. IS Uimpoon Xo 9s508z unpoos xo 0p508z umpoog X1 $\quad 80508 z$ Uimpoog WN 60508z UIMPOOD $\exists N \quad$ BOSOBZ uाMpOOD SN BOSOBZ

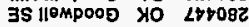
agl llempood iw 91708z Hïмро00 XI SBEOBZ IIImpoog IN SBEOBZ SW 458082

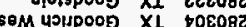
$10 \times 18$ d 4อ, 4วypoog SX 192082 पगायр000 05192082

4 nos sublupoog xo ozzogz

IS 14 हrypoog xo 68108z IN ग4밍oㅇ Xo 891082 Mllod uswpoog of 90108z seujuds poog X1 $\$ 20082$ 3S P000 $X_{1}$ Es008z puog oumlsod Poog N1 Z968LZ 4RON U8WO POOg XI ISBOLZ 18wo poog XI $0268 \angle 2$ उN POOD XI $6888 \angle 2$ Хכกา р009 XI 8S88LZ

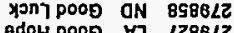

1803 gdoH poog V7 $2286 \angle 2$ OdOH POOg XI 98L6LZ OdOH POOD NI 9620LZ ดdOH POOO Y7 96262Z Aвply poog $X_{1} \quad 59 \angle 6 \angle Z$

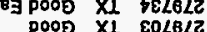

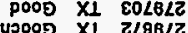

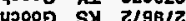

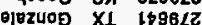
S0|EzU00 WN $1+9622$ S018zuog Y7 it9982z

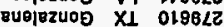

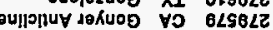
$M N$ ZowOD XI E958LZ $\exists N$ ZOWOפ XI BSSBLZ 4HON ZDWOD XI SSSBLZ

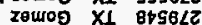
MS Nu100 $\times 0$ LLSBLZ

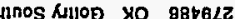
IS A 100 XO S5Y6LZ जER6LZ

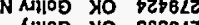
ISOM PBIIOO XI Z9EGLZ MNPVIIOS XI SEEGLZ Pघा|णD $X_{1}$ G928LZ 8Ez6L2 


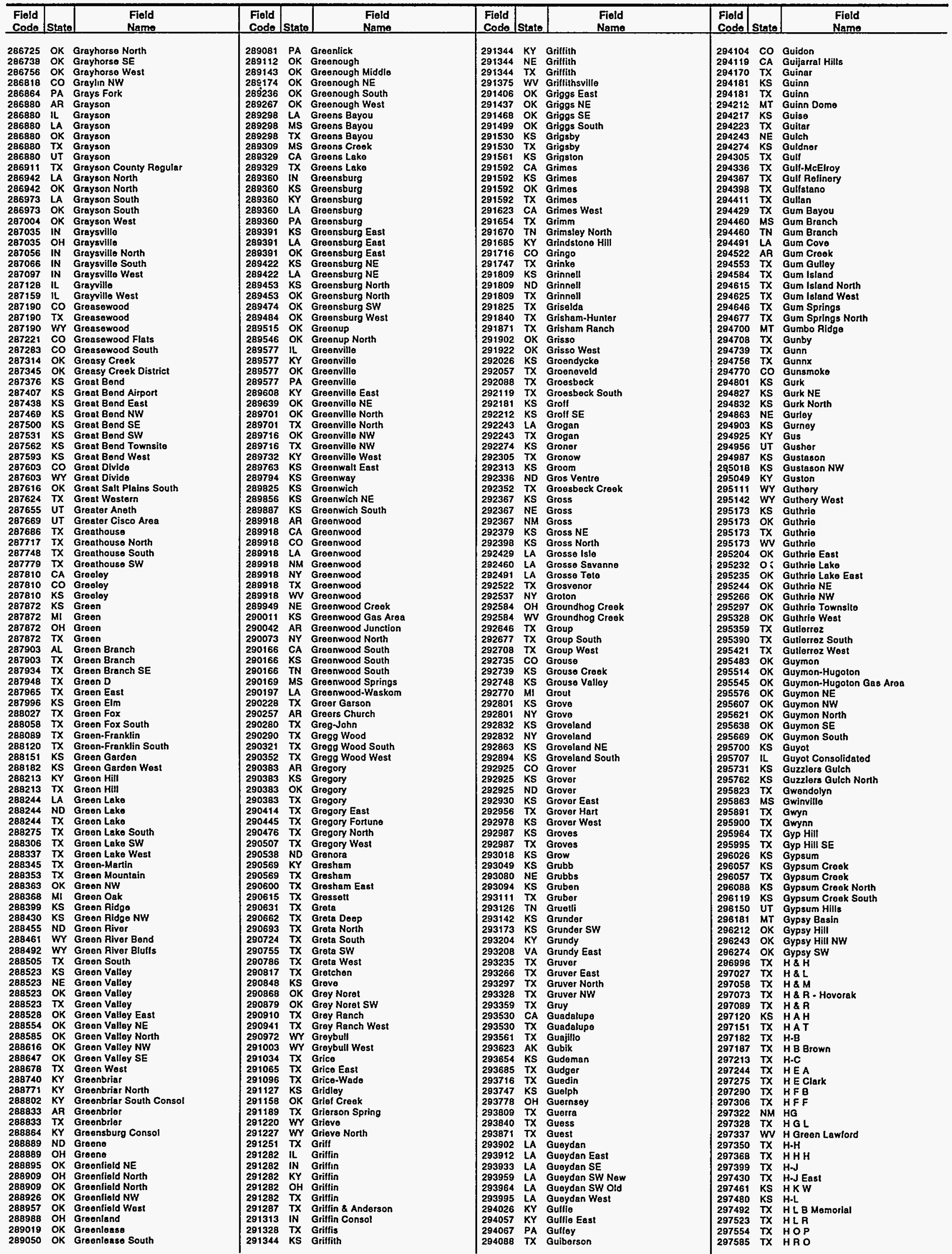




\begin{tabular}{|c|c|c|c|c|c|c|c|c|c|c|c|}
\hline $\begin{array}{l}\text { Flold } \\
\text { Codo } \\
\end{array}$ & Stato & $\begin{array}{l}\text { Flold } \\
\text { Name } \\
\end{array}$ & $\begin{array}{l}\text { Fiold } \\
\text { Code } \\
\end{array}$ & State & $\begin{array}{l}\text { Field } \\
\text { Name } \\
\end{array}$ & $\begin{array}{l}\text { Field } \\
\text { Code }\end{array}$ & State & $\begin{array}{l}\text { Field } \\
\text { Name }\end{array}$ & \begin{tabular}{l|} 
Field \\
Codo \\
\end{tabular} & Stato & $\begin{array}{l}\text { Field } \\
\text { Name } \\
\end{array}$ \\
\hline 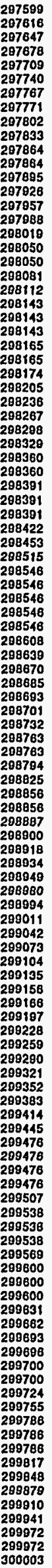 & 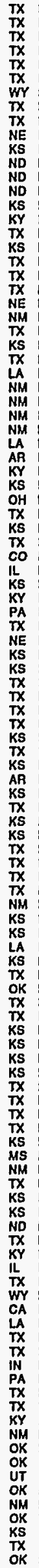 & 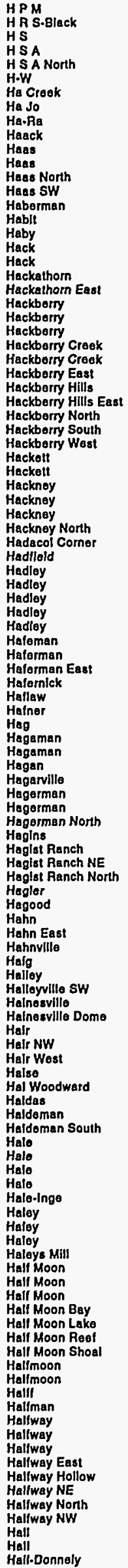 & 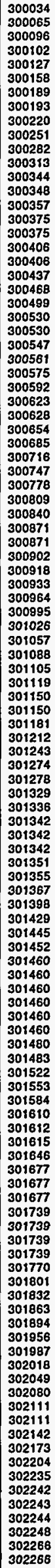 & 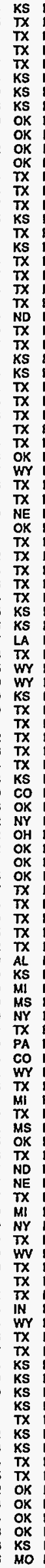 & 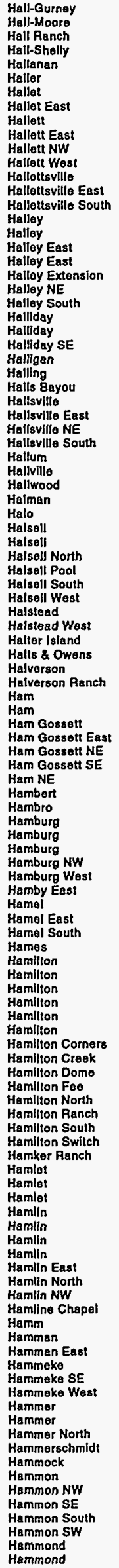 & 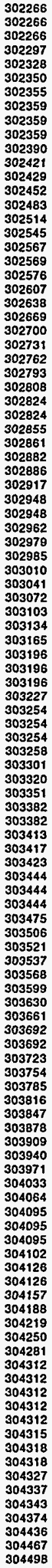 & 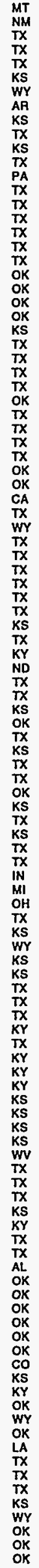 & 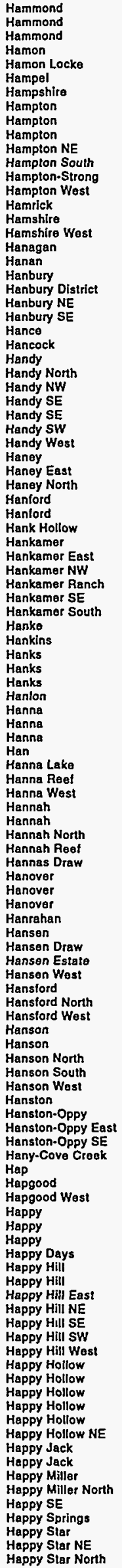 & 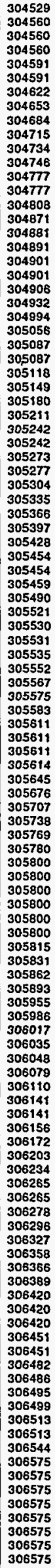 & 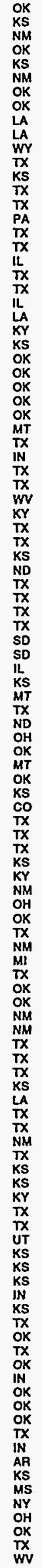 & 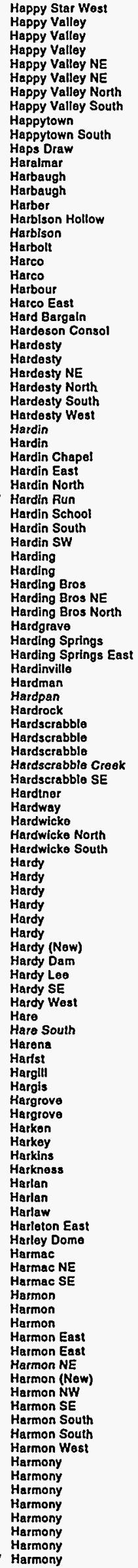 \\
\hline
\end{tabular}




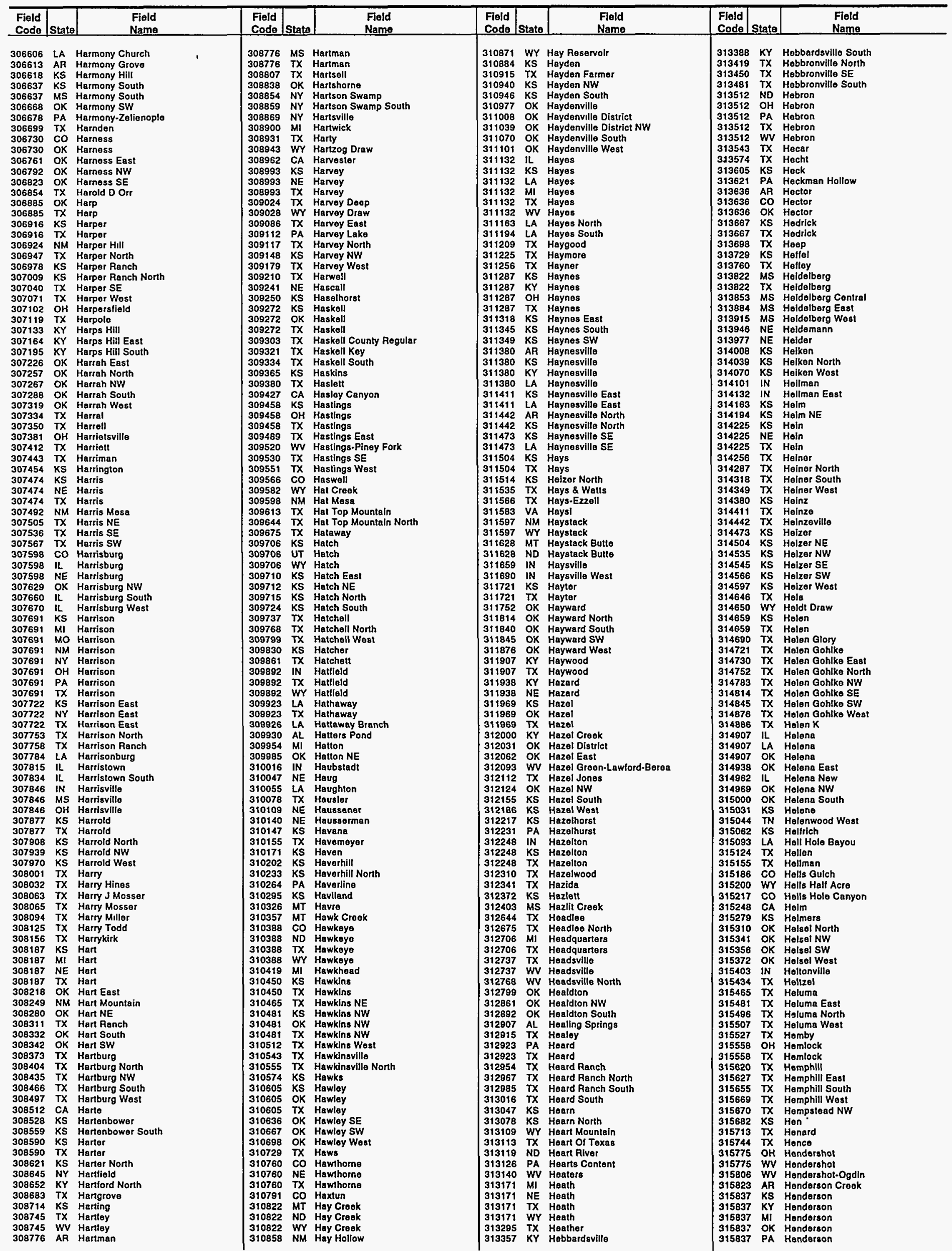




\begin{tabular}{|c|c|c|c|c|c|c|c|c|c|c|c|}
\hline $\begin{array}{l}\text { Fiold } \\
\text { Codo } \\
\end{array}$ & Stato & $\begin{array}{c}\text { Fiold } \\
\text { Namo }\end{array}$ & $\begin{array}{l}\text { Fiold } \\
\text { Codo }\end{array}$ & Stato & $\begin{array}{l}\text { Field } \\
\text { Name }\end{array}$ & $\begin{array}{l}\text { Field } \\
\text { Codo }\end{array}$ & Stato & $\begin{array}{l}\text { Field } \\
\text { Neme }\end{array}$ & \begin{tabular}{|l|} 
Fiold \\
Code \\
\end{tabular} & State & $\begin{array}{l}\text { Field } \\
\text { Name } \\
\end{array}$ \\
\hline 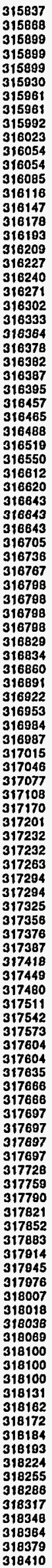 & 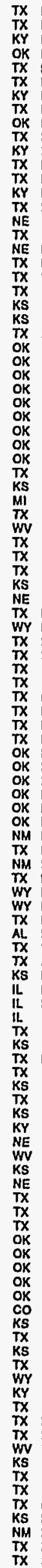 & 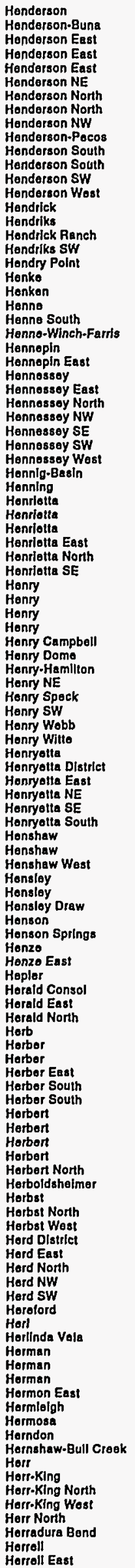 & 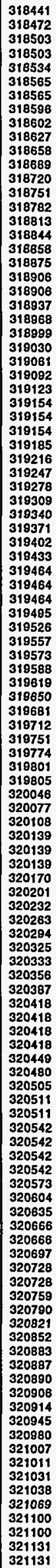 & 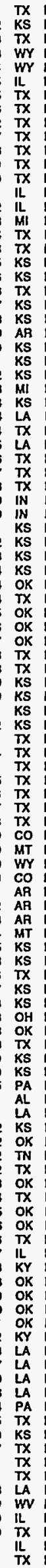 & 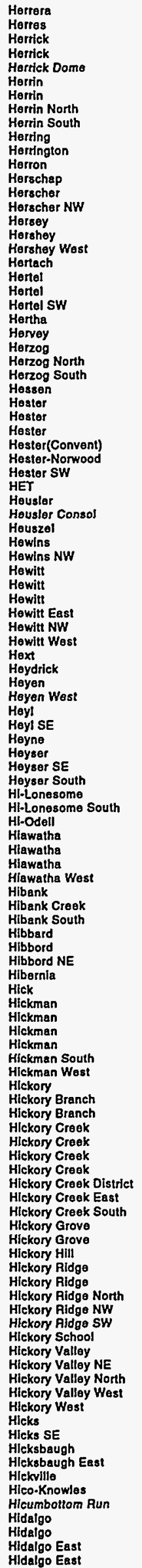 & 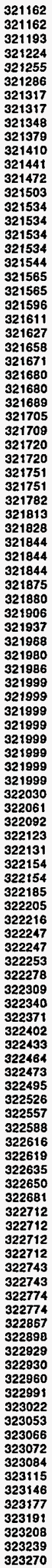 & 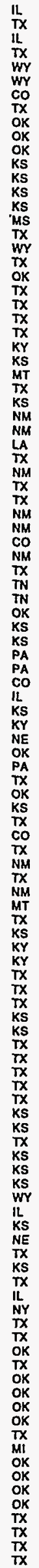 & 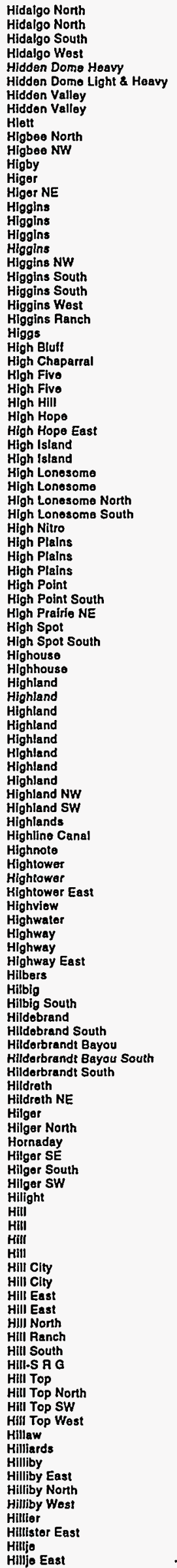 & 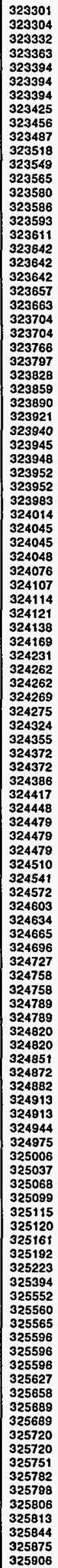 & 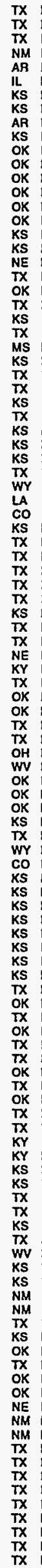 & 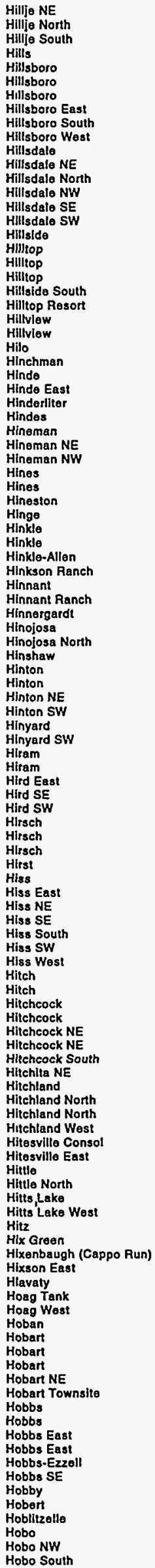 \\
\hline
\end{tabular}




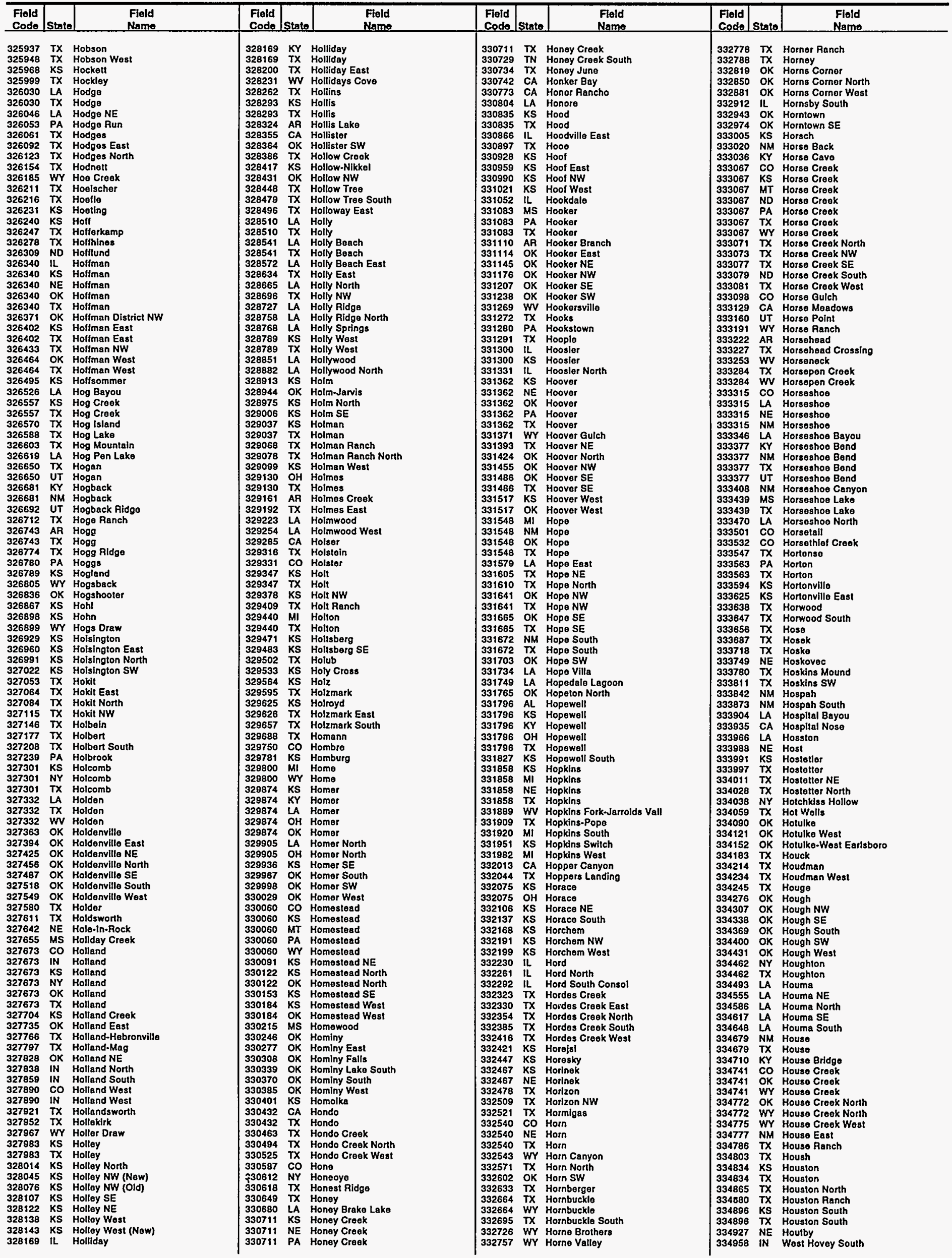




\begin{tabular}{|c|c|c|c|c|c|c|c|c|c|c|c|}
\hline $\begin{array}{l}\text { Flold } \\
\text { Code }\end{array}$ & State & $\begin{array}{l}\text { Field } \\
\text { Name }\end{array}$ & $\begin{array}{l}\text { Fild } \\
\text { Code }\end{array}$ & Istate & $\begin{array}{l}\text { Field } \\
\text { Name }\end{array}$ & $\begin{array}{l}\text { Fiold } \\
\text { Codo }\end{array}$ & State & $\begin{array}{l}\text { Fletd } \\
\text { Namo }\end{array}$ & $\begin{array}{l}\text { Fiold } \\
\text { Codo } \\
\end{array}$ & State] & $\begin{array}{l}\text { Field } \\
\text { Namo }\end{array}$ \\
\hline 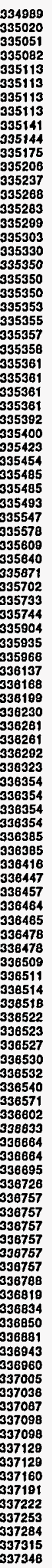 & 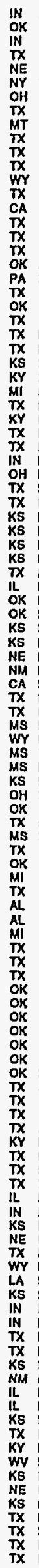 & 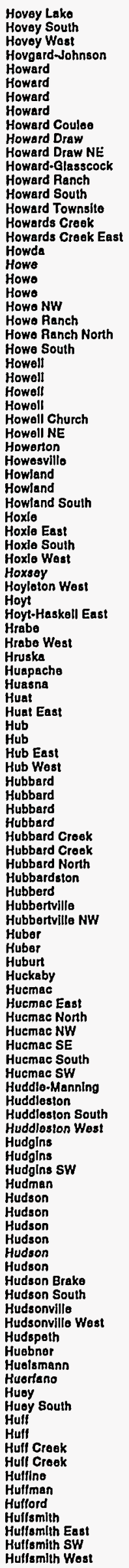 & 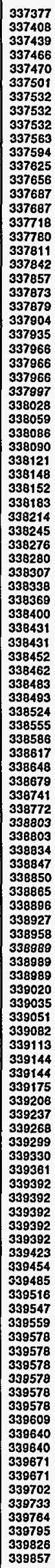 & 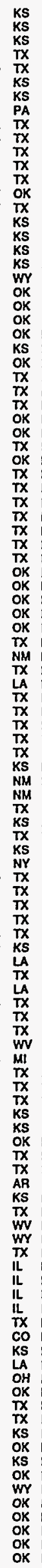 & 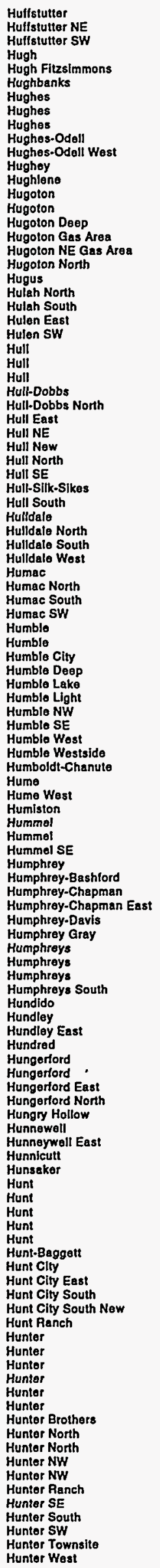 & 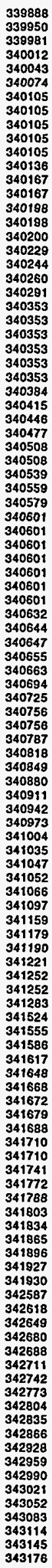 & 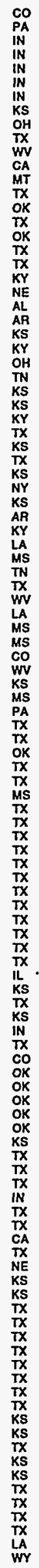 & 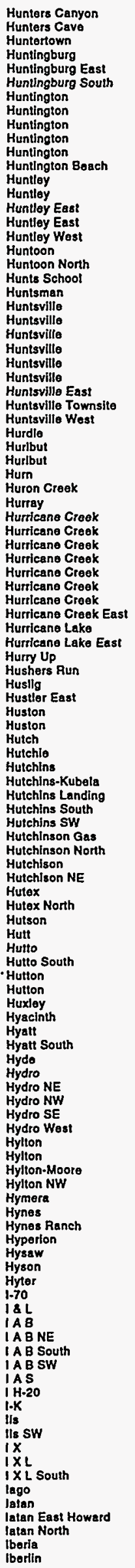 & 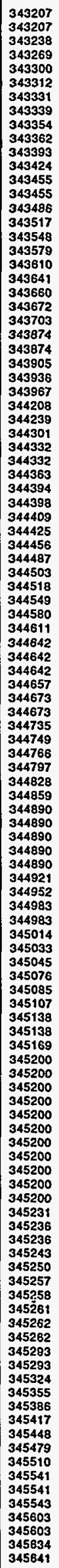 & 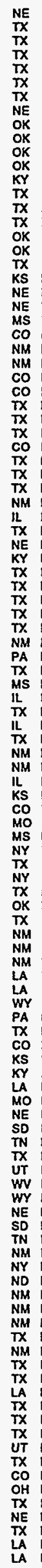 & 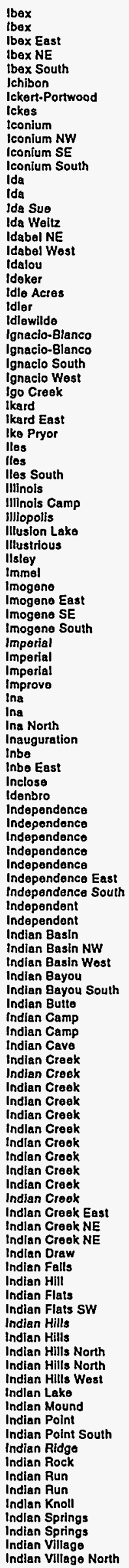 \\
\hline
\end{tabular}




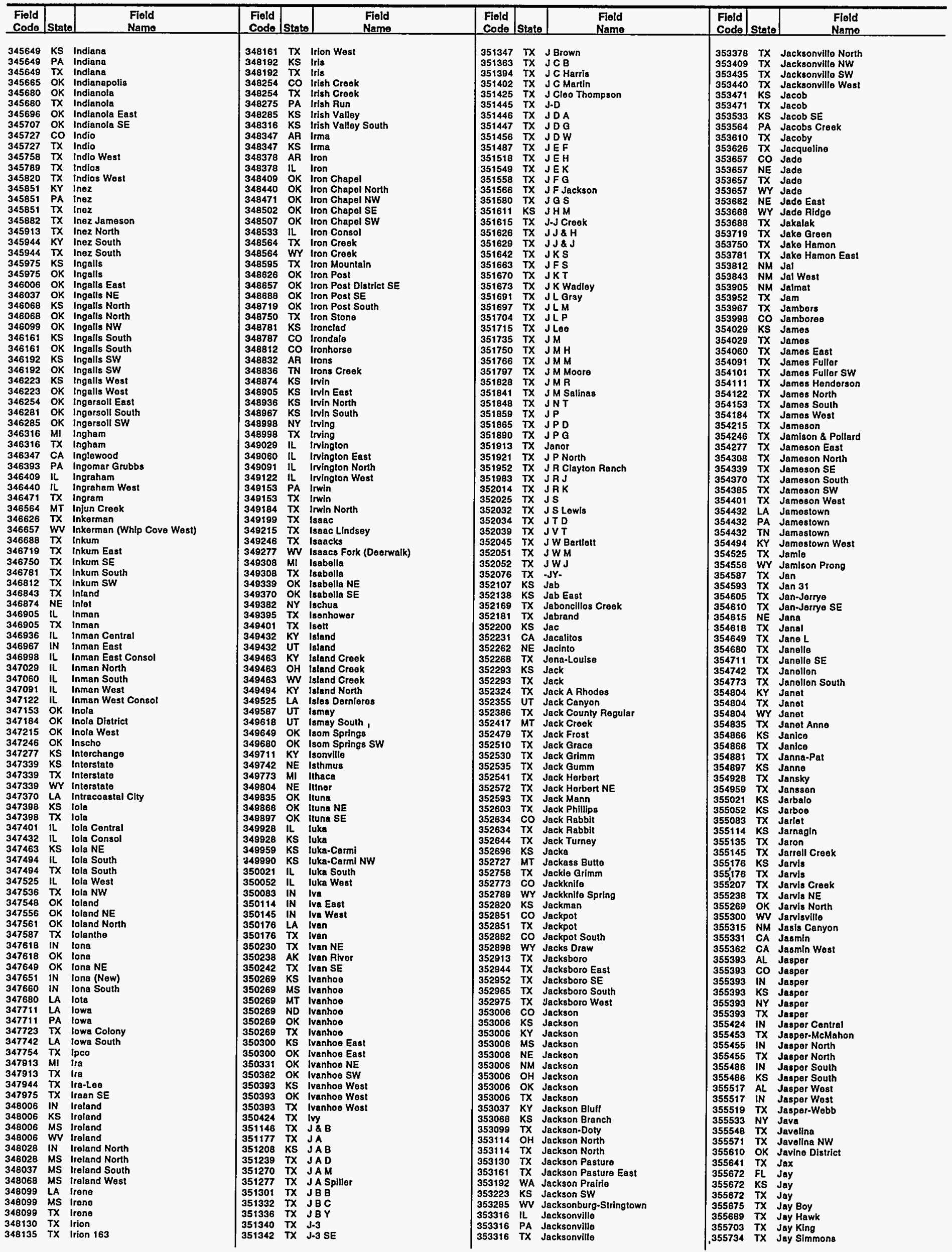




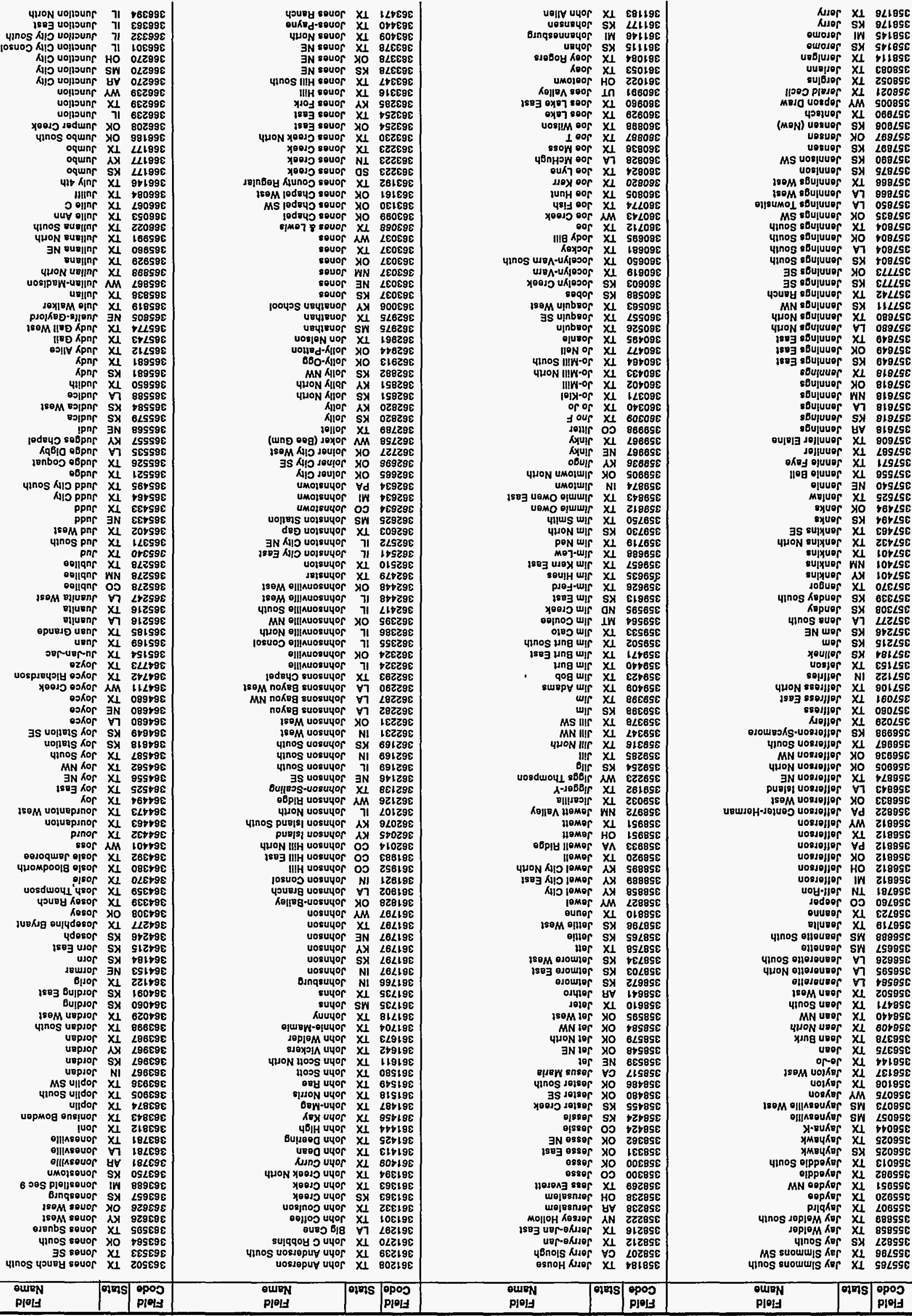




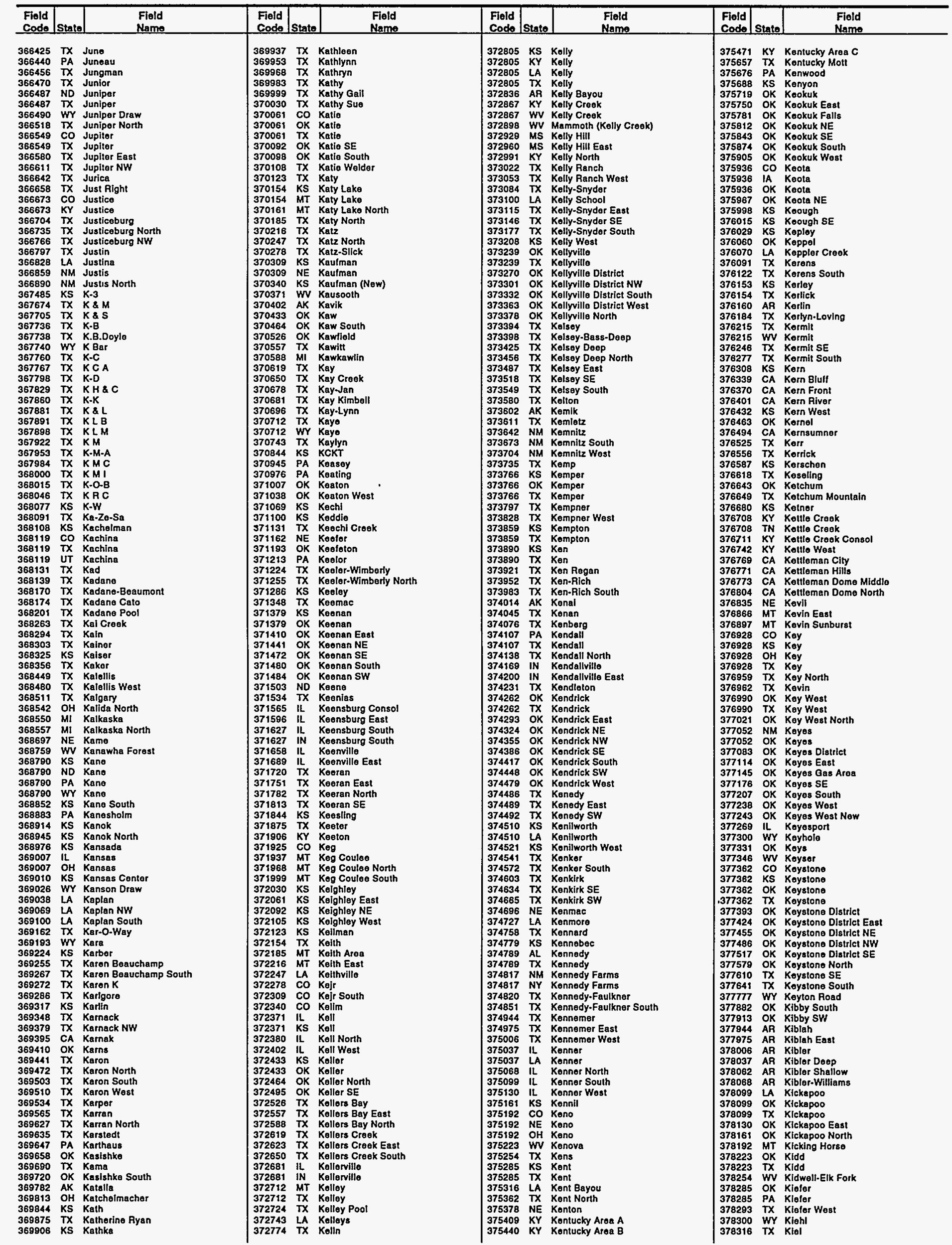




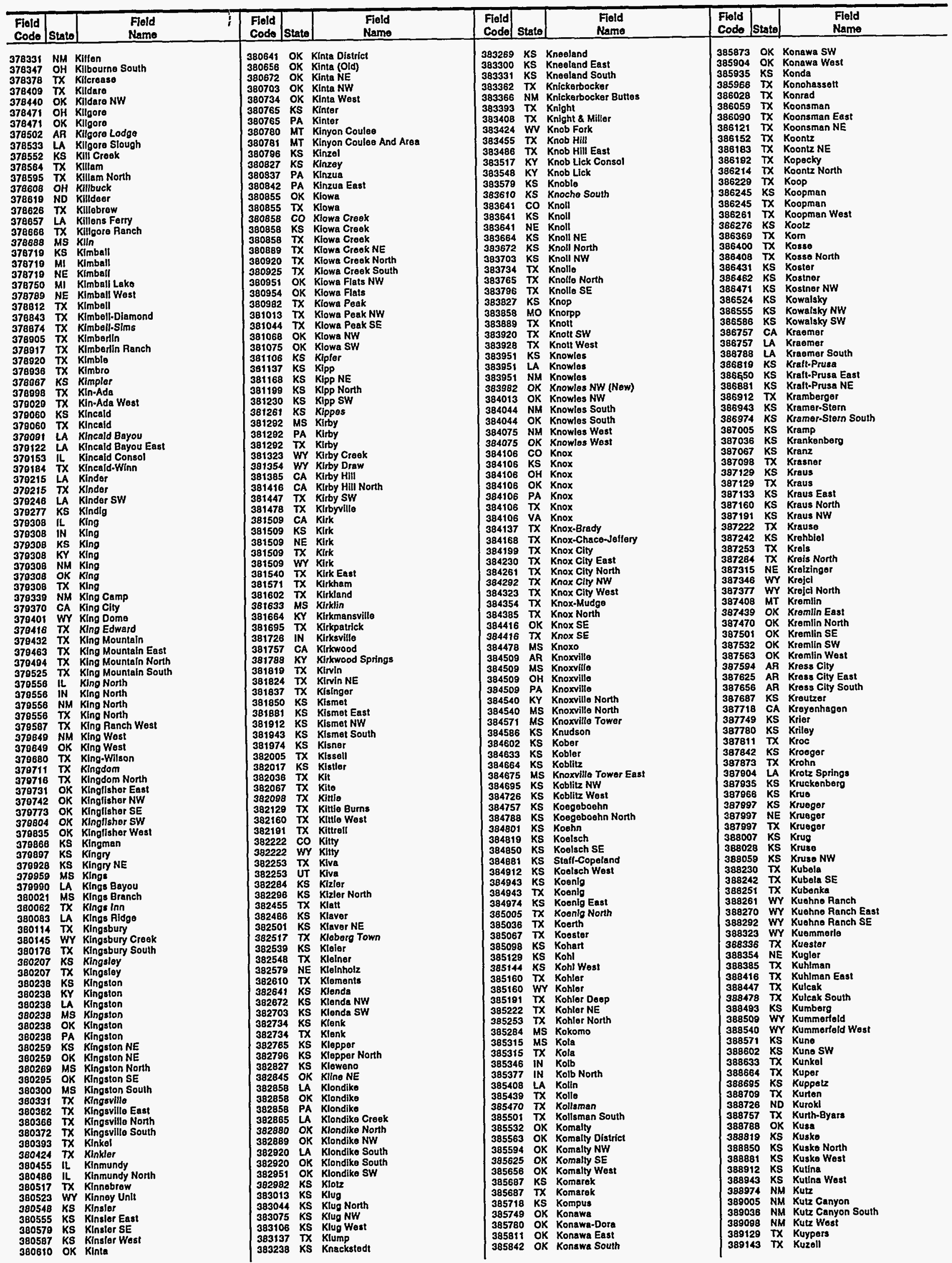




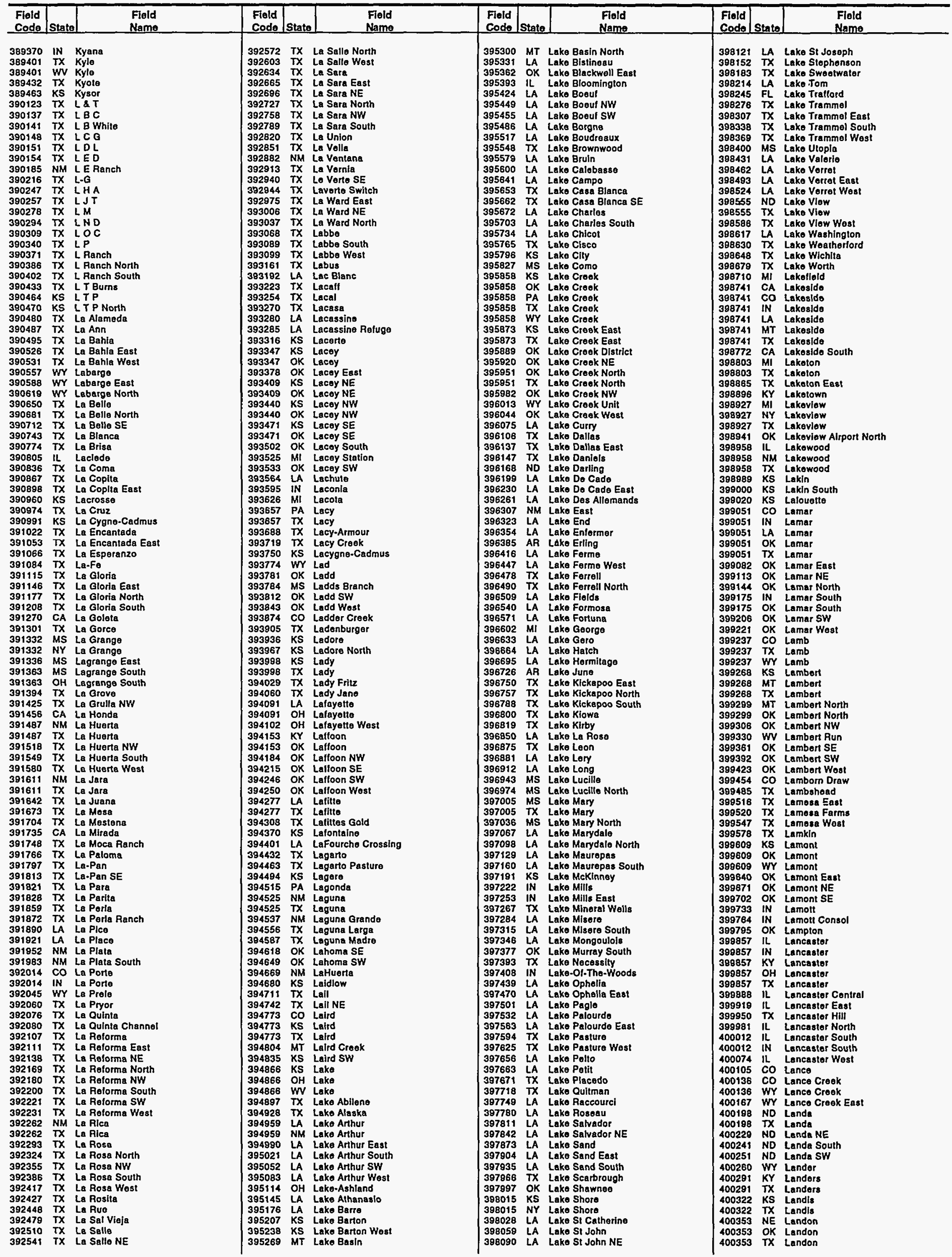




\begin{tabular}{|c|c|c|c|c|c|c|c|c|c|c|c|}
\hline $\begin{array}{l}\text { Fiold } \\
\text { Code }\end{array}$ & State & $\begin{array}{l}\text { Fleld } \\
\text { Name } \\
\end{array}$ & \begin{tabular}{l|l} 
Fild \\
Code
\end{tabular} & State & $\begin{array}{l}\text { Field } \\
\text { Name }\end{array}$ & \begin{tabular}{l|l} 
Fjeld \\
Code
\end{tabular} & State & $\begin{array}{c}\text { Field } \\
\text { Name } \\
\end{array}$ & $\begin{array}{l}\text { Field } \\
\text { Code }\end{array}$ & State & $\begin{array}{l}\text { Field } \\
\text { Namo } \\
\end{array}$ \\
\hline 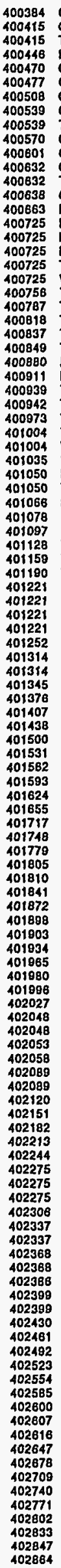 & 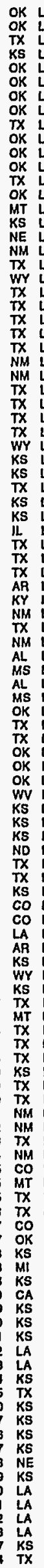 & 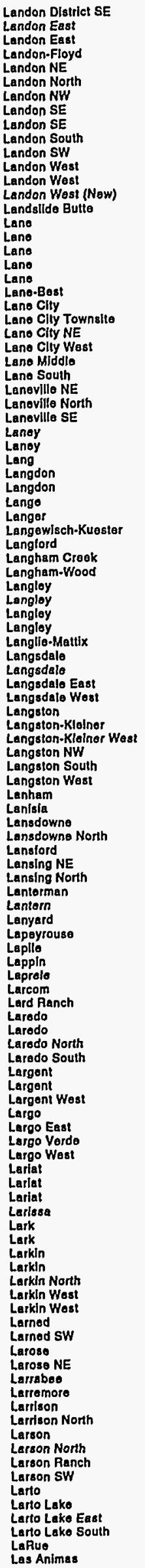 & 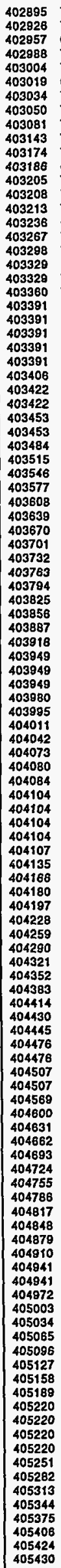 & 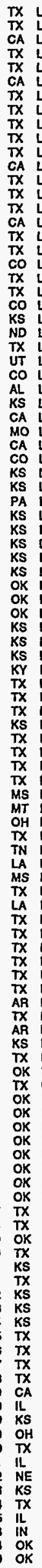 & 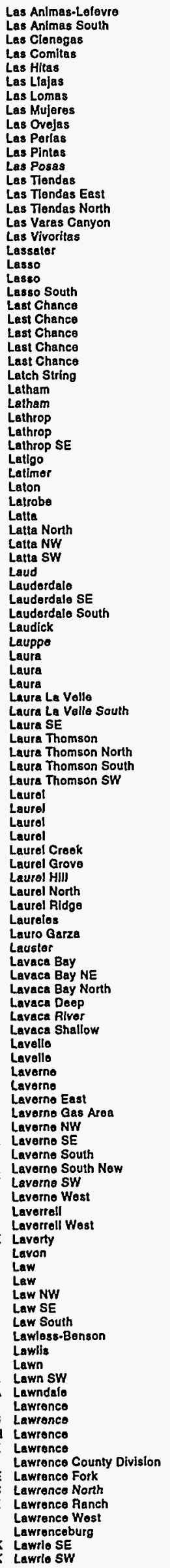 & 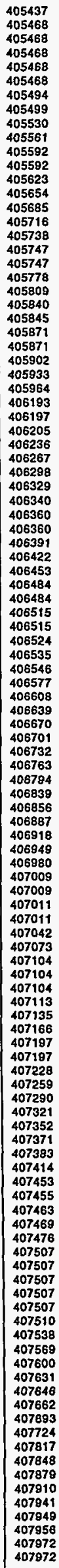 & 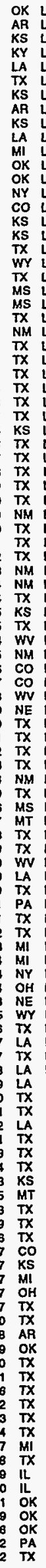 & 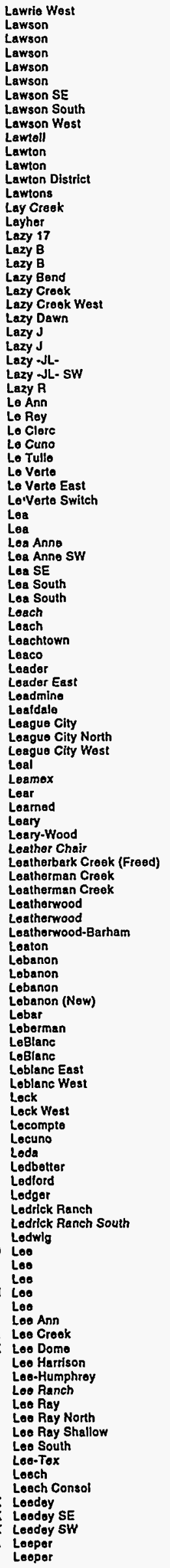 & 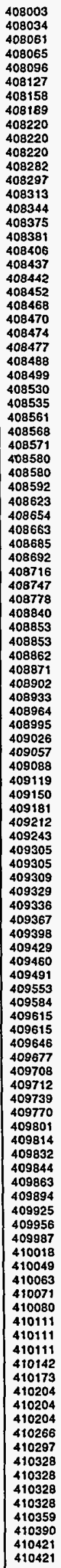 & 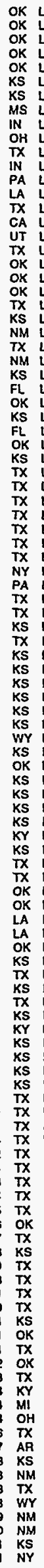 & 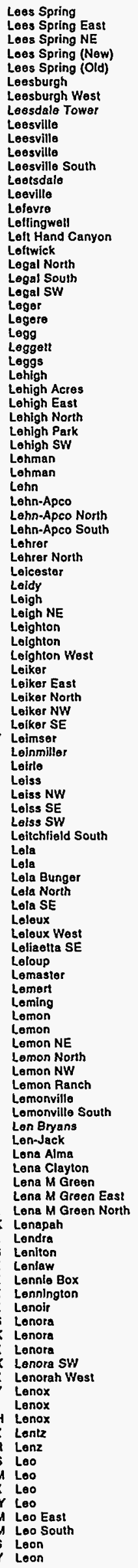 \\
\hline
\end{tabular}




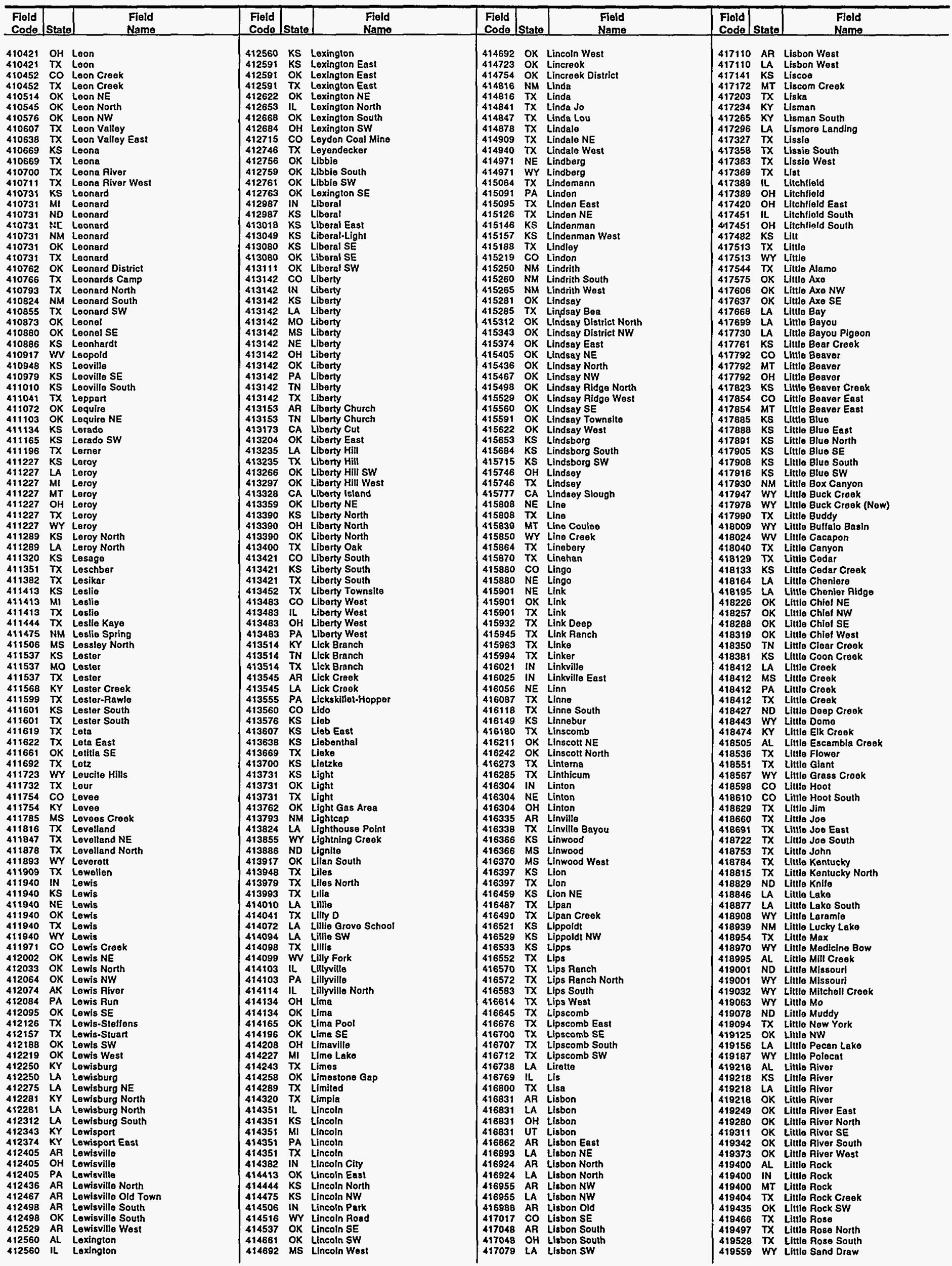




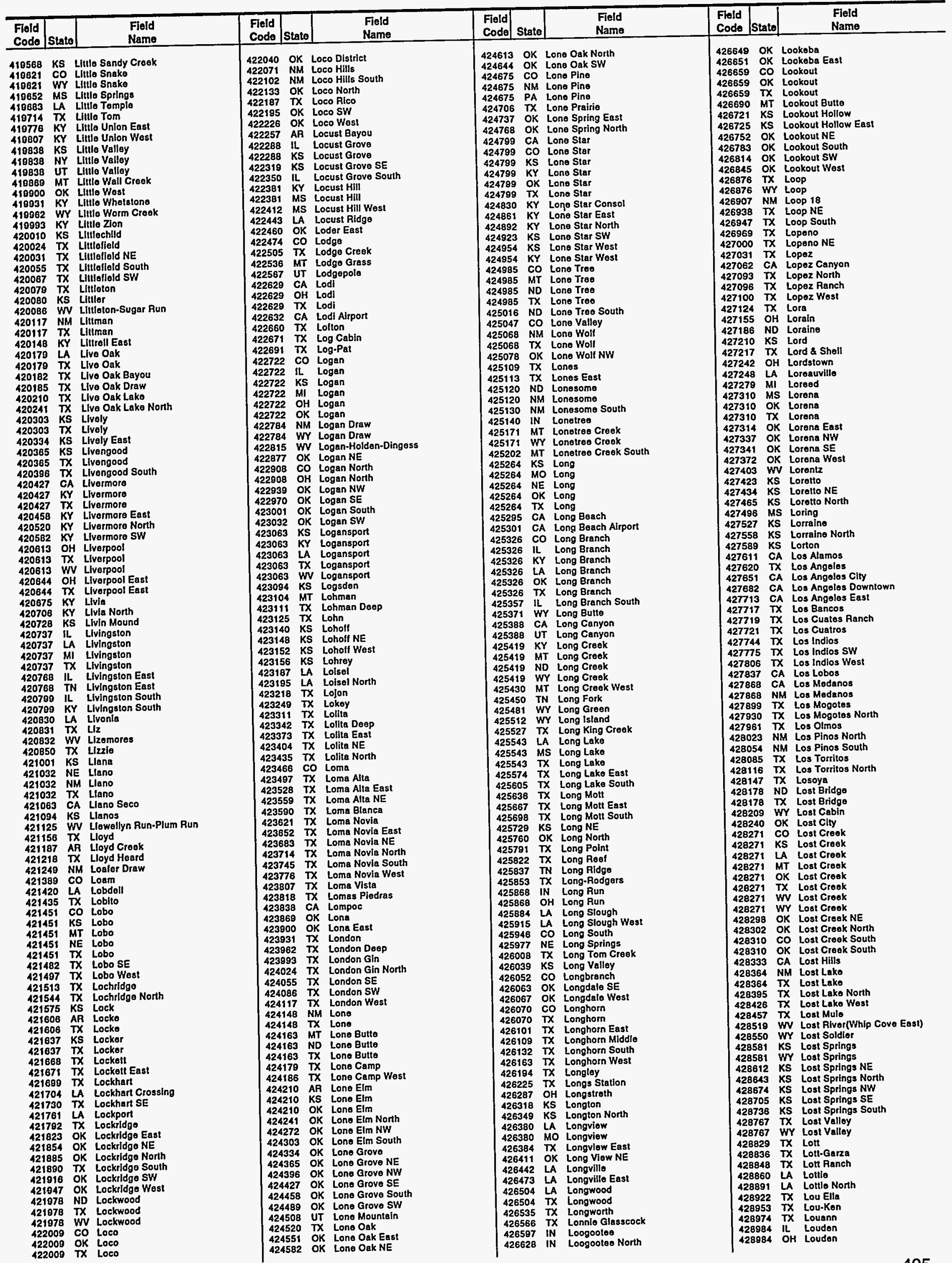




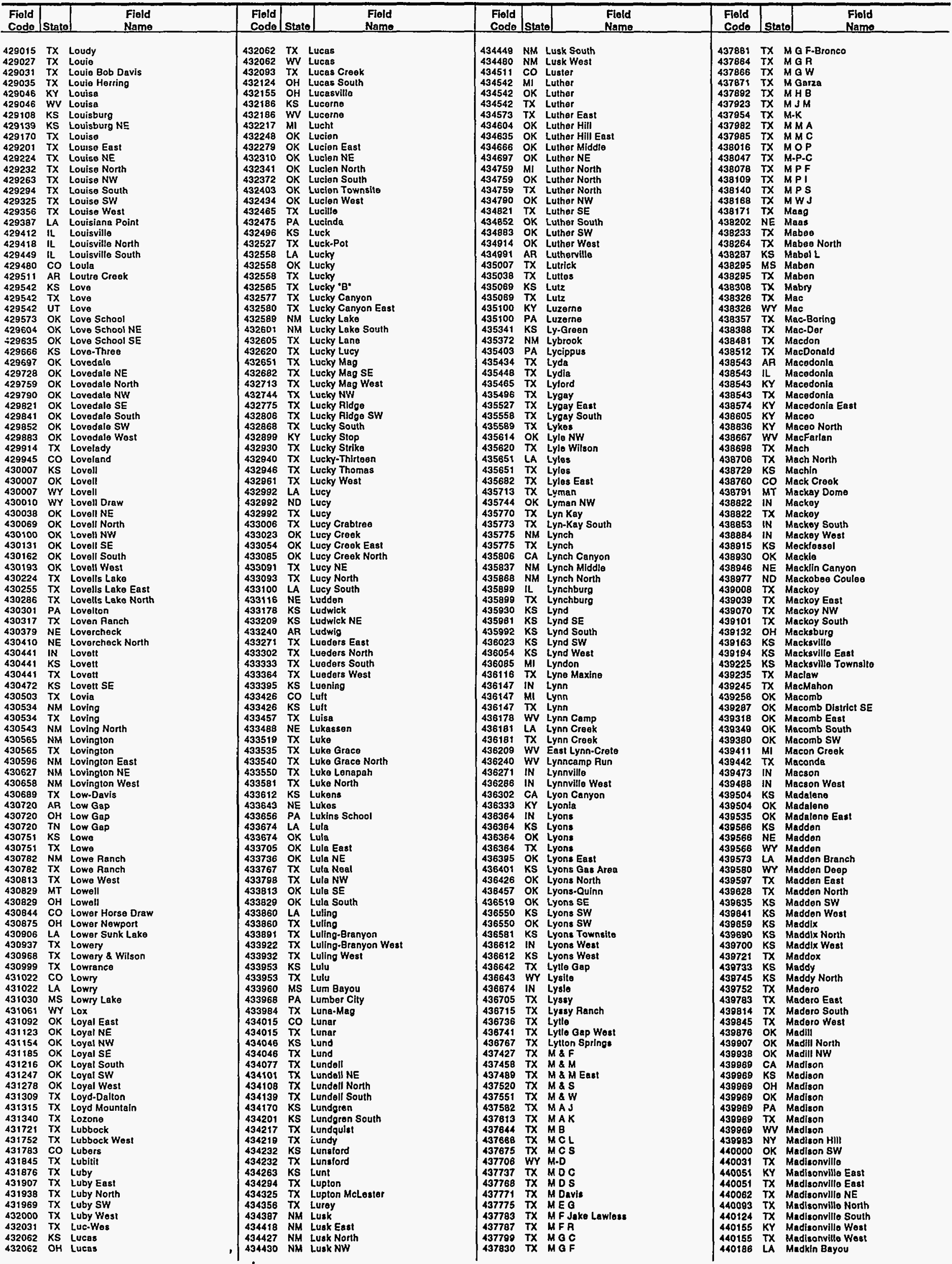




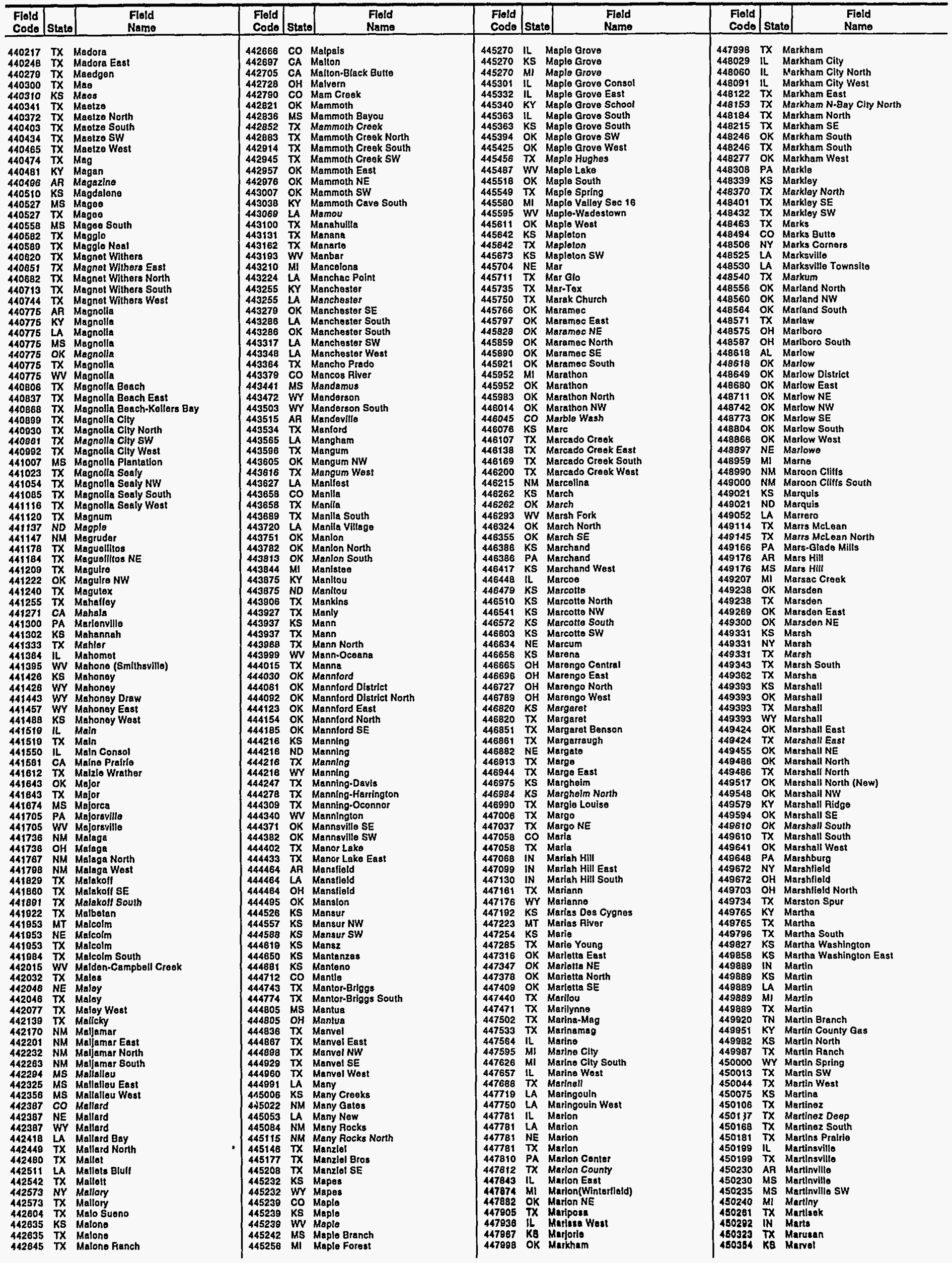




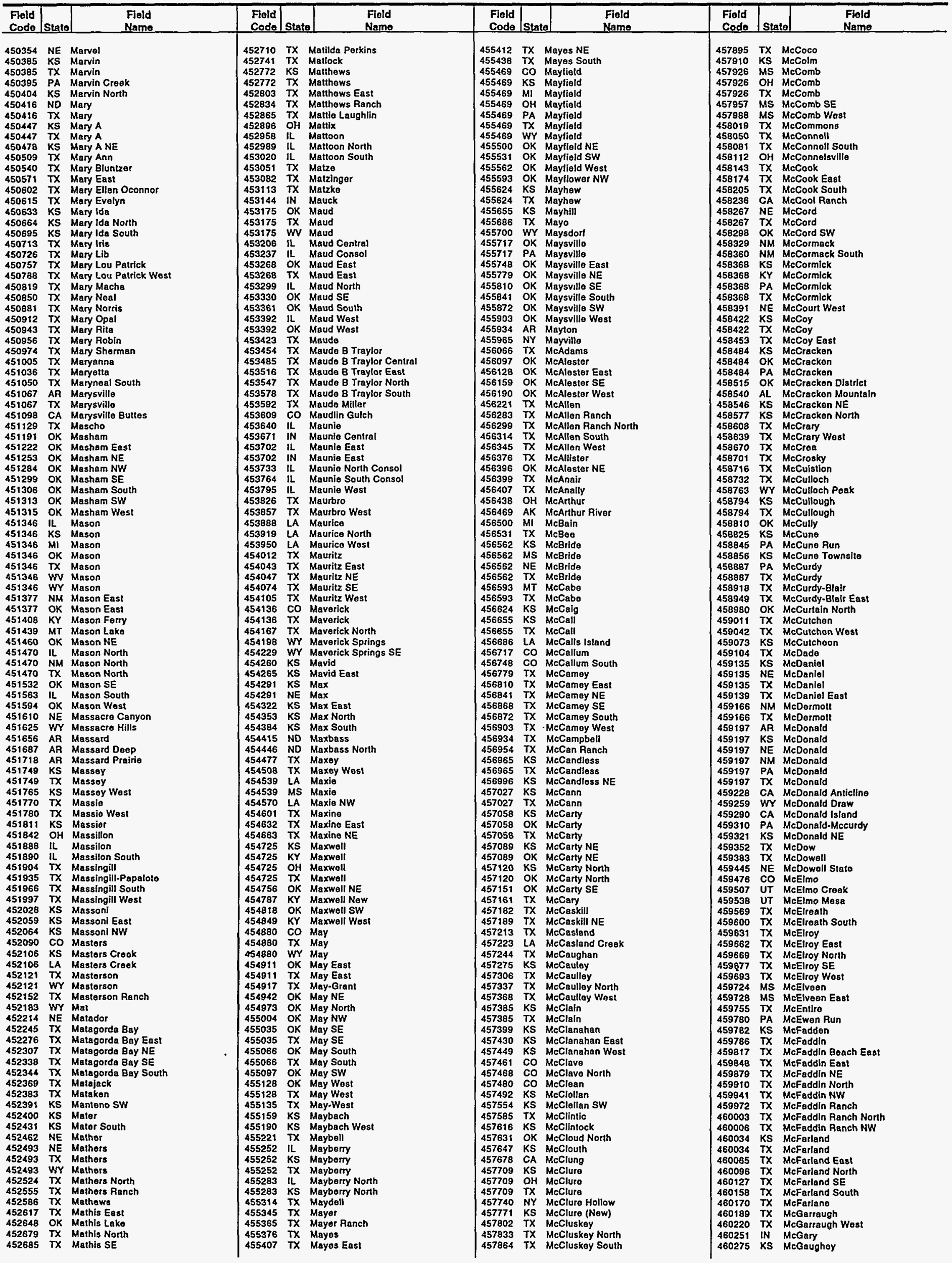




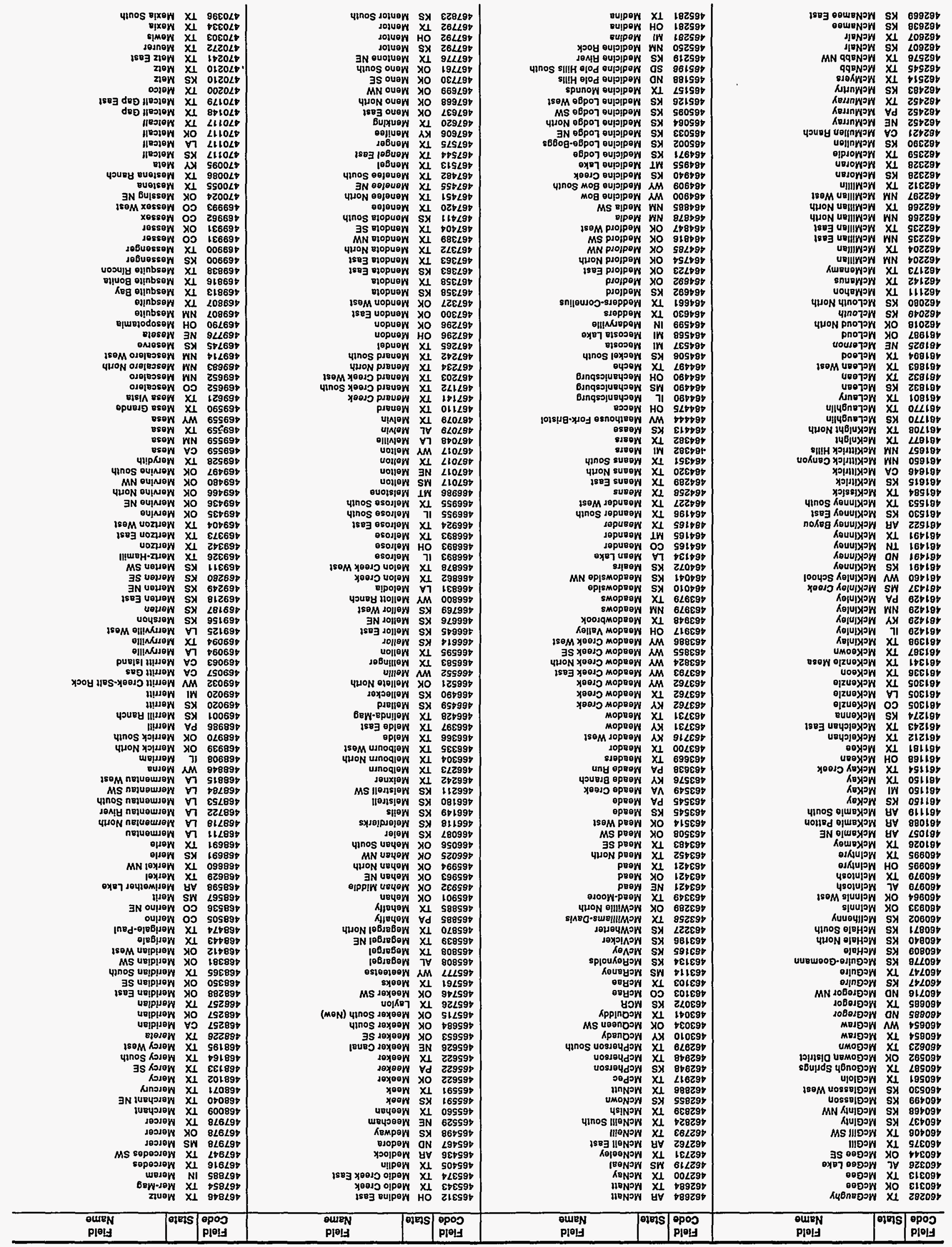




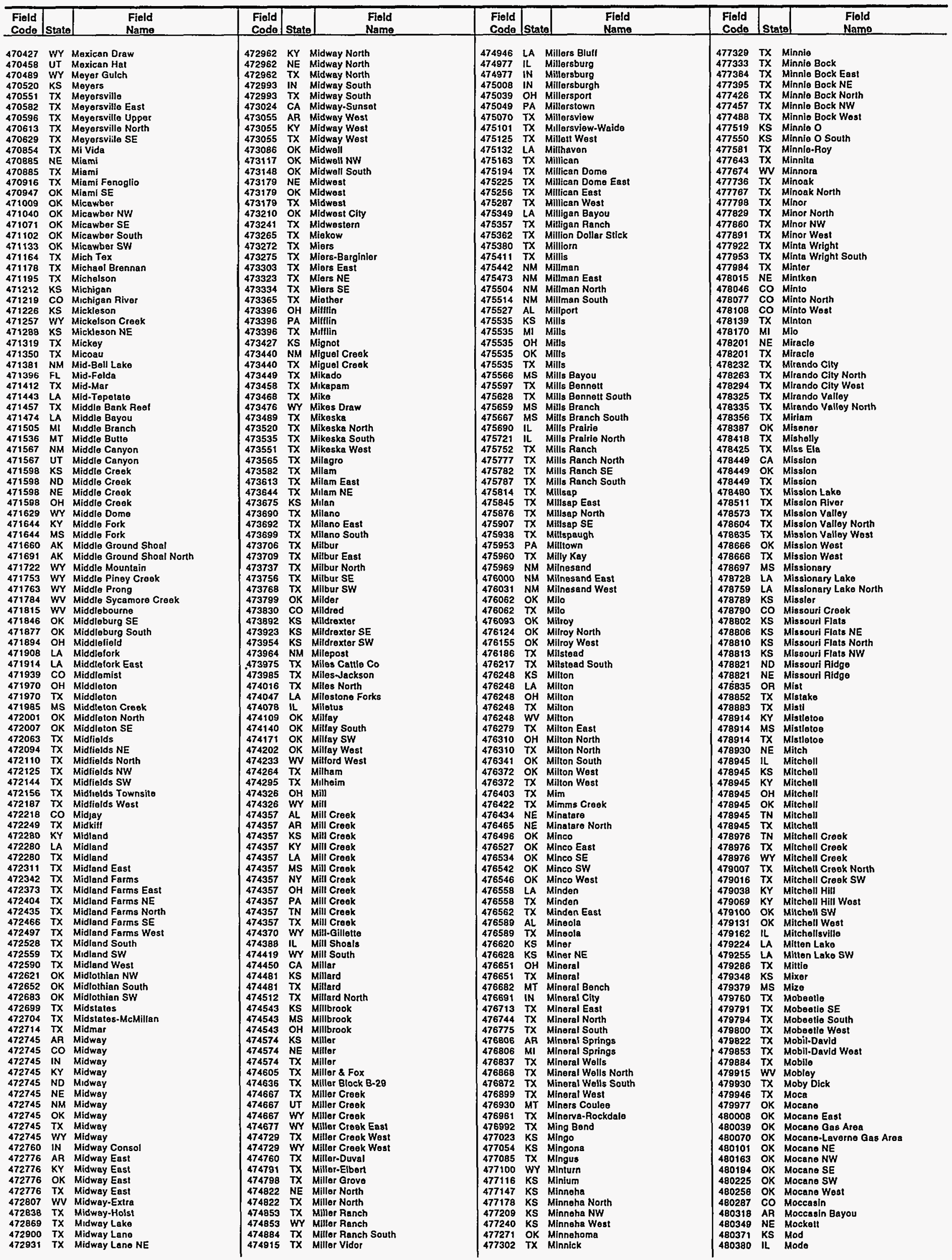




\begin{tabular}{|c|c|c|c|c|c|c|c|c|c|c|c|}
\hline \begin{tabular}{l|l} 
Flold & Codo \\
Cos
\end{tabular} & Stato & $\begin{array}{l}\text { Fleld } \\
\text { Name }\end{array}$ & $\begin{array}{l}\text { Fleld } \\
\text { Code }\end{array}$ & State & $\begin{array}{l}\text { Fiold } \\
\text { Name }\end{array}$ & $\begin{array}{l}\text { Field } \\
\text { Code }\end{array}$ & State & $\begin{array}{l}\text { Fleld } \\
\text { Name }\end{array}$ & $\begin{array}{l}\text { Field } \\
\text { Codo } \\
\end{array}$ & State & $\begin{array}{l}\text { Fleld } \\
\text { Name } \\
\end{array}$ \\
\hline 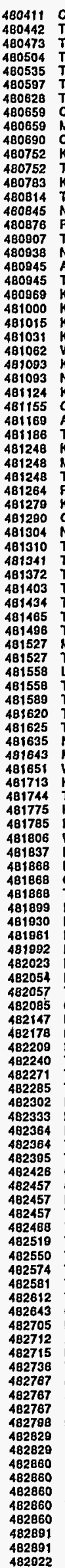 & 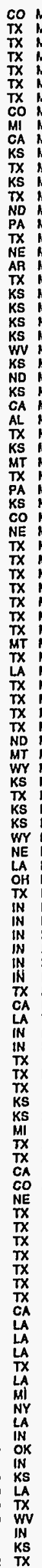 & 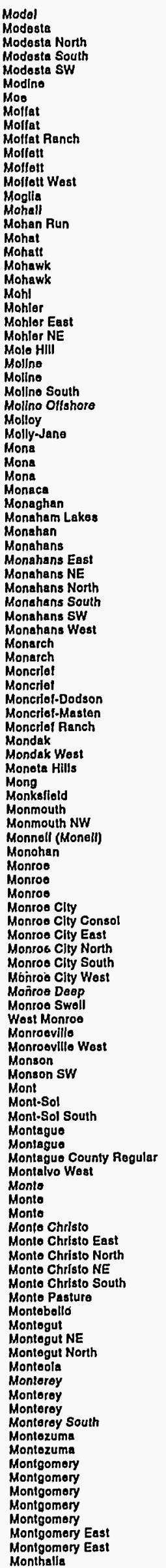 & 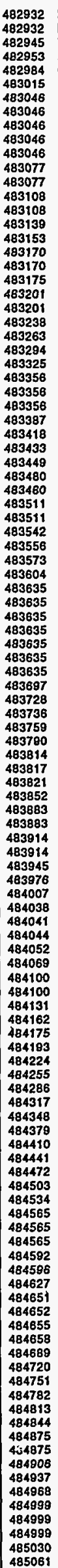 & 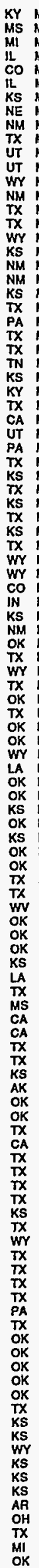 & 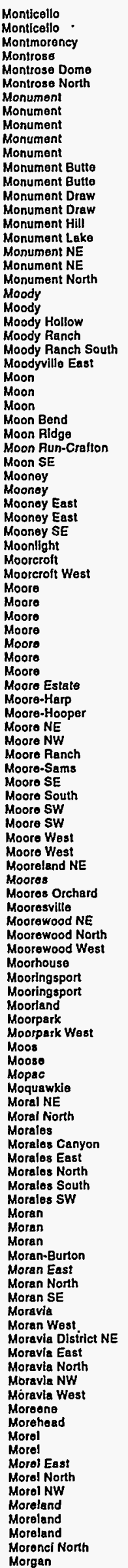 & 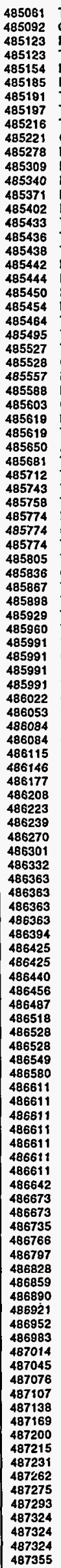 & 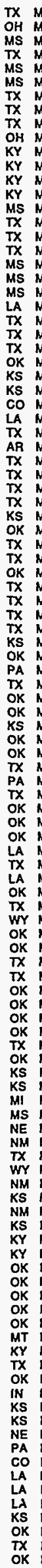 & 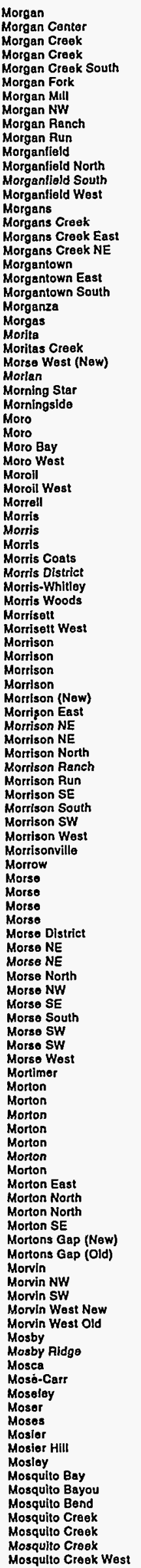 & 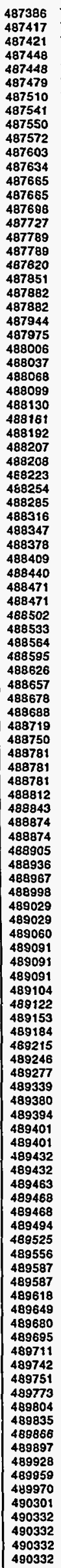 & 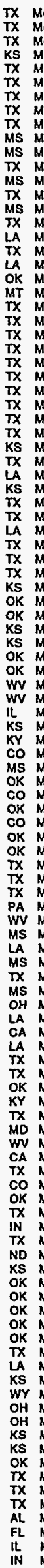 & 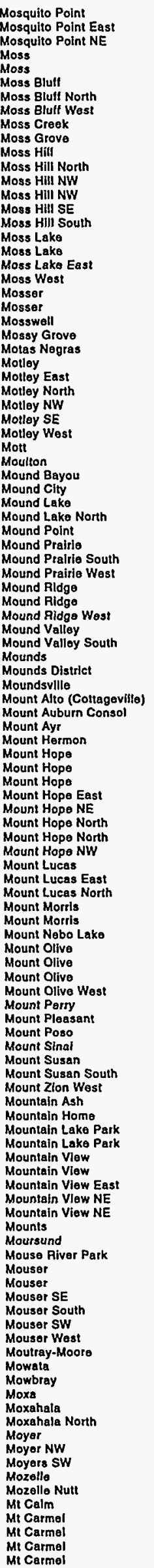 \\
\hline
\end{tabular}




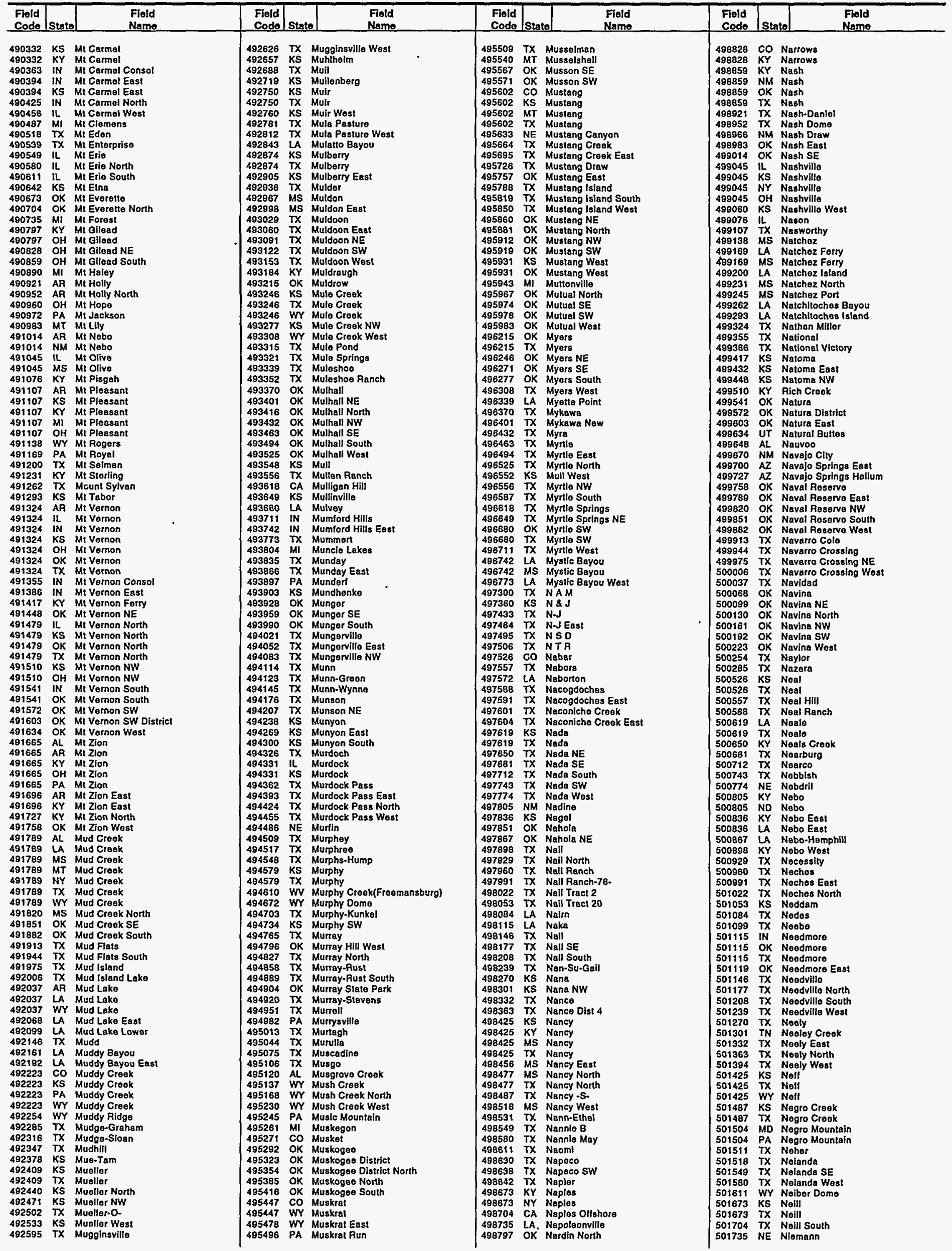


512262

\begin{tabular}{|c|c|c|c|c|c|c|c|c|c|c|c|}
\hline $\begin{array}{l}\text { Field } \\
\text { Code }\end{array}$ & State & $\begin{array}{l}\text { Fleld } \\
\text { Name }\end{array}$ & $\begin{array}{l}\text { Fleld } \\
\text { Code } \\
\end{array}$ & State & $\begin{array}{c}\text { Field } \\
\text { Name }\end{array}$ & $\begin{array}{l}\text { Field } \\
\text { Code }\end{array}$ & State & $\begin{array}{l}\text { Field } \\
\text { Name }\end{array}$ & $\begin{array}{l}\text { Field } \\
\text { Code } \\
\end{array}$ & State & $\begin{array}{l}\text { Field } \\
\text { Name } \\
\end{array}$ \\
\hline 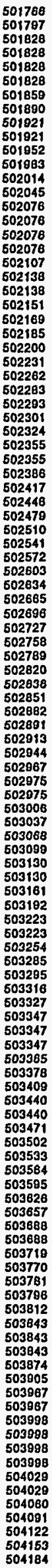 & 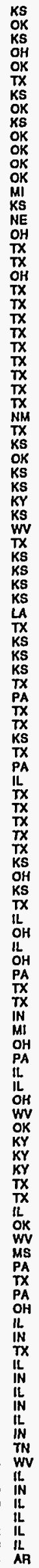 & 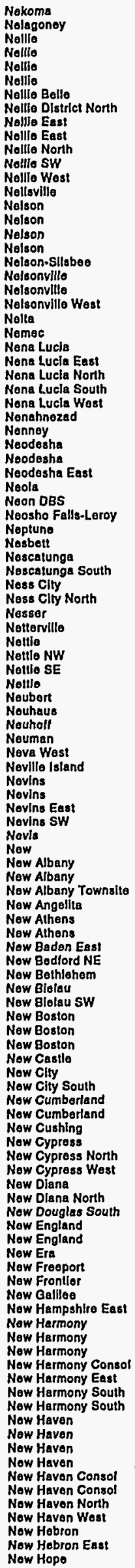 & 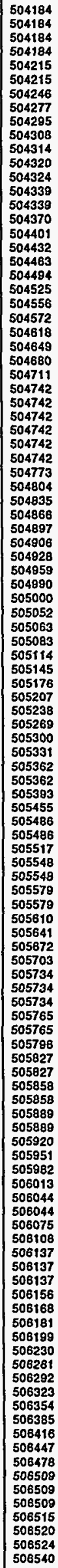 & 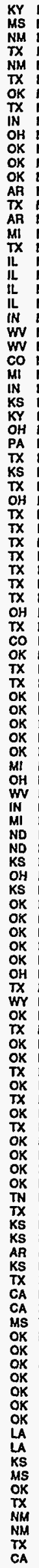 & 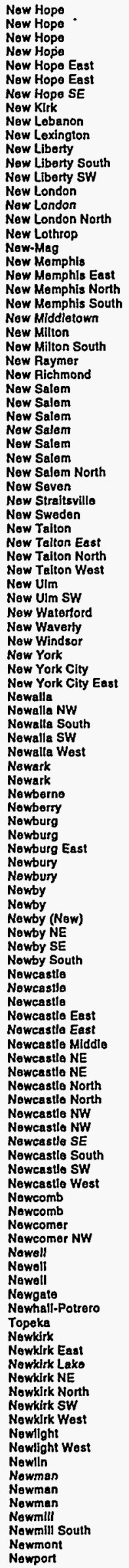 & 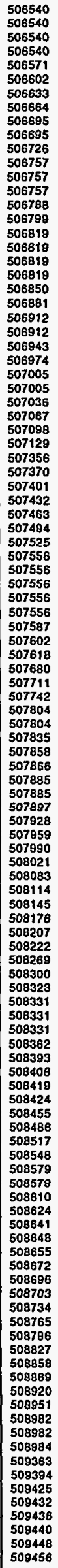 & 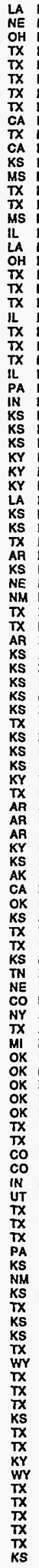 & 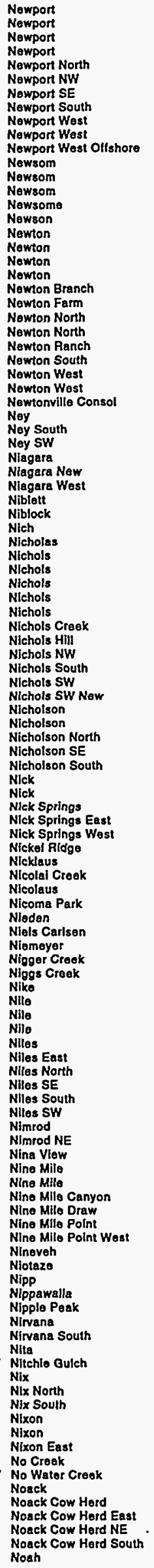 & 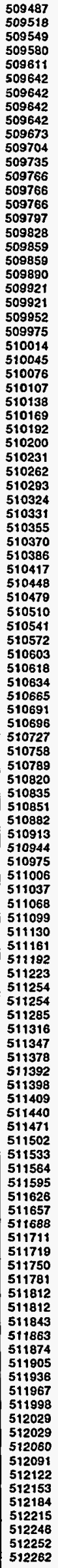 & 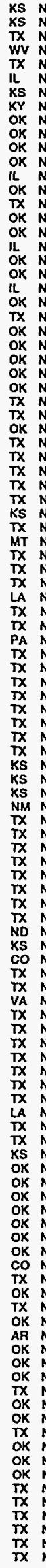 & 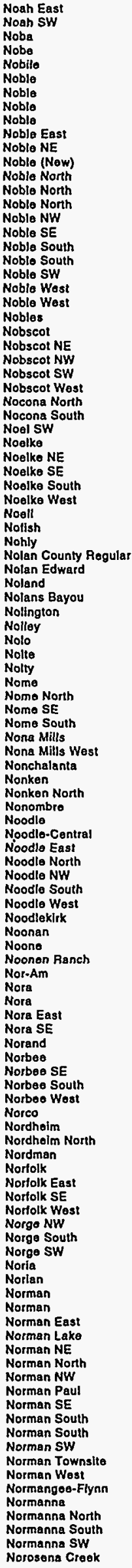 \\
\hline
\end{tabular}

Energy Information Administration/Oll and Gas Field Code Master List 1994 


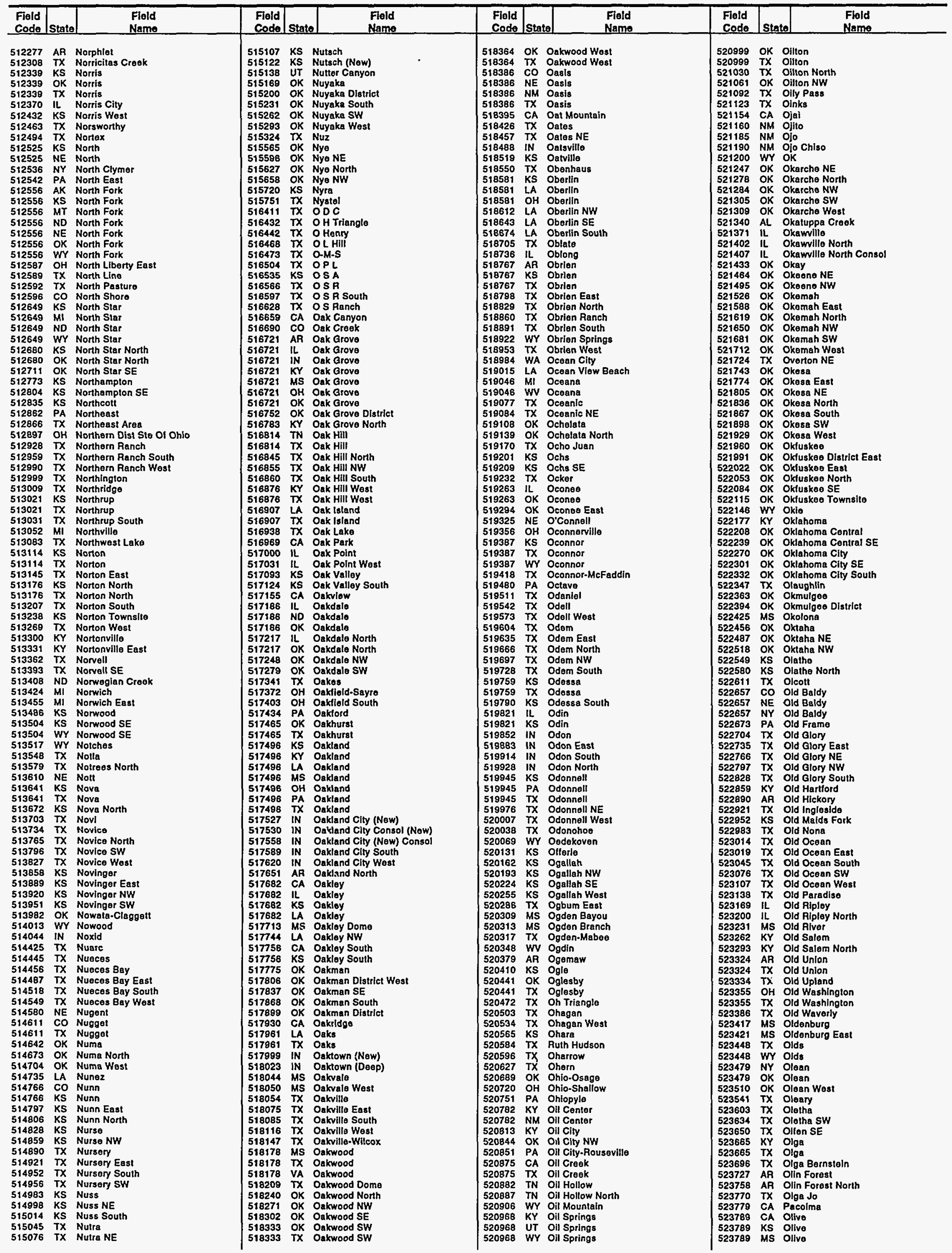




\begin{tabular}{|c|c|c|c|c|c|c|c|c|c|c|c|}
\hline $\begin{array}{l}\text { Flold } \\
\text { Code }\end{array}$ & State & $\begin{array}{l}\text { Fiold } \\
\text { Name } \\
\end{array}$ & $\begin{array}{l}\text { Flold } \\
\text { Codo }\end{array}$ & State & $\begin{array}{l}\text { Fiold } \\
\text { Name } \\
\end{array}$ & $\begin{array}{l}\text { Fleld } \\
\text { Code } \\
\end{array}$ & State & $\begin{array}{l}\text { Field } \\
\text { Name } \\
\end{array}$ & $\begin{array}{l}\text { Fiold } \\
\text { Codo } \\
\end{array}$ & Stato & $\begin{array}{l}\text { Fileld } \\
\text { Name } \\
\end{array}$ \\
\hline 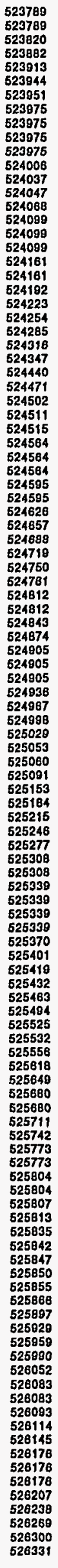 & 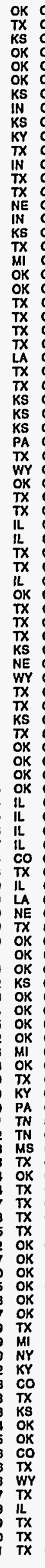 & 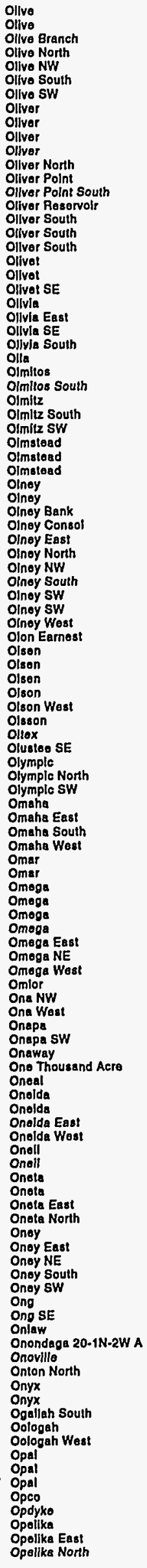 & 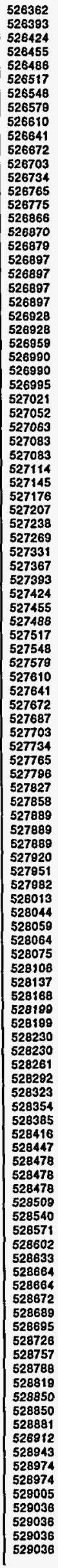 & 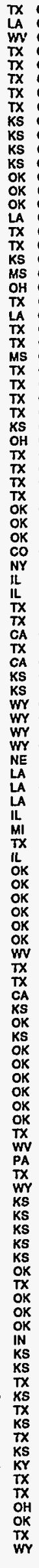 & 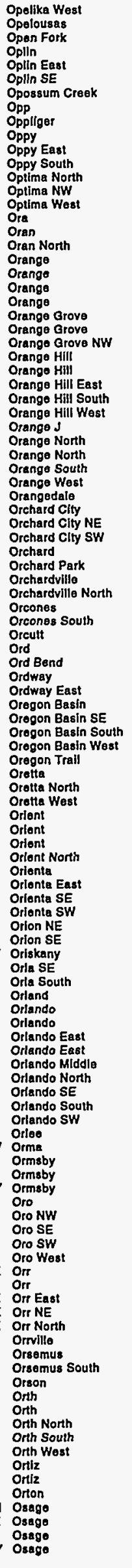 & 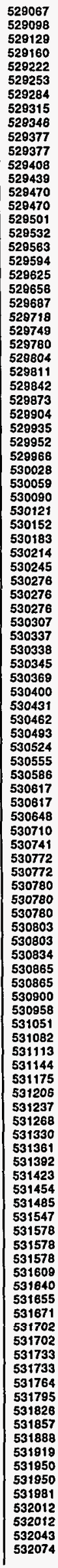 & 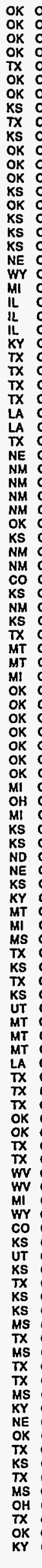 & 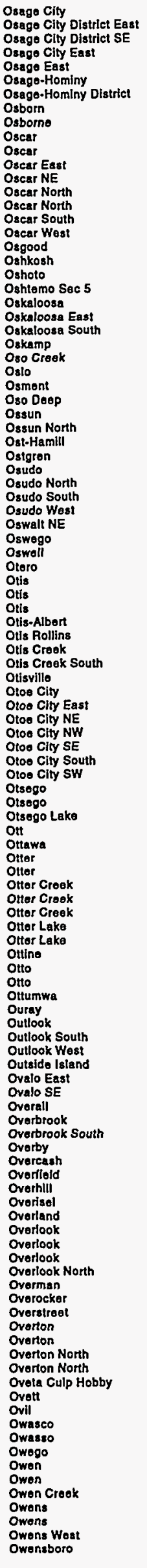 & 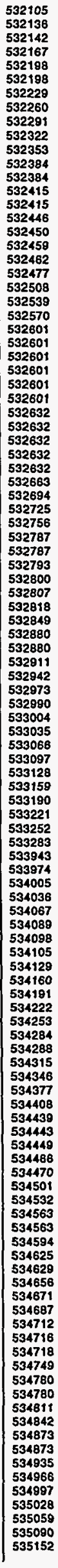 & 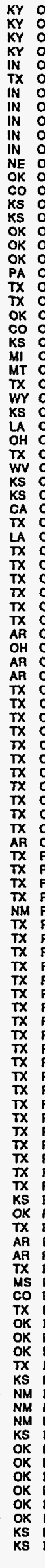 & 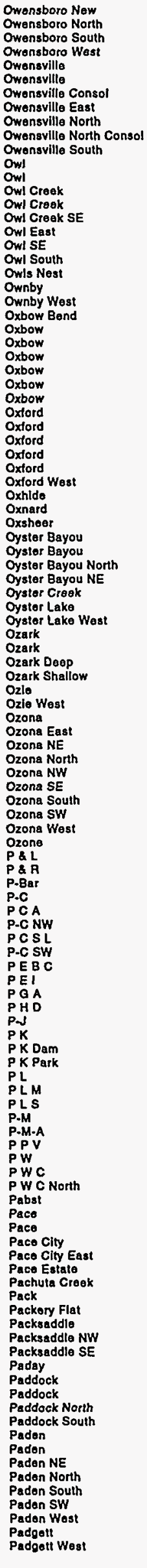 \\
\hline
\end{tabular}




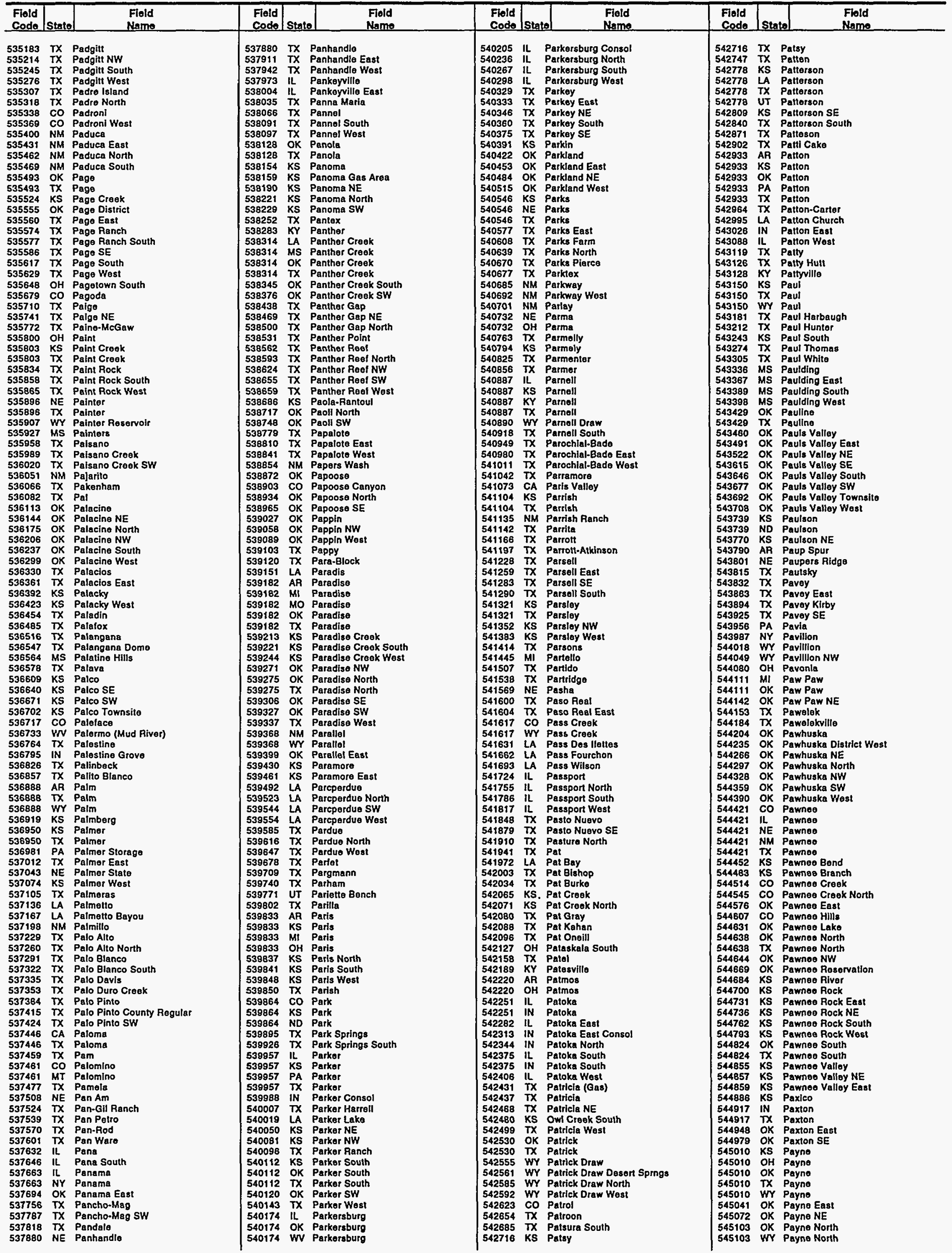




\begin{tabular}{|c|c|c|c|c|c|c|c|c|c|c|c|}
\hline $\begin{array}{l}\text { Flald } \\
\text { Code }\end{array}$ & Stato & $\begin{array}{l}\text { Field } \\
\text { Name }\end{array}$ & $\begin{array}{l}\text { Fleid } \\
\text { Code }\end{array}$ & State & $\begin{array}{l}\text { Field } \\
\text { Namo }\end{array}$ & $\begin{array}{l}\text { Flold } \\
\text { Codo } \\
\end{array}$ & State & $\begin{array}{l}\text { Fiold } \\
\text { Namo }\end{array}$ & $\begin{array}{l}\text { Flold } \\
\text { Code }\end{array}$ & State & $\begin{array}{c}\text { Field } \\
\text { Name } \\
\end{array}$ \\
\hline 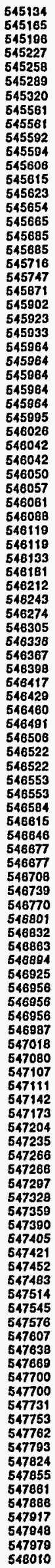 & 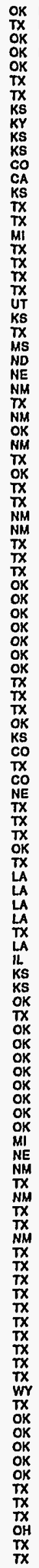 & 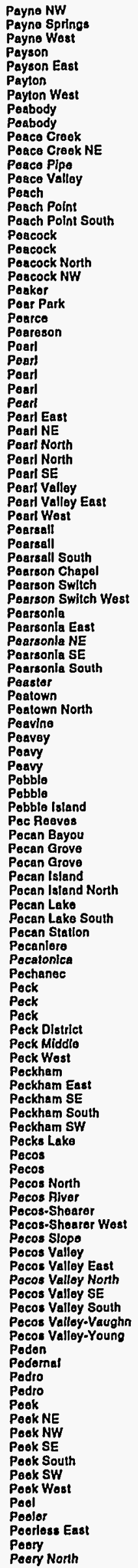 & 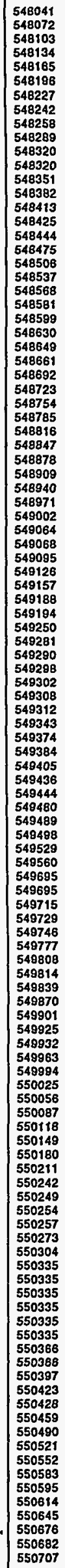 & 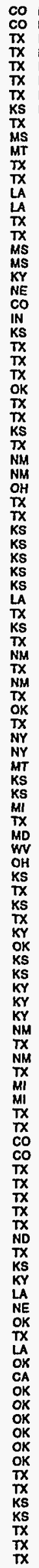 & 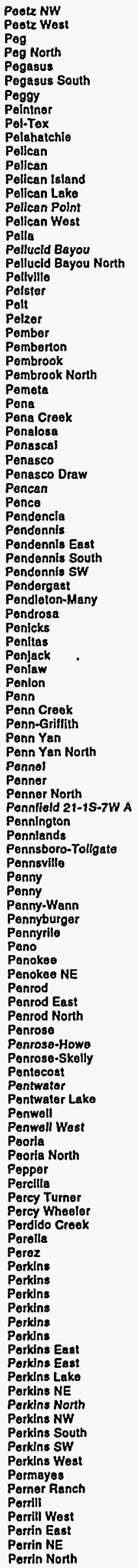 & 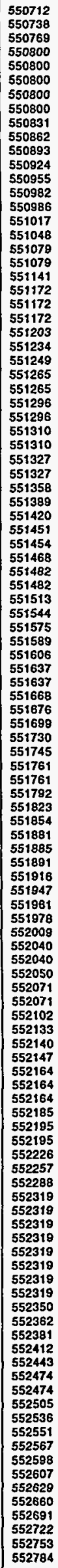 & 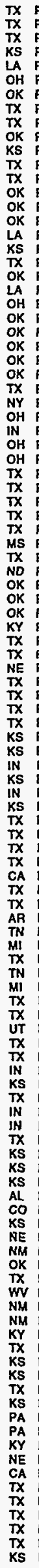 & 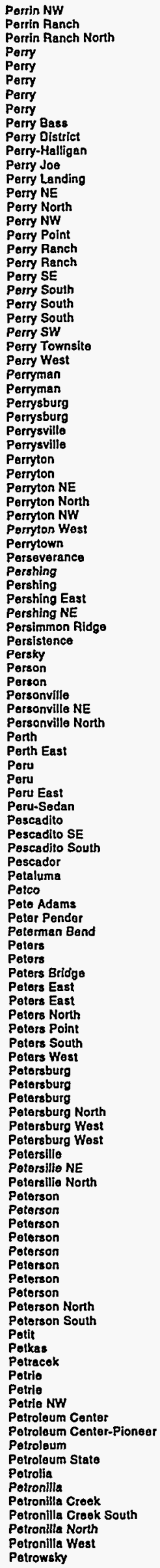 & 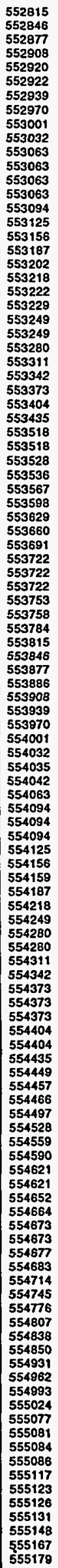 & 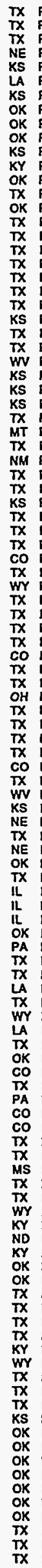 & 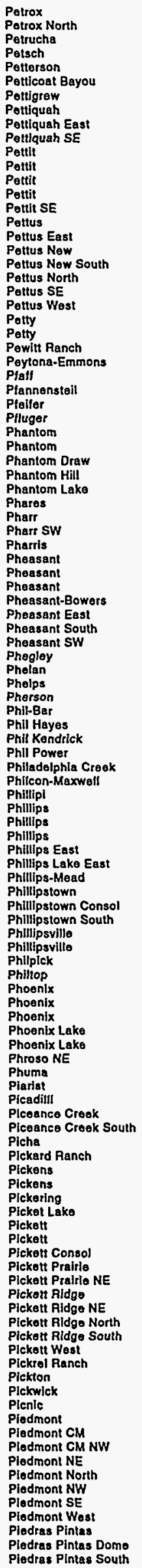 \\
\hline
\end{tabular}




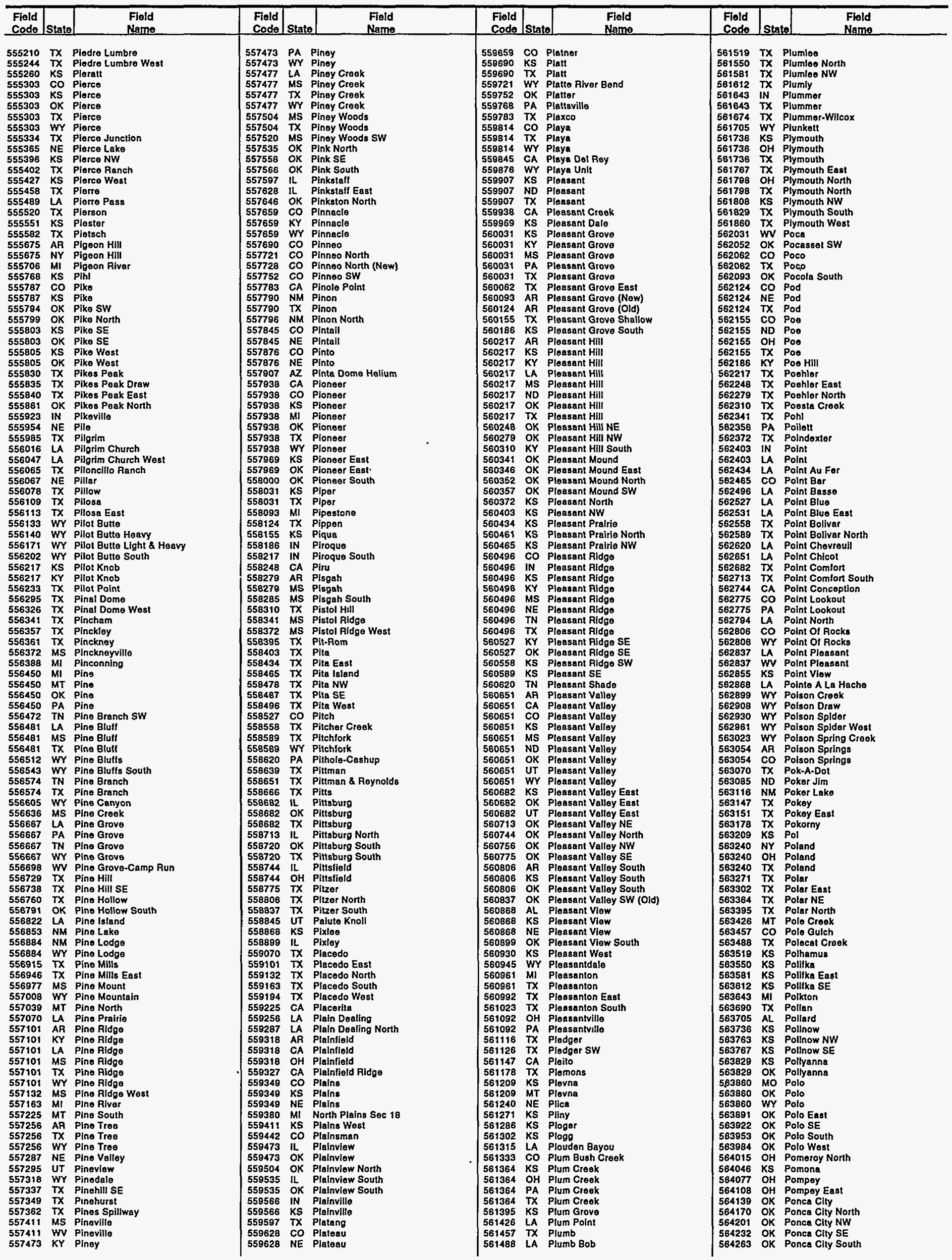




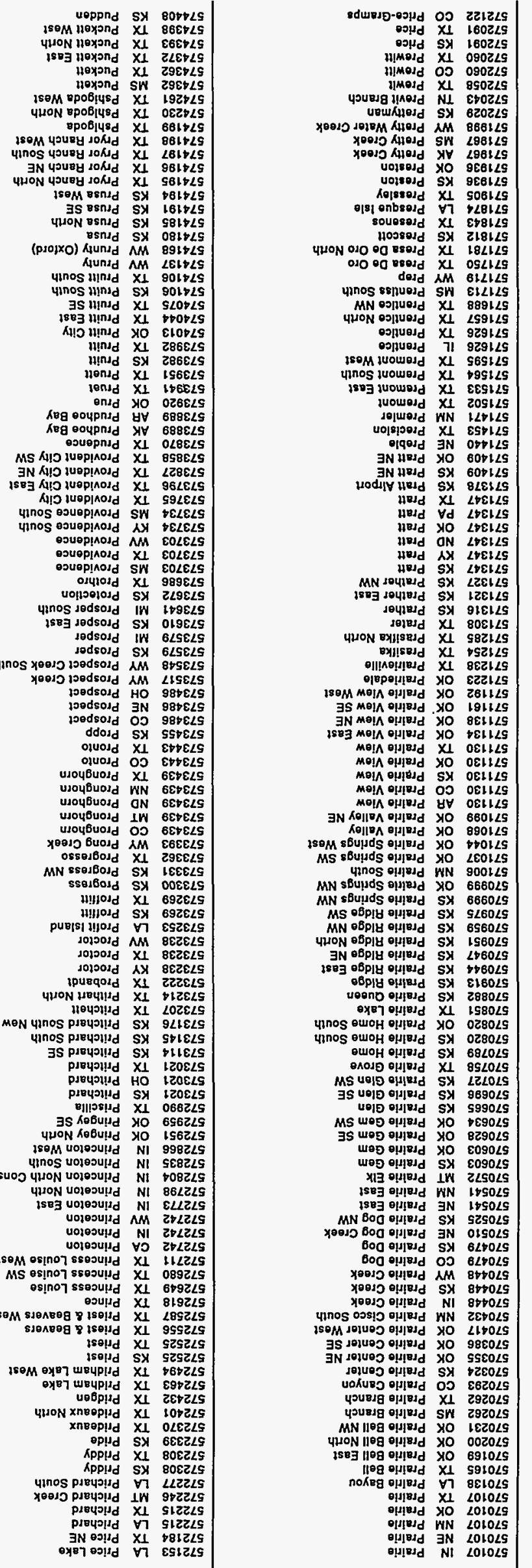

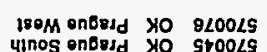

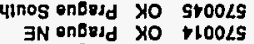

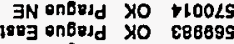
onbesd xo zs889S Joborsd $S x$ t2688s 4lnos opved $X \perp 1068695$ opudd $X_{1}$ 8z889s Y5ü日 sumod XI s8589s somod SX S6s68s

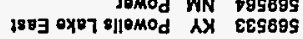

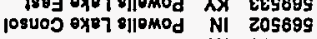
oxp7 s]lemod $N X 1<4699$ 4inos llomod XI Obr6ss 4inos jpMod xo ort89s 2 110MOd MM IlpMOd IM L LE69S "IOMOd SN $\angle$ LE69S IIPMOd OO L4EG9S 150M wouropmod $X 1$ 91869S 4inos wouropmod $X_{1}$ s8z695 IS woynopMod $X_{1}$ t52695 40400pMOd $X_{1}$ EEZ68s $480 M$ LPPMOd OD 191695 sbuinds Jopmod AM OELBos JOMY sopMOd IM BBOBgS 86PIE s0pसOd XI MOM MOd OD 900699 o8ply Y4010d XI SL689S 4LON AHOAOd AX

20IJ AYOAOd SX 186895

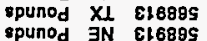

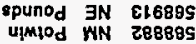
ummiod $S Y$ z8889s

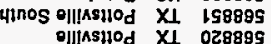
4inos odogsilod $X_{1}$ 68L89S 4HON OJOG\$110 $x_{1}$ 89L899 aroqsilod XI LZL899 silod XI 869899 19841100 $X I$ 8L8895

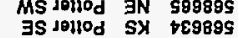

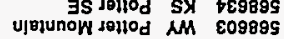
Je110d XI ItS89S JeH10d AN $17589 S$

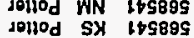

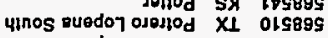
vuedo7 ojostiod XI 6Lr89s olediod y5 8tr895 4inos $18010 d$ XI 786895 I8010d XI s58B9s 4HON $410 \mathrm{~d}$ XI

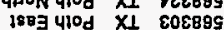
$410 d x_{1}$ E8ze9s 10010d $x_{1}$ 18Z289 orowil|-nnoiod Xo noojod $\times 0$ 891899

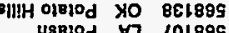

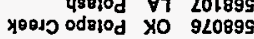
$\exists N$ 비엉 XO 1sp3 ol150d xo +10899 SSOM X0O $380 \mathrm{~d} x_{1} \quad 250299$ MS X8O 180d XI 126299 Xo 180d XI 068295

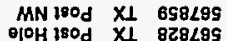
910H 180d $X 1$ 828295

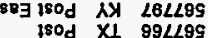

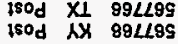
zojumssod $\forall \forall$ SELLOS ebply wnssod XI roLL9S ISOM wopBuUx wnssod XI EL9L9S

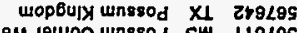

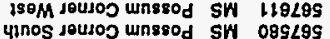
doulog unnsod SW O8SLS9S lousddsod SX Bis 299 Yoejo osod VO $\angle 87299$ ve!pplsod XI 695299

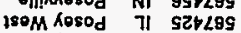

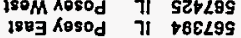
Kosod $S X$ EOELQS 4nnos ussod 71 2E\&L9S 4HON Uasod 71 loc 295 4inos wnנOd Xo St2L9S 4RON wnנod XO 682299

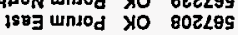
(inors) os!y culod YY SOL $M 12 \angle 9 S$ urupod $\forall d$ gtlL9S

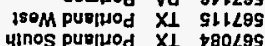
uinos purjuod $S x$ t80L9S
MN puriplod XI $\quad 020 / 99$ 4MNON pupipod XI 190/99

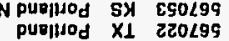

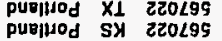

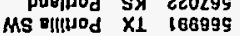

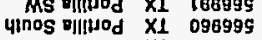

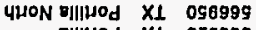
vill $X_{1}$ oz69g9 allow

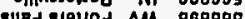
150 Lop X1 288999 opplyod $X_{1}$ Er8999 4HON JOHOd XI 4HON $1000 \mathrm{~d}$ iW so899

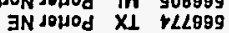

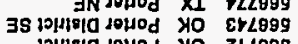
PIIIIIO dOHOd XO 212999 1940d X1 $X_{1}$ OS9999 1240d XO 059999 loulod is 059999 dou1 SX OS9999

$180 M$ soluept10d SX 818999 (0)

180M SoYvoN HOd XI OZS999 4InOS $80450 \mathrm{~N}$ H10d XI OL5899 4HON SOYJON HOd XL S6rg9s soyooN $40 \mathrm{~d} x_{1}$ rgry9s

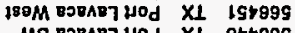
MS BovAv7 Hod XI 9tr99s

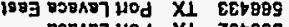

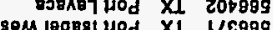

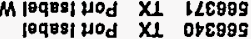
voun HOd IN LYZ899 Lospny Hod V7 lezg9g onveg $10 \mathrm{~d}$ V7 912899

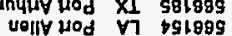
MS seroY MOd XI E己togs 4InOS sorv HOd XI 260995 sojoy Hod $x_{1}$ 190999 oujdored XM 080995 1803 xooso ollnbod $X_{1} \quad 666999$ ollnbod $x_{1}$ \&6es99 obpiy kddod VD toossg $\begin{array}{lll}\text { ddod } & \text { XI } & \text { 9L8S99 } \\ \text { ddod } & S X & \text { SLOSOS }\end{array}$ P|BY Odod AM E98S89 MN Jpidod IW treses OAOJO Jupdod SW El859S 1803 dujdod IW ZOLSOS :D|ARS-0dod XI OZLS 99 odood IN 689999

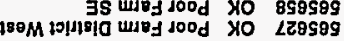
wury jood XO 9BSS9S nog jood XI s9ss99 $150 M$ 에시시 $X_{1}$ rESS99 MS OIIIANAOD $x_{1}$ EOSSOS OlllA100d XI ZLrSOS

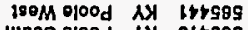
4 nnos ojood 서 1803 O100d AY OLESE9 年 ojo0d AY LLES9S

$190 \mathrm{M}$ 0xy7100d $\forall 7$ 98Z999 อx87 100d V7 s92599 yoolo lood SW Y2Zs9s ssordxy huod OI Eets9s XOPOO KUOd AM ZOIS9S YoDIJ NuOd XI z9ISOS Auod OO ESLS9S

MS oplluod SN ST sqluod Sर्र 001599

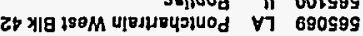

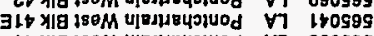

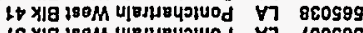

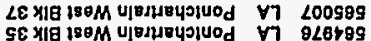
pluod $X 1$ rletgs majued X1 Le8585 48juod XI E88r9s ound $X_{1}$ 25889s o01000 viopuod IW 65Lt99 vsopuod IW ozLtss

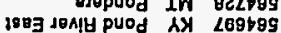
JoAft puod AX 899r9g MS Yyou puod to searas MN YOOSO puod Xo ELSt9S 1583 Y0015 pUOd Yo 08btes yoojs puod yo 6rtt95 xo sud $5 x$. puod XI 8irtos puod SW Bttros

3s oouod Sy oouod SX 9zcrog

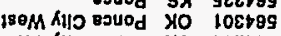
MS Ailo EכUOd XO tozt9s

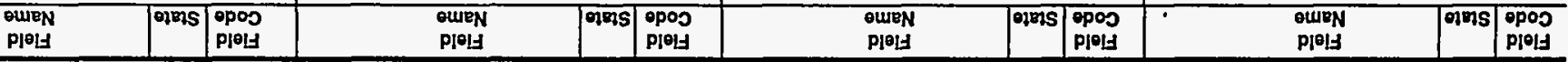




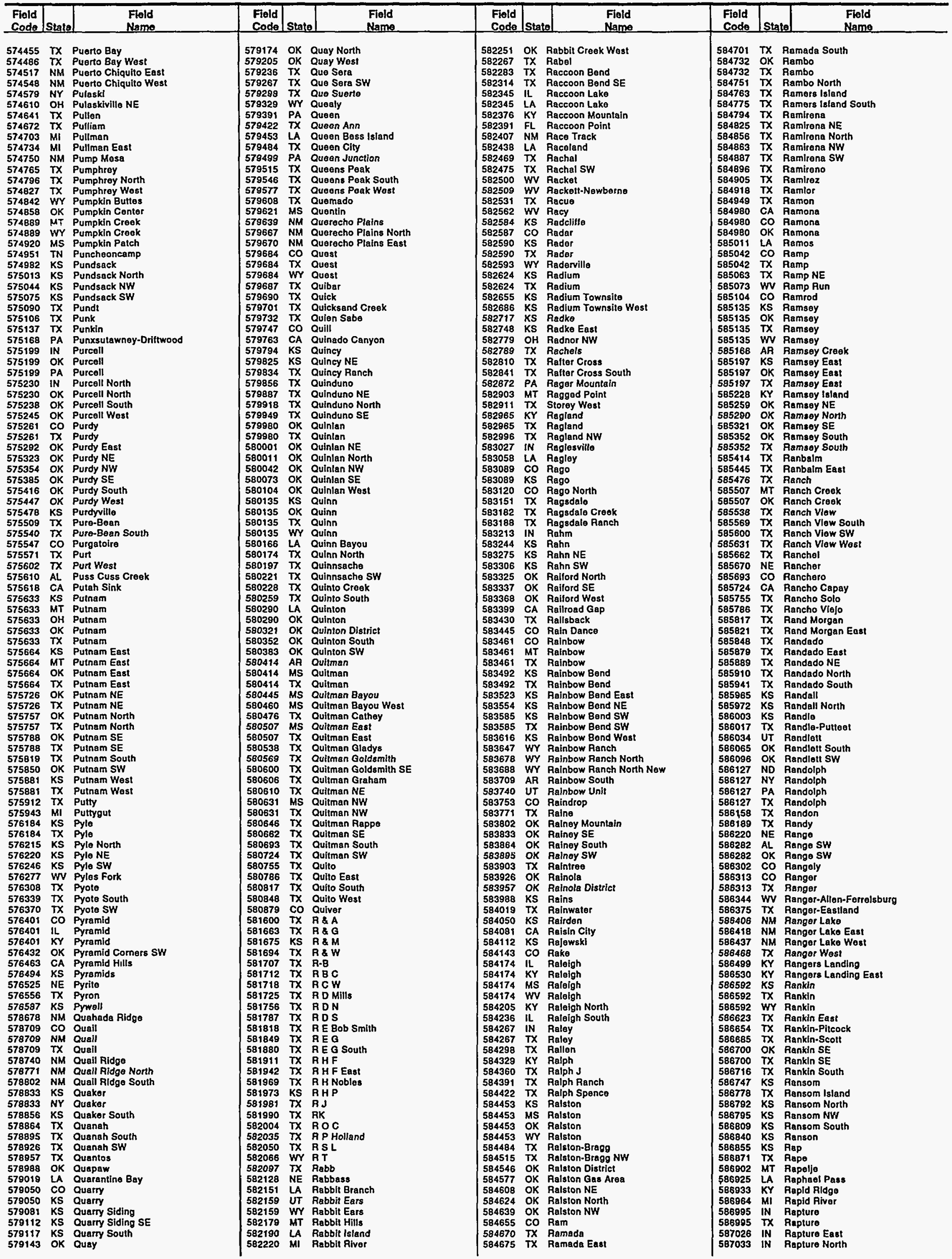




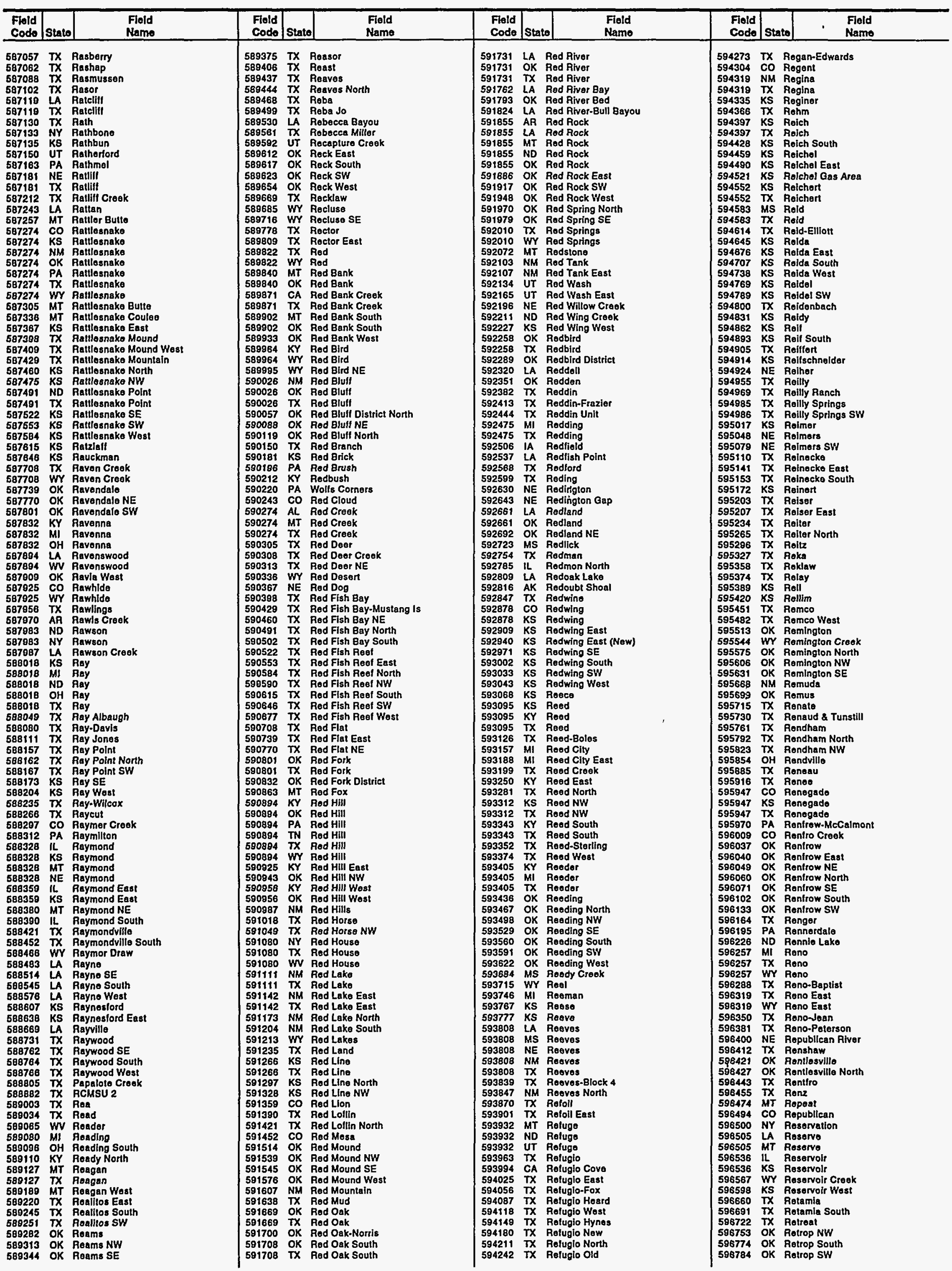




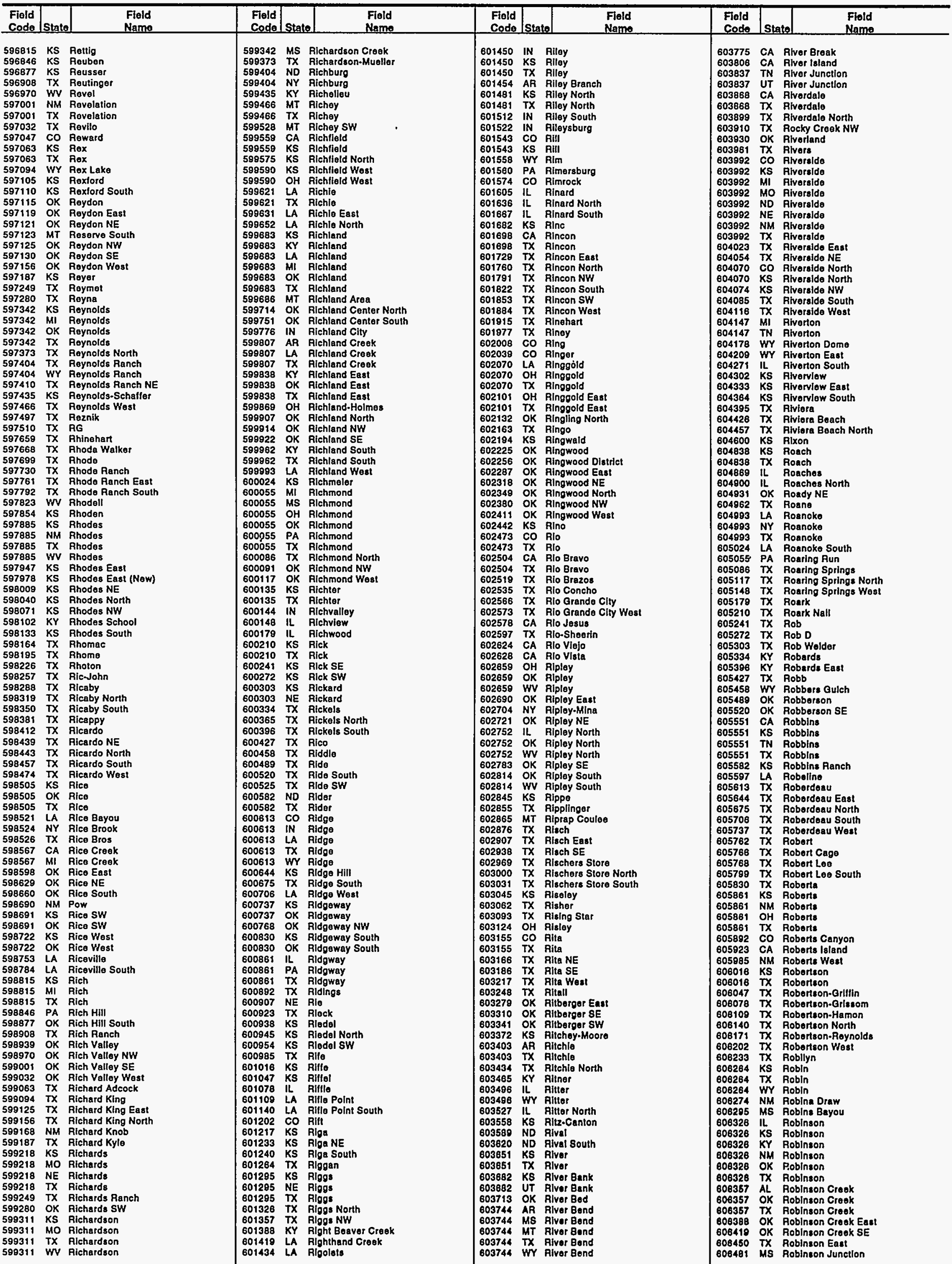




\begin{tabular}{|c|c|c|c|c|c|c|c|c|c|c|c|}
\hline $\begin{array}{l}\text { Fleld } \\
\text { Codo }\end{array}$ & State & $\begin{array}{c}\text { Fleld } \\
\text { Namo }\end{array}$ & $\begin{array}{l}\text { Fiold } \\
\text { Codo }\end{array}$ & State & $\begin{array}{l}\text { Flold } \\
\text { Namo } \\
\end{array}$ & $\begin{array}{l}\text { Flold } \\
\text { Code } \\
\end{array}$ & State & $\begin{array}{l}\text { Field } \\
\text { Name } \\
\end{array}$ & $\begin{array}{l}\text { Flold } \\
\text { Code }\end{array}$ & State & $\begin{array}{c}\text { Field } \\
\text { Name } \\
\end{array}$ \\
\hline 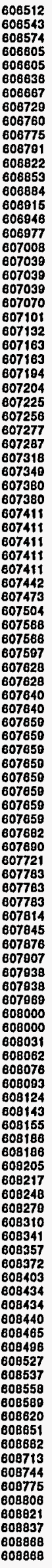 & 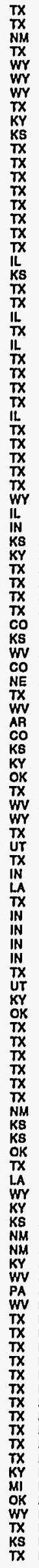 & 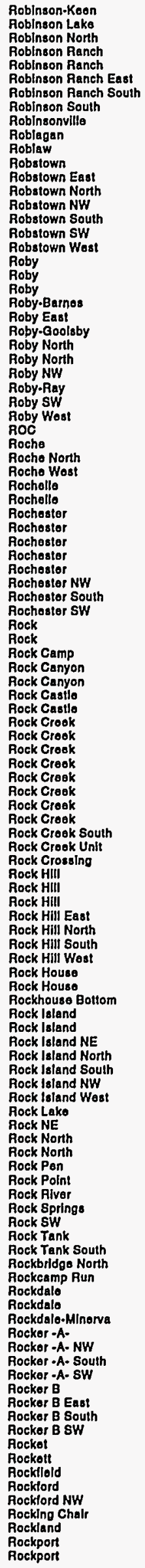 & 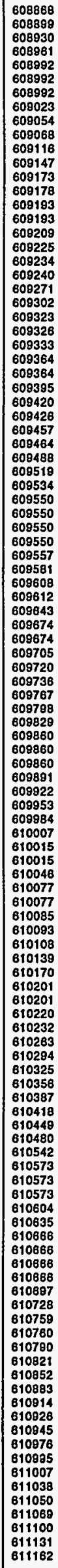 & 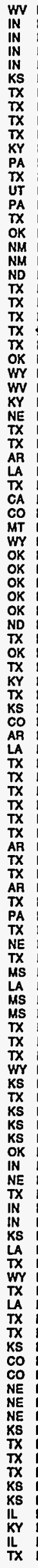 & 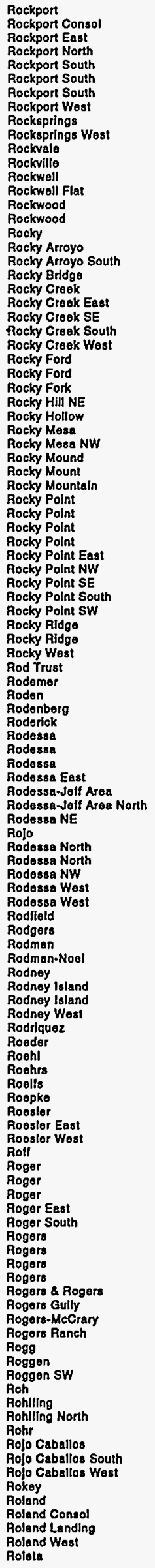 & 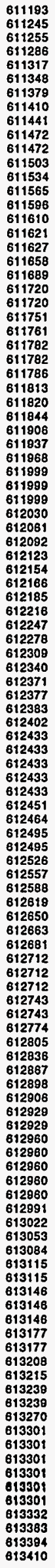 & 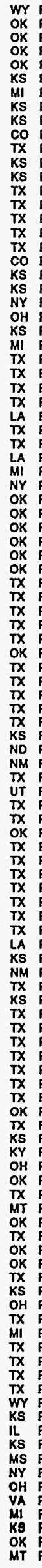 & 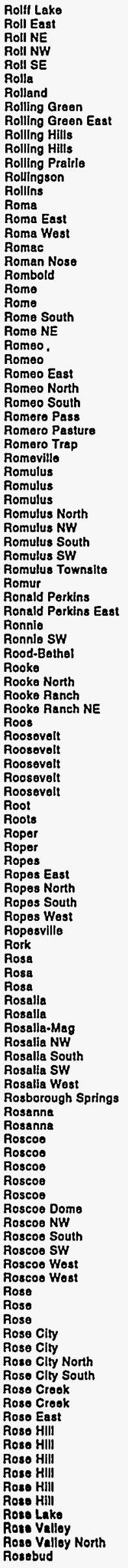 & 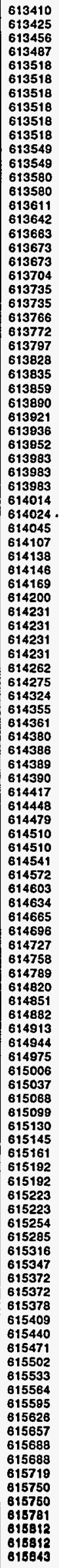 & 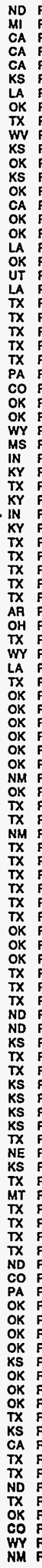 & 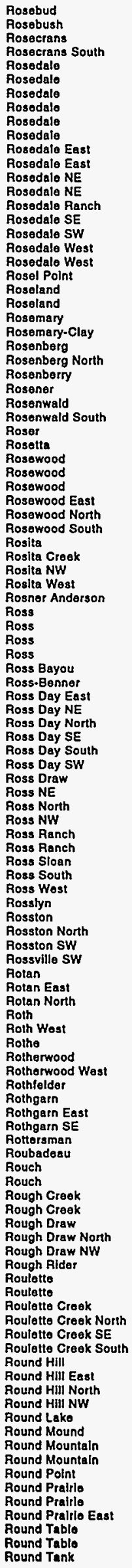 \\
\hline
\end{tabular}




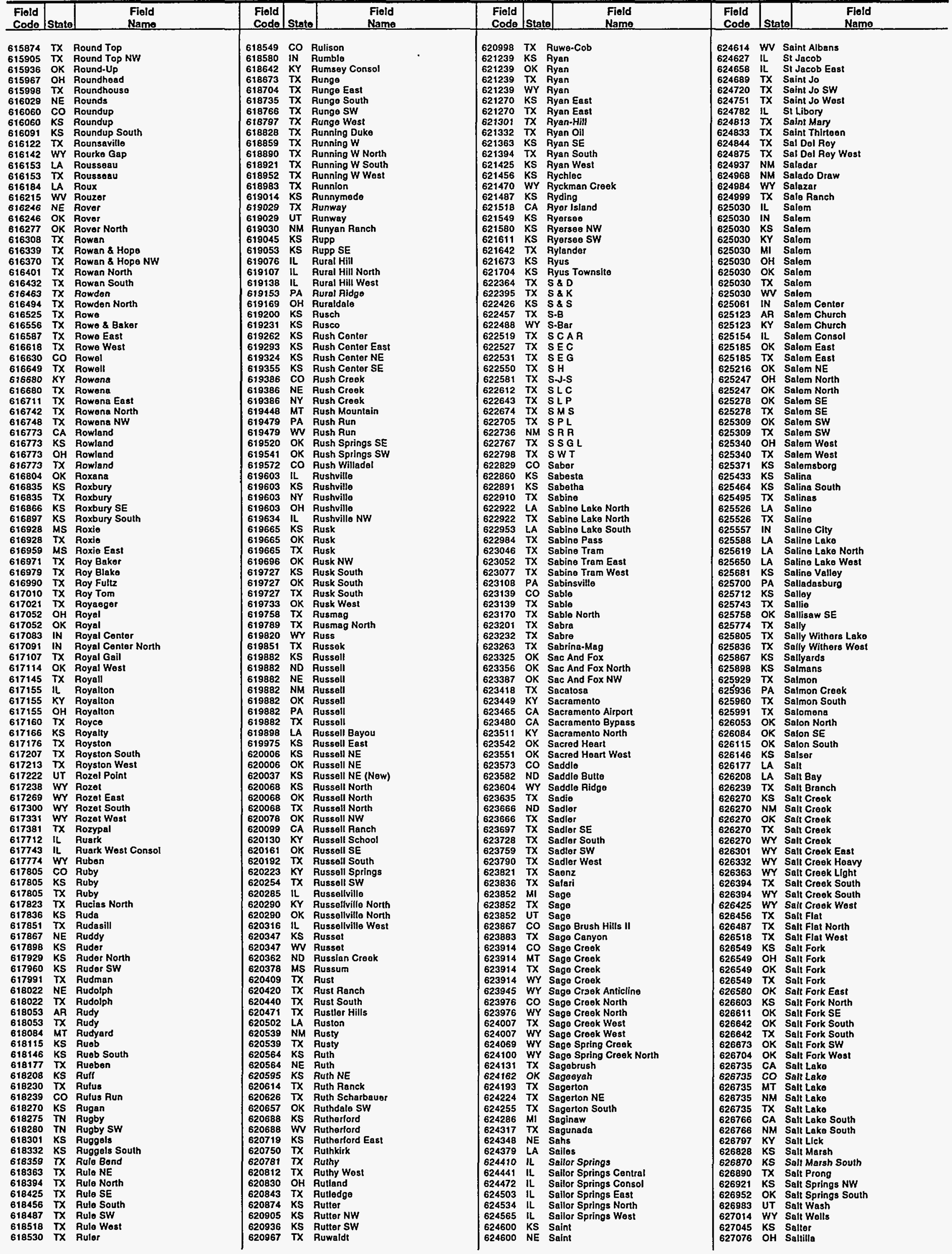




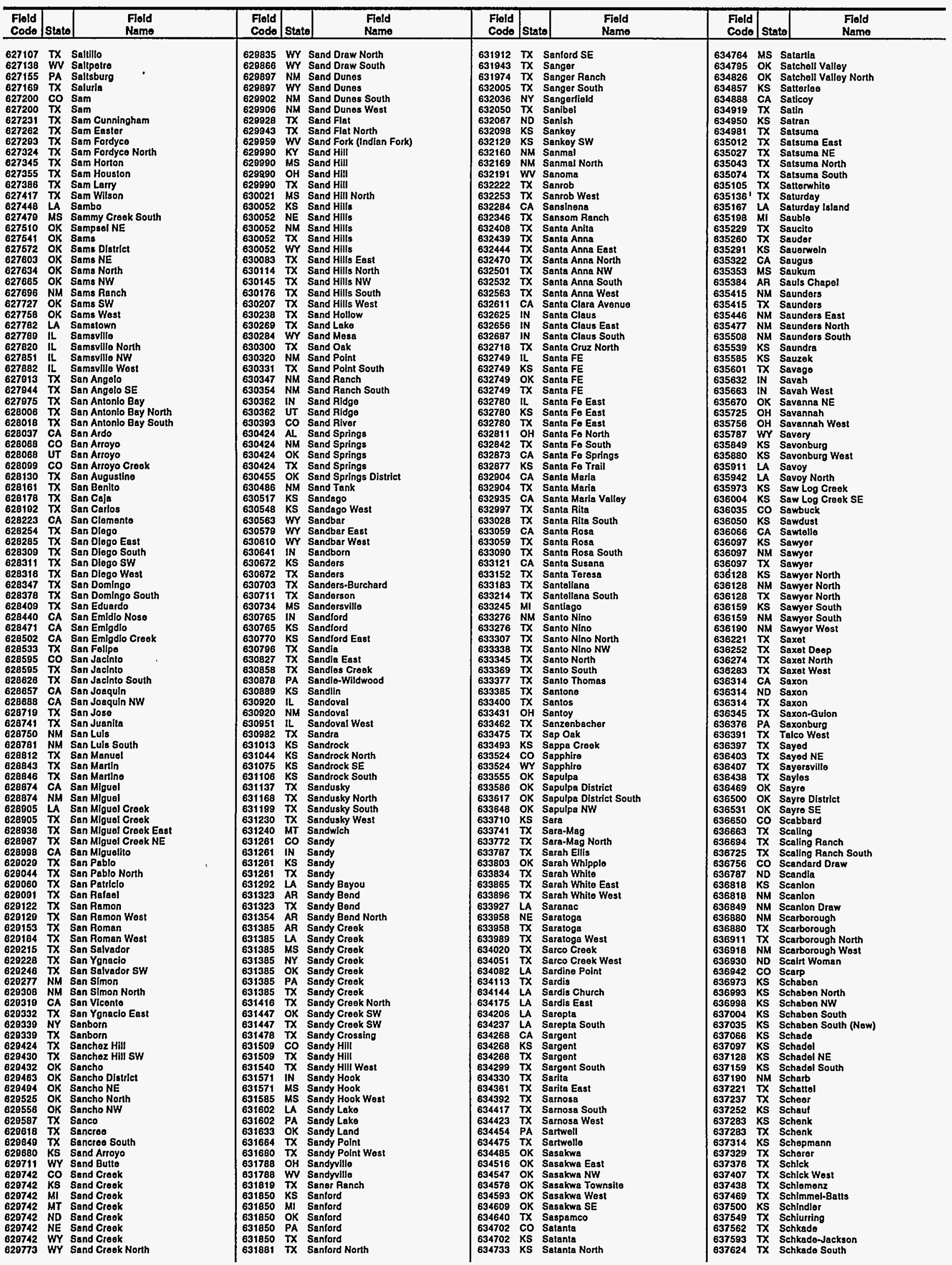




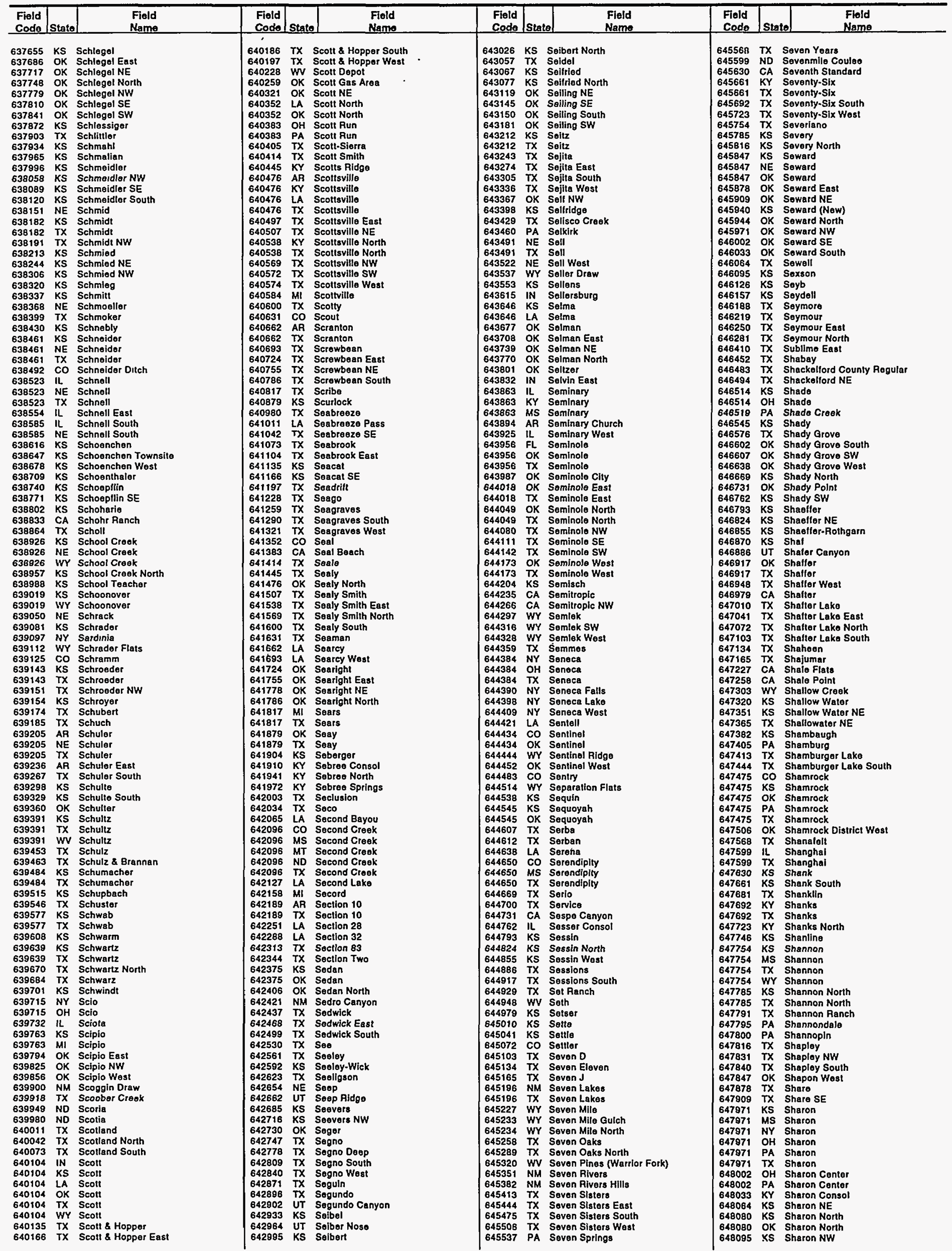




\begin{tabular}{|c|c|c|c|c|c|c|c|c|c|c|c|}
\hline \begin{tabular}{l|} 
Fiold \\
Codo
\end{tabular} & Stato & $\begin{array}{l}\text { Fleld } \\
\text { Name }\end{array}$ & $\begin{array}{l}\text { Field } \\
\text { Code }\end{array}$ & State & $\begin{array}{l}\text { Fiold } \\
\text { Name }\end{array}$ & $\begin{array}{l}\text { Field } \\
\text { Code } \\
\end{array}$ & State & $\begin{array}{l}\text { Fleld } \\
\text { Name } \\
\end{array}$ & $\begin{array}{l}\text { Field } \\
\text { Code } \\
\end{array}$ & State & $\begin{array}{l}\text { Fleld } \\
\text { Name }\end{array}$ \\
\hline 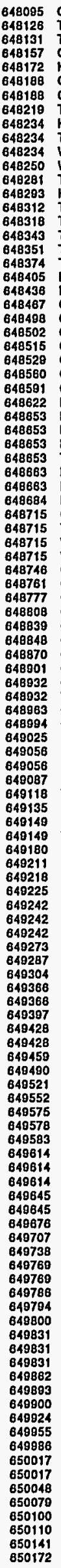 & 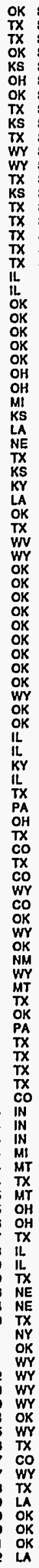 & 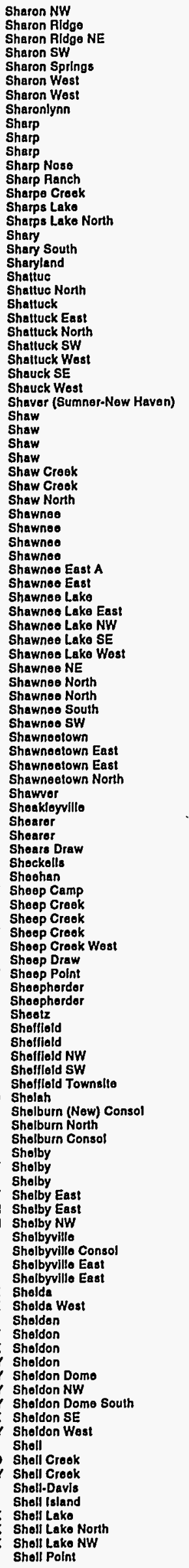 & 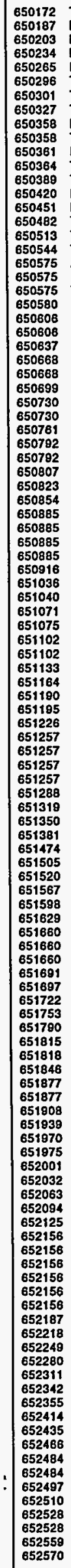 & 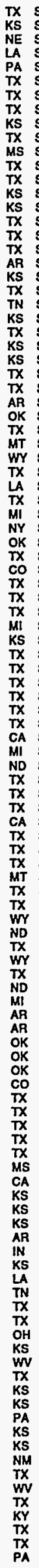 & 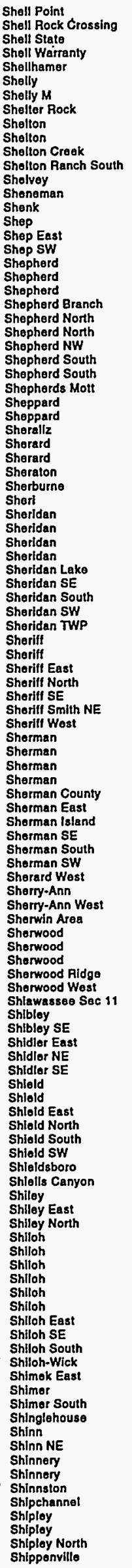 & 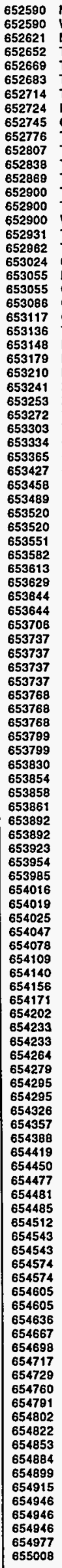 & 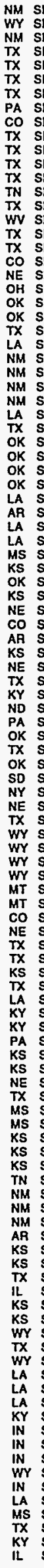 & 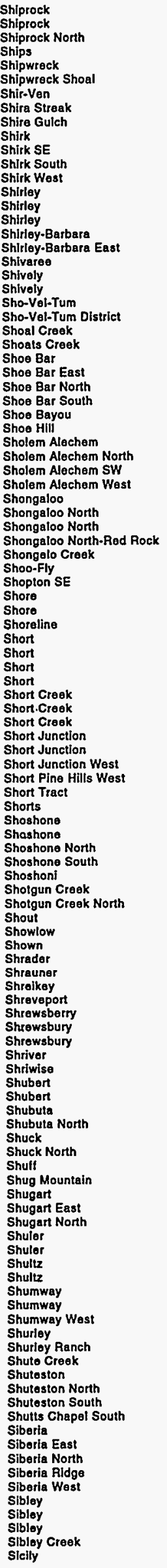 & 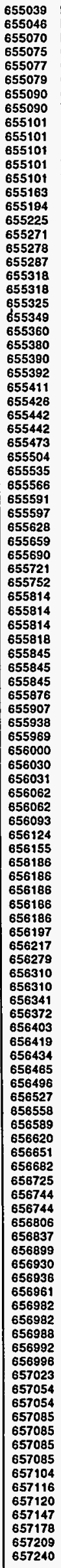 & 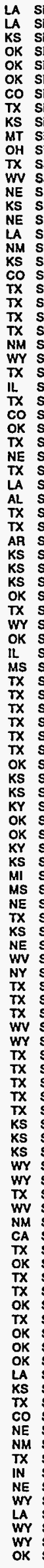 & 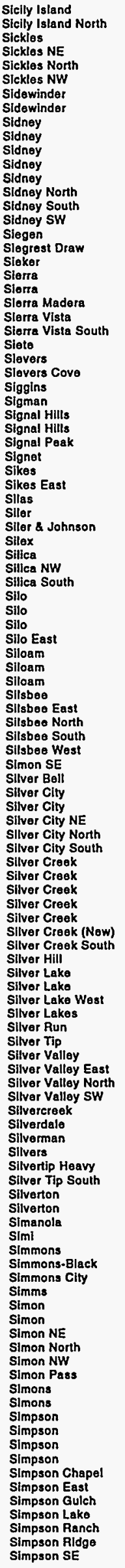 \\
\hline
\end{tabular}




\begin{tabular}{|c|c|c|c|c|c|c|c|c|c|c|c|}
\hline $\begin{array}{l}\text { Field } \\
\text { Code }\end{array}$ & State & $\begin{array}{l}\text { Flold } \\
\text { Name }\end{array}$ & $\begin{array}{l}\text { Field } \\
\text { Code }\end{array}$ & State & $\begin{array}{l}\text { Field } \\
\text { Name }\end{array}$ & $\begin{array}{l}\text { Field } \\
\text { Code }\end{array}$ & IState & $\begin{array}{l}\text { Field } \\
\text { Name }\end{array}$ & $\begin{array}{l}\text { Fiold } \\
\text { Code } \\
\end{array}$ & State & $\begin{array}{l}\text { Fiold } \\
\text { Name }\end{array}$ \\
\hline 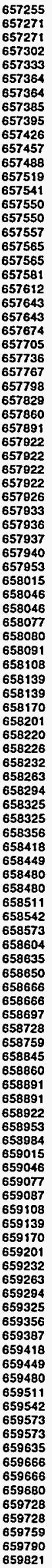 & 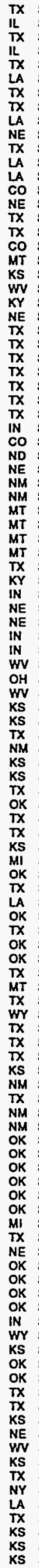 & 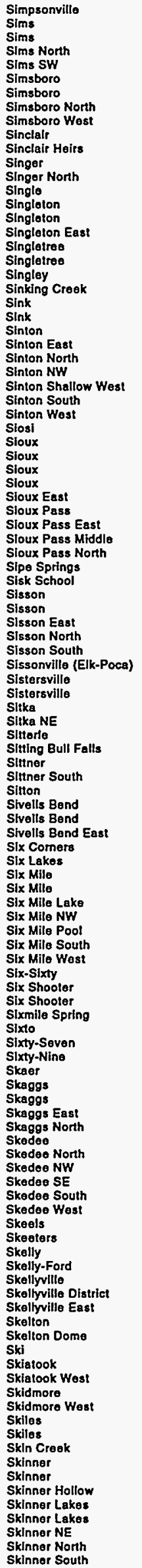 & 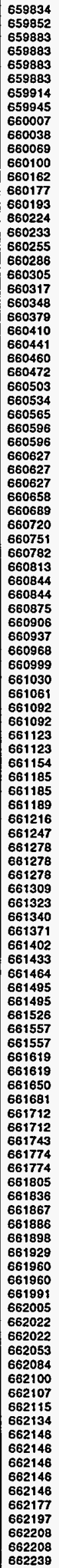 & 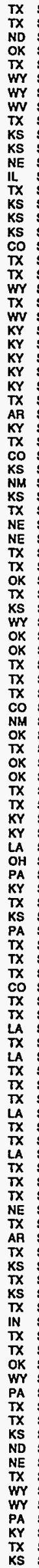 & 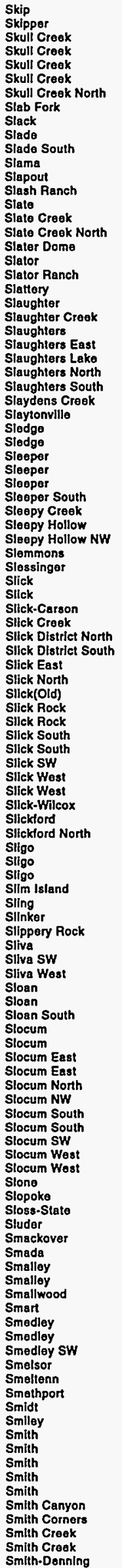 & 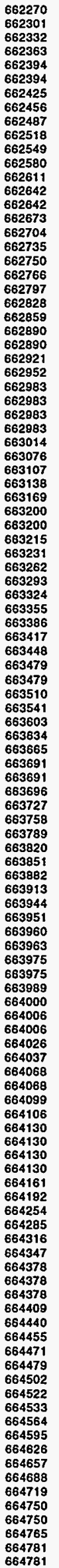 & 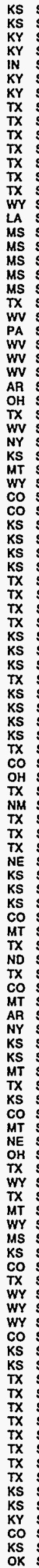 & 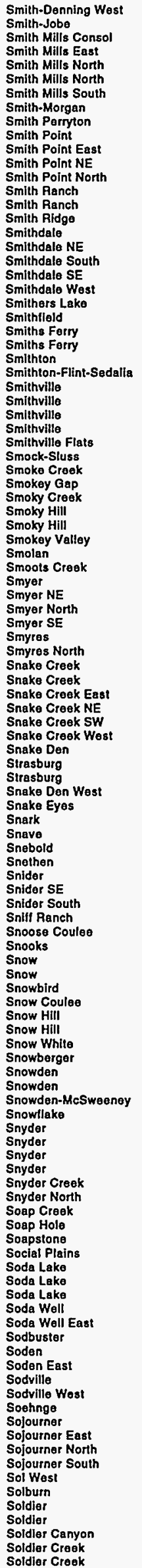 & 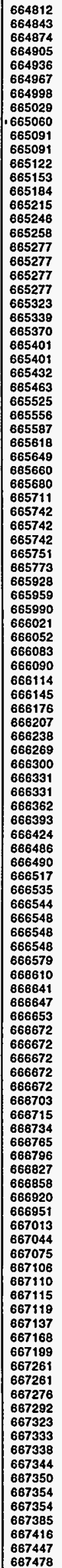 & 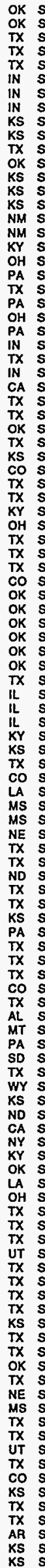 & 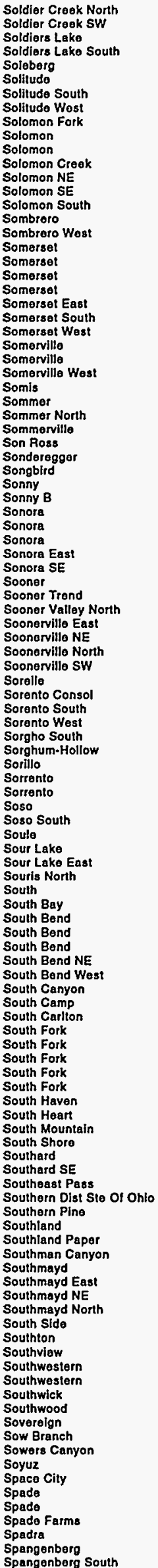 \\
\hline
\end{tabular}


4inOS yonquis ON $9983 \angle 8$

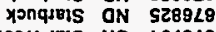
ISOM I8IS XO

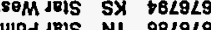
$M N J 83 S$ SY 882929

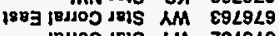
|2DOS IEIS AM $28 \angle 9 \angle 9$ Jiss $X 1102929$ JEIS WN 102929 IEIS SN 102929 JeIs OJ 10L9L9 Jis IV 102929 roddris $X_{1} \quad 0<9929$ ddEIS $X_{1}$ 6E9929 sopdeis $X_{1} \quad 809929$ Uits $x_{1}$ WojuBIS SW LLS9L9 Isem kouves in L25929 (20) 4NoN RejuBis $x_{1}$ sis929

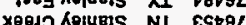
hopuers NI Est929

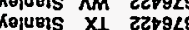
Rojueis Xo zztralo KOJUEIS $\exists N$ zZr9L Kejurls ON टzr9 2 Kojuzis $N X$ zergL2 Kojuris $S x$ zzr9L9

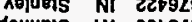
IMON DdoquEIS NX 0079L9 odouvers AX 16E9L2 doyueis $S \times$ I6E9LS

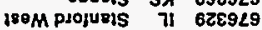
inos pojuels 71862829 pJojulis XI L9Z929 projurs 71 202929 4spuris II 4spuns in 4LON PJPPUEIS $V 7$ tLL929

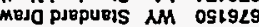
phepueis vi epiglo SOM Sdwrys SW Z219L9 $150 M$ sdWris $\forall Y$ ZLL9LO unnos sdwars $S W$

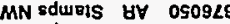
HMON Sduris SW $6109 \angle 9$

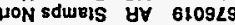

JN SdwEIS $\forall \forall$ 8865L sduris $y \forall$ LS69L9 odwers XI $9265 \angle 9$ sdwols SW 926529 KOIduris SW Sese Loduryis $X 1$ to8s 19 jeduris SY togs opoduris oN $0 S 85 \angle 9$ opoduris IN t58s opodurys oO tsos 29 IsOM puojwars $X_{1} \quad \varepsilon E 8 S \angle 9$ MS projuEls XI 208529 MS pojüEIS XI ILLSL9

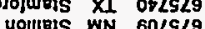
UOIIIESS OD 60LSL9

MS Rө|вIS $X_{1} \quad 8 \angle 95 \angle 9$

Is Ro1 Nets XI 919s29

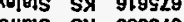

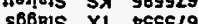

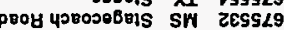
पวR0006вis in EZSSL9 40.00060IS Yd EESSL9 पวR000BEIS AN EZSSLS पว80000Bis OJ EZSSL9 do1S Q6EIS $\mathrm{AM}$ Z6rSL9 do1S $90 \mathrm{BE}$ OD Z6rSL9 IS puE1S 0681S to 19rsL9 püis 08 a H OBEIS IN 898S $\angle 3$ soupdS proj|rIS SW LEES $\angle 9$ MN PHOJIEIS XO OEES 29

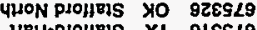

HuH-proj!rls XI

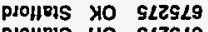
projplis HO sLzsL9 projyls ON SLZSL9 projens $\$$

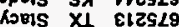
hoEIS Vn EIZS $\angle 9$

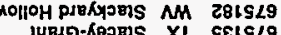

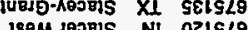
M JOFOIS NI OZLLL9 20581S $\mathrm{NI} 6805 \angle 9$ 2001S Sर 8905 $\angle 9$

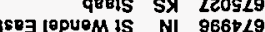
topue Is NI 966019 juosuin is $\mathrm{NI}$ g960\% juesuln is $y$ Y

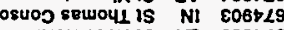
osog is $y 7088729$ osou is $V 7 \quad 2 \angle 8 t \angle 9$

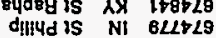

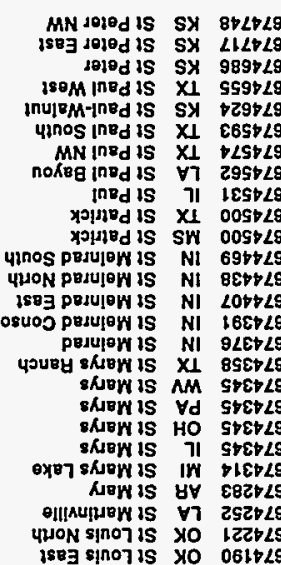

1583 sino is XX $0610 \angle 9$

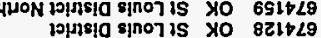
gino is xo $2608 \angle 9$ opnr is $X_{1}$ 0808L9

unnos 4dasor is $\forall 7990 \% \angle 9$ 4hos ydoror is $\lambda x$ g90829 पhoN ydesor is $A X$ SEOrLs puels ydesor is $X l$ EL6EL9 ydosor is $X_{1} \quad 116 \varepsilon \angle 9$ 4desor is $\forall 7$ $116 E 29$ ydesor is Ax 116elg 189M uYor is Yo 088EL9

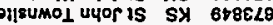
$M N$ ULOP IS XO 818EL MN U4O IS SX 818EL 4HON UYOO IS $S \times$ LBLCLO J58 Uपण is $S X$ 9SLELO uyor is $X \perp$ GZLEL9 u4O is u4or is vi szeces uYOr is SX SZLELO MS sowre is XX $\forall 69 \varepsilon \angle 9$ is sowr is v7 zese $\angle 9$ soure is NI ZC9EL9 sowve is 71 ZE9E $\angle 9$ NU⿻H is HO BESELS Nu०H is NI 6ESEL

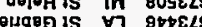

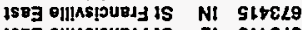
is8

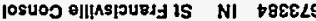

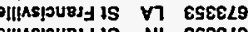
Ollispurdy is NI ESEEL Oif Aspurd is 71 ESEELS

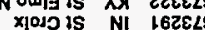
JEוD is X1 192EL9 गEIO IS HO เ9ZELS

JSOM SOHEYO IS NN $\quad$ BZZELS 4NON SOpByD is XI L9IELS

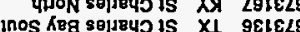

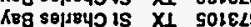
sol 1840 is $X I \$ \angle O E \angle O$

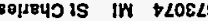
SopruD is $\lambda X \quad \nabla \angle 0 E L 9$ uinos Auouluy is $X_{1} \quad 186229$ Ruounuy is $X_{1}$ OSBZLS kuopury is $\mathrm{Nl}$ 056zL9 ह! SOIRnbS WN $87 L 2 L 9$ unnos ye8jo tounnbs Xo LILZL

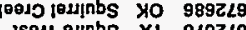
ounbs $x_{1} \quad 8 \angle 9 z \angle 9$ $150 \mu$ yoo 01 nhbs $X 1$ SS9ZLS

$\exists N$ yovJj menbs XO $6 Z 92 \angle 2$ YoerJ MBnbS $\mathrm{NM} \nabla 292 L 9$

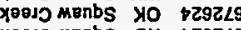
Xoojoments ON TZ9ZLS X00J MEnbs OS DZ9ZL OejnoO MEnbS IN EBSEL OHn8 MEnbs hM Z8SZL MOllOH 4\&EnbS $X I$ Z9SZLS 4RON OX87 odunbs WN IESEL ex日7 erenbs WN O0SZL oung erenbs ON 69tZL yinos sinds XI LOtZLO Je!unds NI 16EZL

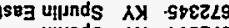
uाjunds $\lambda$ त्र tlezL

4HON uibinds 05 E8zZL

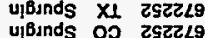
ujBunds OO ZszZL9 1883 206inds $X_{1} \quad 72 z 2 \angle 9$ isom voosinds $\mathrm{NI}$ 0612L9 osuog uoobinds NI GS1ZLO yoojo inds in BzizL

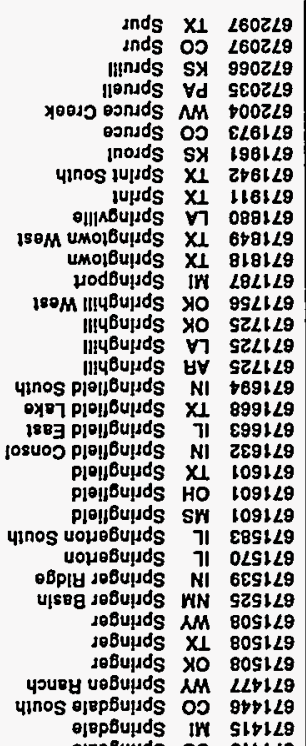

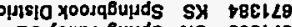

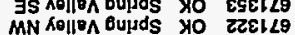

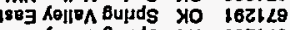

Ko|lB $\wedge$ Bupds in 09zLL29

Kellib

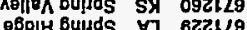

ebpy buts

unnos puod BuHdS SW SOZZL9

puod Buyds SW 8BLLL9

oxi7 Bupds IW L8LLL9

IIIH Bupds SW 981129

IIIH BupdS SN 9ELLL

Aling Bunds $\mathrm{NI}$ sotilg

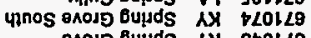

OAOLO Buids NA EtOLL9

4inos xo010 Bujds in $2100 \angle 9$ 4 Inos ye015 Bupds XI 210128 4นON X9010 BujJds XI $1860 \angle 9$ 2883 XOOJO Buj]S $X_{1} 0960 \angle 9$ X0010 Bupds AM BL60L9 xo010 Bulsts XI 616029 yoorg buyds NI 616028

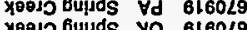
toojo bupds yo 6t60L8 xoojo bujds $3 N$ 6160L9 xoujounds ON 616029 *oro Bulds V7 816029 xodo builds SY 616029 xoorJ buyds OO 616029 xomo ouyds ON 888029 (1) 888029

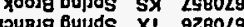
Trusg uljds XI 928029 Burds if 988029 Buinds $X_{1} \quad 982029$ Bupds Xo $58 \angle 0 \angle 9$ Builds SY SBLOL9 urojds XI BLLOLS opyurds $\forall \mathrm{V}$ t920L9 onBzids XI EELOLO dOOC ISOM NuBQqBJdS XI $20 \angle 0 \angle 9$ SOM Asequeds XI $\quad$ 1L9029 1503 pary puell Kuseqrids XI 069029

vorV PueJl Nueqzids XI $\quad 609029$

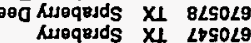
Aueques $X_{1} \quad$ L 25029

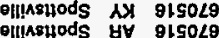
Ollisuods HY $915020^{\circ}$ ISOM uods XO $5870 \angle 9$ MS ujods Xo t9t0L9 unos wods xo Eztols

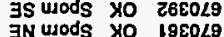
1803 usods Xo 088029 wods Y्र० $6820 \angle 9$ Kuoods XI 8920L9 4nnos eBunids SW 592029 MS doulds SX StzoLO Jeillds SX LEZOLO MS EJopuejds XI 4inos ruopuejds $X_{1} \quad 921029$ 1883 घjopuejs $\mathrm{XI}_{1} 811029$

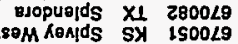
IS Ranids Sx 020029 MN AOAldS SX 686699 \|1889-8qDJO-RoAdSS SX 8S6699

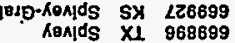
hanlds $X_{1} \quad 968699$ Is oujds yo 998699

4मON OUdS YO odjs xo RLL699 $180 M$ ids $X 1$ L1 soulds XI 6LQ699

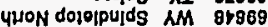
4MON doloipulds XI 8r969 dojepulds AM $\angle 1009$ dojelpujds XI LLO899 ofpulds OD 80068

IS 40oulds $X_{1} \quad 009899$

MN YOBUIdS $X_{L}$ 86569 40001ds XI 865699 xHytilds $x_{1}$ 985699 olllds $X_{1}$ ss5698 IIIdS X1 tZS699 MS oxyds $x_{1} \quad$ E6p699 4HON Oxj]S XI 297699 $80 \exists$ oxids XI let69g

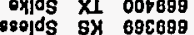
lepids XI 208699 opids $r$ Locesg 10j0s X1 92z699 huds $X_{1}$ srzes9

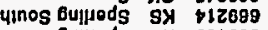
bujpods SY col699

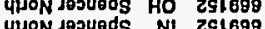
puls Druo joauds 政 J09uods $X 1$ 82069 dovuods $x_{0} 02069$ daguods 40 sooueds VIN 8Z0699

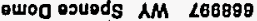
osuods XI $\mathrm{x} 86890$

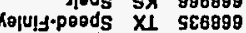
Xelujoods $Y_{d}$ tzes89

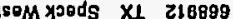




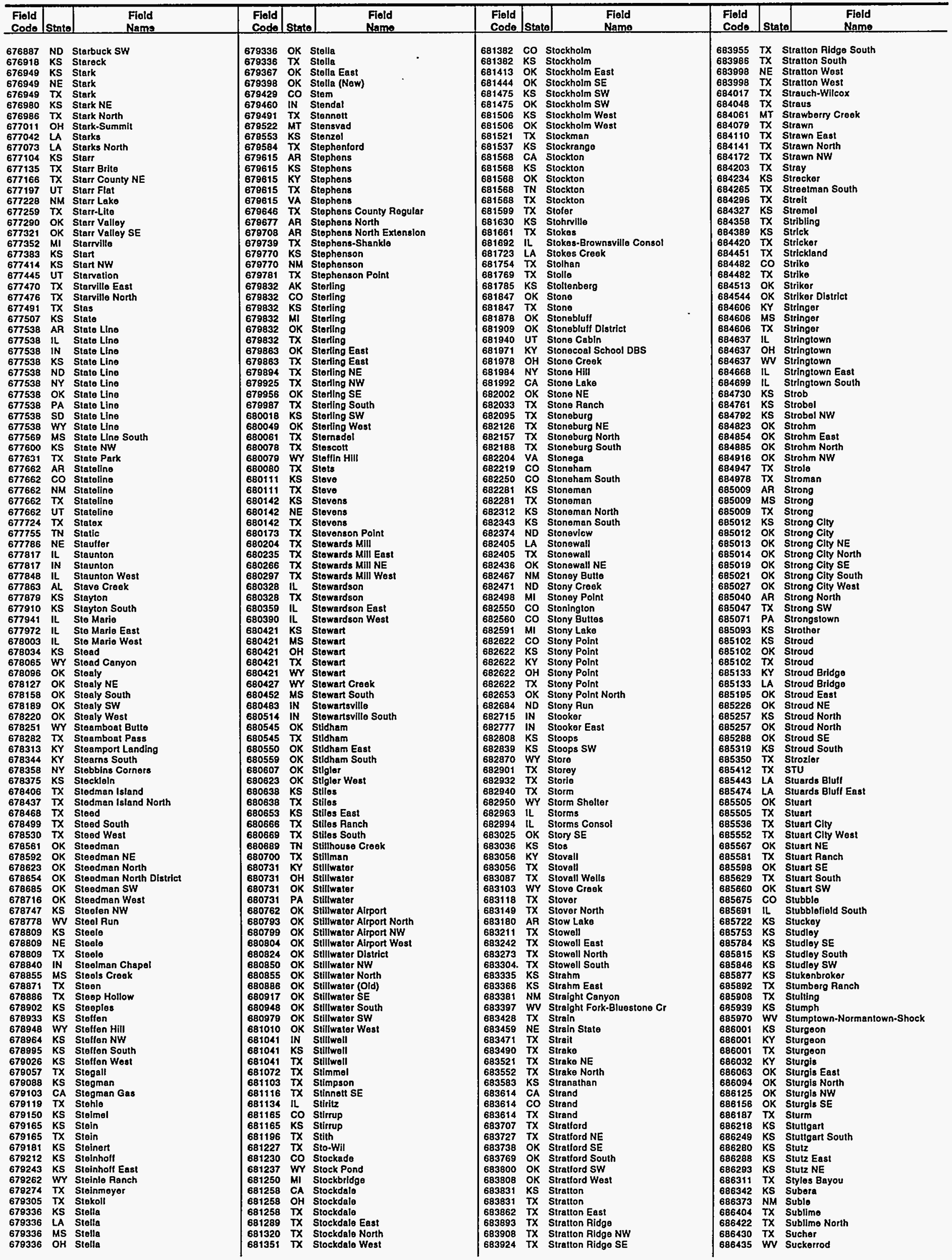




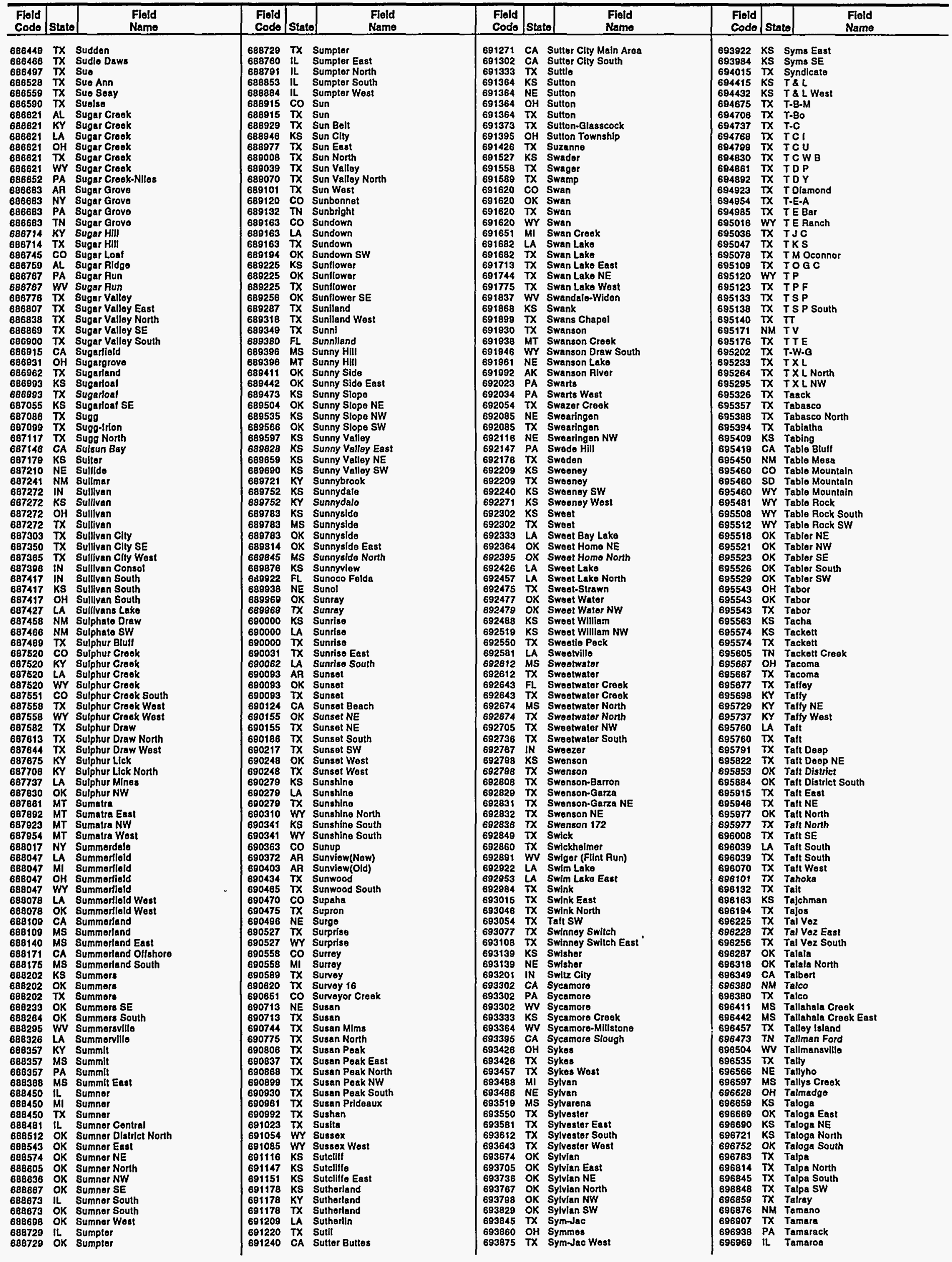

Energy Information Administration/Oil and Gas Field Code Master List 1994 


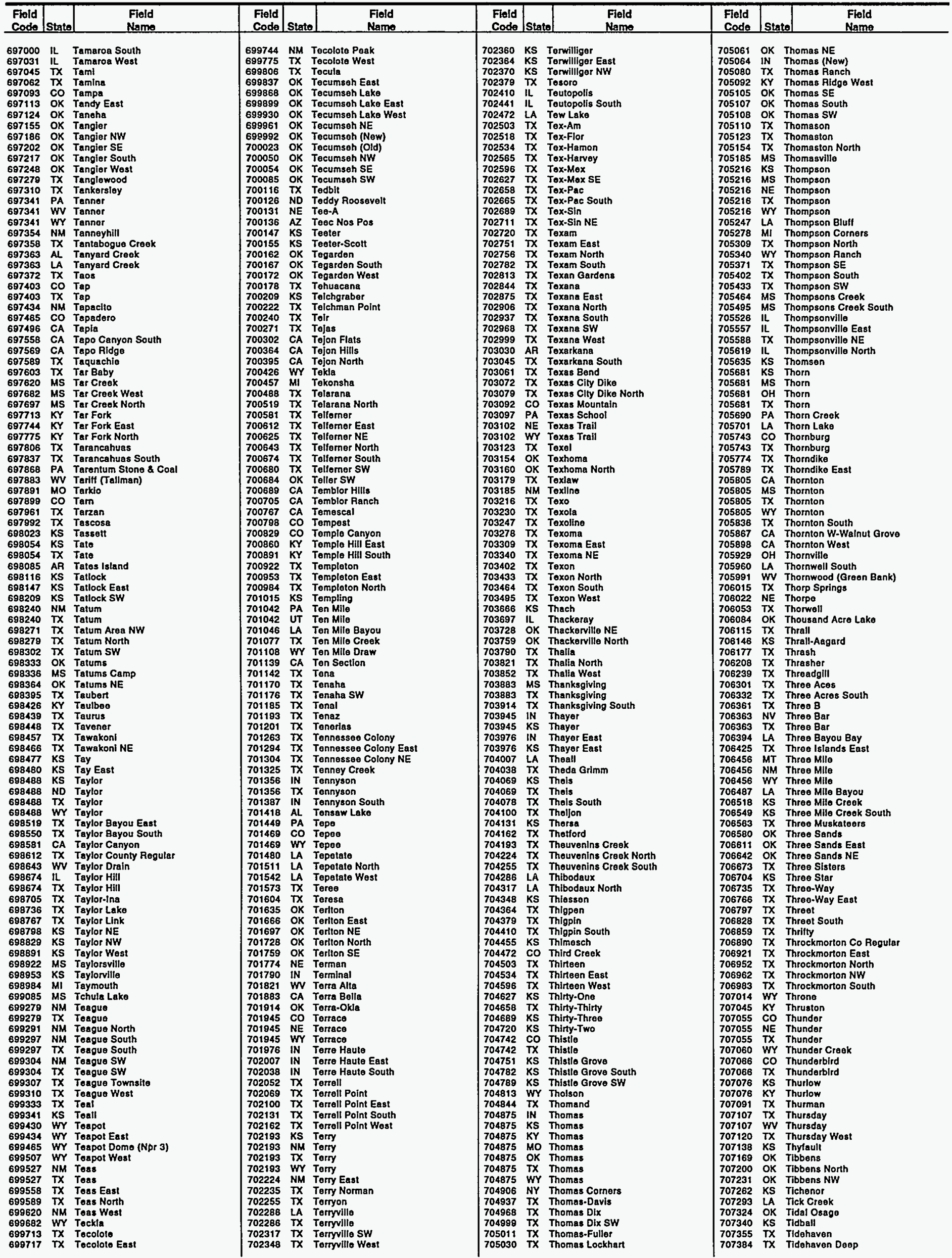




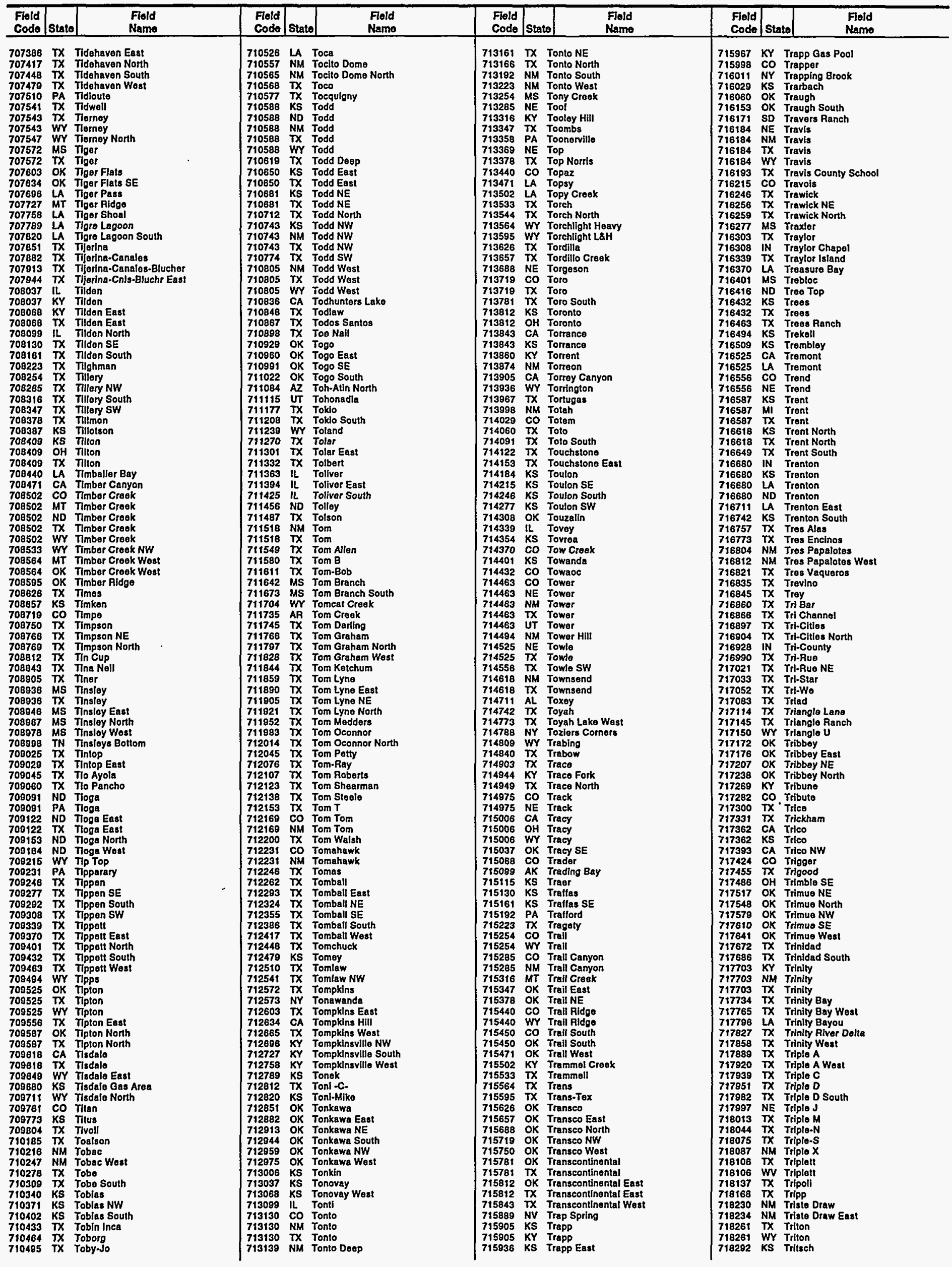




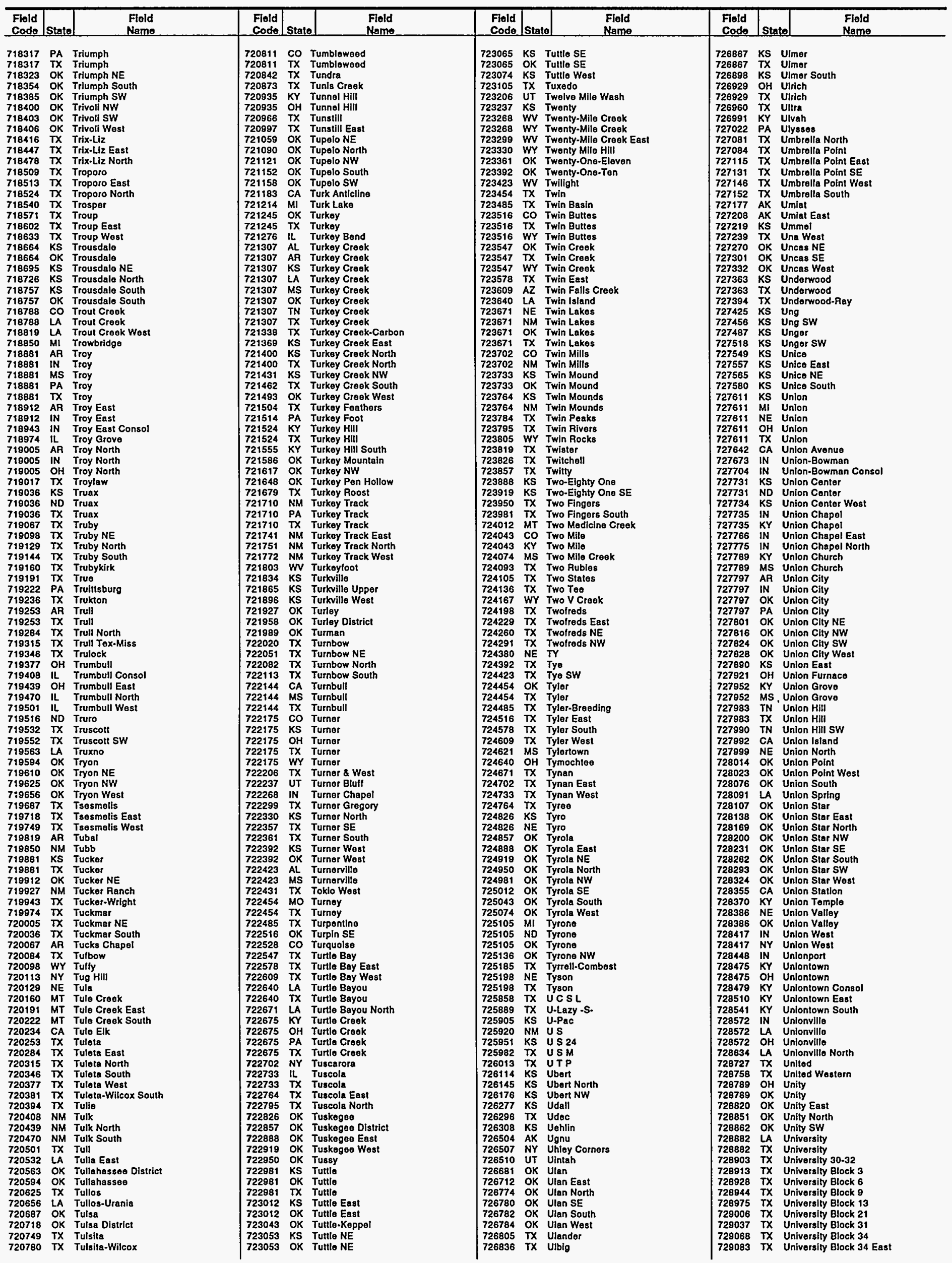




\begin{tabular}{|c|c|c|c|c|c|c|c|c|c|c|c|}
\hline $\begin{array}{l}\text { Fleld } \\
\text { Codo }\end{array}$ & State & $\begin{array}{l}\text { Fleld } \\
\text { Name } \\
\end{array}$ & $\begin{array}{l}\text { Field } \\
\text { Code }\end{array}$ & State & $\begin{array}{l}\text { Field } \\
\text { Name }\end{array}$ & $\begin{array}{l}\text { Fleid } \\
\text { Codo } \\
\end{array}$ & State & $\begin{array}{l}\text { Field } \\
\text { Name } \\
\end{array}$ & $\begin{array}{l}\text { Fiold } \\
\text { Code }\end{array}$ & State & $\begin{array}{l}\text { Field } \\
\text { Name }\end{array}$ \\
\hline 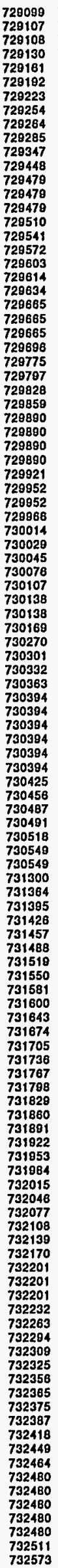 & 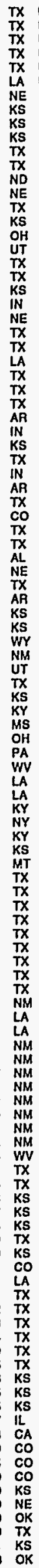 & 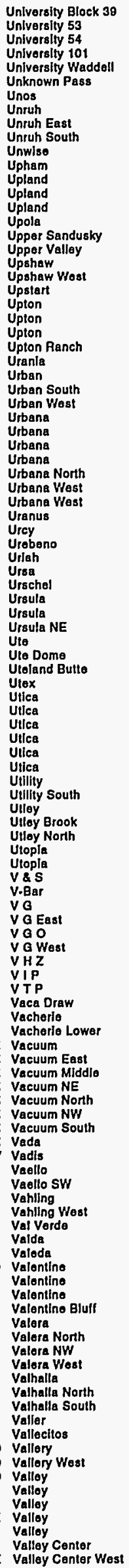 & 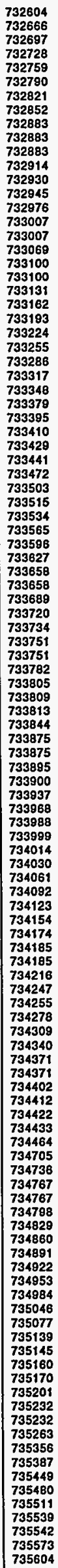 & 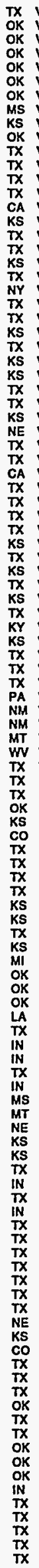 & 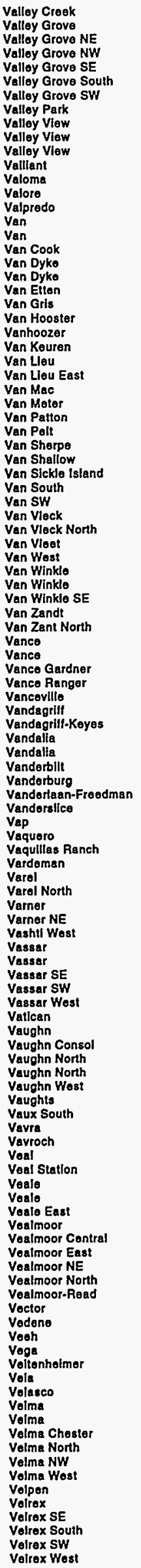 & 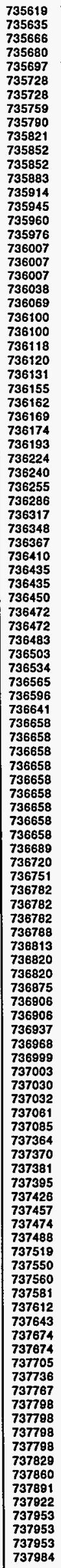 & 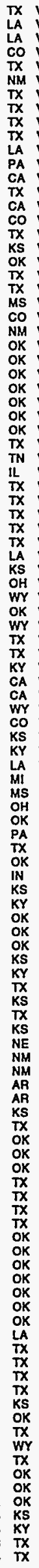 & 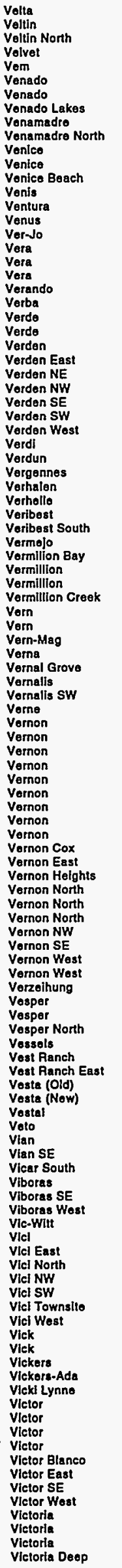 & 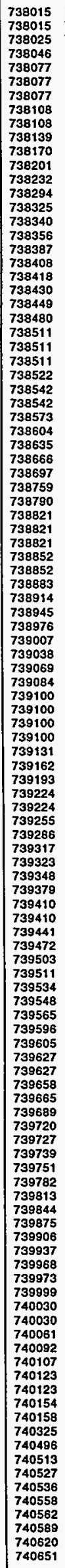 & 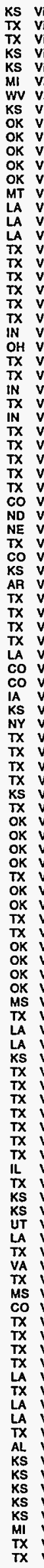 & 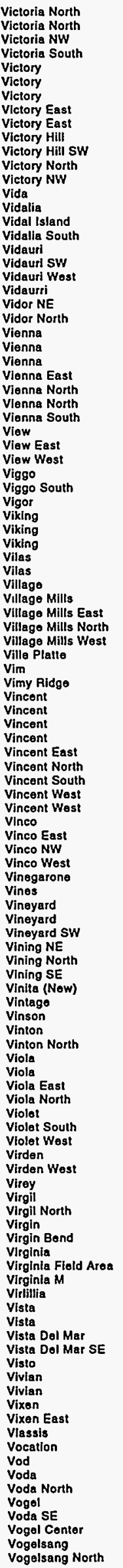 \\
\hline
\end{tabular}

Energy Information Administration/OIl and Gas Field Code Master List 1994 


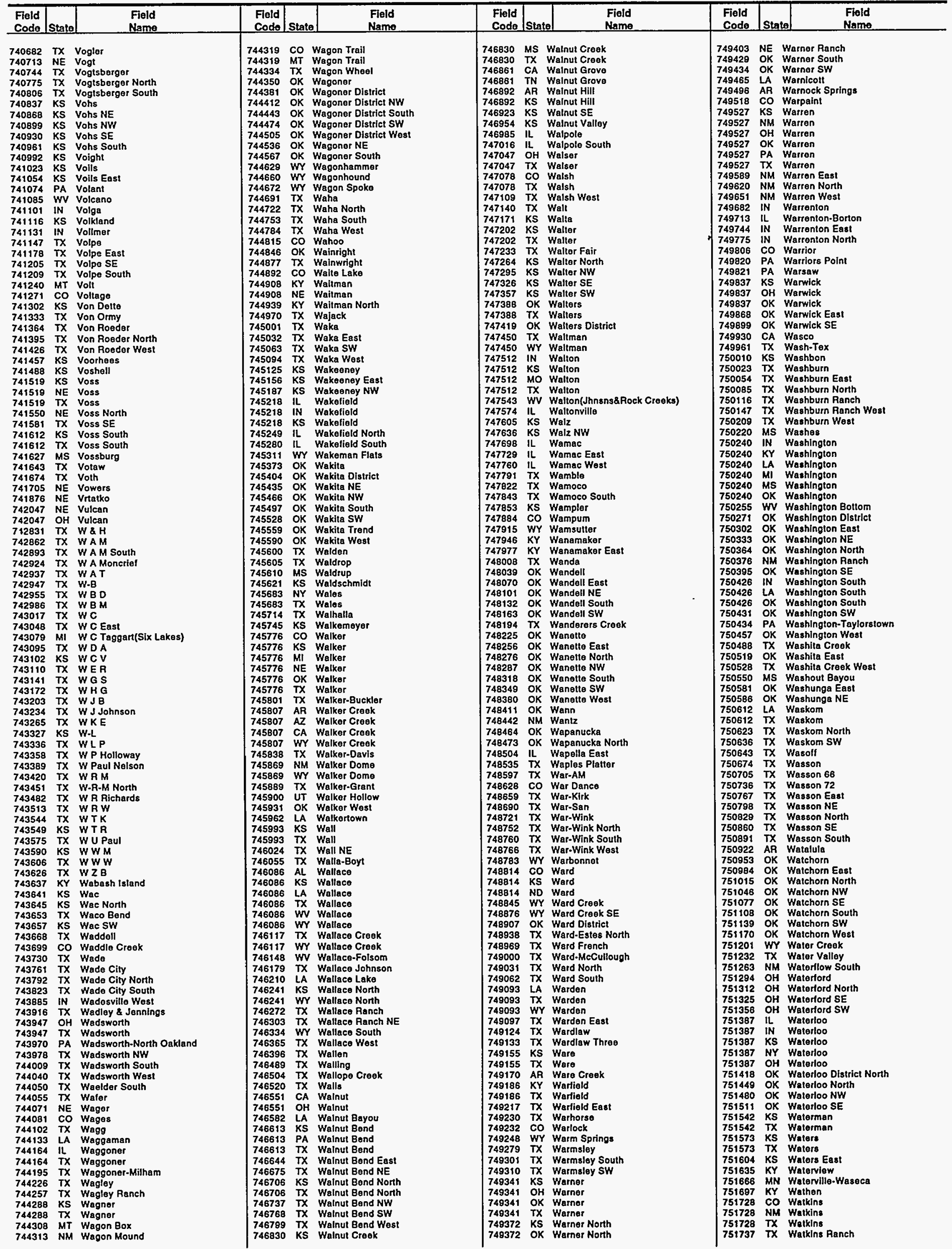




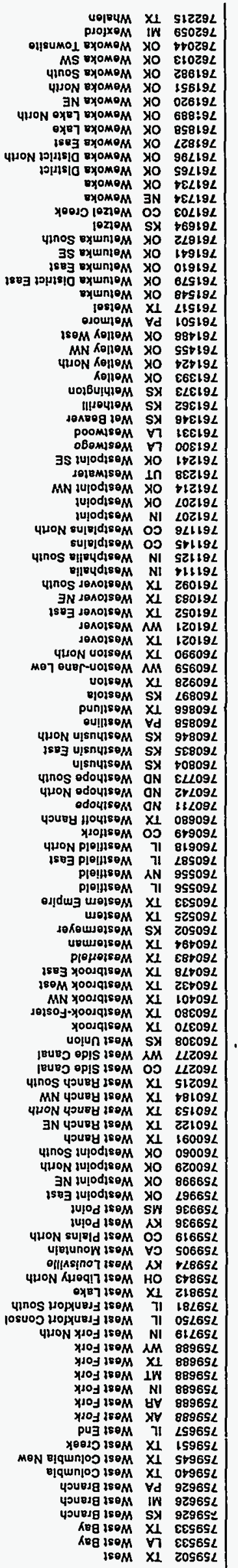

4HON $18085 \mathrm{M}$ SS vossoM $\forall Y$ L $12069 L$ uosse M XI

4LON $10850 M$ SX 607692

poop AossoM $S X$

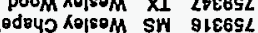
podвy Ropsem it oicesL AojsoM XI sazesL heisem $\forall \mathrm{d}$ S8265L hepseM Xo 582652

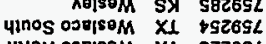
पLON OJPRSOM XI EZZESL

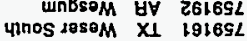
JesoM XI oel6sL म0588M XI 660692 THOM IM LEOESL पमON 4मOM SY 20065 4म0M Sx $52689 L$ $1904-10010 M$ SX $28889 L$

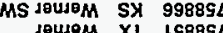
JQUנOM ON 10u+M $S x$ L $780 M$ 7140M $X_{1}$ Oz88SL 4InOS zuUOM $X 1$ 68L8SL 1589 Z7UPM $X_{1}$ 8SLSSL nuणM $\times 1$ L2L8SL 27U0M YO LZLQSL oxsuaM $X_{1}$ 96985L

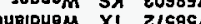

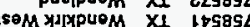

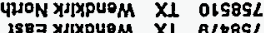

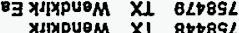

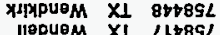
UlppueM XI $21+852$ U!llopuoM HO 988852

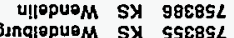
Bincispue $M$ SX $59889 L$

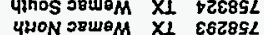
овшоM $X_{\perp}$ zgr8sL $\mid X_{\perp}$-poom|aM $X_{1}$ LEZBSL Jos!now-pomioM XI Doz8s

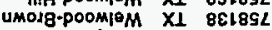
${ }_{150 M}$ All10M Y MS ANIOM YO 9LO8SL

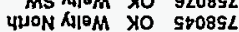
IN AllaM YO

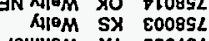

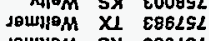
JowyjeM sx E86LSL 48I0M Y7 RERSL

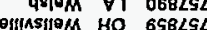

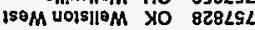

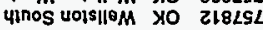

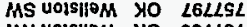
MN UOBSIIOM Yo 99LLSL पHON UO3SIIOM Y्रO SELLSL

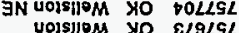
(N) piosity

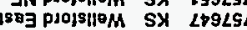
pJojs|loM SX Z79LSL OJnq8\|BM $M M$ HISLSL

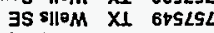
$\exists N$ SIIOM XI BISLSL MEJO S\|OM In SGTLSL Y्र0015 S\|OM SW $287 \angle S L$

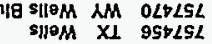
SIIOM HO 9StLSL SIPM SW 9STLSL

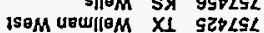
MS UBயIIOM XI DEELSL $M N$ URயU|ण M XI ESELSL

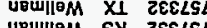
IN UO:BU!liOM SY LOELSL

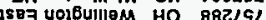
UOJBU!llOM HO OLZLSL voibunirM SY OLZLSL पo:BU\|IIIM OO OLZLSL IIIOM $5 X$ GERLSL Ning

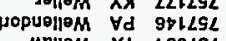

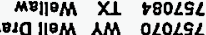
X0150 $110 M$ MM peYiOM $X_{1}$ ESOLSL मөाOM $X_{1}$ ZZOLSL

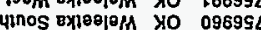
IS Ex10010M YO tS699L

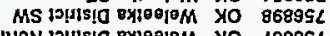

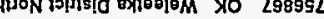
כמנs

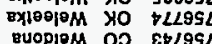
$\begin{array}{lll}\text { uopleM } & \text { OD } & 87295 L \\ \times 1 & 18999 L\end{array}$
uopleM IW L899sL

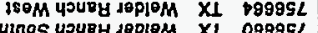

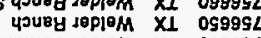
weunng-JopjoM $x_{1}$ 6Lg9sL деpieM XI $88999 L$ члпо

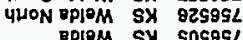

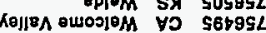
OWOH OWOәOM VI BLPSSL awo5pM Ho t9r9sL

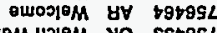

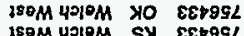
IEDM 4әPM SN EEr9SL 4inos IS 4ग0M $X_{1}$ BLESSL 4HON YOIOM XI OOE9SL

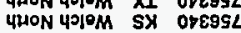

MS J4०W-4गणM SY IEEgSL ग40\%-पग10M SX 508952 3ppoप4108-4ग0M SX LPZ9SL 4ग10 MM 912992

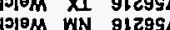
4ग0M WN 9L29SL losuoO YHON UOOIOM NI COZ9GL losuog UJoq1iOM SX S8189L U. woqiem $\mathrm{N1}$ t919s IN |өZ]|⿴囗十 SX EZIQS |0Z2I0M SX 26095: J86uाlipM XI 190992 sioM $\times 1$ L865s

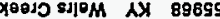

IS89 IOM WN LEGSSL

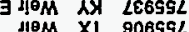
JOM WN 900SSL IOM $N$ N

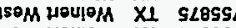
MS मou; 4HON HOUIOM XI Z8LSSL मөuाOM XI $X_{1}$ เSLSSL

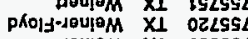
18U10M $X_{1}$ 68955

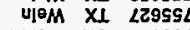

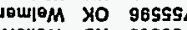
मणनM sर win $x_{1}$ tessgh 4 Hnos $1503110 M X 1$ totss ${ }_{10 M} X_{1}$ ortssL LexIOM SY Otrss

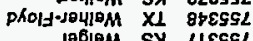
Prob $5 \times$ L LESgL BUBBIOM XI sgzss uвะuryeM $S Y$ tZZSS ISOM $1000 M$ XI E6ISSL MS JOBOM XI 98ISSL HON JEBOM $x_{1}$ 2915SL

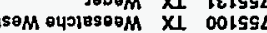
MS 84ว88500M X1 $X_{1}$ 6905SL

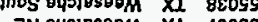
IN OUगBESOOM XI LOOSSL Oपग3ESOOM XI 9L6tSL sworM XI St6ts.

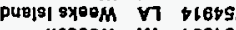
nopeop X1 to8bs 2 บолвчрөоM XI E88rsL ynos pooM XI zsBrsL ises poom XI lests pooM XI GSLSSL IOPOM $5 X \quad 8 Z \angle t S L$

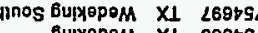

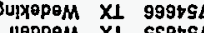
IIөppoM Xl seats 2 10.0\% $X_{1}$ rogrsL JO15qOM XI zbsts solsqoM $\forall d$ zrspsL

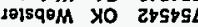
Jग8sq9M $\mathrm{SX}$ zrstsL MS SqOM SY UISPSL sqom sy opts $150 M$ Loq0 $x$ $100 d$ Jegon $X 1$ gibrsh $\exists N$ Jeq०M XI Bew-JPqOM XI GSErsL JO9OM $\times \perp$ t6ZrSL JaqqeM $S x$ EgzrsL $380 M$ 99०M Y्र० 2ह275L 4inos qq०M $X_{1}$ lozrs2 IS KBy q9०M XI OLLFSL Hilod $990 M$ XI

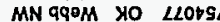

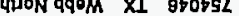
4HON $990 M$ YO 95O6GL 4RON qGOM SX 9b0tsL
A110 990M 바 $\$ 8689 L$ 99PM XI ES6ESL q9OM YO ES6ESL qqeM Sत्र ES6EgL บวUВН UOS1O \& JOAEOM XI LGBESL JoAgoM XI 098EsL 10ABOM HO O98ESL $\triangle 0 A B O M$ 3N O9BEGL JPABOM SY 098ESL

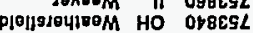

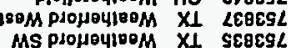

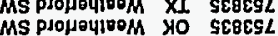
IInOS POOHOUtEOM Y YO ZEEESL 35 p10 $041200 \mathrm{M}$ XI 3S PJoн MN Propa

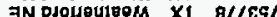

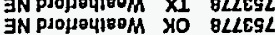
P.0म04180M XI L9LESL

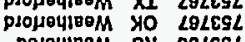
posoufrem $5 \times$ OELEgL

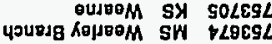
OABOM IN zIOESL Uерi7eM $X_{1}$ 189EsL OdOH OM XI OOEESL lood OPFSKOM Xo OrEEs $L$

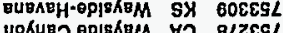
UOKUED epISKOM YD QLZCGL oppsR日M XI LZZESL eppgROM SW LPEESL 80M ExOUXDM YO 9LZEsL 4n NOS EXOUKBM YO SQIESL IS ExoukPM Yo 991ESL 4HON छyoukBM Xo tglesL IN EXOUKBM XO EZLESL 1583 Ex०UКBM Y्र० $26059 L$

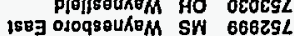
MS OUKOM YO B90ZSL 4InOS OUKBM YO 996ZSL 4TnOS OUROM SY 998ZSL MNN OURM YO 198ZSL MN ouxam Sx lobasL 4wo ourom Sh bozs 4MON

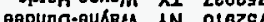
OUKBM XI 8062SL oukp OUKBM SN 900928

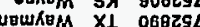

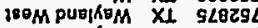

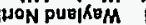
puE|KaM XI E18zsL puejkeM IF ElazsL OX87 X8M $\forall 7$ OOBZsL

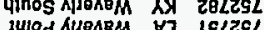
व लrone R ALPABM $A Y$ OZLZSL XIPAABM 71 OZLZSL ATJ日ABM OD OZLzgL pUBjoAg/A SW 689ZSL

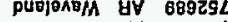

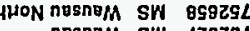

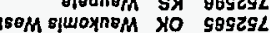
MS siuoynom yo teszsL

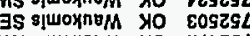
MN SiwoxneM YO ZLLZSL 4row swoxnom xo irrash meN. g goxnop xo oLECSL

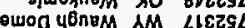
4 BñM $M M$ SBzzsL ${ }_{460 \mathrm{nOM}}^{4} \mathrm{SX}$ 98zzgL 4hnos silm $x 1$ gsash

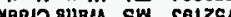
Y00Jว ร110 SW SHEM XI Z9LESL

BraquenteM oD IEIzSL iseM UOSIOM 71 DorzsL 4วuвy UOSIOM $X_{L}$ zSOzSL Buluna-Uosidy $\forall \dot{0}$ grozsL

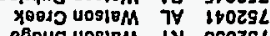
e6pHE vosibM $A X$ BEozsL vosigM XI LO0ZsL UOSIOM HO LOOZSL uosigM IN LO0ZsL UosipM V7 LO0zgL บOSIEM 71 LO0ZSL 150M SBUOIYM Xo 9LO1SL

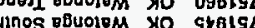
作 MN घ6uoleM YO 8B8tgL IN EBUOIDM YO IEBISL

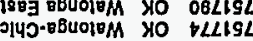

ouren




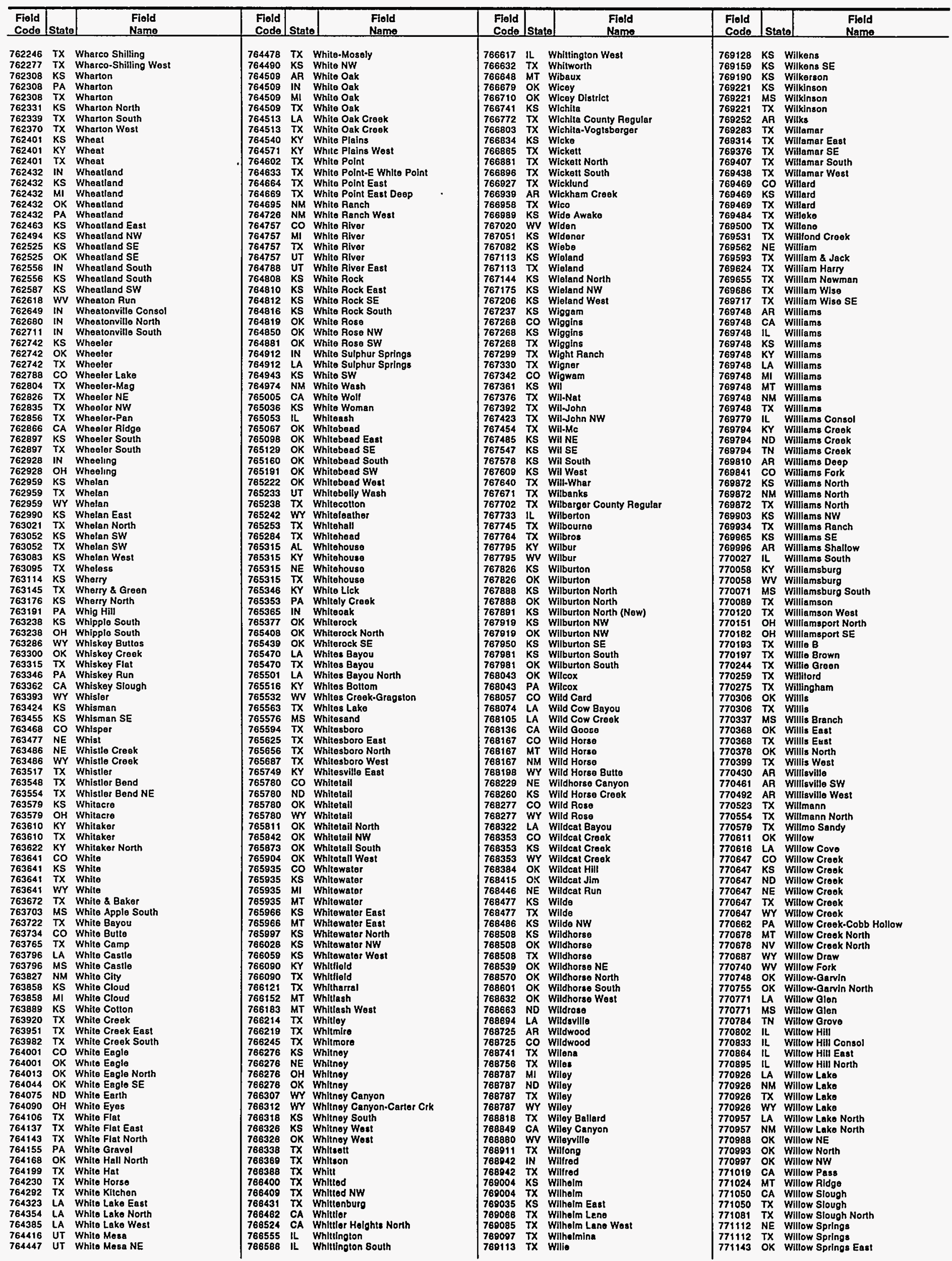




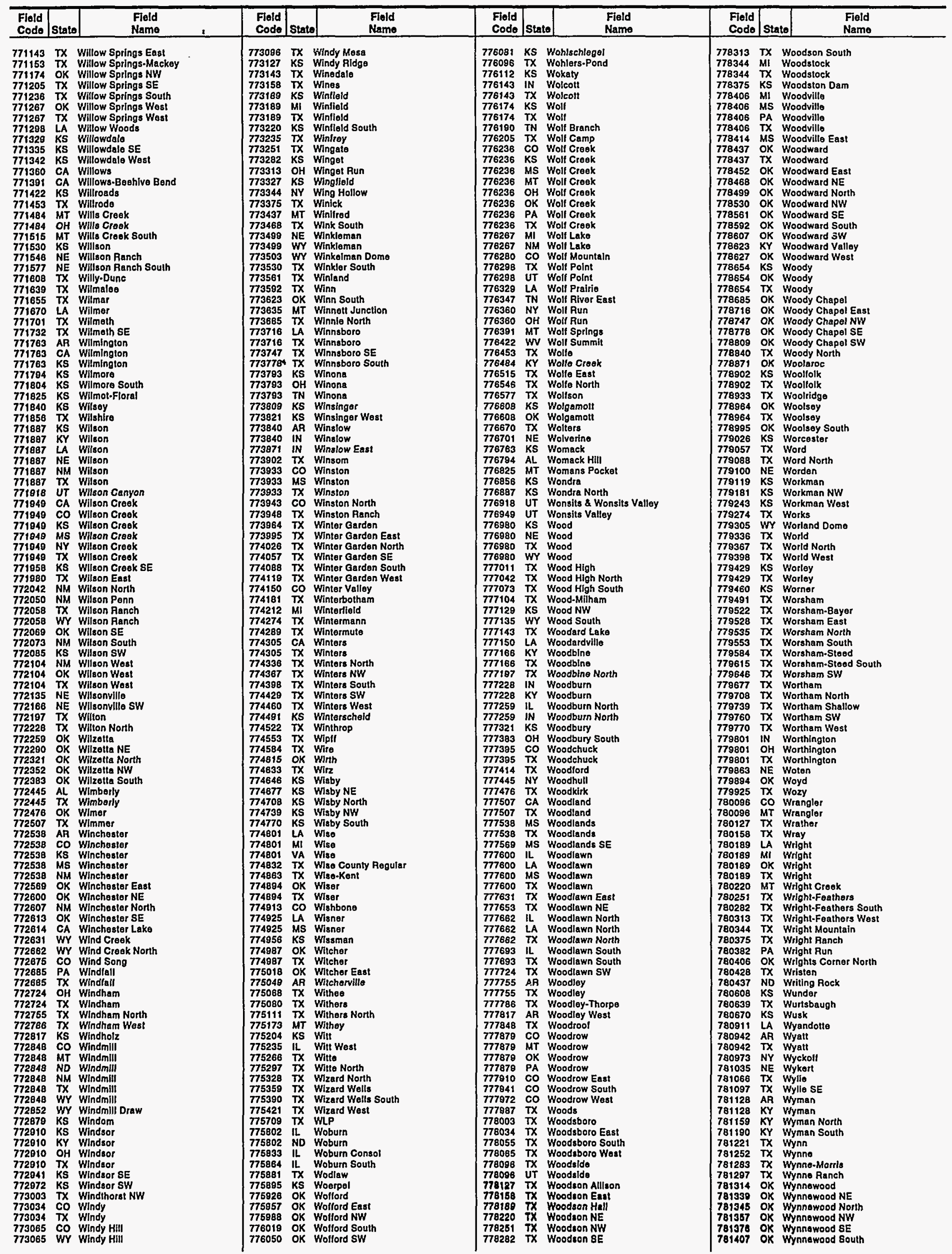




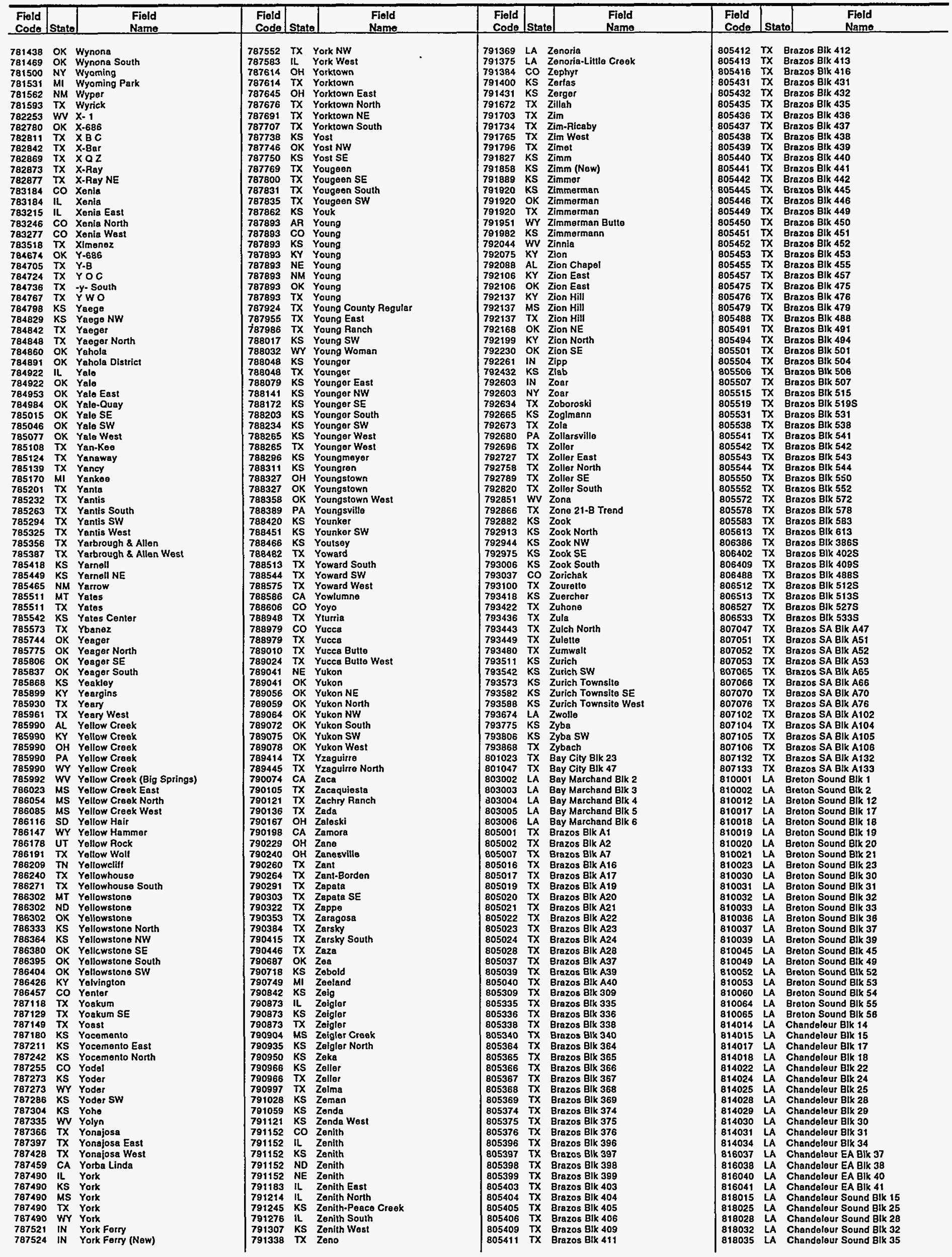




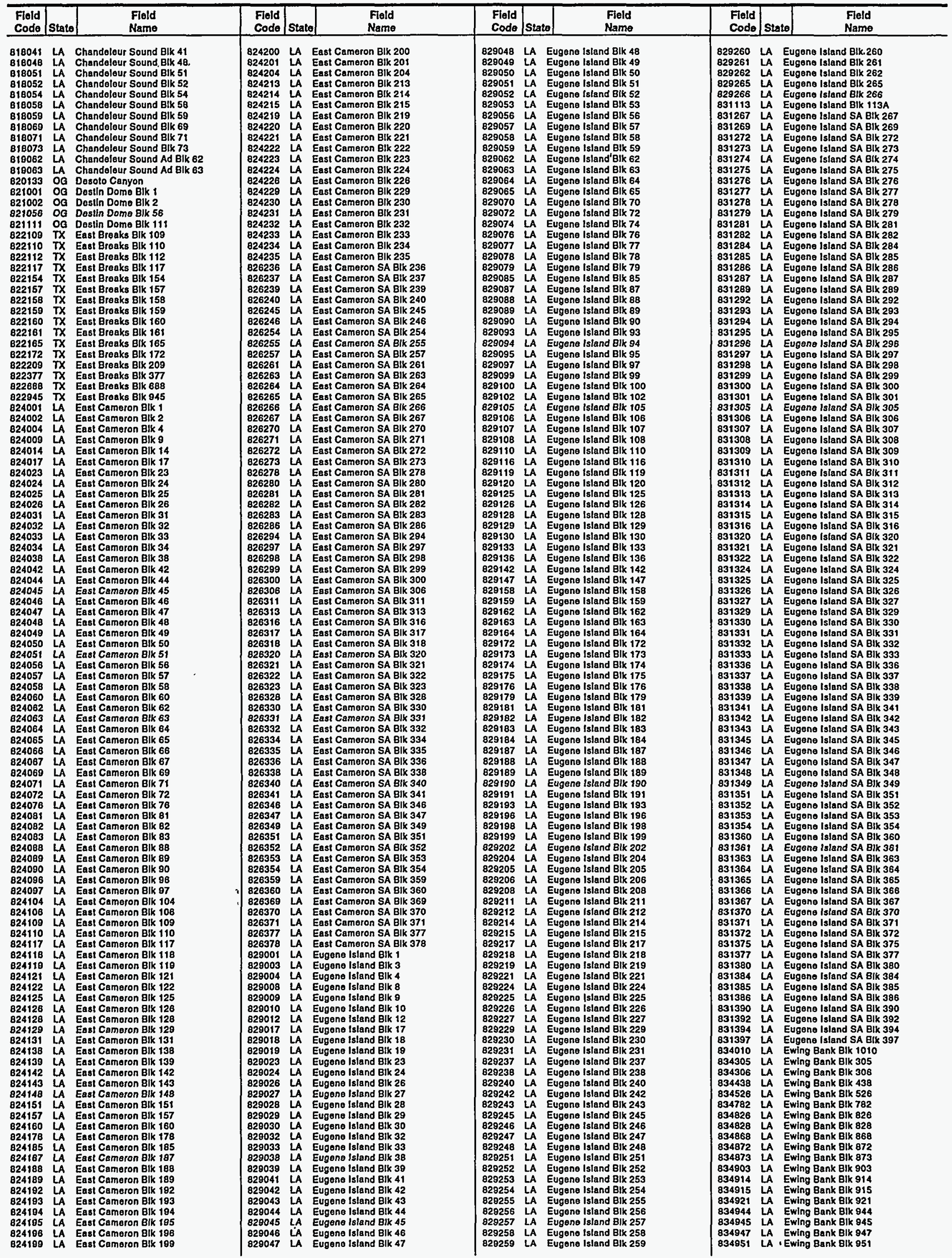




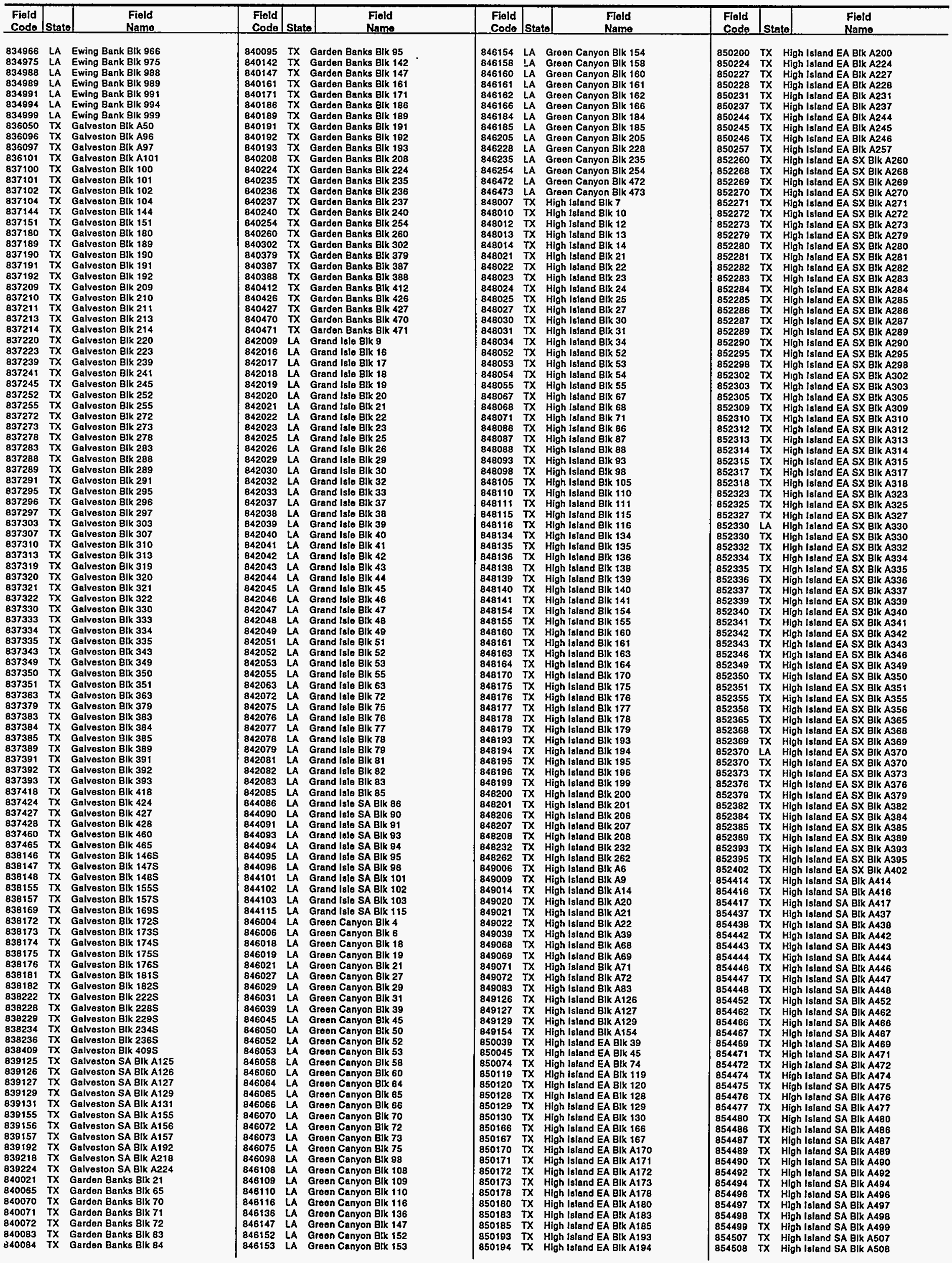




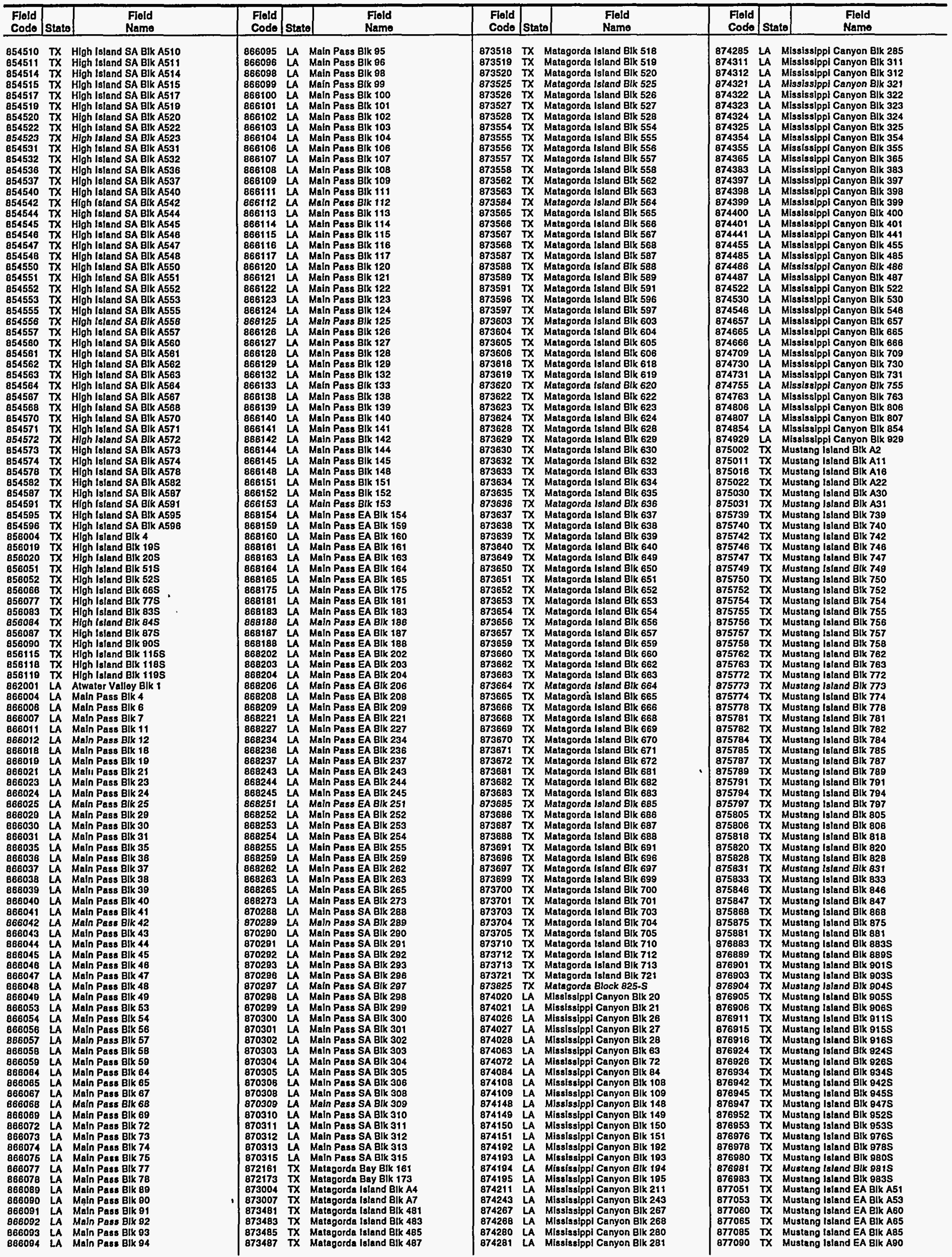


SLl X18 VS \&1 49נEW 4!nOS $\forall 7 \quad$ SL5106

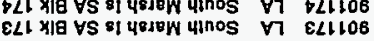

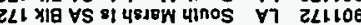

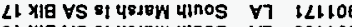

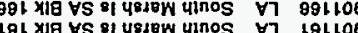
L9l XIB VS

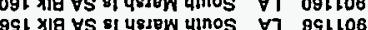

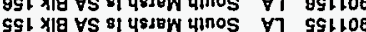

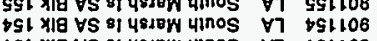

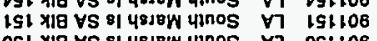
GH

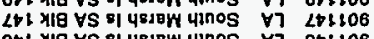

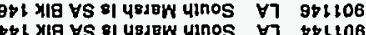

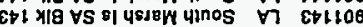

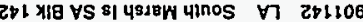

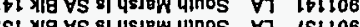

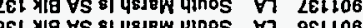
Q

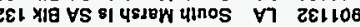

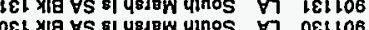

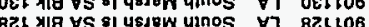

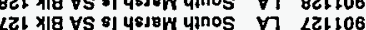

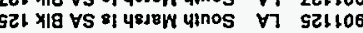

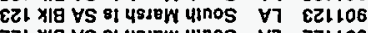

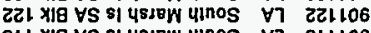

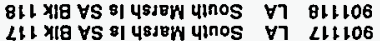

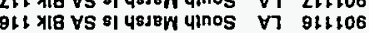

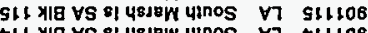

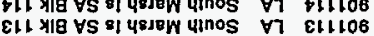

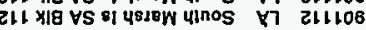

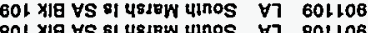

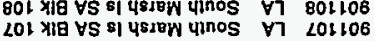

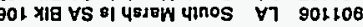
sob (3) SB 1 18 VS $914818 W$ uाnOS $V 7560108$

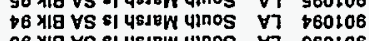
OB ${ }_{18} \forall S$ \&1 48Jew utnos $\forall 7060106$

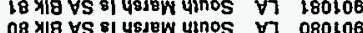

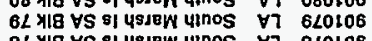

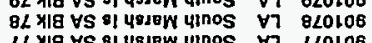

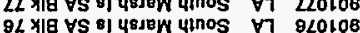

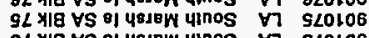

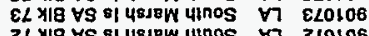

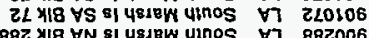

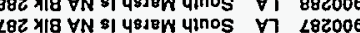
$18 z \times 18 \forall N 914918 W$ Linos $\forall 7182006$ 082 118 VN 81 4818W 4210S $V 7 \quad 082006$ SLZ YIB VN SI LSIEW 4INOS $V 7$ SLZOD6

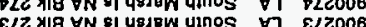

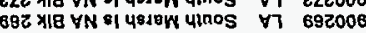

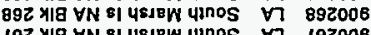

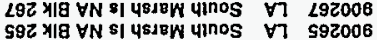

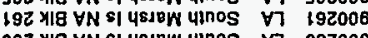

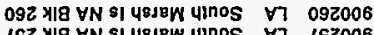

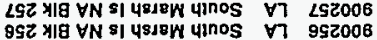

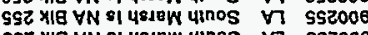

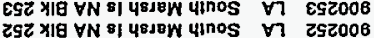
OSZ XIG $\forall N$ si y9JeW uाnOS $\forall 7$ osz006

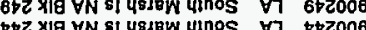

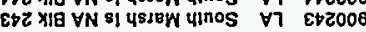

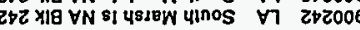

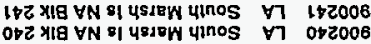

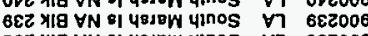

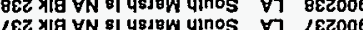

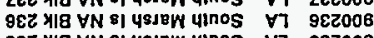
SEZ XIR YN 81 4SJEW 4INOS $V 7$ SEZOOB

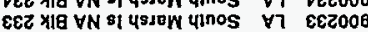

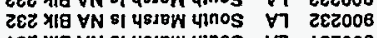

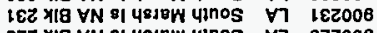

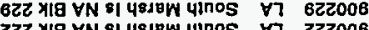

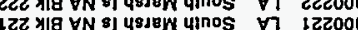

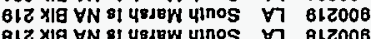

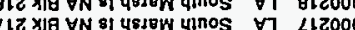
S1Z X18 VN $914820 \mathrm{~W}$ yाnOS $V 7$ s12000

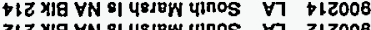
दน $B 0 Z$ भाa $\forall N$ \&

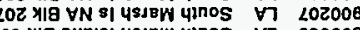

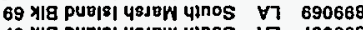

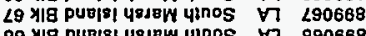

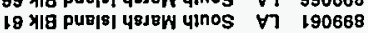

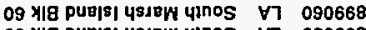

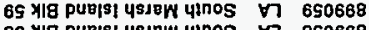

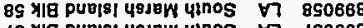

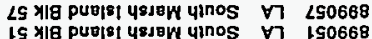

os xis puajs! 4s18w yinos $\forall 7 \quad 050668$

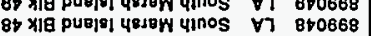

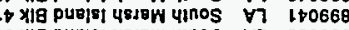

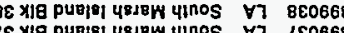

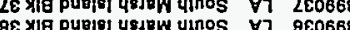

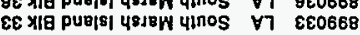

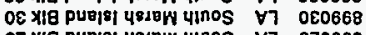

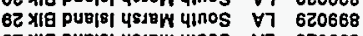

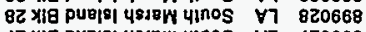
$\angle Z$ श

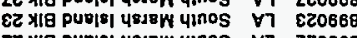

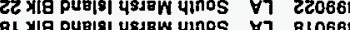
ol X⿻一丿

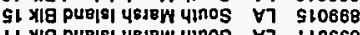

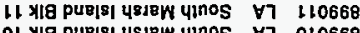

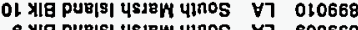
6 심 puㅂ.

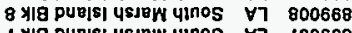

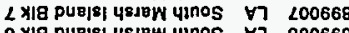

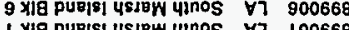

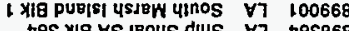

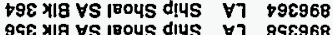

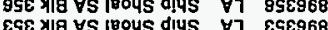
४SE ISE XIB VS jBous dils $\forall 7$ ISc968 OSE XIB $\forall S$ jBOUS di4S $\forall 7$ 0SE968

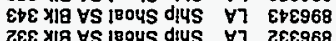

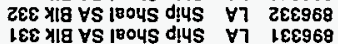

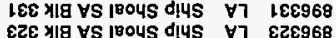

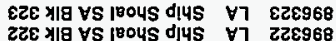

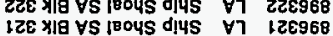

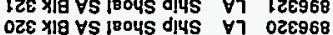

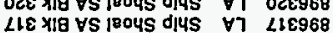
918 x: $\forall S$ irous di4S V7 918968

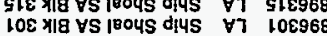

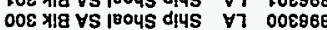
662 yI $\forall S$ joous di4S $\forall 7$ 68298 962 118 VS |804S duS $\forall 7 \quad 962968$

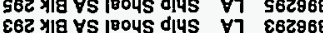

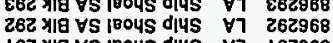

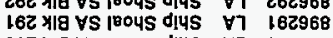
062 yו8 vs pous dius $\forall 7062968$ $8 \angle 2$ गI8 $\forall S$ j804S di4s $\forall 7$ 8LZ968

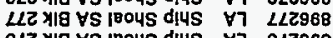
$9 \angle 2$ ฟI8 $\forall S$ joous d!uS V7 $9 \angle 2968$

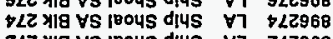
ZLZ YIE VS $1804 S$ dIUS $\forall 7$ ZLZ868

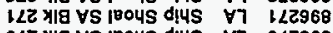
OLZ Y

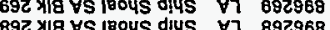
$99 Z$ 시 $\forall S$ j804S dịS $\forall 7992968$ E9Z Xil VS 18045 dils V7 892968

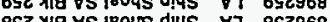
$992 \times 18$ VS ESE ZSZ

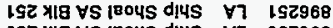

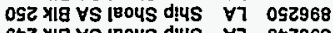

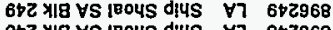

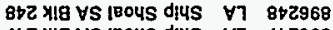

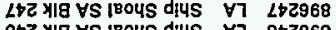

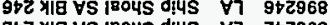
$142 \times 18$ ys 18045 di4s $\forall 7$ toz

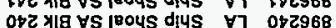

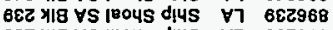
BEz $\forall \varepsilon 11 \times 18$ 1804S di4s v7 ह11968

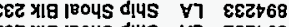

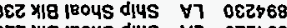
sZz

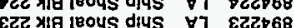
टटट XIO $612 \times 1818045$ di4s v7 $612+68$ 8I2 xाB 18045 dius $\forall 7$.

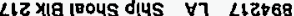

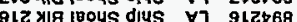
SIZ \18 1804S di4s $\forall 7$ sizb68 602 xil 1804S di4s $\forall 7$ 602768

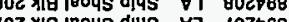

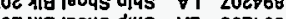
(1) s0z xाa boys dits $\forall 7$ sozb68 20z $102 \times 18$ l8ous dus $\forall 7102768$ 661 11 1804s dits $\forall 7$ 681768 861 ×11 18045 dits $v 7$ 86t788

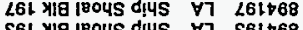

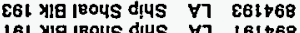
$161 \times 118$ loo4s d4S $\forall 7161+68$

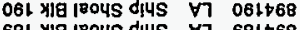

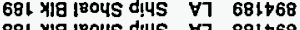

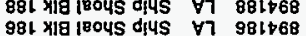

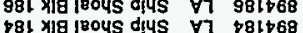

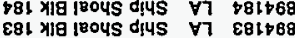

281 ห18 18045 dis $\forall 7 \quad 281768$ 081 xा8

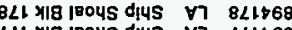

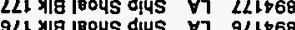

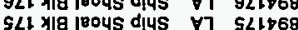

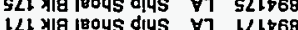

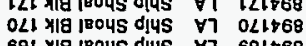

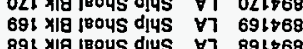

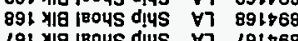
29l x/8 j804S di4S V7 291768 S9! $\times 19$ joous di4s V7 s9เt68

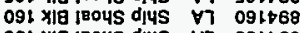
8St $\times 18$ 1004S di4S $\forall 7$ 8S 1768 2Sl xla yous dj4S V7 LSt668

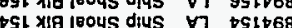
ost xig 1804s di4s v7 0stb68 6rl xila 18045 dits v7 6rlt68

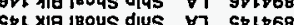

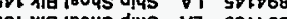

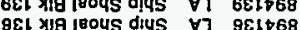
9el xis jous dits $\forall 7$ 9ett68 sel EEl

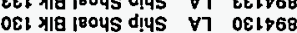
$62 t$ xla l804S djus v7 621+68

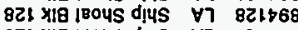
0ZL 119 1804s di4s $\forall 7$ 0ट1768

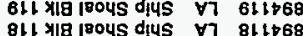

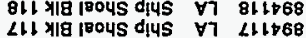

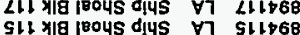
111 $x 18$ 1804s dius v7

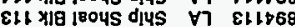

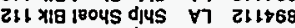

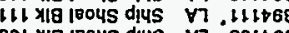
101 रा sol yia joous dius vi solt68 101 x|8 |в04S dius $v 7101768$ 003 x/8 $18045 d 45$ v7 001768 66 ×18 18045 di4s V7 680068 $86 \times 1818045$ ditus $\forall 7860 t 68$ 26 xil 18045 dus 260568

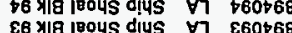

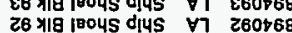

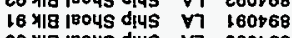
08 xा8 18045 di4s $v 7060+68$ 28 118 18045 di4s 77 280t68 $98 \times 118$ 18045 di4s $\forall 7.800768$ 08 xis la04S dius V7 080768 $62 \times 18$ l804S di4S V7 620768

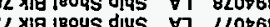
$2 \angle x \mid 1$ lBous dius $\forall 7$

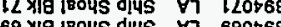
89 xil ibous di4s v7 690168 48 1804s dus 77890868 99 4 18 1804s dys $\forall 7$ 990568

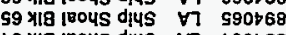
69 xil loous di4s $\forall 7$ b90768 89 ×18 18045 dquS $\forall 7$ 890t68

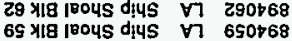

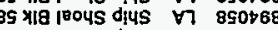
zs xis loous di4s vi 250768

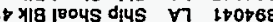
18

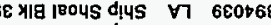
145 $880 t 68$

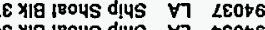
हe $x 18$ l804s dus vi E20t68

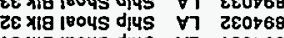
IE 118 joous di4s $\forall 7$ 120068

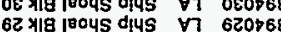
Q己 $\angle 2 \times 18$ jEo4s di4s $\forall 7$ 120068 $92 \times 18$ iвous dius $\forall 7$ 920 68 $S z$ xis loous divs $\forall 7$ szot68 \&Z गा 9l ग18 joous dus V7 910768 si xi8 1804s dus $\forall 7$ stop68

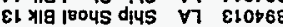

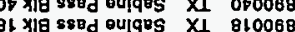
$\angle 4 \times 18$ 880d OUIq8S XI $\angle 10068$

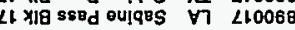

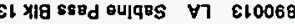

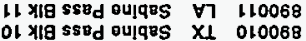

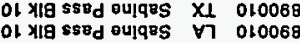

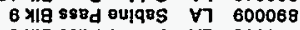
8 xil s88d oujges $\forall 7$ 800068 L XII ssed oulq日s $\forall 7 \quad 200068$ 188 Y18 घ100*sued DO $288 \angle 88$ $166 \times 18$ ग!। Q0W $00 \quad 166988$ 066 >18 өा140\% 90066988 196 118 өाlqOW DO 19698

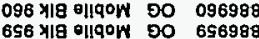

856 หเด S56 $\times 180190$

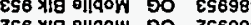
458

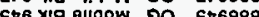
$\angle 16 \times 18$ ollow 00 $218 \times 18$ ongow

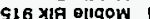
SI6 606 \11 806 भा $\angle 06$ xा 906 มाв Өा190W 50906988

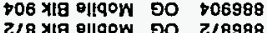

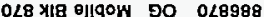




\begin{tabular}{|c|c|c|c|c|c|c|c|c|c|c|c|}
\hline $\begin{array}{l}\text { Field } \\
\text { Codo }\end{array}$ & State & $\begin{array}{l}\text { Field } \\
\text { Name } \\
\end{array}$ & $\begin{array}{l}\text { Field } \\
\text { Code }\end{array}$ & State & $\begin{array}{l}\text { Field } \\
\text { Name }\end{array}$ & $\begin{array}{l}\text { Field } \\
\text { Code } \\
\end{array}$ & State & $\begin{array}{l}\text { Field } \\
\text { Name } \\
\end{array}$ & $\begin{array}{l}\text { Field } \\
\text { Code }\end{array}$ & Stat & $\begin{array}{l}\text { Field } \\
\text { Name }\end{array}$ \\
\hline 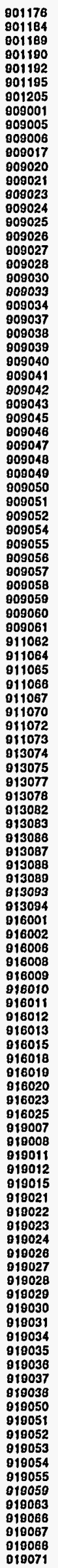 & 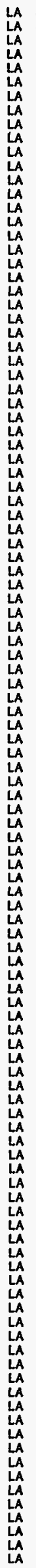 & 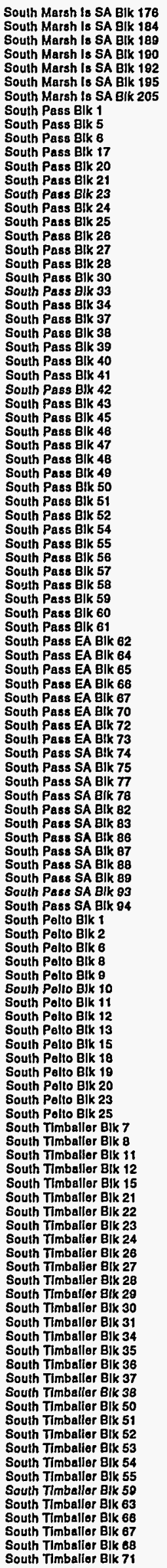 & 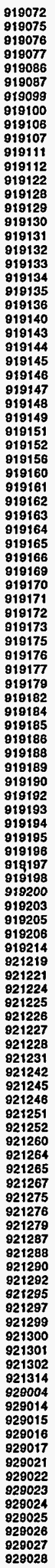 & 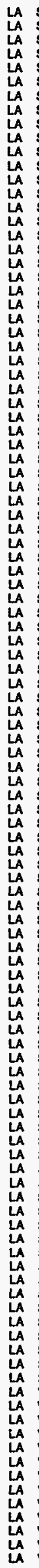 & 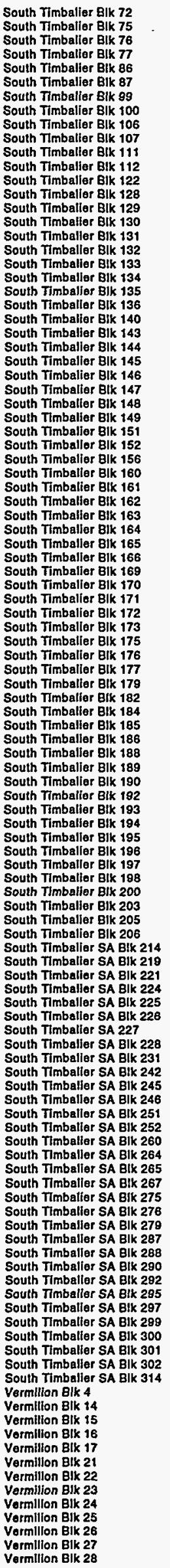 & 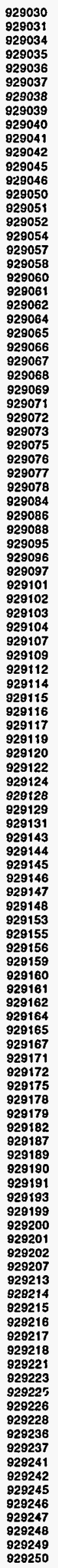 & 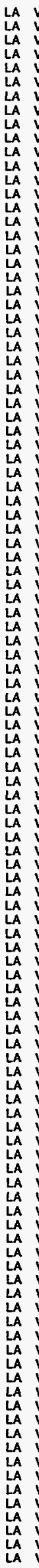 & 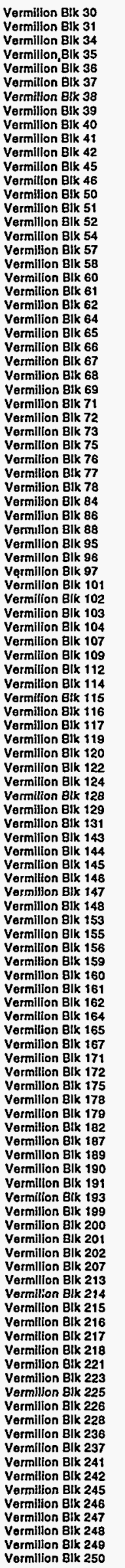 & 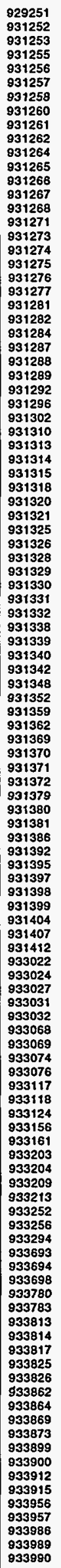 & 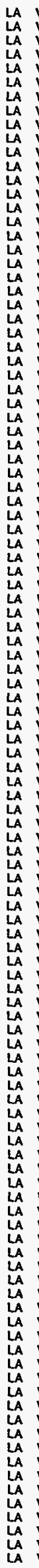 & 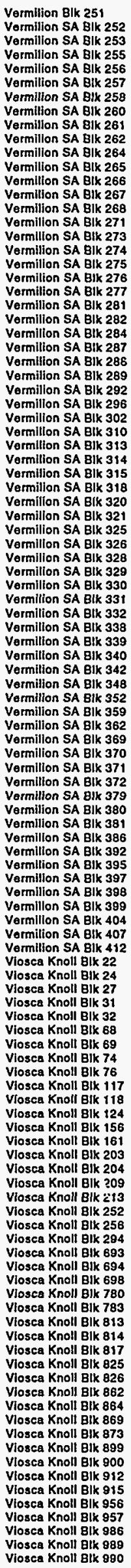 \\
\hline
\end{tabular}


935001

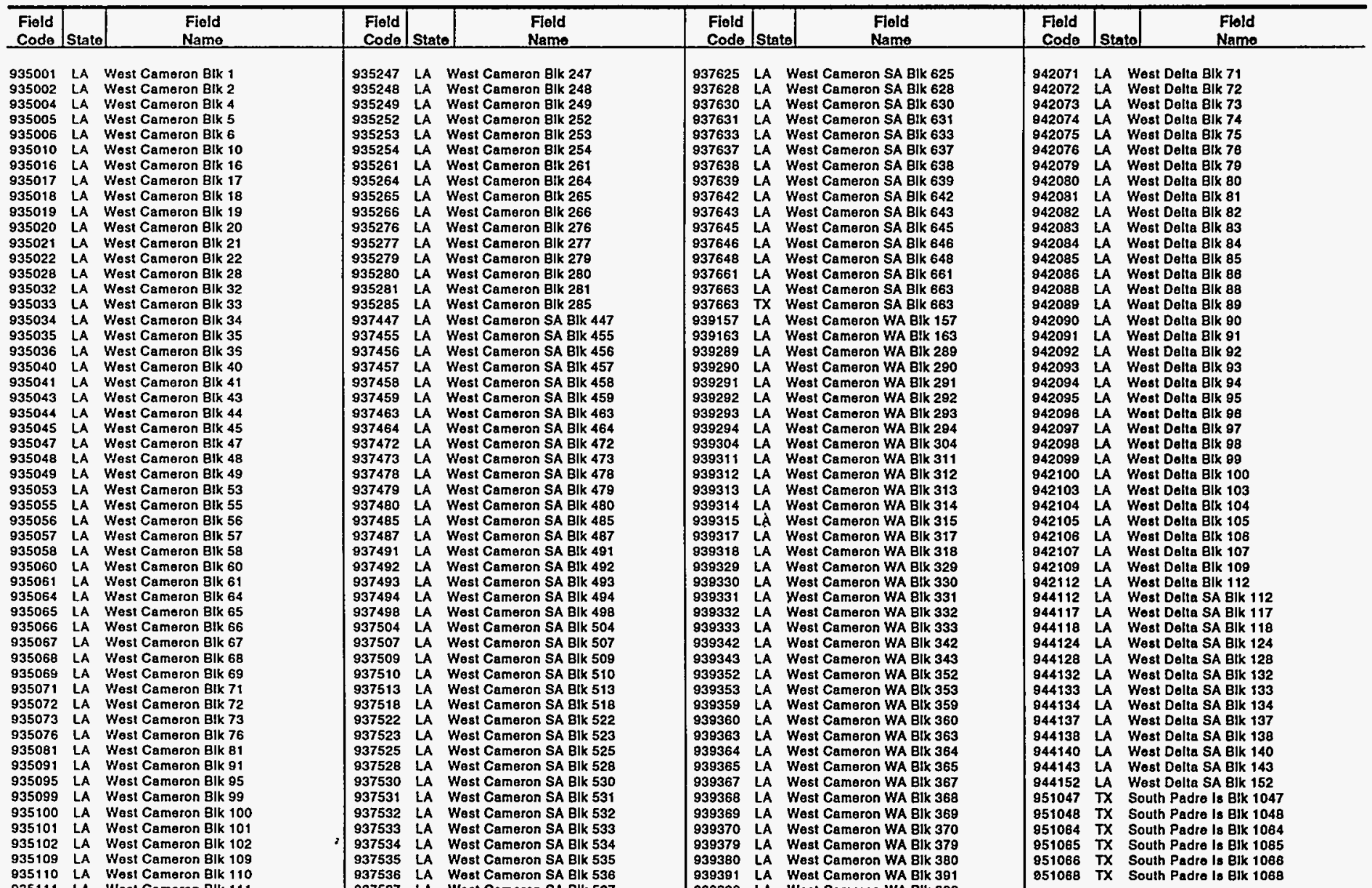

93511 LA West Cameron Blk 111

935116 LA West Camaron Blk 116

935132 LA West Cameron Blk 132

935135 LA West Cameron Blk 135

935142 LA West Camaron B!k 142

935143 LA West Cameron Blk 143
935144 LA West Cameron Blk 144

935145 LA West Cameron BlK 145

935146 LA West Cameron Blk 146

935149 LA West Cameron Blk 149

935150 LA West Cameron Blk 150

935153 LA West Cameron Blk 153

935165 LA West Cameron Blk 165

935168 LA West Cameron Blk 168

935170 LA West Cameron Blk 170

935172 LA Wast Cameron Blk 172

935173 LA West Cameron Blk 173

935174 LA West Cameron Blk 174

935176 LA Wost Cameron Blk 176

935178 LA Wost Cameron BIk 178

935180 LA West Cameron BIK 180

935181
935182 LA West Cameron Blk 181

935182
835187 LA West Cameron Blk 182

835187
935188 LA West Camaron Blk 187
Lest Cameron Blk 188

935192 LA West Cameron Bjk 192

935193 LA West Cameron Blk 193

935194 LA West Cameron Blk 194

935197 LA West Cameron BIk 197

935198 LA West Cameron BIk 198

935199 LA West Cameron Blk 199

935201
835202 LA West Cameron Blk 201

935204 LA West Cameron Blk 204

935205 LA West Cameron Blk 205

935206 LA West Cameron Bik 206

935212 LA West Cameron Blk 212

935215 LA West Cameron Blk 21

935222 LA West Cameron Blk 222

935222 LA West Cameron Blk 222

935226 LA West Cameron Blx 226

935228 LA West Cameron BlK 228

935230 LA West Cameron Blk 230

935230 LA West Cameron Blk 230

935231 LA West Cameron Blk 231

935236
935237
LA West Cameron Blk 237

935237
935238
LA We West Cameron BlK 237
Waneron Blk 238

935239 LA West Camaron Blk 238

935241 LA West Cameron Blk 241

937536 LA West Cameron SA Blk 536
$\mathbf{9 3 7 5 3 7}$ LA West Cameron SA Blk 537

937538 LA West Cameron SA Blk 53

937539 LA West Cameron SA Blk 539

937540 LA West Cemeron SA Blk 540

937541 LA West Cameron SA Blk 541

$\mathbf{9 3 7 5 4 2}$ LA West Cameron SA Blk 542

937543 LA West Cameron SA BIK 543

937544 LA West Cameron SA BIK 544

937547 LA West Cameron SA BIk 547

937548 LA West Cameron SA BlK 548

937551 LA West Cameron SA Blk 551

937552 LA Wost Cameron SA Blk 552

937553 LA West Cameron SA Blk 553

937554 LA West Camaron SA Blk 554

937555 LA West Cameron SA Blk 555

937556 LA West Cameron SA Blk 55

937557 LA West Cameron SA Blk 557

937560 LA West Crmeron SA Blk 580

937561 LA West Cameron SA Blk 561

937563 LA West Cameron SA Blk 563

937564 LA West Cameron SA Blk 564

937565 LA West Cameron SA Blk 565

937566 LA West Cameron SA Blk 586

937570 LA West Cameron SA Blk 570

937571 LA West Cameron SA Blk 57

937572 LA West Cameron SA Blk 572

937573 LA Wast Cameron SA Blk 573

937574 LA West Cameron SA Blk 574

937575 LA West Camoron SA Blk 575

937576 LA West Cameron SA Blk 576

937583 LA West Cameron SA Blk 583

937584 LA West Cemaron SA Blk 584

937586 LA West Cameron SA Blk 586

937587 LA West Cameron SA Blk 587

937588 LA West Cameron SA Blk 588

937589 LA West Camaron SA Blk 589

937592 LA West Cameron SA Blk 592

93759 LA Wost Camoron SA Blk 593

937594 LA West Camoron SA Blk 594

93755 LA Wost Camoron SA Blk 595

937587 LA Wost Camoron SA Blx 597

937600 LA Wost Camom SA BK 600

937601 LA West Camoron SA Blk 601

937605 LA West Camoron SA BIK 604

93700 LA Wost Camoron SA BIK 605

93706 LA West Camoron SABIk 806

937608 LA West Cameron SA Blk

037000 LA West Camoron SABK 609

937610 LA Wost Cameron SABIK 610

037612 LA West Cameron SA BIK B12

937013 LA Wost Camoron SA BIK B13

937616 LA West Camor SA BK B10

937617 LA Wost Camoron SA BIX 619

037618 LA West Cameron SA BK B18

937619 LA West Cameron SA

937620 LA West Camoron SA BIK B19

937620
937624
LA West Camoron SA BIk 624
939391 LA West Cameron WA Blk 391
939398 LA West Cameron WA Blk 398

939401 LA West Cameron WA Blk 401

939405 LA West Gameron WA Blk 405

939406 LA West Cameron WA Blk 405

939408 LA West Cameron WA BlK 408

939409 LA West Cameron WA Blk 409

939414 LA West Cameron WA BlK 414

939421 LA West Cameron WA Blk 421

939425 LA Wast Cameron WA BIK 425

939426 LA West Cameron WA BlK 426

939427 LA West Cameron WA Blk 427

939431 LA West Cameron WA Blk 431

239432 LA Wast Camaron WA Blk 432

LA West Cameron WA Blk 433

939437 LA West Cameron WA Blk 437

942017 LA West Delta Blk 17

942018 LA West Delta Blk 18

942020 LA West Delta Blk 20

LA West Delta 8lk 2

942023 LA West Delta Blk 23

942024 LA West Delta Blk 24

942025 LA West Delta Blk 25

942026 LA West Delta Blk 26

942027 LA West Delta Blk 27

942028 LA Wost Delta Blk 2

942029 LA West Delta Blk 29

942030 LA West Delta Blk 30

942031 LA West Dolta Blk 31

942032 LA West Dolta 8lk 32

942033 LA West Delta Blk 33

942034 LA West Delte Blk 34

942035 LA West Delte Blk 35

942036 LA West Deita Blk 38

942038 LA West Delta Blk 38

942040 LA Wost Dolta Blk 40

942041 LA West Deita Blk 41

942042 LA Wost Delta Blk 42

942043 LA West Deite BlK 43

942044 LA Wast Delta Blk 44

942045 LA Wast Dalla BJk 45

842050 LA Wost Della BIk 50

942052 LA Wost Delta Blk 52

2053 LA Wosl Dalla Blk 53

942055 LA Wast Dalta Blk 55

42056 LA Wost Della Blk 5

205 LA Wor Dolla BIK 57

2050 LA Wos Dolle BlK 5B

942081 LA Wost Delta Blk

O4208r LA Wost Delta Blk 61

42062 LA West Della Blk

942084 LA West Delte Blk 64

942084 LA Wost Dolta Blk 64

2007 LA Wost Della Blk

942088 LA West Delta Blk 68

842070 LA West Dolta Blk 70

951068 TX South Padro ls BIk 1068 
Oil and Gas
Invalid Field
Record List 
CALIFORNIA

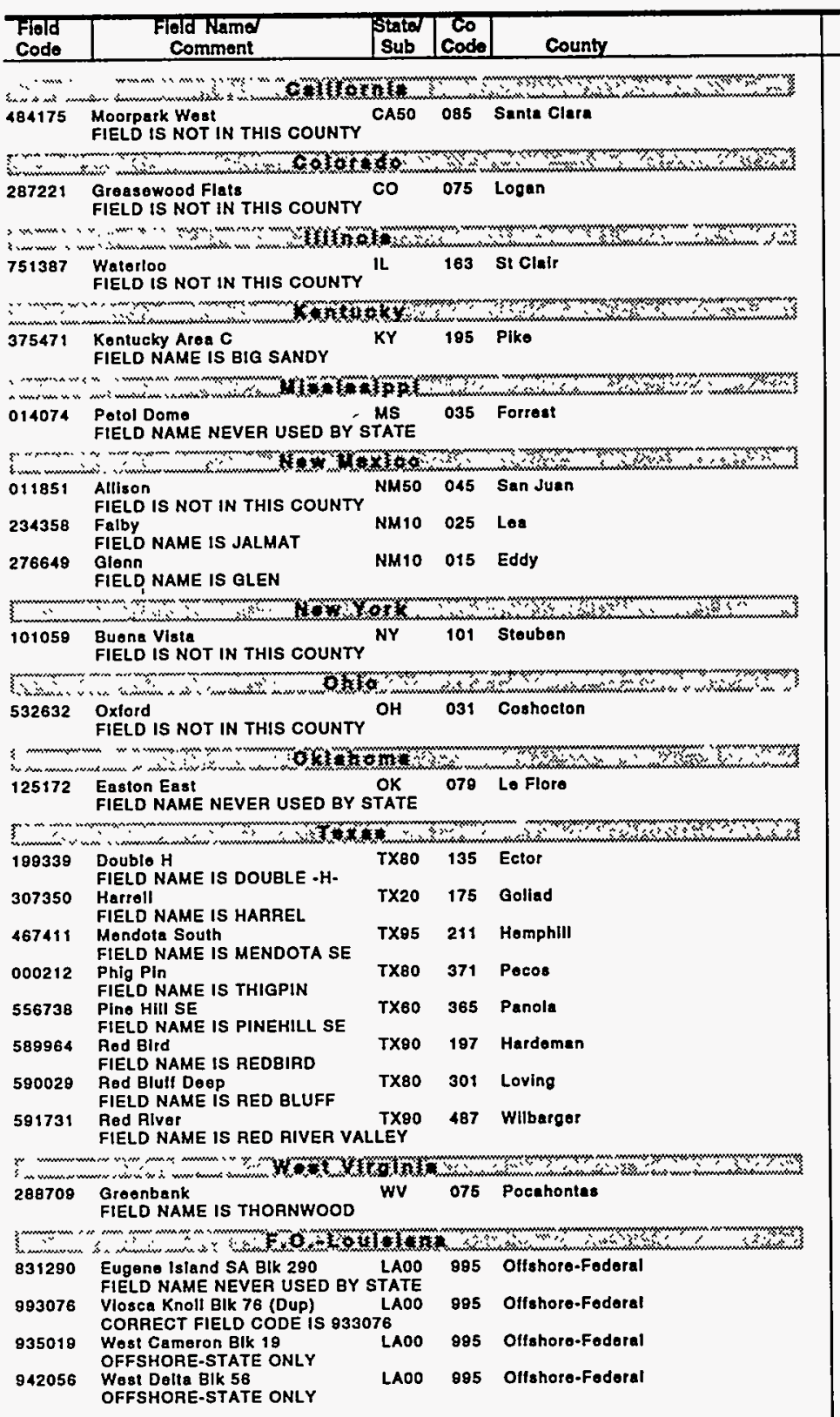
Moorpatk West

Ant 